\title{
Course Notes: United States Particle Accelerator School \\ Beam Physics with Intense Space-Charge
}

\author{
J.J. Barnard and S.M. Lund \\ Lawrence Livermore National Laboratory \\ Livermore, CA 94550 \\ and \\ Accelerator Fusion Research Division \\ Ernest Orlando Lawrence Berkeley National Laboratory \\ Berkeley, CA 94720
}

June 2008

Presented at

Annapolis MD, 16-27 Jnue 2008

This work was supported by the Director, Office of Science, Office of Fusion Energy Sciences, of the U.S.

Department of Energy under Contract No. DE-AC02-05CH11231, and the U.S. Department of Energy, Lawrence Livermore National Laboratory under Contract DE-AC52-07NA27344 


\section{LLNL-AR-407617}

Beam Physics

with Intense Space-Charge

Lecturers:

John J. Barnard and Steven M. Lund Lawrence Livermore National Laboratory Lawrence Berkeley National Laboratory

\section{Grader:}

Christos Papadopoulous

University of Maryland

\section{Course Notes:}

United States Particle Accelerator School Held 16-27 June, 2008

Annapolis, Maryland

Sponsored by University of Maryland at College Park

Prepared under the auspices of the US Department of Energy at the Lawrence Livermore and Lawrence Berkeley National Laboratories under Contracts No. DE-AC52-07NA27344 and DE-AC02-05CH11231. 


\section{Beam Physics with Intense Space Charge}

Instructors: John Barnard and Steven Lund, Lawrence Livermore National Laboratory

\section{Purpose and Audience}

The purpose of this course is to provide a comprehensive introduction to the physics of beams with intense space charge. This course is suitable for graduate students and researchers interested in accelerator systems that require sufficient high intensity where mutual particle interactions in the beam can no longer be neglected. Prerequisites: undergraduate level Electricity and Magnetism and Classical Mechanics. Some familiarity with plasma physics, special relativity, and basic accelerator physics is recommended but not required.

\section{Objectives}

This course is intended to give the student a broad overview of the dynamics of beams with strong space charge. The emphasis is on theoretical and analytical methods of describing the acceleration and transport of beams. Some aspects of numerical and experimental methods will also be covered. Students will become familiar with standard methods employed to understand the transverse and longitudinal evolution of beams with strong space charge. The material covered will provide a foundation to design practical architectures.

\section{Instructional Method}

Lectures will be given during morning sessions, followed by afternoon discussion sessions, which will engage the student on the material covered in lecture. Daily problem sets will be assigned that will be expected to be completed outside of scheduled class sessions. Problem sets will generally be due the morning of the next lecture session. A final take home exam will be given on the second Thursday, and will cover the contents of the entire course. Two instructors will be available for guidance during evening homework sessions.

\section{Course Content}

In this course, we will introduce you to the physics of intense charged particle beams, focusing on the role of space charge. The topics include: particle equations of motion, the paraxial ray equation, and the Vlasov equation; 4-D and 2-D equilibrium distribution functions (such as the Kapchinskij-Vladimirskij, thermal equilibrium, and Neuffer distributions), reduced moment and envelope equation formulations of beam evolution; transport limits and focusing methods; the concept of emittance and the calculation of its growth from mismatches in beam envelope and from space-charge non-uniformities using system conservation constraints; the role of space-charge in producing beam halos; longitudinal space-charge effects including small amplitude and rarefaction waves; stable and unstable oscillation modes of beams (including envelope and kinetic modes); the role of space charge in the injector; and algorithms to calculate space-charge effects in particle codes. Examples of intense beams will be given primarily from the ion and proton accelerator communities with applications from, for example, heavy-ion fusion, spallation neutron sources, nuclear 
waste transmutation, etc.

\section{Reading Requirements}

Extensive class notes will be provided that will serve as the primary reference. (To be provided by the USPAS) "The Theory and Design of Charged Particle Beams" Second Edition, Updated and Expanded by Martin Reiser, Wiley \& Sons 2008.

\section{Credit Requirements}

Students will be evaluated based on performance: final exam (20\% of course grade), homework assignments (80\% of course grade). 


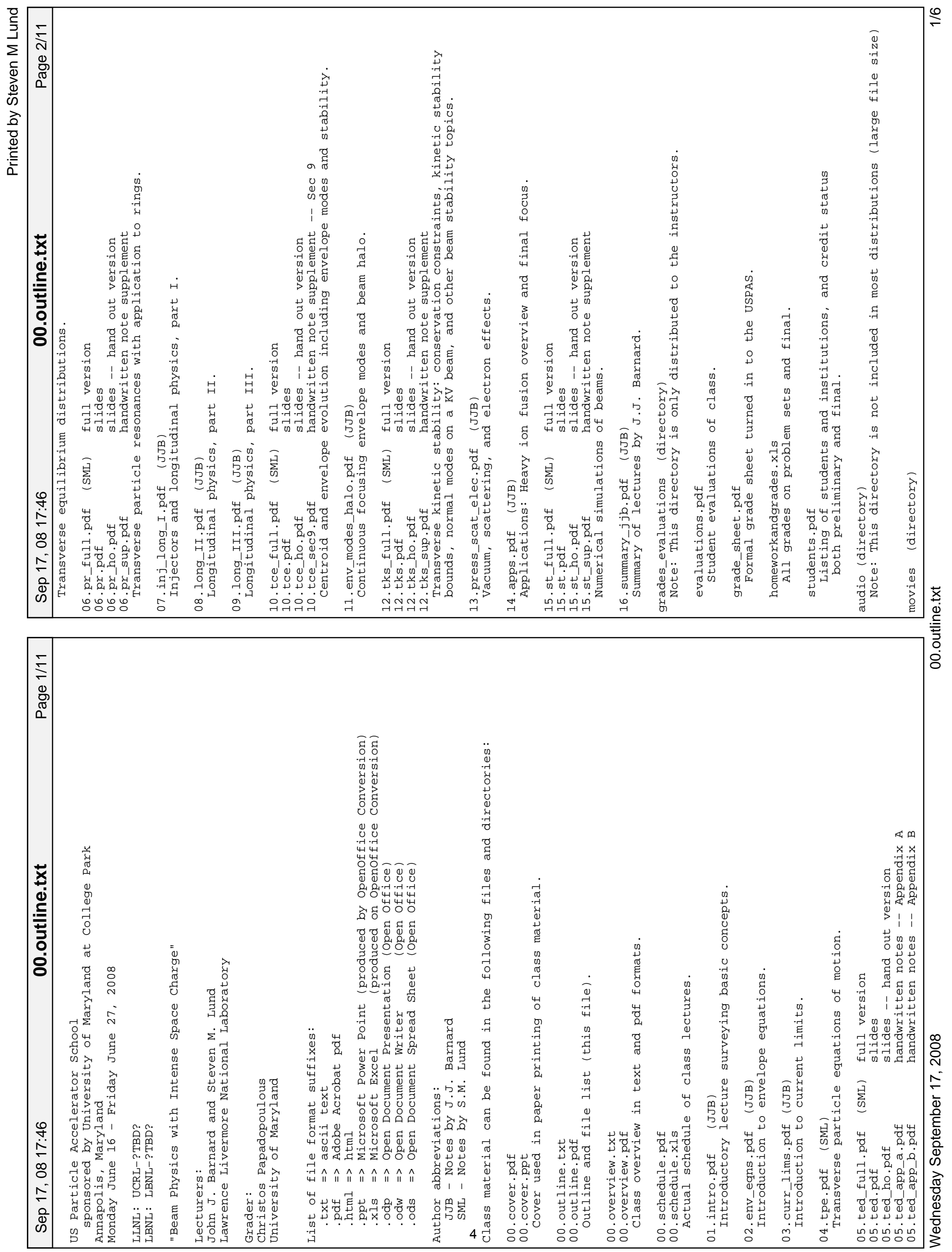




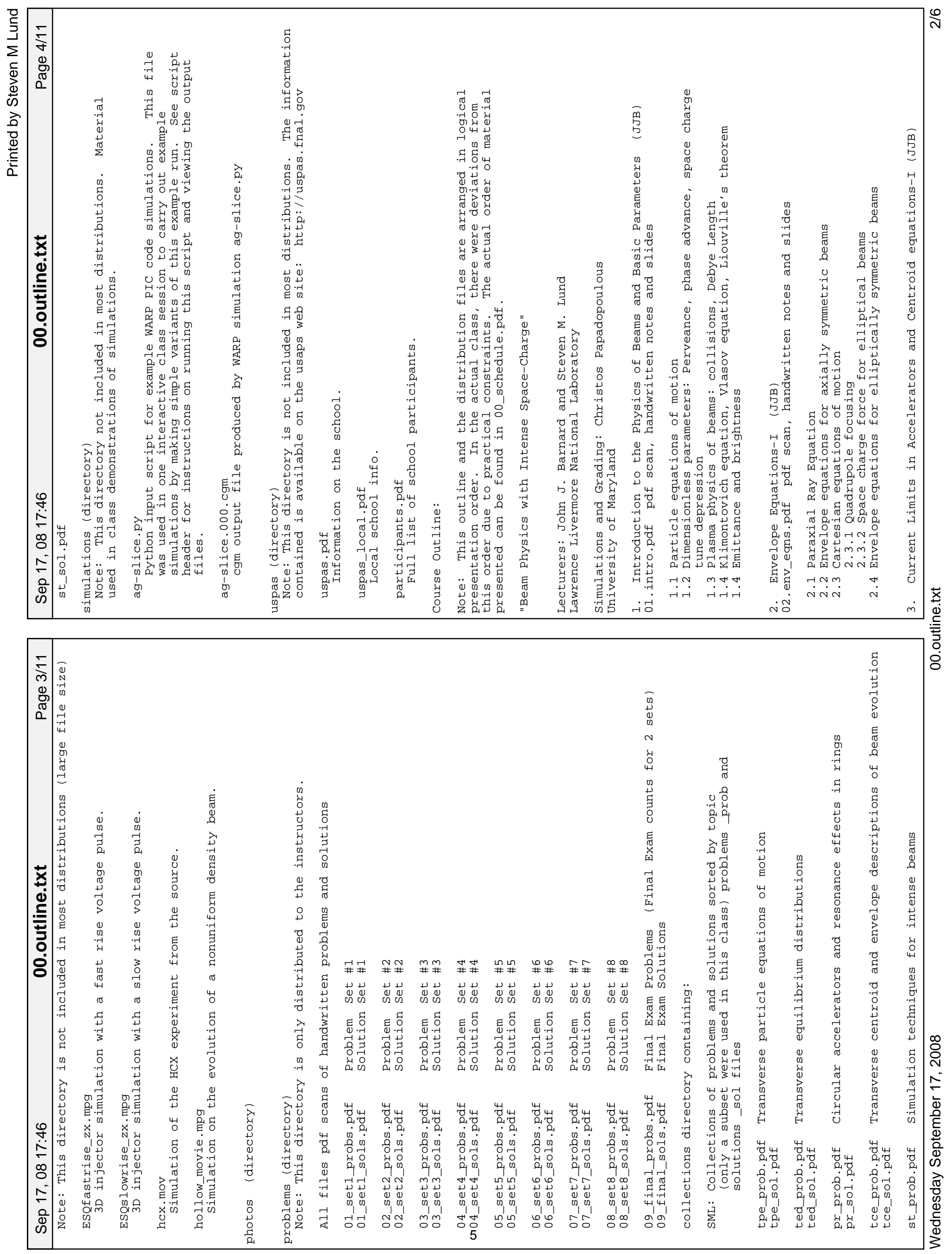



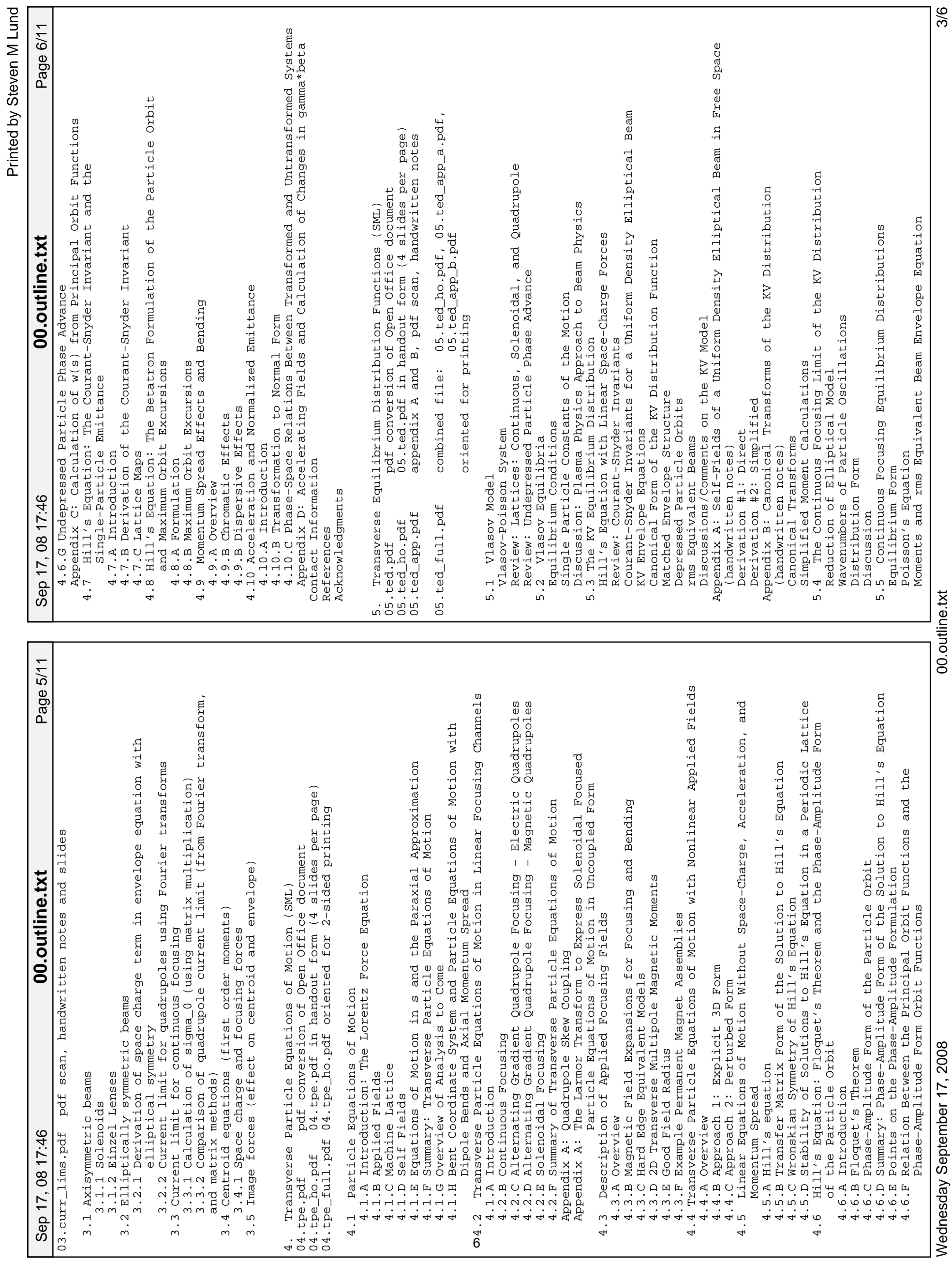

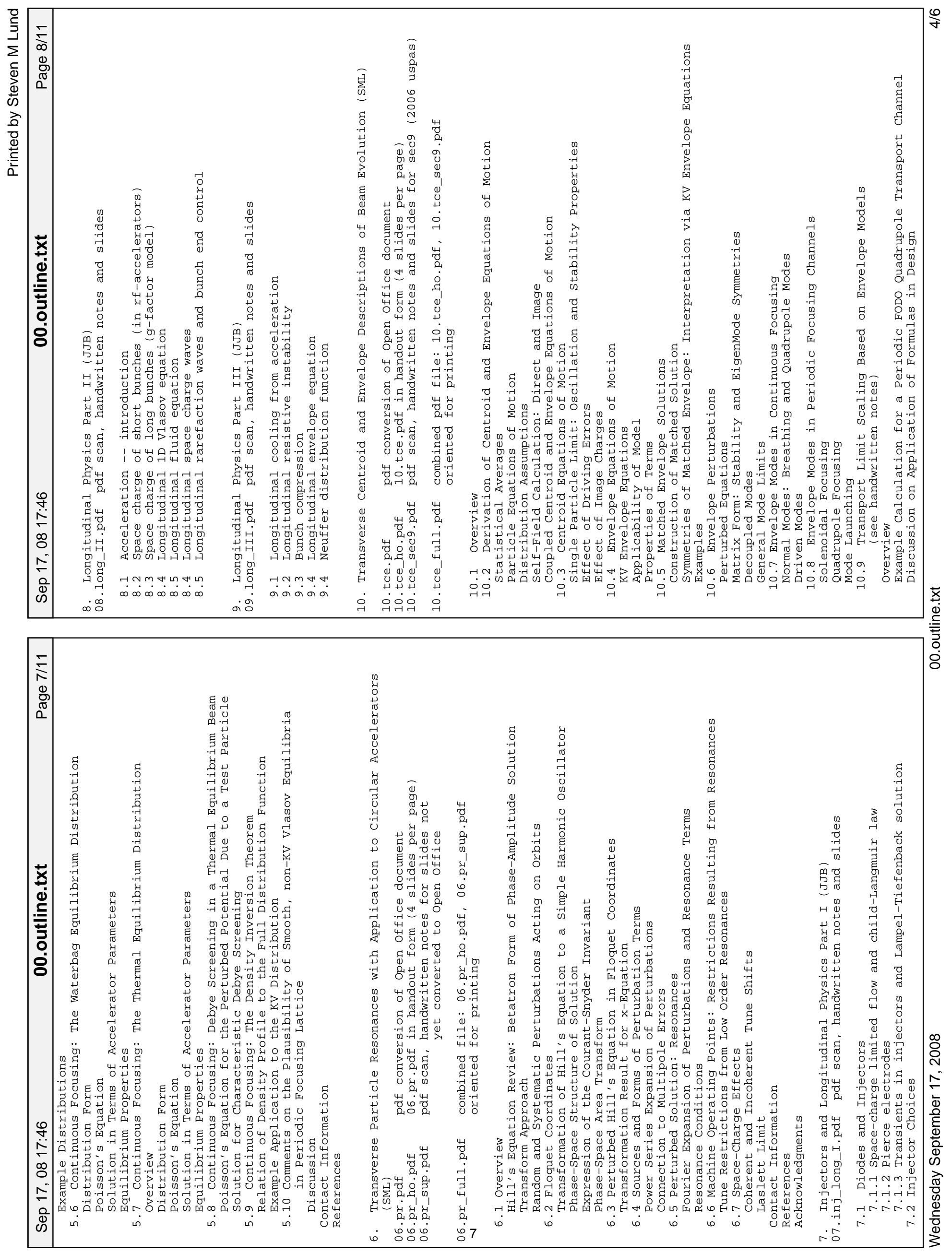

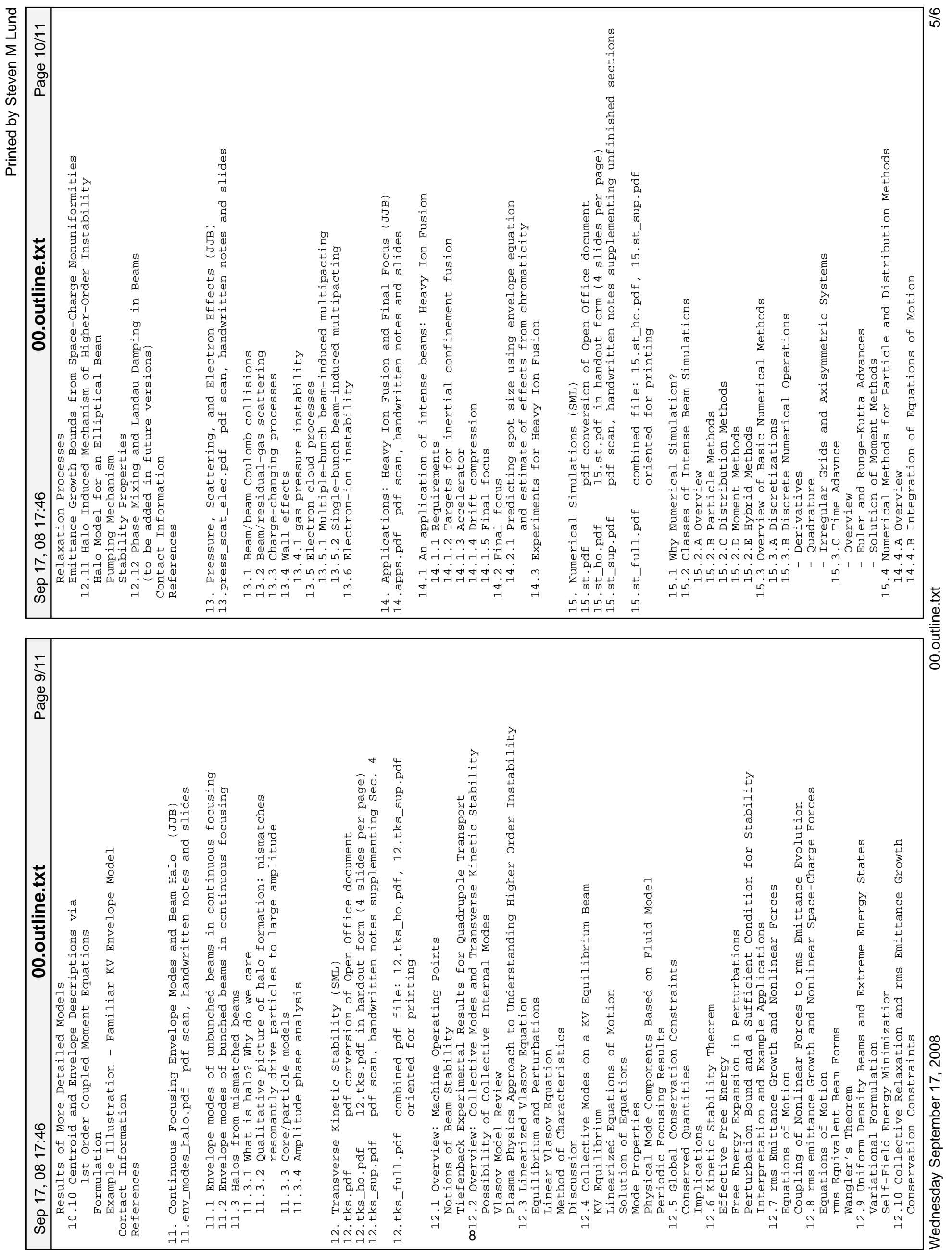


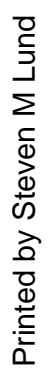

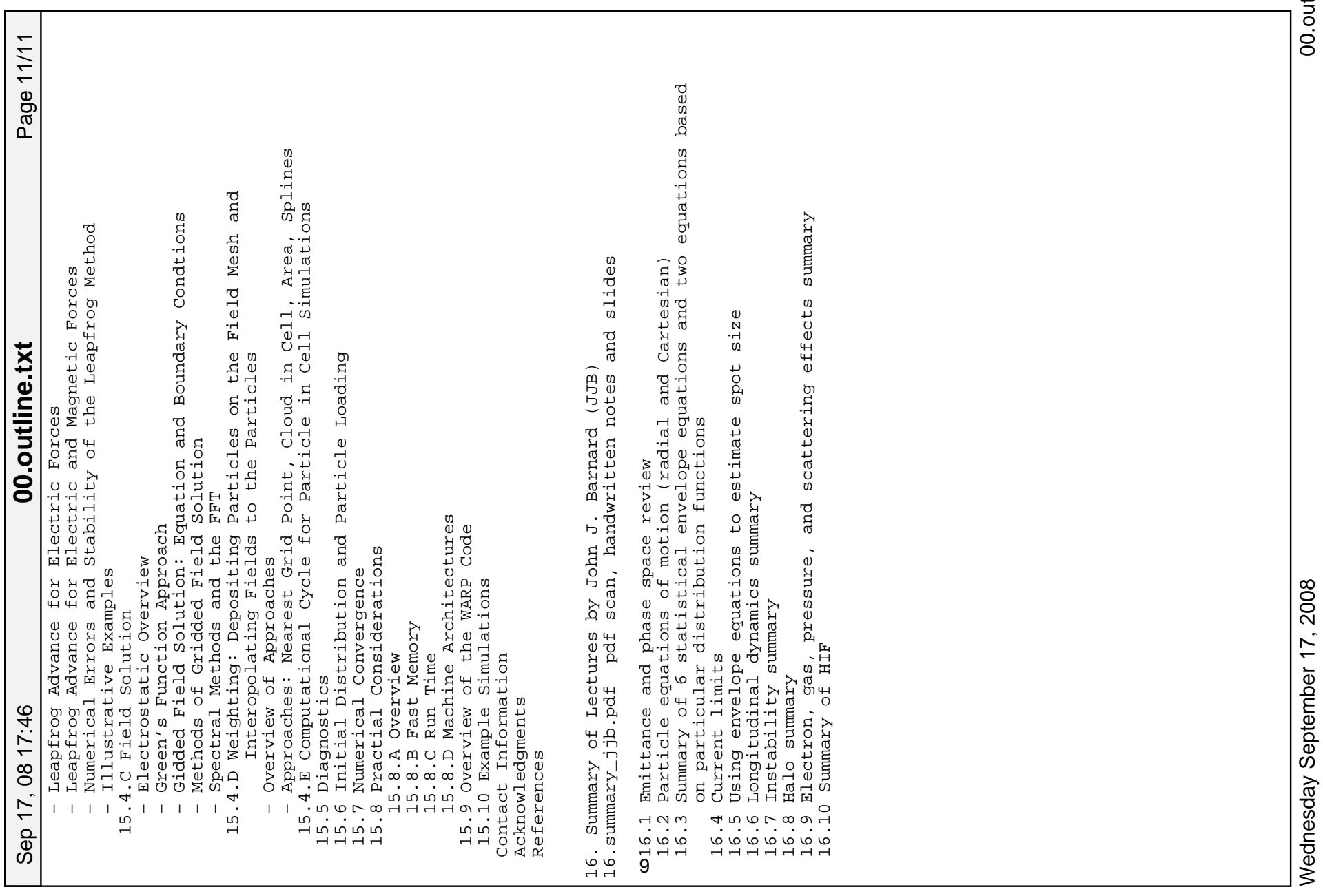



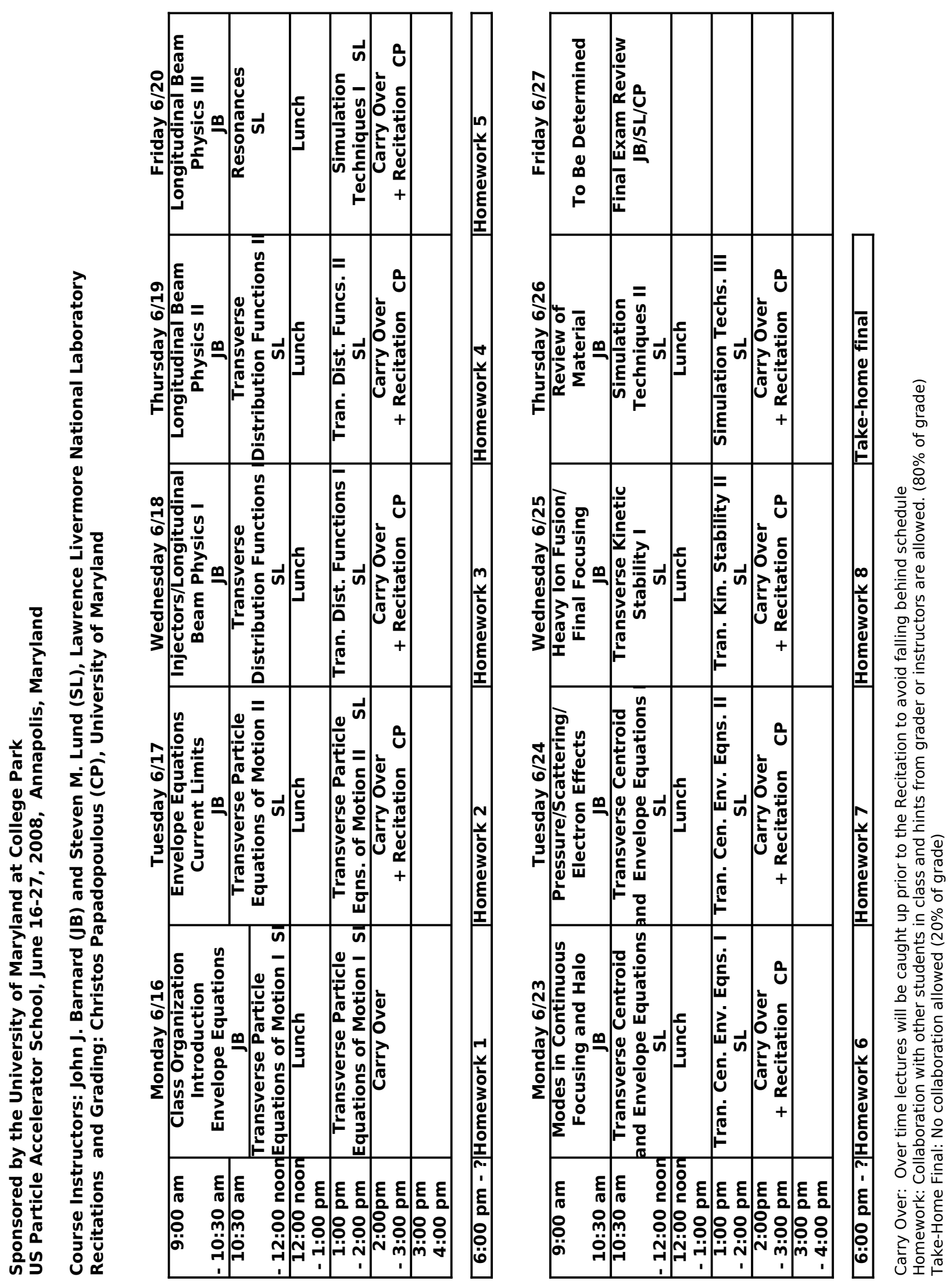


\section{John Barnard Steven Lund USPAS \\ June 2008}

I. Introduction

(related reading in parentheses)

Particle motion (Reiser 2.1)

Equation of motion (Reiser 2.1)

Dimensionless quantities (Reiser 4.2)

Plasma physics of beams (Reiser $3.2,4.1$ )

Emittance and brightness (Reiser 3.1

- 3.2) 


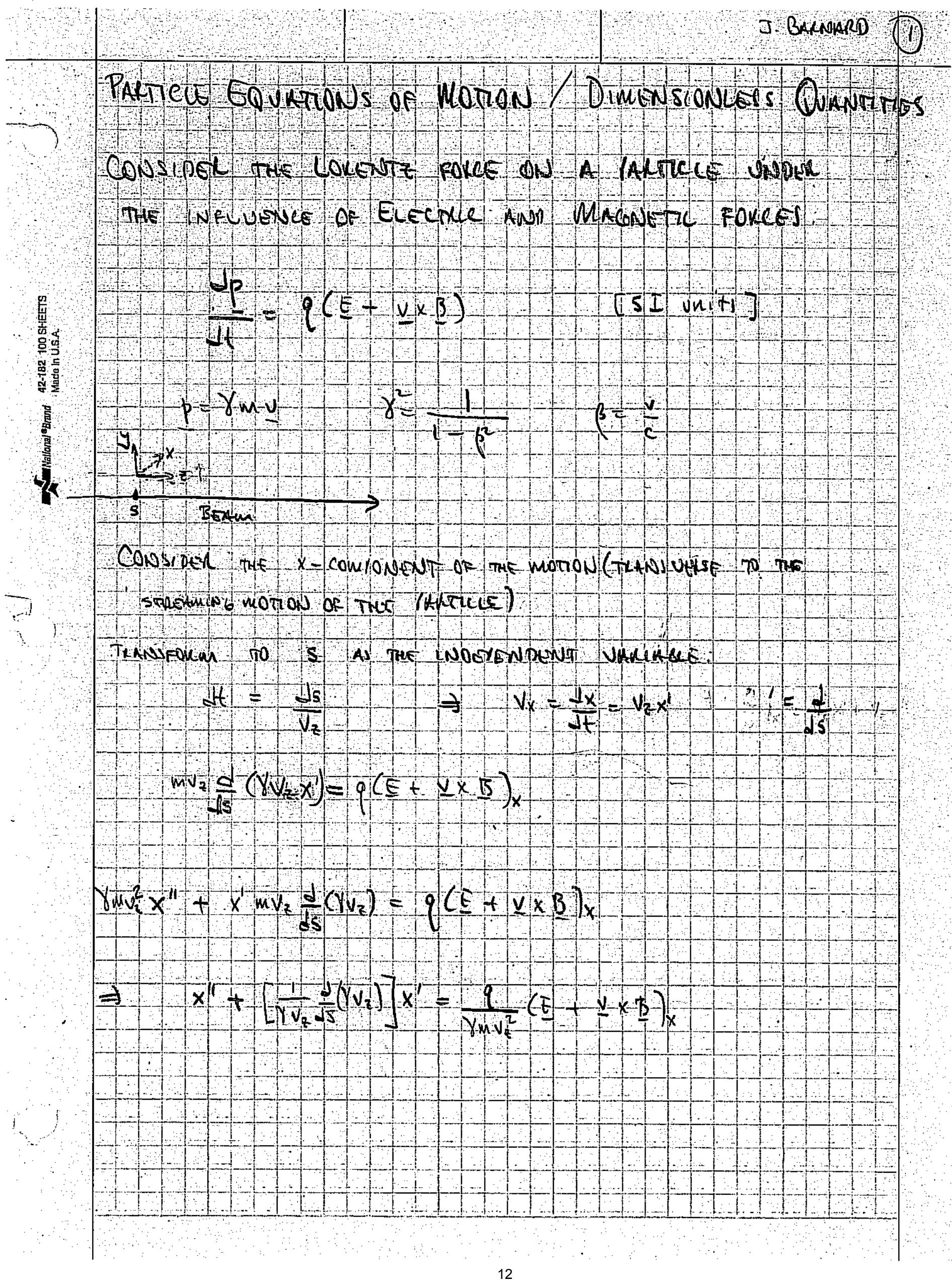




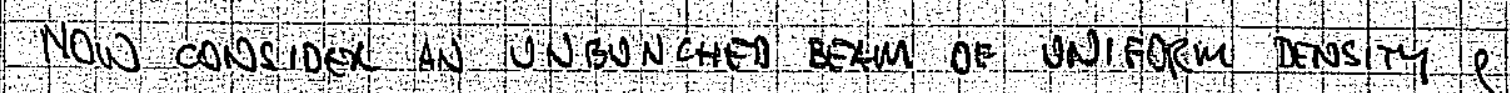

Ho. Cincount Closs secRON

$$
\begin{aligned}
& \text { Q } E=\varepsilon_{0} \\
& 2 \pi r E=\frac{\pi r^{2} \rho}{g} \text { (Gavss noorew ) }
\end{aligned}
$$

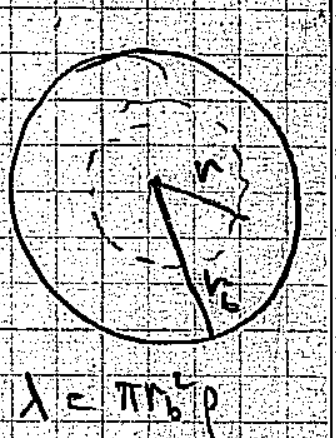

$$
\left.E_{r}=\frac{\rho}{2 \varepsilon_{0}} r\right)=\frac{\lambda}{2 \pi \varepsilon_{0}} \frac{r}{r_{b}^{2}} \quad E_{x}=E_{r} \cos \theta=\theta_{r}\left(\frac{x}{r}\right)=\frac{\lambda}{2 \pi \varepsilon_{0}} \frac{x}{v_{b}}
$$

Sinlant $0 \times B=\mu_{0} \mathrm{I}$

$$
2 \pi r B_{\theta}=\mu_{0} \rho v_{7} \pi r^{2} \text { (stokes thobrem }
$$

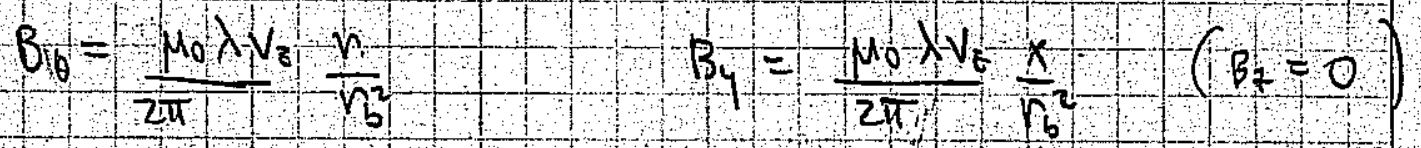

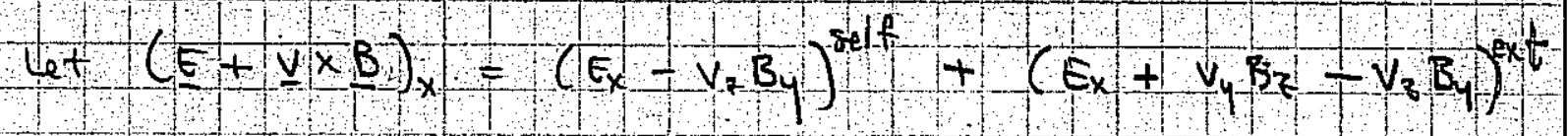

$$
\text { Using } \mu_{0} \epsilon_{0}=\frac{1}{c^{6}}
$$

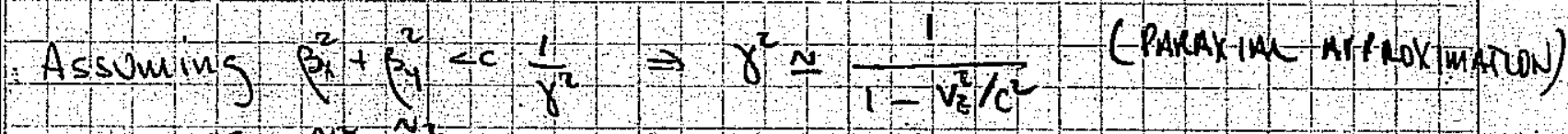

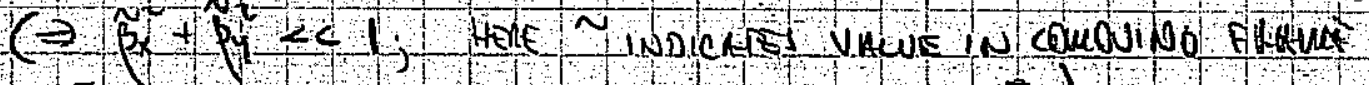

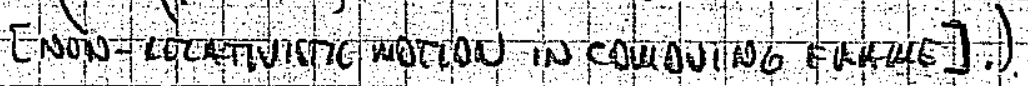

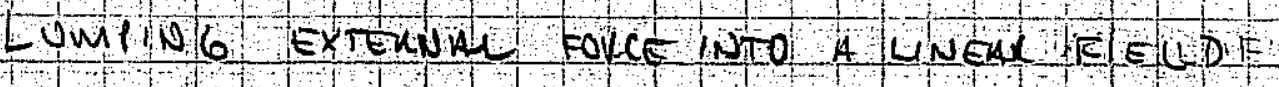




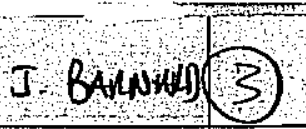

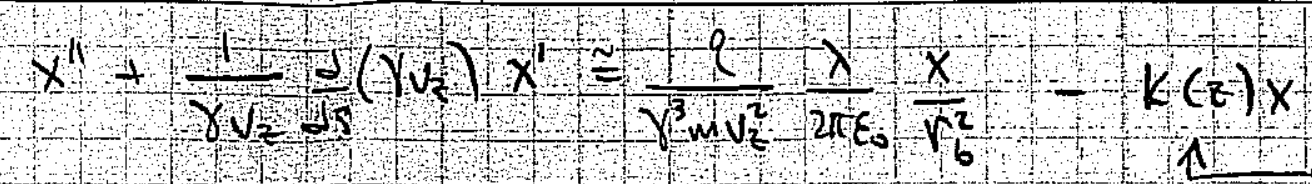

$$
\begin{aligned}
& =Q \frac{x}{n_{b}^{2}}-k(z) x
\end{aligned}
$$

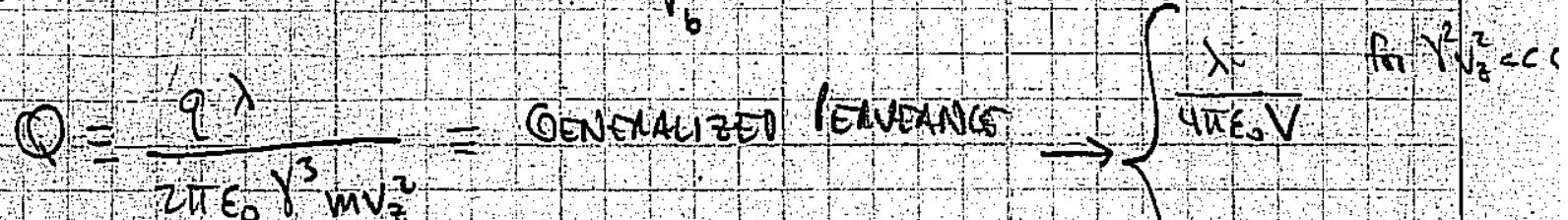

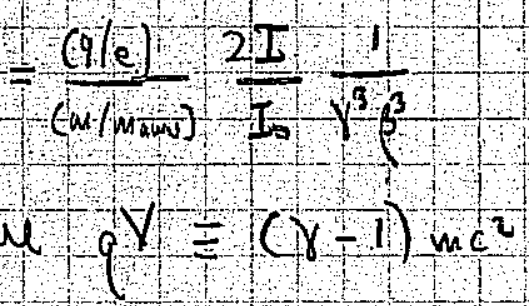

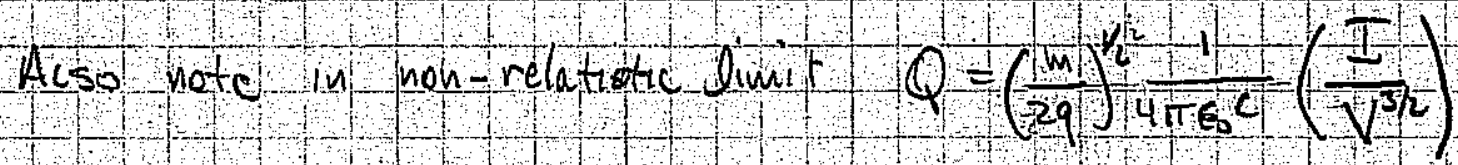

(same scaling a origual term peroeance chanagiagum up pckus).

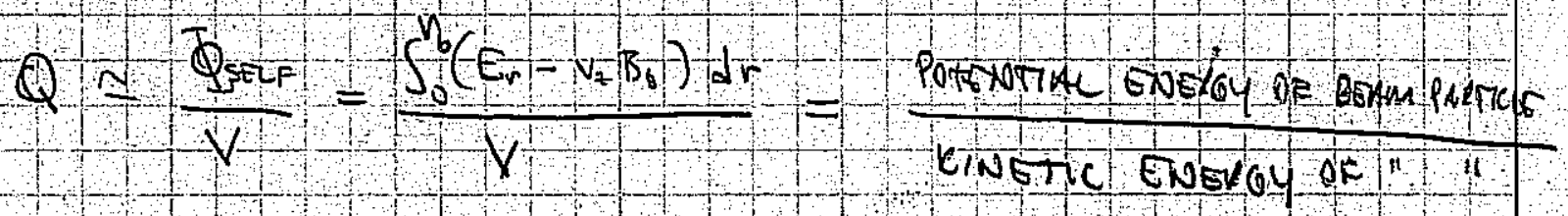

SOMETINES PELIODKC FOCUS ING IS EMHLOUED

$$
\begin{aligned}
& K(z)=K(z+S) \\
& S=P=10 D
\end{aligned}
$$

HOL SOME RuNT OSES a SU LTABLE conosTANT

CAN BE FOONO WHICH CAVTORSS SLOW VARIMTION

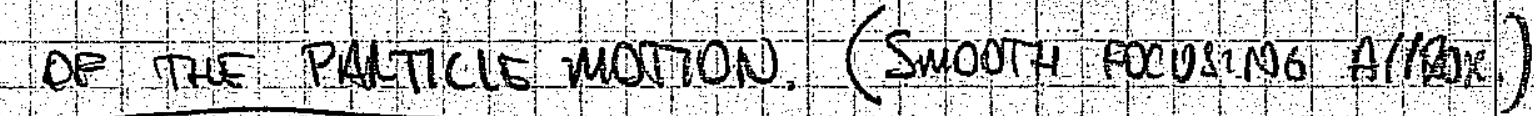

$$
\begin{aligned}
& \Rightarrow x^{2}+\frac{1}{v_{z}} \frac{d}{s}\left(\gamma_{z}\right) x^{\prime}=Q \frac{x_{1}}{v_{b}^{2}}-k_{0} x
\end{aligned}
$$

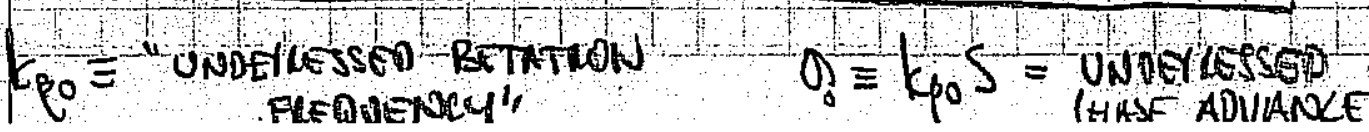




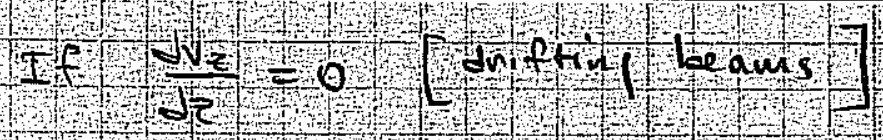

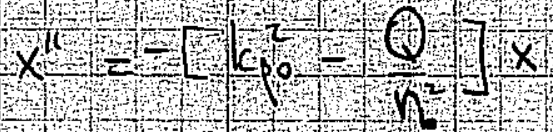

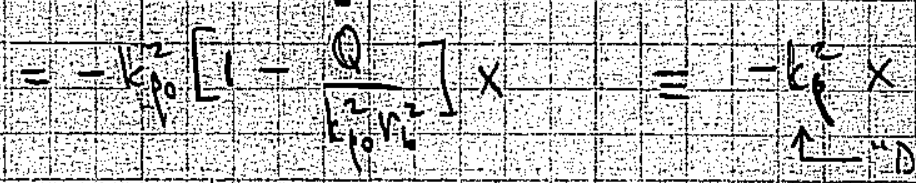

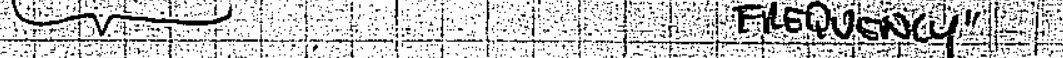

$$
\left.=\left(\frac{0}{0 .}\right)^{2}\right)=(\text { Che periession }
$$

\section{EFEG of stale Gurge is to cougth}

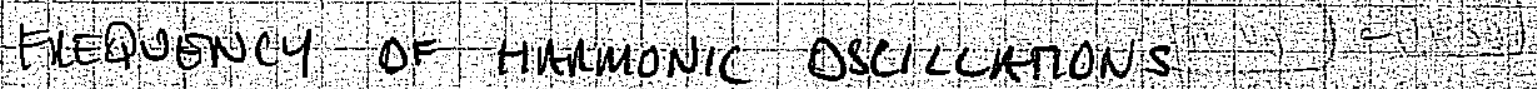

$\frac{0}{0 .}=0 \stackrel{\Rightarrow}{\Rightarrow}$ PULG TUNE PELLESEO

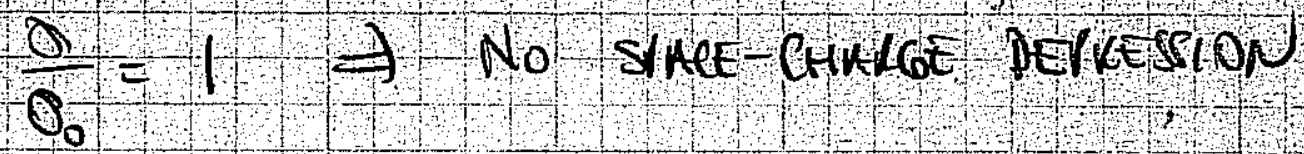


J. Barnard (5)

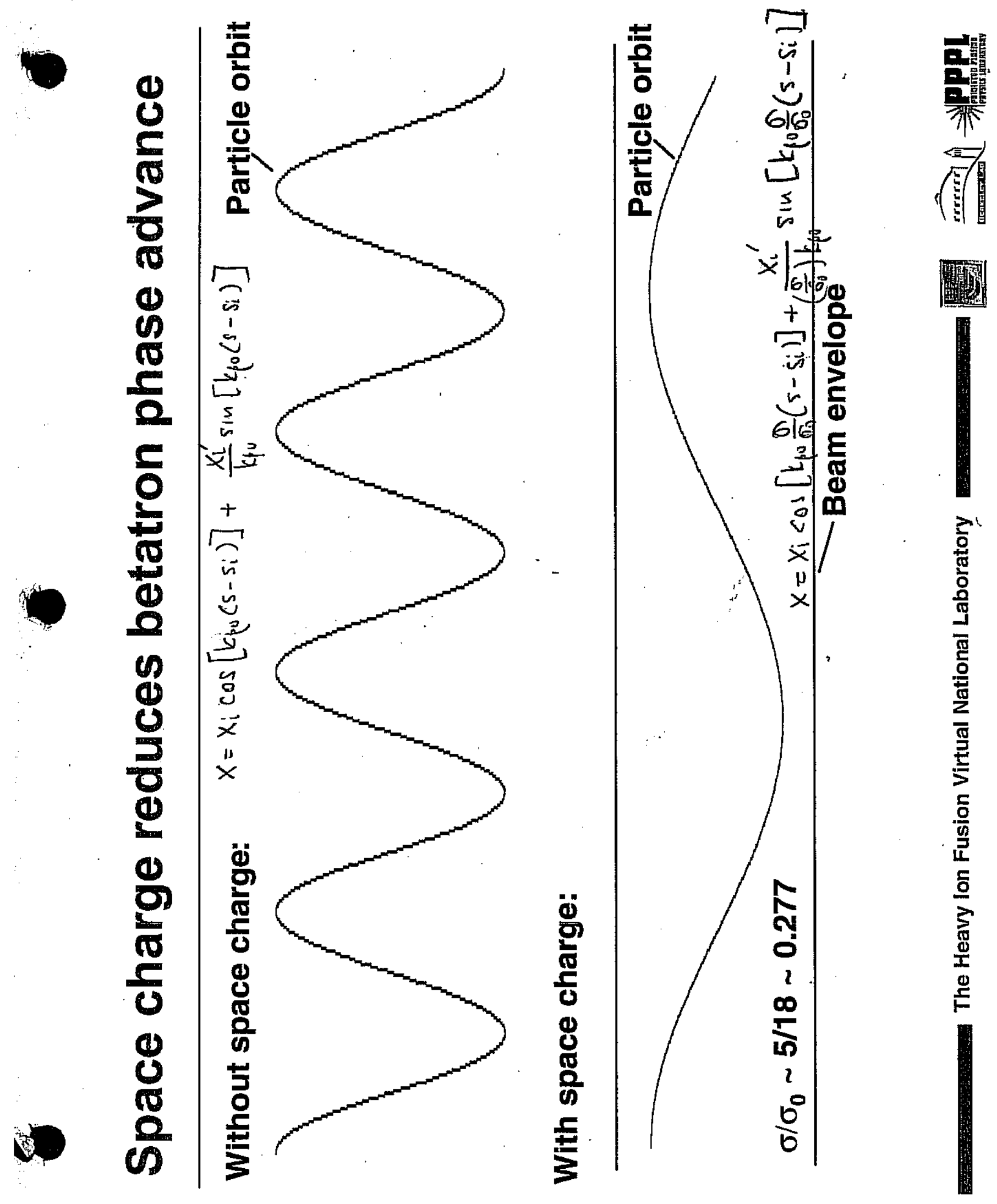




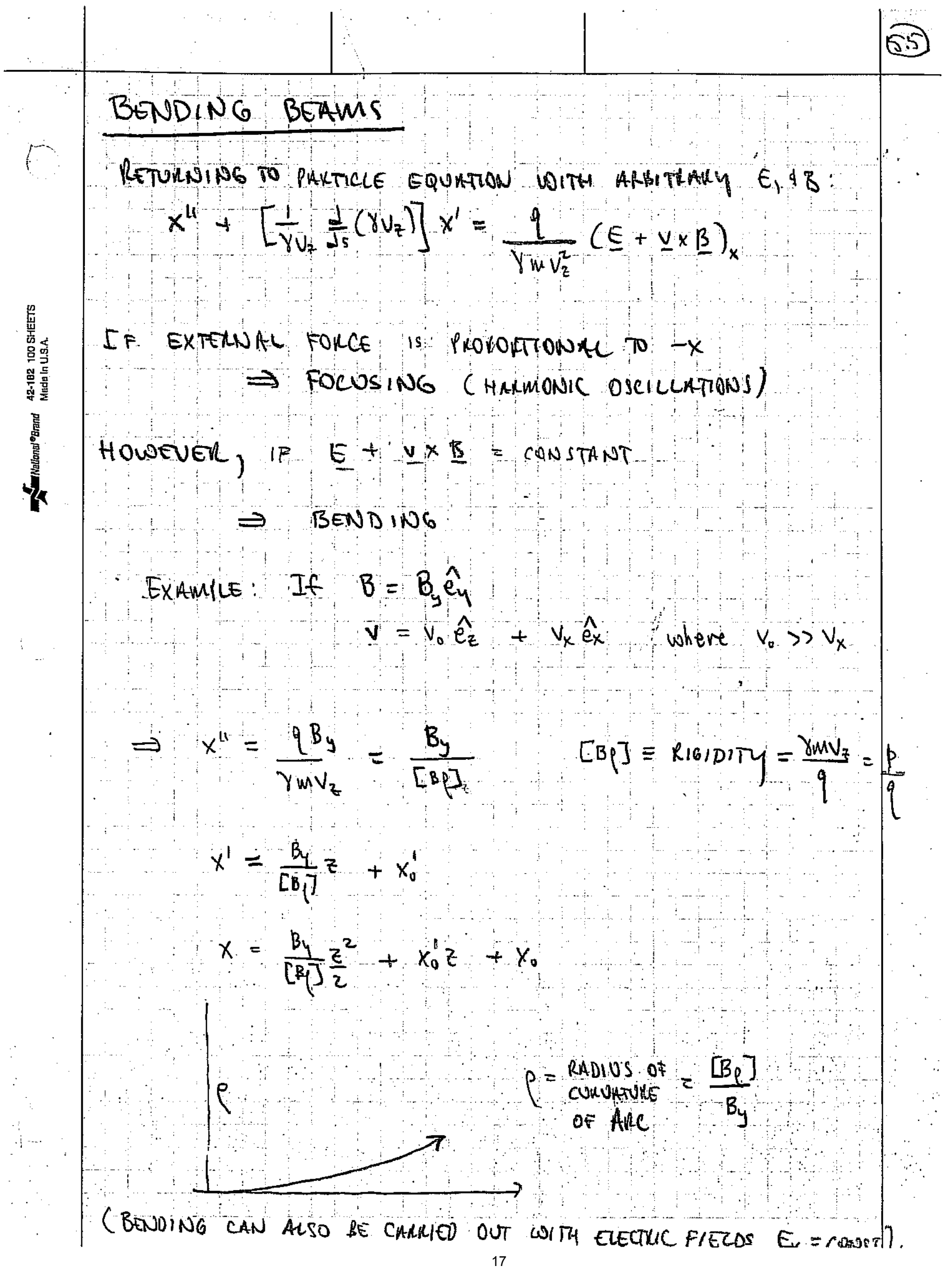




\section{Pinting taysios of Beams}

\section{$3+B$ and wates (b)}

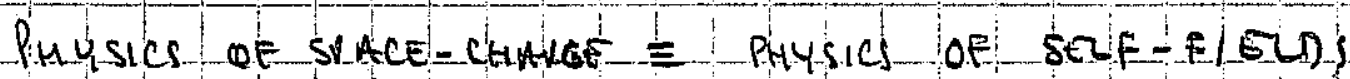

E puanma raysics of pakticle batans

\section{PLACMA IANATMETSL}

$q g_{J P}=\frac{1}{4 \pi \varepsilon_{0}} \frac{9^{2}}{r}$

$\simeq \frac{1}{4 \pi \varepsilon_{0}} n_{0}^{1 / 3} q^{2}$
Avexuge porenture enekata

OE INATRLLE DUE TO ITS NEAKEST

NGTG HOR

$(q=$ change of panticl $)$

If $q \Phi_{I P}<k_{T} T \Rightarrow$ lutima (wealdy coviled flasma)

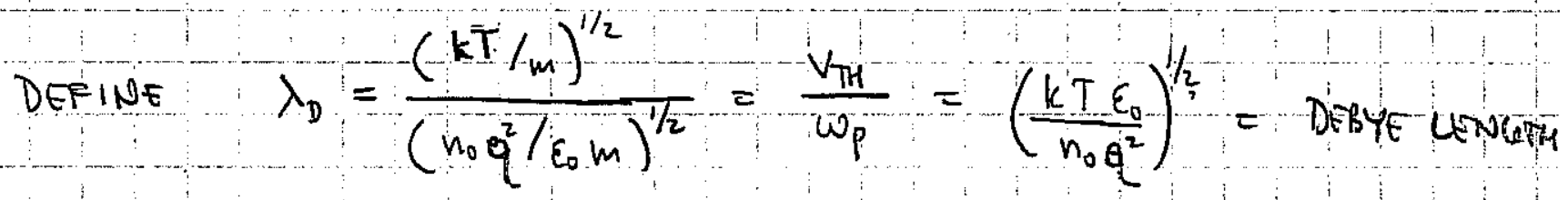

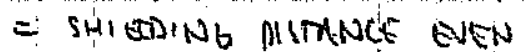

IN NON-NEUTRRE MANMA

DEFINE $\Lambda=\frac{4 \pi}{3} u_{0} \lambda_{0}^{3} \equiv$ Kasma Panametox

$$
\sim\left(\frac{\log T}{q}\right)^{3 / 2}>1
$$


Kumantanuen Eountion

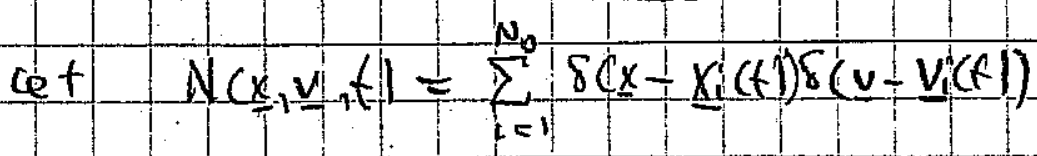

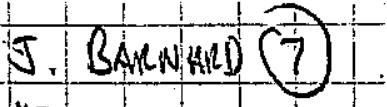

REE. "INTRO TO PLS'SUMA

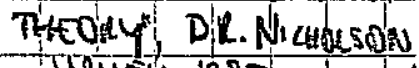
Witey 1983!

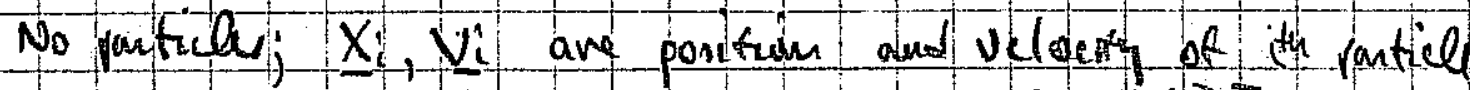

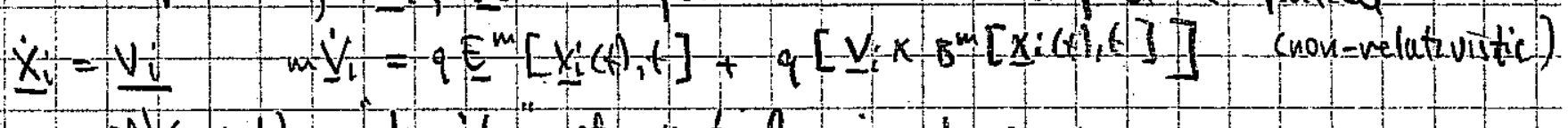

$N(x, v, t)=$ Jensity of particle in hou space

$$
\int N^{3} \times d N=N_{0}
$$

Let $u=x-x(t)$

Takiy denorial

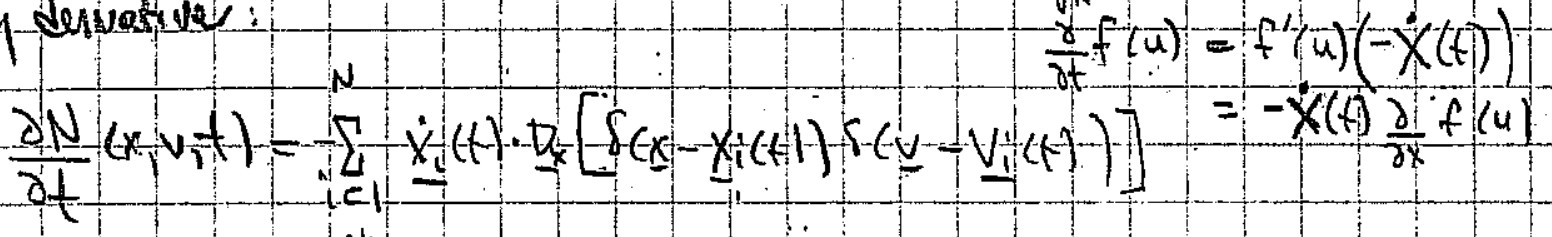

$$
\begin{aligned}
& -\sum_{i=1} V_{i}(f) \cdot \nabla_{v}\left[\delta\left(x-X_{i}(t)\right) \delta\left(v-V_{i}(t)\right]\right.
\end{aligned}
$$

MANWELS

Equarion ow.

$$
\text { Lem }
$$

$$
\begin{aligned}
& \nabla E^{m}=(\theta) q d v N(x, v) \quad \nabla \cdot B^{m}=0 \\
& \nabla \times E^{m}=\frac{\partial B^{m}}{\partial t} \quad \nabla \times B^{m}=\mu_{0} \int \frac{v-v-N\left(x-v_{1} t\right)}{J v^{m}}+\frac{\partial E^{m}}{\partial t} \\
& \Rightarrow \frac{\partial N}{\partial t}\left(x_{T}-t\right)=-\sum_{i=1}^{W_{0}} V_{i}(t): \nabla_{x}\left[\delta\left(\underline{L}-\underline{X}_{i}(t)\right) \delta\left(v-V_{i}(t)\right)\right]
\end{aligned}
$$

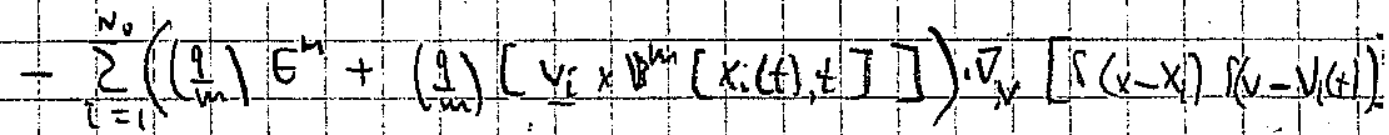

Note that $\underline{v}_{i}(t) \delta\left(\underline{v}-V_{i}(t)\right)=v \delta\left(v-V_{i}(t)\right)-50$

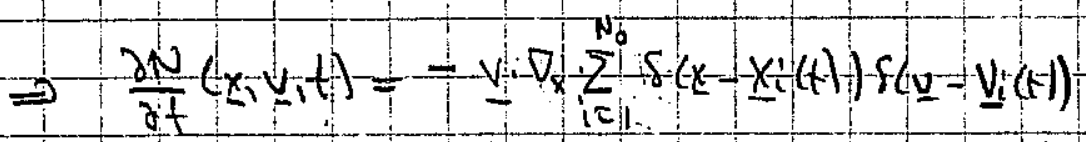

$$
\begin{aligned}
& -\left(\frac{g}{m} \epsilon^{m}\left(x_{1}, t\right)+\frac{g}{m}\left(v \times \underline{\beta}^{m}(\underline{x}, t)\right)\right)+\nabla v \sum_{i=1}^{N} \delta\left(x-x_{i}(t) \delta\left(v-V_{i}(t)\right)\right.
\end{aligned}
$$

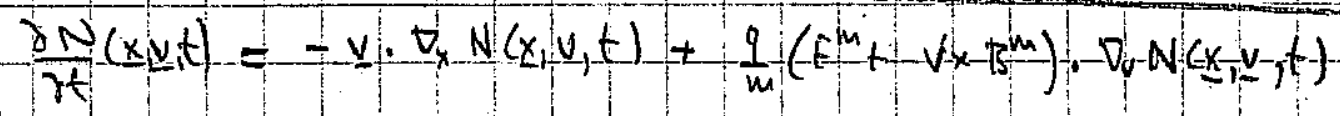$$
\nabla_{x}=\text { siatipia }
$$$$
\text { guadieu }
$$$$
\nabla_{v}=g_{w, i r t}^{n a} d i c u
$$$$
3 \text { velocit }
$$

Climbinush fquation

19 
Total devinateve alous an oubit:

J. BARNit(2) (8)

$$
\begin{aligned}
& \frac{D}{\partial t}=\frac{\partial}{\partial t}+\frac{J_{x}}{\partial t} \cdot \nabla_{x} \\
& \left.\frac{\omega_{v}}{d H}\right|_{\text {Duज }} \nabla_{\bar{v}} \\
& \Rightarrow \quad \frac{\theta}{\partial t} N(\underline{v},+)=0 \\
& f(x, v, t)=\frac{\int N\left(x-v v^{3}+1\right) d v}{\Delta x^{3} \Delta v^{3}} \\
& E\langle N(\underline{x}, \underline{v}, t)\rangle
\end{aligned}
$$

Note that NEC or a nothin in. betwelu.

over some oon in hase syace $\Delta x+\Delta v$ and thal siz of box

Assume $n^{-1 / 3}<\Delta x<\lambda_{D}$ so that $f(x, v, f)$ is suresta functiven.

Then

$$
\begin{array}{c|c|c}
N=f+\delta f & f=\langle N\rangle & \langle\delta f\rangle=0 \\
E^{m}=E+\delta E & E=\langle E\rangle & \langle\delta E\rangle=0 \\
B^{m}=B+\delta B & B=\left\langle B^{m}\right\rangle & \langle\delta B\rangle=0
\end{array}
$$

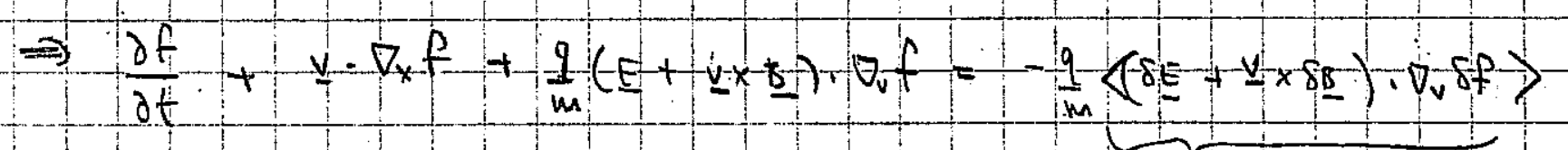

Average of s/Lrey QuAntites "Discrete /ancL efeEct. QR. "COULSIONI"

If collisions are neqlected (set RHS to zeno):

Vlarov-EQOAron

$$
\frac{\partial f}{\partial t}+v \cdot \nabla f f \frac{q}{m}(E+\underline{v} \times \underline{s}) \cdot \nabla f=0
$$

$\Rightarrow \frac{D_{1} f}{D_{t}}=0$ P PHAE SHe DeThM CONSTANOT ON THESTOHES. CLOOUVILLE'S THEOROW 
J.BARnARI (9)

THE RHS is DUE to COLISIONS WITH NON-SMOOTH FIELPS:

VERY HEURISTICALLY

$$
\begin{aligned}
& -\frac{q}{m}\left\langle(\delta E+\underline{v} \times \delta B) \cdot \nabla_{v} \delta f\right\rangle \sim \nu_{c} f \\
& \nu_{c} \sim \sigma_{n v} .
\end{aligned}
$$

$\sigma \sim \pi r_{c}^{2}$ when $r_{c}$ is given by $k T \sim \frac{q^{2}}{4 \pi \varepsilon_{0} r_{c}}$

(for lavpe auple collicions)

$$
\Rightarrow \nu_{c} \sim \pi\left(\frac{q^{2}}{4 \pi \varepsilon_{0} k T}\right)^{2} n_{0}\left(\frac{k T}{m}\right)^{1 / 2}
$$

On lhS of Vlasov equatom: (VONY ROOOH, BOT MAIN SCALING IS COAHECT, WO ITH FACTOM!)

$$
\frac{q}{m} \in \nabla_{\Delta} f \sim\left(\frac{g \lambda_{D} n_{0}}{\varepsilon_{0}}\right) \frac{f}{V_{T_{H}}}
$$

whene $V_{T H} \sqrt{\frac{l e T}{m}}$

$\frac{\text { COUISION TEMm }}{\text { LHS }} \sim \frac{1}{16 \cdot \lambda_{0}^{3} n_{0}}=\frac{1}{16 \Lambda}$

21 


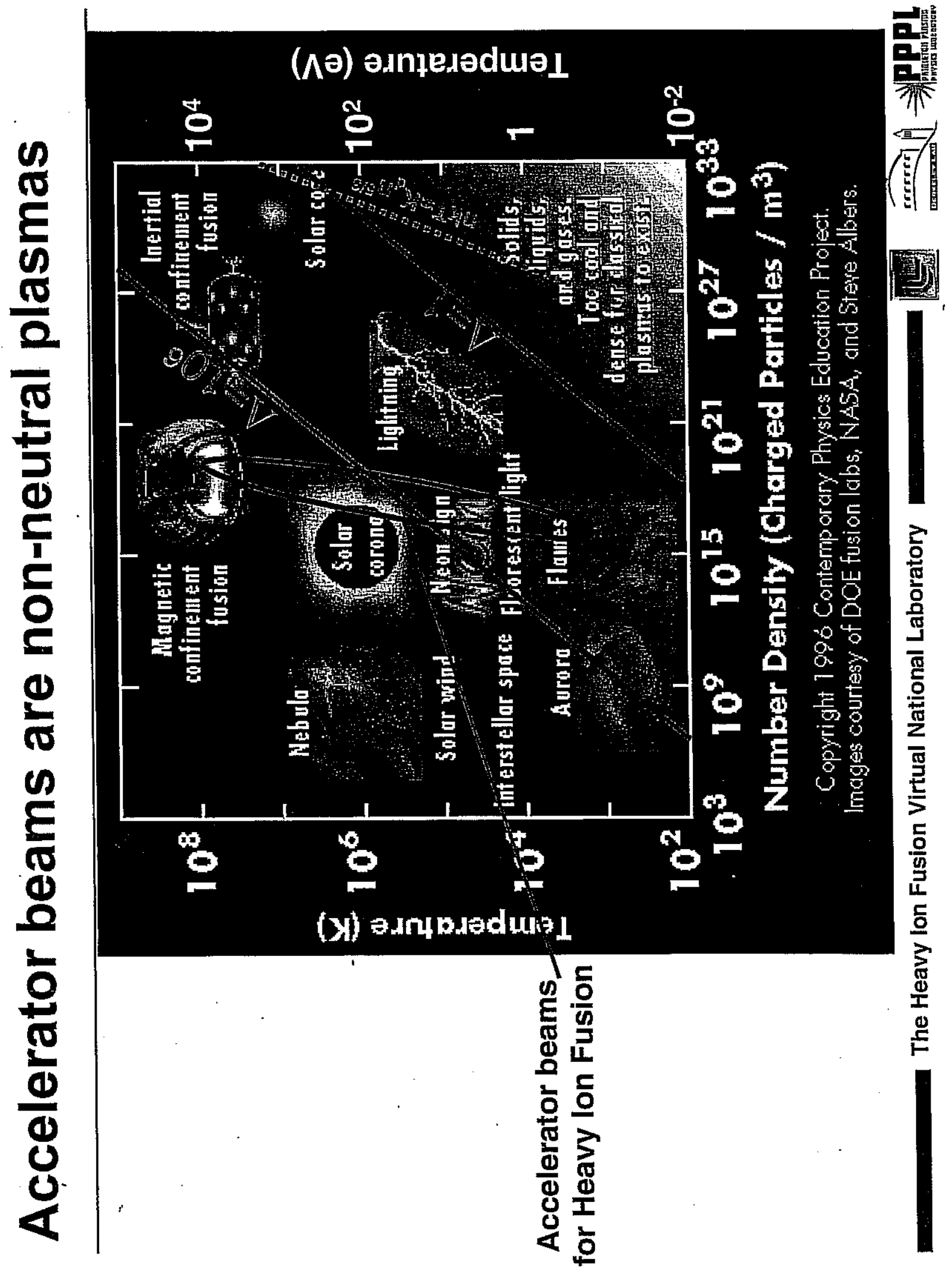


$T$

J. BanNato (it)

Deschirtions of THE BEAM

Liouviues Theorem: $\frac{d f}{d t}=0$ along a trajectory in phase space,

Let $d N=f d x d y d z d p_{x} d_{p_{1}} d p_{z}$

The continuity equation in phase space is:

$$
\frac{\partial f}{\partial t}+\underline{\nabla}(f \underline{v})=0
$$

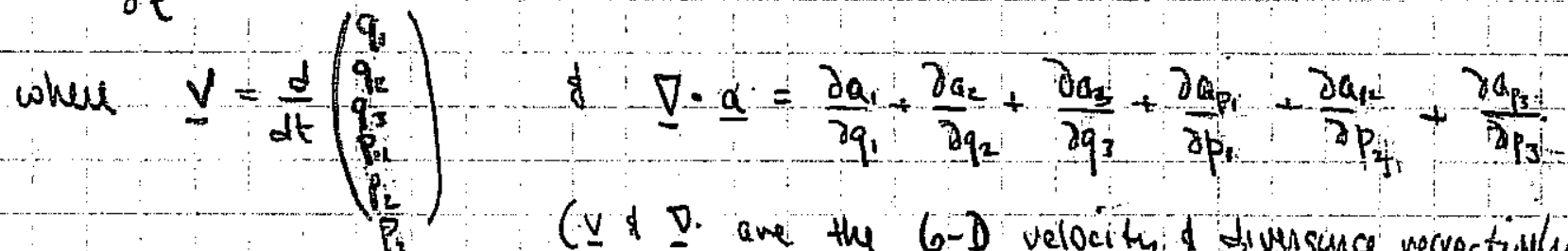

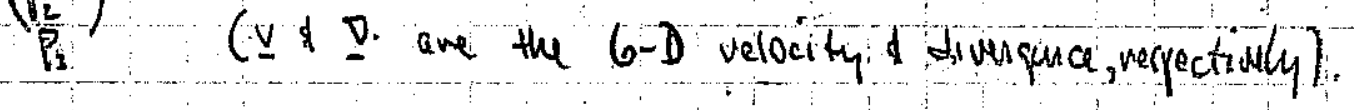

If the system is governed by a Hamiltonian $4(q, p, t)$

$$
\begin{aligned}
& \dot{q}_{i}=\frac{\partial H}{\partial p_{i}} \quad \dot{p}_{i}=-\frac{\partial H}{\partial q_{i}} \\
& \text { Now, } \nabla \cdot v=\sum_{i=1}^{3} \frac{\partial q_{1}}{\partial q_{i}}+\frac{\partial \dot{p}_{i}}{\partial r_{i}}=\sum_{i=1}^{3} \frac{\partial^{2} H}{\partial q_{i} \partial \psi_{i}}-\frac{\partial^{2} H}{\partial p_{i} \partial q_{i}}=0 \\
& \Rightarrow \frac{\partial f}{\partial t}+f \underline{\underline{v}} \underline{y}+\underline{v} \cdot \underline{\underline{f}}=0
\end{aligned}
$$

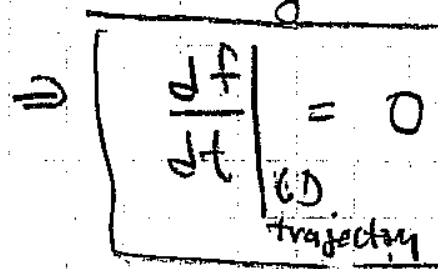

23 


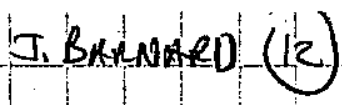

Enittance \& Bucatiness

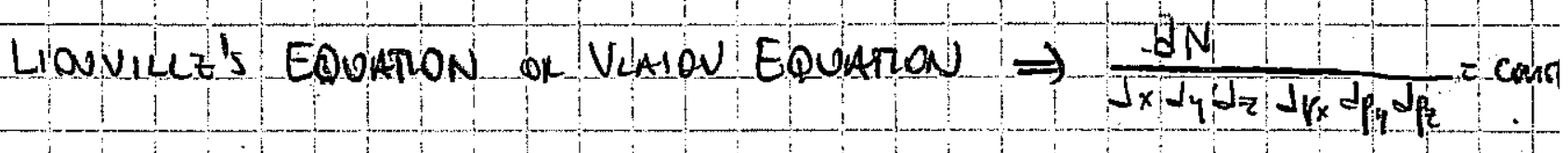

If $x^{\prime \prime}=f(x)$ AN No Funtrous $(y+z)$

$$
\begin{aligned}
& y^{n}=f(y)=\text { in } i \quad(x, y) \\
& z^{\prime \prime}=f(z)=1-1-4(x, d y)
\end{aligned}
$$

THeN $\frac{d N}{d x d p_{x}}=$ const, $\frac{d N}{d y d p_{y}}=$ coust $\& \frac{d N}{d z d P}=$ coust

selarately.

1ST DGP I TION:

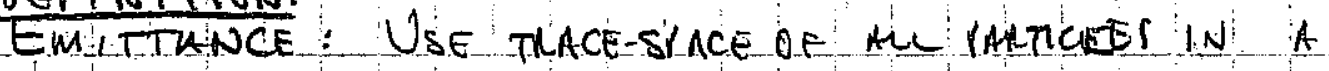
GIVEN SLCE OF WOAM.

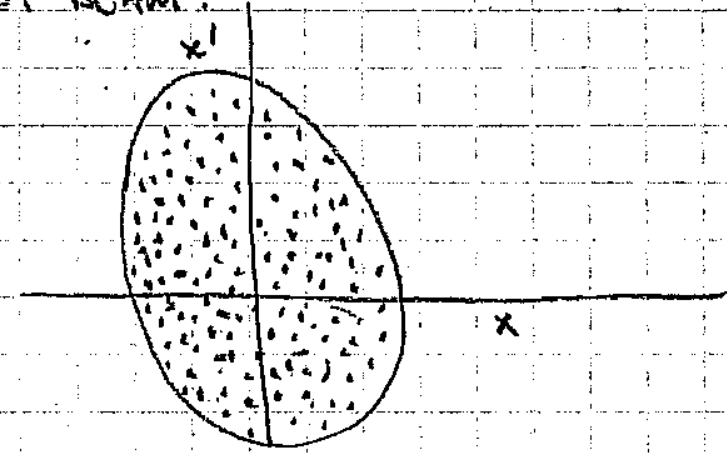

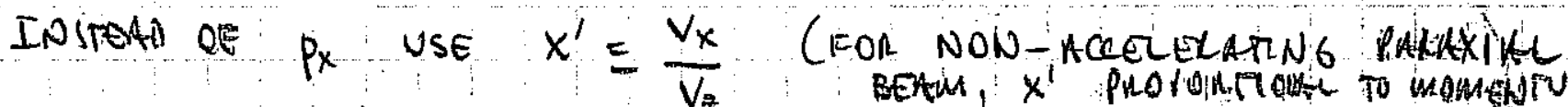

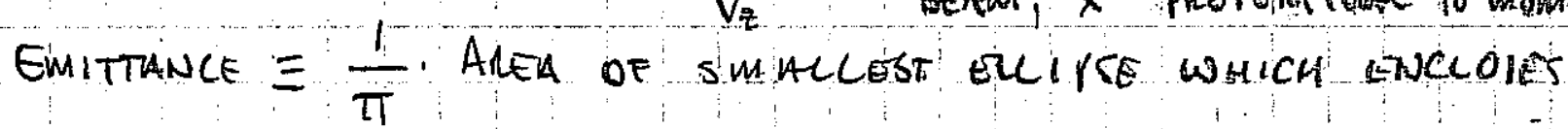
ALU AARTCLES. (TLAE-SIHE DGEINITON)

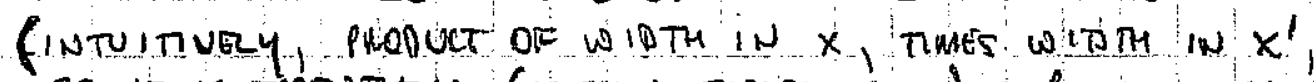

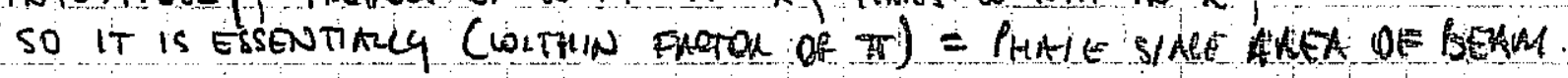

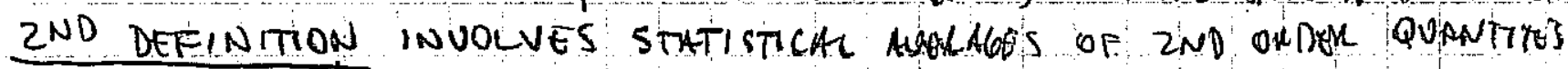
(such A rms).

$$
\epsilon_{x} \equiv 4\left(\left\langle x^{2}\right\rangle\left\langle x^{12}\right\rangle-\left\langle x x^{\prime}\right\rangle^{2}\right)^{1 / 2}
$$

For an uivight uniform beam (in rhase space): $\left\langle x^{2}\right\rangle=\frac{h^{2}}{4} \quad\left\langle x^{12}=\frac{x_{\text {max }}^{2}}{4}\right.$

$$
\Rightarrow \epsilon_{x}=V_{x} x_{\max }=\frac{\text { Area }}{\pi}
$$$$
+\left\langle x x^{i}\right\rangle=0
$$

24 
Nongualized EmLitrance

Fo a bean that i accelenating return to $x, \beta_{x}$ an sefinition of phive spuce ama:

$$
\begin{aligned}
& p_{k}=\gamma m v_{x}=\gamma m v_{z} x^{\prime} \quad A g a w_{1} \text { assumin } v \cong v_{z}
\end{aligned}
$$

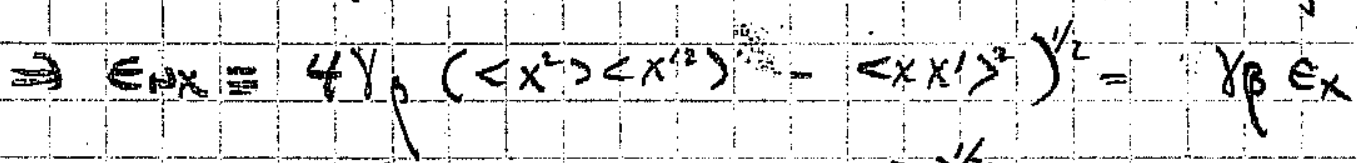

$$
\begin{aligned}
& =\frac{4}{m}\left(\left\langle x^{2}\right\rangle\left\langle p_{x}^{2}\right\rangle-\left\langle x p_{x}\right\rangle^{2}\right)^{1 / 2}
\end{aligned}
$$

13

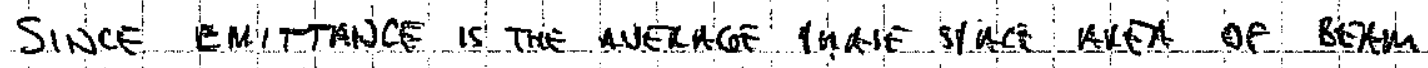

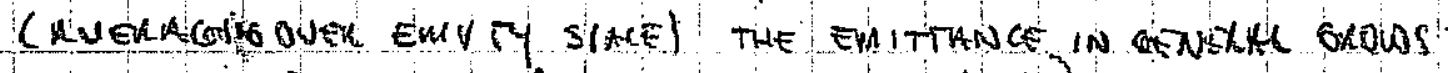

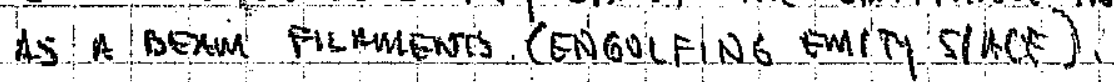

BMOHTNES

The dROSIYY of lanteles in 6-D pHASE SIACE IS:

$$
\frac{d N}{d x d y d z d x d y d \sqrt{z}}=f
$$

Microscoric nENOSIY

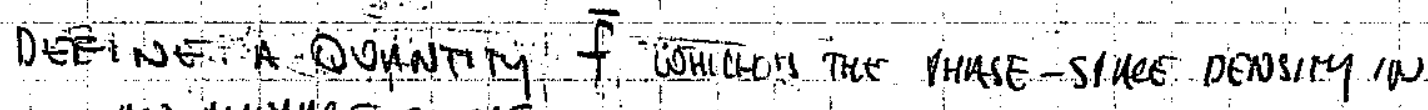
AN HUTHALE SENOSE

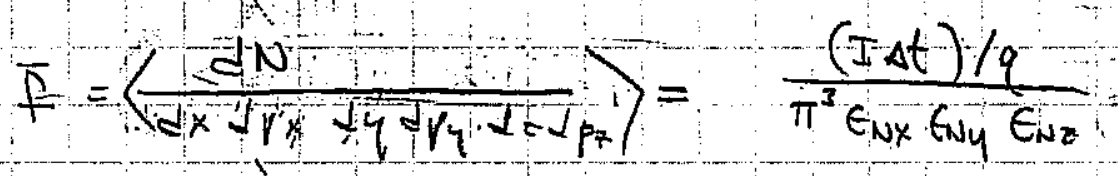

Note $f(x, l)=$ conitant aloug a hapectory, whereas $\bar{f}$ isvally is a tecreaing fouction of $z$.

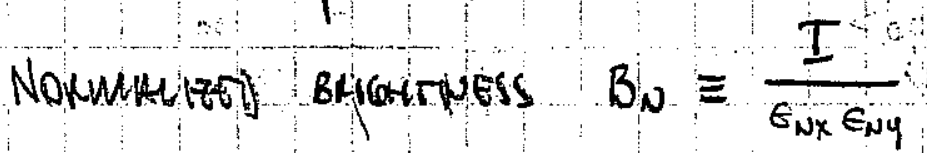

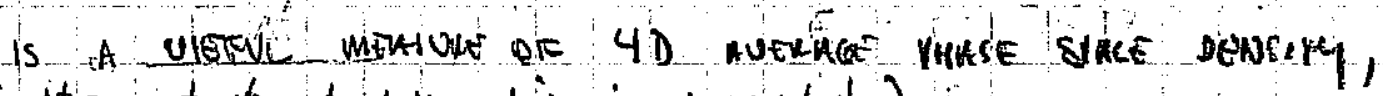

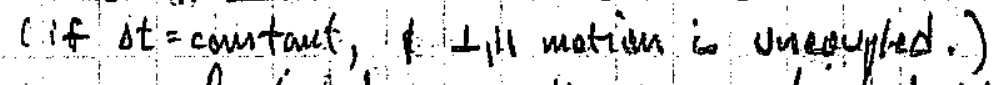

Fo non-acceleratug beams the unnormalized buightues $B$ calso if it a canst.

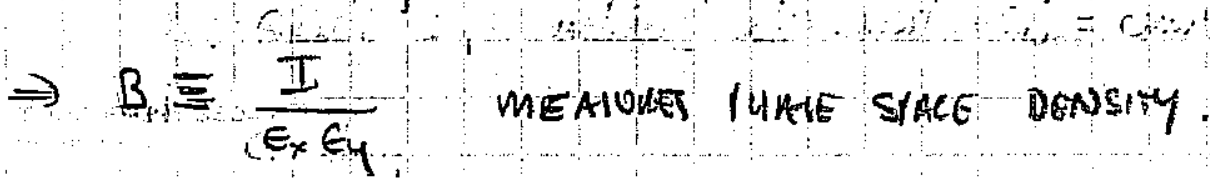
$q$ I.ll motidu uncoupled .

25 

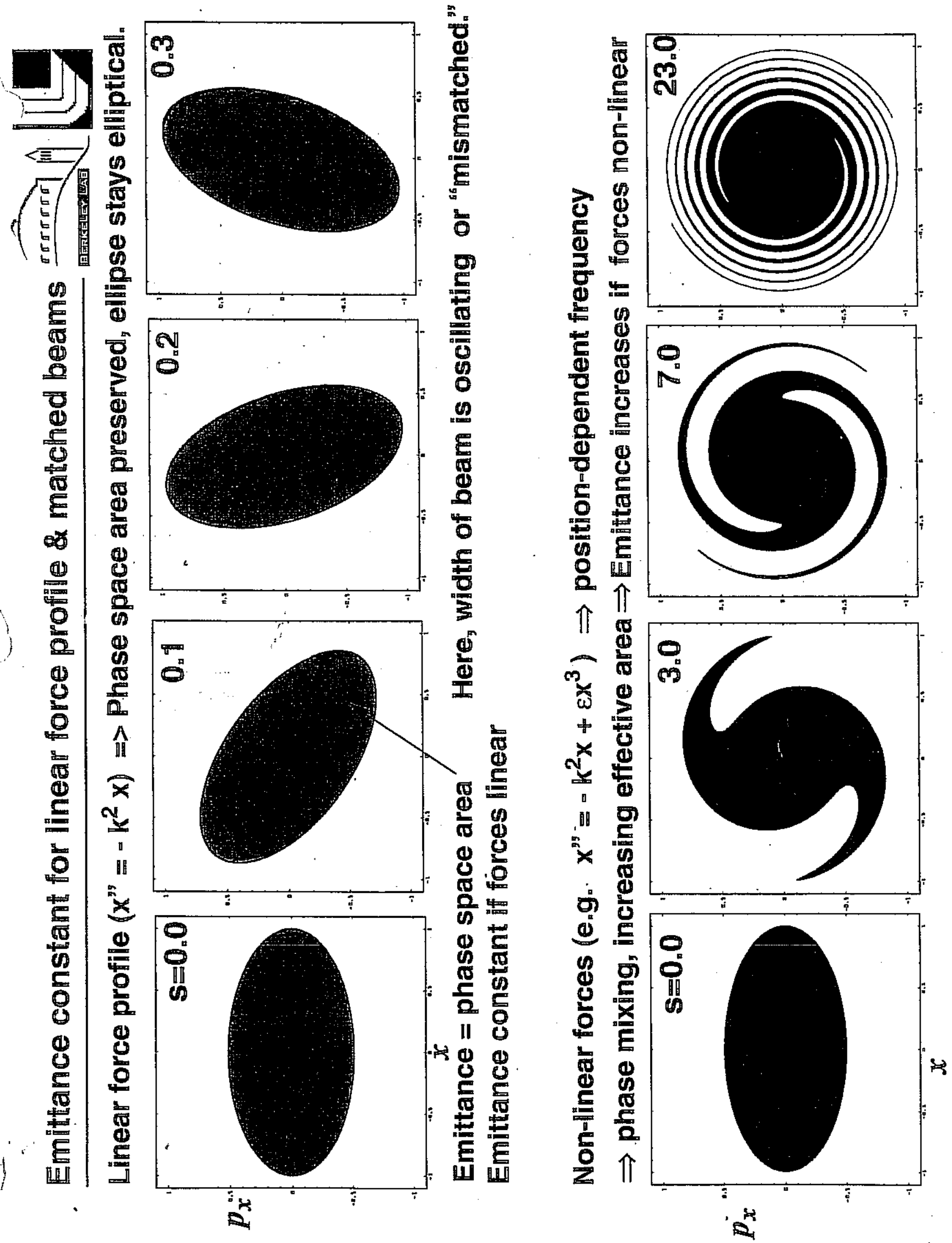


\section{John Barnard Steven Lund USPAS June 2008}

\section{Envelope Equations}

Paraxial Ray Equation

Envelope equations for axially symmetric beams

Cartesian equation of motion

Envelope equations for elliptically symmetric beams 


\section{John Barnard Steven Lund \\ Roadmap: \\ USPAS \\ June 2008}

Single particle equation with Lorentz force $q(E+v \times B)$

Make use of:

1. Paraxial (near-axis) approximation $\left(r<1 / k_{\beta 0}\right.$ and $\left.x^{\prime}=v_{x} / v_{z}<<1\right)$

2. Conservation of canonical angular momentum

3. Axisymmetry $f(r, z)$

Paraxial Ray Equation for Single Particle

Next take statistical averages over the distribution function

$\Rightarrow$ Moment equations

Express some of the moments in terms of the rms radius and emittance

$\Rightarrow$ Envelope equations (axi-symmetric case)

Some focusing systems have quadrupolar symmetry Rederive envelope equations in cartesian coordinates $(x, y, z)$ rather than radial $(r, z)$ 


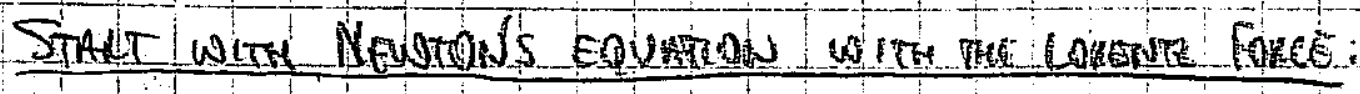

STARNARD Q

$$
\frac{d p}{d t}=q(E+v \times B)
$$

In conteizain coordenate:

$$
\begin{aligned}
& \frac{d}{d t}(\gamma m \dot{x})=\gamma m x+\gamma m x=q\left(E x+y B_{z}-\varepsilon B_{\eta}\right) \\
& \frac{d}{d t}\left(\gamma_{m} y\right)=\gamma_{m y}+\gamma_{m y}=q\left(E_{y}+z B_{x}-\gamma_{B}\right) \\
& \frac{\partial}{H}(\gamma m)=\dot{\gamma} m+y_{m z}-q\left(E_{z}+\dot{x} B_{y}-\dot{y} B x\right)
\end{aligned}
$$

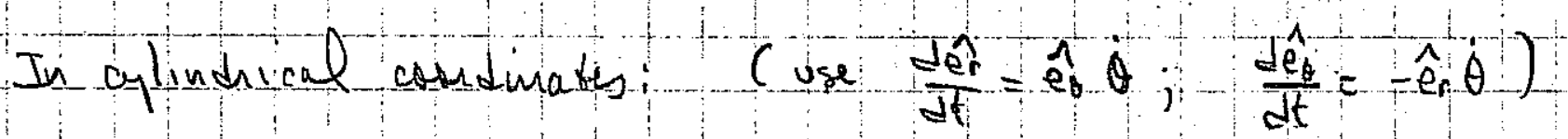

$$
\begin{aligned}
& \frac{d}{d t}(\gamma m \dot{r})-\gamma \mu \theta^{2}=g\left(f_{r}+r \theta B z-z B\right) \\
& \frac{1}{r} d(\sqrt{w} r \theta)-q\left(E_{\theta}+\frac{1}{z} B r-r B z\right) \\
& \frac{d}{d t}\left(V_{m}\right)-q\left(E_{z}+r b_{b}-r b_{r}\right)
\end{aligned}
$$

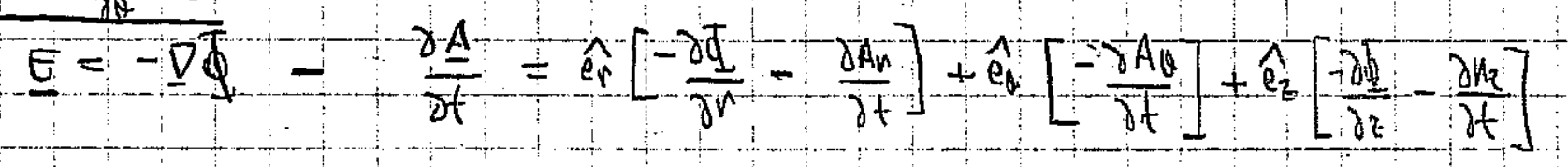

$$
\begin{aligned}
& \underline{B}=\nabla \times A=\hat{\theta}\left[-\frac{\partial}{\partial z} A_{\theta}\right]+\hat{\theta}_{\theta}\left[\frac{\partial A_{r}}{\partial z}-\frac{\partial A_{z}}{\partial r}\right]+\hat{\theta}_{\theta}\left[\frac{1}{r} \frac{\partial r}{\partial r} A_{\theta}\right] \\
& q r\left(b_{\theta}+e b_{r}-\dot{r} B_{z}\right)+q\left(-\frac{\partial \omega A_{\theta}}{\partial t}-\dot{\partial} w_{\theta}-r \frac{\partial}{\partial r}\left(r A_{\theta}\right)\right) \\
& =-q\left[\frac{\partial r}{\partial t}+v-\frac{\partial}{\partial x}\right]\left(n A_{\theta}\right) \\
& =-9 \frac{d\left(r A_{\theta}\right)}{d t}
\end{aligned}
$$

Eqtu $\pi \Rightarrow$ \& IV

$$
\frac{d}{d t}\left(8 m r^{2} \theta+q r A_{\theta}\right)=0
$$

29 
(1.5)

$$
\begin{aligned}
& \underline{p}=p_{r} \hat{e}_{r}+p_{\theta}^{*} \hat{e}_{\theta}+p_{z} \hat{e}_{z} \\
& \text { whene } p_{v}=\gamma_{m i} \dot{r} \\
& r^{*}=\gamma_{m} r \dot{\theta} \\
& p_{z}=\gamma_{m} \dot{z} \\
& \frac{d p}{d t}=\dot{p}_{v} \hat{e}_{v}+p_{r} \hat{e}_{n}+p_{*}^{*} \hat{e}_{u}+p_{\hat{\theta}}^{*} \hat{e}_{n}+p_{r} \hat{e}_{z} \\
& \Rightarrow \frac{d p}{d t}=\left(p_{v}-p_{\theta}^{*}\right) \hat{e}_{r}+\left(p_{\theta}+p_{\theta}^{*}\right) \hat{e}_{\theta}+p_{z} \hat{e}_{z}
\end{aligned}
$$

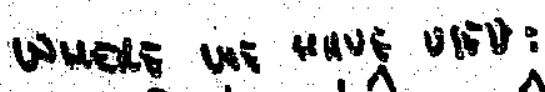

$$
\begin{aligned}
& \frac{J \hat{e}_{r}}{d t}=\hat{e}_{0} \dot{\theta} \quad \frac{J \hat{e}_{t}}{d t}=-\hat{e}_{r} \dot{\theta} \\
& \Rightarrow \frac{d p}{d t}=\left[\frac{d}{d t}\left(\gamma_{m i}\right)-\frac{d}{d t}\left(\gamma_{\min } \dot{j}^{2}\right)\right] \hat{e}_{r} \\
& +\left[\gamma_{\text {mir }} \dot{\theta}+\frac{d}{d t}\left(\gamma_{m r} \dot{\theta}\right)\right] \hat{e_{\theta}} \\
& =\frac{1}{r} \frac{1}{d t}\left(\gamma_{\min }^{2} \dot{\theta}\right) \\
& +\frac{d}{d t}\left(\gamma_{m z}\right) \hat{e}_{z}
\end{aligned}
$$

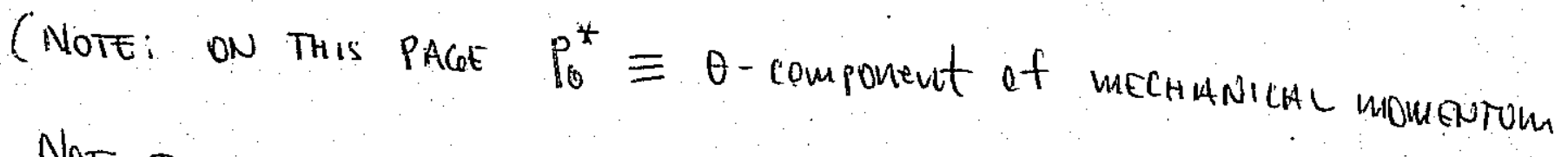

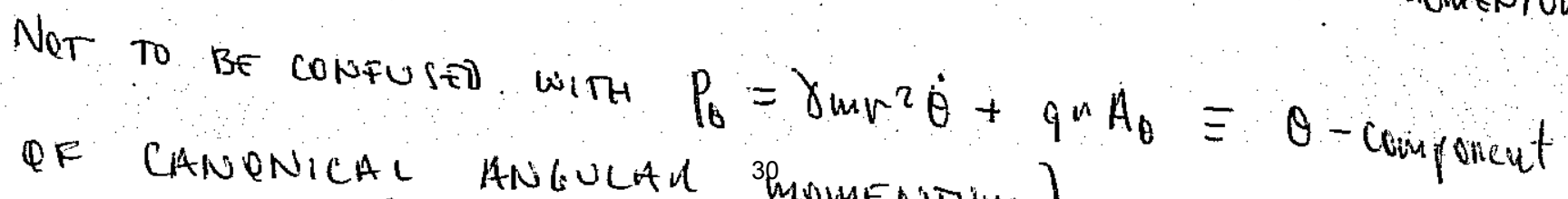
pr canenical hangulan shomentum) 


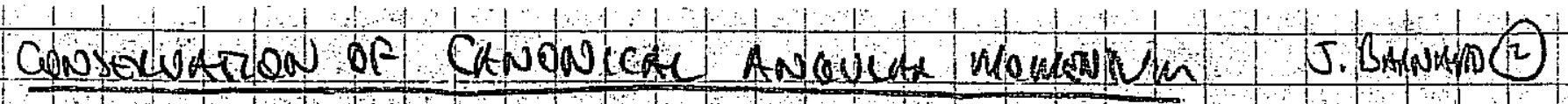

$$
\begin{gathered}
\text { DefNe } p_{0}=\gamma_{m r^{2}} \theta+q r A_{0} \\
\frac{d}{J t} P_{\theta}=0
\end{gathered}
$$

(consonvarous ore

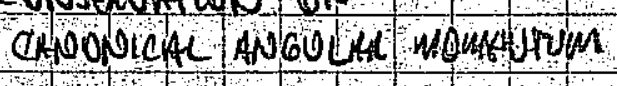

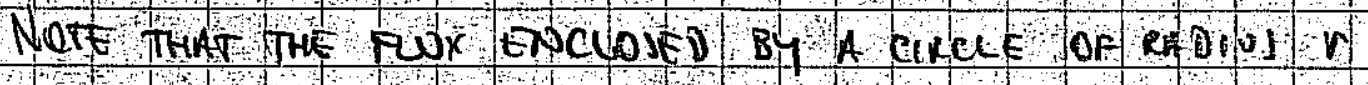

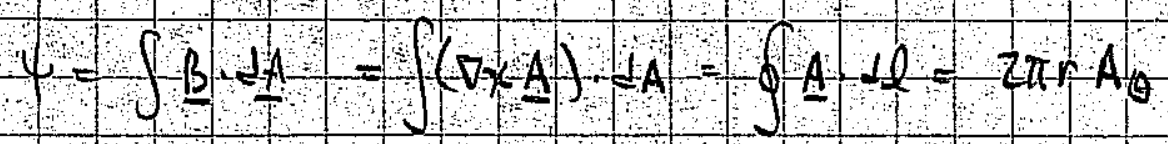

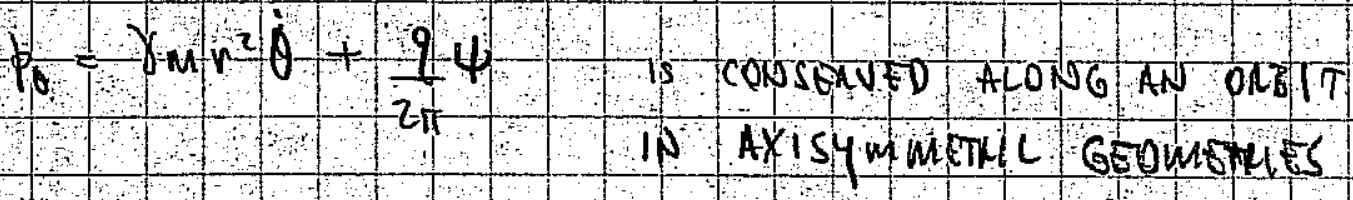


Extonor

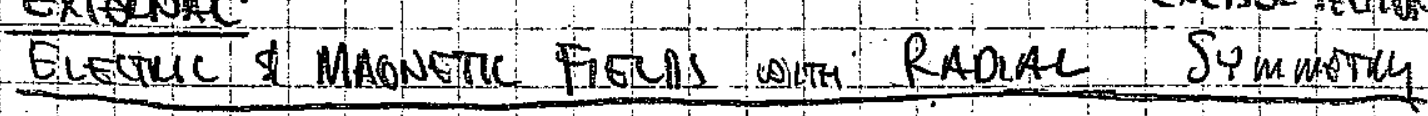

S.BACASAD

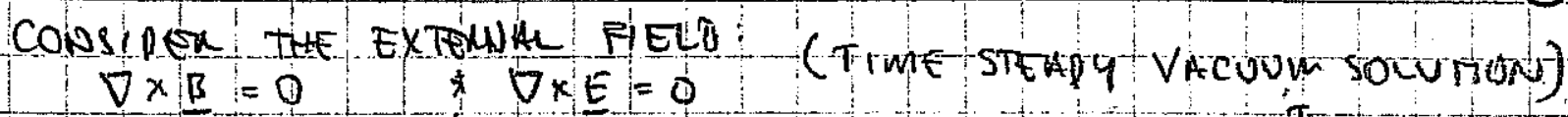

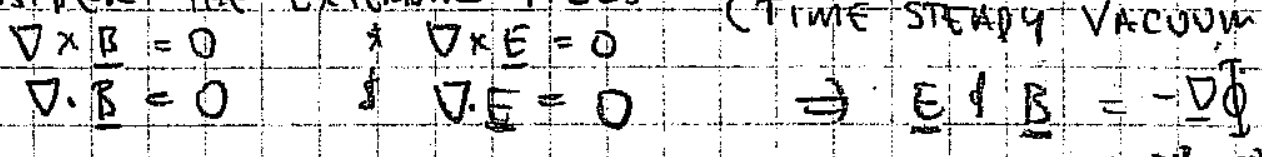

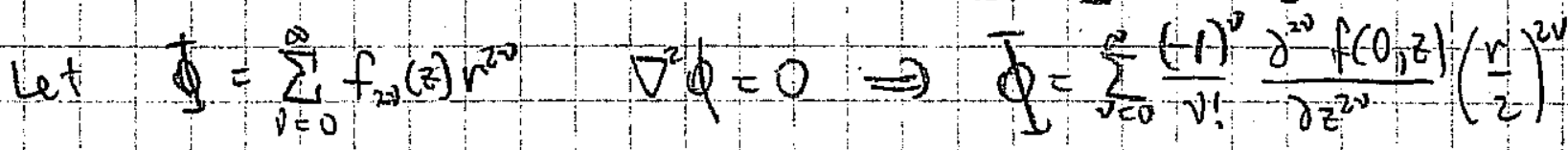

$$
\Rightarrow \phi=\Phi(0, z)-\frac{\partial^{2} \Phi(0, z)}{\partial z^{2}} r^{2}+\frac{1}{64} \frac{\partial^{4} \phi(0, z)}{\partial z^{4}} r 4
$$

Let $B_{z}(0, z)=B(z) \quad \&$ et $\Phi(0, z)=V(z)$

$$
\begin{aligned}
& B_{z}(r, z)=B+\frac{r^{2}}{4} \frac{\partial B}{\partial z^{2}}+\frac{r^{2}}{6 t} \frac{\partial^{4} B}{\partial z^{4}} \\
& B r(r z)=-\frac{r}{z}+\frac{\partial}{16} \frac{r^{3}}{\partial z^{3}}+\cdots \\
& \Phi(r, z)=V(z)=\frac{1}{4} V^{n} r^{2}+\frac{r}{64} \frac{1}{\pi t} \\
& \Rightarrow \quad E_{r}=\frac{1}{2} V^{r} r-\frac{r^{3}}{16} \frac{\partial^{4} V}{\partial z^{4}}
\end{aligned}
$$

$A-150, \psi=\pi r^{2} B(z)$

32 
(3.)

$$
\begin{aligned}
& \nabla^{2} \phi=\frac{1}{r} \frac{\partial}{\partial r}\left(r \frac{\partial d}{\partial v}\right)+\frac{\partial^{2} \phi}{\partial z^{2}}=0 \\
& f(r, z)=\sum_{v=0}^{N} f_{2 v}(z) r^{2 v}=f_{0}+f_{2} r^{2}+f_{u} r \\
& \sum_{v=1}^{\infty}[2 v+2 v(v-1)] f_{2 v} r^{2 v-2}+\sum_{v=0}^{\infty} f_{2 v}^{\prime \prime} r^{2 v}=0
\end{aligned}
$$

33 
(4)

Paraxial Ray Equation

$$
\begin{aligned}
& (I) \Rightarrow \frac{d}{d t}\left(\gamma_{m} \dot{r}\right)-\gamma_{m r} \dot{\theta}^{2}=q\left(\frac{V^{\mu}}{2} r+r \dot{\theta} B\right)+q\left(E_{r}^{\text {elf }}-V_{\tau} B_{\theta}^{\text {ste }}\right) .
\end{aligned}
$$

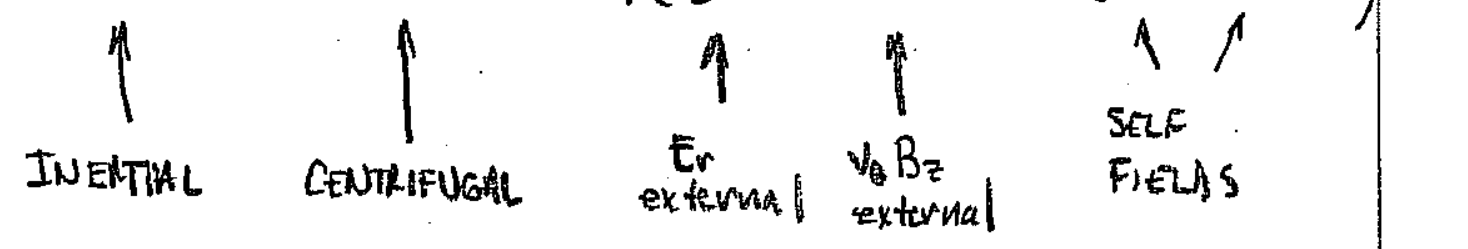

Now use $s$ ac independent variable $v_{z} d t=d s$

$$
v_{z} \frac{d}{d s}\left(\gamma_{m} v_{z} r^{\prime}\right)-\gamma_{m} v_{z}^{2} r \theta^{\prime 2}=q\left(v^{\prime \prime} r+r v_{z} \theta^{\prime} B\right)+q\left(t_{r}^{g l t}-v_{z} b_{z}^{d f f}\right)
$$

Expanding IT $^{\text {ST }}$ term and $v_{z} \cong v_{j}$ and DIVIDING By $\gamma_{m} v^{2}$ :

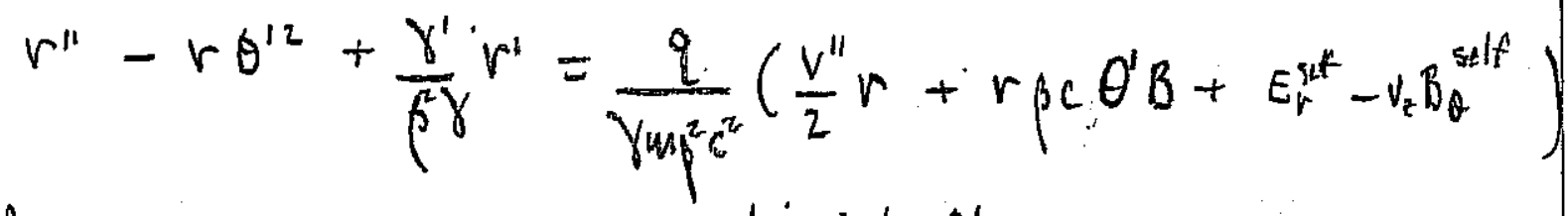

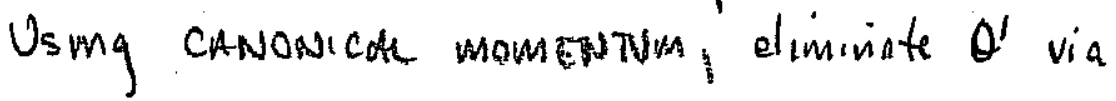

(Pi)

$$
\theta^{\prime}=\frac{p_{\theta}-\frac{q \varphi}{2 \pi}}{\gamma m r^{2} \beta c}=\frac{p_{\theta}}{\gamma m r^{2} \beta c}-\frac{q^{\beta}}{2 \gamma_{\beta m c}} \equiv \frac{p_{\theta}}{\gamma_{m r^{2} \beta c}}-\frac{\omega_{b}}{2 \gamma_{\beta c}}
$$

where we define $\omega_{c} \equiv \frac{q B}{m}$.

ADDING THE TWO O' TELLS IN THE EQUATOR (MI)

$$
\begin{aligned}
-r \theta^{2}-\frac{r \omega_{c} \theta^{\prime}}{\gamma \beta c}=\frac{-p_{\theta}^{2}}{\gamma^{2} m^{2} r^{3} \beta^{2} c^{2}} & +\frac{\beta_{\theta} \omega_{c}}{\gamma^{2} m \beta^{2} c^{2} r}-\frac{r \omega_{c}^{2}}{4 \gamma^{2} \beta^{2} c^{2}} \\
& -\frac{p_{b} \omega_{c}}{\gamma^{2} m \beta^{2} c^{2} r}+\frac{r \omega_{c}^{2}}{2 \gamma^{2} \beta^{2} c^{2}} \\
& =\frac{-p_{\theta}^{2}}{\gamma^{2} m^{2} \gamma^{3} \beta^{2} c^{2}}+\frac{r \omega_{c}^{2}}{2 \gamma^{2} \beta^{2} c^{2}}
\end{aligned}
$$

34 
(5)

So equation (PI) becomes:

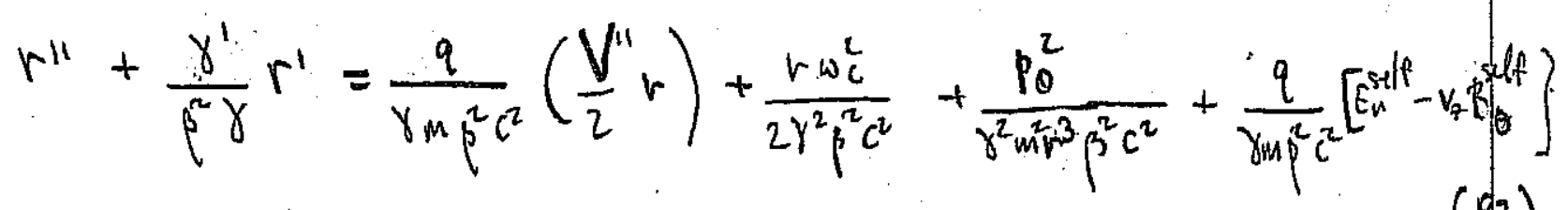

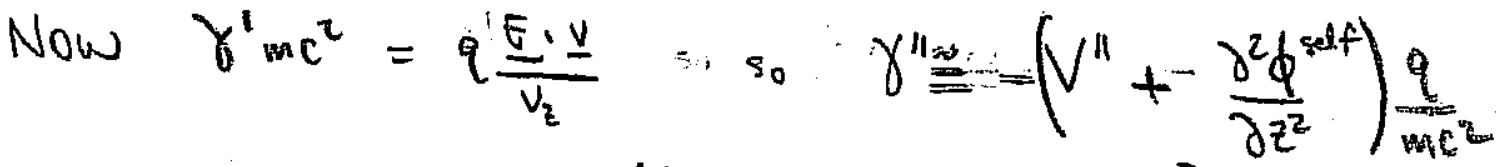

Calculating $\frac{q}{\gamma_{m \beta^{2}} c^{2}}\left[\frac{V^{\prime \prime}}{2} r+E_{r}^{\text {self }}-v_{2} B_{\theta}^{\text {self }}\right]$ :

$$
\begin{aligned}
& \nabla^{2} \phi^{\text {self }}=\frac{-\rho}{\varepsilon_{0}} \Rightarrow \frac{1}{r} \frac{\partial}{\partial r}\left(r \frac{\partial \phi}{\partial r}\right)=\frac{-f}{\varepsilon_{0}}-\frac{\partial^{2} \phi^{s+1}}{\partial t^{2}} \\
& \Rightarrow \frac{\partial}{\partial r}\left(r \frac{\partial \phi}{\partial v}\right)=\frac{-r \rho(r)}{\varepsilon_{0}}-\frac{r^{2} d^{2} \varphi^{l f}}{\partial z^{2}} \\
& r \frac{\partial \phi}{\partial r}=-\frac{1}{2 \pi \varepsilon_{0}} \int_{0}^{r} 2 \pi r(r) d r-\frac{r^{2}}{2} \frac{\partial^{2} \phi}{\partial z^{2}} \\
& =-\frac{1}{2 \pi \varepsilon_{0}} \lambda(r)-\frac{r^{2}}{2} \frac{\partial^{2} \phi_{s e l f}}{\partial z^{2}} \\
& \Rightarrow \quad E_{r}^{\text {self }} \doteq \frac{\lambda(r)}{2 \pi \varepsilon_{0} r}+\frac{r}{2} \frac{\partial^{2} e^{\operatorname{self}}}{\partial z^{2}} \\
& \nabla \times \underline{B}=\mu_{0} J \Rightarrow 2 \pi r B_{\theta}=\mu_{0} \int_{0}^{r} 2 \pi r J_{i}(r) d r=\mu_{0} v_{z} \lambda(r) \\
& B_{\theta}^{\text {self }}=\frac{\mu_{0} V_{z} \lambda(r)}{\varepsilon_{\pi} r}=\frac{V_{z}}{c^{2}} \frac{\lambda(r)}{2 \pi \varepsilon_{0} r}
\end{aligned}
$$

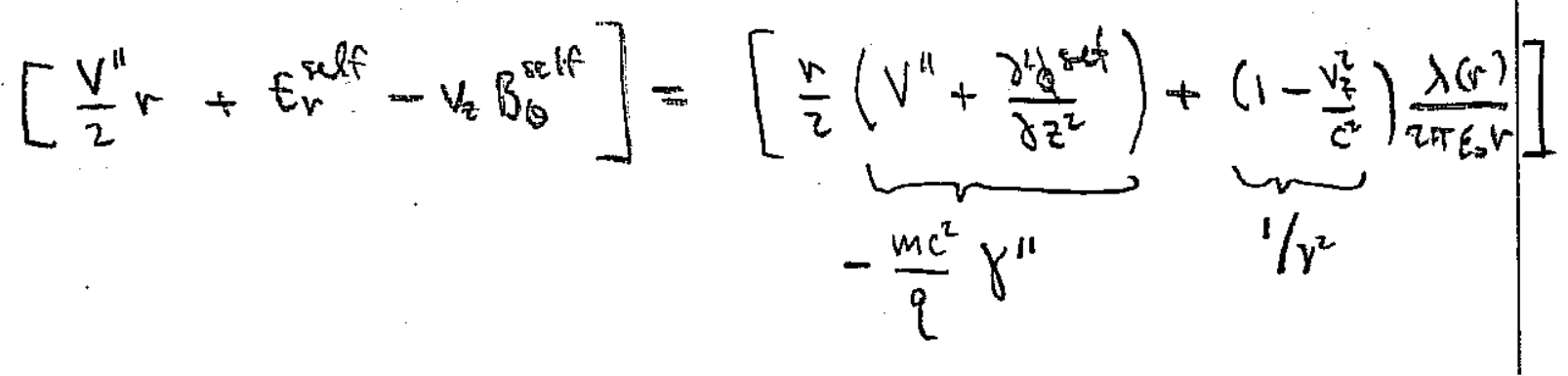

35 


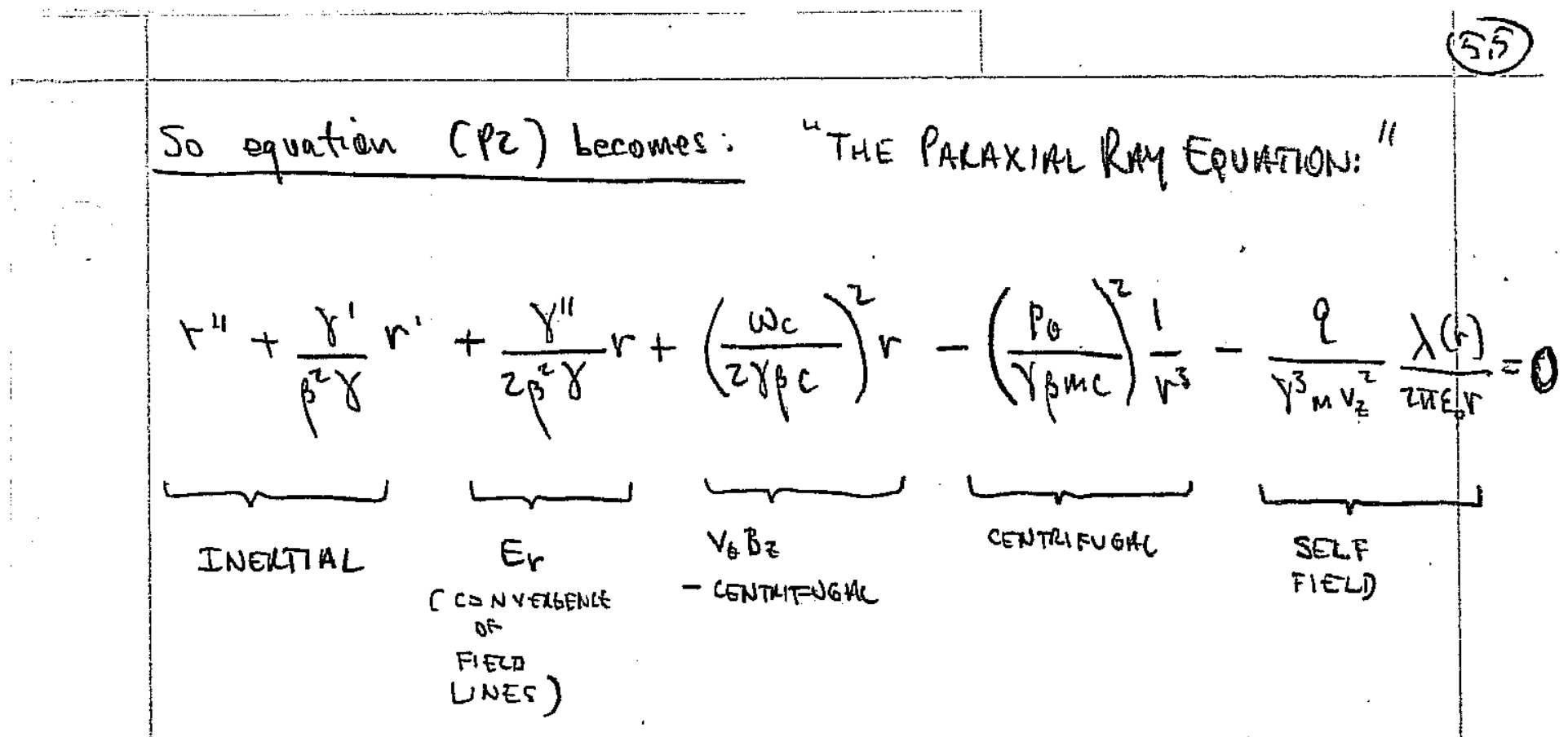

ㄹivis

36 
5. BASNOASAD

Moment EQuations

(b)

Vlasev eqtu: $\frac{\partial f}{\partial s}+x^{\prime} \frac{\partial f}{\partial x}+x^{\prime \prime} \frac{\partial f}{\partial x^{\prime}}+y^{\prime} \frac{\partial f}{\partial y}+y^{\prime \prime} \frac{\partial f}{\partial y^{\prime}}=0$

Let $g=g\left(x, x^{\prime}, y, y^{\prime}\right) ; \quad N=\iiint \int f d x d x^{\prime} d y d y^{\prime}$

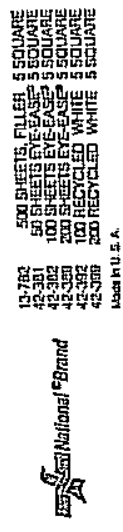

MULTIRLY VLAOU equation by $g \$ \frac{1}{N} \iiint \int d x d x^{\prime} d y^{\prime} d y^{\prime}$

$$
\int d x d x^{\prime} d y d y\left[g \frac{\partial f}{\partial s}+g x^{\prime} \frac{\partial f}{\partial x}+g x^{\prime \prime} \frac{\partial f}{\partial \vec{k}^{\prime}}+g y^{\prime} \frac{\partial f}{\partial y}+g y^{\prime \prime} \frac{\partial f}{\partial y^{\prime}}\right]=0
$$

$$
\begin{aligned}
& \text { INTEGLAF DY INATS }
\end{aligned}
$$

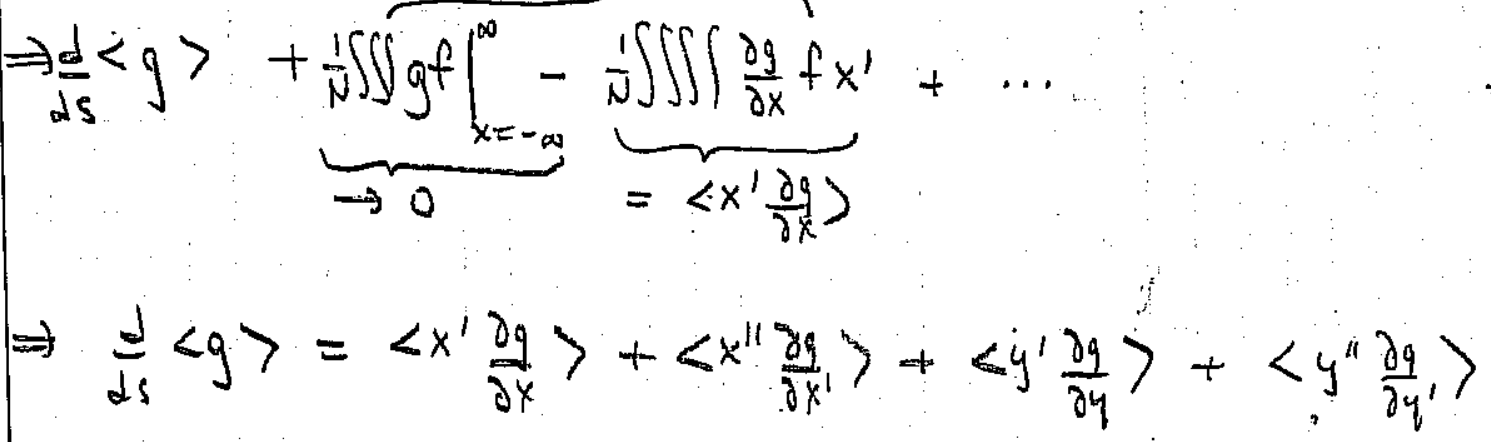

$$
\begin{aligned}
& \text { But } \frac{d g}{d s}=\frac{\partial g}{\partial x} \dot{x}^{\prime}+\frac{\partial g}{\partial x^{\prime}} x^{\prime \prime}+\frac{\partial g}{\partial y} y^{\prime}+\frac{\partial g}{\partial y^{\prime}} y^{\prime \prime} \\
& \Rightarrow \frac{d}{d i}\langle g\rangle=\left\langle g^{\prime}\right\rangle \\
& =0
\end{aligned}
$$

So $\frac{d}{\sqrt{5}}\left\langle x^{2}\right\rangle=2\left\langle x x^{\prime}\right\rangle$

$$
\begin{aligned}
& \frac{d}{d s}\left\langle x^{\prime 2}\right\rangle=2\left\langle x^{\prime} x^{\prime \prime}\right\rangle \quad \text { off } \ldots \\
& \frac{d}{d s}\left\langle x x^{\prime}\right\rangle=\left\langle x^{\prime \prime}\right\rangle+\left\langle x^{\prime c}\right\rangle
\end{aligned}
$$

37 


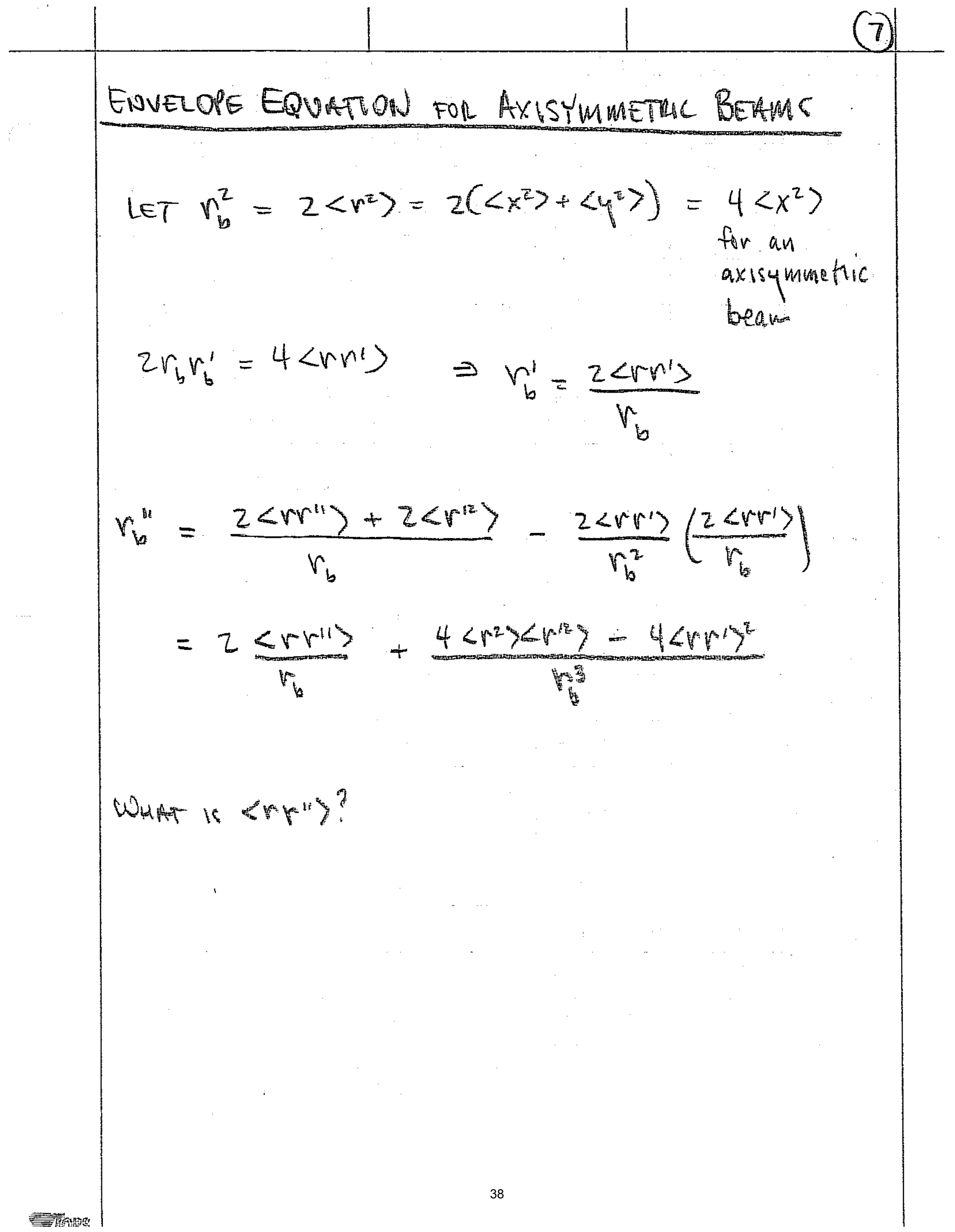




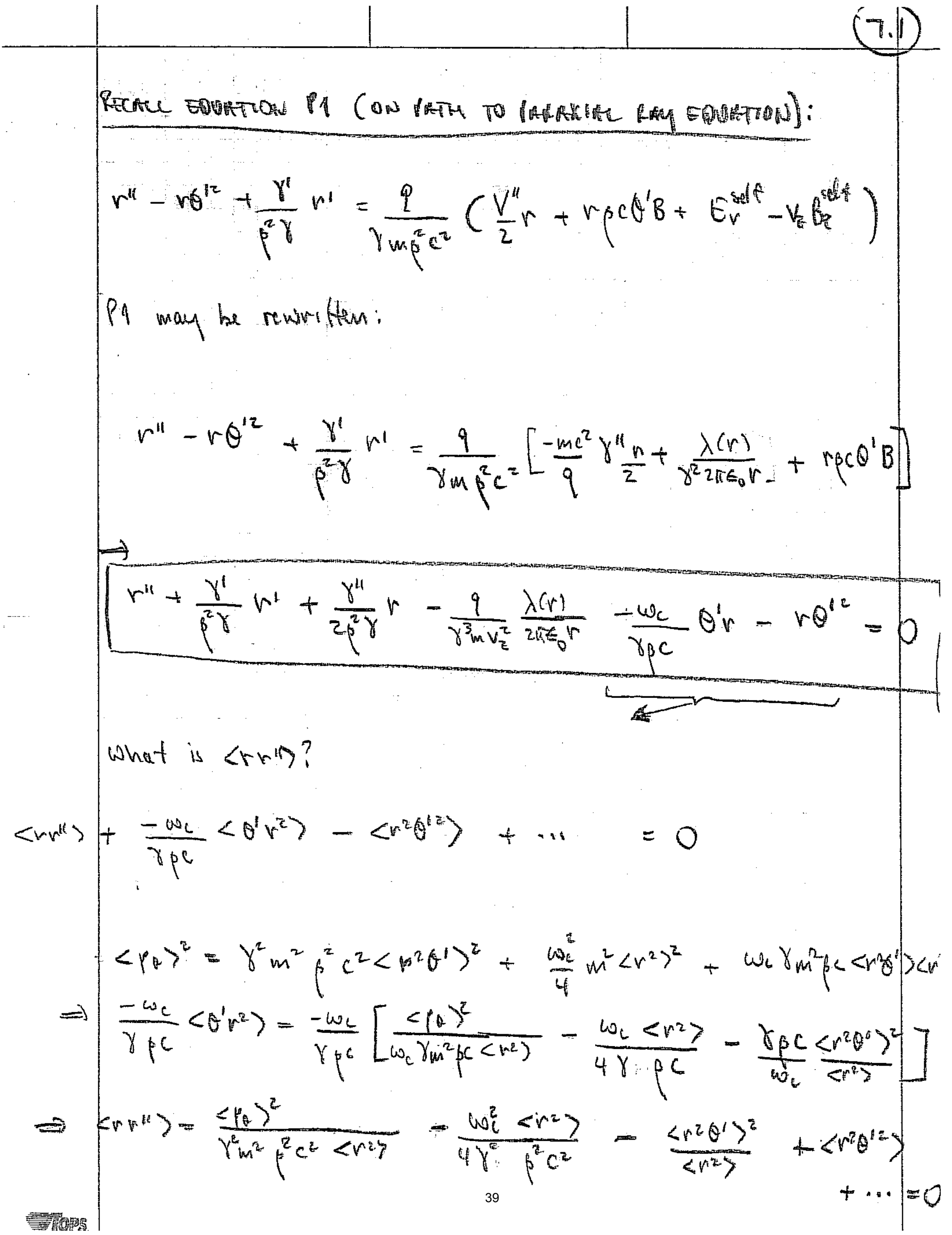




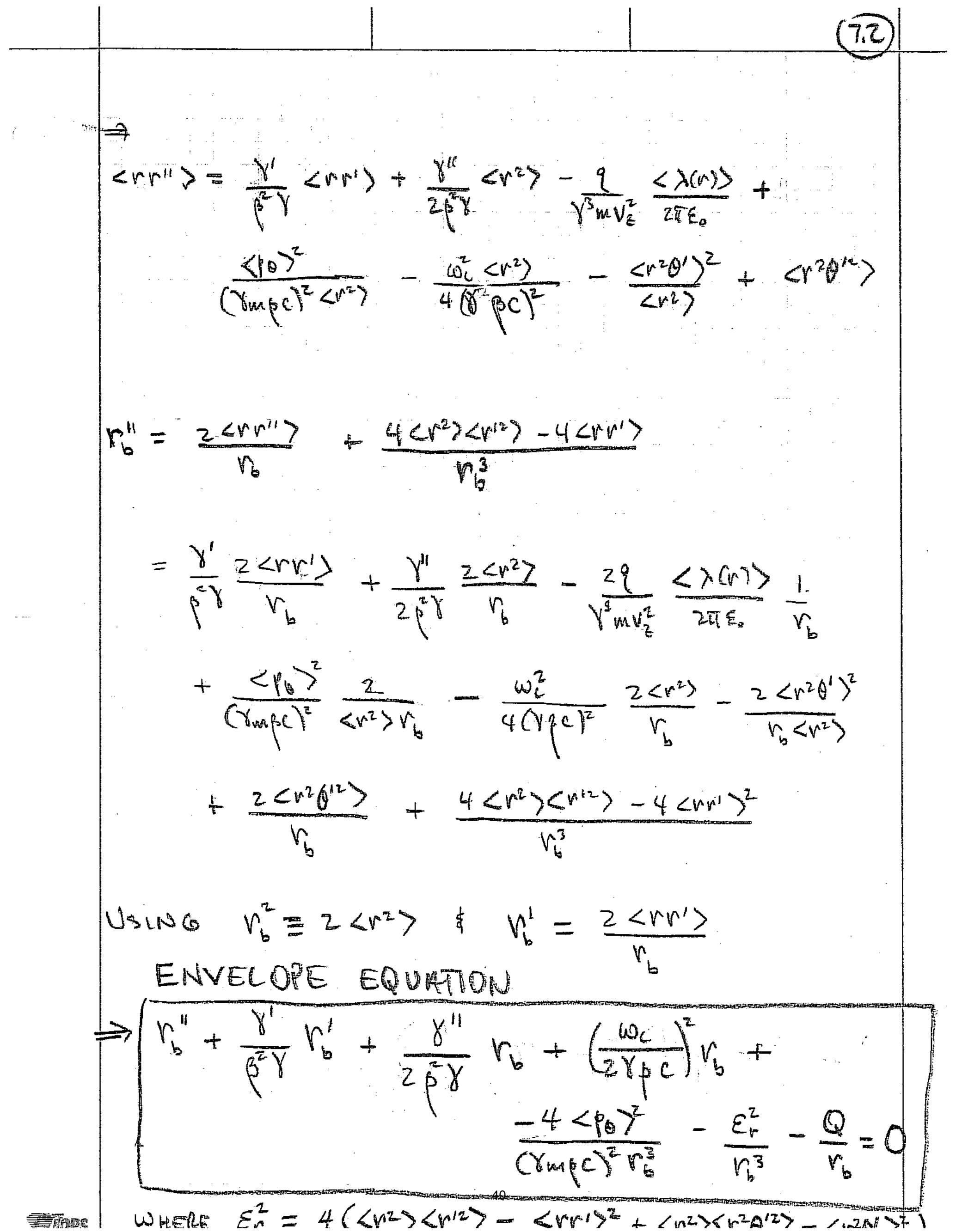




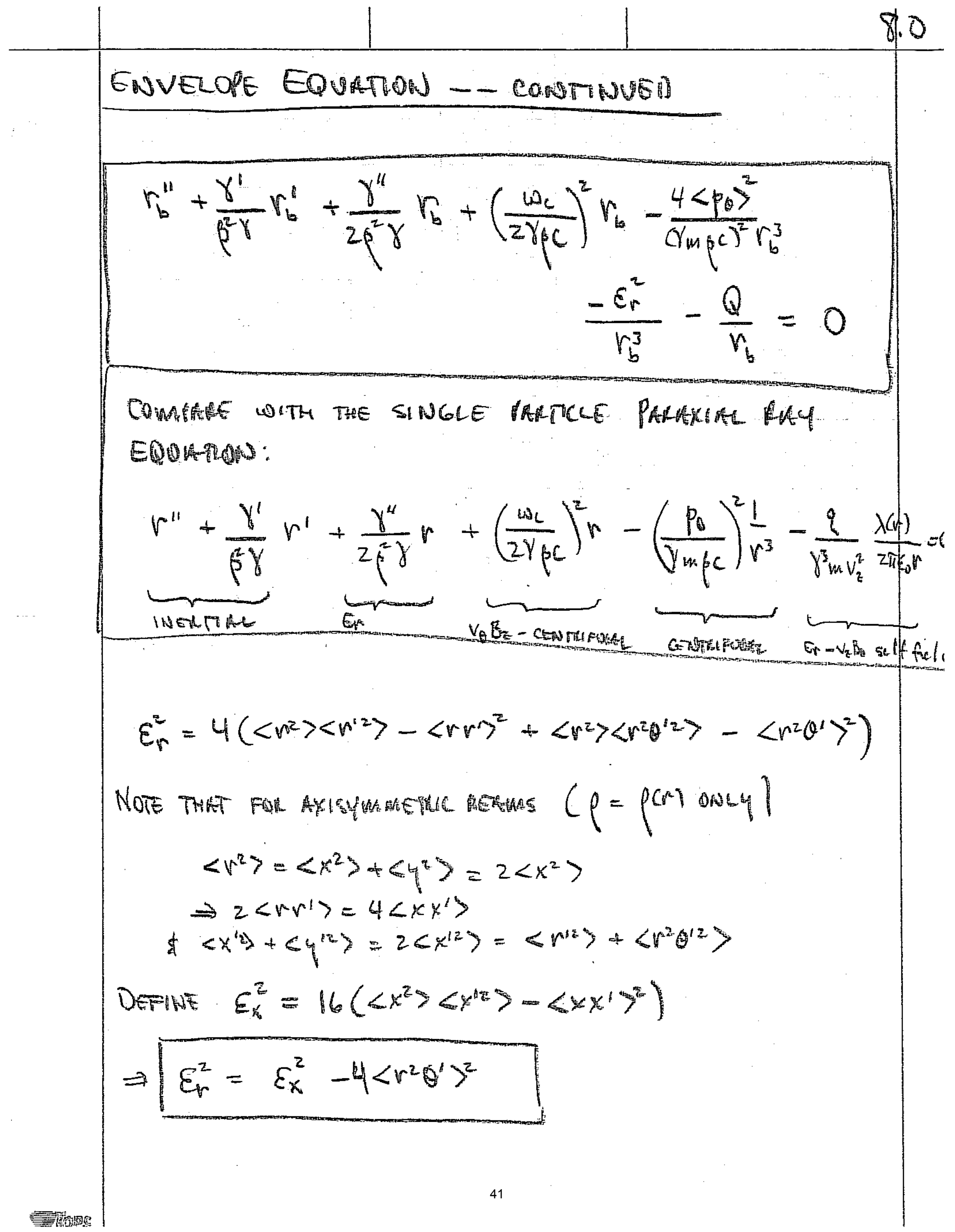




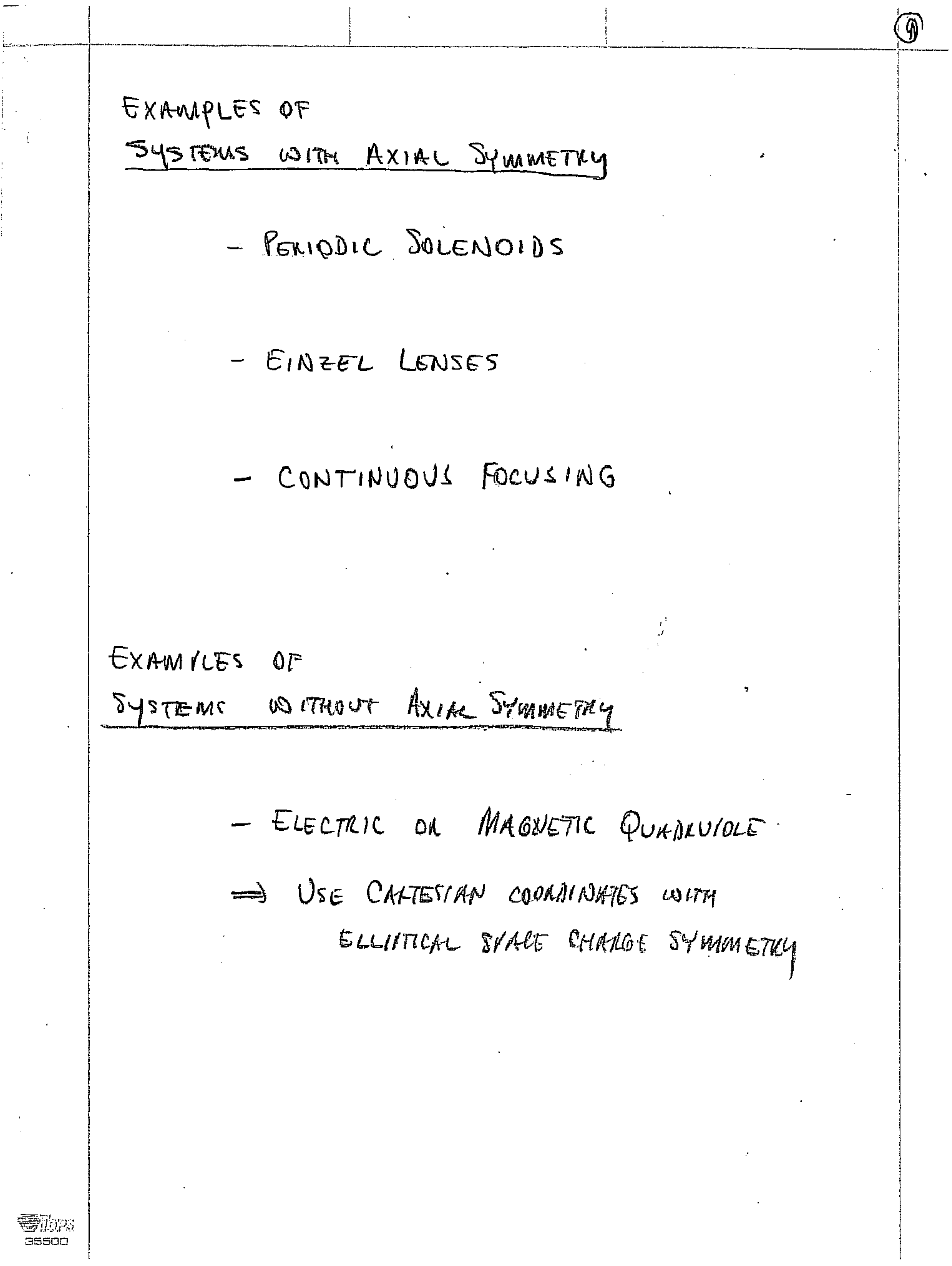




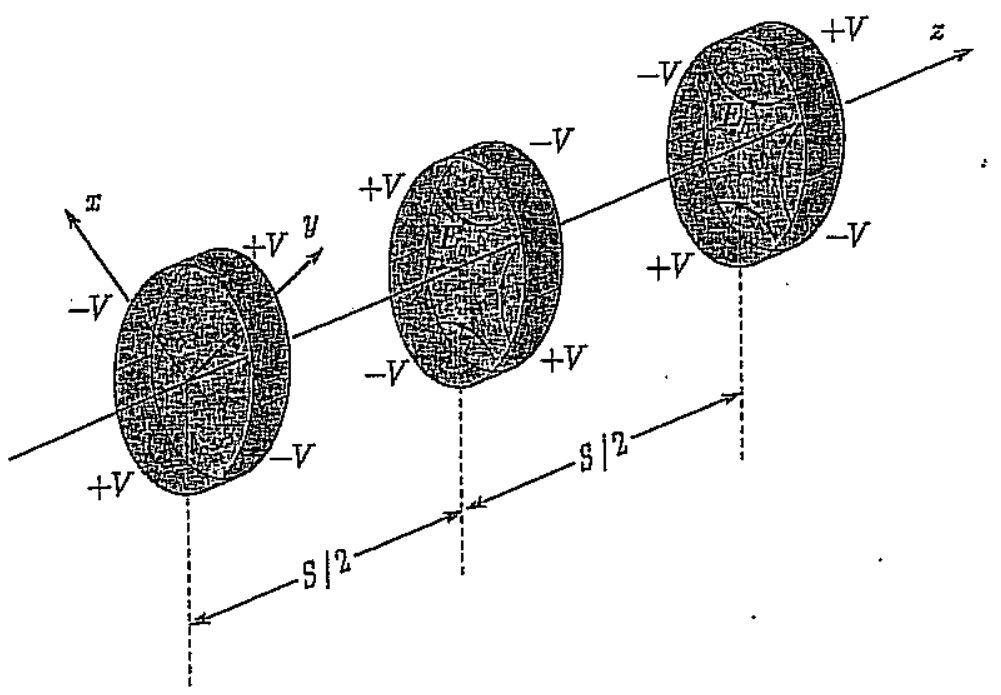

Figure 3.3. Schematic of conductor configuration with applied voltages producing an alternating-gradient quadrupole electric field with axial periodicity length $S$.

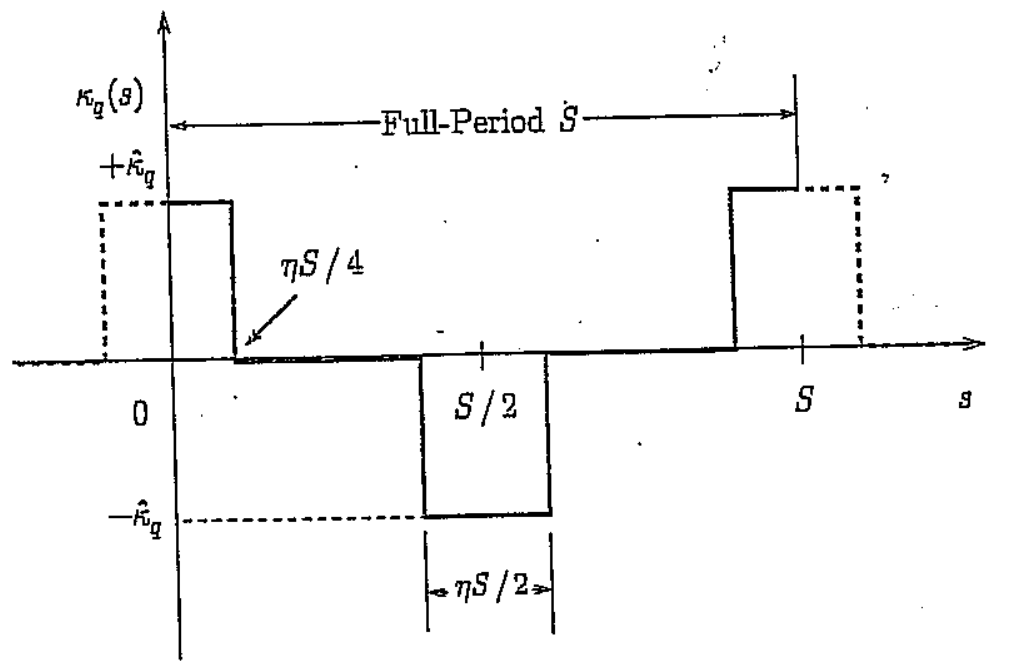

Figure 3.7. Alternating step-function model of a periodic quadrupole lattice with filling factor $\eta$ for the lens elements. The figure shows a plot of the quadrupole coupling coefficient $\kappa_{q}(s)$ versus $s$ for one full period $(S)$ of the lattice. Such a configuration is often called a FODO transport lattice (acronym for focusing-offdefocusing-off). 
figure tham

Davidison a 4 in, zanz.

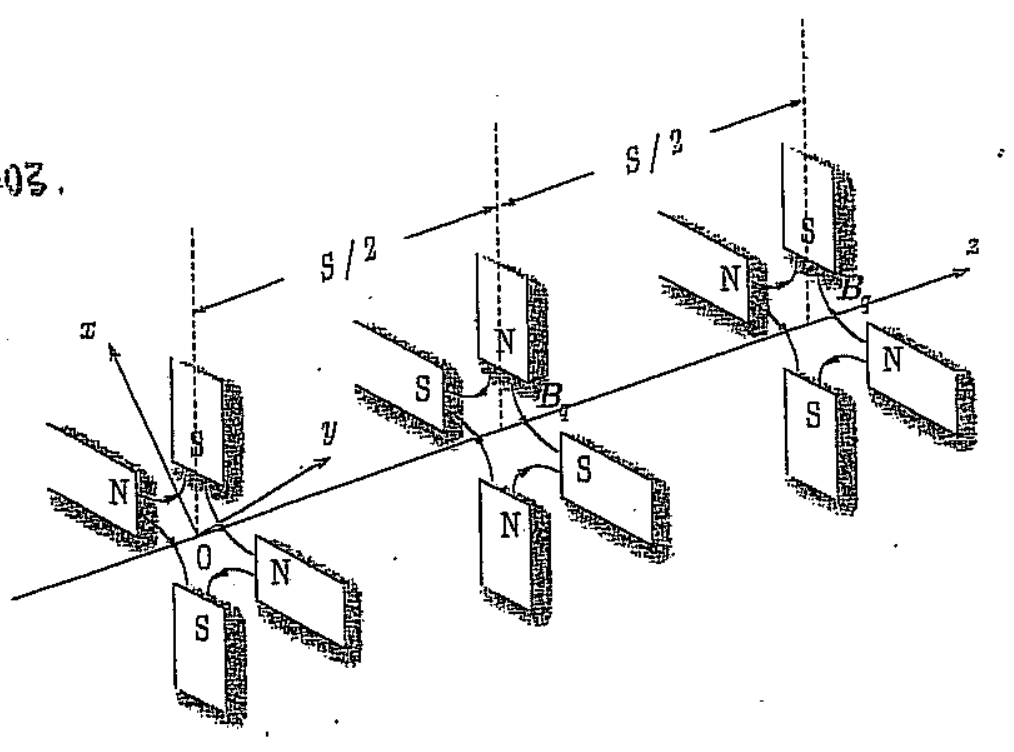

Figure 3.1. Schematic of magnet sets producing an alternatinggradient quadrupole field with axial periodicity length $S$. 


\section{OJ. BARNAEND}

(B)

$2 \equiv$ BEAM OPTICS AND FOCUSING SYSTEMS WITHOUT SPACE CH

From

Reiser, p.llz

$$
\begin{aligned}
& E_{x}=-E^{\prime} x \\
& E_{y}=E^{\prime} y
\end{aligned}
$$

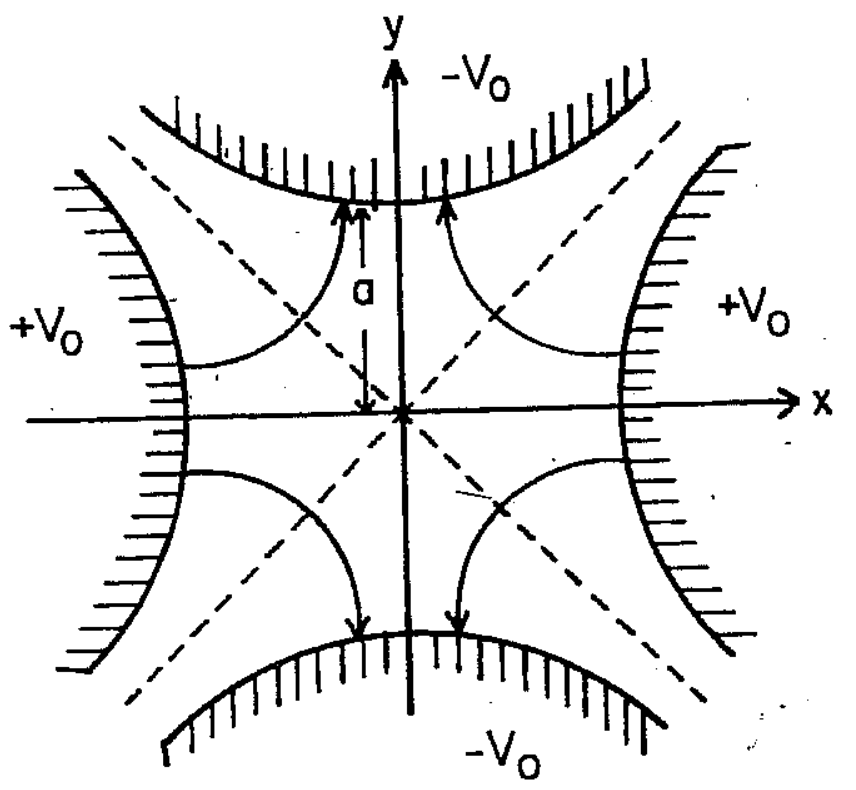

$$
\begin{aligned}
& F_{x}=-q E^{\prime} x \\
& F_{y}=q E^{\prime} y
\end{aligned}
$$

Electrostatic Quás

Figure 3.15. Electrodes and force lines in an electrostatic quadrupole.

$$
\begin{array}{lll}
B_{x}=B^{\prime} y & F_{x}=-q \cdot v_{z} B^{\prime} \cdot x \\
B_{y}=B^{\prime} x & \text { y } & F_{y}=q V_{x} B^{\prime} y
\end{array}
$$

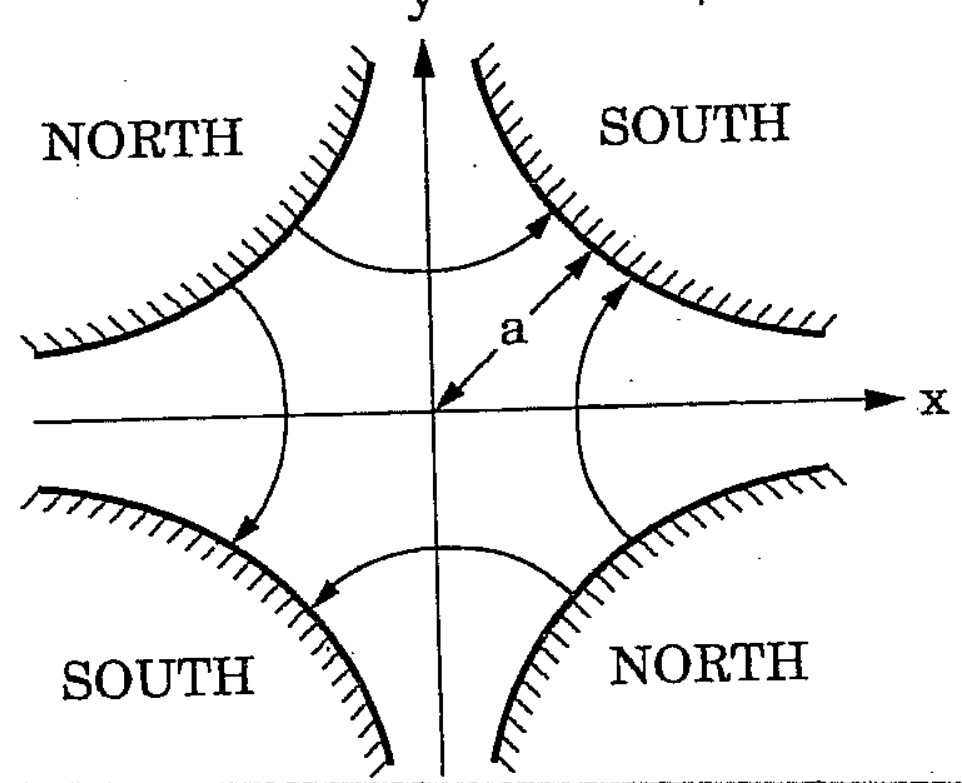

MAGNETIC

QuRes 
J.DAdNatio

Quadnutole Focusing

(14)

Now, relax radial symmetly:

For $\nabla \cdot E=0$ or $\nabla \times B=0$

Exyano field in chlinpuical "multroles":

$$
\begin{aligned}
& E_{r,} B_{r}=\sum_{n=1}^{\infty} f_{n} r^{n-1} \cos (n \theta)
\end{aligned}
$$

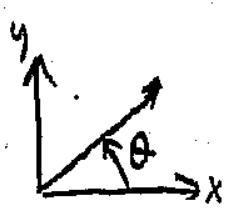

$$
\begin{aligned}
& E_{\theta}, B_{\theta}=\sum_{n=1}^{\infty}-f_{n} r^{n-1} \sin (n \theta) \\
& E_{x}=E_{r} \cos \theta-E_{\theta} \sin \theta \\
& \dot{E}_{y}=E_{r} \sin \theta+E_{\theta} \cos \theta \\
& n=1 \Rightarrow \text { dipole }\left\{\begin{array}{l}
E_{n}=f_{1} \cos \theta \\
E_{\theta}=f_{1} \sin \theta
\end{array} \Rightarrow \begin{array}{l}
E_{x}=f_{1} \\
E_{y}=0
\end{array}\right. \\
& n=2 \Rightarrow \text { quadrupule }\left\{\begin{array}{l}
E_{r}=f_{2} r \cos 2 \theta \\
E_{\theta}=f_{2} r \sin 2 \theta
\end{array} \Rightarrow \begin{array}{l}
E_{x}=f_{2} x \\
E_{y}=-f_{2} y
\end{array}\right.
\end{aligned}
$$

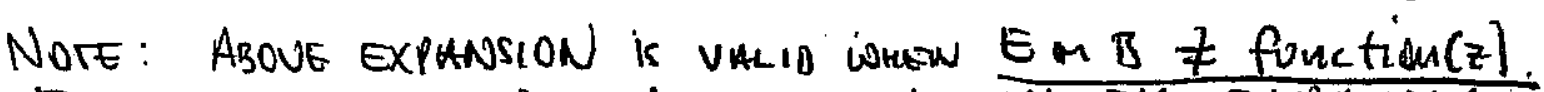

FOR MAGAETS OF FINITE AXISL EXTENT, FQL EACH FUNOAMESNTAL

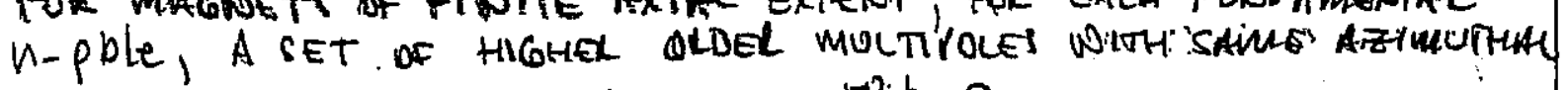

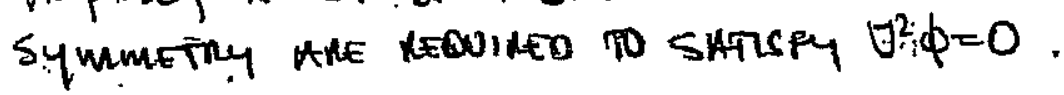

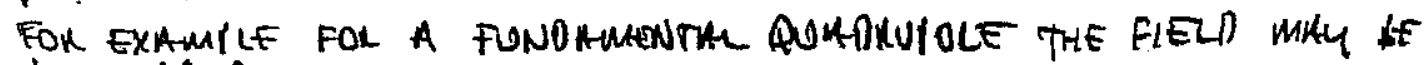
EXIANAER:

$$
\begin{aligned}
& E_{r}=\sum_{\nu=0}^{\infty} f_{2,}(z)[1+\nu] r^{1+2 v} \cos [z \theta] \\
& E_{v}=\sum_{\nu=0}^{\infty}-f_{2, \nu}(z) r^{1+2 \nu} \sin [2 \theta] . \\
& E_{z}=\sum_{\nu=0}^{\infty} \frac{1}{2} \frac{d f_{\nu \nu} \nu}{d z} r^{2+2 \nu} \cos 2 \theta
\end{aligned}
$$

wisth $f_{z p^{\nu}+1}(z)=\frac{-1}{4(v+1)(v+3)} \frac{d^{2} f_{z v}(z)}{d z^{2}}$

SEE LUND, S.M. (Cia96)

Foll ERAtulle. HIF uore gh- 
(16)

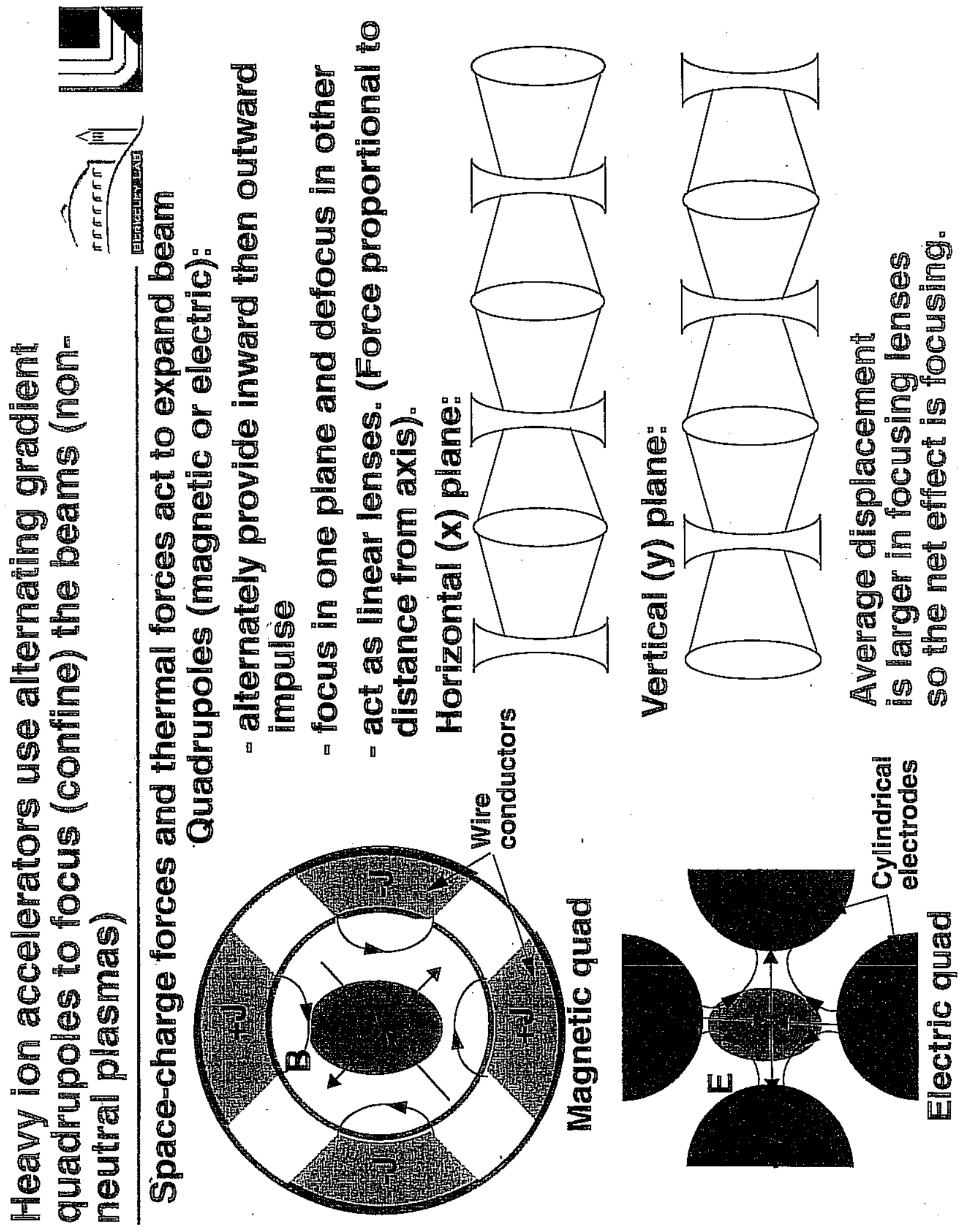




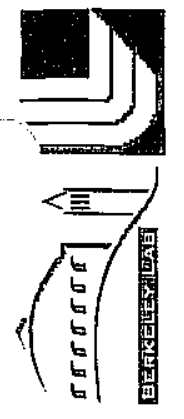

造)

(1)

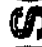

8

2

(

?

$\pi$

(1)

응

0

8

U

8

(1)

(1)

()

\%

5

(9)

0

0

(D)
ต

ד

$\sigma 8$

o 5

요

严寻

(0)

10 .

$1 N$

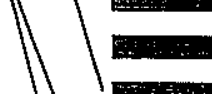

ras

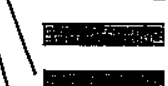

-

-

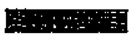

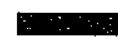

ratis

and..

(1)

8

พั้

Hain

and

逢

nam

(2)

$\Phi$

(5)

.

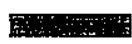

을

$\frac{8}{4}$

뇰

0

I

$\sum^{2}$

3

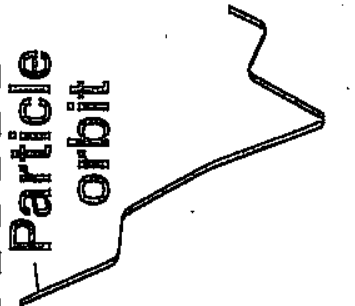

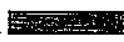

nam

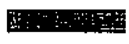

B.....

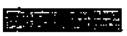

․․

an

s.m.

\&an

$-1$

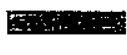

$-$

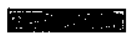

$\therefore$

풀

$+\infty$

표

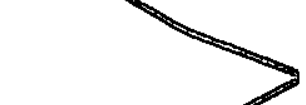

-

팔

D...

-

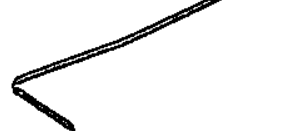

.......

rach

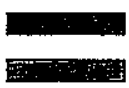

(1):

Fin

ins.

䨍

제

5⿴囗玉

…

Eac

aㅡㅁㅇ

स.

Ban.

-

menc

Hes

nala

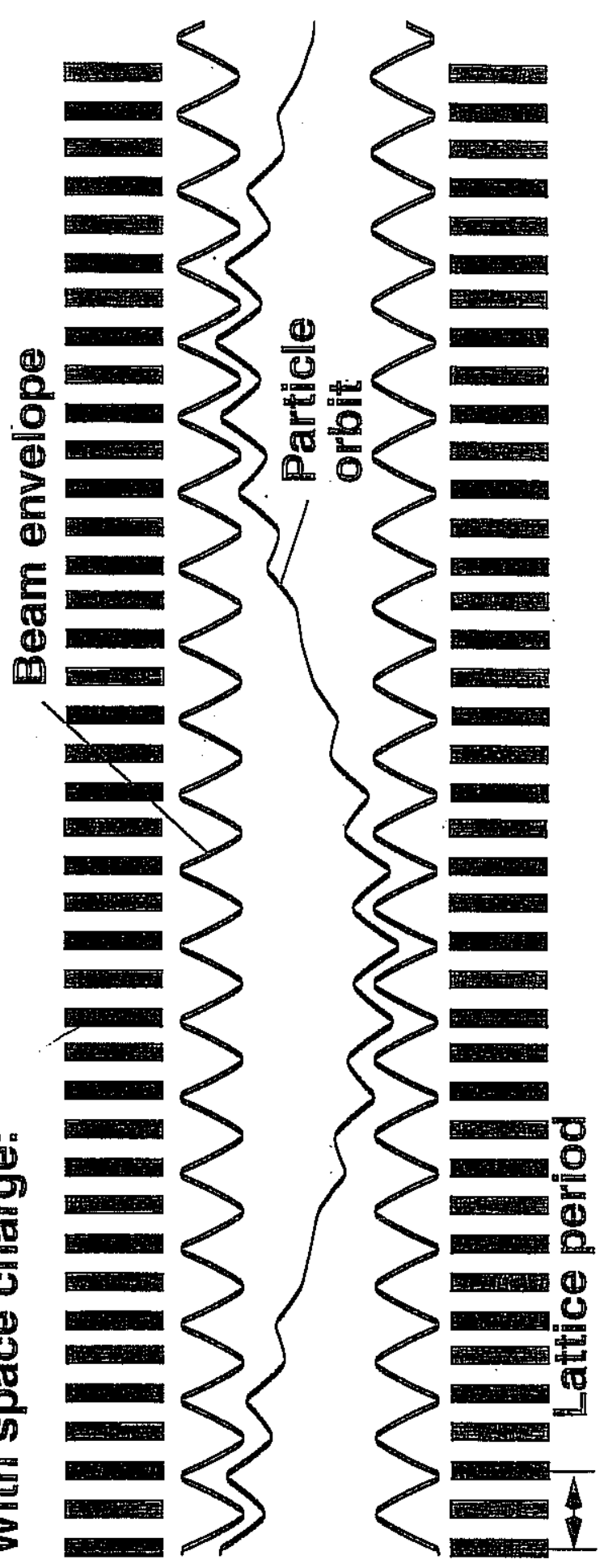




\section{John Barnard Steven Lund \\ USPAS \\ June 2008}

\section{$\underline{\text { Current limits }}$}

A. Axisymmetric

1. Solenoids

2. Einzel lens

B. Quadrupolar

1. Derivation of envelope equations with elliptic symmetry

2. Current limit using fourier transform method 


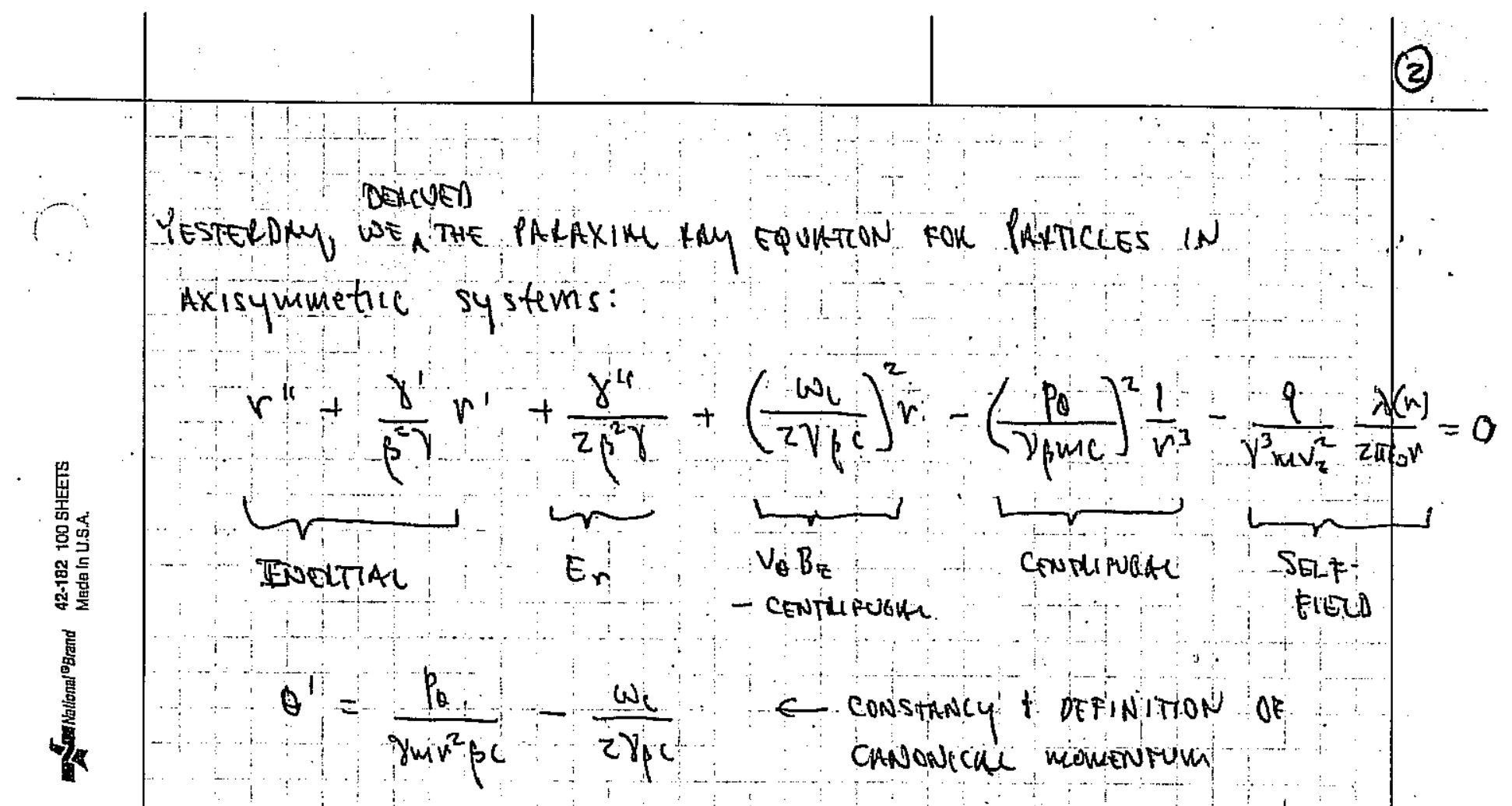

ENUETOL EQUATION BOR AXISYMmEmIC BEAM

$$
\begin{aligned}
& r_{b}^{1+}+\frac{\gamma^{\prime} v_{b}^{\prime}}{\beta^{2} \gamma^{\prime}}+\frac{\gamma^{\prime \prime}}{2 \beta^{2} \gamma} r_{b}+\left(\frac{\omega_{c}}{2 \nu_{j c}}\right)_{b}^{2}-\frac{4\left\langle\gamma_{b}\right\rangle^{2}}{\left(\gamma_{m} c^{2}\right)^{2} r_{b}^{3}}-\frac{\varepsilon_{v}^{2}}{r_{b}^{3}}-\frac{Q}{n_{b}}=0 \\
& \varepsilon_{r}^{2}=4\left(\left\langle r^{2}\right\rangle\left\langle r^{i}\right\rangle-\left\langle r r^{\prime}\right\rangle^{2}+\left\langle r^{2}\right\rangle\left\langle r^{2} \theta^{2}\right\rangle-\left\langle r^{2} \theta^{2}\right)^{2}\right)
\end{aligned}
$$




\section{Periodic Einzel lenses}

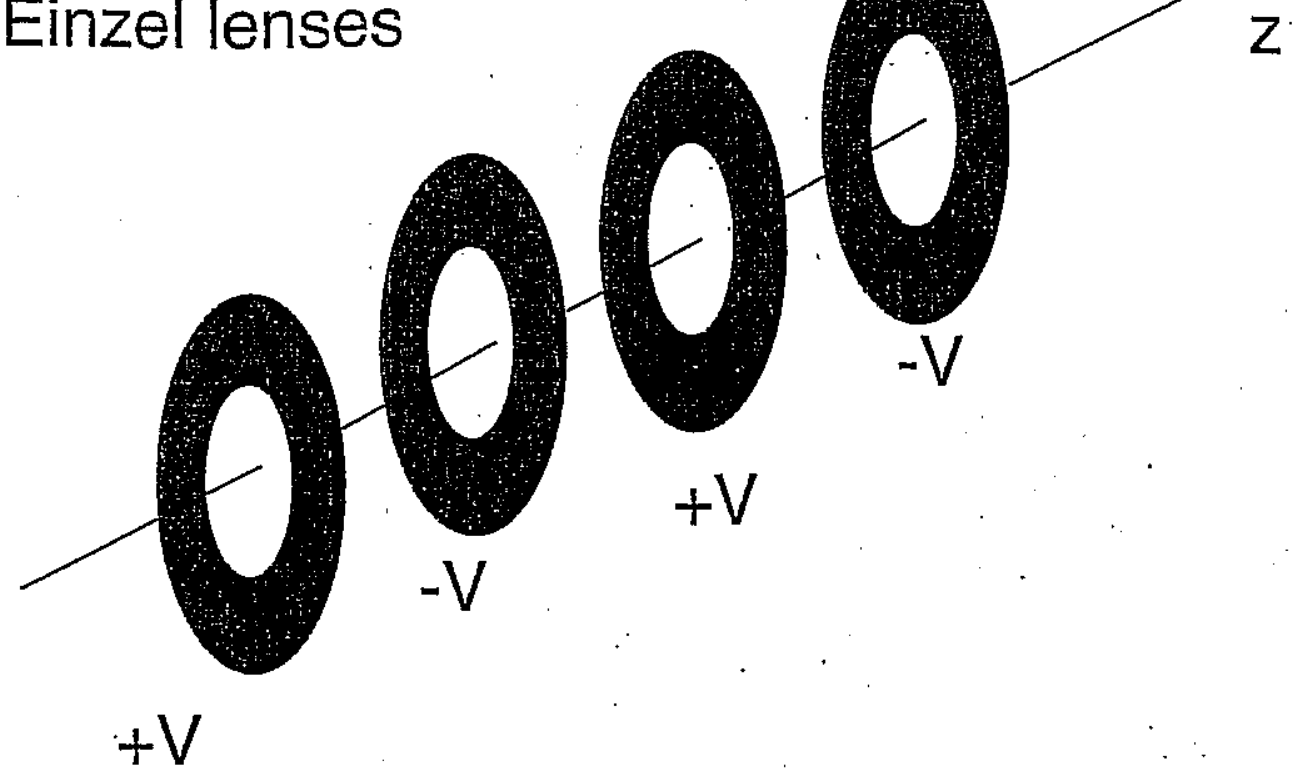

\section{Pellodic Solendidos}

(a)<smiles></smiles>

$s$

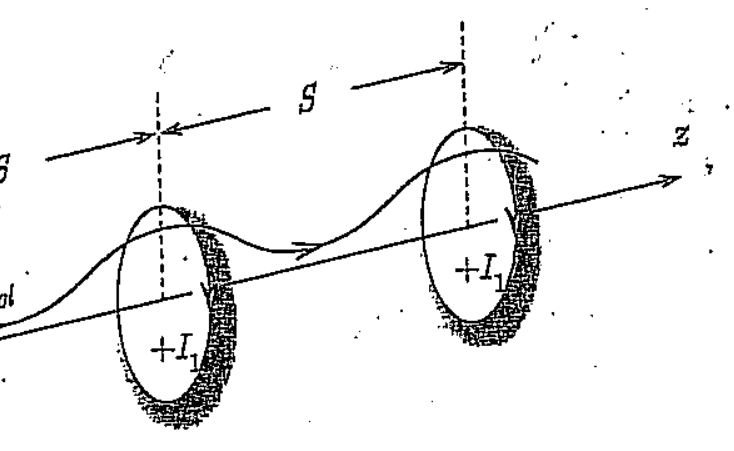

(b)

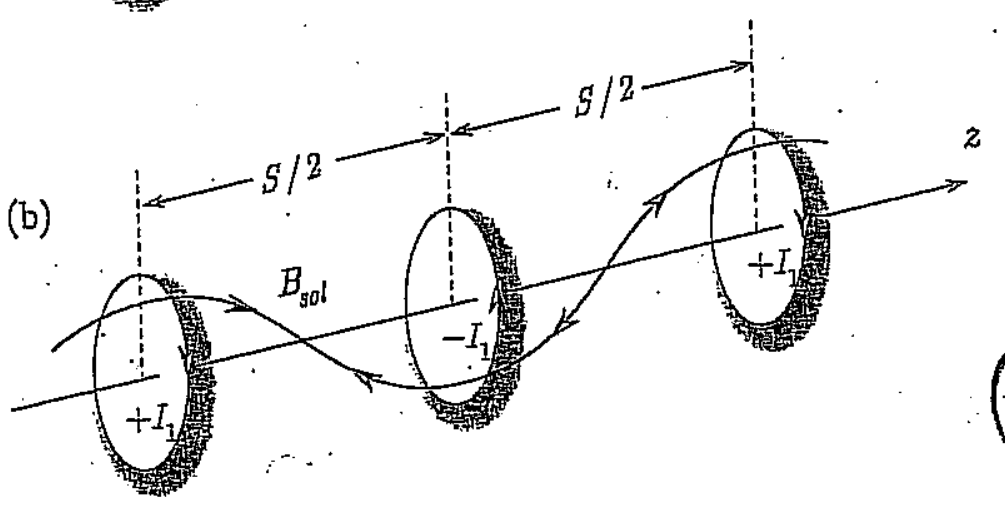

Figure 3.2. Schematic of magnet sets producing a periodic focusing solenoidal field with axial periodicity length $S$. In Fig. 3.2 (a), successive coils are apaced by $S$ and have the same current polarity $+I_{1},+I_{1}, \cdots$. In Fig. 3.2 (b), successive coils are spaced by $S / 2$ and have alternating current polarities $+I_{1},-I_{1}, I_{1}, \cdots$.
Tloukt From Davidian H Qin, 2003 ) P. 55 "Physics of INTENSE (HANLGA) Patticar hatum! iv Hlam ENEL" Accerexisuls" 


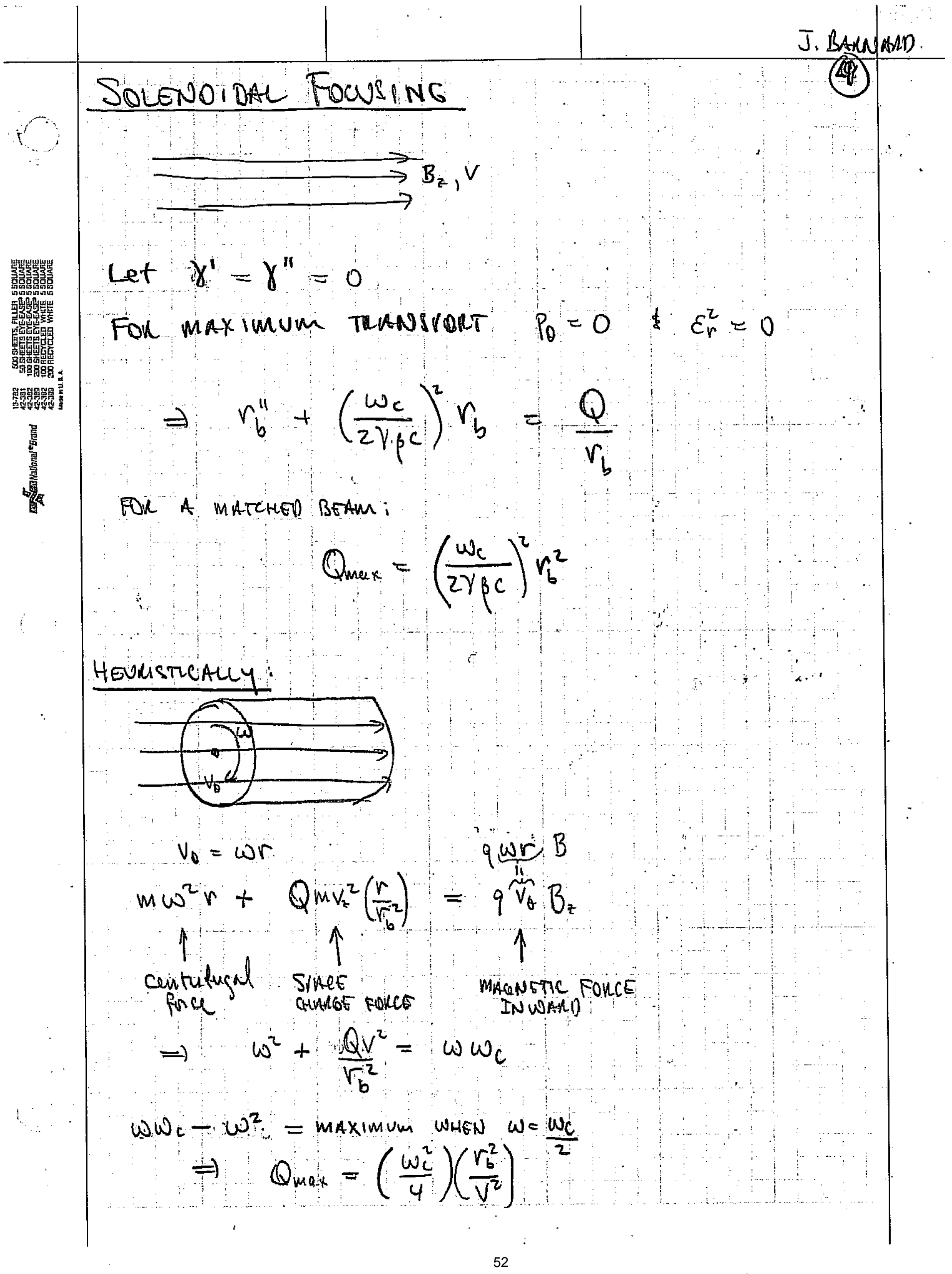




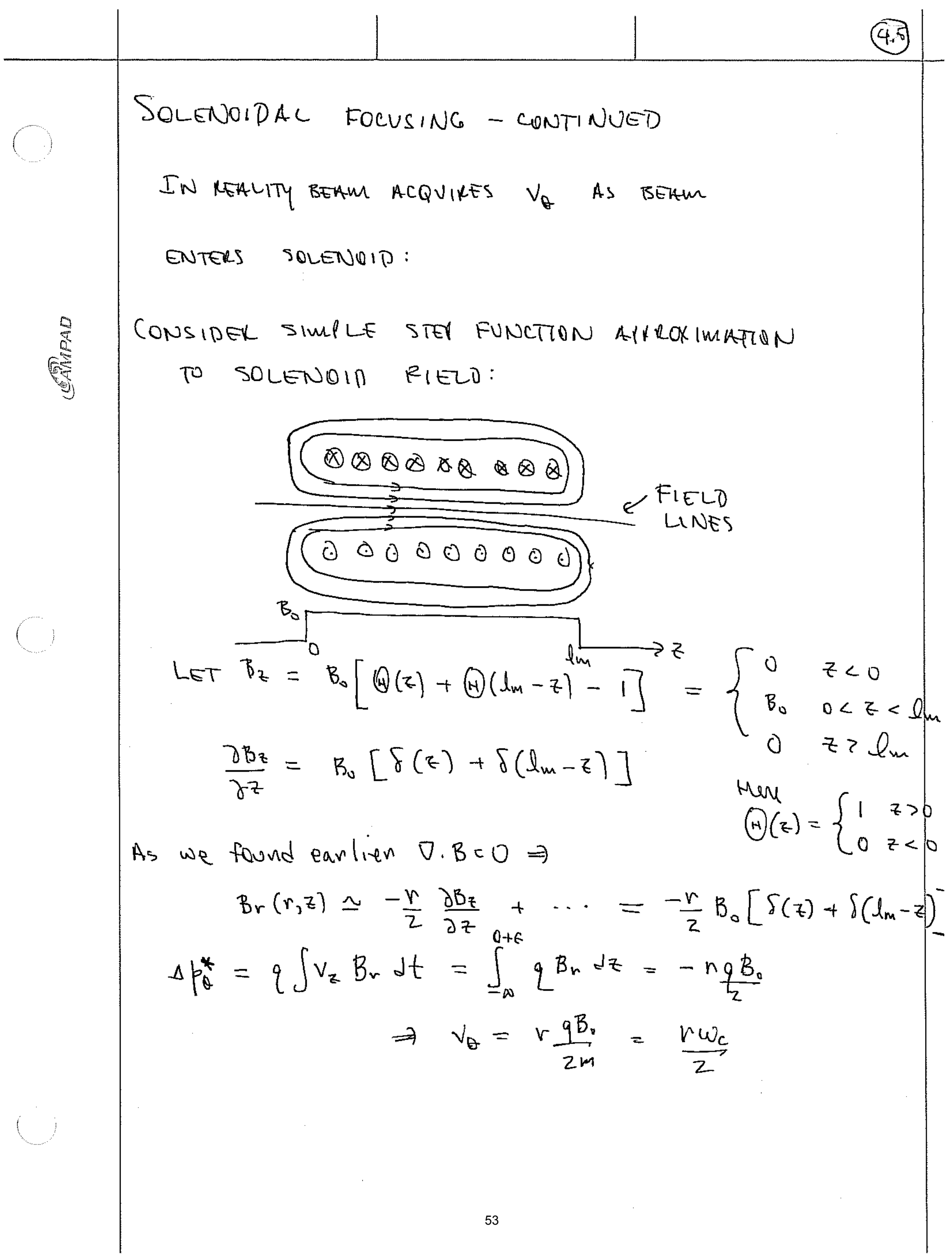


EINZEL LSNS

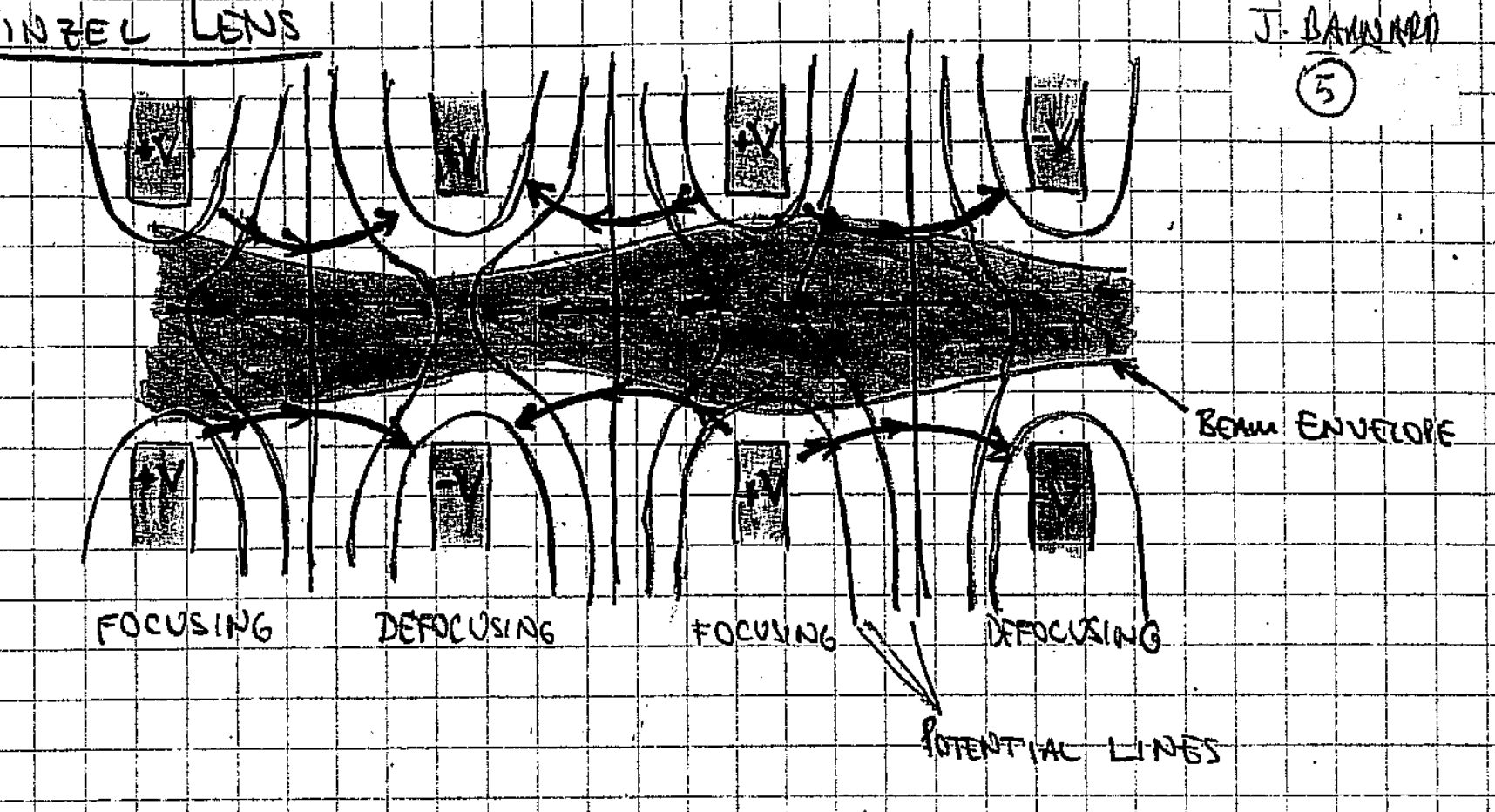

Focusing occuns at large radios than detocusing

$\Rightarrow$ NeT INWANO FORE 


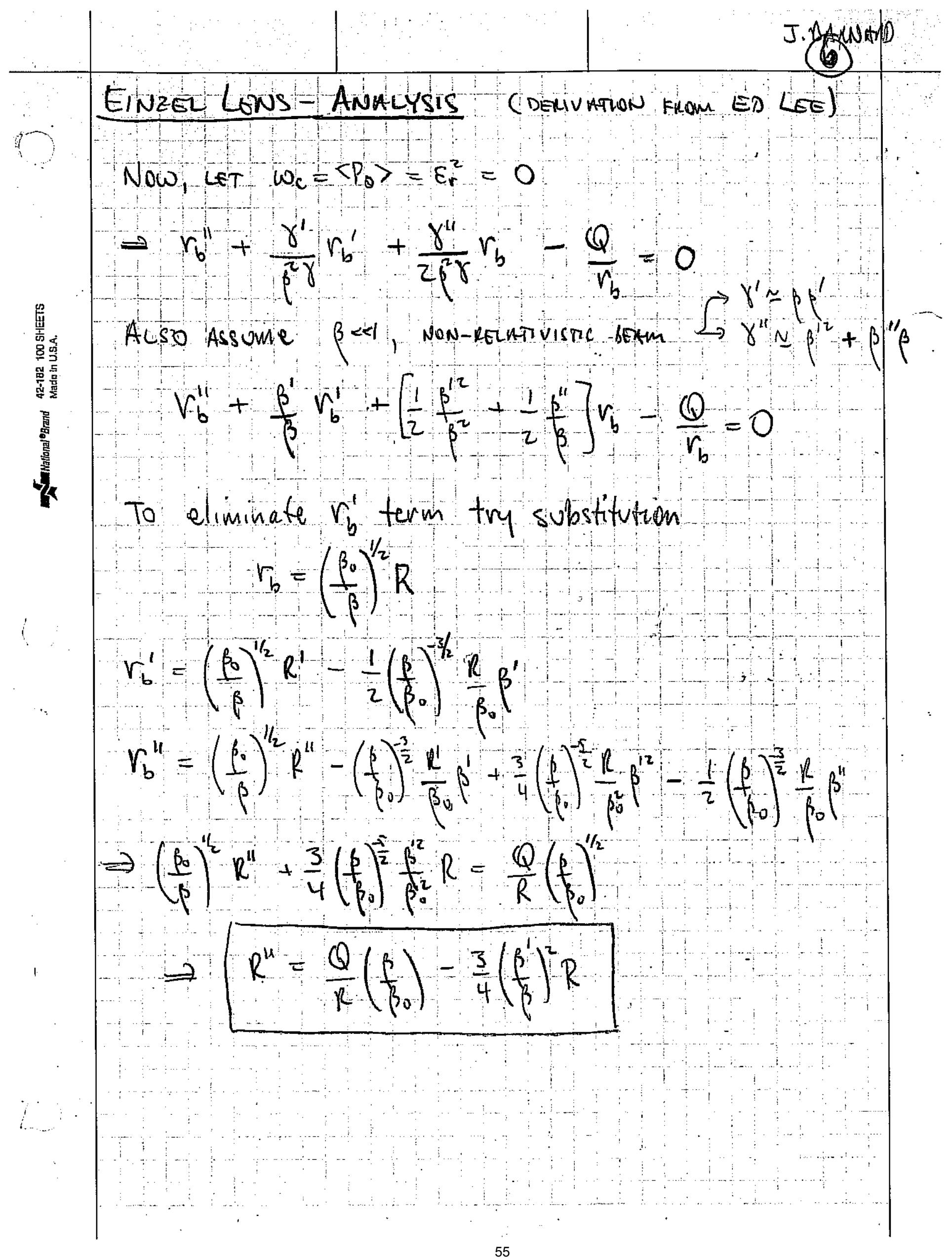




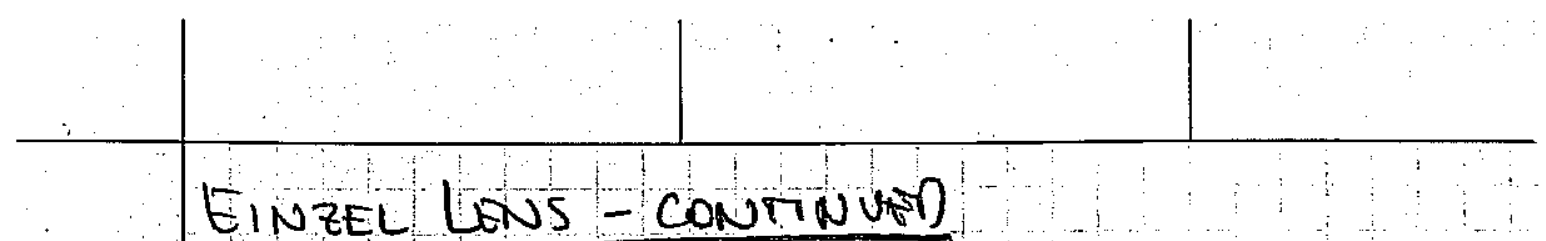

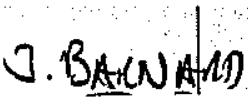

(7)

Mones

LET, $Q=\phi_{0} \cos \left(\frac{\pi z}{L}\right)$

$$
\frac{1}{2} m v^{2}+q \phi=\text { contant }
$$

$$
\begin{array}{r}
\vec{T} v^{2}=v_{0}^{2}+\frac{2 g \phi}{m} \cos \left(\frac{\pi z}{L}\right) \\
v^{\prime}=-\frac{9 \phi_{0}}{m v}\left(\frac{\pi}{L}\right) \sin \left(\frac{\pi z}{L}\right)
\end{array}
$$

If $\left(\frac{2 q \phi_{0}}{m}\right)<v_{0}^{2} \quad\left(\frac{\beta^{\prime}}{\beta}\right)^{2} \simeq\left(\frac{q \phi_{0}}{m v_{0}^{2}}\right)^{2}\left(\frac{\pi}{\tau}\right)^{2} \sin \left(\frac{\pi z}{L}\right)$

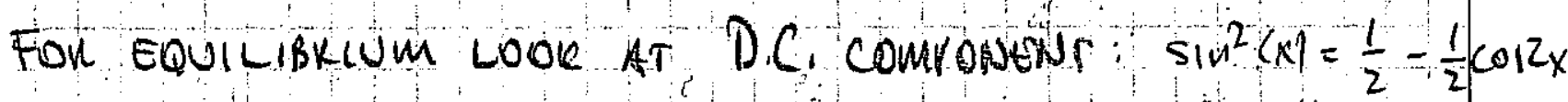

$$
\begin{aligned}
& R R^{l t}=0 \Rightarrow \frac{Q}{\bar{R}}=\frac{3}{4}\left(\frac{\beta^{\prime}}{\beta}\right)^{2} \bar{R} \\
& R E\left(\frac{\beta}{\beta_{0}}\right)^{1 / 2} r_{b} \Rightarrow \bar{R}=r_{b} \\
& \frac{\left(\frac{\beta}{\beta}\right)^{2}}{r^{\prime}}=\frac{1}{2}\left(\frac{9 Q_{0}}{m v_{0}^{2}}\right)^{2}\left(\frac{\pi}{L}\right)^{2} \\
& \Rightarrow Q_{\text {max }}=\frac{3 \pi^{2}}{8}\left(\frac{q Q_{b}}{m v_{0}^{2}}\right)^{2}\left(\frac{r_{b}}{L}\right)^{2}
\end{aligned}
$$


OJ. BANANA

(15.5)

$!$

BEAM OPTICS AND FOCUSING SYSTEMS WITHOUT SPACE CH

From

RaIser, p.112

$$
\begin{aligned}
& E_{x}=-E^{\prime} x \\
& E_{y}=E^{\prime} y
\end{aligned}
$$

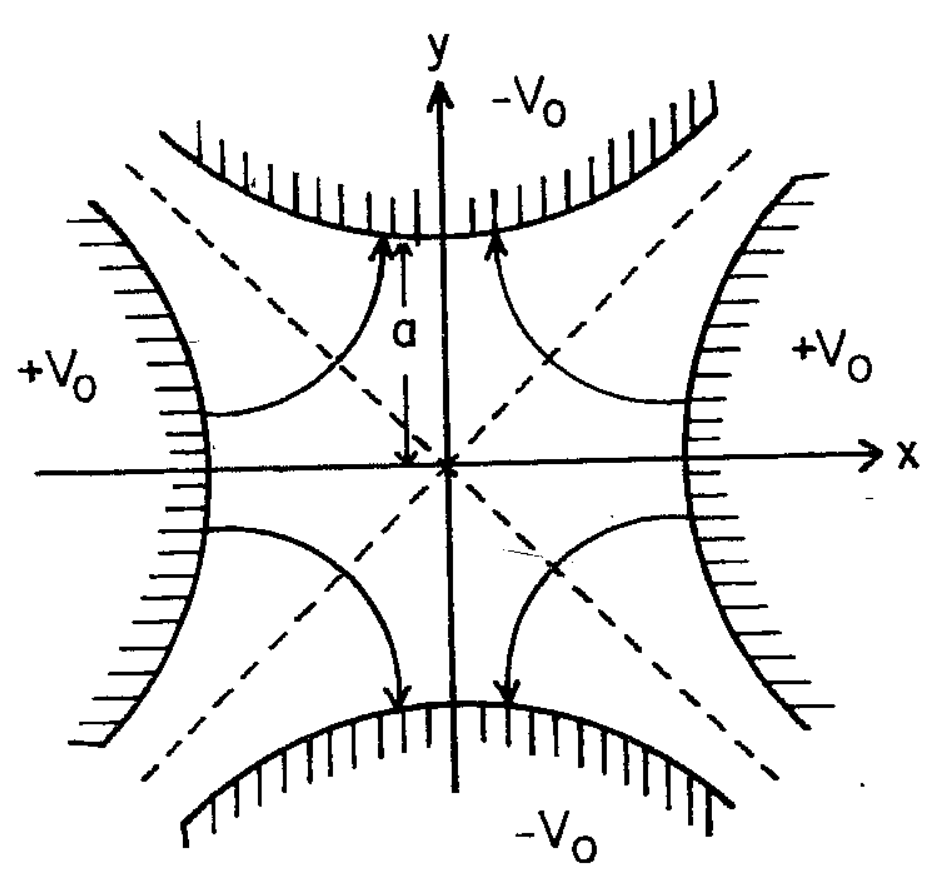

$$
\begin{aligned}
& F_{x}=-q E^{\prime} x \\
& F_{y}=q E^{\prime} y
\end{aligned}
$$

Electrostatic

Quads

Figure 3.15. Electrodes and force lines in an electrostatic quadrupole.

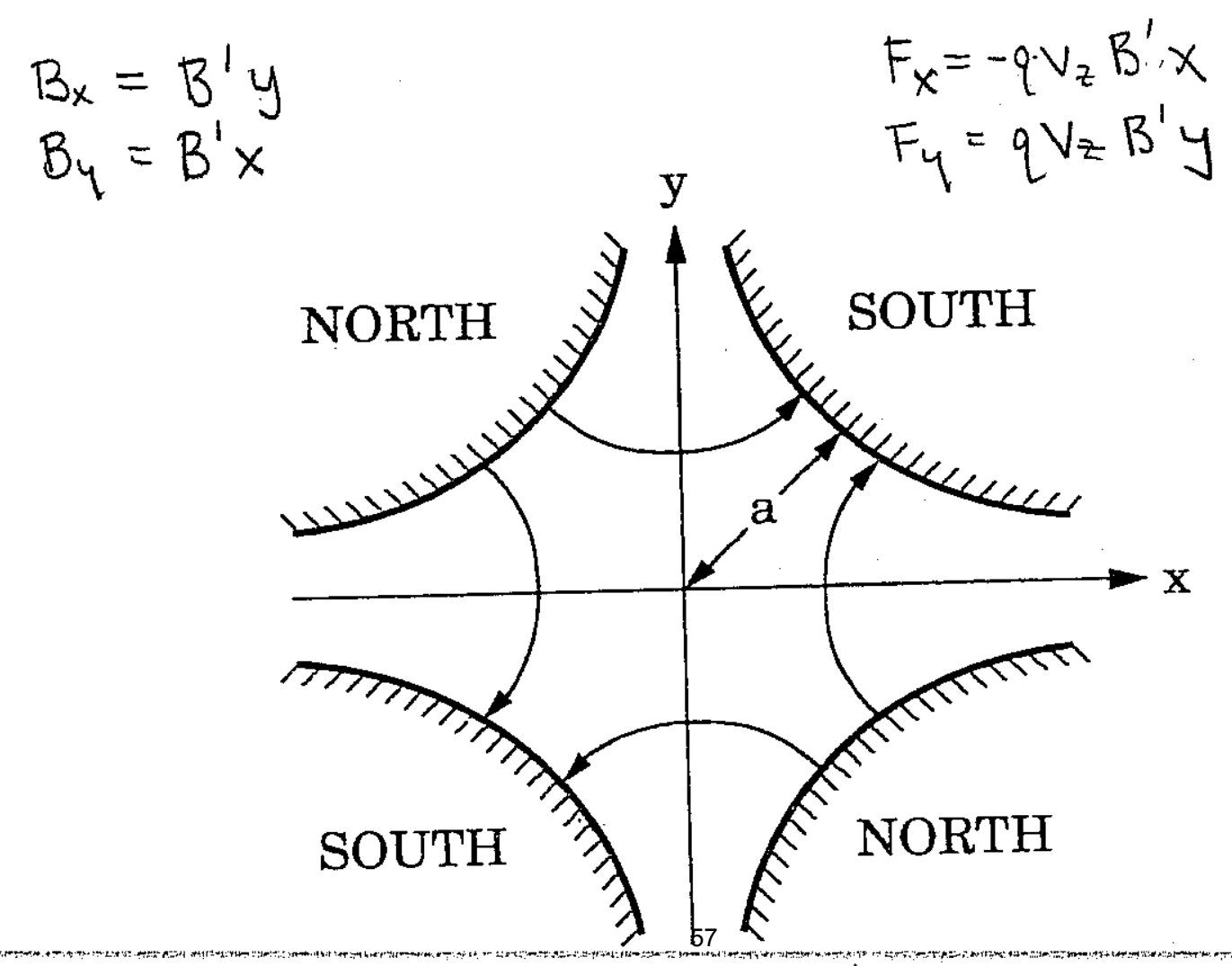

MAGNETS

QuADS 


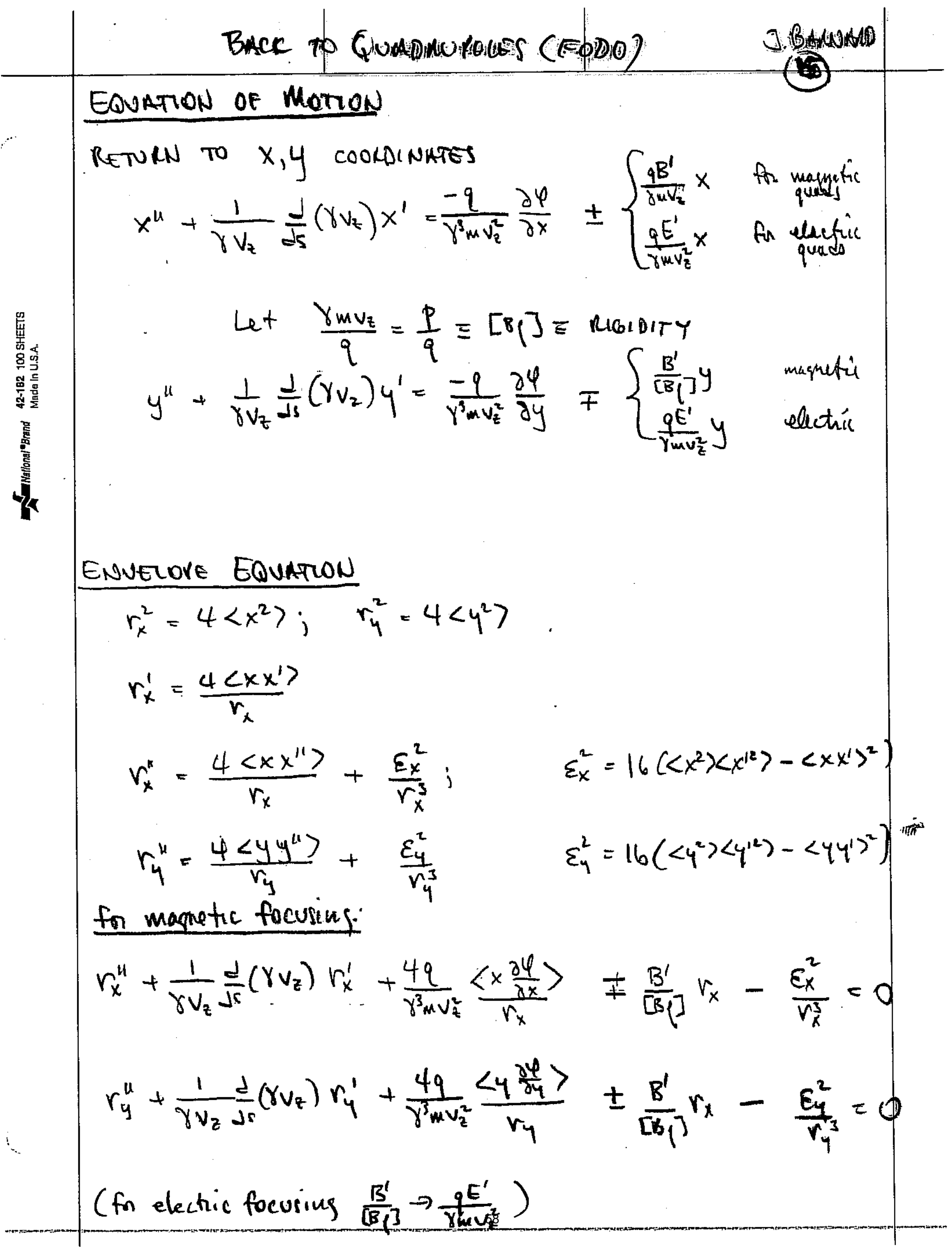




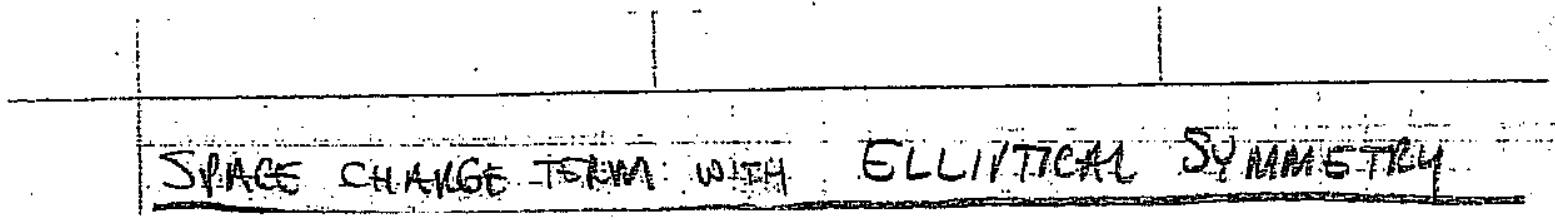
$i^{\prime}$

HW: ELLIMCAL SUWAEERY: $\rho=\rho\left(\frac{x^{2}}{x^{2}}+\frac{y^{2}}{r_{y}^{2}}\right)$

(9)

SPACE CHARG TIMA WIA ELLITTCA SUMHETH

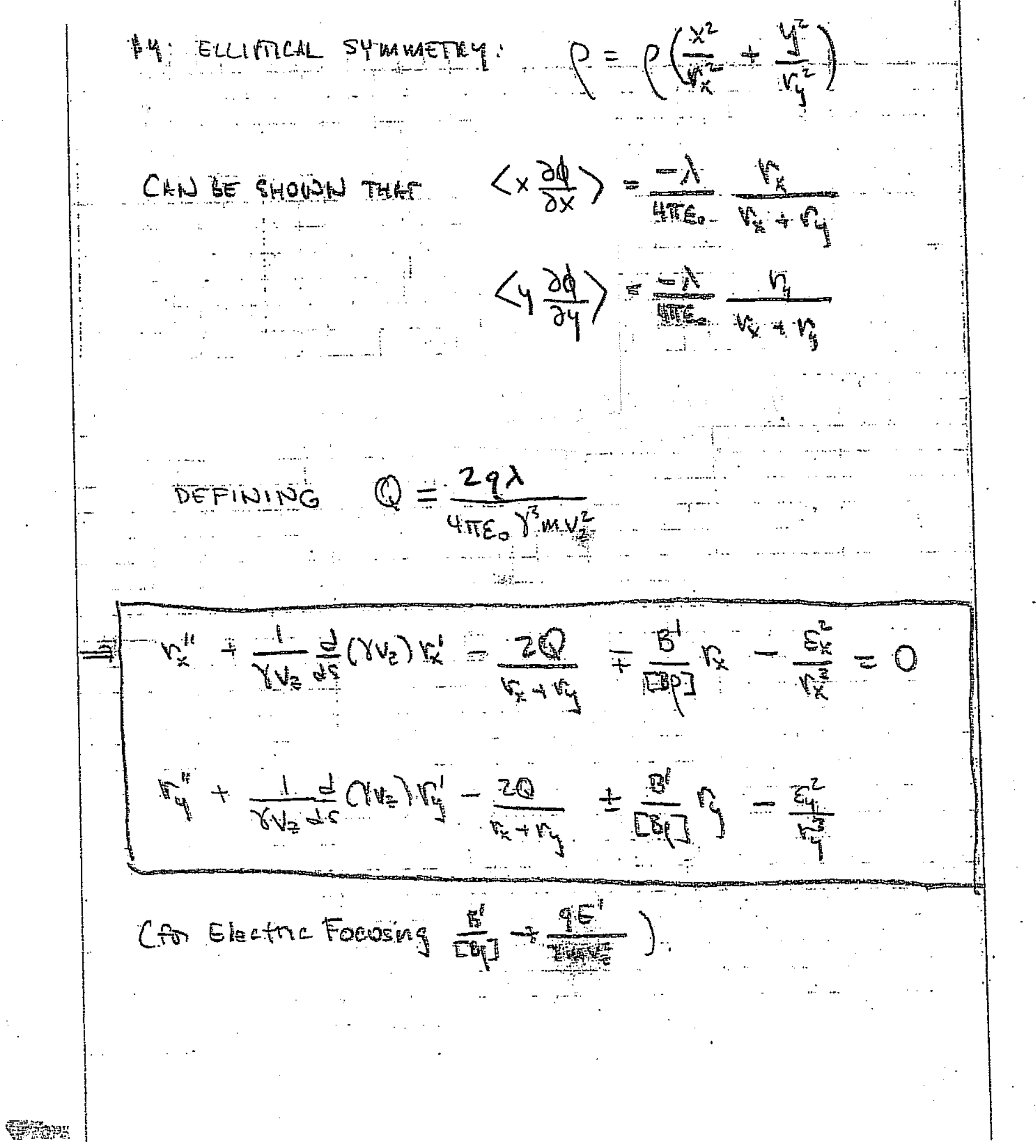

59 


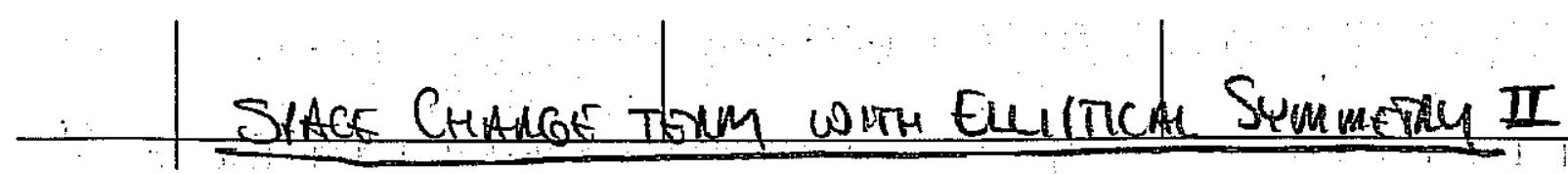

5. BaAnOND

(10)

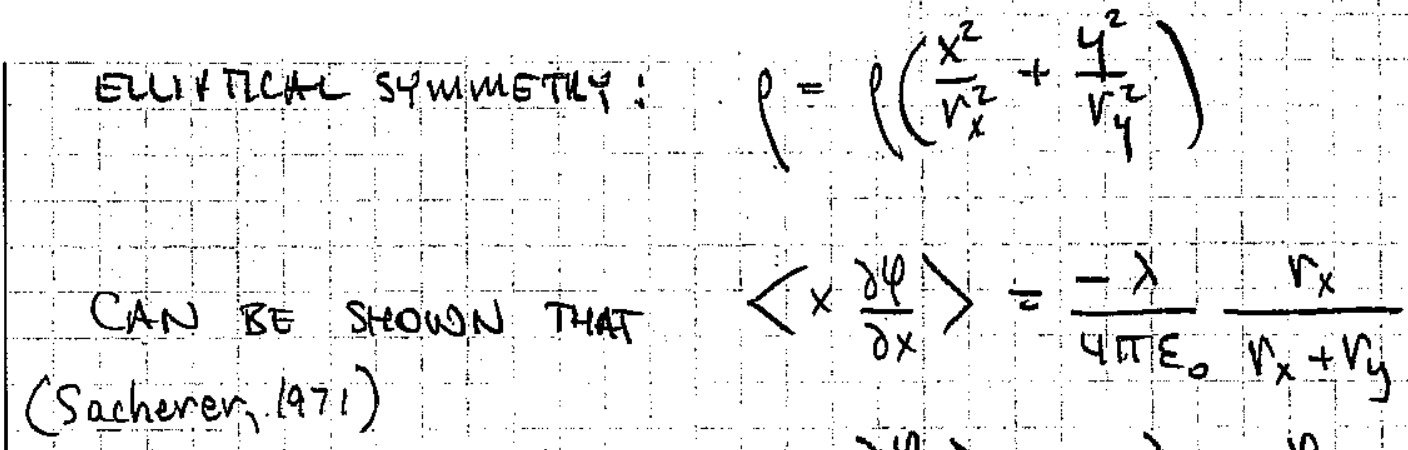

$$
\$ \quad\left\langle y \frac{\partial \varphi}{\partial y}\right\rangle=\frac{-\lambda}{4 \pi \varepsilon_{0}} \frac{r_{y}}{r_{x}+r_{y}}
$$

Outline of kloof:

(from R. Rune)

Let $x=\frac{x^{2}}{r_{x}^{2}+5}+\frac{y^{2}}{r_{y}^{2}+5}$

Define $\quad \eta(x)$ such that $p(x, y)=\left.\frac{d \eta(x)}{d x}\right|_{s=0}=\left.\hat{p}(x)\right|_{s=0}$

$$
\text { So } \rho=\hat{\rho}\left(\frac{x^{2}}{r_{x}^{2}}+\frac{y^{2}}{r_{y}^{2}}\right)=\left.\hat{\rho}(x)\right|_{s=0}
$$

Define $\Psi(x, y)=\frac{-n r_{y}}{4 \varepsilon_{0}} \int_{0}^{\infty} \frac{\eta(x) d s}{\sqrt{r_{x}^{2}+s} \sqrt{r_{y}^{2}+s}}$

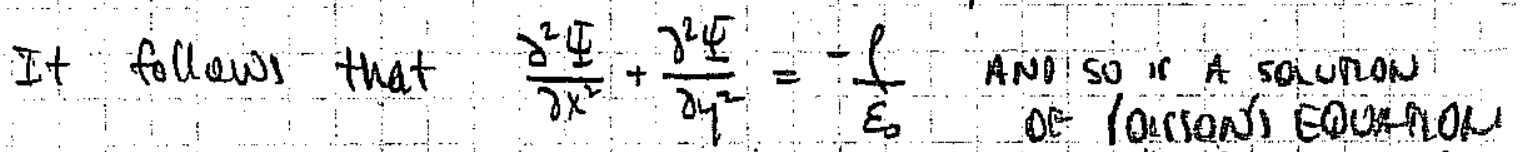
WHAT is $\left.<\times \frac{\partial \psi}{\partial x}\right\rangle$ ? ( since $\Psi \rightarrow 0$ as $x_{14} \rightarrow \infty$ )

$$
\begin{aligned}
& \left\langle x \frac{\partial \Psi}{\partial x}\right\rangle=\frac{-r_{x} r_{4}}{\varphi \lambda \varepsilon_{0}} \int_{-\infty}^{\infty} d_{x}^{\infty} \int_{-\infty}^{\infty} d x \rho(x, y) \int_{0}^{\infty} \frac{\eta \frac{\partial x}{\partial x} d s}{\sqrt{x_{x}^{2}+s} \sqrt{r_{j}^{2}+s}} \\
& \text { where } \lambda=\int_{-\alpha}^{\infty} d_{x} \int_{-N}^{\infty} d y f(x, y)
\end{aligned}
$$

60 


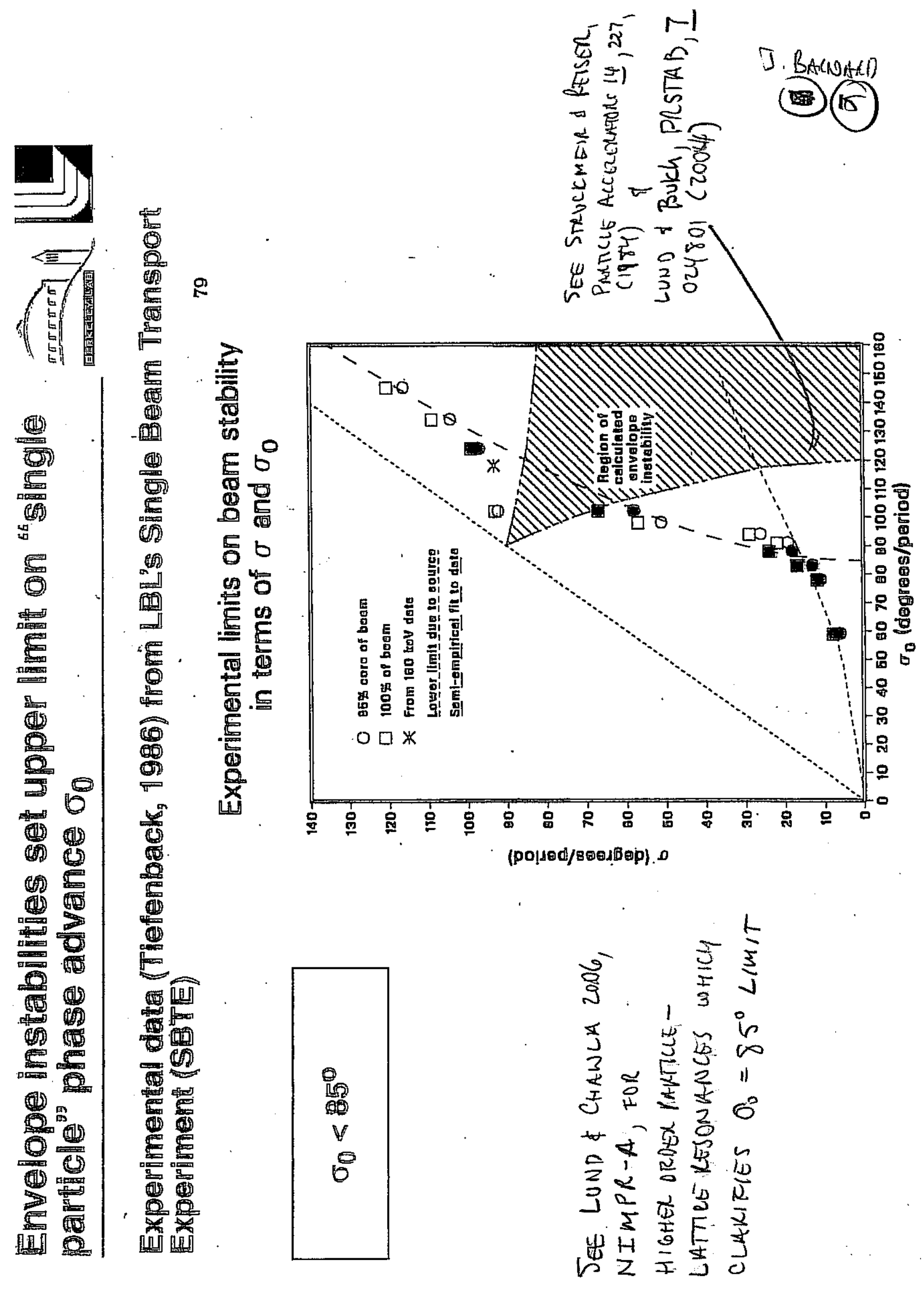





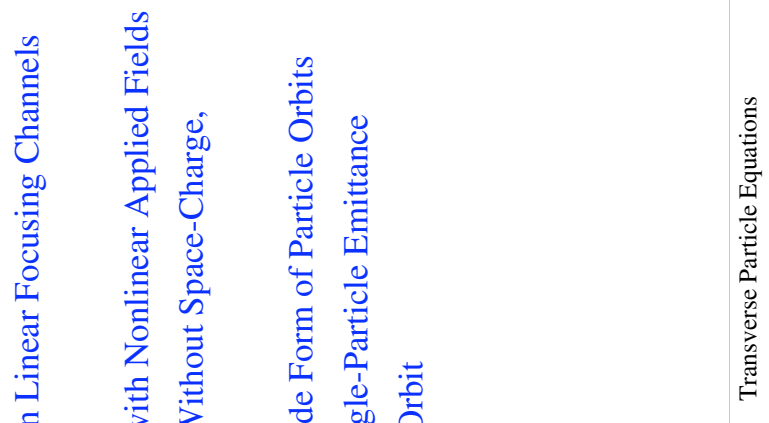

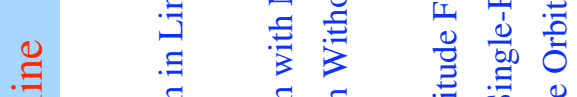

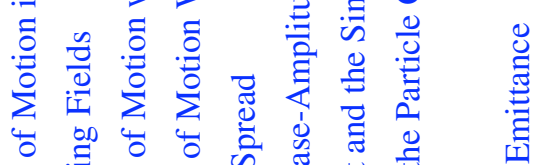

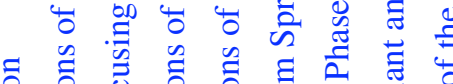

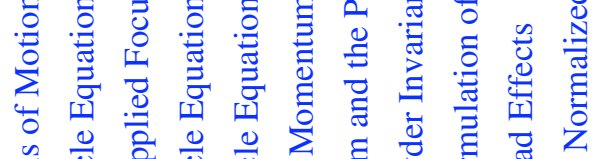

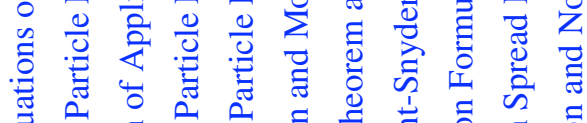

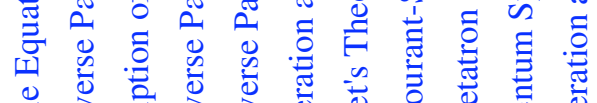

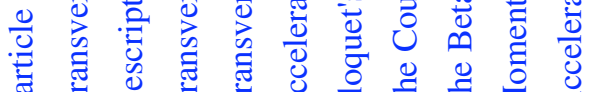

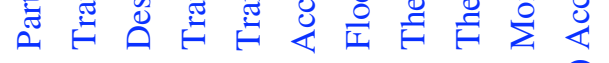

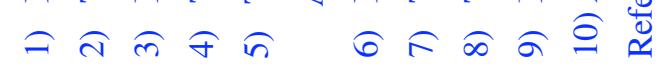
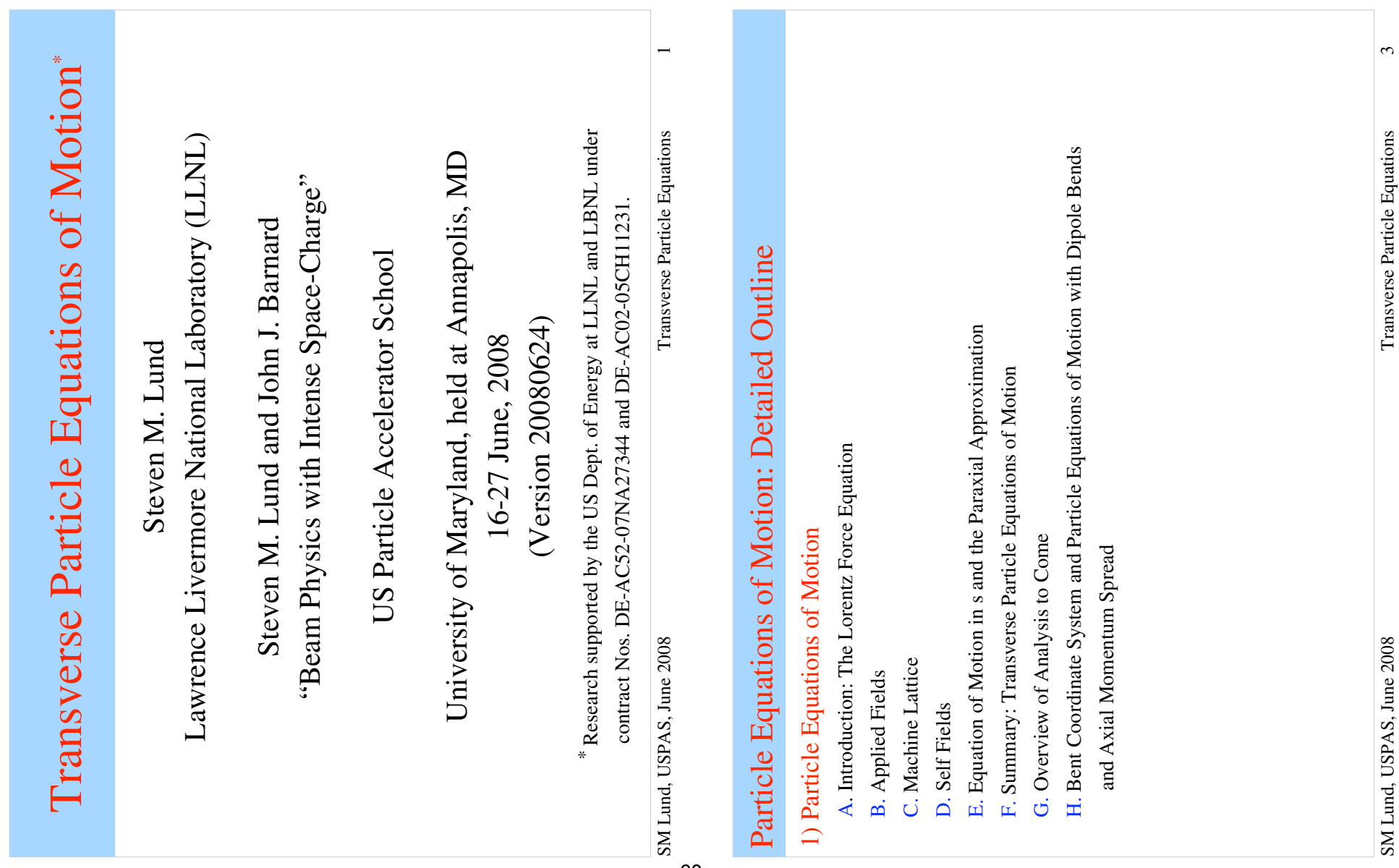

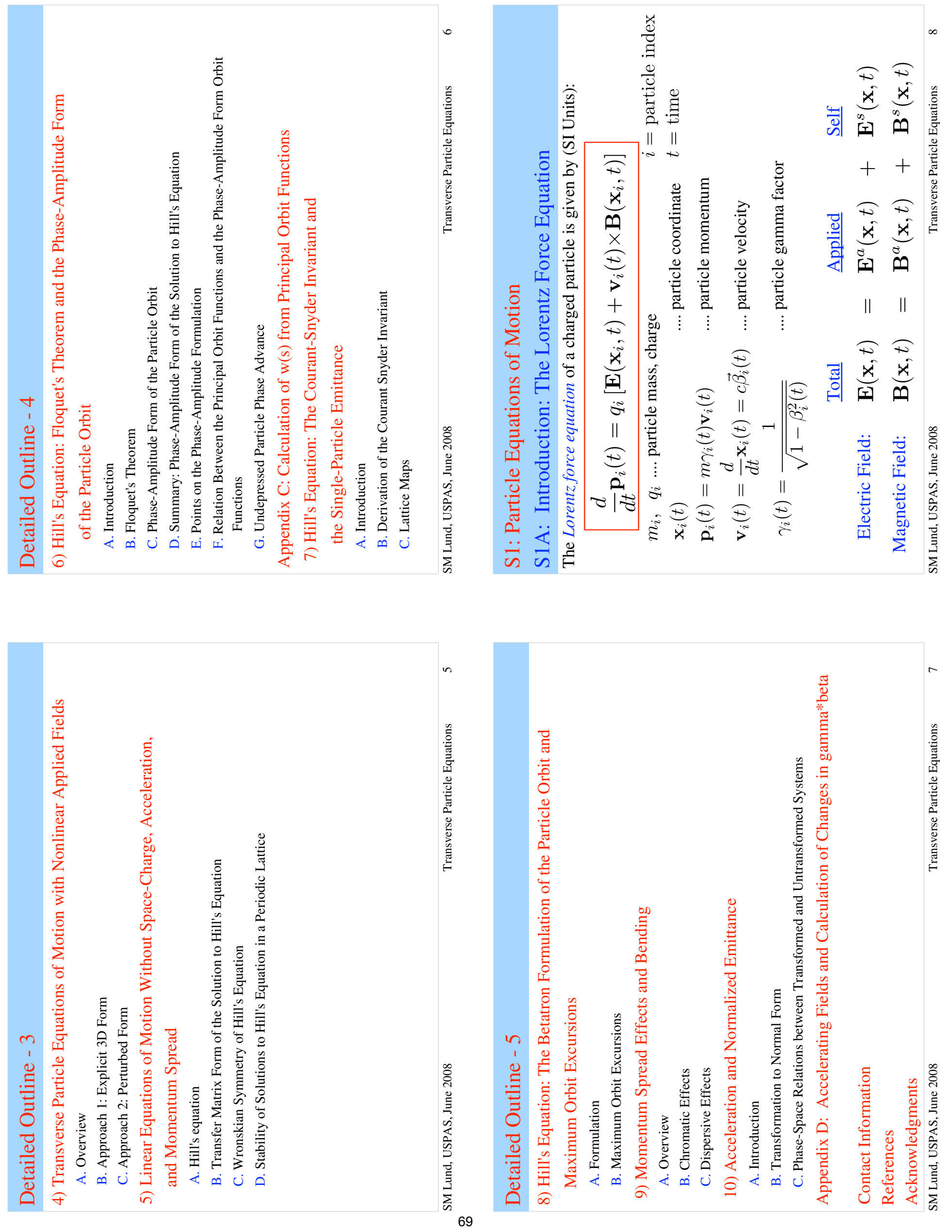

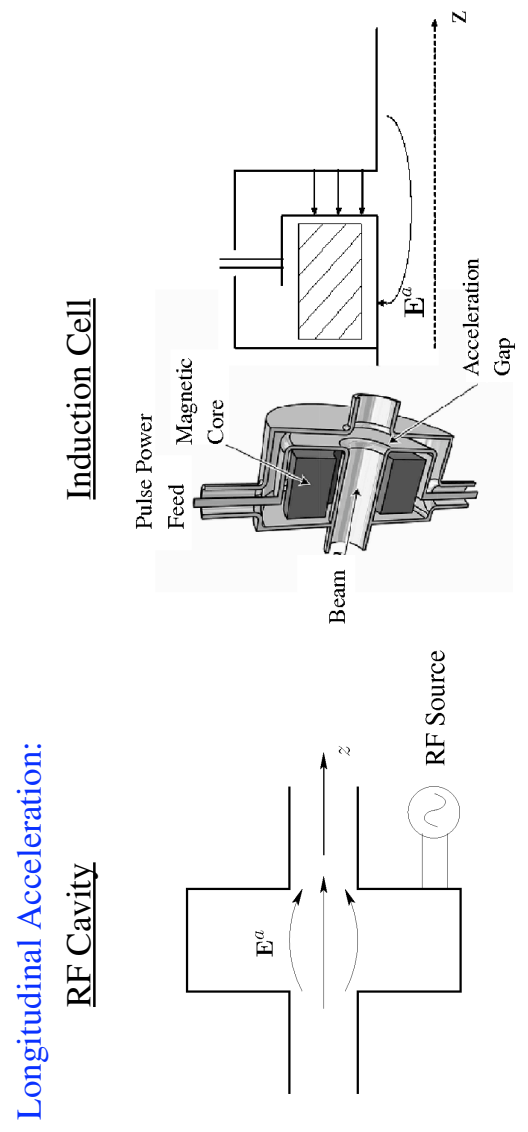

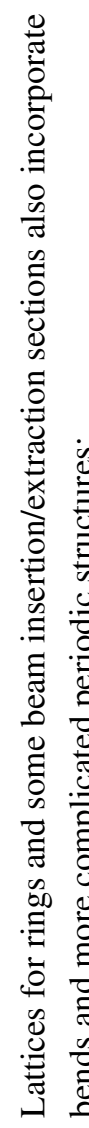

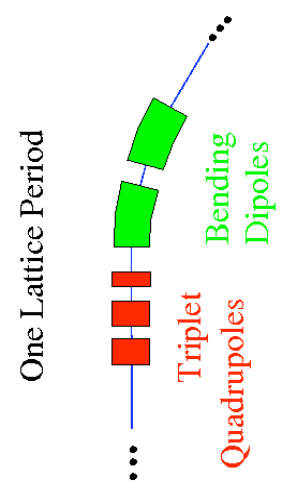

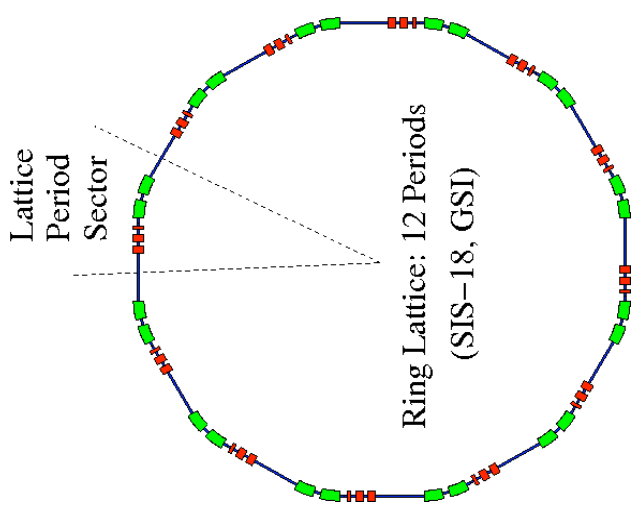

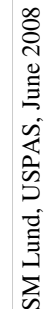
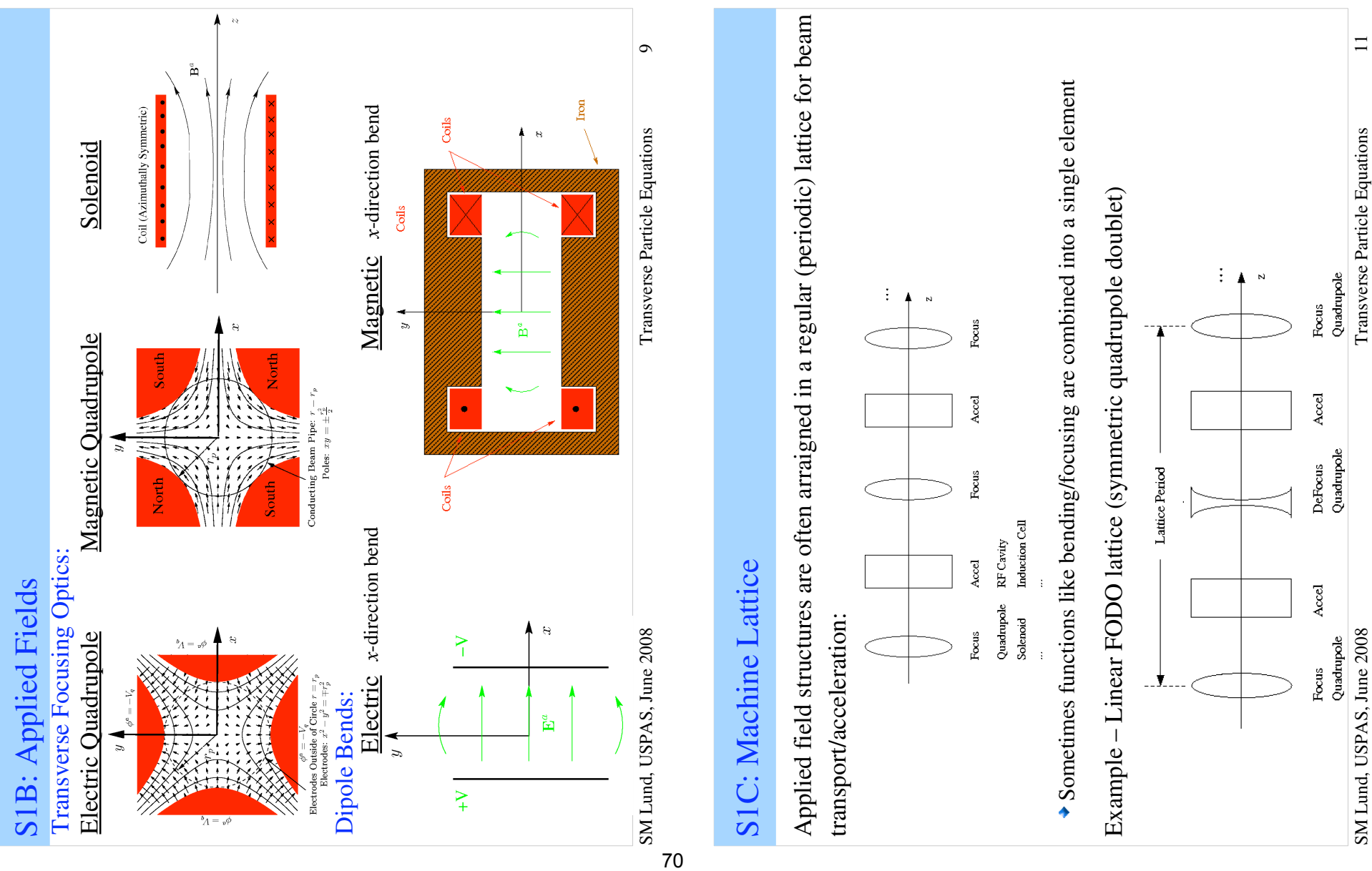

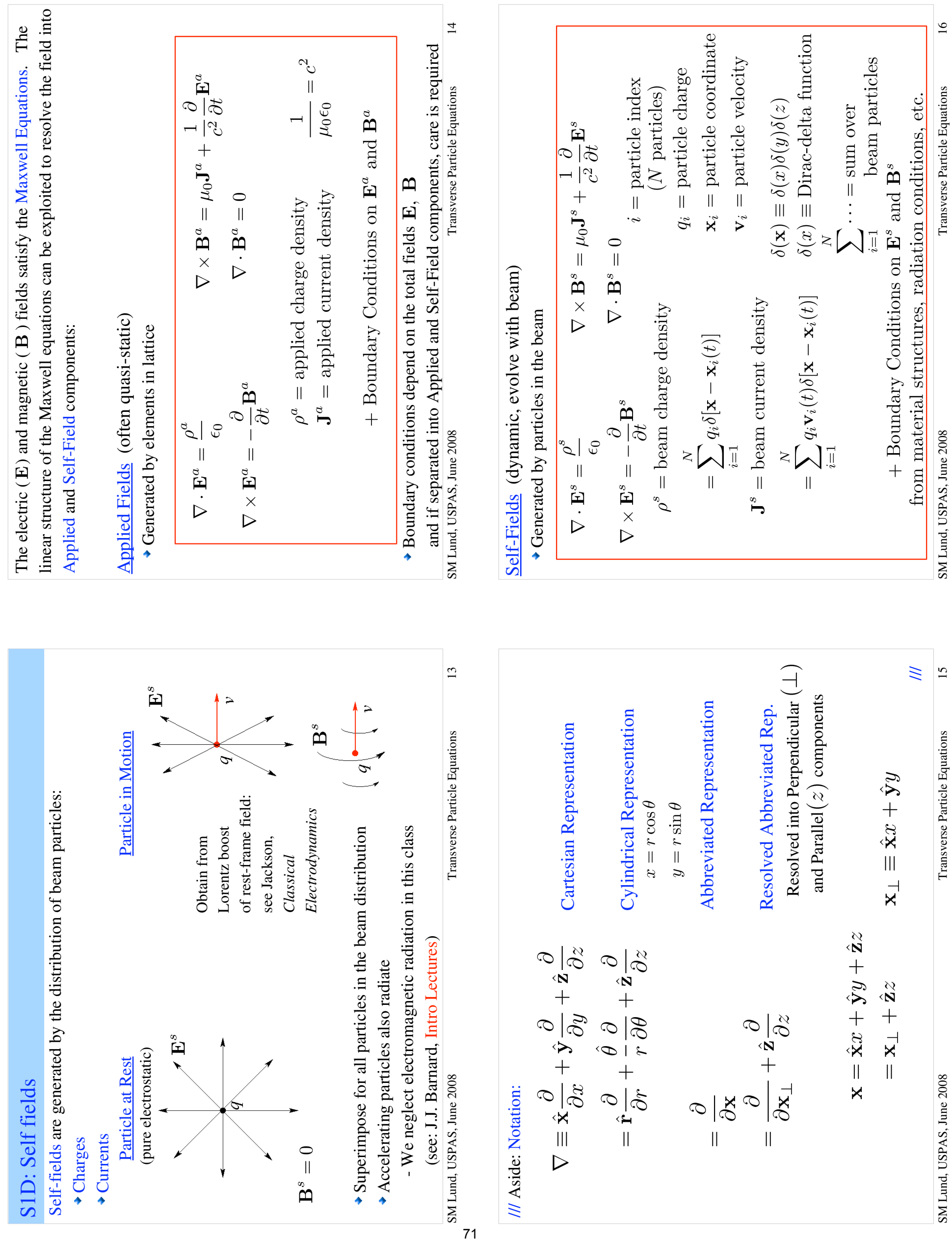


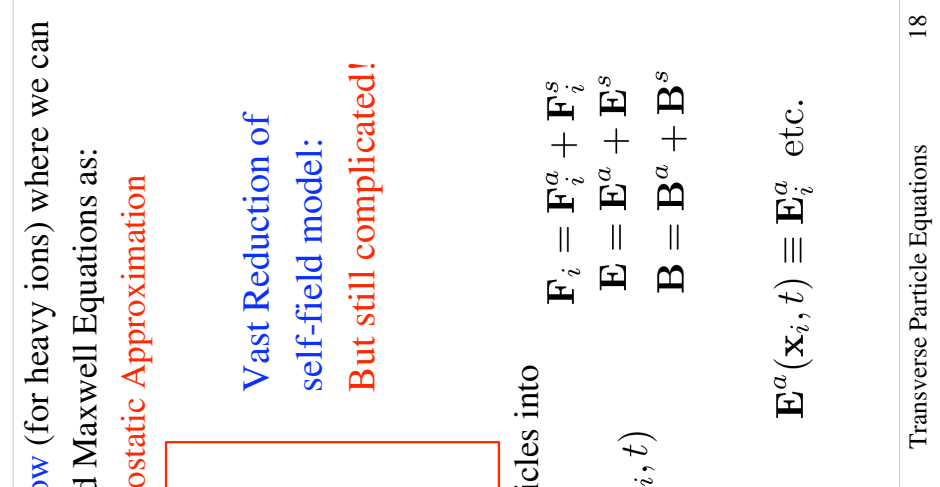

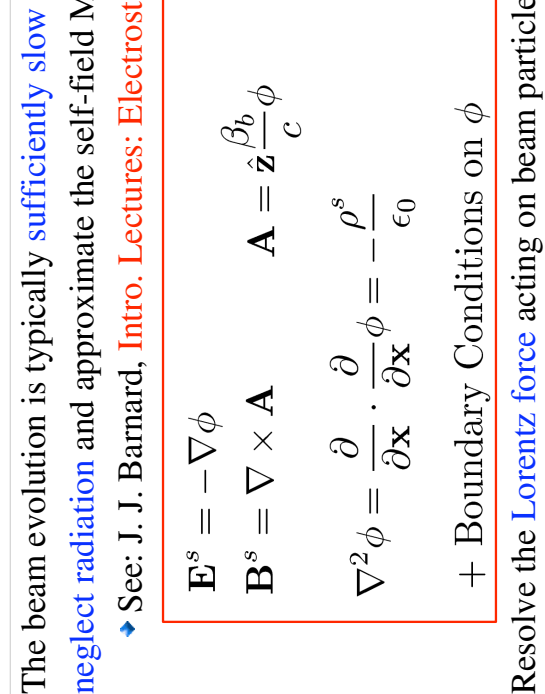

$\underset{\vec{x}}{i \vec{x}}$

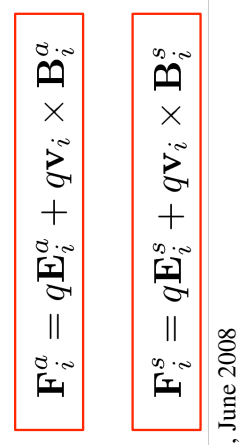

क

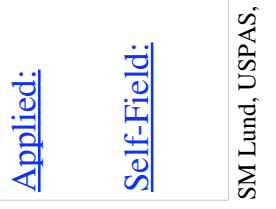

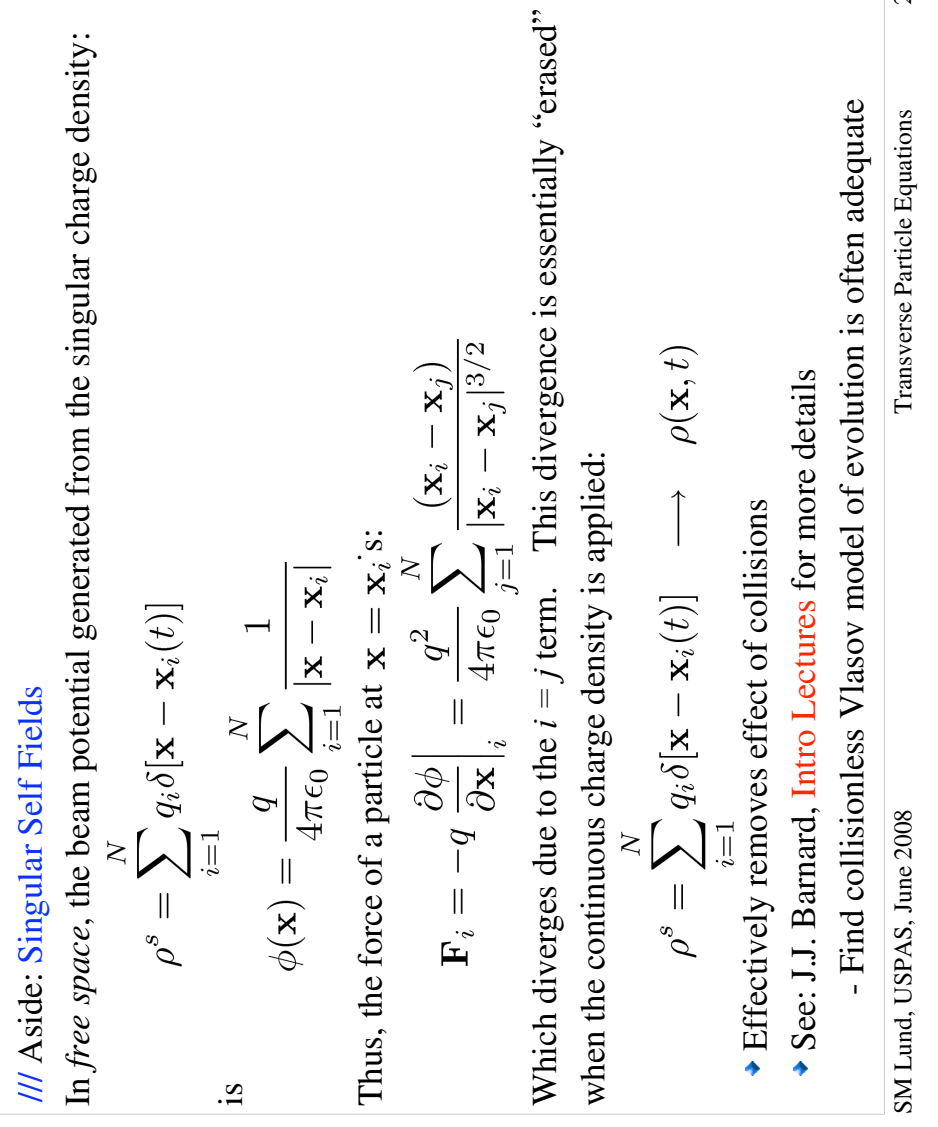



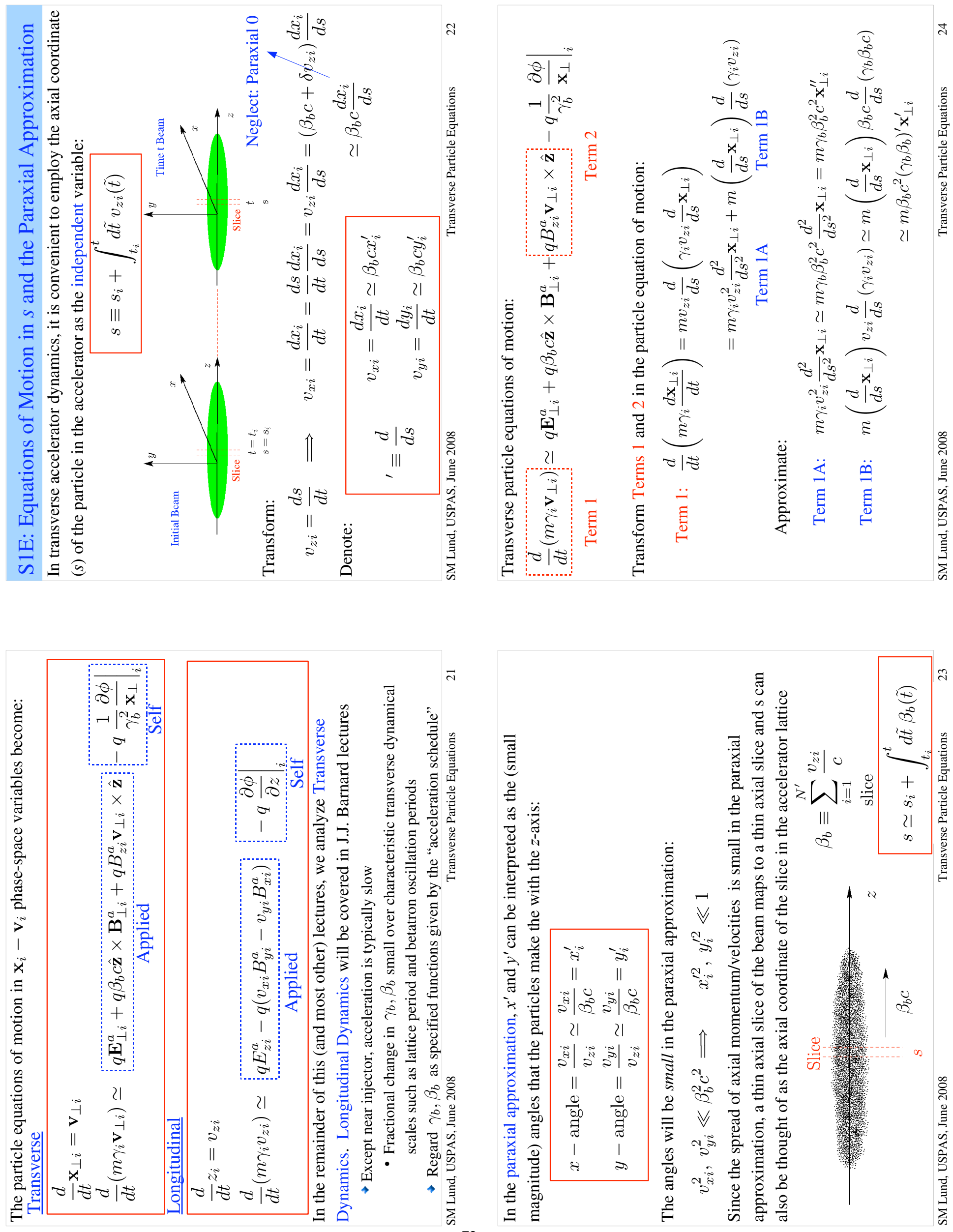

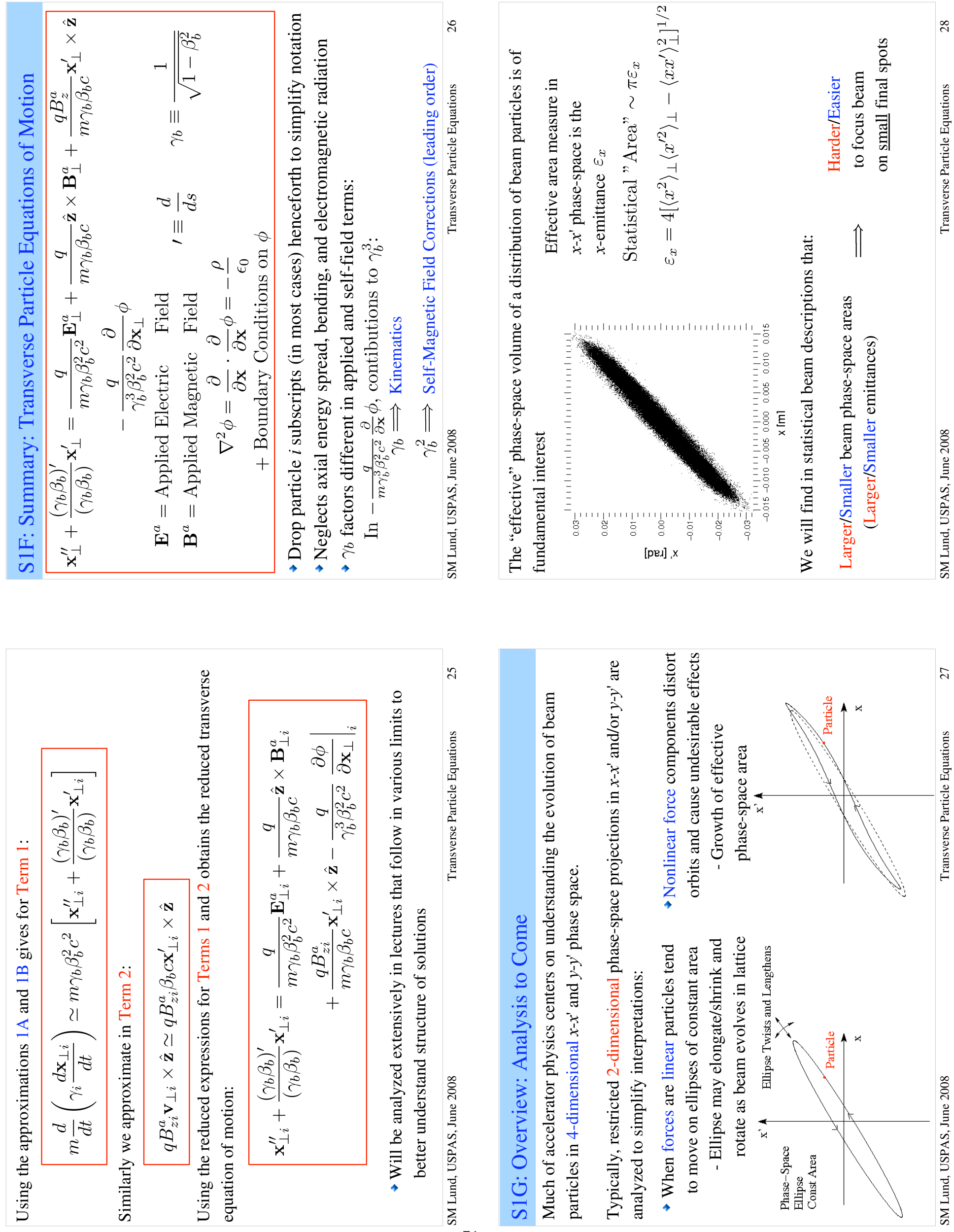

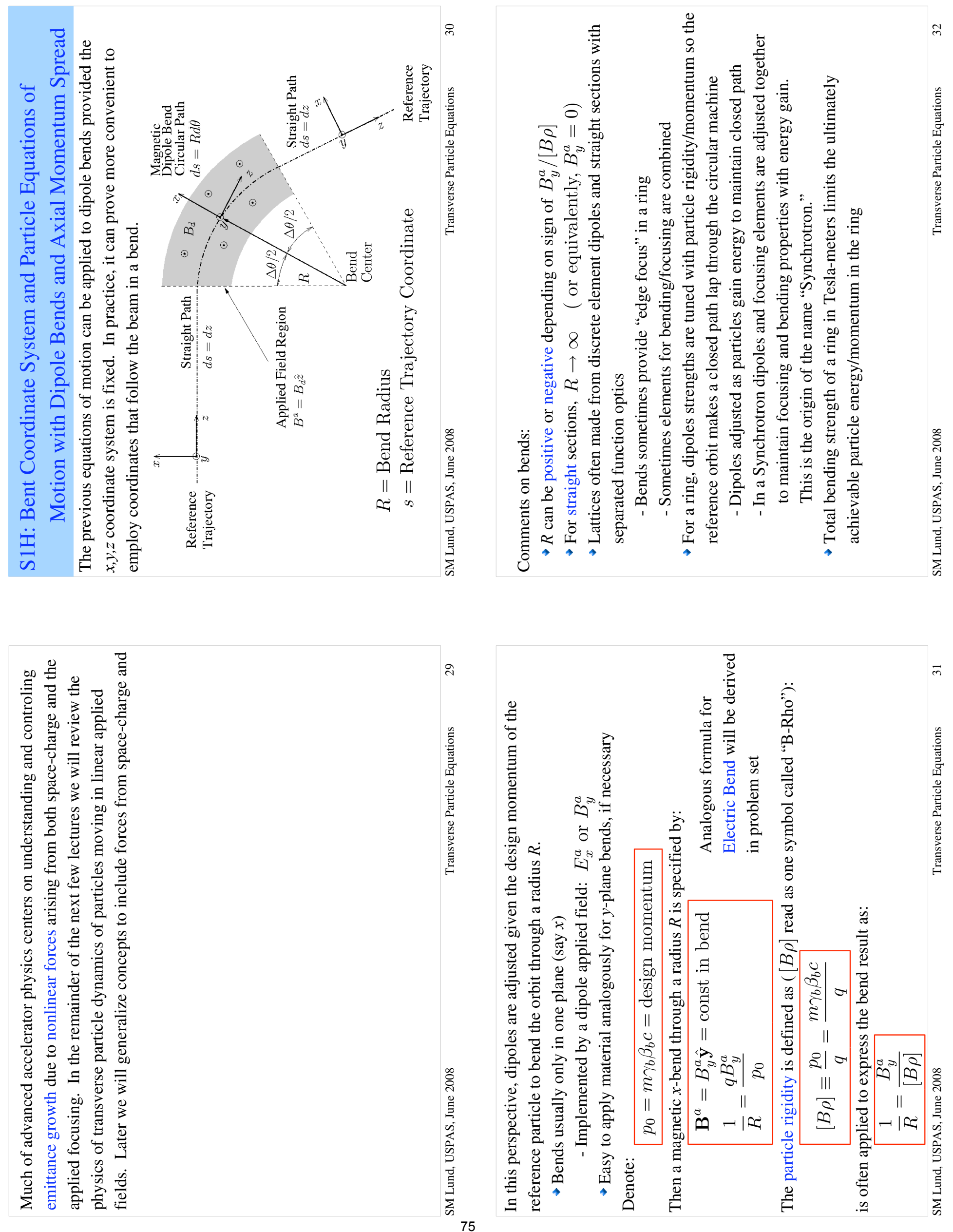

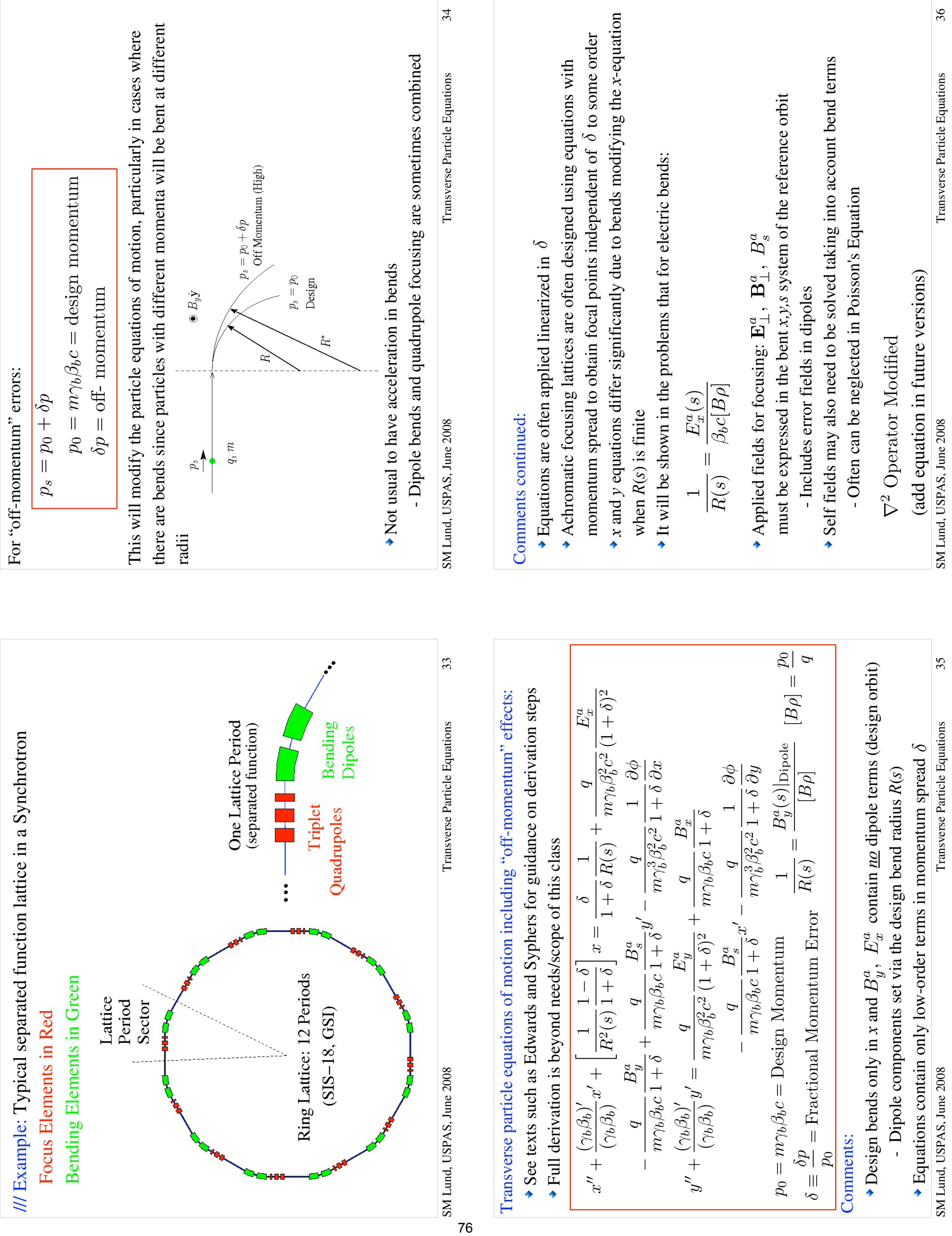

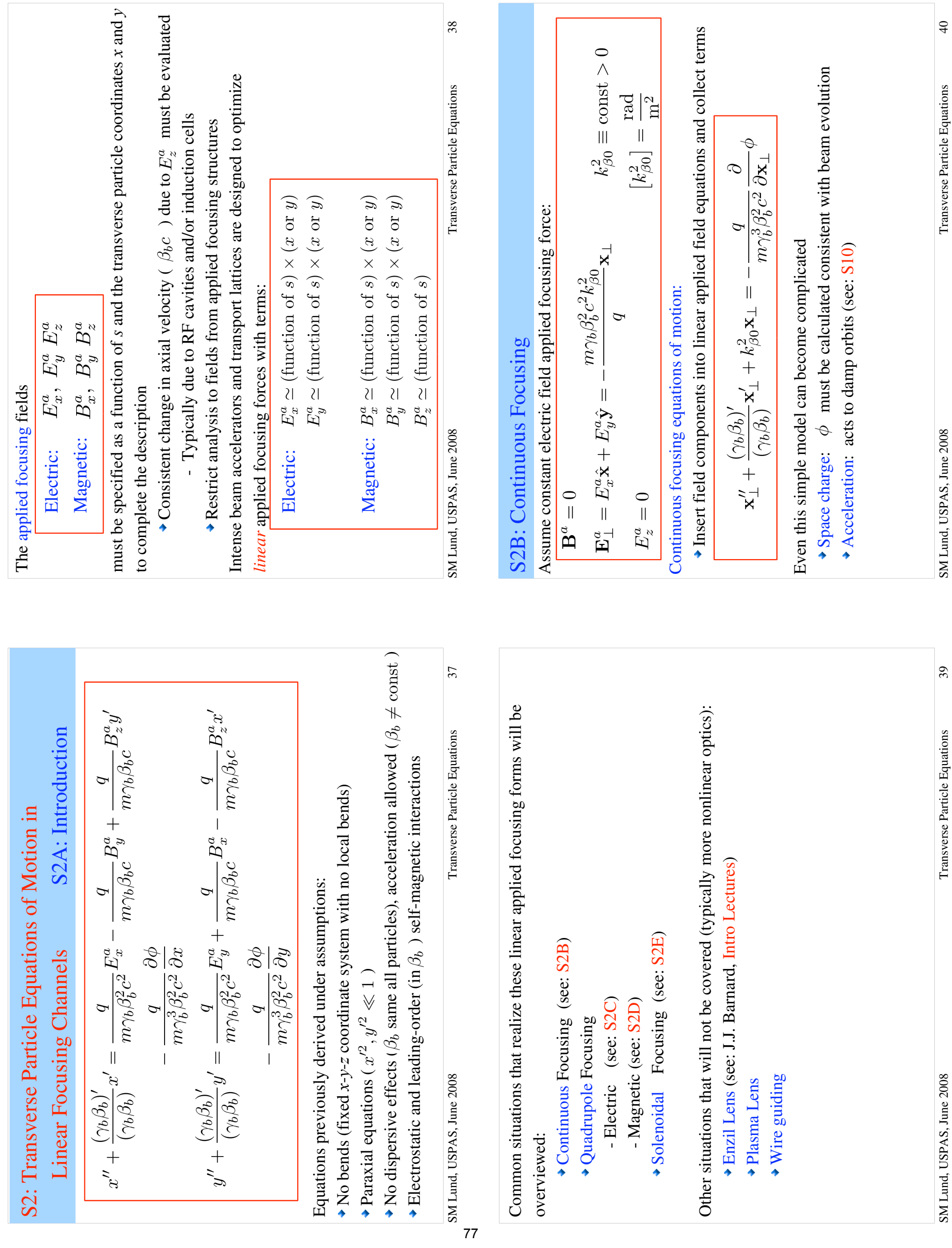

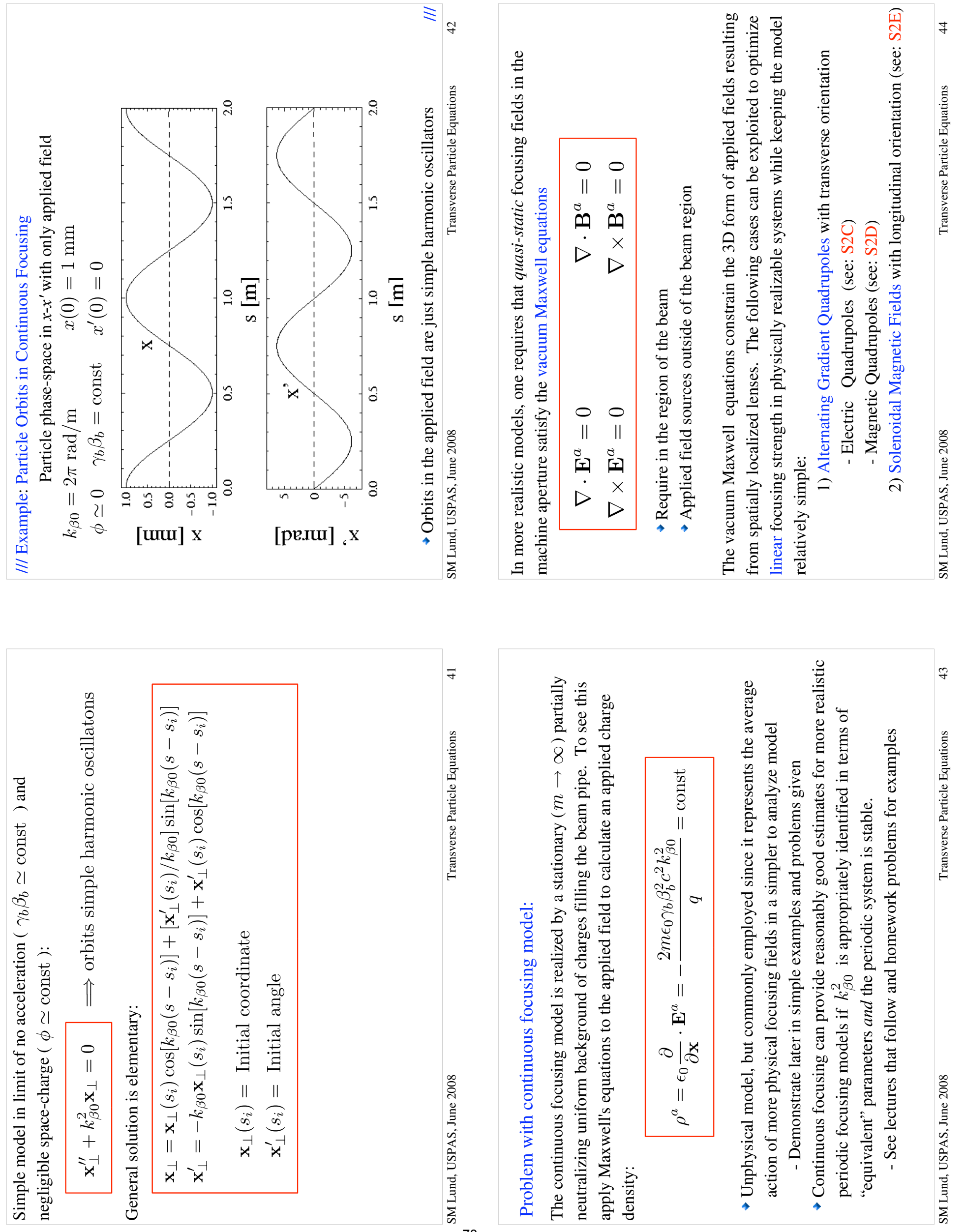


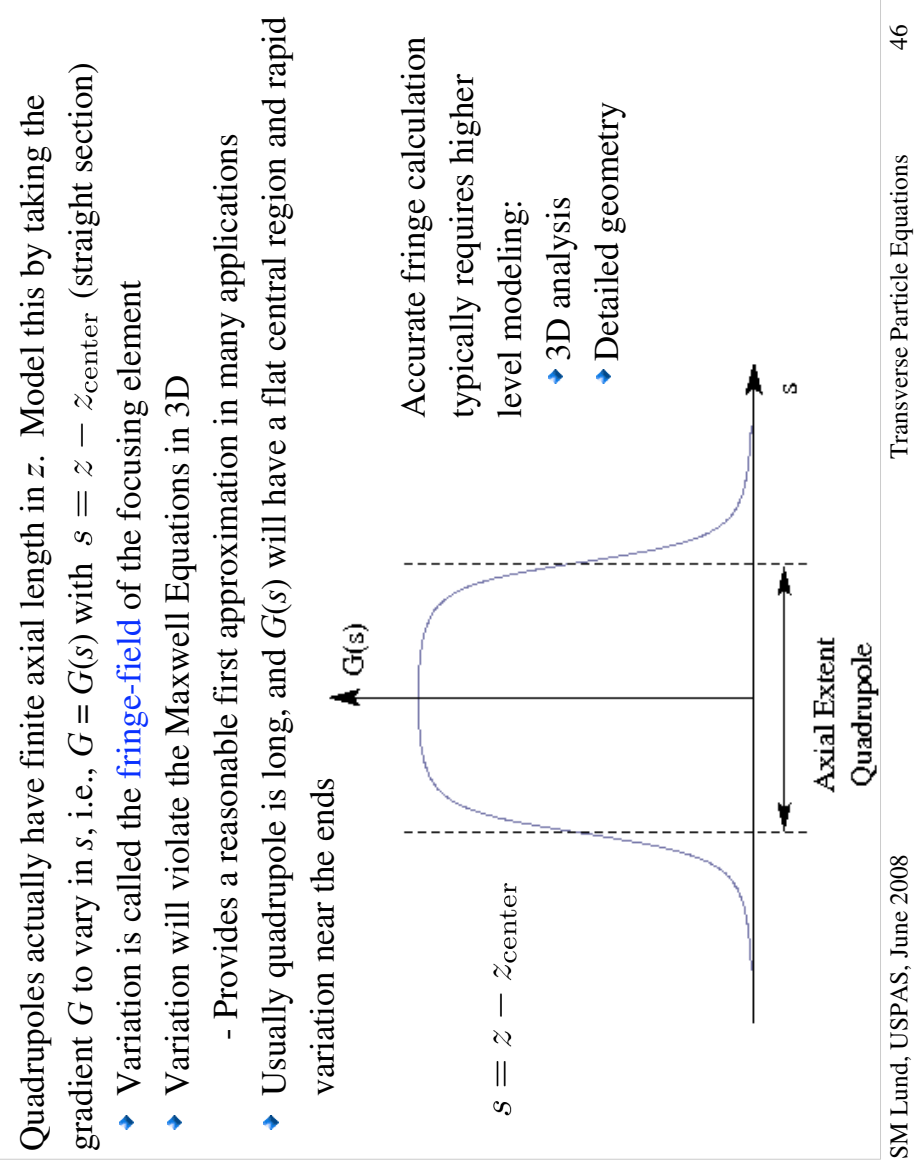

+
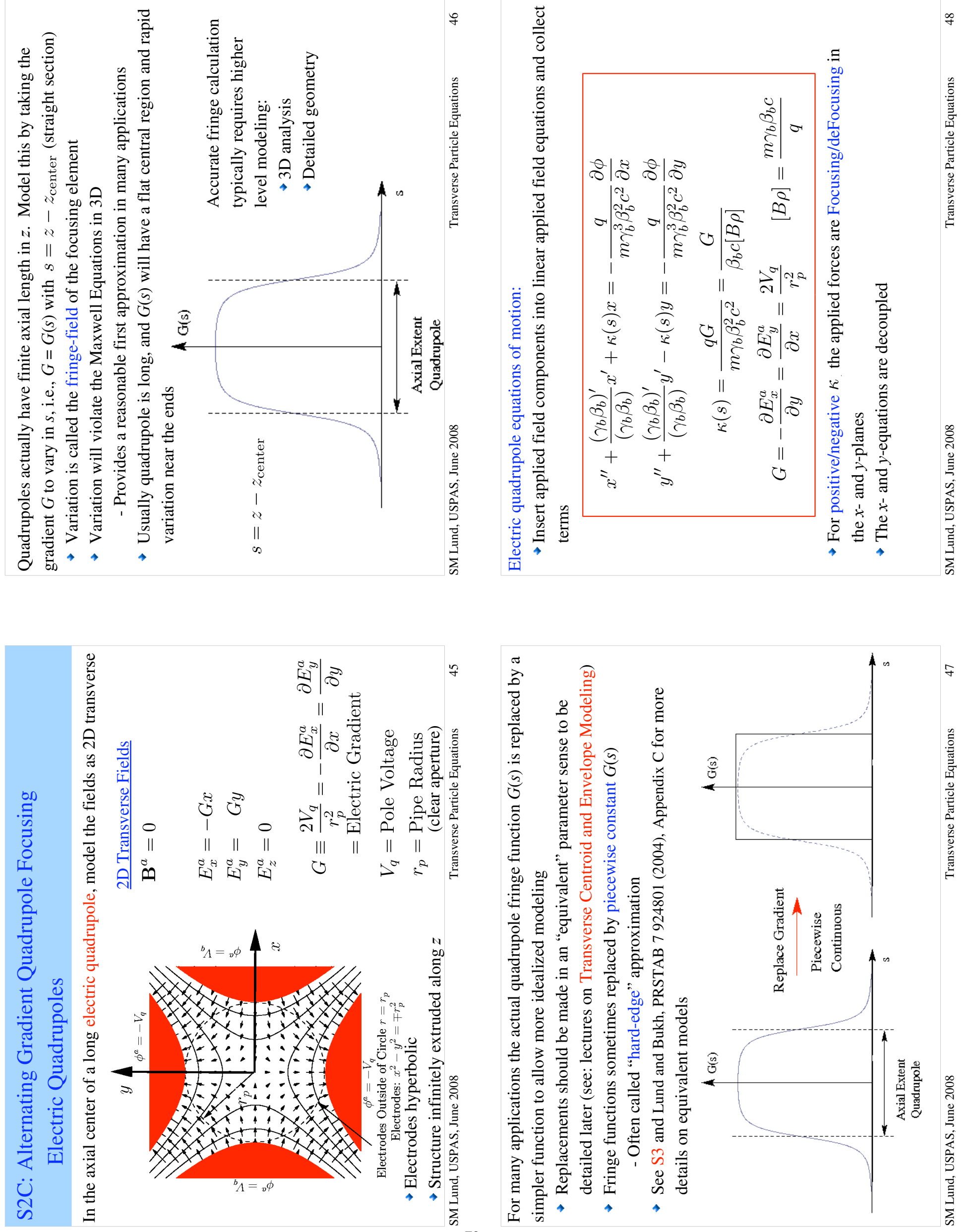

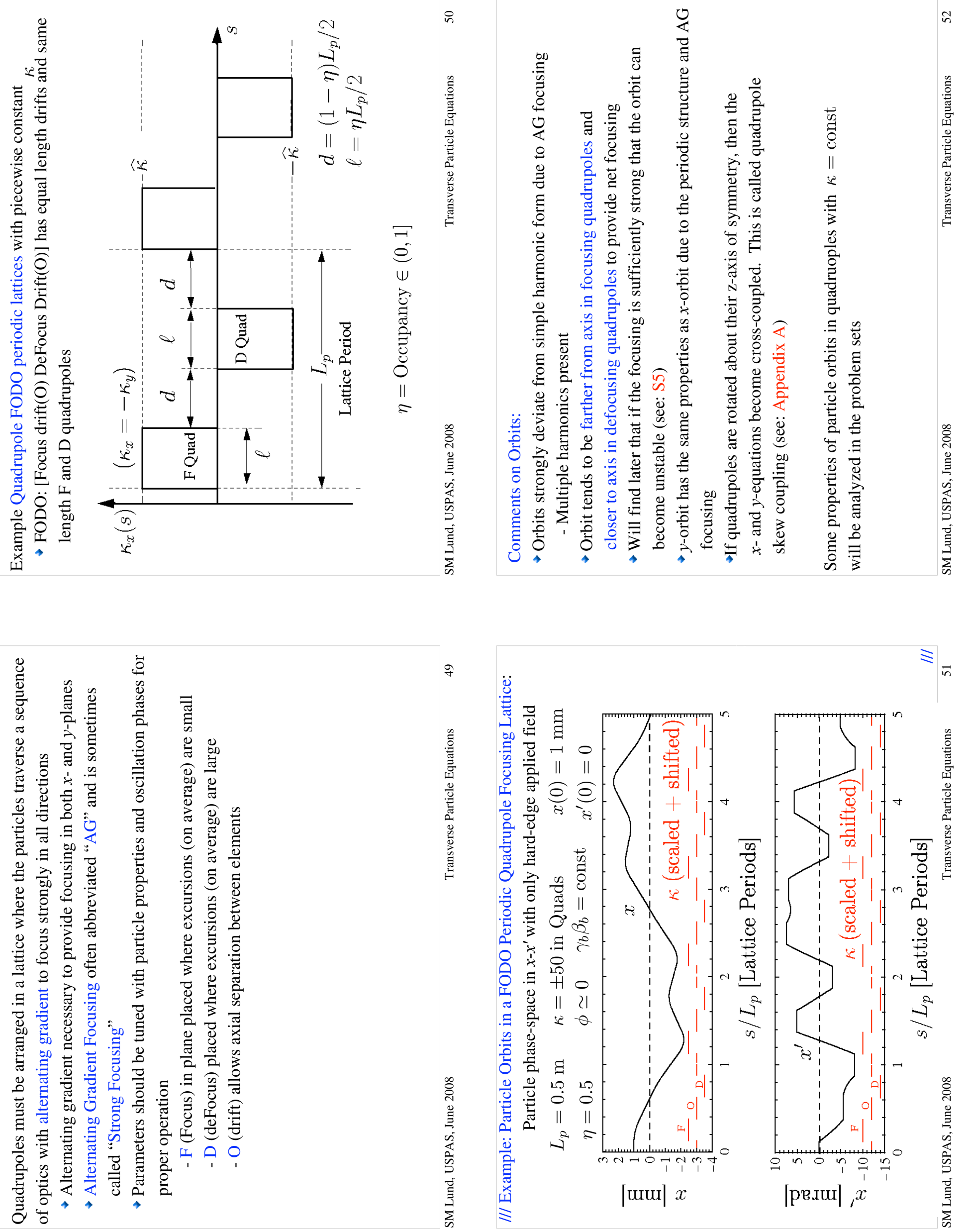

g

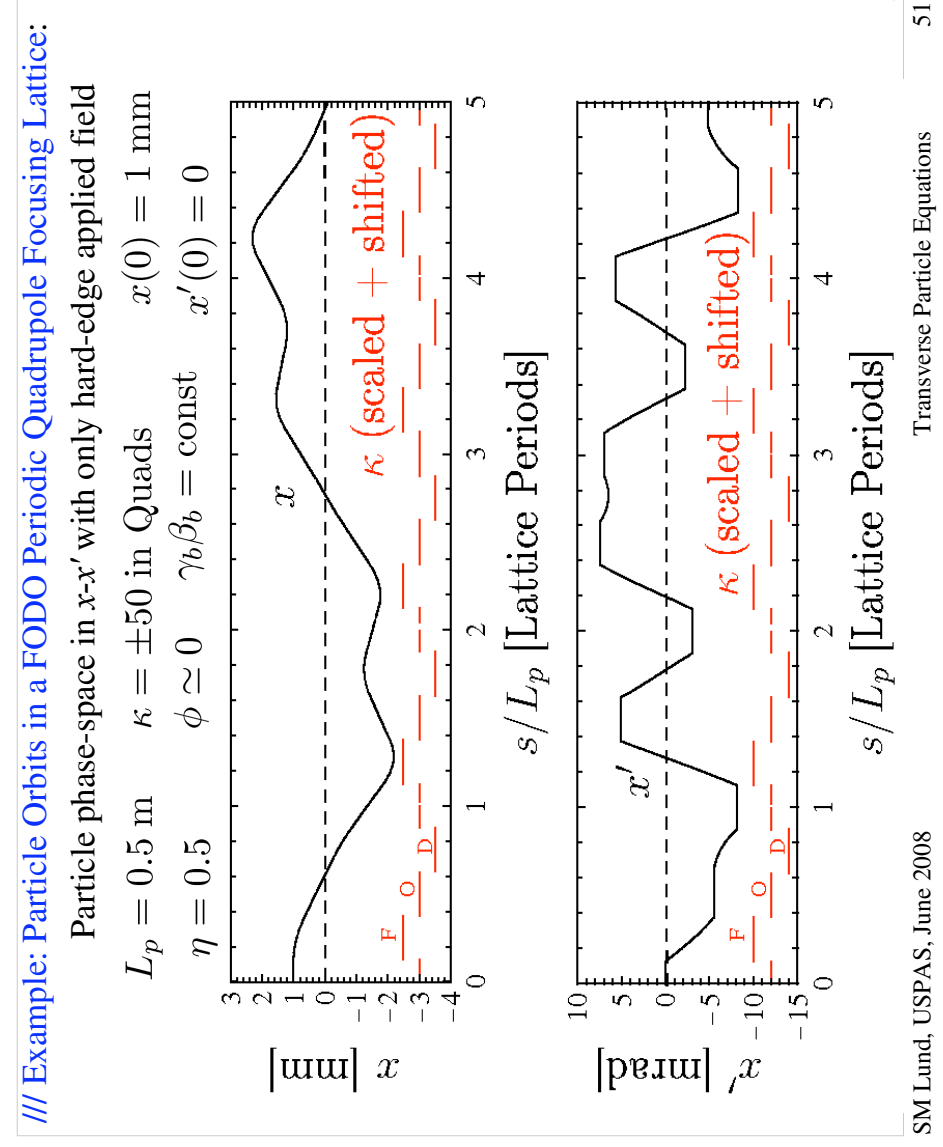



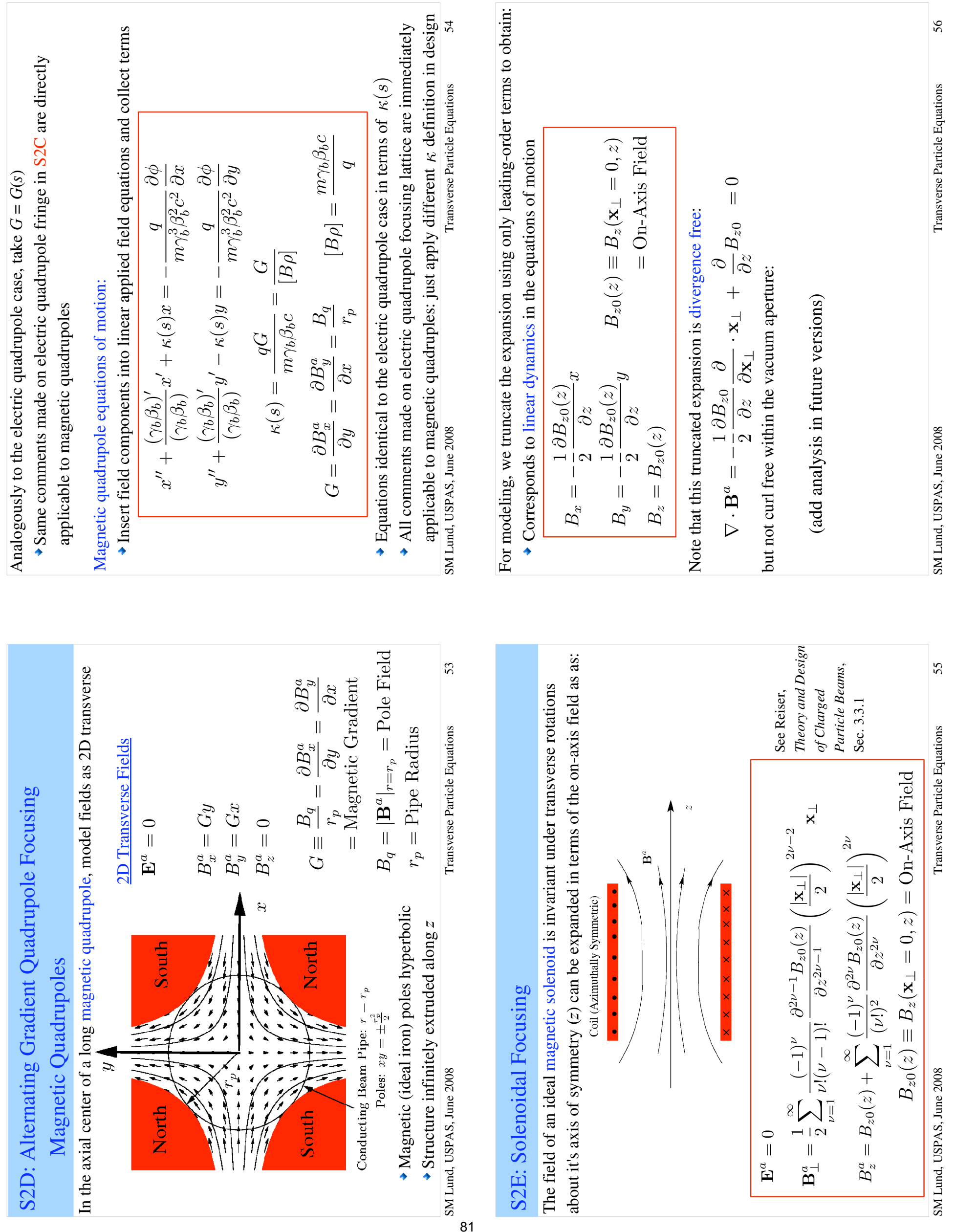

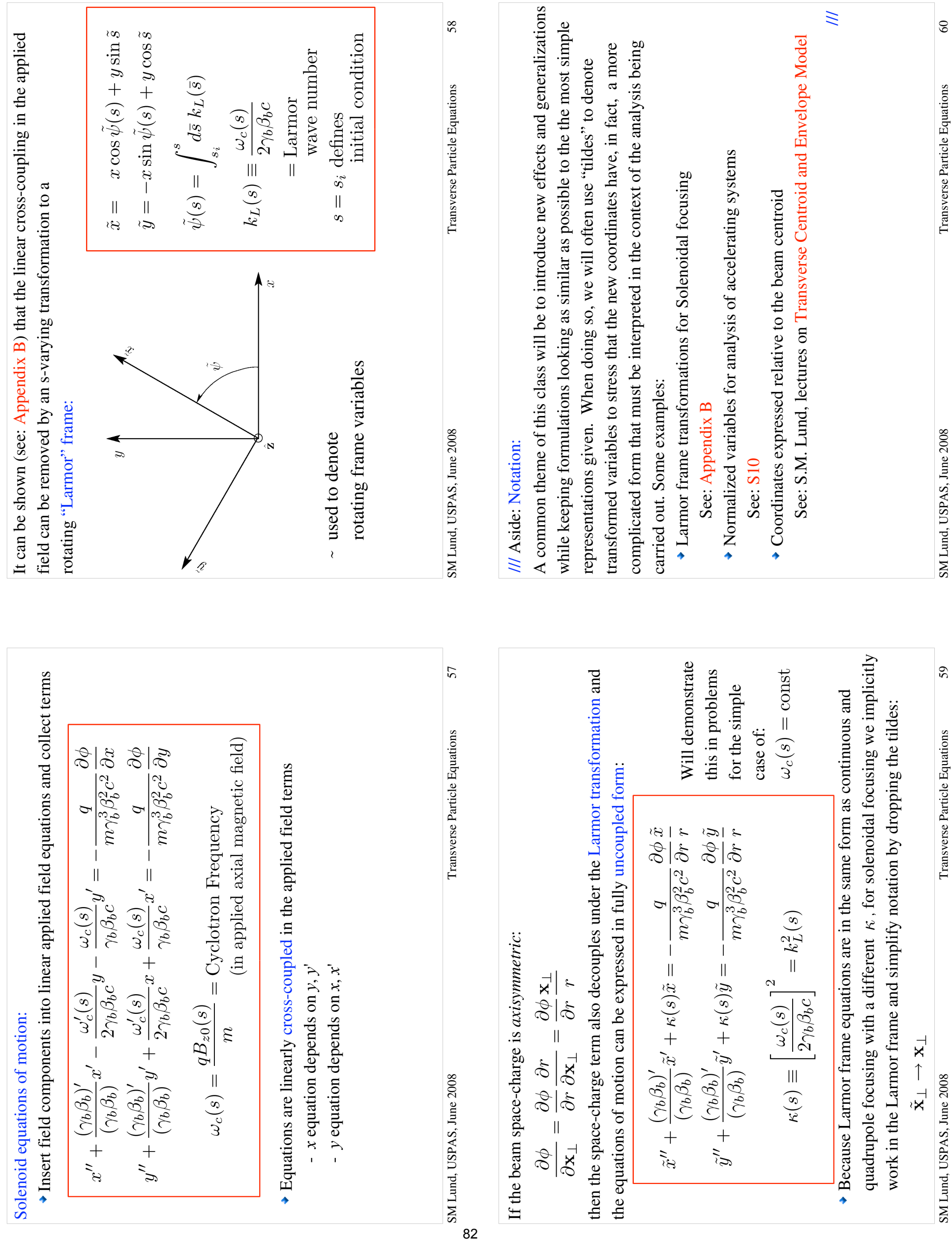

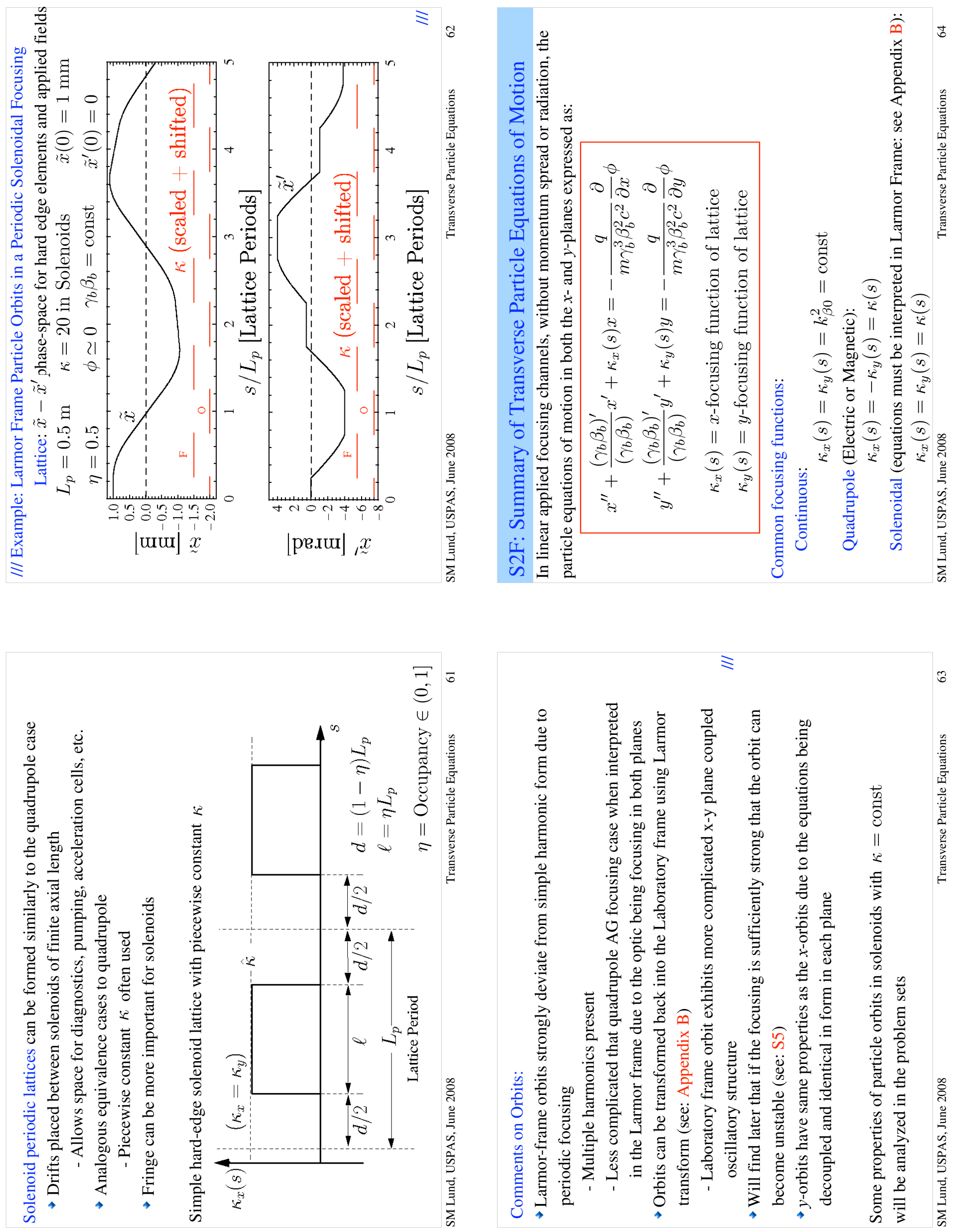

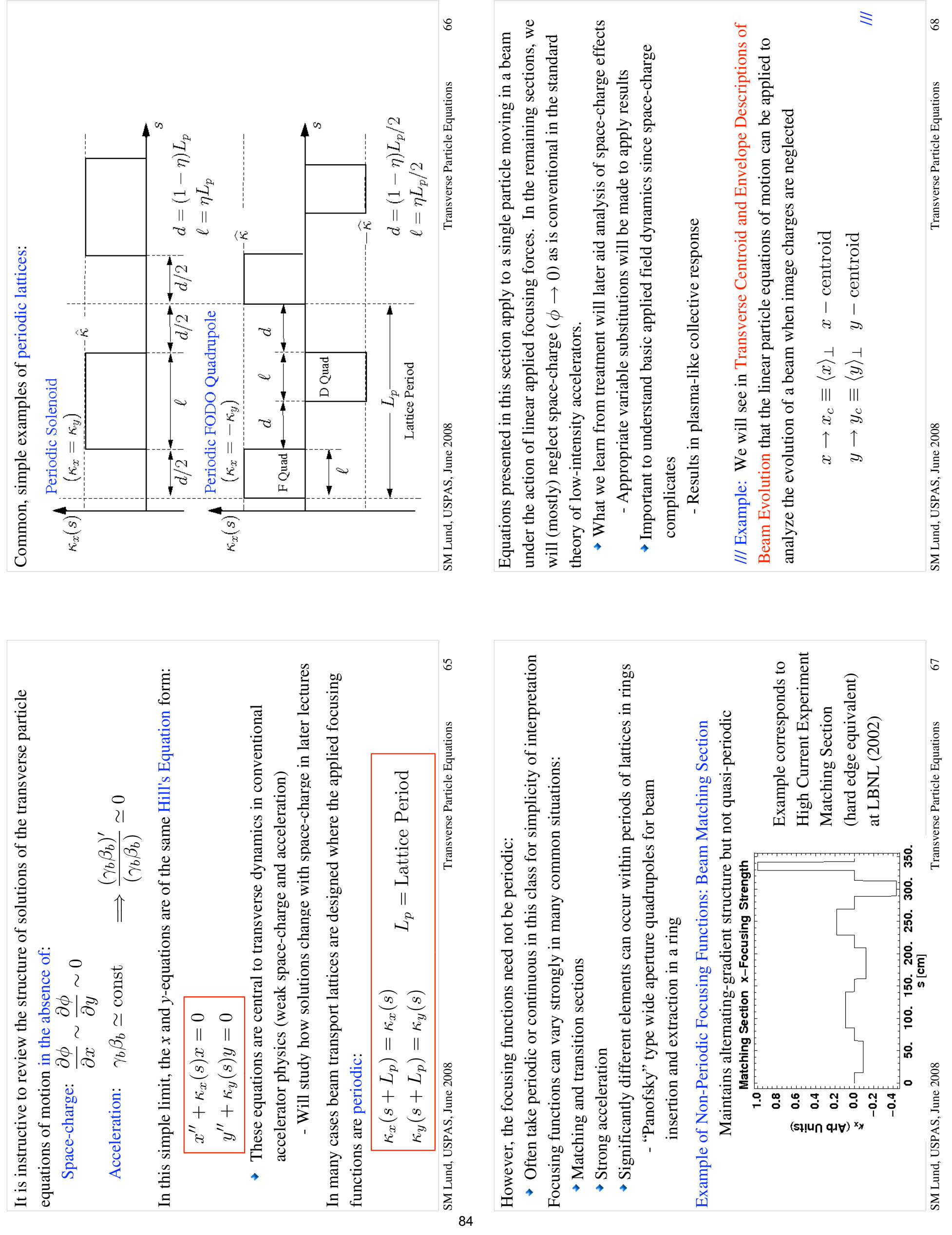


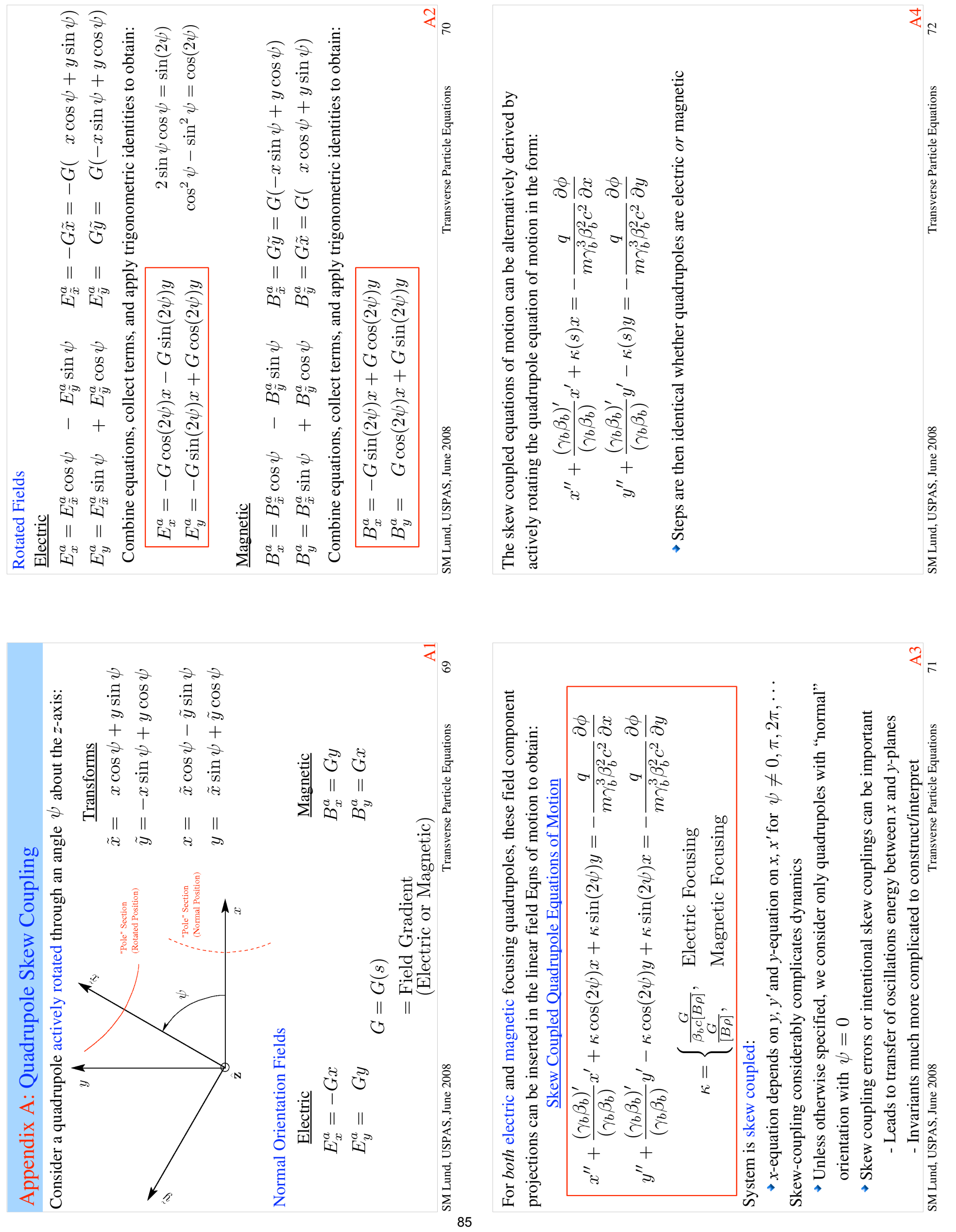



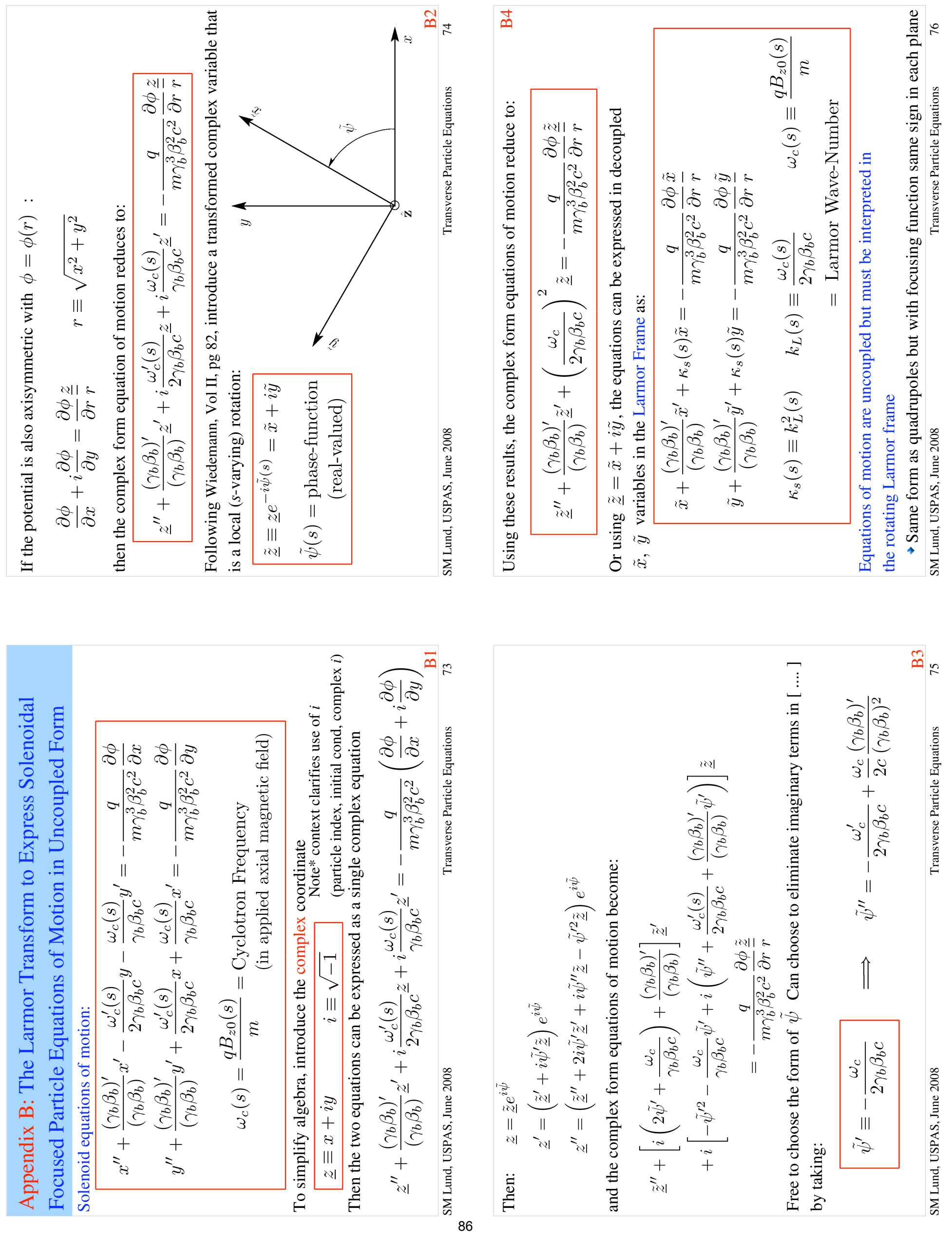

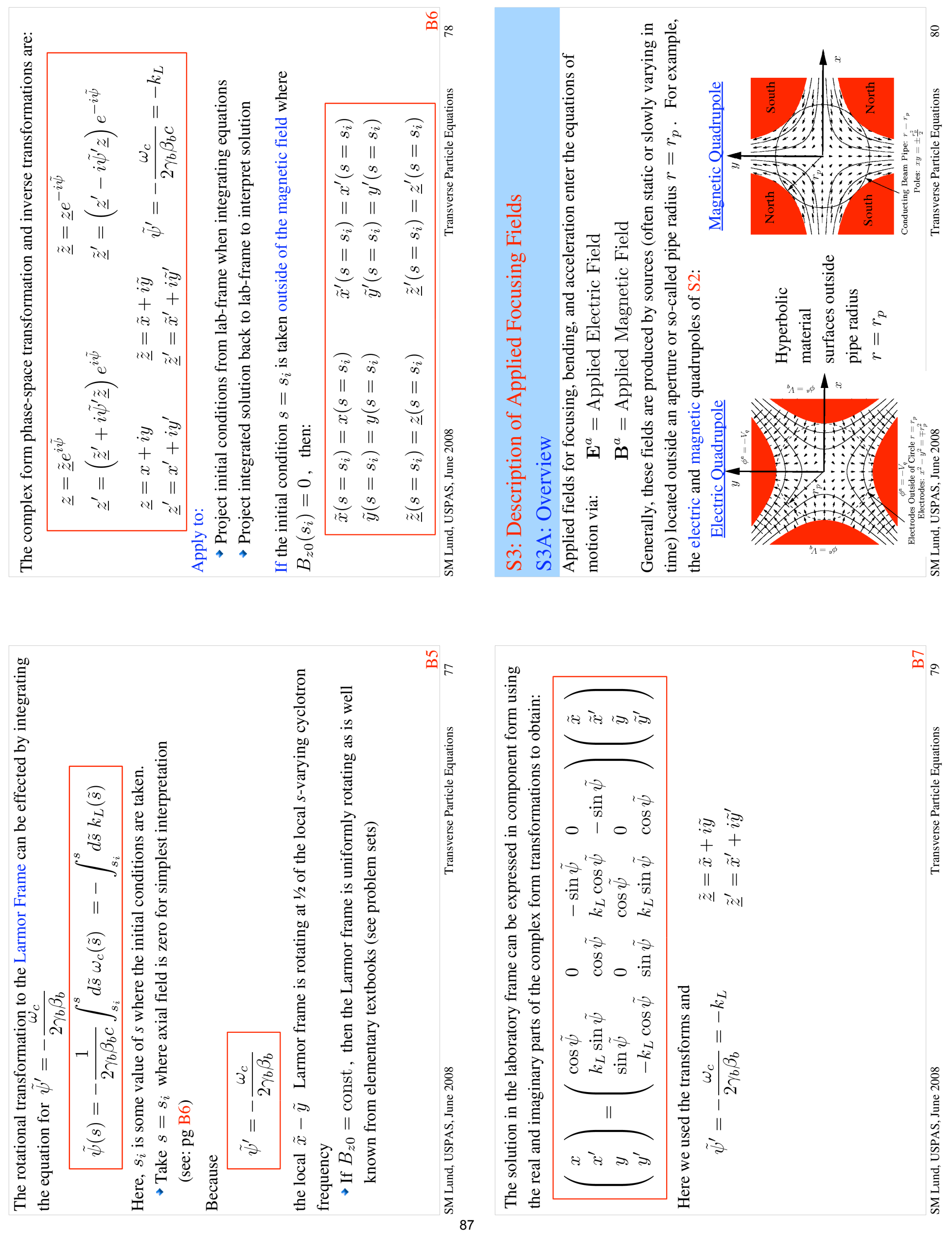

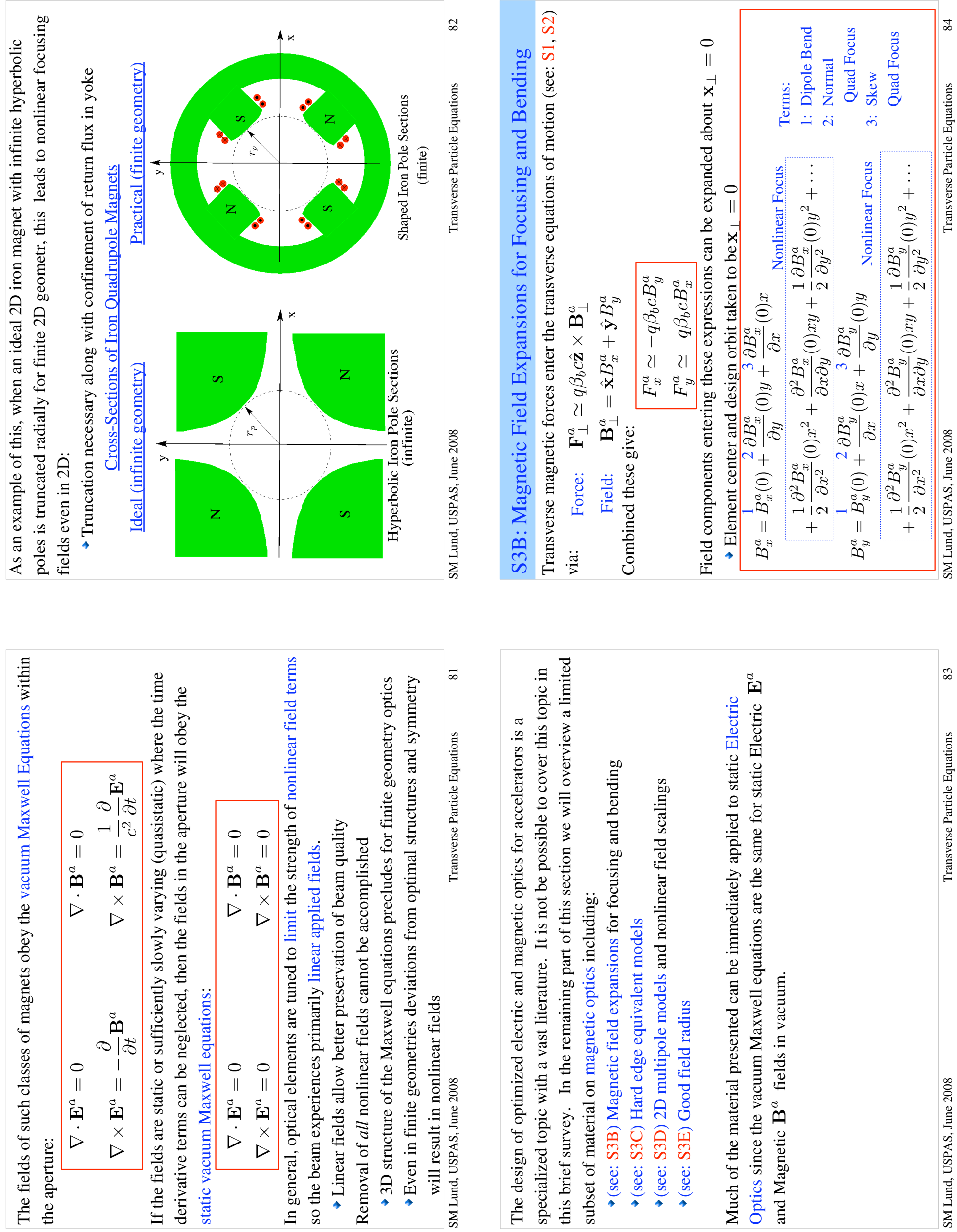

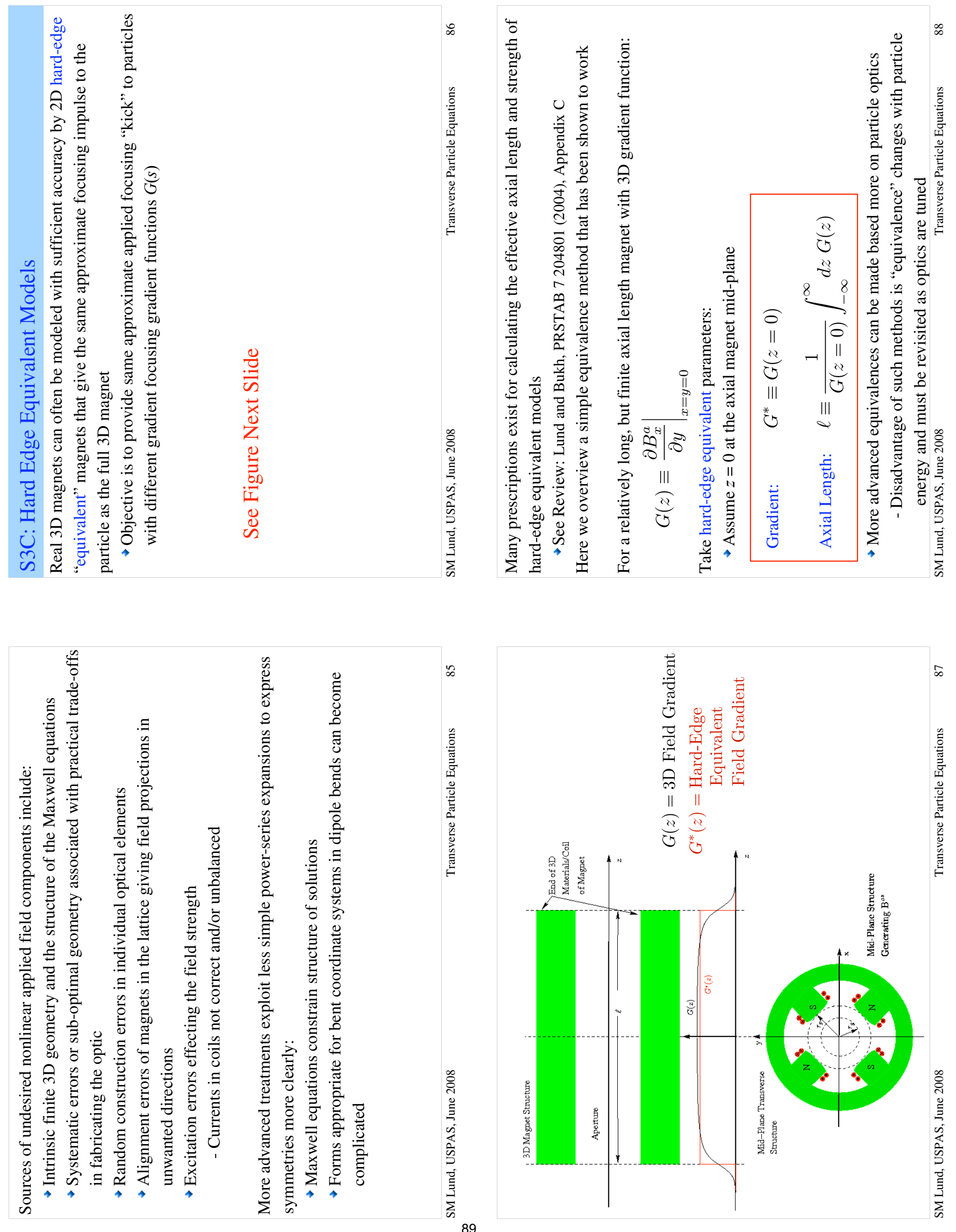

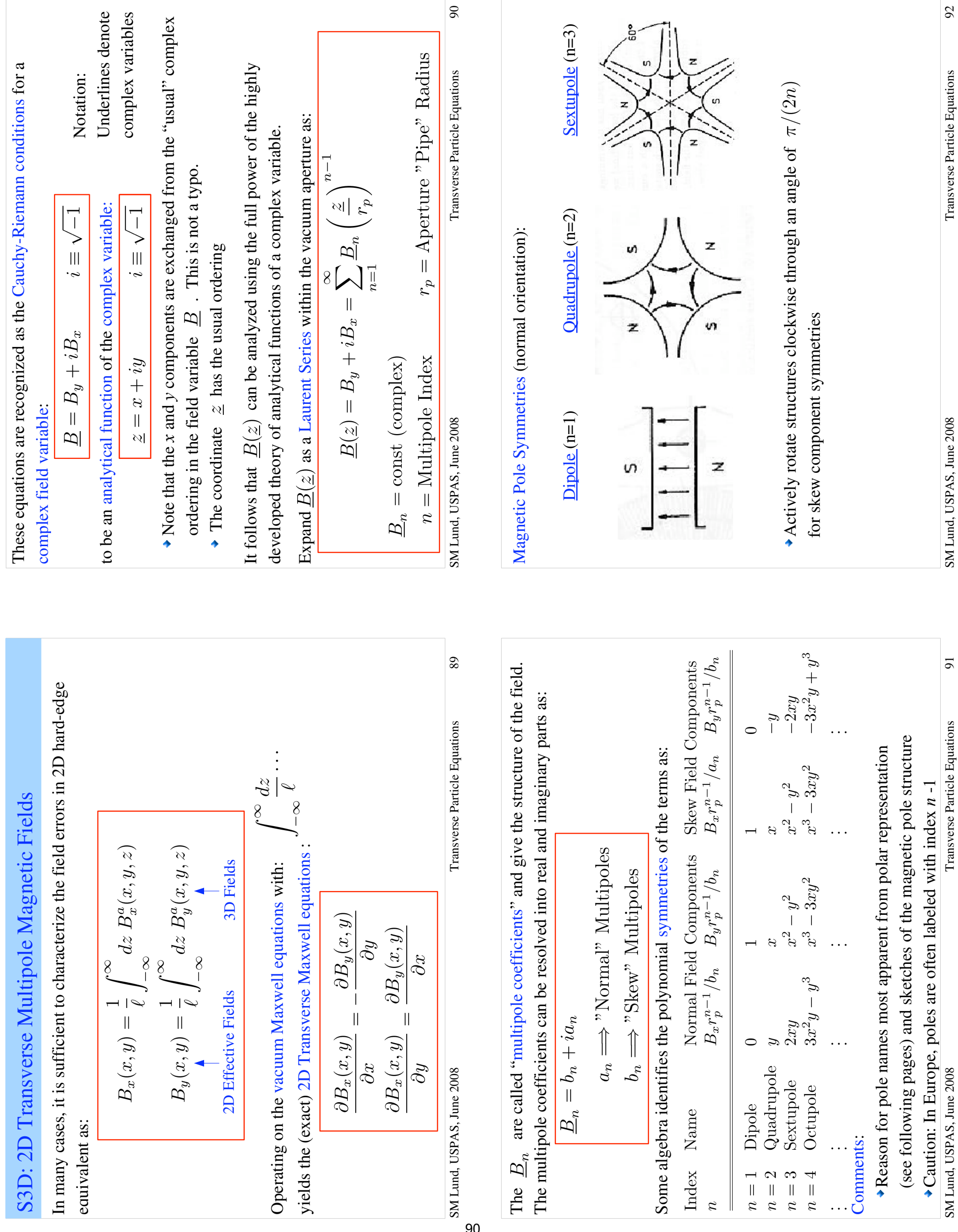

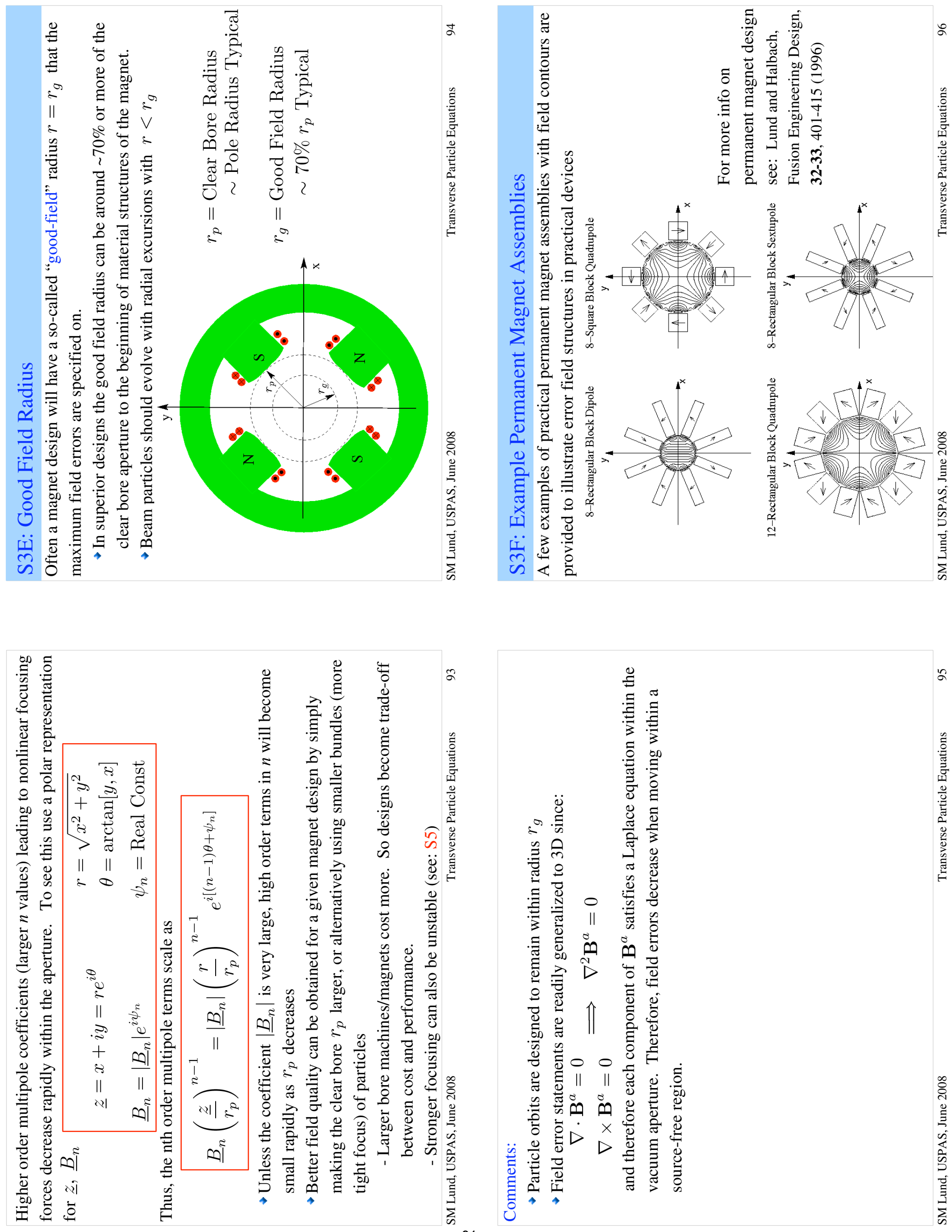

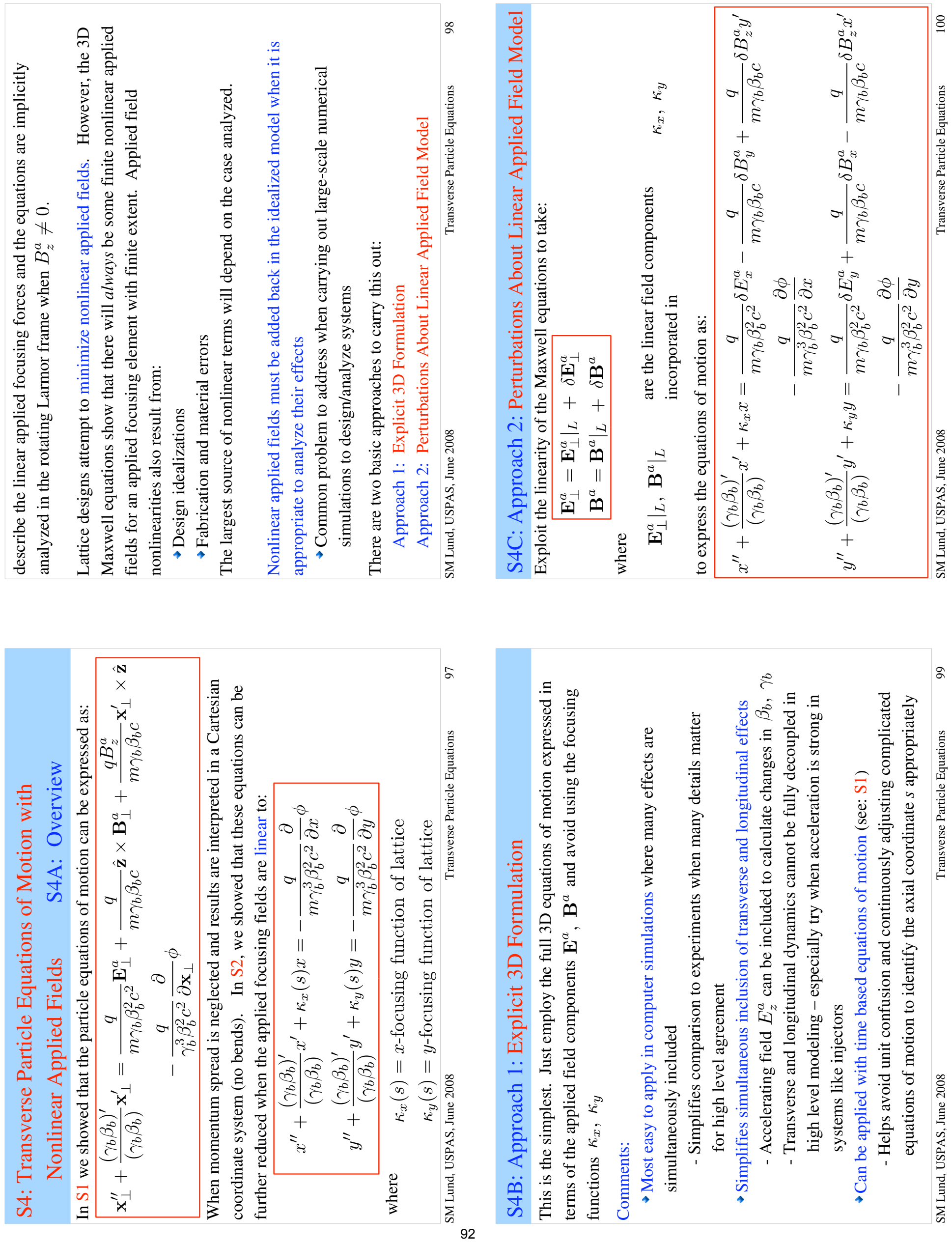


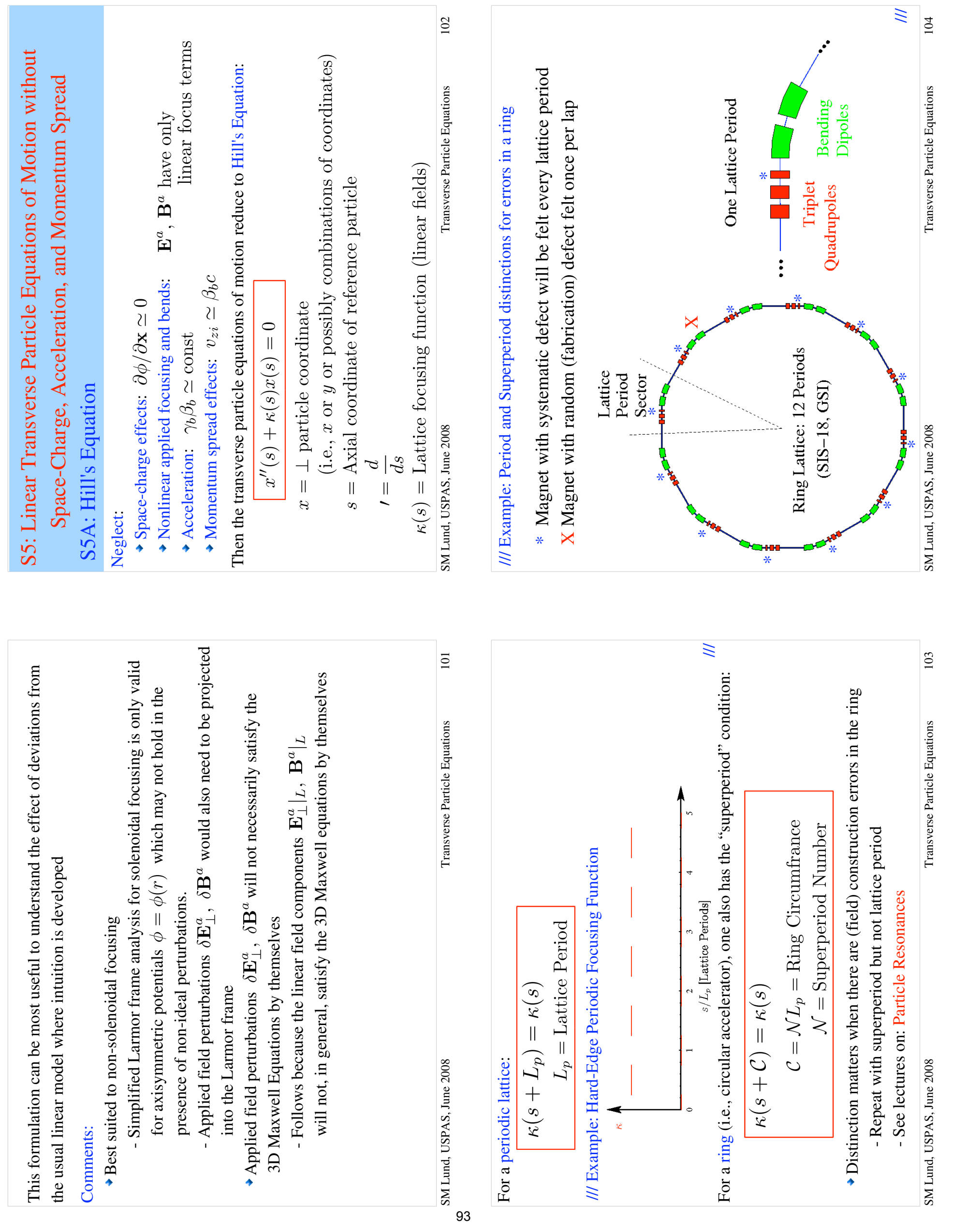



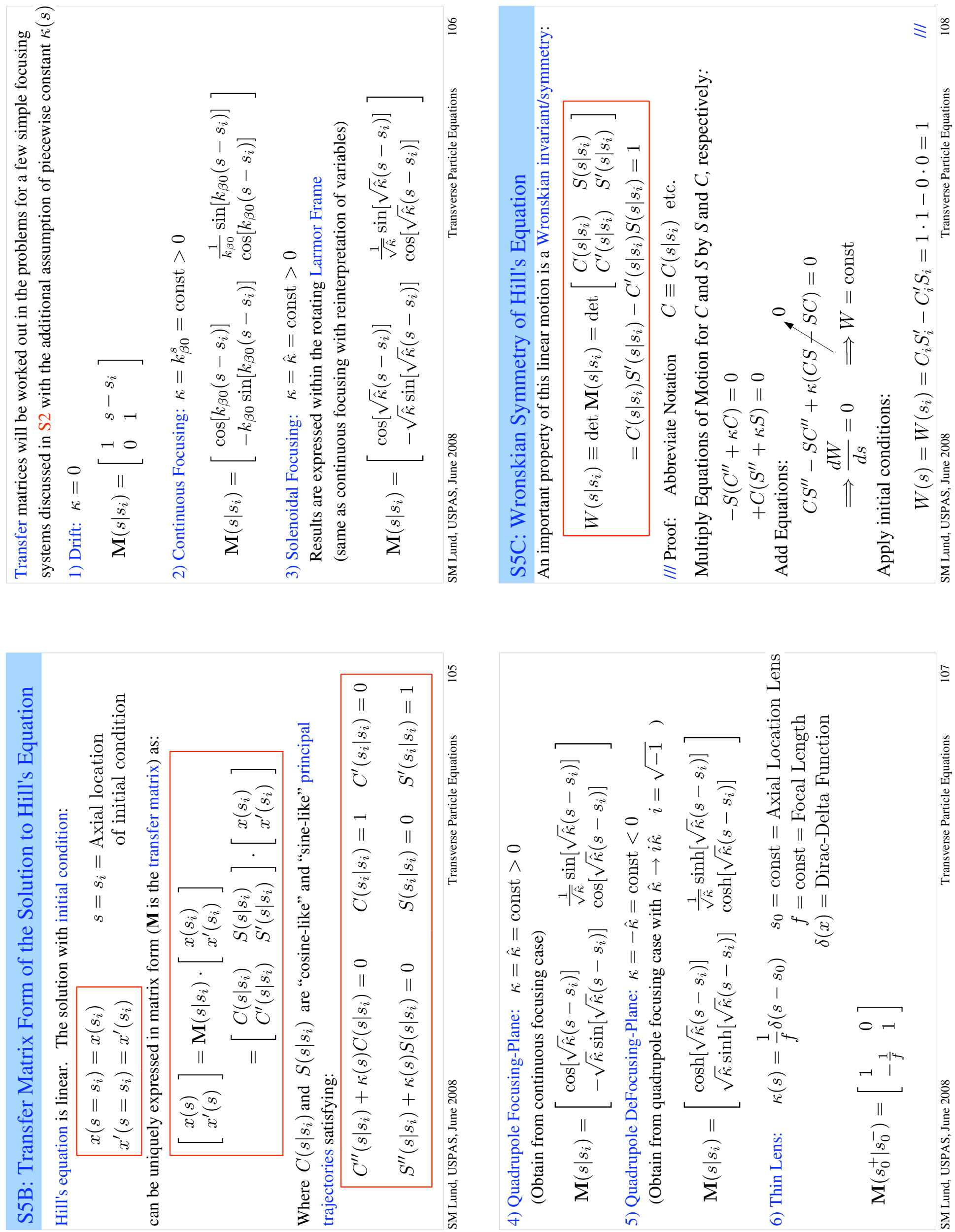

$\stackrel{\circ}{\circ}$

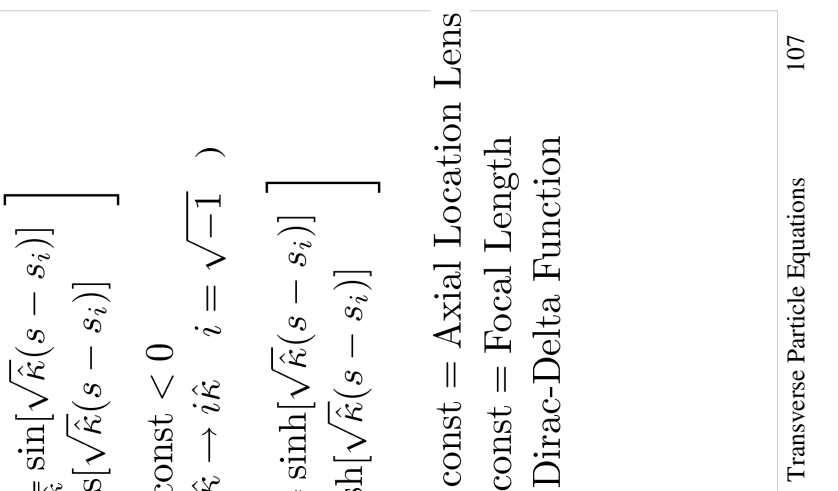

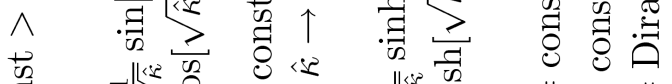

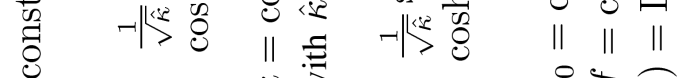

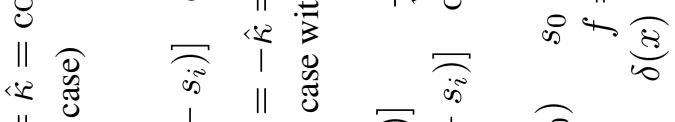

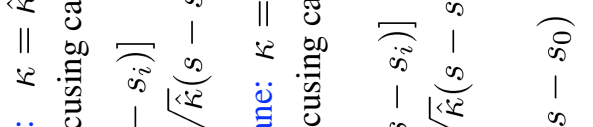

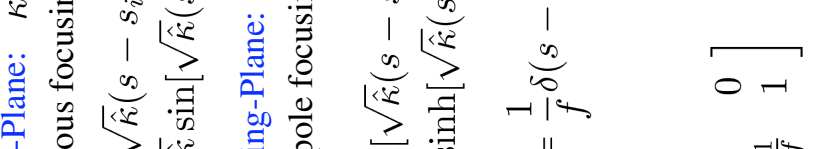

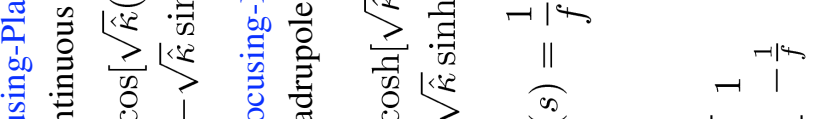

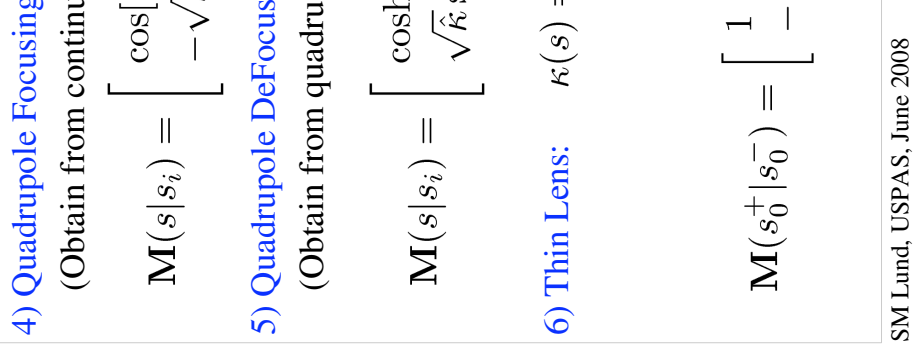



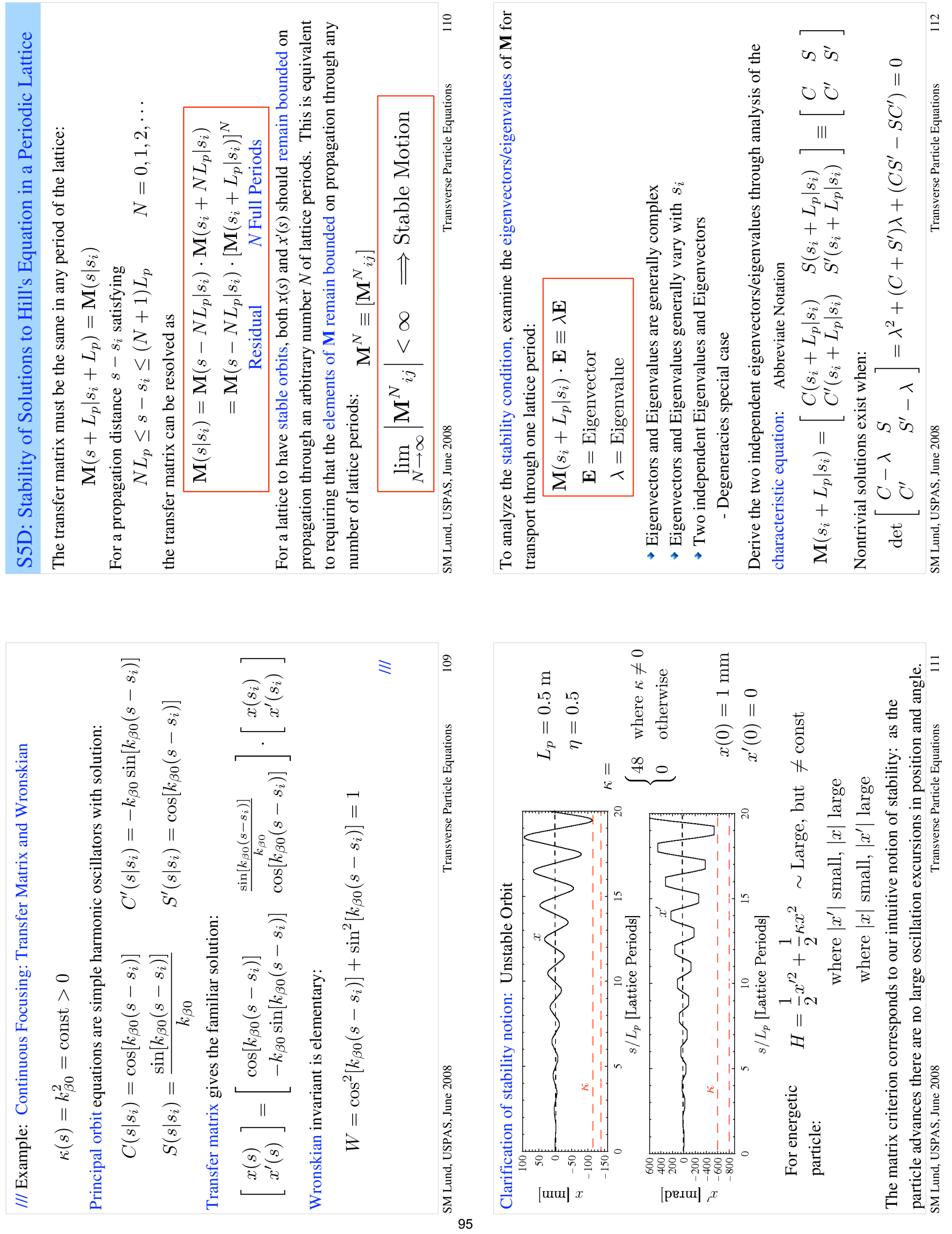

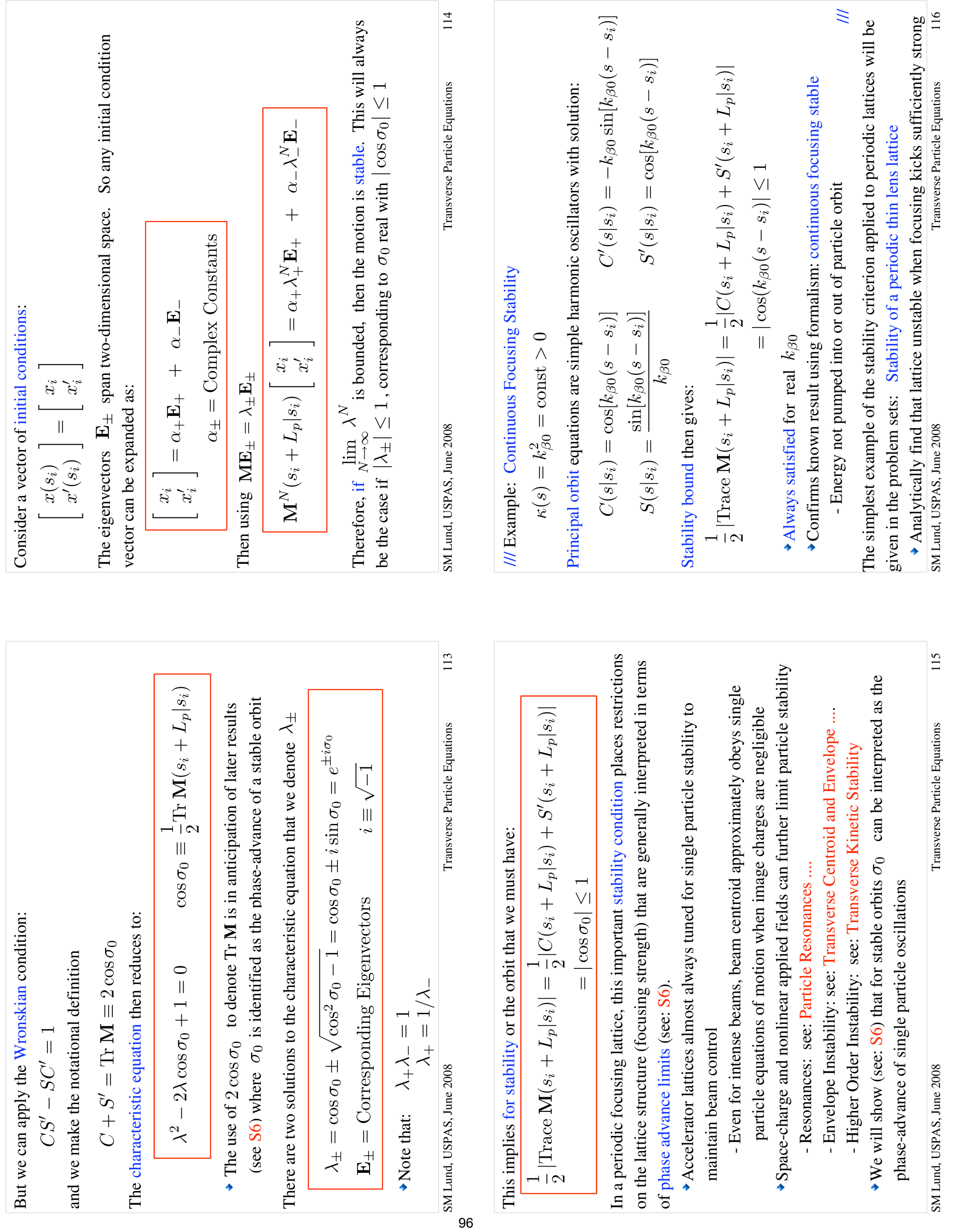

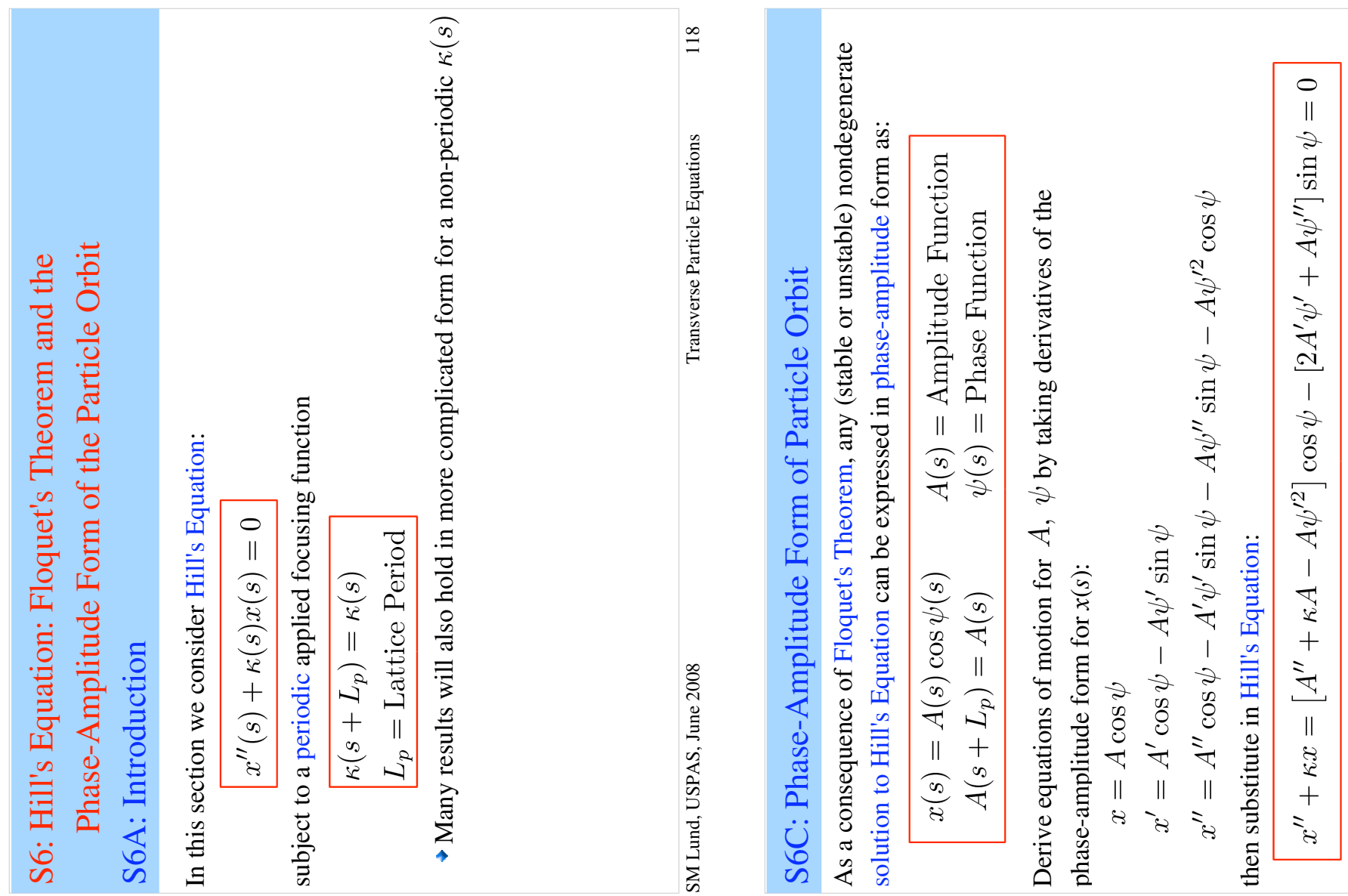

ฐ

$<$
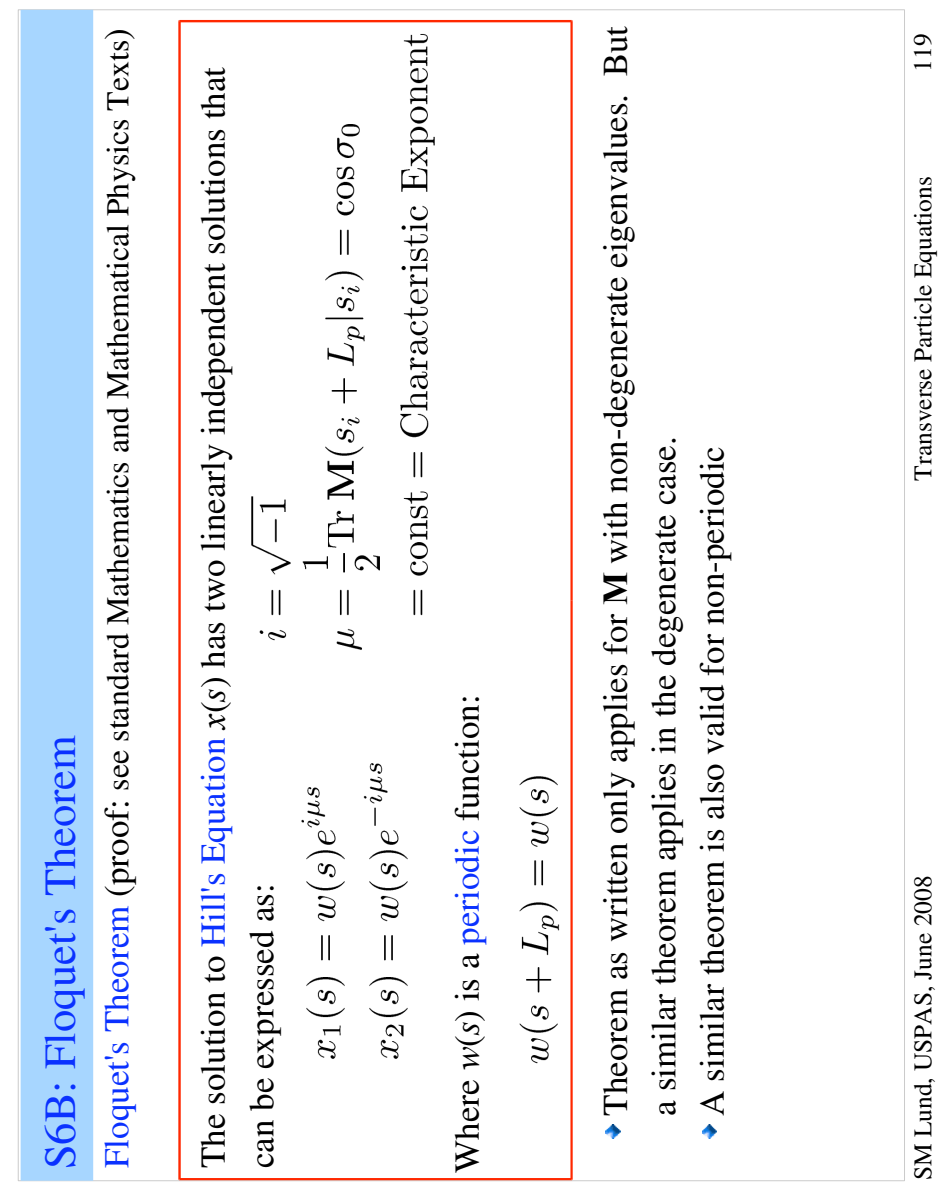

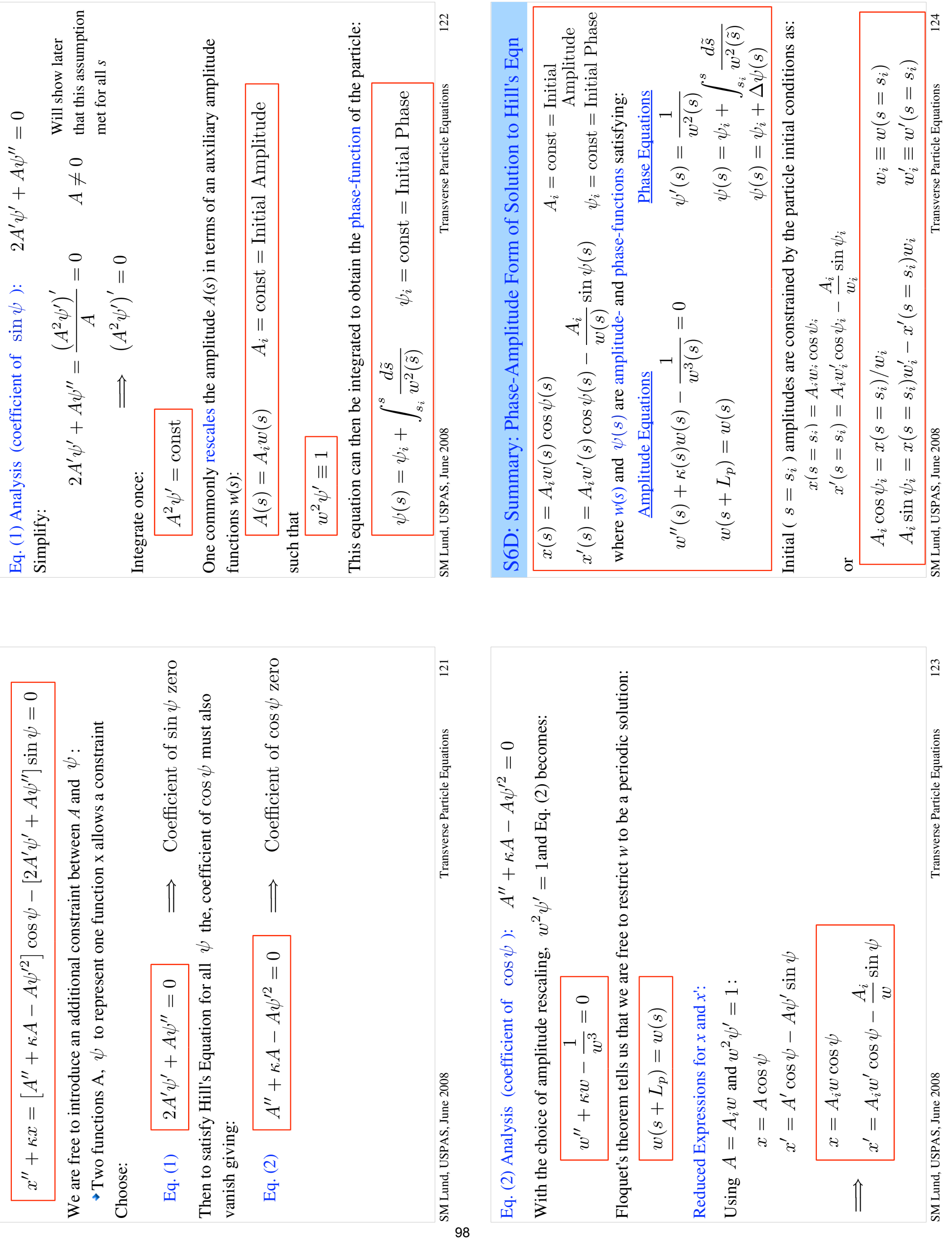

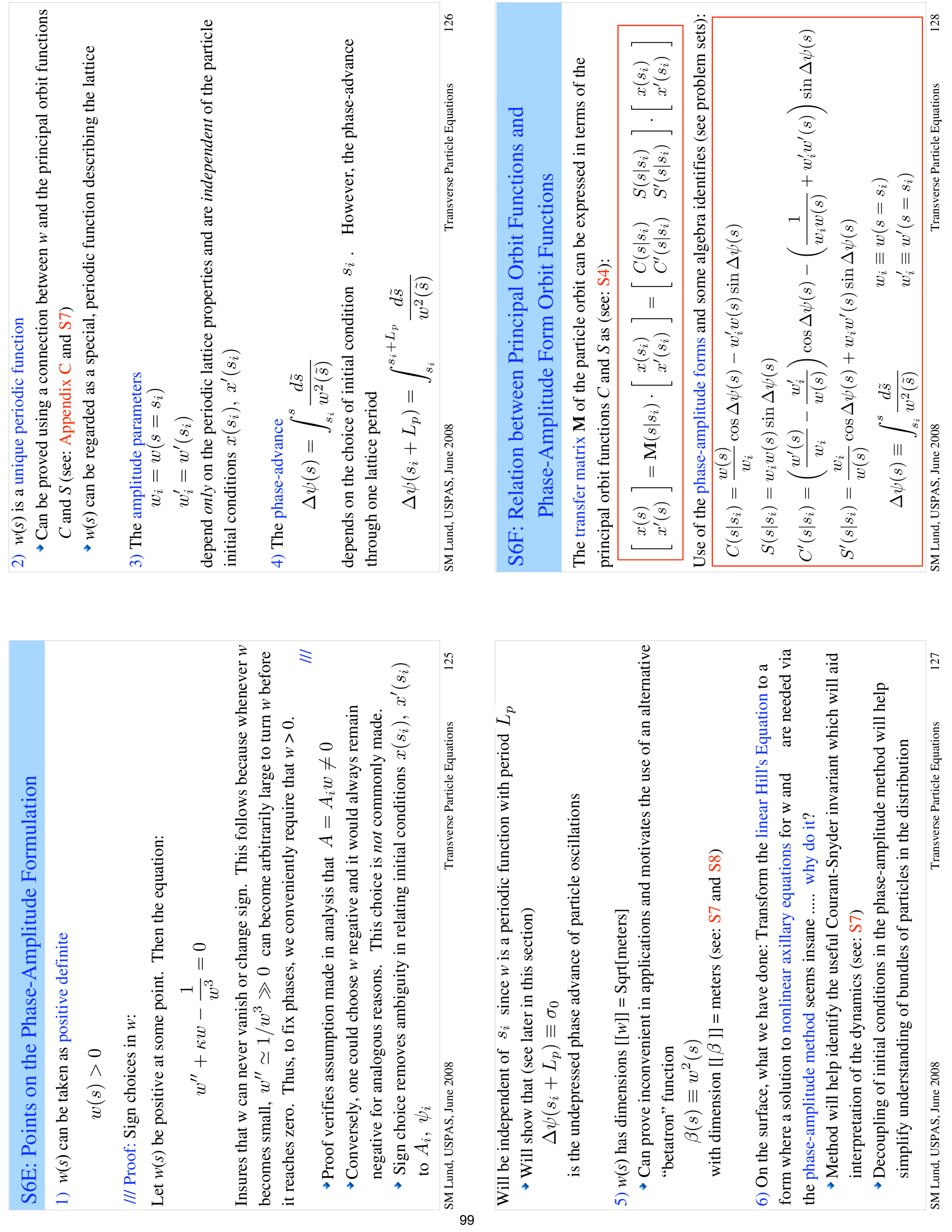
$\leqslant$

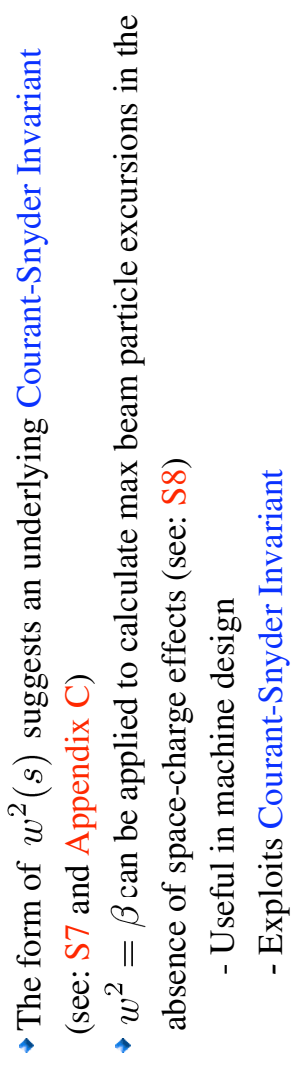

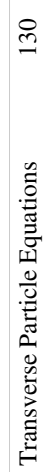

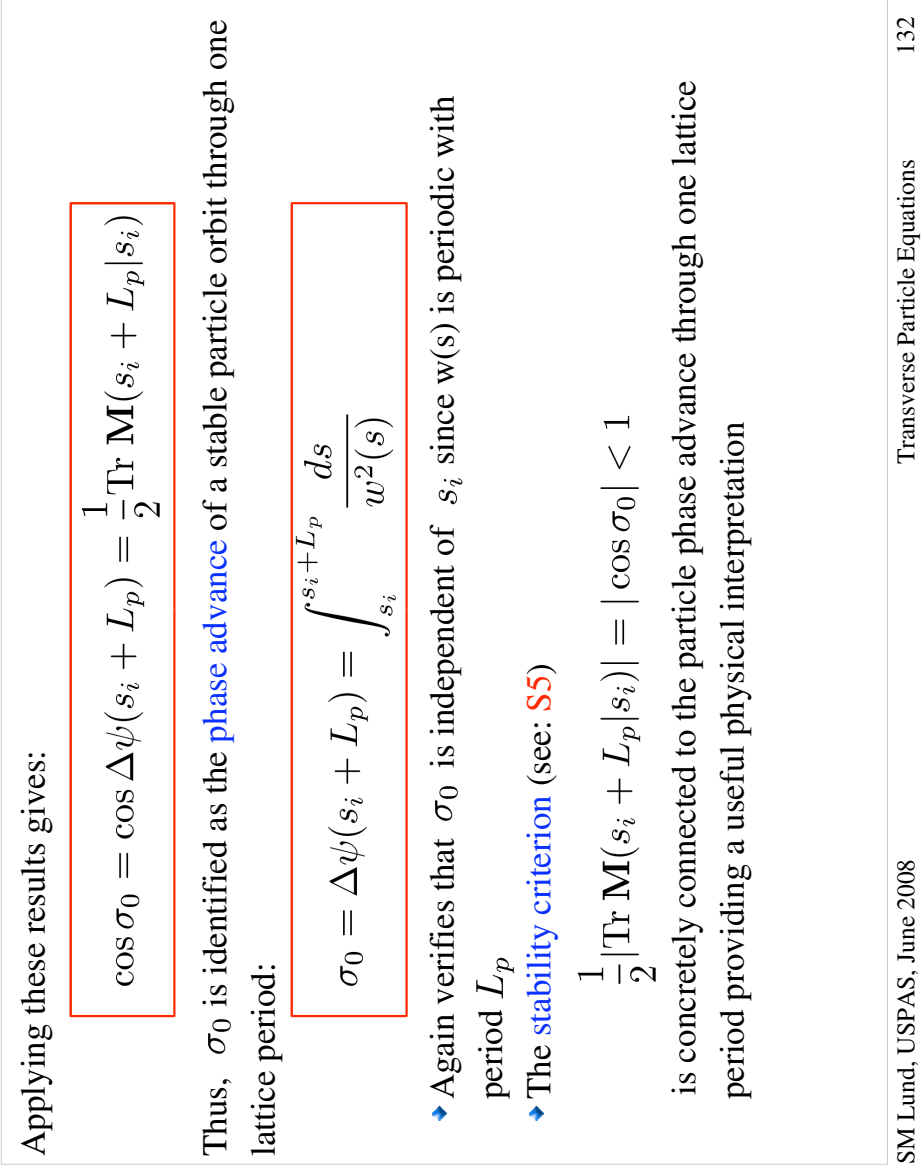

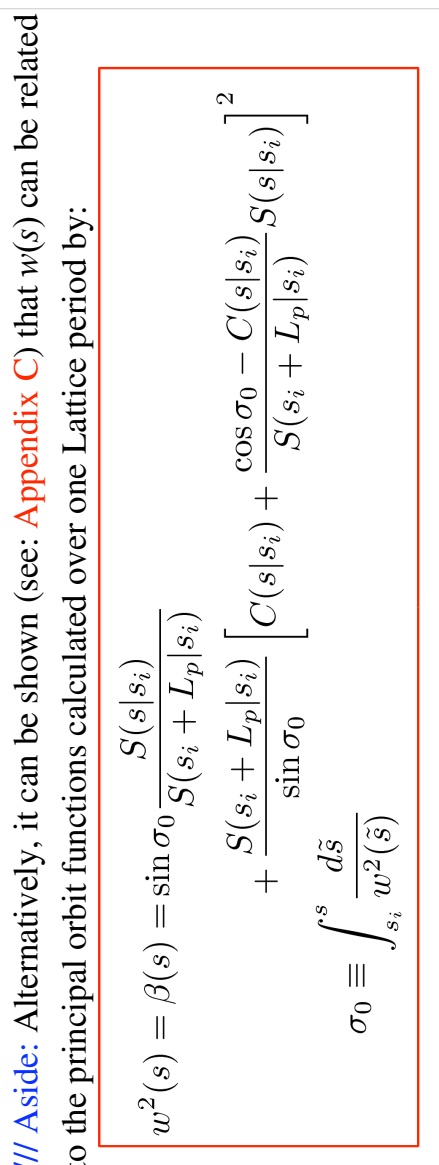

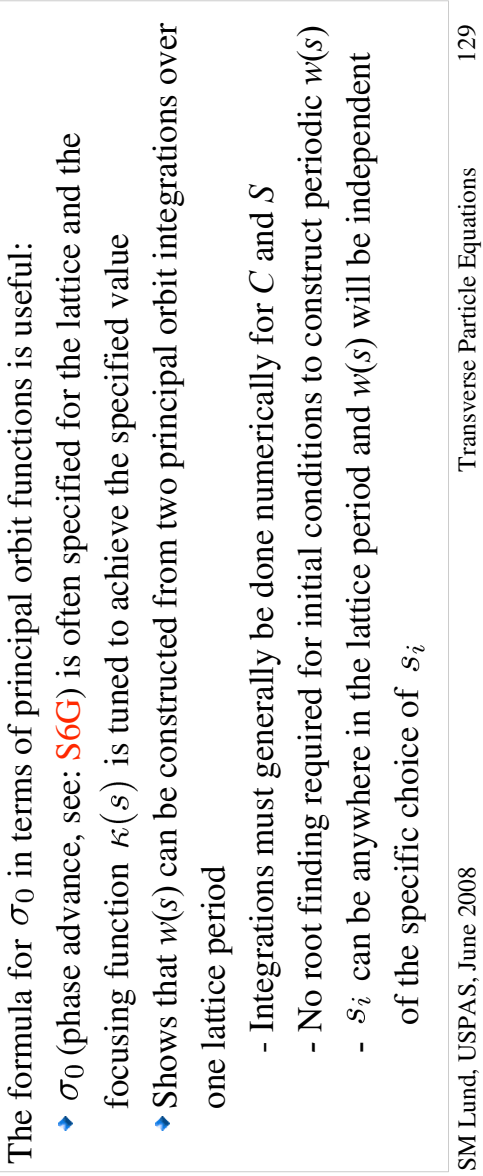

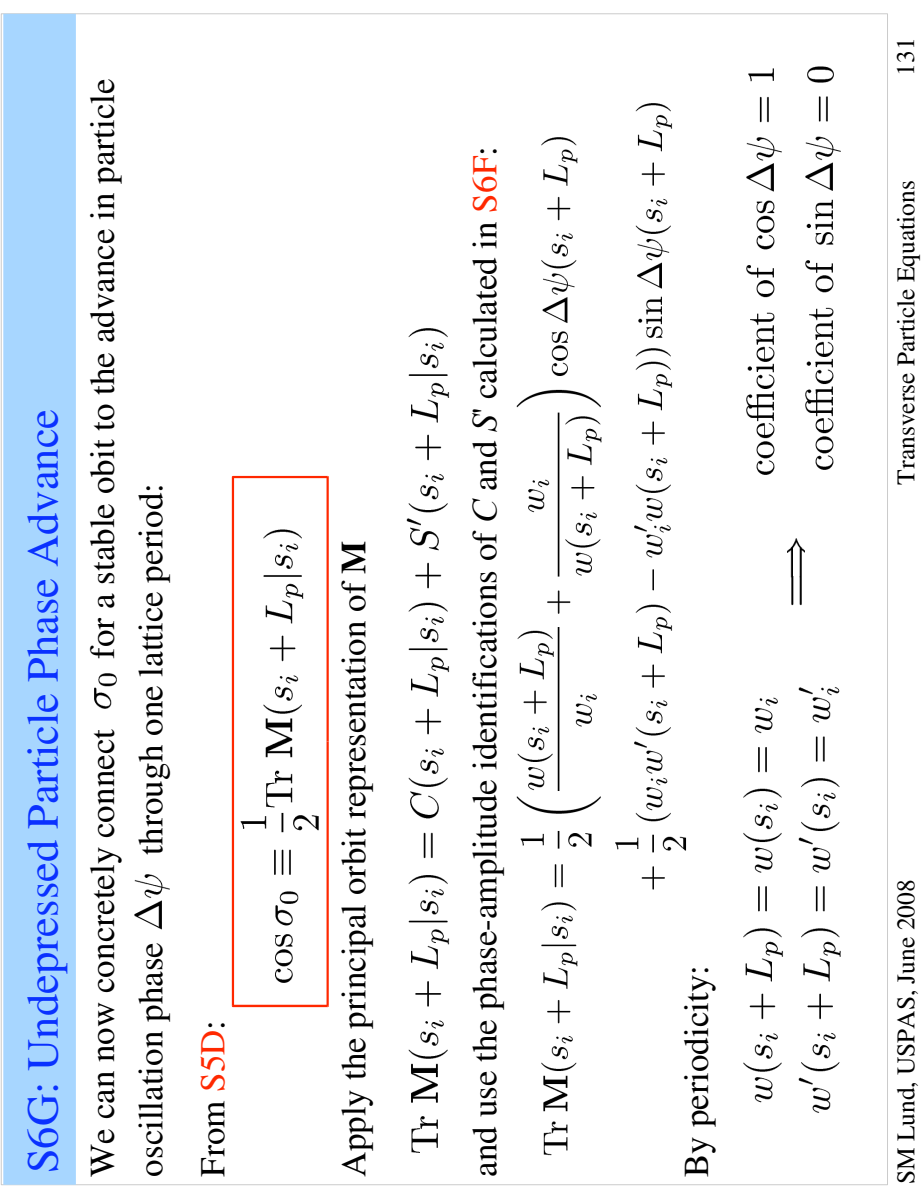



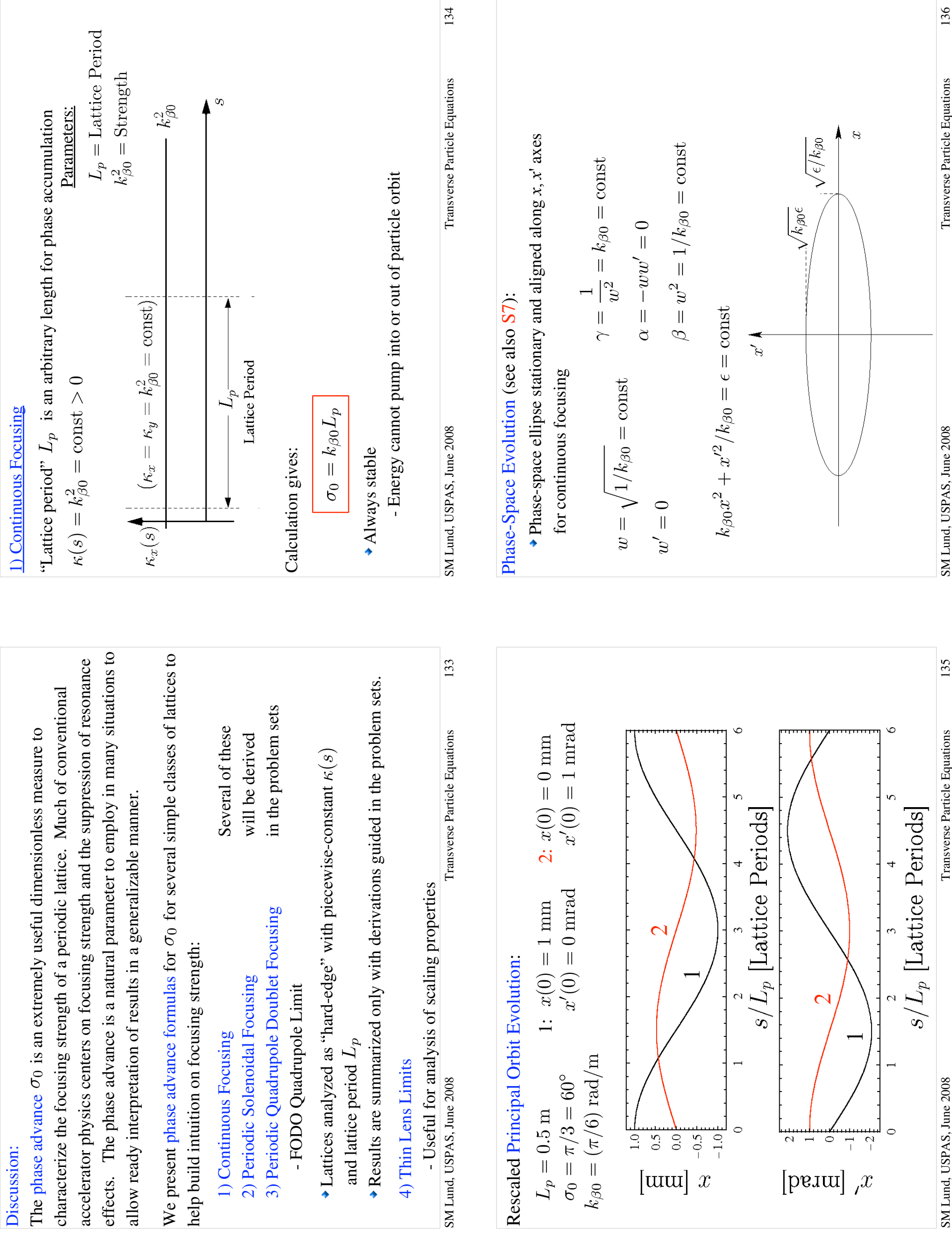

$\hat{\Omega}$
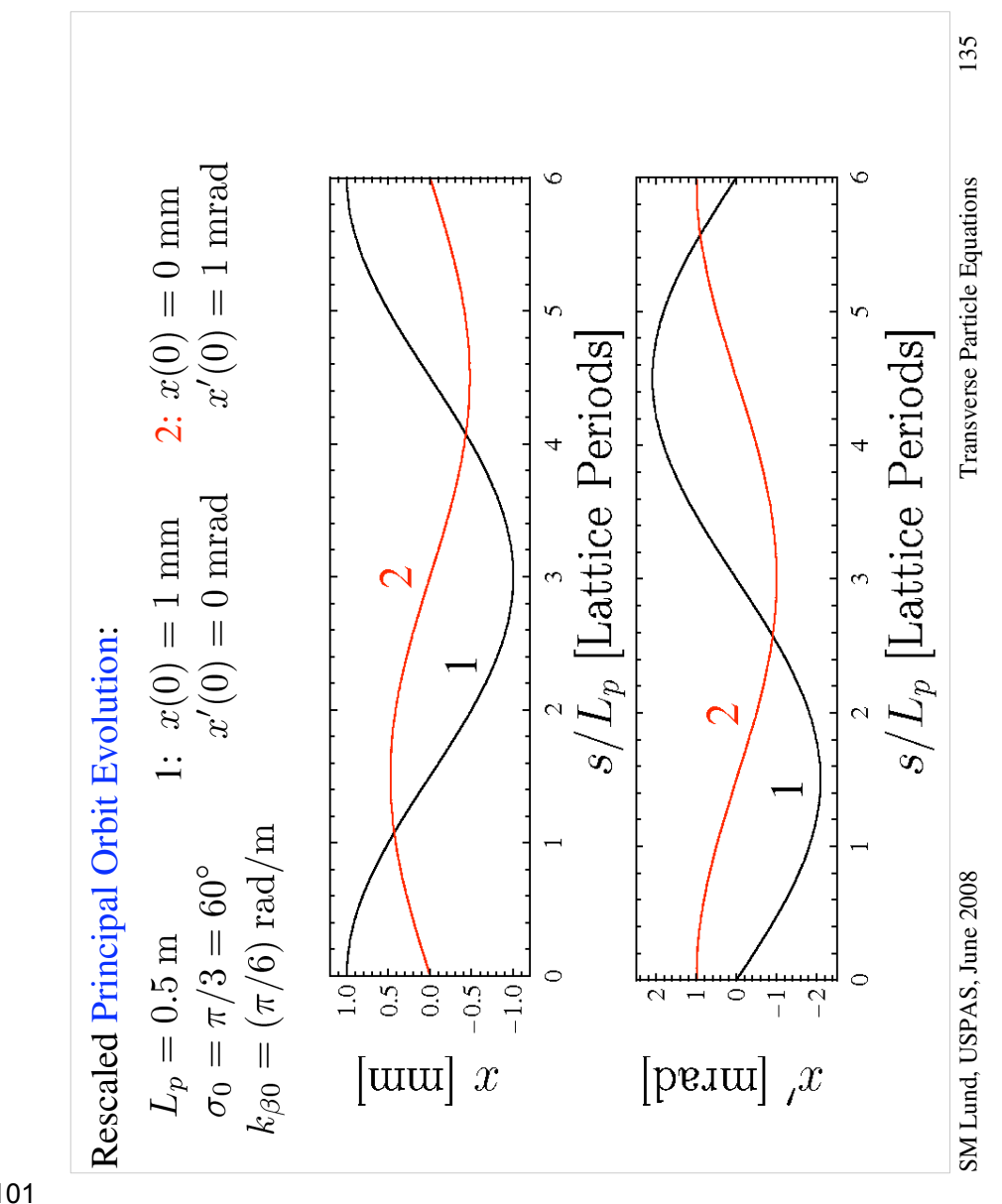

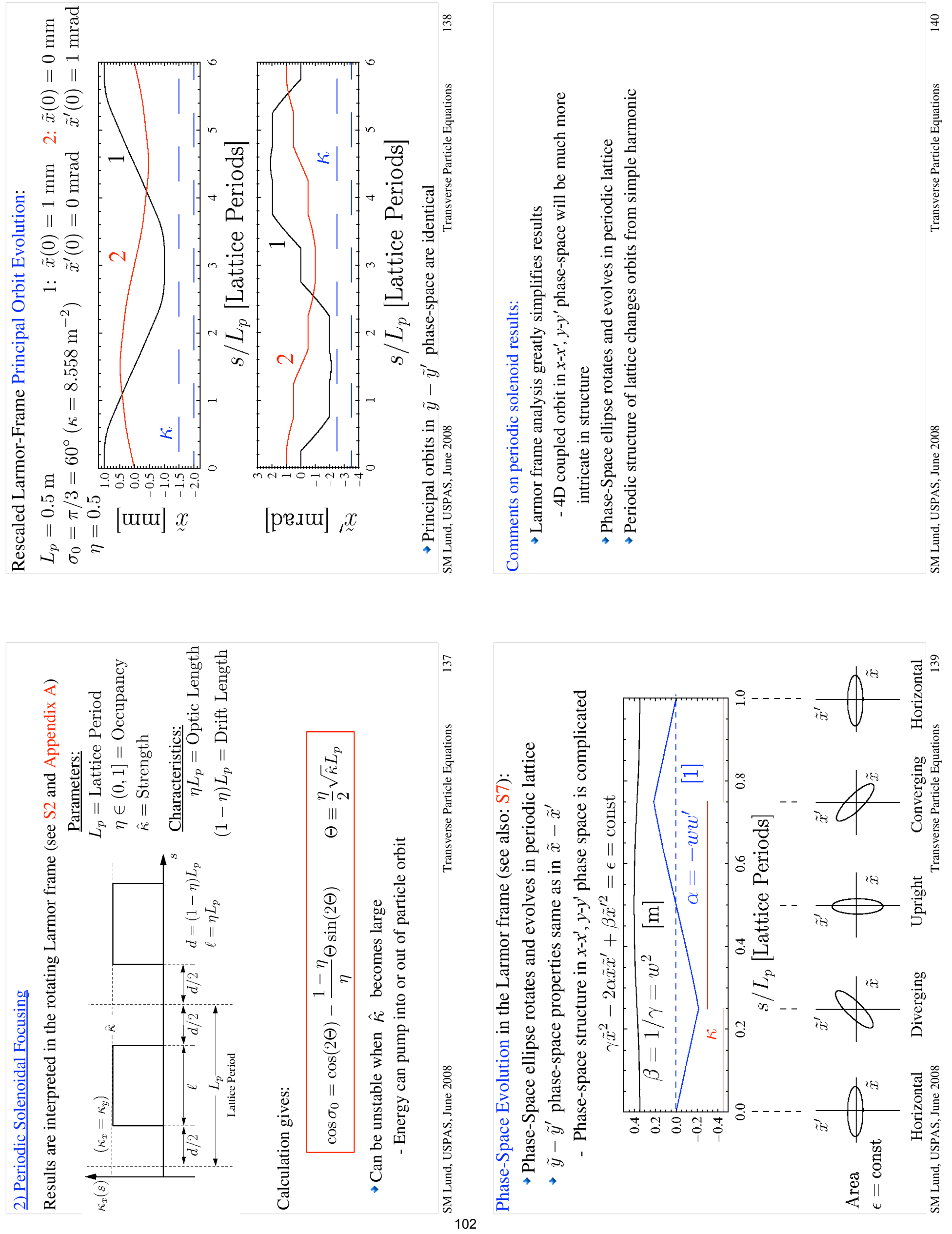

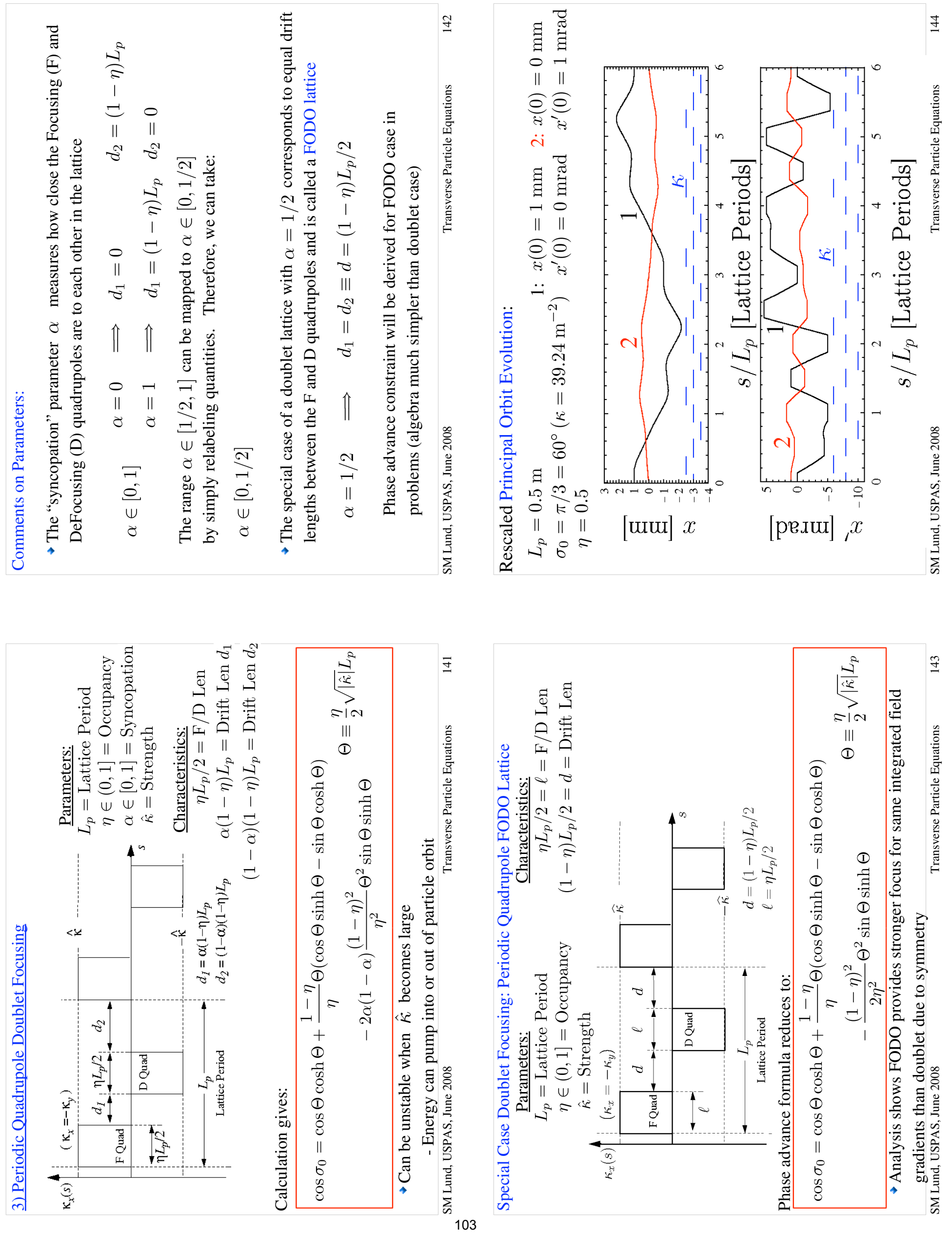

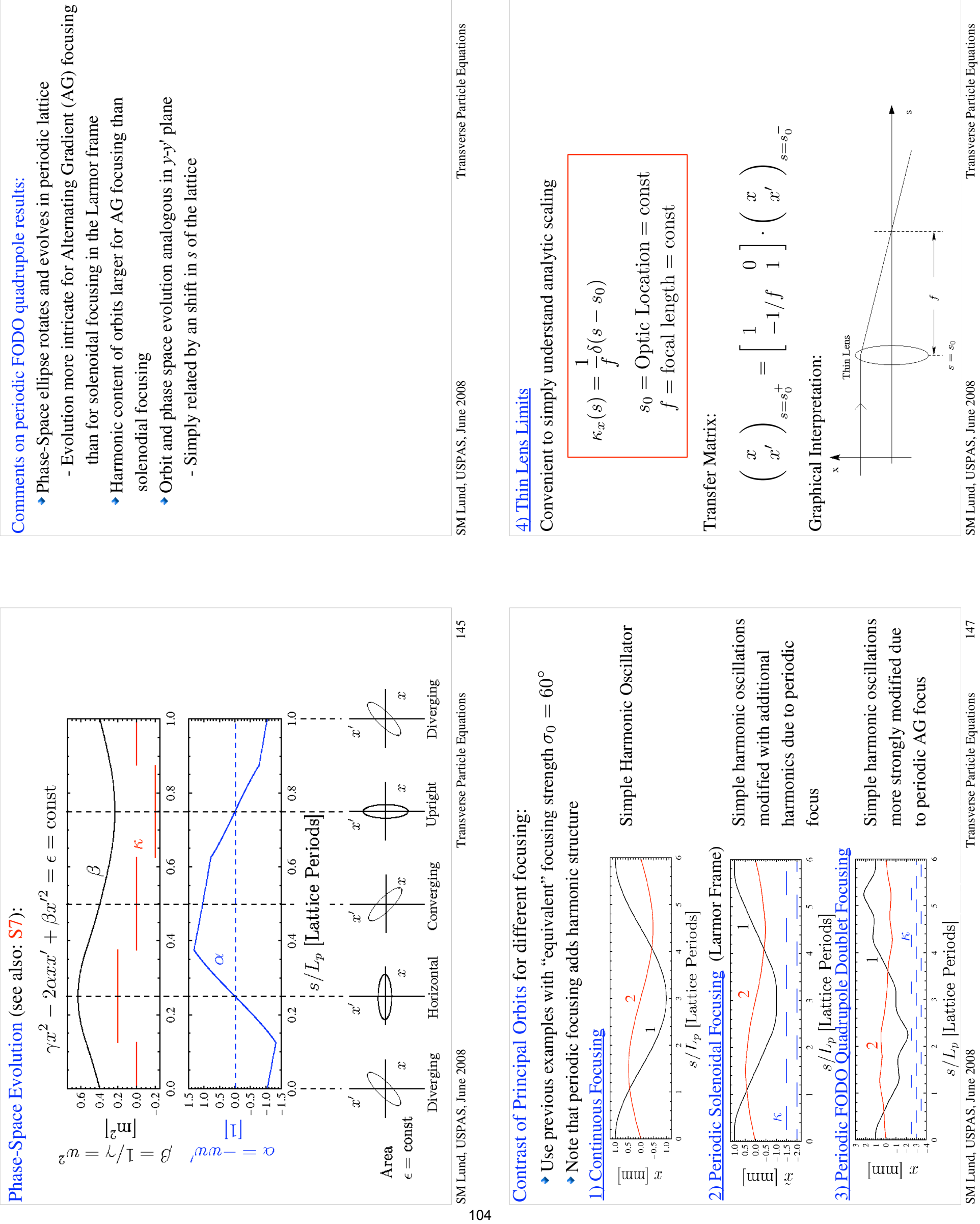

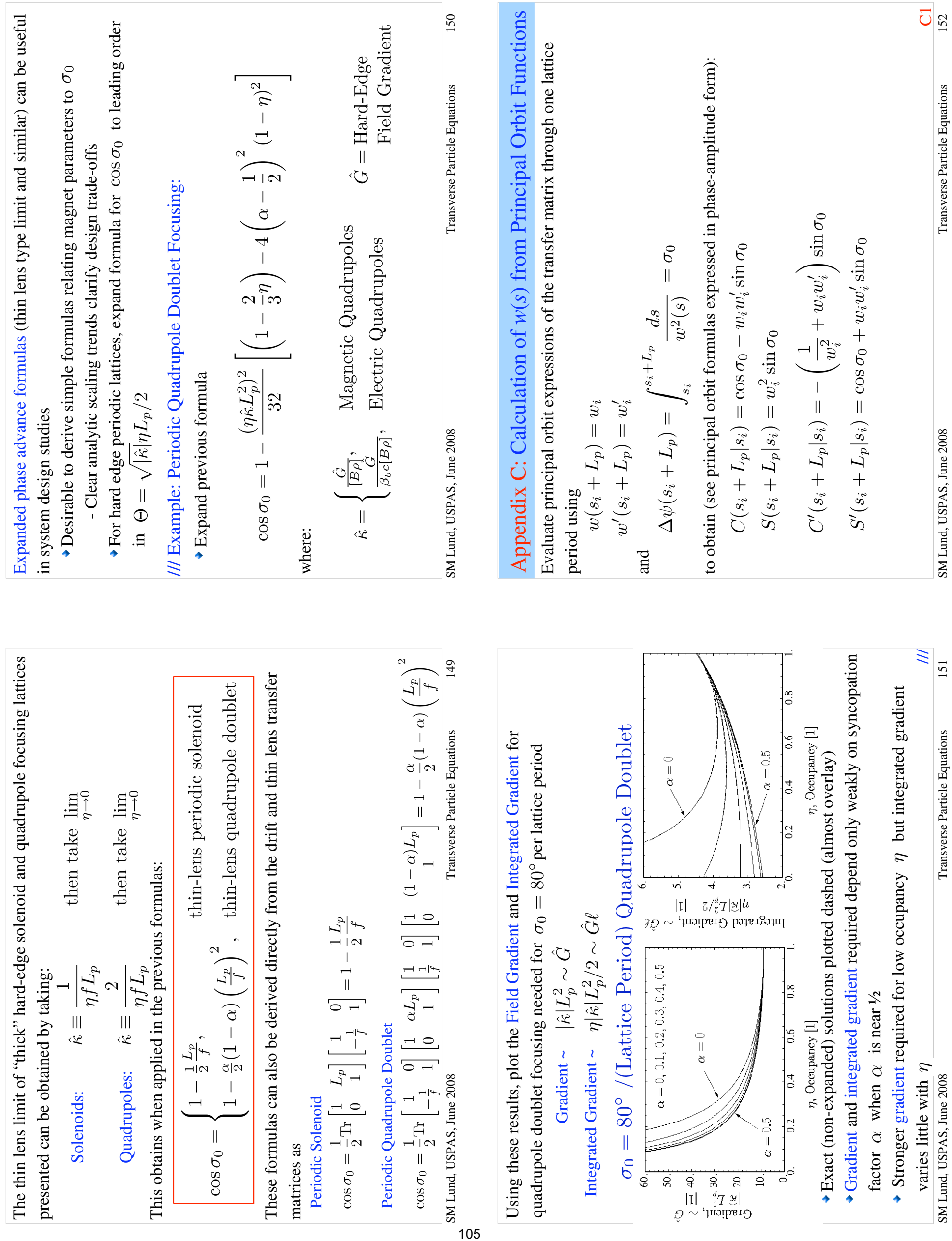

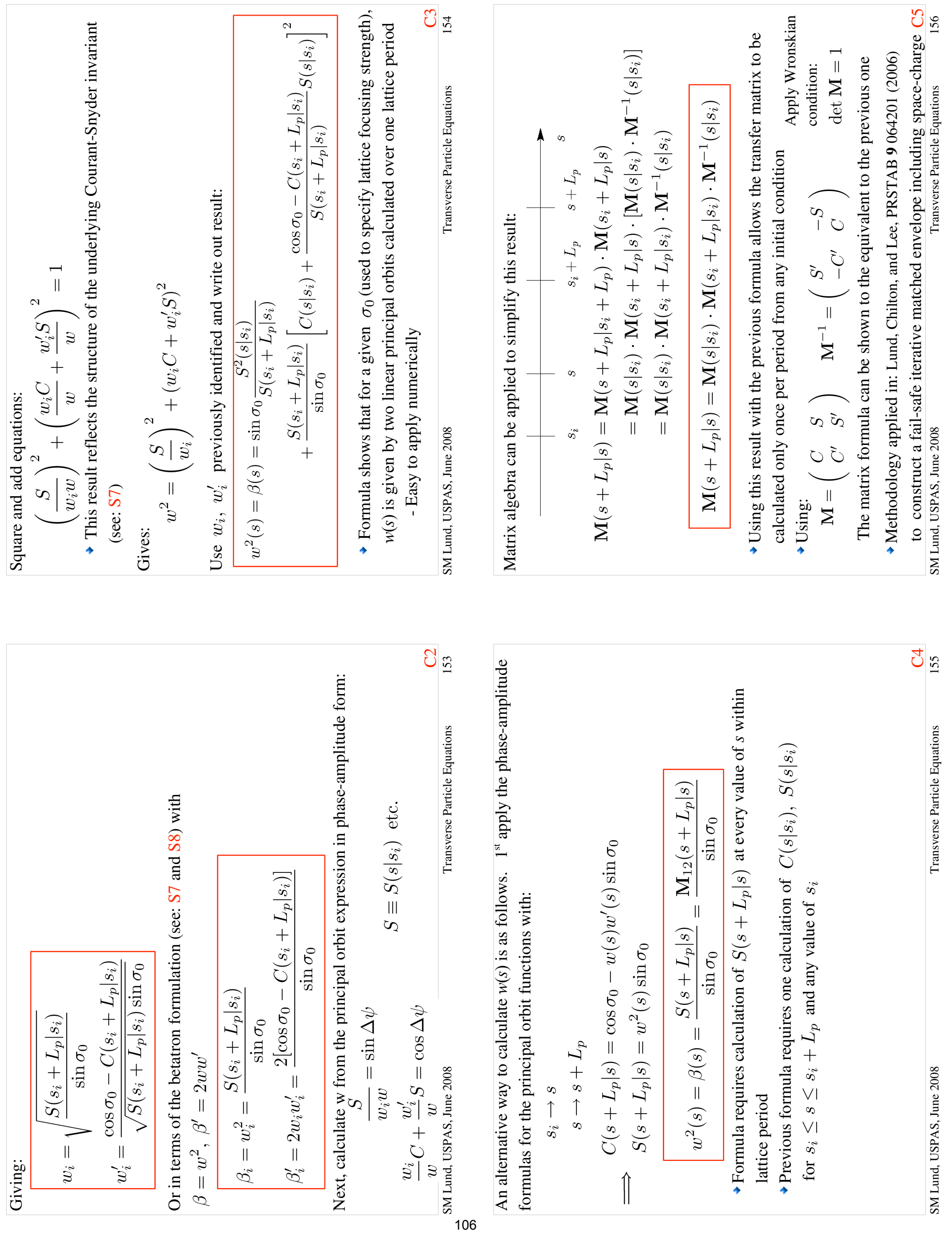

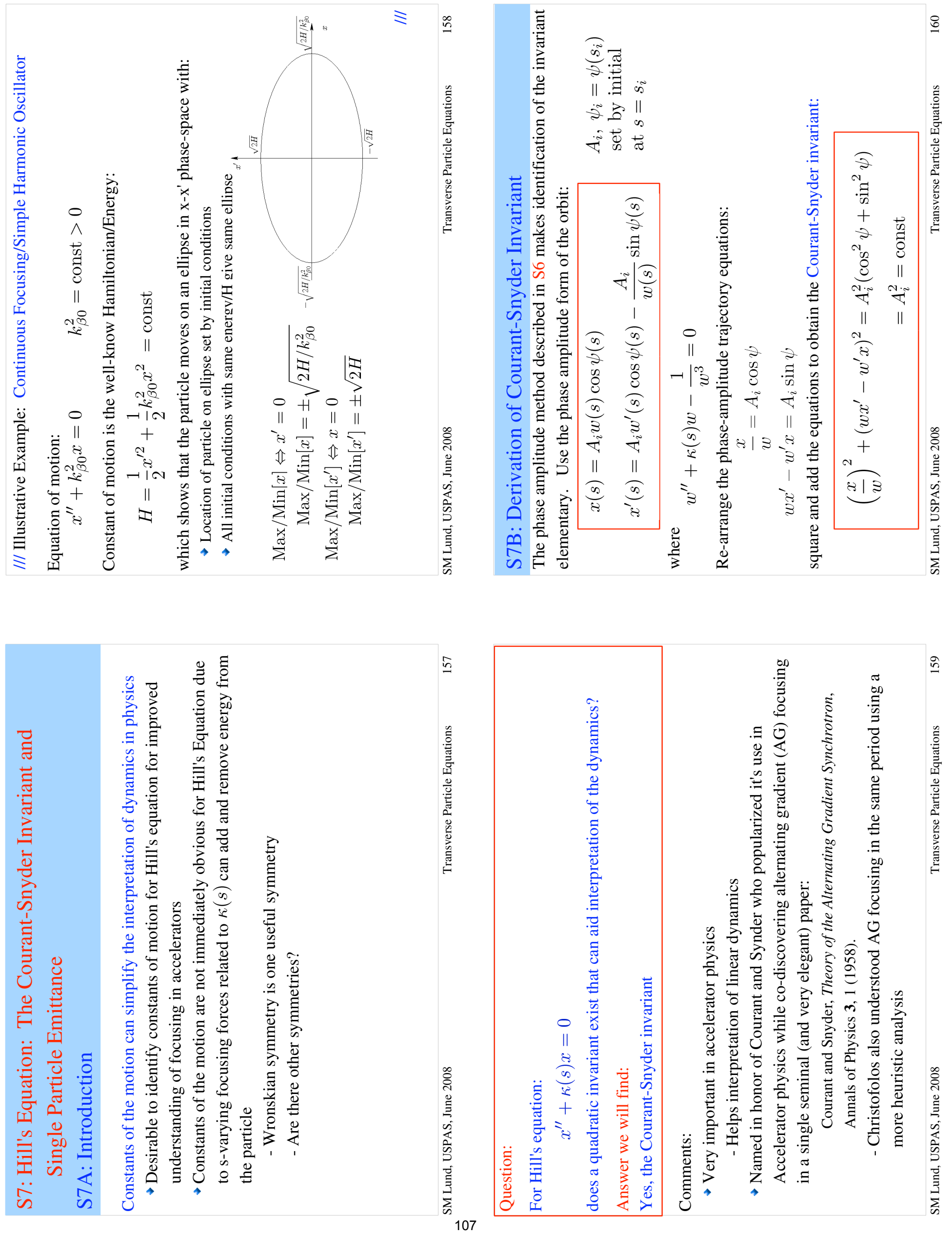

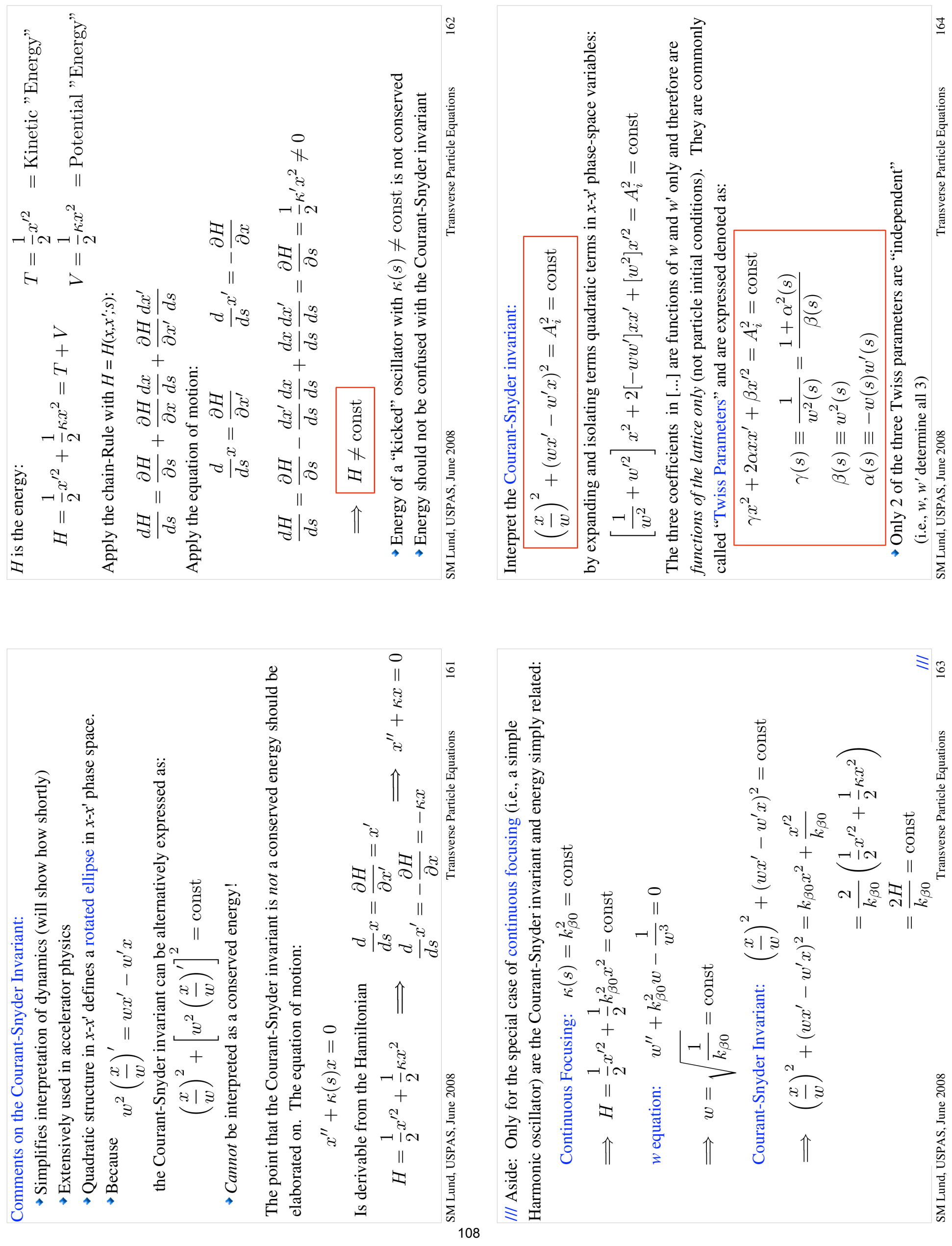

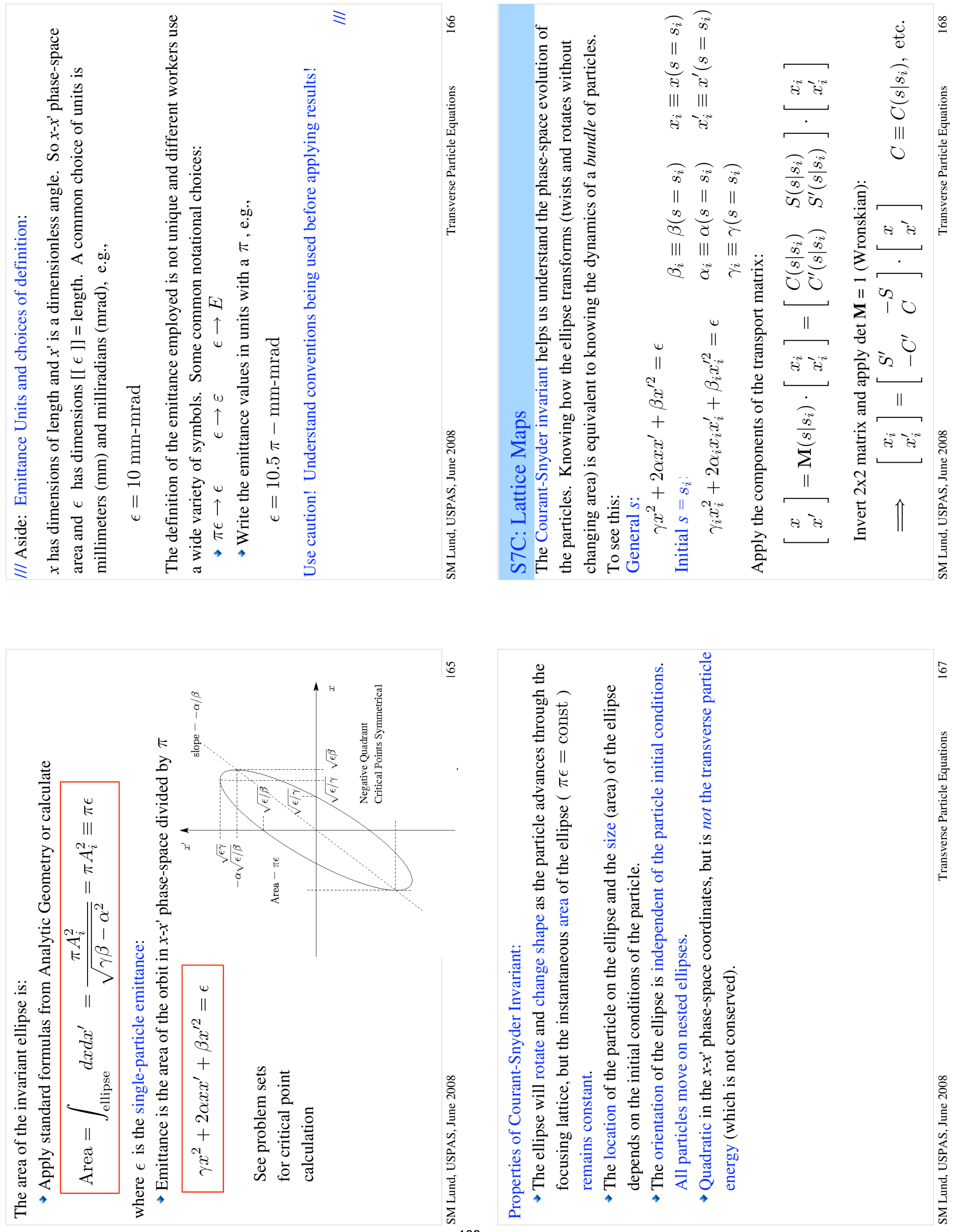

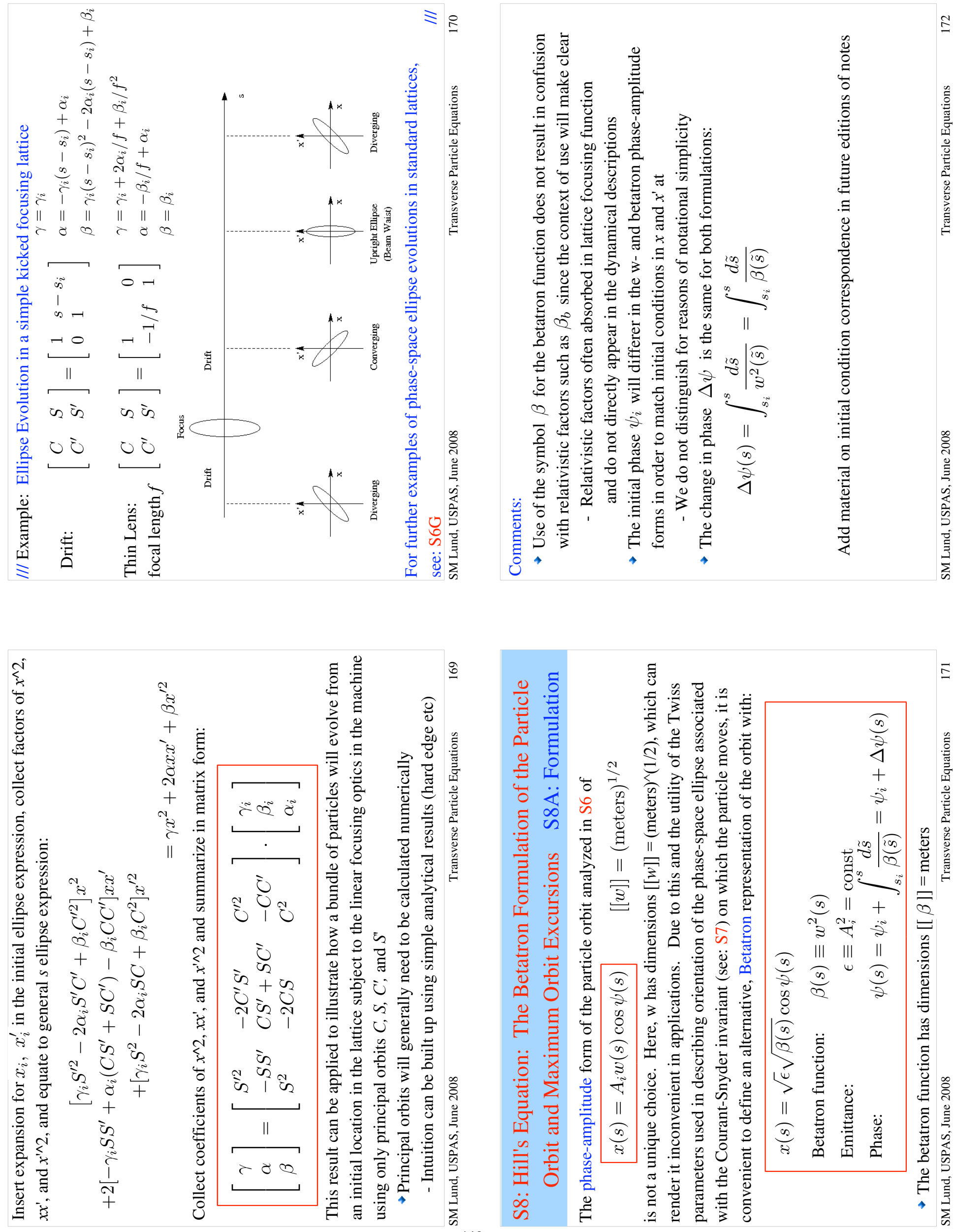


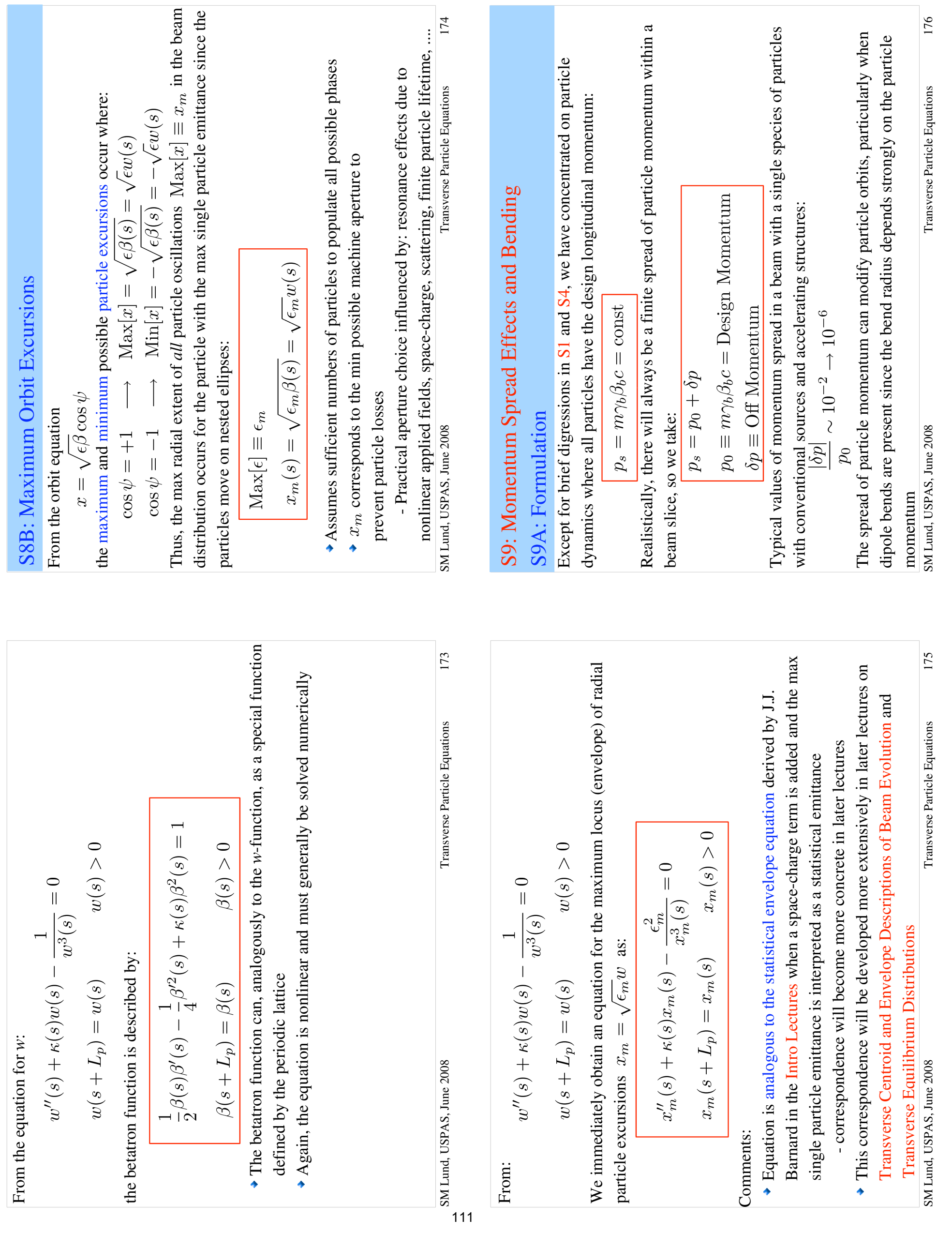



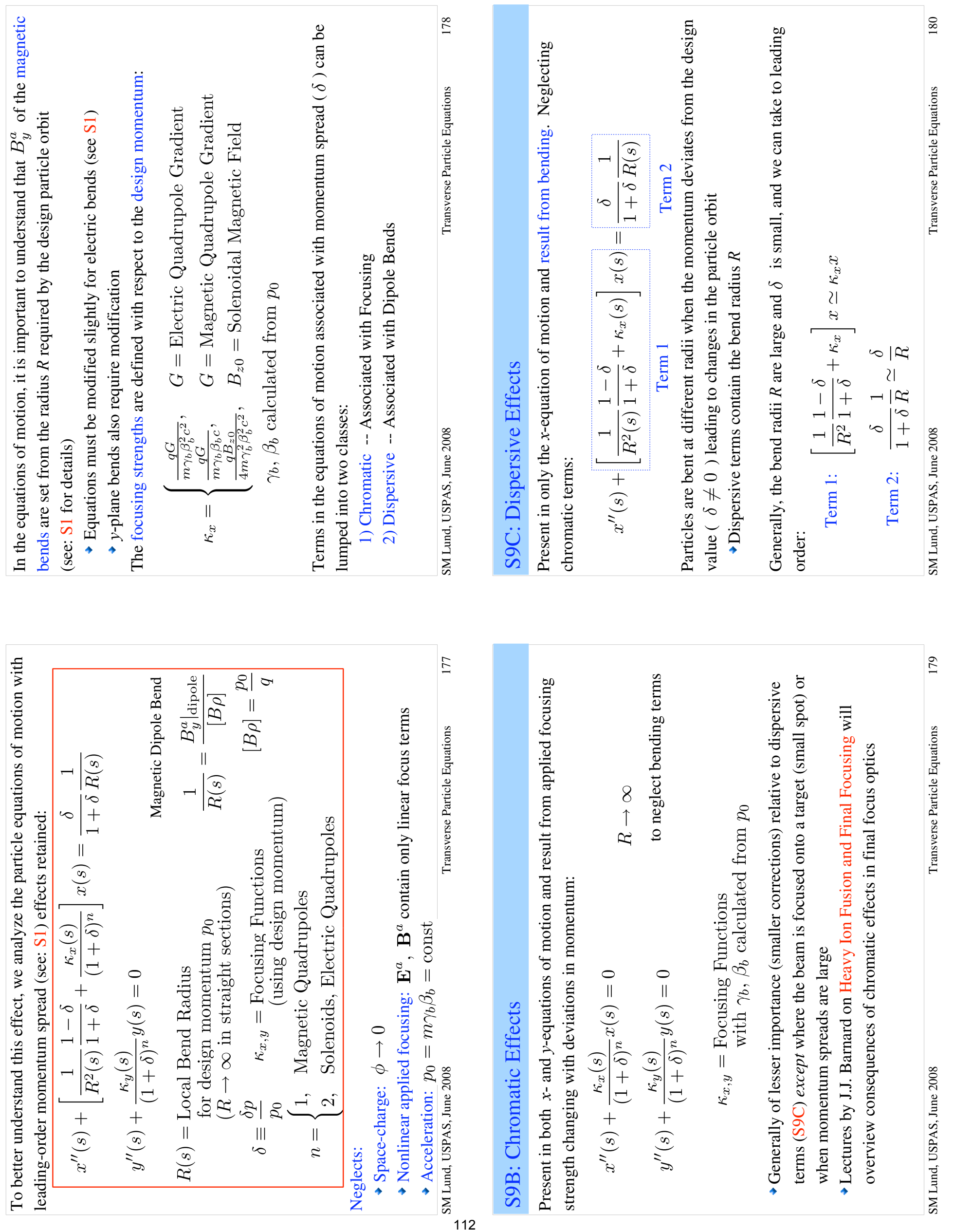

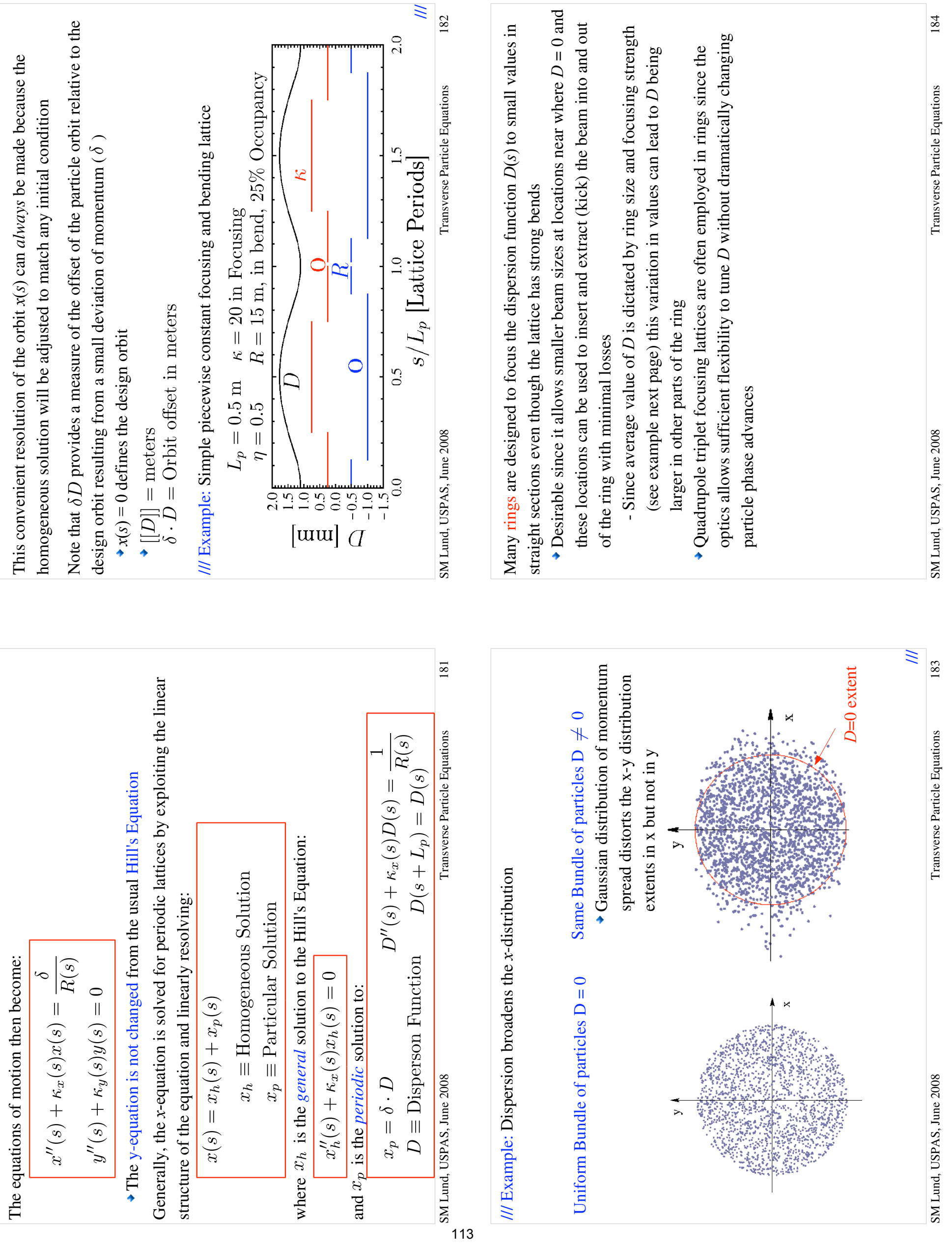

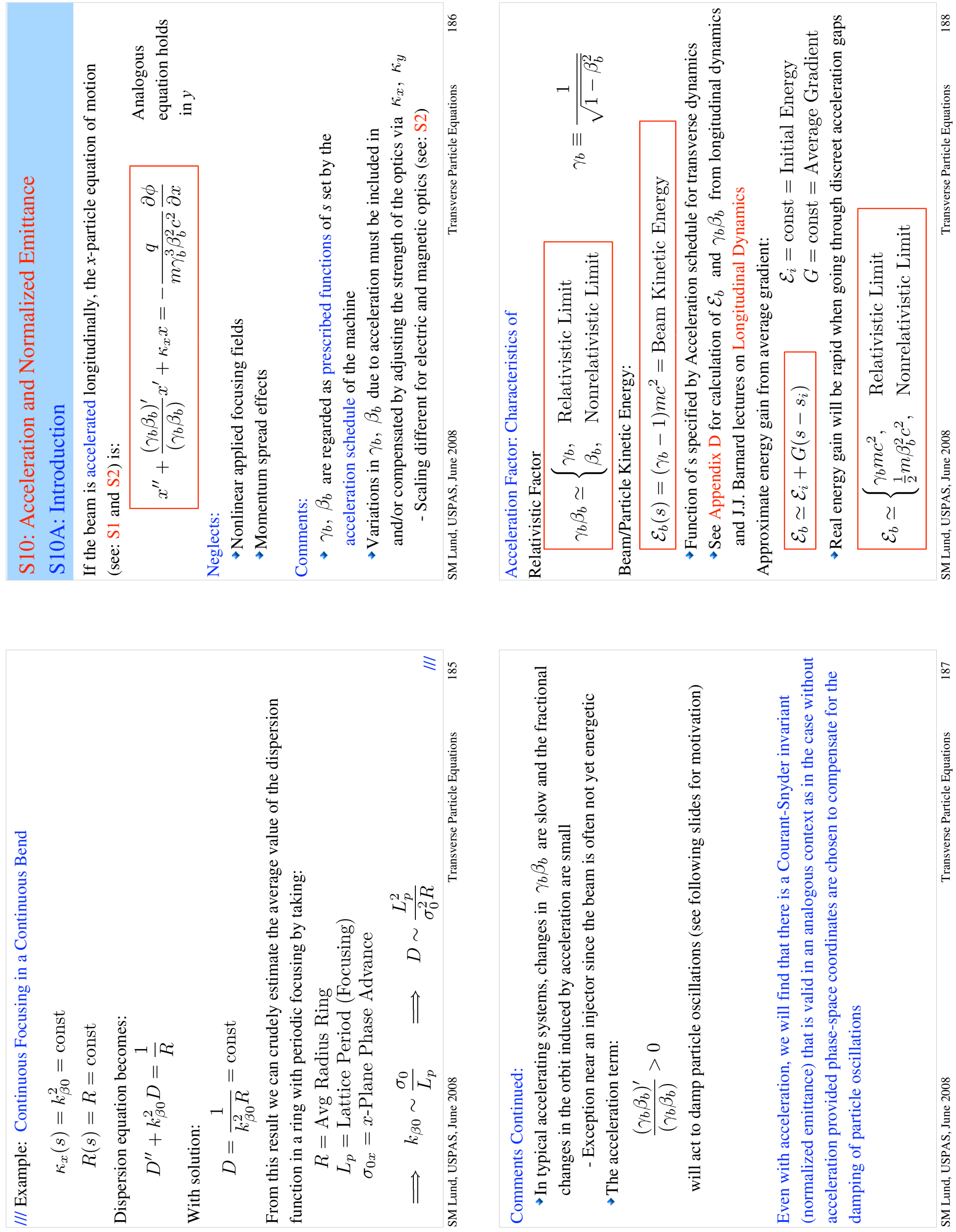

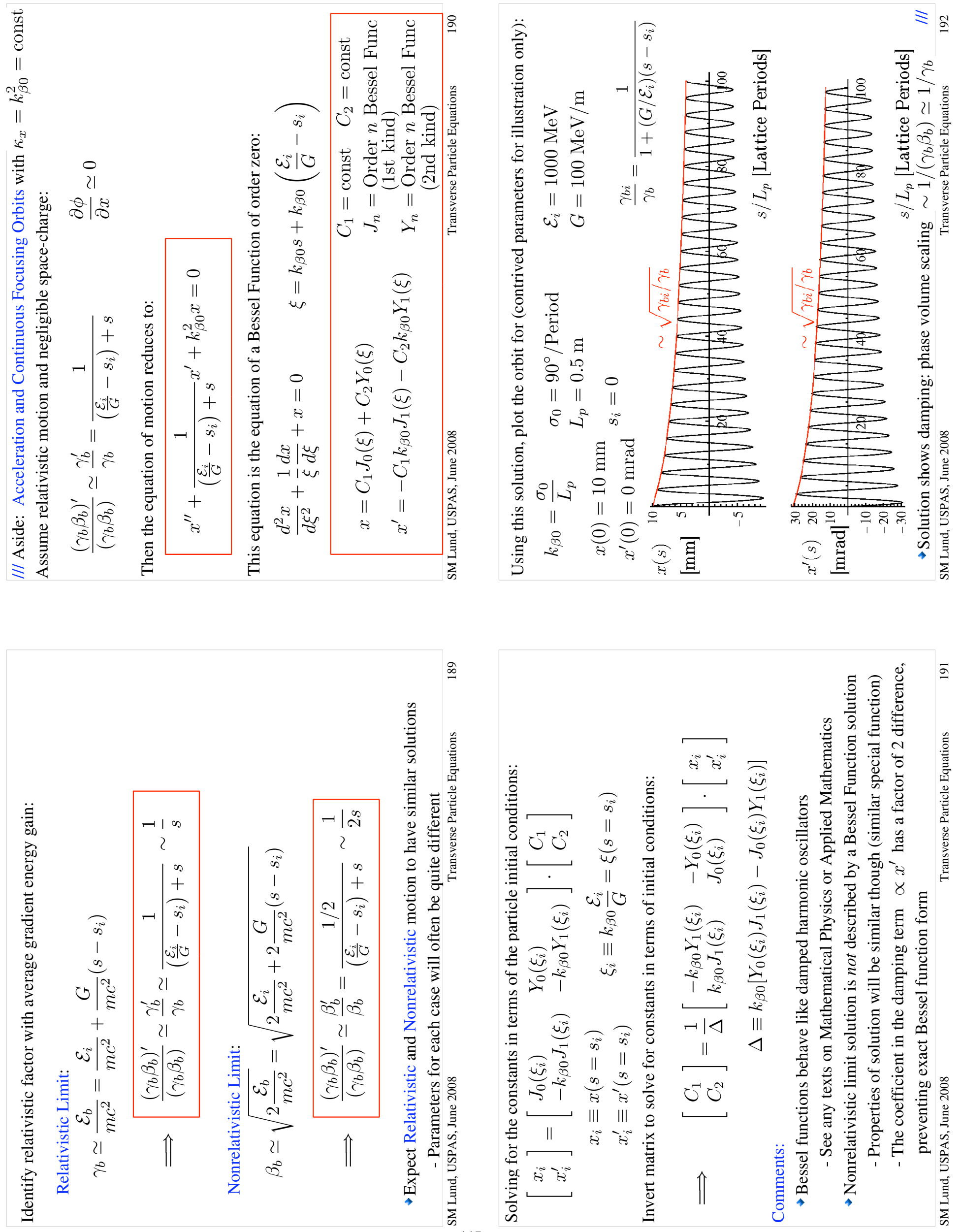

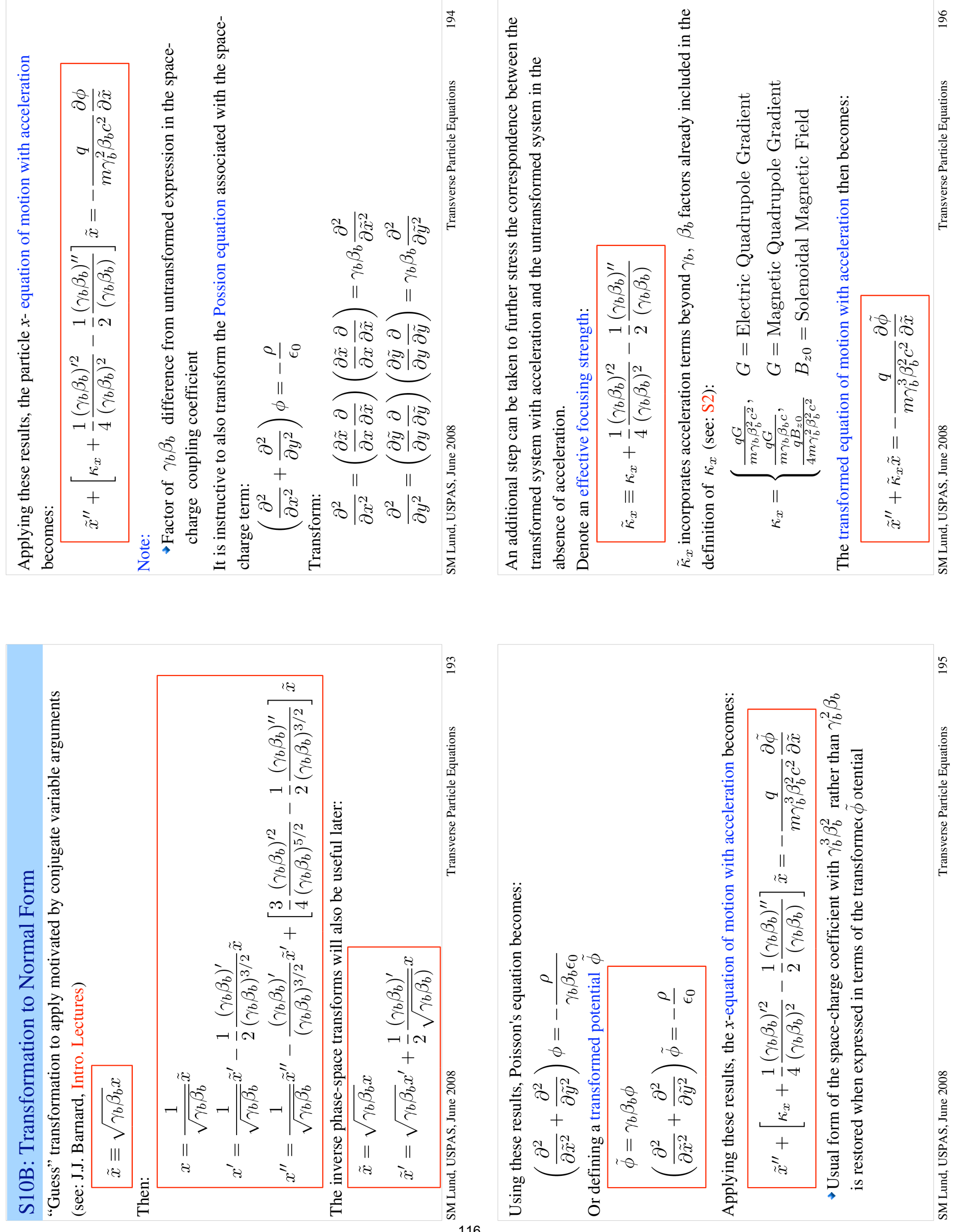

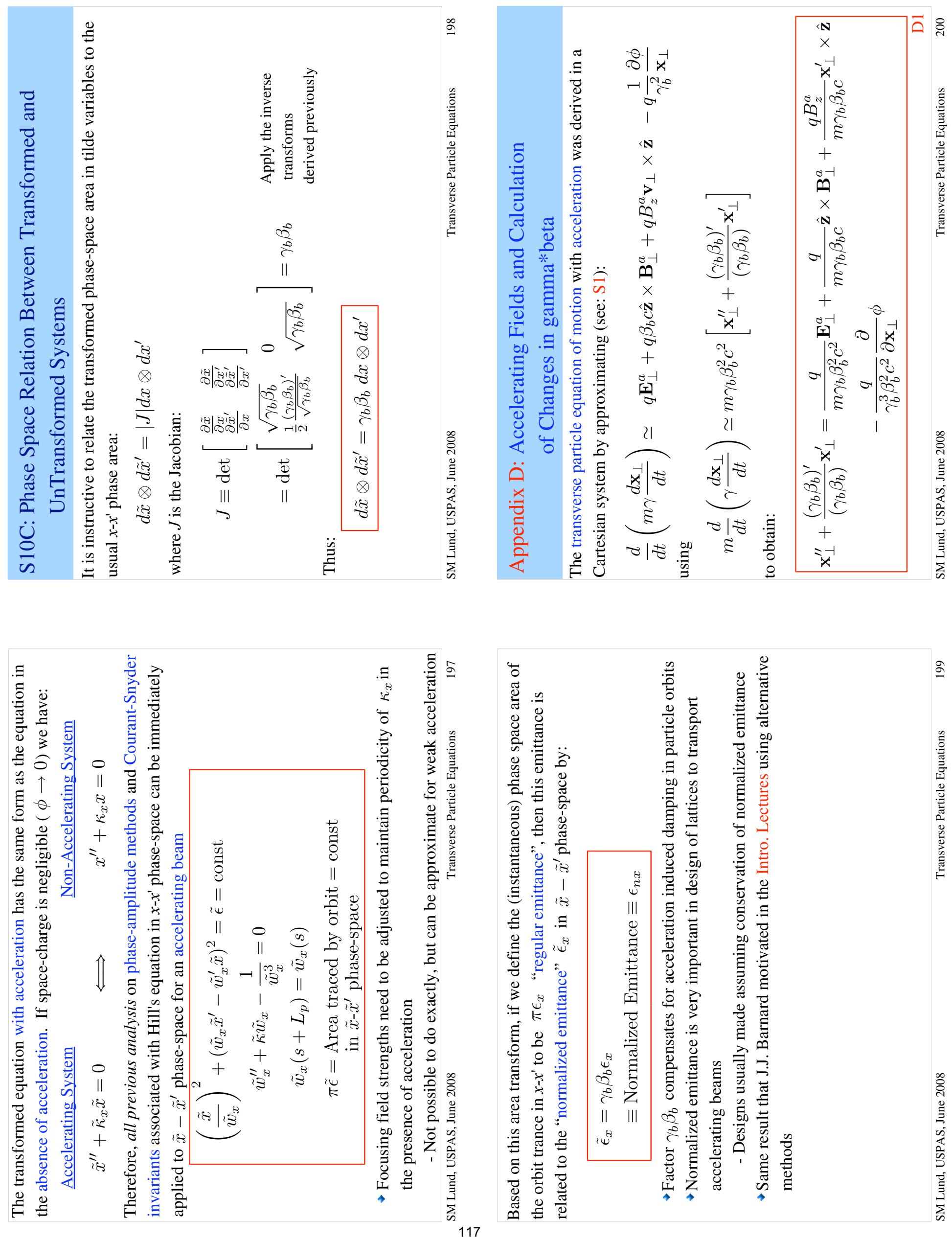


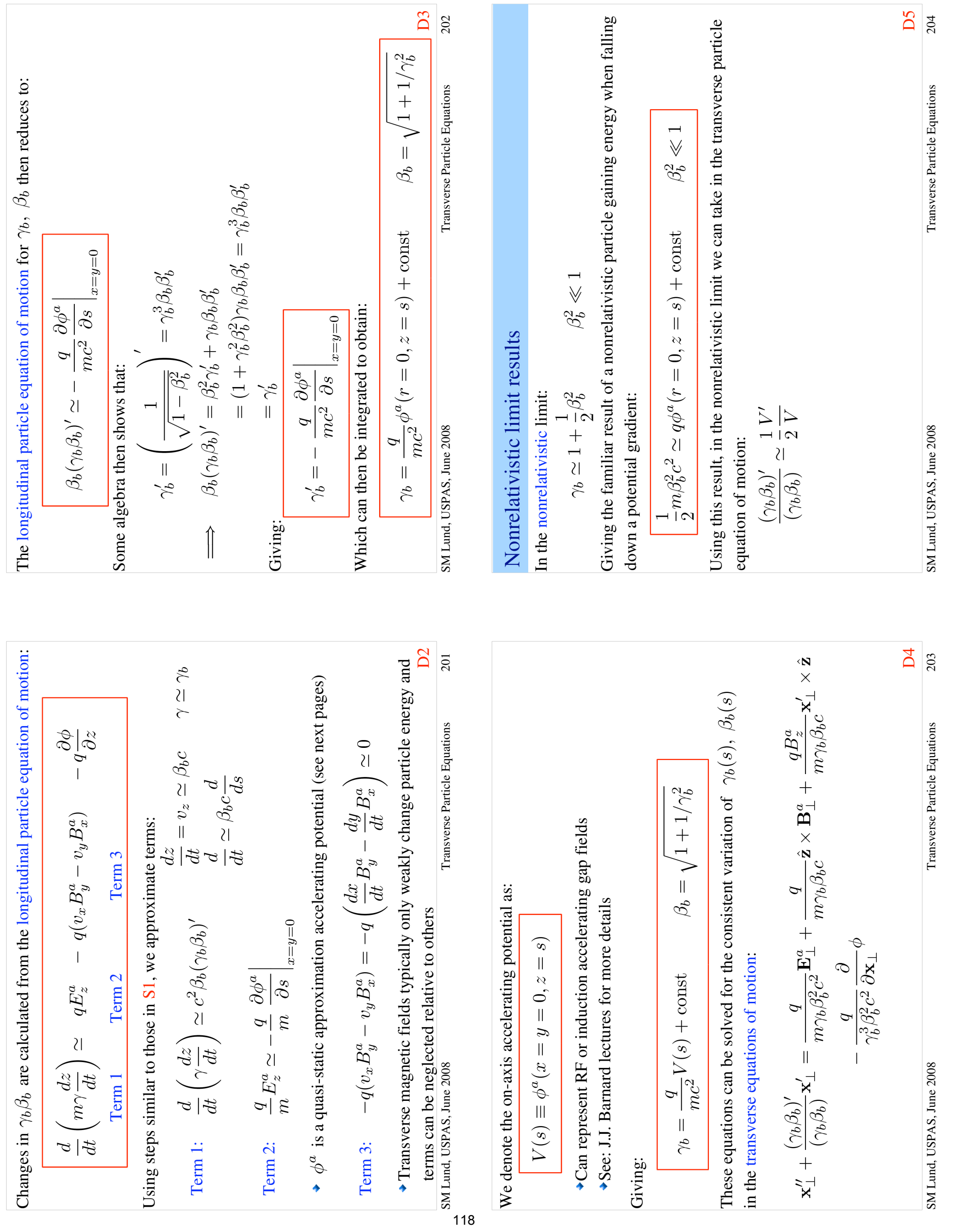



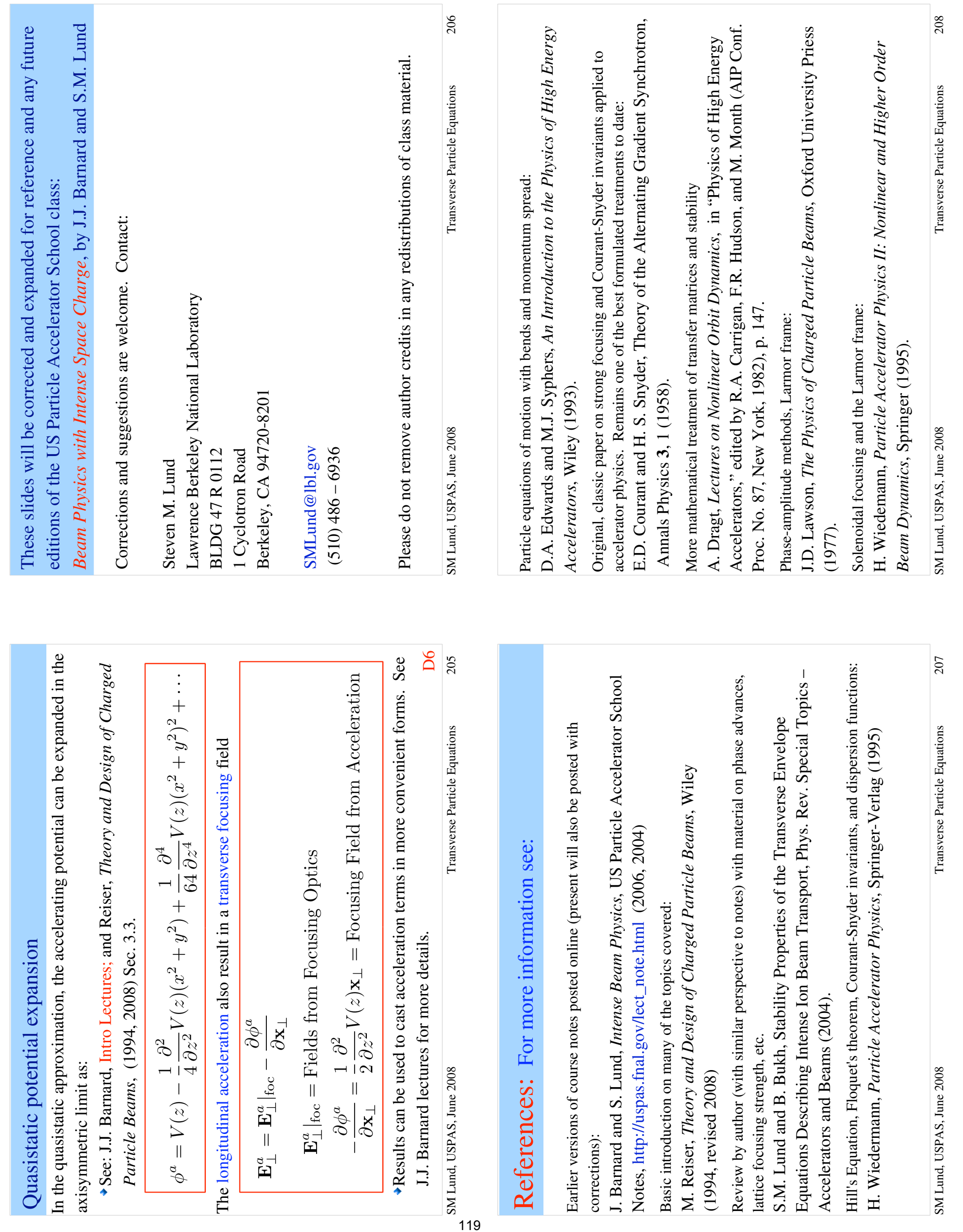


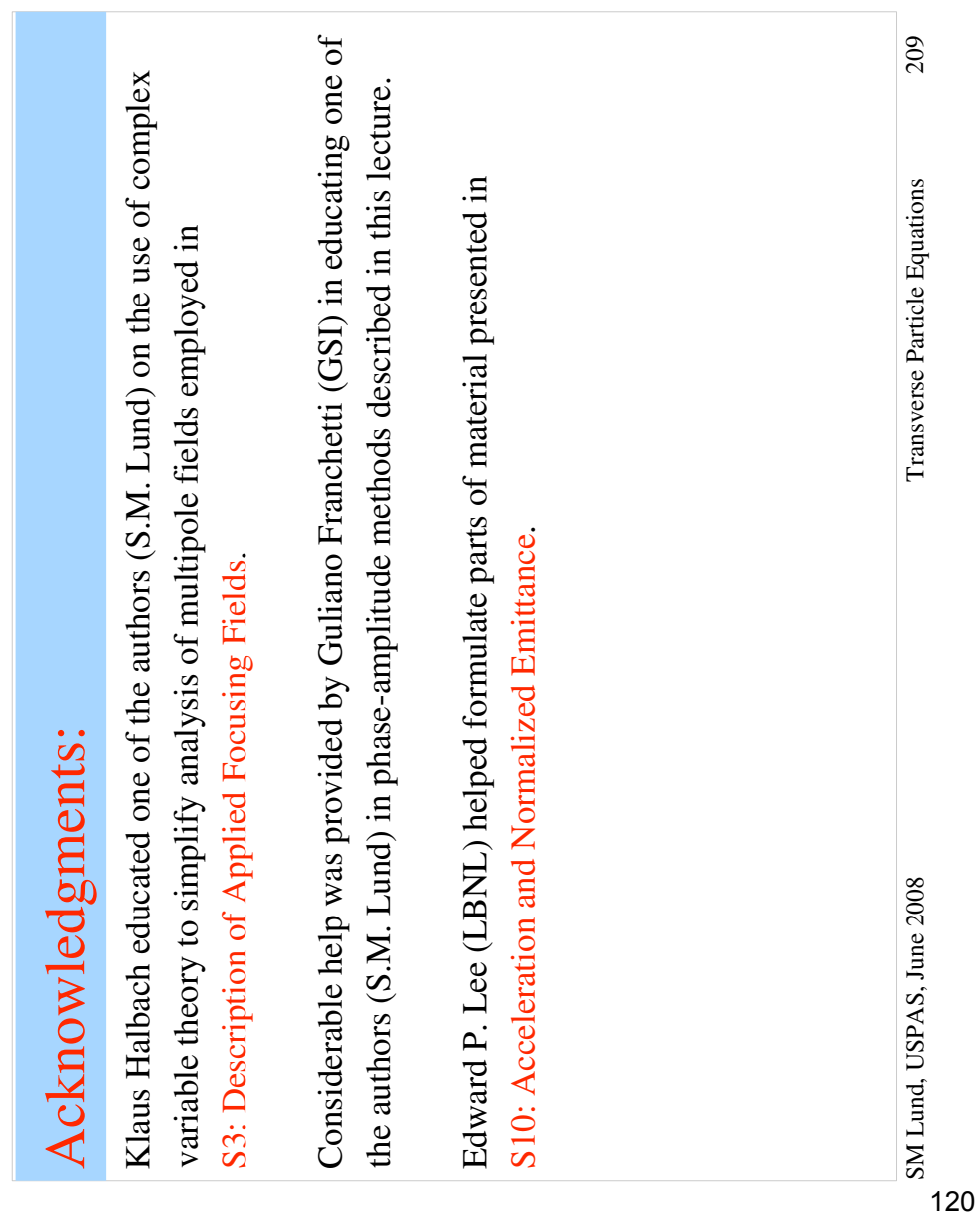



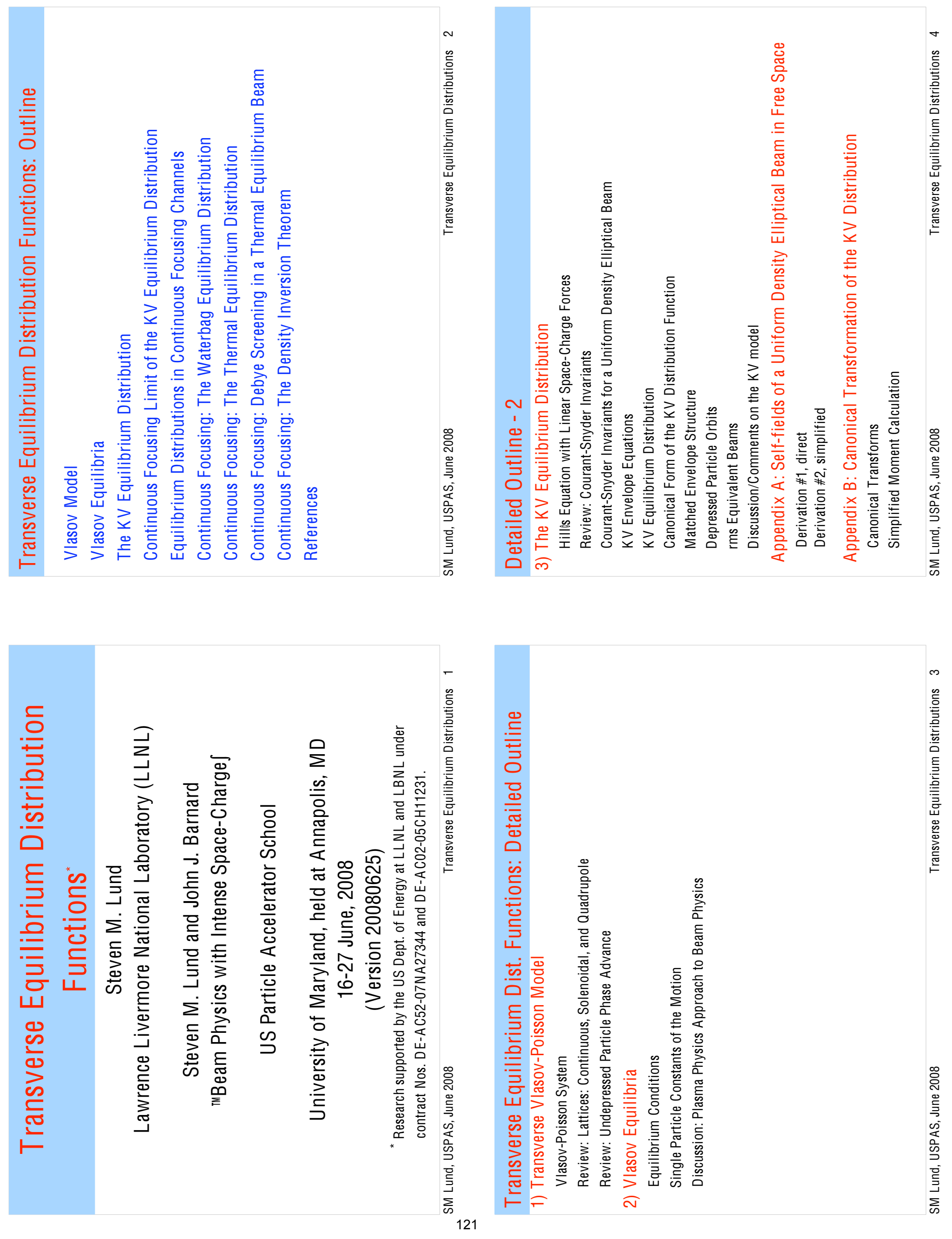

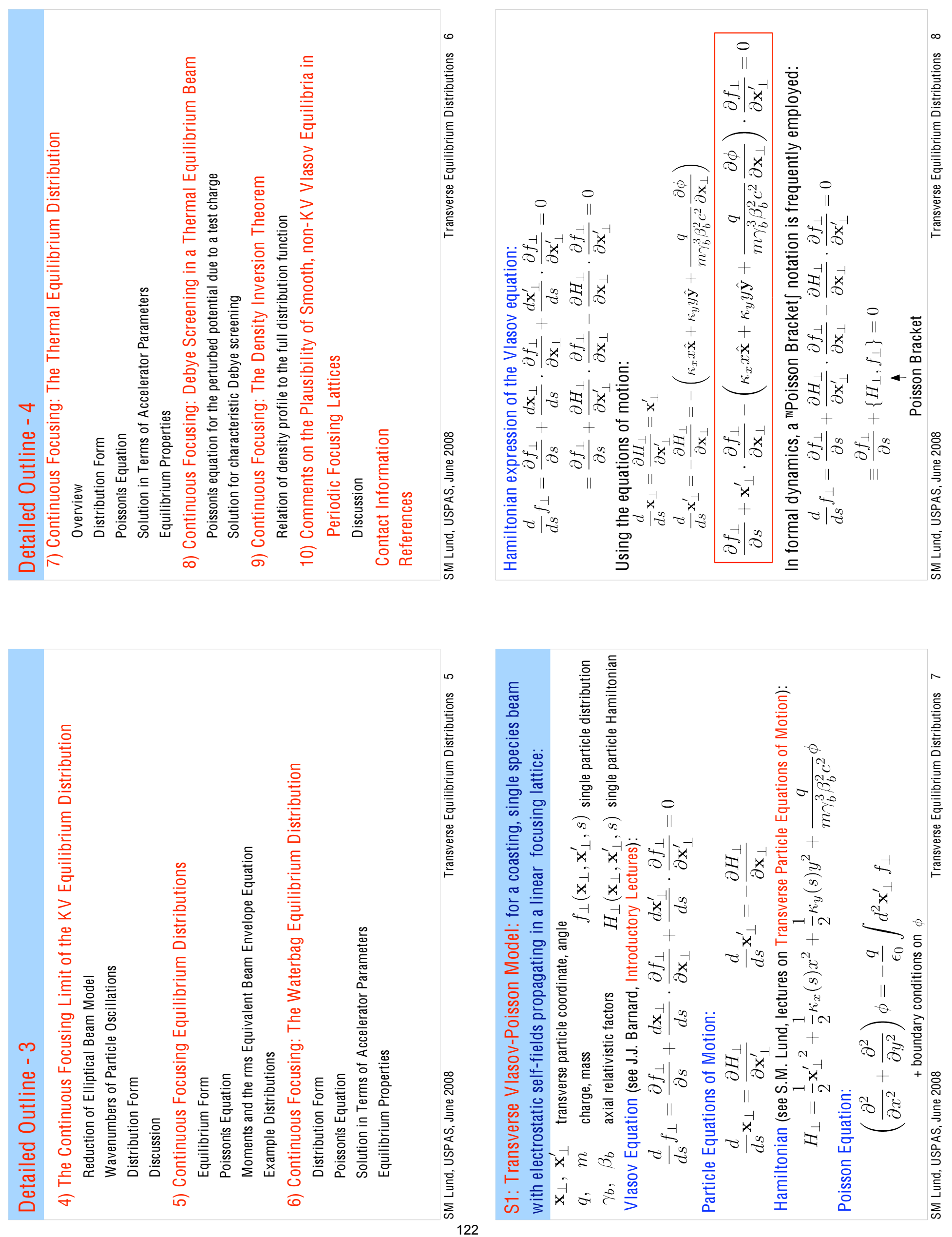

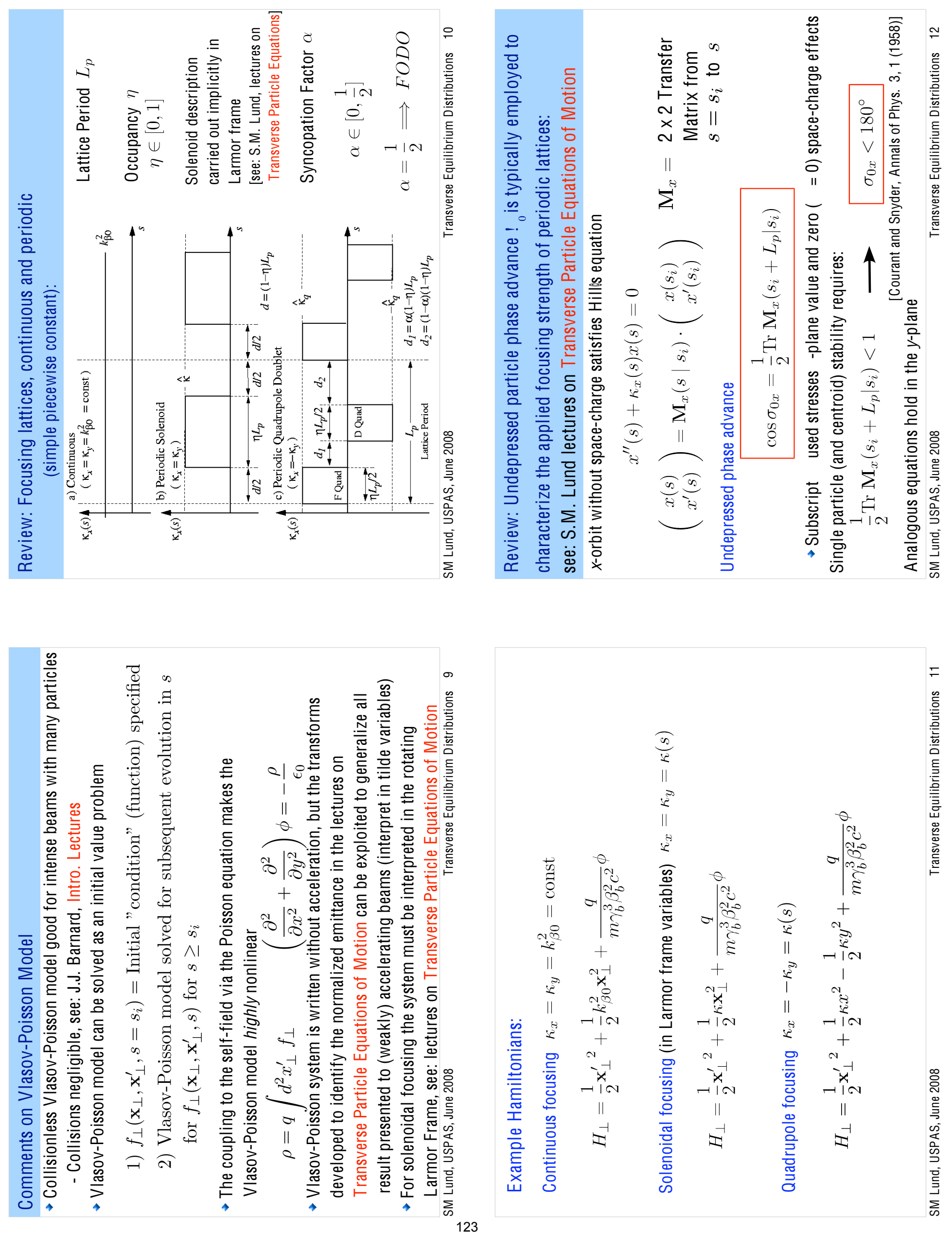

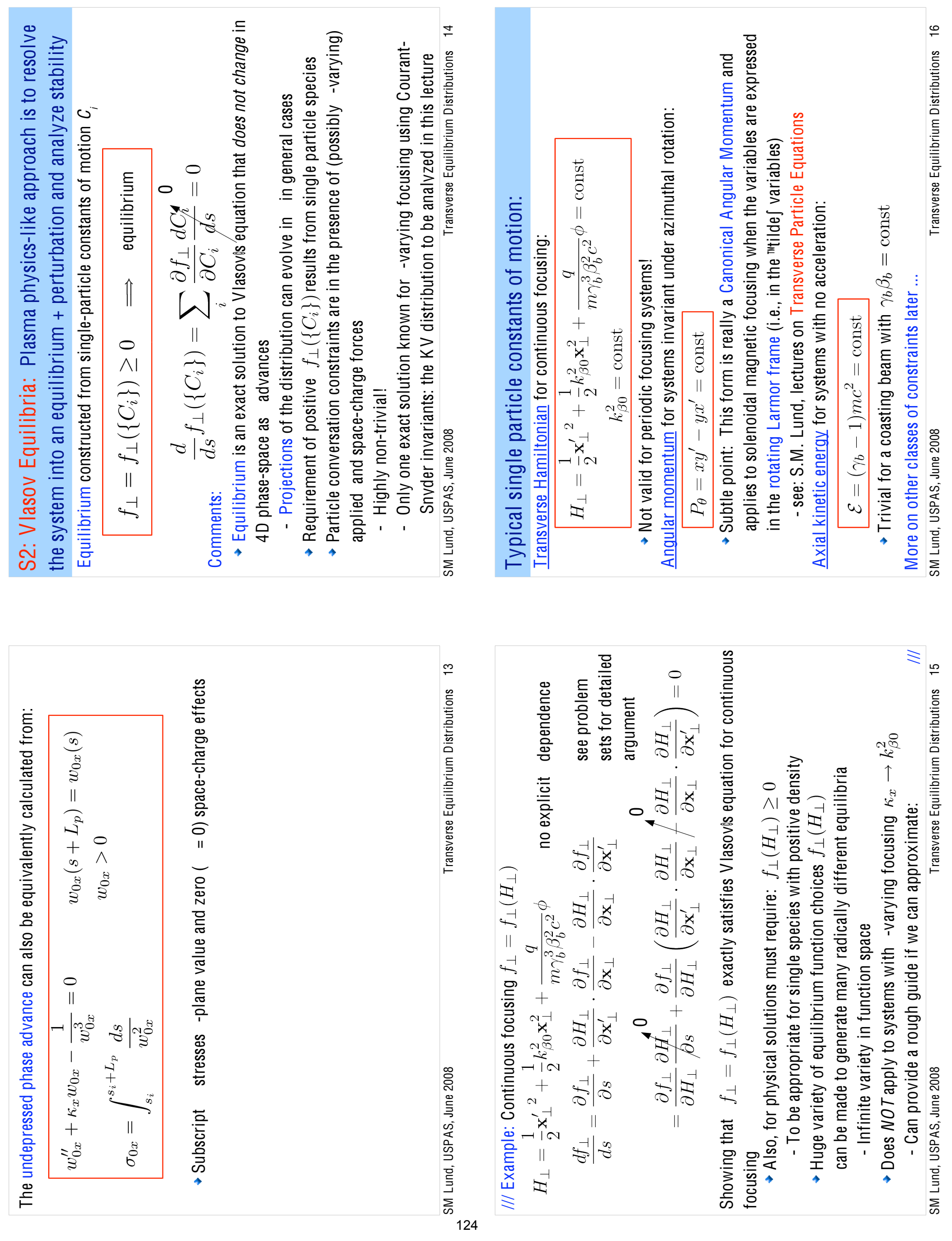


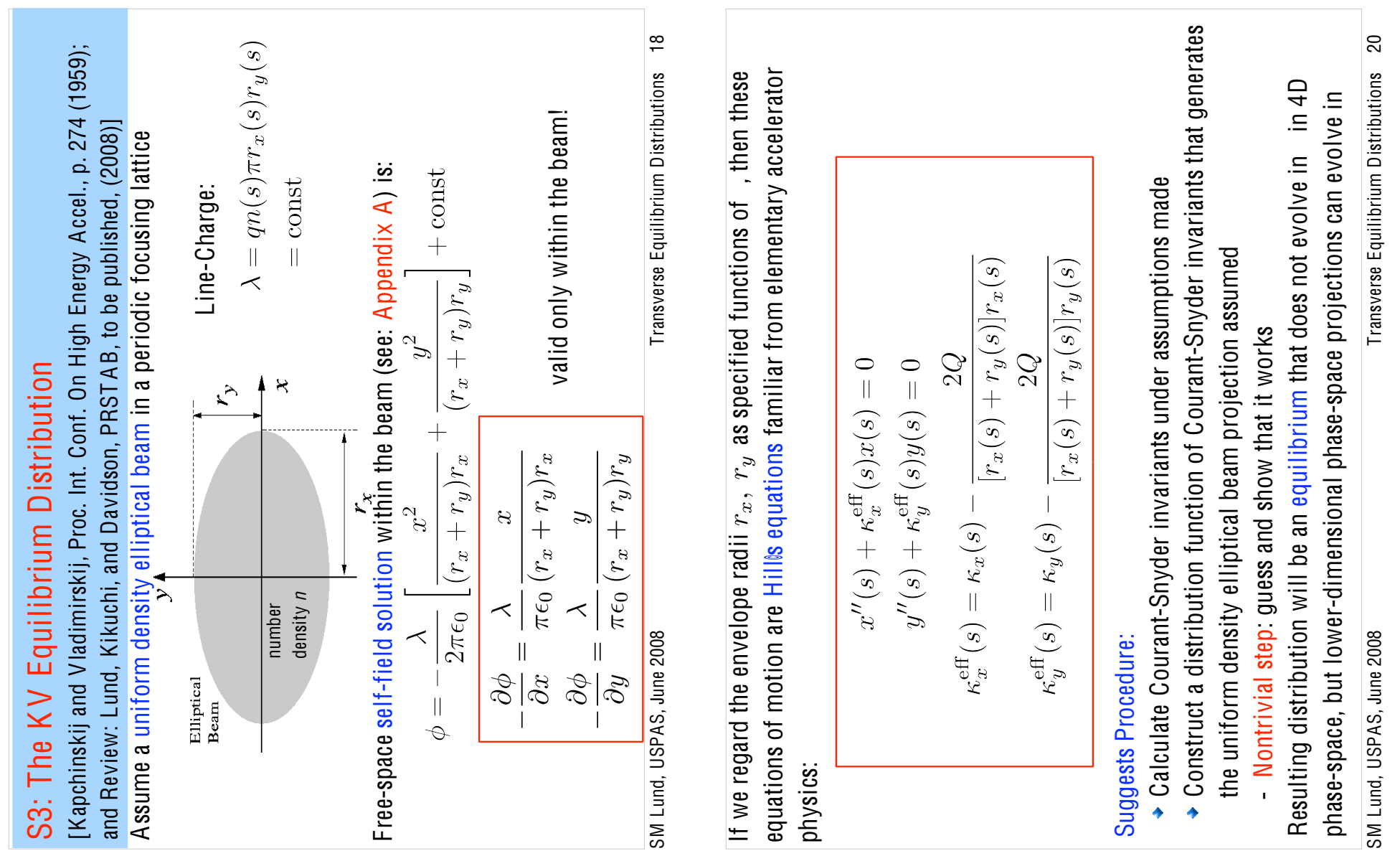

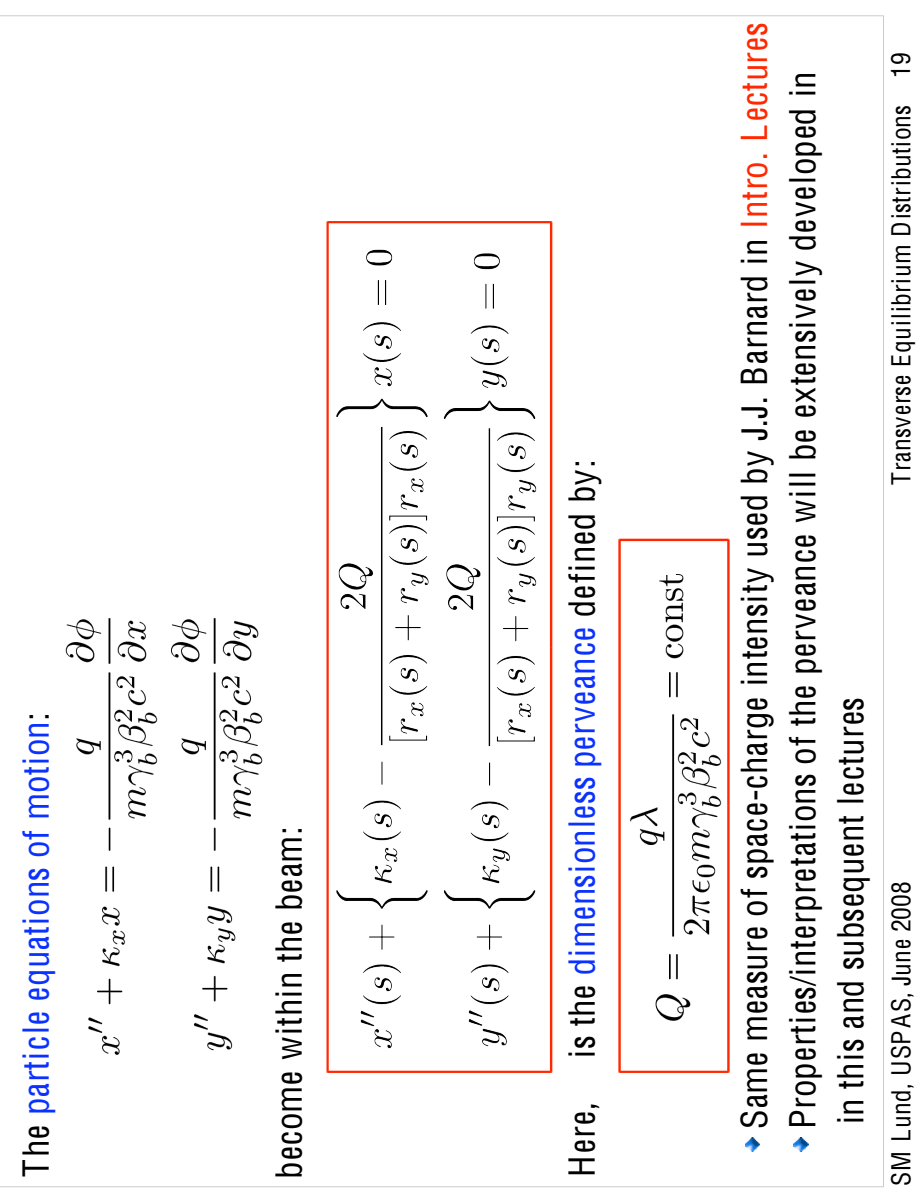



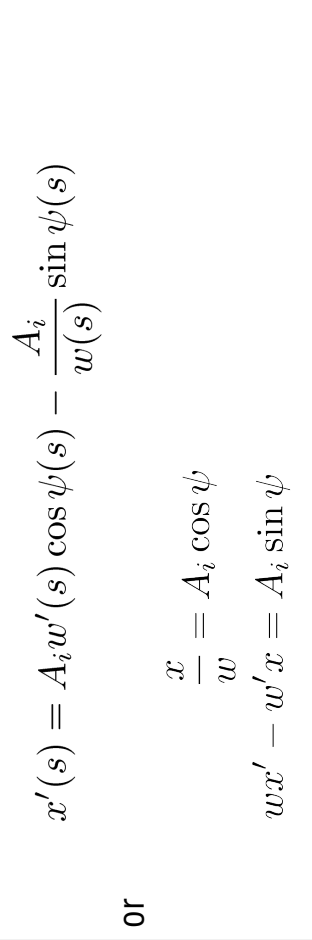
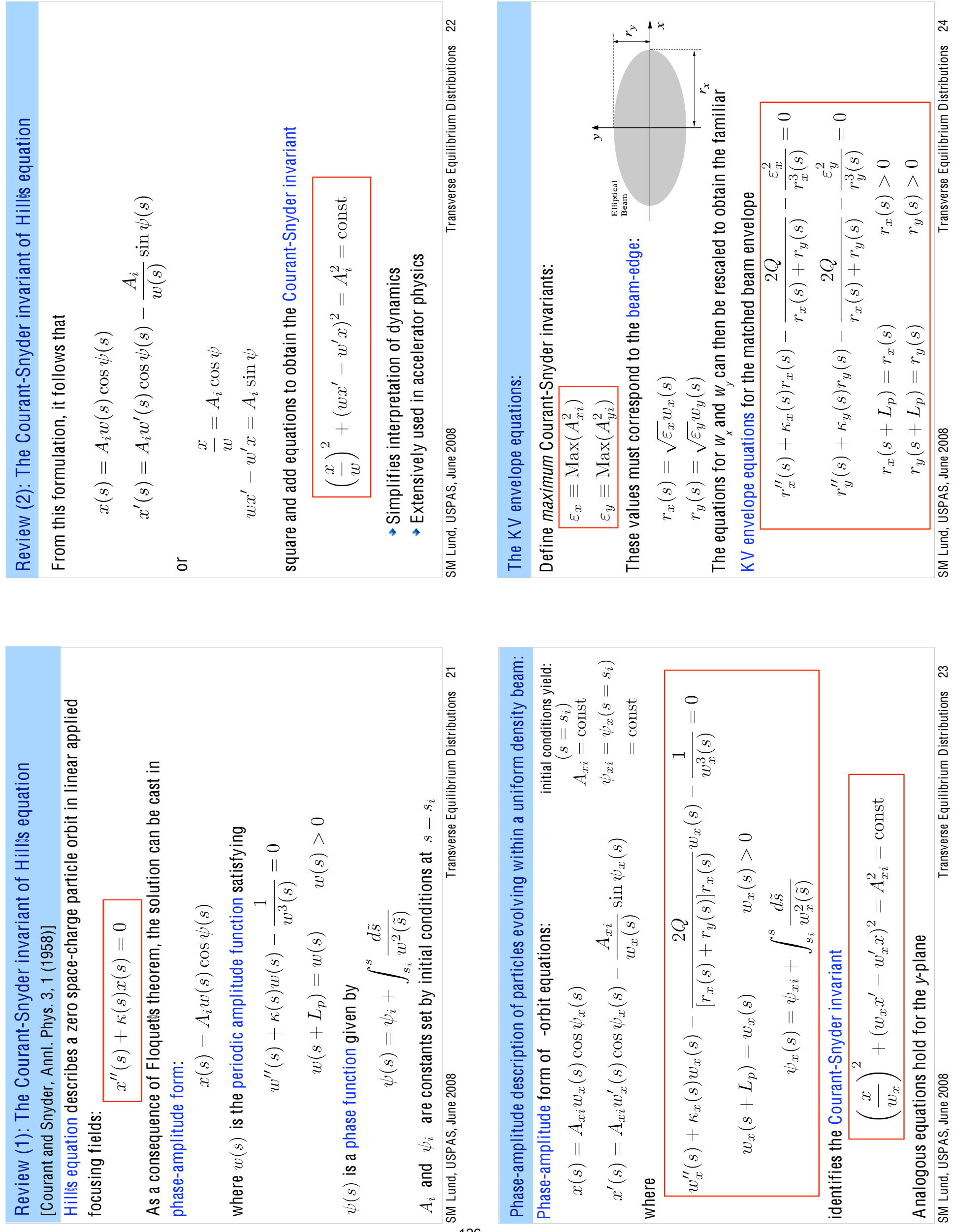

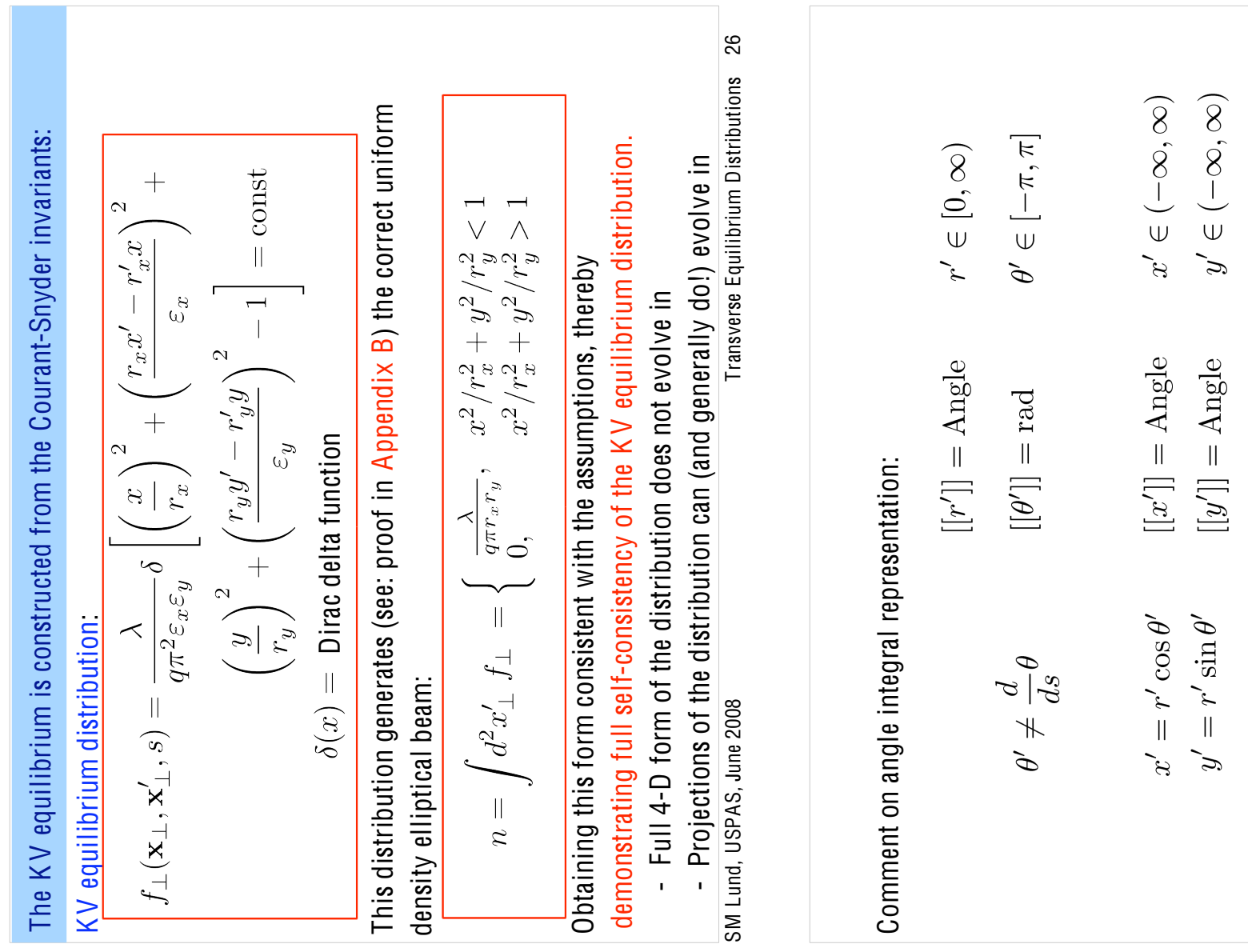

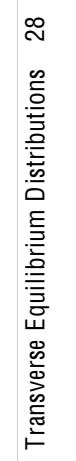
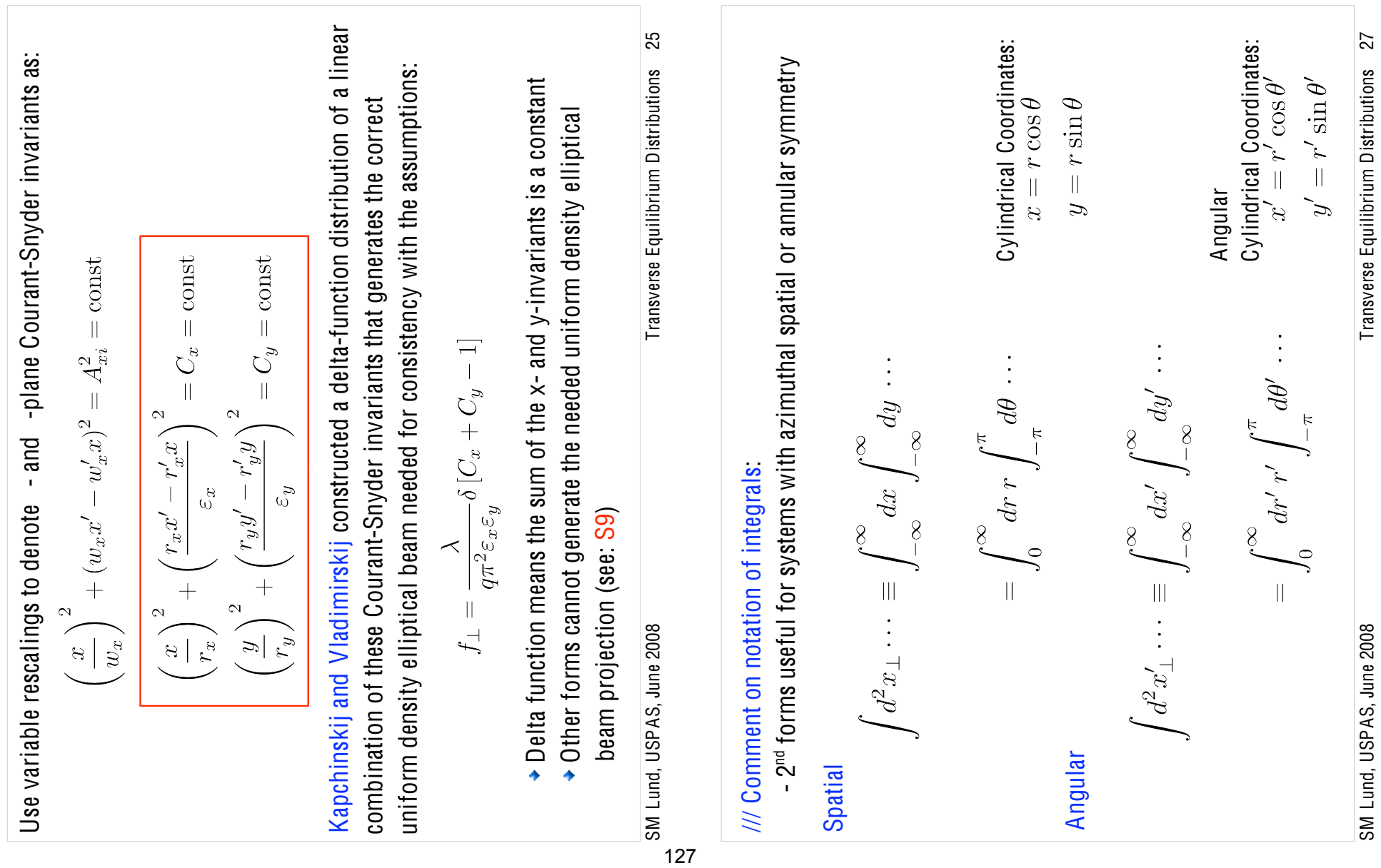

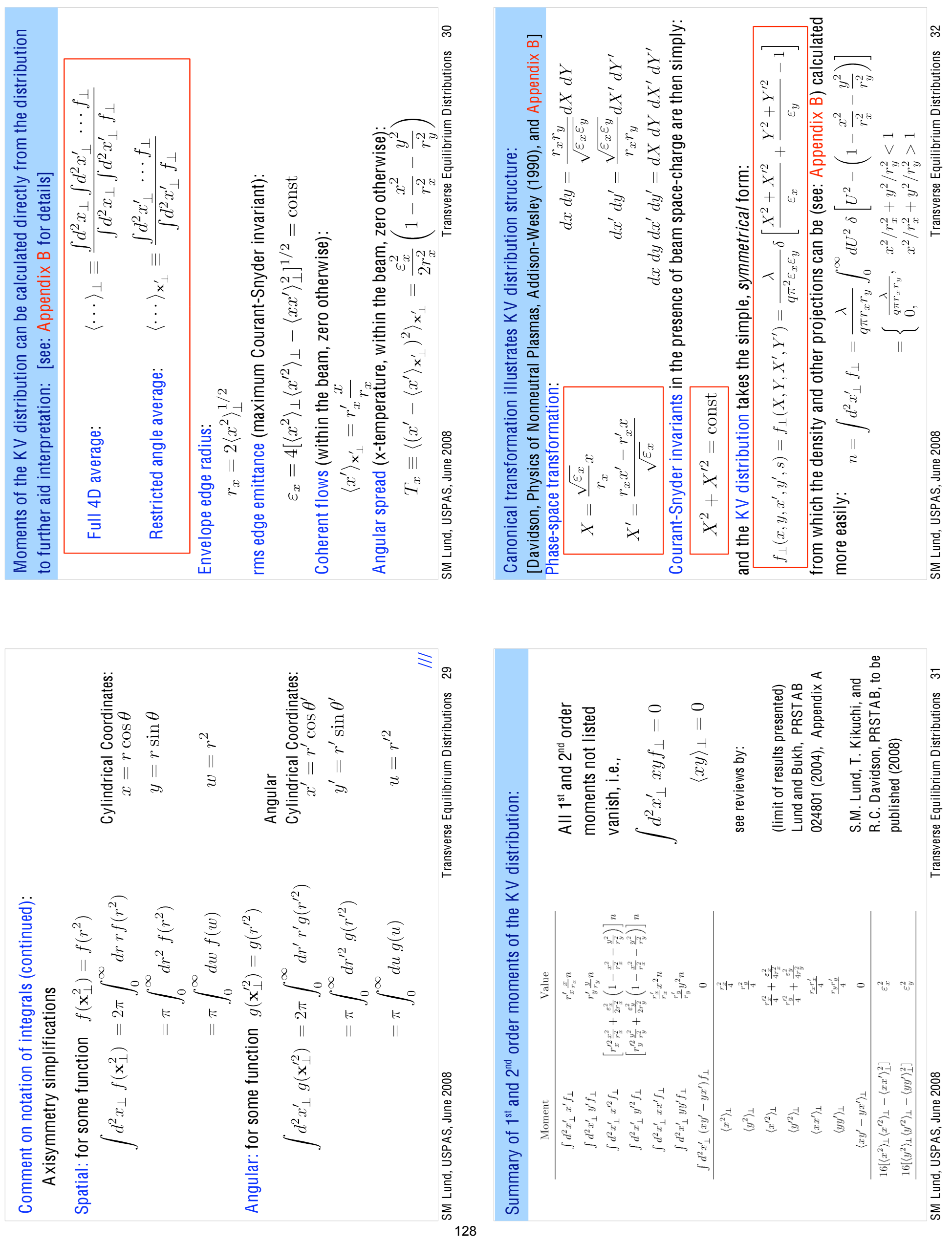

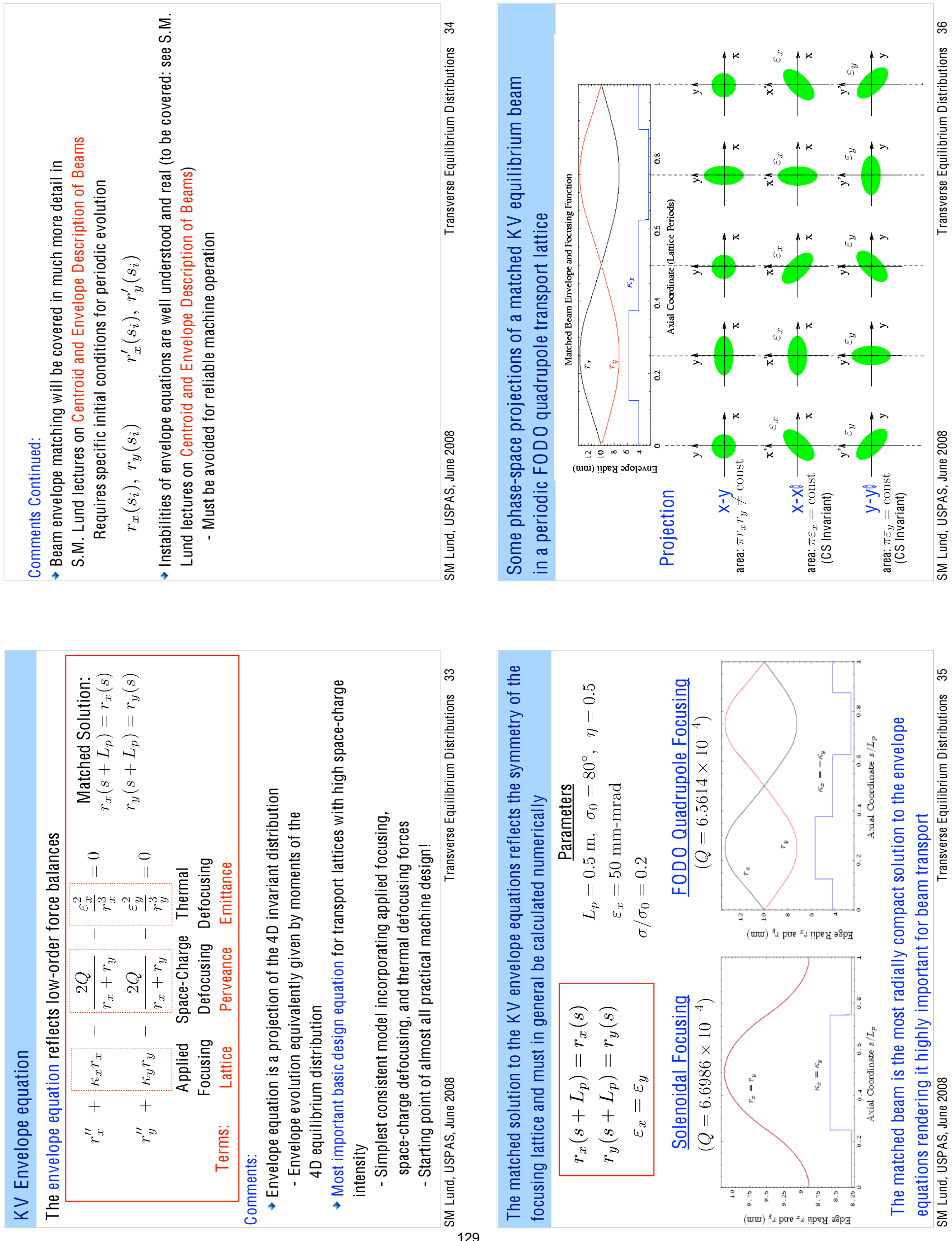

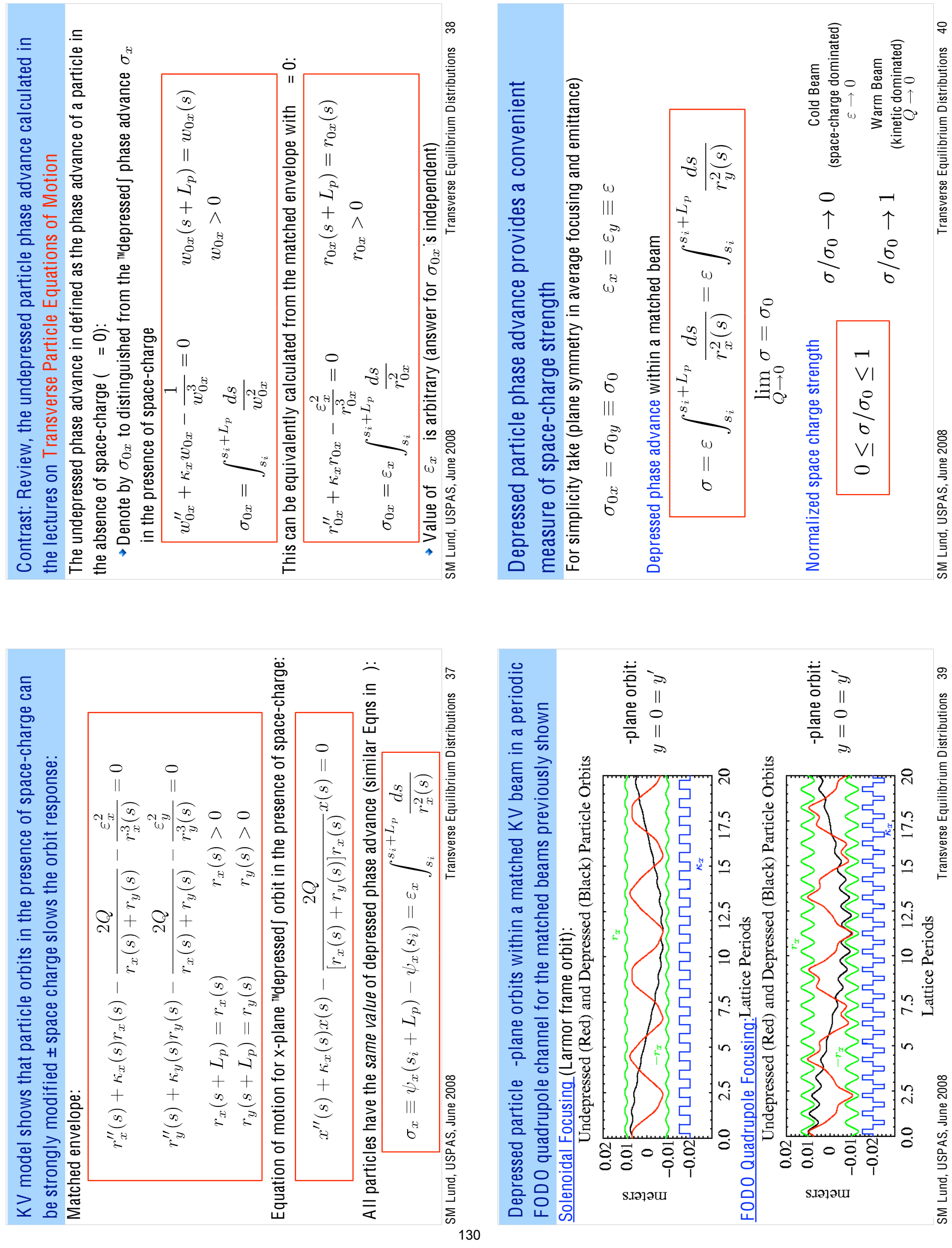

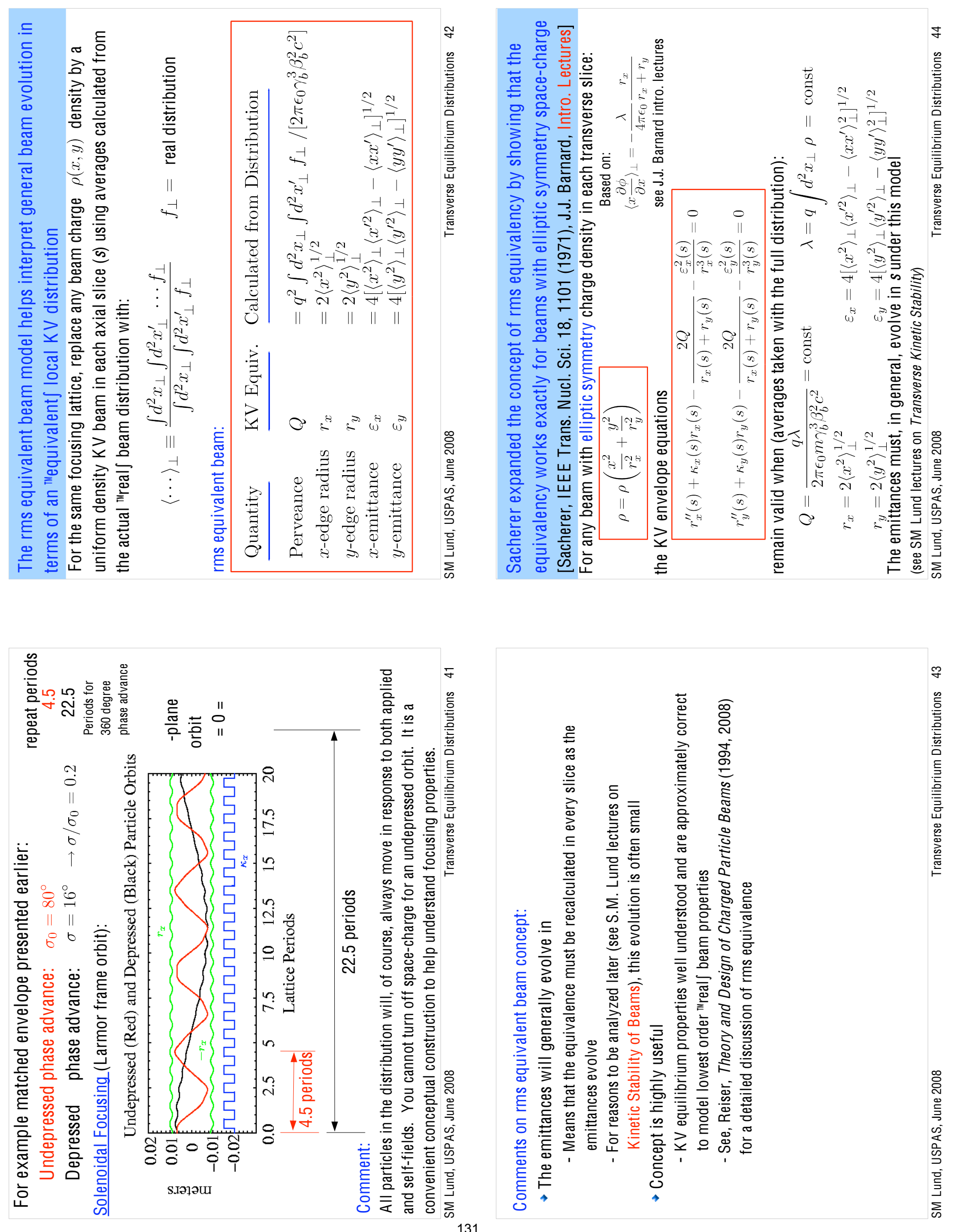

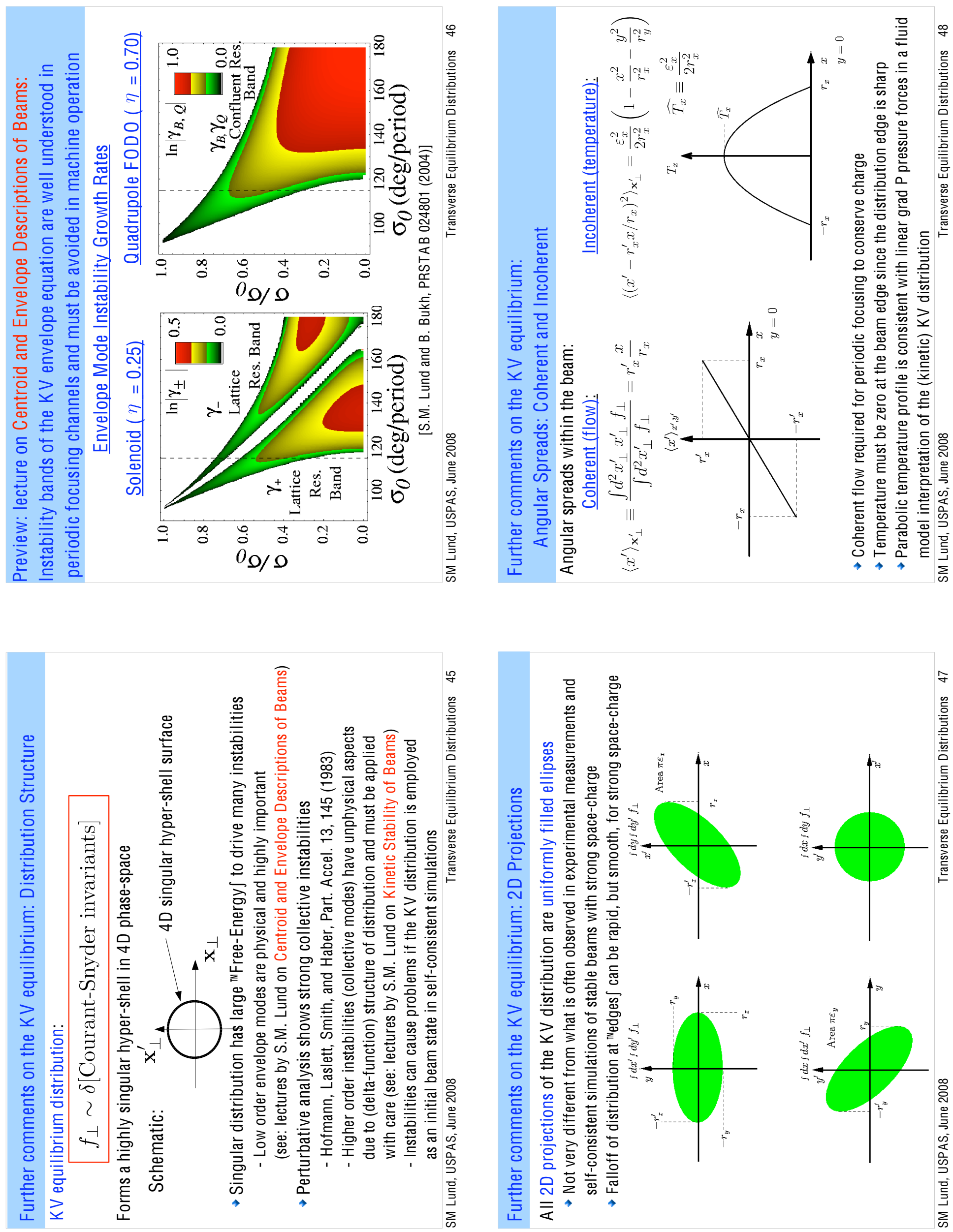

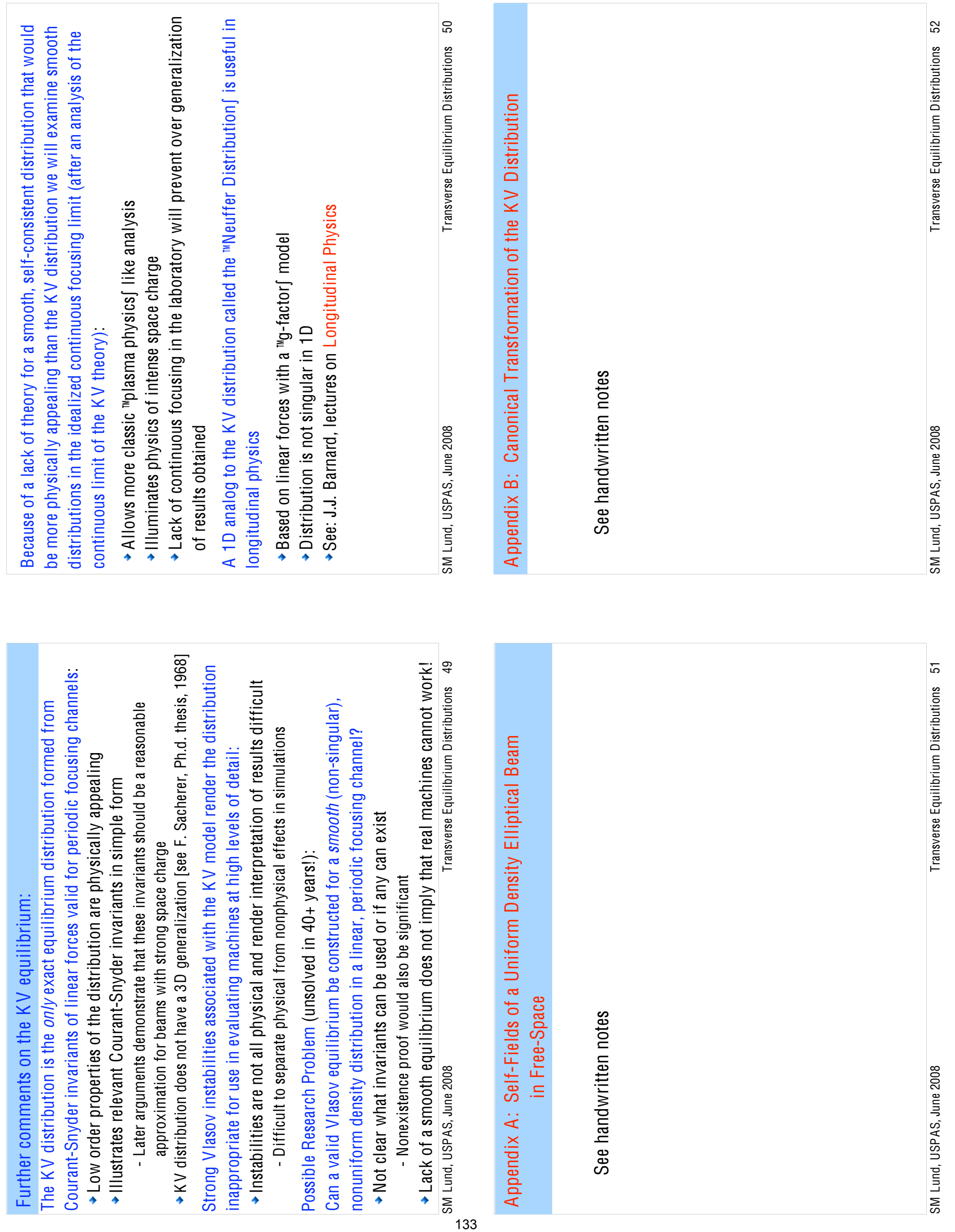

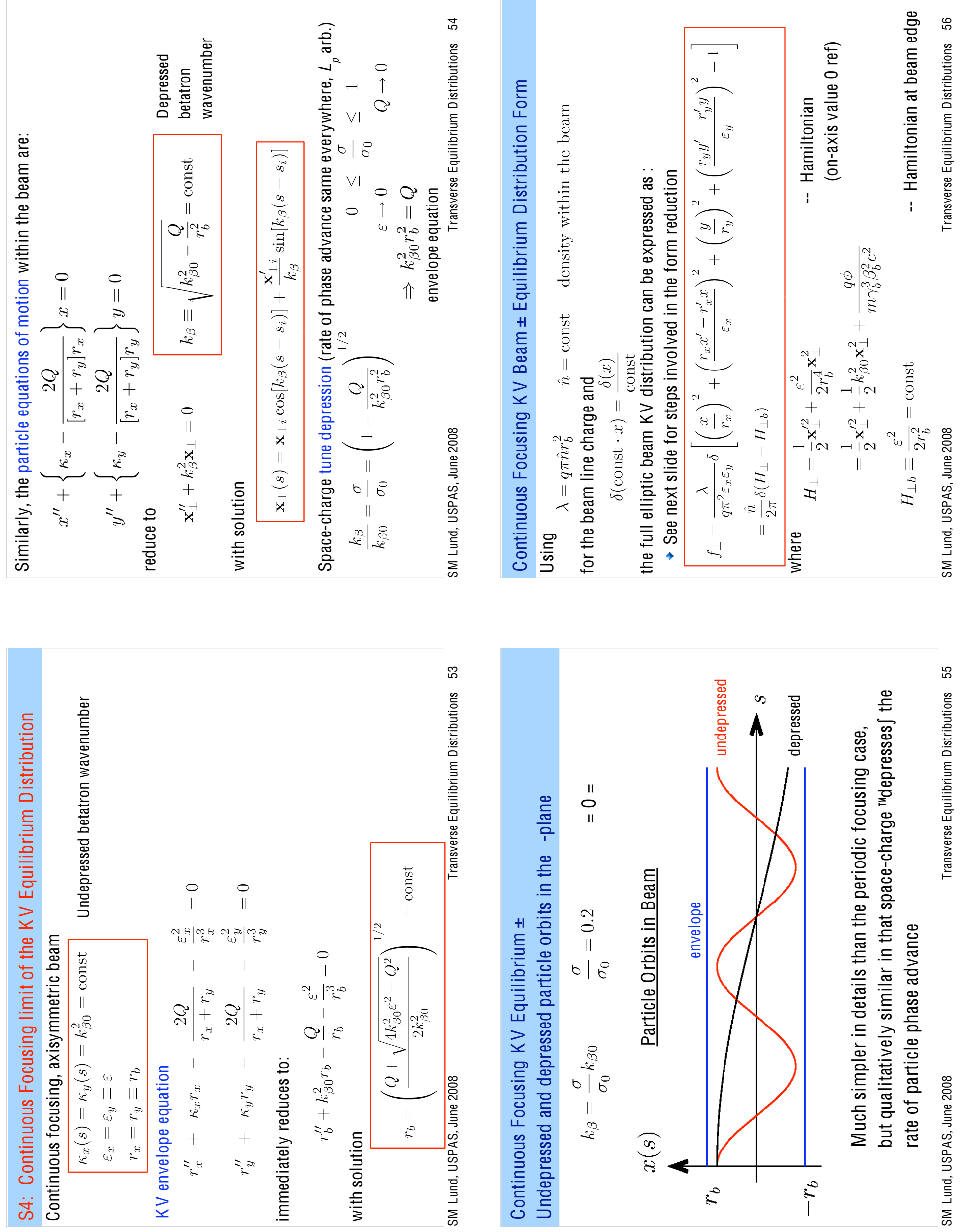


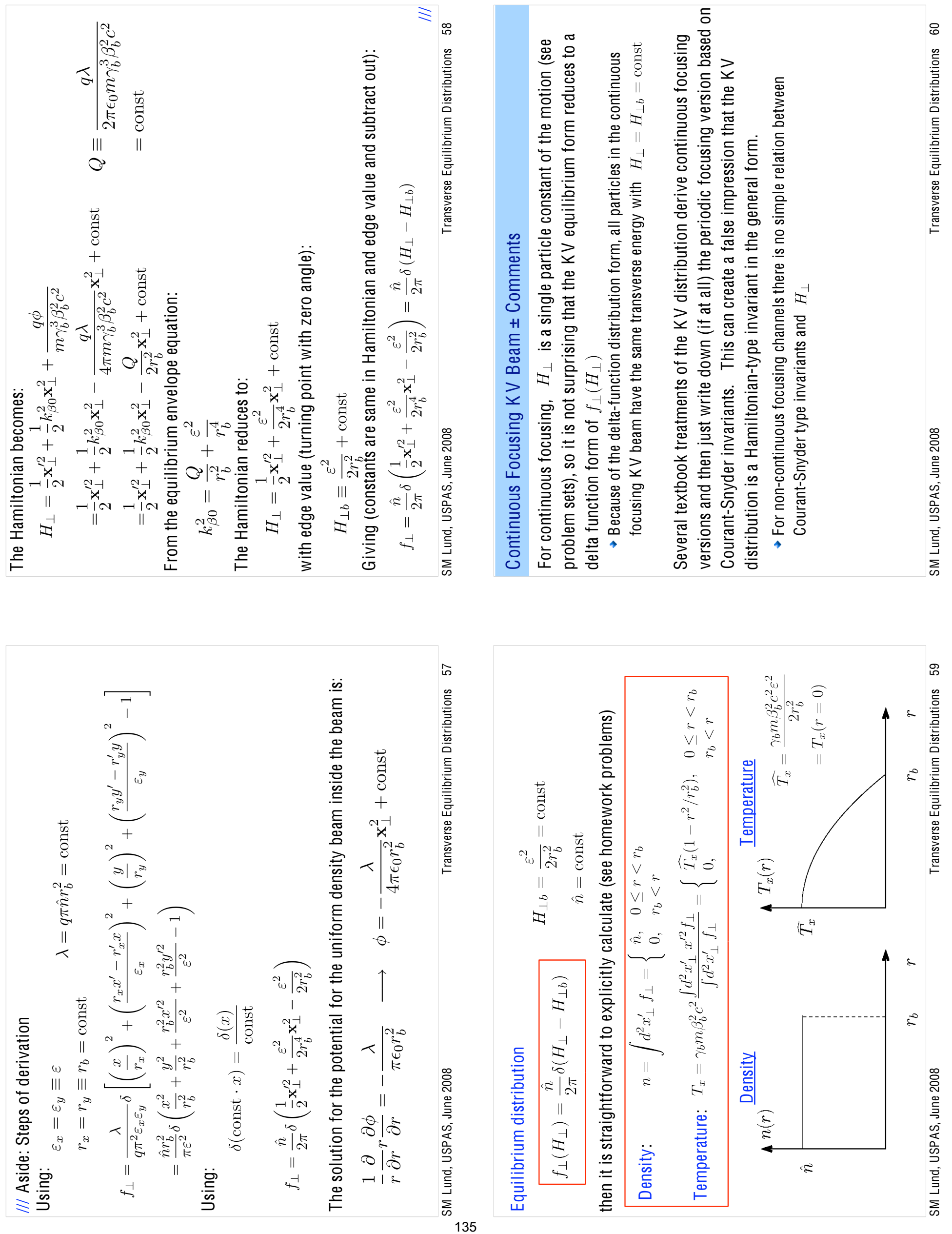



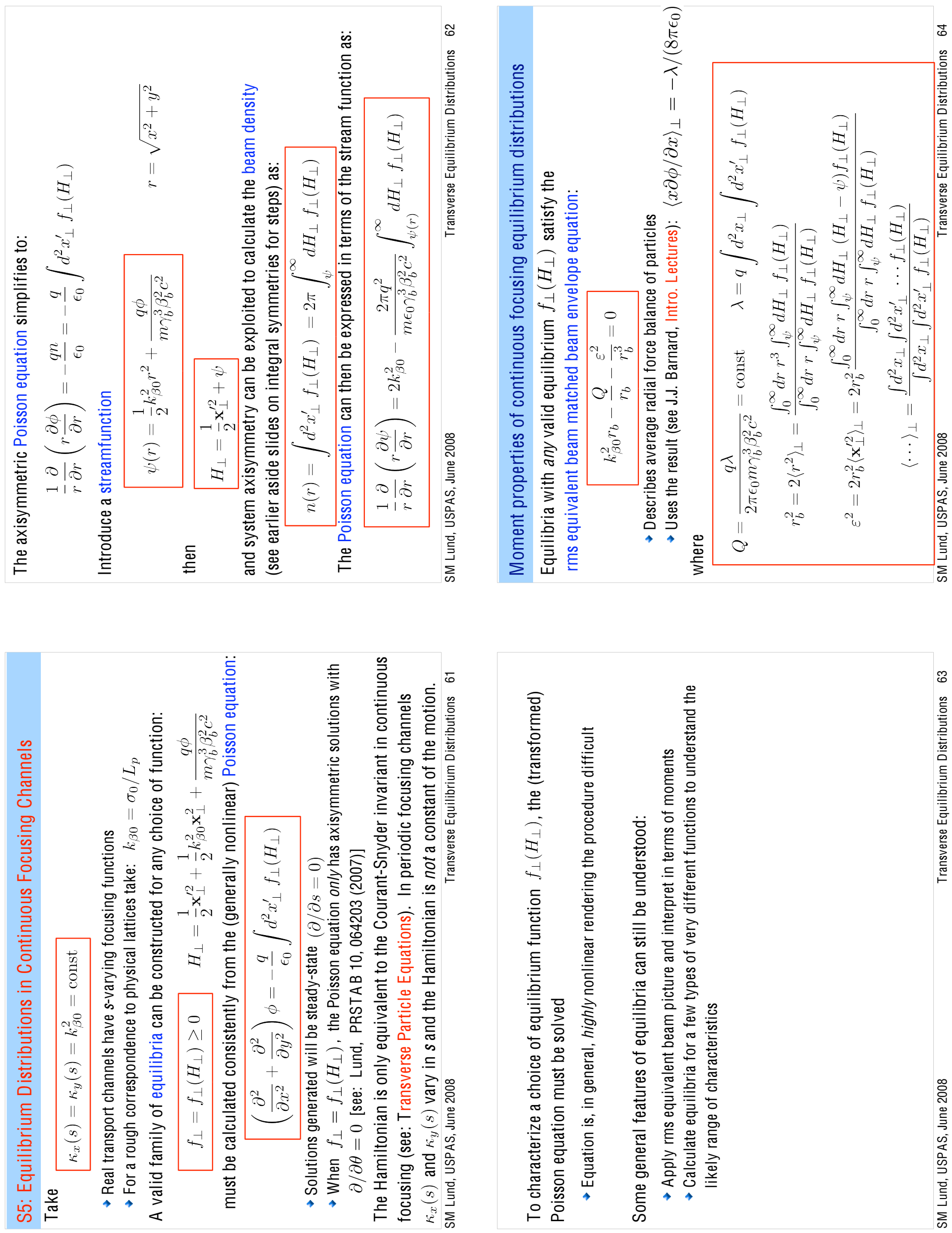

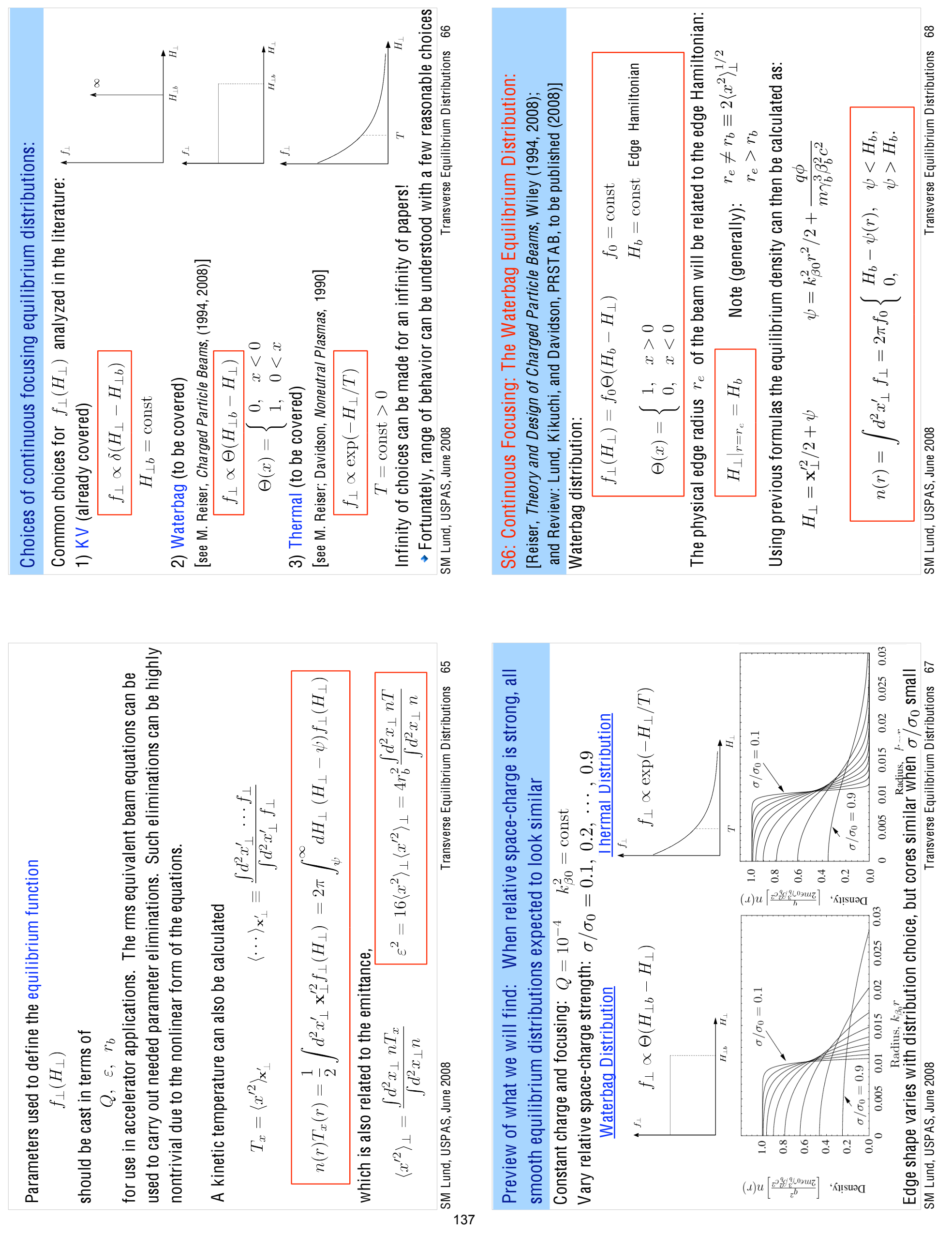

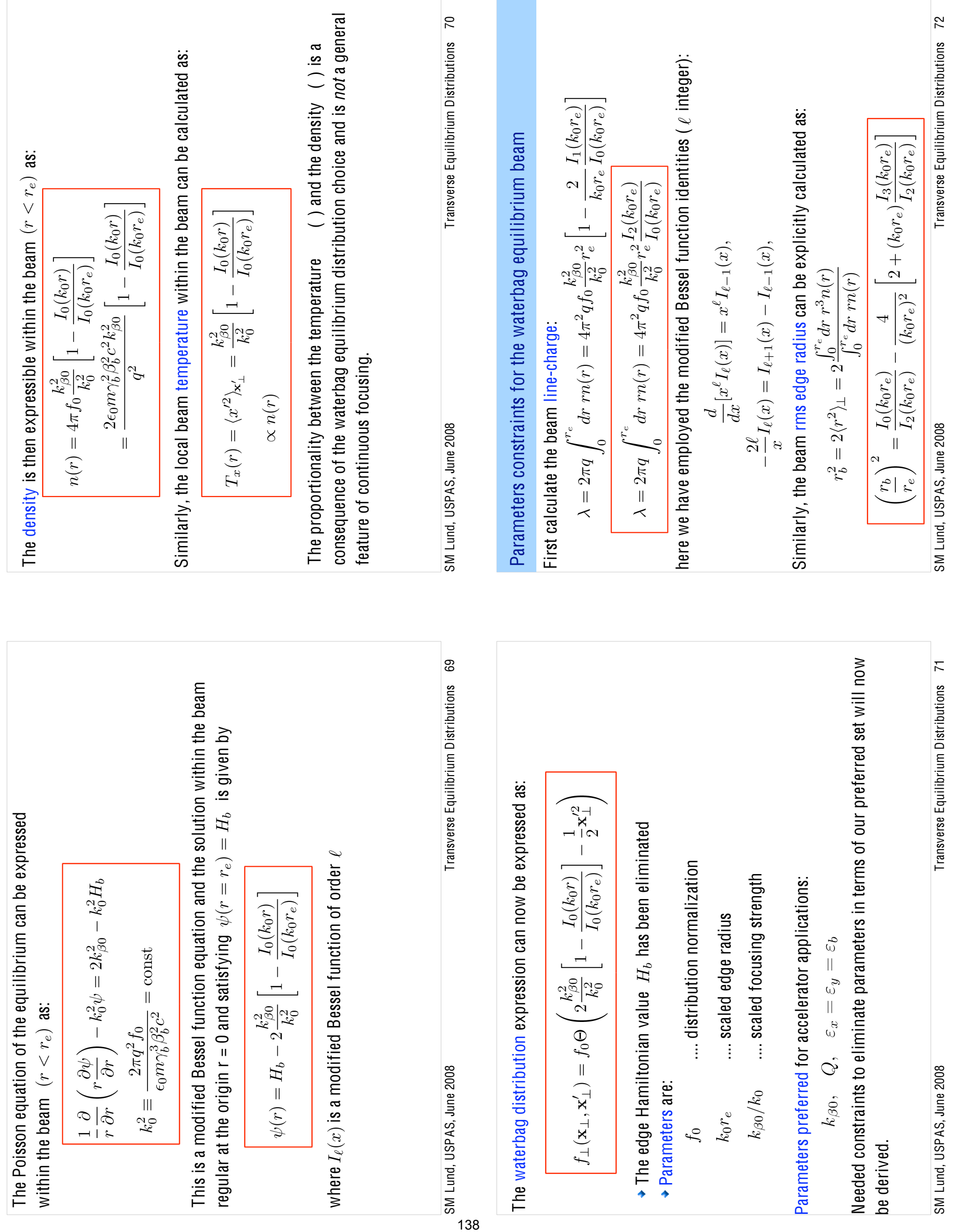

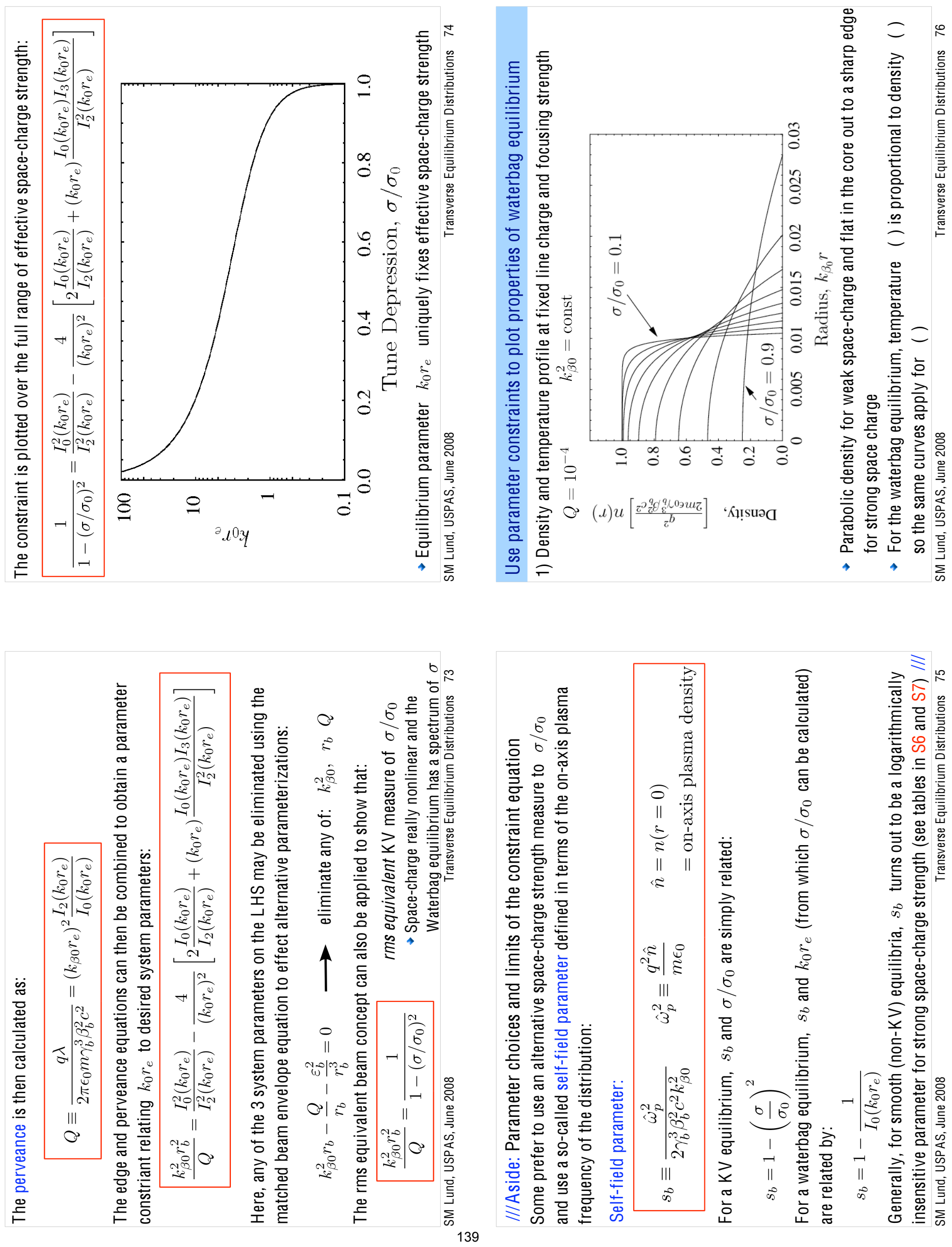

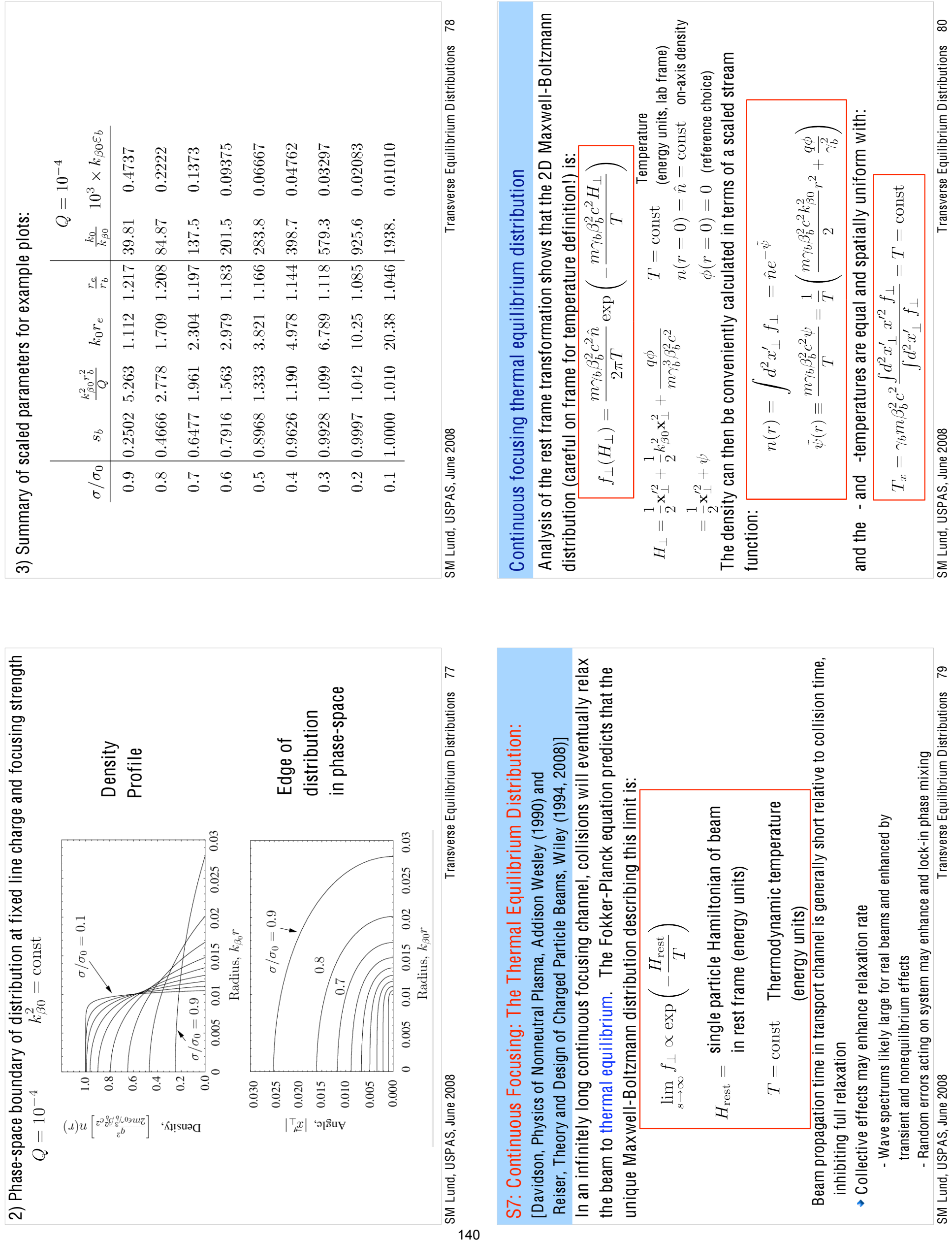

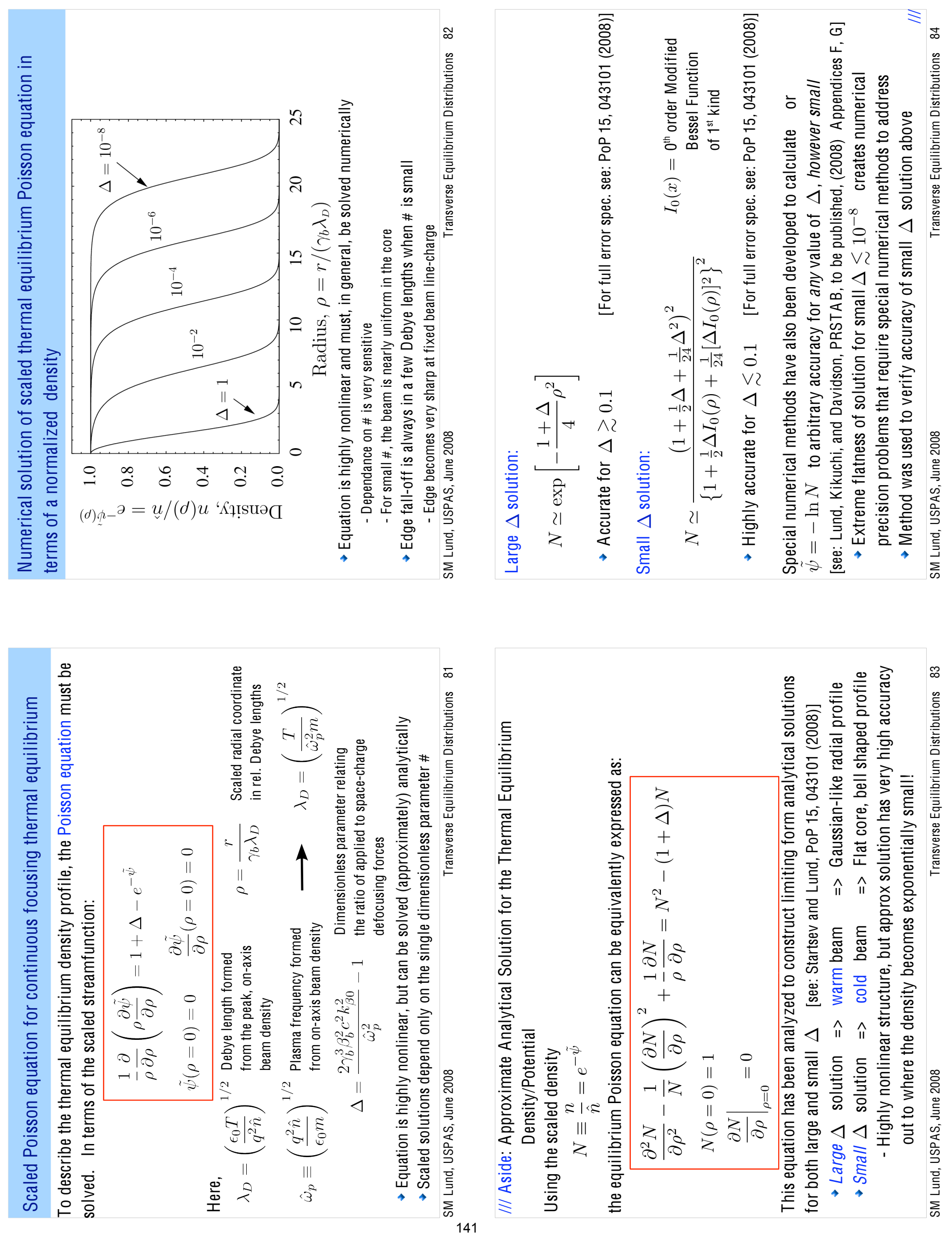

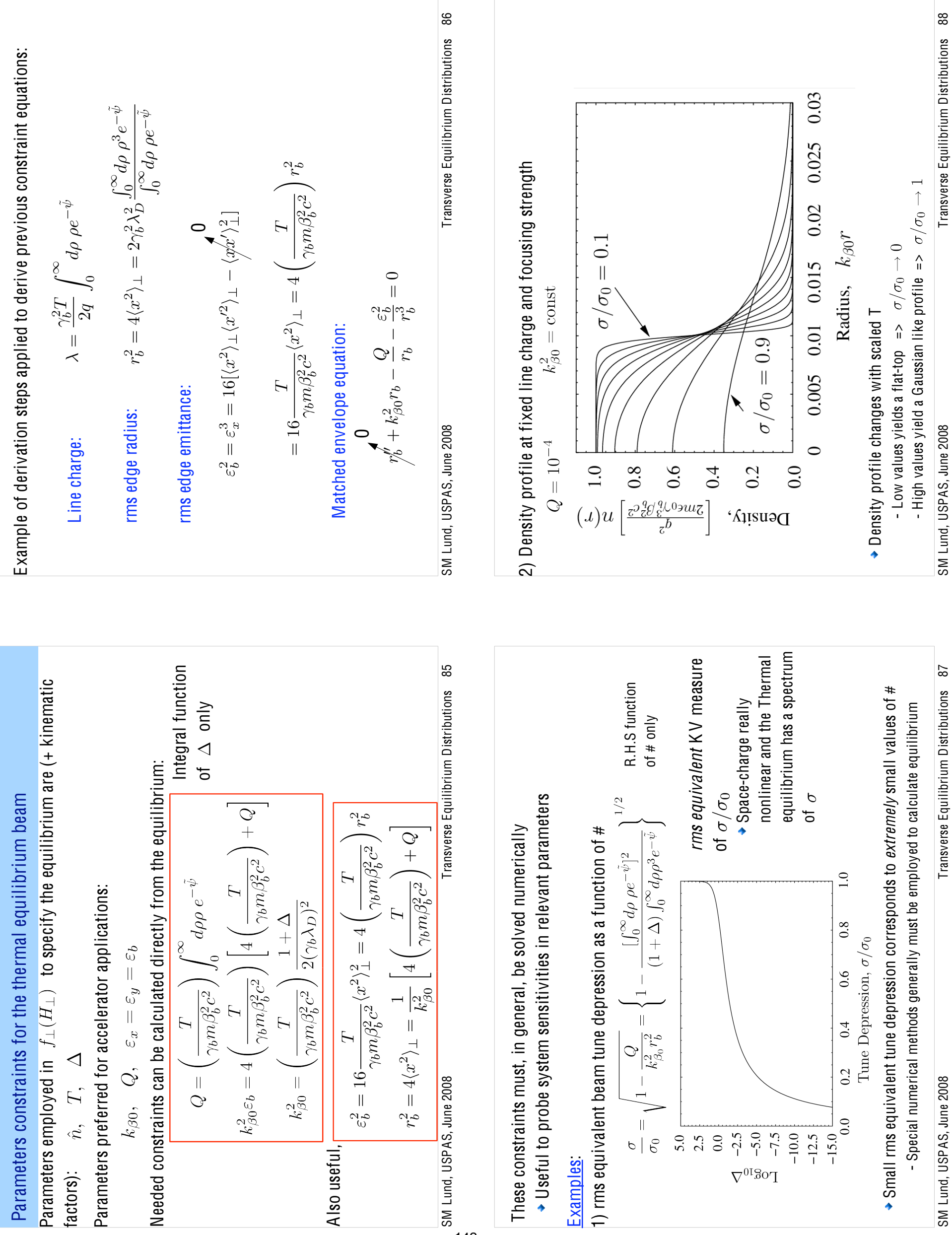


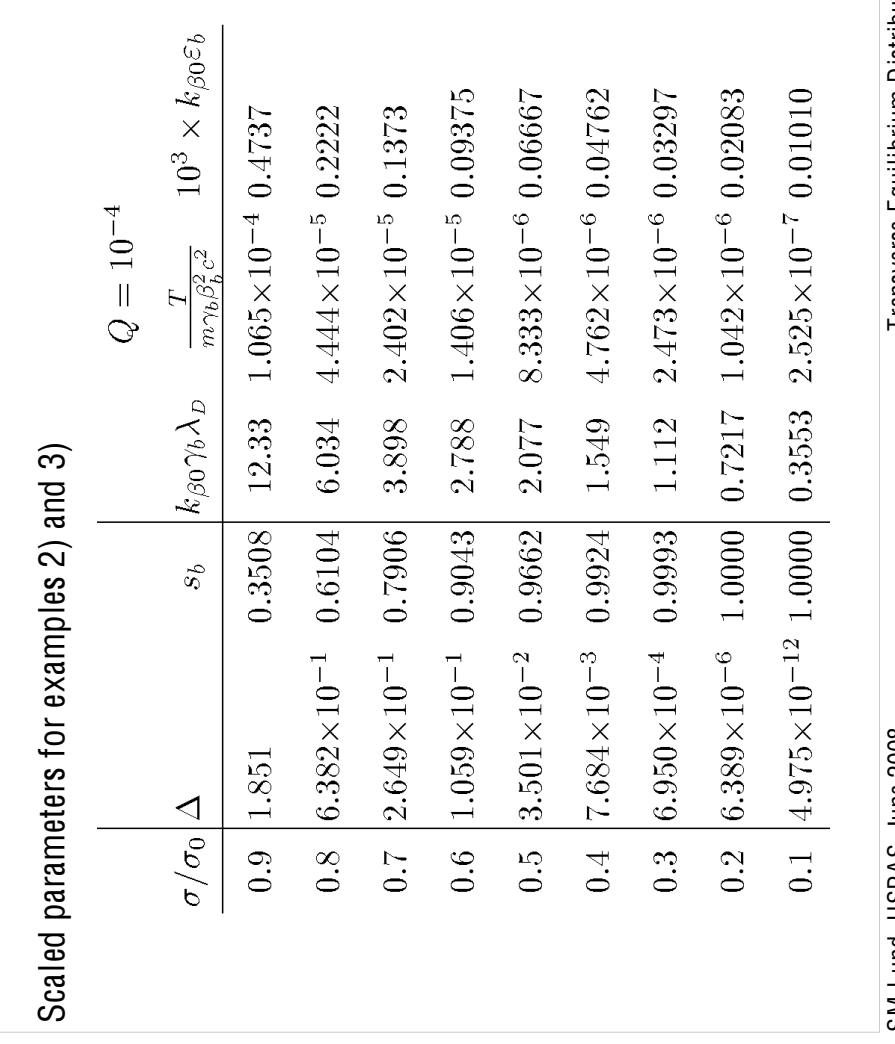

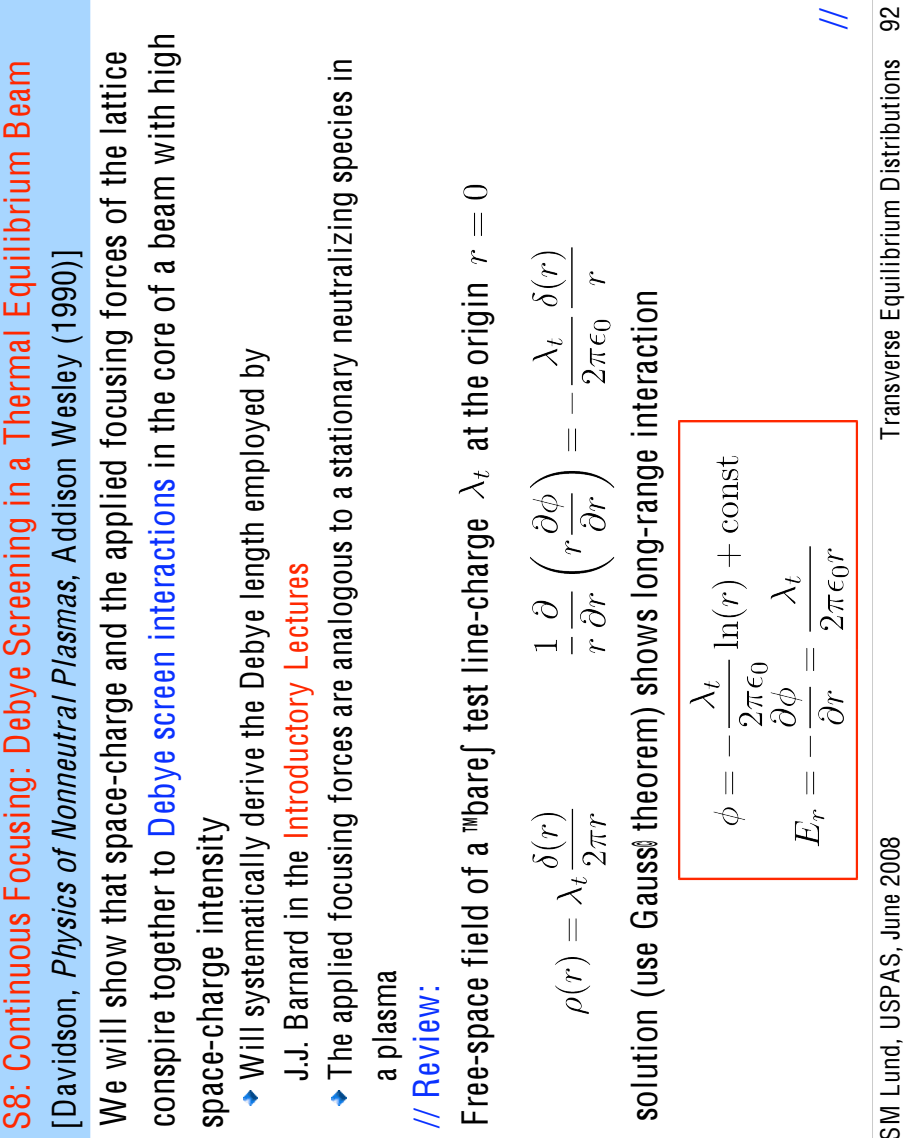
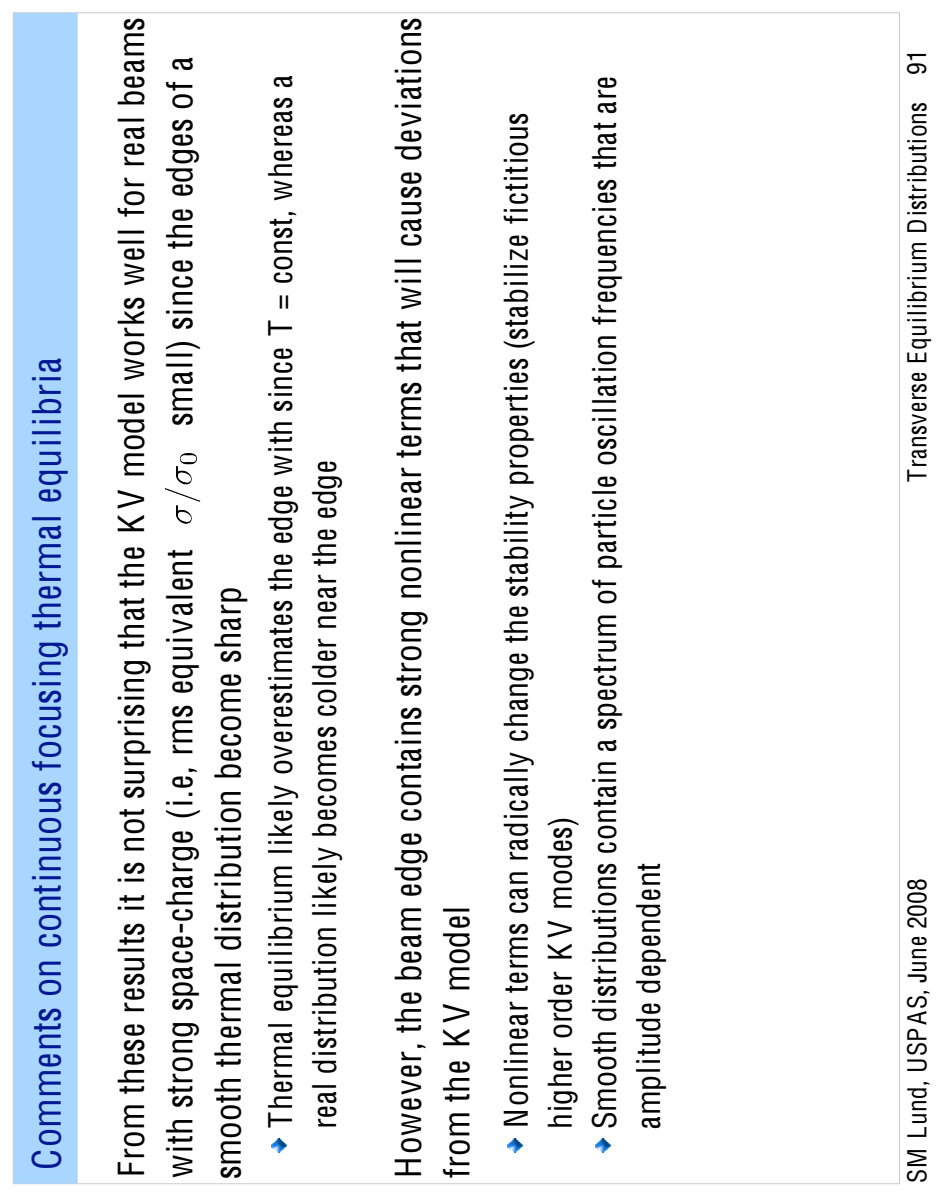

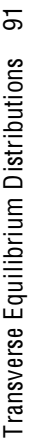
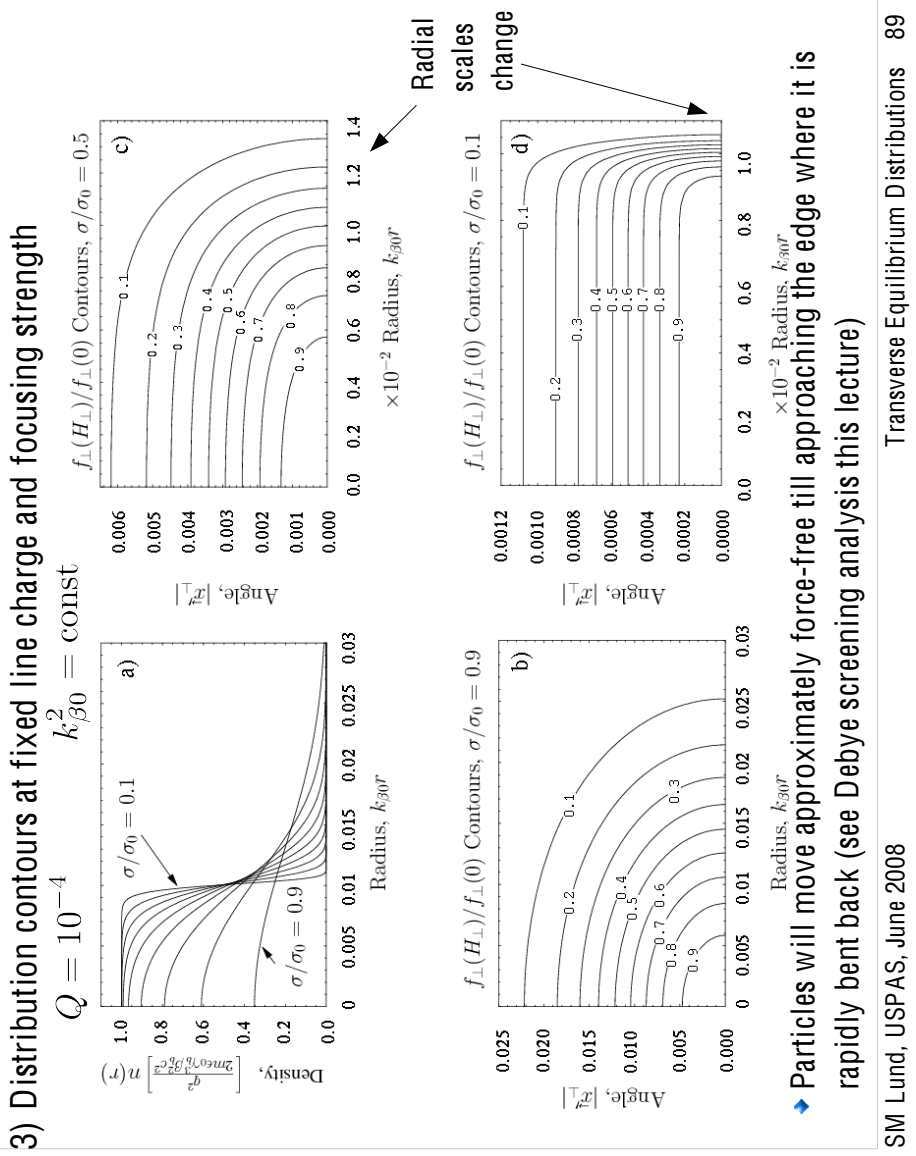

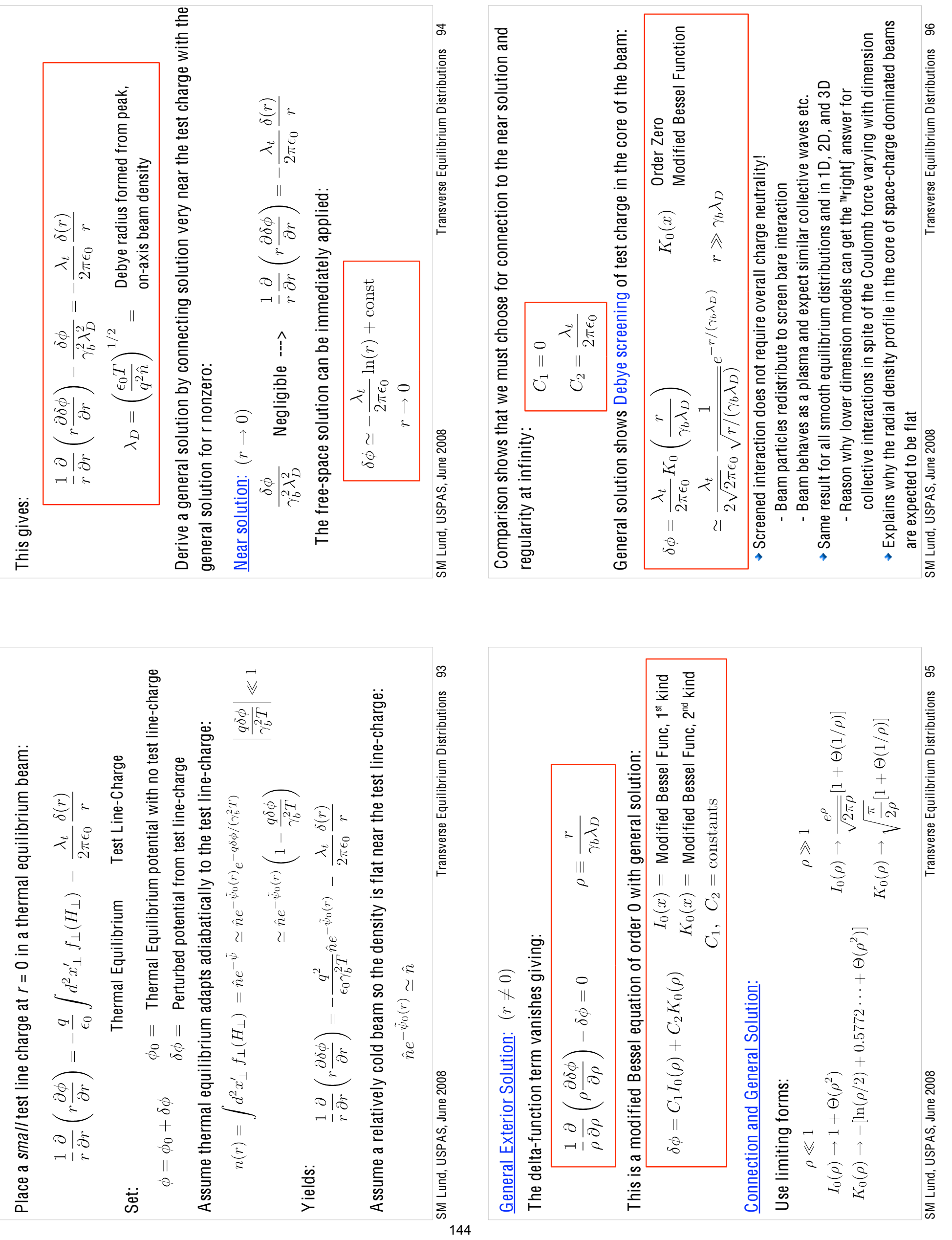

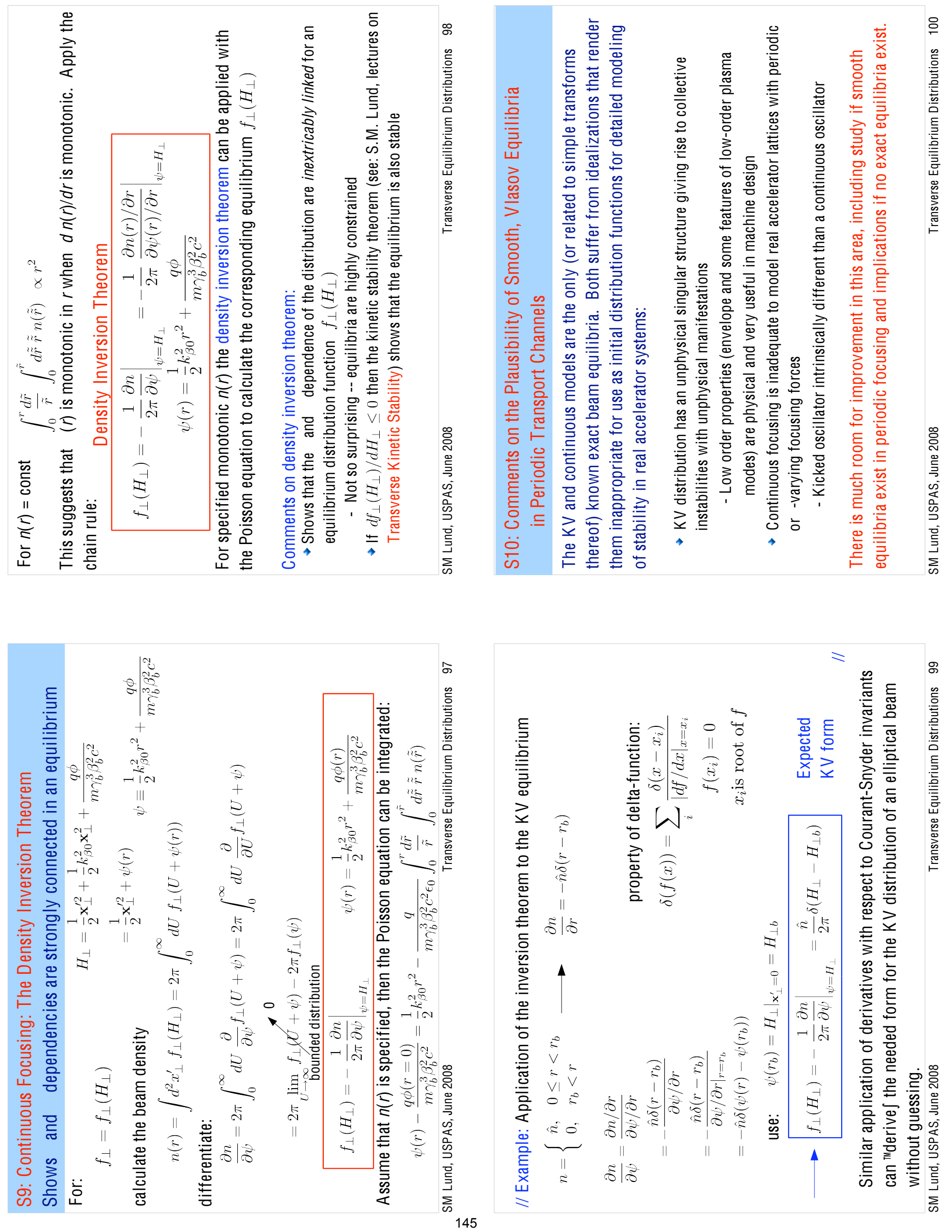

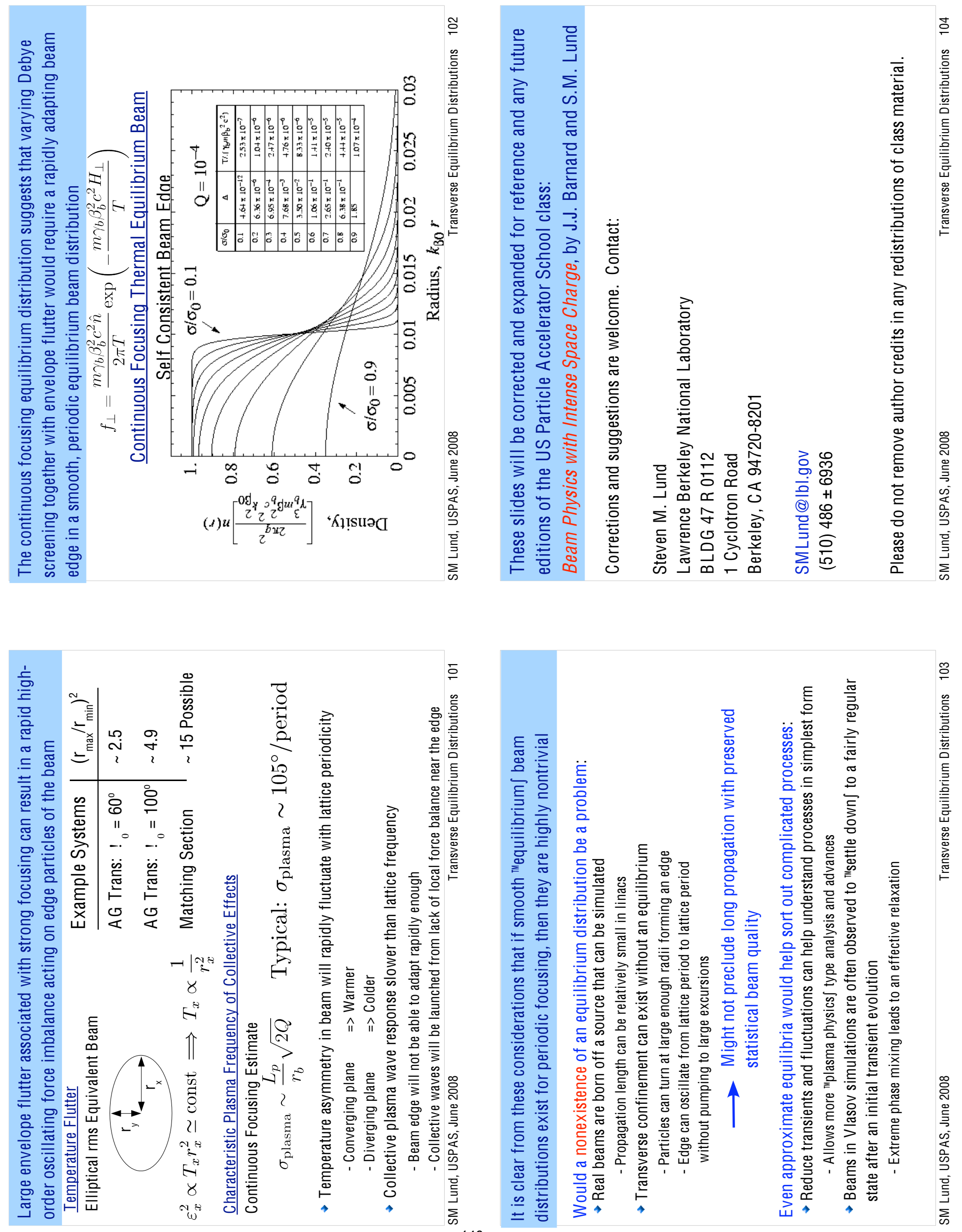


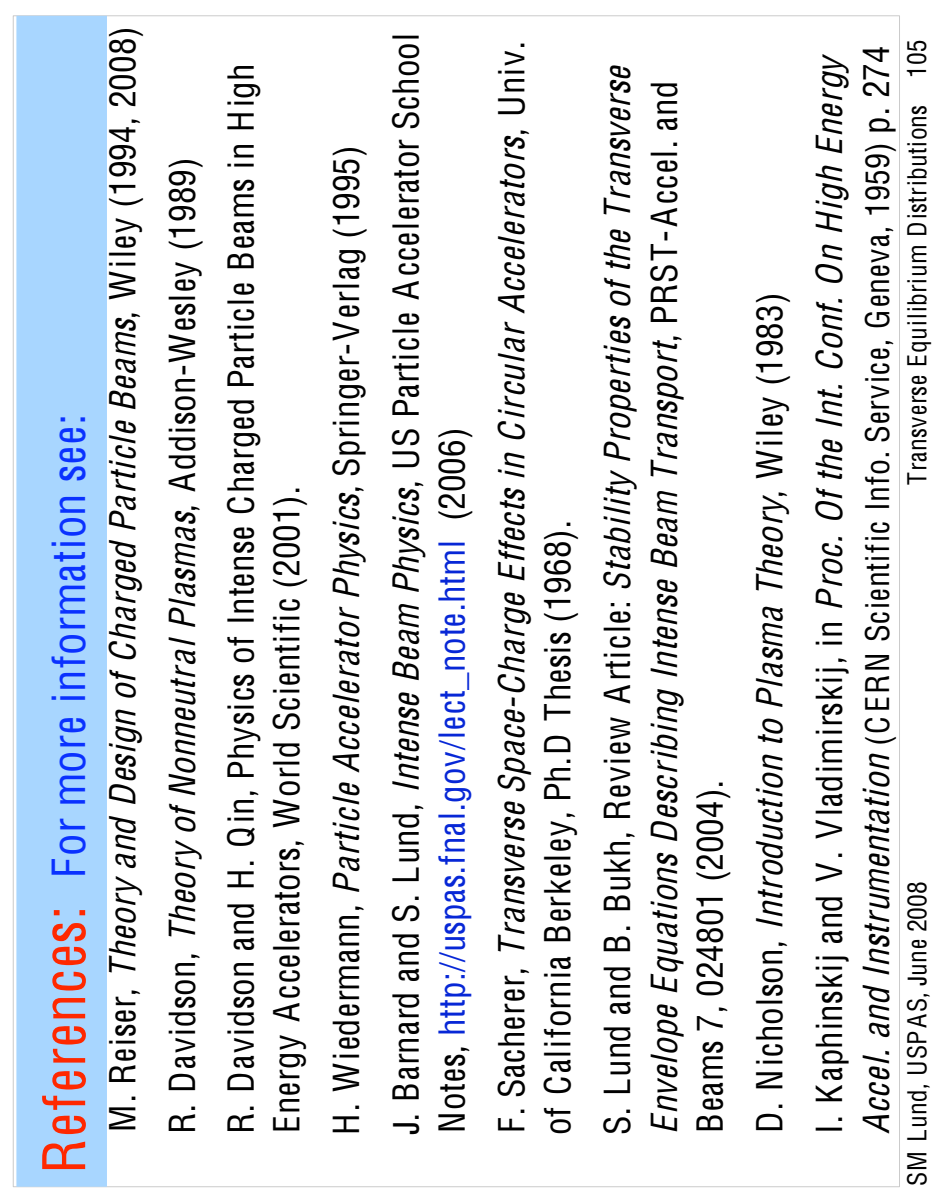


J.J. Barnard and S,M. Lind USPAS June 2008 Appendix A

Self Fields of a Uniform Density Elliptical Beam in Free Space

$$
\begin{aligned}
\left(\frac{\partial^{2}}{\partial x^{2}}+\frac{\partial^{2}}{\partial y^{2}}\right) \phi & =\left\{\begin{array}{cl}
-\frac{\lambda}{\pi \varepsilon_{0} \Gamma_{x} r_{y}} ; & \frac{x^{2}}{\sqrt{x}}+\frac{y^{2}}{\sqrt{y^{2}}}<1 \\
0 ; & \frac{x^{2}}{r_{x}^{2}}+\frac{y^{2}}{r_{y}^{2}}>1
\end{array}\right. \\
\frac{\partial \phi}{\partial r} & \sim \frac{\lambda}{2 \pi \varepsilon_{0} r} \text { as } r \rightarrow \infty .
\end{aligned}
$$

The solution to this system to an arb. constant has been formally constructed by Landau. \& Litshitz and others (gravitational field analog) as:

$$
\phi=-\frac{\lambda}{4 \pi \varepsilon_{0}}\left\{\int_{0}^{\xi} \frac{d s}{\left[\left(r_{x}^{2}+s\right)\left(r_{y}^{2}+s\right)\right]^{1 / 2}}+\int_{\xi}^{\infty} \frac{d s}{\left[\left(r_{x}^{2}+s\right)\left(r_{y}^{2}+s\right)\right] / 2}\left(\frac{x^{2}}{r_{x}^{2}+s}+\frac{y^{2}}{r_{y}^{2}+s}\right)\right\}
$$

where + canst.

$\int \xi=0: 5$ when $\left(x / F_{x}\right)^{2}+\left(y / h_{y}\right)^{2}<1$

$\sum \sum_{\text {root of }} \frac{x^{2}}{r_{x}^{2}+\xi}+\frac{y^{2}}{r_{y}^{2}+\xi}=1$, when $\left(x / x_{x}\right)^{2}+\left(y / T_{y}\right)^{2}>1$

Trivially for $x=y=0$

$$
\phi(x=y=0)=\text { canst. }
$$

Calculate:

$$
\begin{aligned}
\frac{\partial \phi}{\partial x}=-\frac{\lambda}{4 \pi \varepsilon_{0}} & \left\{\int_{\xi}^{\infty} \frac{d s}{\left[\left(r_{x}^{2}+s\right)\left(r_{y}^{2}+s\right)\right]^{1 / 2}} \frac{2 x}{r_{x}^{2}+s}\right. \\
& \left.-\frac{1}{\left[\left(r_{x}^{2}+\xi\right)\left(r_{y}^{2}+\xi\right]^{1 / 2}\right.}\left[1-\frac{x^{2}}{r_{x}^{2}+\xi}-\frac{y^{2}}{r_{y}^{2}+\xi}\right] \frac{\partial \xi}{\partial x}\right\}
\end{aligned}
$$

If $\begin{aligned} \xi \neq 0 & \Rightarrow 1=\frac{x^{2}}{\Gamma_{x}^{2}+\xi}+\frac{y^{2}}{r_{y}^{2}+\xi} \\ \xi=0 \Rightarrow \frac{\partial \xi}{\partial x}=0 & \Rightarrow \Rightarrow \begin{array}{l}\text { Ind form } \\ \text { vanishes }\end{array}\end{aligned}$ 
SiM. Lond Az/

$$
\frac{\partial \phi}{\partial x}=-\frac{\lambda}{2 \pi \varepsilon_{0}} \int_{\xi}^{\infty} \frac{d s}{\left[\left(r_{x}^{2}+s\right)\left(r_{y}^{2}+s\right)\right]^{1 / 2}} \frac{x}{r_{x}^{2}+5}
$$

by symmetry

$$
\frac{\partial \phi}{\partial y}=-\frac{\lambda}{2 \pi \varepsilon_{0}} \int_{\xi}^{\infty} \frac{d s}{\left[\left(r_{x}^{2}+s\right)\left(r_{y}^{2}+s\right)\right]} \frac{y}{r_{y}^{2}+s}
$$

Differentiating again and using the chain role:

$$
\begin{aligned}
\left(\frac{\partial^{2}}{\partial x^{2}}+\frac{\partial^{2}}{\partial y^{2}}\right) \phi & =-\frac{\lambda}{2 \pi \varepsilon_{0}}\left\{\int_{\xi}^{\infty} \frac{d s}{\left[\left(x^{2}+s\right)\left(r_{y}^{2}+s\right)\right]^{1 / 2}}\left[\frac{1}{r^{2}+s}+\frac{1}{r_{y}^{2}+s}\right]\right. \\
& \left.-\frac{1}{\left[\left(\left(x^{2}+\xi\right)\left(r_{y}^{2}+\xi\right)\right]^{1 / 2}\right.}\left[\frac{x \partial s / \partial x}{r_{x}^{2}+\xi}+\frac{y \partial \xi / \partial y}{r^{2}+\xi}\right]\right\}
\end{aligned}
$$

Must show that the rites. reduces to the needed forms for: case l exterior $\xi$ satisfies: $\frac{x^{2}}{x_{x}^{2}+\xi}+\frac{y^{2}}{r^{2}+\xi}=1$ case interior $\xi=0$

case l (exterior: $\left.x^{2} / r_{x}^{2}+y^{2} / r^{2}>1\right)$

Distrentiate $\quad \frac{x^{2}}{\sqrt{x^{2}+\xi}}+\frac{y^{2}}{\sqrt{y^{2}+\xi}}=1$

$$
\begin{aligned}
& \Rightarrow \quad \frac{\partial \xi}{\partial x}=\frac{2 x}{\left(x_{x}^{2}+\xi\right)\left[\frac{x^{2}}{\left(x^{4}+\xi\right)^{2}}+\frac{y^{2}}{\left(x^{2}+\xi\right)^{2}}\right]} \\
& \frac{\partial \xi}{\partial y}=\frac{2 y}{\left(x_{x}^{2}+\xi\right)\left[\frac{x^{2}}{\left(x^{2}+y^{2}\right)^{2}}+\frac{y^{2}}{\left(y^{2}+\xi\right)^{2}}\right]} \\
& \Rightarrow \frac{x \partial \xi / \partial x}{r_{x}^{2}+\xi}+\frac{y \cdot \partial \xi \partial y}{r^{2}+\xi}=2\left[\frac{x^{2}}{\left(r_{x}^{2}+\xi\right)^{2}}+\frac{y^{2}}{\left.\left(r_{y}^{2}+\xi\right)^{2}\right]} \frac{1}{\left[\frac{x^{2}}{\left(r_{x}^{2}+\xi\right)^{2}}+\frac{y^{2}}{\left(y_{y}^{2}+\xi\right)^{2}}\right]}=2\right.
\end{aligned}
$$

Also need integrals like: $\quad W^{2}=s+y^{2} \infty$

$$
I_{x}(\xi) \equiv \int_{\xi}^{\infty} \frac{d s}{\left[\left(r_{x}^{2}+s\right)\left(r_{y}^{2}+s\right)_{4}\right]^{1 / 2}} \frac{1}{\Gamma_{x}^{2}+s}=2 \int_{\sqrt{r_{x}^{2}+\xi}}^{\infty} \frac{d w}{\left(r_{x}^{2}-r_{y}^{2}+w^{2}\right)^{3 / 2}}
$$


S.M. Lond

As)

This integral can be done using tables:

$$
I_{x}(\xi)=\left.\frac{z W}{\left(r_{x}^{2}-r_{y}^{2}\right) \sqrt{r_{x}^{2}-r_{y}^{2}+w^{2}}}\right|_{w=\sqrt{r_{x}^{2}+\xi}} ^{w \rightarrow \infty}=\frac{2}{r_{x}^{2}-r_{y}^{2}}-\frac{2 \sqrt{r_{y}^{2}+\xi}}{\left(r_{x}^{2}-r_{y}^{2}\right) \sqrt{\sqrt{r_{x}^{2}+\xi}}}
$$

Similarly:

$$
\begin{aligned}
& I_{y}(\xi) \equiv \int_{\xi}^{\infty} \frac{d s}{\left[\left(r_{x}^{2}+s\right)\left(r_{y}^{2}+s\right)\right]^{1 / 2}} \frac{1}{\left(r_{y}^{2}+s\right)}=\frac{2}{r_{y}^{2}-r_{x}^{2}}-\frac{2 \sqrt{r_{x}^{2}+\xi}}{\left(\sigma r y_{y}^{2}-\sigma_{x}^{2}\right) \sqrt{r_{y}^{2}+\xi}} \\
& \begin{aligned}
\int_{0}^{\infty} \frac{d s}{\left[\left(r_{x}^{2}+s\right)\left(r_{y}^{2}+s\right)\right]^{2 / 2}}\left[\frac{1}{r_{x}^{2}+s}+\frac{1}{r_{y}^{2}+s}\right]=I_{x}(\xi)+I_{y}(\xi) \\
=\frac{2}{\sigma_{x}^{2}-r_{y}^{2}}\left(\frac{\sqrt{r_{x}^{2}+\xi}}{\sqrt{r_{y}^{2}+\xi}}-\frac{\sqrt{r_{y}^{2}+\xi}}{\sqrt{r_{x}^{2}+\xi}}\right)=\frac{2}{\left[\left(r_{x}^{2}+\xi\right)\left(r_{y}^{2}+\xi\right)\right]^{1 / 2}}
\end{aligned}
\end{aligned}
$$

Using these results:

$$
\begin{aligned}
\left(\frac{\partial^{2}}{\partial x^{2}}+\frac{\partial^{2}}{\partial y^{2}}\right) \phi & =\frac{\lambda}{2 \pi \varepsilon_{0}}\left\{\frac{2}{\left\{\left[\left(r_{x}^{2}+\xi\right)\left(r_{y}^{2}+\xi\right)\right]^{1 / 2}\right.}-\frac{2}{\left.\left[\left(r_{x}^{2}+\xi\right)\left(r_{y}^{2}+\xi\right)\right]^{1 / 2}\right\}}\right. \\
& =0 \quad \text { checks. }
\end{aligned}
$$

case z (interior: $\left.x^{2} / r^{2}+y^{2} / r^{2}<1\right)$

$$
\begin{gathered}
\xi=0 \Rightarrow \frac{x \partial \xi / \partial x}{r_{x}^{2}+\xi}+\frac{y j / \partial y}{r_{y}^{2}+\xi}=0 \\
\Rightarrow I_{x}(\xi=0)=I_{y}(\xi)=\frac{2}{\left(\Gamma_{x}+r_{y}\right) r_{x}} \text { and } I_{y}(\xi=0)=\frac{2}{\left(\Gamma_{x}+r_{y}\right) r_{y}}
\end{gathered}
$$

Using these results:

$\left(\frac{\partial^{2}}{\partial x^{2}}+\frac{\partial^{2}}{\partial y^{2}}\right) \phi=-\frac{\lambda}{2 \pi \varepsilon_{0}}\left\{\frac{2}{\sqrt{x} r_{y}}-0\right\}=\frac{-\lambda}{\varepsilon_{0} \pi \sqrt{x} \sqrt{y}_{y}}=\frac{\text { checks }}{\frac{\tilde{n} \hat{n}}{\varepsilon_{0}}}$

Finally, check the limiting form outside the beam for $r$ large $\Rightarrow 3$ large.

$$
\begin{array}{ll}
-\frac{\partial \phi}{\partial x}=\frac{\lambda}{2 \pi \varepsilon_{0}} \times I_{x}(\xi) & \lim _{r \rightarrow \infty} I_{x}(\xi)=\frac{1}{\xi}=\frac{1}{r^{2}} \\
-\frac{\partial \phi}{\partial y}=\frac{\lambda}{2 \pi \varepsilon_{0}} y I_{y}(\xi) & \lim _{r \rightarrow \infty} I_{y}(\xi)=\frac{1}{\xi}=\frac{1}{r^{2}}
\end{array}
$$


S.M. Lond

AS.

Thus:

$$
\begin{aligned}
& \lim _{r \rightarrow \infty}-\frac{\partial \phi}{\partial x}=\frac{\lambda}{2 \pi \varepsilon_{0}} \frac{x}{r^{2}} \\
& \lim _{r \rightarrow \infty}-\frac{\partial \phi}{\partial y}=\frac{\lambda}{2 \pi \varepsilon_{0}} y
\end{aligned}
$$

These have the correct / smiting forms for a line charge at the origin. Completing the verification of the general formula.

In the beam $\left(x^{2} / x^{2}+y^{2} / y^{2}<1, \xi=0\right)$, the formula reduces to:

$$
\begin{aligned}
\phi & =-\frac{\lambda}{4 \pi \varepsilon_{0}}\left\{x^{2} I_{x}(\xi=0)+y^{2} I_{y}(\xi=0)\right\}+\text { cons. } \\
& =-\frac{\lambda}{4 \pi \varepsilon_{0}}\left\{\frac{2 x^{2}}{r_{x}\left(r_{x}+r_{y}\right)}+\frac{2 y^{2}}{\left.r_{(1 / x}+r_{y}\right)}\right\}+\text { cons. } \\
\phi & =-\frac{\lambda}{2 \pi \varepsilon_{0}}\left\{\frac{x^{2}}{\Gamma_{x}\left(r_{x}+r_{y}\right)}+\frac{y^{2}}{r_{y}\left(r_{x}+r_{y}\right)}\right\}+\text { cont. }
\end{aligned}
$$

The case of an axisymmetric beam with

$$
r_{x}=r_{y}=r_{b}
$$

is easy to construct explicitly and is included in the homework problems.

There is also an alternative way to do this tied calculation, that is less direct but more efficient, We carry out this proof how since steps involved are useful for other 15purposes. 
$S_{1} M_{1}$ Land

AS)

A density profile with elliptic symmetry can be expressed as:

$$
n(x, y)=n\left(\frac{x^{2}}{\sqrt{x}}+\frac{y^{2}}{1 y^{2}}\right)
$$

Here we do not assume a specific uniform density profile and we leave $n\left(x^{2} / r_{x}^{2}+y^{2} / r_{y}^{2}\right)$ arbitrary outside of having elliptic symmetry. The solution to the 2D Poisson equation in tree-space $+1$

$$
\left(\frac{\partial^{2}}{\partial x^{2}}+\frac{\partial^{2}}{\partial y^{2}}\right) \phi=-\frac{g n}{2 \varepsilon_{0}}
$$

is then given by

$$
\phi=-\frac{q x}{4 \varepsilon_{0}} \int_{0}^{\infty} \frac{d \xi \frac{2(z)}{\sqrt{1 x_{x}^{2}+\xi} \sqrt{r_{y}^{2}+\xi}}}{\partial \equiv=\frac{x^{2}}{\sqrt{x^{2}+\xi}}+\frac{y^{2}}{y^{2}+\xi}}
$$

where. $\eta(8)$ is a function defined such that:

$$
n(x, y)=\left.\frac{d \eta(z)}{d \nu}\right|_{\xi=0}
$$

This choice for $\eta(x)$ can always be made.

152 
S.M. Lond AG

We first prove that this solution is valid by direct substitution:

$$
\begin{aligned}
& \mathscr{X}=\frac{x^{2}}{r_{x}^{2}+\xi}+\frac{y^{2}}{r_{y}^{2}+\xi} \Rightarrow \frac{\partial y}{\partial x}=\frac{2 x}{r_{x}^{2}+\xi} ; \frac{\partial^{2} \mathscr{y}}{\partial x^{2}}=2 \\
& \frac{\partial D}{\partial y}=\frac{2 y}{r_{y}^{2}+\xi} ; \frac{\partial^{2} y}{\partial y^{2}}=\frac{2}{1 y^{2}+\xi}
\end{aligned}
$$

Substitute in Poisson's equation and use the chain rule and results above:

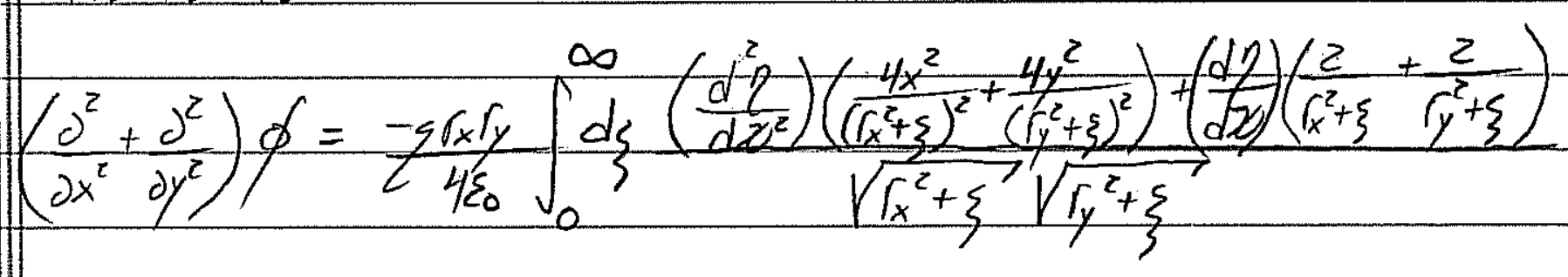

Note: $\quad d x=-\left[\frac{x^{2}}{\left(\Gamma_{x}^{2}+\xi\right)^{2}}+\frac{y^{2}}{\left(\Gamma_{y}^{2}+\xi\right)}\right] d \xi$

so the first integral can be simplified by partial integration:

$$
\begin{aligned}
& \int_{0}^{\infty} \frac{d \xi\left(\frac{d^{2} y}{d x^{2}}\right)\left(\frac{4 x^{2}}{\left(x_{x}^{2}+\xi^{2}\right.}+\frac{4 y^{2}}{\left(\sqrt{y^{2}}+\xi\right)^{2}}\right)}{\sqrt{\sqrt{x}^{2}+\xi \sqrt{r_{y}^{2}+\xi^{2}}}}=-4 \int_{0}^{\infty} \frac{d \xi}{\frac{d^{2} y}{d y^{2}} \frac{d y}{d \xi}}
\end{aligned}
$$

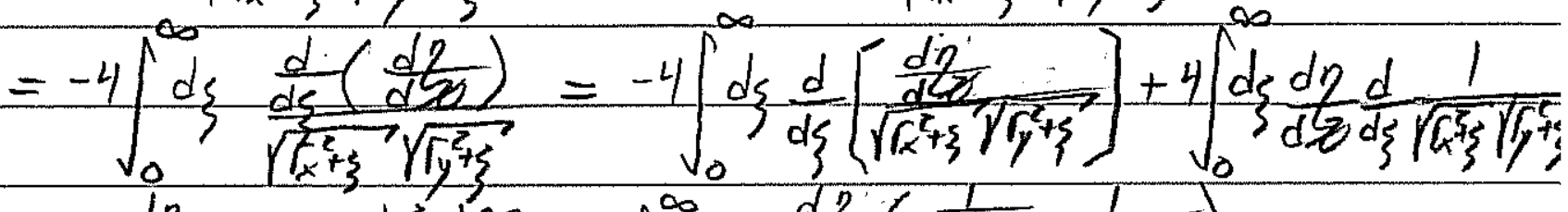

$$
\begin{aligned}
& \left.=\left.\frac{-4 \frac{d \eta}{d x}}{\sqrt{x_{x}^{2}+\xi \sqrt{r_{y}^{2}+\xi}}}\right|_{\xi \rightarrow 0} ^{\xi \rightarrow \infty}-2 \int_{0}^{\infty} \frac{d \xi}{\frac{d \eta}{d x}\left(\frac{1}{r_{x}^{2}+\xi}+\frac{1}{r_{y}^{2}+\xi}\right.}\right)
\end{aligned}
$$

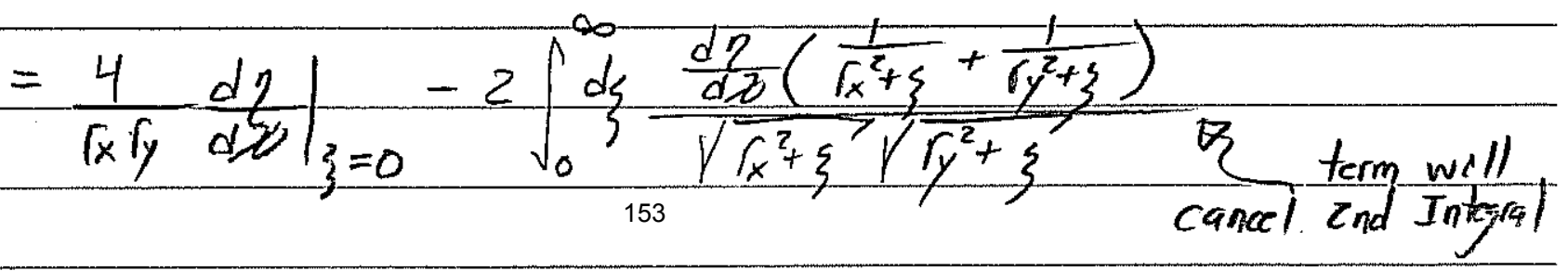


S.M. Lond A7/

Thus:

$$
\left(\frac{\partial^{2}}{\partial x^{2}}+\frac{\partial^{2}}{\partial y^{2}}\right) \phi=-\left.q x_{y \varepsilon_{0}} \frac{y}{x+y} \frac{d \eta(x)}{d x}\right|_{\xi=0}
$$

But $\left.\frac{d \eta(x)}{d x}\right|_{\xi=0}=n(x, y)$ by definition.

$$
\Rightarrow\left(\frac{\partial^{2}}{\partial x^{2}}+\frac{\partial^{2}}{\partial y^{2}}\right) \phi=-g n(x, y) \quad \text { verifying the result. }
$$

For a uniform density ellipse we take:

$$
\eta(x)=\frac{\lambda}{q \pi r_{x} r_{y}}\left\{\begin{array}{ll}
x ; & ; x<1 \\
1, x>1
\end{array} \Rightarrow \frac{d \eta(x)}{d x}=\left\{\begin{array}{l}
\frac{\lambda}{\varepsilon \pi r_{x} ;} ; x<1 \\
0 ; x>1
\end{array}\right.\right.
$$

Thus

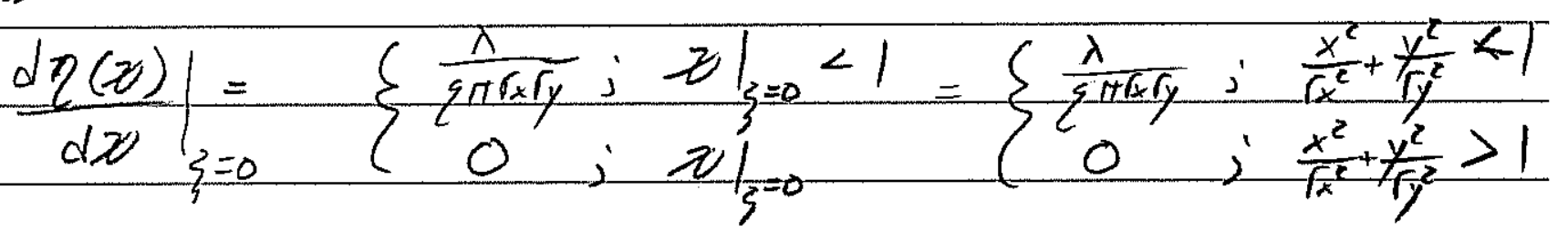

$\left.\therefore \quad \frac{d \eta(x)}{d x}\right|_{z=0}=n(x, y)$ for a uniform density $d x \quad \xi=0 \quad$ elliptical beam. with radii

Apply these results to calculate $\phi\left[\sigma_{x}, r_{y}\right.$ and density $\lambda\left(g \pi \sigma_{x} r_{y}\right)$

$$
\phi=-q \frac{g_{x} r_{y}}{4 \varepsilon_{0}} \int_{0}^{\infty} \frac{d y(z)}{\sqrt{r_{x}^{2}+\xi \sqrt{r_{y}^{2}+\xi}}} \text { density elliptical beam. }
$$

$$
\begin{aligned}
& X=\frac{x^{2}}{x_{x}^{2}+\xi}+\frac{y^{2}}{r_{y}^{2}+\xi} \quad \text { if } \frac{x^{2}}{x_{x}^{2}}+\frac{y^{2}}{x^{2}}<1, \text { then } \\
& \qquad D=\frac{x^{2}}{x_{x}^{2}+\xi}+\frac{y^{2}}{y^{2}+\xi}<1 \text { for all }
\end{aligned}
$$

Using this and the result above for $q(x)$, o inside the elliptical beam is:

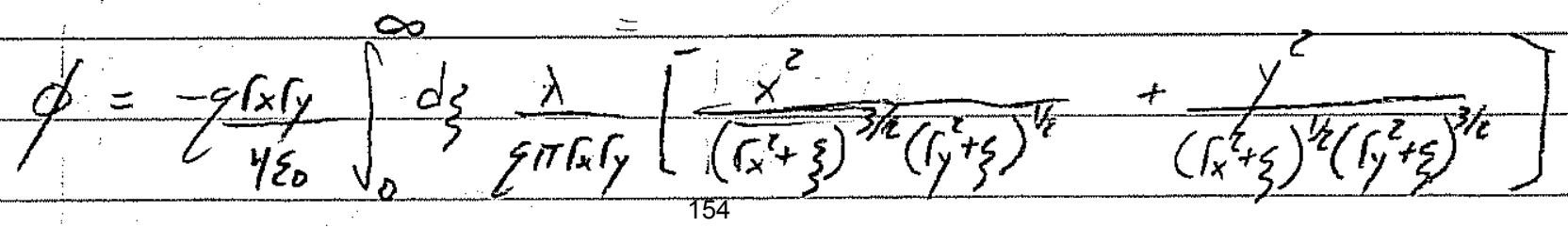


S. M. Lond AB/

$$
\phi=-\frac{\lambda}{4 \pi \varepsilon_{0}}\left\{x^{2} \int_{0}^{\infty} \frac{d}{\left(r_{x}^{2}+\xi\right)^{3 / 2}\left(r_{y}^{2}+\xi\right)^{1 / 2}}+y^{2} \int_{0}^{\infty} \frac{d}{\xi} \frac{1}{\left(r_{x}^{2}+\xi\right)^{1 /(}\left(r_{y}^{2}+\xi\right)^{3 / c}}\right\}
$$

Using Mathematic or Integral tables:

$$
\begin{aligned}
& \int_{0}^{\infty} \frac{d}{\xi \xi} \frac{1}{\left(r_{x}^{2}+\xi\right)^{3 / 3}\left(r_{y}^{2}+\xi\right)^{1 / 2}}=\frac{2}{\Gamma_{x}\left(r_{x}+r_{y}\right)} \\
& \int_{0}^{\infty} \frac{1}{\left(r_{x}^{2}+\xi\right)^{1 / 2}\left(r_{y}^{2}+\xi\right)^{3 / 2}}=\frac{2}{r_{y}\left(r_{x}+r_{y}\right)}
\end{aligned}
$$

Hence

$$
\phi=-\frac{\lambda}{2 \pi \varepsilon_{0}}\left\{\frac{x^{2}}{r_{x}\left(r_{x}+r_{y}\right)}+\frac{y^{2}}{r_{y}\left(r_{x}+r_{y}\right)}\right\}+\text { cons }
$$

since an overall constant can always be added to $\varnothing$ (The integral has a reference choice $\phi(x=y=0)=0$ built in.).

The steps introduced in this proof can also be used to show that:

$$
\begin{array}{ll}
\left\langle x \frac{\partial \phi}{\partial x}\right\rangle_{1}=\frac{-\lambda}{4 \pi \varepsilon_{0}} \cdot \frac{r_{x}}{r_{x}+r_{y}} & \\
\left\langle y \frac{\partial \phi}{\partial y}\right\rangle_{1}=\frac{-\lambda}{4 \pi \varepsilon_{0}} \quad \frac{r_{y}}{r_{x}+r_{y}} & \lambda=g \int d^{2} x n \\
& r_{x} \equiv 2\left\langle x^{2}\right\rangle^{1 / 2} \\
& r_{y} \equiv 2\left\langle y^{2}\right\rangle^{1 / 2}
\end{array}
$$

for any elliptic symmetry density profile $n(x, y)=n\left(x^{2} / x_{x}^{2}+y^{2} / y_{y}^{z}\right)$. In the intro. lectures these results were employed to show that the kV envelope equations with evolving remittances can be applied to elliptic symmetry beams. This result was first demonstrate by Sacherer: [IEEE Trans Nock Sci. $\left.{ }_{155}^{18}, 1105(1971)\right]$ 
Jive Barnard and S,M Lond USPAS June 2008 Appendix B

$B 1 /$

Canonical Transformation of the TC V Distribution

The single - particle equations of motion can be derived from the Hamiltonian: $\left(\frac{d \overrightarrow{x_{1}}}{d s}=\frac{\partial H}{\partial \vec{x}_{1}^{\prime}}, \frac{d \vec{x}_{1}^{\prime}}{d s}=-\frac{\partial H}{\partial \vec{x}_{2}}\right)$

$$
\begin{aligned}
H_{1}\left(x, y, x^{\prime}, y^{\prime}, s\right)= & \frac{1}{2} x^{\prime 2}+\left[R_{x}(s) \cdots-\frac{2 Q}{\Gamma_{x}(s)\left[\Gamma_{x}(s)+\Gamma_{y}(s)\right]}\right] \frac{x^{2}}{2} \\
& +\frac{1}{2} y^{\prime 2}+\left[r_{y}(s)-\frac{2 Q}{\Gamma_{y}(s)\left[F_{x}(s)+\Gamma_{y}(s)\right]}\right] \frac{y^{2}}{2}
\end{aligned}
$$

Perform a canonical transform to new variables $Y, Y, \bar{X}^{\prime}, Y^{\prime}$ using the generating function

$$
F_{r}\left(x, y, X^{\prime} Y^{\prime}\right)=\frac{x}{w_{x}}\left[X^{\prime}+\frac{x w_{x}^{\prime}}{2}\right]+\frac{y}{w_{y}}\left[Y^{\prime}+\frac{y w_{y}^{\prime}}{z^{\prime}}\right]
$$

Then:

$$
\begin{aligned}
& \bar{X}=\frac{\partial F_{z}}{\partial I^{\prime}}=\frac{x}{W_{x}} \\
& Y=\frac{\partial F_{z}}{\partial Y^{\prime}}=\frac{y}{W_{y}} \\
& \dot{x}^{\prime}=\frac{\partial F_{z}}{\partial \dot{X}}=\frac{1}{W_{x}}\left(\underline{X}^{\prime}+x w_{x}^{\prime}\right) \\
& \dot{y}^{\prime}=\frac{\partial F_{z}}{\partial y}=\frac{1}{w_{y}}\left(Y^{\prime}+y w_{y}^{\prime}\right)
\end{aligned}
$$

Ref:

R.C. Davidson,

"Phys ic s of Nonneutra / Plasmas" Addison-Westeys 1990

comment!

Here, $\Sigma^{\prime} \neq$ $\frac{d}{d s} \bar{X}, X^{\prime}$ merely denotes the conjugate

and solving for $X^{\prime}, Y^{\prime}$ : variable to $X$

$$
\begin{aligned}
& I^{\prime}=w_{x} x^{\prime}-x w_{x}^{\prime} \\
& I^{\prime \prime}=w_{y} y^{\prime}-y w_{y}^{\prime}
\end{aligned}
$$

Also, $\mathbb{X}, \mathbb{X}^{\prime}$ both have dim. meters $1 / 2$

The Courante - Snyder invariants are then simply expressed:

$$
\begin{aligned}
& X^{2}+X^{\prime 2}=\text { cont } \\
& Y^{2}+Y^{\prime 2} \pm 56 \text { cons }
\end{aligned}
$$


S.M. Lond

BL

One can show from the transformations that:

$$
\begin{aligned}
& d x d y=w_{x} w_{y} d X d Y \\
& d x^{\prime} d y^{\prime}=\frac{d \underline{X}^{\prime} d Y^{\prime}}{w_{x} w_{y}} \\
& d x d y d x^{\prime} d y^{\prime}=d X d Y d I^{\prime} d Y^{\prime} \quad \begin{array}{l}
\text { transforms } \\
\text { in general- } \\
\text { Results from } \\
\text { structure of }
\end{array} \\
& \text { structure of }
\end{aligned}
$$

Therefore, the distribution in trans formed phase space variables is the same as for the original variables:

$$
\begin{aligned}
& f_{1}\left(\bar{X}, Y, X^{\prime}, Y^{\prime}, s\right)=f_{i}\left(x, y, x^{\prime}, y^{\prime}, s\right) \\
& =\frac{\lambda}{\varepsilon \pi^{2} \varepsilon_{x} \varepsilon_{y}} \delta\left[\frac{X^{2}+X^{\prime 2}}{\varepsilon_{x}}+\frac{Y^{2}+Y^{\prime 2}}{\varepsilon_{y}}-1\right]
\end{aligned}
$$

Now examine the density:

$$
\begin{aligned}
& n(x, y)=\int d x^{\prime} d y^{\prime} f_{1}=\int \frac{d X^{\prime} d Y^{\prime}}{w_{x} w_{y}} f_{1}= \\
& U_{x}=\bar{X}^{\prime} / \sqrt{\varepsilon_{x}}, \quad U_{y}=I^{\prime} / \sqrt{\varepsilon_{y}} \\
& \Gamma_{x}=\sqrt{\varepsilon_{x}} W_{x}, \Gamma_{y}=\sqrt{\varepsilon_{y}} W_{y} \\
& d U_{x} d U_{y}=\frac{d \bar{X}^{\prime} d Y}{\sqrt{\varepsilon_{x} \varepsilon_{y}}} \\
& n=\frac{\lambda}{\varepsilon \pi^{2} r_{x} r_{y}} \int d U_{x} d U_{y} \delta\left[U_{x}^{2}+U_{y}^{2}-\left(1-\frac{\bar{X}^{2}}{\varepsilon_{x}}-\frac{Y^{2}}{\varepsilon_{y}}\right)\right]
\end{aligned}
$$


S.M. Lond B3

Explat the cylindrical symmetry:

$$
\begin{gathered}
U_{1}^{2}=U_{x}^{2}+U_{y}^{2} \\
d U_{x} d U_{y}=d \psi U_{1} d U_{1}=d \psi \frac{d U_{1}^{2}}{2} \\
n(x, y)=\frac{\lambda}{q \pi^{2} r r_{y}} \int_{0}^{2 \pi} d \psi \int_{0}^{\infty} \frac{d U_{1}^{2}}{2} \delta\left[U_{1}^{2}-\left(1-\frac{x^{2}}{\sqrt{x}}-\frac{y^{2}}{r_{y}^{2}}\right)\right]
\end{gathered}
$$

Thus:

$$
\begin{aligned}
n(x, y) & =\frac{\lambda}{2 \pi r_{x} r_{y}} \int_{0}^{\infty} d V_{1}^{2} \delta\left[U_{1}^{2}-\left(1-\frac{x^{2}}{r_{x}^{2}}-\frac{y^{2}}{r_{y}^{2}}\right)\right] \\
& = \begin{cases}\frac{\lambda}{g \pi r_{x} r_{y}}=\hat{n} ; & \frac{x^{2}}{r_{x}^{2}}+\frac{y^{2}}{r_{y}^{2}}<1 \\
0 \quad & \frac{x^{2}}{r_{x}^{2}}+\frac{y^{2}}{r_{y}^{2}}>1\end{cases}
\end{aligned}
$$

Showing that the singular kv distribution yields the required uniform density beam of elliptical cross-section.

Note

$$
\hat{n}=\frac{\lambda}{q \pi r_{x} r_{y}}
$$

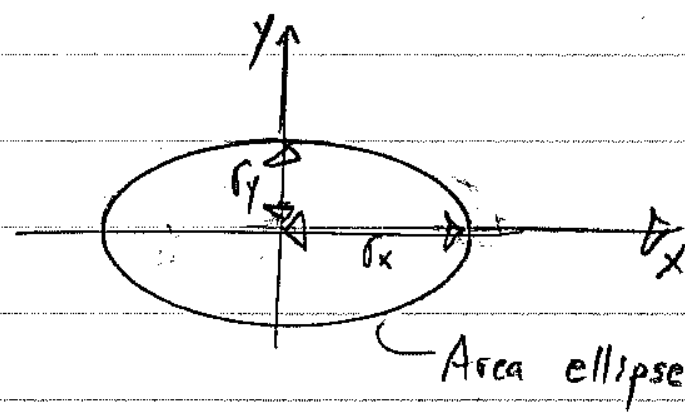

$=\pi r_{x} r_{y}$

$\lambda=g \hat{n} \pi r_{x} r_{y}$

for uniform density. 
S.M. Lond BY

An interesting footnote to this append is that an infinity of Generating functions can be used to transform the $\mathrm{kV}$ distribution in standard quadratic form:

$$
f \pm \sim \delta\left[X^{2}+Y^{\prime 2}+X^{2}+Y^{2}-\text { cons }\right]
$$

to other sets of variables. This will generate other distributions with $K V$ form for skew coupling and other effects. It would not be logical to label such distributions as "new" as has been done in the literature. However, identifying physically relevant transforms has practical value. 
S.M. Lund B5

Inverse transform:

$$
\begin{aligned}
& x=w_{\alpha} \underline{Z} \\
& w_{x} x^{\prime}=\bar{X}^{\prime}+x w_{x}^{\prime} \Rightarrow x^{\prime}=\frac{\bar{X}^{\prime}}{w_{x}}+w_{x}^{\prime} X \\
& x=w_{x} \bar{X} \quad x^{\prime}=\bar{X}^{\prime} / w_{x}+w_{x}^{\prime} \bar{X} \\
& y=w_{y} Y \quad y^{\prime}=I^{-1} / w_{y}+w_{y}^{\prime} Y
\end{aligned}
$$

Next,

$$
\begin{aligned}
\frac{d}{d s} \bar{X} & =\frac{x^{\prime}}{W_{x}}-\frac{x}{W_{x}^{2}} W_{x}^{\prime} \\
& =\frac{\underline{X}^{\prime}}{W_{x}^{2}}+\frac{W_{x}^{\prime} \not}{W_{x}}-\frac{W_{x}^{\prime} X X}{W_{x}}=\frac{X^{\prime}}{W_{x}^{2}}
\end{aligned}
$$

Thos,

$$
\begin{aligned}
& \begin{array}{l}
\frac{d}{d s} \bar{X}=\frac{I^{\prime}}{W_{x}^{2}} \\
\frac{d}{d s} I=\frac{I^{\prime}}{W_{y}^{2}}
\end{array} \\
& \frac{d}{d s} X^{\prime}=W_{x}^{\prime} x^{\prime}+W_{x} x^{\prime \prime}-x^{\prime}+1 x^{\prime \prime}+w_{x}^{\prime \prime}
\end{aligned}
$$

$$
\Rightarrow \begin{aligned}
& x^{\prime \prime}=\frac{\frac{d}{d s} \nabla^{\prime}}{W_{x}}+w_{x}^{\prime \prime} X \\
& y^{\prime \prime}=\frac{\frac{d}{d s} I^{\prime}}{W_{y}}+w_{y}^{\prime \prime} I
\end{aligned}
$$

160 
S.M. Lund

B6/

Apply in Eqn of motion:

$$
\begin{aligned}
& x^{\prime \prime}+R_{x} x-\frac{2 Q x}{\left(R_{x}+\Gamma_{y}\right) \Gamma_{x}}=0 \\
& \frac{\frac{d}{d s} X^{\prime}}{w_{x}}+w_{x}^{\prime \prime} \underline{X}+R_{x} w_{x} X-\frac{1}{w_{x}^{\prime}}-\frac{2 Q w_{x} X}{\left(k_{x}+r_{y}\right) r_{x}}=0 \\
& \text { " } 1 \frac{1}{w_{x}^{\frac{3}{x}}} \text { from } w_{x} \text { equ } \\
& \frac{d}{d s} \bar{X}^{\prime}+W_{x}\left(W_{x}^{\prime \prime}+R_{x} W_{x}-\frac{2 Q W_{x}}{\left(F_{x}+r_{y}\right) \Gamma_{x}}\right) Z=0 \\
& \frac{d}{d s} \bar{X}^{\prime}+\frac{1}{W_{x}^{2}} \bar{X}=0, \quad \frac{d}{d s} \bar{X}=\frac{X^{\prime}}{w_{x}^{2}} \\
& \frac{d}{d s} Y^{\prime}+\frac{1}{W_{y}^{2}} \bar{Y}=0, \quad \frac{d}{d s} Y^{\prime}=\frac{Y^{\prime}}{W_{y}^{2}}
\end{aligned}
$$

Following Diuidson, these equs can be solved

$$
\begin{gathered}
I(s)=\bar{X}_{i} \cos \Psi_{x}(s)+\bar{X}_{p}^{\prime} \sin \psi_{x}(s) \\
\Psi_{x}(s)=\int_{s_{1}}^{s} \frac{d \tilde{s}}{W_{x}^{2}(s)} ; \Psi_{x}^{\prime}(s)=\frac{1}{W_{x}^{2}(s)}
\end{gathered}
$$

etic = This also demonstrates explicity the C.S. invariant.

$$
\underline{X}^{2}+\mathbb{X}^{\prime 2}=\text { const. }
$$

Note:

161

$$
\frac{d}{d s} \bar{X}=\frac{\bar{X}^{\prime}}{W_{x}^{2}}
$$


S.M. Lond

BF/

Transformed Aamdlomian:

$$
\begin{aligned}
& \frac{d}{d s} X \cdot \frac{\partial \tilde{H}}{\partial \bar{X}^{\prime}}=\frac{X^{\prime}}{w_{x}^{2}} \\
& \frac{d}{d s} Y^{\prime}=\frac{\partial \tilde{H}}{\partial \underline{Y}^{\prime}}=\frac{\Psi^{\prime}}{w_{y}^{2}} \\
& \frac{d}{d s} \bar{X}^{\prime}=\frac{-\partial \tilde{H}}{\partial \bar{X}}=-\frac{1}{w_{x}^{2}} \bar{X} \\
& \tilde{H}=\tilde{H}\left(\bar{X}, Y, X^{\prime}, Y^{\prime}\right) \\
& \frac{d}{d s} \underline{I}^{\prime}=-\frac{\partial \tilde{H}}{\partial \Psi}=-\frac{1}{W_{y}^{2}} \tilde{Y} \\
& \Rightarrow \quad H^{2}=\frac{1}{2 w_{x}^{2}} \bar{X}^{\prime 2}+\frac{1}{2 w_{y}^{2}} Y^{\prime 2}+\frac{1}{2 w_{x}^{2}} \bar{X}^{2}+\frac{1}{2 w_{y}^{2}} Y^{2}+\text { cost }
\end{aligned}
$$

Note that $\tilde{H}$ is still explicitly $s$-dependant based on $w_{x}$ and wy raffle functions. This Hamettondan can also be found usia results from the theory of canonical transforms

$$
\Rightarrow \widetilde{H}=H+\frac{\partial F_{2}}{\partial s}
$$

and the coordinate transform expressions.

162 

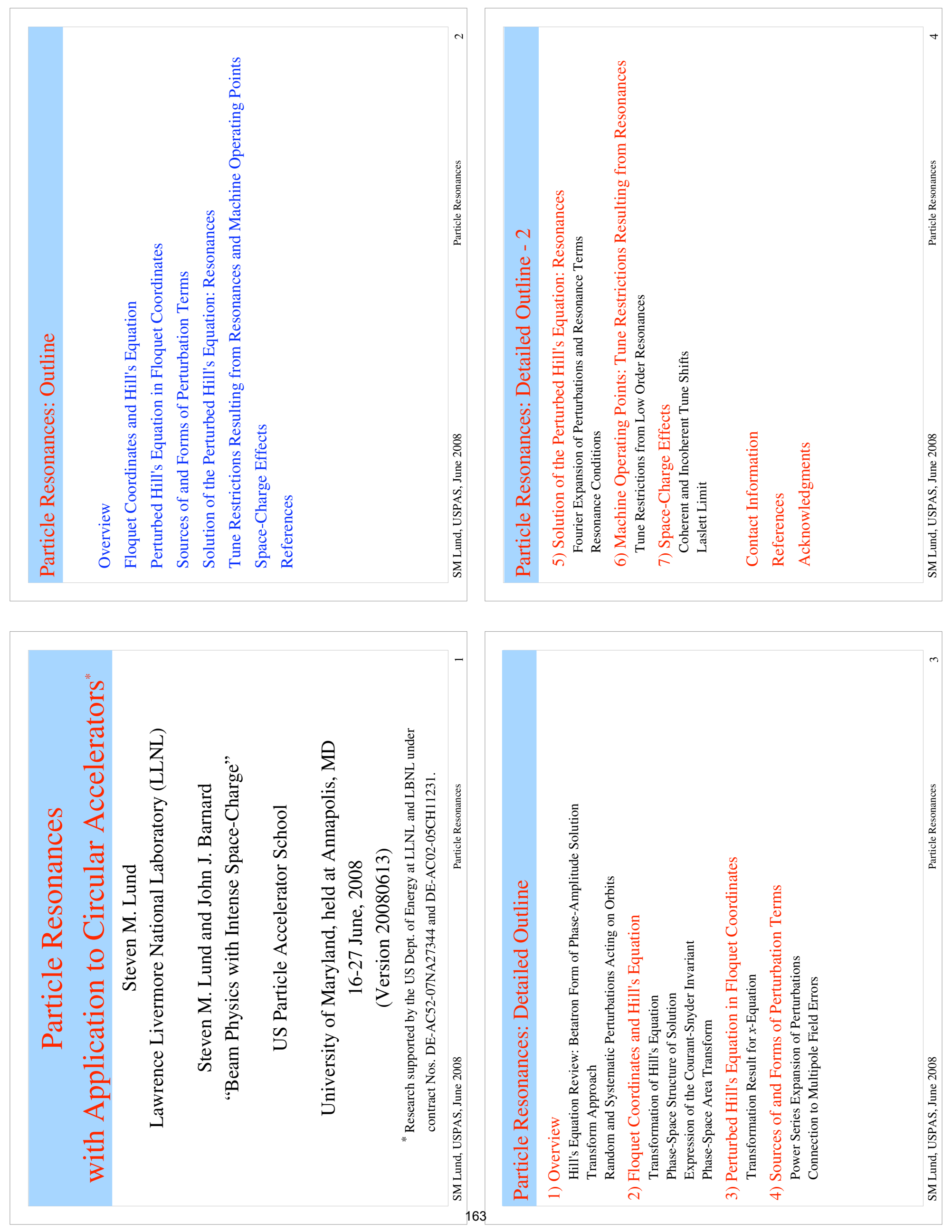

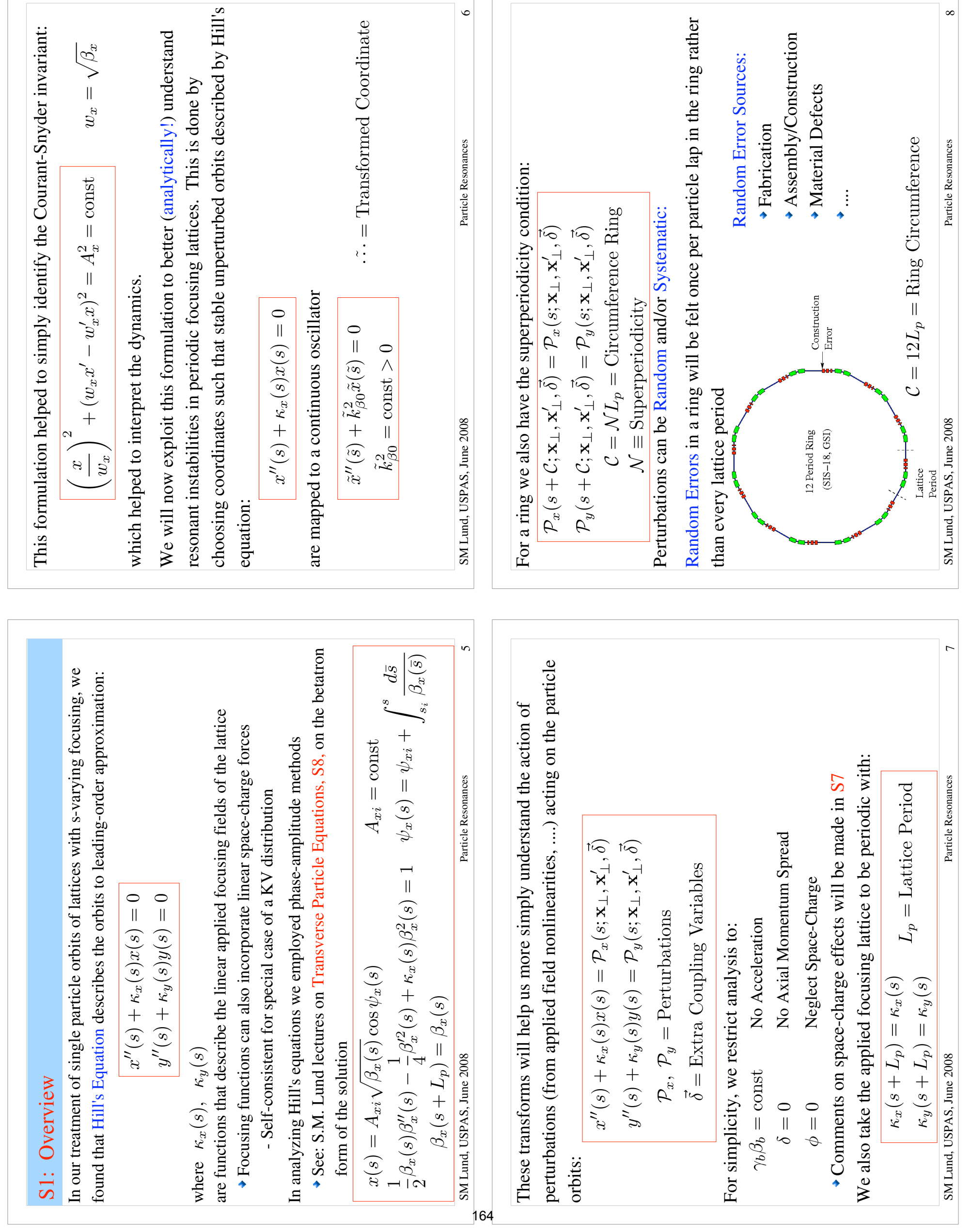

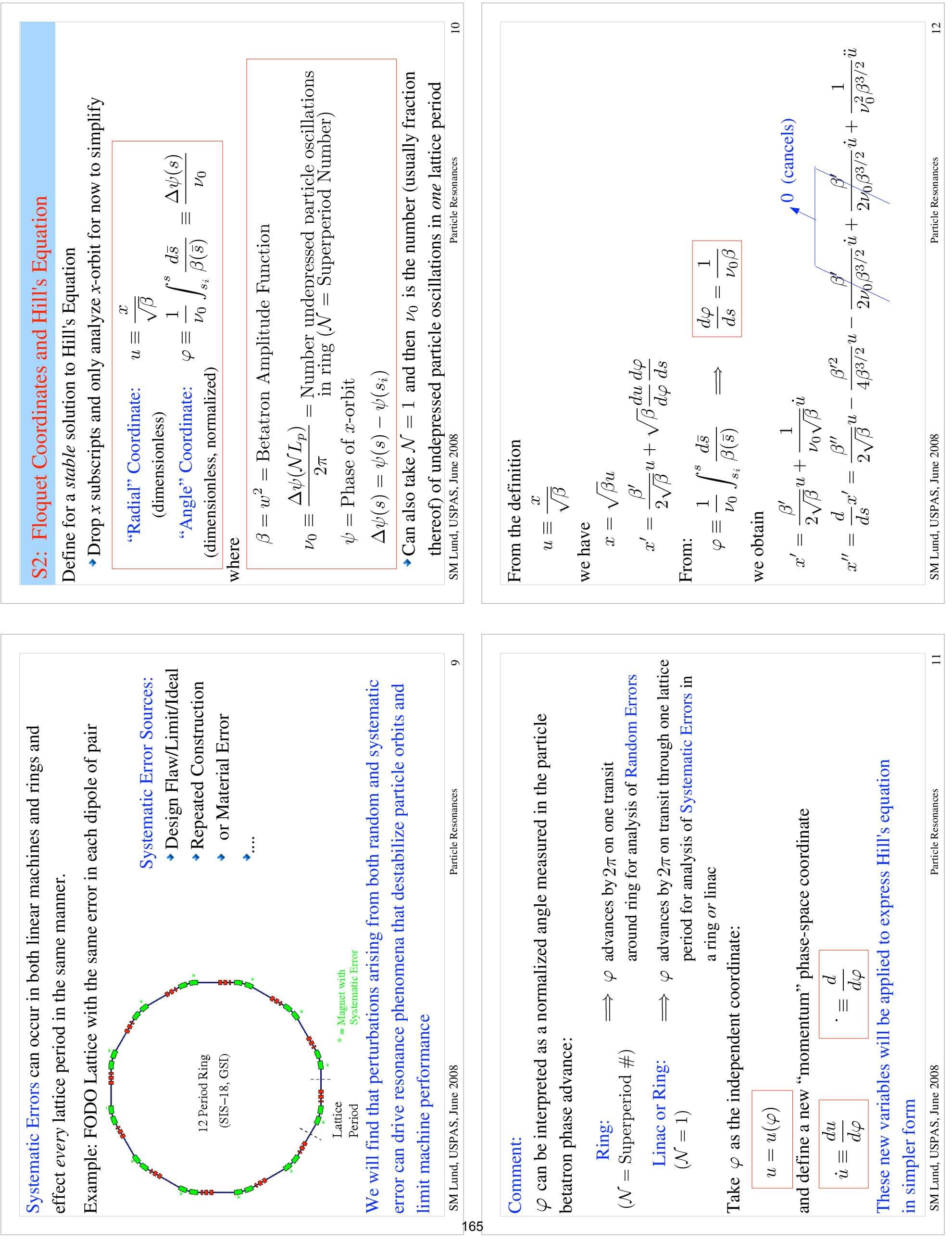

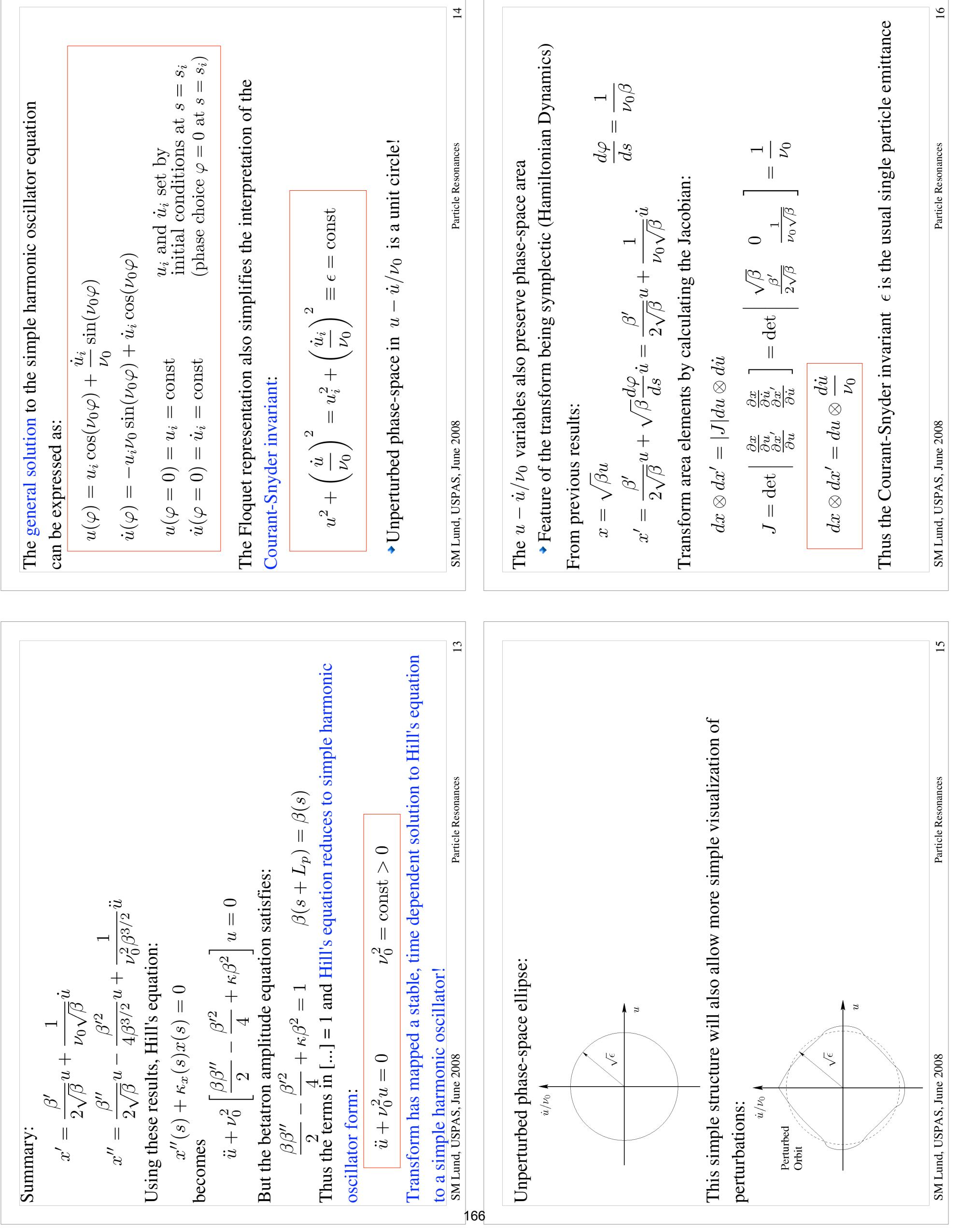

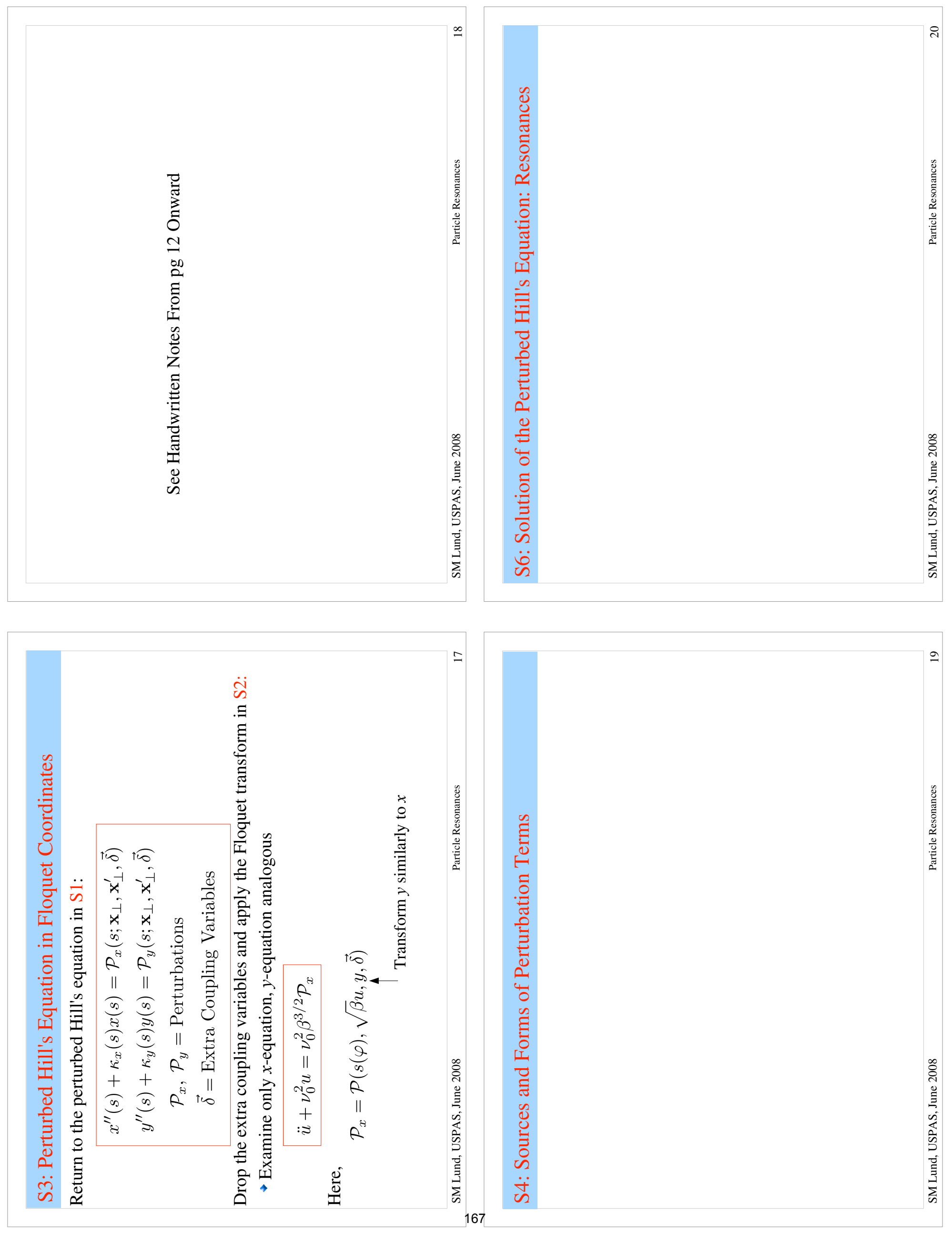


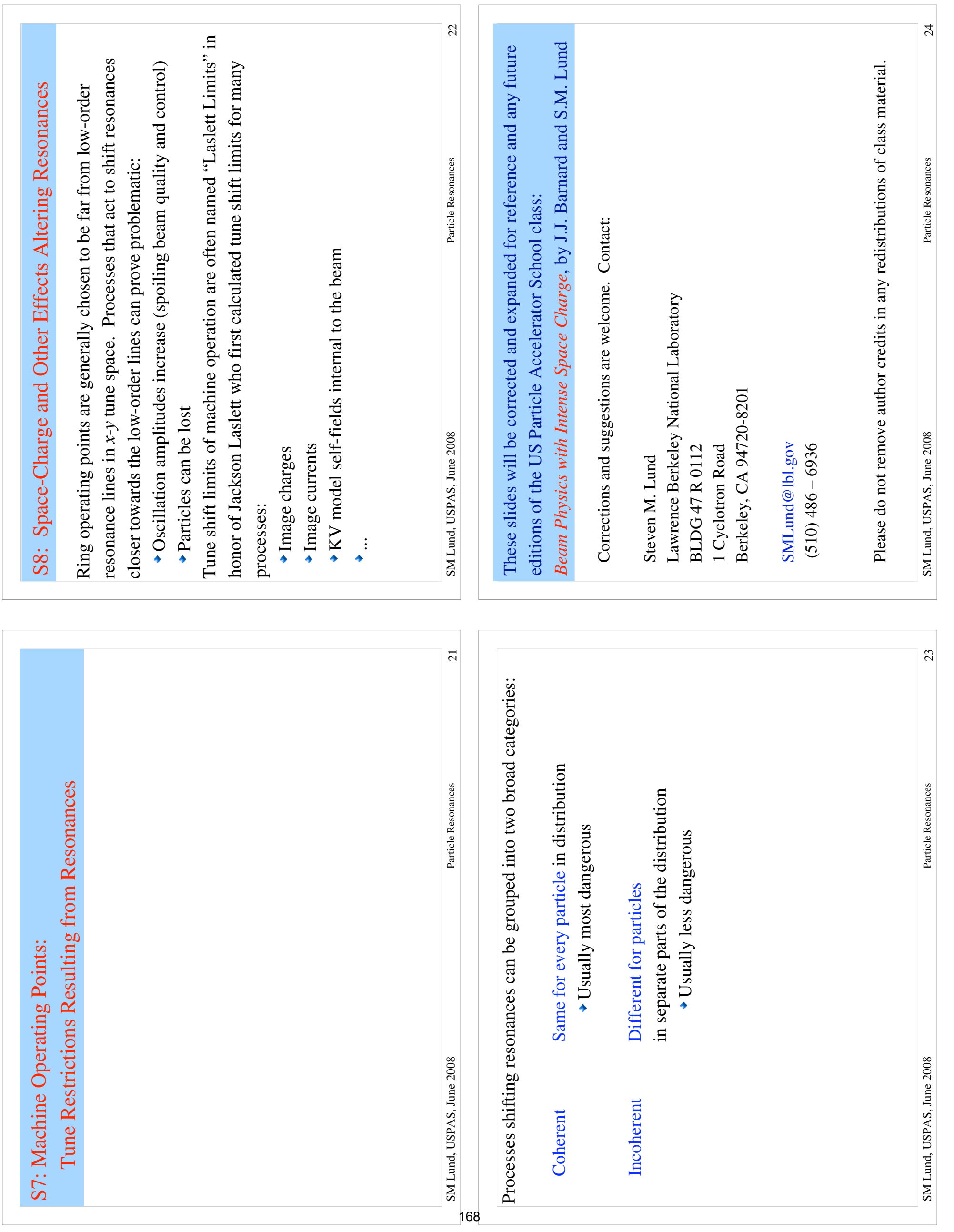



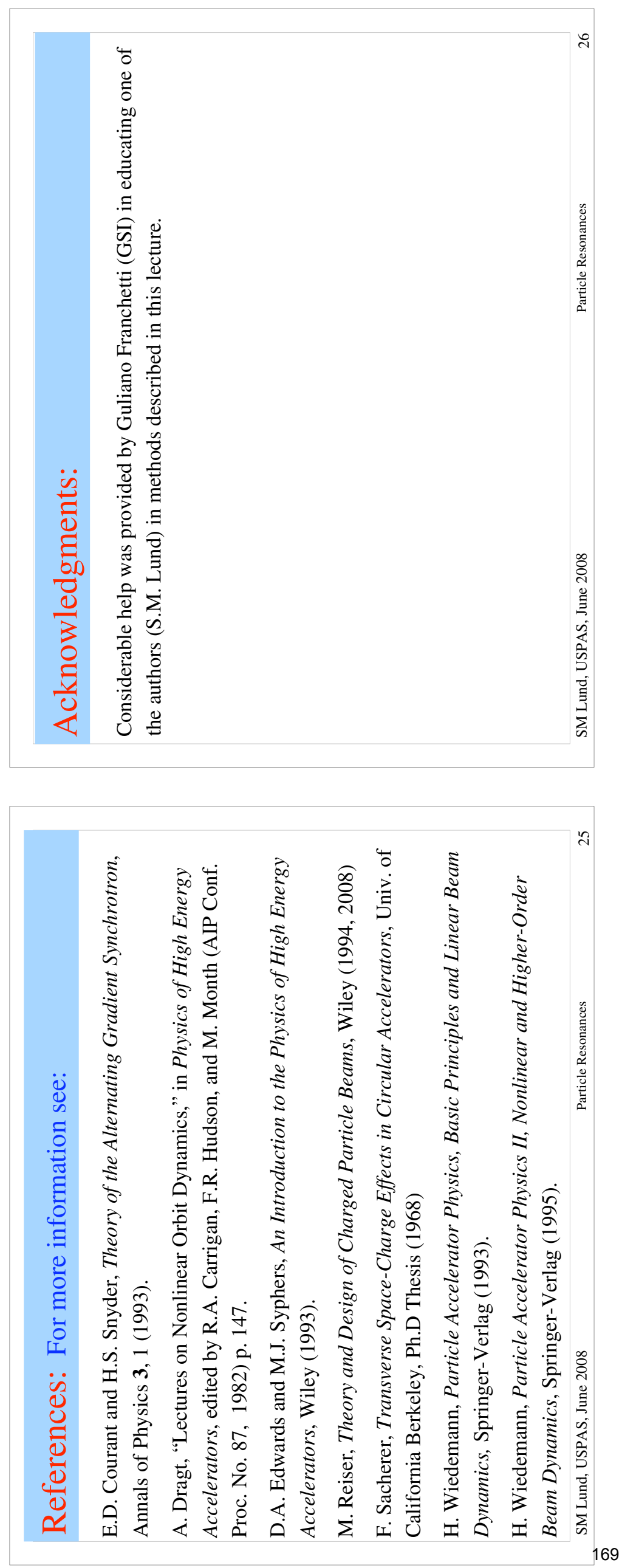
Us Particle Accel School

S,M, Lind

12)

Particle Resonance Notes - Supplement.

We now analyze the effects of perturbations on the dynamics

$$
\ddot{U}+\nu_{0}^{2} U=\nu_{0}^{2} \beta^{3 / 2} P(x, y, s)
$$

Expand the perturbation in a power series - Can always be done for all physical applied field
perturbations.

$$
\begin{aligned}
P_{(}(x, y, s) & =\underline{P}_{0}(y, s)+\underline{P}_{1}(y, s) x+\underline{P}_{2}(y, s) x^{2}+\cdots \\
& =\sum_{n=0}^{\infty} P_{n}(y, s) x^{n}
\end{aligned}
$$

and take

* Prepresents a perturbation due to:

- Systematic or random

$$
x=\sqrt{\beta} u
$$

field errors in magnets.

to obtain

- Alignment error induced

$$
\ddot{u}^{0}+\nu_{0}^{2} U=\nu_{0}^{2} \sum_{n=0}^{\infty} \beta^{\frac{n+3}{2}} P_{n}(y, 5) U^{n}
$$

etc.

To more simply illustrate resonances we will take the particle to move in the x-plane only $(y=0$ for all s). If this is not the case the formalision can be generalized by expanding. the $p_{n}(y, s)$ in a power series in $y$ and generalizing the notation. for the Floguet coordinates to distinguish between the $x$-and $y$-planes pete, The essential character of the more general analysis is Illustrated by this simple case.

$y(s)=\equiv_{70} 0$ to simplify picture 
S.M. Lond

13.

In this special case $(y=0)$ we expand each coefficient in the power series in a Fourier series as:

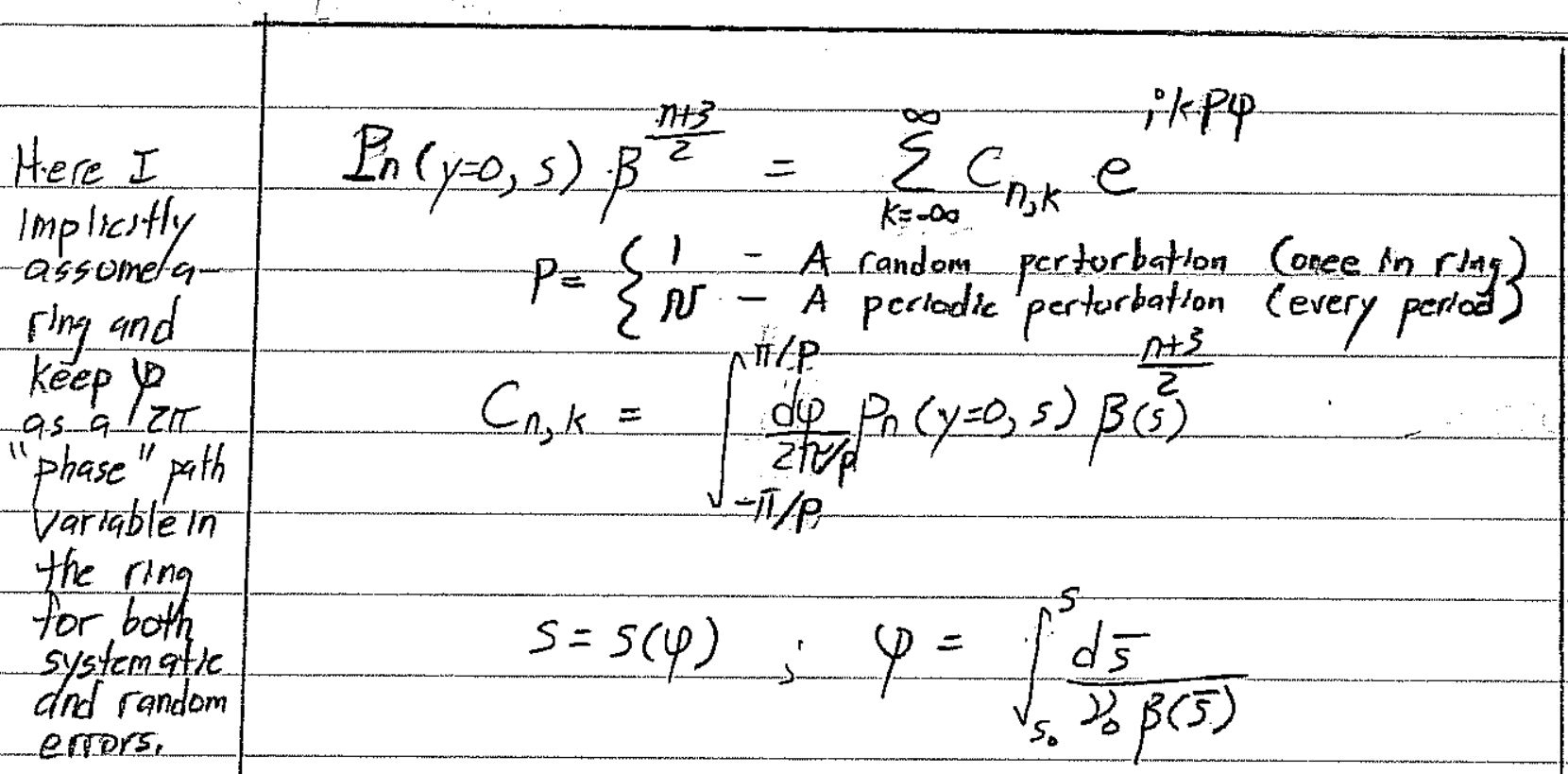

Then the perturbed equation of motion becomes:

$$
\ddot{u}+\nu_{0}^{2} U=\nu_{0}^{2} \sum_{n=0}^{\infty} \sum_{k=-\infty}^{\infty} c_{n_{3}} e^{i k p \varphi} U^{n}
$$

For the case of small amplitude perturbations this equation can be analyzed perturbatively to linear order as:

where!

$$
U=U_{0}+\delta U \quad ;\left|U_{0}\right|>>|\delta U|
$$

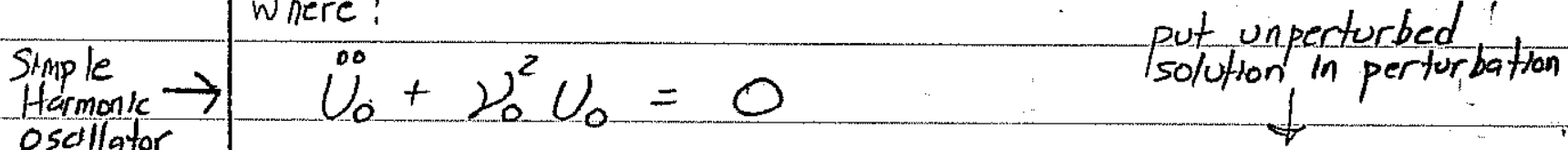

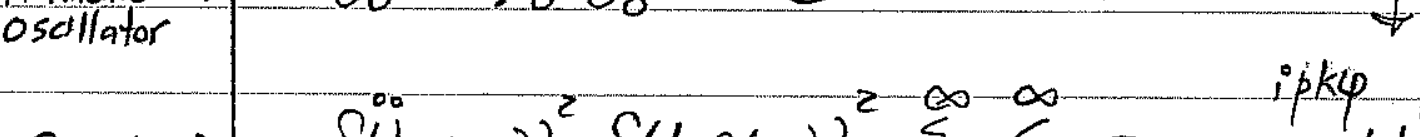

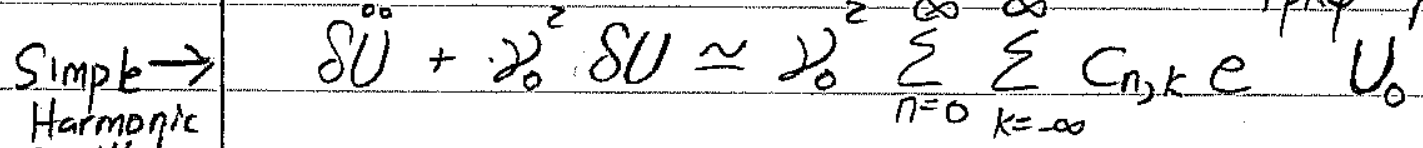

$$
\begin{aligned}
& \text { osdllator } \\
& \text { with } \\
& \text { driving terms. }
\end{aligned}
$$


S.M. Lond 14

Wo represents the unperturbed particle orbit. The general solution to the equation for $U_{0}$

$$
U_{0}=U_{0 i} \cos \left(\nu_{0} \varphi+\psi_{i}\right)
$$

U oi, $\psi_{i}$ initial conditions (constants)

Then,

$$
\begin{aligned}
U_{0}^{n} & =U_{0 i}^{n}\left(\frac{e^{i\left(\nu_{0} \varphi+\psi_{i}\right)}+e^{-i\left(\nu_{0} \varphi+\psi_{i}\right)}}{2}\right)^{n} \\
& =\frac{U_{0 i}^{n}}{2^{n}} \sum_{m=0}^{n}\left(\begin{array}{l}
n \\
m
\end{array}\right) e^{\left.i(n-m) \nu_{0} \varphi+\psi_{i}\right)} e^{-i m\left(\nu_{0} \varphi+\psi_{i}\right)}
\end{aligned}
$$

Here, $\quad\left(\begin{array}{l}n \\ m\end{array}\right) \equiv \frac{n ?}{m !(n-m) !}$ is a binomial coefficient.

$$
=\frac{U_{0 i}^{n}}{2^{n}} \sum_{m=0}^{n}\left(\begin{array}{c}
n \\
m
\end{array}\right) e^{i(n-2 m)-y_{0} \varphi} e^{i(n-2 m) \psi_{i}}
$$

and the perturbed equation becomes:

$$
\begin{gathered}
\delta \dot{U}+\nu_{0}^{2} \delta U \simeq \nu_{0}^{2} \sum_{n=0}^{\infty} \sum_{k=\infty}^{\infty} \sum_{m=0}^{n}\left(\begin{array}{l}
n \\
m
\end{array}\right) C_{n, k} e^{i\left((n-2 m) \psi_{0}+p k\right] \varphi} \\
x e^{i(n-2 m) \psi_{i}}
\end{gathered}
$$

In general, can take $\delta U=\delta\left(u_{h}+\delta u_{p}\right.$ where $\delta u_{h}^{\circ}+\nu_{0}^{2} \delta U_{h}=0$ but in this case we can take $\delta \nu_{h}=0$ since this part of the perturbative solution is contained in No. Thus only the particular solution need be found. From the properties of a driven harmonic oscillator, we know that z no stable solution exists 
S.M. Lond

15)

whenever the the frequency of the driving force equals that of the restoring force (resonant exchange). Thus we have the resonance condition: see Homework?

$$
\begin{aligned}
& (n-Z m) \nu_{0}+p k= \pm \nu_{0} \\
& n=1,2,3, \ldots, \quad m=0,1,2, \cdots \cdots \\
& k=-\infty, \ldots, 0, \ldots ., \infty \\
& p=\left\{\begin{array}{ll}
1 & \text { A random perturbation (one per ring) } \\
\text { IN A periodic perturbation (every lattice pordod) }
\end{array}\right)
\end{aligned}
$$

For yo satisfying this condition the perturbation will grow in amplitude. If the growth rate is sufficiently large, such tunes will be unreliable operating points of the machine and the corresponding perturbation must be corrected. Since this is a linear analysis, the perturbations may be analyzed in furn:

Examples:

$n=0$ (Dipole Perturbation)

$n=0 \Rightarrow m=0$ and the resonance condition becomes:

$$
\nu_{0}= \pm p k \quad p \cdot k=\text { integer }
$$

Therefore:

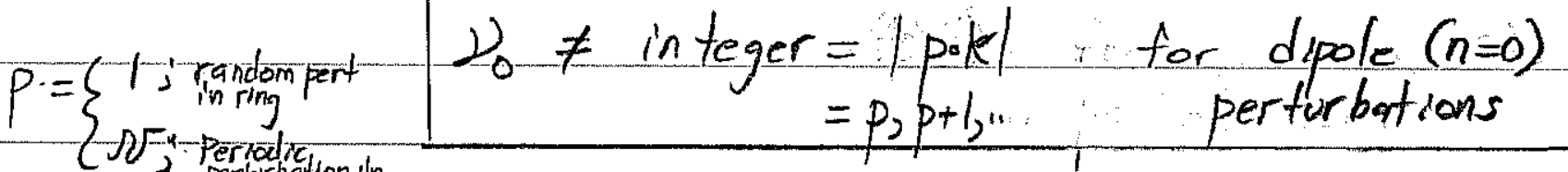

$$
\begin{aligned}
& \text { perturbation in } \\
& \text { perturbation. } \\
& p=1 \mathrm{~N} \cdot \begin{array}{c}
\text { Random error in ring } \\
\text { (once per lap. }
\end{array} \quad \text { yo } \neq 1,2,3, \ldots . . \\
& p=\Omega: \text { Systematic error } \\
& \nu_{0} \neq N, 2 \Omega, 3 /, \ldots
\end{aligned}
$$

Sysfematiou errors less restrictive. 
S.M, Lond

16)

$n=1$ Quadrupole Perturbation

The resonance conditions give: $\Rightarrow n=1, m=0,1$

$$
\begin{aligned}
\nu_{0}+p k= \pm \nu_{0} & (n=1, m=0) \\
-\nu_{0}+p k= \pm \nu_{0} & (n=1, m=1)
\end{aligned}
$$

$p K+\nu_{0}=\nu_{0}$ represents a special case that can be eliminated by "renormalizing" the driving force of the oscillator and $p K=2 \nu_{0}$ implies that:

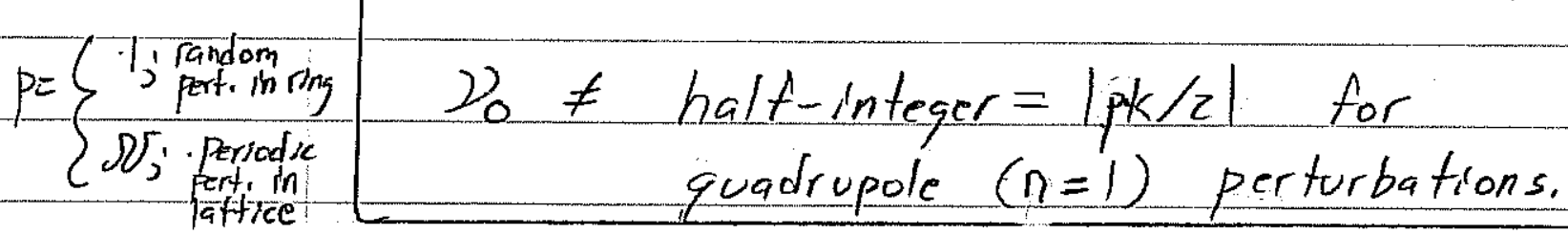

$n=2$ Sextupole Perturb Cations $\Rightarrow n=2, m=0,1,2$

The resonance conditions glue:

$$
\begin{aligned}
2 \nu_{0}+p k & = \pm \nu_{0} \\
p k & = \pm \nu_{0} \\
-2 \nu_{0}+p k & = \pm \nu_{0}
\end{aligned}
$$

These conditions are equivalent to:

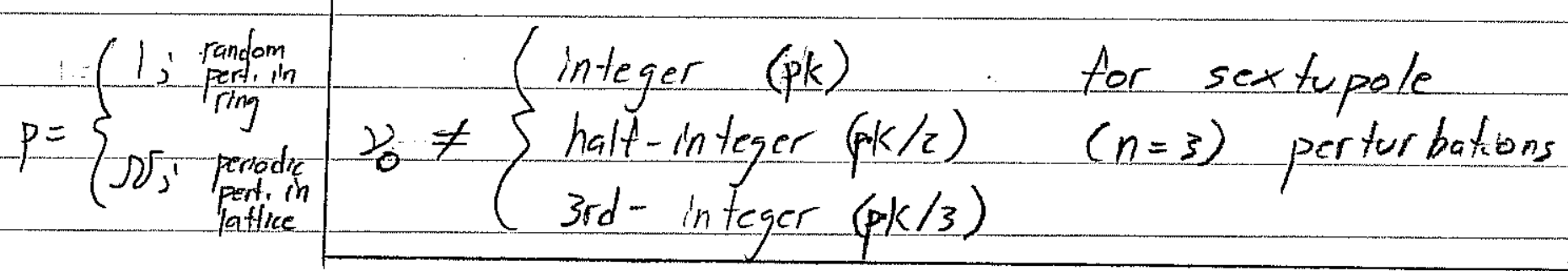

The integer and halt -integer restrictions were already obtained with respect to the dipole and quadrupole cases. The Bid integer is a new restriction.

Other cases similar 
S.M. Lond

$17 /$

Aside Interpretation of low -order resonance conditions:

Dipole Errors

Consider a ring with 1 dipole error. along the azimuth of a ring:

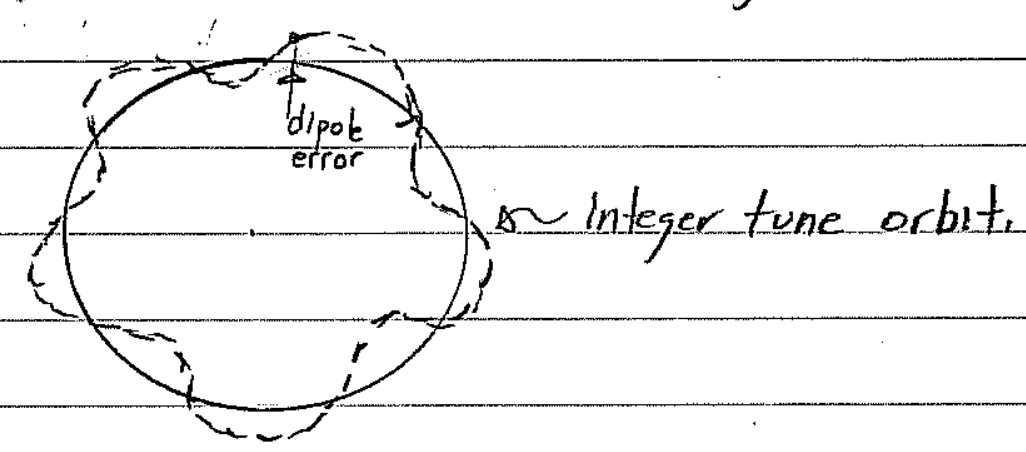

If the particle is oscillating with integer tune, then the particle will experience the error each time with the same phase if and the particle trajectory will "wal k-off" lap to -lap in phase -space, Since the machine aperture is finite the particle will be lost.

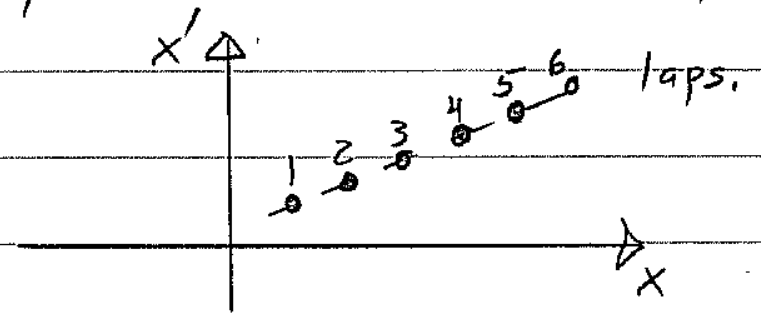

Quadrupole Errors

For a single quadrupole error along the azimuth of a ring, a similar qualitative argument leads one to conclude that if the particle oscillates with 1/2 integer tune that the orbit can "walk-off" lap-to-lap w. phase space patterns as shown here:

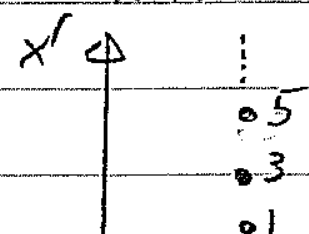

175

$4^{\circ}$ 
S.M. Lond 18/

The general resonance condition for x-plane motion can be summarized as:

$$
M \Sigma_{0}=N
$$

M, $N$ integers of the same sign.

$M=$ order resonance

Generally higher order numbers are less dangerous. - Longer coherence path for validity of theory and coefficient ts In the general case particle motion is not restricted to the $x$-plane $(y \neq 0)$ and a more general resonance analysts shows that:

$$
\begin{aligned}
& M_{x} \nu_{o x}+M_{y} \nu_{0 y}=N \\
& \nu_{0 x}=x \text {-plane tune } \\
& \nu_{0 y}=y \text {-plane tune }
\end{aligned}
$$

$M_{x}, M y, N$ integers of the same sign.

$$
\left|M_{x}\right|+|M y|=\text { order resonance. }
$$

These restrictions are plotted order-by-order in $2 x-2 y$ plots to find allowed tunes where the mach he can safely operate. Generally, lower order resonances are more dangerous, since small effects can invalidate the ideal analysis and "wash out" higher order resonances.

176 
S.M. Lond

$19 /$

Typical tune plots for up to 3 rd order resonances: $\Omega=1$ Superperiodicity

Mach ing operating point = chosen to aver. resonance lines.

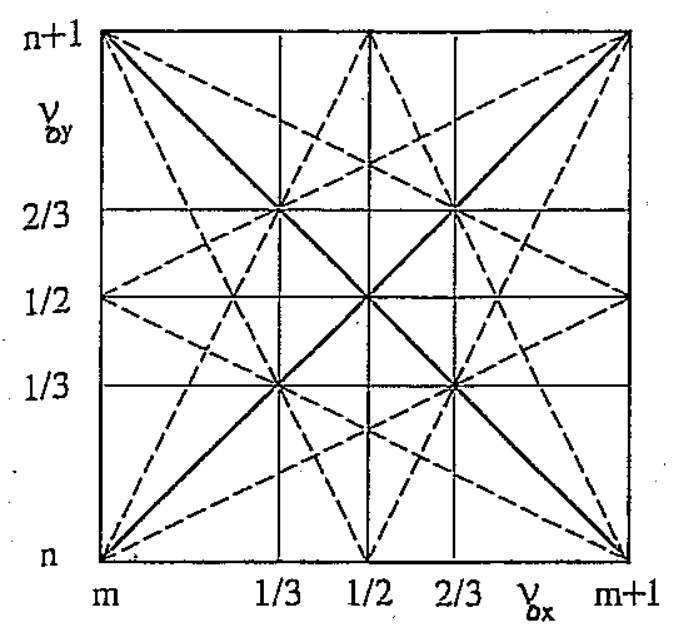

Atipplicable to: field errors from random construction errors,

From Wiedemann

$$
N=4 \text { Superperiodicity }
$$

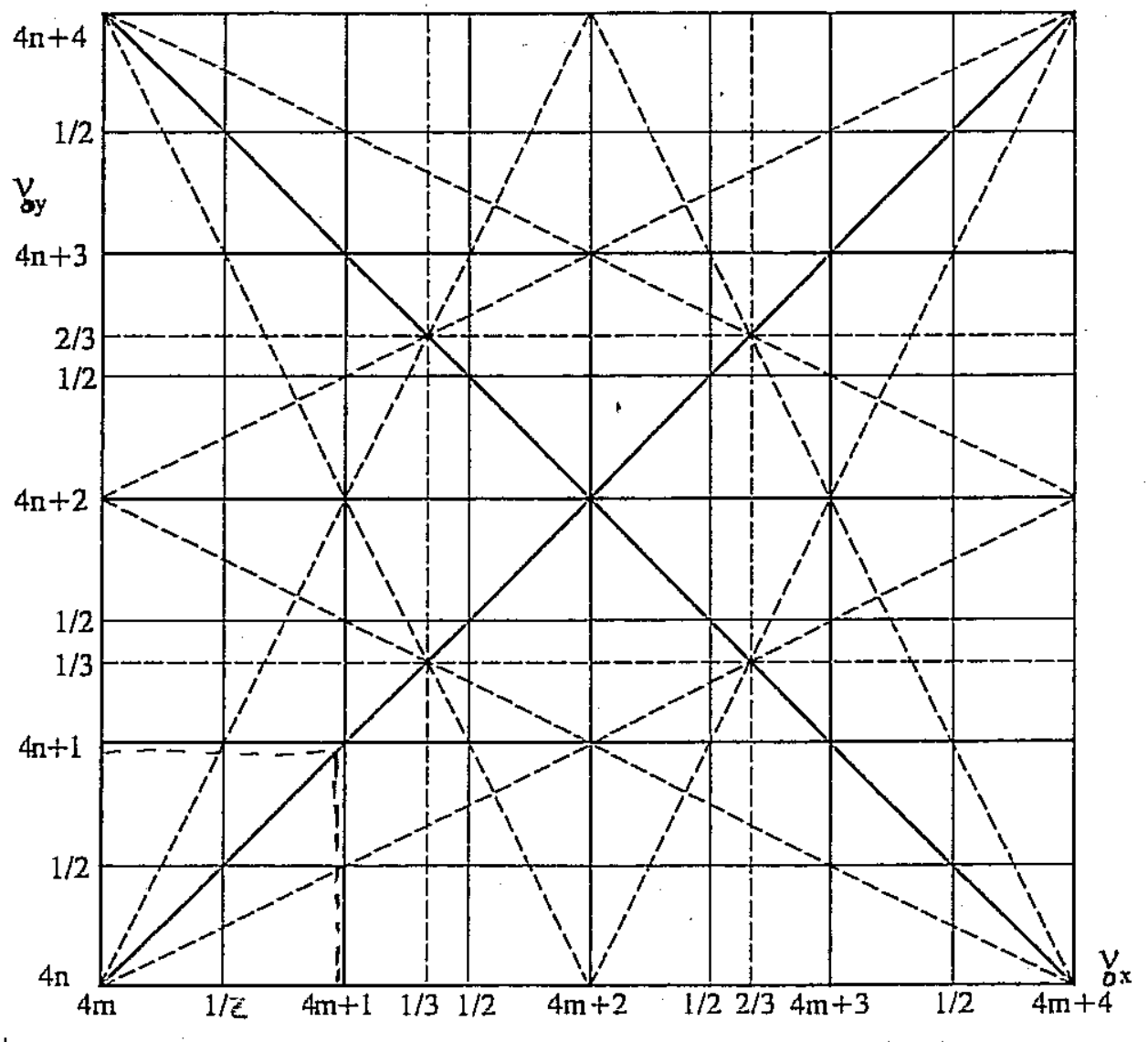

Applicable to systematic lattice $\frac{\text { Fiekterrors }}{\text { in } a 4-\text { period }}$ ring.

Note lesser density of resonance Ines than in the $\Omega=1$ case. 
S.M. Land

$20 /$

Distinguishing between field errors from random construction $(p=1)$ and systematic errors on the lattice $(p=(N)$ is important.

Random errors $(p=1)$

- Errors always present and can give low order resonaces.

- Usually have weak amplitude coefficients and may be corrected.

Systematic errors $(p=\Omega)$

- Lead to higher order resonaces for large $\Omega$ and a lower density of resonance Ines

- Lane symmetric rings (N large) have lesser restrictions from systematic errors

- Practical issues such a construction cost and getting the beam into and out of the ring dead to smaller $\Omega$

- Amplitude coefficients can be large and the systematic resonances can be strong and thus can be dangerous.

In practice, resonances higher than sro order need rarely be considered.

- Effects outside model tend to wash out higher order resonates. 
SiM. Lond z//

Effects of Space Charge on Resonances

Machine operating points are generally chosen far from low -order resonance lines, a coherent processes that shaft tune values towards a low-order resonace are dangerous.

Coherent - same for each particle in ter ....... distribution.

incoherent-difterent (Fandom) for each particle in distribution.

Tone, shift limits are often called "Laslett Limits" because hen firstculated such limits for many processes

- image charges

- image currents

- space charge

$:$

For space-charge, the so-called Laslett limit is taken as:

$$
\nu_{0}-\nu=\Delta \nu<1 / 4
$$

$$
\begin{aligned}
& D_{0}= \text { tune af zero } \\
& \text { space charge } \\
& \nu= k V \text { distribution } \\
& \text { tune }
\end{aligned}
$$

This "is probably over idealized and restrictive but is widely used to set ring current
limits.

179 
S.M. Lond

The Lasleft condition is probably overly restrictive:

- Real space-charge is not coherent like a $\mathrm{kV}$ model

- spectrum of $\beta$-tunes,

- no equilibrium beam and oscillations may crack resonances.

- Simulations indicate that $10 s \rightarrow 100 s$ of laps may pose little problems for strong space charge.

- Univ Maryland Ring may soon ( 2007t) operate in such regime to test in the lab.

This could be a good research area with new physics.

180 


\section{John Barnard Steven Lund USPAS June 2008}

\section{Injectors and longitudinal physics -- I}

1. Child-Langmuir Law

(Reiser 2.5.2, Appendix 1)

2. Pierce electrodes

3. Transients in injectors

4. Injector choices 


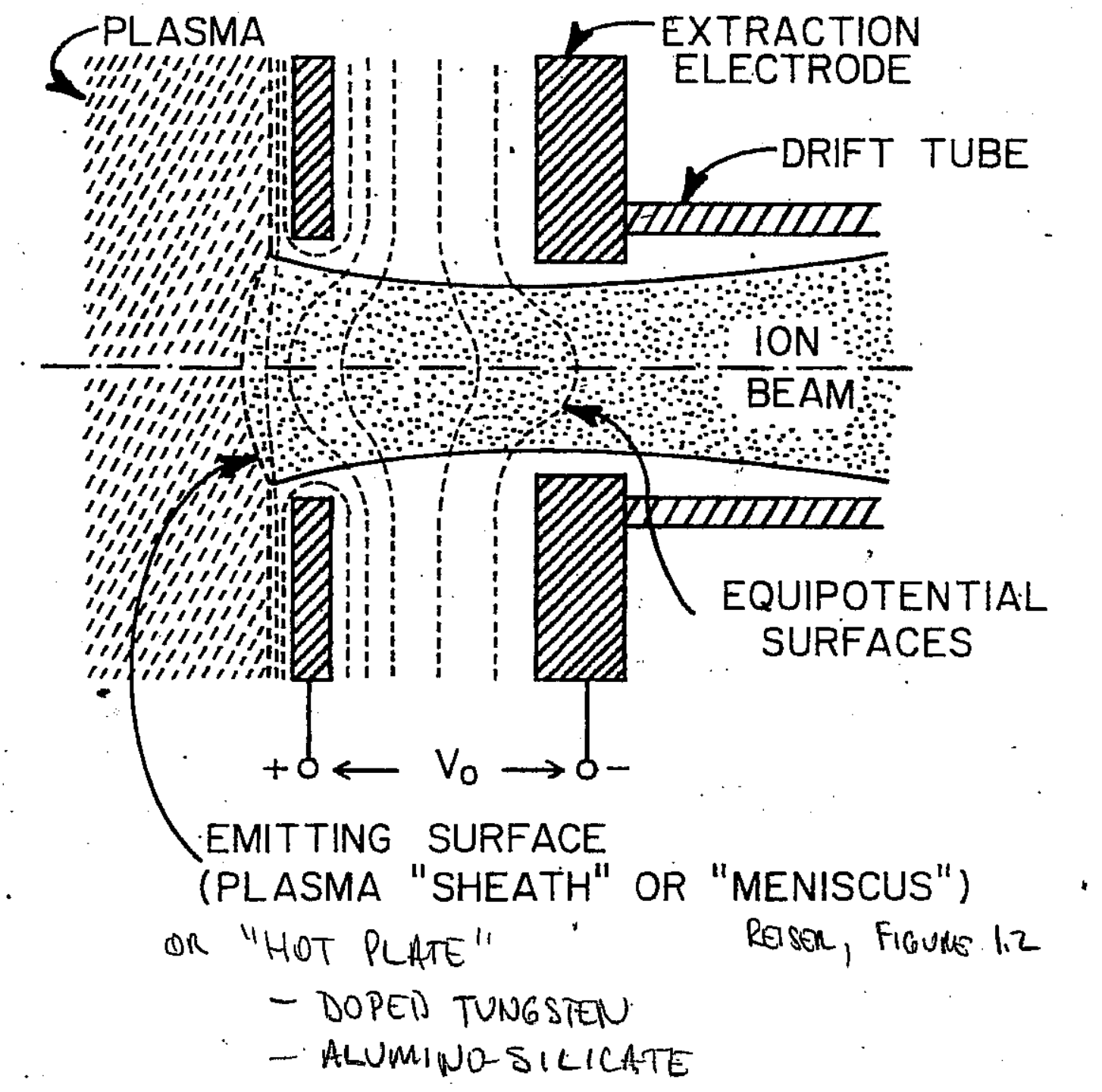




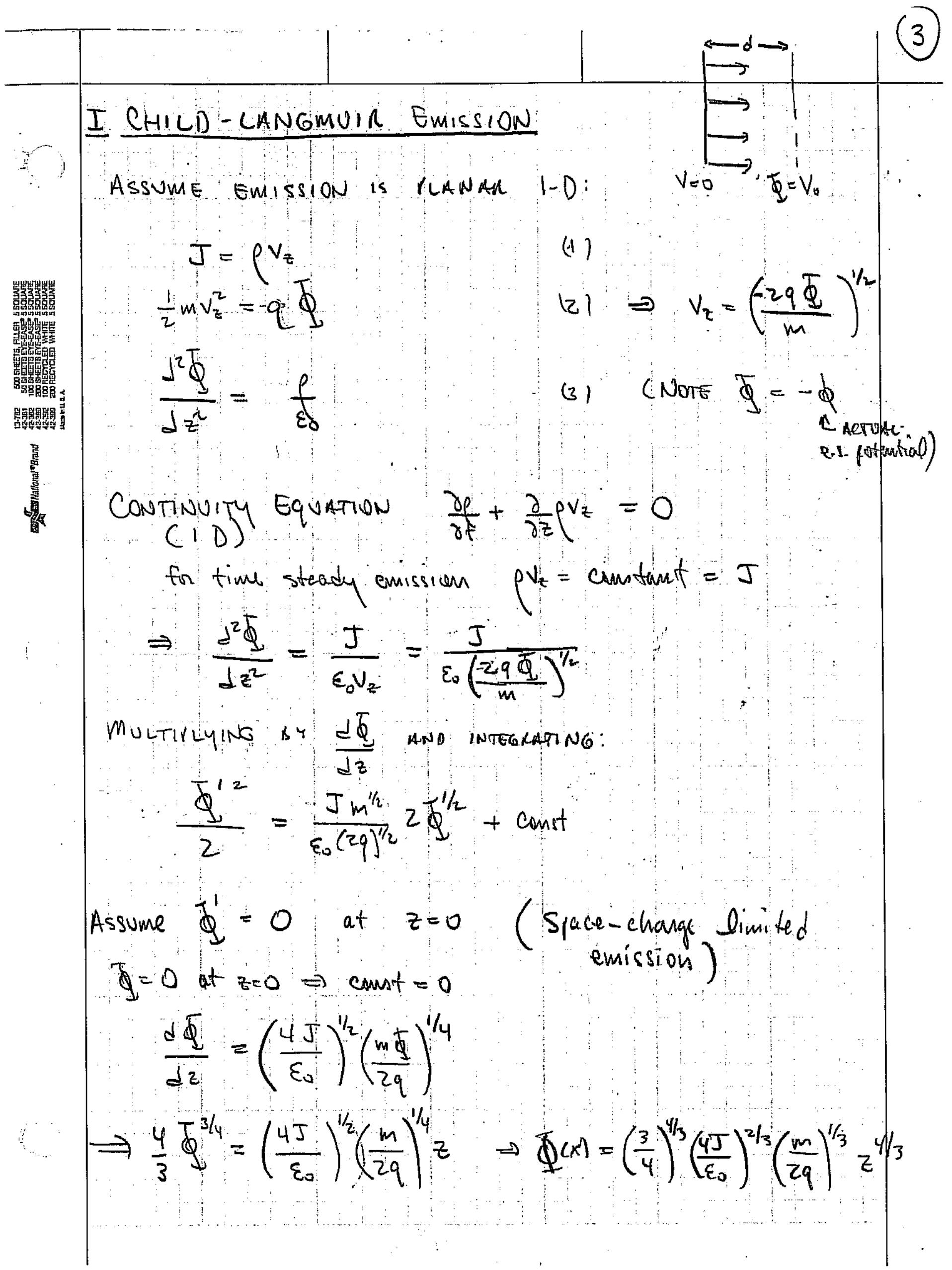




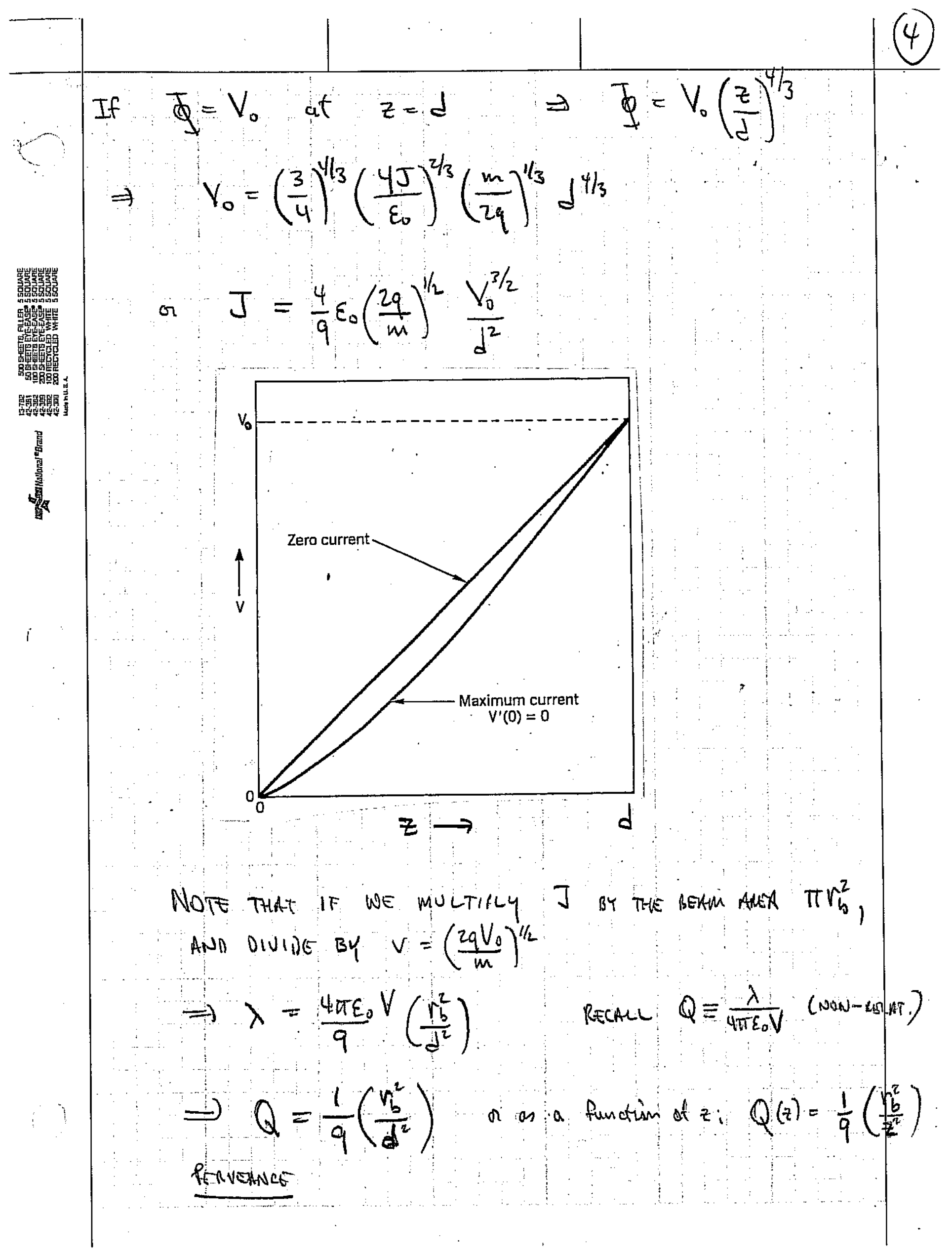

NOTE THAT IF WE MULTIpLY J BY THE BERM ALAN $\pi r_{b}^{2}$, AND DIVIDE BY $V=\left(\frac{2 q V_{0}}{m}\right)^{1 / 2}$

$\Rightarrow \lambda=\frac{4 \pi \varepsilon_{0} V}{9}\left(\frac{r_{b}^{2}}{d^{2}}\right)$

ReCALL $Q \equiv \frac{\lambda}{4 \pi \varepsilon_{0} V}$ (NON-USMT)

$\Rightarrow Q=\frac{1}{9}\left(\frac{r_{b}^{2}}{d^{2}}\right)$ or as a function of $z_{1} Q(z)=\frac{1}{q}\left(\frac{r^{2}}{z^{2}}\right)$

fENEANGE

184 
- Pierce Column

$$
\begin{aligned}
& V: \sim z^{1 / 3} \\
& E \sim z^{1 / 3}
\end{aligned}
$$

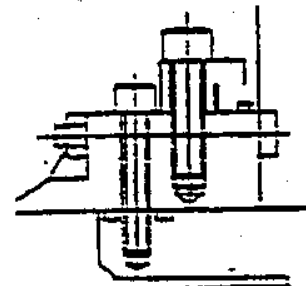
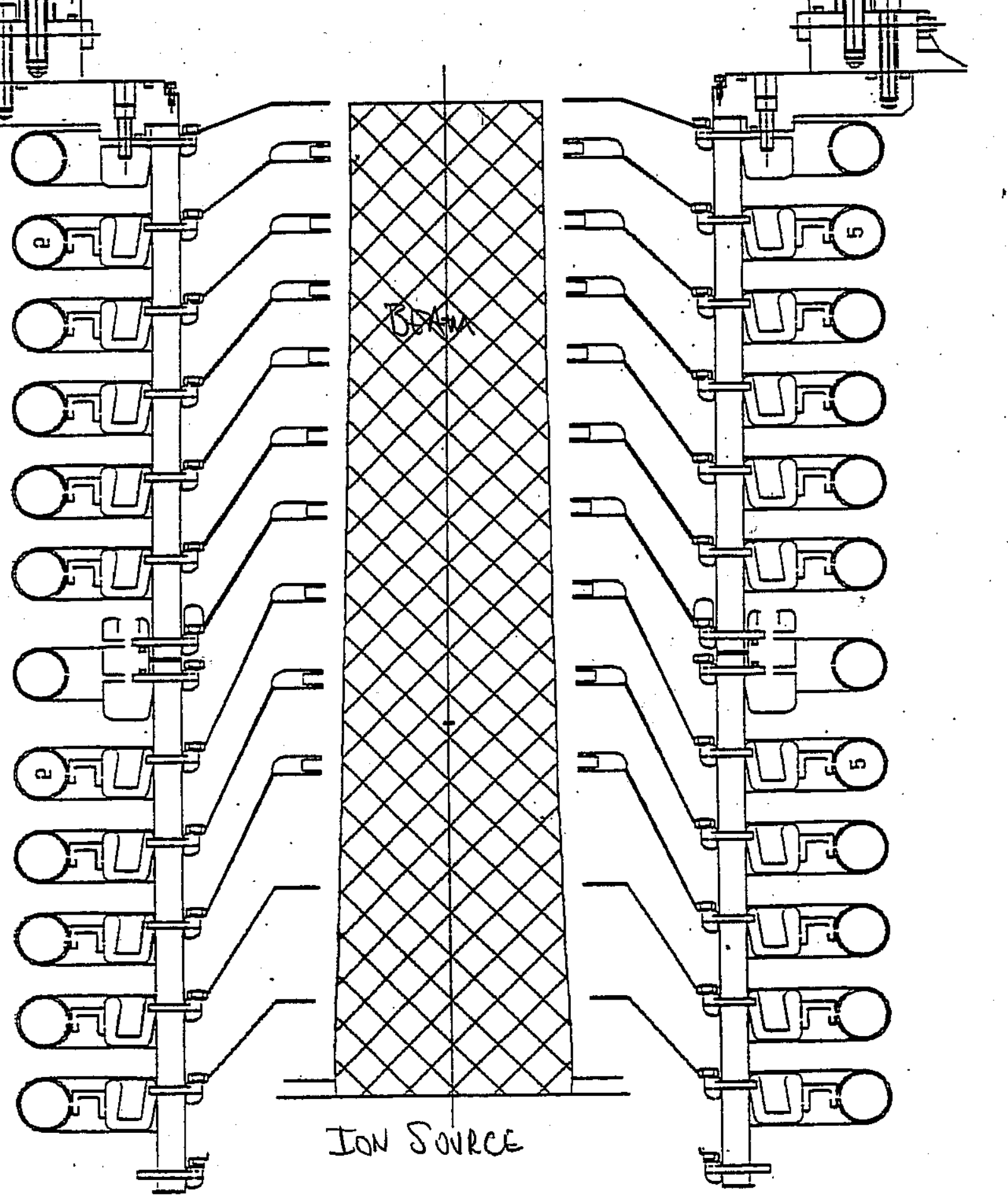

i

(3) 


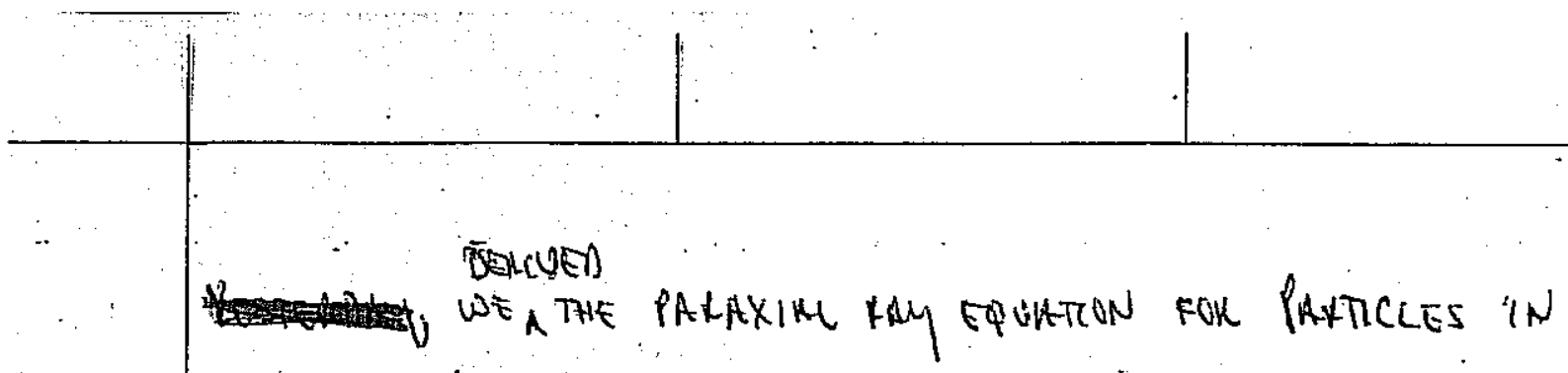

Axisymmetice systems:

(6)

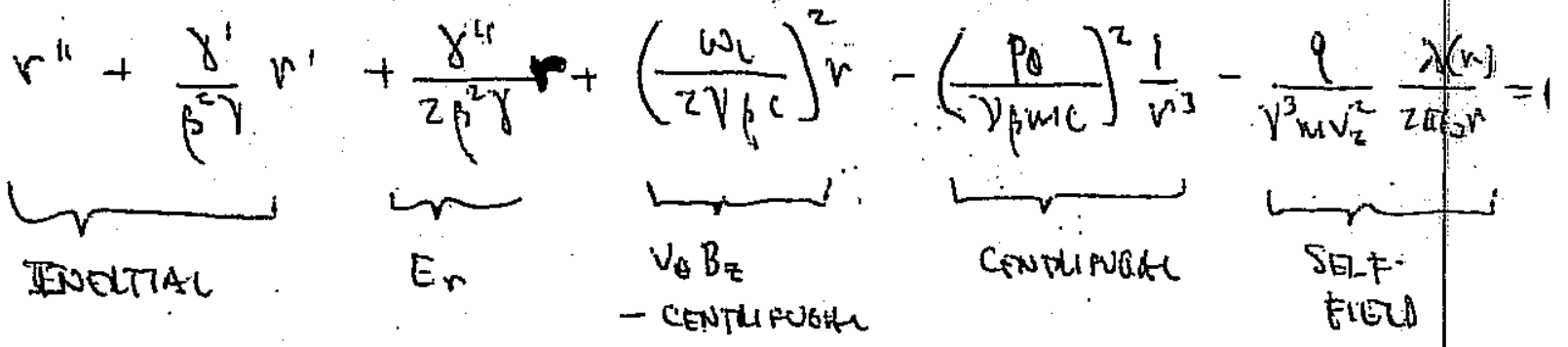

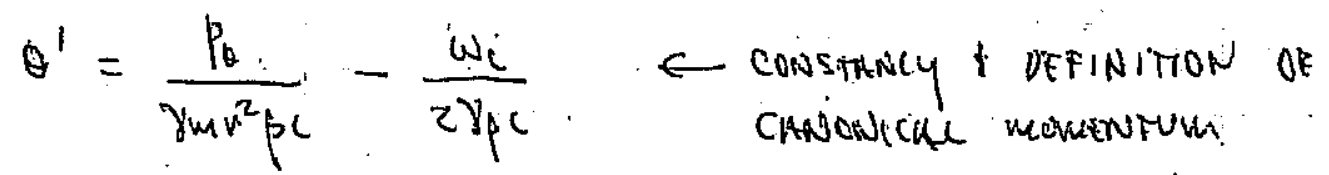

enverole equaton for Axisymmetnic Betm

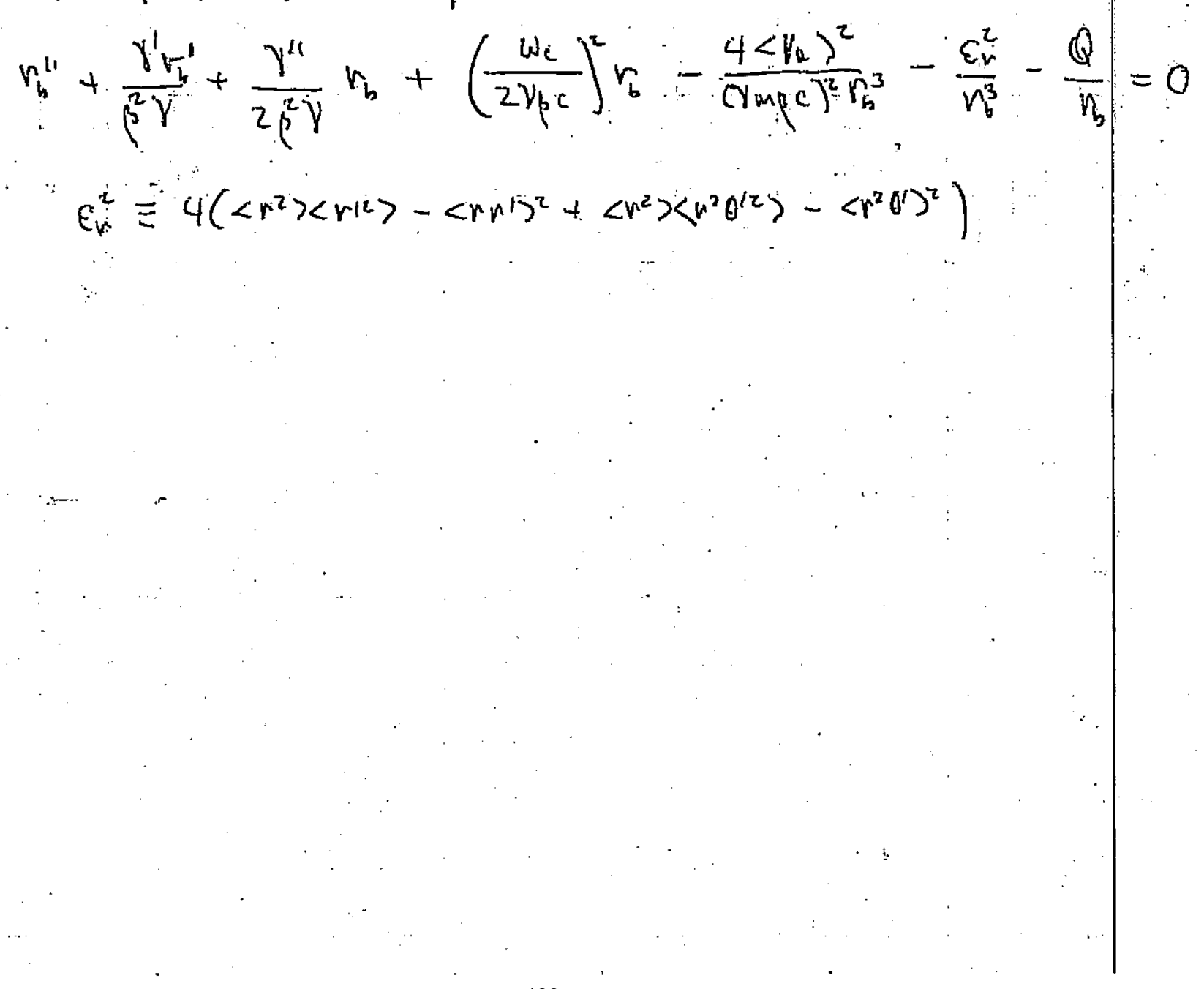

186 


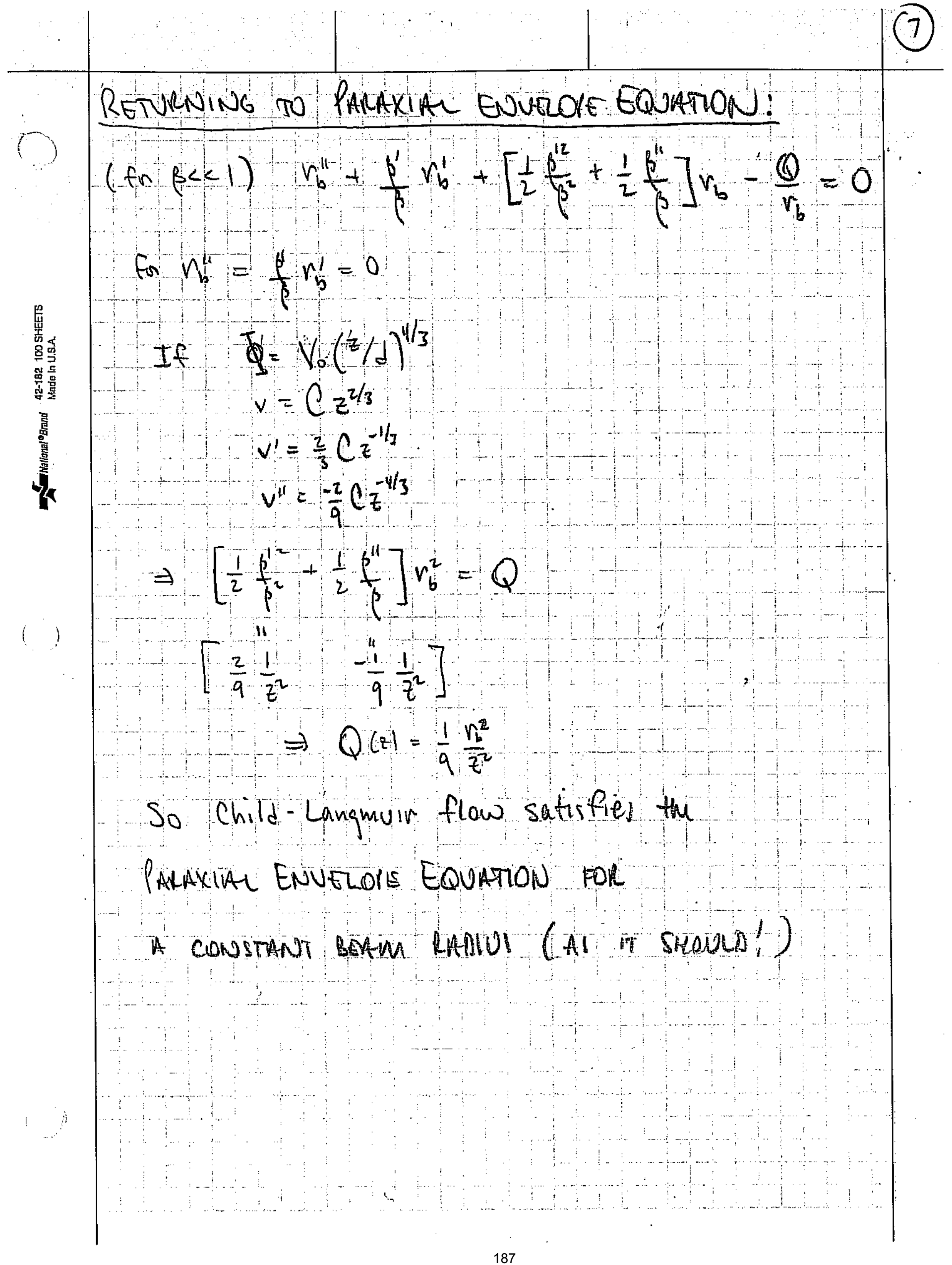




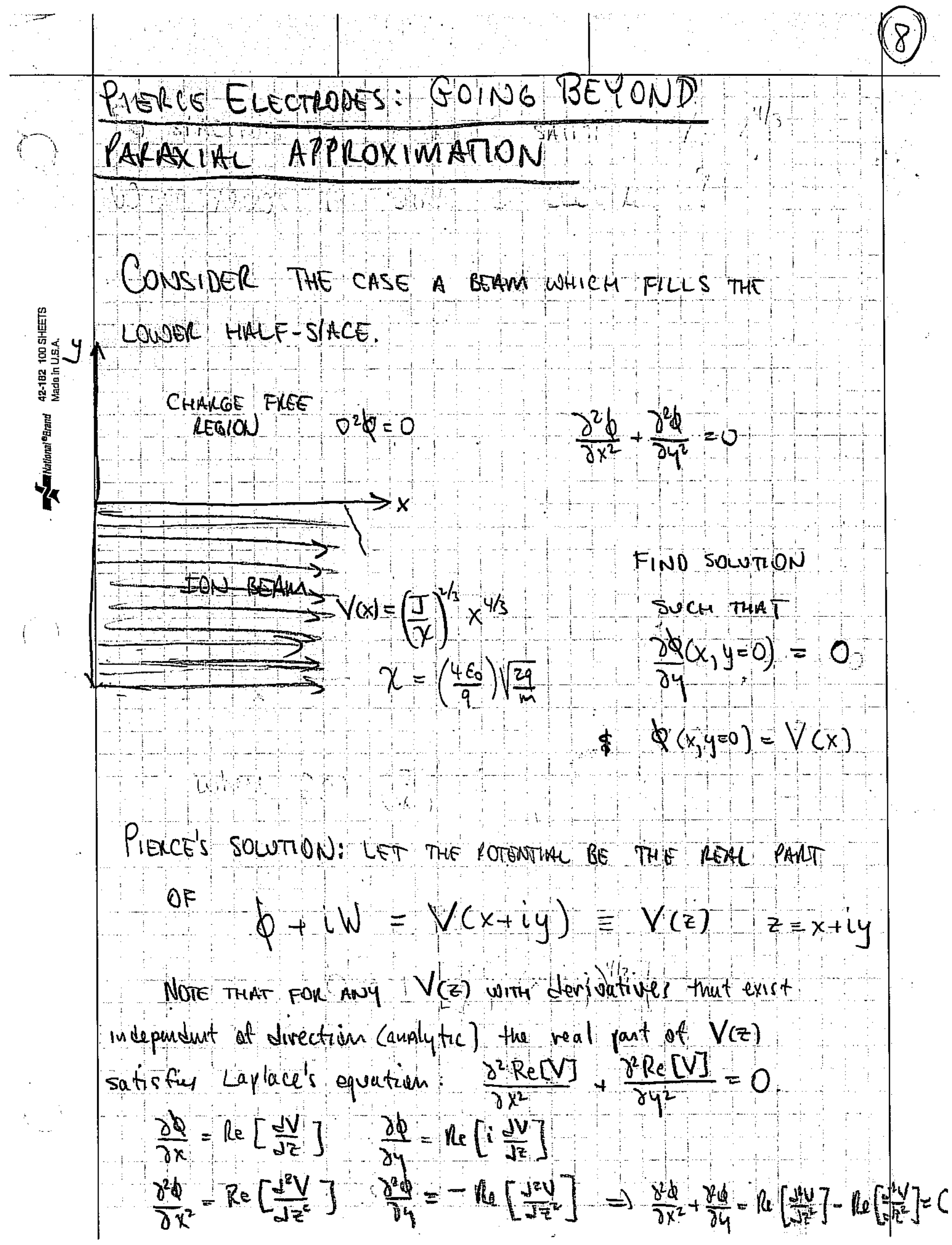




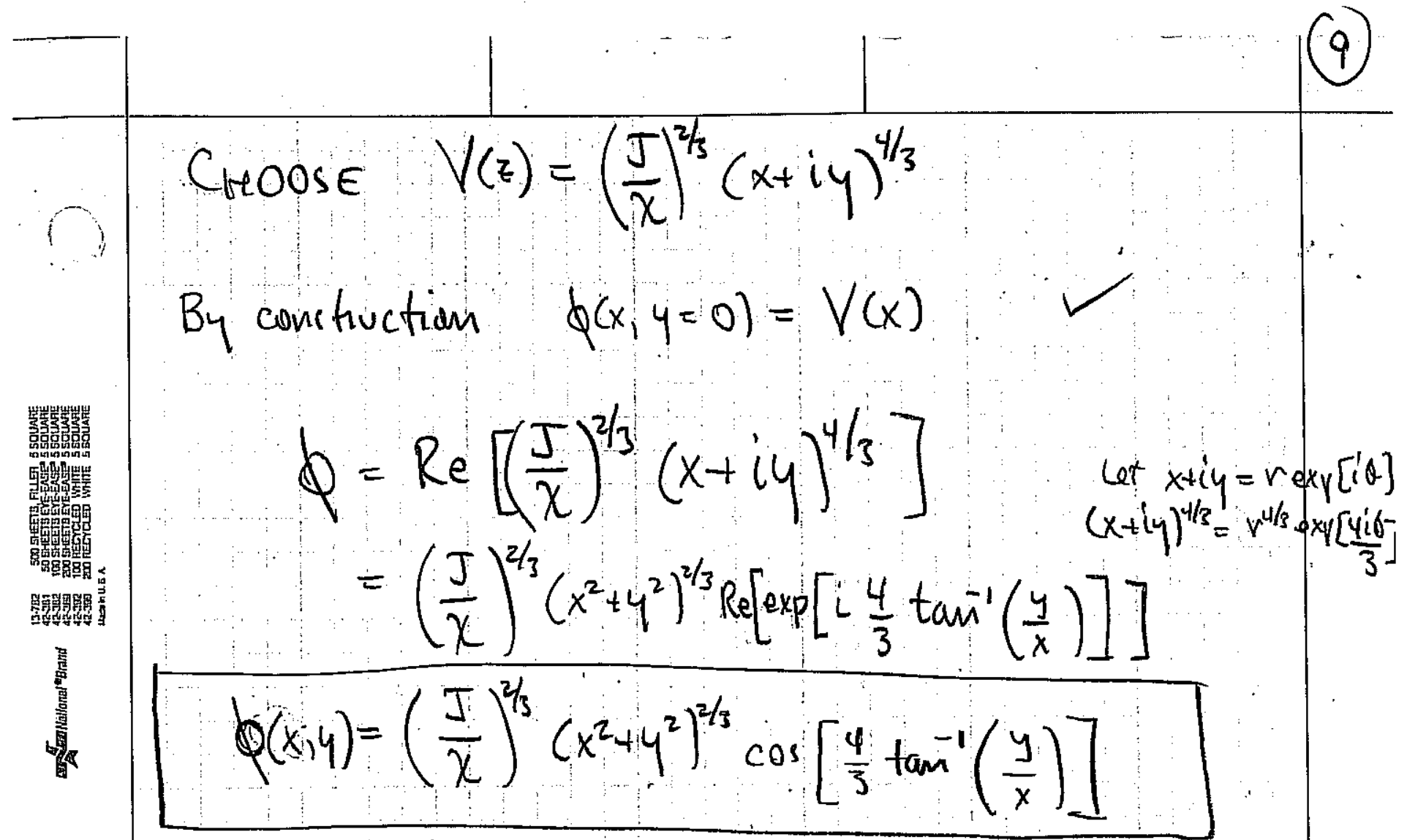

Note that $\phi(x, y)=\phi(x,-y) \Rightarrow \frac{\partial \phi}{\partial y}(x, y=0)=0$

$$
\begin{aligned}
\varphi=0 \text { EqUIPOTENTIAL: } \\
\Rightarrow 0=\cos \left[\frac{4}{3} \tan ^{-1}\left(\frac{y}{x}\right)\right] \\
\Rightarrow \tan ^{-1}\left(\frac{y}{x}\right)=\frac{3}{4}\left(\frac{\pi}{2}\right)=67.5^{\circ}
\end{aligned}
$$

For a goñrRe equivotantial PASSING THMOUGH $X_{0}$ :

$$
x_{0}^{y / 3}=\left(x^{2}+y^{2}\right)^{2 / 3} \cos \left[\frac{4}{3} \tan ^{-1}\left(\frac{y}{x}\right)\right]
$$

189 


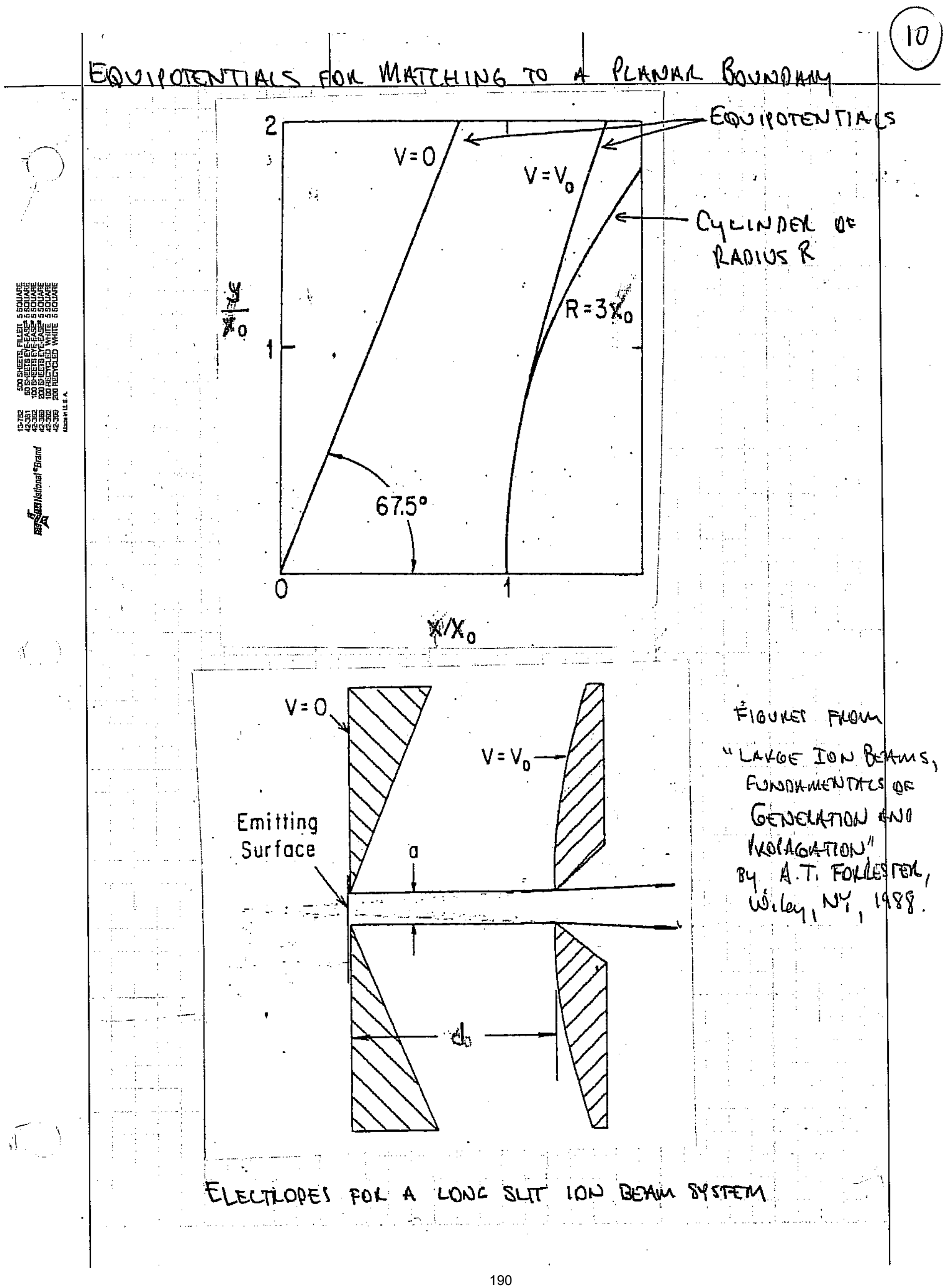




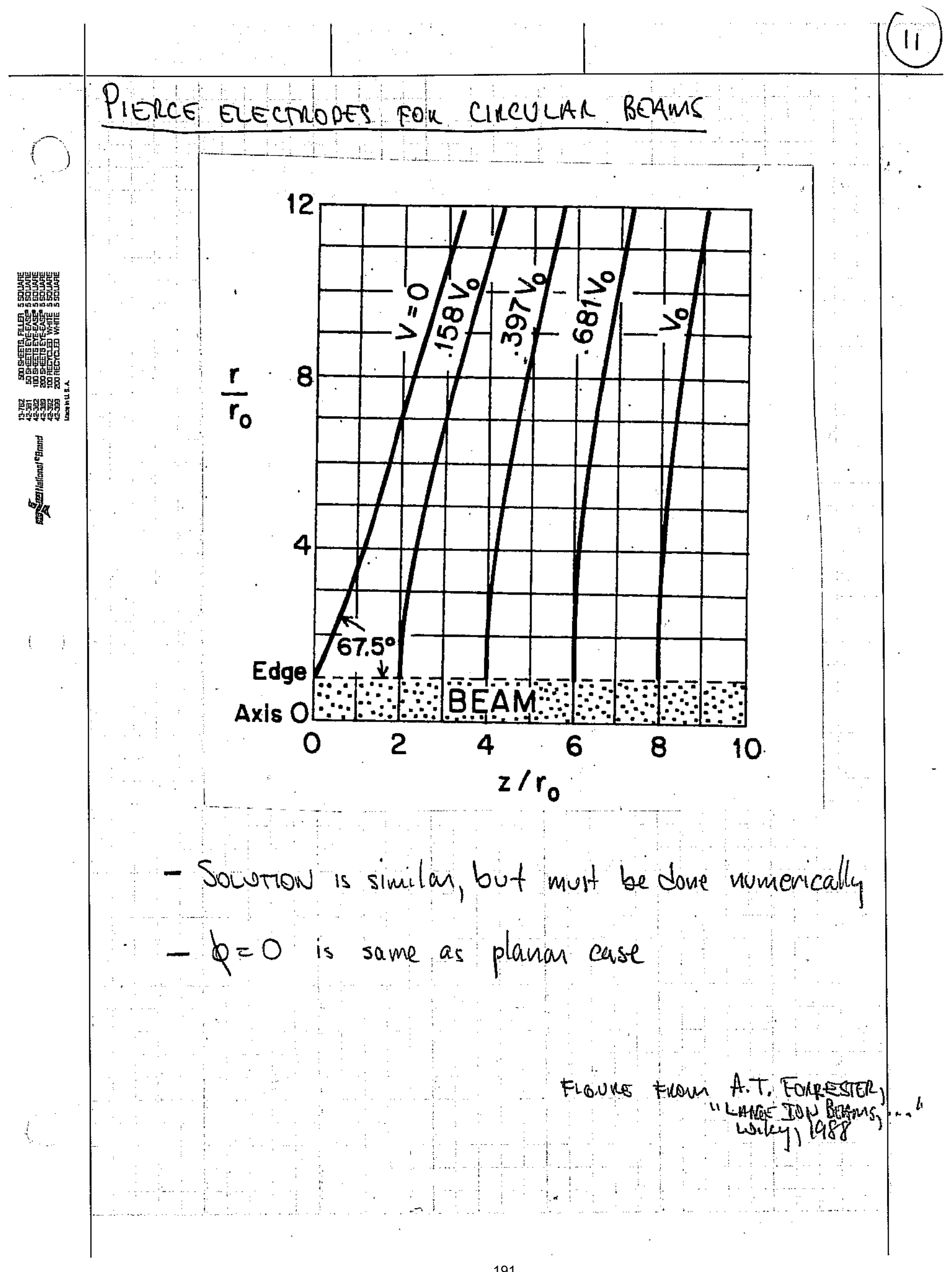

19 


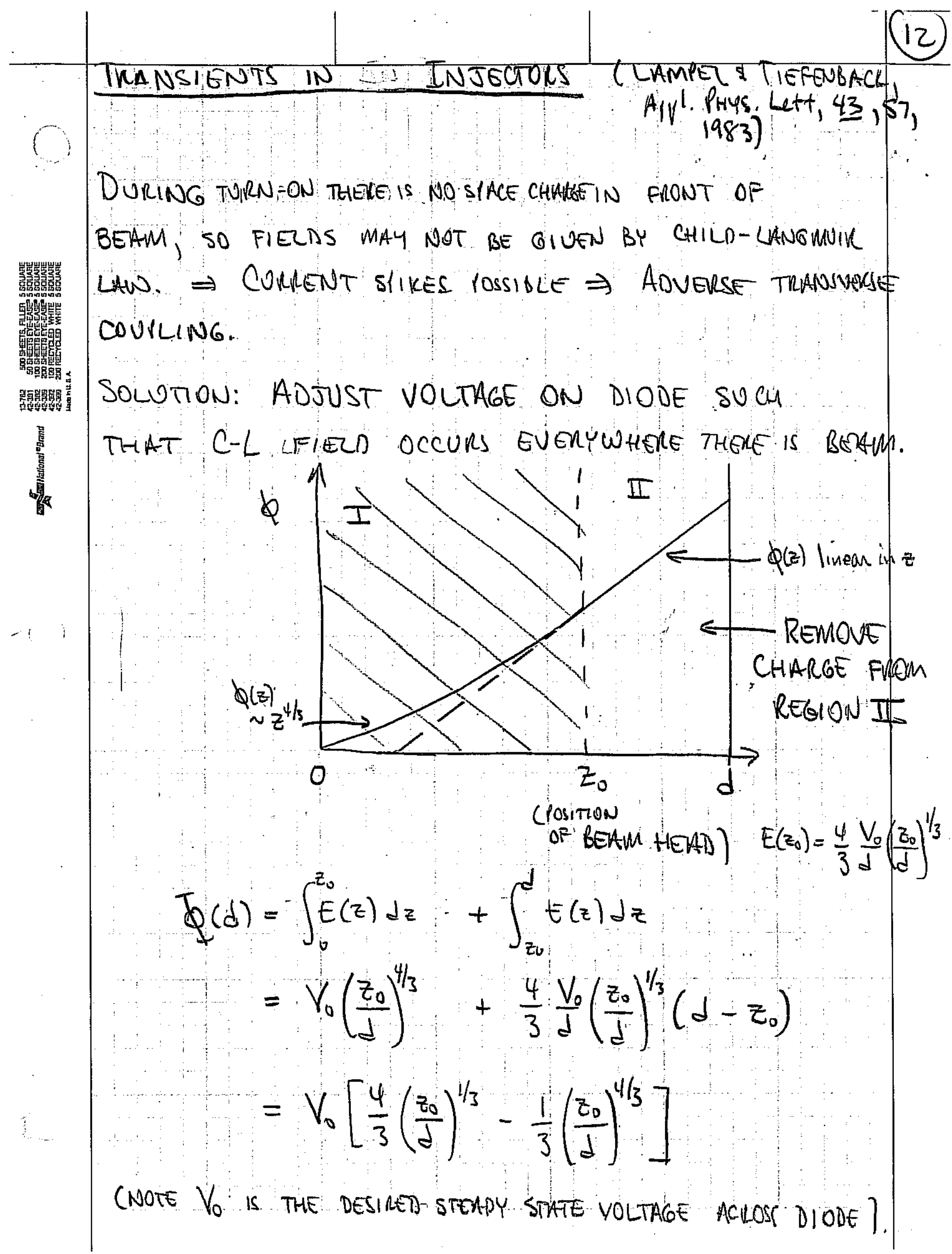




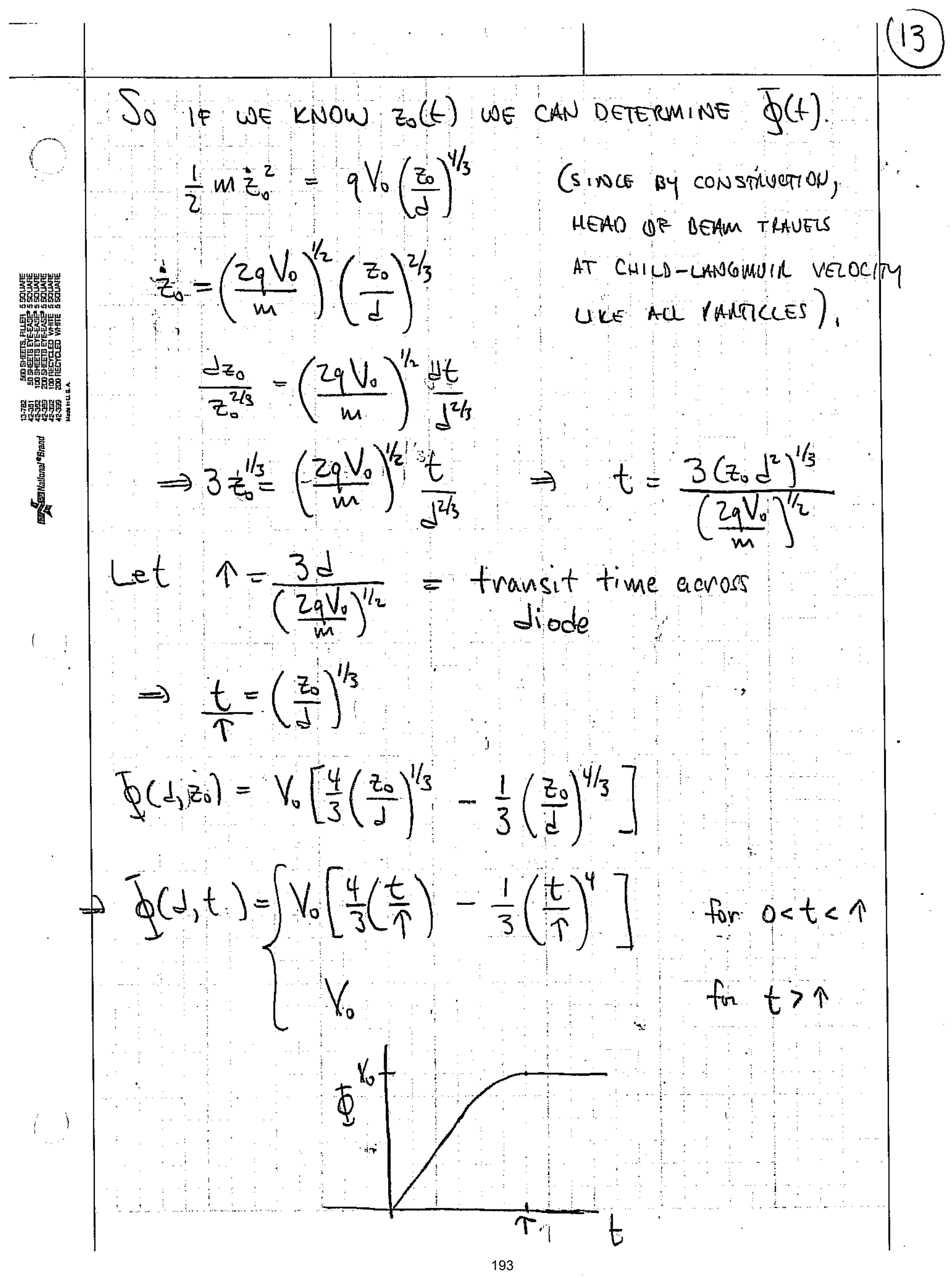




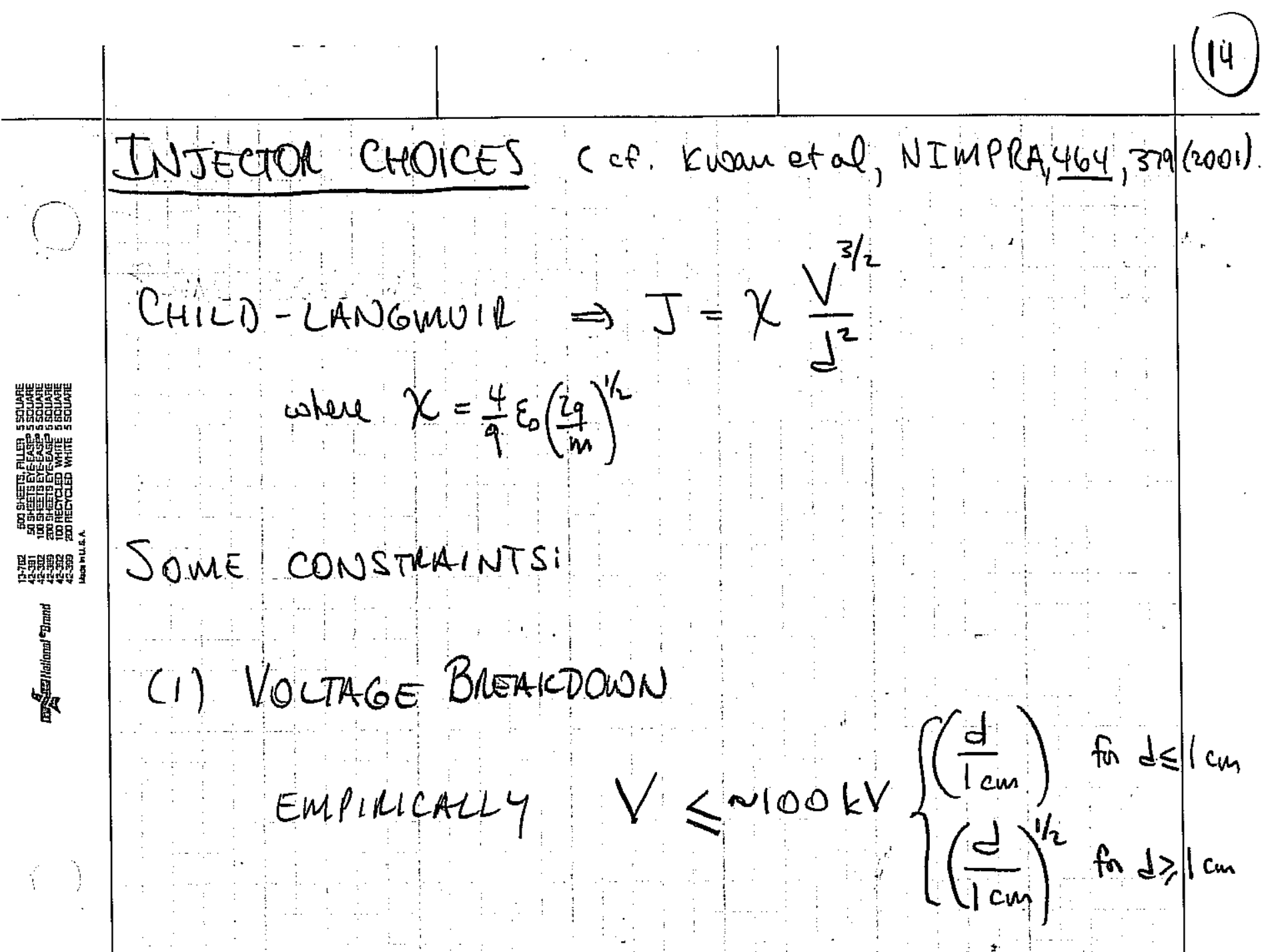

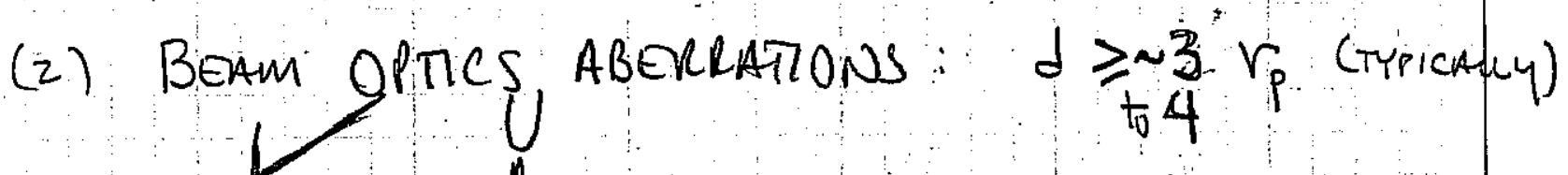

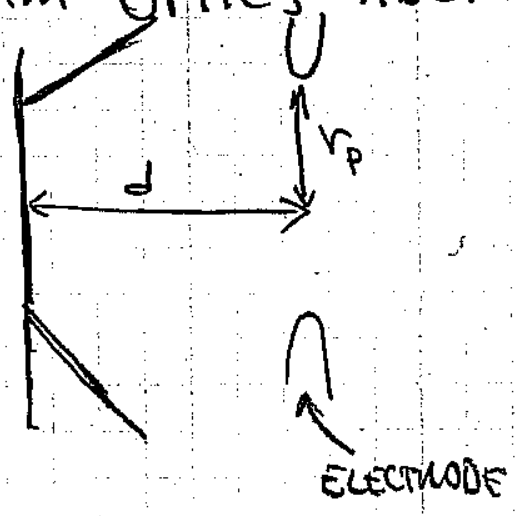

Note tHAT

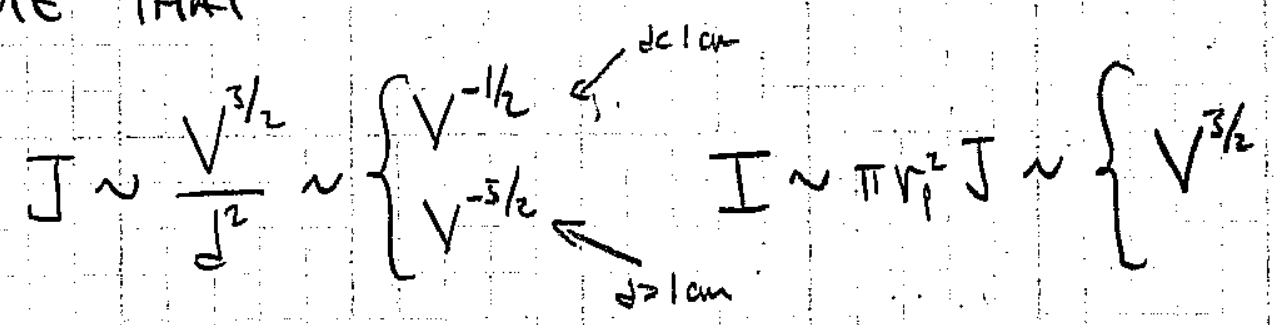

Thus current density decrelices with sire and uso too, but I increases.

194 


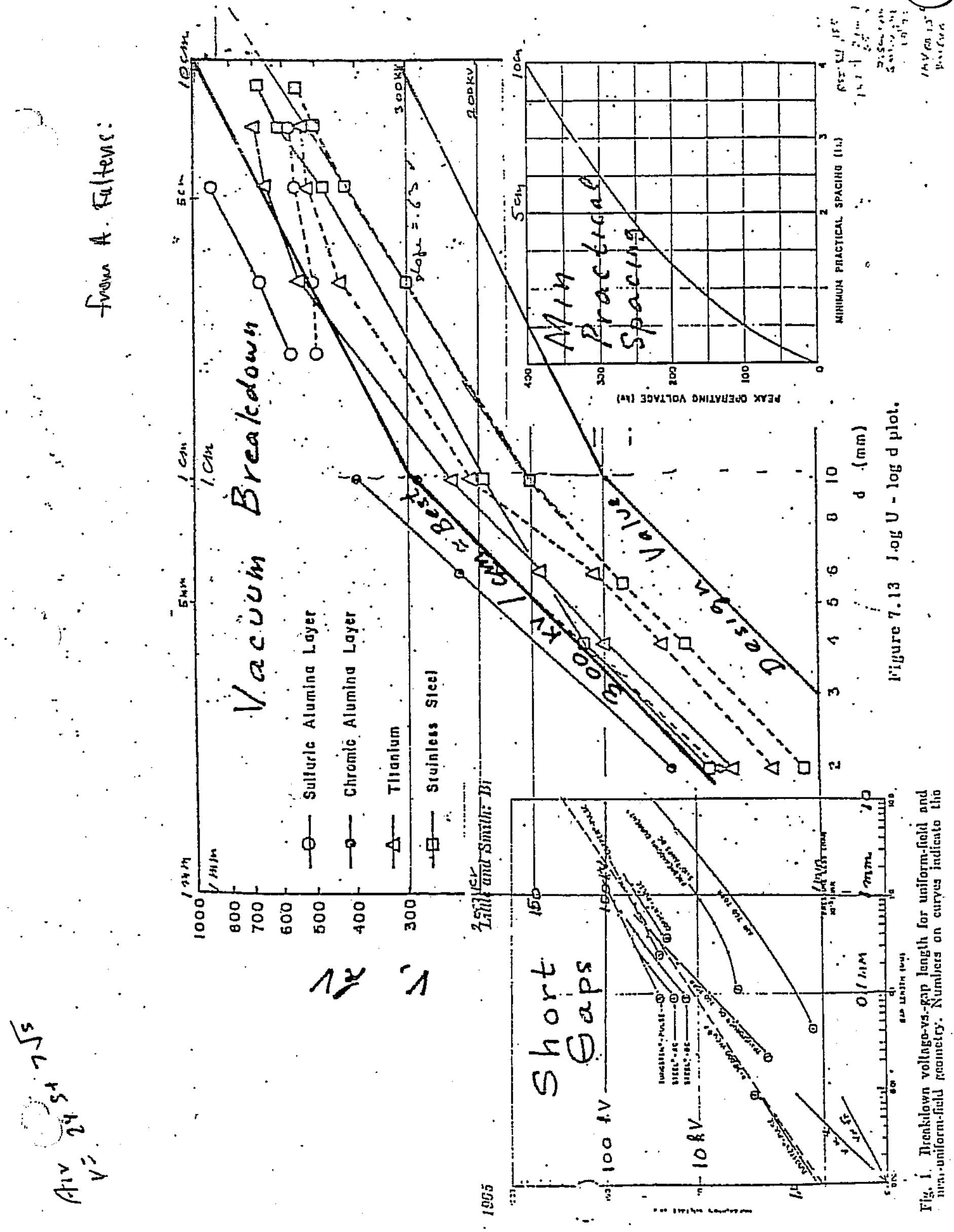




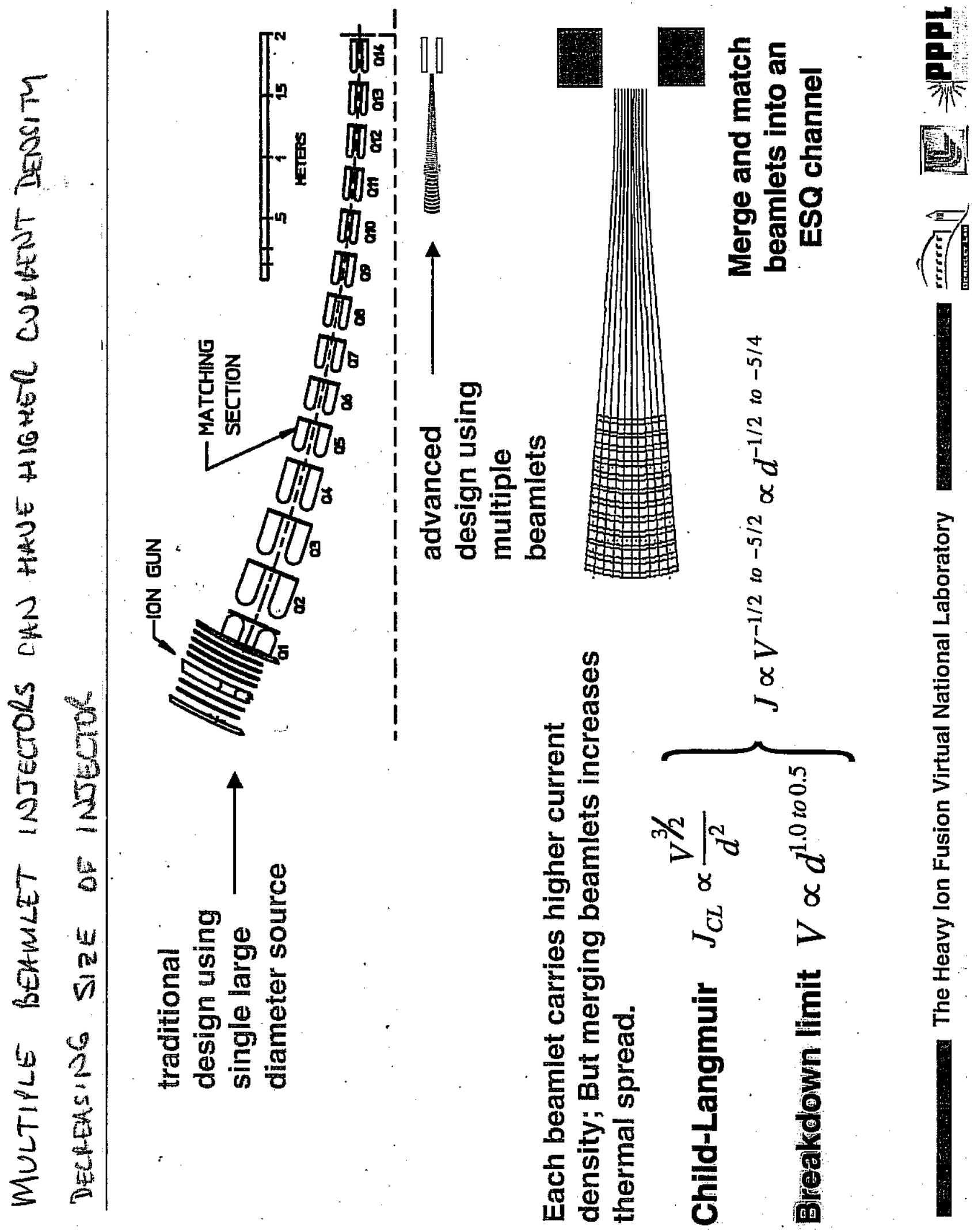



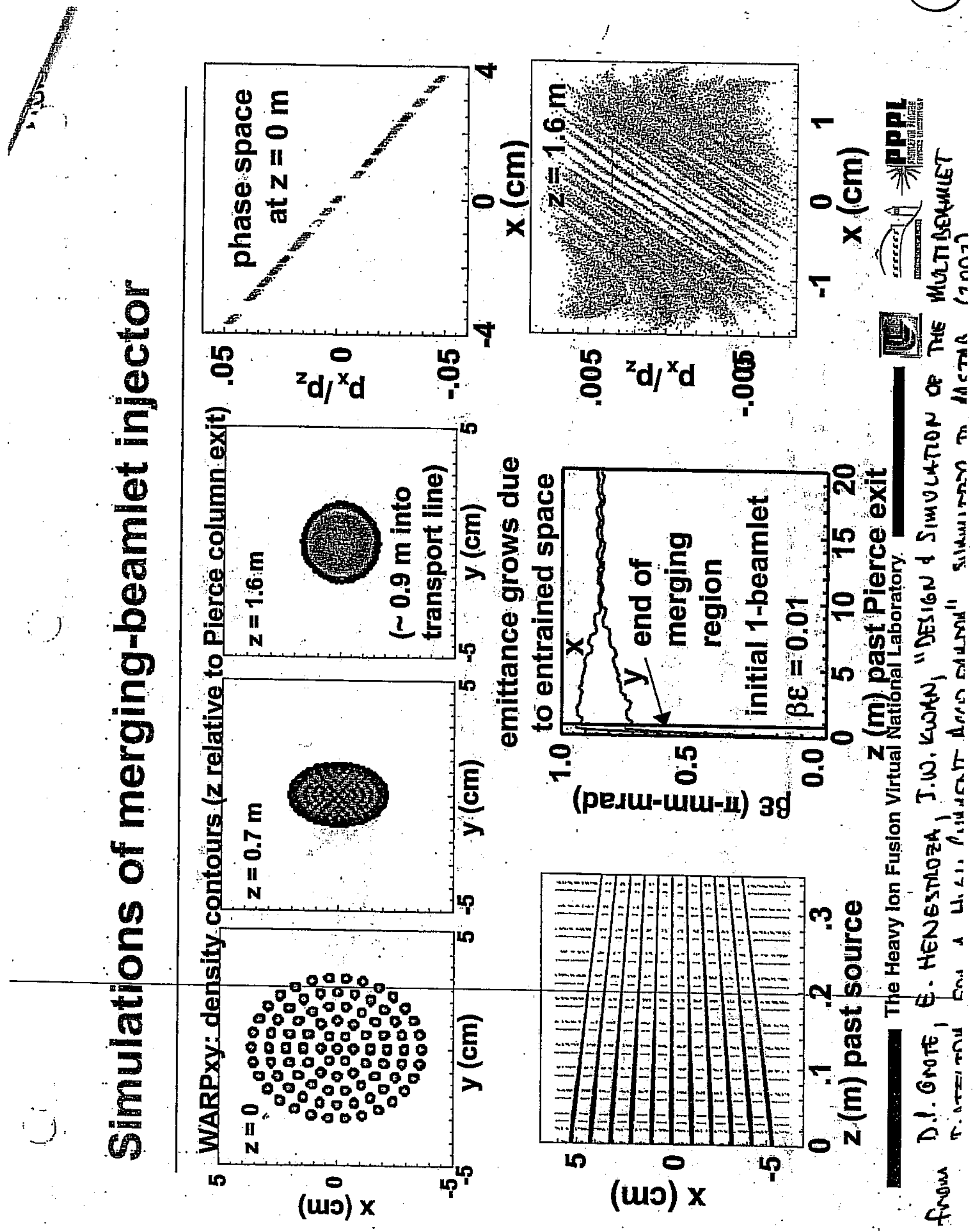


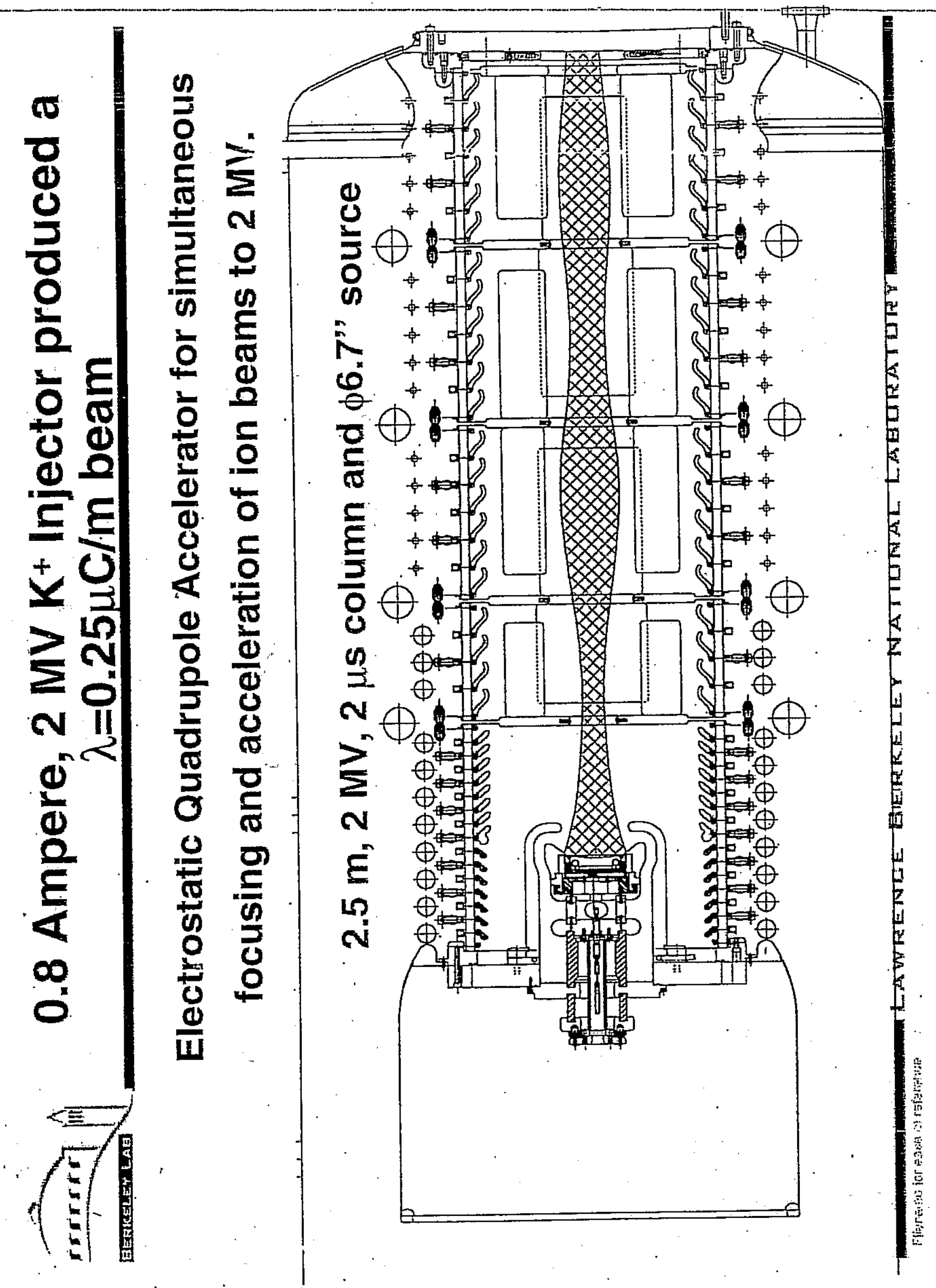


(19)

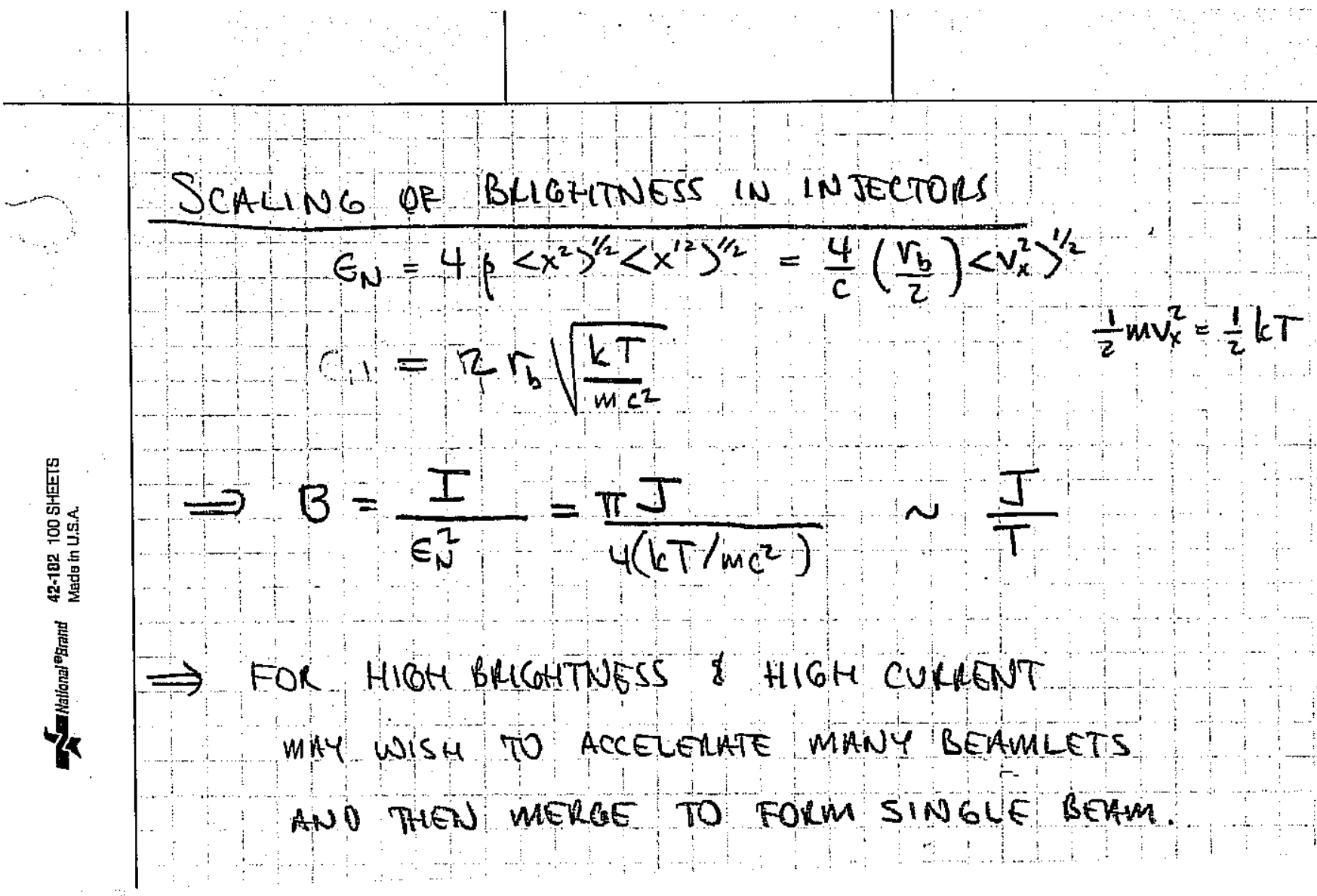

1. $\mid$

199 


\section{John Barnard Steven Lund \\ USPAS \\ June 2008}

\section{Injectors and longitudinal physics -- II}

1. Acceleration - introduction

2. Space charge of short bunches (rf)

3. Space charge of long bunches

4. Longitudinal space charge waves

5. Longitudinal rarefaction waves and bunch ends 


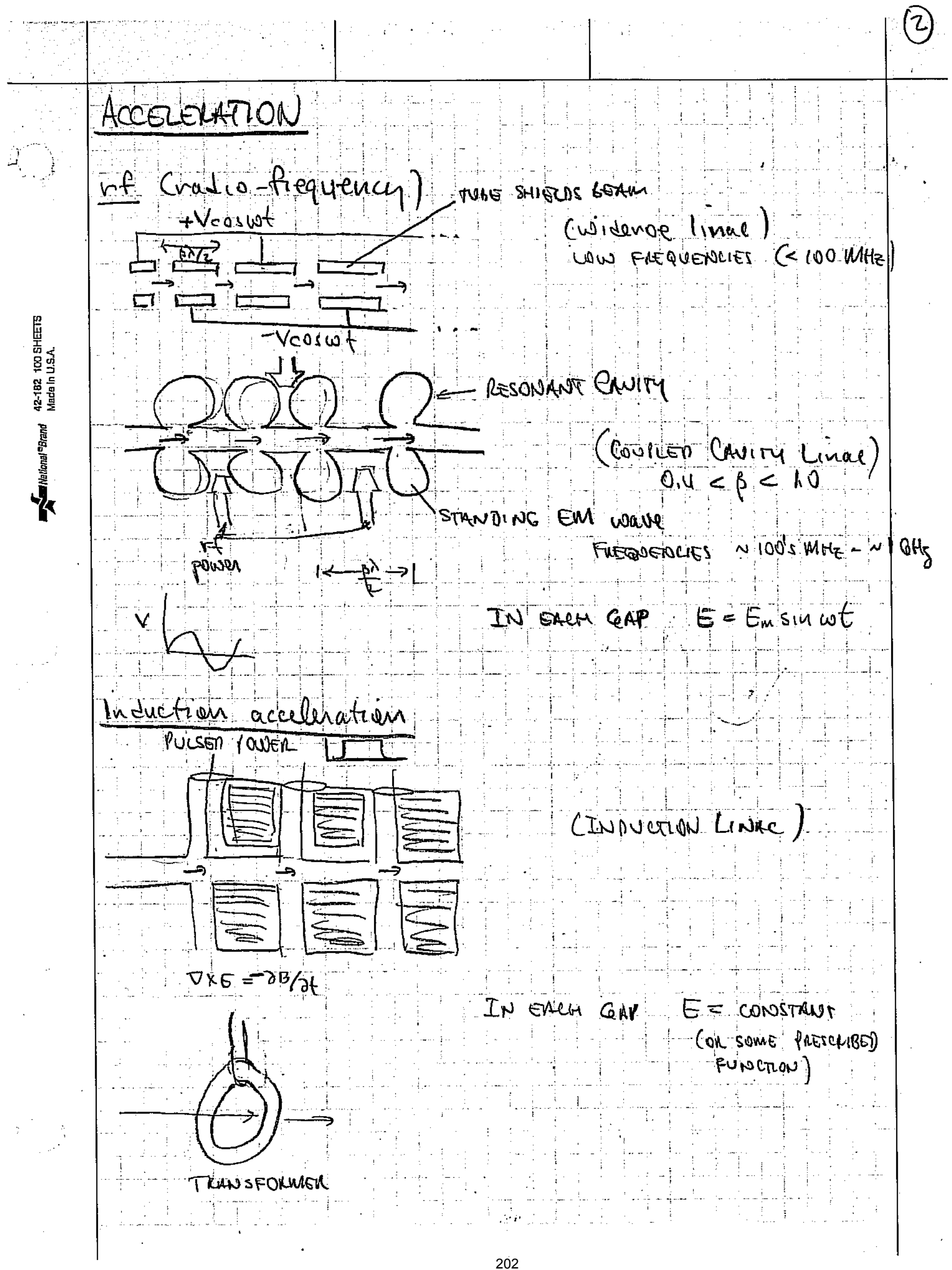




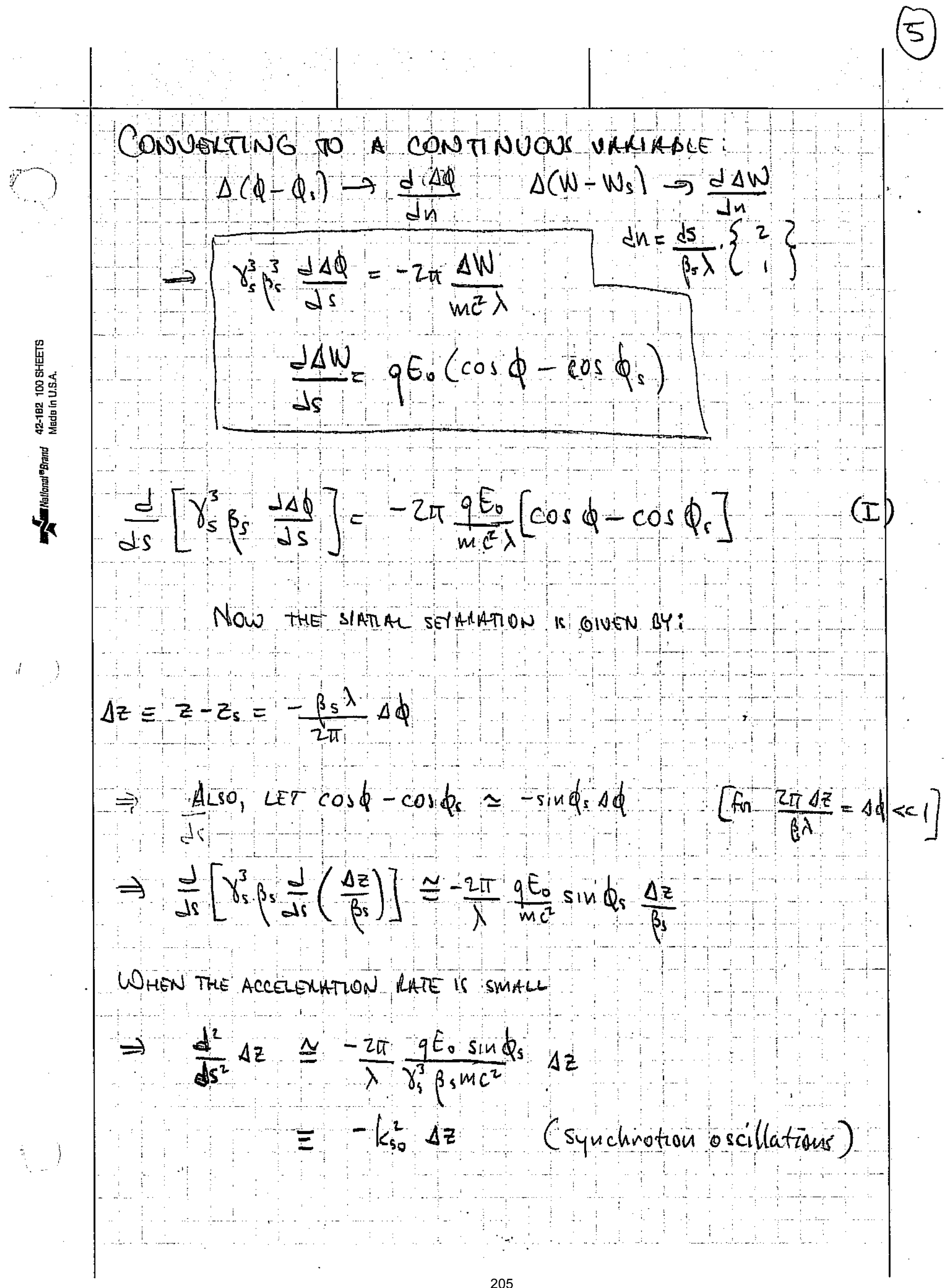


RETUKNING TO $\Delta W-Q$ NOTATTON

(6)

Let $\omega=\frac{\Delta W}{m c^{2}} \quad A=\frac{2 \pi}{\beta_{s}^{3} \gamma_{s}^{3} \lambda}$

$$
B=\frac{q 50}{m c^{2}}
$$

$$
\begin{aligned}
& \Rightarrow \omega^{\prime}=B\left(\cos \phi-\cos \phi_{s}\right) \\
& \phi^{\prime}=-A \omega \\
& \phi^{\prime \prime}=-A B\left(\cos \phi-\cos \phi_{s}\right)
\end{aligned}
$$

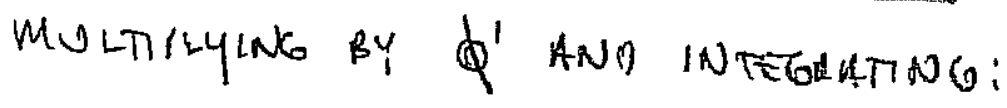

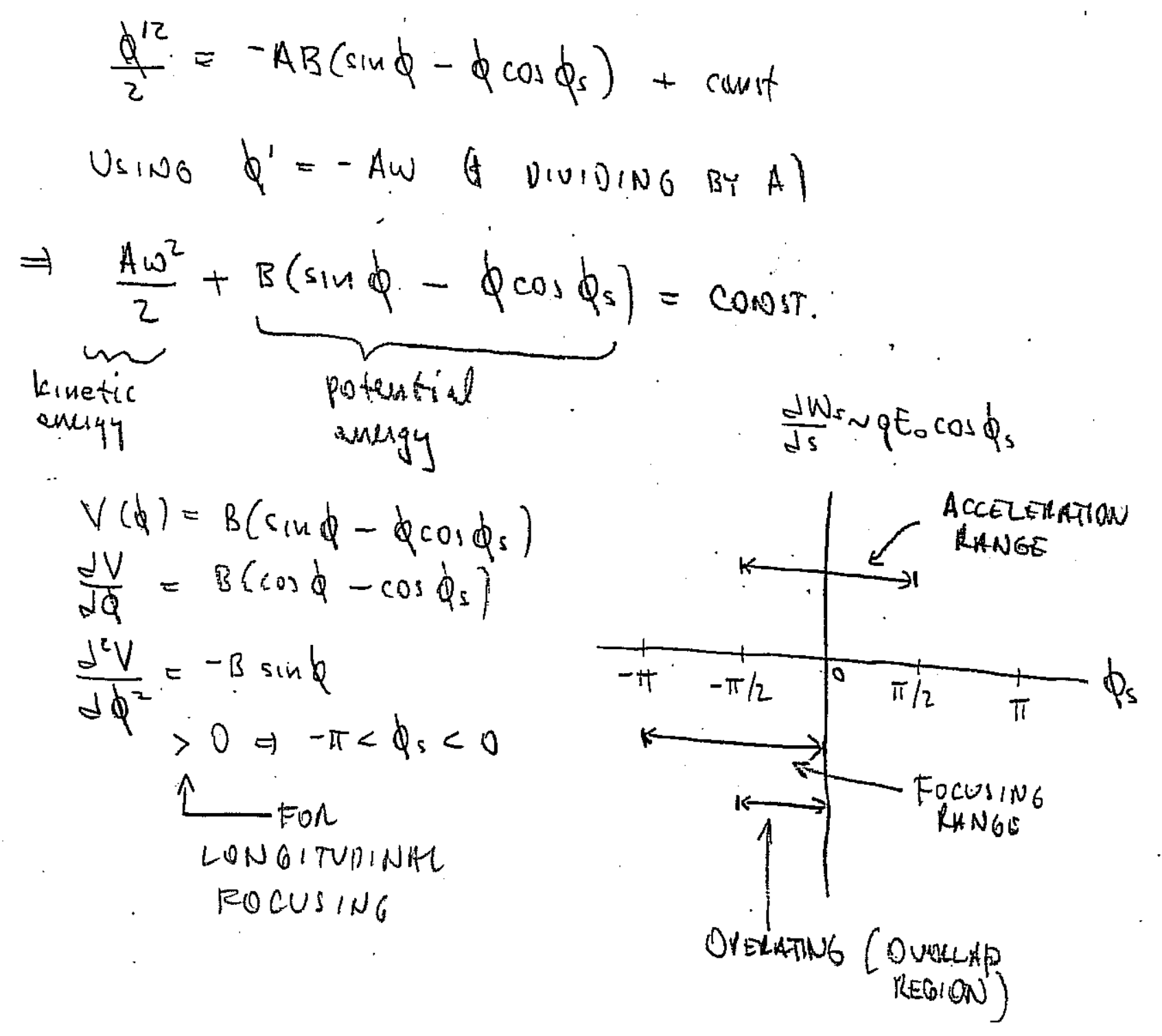

206 
4

\section{$1 \%$}

IGIUDINAL MOTION WHEN ACCELERATION RATE IS SMALL $\equiv 177$

indultaneous acceleration and a potential well when $-\pi / 2 \leq \phi_{s} \leq 0$. The siable region for the phase moion extumericaily by solving for $\phi_{2}$ using lower phase limit $\phi_{2}$ can be 6.3 . shows longitudinal phase space and the $H_{\phi}\left(\phi_{2}\right)=H_{\phi}\left(-\phi_{s}\right)$. Figur at the potential maximum, where $\phi=-\phi_{s}$, we

the

the

$<0$.

ains
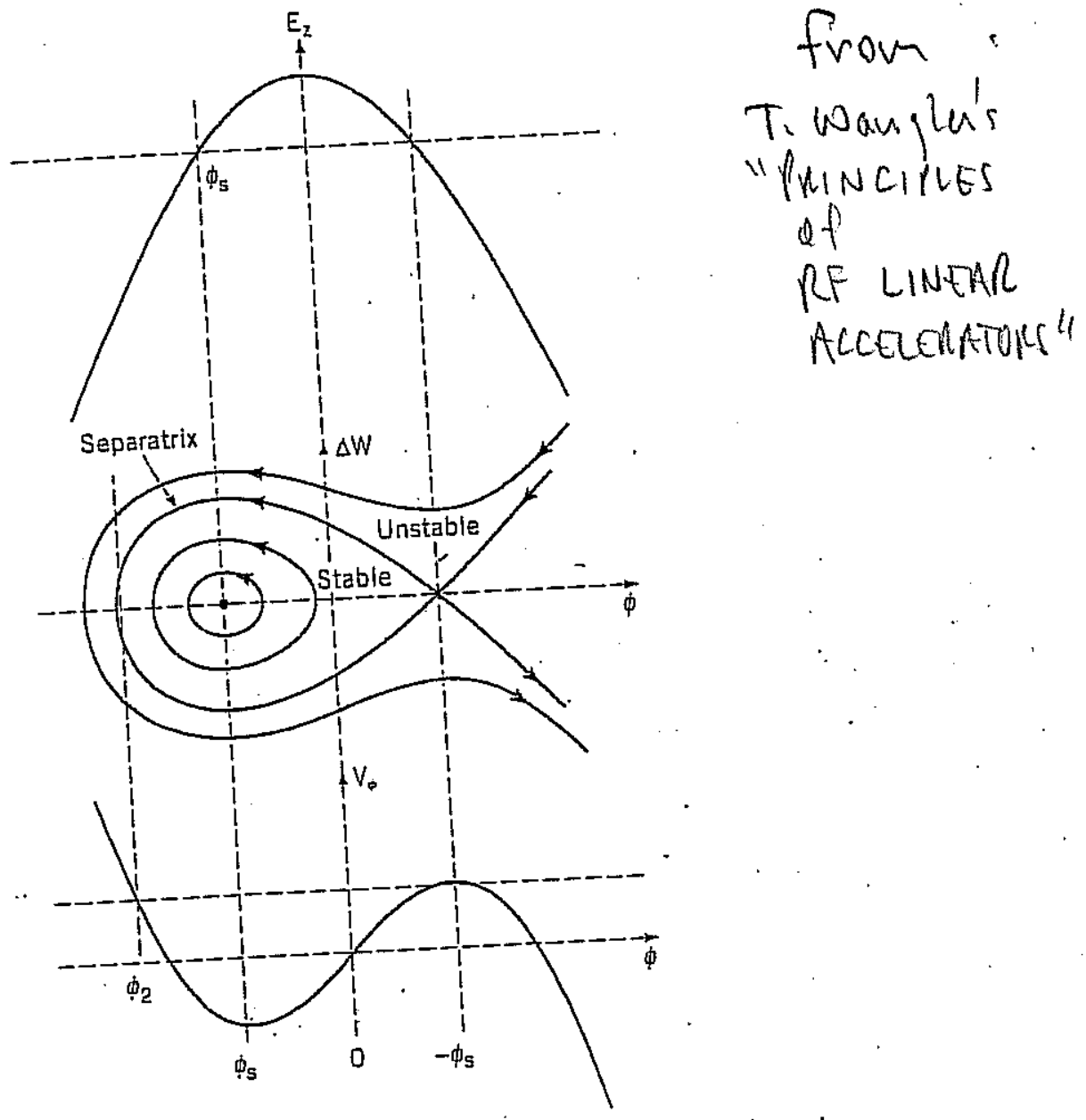
Figure 6.3. As the top, the accelerating field is shown as a cosine funtion of the phase; the synchronous phase $\phi_{3}$ is shown as a negalve number, which lo linal phase-space trajectories, the field is rising in time. The middle plot shows some which passes through the unstable fixed including the separatrix, the limiting stable trojectory, when at $\Delta W=0$ and $\phi=\phi_{1}$, where the point at $\Delta W=0$, and $\phi=-\phi_{1}$. The stable fixed point in the bottom plat.

ongitudinal potential well has its minimum, as shown in the bollom
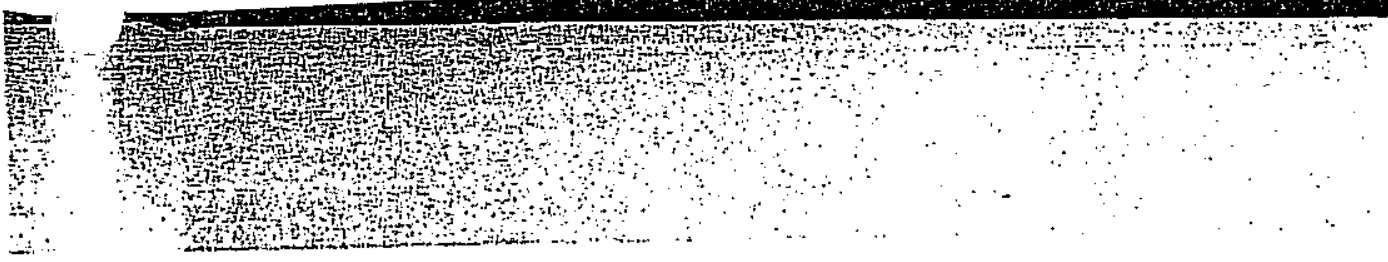
(8)

SPACE-CHAHEE FIELO DF BUNGHE BOHMS

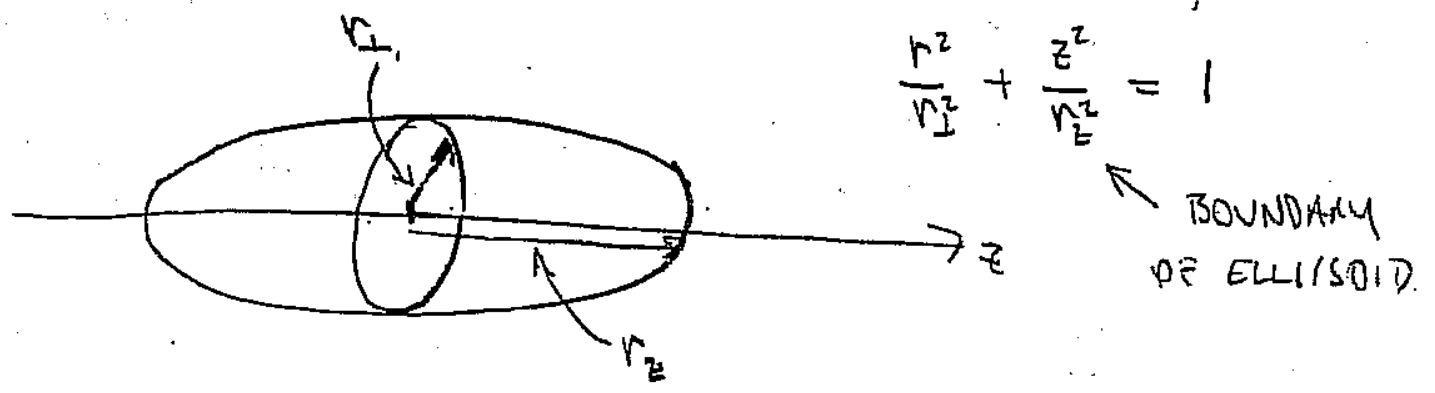

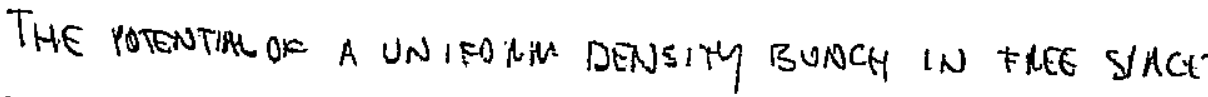

(a maclaurin spmeroid) is GIVEN by:

$$
\varphi=\frac{\rho}{4 \varepsilon_{0}}\left(\alpha_{i} r^{2}+\alpha_{11} z^{2}-\delta\right)
$$

Cof Landas $\$$ Lifshity, Clasicis) Thesiy of

whene $\alpha_{L}=r_{\perp}^{2} r_{z} \int_{0}^{\infty} \frac{d s}{\left(r_{1}^{2}+s\right) \Delta}$

$$
\begin{aligned}
& \alpha_{11}=r_{\perp}^{2} r_{z} \int_{0}^{\infty} \frac{d s}{\left(r_{z}^{2}+s\right) \Delta} \\
& \delta=r_{\perp}^{2} r_{z} \int_{0}^{\infty} \frac{d s}{\Delta}
\end{aligned}
$$

where $\Delta^{2}=\left(r_{1}^{2}+5\right)^{2}\left(r_{z}^{2}+5\right)$

FOR NON-LELATIVISTIC BEAM:

$$
\begin{gathered}
E_{z}=-\frac{\partial \phi}{\partial z}=f \frac{\rho}{\varepsilon_{0}} z \\
E_{r}=-\frac{\partial \phi}{\partial r}=\frac{(1-f)}{2} \frac{f}{\varepsilon_{0}} r \\
f=f(\alpha)=\left\{\begin{array}{lll}
\frac{\alpha^{2}}{1-\alpha^{2}}\left[\frac{1}{\sqrt{1-\alpha^{2}}} \tanh ^{-1} \sqrt{1-\alpha^{2}}-1\right] & \alpha<1 \quad \alpha \equiv \frac{r_{1}}{r_{z}} \\
\frac{\alpha^{2}}{\alpha^{2}-1}\left[1-\frac{1}{\sqrt{\alpha^{2}-1}} \tan ^{-1} \sqrt{\alpha^{2}-1}\right] & \alpha>1
\end{array} \quad\right.
\end{gathered}
$$


(Q))

FOK RELNTUISTIC bEAM

(cf. Luvid \& BARN ARD 1997.)

$$
\begin{aligned}
& \frac{d^{2} \underline{x}_{L}}{d s^{2}}=\frac{F_{1}}{\gamma, p_{s}^{2} m c^{2}} \\
& F_{1 s}=\frac{-q}{\gamma_{1}^{2}} \frac{\partial \phi}{\partial x_{1}}=\frac{q \rho}{2 \gamma_{s}^{2} \epsilon_{0}}[1-f(x)] \underline{x}_{1} \\
& \frac{d^{2} \Delta z}{d s^{2}}=\frac{F_{z}}{\gamma_{s}^{3} \beta_{r}^{2} m c^{2}} \\
& F_{z s}=\frac{-q}{\gamma_{s}^{2}} \frac{\partial \phi}{\partial z}=\frac{q p}{\epsilon_{0}} f(\alpha) \Delta z \\
& \alpha=\frac{r_{1}}{\gamma r_{z}} \quad\left[\alpha=\frac{r_{\perp}}{\left(r_{z} \text { in comoving f(tame }\right)}\right]
\end{aligned}
$$

COMBINENG FOCUSING + SELF FIELDS

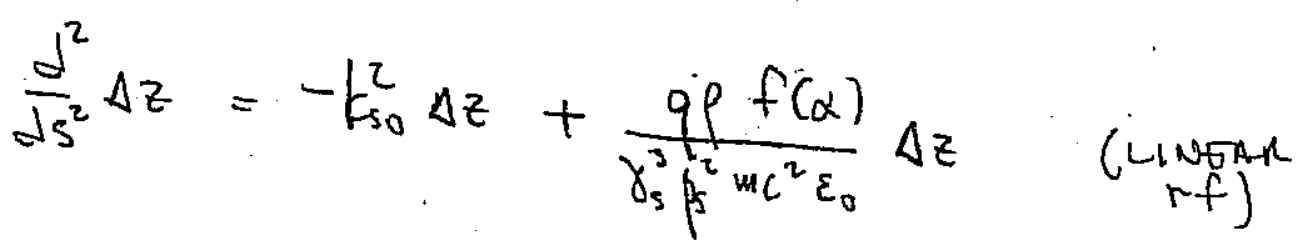

209 


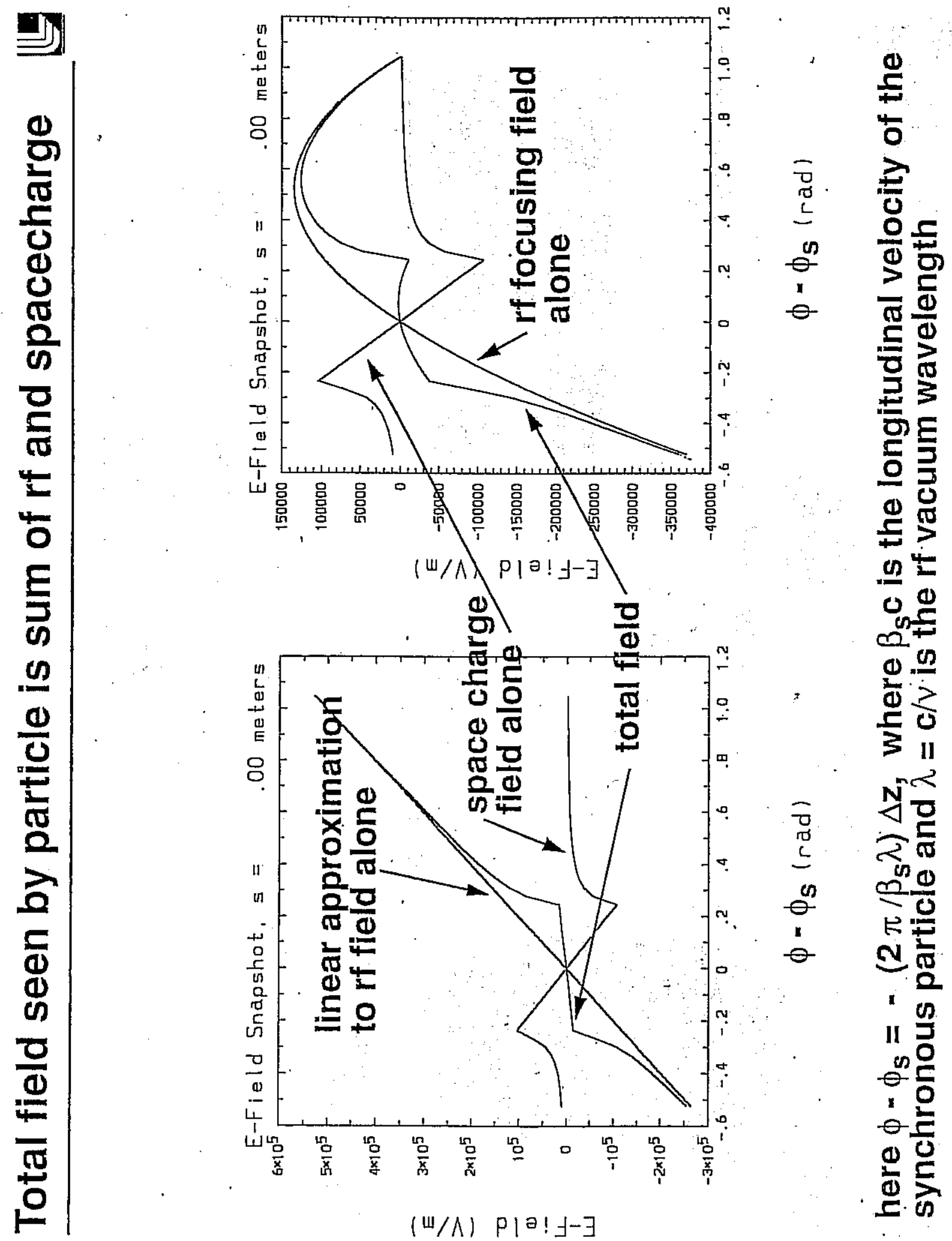



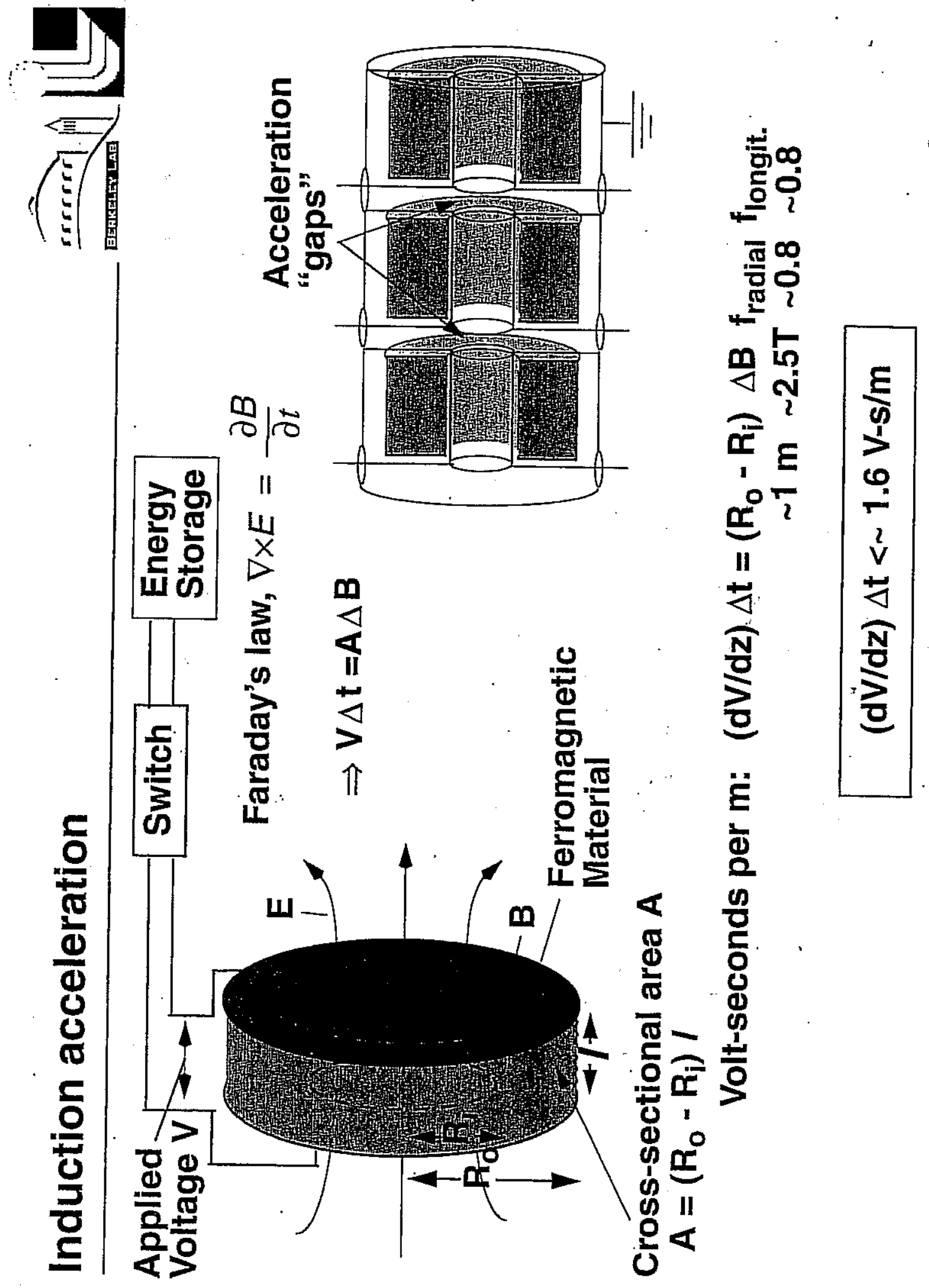


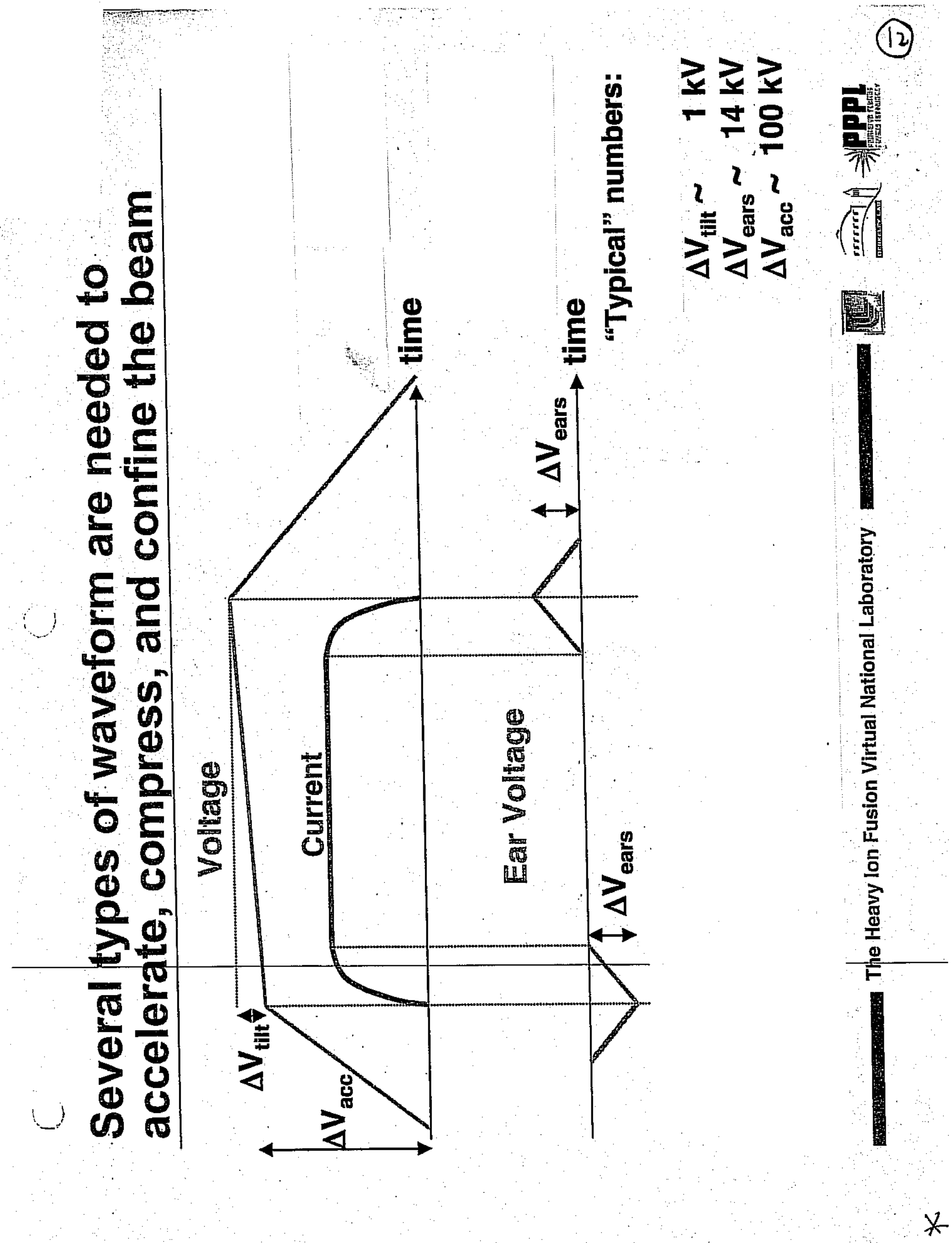




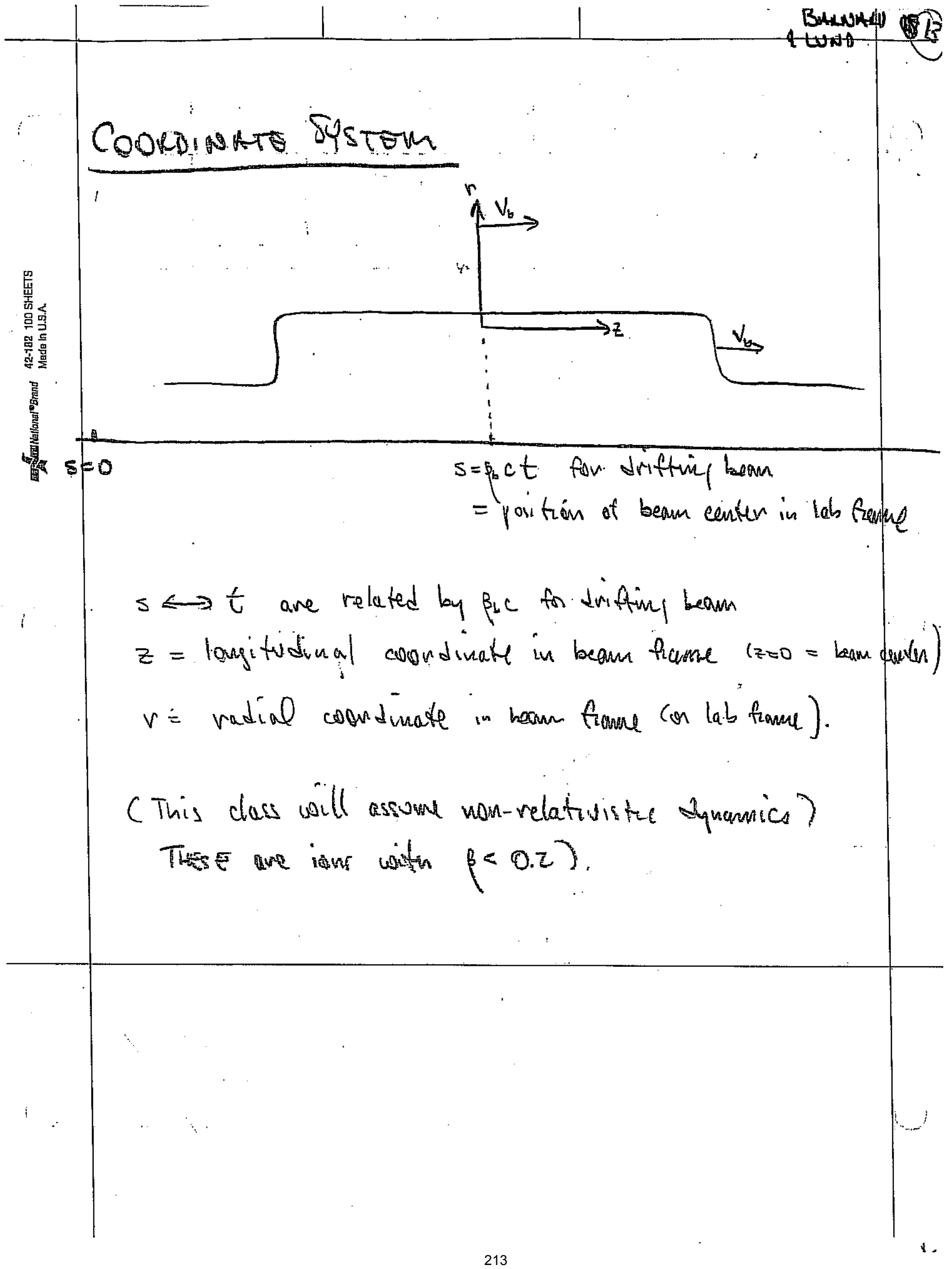




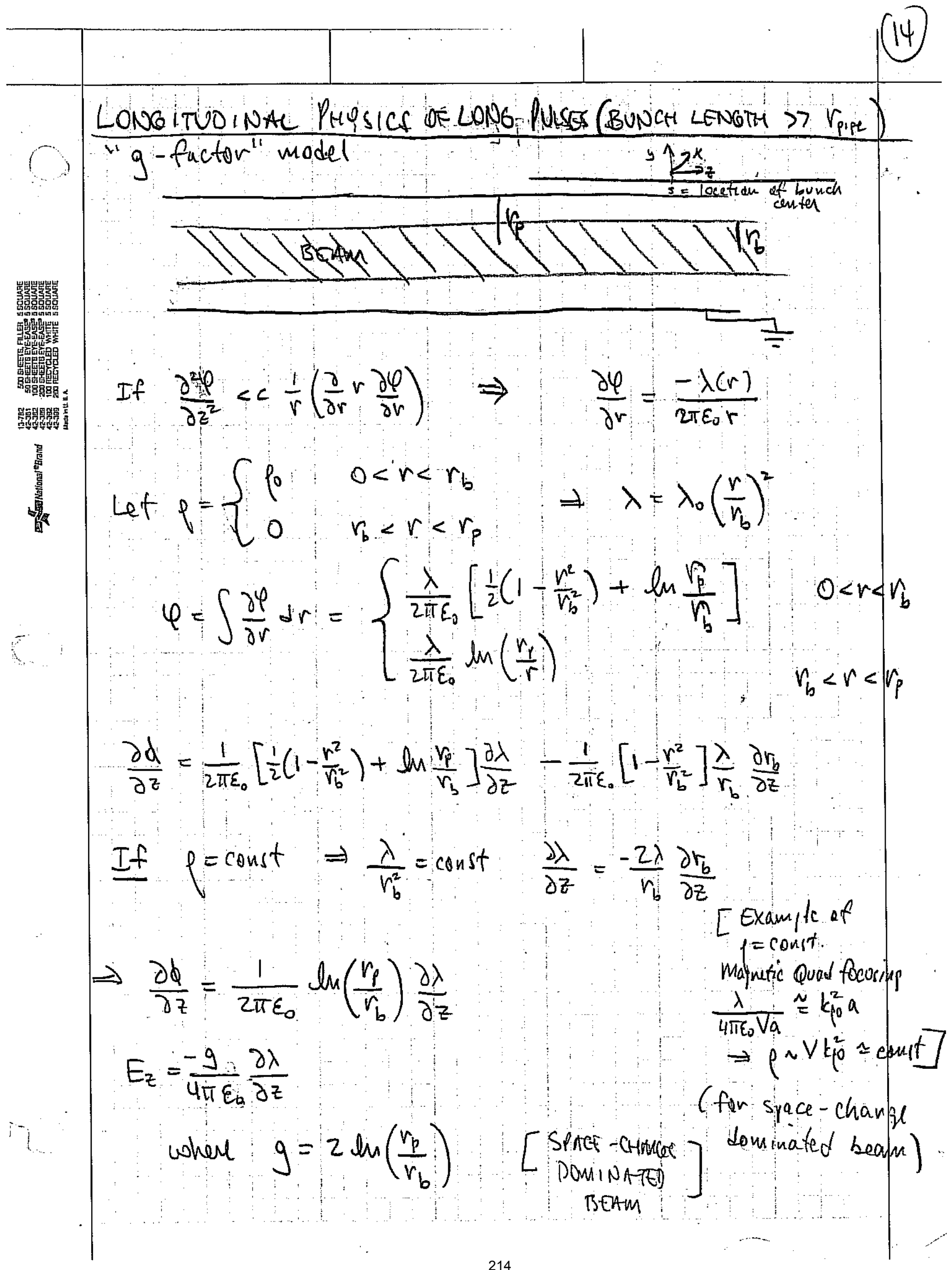


For emittance dominated beAms:

RADIUS NOT DETERMINED BY $\lambda$

so $\frac{\partial \sqrt{b}}{\partial z} \simeq 0$

$$
\begin{gathered}
\left\langle\frac{\partial \phi}{\partial z}\right\rangle=\frac{1}{2 \pi \varepsilon_{0}}\left[\frac{1}{2}\left(1-\left\langle\frac{r^{2}}{r_{b}^{2}}\right\rangle\right)+\ln \frac{r_{p}}{r_{b}}\right] \frac{\partial \lambda}{\partial z} \\
\Rightarrow g=2 \ln \left(\frac{r_{p}}{r_{b}}\right)+\frac{1}{2}^{1 / 2} \quad \text { (EMITTANCE DOMINATE) } \\
\text { BEAMS) }
\end{gathered}
$$

(SEe raiser; SECtion 6.3 for discussion on $g$-factor).

215 


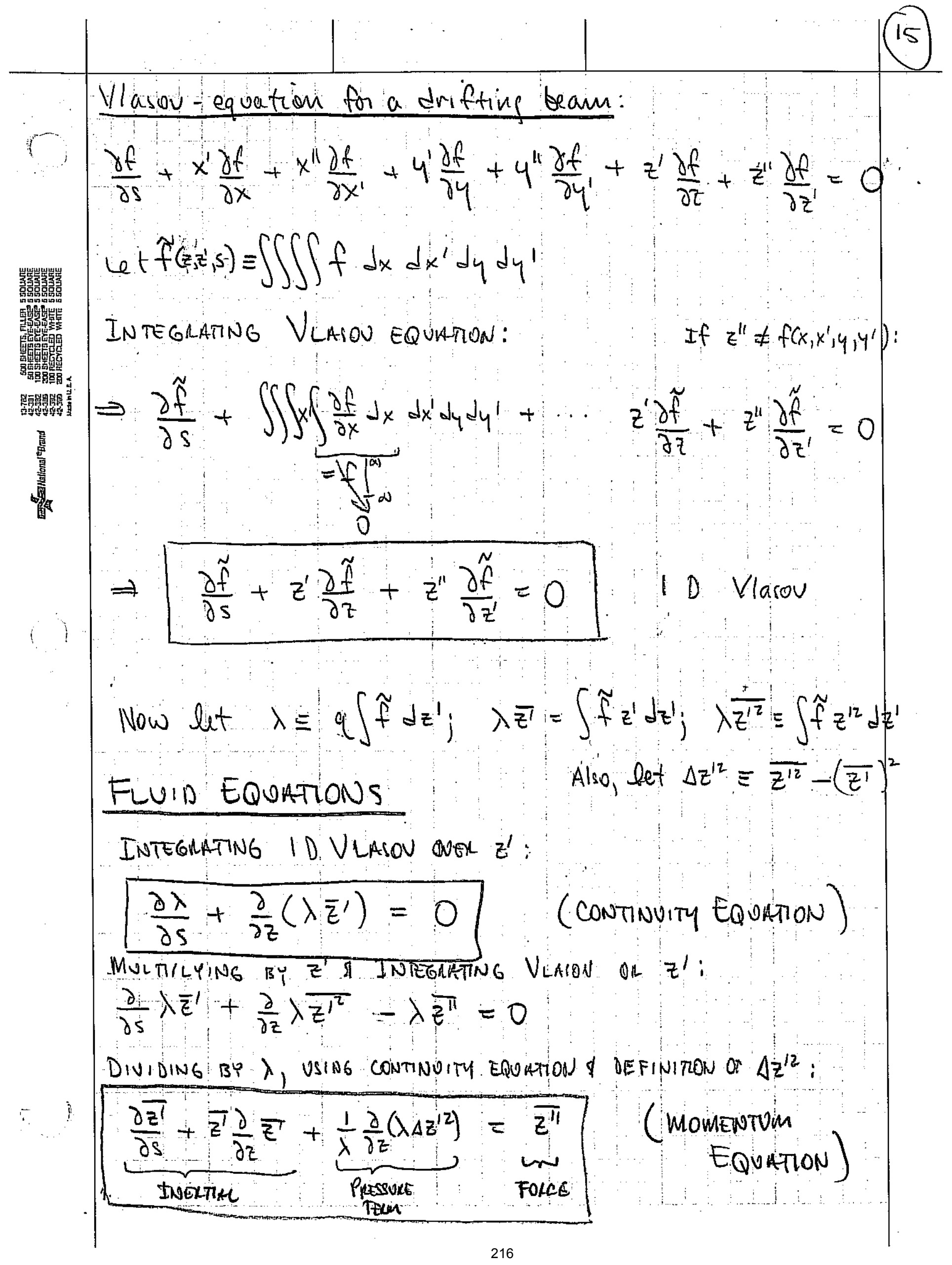




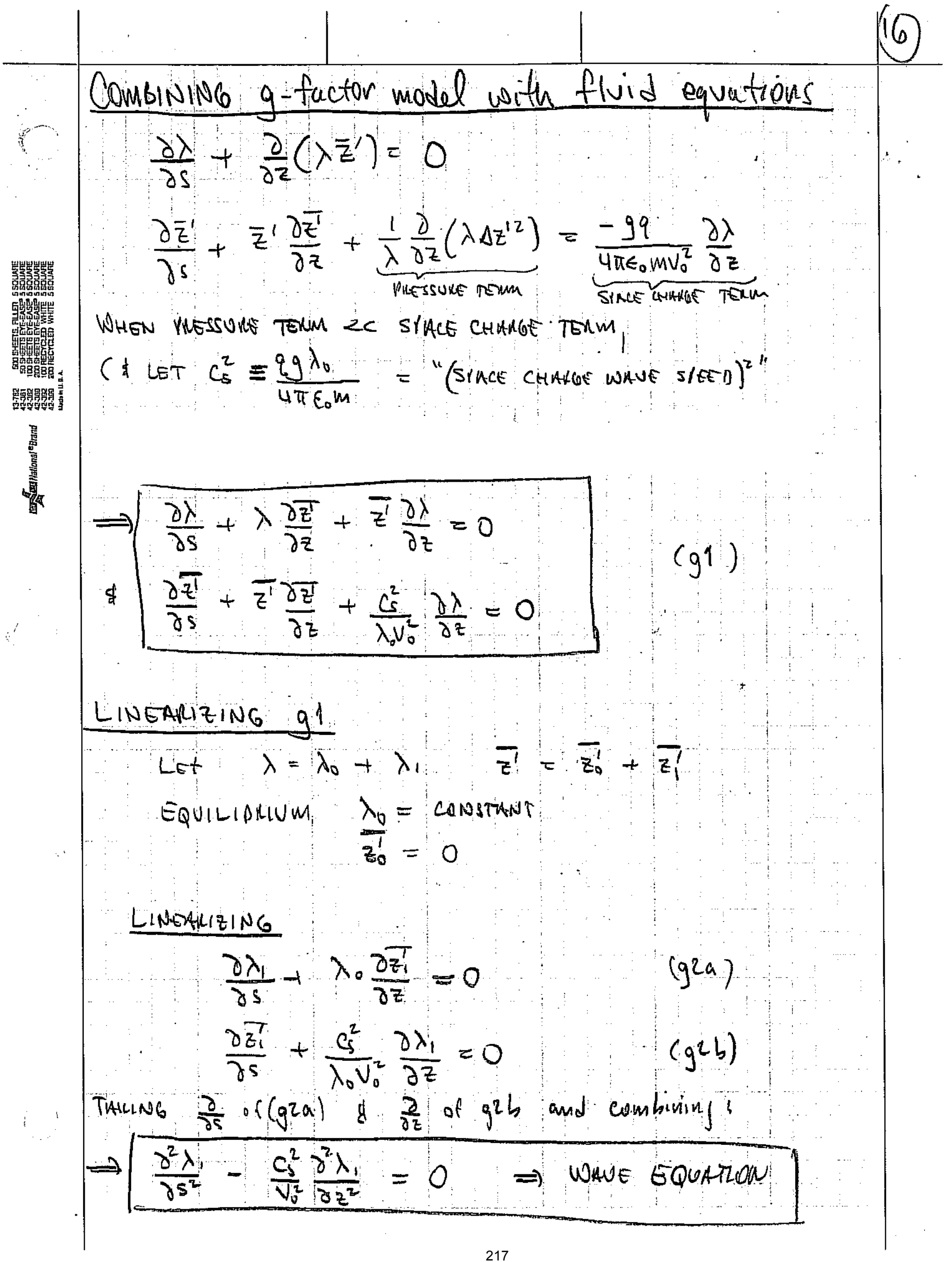




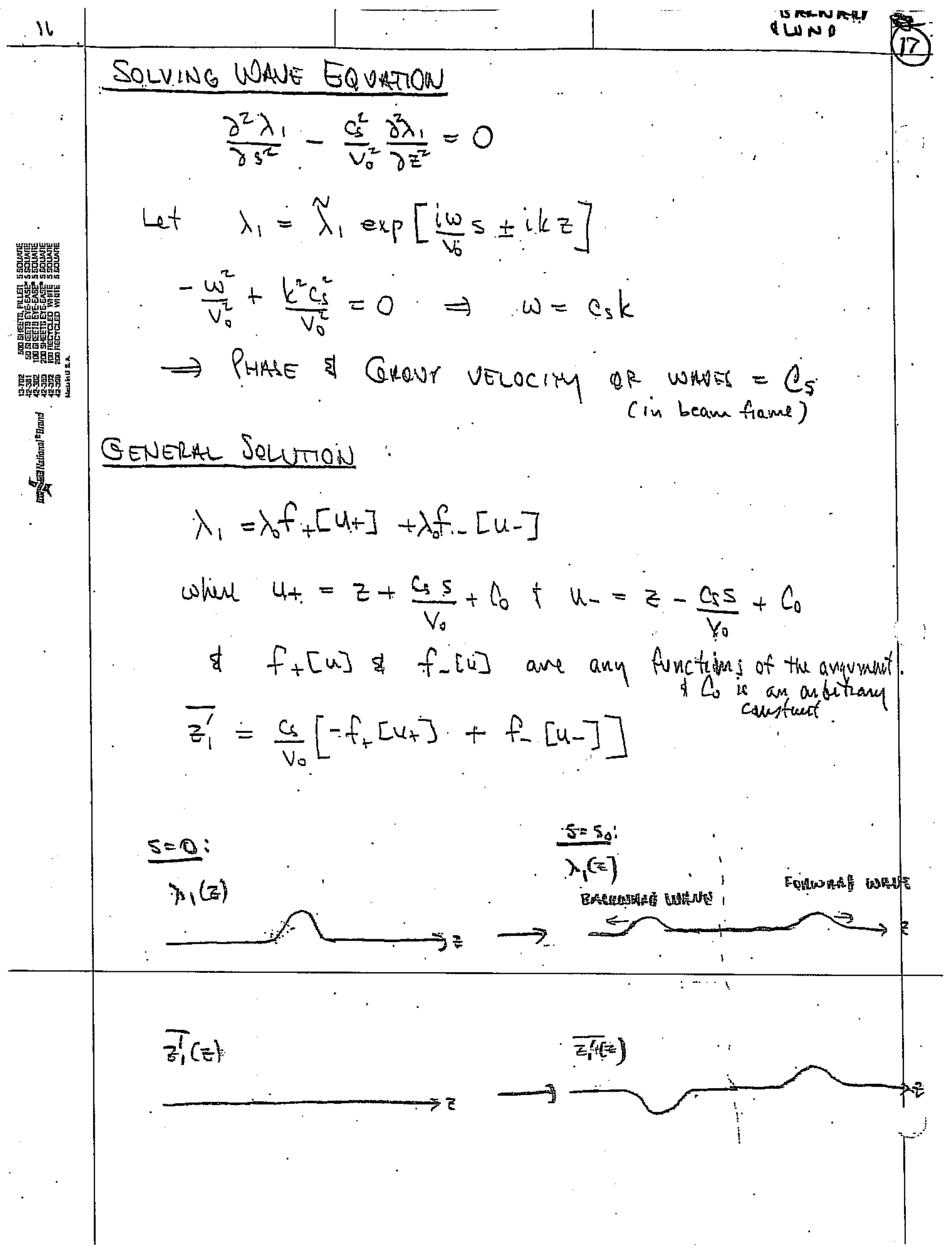

218 
BOAMENDS \& Rareaction WAVES (FAuTENS tLEE) BEAM ENDS \& RAREFACTION WAVES J. AN. PMYS. 61, 5:19) (Alo Landias qLifshles,

SORPOSE YOU STANT WITM A VULSE TALT

ENDS WITH A STER FUNCTION IN $\lambda$. WhHT

HAIVENS TO THE END?
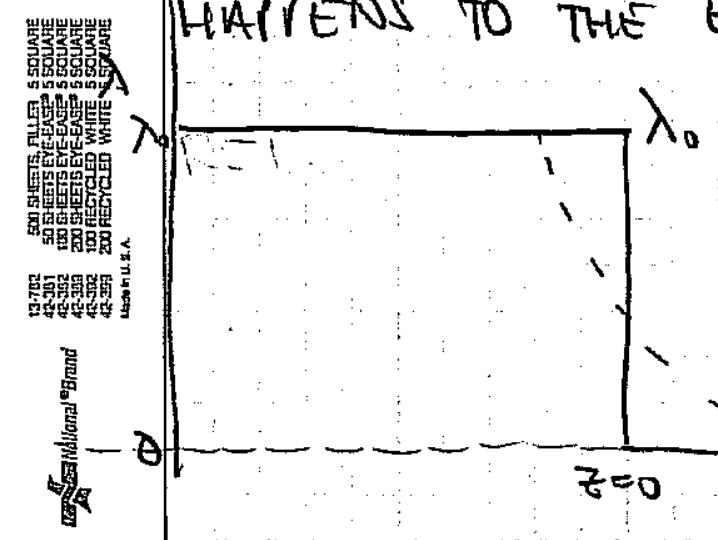

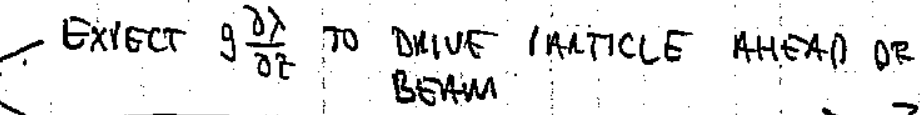

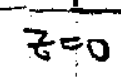

TO ANALYZE: RETURN TO NON-LINEAR FLUID EQUATIONS (SINCE $\left.\delta: \lambda \sim \lambda_{0}\right)(g 1)$ :

$$
\begin{array}{ll}
\frac{\partial \lambda}{\partial s}+\lambda \frac{\partial \bar{z}}{\partial z}+\overline{z^{\prime}} \frac{\partial \lambda}{\partial z}=0 & \text { (Contnuim) } \\
\frac{\partial z^{2}}{\partial s}+\overline{z^{\prime}} \frac{\partial \bar{z}}{\partial z}+\frac{c^{2}}{\lambda_{0} v_{0}^{2}} \frac{\partial \lambda}{\partial z}=0 & \text { (momentum) }
\end{array}
$$

IST IT IS CONUENIENT to DERINE: $\Lambda \equiv \lambda / \lambda_{0}$

$$
\begin{aligned}
& \left(c_{s}^{2} \equiv \frac{q}{m} \frac{g \lambda_{0}}{4 \pi \varepsilon_{0}}\right) \\
& V \equiv \frac{V_{0}}{c_{s}} \overline{z^{\prime}} \\
& z=\frac{v_{0}}{c_{s}} z
\end{aligned}
$$

$\Rightarrow \quad \frac{\partial \Lambda}{\partial s}+\Lambda \frac{\partial V}{\partial z}+V \frac{\partial \Lambda}{\partial z}=0$

(contrnuary)

$\frac{\partial V}{\partial s}+V \frac{\partial V}{\partial z}+\frac{\partial A}{\partial z}=0$

\section{$(r 1)$}

comomention) 


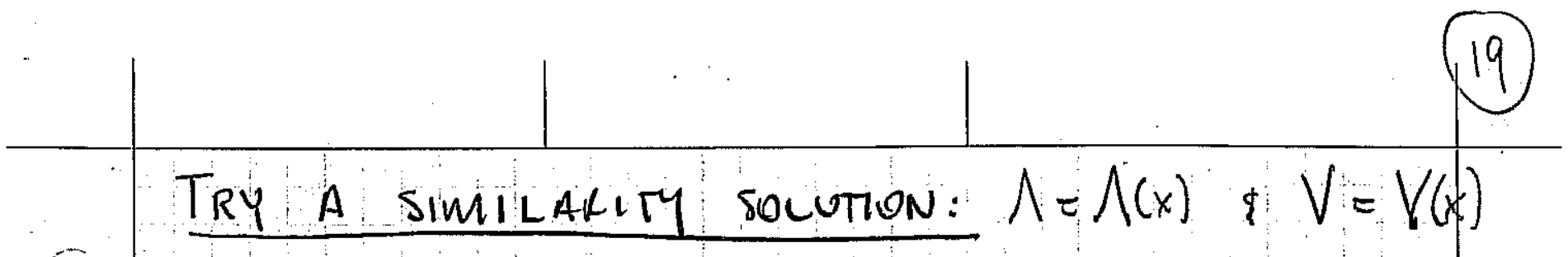

WHERE $\quad x=\frac{z}{s}=\left(\frac{V_{0} z}{c_{S}}\right)$

$$
\begin{aligned}
& \frac{\partial x}{\partial s}=-\frac{x}{s} \quad \frac{\partial x}{\partial z}=\frac{x}{z} \\
& \frac{\partial \Lambda}{\partial s}=\frac{\partial \Lambda}{\partial x} \frac{x}{s} \quad \frac{\partial \Lambda}{\partial z}=\frac{d \Lambda}{\partial x} \frac{x}{z} \quad \frac{\partial V}{\partial s}=-\frac{d V}{d x} \frac{x}{s} \quad \frac{\partial V}{\partial z}=\frac{j V}{d x} \frac{x}{z} \\
& {\left[-\frac{d \Lambda}{d x} \frac{x}{5}+\Lambda \frac{d V}{d x} \frac{x}{z}+V \frac{d \Lambda}{d x} \frac{x}{z}\right]=0} \\
& {\left[-\frac{d V}{d x} \frac{x}{s}+V \frac{d V}{d x} \frac{x}{z}+\frac{d \Lambda}{d x} \frac{x}{z}\right]=0} \\
& \text { Continvity) } \\
& \text { (momentum) } \\
& \text { muitivicu by zols a qatime temis): } \\
& \Rightarrow\left[\begin{array}{cc}
V-x & \Lambda \\
1 & V-x
\end{array}\right]\left[\begin{array}{l}
d V / J x \\
d V / d x
\end{array}\right]=0
\end{aligned}
$$

FOE NON-TLIVIAL SQLUTTON DETOLMINANAT MUIT UANISH:

$$
\begin{aligned}
& \Lambda=[V-x]^{2} \\
\Rightarrow & \frac{d \Lambda}{d x}=2[V-x]\left[\frac{d V}{d x}-1\right] \quad d \frac{d \Lambda}{d x}=-[V-x] \frac{d V}{d x} \\
\Rightarrow & -\frac{d V}{d x}-2 \frac{d V}{d x}-2 \Rightarrow \frac{d V}{d x}=\frac{2}{3} \\
\Rightarrow & V=\frac{2}{3} x+C \\
& \Lambda=\left[-\frac{1}{3} x+C\right]^{2}
\end{aligned}
$$

220 


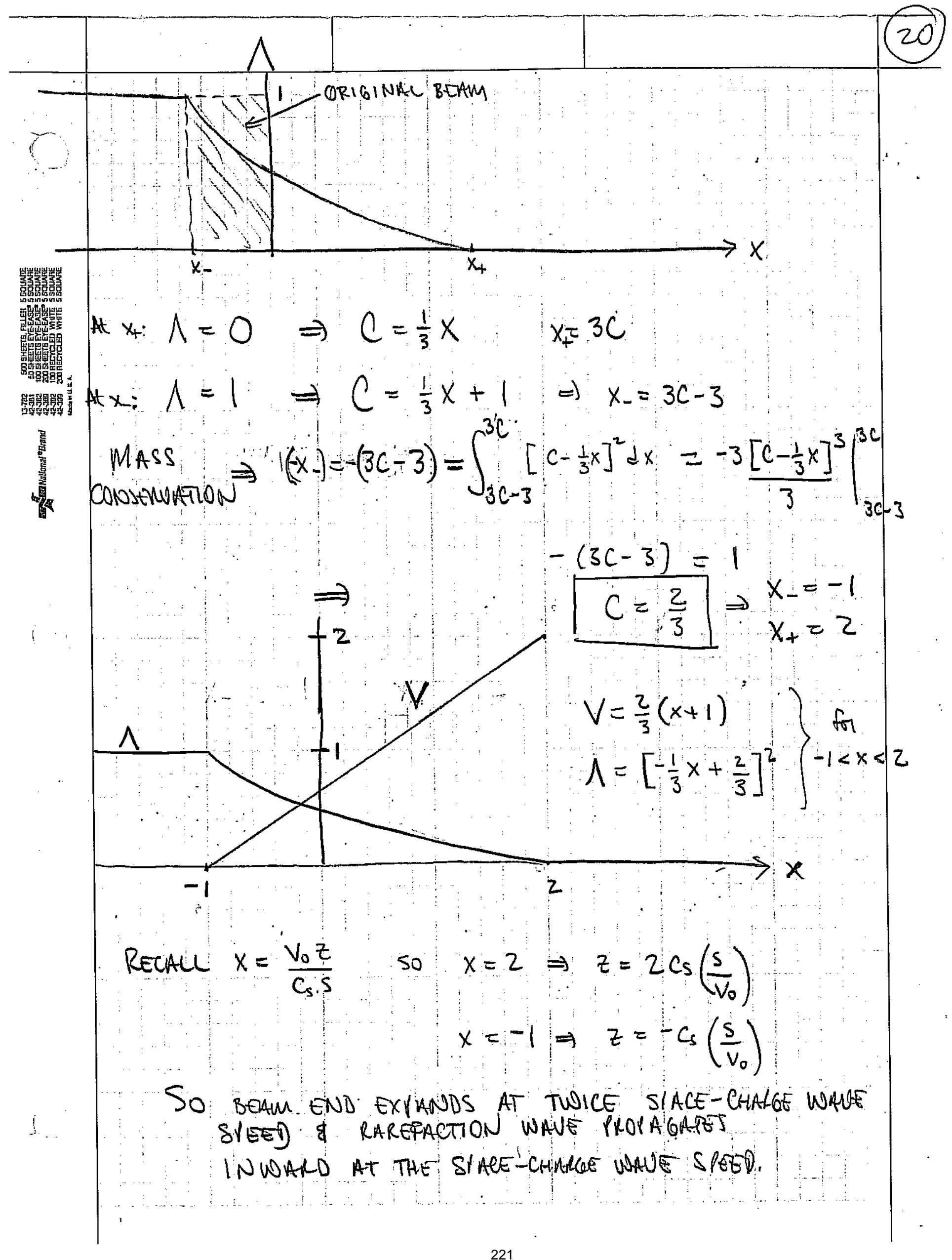




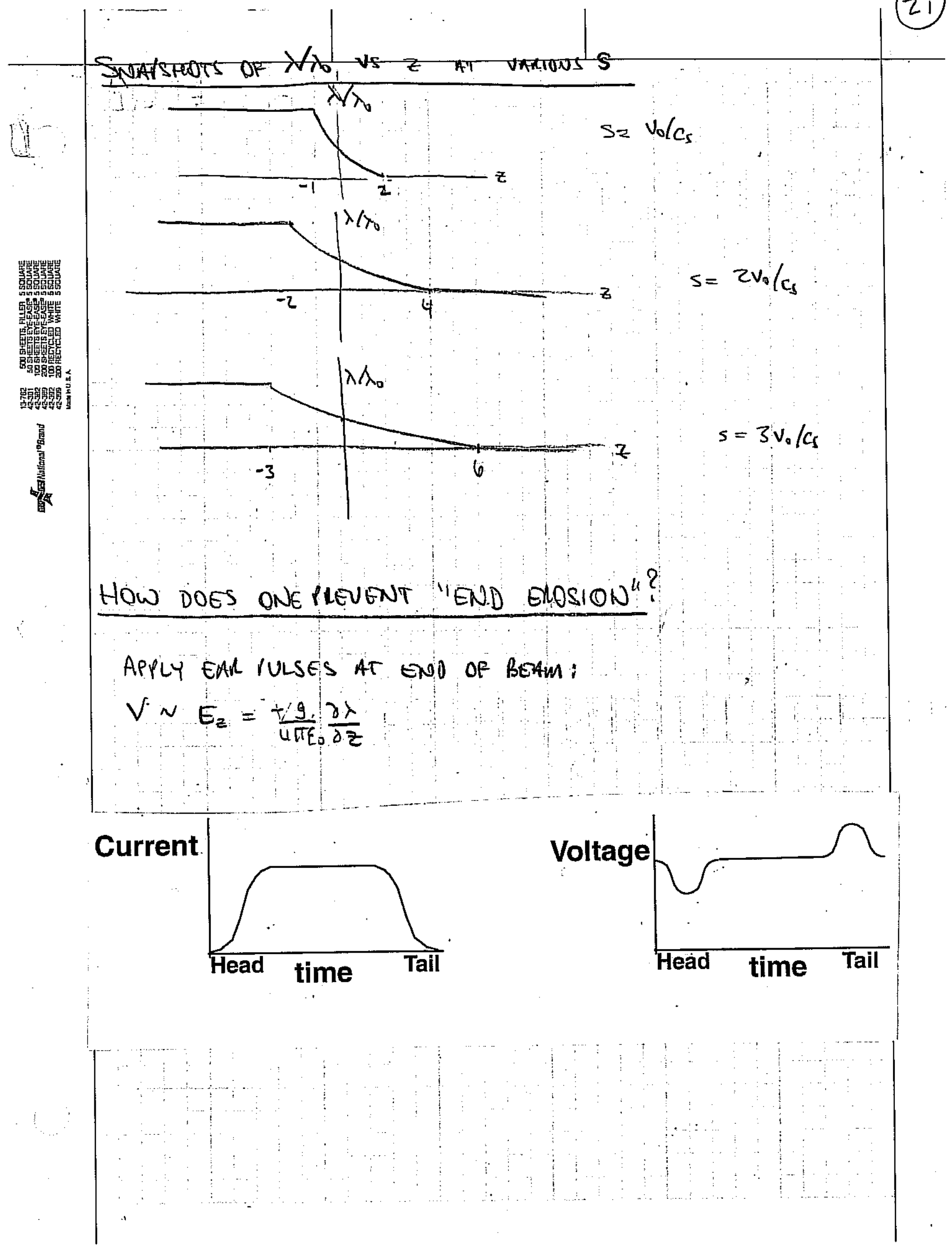


C. CHAPTER 6. INTERMITTENTLY-APPLIED AXIAL CONFINING FIELDS 98

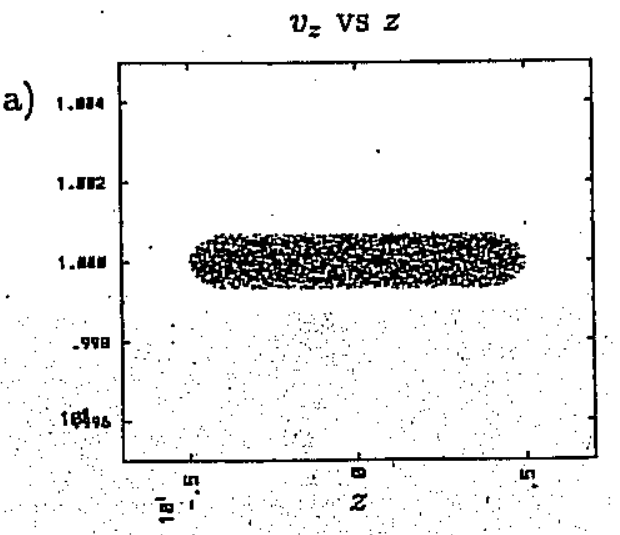

Ear Field vs $z$

c)

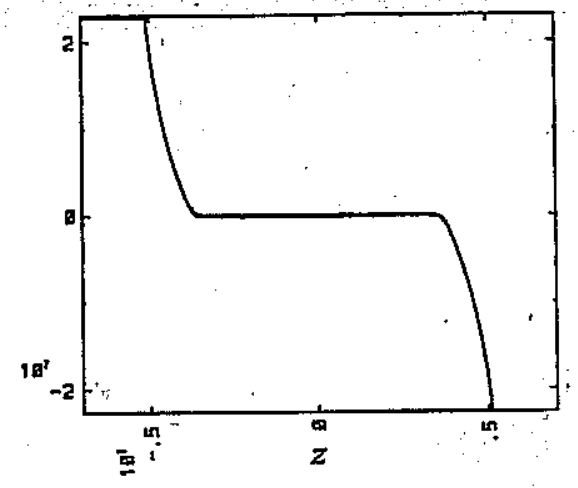

$v=\operatorname{VS} z$

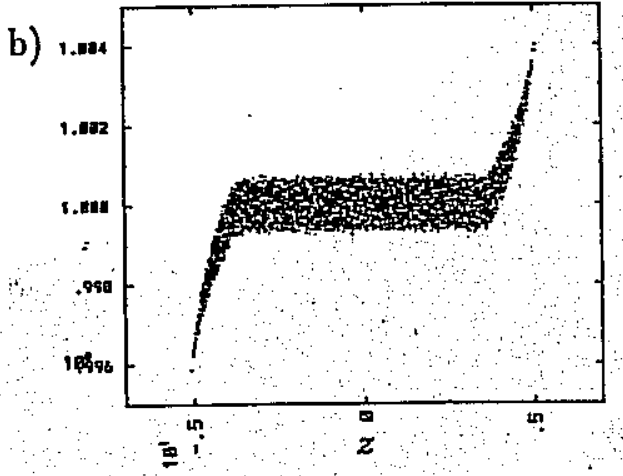

$v_{z}$ vs $z$

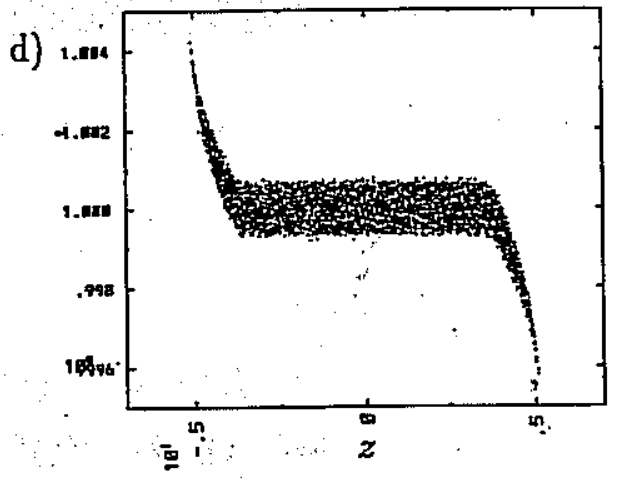

$v_{z}$ vs $z$

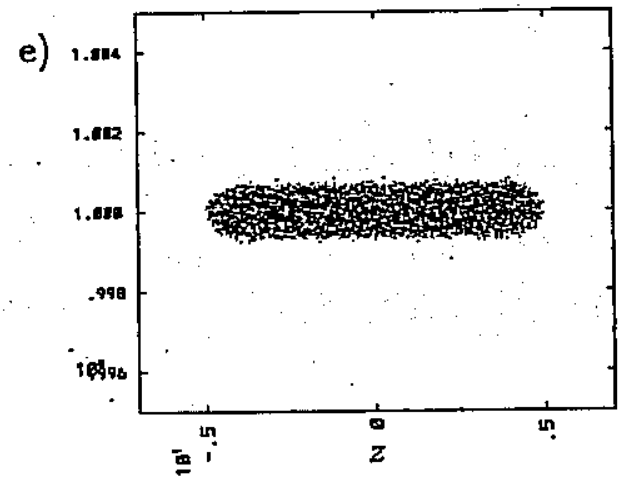

Figure 6.4: One cycle of intermittently-applied ears. (a) Initial phase space (b) Beam expands (c) Ear Field is applied (d) Beam is compressed (e) Beam expands back to its initial state

$$
\begin{aligned}
& \text { from D. Callahan Willen } \\
& \text { PUB thosic, U.C. Davis, } 1994 .
\end{aligned}
$$




\section{John Barnard Steven Lund USPAS June 2008}

\section{Injectors and longitudinal physics -- III}

1. Longitudinal cooling from acceleration

2. Longitudinal instability

3. Bunch compression

4. Neuffer distribution 


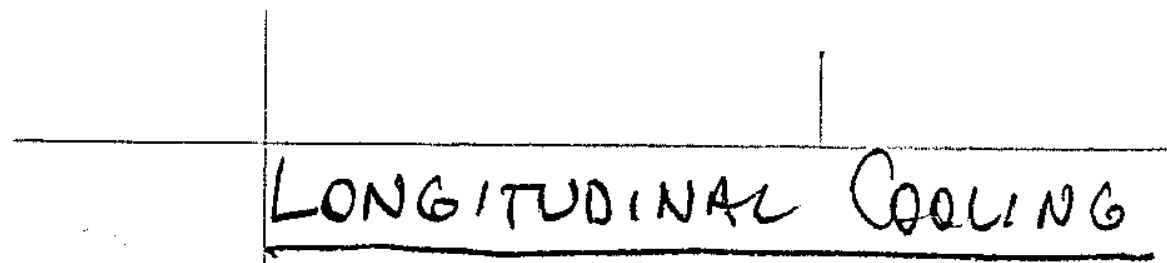

(2)

1. During injection betm undergoes cakge longitupinal exiansion

2. $T_{\perp 0}=T_{110}$ AT SOUKCE, BUT $T_{\perp} \neq T_{11}$ AETER accerelation

3. ImplichtLons ror botm STABILITY and emitTANCE EVOLUTION

CoNsioer I pIODE:

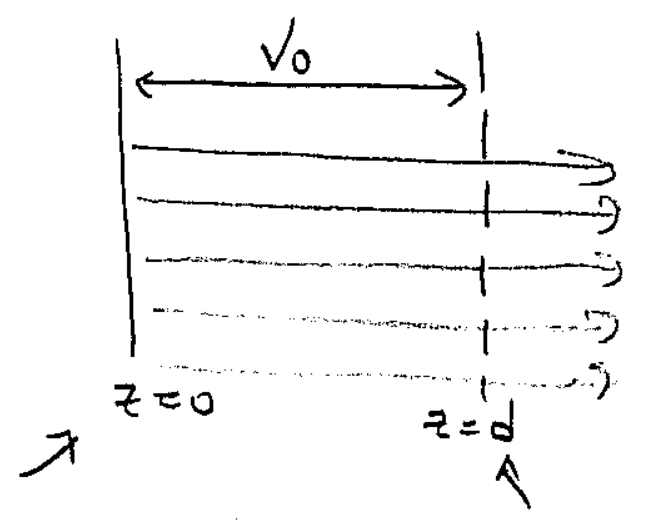

AT SOURCE

$$
\begin{aligned}
E_{0} & =\frac{p_{z 0}^{2}}{2 m} \\
\Delta E_{110} & \equiv \frac{\left\langle p_{z 0}^{2}\right\rangle}{2 m}=\frac{1}{2} k T_{110}
\end{aligned}
$$

AT END OF DIODE

$E_{f}=q V_{0}+\frac{p_{z 0}^{2}}{2 m}=\frac{p_{z s}^{2}}{2 m}$

SINGE $E_{11}=\frac{p_{t}^{2}}{2 m} \Rightarrow \Delta E_{11}=\frac{2 p_{z} \Delta p_{z}}{2 m}$

$\frac{\Delta E}{E}=\frac{2 \Delta p_{z}}{p_{z}}$

$\frac{1}{2} k T_{f} \cong \frac{\Delta p_{z f}^{2}}{2 m}=\left(\frac{p_{z f} \Delta E_{f}}{2 E_{f}}\right)^{2} \frac{1}{2 m}=\frac{\Delta E_{f}^{2}}{4 E_{f}}=\frac{k T_{0}}{2}\left[\frac{1}{2} \frac{k T_{0}}{q V_{0}}\right]$

$\Rightarrow k T_{f}=\frac{1}{2} k T_{0}\left[\frac{k T_{0}}{q V_{0}}\right]$ 


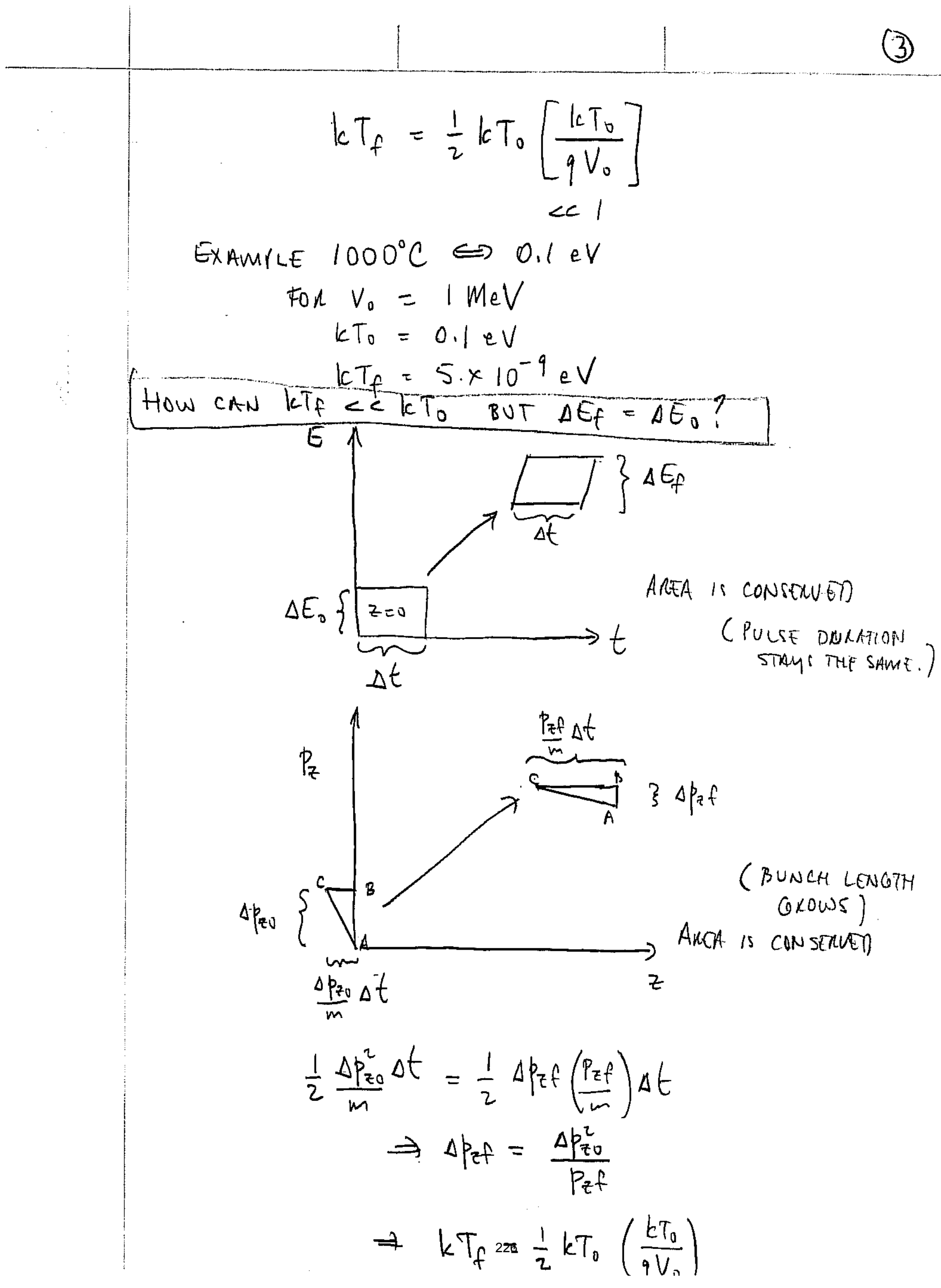


(C) Note: $\quad \overline{z^{\prime}} \equiv\left\langle\frac{d z}{d s}\right\rangle ; \quad s=v_{0} t$

Let $\quad u=\left\langle\frac{d z}{d t}\right\rangle$; then $u=v_{0} z^{1}$

- fluid velocity in comoving frame

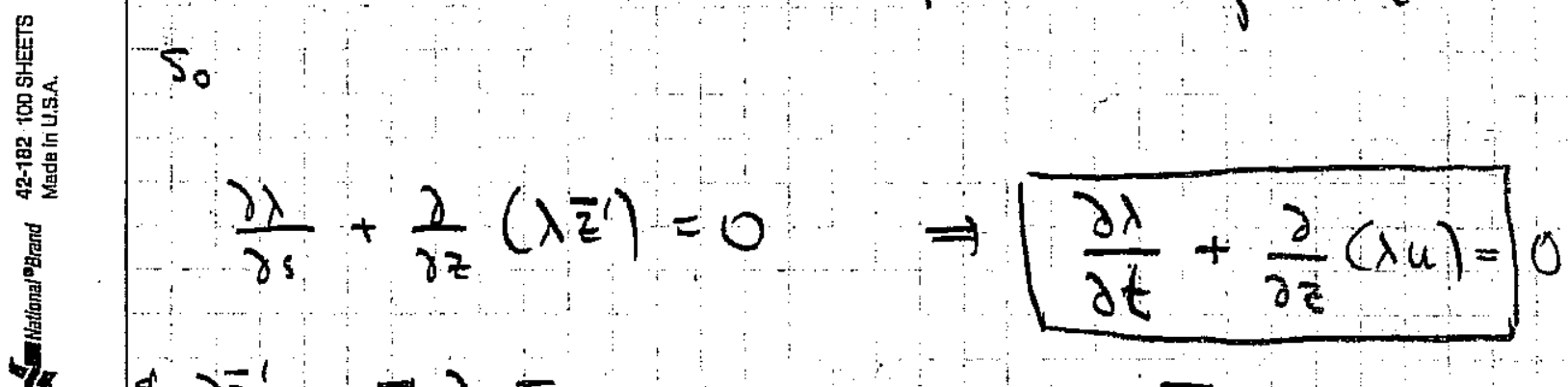

$\frac{f^{\prime}}{\partial s}+\frac{\partial}{z^{\prime}} \frac{\partial}{\partial z}+\frac{1}{\lambda} \frac{\partial}{\partial z}\left(\lambda \Delta z^{\prime z}\right)=\bar{z}^{\prime \prime}$

$\Rightarrow \frac{\partial u}{\partial t}+\frac{\partial u}{\partial z}+\frac{1}{\lambda} \frac{\partial}{\partial z}\left(\lambda\left[\left\langle v_{z}^{2}\right\rangle-u^{2}\right]\right)=\frac{\pi}{z}$

$$
\text { Since } p_{z}=m \int_{-\infty}^{\infty} n\left[v_{z}^{2}-u^{2}\right] d v_{z}=\text { whene } n=\frac{\lambda}{\pi r_{b}^{2}}
$$

$\Rightarrow \frac{\partial u}{\partial t}+u \frac{\partial u}{\partial z}+\frac{\pi r_{b}^{2}}{m \lambda} \frac{\partial}{\partial z} p_{z}=\overline{\bar{z}}$

where $z=\frac{d^{2} z}{d t^{2}}$

$$
=\frac{F_{z}}{m}
$$




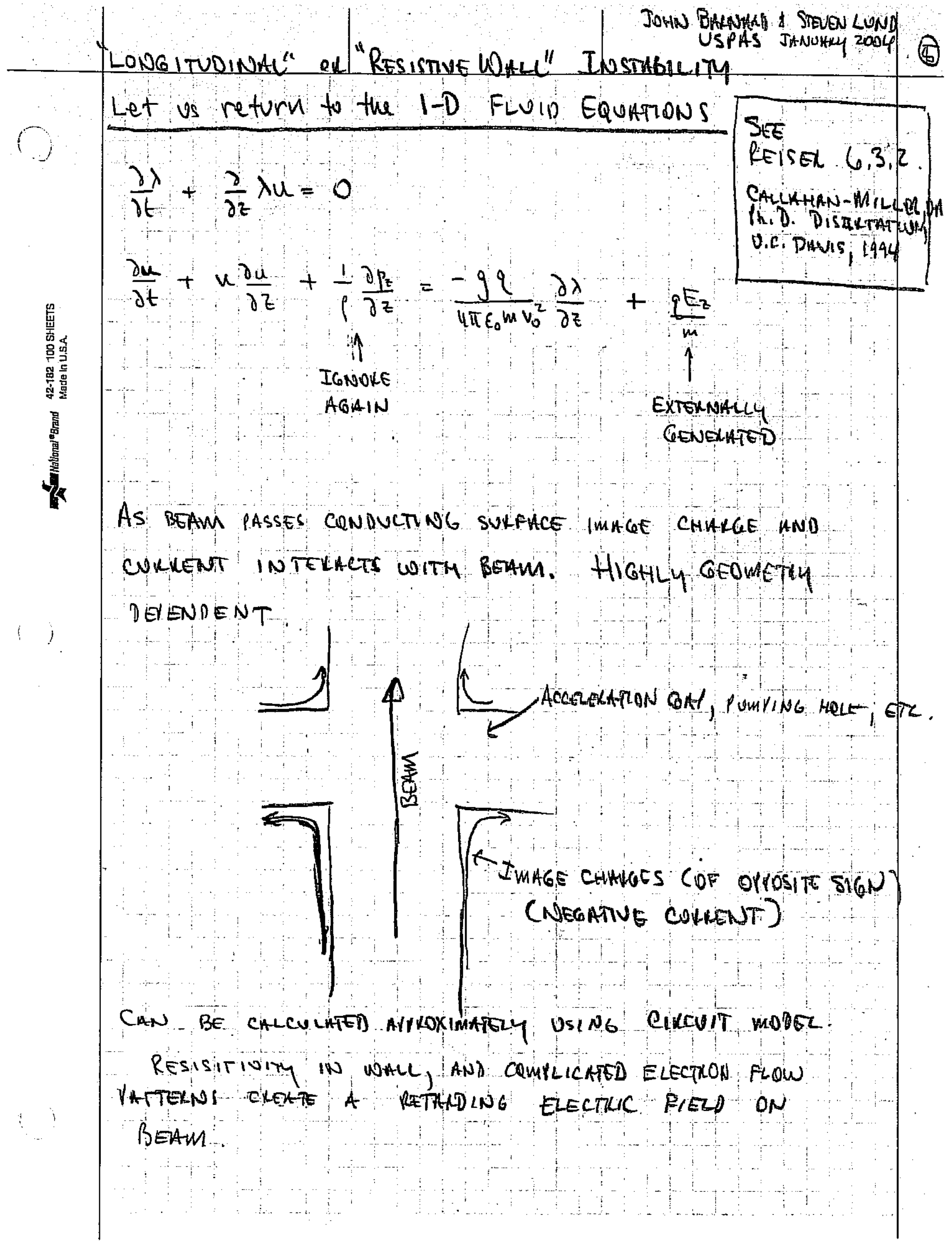

228 


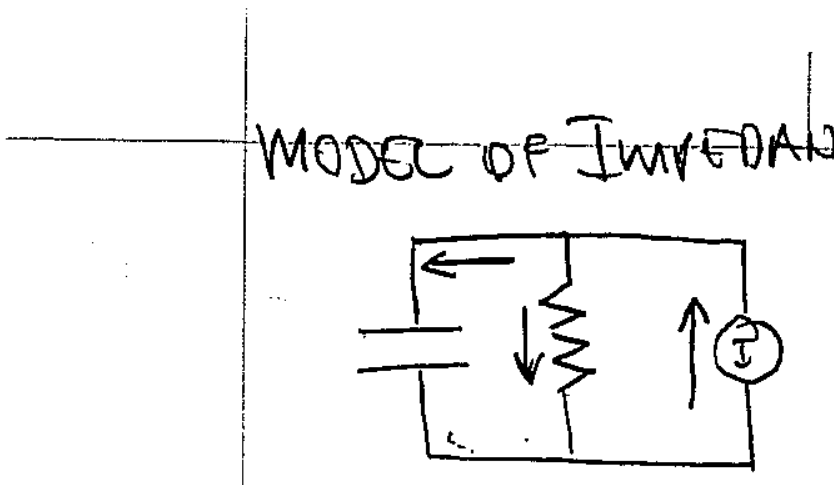

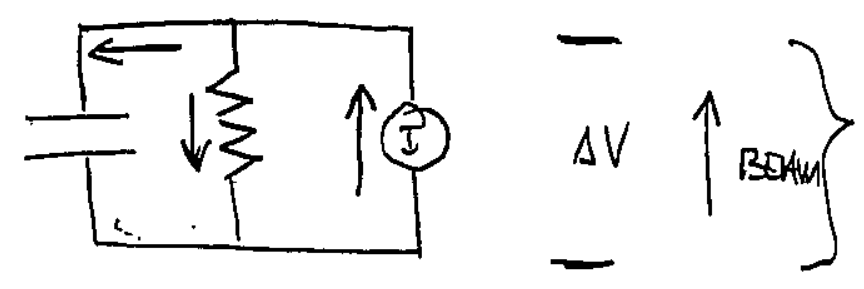

$$
\begin{aligned}
& \text { ONE MODULE OF } \\
& \text { MANY, EACH SEPARATED) } \\
& \text { BY DISTANCE } L \\
& I=C \frac{d \Delta V}{d t}+\frac{\Delta V}{R} \\
& I=[C L] \frac{d \Delta V / L}{d t}+\frac{\Delta V / L}{R / L} \\
& E=-\frac{\Delta V}{L} \quad C^{+}=C L \quad R^{*}=\frac{R}{L} \\
& I_{1}=i \omega C^{+} E_{1}-\frac{E_{1}}{R^{*}} \\
& \Rightarrow E_{1}=\frac{-R^{*}}{1-i \omega C^{+} R^{*}} I_{1} \quad Z^{*} \equiv-\frac{E_{1}}{I_{1}}=\frac{R^{*}}{1-i \omega C^{+} R^{*}}
\end{aligned}
$$

RETURNING TO THE ID FLUID EQUATIONS

$$
\frac{\partial \lambda}{\partial t}+\frac{\partial}{\partial z} \lambda v=0 \quad \frac{\partial u}{\partial t}+u \frac{\partial u}{\partial z}=\frac{-q g}{4 \pi \varepsilon_{0} m} \frac{\partial \lambda}{\partial z}+\frac{q \varepsilon_{z}}{m}
$$

Let $\lambda=\lambda_{0}+\lambda_{1} \exp [i(k z-\omega t)]$

$$
u=v_{0}+u_{1} \exp [i(k-\omega t)]
$$

229 


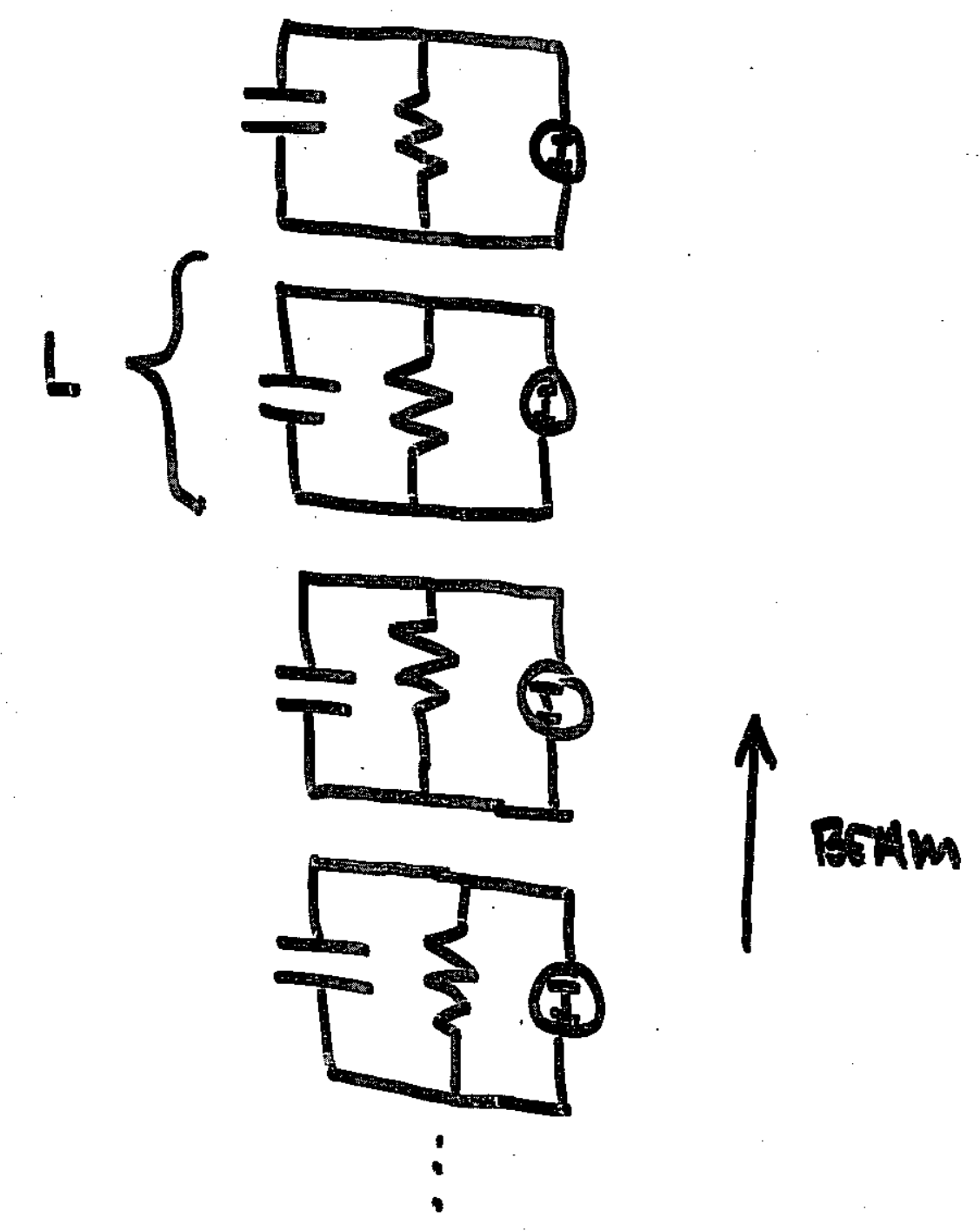

Continuous GIMIT:

$$
\begin{aligned}
& R^{*}=R / L \text { Rasutance jevo wint Rength } \\
& C^{t}=C L \quad C^{-1} \text { per unist oungth } \\
& E=\frac{\text { av Aurmar clectic }}{l}
\end{aligned}
$$

230 


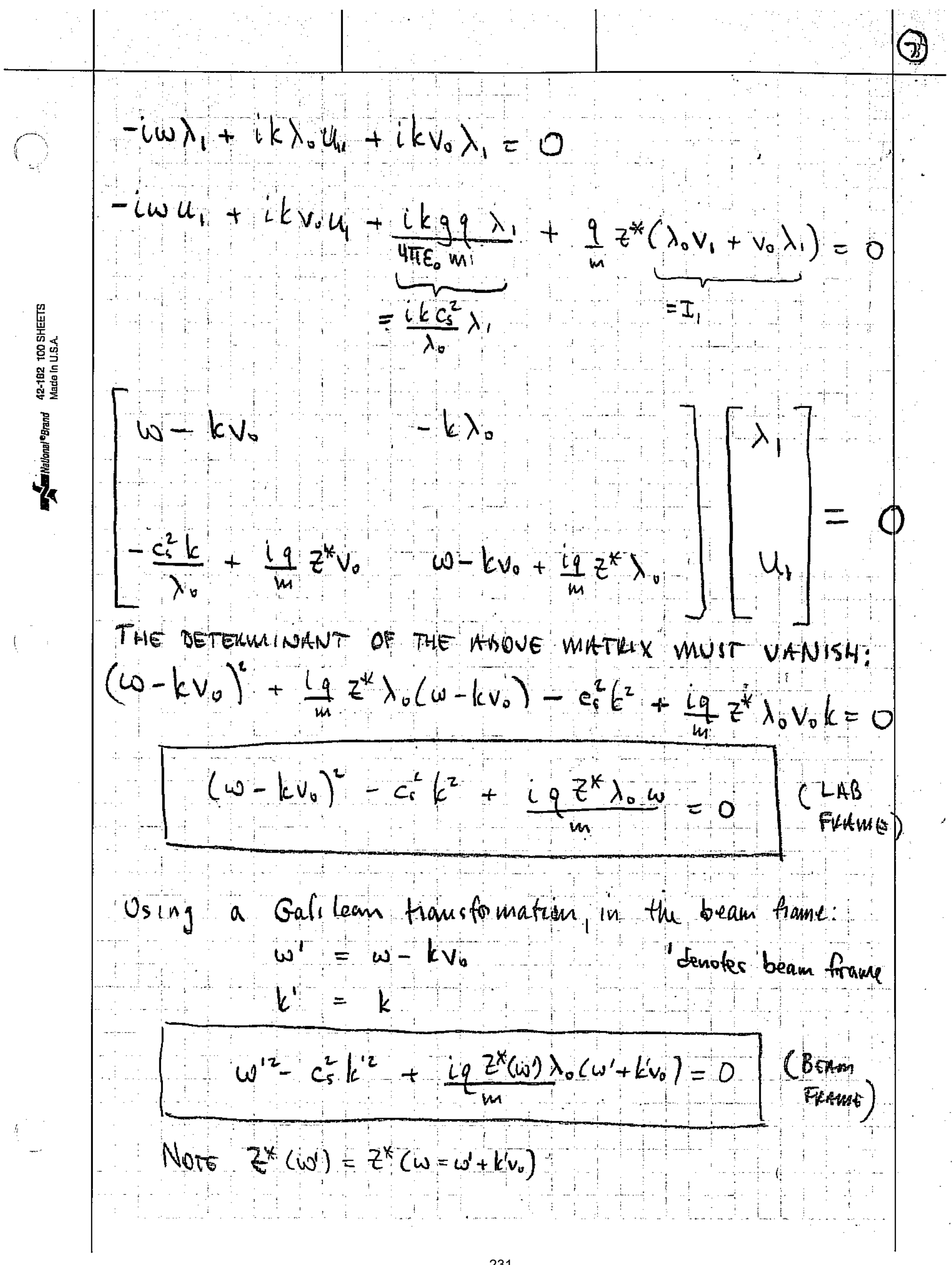

231 


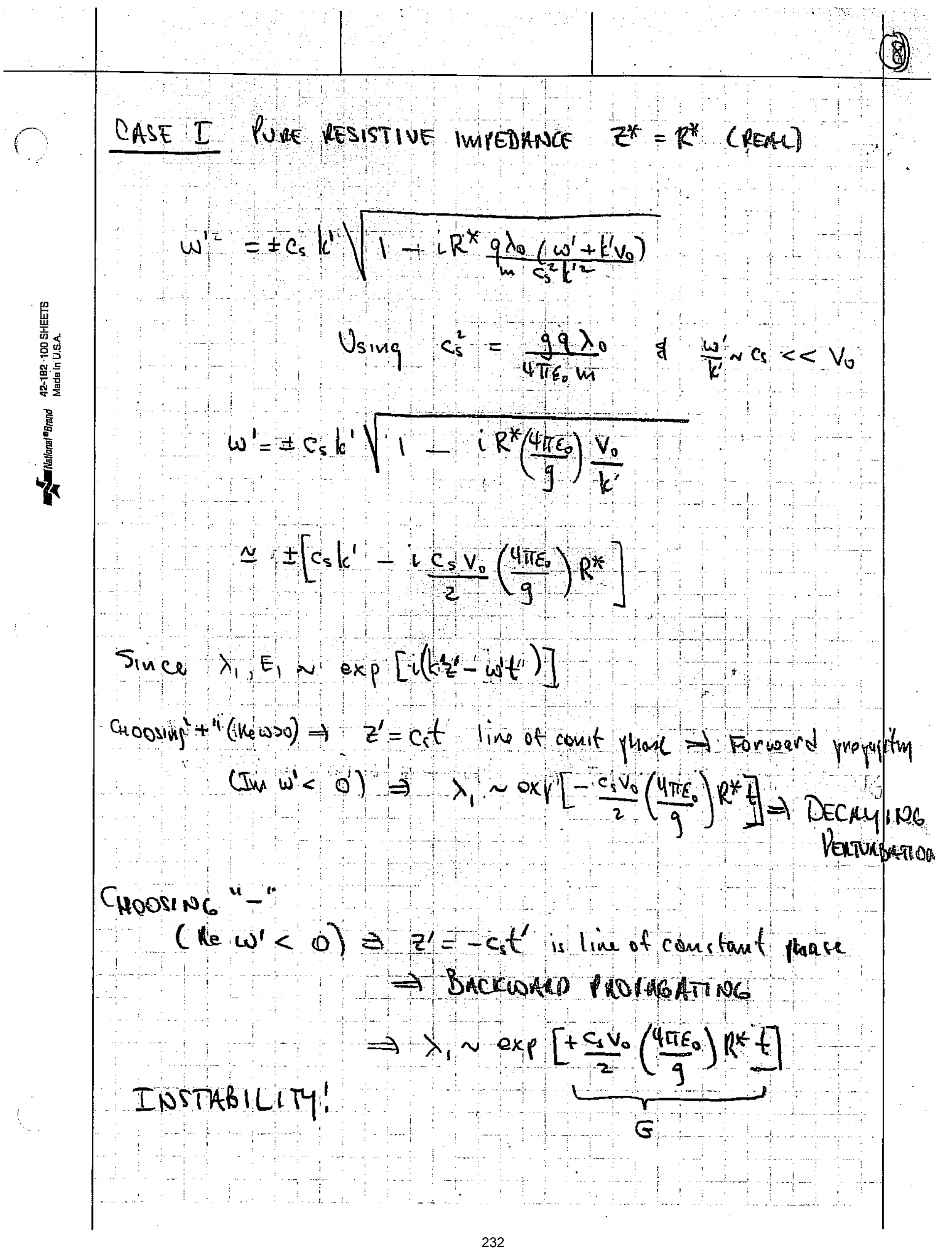




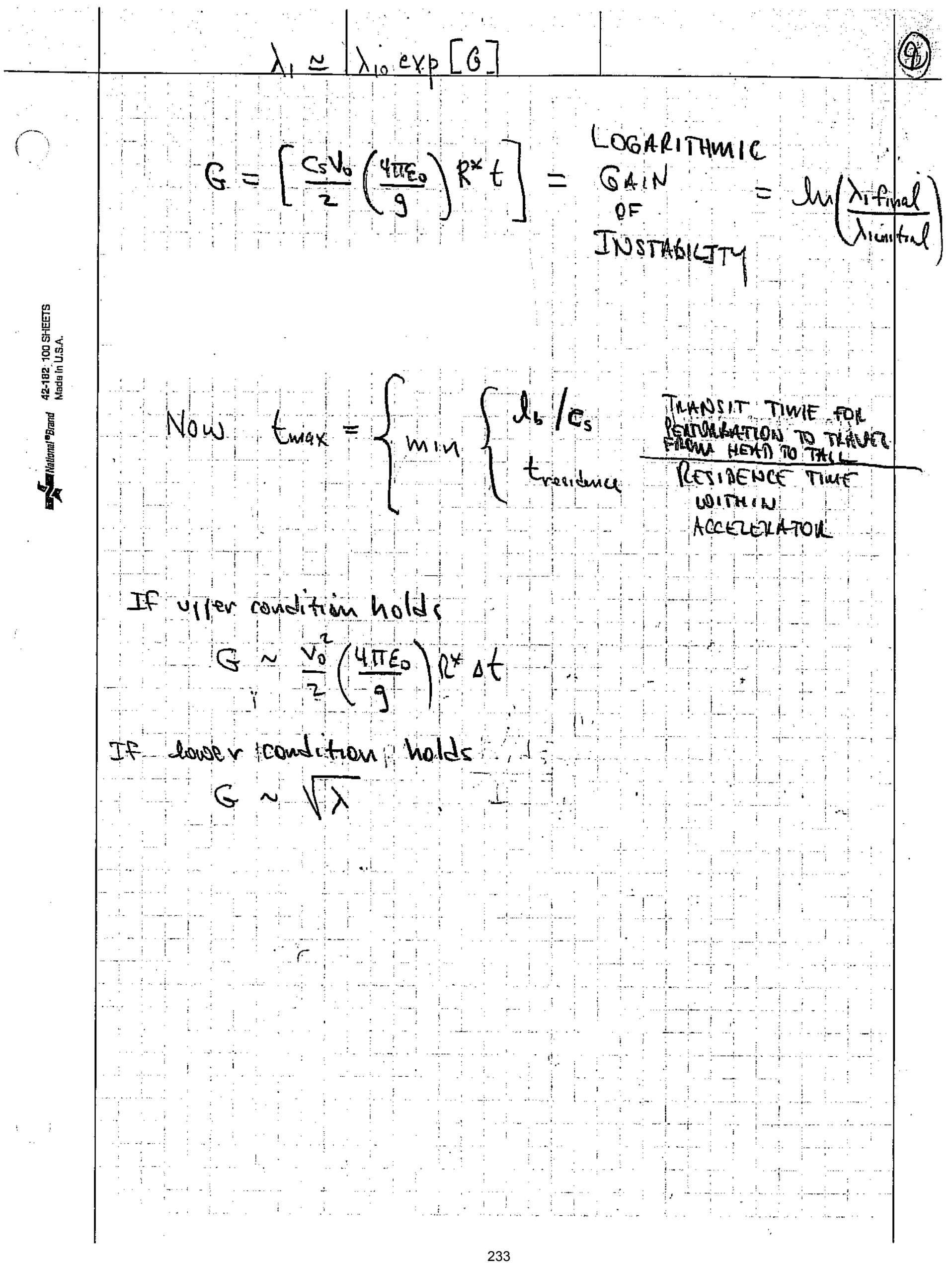




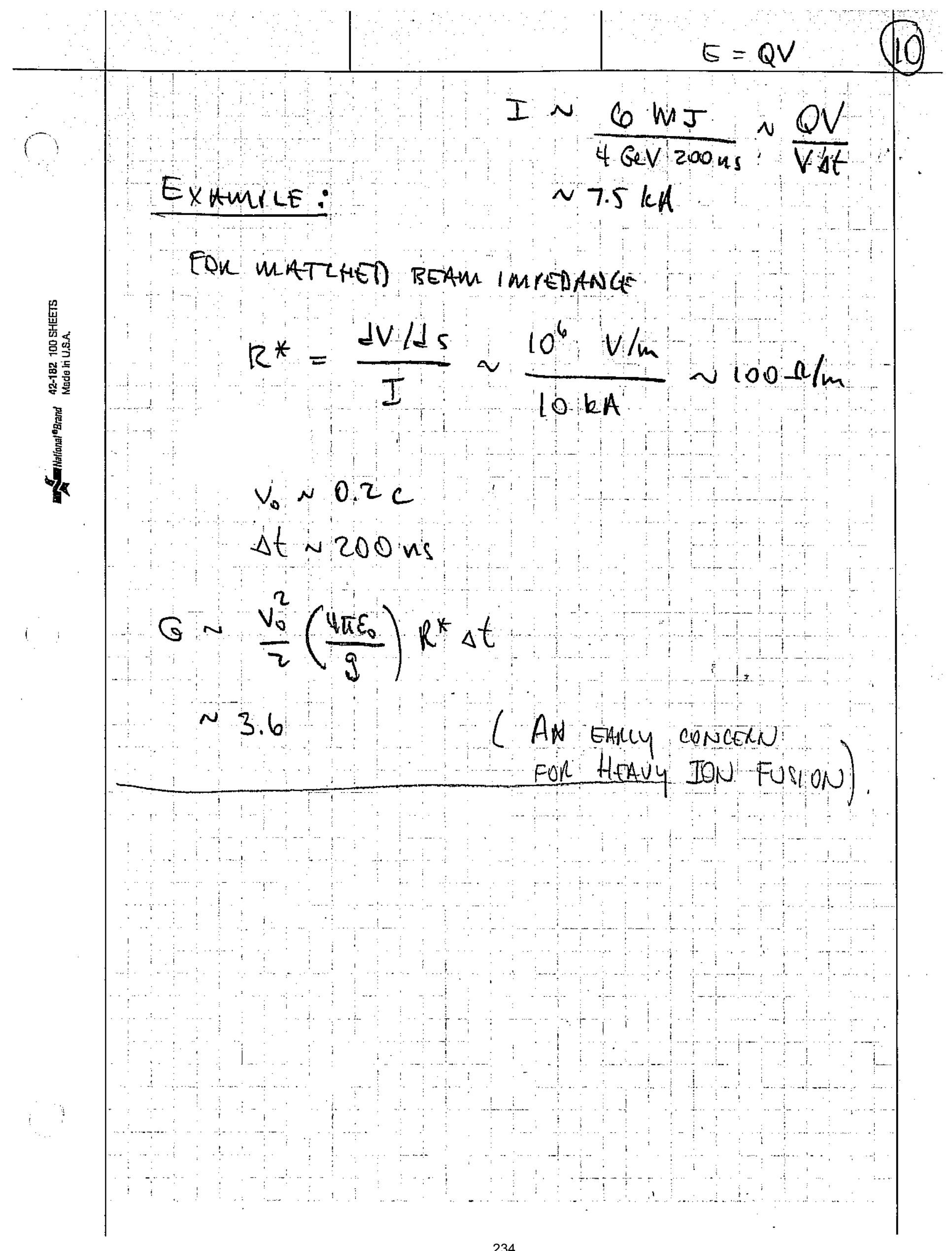




$$
R^{*}=100 \Omega / m
$$

FOR ALL SIMVLARIONS $(p 11-15)$

$V_{0}=c / 3$ $I=3 \mathrm{kA}$ $l_{b}=10 \mathrm{~m}$ $\frac{n_{p}}{r_{p}}=0.4$

a)

Electrostatic Potential on Axis vs $z$
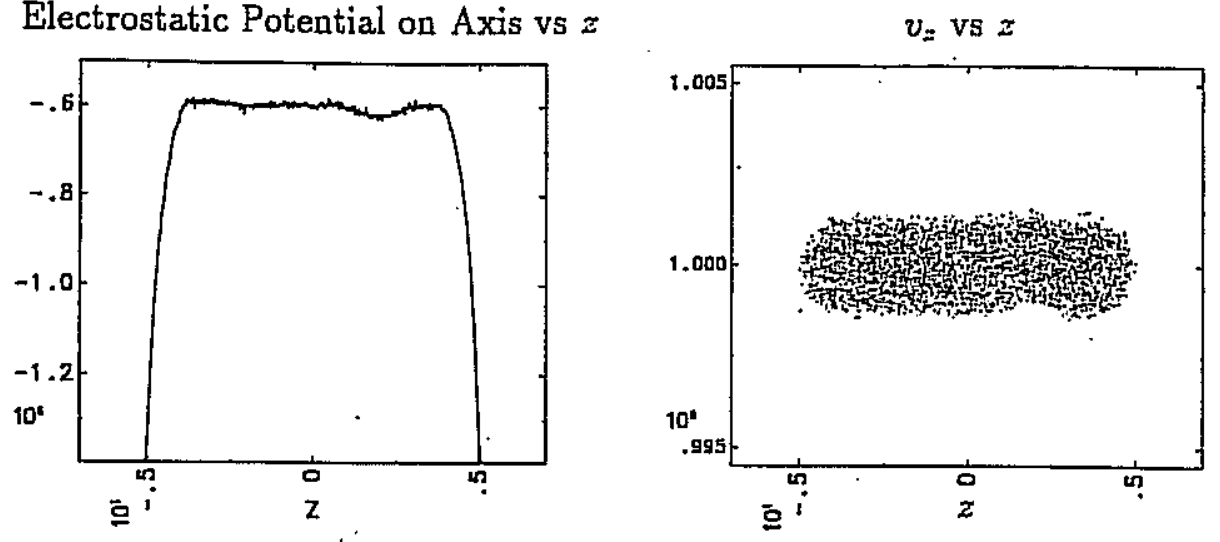

Electrostatic Potential on Axis vs $z$

$k T_{\perp}=k T_{11}=10 \mathrm{kel}$
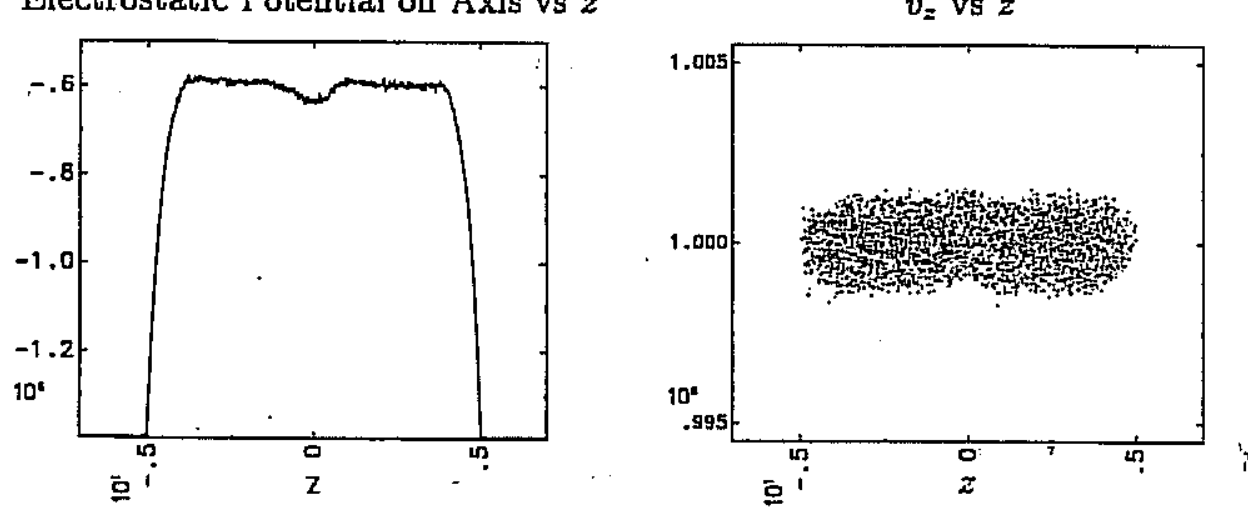

Electrostatic Potential on Axis vs $z$

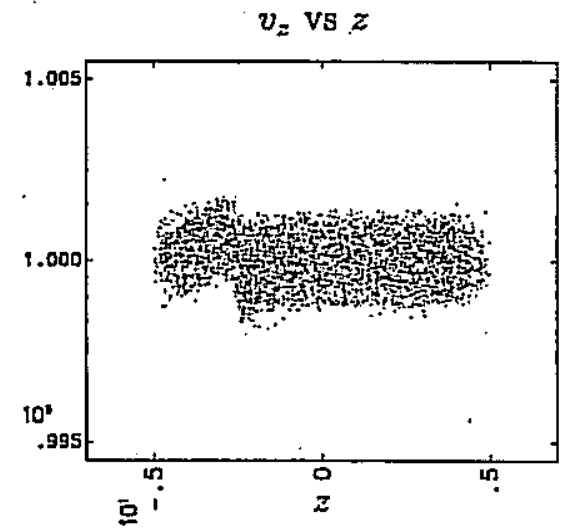

Figure 4.2: A simulation with $100 \Omega / \mathrm{m}$ resistance shows moderate growtl. (a) 6.6 $\mu \mathrm{s}$, (b) $10.9 \mu \mathrm{s}$, (c) $17.5 \mu \mathrm{s}$

frow D.A.Callahan Milles, Ih. D. Theris U.C. Davis, 1994 


$$
R^{*}=100 \Omega / \mathrm{m}
$$

a)

Electrostatic Potential on Axis vs $z$

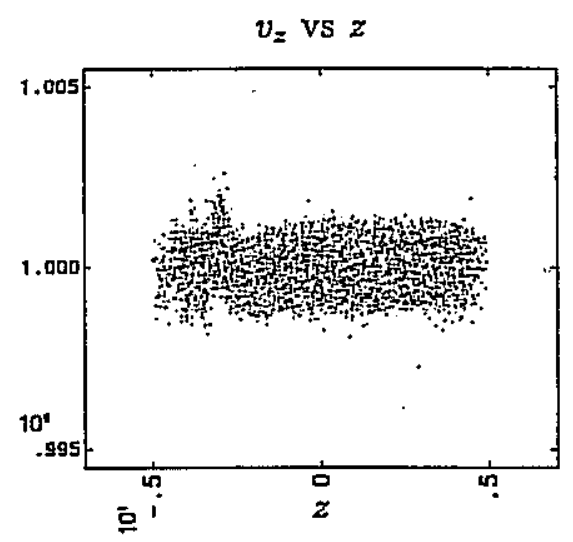

Electrostatic Potential on Axis vs $z$

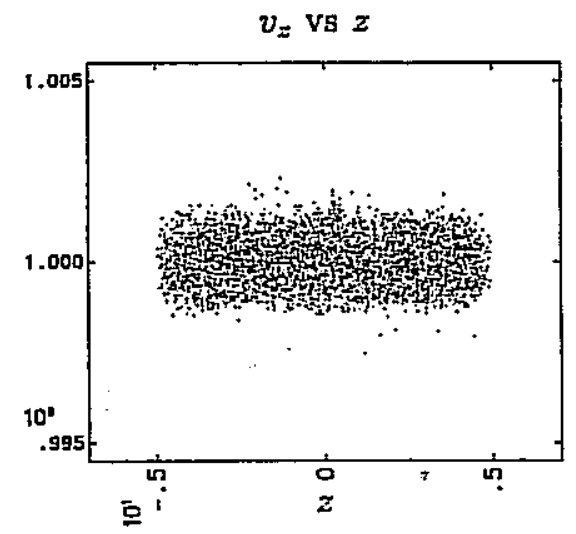

Electrostatic Potential on Axis vs $\mathcal{z}$

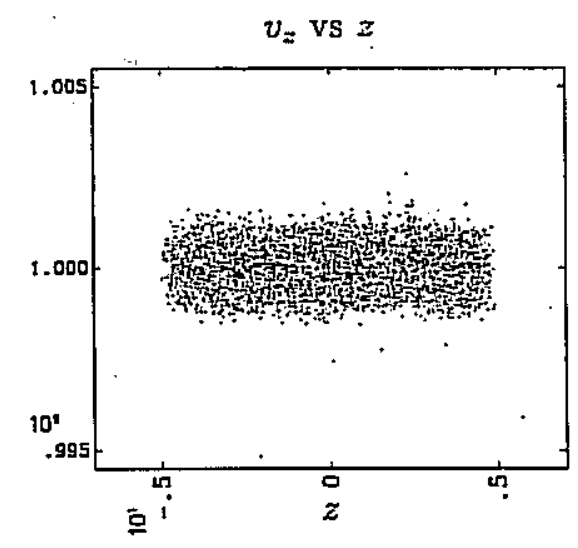

b)

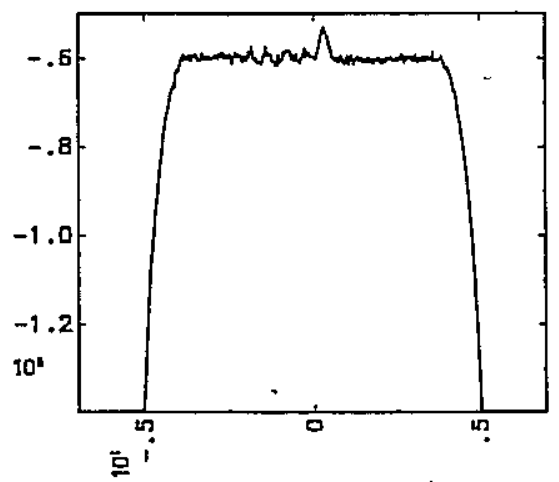

c)

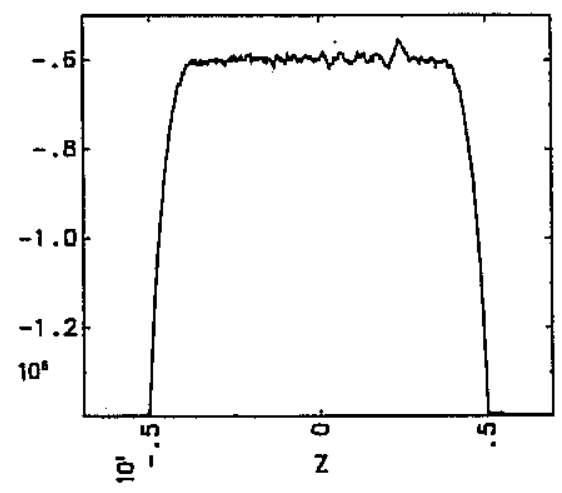

Figure 4.3: The perturbation reflects off the beam end and decays as it travels forward.

(a) $28.4 \mu \mathrm{s}$, (b) $35.0 \mu \mathrm{s}$, (c) $39.4 \mu \mathrm{s}$

$$
\begin{aligned}
& \text { fom D.A. Callaham Milles, Th. D. Thesis } \\
& \text { U.C. Daves, } 1994 \\
& \text { (AOKWHAD WRVE) }
\end{aligned}
$$




$$
R^{*}=200 \Omega / m
$$

a)

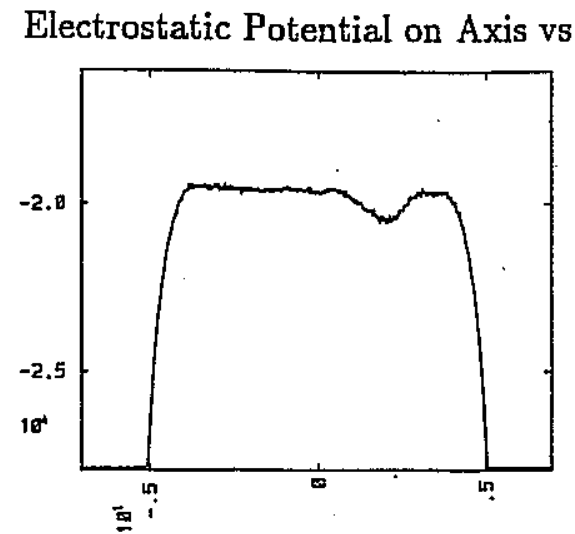

Electrostatic Potential on Axis vs $z$
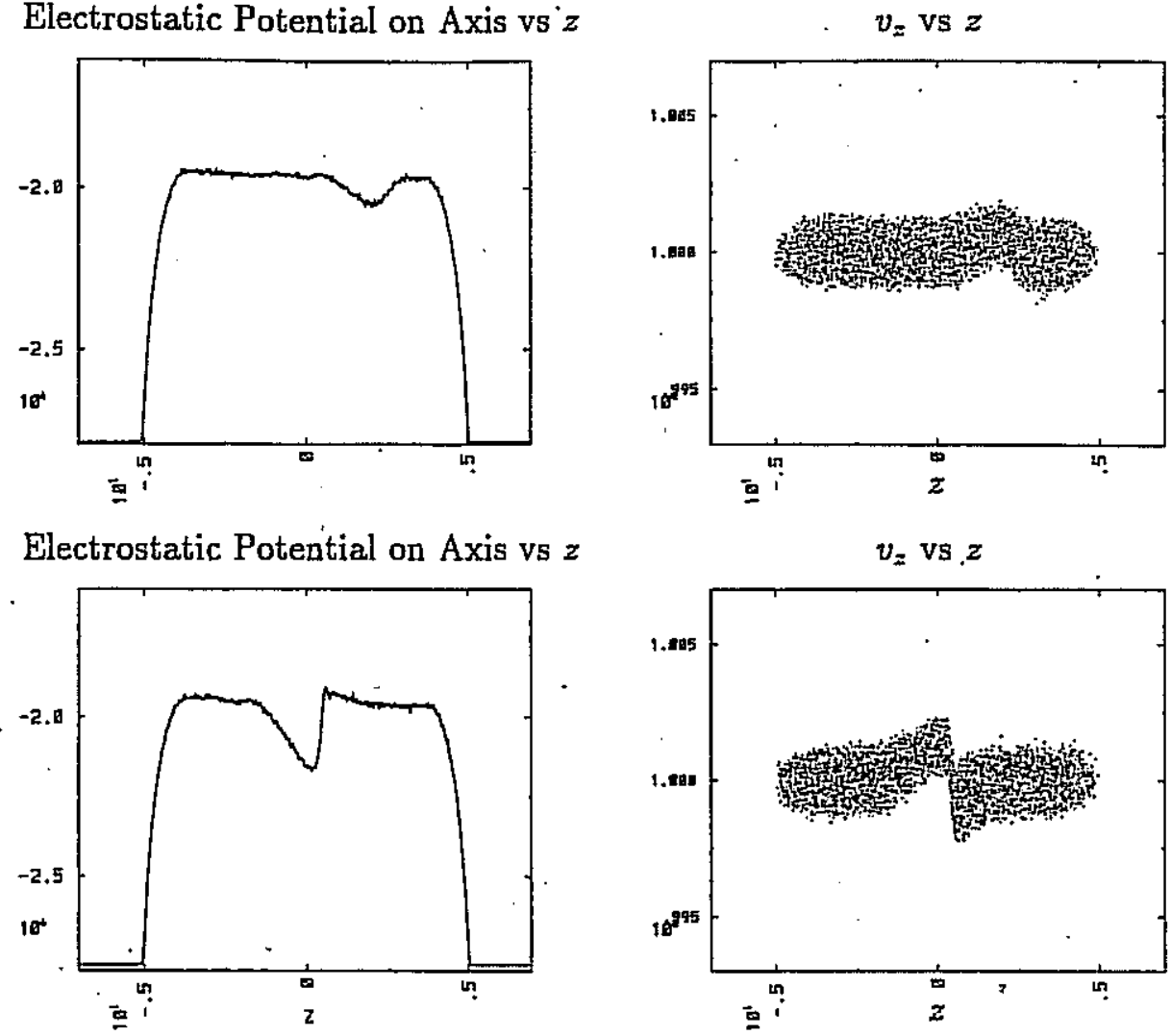

Electrostatic Potential on Axis vs $\boldsymbol{z}$

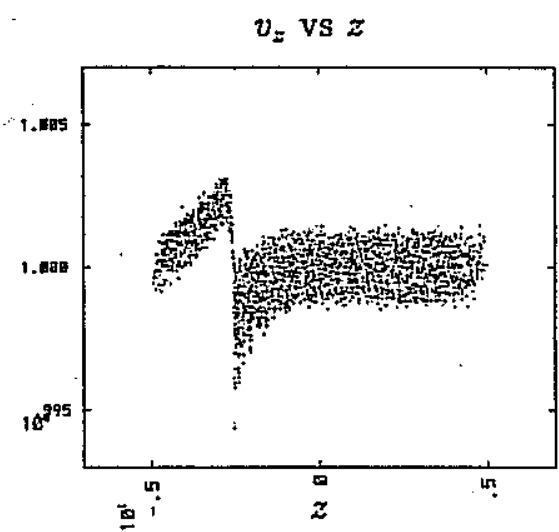

Figure 4.1: A simulation with $200 \Omega / \mathrm{m}$ resistance shows large amounts of growth.

(a) $6.6 \mu \mathrm{s}$, (b) $10.9 \mu \mathrm{s}$, (c) $17.5 \mu \mathrm{s}$

$$
\begin{aligned}
& \text { from D.A.Callaham Miller, Ph. D. Thesiss } \\
& \text { U.C. Davis, } 1994
\end{aligned}
$$




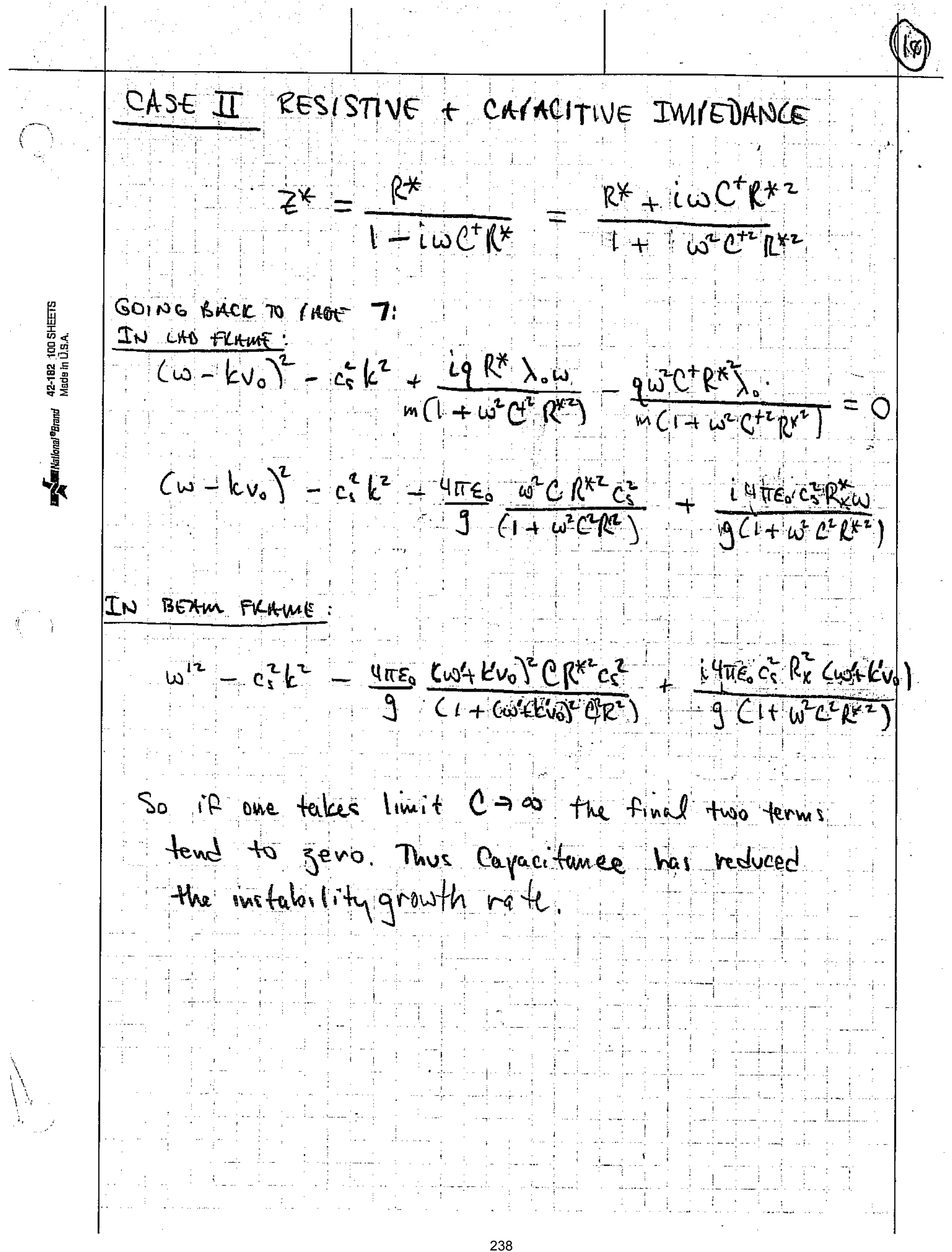




$$
\begin{aligned}
& R^{*} C^{*}=2 \times 10^{-8} \mathrm{~s} \\
& R^{*}=100 \Omega / \mathrm{m}
\end{aligned}
$$

a)

Electrostatic Potential on Axis vs $z$

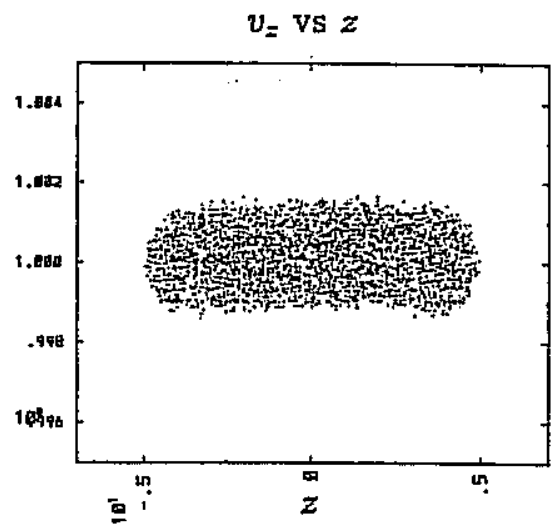

Electrostatic Potential on Axis vs $z$

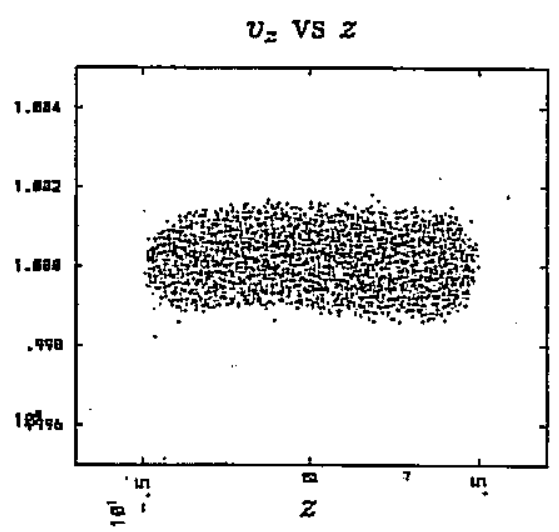

b)

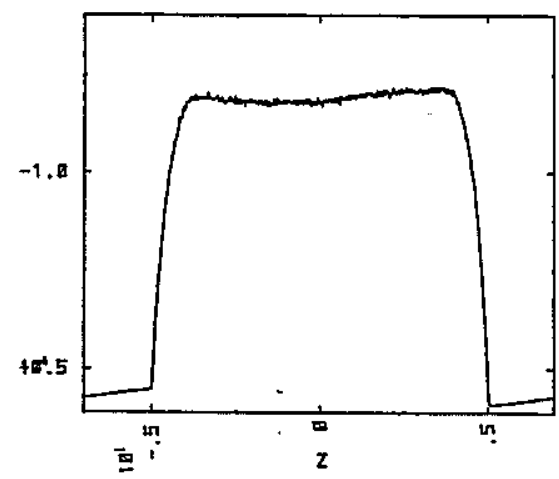

Electrostatic Potential on Axis vs $z$

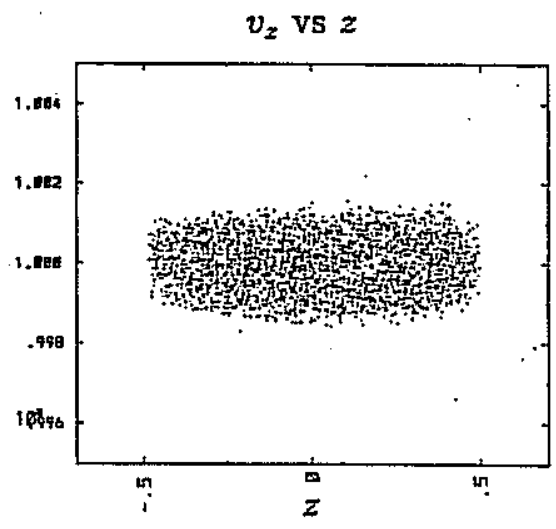

Figure 4.6: When capacitance is added to the system, a larger perturbation is launched, but little growth occurs (a) $6.6 \mu \mathrm{s}$, (b) $10.9 \mu \mathrm{s}$, (c) $17.5 \mu \mathrm{s}$

from D.A.Callahan Miller, Ph. D. Thesis, U.C. Davis, 1994 


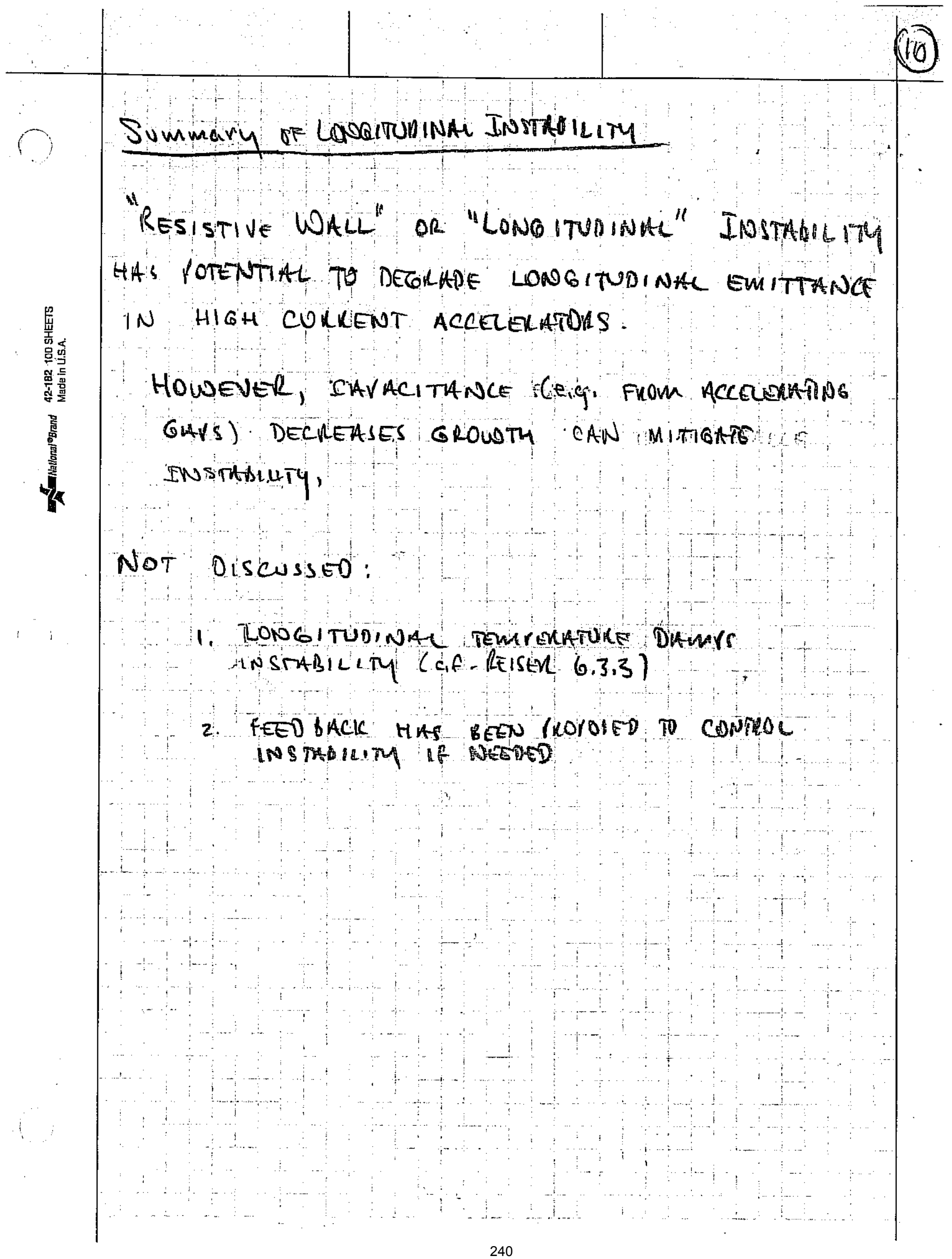


Salnete

(1)

Duft Comrression

Objects:

AIPLY a HEAD-TO-THIL VELOCIMY TLT TO increate curllent by decreasing iulse iuthtion

During compression "eArs" aRE nOt requineo

AT END OF DLIFT COMVREISION, VELOCIMY "TILT" SHOULD BE MINIMIZED, SO THAT CHROMLAC ADERLATONS IN FINAL FOCOS ALE MIUIMIZEO.

241 

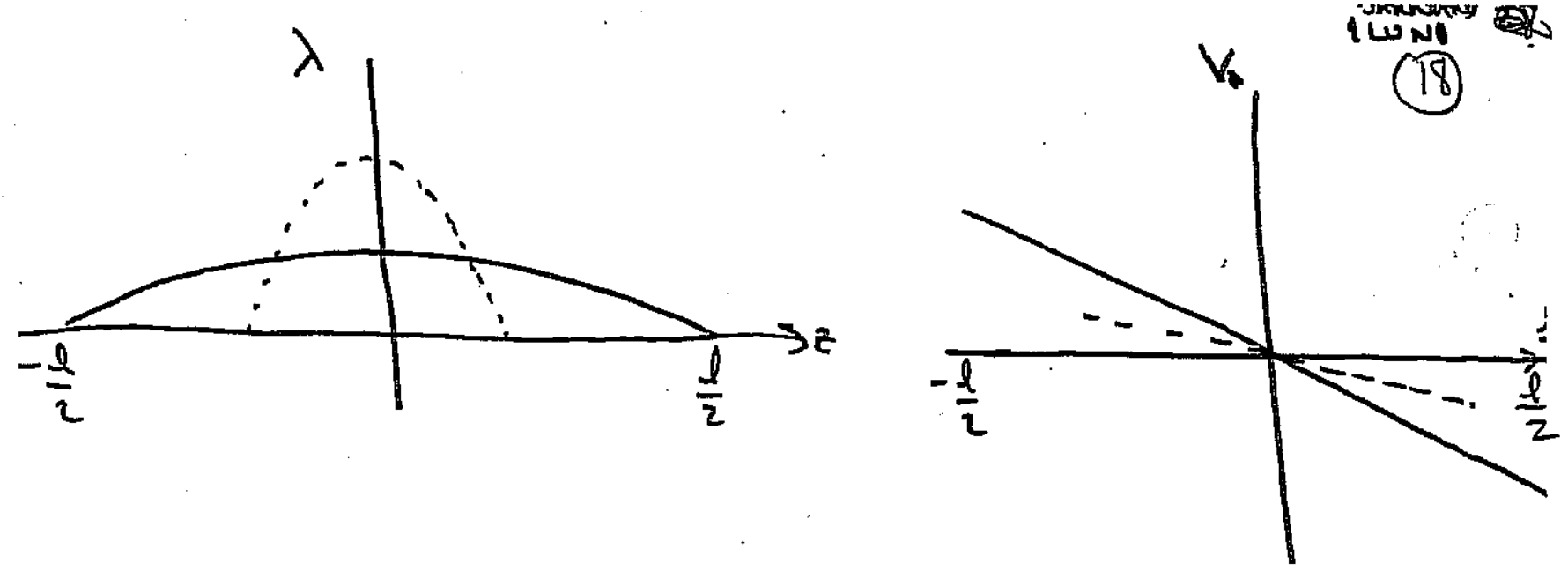

$$
\begin{aligned}
& \frac{\partial \lambda}{\partial t}+\frac{\partial}{\partial z} \lambda v=0 \quad \text { CONTINUITY ERUpTION } \\
& \frac{\partial v}{\partial t}+v \frac{\partial v}{\partial z}=\frac{-q g}{m 4 \pi \epsilon_{0}} \frac{\partial \lambda}{\partial z} \quad \text { MOMENTUM EquATiON } \\
& \text { Let } \lambda=\lambda_{0}(t)\left(1-\frac{4 z^{2}}{l^{2}(t)}\right) \Leftarrow \text { ParabOLIC LINE } \\
& V=-\Delta V(t) \frac{z}{l(t) \quad \leftarrow \text { LINEAR VELOCITy }}
\end{aligned}
$$

(1) Mass conservation:

$$
Q_{c}=\int_{-l / 2}^{l / 2} \lambda d z=\lambda_{0} \int_{-\frac{l}{2}}^{l / 2}\left(1-\frac{4 E^{2}}{l^{2}}\right) d z=\frac{2}{3} \lambda_{0} l=\begin{aligned}
& \text { constant } \\
& \text { (but } \\
& \lambda_{0}=\lambda_{0}(t) \\
& l=l(t))
\end{aligned}
$$

242 
calculating Partial Delivativet:

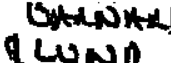

$$
\begin{aligned}
& \frac{\partial \lambda}{\partial t}=\dot{\lambda}_{0}\left(1-\frac{4 z^{2}}{l^{2}}\right)+2 \lambda_{0}\left(\frac{4 z^{2}}{l^{3}}\right) \dot{l} \\
& \frac{\partial \lambda}{\partial z}=\frac{-8 z}{l^{2}} \lambda_{0} \\
& \frac{\partial V}{\partial t}=-\Delta V\left(\frac{z}{l}\right)+\frac{\Delta V z}{l^{2}} l \\
& \frac{\partial V}{\partial z}=-\frac{\Delta V}{l}
\end{aligned}
$$

(19)

$$
\begin{aligned}
& \text { FKon DERINITION OF } \\
& \Delta V \& d: \\
& \Delta Y=-\dot{l}
\end{aligned}
$$

(2) CONTINUITY EquhTION $\Rightarrow\left(1-\frac{4 z^{2}}{l^{2}}\right)\left(\dot{\lambda}_{0}-\frac{\Delta V \lambda_{0}}{\ell}\right)=0$

(3) Momentum EquRTION $\Rightarrow\left(\frac{z}{l}\right)\left[-\Delta \dot{V}+\frac{\dot{l} \Delta V}{l}+\frac{\Delta v^{2}}{l}+\frac{8 q g}{m 4 \pi \epsilon_{0}} \frac{\lambda_{0}}{l}\right]=0$

(1) $1(2) \Rightarrow \frac{\dot{\lambda_{0}}}{\lambda_{0}}=\frac{\Delta v}{l}=\frac{-\dot{l}}{l}$

(3) $\$$ (4)

$$
\Rightarrow \quad \ddot{l}-\frac{1299}{4 \pi \varepsilon_{0} m} \frac{Q_{c}}{l^{2}}=0
$$

where $Q=\frac{2}{3} \lambda_{0} l=$ canit.<smiles>CC(C)N1CCN(C)C1</smiles>
IUNCH CNOT

LONGITUOINAL "ENUELORE" EQUATIN Ponvonute (WITHOUT EMITTHNCE)

243 
DANANELC

MuLtirLY BY $l$ \& INTEGLATE:

(20)

$$
\begin{aligned}
& \frac{l^{2}}{2}+\frac{12 q g}{4 \pi \varepsilon_{0} m} \frac{Q_{L}}{l}=\frac{l_{f}^{2}}{l_{0}^{2}}+\frac{12 q g}{4 \pi \varepsilon_{0} m} \frac{Q_{c}}{l_{f}} \\
& \text { HERE SUBSCKIPT "f" } \\
& \text { = "final" } \\
& \Rightarrow \dot{l}_{0}=\sqrt{\frac{16 q g}{4 \pi \varepsilon_{0} m} \lambda_{f}\left[1-\frac{l_{f}}{J_{0}}\right]} \\
& \text { q susscist. " } 0 \text { " } \\
& \text { = origival a initial }
\end{aligned}
$$

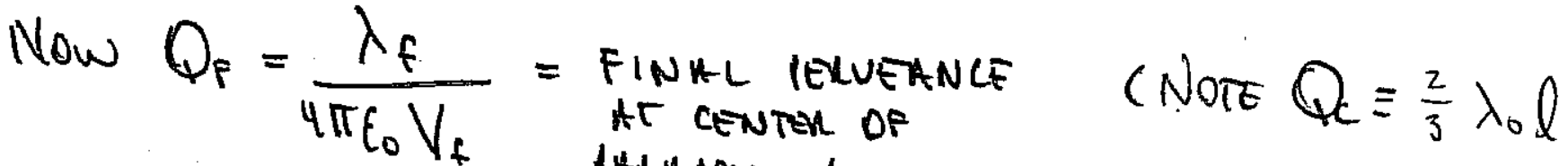

$$
\begin{aligned}
& \text { InathoOUL TULSE } \\
& =\text { CHAKGE }
\end{aligned}
$$

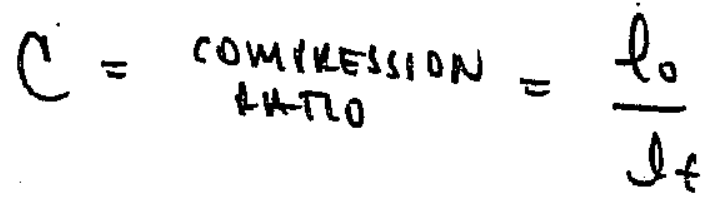

$$
\begin{aligned}
& \text { whereas } Q_{F} \equiv \text { PERVEANCE } \\
& \text { CDMENIIONLESI } \\
& \frac{\Delta v}{v_{0}}=\underset{\text { velocity }}{t_{1} \mid t}=\frac{|i|}{v_{0}} \\
& \rightarrow \quad \frac{\Delta v}{v}=\sqrt{8 g Q_{f}\left[1-\frac{1}{C}\right]} \\
& \text { for } Q_{f}=10^{-4} \\
& g=1.1 \\
& c=20 \\
& \Rightarrow \frac{\Delta v}{v}=0.029 \\
& \text { DUFT LENGTH } \cong \frac{l}{A V} V_{0}=\frac{l}{A V / v}=345 \mathrm{~m} \text { for } l=10 \mathrm{~m}
\end{aligned}
$$

244 
Vlasov-equation for a drifting beam:

$\frac{\partial f}{\partial s}+x^{\prime} \frac{\partial f}{\partial x}+x^{\prime \prime} \frac{\partial f}{\partial x^{\prime}}+y^{\prime \prime} \frac{\partial f}{\partial y}+y^{\prime \prime} \frac{\partial f}{\partial y^{\prime}}+z^{\prime} \frac{\partial f}{\partial t}+z^{\prime \prime} \frac{\partial f}{\partial z^{\prime}}=0$

Let $\tilde{f}^{\prime}\left(x^{\prime}, x^{\prime}, s\right) \equiv \iiint \int f d x d x^{\prime} d y d y^{\prime}$

integrating. Vlatou equation:

If $z^{\prime \prime} \neq f\left(x, x^{\prime}, y, y^{\prime}\right)$ :

$\Rightarrow \frac{\partial \tilde{f}}{\partial s}+\iiint \int_{=f \int_{-\infty}} \frac{\partial f}{\partial x} d x d x^{\prime} d y d y^{\prime}+\cdots z^{\prime} \frac{\partial \tilde{f}}{\partial z}+z^{\prime \prime} \frac{\partial \tilde{f}}{\partial z^{\prime}}=0$

$\Rightarrow \quad \frac{\partial \tilde{f}}{\partial s}+z^{\prime} \frac{\partial \tilde{f}}{\partial z}+z^{\prime \prime} \frac{\partial \tilde{f}}{\partial z^{\prime}}=0$ i D Vlasov

Now lit $\lambda \equiv q \int \tilde{f} d z^{\prime} ; \lambda \overline{z^{\prime}}=\int \tilde{f} z^{1} d z^{\prime} ; \lambda \overline{z^{\prime z}} \Xi \int \tilde{f} z^{\prime 2} d z^{\prime}$

Fluid Equations Also, let $\Delta z^{12} \equiv \overline{z^{12}}-\left(\overline{z^{\prime}}\right)^{2}$

Integating 'ID Vlasov over $z$ ':

$\frac{\partial \lambda}{\partial s}+\frac{\partial}{\partial z}\left(\lambda \bar{z}^{\prime}\right)=0$ (CONTINUITY EQUATION)

MULTILYING BY $Z^{\prime}$ INEGGRTNG VLAIN OL $z^{\prime}:$

$\frac{\partial}{\partial s} \lambda \bar{z}^{\prime}+\frac{\partial}{\partial z} \lambda \overline{z^{\prime 2}}-\lambda z^{\prime \prime}=0$

Dividing Bi $\lambda$, using continuitry equation \& definiton a $\Delta z^{12}$;

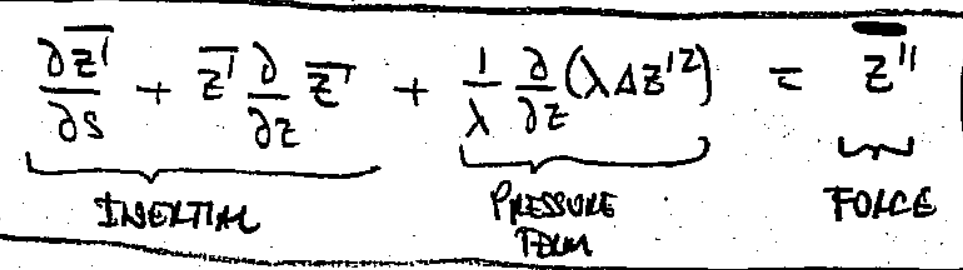

(MOMENTUM EQUnTION) 


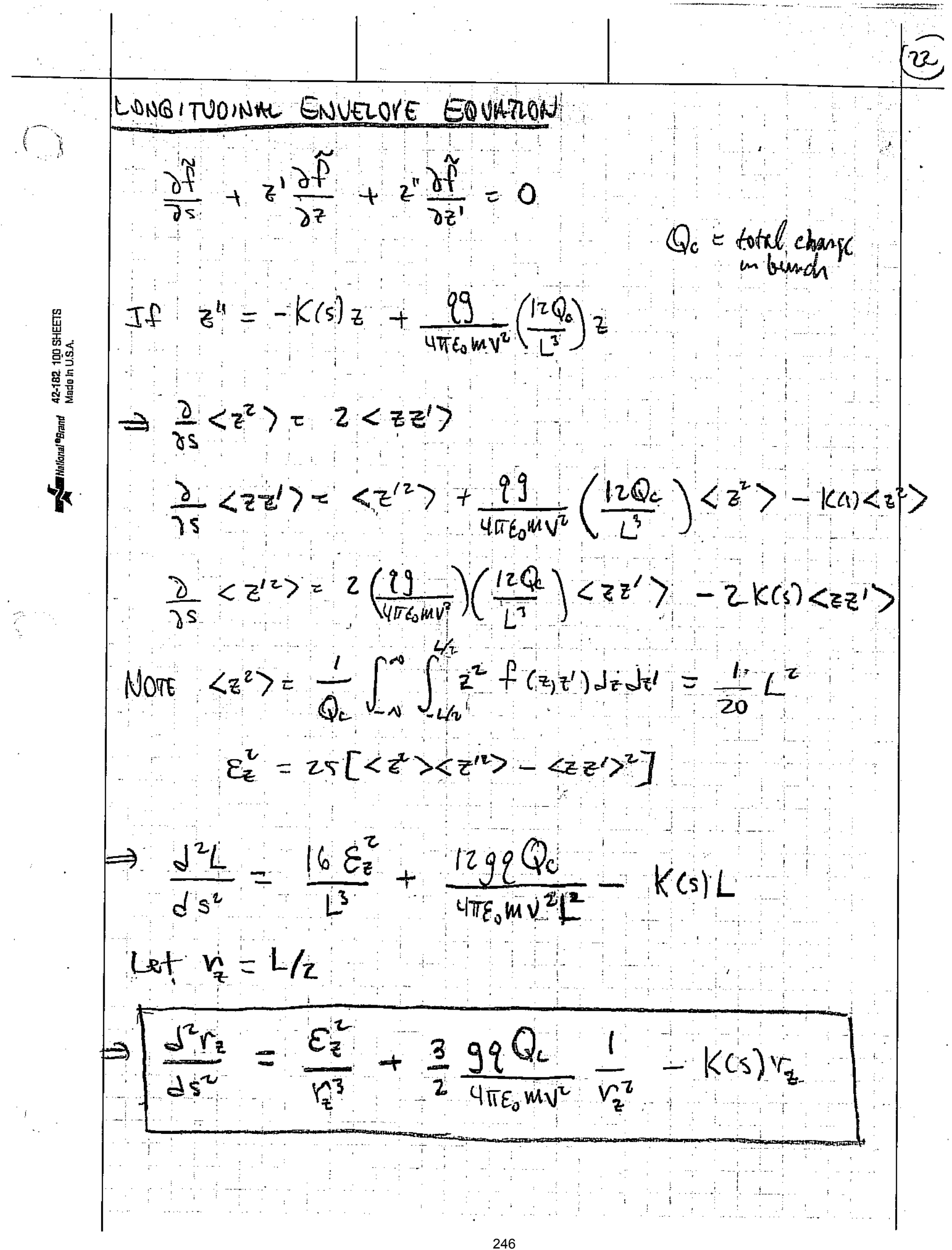




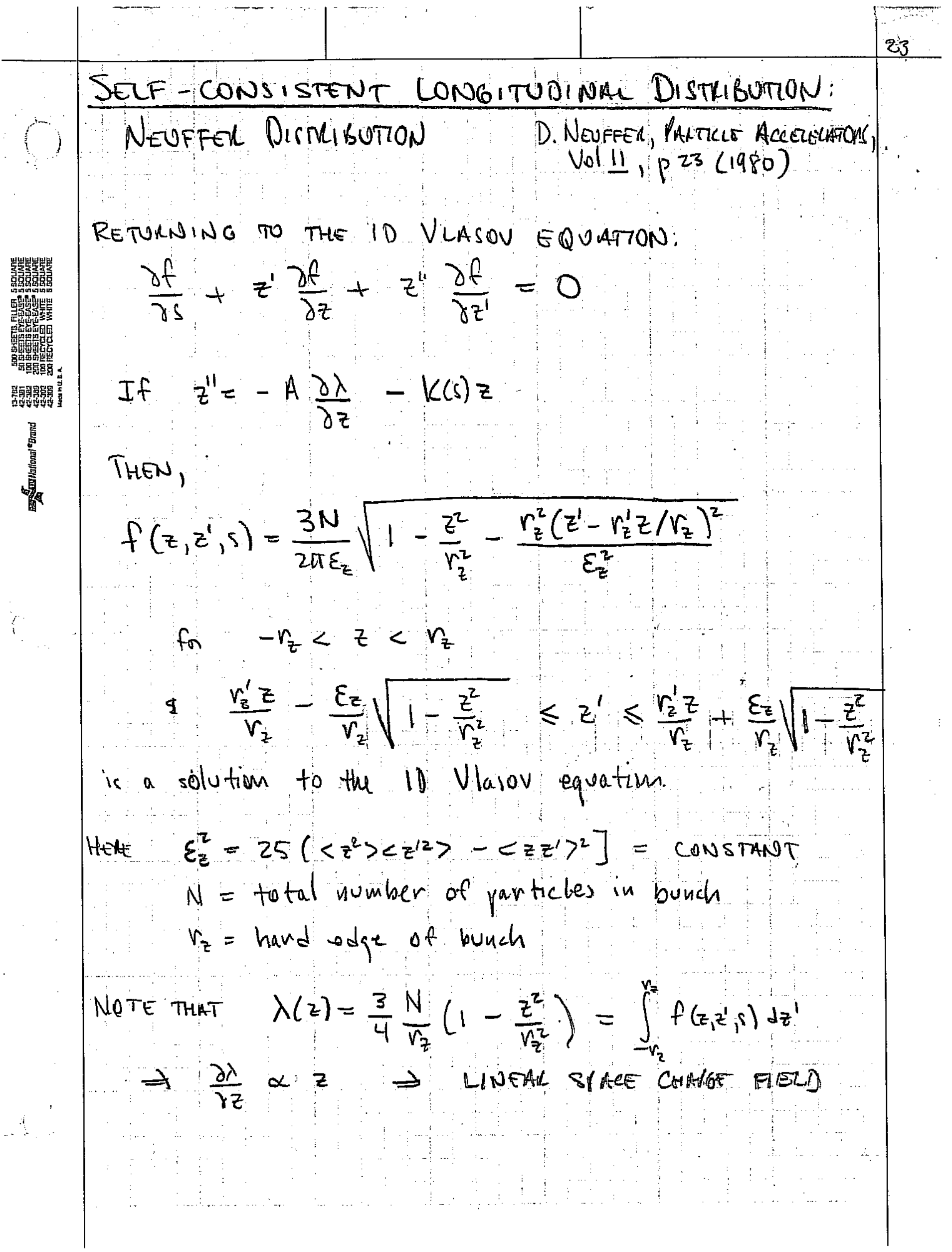

247 
(24)

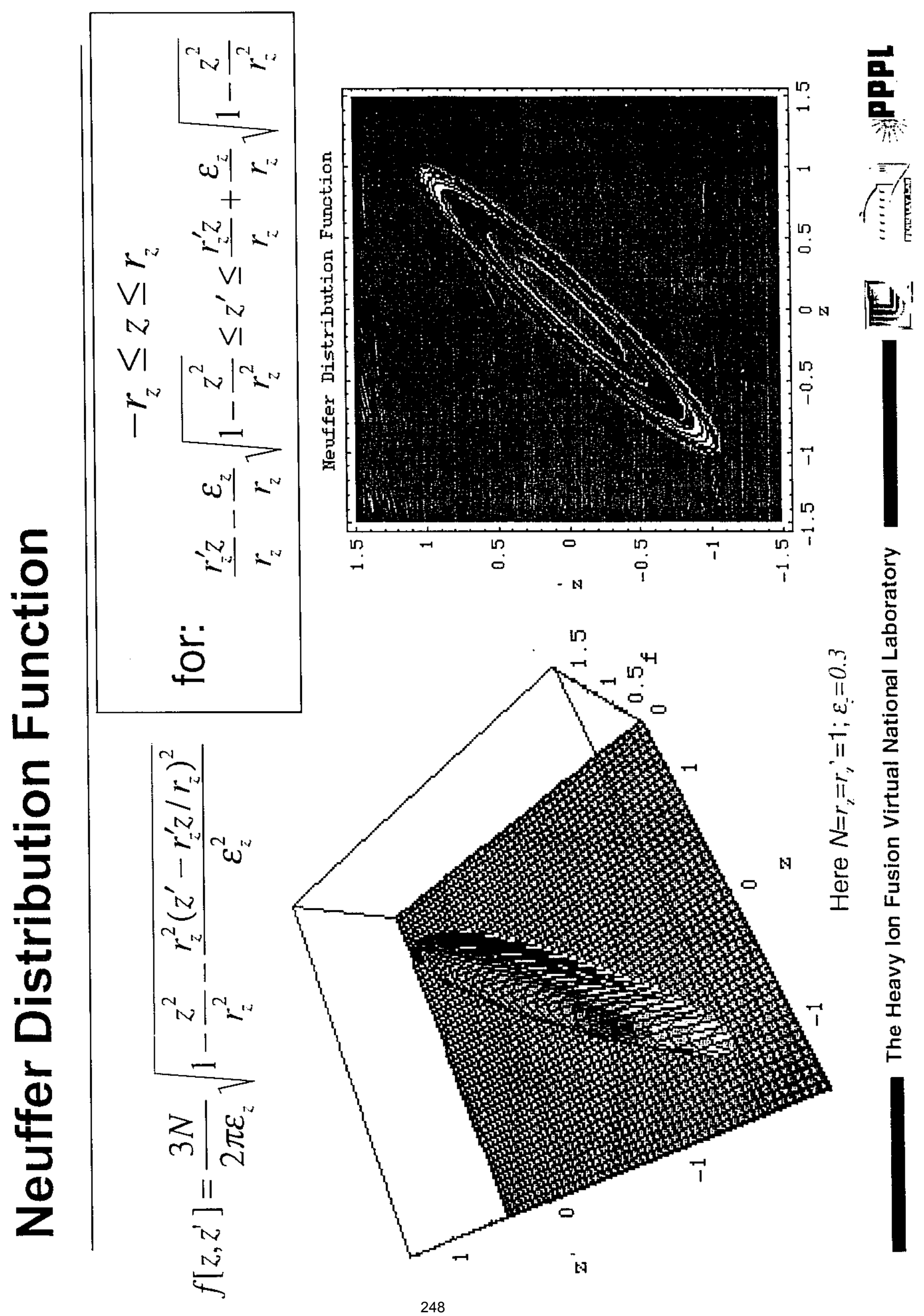


逢

(25)

NOTE:

- Distribution function has elliptical boundary IN $Z$-Z' PHASE SPACE

- II $\varepsilon_{z}$ is area of ellipse and $k$ constant

- Analogous to K-V distribution WITH LINEAR SHALE CHARGE FORCE AND SECOND ORDER ENVELOPE EQUATION TO DESCRIBE THE MOTION DE THE DISBMIBUTTON:

$$
\frac{d^{2} r_{z}}{d s^{2}}=\frac{\varepsilon_{z}^{2}}{r_{z}^{3}}+\frac{3}{2} \frac{A N}{r_{t}^{2}}-K(s) r_{z}
$$

Note also that neuffer function can be USED FOR BUNCHED BEAMS IN WHICH $E_{z} \propto i$, AS IN A UNIFORM DENSITY ELLIPSOID.

249 


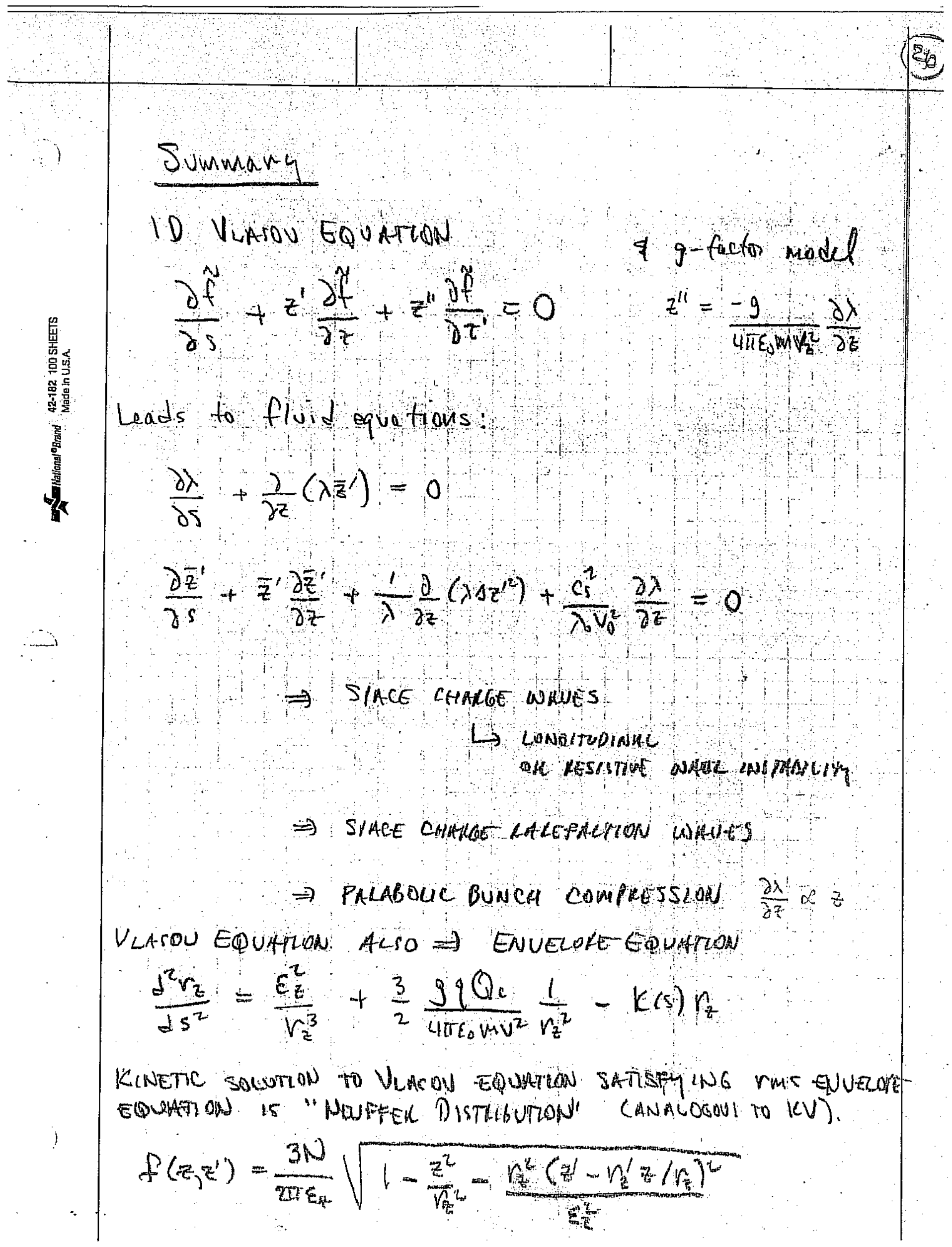



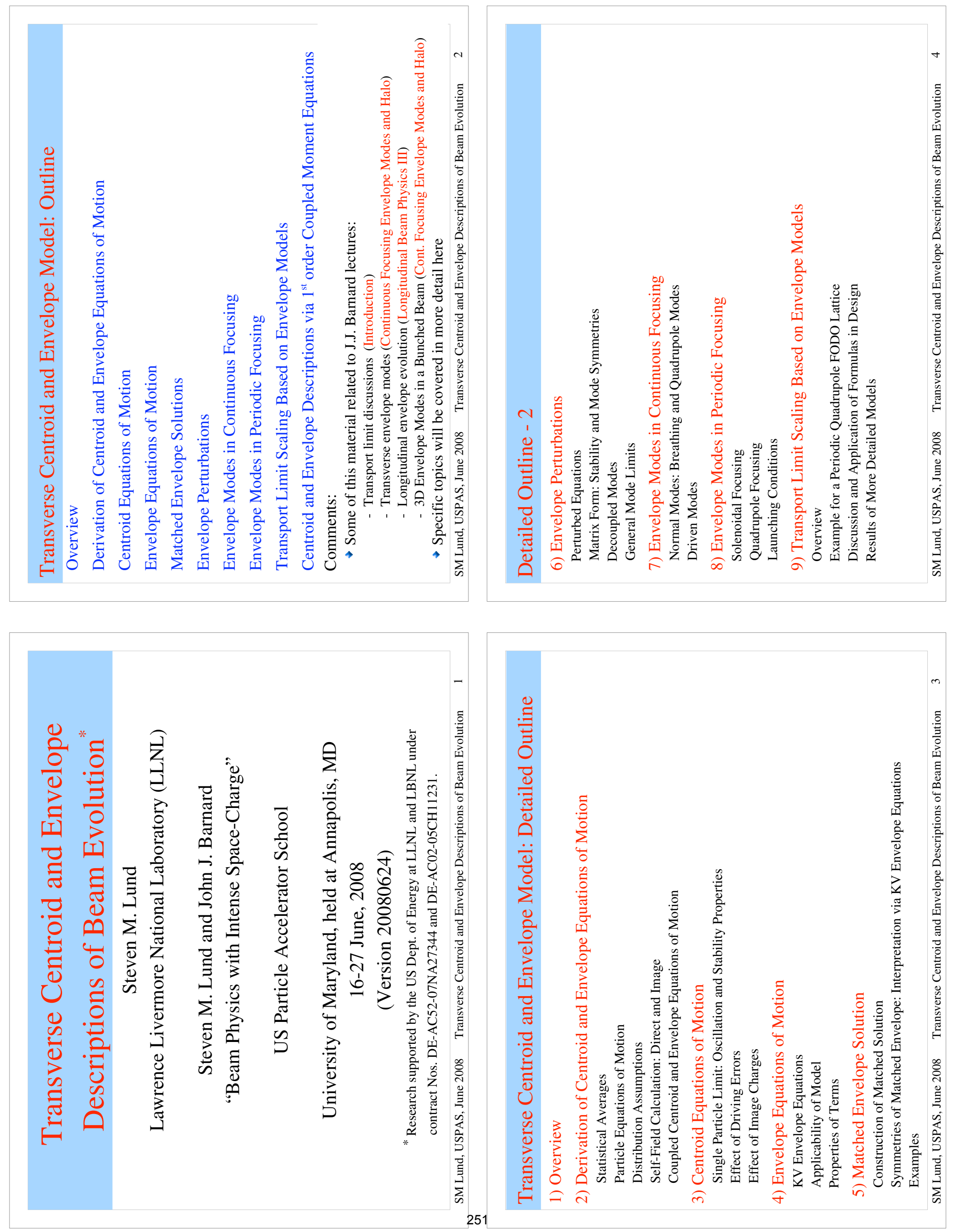

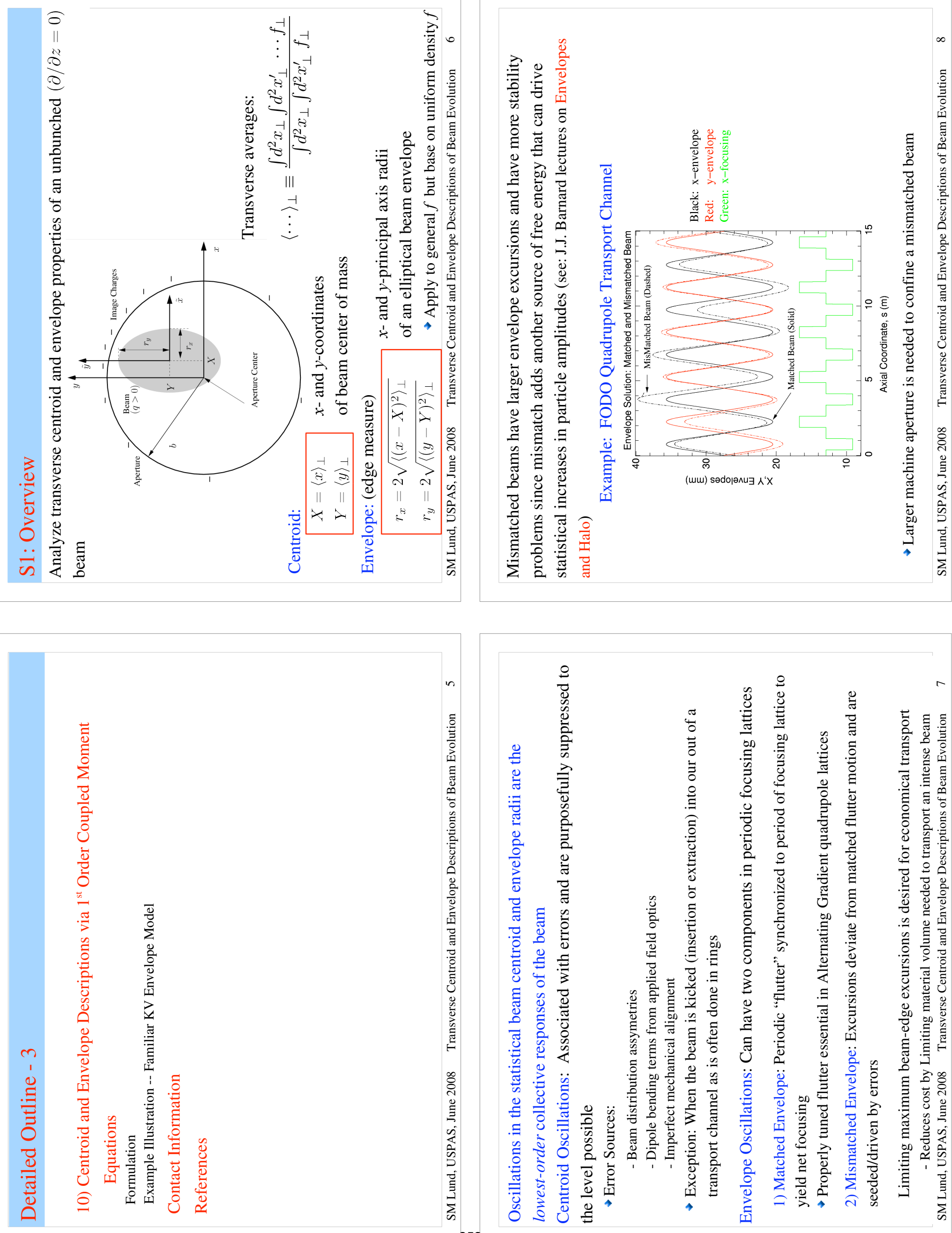

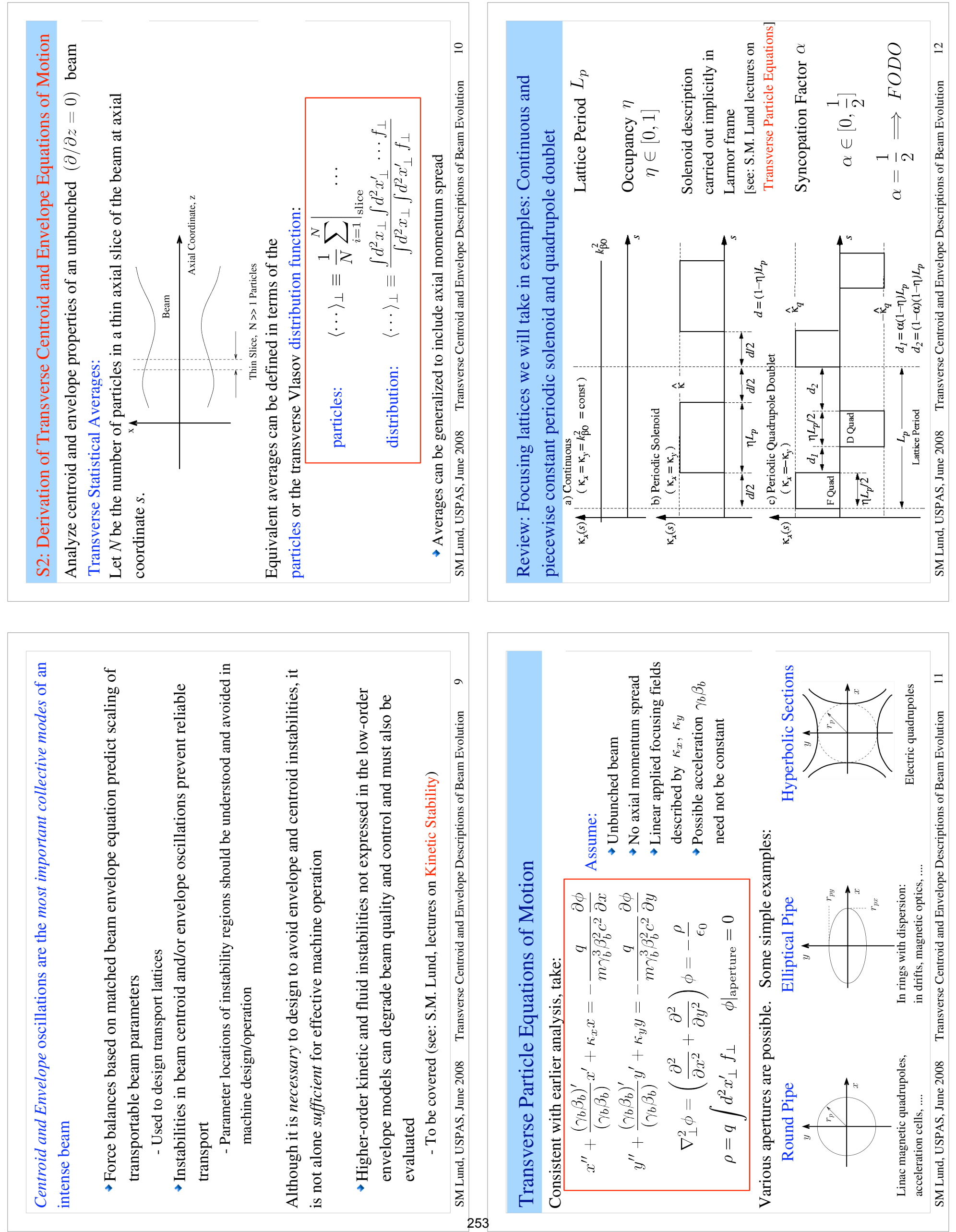

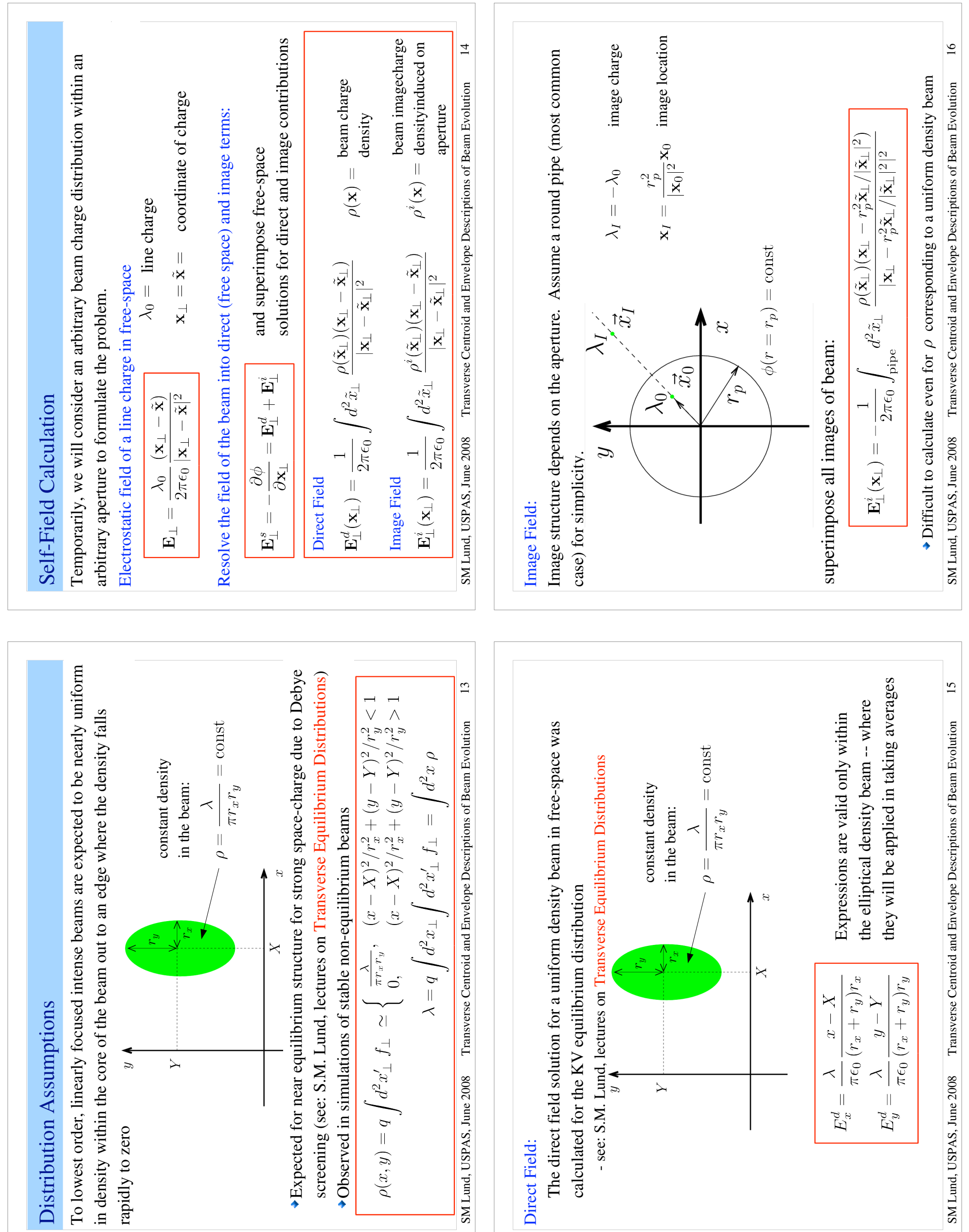

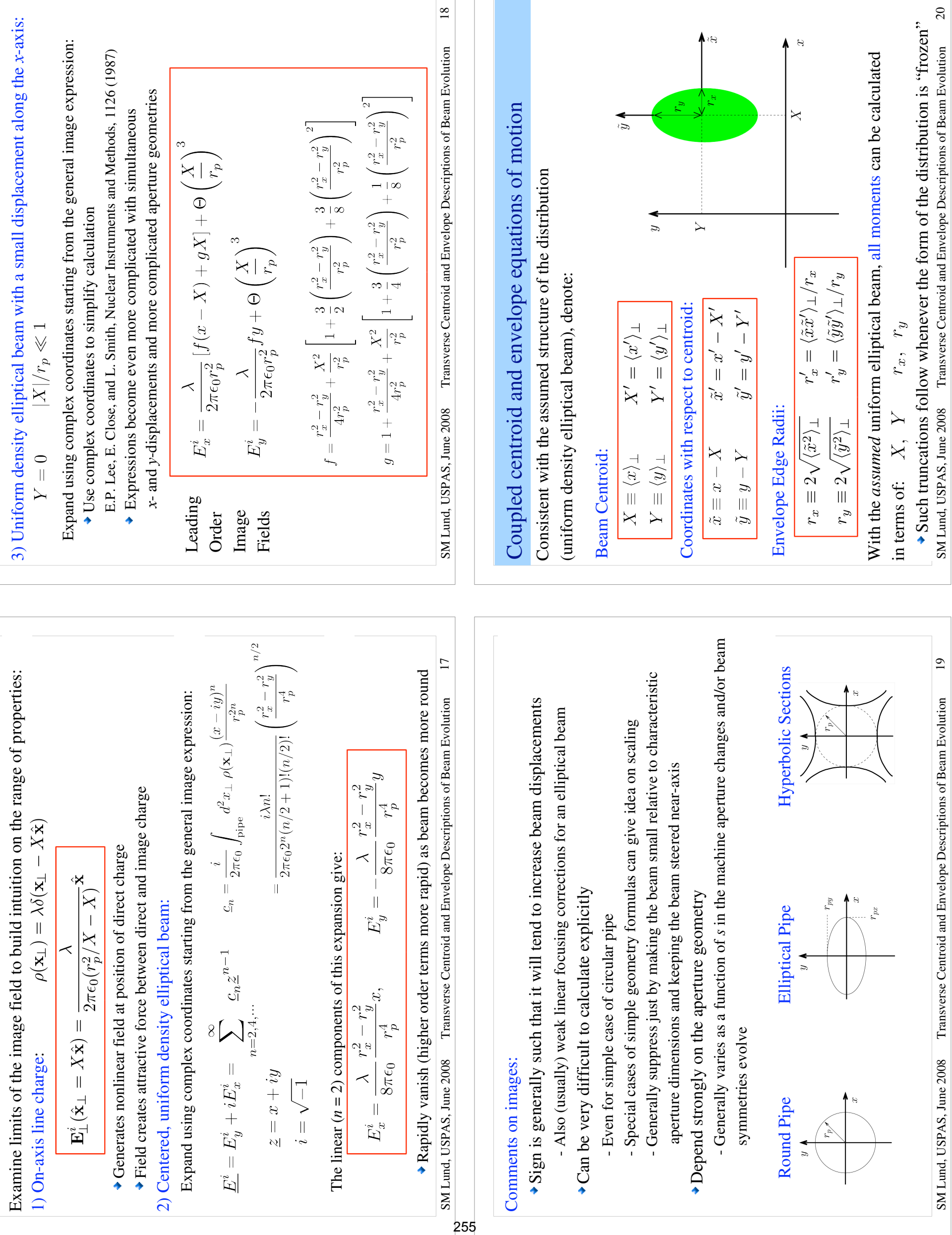

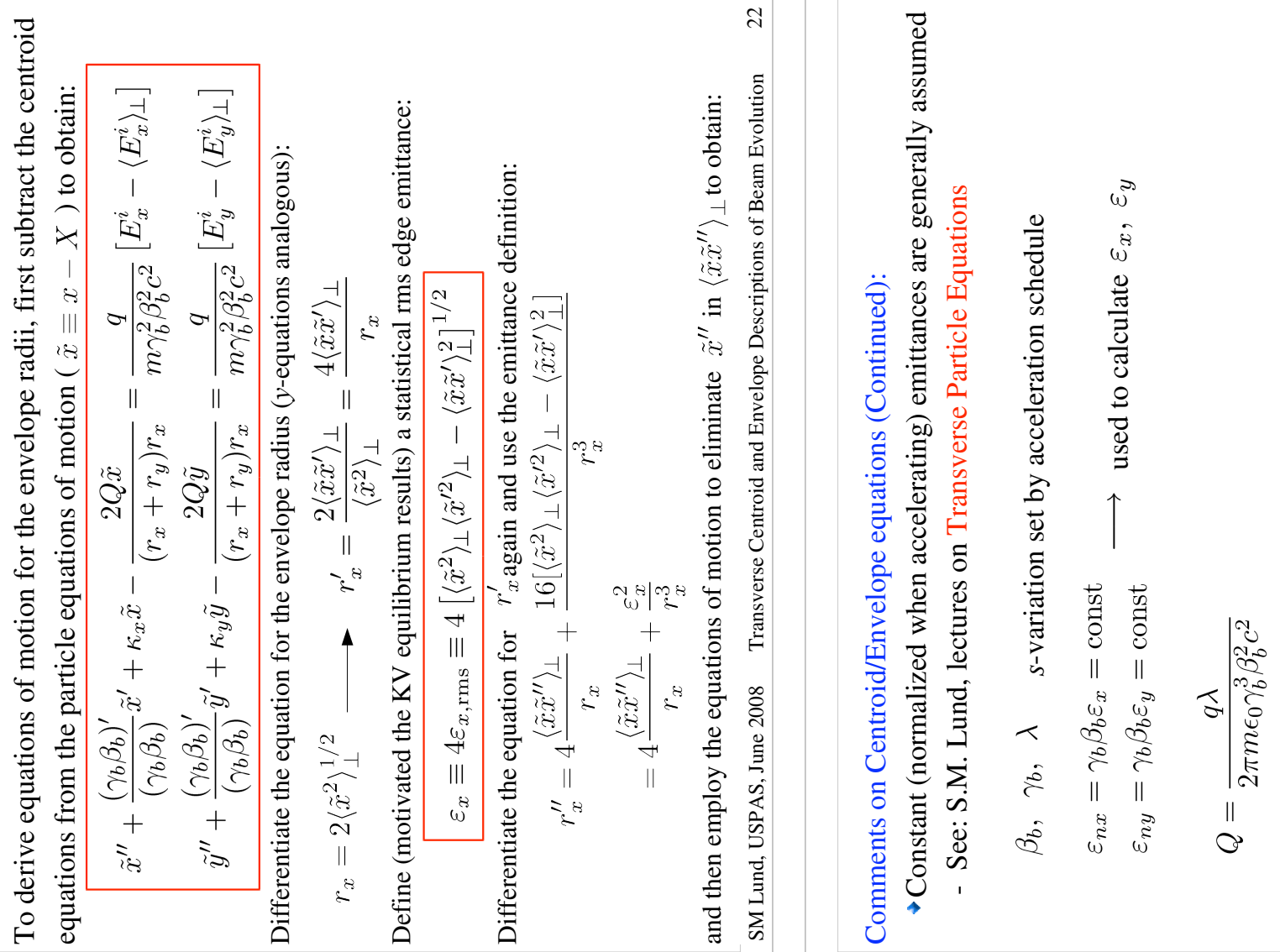

3
$\frac{2}{0}$
0

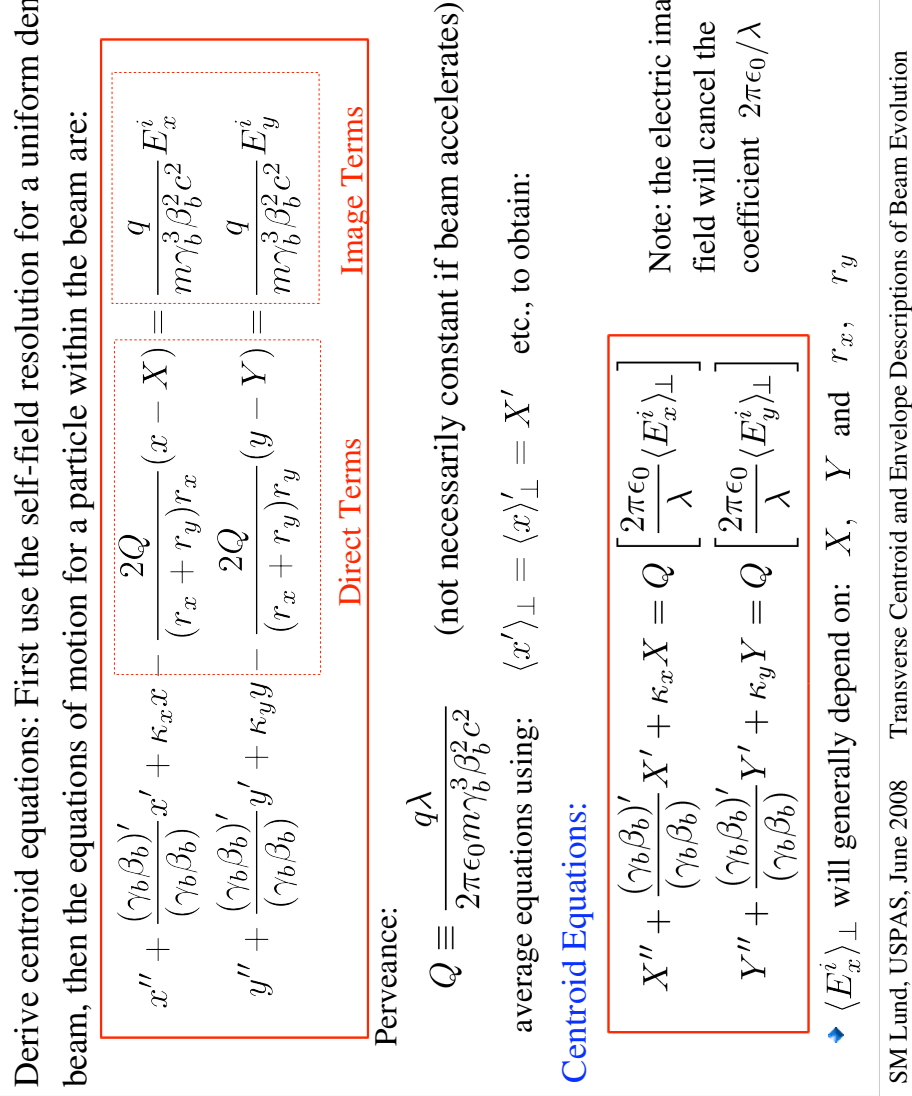

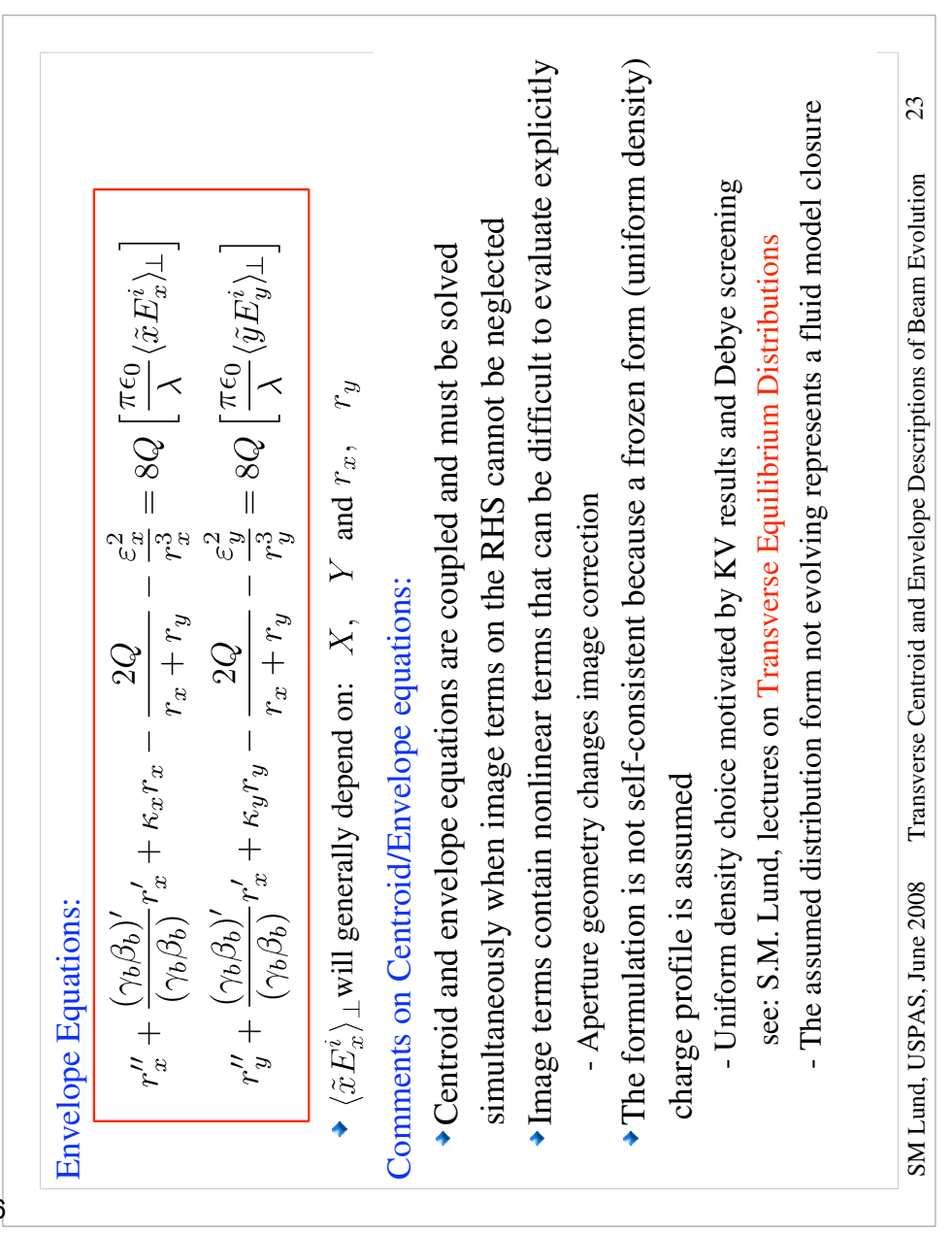



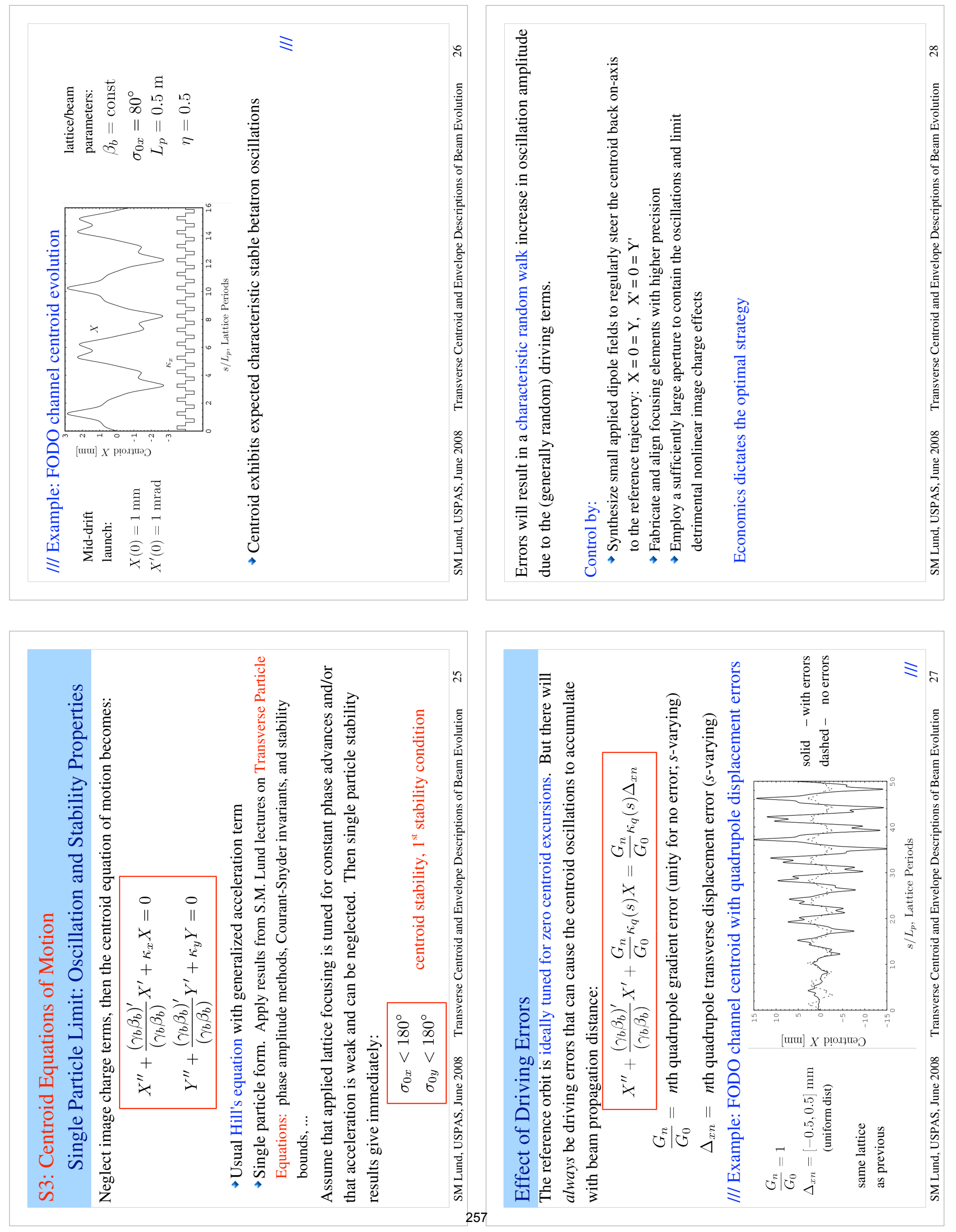

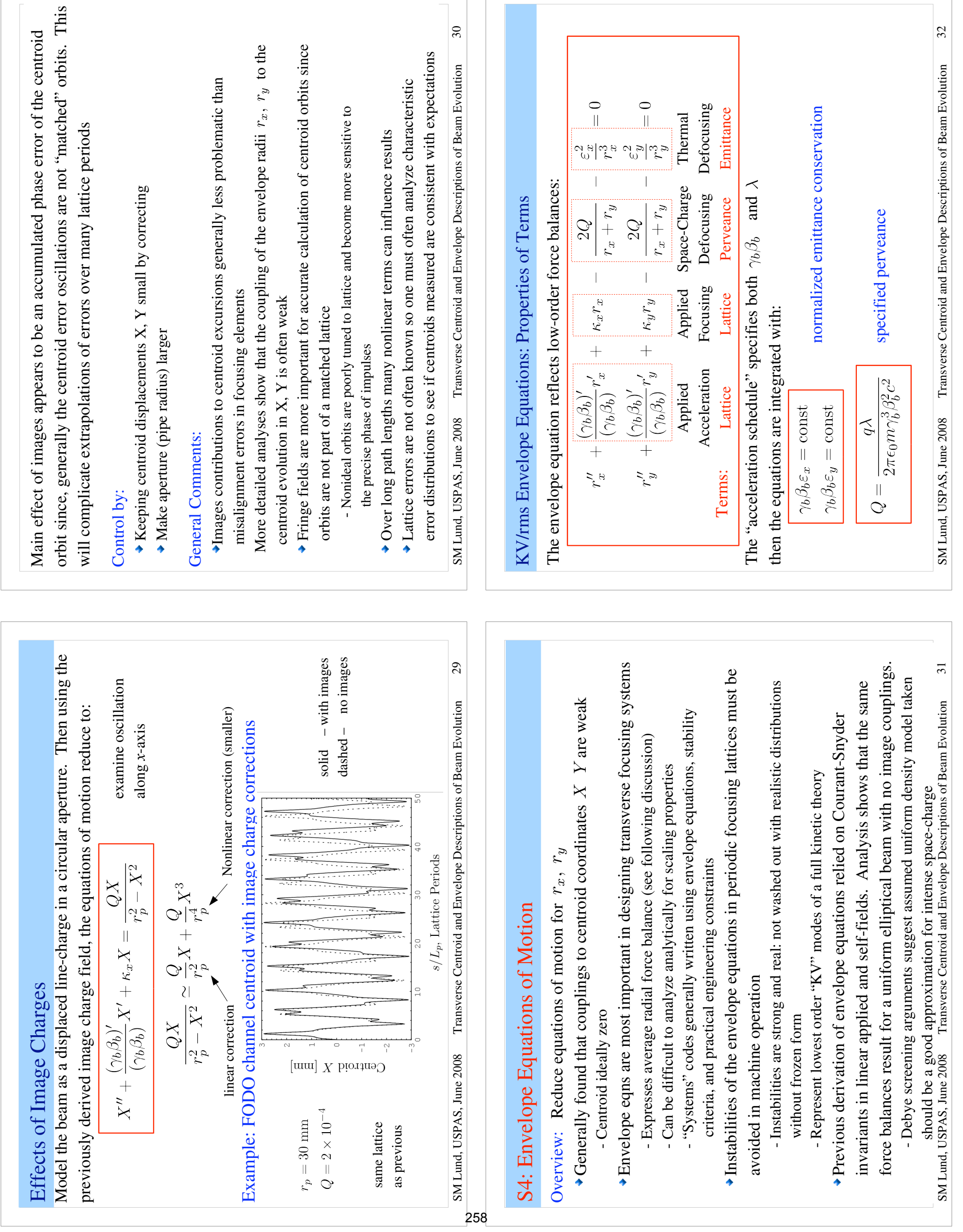

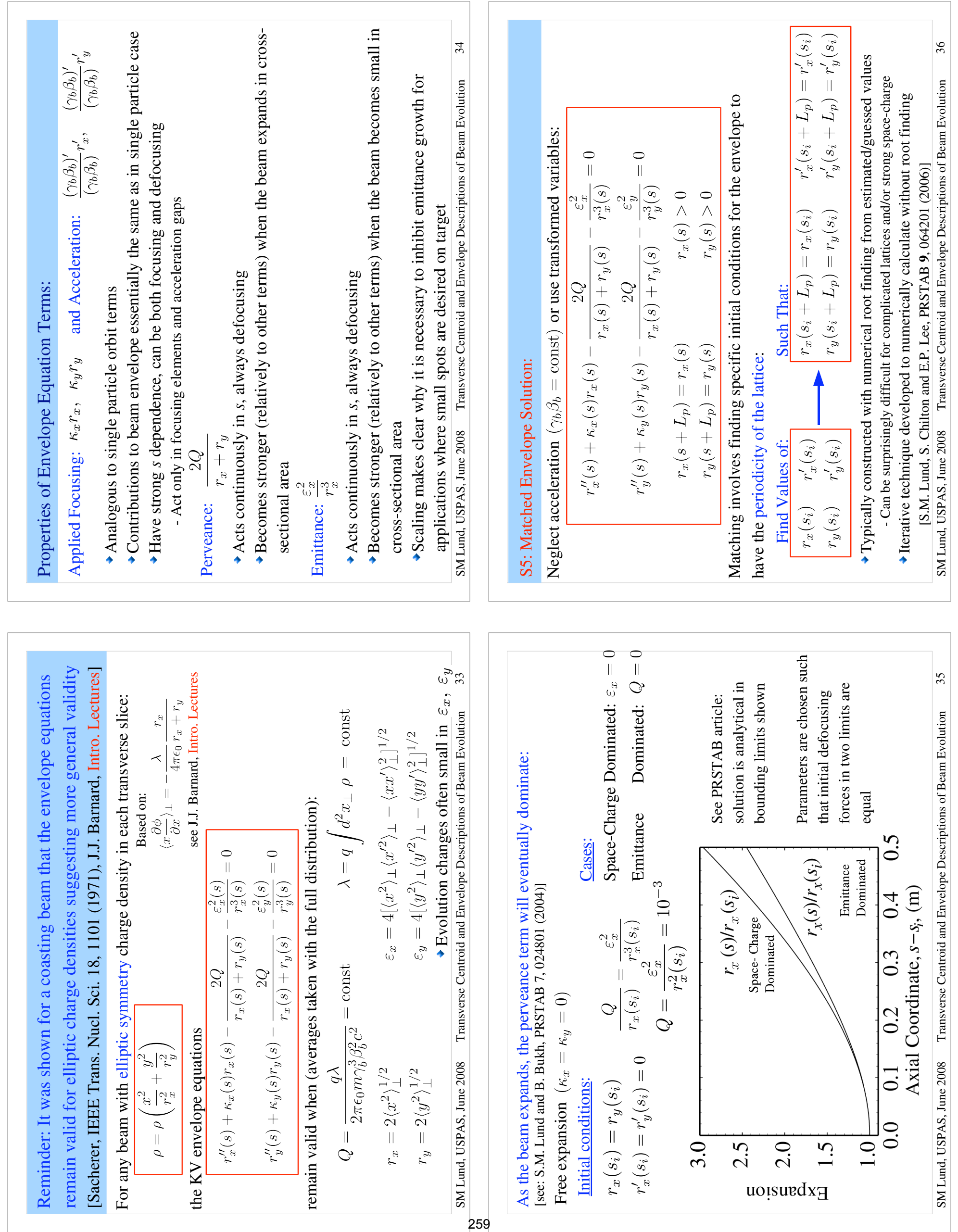

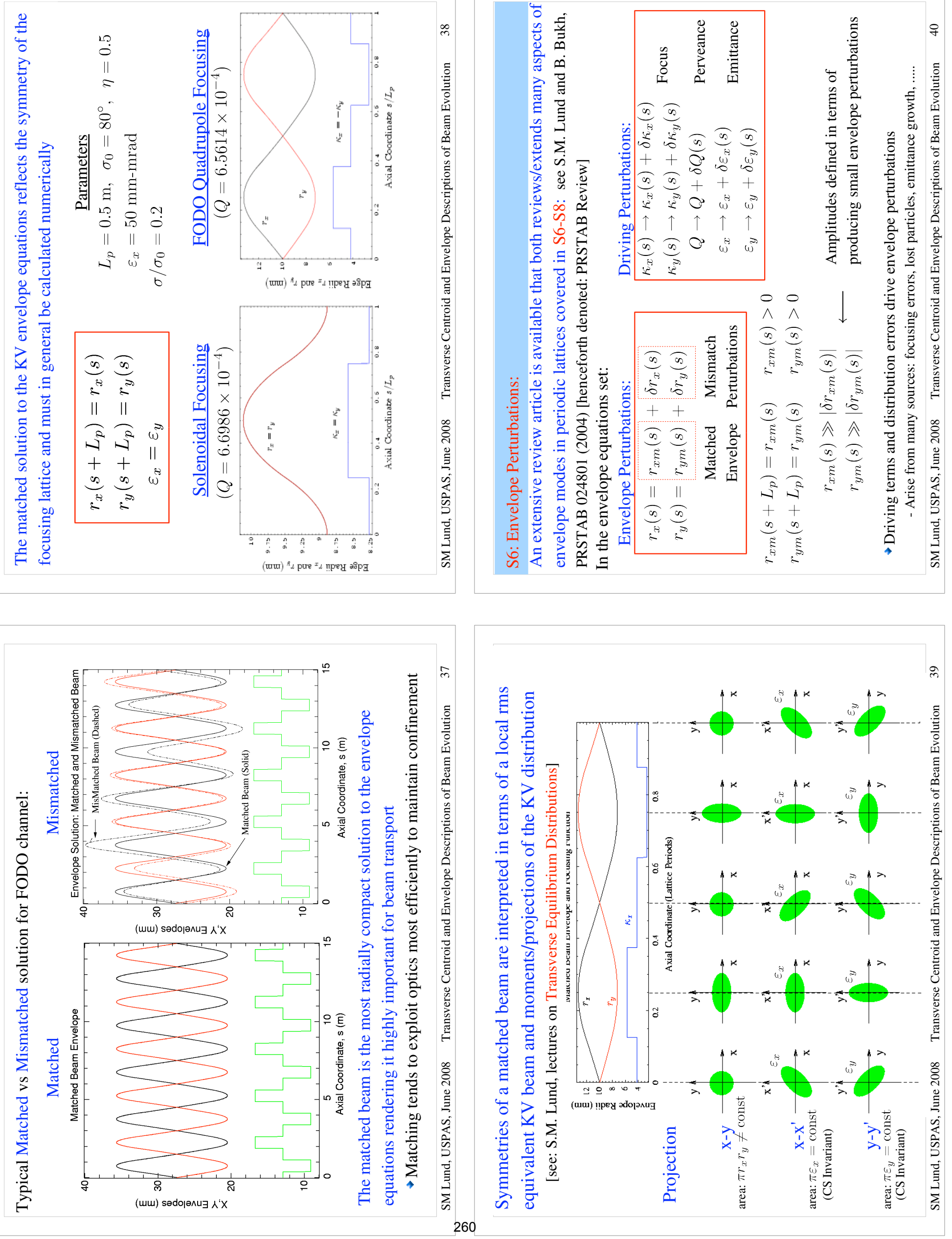

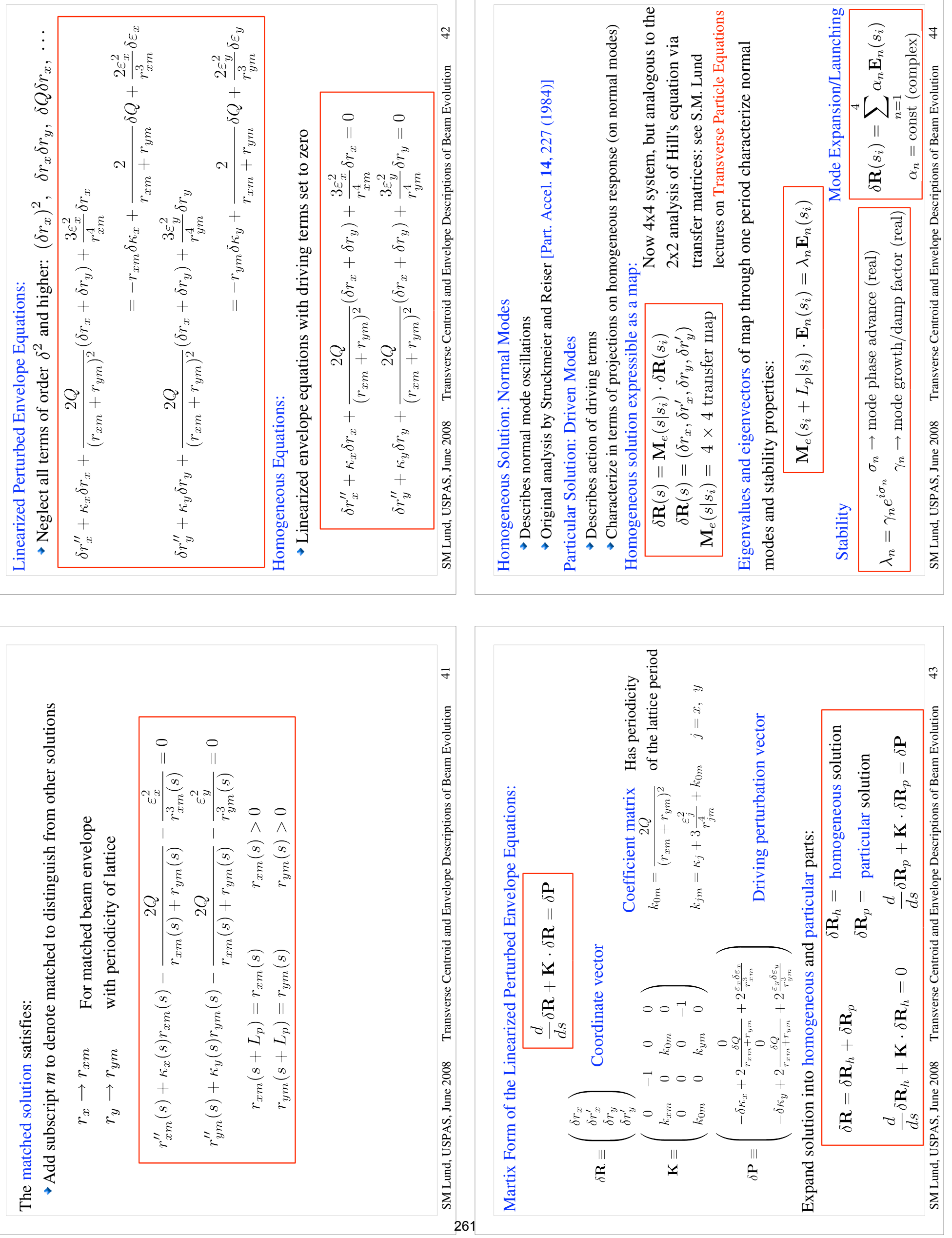

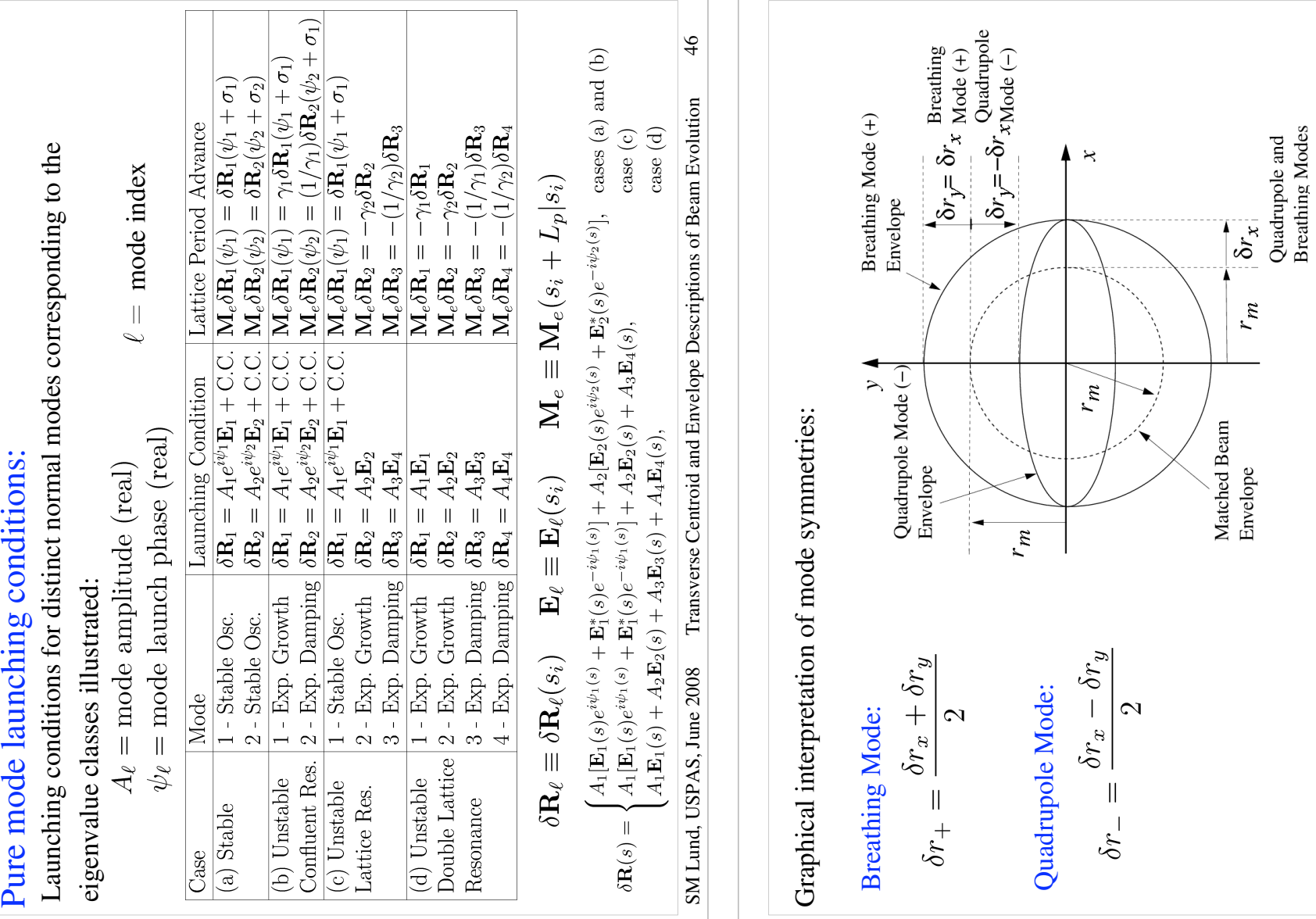

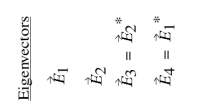
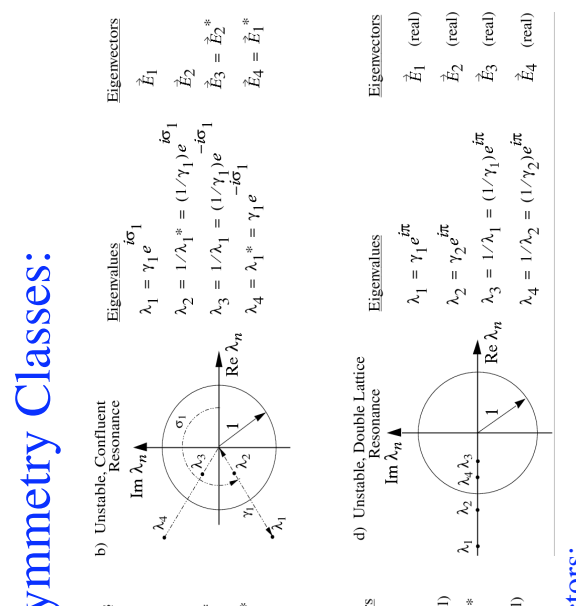

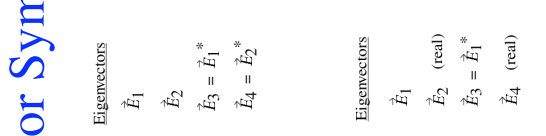

要

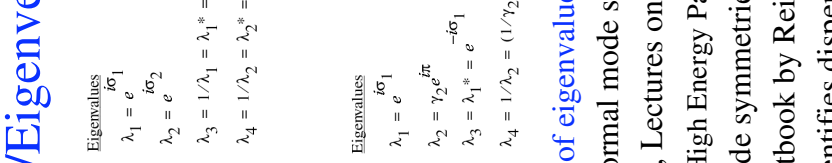

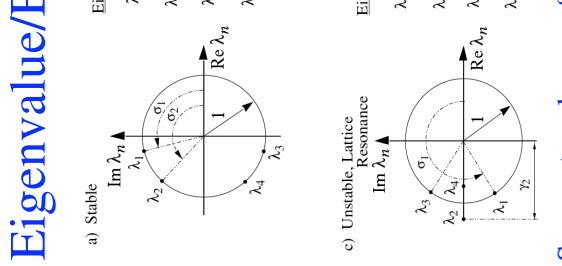

q

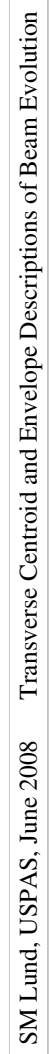

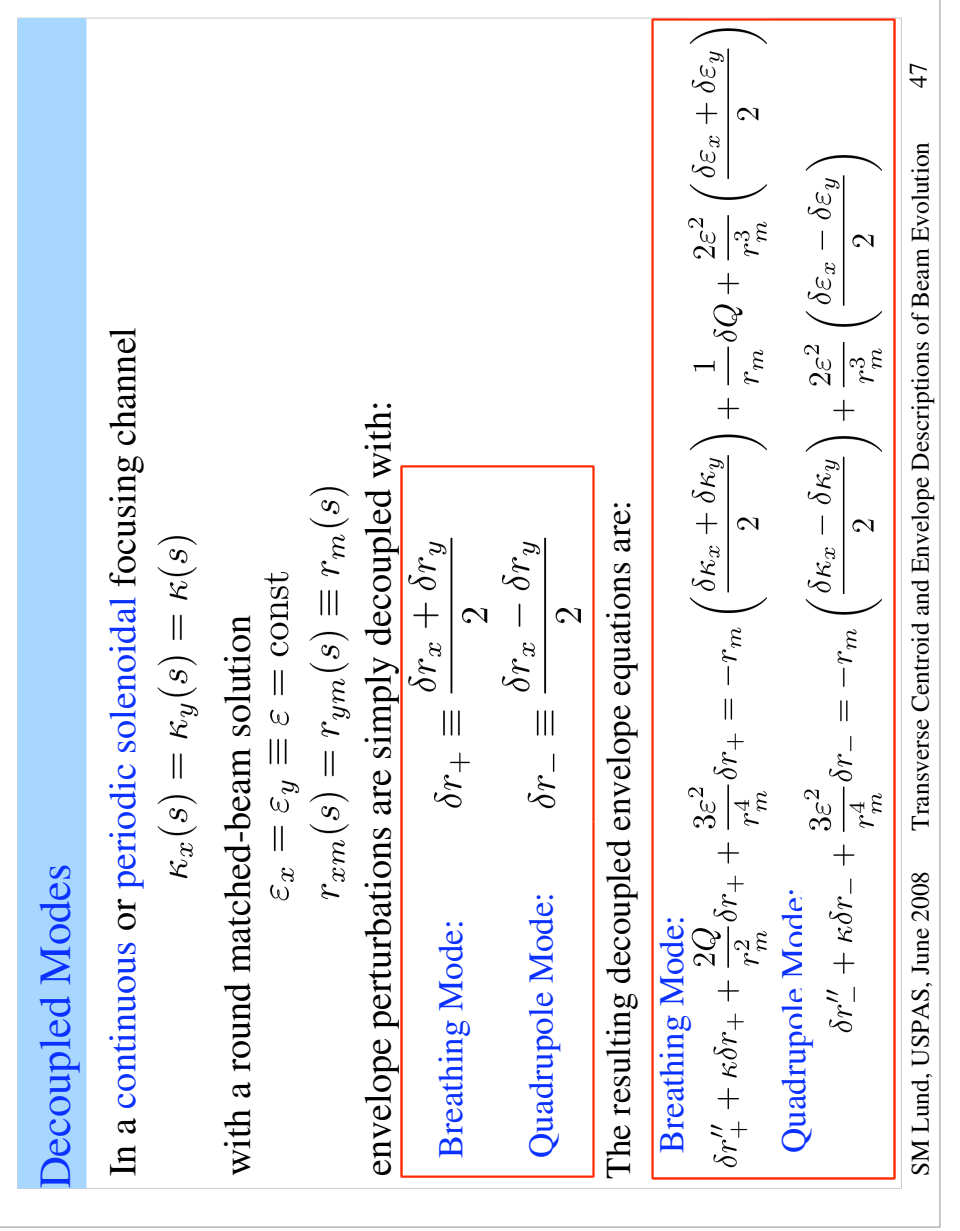



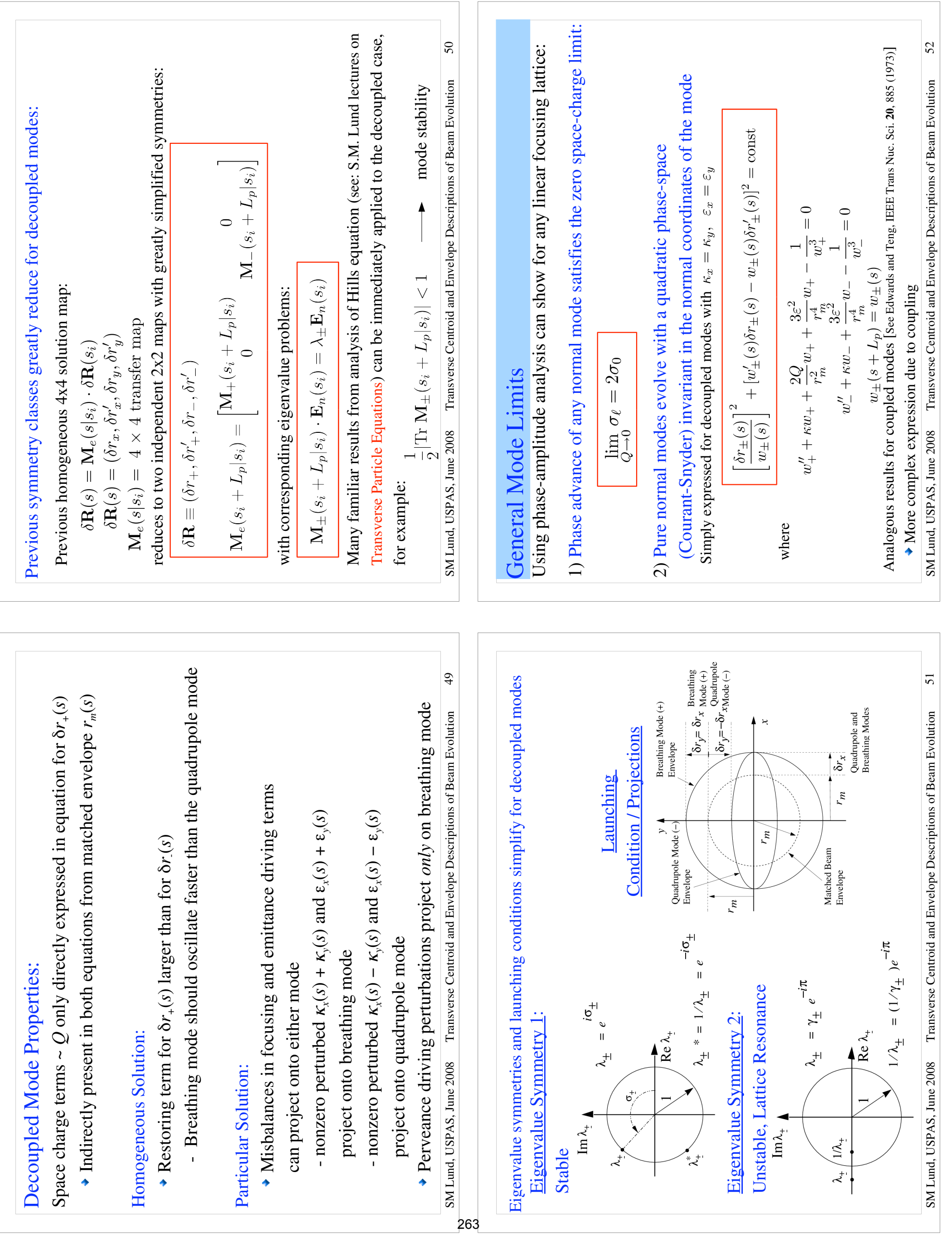

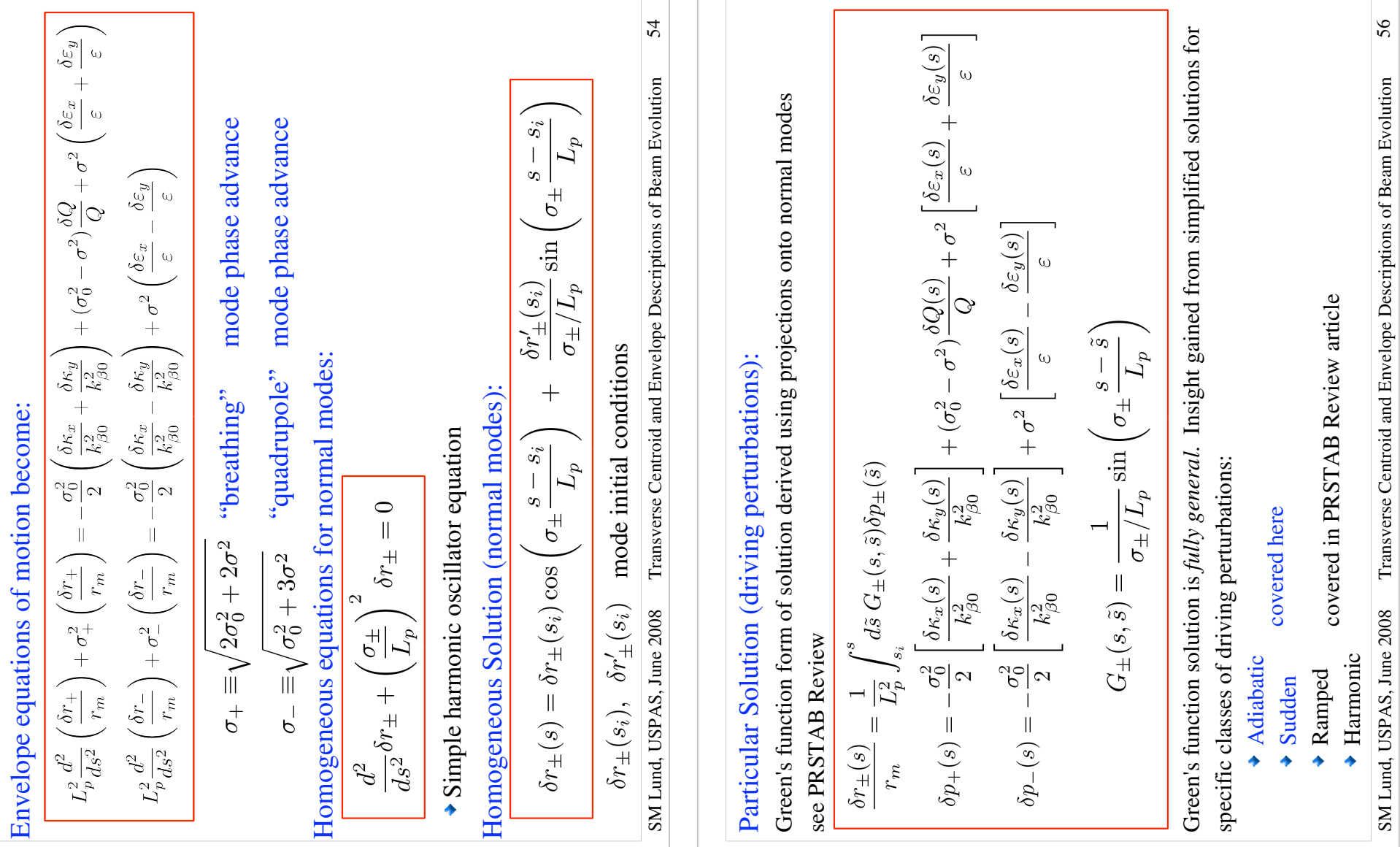

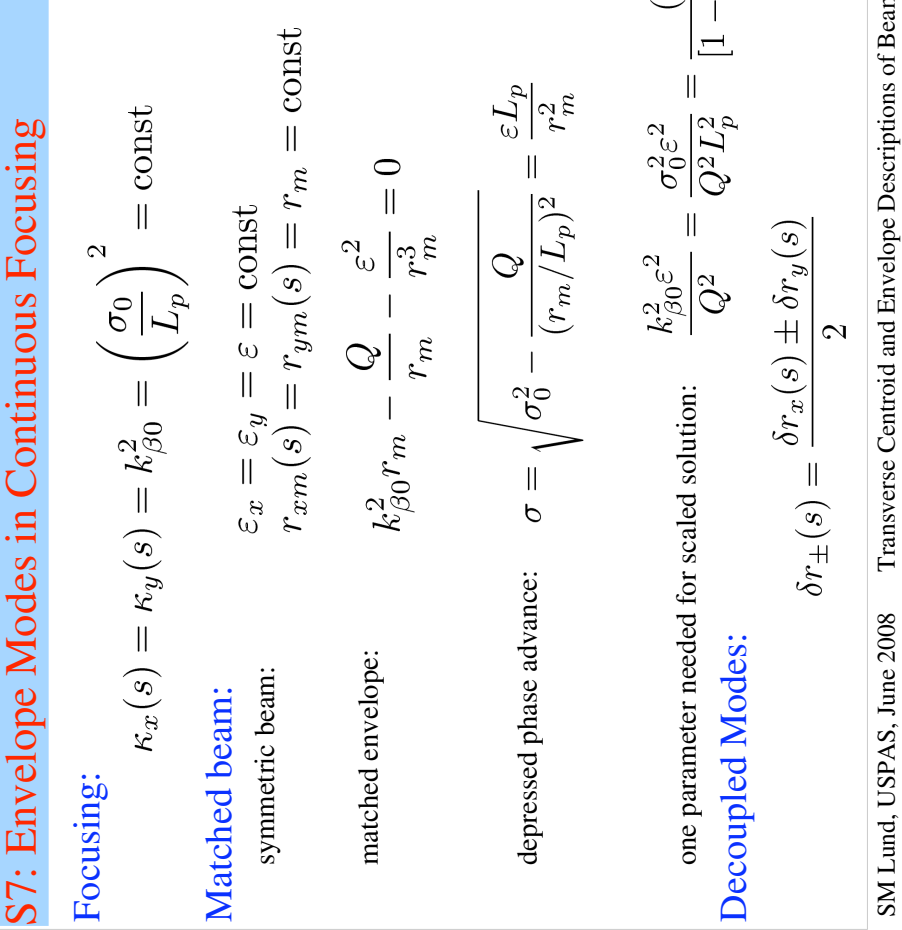

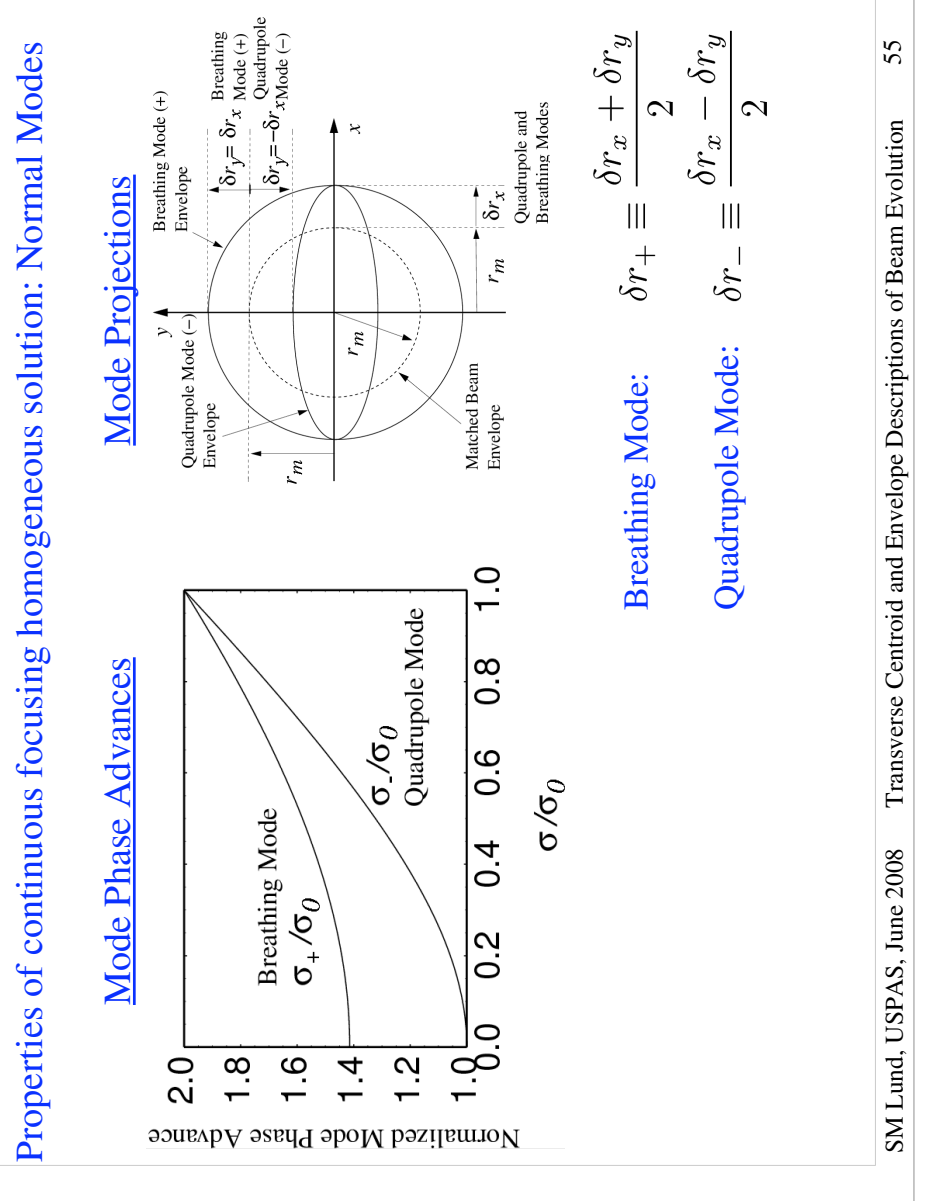



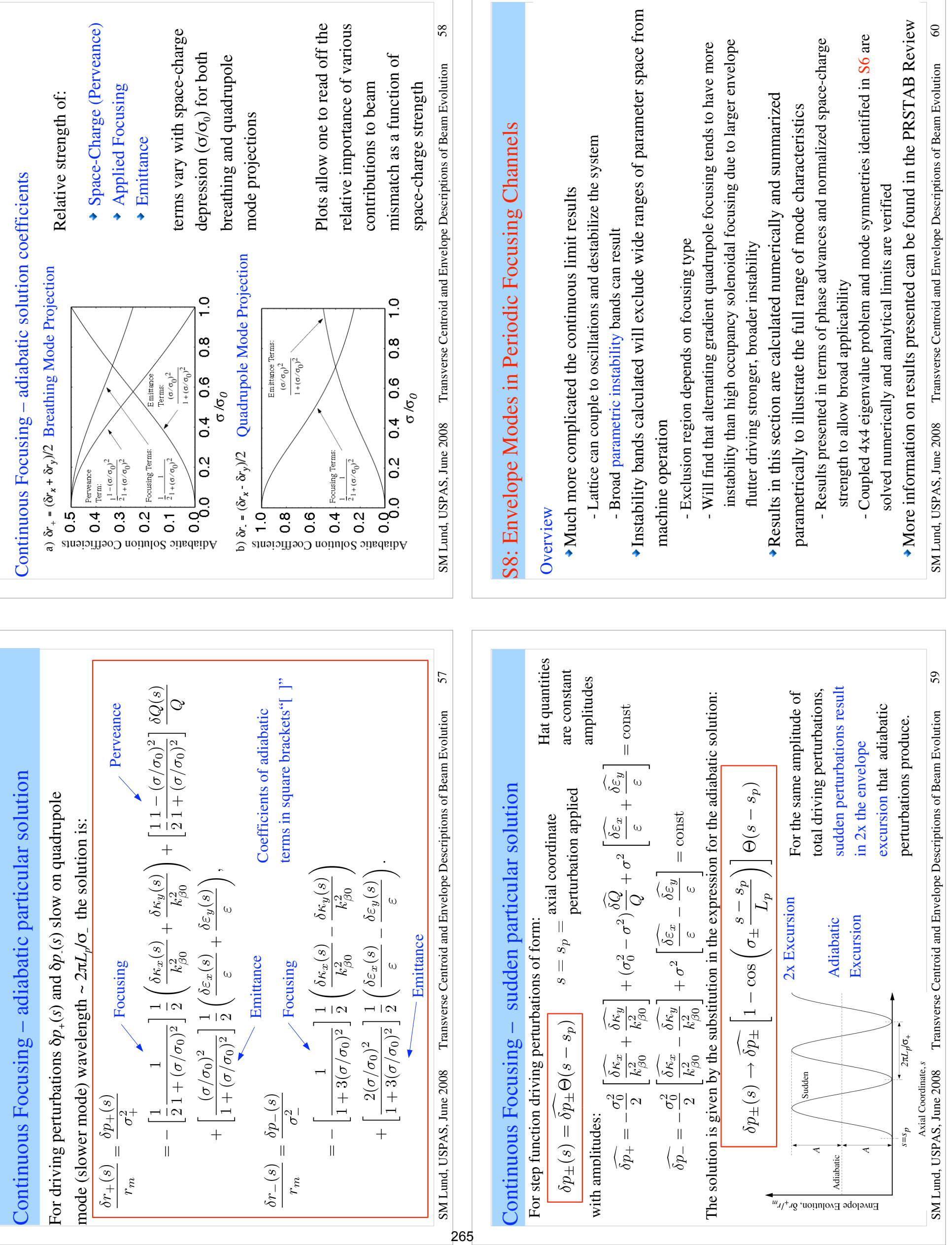

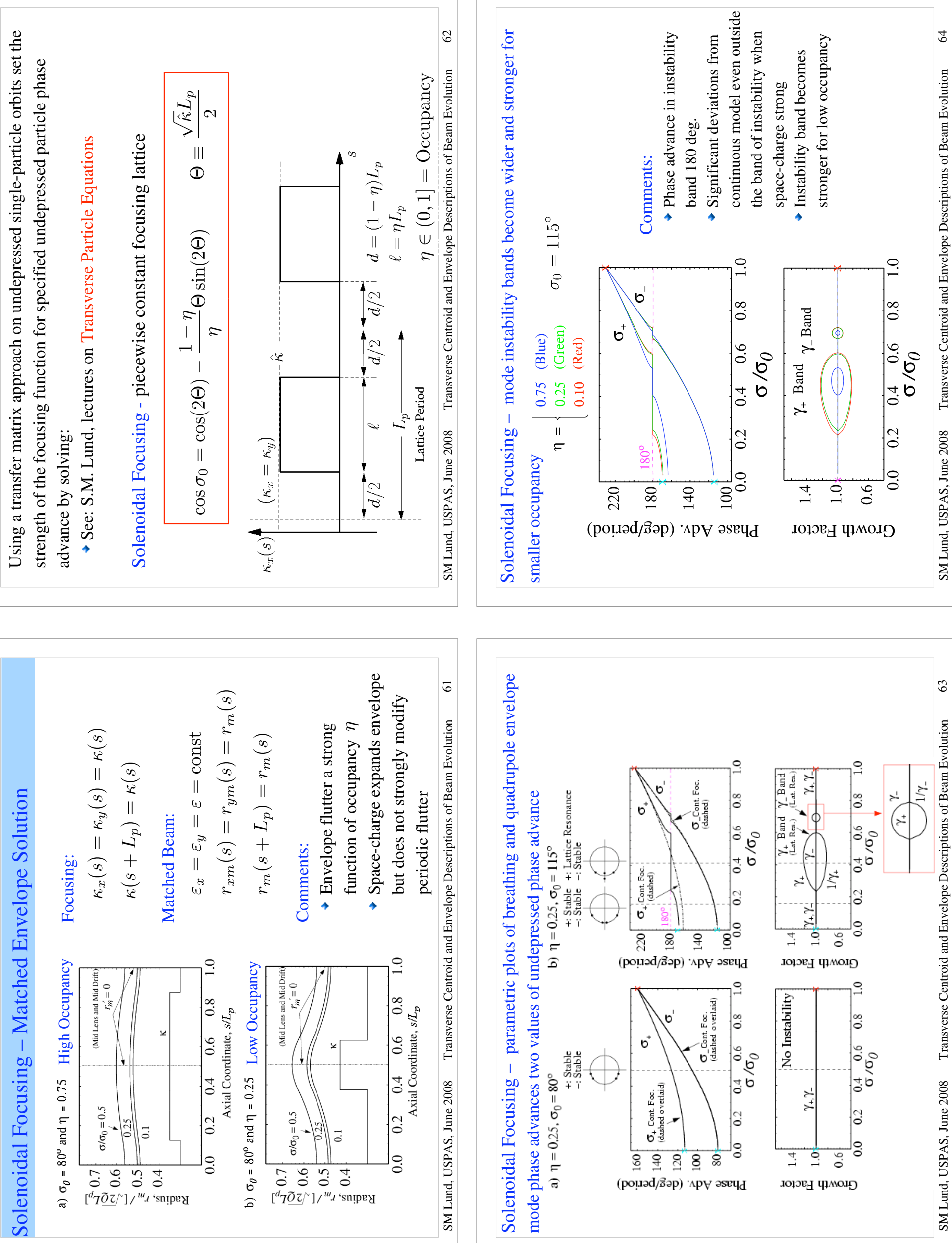

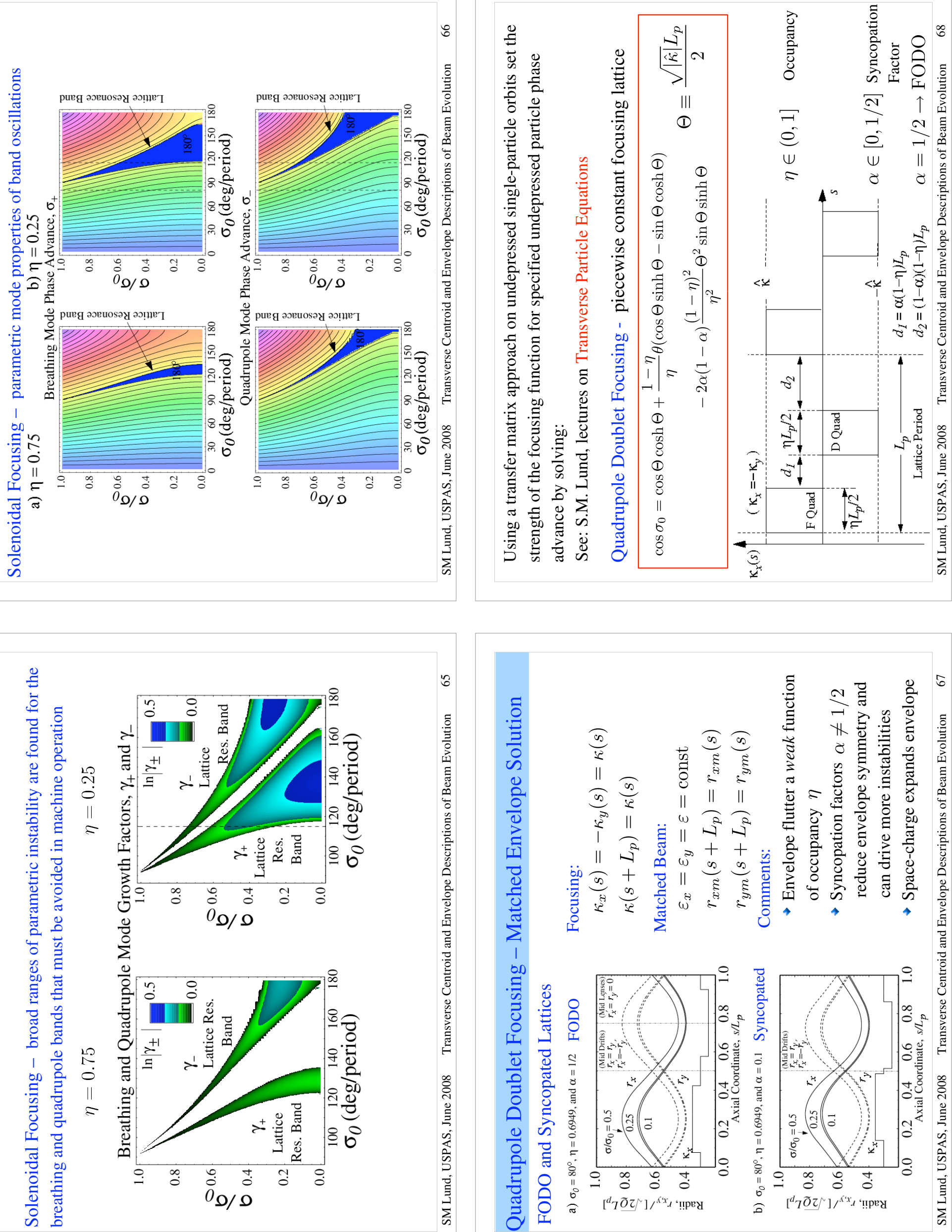

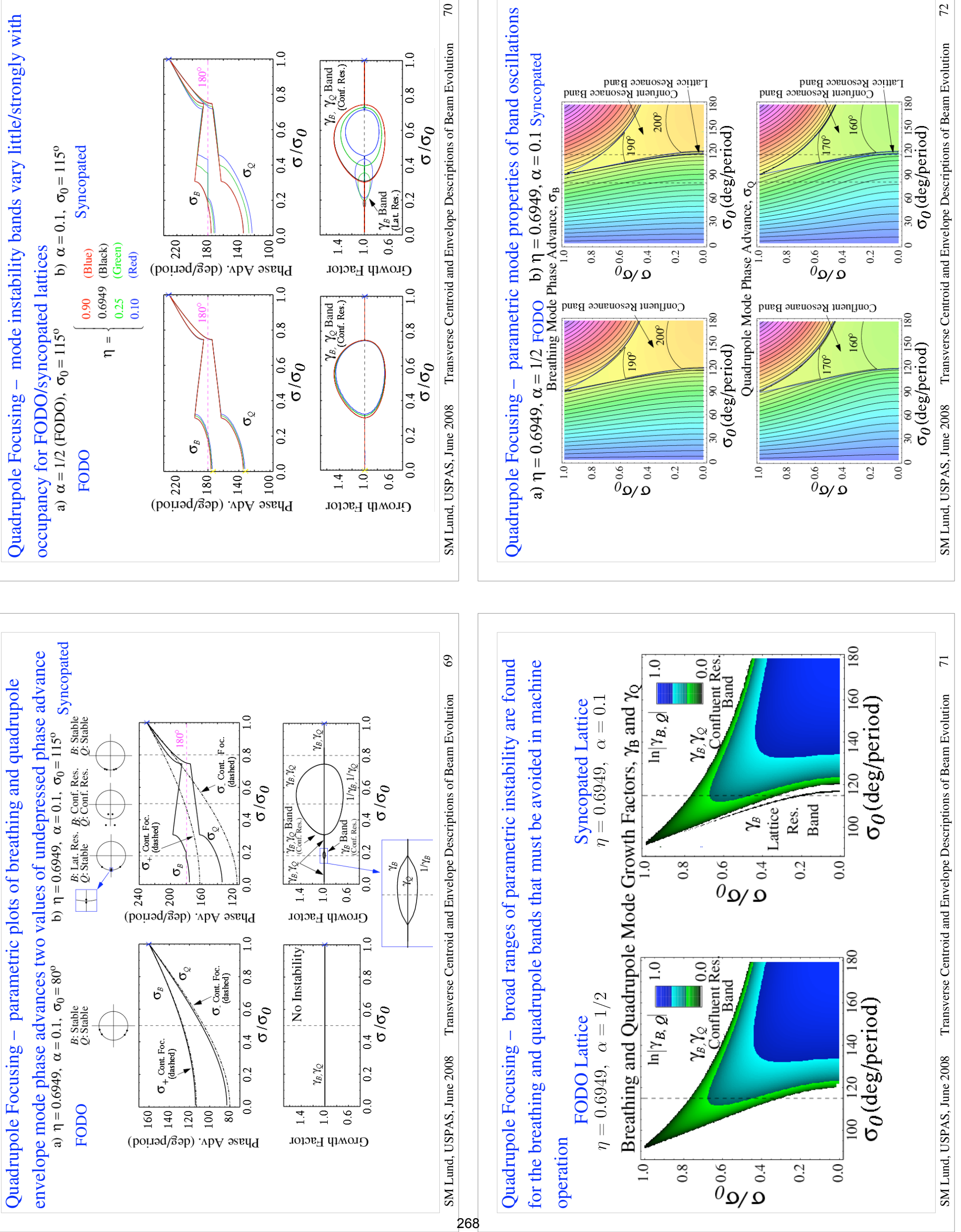


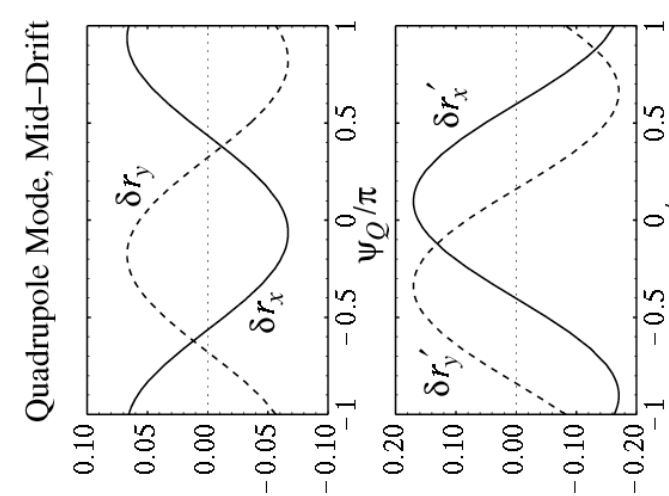

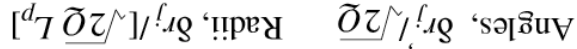

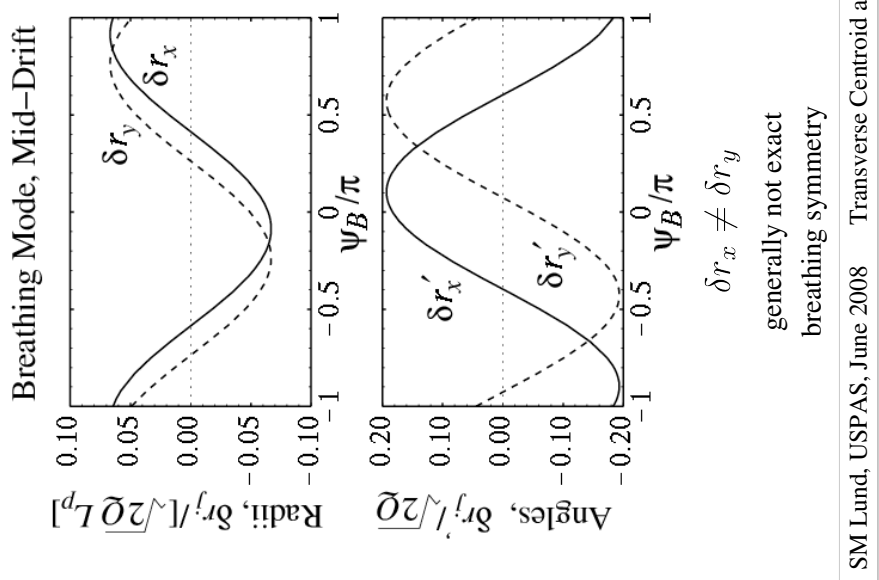

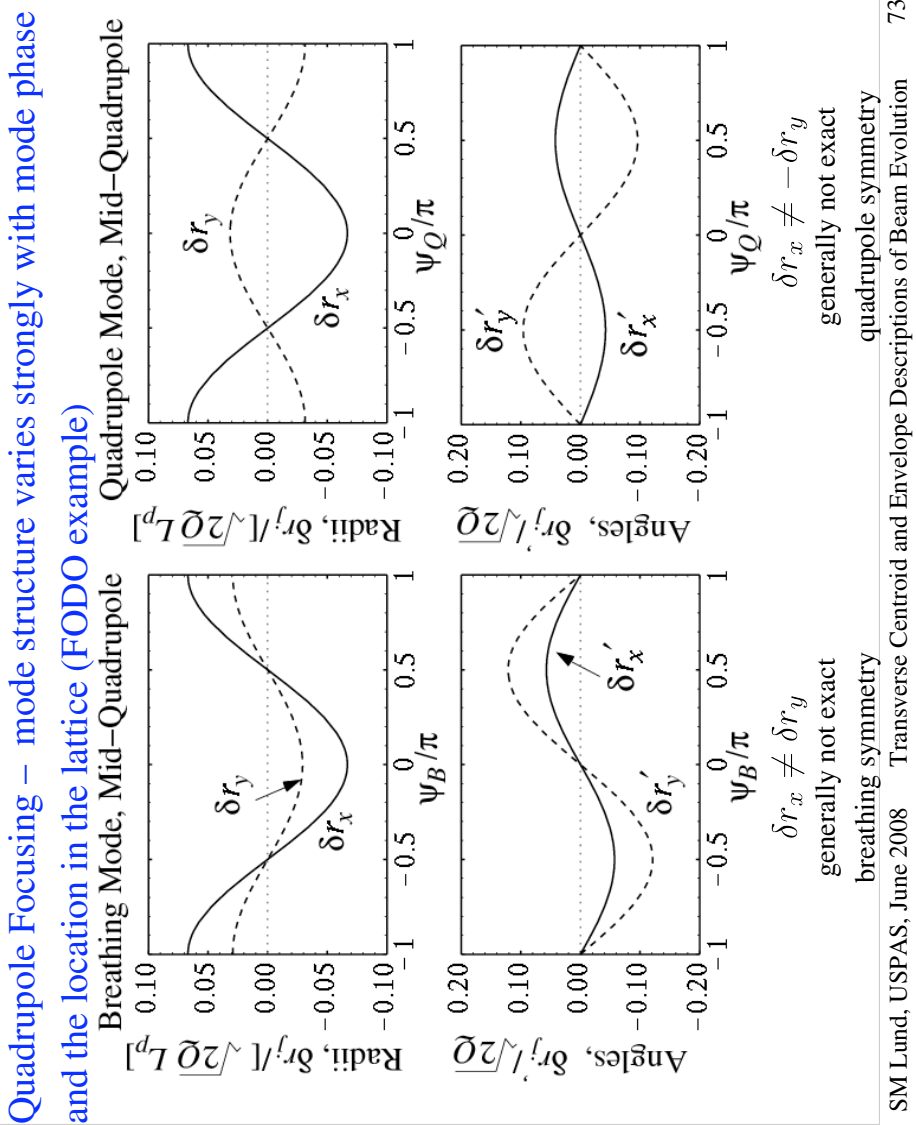

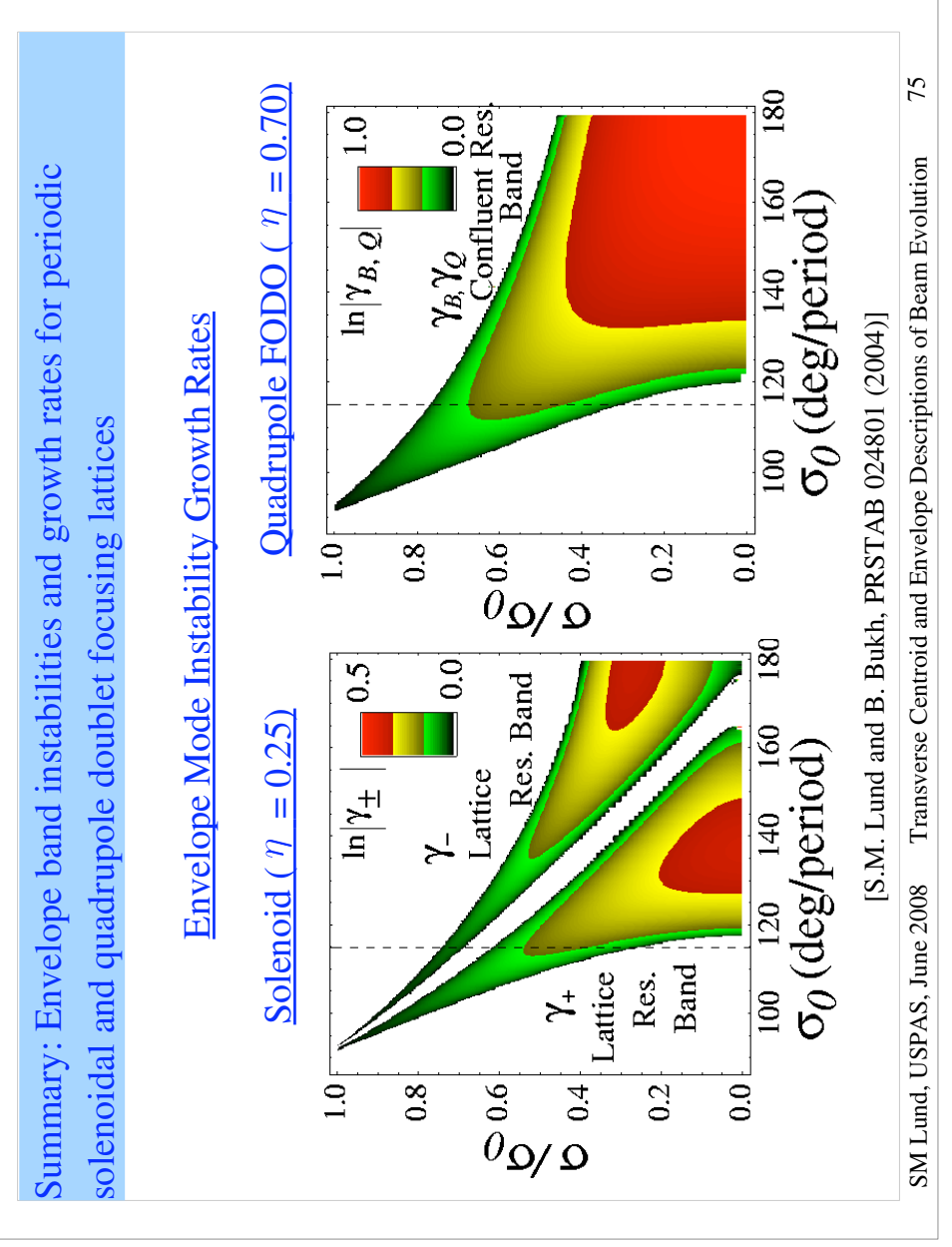



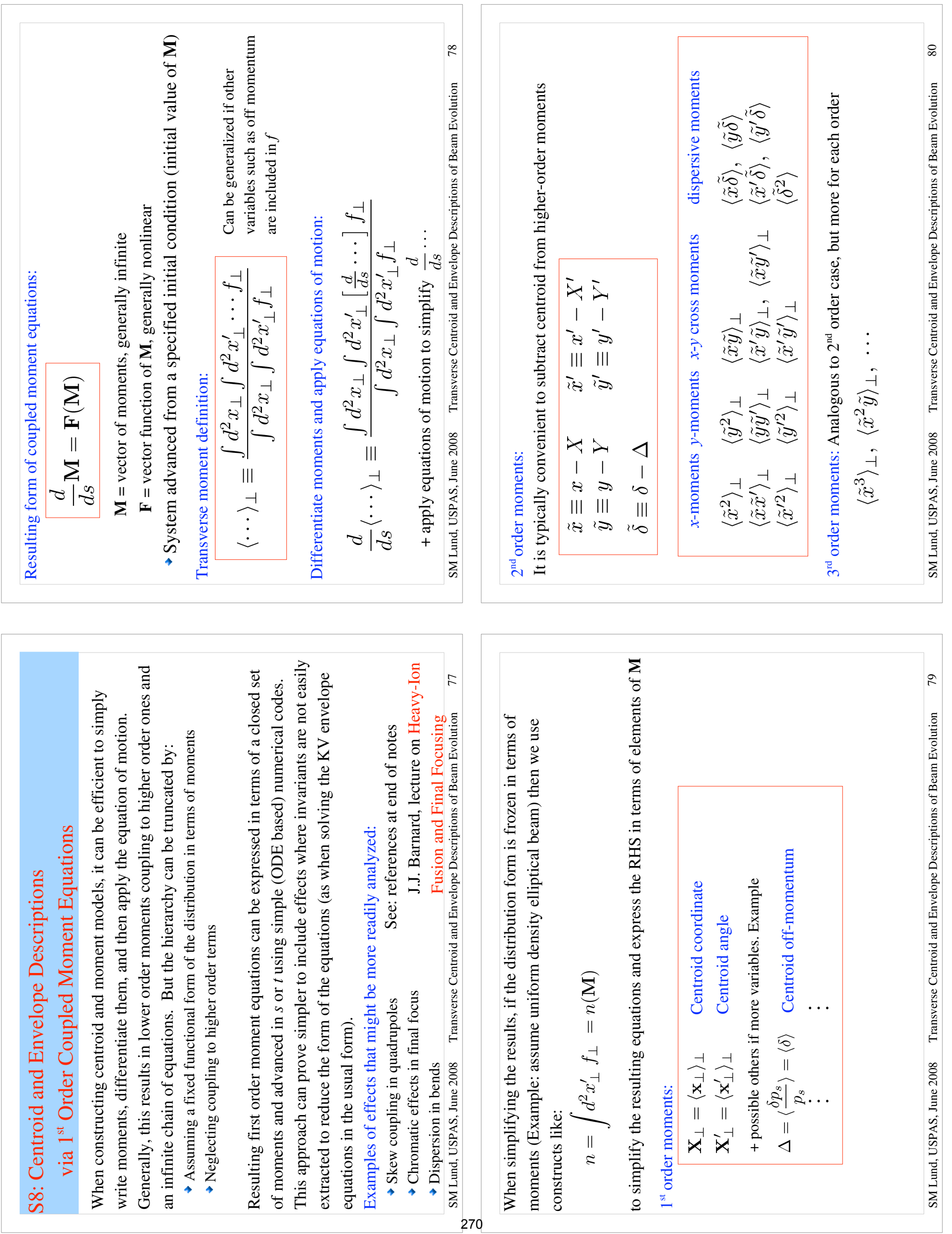

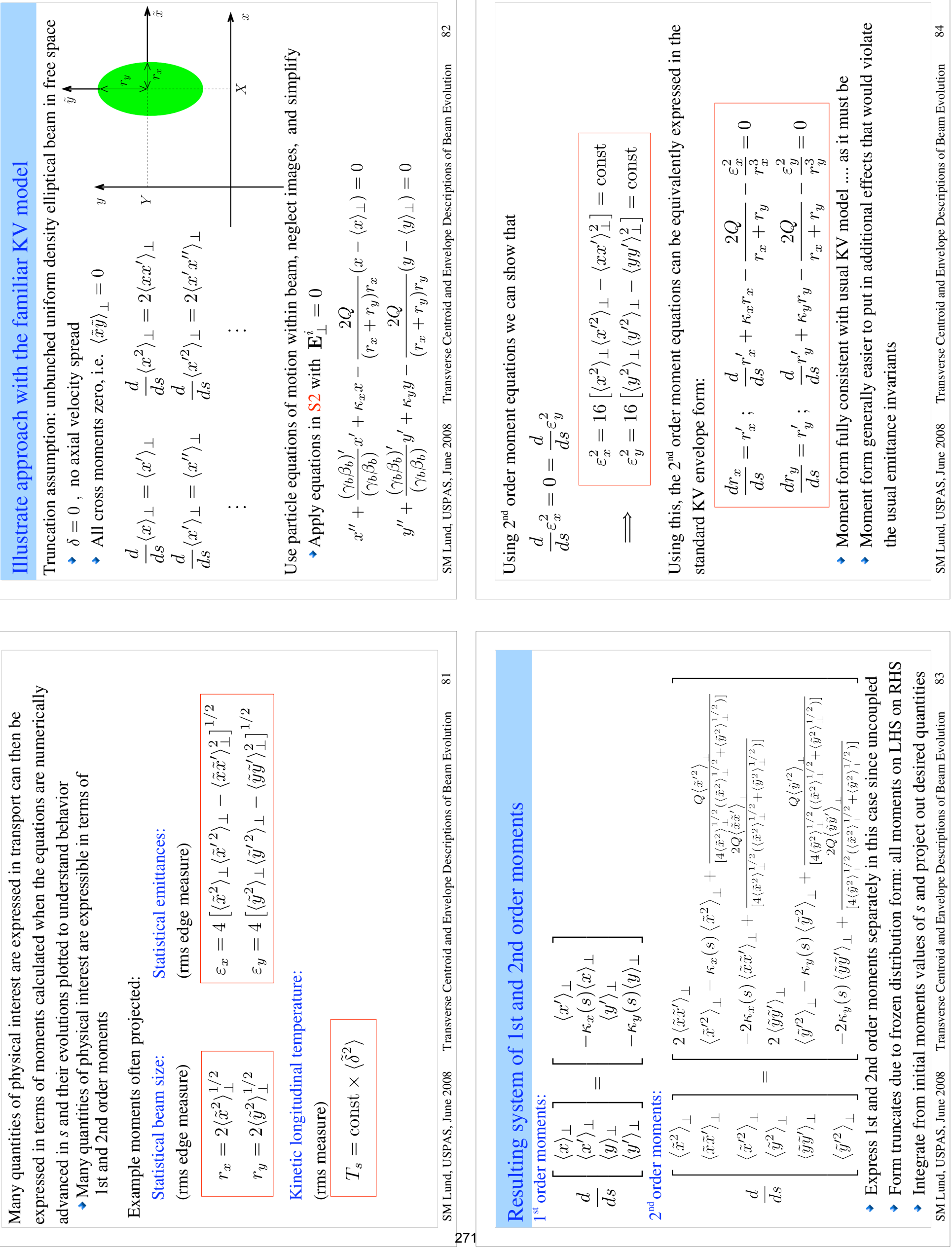

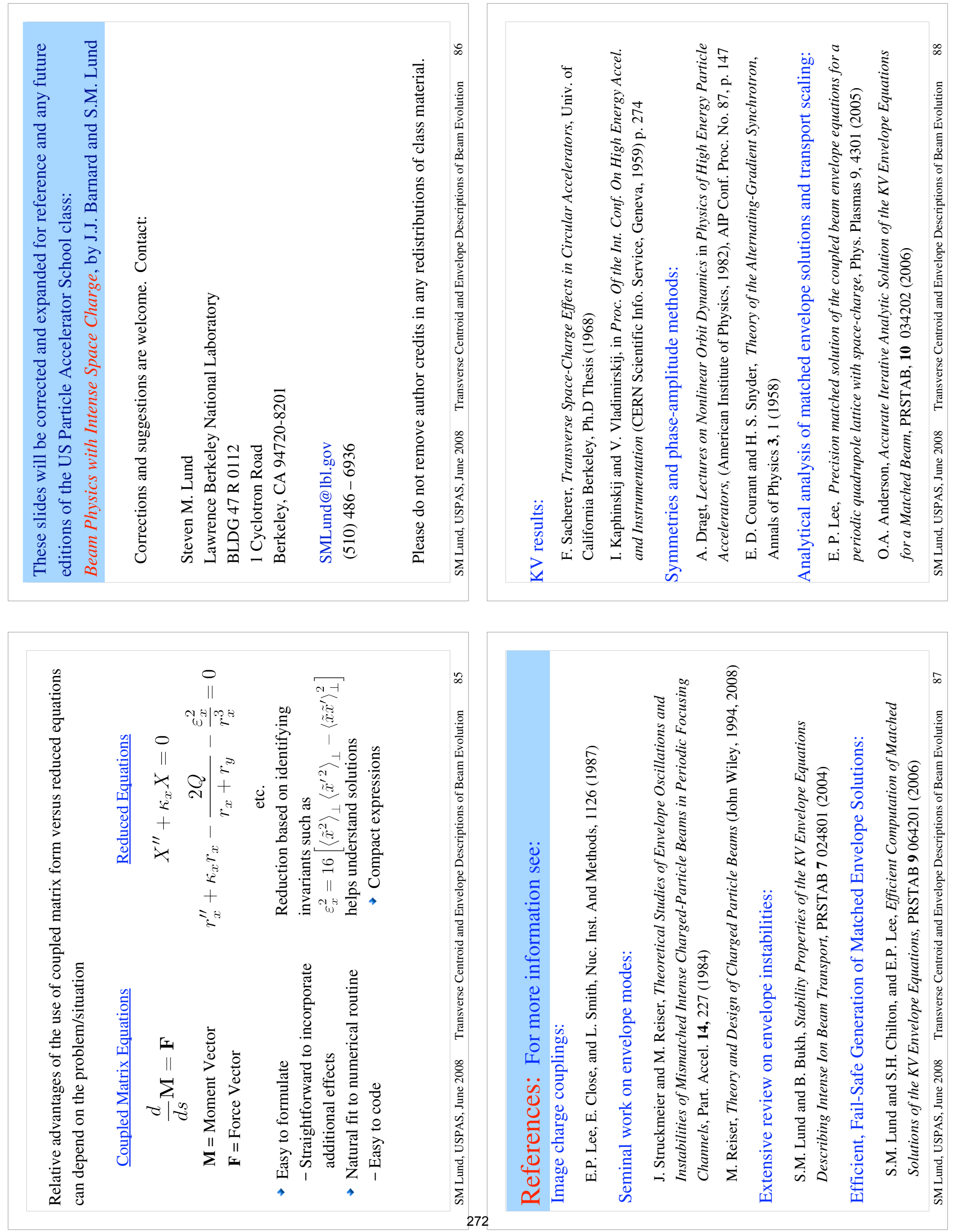


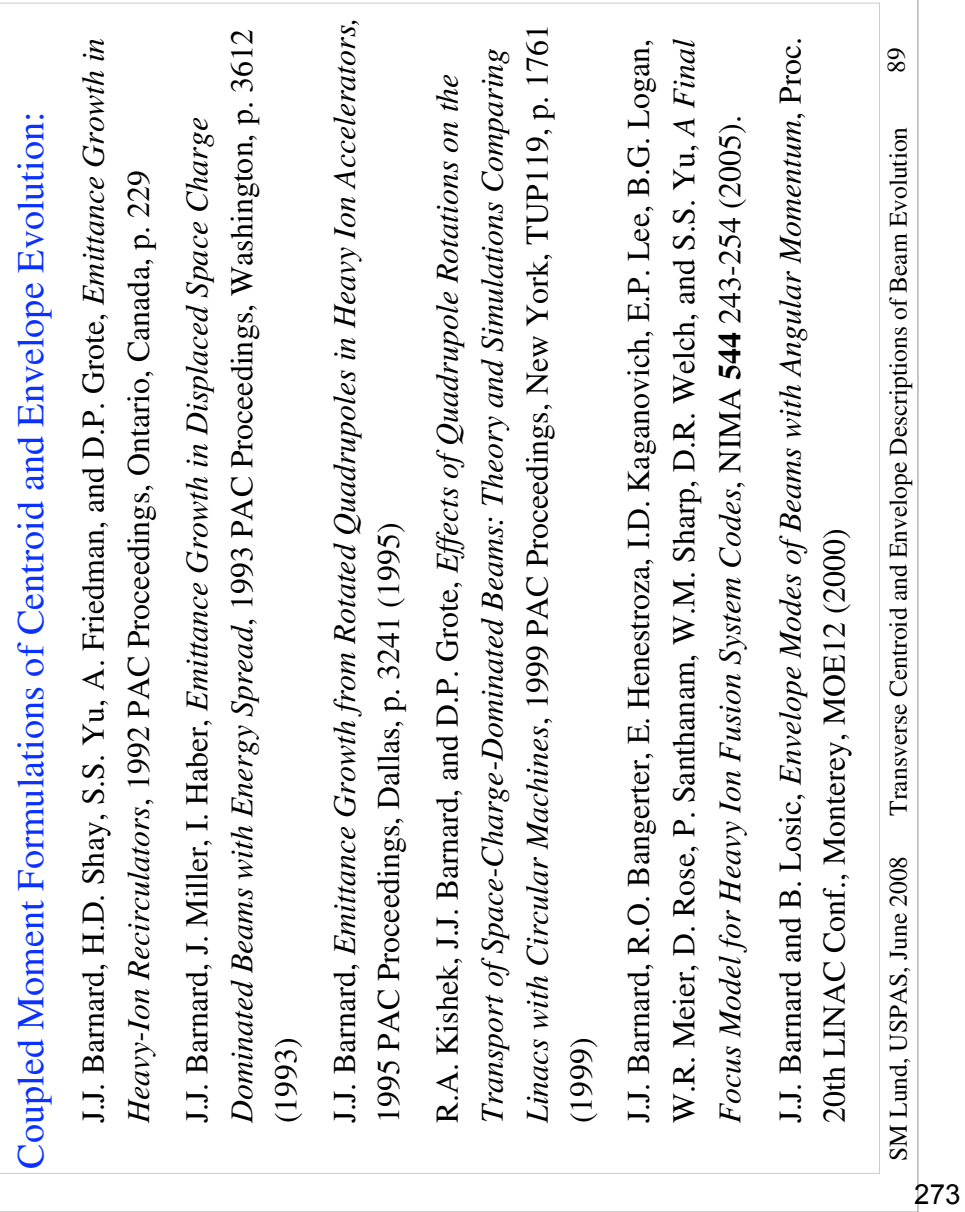


VJ. Barnard and S.M. Lond USPAS, June 2008

99 Transport Limit Scaling Based on the

Matched Beam Envelope Equations

for Periodic Focusing Channels

The scaling of the maximum beam currents or equivalently, the maximum perveance $Q$ that can be transported at a given energy. with a specified focusing technology and lattice is of critical importance in designing optimal transport and acceleration channels. Needed equations can be derived from approximate. analytical solutions to the matched beam envelope equations for a given lattice. Alternatively, numerical solutions of the envelope equations can be evaluated. But analytical solutions are preferable to understand scaling and enable rapid evaluation of design tradeotts.

As a practical matter, equations derived must be applied to regimes where technology is feasible.

- Magnet Field Limits

- Electron breakdown

- Vacuum

Transport limits are inextricably linked fo technology. Moreover, higher order stability constraints etc. must also be respected. Treatments of these topics are beyond the scope of this class. Here we present simplified treatments to ${ }^{274}$ highlight issues and methods. 
S.M. Lond $2 /$

First review an example sketched by J.J. Barnard in the Intro, lectures.

Transport Limits of a Periodic FODO Quadrupole Transport Channel

$$
\begin{aligned}
& \Gamma_{x m}^{\prime \prime}+\frac{\left(\gamma_{b} \beta_{b}\right)^{\prime}}{\left(\gamma_{b} \beta_{b}\right)} \Gamma_{x m}^{\prime}+\beta_{x} \Gamma_{x m}-\frac{2 Q}{\Gamma_{x m}+\Gamma_{y m}}-\frac{\varepsilon_{x}^{2}}{\sigma_{x m}^{3}}=0 \\
& \Gamma_{y m}^{\prime \prime}+\frac{\left(\gamma_{b} \beta_{b}\right)^{\prime}}{\left(\gamma_{b} \beta_{b}^{\prime}\right)} r_{y m}-\kappa_{x} \Gamma_{y m}-\frac{2 Q}{r_{x m}+r_{y m}}-\frac{\varepsilon_{y}^{2}}{\gamma_{y m}^{3}}=0 \\
& r_{x m}\left(s+L_{p}\right)=r_{x m}(s) ; r_{y m}\left(s+L_{p}\right)=r_{y m}(s) \\
& \text { Lix }(s)=-C_{y}(s) \\
& L=\text { Half-Period } L=L_{p} / 2 \\
& \eta=Q \text { quadrupole "occupancy" } 0<\eta \leqslant 1
\end{aligned}
$$

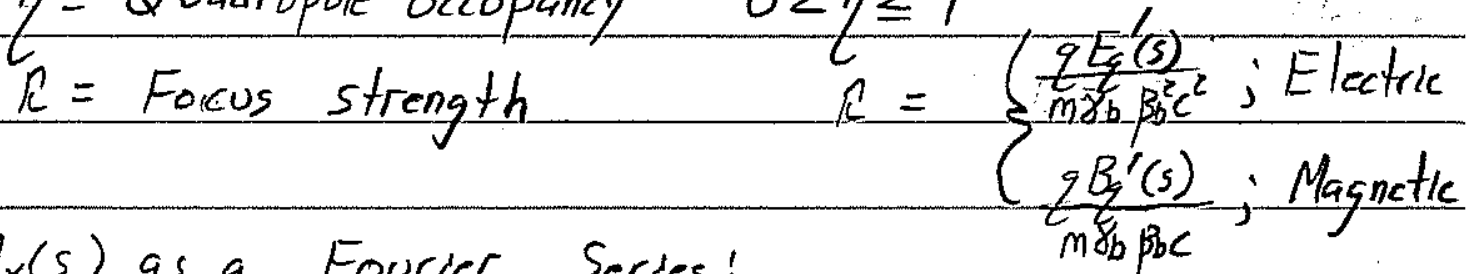

$$
\begin{aligned}
& R_{x}(s)=\sum_{n=1}^{\infty} R_{n} \cos \left(\frac{n \pi s}{L}\right) \\
& \left.\kappa_{n}=\frac{1}{L} \int_{0}^{2 L} \kappa_{x}(s) \cos \left(\frac{n \pi s}{275} L\right)=\frac{2 \hat{k}}{n \pi}\left[1-(-1)^{n}\right] \sin \left(\frac{n \pi \eta}{2}\right)\right]
\end{aligned}
$$


S.M. Lond

And expand the periodic matched beam envelope by:

$$
\begin{aligned}
\Gamma_{x m}= & \Gamma_{b}\left[1+\Delta \cos \left(\frac{\pi S}{L}\right)\right]+\sum_{n=2}^{\infty} \Delta_{x n} \cos \left(\frac{n \pi s}{L}\right) \\
\Gamma_{y m}= & \Gamma_{b}\left[1-\Delta \cos \left(\frac{\pi S}{L}\right)\right]+\sum_{n=i}^{\infty} \Delta_{y n} \cos \left(\frac{n \pi s}{L}\right) \\
& \Gamma_{b}=\text { cont }=\text { avg, beam radius. } \\
& |\Delta|=\text { canst } \angle L 1 \\
& \Delta_{x n} \text { constants with }\left|\Delta_{x n}\right|<L|\Delta|
\end{aligned}
$$

Take:

- $\left(\gamma_{b} \beta_{b}\right)^{\prime}=0 \quad \Rightarrow$ coasting beam

- $\varepsilon_{x}=\varepsilon_{y}=\varepsilon \quad \Rightarrow$ isotropic beam

and insert these expansions in the envelope equations. Neglect:

- All terms $\theta\left(\Delta^{2}\right)$ and higher

- Fast oscillation terms $\sim \cos \left(\frac{n \pi s}{L}\right)$ with $n \geq z$.

to obtain two independent constraint equations:

$$
\text { Avg : } \quad \frac{2 \Delta \hat{\kappa}}{\pi} \Gamma_{b} \sin \left(\frac{\pi \eta}{2} \tau\right)-\frac{Q}{r_{b}}-\frac{\varepsilon^{2}}{r_{b}^{3}}=0
$$

Fundamental.

$$
e_{c} \cos \left(\frac{\pi s}{L}\right):-\Delta\left(\frac{\pi}{L}\right)^{2} r_{b}+\frac{4 \hat{R} r_{b}}{\pi} \sin \left(\frac{\pi \eta}{2^{2}}\right)+\frac{3 \Delta \varepsilon^{2}}{r_{b}^{3}}=0
$$

276 
S.M. Lond

These equations can be solved to express the maximum beam edge excursion as

$$
\operatorname{Max}\left[\Gamma_{x m}\right]=\operatorname{Max}\left[r_{y m}\right] \cong \Gamma_{b}(1+|\Delta|)=\Gamma_{b}\left(1+\frac{4|\hat{R}| L^{2} \sin \left(\frac{\pi \eta}{2}\right)}{\pi^{3}\left(1-3 \frac{L^{2} \varepsilon^{2}}{\pi^{2} T_{b}^{2}}\right)}\right\}
$$

and the beam Perveance as:

$$
Q=\frac{2}{\pi^{2}}\left[\frac{\sin \left(\frac{\pi \eta}{2}\right)}{\left(\frac{\pi q}{2}\right)}\right]^{2} \frac{\eta^{2} \hat{\Omega}^{2} L_{b}^{2}}{\left(1-\frac{3 L^{2} \varepsilon^{2}}{\pi^{2} T_{b}^{4}}\right)}-\frac{\varepsilon^{2}}{\Gamma_{b}^{2}}
$$

Design Strategy:

1) Choose a lattice period 2L, quadrupole occupancy ? , and clear machine "pipe" radius ip consistent with focusing technology employed.

2) Choose the largest possible focus strength $\hat{n}$ (quadrupole current or voltage excitation) for beam energy with undepressed particle phase advance:

$$
\delta_{0} \leqslant 80^{\circ} / \text { period. "Tiefenback Limit" }
$$

- Larger phase advances correspond to stronger focus and smaller beam cross-sectional area for green values of $Q, \varepsilon$.

- Weaker phase advance suppresses various particle, envelope, and collective instabilities for rel able transport: [Ret: Me Ge Tiefonback, "Spar e-Charge Limits on the Transport of Ion Beamy, WC. Berkeley P77.d Thesis, 1986 LBL-22465 
S.M. Lond

5

3) Choose a suitable beam-edge to aperture clearance factor:

$$
\begin{gathered}
\Gamma_{p}=\operatorname{Max}\left[\sigma_{x m}\right]+\Delta_{p} \\
\Delta_{p}=\text { Clearance. }
\end{gathered}
$$

to allow for misalignments, limit scraping of halo particles outside the beam core, reduce image charges, gas propagation times from the aperture to the beam, and other nonideal effects.

4) Evaluate choices made using higher-order theory numerical simulations etc. Iterate chores made to reoptimize when evaluating cost.

Effective application of this formulation requires extensive practical knowledge!

- Nonideal effects collective instabilities, halo, electron and gas interactions (species contamination), 1....

- Technology limits. Voltage breakdown, vacuum, superconducting magnets, ......

278 
S.M. Lond 6/

In practice, for intense beam transport, the emittance terms $\varepsilon_{x}$, $\varepsilon_{y}$ can often be neglected for. the purpose of obtaining simpler. sealing relations that are more easily understood.

$$
\begin{aligned}
& \lim _{\varepsilon_{x} \rightarrow 0} \delta_{x}=0 \\
& \lim _{\varepsilon_{y} \rightarrow 0} \delta_{y}=0
\end{aligned}
$$

$\Rightarrow$ Foll space charge depression

In this limit $Q \rightarrow Q_{\max }$, the maximum transportable perveance,

For our previous example for FODO guadrupoles; the $\varepsilon>0$ limit obtains:

$$
\begin{aligned}
& \lim _{\varepsilon \rightarrow 0} \operatorname{Max}\left[\Gamma_{x m}\right]=r_{b}\left\{1+\frac{4|\hat{\kappa}| L^{2}}{\pi^{3}} \sin \left(\frac{\pi}{2} \eta\right)\right\} \\
& \lim _{\varepsilon \rightarrow 0} Q=Q_{\text {max }}=\frac{2}{\pi^{2}}\left[\frac{\sin \left(\frac{\pi}{2} \eta\right)^{2}}{\left(\frac{\pi}{2} \eta\right)}\right] \eta^{2} \hat{R}^{2} L^{2} r_{b}^{2}
\end{aligned}
$$

279 
S.M. Lond

7/

Unfortunately, the method introduced before are inadequate for lattices with lesser degrees of symmetry such as syncopated quadrupole doublet lattices. However, methods introduced by Lee [E.P .Lee, Physics of Plasmas 9, 4301 (z00z)]. can be applied in this situation and also obtain more accurate results. It is beyond the scope of this class to carry out derivations with these methods, but we summarize results derived.

Quadrupole Doublet Lattice

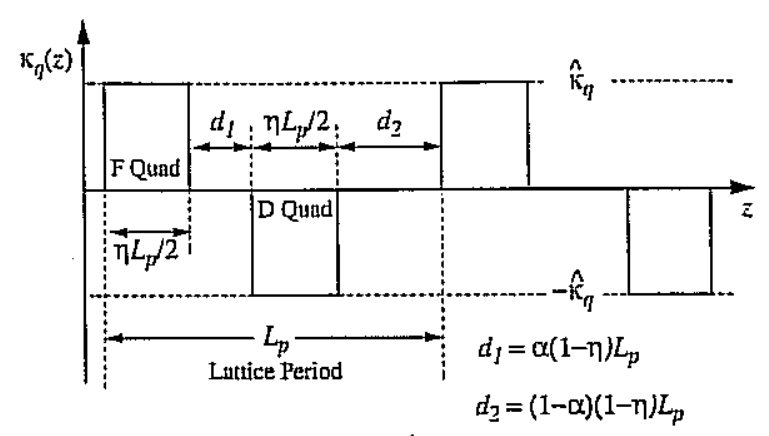

Denote:

Avg Radius: $\quad \overline{\Gamma_{m}}=\int_{0}^{L_{p}} \frac{d s}{L_{p}} \Gamma_{x m}(s)=\int_{0}^{L_{p}} \frac{d s}{L_{p}} \Gamma_{y m}(s)$

$\operatorname{Max}$ Excursion: $\operatorname{Max}\left[\Gamma_{m}\right] \equiv \operatorname{Max}\left[\Gamma_{x m}, r_{y m}\right]$ in period

280 


$$
\cos \sigma_{0}=1-\frac{\left(\eta \widehat{\kappa_{q}} L_{p}^{2}\right)^{2}}{32}\left[\left(1-\frac{2}{3} \eta\right)-4\left(\alpha-\frac{1}{2}\right)^{2}(1-\eta)^{2}\right]
$$

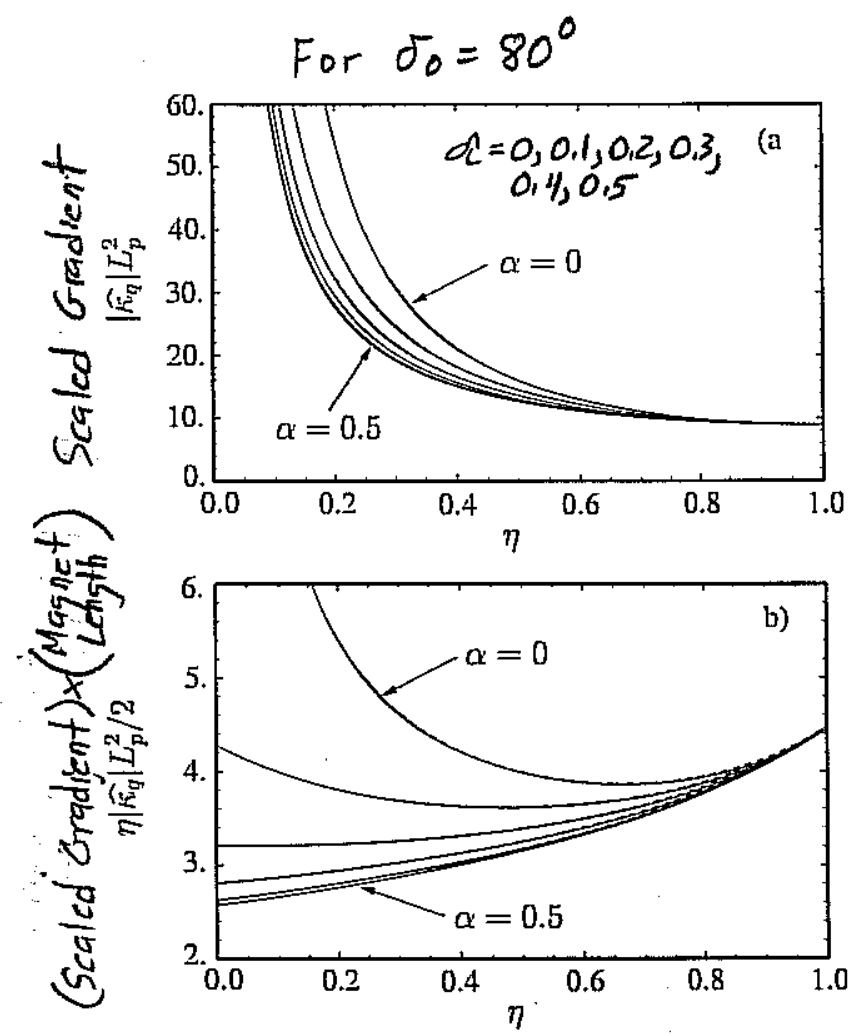

Envelope Flutter

$$
\frac{\operatorname{Max}\left[r_{m}\right]}{\overline{r_{m}}}-1=\frac{\left(1-\cos \sigma_{0}\right)^{1 / 2}(1-\eta / 2)\left[1-4(\alpha-1 / 2)^{2}(1-\eta)^{2}\right]}{2^{3 / 2}\left[(1-2 \eta / 3)-4(\alpha-1 / 2)^{2}(1-\eta)^{2}\right]^{1 / 2}}
$$

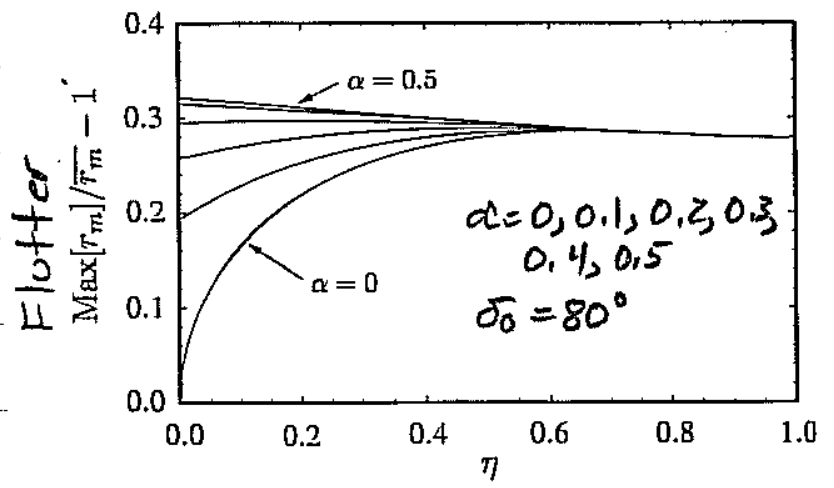


9)

Relations Connecting Max Transportable Perveance Qmax and Lattice Parameters

$$
\begin{aligned}
& Q_{\max }=\frac{\left(1-\cos \sigma_{0}\right)^{1 / 2}}{2^{3 / 2}} \frac{\eta\left[(1-2 \eta / 3)-4(\alpha-1 / 2)^{2}(1-\eta)^{2}\right]^{1 / 2}}{\left(\operatorname{Max}\left[r_{m}\right] / \overline{r_{m}}\right)^{2}}\left|\widehat{\kappa_{q}}\right| \operatorname{Max}\left[r_{m}\right]^{2} \\
& =\frac{\left(1-\cos \sigma_{0}\right)^{1 / 2}}{2^{3 / 2}} \frac{\eta\left[(1-2 \eta / 3)-4(\alpha-1 / 2)^{2}(1-\eta)^{2}\right]^{1 / 2}}{\left\{1+\frac{\left(1-\cos \sigma_{0}\right)^{1 / 2}(1-\eta / 2)\left[1-4(\alpha-1 / 2)^{2}(1-\eta)^{2}\right]}{2^{9 / 2}\left[(1-2 \eta / 3)-4(\alpha-1 / 2)^{2}(1-\eta)^{2}\right]^{1 / 2}}\right\}^{2}}\left|\widehat{\hbar_{q}}\right| \operatorname{Max}\left[r_{m}\right]^{2} . \\
& \frac{\operatorname{Max}\left[r_{m}\right]}{L_{p}}=\sqrt{\frac{Q_{\operatorname{mnx}}}{2\left(1-\cos \sigma_{0}\right)}}\left(\frac{\operatorname{Max}\left[r_{m}\right]}{\bar{r}_{m}}\right) \\
& =\sqrt{\frac{Q_{\max }}{2\left(1-\cos \sigma_{0}\right)}}\left\{1+\frac{\left(1-\cos \sigma_{0}\right)^{1 / 2}(1-\eta / 2)\left[1-4(\alpha-1 / 2)^{2}(1-\eta)^{2}\right]}{2^{3 / 2}\left[(1-2 \eta / 3)-4(\alpha-1 / 2)^{2}(1-\eta)^{2}\right]^{1 / 2}}\right\},
\end{aligned}
$$

Derivation and application of scaling relations can be complicated. They are often applied in systems codes to generate plots that can be interpreted more readily,

282 


\section{John Barnard Steven Lund USPAS \\ June 2008}

Mismatched Beams and Beam Halo

Envelope modes of beams in continuous focusing

Envelope modes of bunched beams in continuous focusing

Halos from mismatched beams 
Barmiall

(2)

Smooth Focusing Envelope MODES

If $\frac{d}{d s} \gamma_{\beta}=0 \& K(s)=k_{p 0}^{2}=$ constant \& $\epsilon_{x}=\epsilon_{y}$

$$
\begin{array}{r}
\Rightarrow \quad r_{x}^{\prime \prime}+k_{p 0}^{2} r_{x}-\frac{2 Q}{r_{x}+r_{y}}-\frac{\epsilon^{2}}{r_{x}^{3}}=0 \\
r_{y}^{\prime \prime}+k_{p 0}^{2} r_{y}-\frac{2 Q}{r_{x}+r_{y}}-\frac{\epsilon^{2}}{r_{y}^{3}}=0
\end{array}
$$

THE EQUILIBRIUM OCCURS WHEN $r_{x}^{\prime \prime}=r_{y}^{\prime \prime}=0$

$$
\begin{aligned}
& \& r_{x}=r_{y}=r_{b} \\
& \Rightarrow k_{p 0}^{2} r_{b}-\frac{Q}{r_{b}}-\frac{\epsilon^{2}}{r_{b}^{3}}=0
\end{aligned}
$$

ThIS is easily souter for $r_{b}$ :

$$
r_{b}=\frac{Q^{1 / 2}}{k_{p_{0}}}\left[\frac{1}{2}+\frac{1}{2} \sqrt{1+4 k_{0}^{2} \epsilon^{2} / Q^{2}}\right]^{1 / 2} \rightarrow\left\{\begin{array}{l}
\frac{Q^{1 / 2}}{k_{p_{0}}} ; \frac{2 k_{0} \epsilon}{Q} \ll 1 \\
\frac{\epsilon^{1 / 2}}{k_{p_{0}}^{1 / 2}} ; \frac{2 k_{0} \epsilon}{Q} \gg 1
\end{array}\right.
$$

Let $\quad r_{x}=r_{b}+\xi(s)$

$$
r_{y}=r_{b}+\eta(s)
$$

284 
Bi+unath (3)

LINEARIZING ( 51 )

$$
\begin{aligned}
& (s 1) \Rightarrow 0=\xi^{\prime \prime}+k_{p_{0}}^{2}\left(r_{b}+\xi\right)-\frac{2 Q}{2 r_{b}}\left(1-\frac{\xi}{2 r_{b}}-\frac{\eta}{2 r_{0}}\right)-\frac{\epsilon^{2}}{r_{b}^{3}}\left(1-\frac{3 \xi}{r_{0}}\right) \\
& 0=\eta^{4}+k_{p_{0}}^{2}\left(r_{b}+\eta\right)-\frac{2 Q}{2 v_{b}}\left(1-\frac{\xi}{2 r_{b}}-\frac{\eta}{2 v_{b}}\right)-\frac{\epsilon^{2}}{\eta_{b}^{3}}\left(1-\frac{3 \eta}{v_{b}}\right)
\end{aligned}
$$

$$
\begin{aligned}
& \xi^{\prime \prime}+\left(k_{p o}^{2}+\frac{Q}{2 v_{b}^{2}}+\frac{3 \epsilon^{2}}{n_{b}^{4}}\right) \xi+\frac{Q}{2 n_{b}^{2}} \eta=0 \\
& \eta^{\prime \prime}+\left(k_{p_{0}}+\frac{Q}{2 V_{b}^{2}}+\frac{3 \epsilon^{2}}{n_{b}^{4}}\right) \eta+\frac{Q}{2 n_{b}^{2}} \xi=0
\end{aligned}
$$

$U \sin \theta \quad k_{\beta}^{2}=k_{p o}^{2}-\frac{Q}{r_{b}^{2}}=\frac{\epsilon^{2}}{r_{b}^{4}}$

$$
\begin{array}{r}
\Rightarrow \xi^{\prime \prime}+\left(\frac{3}{2} k_{p 0}^{2}+\frac{5}{2} k_{p}^{2}\right) \xi+\left(\frac{1}{2} k_{p o}^{2}-\frac{1}{2} k_{p}^{2}\right) \eta=0 \\
\eta^{u}+\left(\frac{3}{2} k_{p 0}^{2}+\frac{5}{2} k_{p}^{2}\right) \eta+\left(\frac{1}{2} k_{p o}^{2}-\frac{1}{2} k_{p}^{2}\right) \xi=0 .
\end{array}
$$

LET $\varphi_{1}=\xi-\eta$ SUBtRACTNG (B) From (A)

$$
\rho_{1}^{\prime \prime}+k_{1}^{2} \rho_{1}=0
$$

where $k_{1}^{2}=\left(\frac{3}{2} k_{\beta_{0}}^{2}+\frac{5}{2} k_{p}^{2}-\frac{1}{2} k_{p_{0}}^{2}+\frac{1}{2} k_{\beta}^{2}\right)=k_{\beta 0}^{2}+3 k_{\beta}^{2}$

LET $\varphi_{2}=\xi+\eta \quad \operatorname{ADONO} G$ (A) \& (B):

$$
\varphi_{2}^{\prime \prime}+k_{2}^{2} \varphi_{2}=0
$$

where $k_{2}^{2}=\left(\frac{3}{2} k_{10}^{2}+\frac{5}{2} k_{\beta}^{2}+\frac{1}{2} k_{\beta 0}^{2}-\frac{1}{2} k_{\beta}^{2}\right)=2 k_{\beta 0}^{2}+2 k_{\beta}^{2}$

285 
(3.5)

Letting $\xi=\xi_{0} e^{i k_{1} s} \quad \eta=\eta_{0} e^{i k, s}$ where $k_{1}^{2}=k_{p o}^{2}+3 k_{p}^{2}$

(A) $\Rightarrow$

$$
\begin{aligned}
& -\left(k_{p o}^{2}+4 k_{j}^{2}\right] \xi_{0}+\left(\frac{3}{2} k_{p 0}^{2}+\frac{5}{2} k_{\beta}^{2}\right) \xi_{0}+\left(\frac{1}{2} k_{p o}^{2}-\frac{1}{2} k_{p}^{2}\right) \eta_{0}=0 \\
& \Rightarrow \quad \frac{1}{2}\left(k_{p-1}^{2}-k_{\beta}^{2}\right)\left[\xi_{0}+\eta_{0}\right]=0 \quad\left(A^{\prime}\right)
\end{aligned}
$$

$(\beta)$

$$
\begin{aligned}
& -\left(k_{p_{0}}^{2}+3 k_{p_{1}}^{2}\right) \eta_{0}+\left(\frac{3}{2} k_{p_{0}}^{2}+\frac{5}{2} k_{p}^{2}\right) \eta_{0}+\left(\frac{1}{2} k_{p_{0}}^{2}-\frac{1}{2} k_{p}^{2}\right) \xi_{0}=0 \\
& \Rightarrow \frac{1}{2}\left(k_{p o}^{2}-k_{p}^{2}\right)\left[\xi_{0}+\eta_{0}\right]=0 \quad\left(B^{\prime}\right)
\end{aligned}
$$

Simicamy for $\xi=\xi_{0} e^{i k_{2} s}$ and $y=\eta_{0} e^{i k_{2} s}$

$$
\begin{aligned}
& (A) \Rightarrow \\
& -\left(2 k_{p 0}^{2}+2 k_{p}^{2}\right) \xi_{0}+\left(\frac{3}{2} k_{p o}^{2}+\frac{5}{2} k_{p}^{2}\right) \xi_{0}+\left(\frac{1}{2} k_{p 0}^{2}-\frac{1}{2} k_{p}^{2}\right) \eta_{0}=0 \\
& \Rightarrow \frac{1}{2}\left(k_{p 0}^{2}-k_{p}^{2}\right)\left[-\xi_{0}+\eta_{0}\right]=0 \quad\left(A^{\prime \prime}\right) \\
& (B) \Rightarrow \\
& -\left(2 k_{p 0}^{2}+2 k_{p}^{2}\right) \eta_{0}+\left(\frac{3}{2} k_{p 0}^{2}+\frac{5}{2} k_{p}^{2}\right) \eta_{0}+\left(\frac{1}{2} k_{p 0}^{2}-\frac{1}{2} k_{p}^{2}\right) \xi_{0}=0 \\
& \frac{1}{2}\left(k_{p 0}^{2}-k_{p o}^{2}\right)\left[-\eta_{0}+\xi_{0}\right]=0 \quad\left(B^{\prime \prime}\right)
\end{aligned}
$$

286 
Banalatel 4

THE SOLUTIONS ARE:

$$
y_{1} \sim e^{i k_{1} s} \Rightarrow \xi \leqslant \eta \sim e^{i k_{1} s}
$$

EQUATIONS (A') \& (B') CAN OE WRITTEN

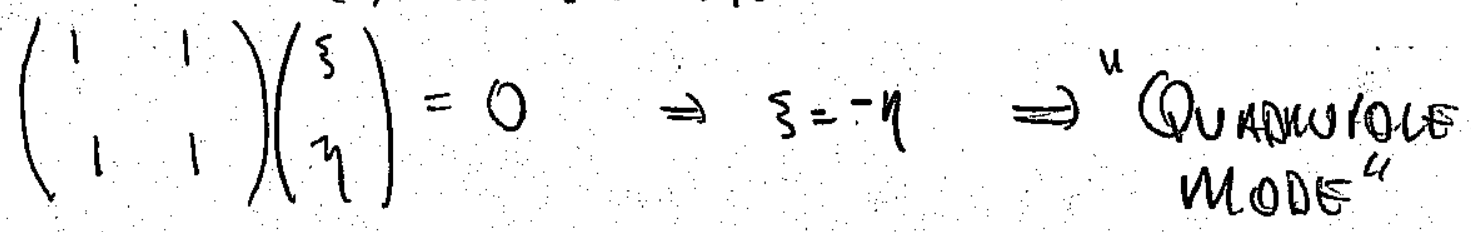

For $J_{2} \sim e^{i k_{2} S}$ THE MARL $=\left(A^{\prime \prime}\right) d\left(B^{\prime \prime}\right)$

$$
\left(\begin{array}{cc}
-1 & 1 \\
1 & -1
\end{array}\right)\left(\begin{array}{l}
\xi \\
\eta
\end{array}\right)=0 \Rightarrow \xi=\eta \Rightarrow \begin{gathered}
\text { BREATHING } \\
\text { MAE" }
\end{gathered}
$$

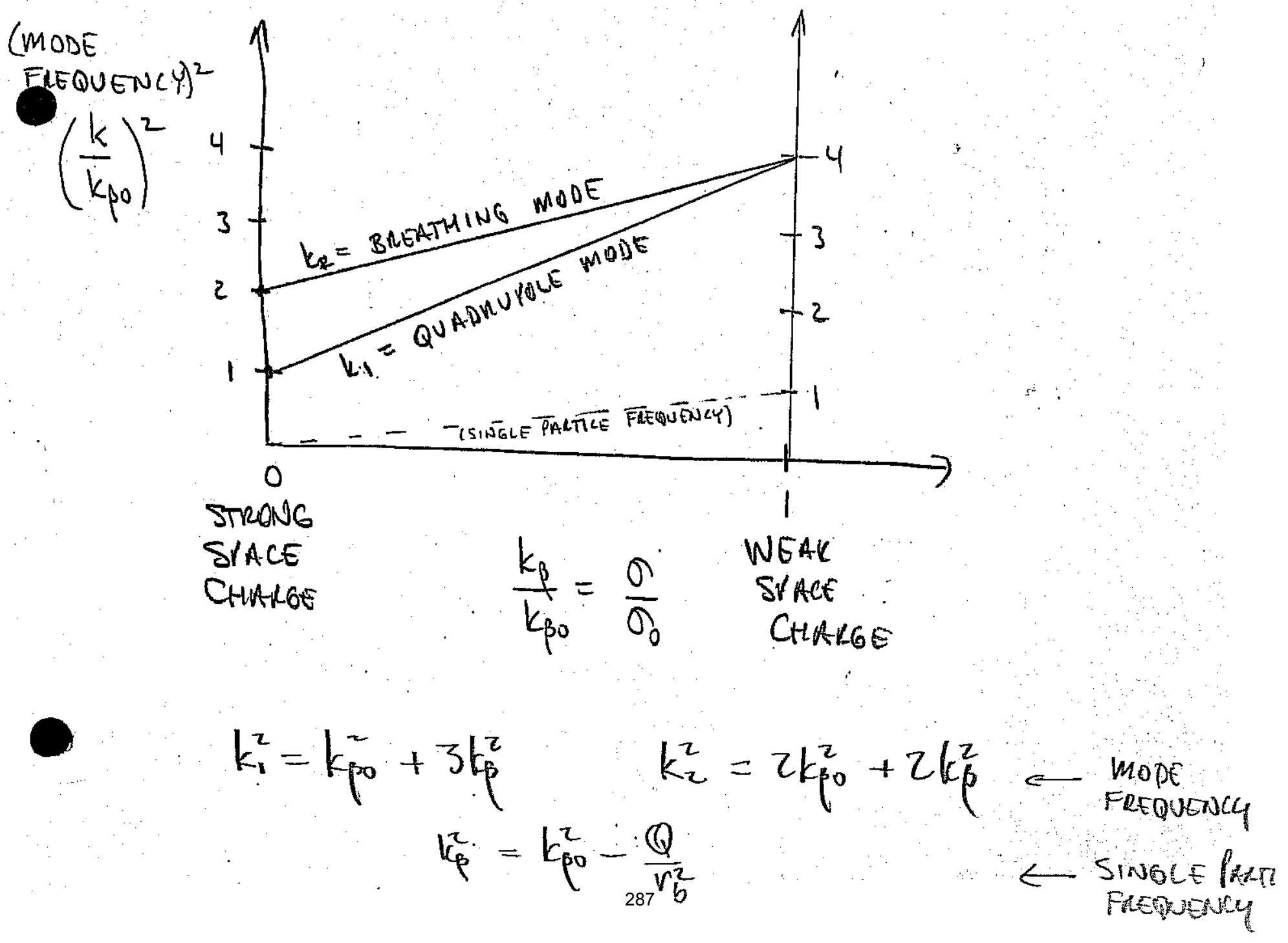




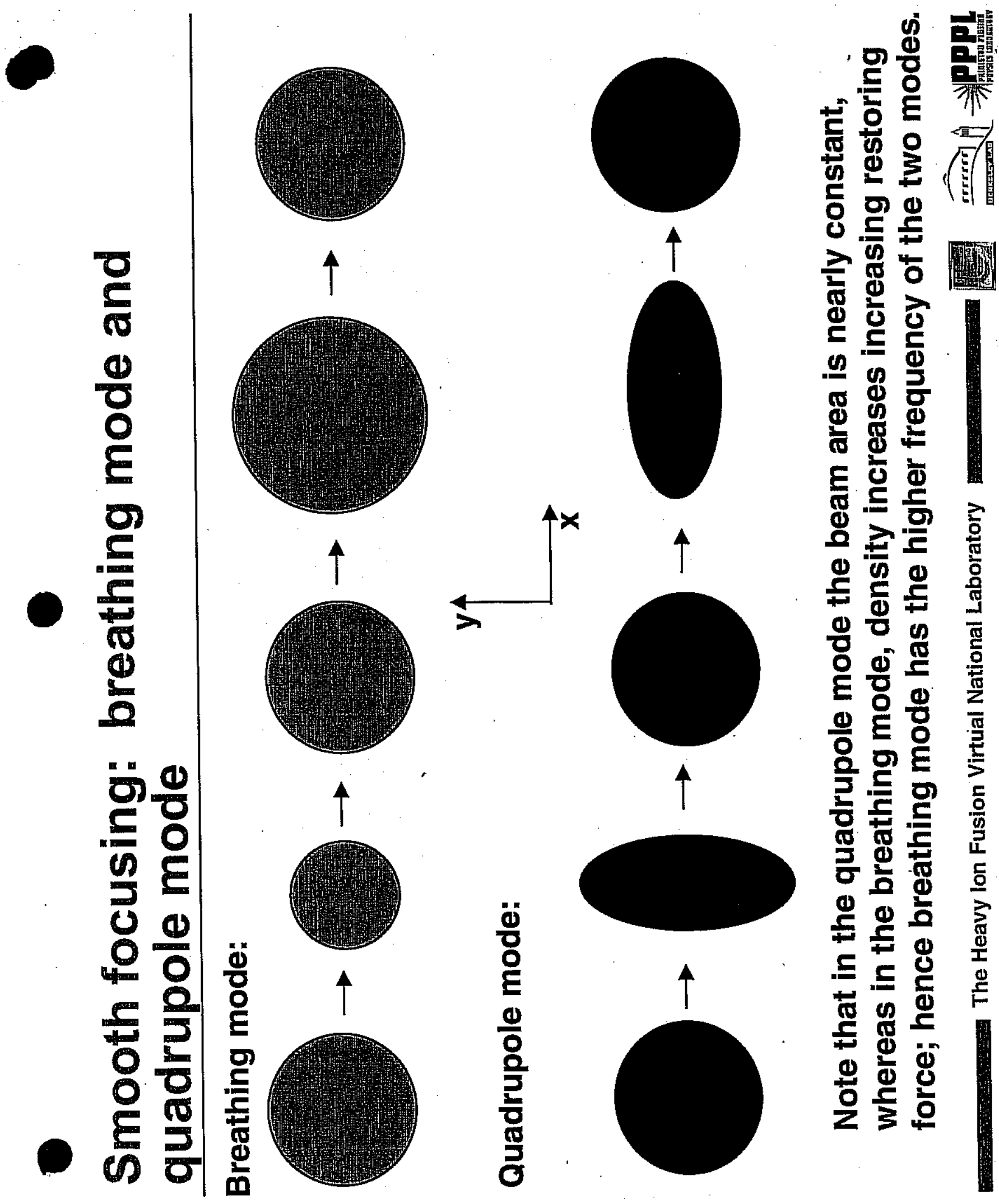


TOHNBHNALS

I STEVGN LUND

USits

JANUKYY 2004

II GNUELOTE MODES OR BuNGHED BaHMS

IN CONTHUONUI FOCUIING CHANNEZS 

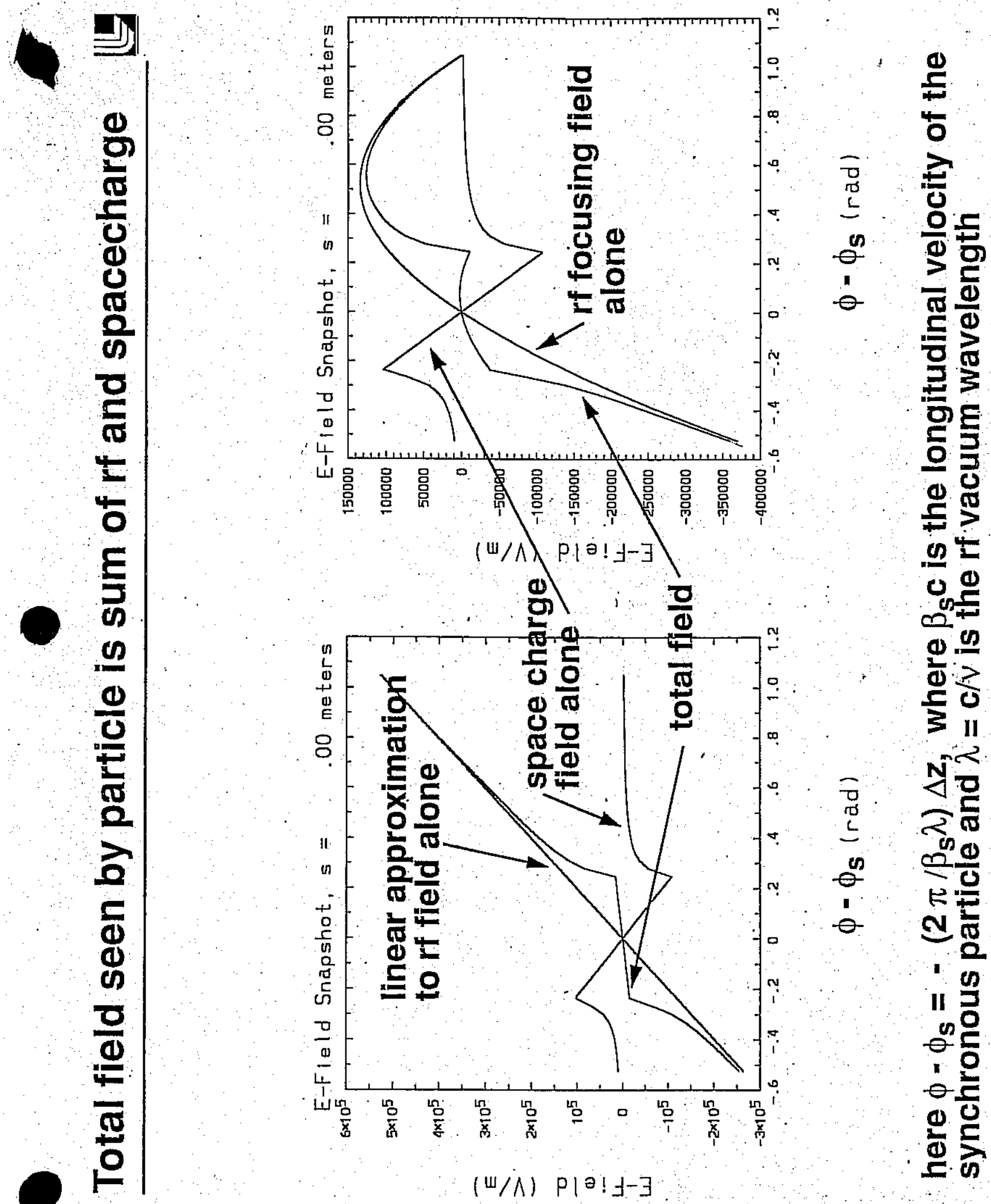
(8)

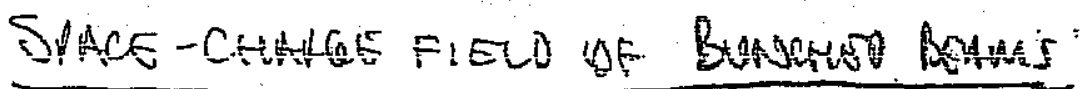

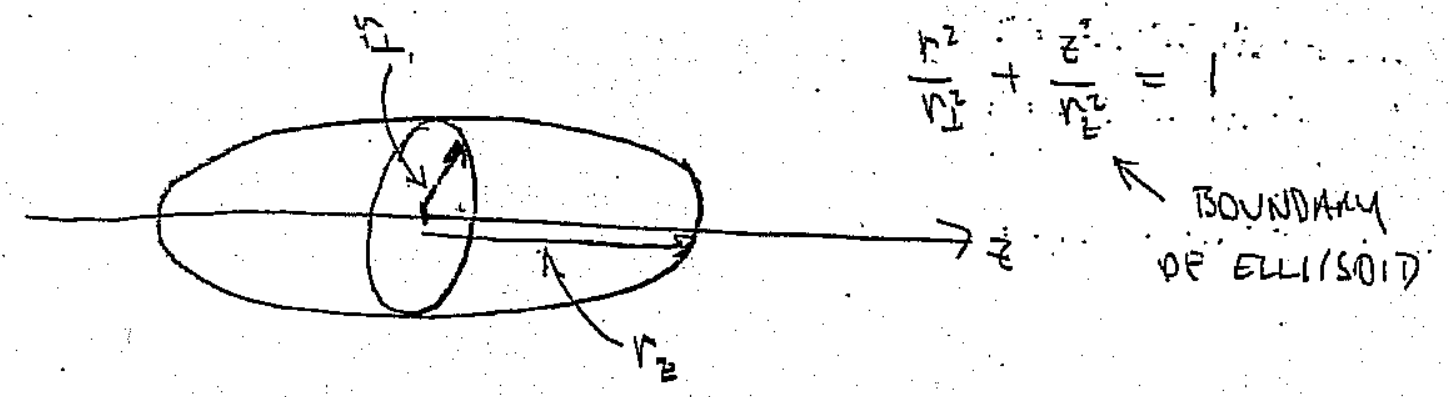

The rotentiac de a un ieO wim density bunch in fire siace

(a macLRURIN SPHEROID) IS GIVEN by:

$$
\varphi=\frac{\rho}{4 \varepsilon_{0}}\left(\alpha_{i i} r^{2}+\alpha_{11} z^{2}-\delta\right)
$$

Coff Landaus Lifskity, Classical Thesy of Fiers, 1.297$)$

where $\alpha_{L}=r_{\perp}^{2} r_{E} \int_{0}^{\infty} \frac{d s}{\left(\dot{x}_{1}^{2}+s\right) \Delta}$

$$
\begin{aligned}
& \alpha_{11}=r_{L}^{2} r_{z} \int_{0}^{\infty} \frac{d s}{\left(r_{z}^{2}+5\right) \Delta} \\
& \delta=r_{\perp}^{2} r_{z} \int_{0}^{\infty} \frac{d s}{\Delta}
\end{aligned}
$$

where $\Delta^{2}=\left(r_{1}^{2}+5\right)^{2}\left(r_{2}^{2}+5\right)$

FOX NON-LELATVISTIC SERM:

$$
\begin{aligned}
& E_{z}=-\frac{\partial \phi}{\partial z}=f \frac{\rho}{\varepsilon_{0}} z \\
& E_{r}=\frac{-\partial d}{\partial r}=\frac{(1-f)}{2} \frac{f}{f_{0}} r
\end{aligned}
$$

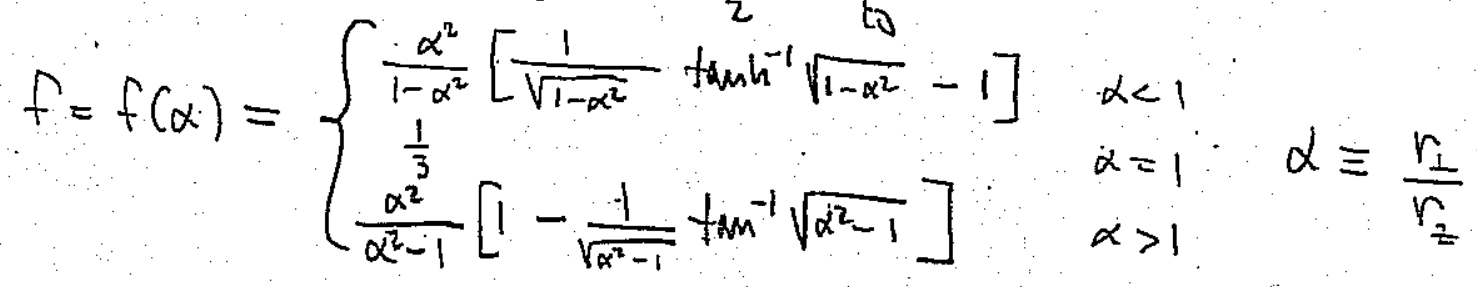


(9)

For heUATVISTIC BEAM

(cf, Lund BARENARD 1997)

$$
\begin{aligned}
& \frac{d^{2} x_{L}}{d s^{2}}=\frac{F_{L}}{\gamma, s_{s}^{2} m c^{2}} \\
& F_{1 s}=\frac{-q}{Y_{s}^{2}} \frac{\partial \phi}{\partial X_{L}}=\frac{9 \rho}{2 \gamma_{1}^{2} \epsilon_{0}}\left[1-f\left(x_{0}\right)\right] \underline{X_{L}} \\
& \frac{d^{2} \Delta z}{d s^{2}}=\frac{F_{z}}{Y_{s}^{3} p_{r}^{2} m c^{2}} \\
& F_{z s}=\frac{-q}{\gamma_{s}^{2}} \frac{\partial \phi}{\partial z}=\frac{q p}{\epsilon_{0}} f(\alpha) \Delta z \\
& \alpha=\frac{r_{\perp}}{\gamma r_{z}} \quad\left[\alpha=\frac{r_{\perp}}{\left(r_{z} \text { in comoving thame }\right)}\right]
\end{aligned}
$$

Combineng Focusing + SELF FIELdS

$$
\begin{aligned}
& \frac{d^{2}}{d s^{2}} \Delta z=-k_{s 0}^{2} \Delta z+\frac{g \rho f(\alpha)}{\gamma_{s}^{3} \beta_{s}^{2} m c^{2} \varepsilon_{0}} \Delta z \quad \text { (unszarc) } \\
& \frac{d^{2}}{d s^{2}} \underline{x}_{1}=-k_{0}^{2} \underline{x}_{1}+\frac{q \rho\left[1-f \cdot\left(\alpha^{\prime}\right)\right]}{2 \gamma_{s}^{3} \beta_{s}^{2} m c^{2} \varepsilon_{0}} x_{1} \\
& \rho=\frac{3 I \lambda_{r f}}{4 \pi r_{\perp}^{2} r_{z} c}
\end{aligned}
$$

292 


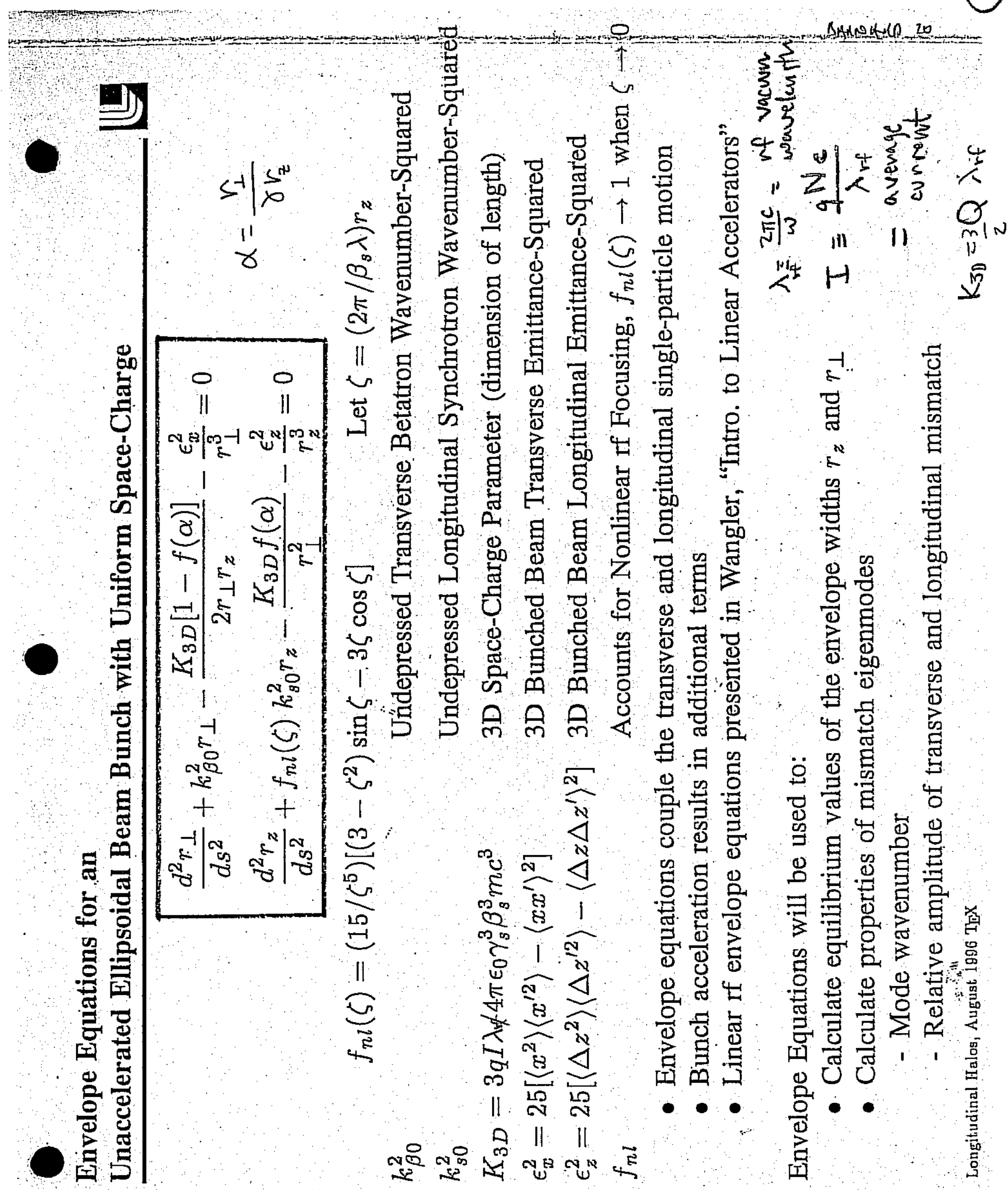




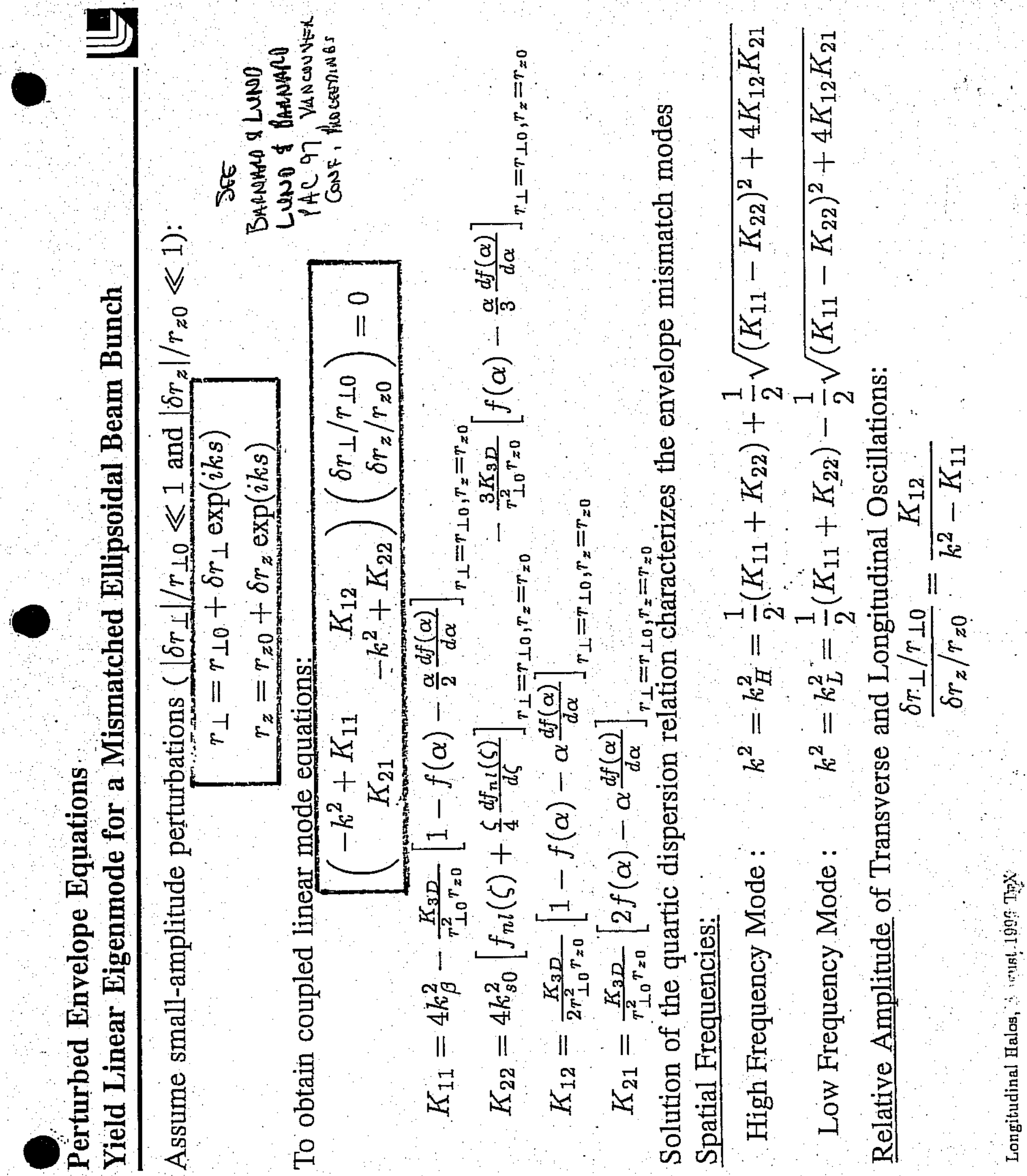


H
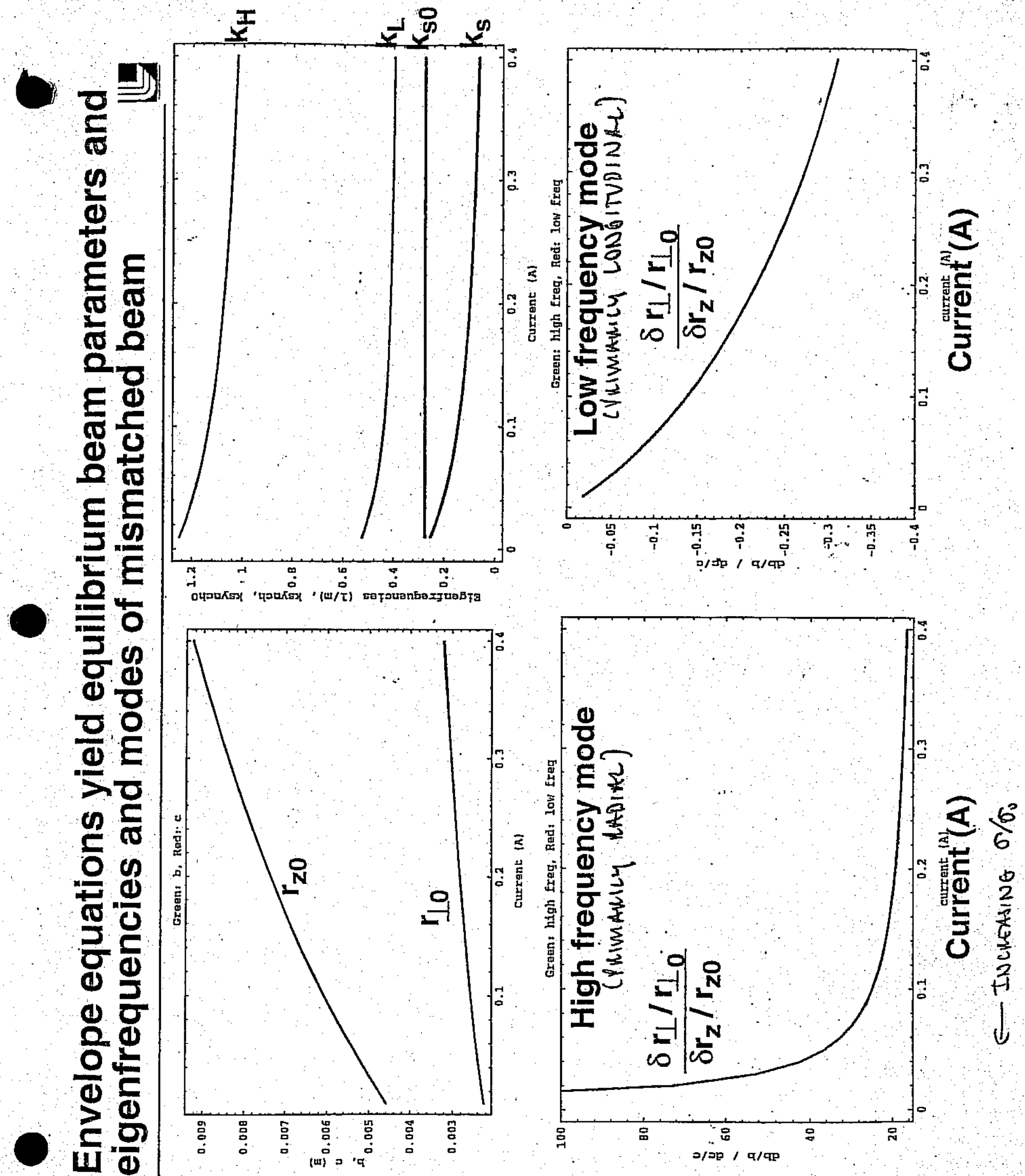
JoHN BAKNGMD

(13)

\$ STEVEN LUNA USPAS JanNa 2004

III HALOS

1. What is Halo? Why do we care?

2. Qualitative picture or halo formation: mismatches resonate dave particles to large amplitude

3. core/parttcle models

4. Amrlitude/Puase Analysis

296 


\section{FULLY SELF-CONS ISTENT PIC CODE RESULTS}

From

"Bexm Loss $\$$ Bexm

HALO SUVDes" RODOH RYNE, 1995

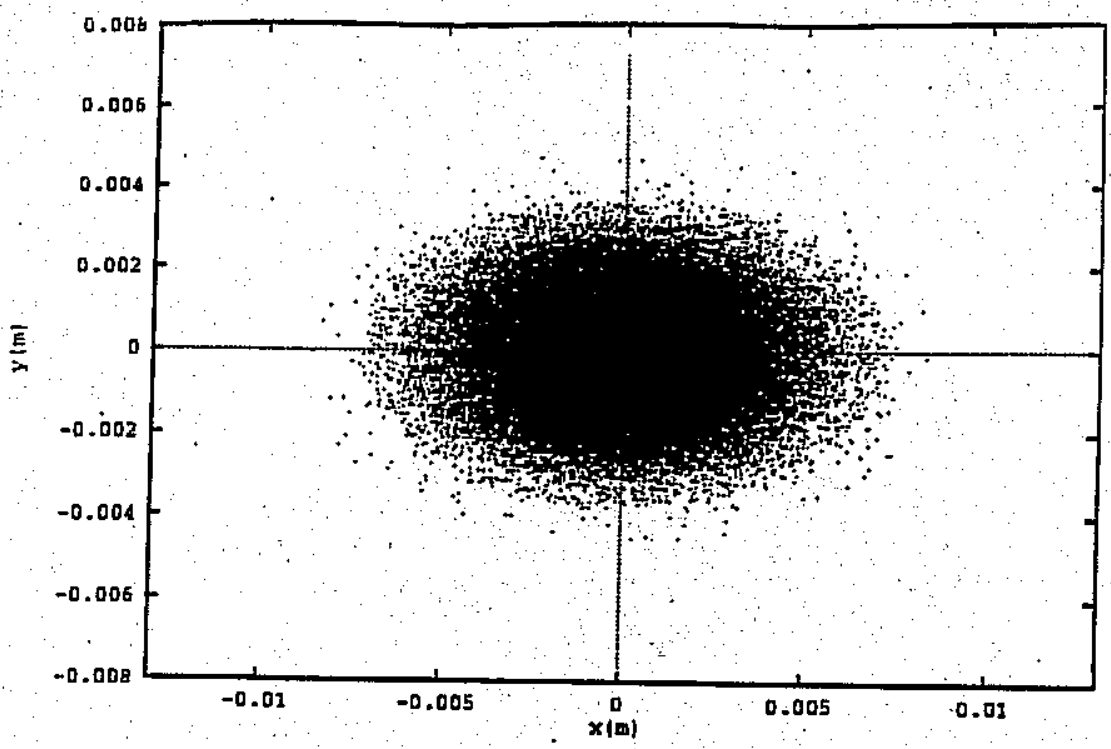

MATCHED Bexm

Figure 5: Beam halo after 22 focusing periods in a FODO channel. The initial distribution is an $r m s$ matched Gaussian beam. ( $\left.\sigma_{0}=70 \mathrm{deg}, \sigma=35 \mathrm{deg}\right)$

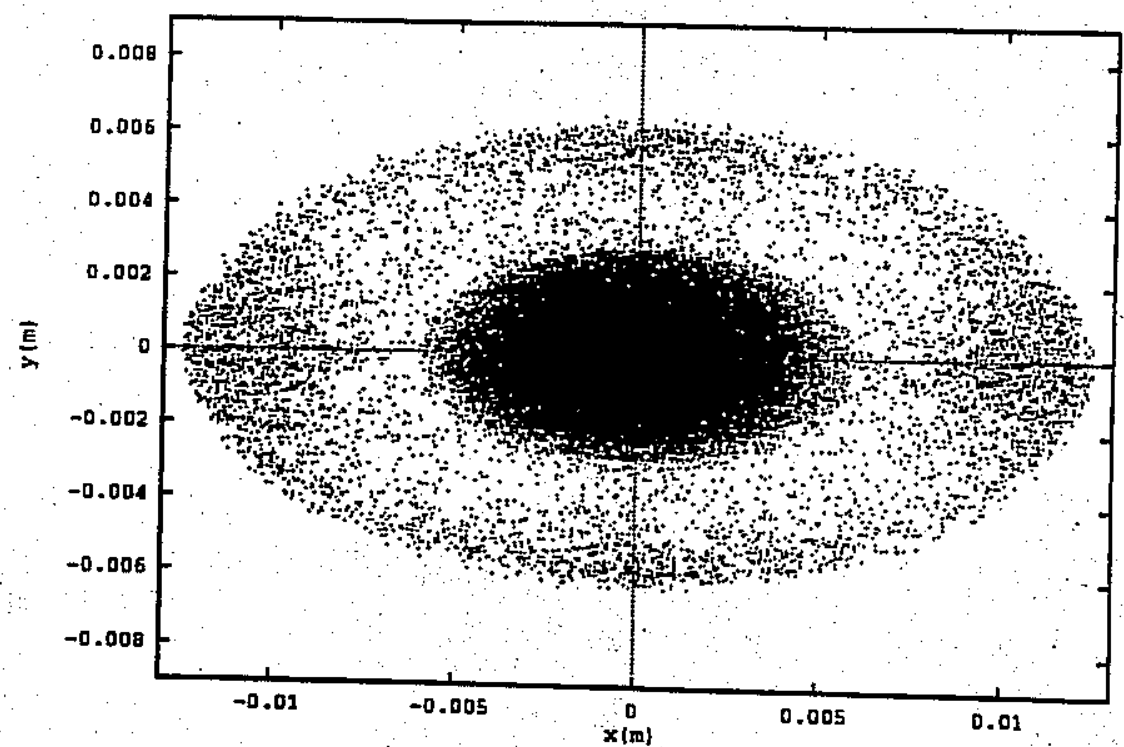

mismiatziOO

DEAM

Figure 6: Beam halo after 22 focusing periods in a FODO channel. The initial distribution is an rms mismatched Gaussian beam. ( $\left.\sigma_{0}=70 \mathrm{deg}, \sigma=35 \mathrm{deg}\right)$ 
BAINGHD (15)

WHY Y DO WE CARE?

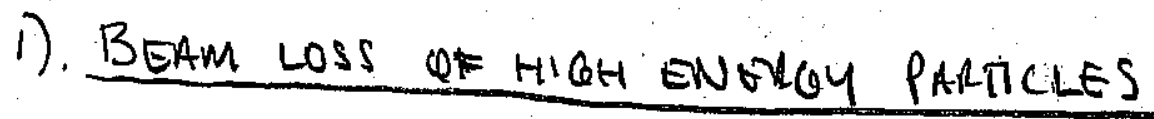
$\Rightarrow$ ACCELERATOR ACTUATION

EXAMPLE: For tHE $1 \mathrm{GeV}, 100 \mathrm{~mA}$ Accelerator PRODUCtION OF TRITIUM (APT) PANHMETRS $1 \mathrm{nA} / \mathrm{m}$ of BEAm LOSS ALLOWED FON

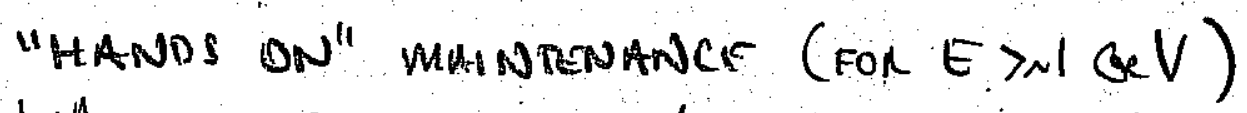
$\left(\frac{\ln A}{m} m \times 1000 m \Rightarrow 10^{-6}\right.$ A loss allowed $)$

$$
\Rightarrow \frac{10^{-6} A}{0.1 A} \Rightarrow 10^{-5} \text { fractional beam loss }
$$
allowed!!

2). BEAM loss ON WALLS

$\Rightarrow$ ELETION/GAS EMISSION FROM WALLS

FOR LONG pULSE OR DC MACHINES, OR STORAGE LINGS, WHERE ELECTRONS CAN BE DRAWN INTO BEAM AND ACCUMULATION $\Rightarrow$ INSTABILITIES AND NONLINEAR FIELDS COULD DESTROY BEAM DMITTHNCS QR EVEN DISRUPT BEAM:

3). IncREASE in emitTANCE

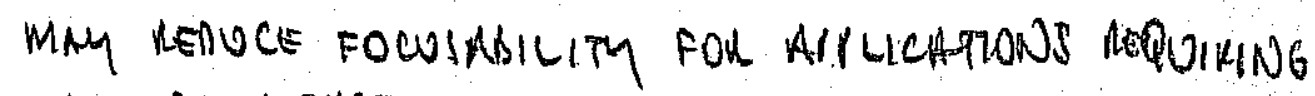
A SMALL DEAL SHOT.

298 
BATNGHC 16

What is THE BASIC YHYSICS?

RECALL DISPERSION RELATION FOR GNUELOVE MODES:
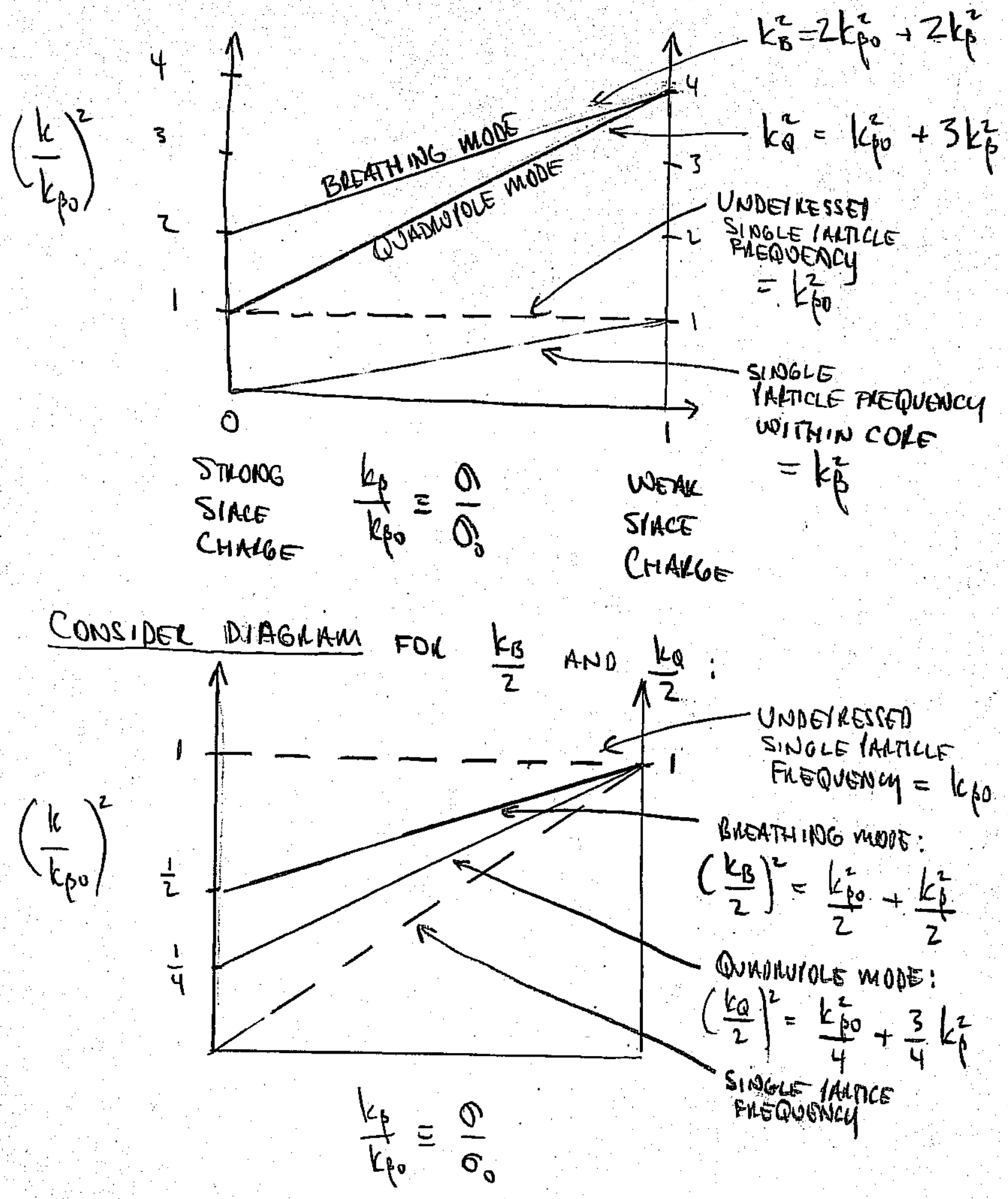

299 
BASCOALO (17)

Pantiles in orbits fall from cole oscillate AT FREQUENCY $k_{\text {BO }}$, WHEREAS THOSE WITH N CORE OSCILLATE at fRequency kP.

At intermediate raDiI, particle fREQUENCY = envelOP FrequencY $/ 2$

$\Rightarrow$ Possibility of Resonance

$\Rightarrow$ Mismatches (excitation or envelope MODES) TOGETHER WITH COMMENSURATE particle fRequency and environ hale-flequency ANE THE SOURCE OF HALO.

300 
BarNaRd (18)

Suggests using a core/ test particle mode TO EXPLORE THESE EFFECTS (O'CONNGL, WangleR, JAMESON, RUNE,...)

ASSUME KV RENVELOVE OSCILLATING AT a MODE FREQUENCY.

USE KNOWN ANALYTIC FORMULAS INTERIOR ANA EXTERIOR TO BEAM.

For eXAmPLE, for circular beAm

$$
x^{\prime \prime}= \begin{cases}-\left[k_{\beta_{0}}^{2}-\frac{\mathbb{Q}}{r_{b}^{2}}\right] x & \text { for } r<r_{b} \\ -\left[k_{\beta_{0}}^{2}-\frac{\mathbb{Q}}{r^{2}}\right] x & \text { for } r>r_{b}\end{cases}
$$

similarly for $y$.

$$
r_{b}=r_{b o}+\delta r_{b} \cos \left(k_{B} s+\phi\right)
$$

Distribute test particles in phase since AND FOLLOW EVOLUTION OF ORBITS. ALLOWS PHYSICS STUDIES, WHICH CAN BE FOLLWED OP WITH SELF-CONSISTENT PIC RUNS.

301 
BANNWLO (19)

NOTE THAT THE SAME TYKE OP RESONANCES OCCUR

LONGITUDINALLY, IN BUNCHED BEAMS.

BETATRON FREQUENCY $\rightarrow$ SYNCHROTRON FREQUENCY

$$
k_{\beta_{0}} \rightarrow k_{\text {so }}
$$

$k_{\beta} \rightarrow k_{S}$ (DEPAESST) SYNCALOTEOI FREQUENCY)

BREATHING
MODE $k_{B} \rightarrow k_{L}$ LOW

RESONANCE CONDITION

$$
k_{\beta}<\frac{k_{B}}{2}<k_{\beta 0} \quad \rightarrow \quad k_{s}<\frac{k_{L}}{2}<k_{s 0}
$$

ADDITIONAL COMPLICATION:

Mi

INTRINSIC NON-LINGARTY OF $r f$ BUCKET

302 


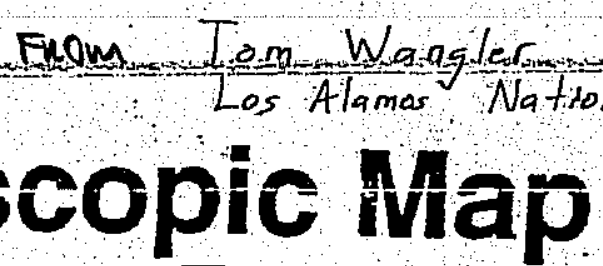 \\ Stroboscopic Map
(The Peanut Diagram)}

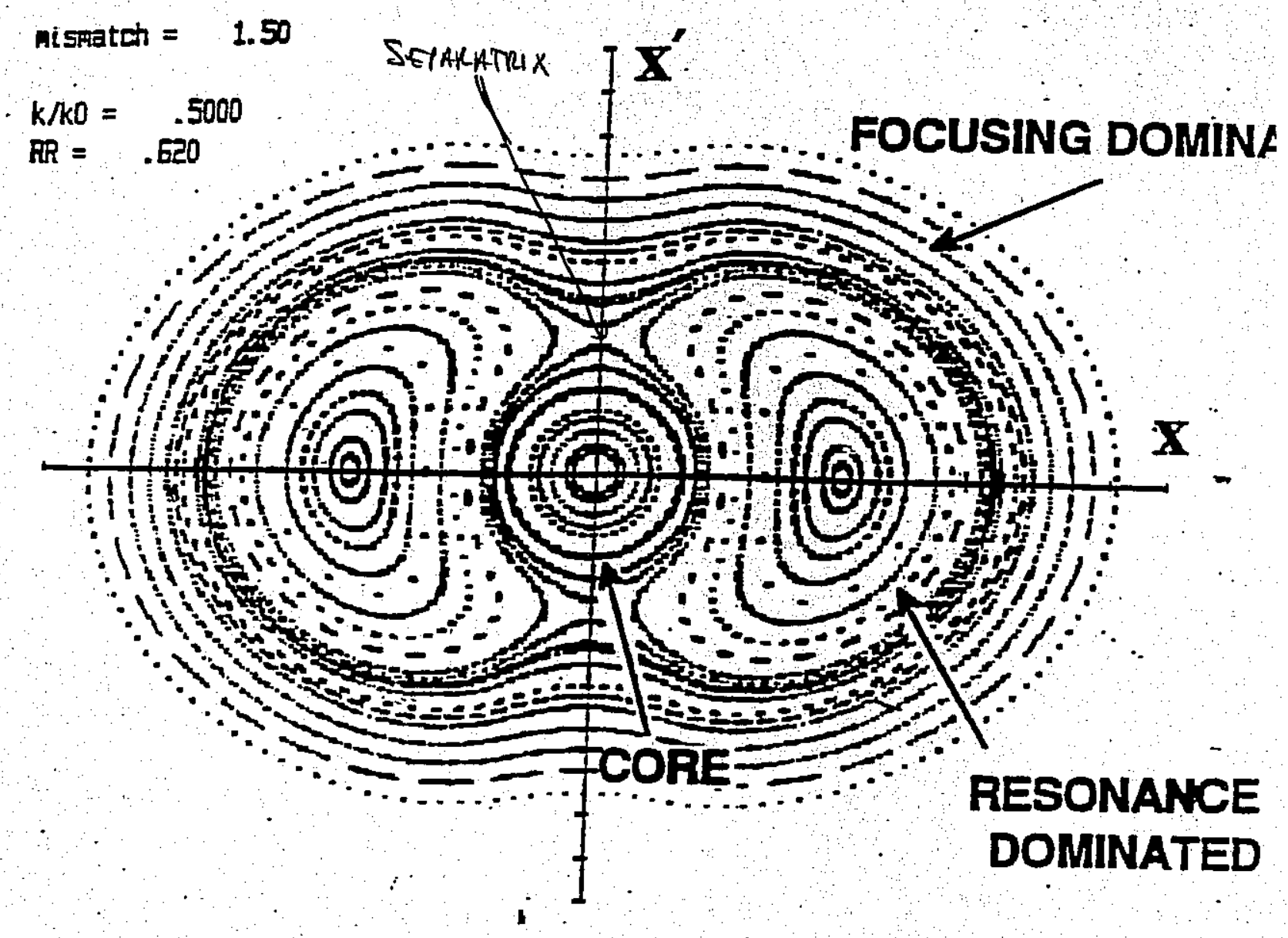

- Accumulate Many Snapshots of Phase Space Taken at Minimum Amplitude of Core Oscillation. Cox nuy pAtriculat

- Follow an Array of Particles to Obtain a "Trajectory Field".

- Regular rrajectories Appear as Smooth Curves.

- Chaotic Trajectories Appear as Stochastic Scatter.

- Initial positions of particles in maAs s/ace wolle equaly SPACE AlONG $X$ \& $X^{\prime}$ aXes. 

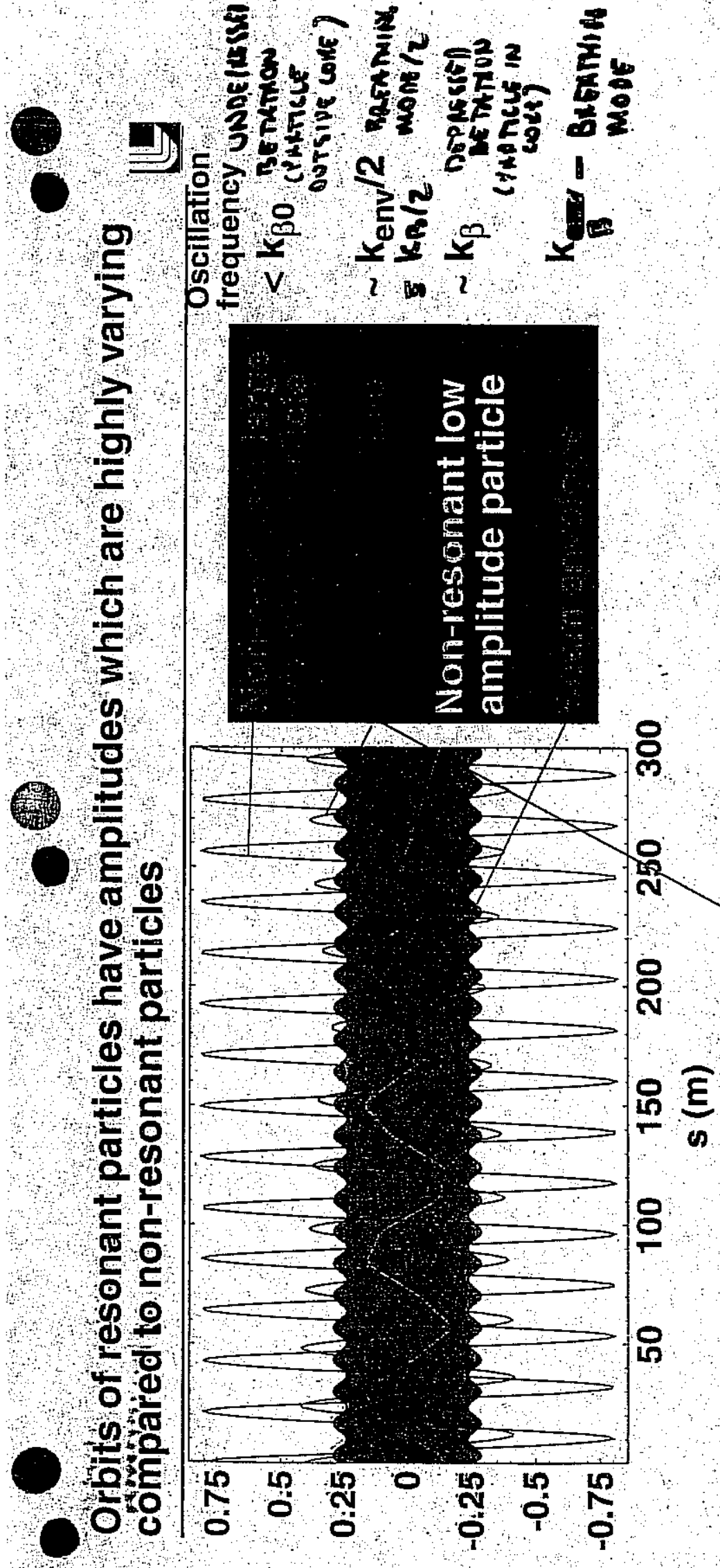

uolfisod- $x$

0

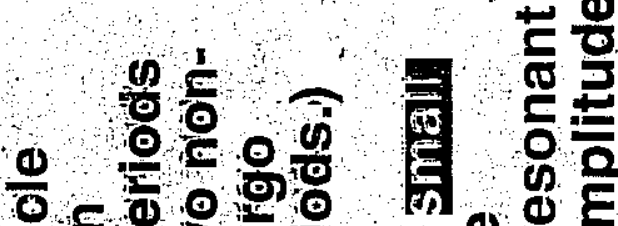

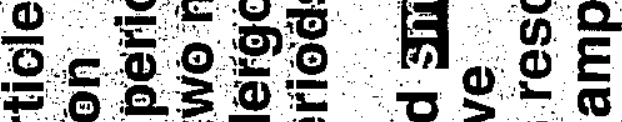
- 000

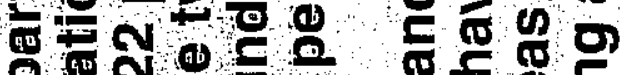
- HNEJE U

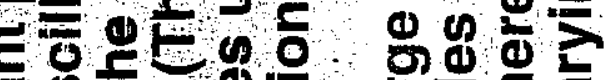
- $0=0+0$

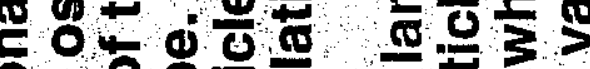

$0.9-40=0$

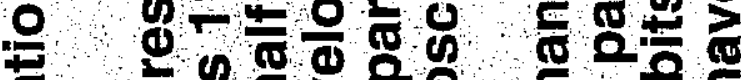

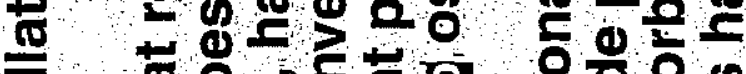

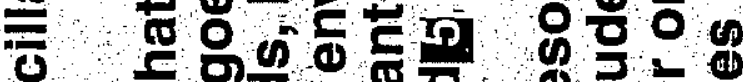

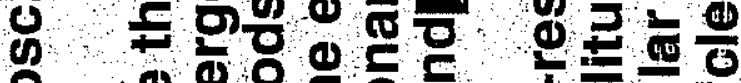

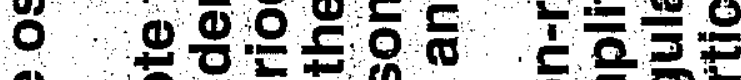
o 의워 ᄋ Z

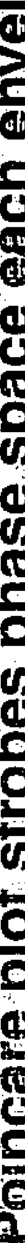

\begin{tabular}{l}
2 \\
\hline 0 \\
0 \\
0 \\
0 \\
0 \\
0 \\
0 \\
0 \\
0 \\
0 \\
0 \\
0 \\
0 \\
0 \\
0 \\
0 \\
0 \\
0 \\
0 \\
0 \\
0 \\
0
\end{tabular}

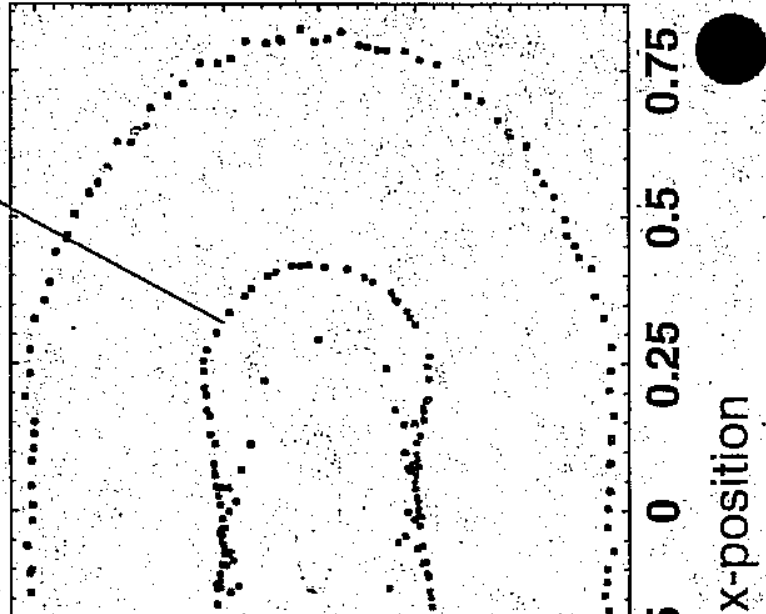




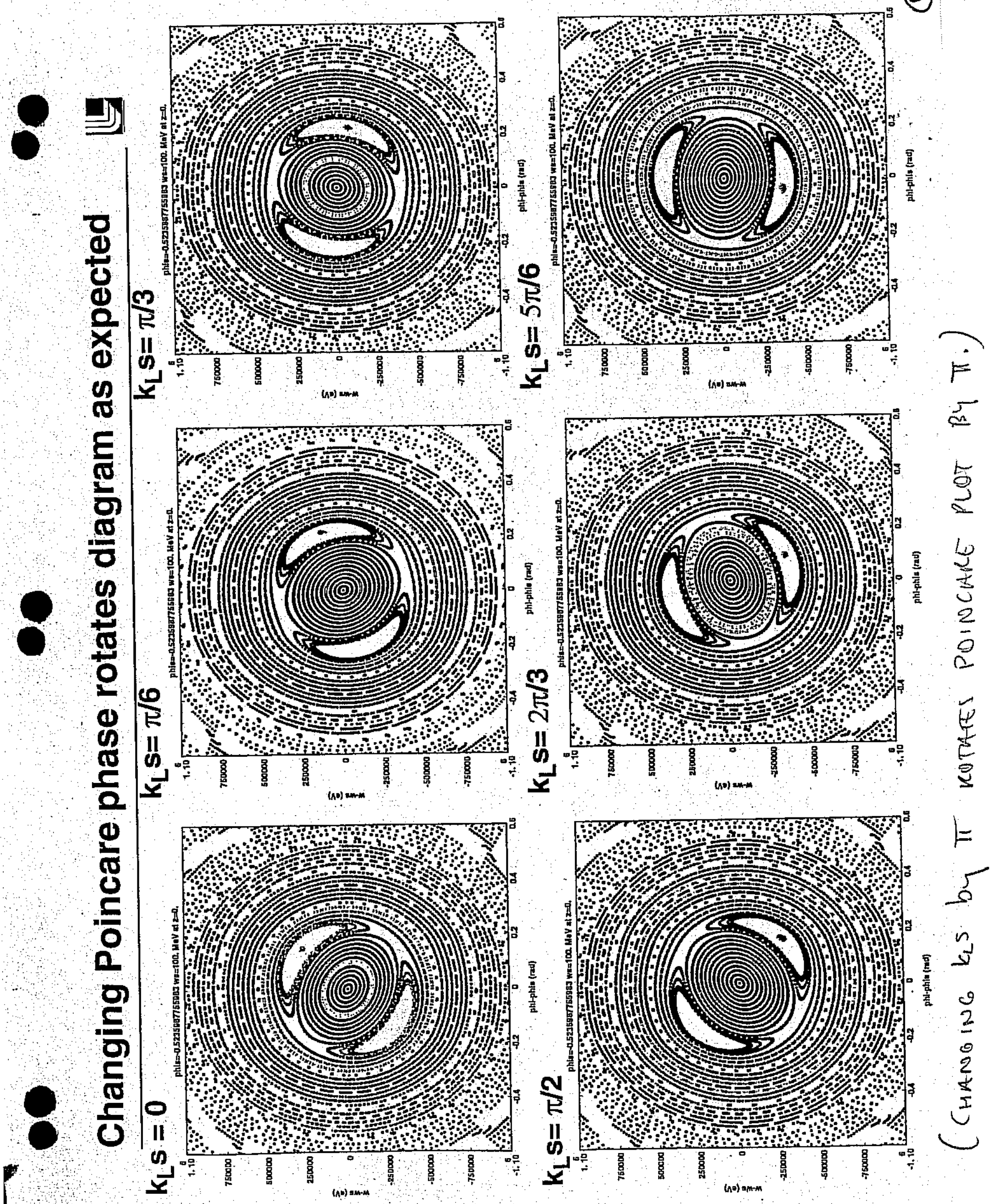




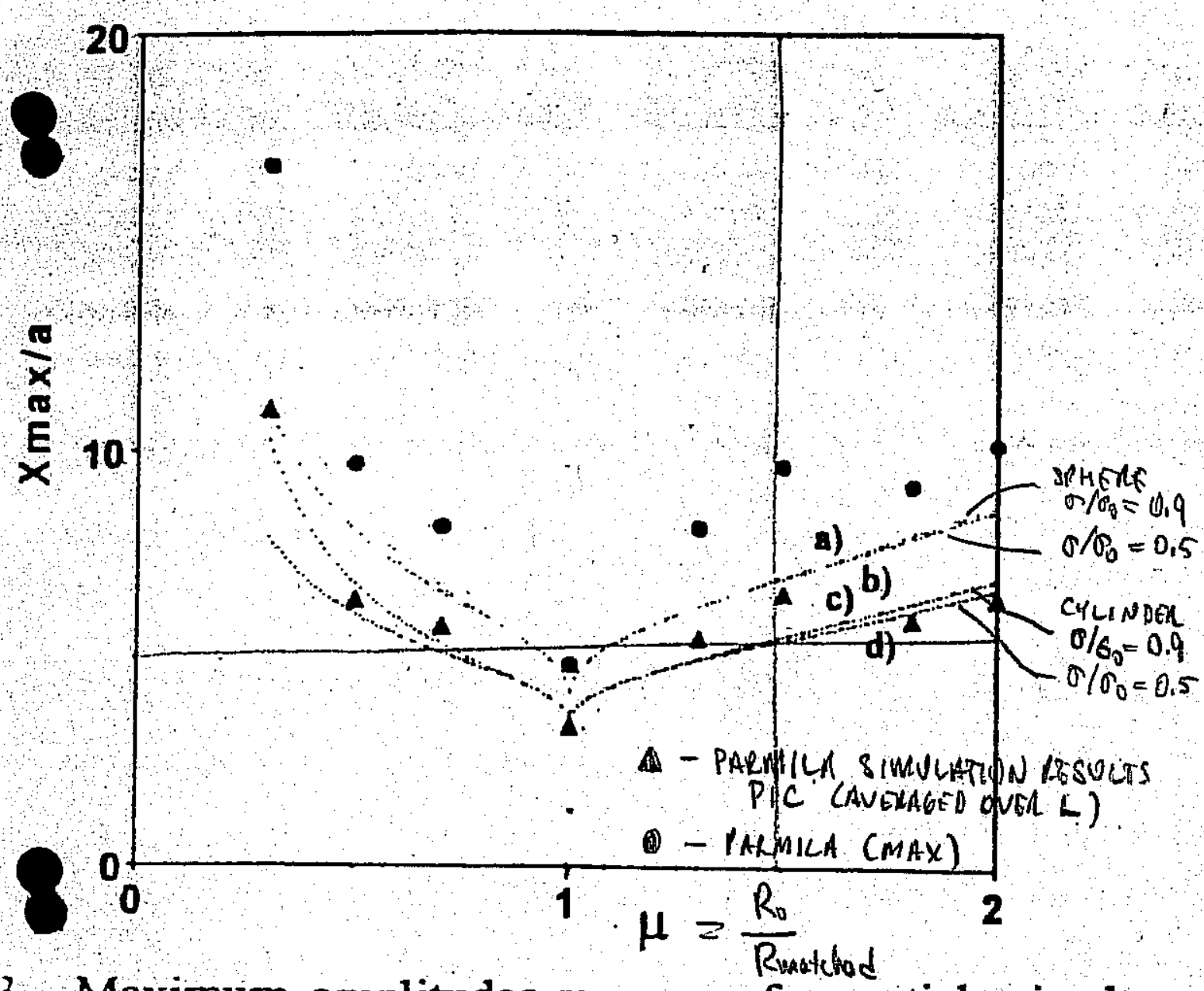

3. Maximum amplitudes versus $\mu$ for particles in the nance regions for the cylinder, and sphere models: a) re, $\eta=0.9, b$ ) sphere, $\eta=0.5, c)$ cylinder, $\eta=0.9$, and d) ider, $n=0.5$. The triangles represent the smoothed MILA simulation results for comparison with the els, and the dots represent the maximum amplitudes ding quadrupole flutter.

a real linac, additional effects that are not included in the ble-core model, must be accounted for, such as beamope flutter associated with a quadrupole focusing system, eration and the influence of other modes of the a hed beam. We have conducted a test of the predictions ie particle-core model, by carrying out PARML A ations, using an $\mathrm{r}-\mathrm{z}$ space-charge mesh with individual it $1 \mathrm{~A} 5$ man 1 .
Work du that beam 1 the new li1 mainly resF models to I formed. Thi and one for limited to a the strengt predictions. bounds of $t$ a linac. Sin produce sn consistent v that the brea the halo. To as well as 1 keeping $\varepsilon$ si can contribı beam-residi However, th than beam $\mathrm{n}$

The author Gluckstem a

* Work comn 
From WANGLE et al

(24)

XVIIII Int' LINAC CONF. GENOYN, SWITEEXLANAI

Wangler's core/test particle nesults

$$
\frac{X_{\max }}{\left\langle x^{2}\right\rangle^{1 / 2}}=A+B|\ln \mu|
$$

$$
\begin{aligned}
& \mu=\left(\frac{r_{b i}}{r_{b 0}}\right) . \\
& =\text { INTTAL }
\end{aligned}
$$

For

$\begin{array}{llcc} & 0 / 00 & A & B \\ \text { CYLINDER } & 0.5 & 3.97 & 3.83 \\ \text { CYLINDER } & 0.9 & 3.91 & 4.25 \\ \text { SPHERE } & 0.5 & 4.87 & 5.30 \\ \text { SPHERE } & 0.9 & 4.81 & 5.56\end{array}$

307 
(25)
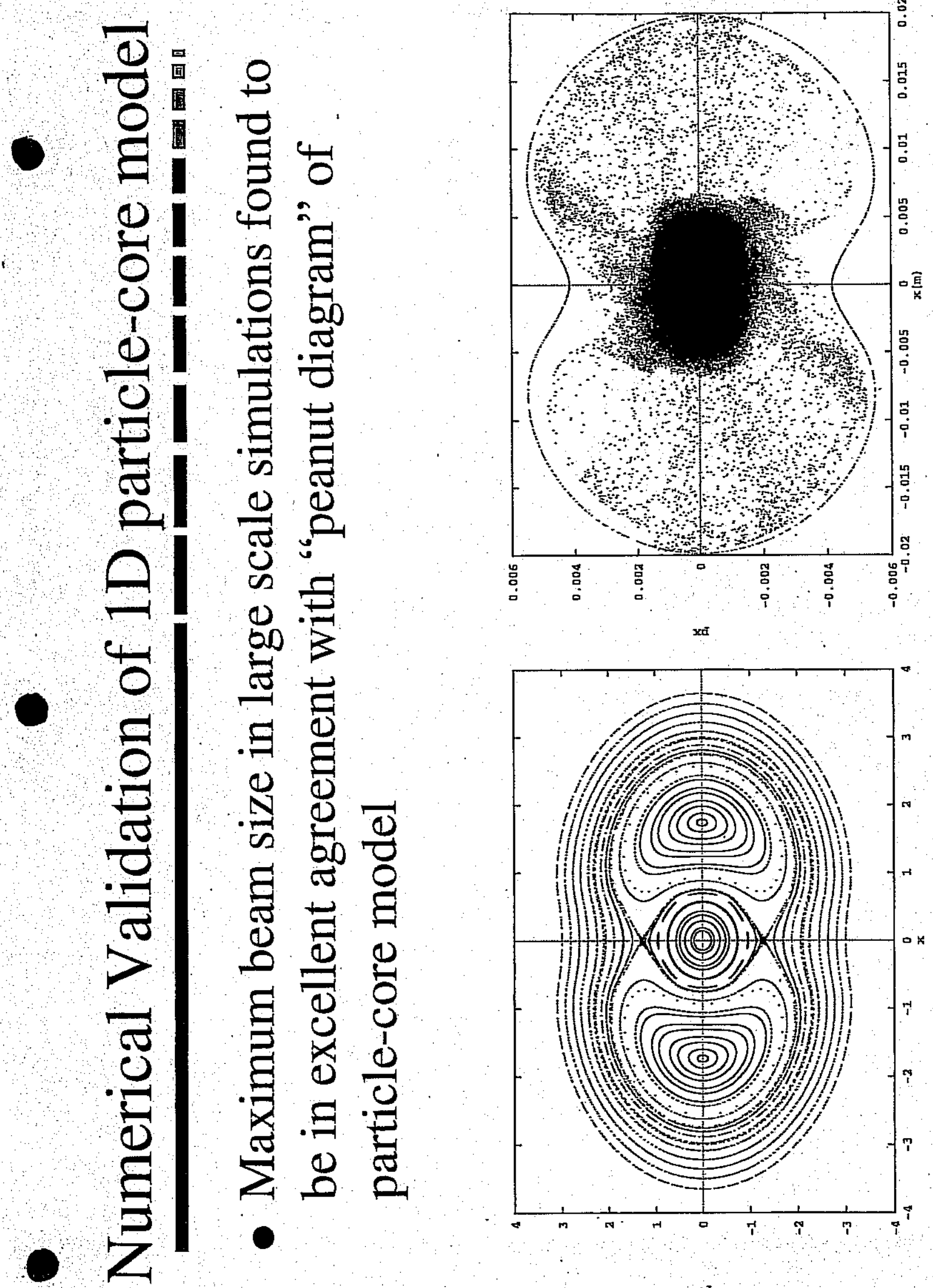

$\mathbf{x t \tilde { T }}$

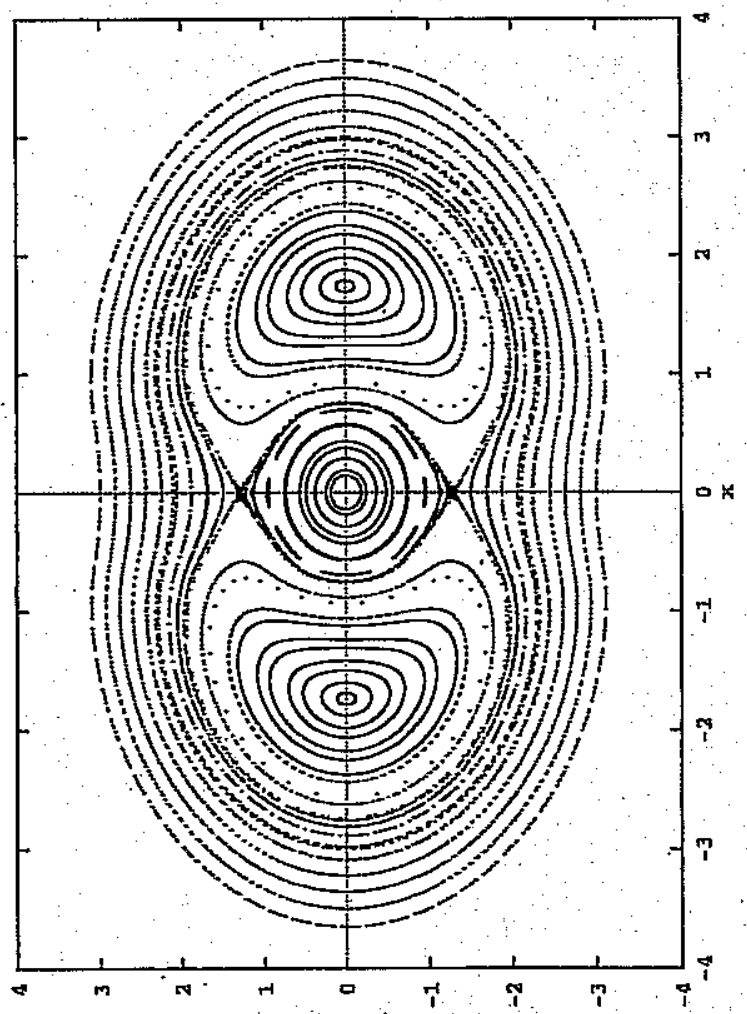

xत्C 
14 -

(26)
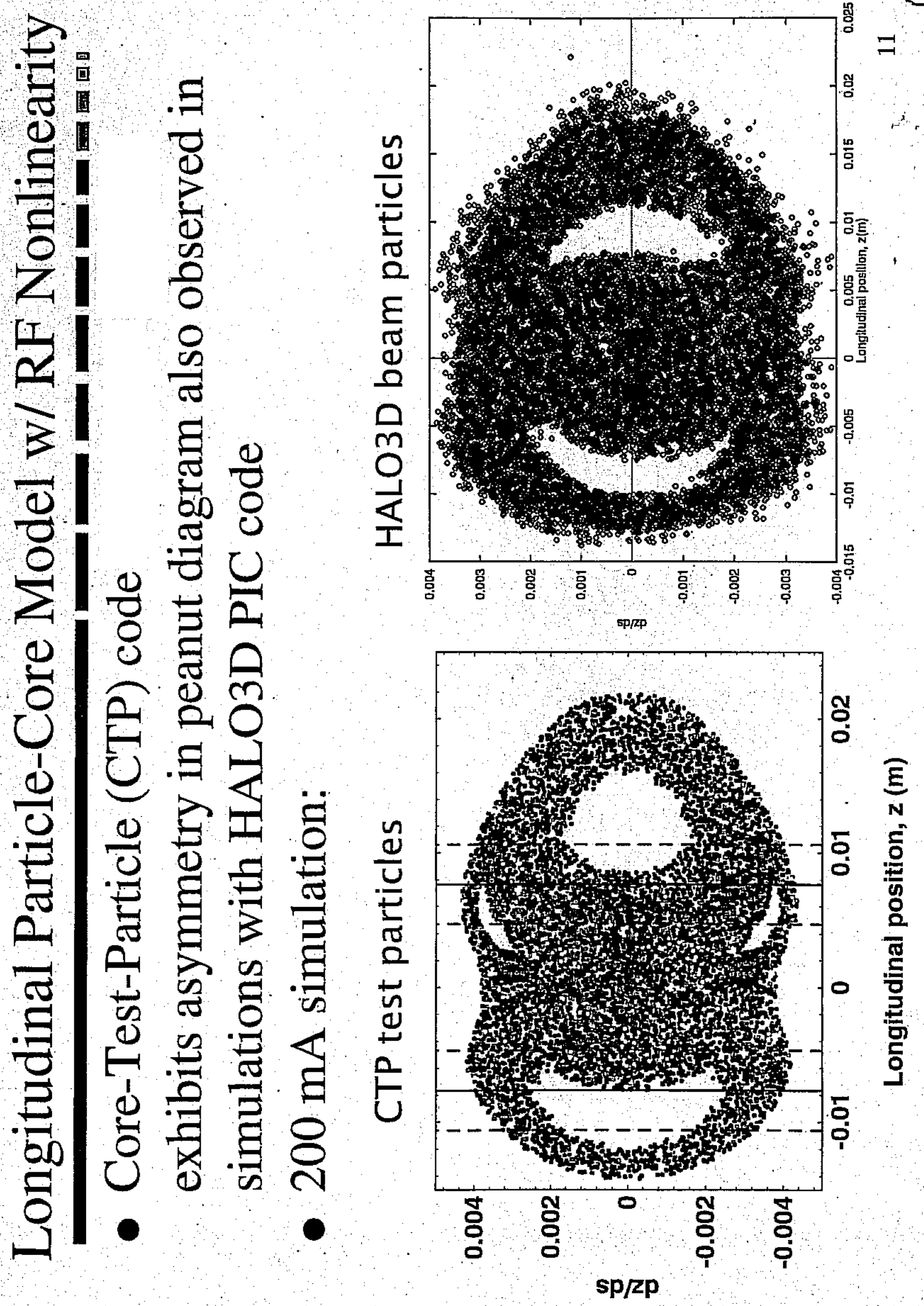
(27)

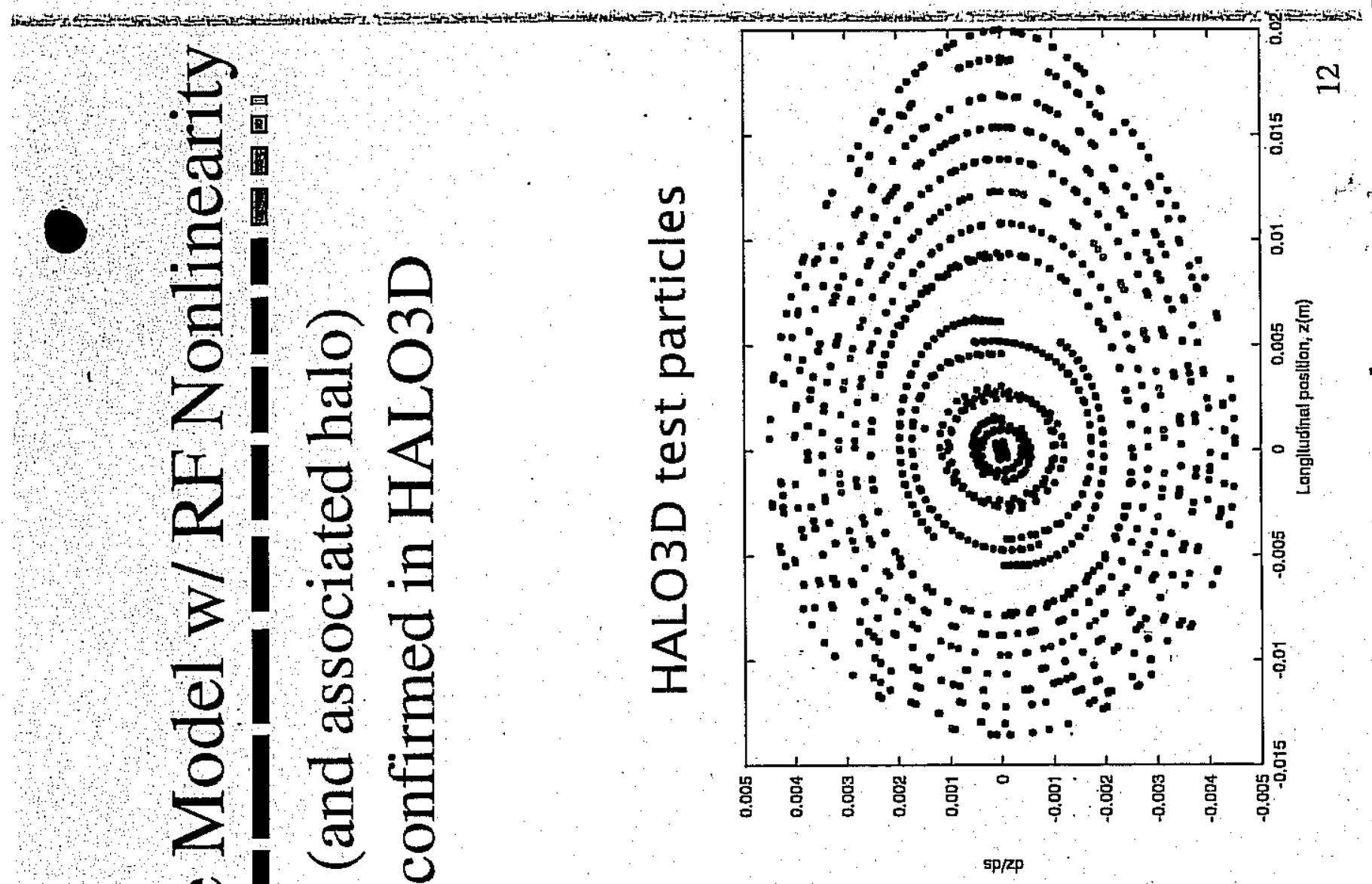



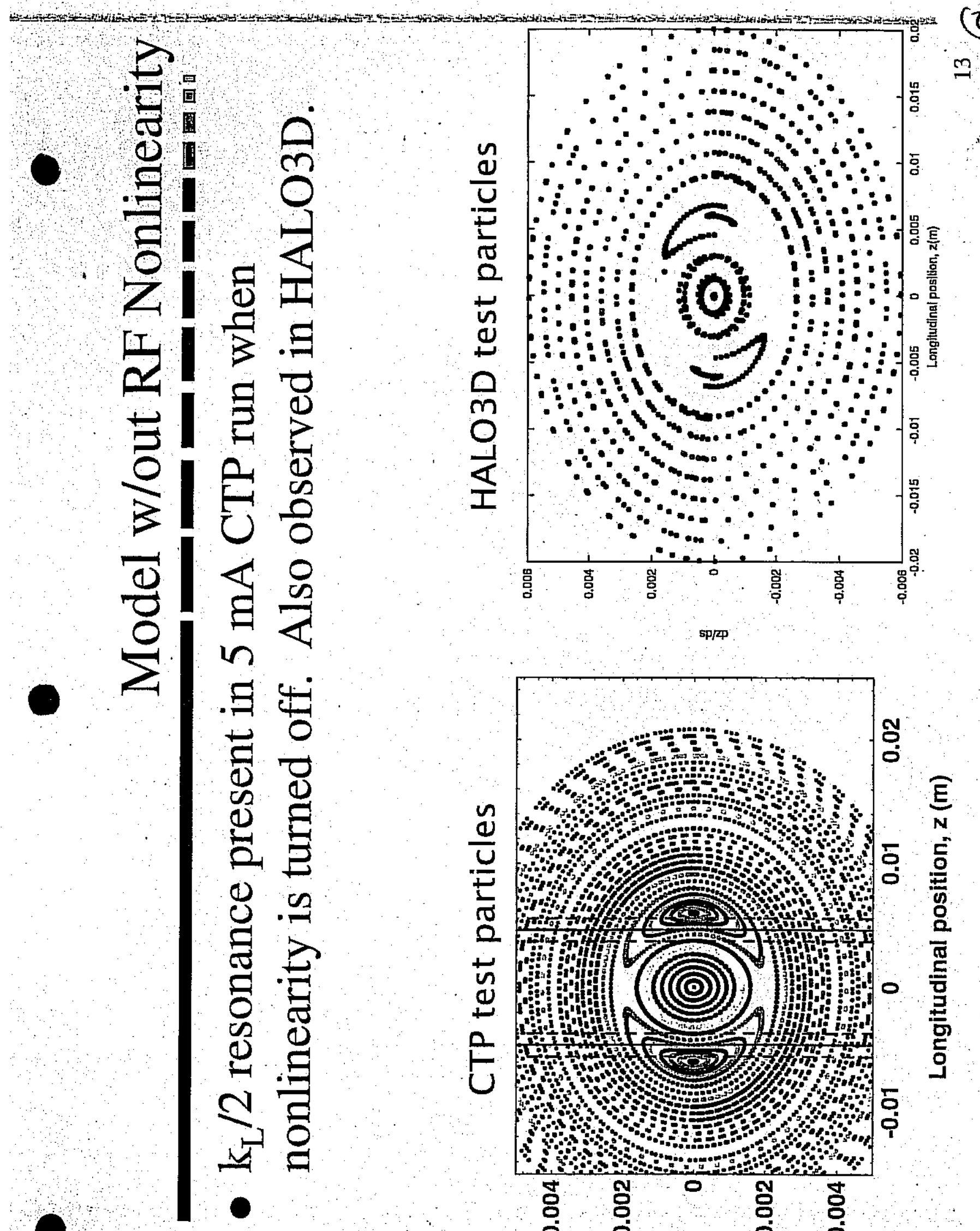

(28)

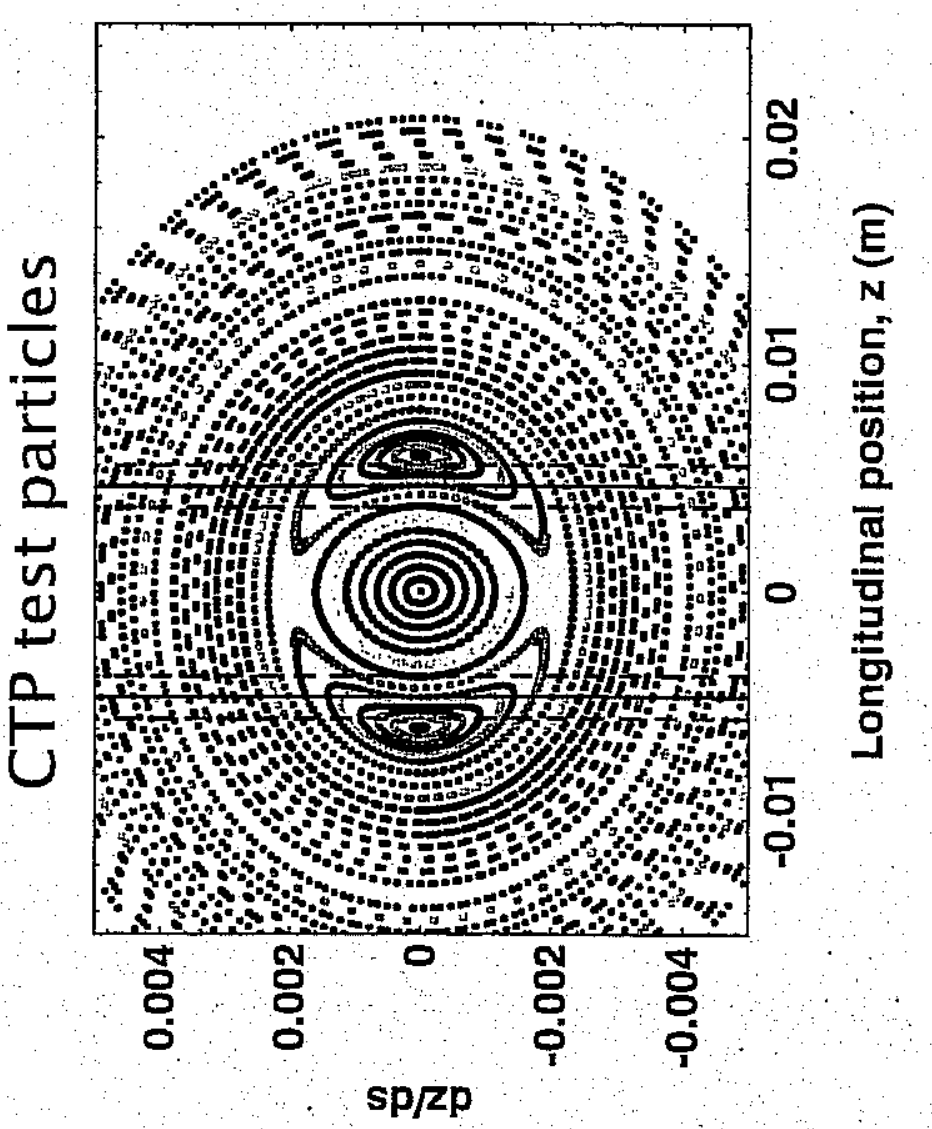




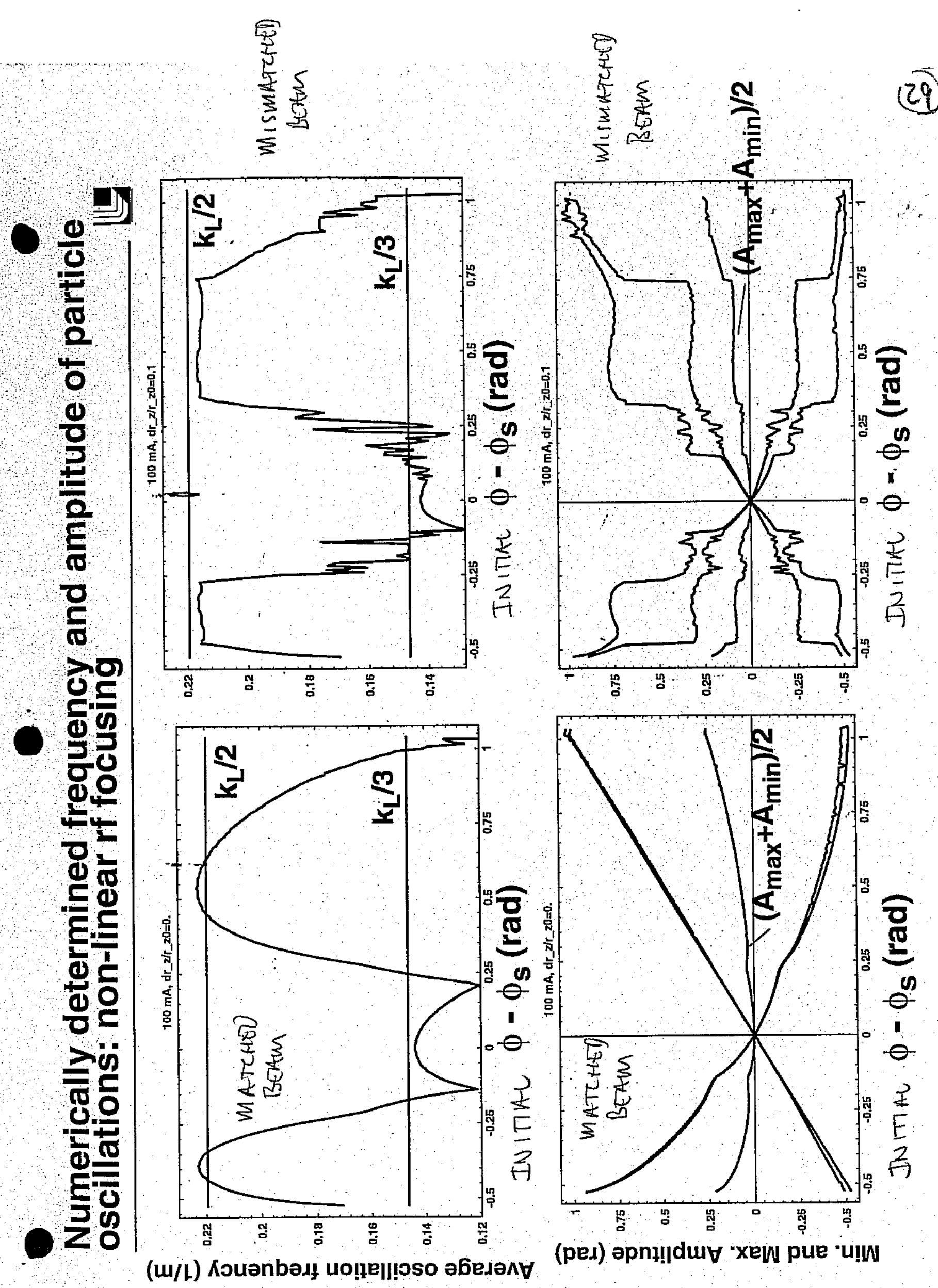


Ð

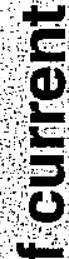

ธ

0

है

(2)

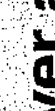

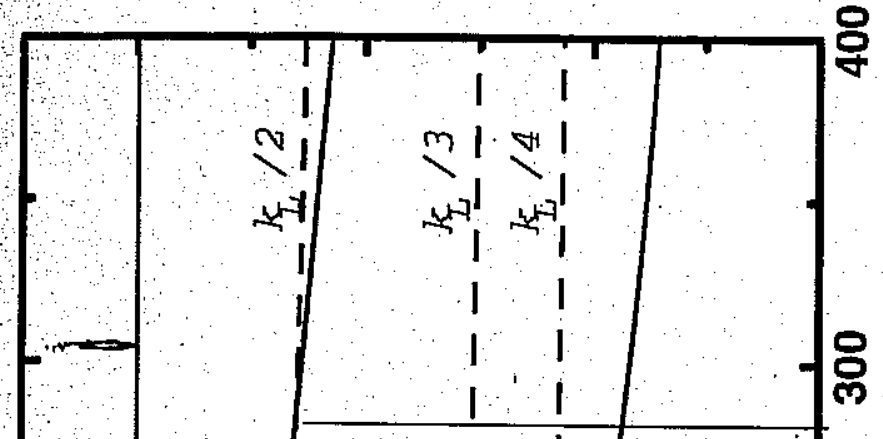

용

ह

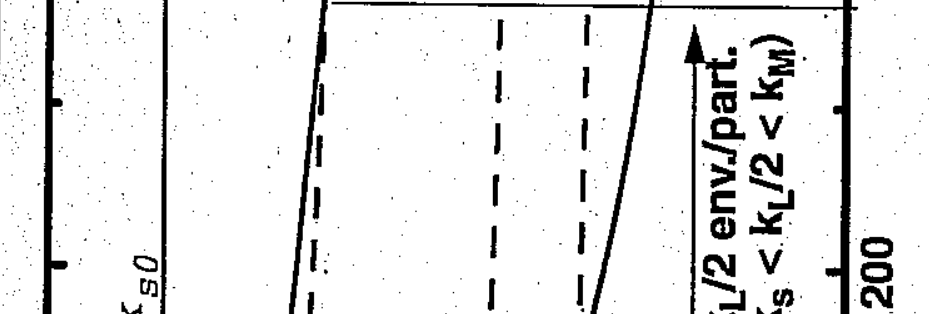

.

-

0

8

8

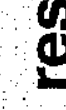

ง

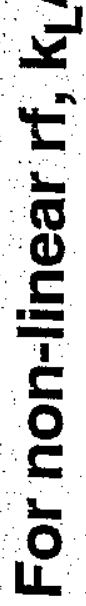

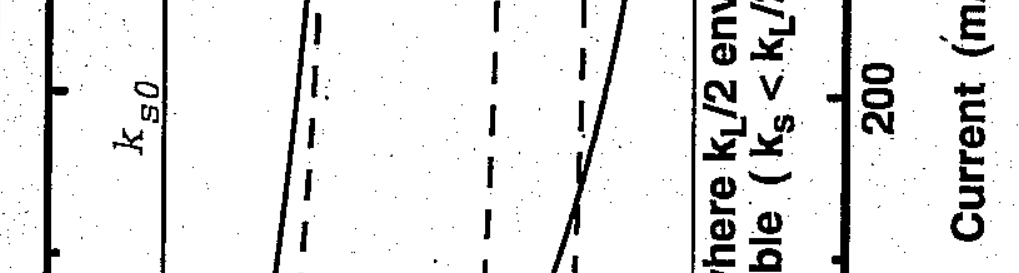

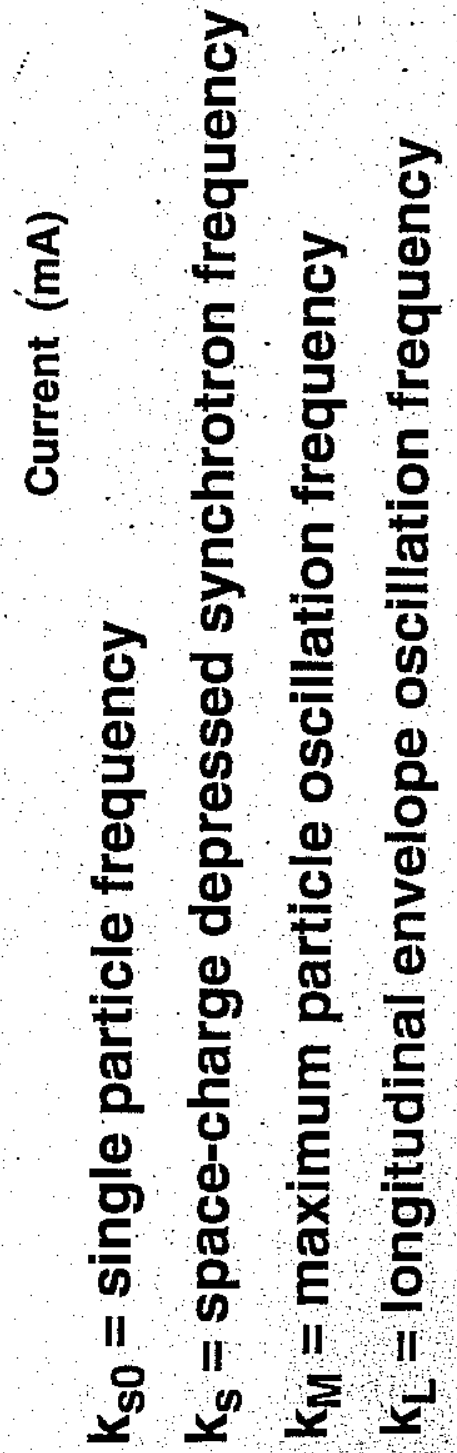


(3)

Amplitude Prase Analysis Gluclestown, Phys. Rev. Letters 73, $1247 \cdot 1994$

$$
x^{\prime \prime}+k_{p 0}^{2} x= \begin{cases}\frac{Q}{r_{b}^{2}} x & \text { fo } r<r_{b} \\ \frac{Q}{r^{2}} & \text { for } r>r_{b}\end{cases}
$$

Assume $r_{b}=r_{b o}+\epsilon \cos \left(k_{B} s\right)$

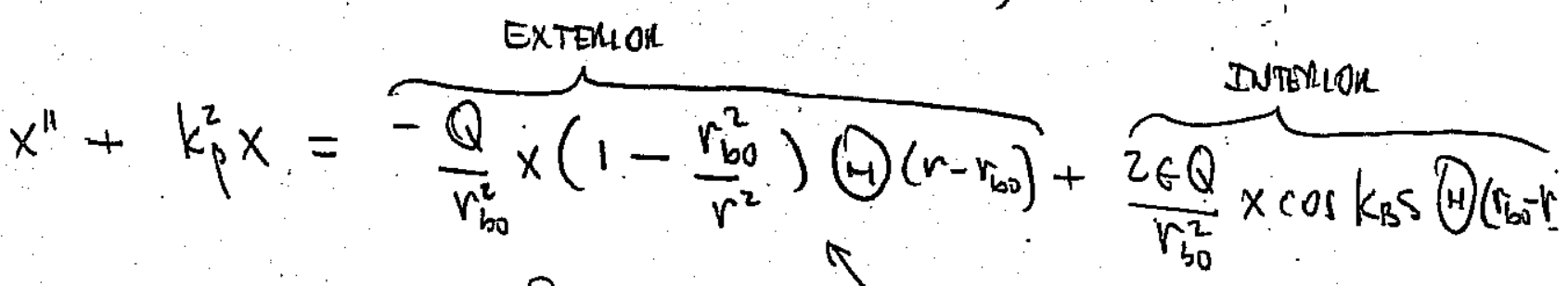

$$
\begin{aligned}
& H(x)= \begin{cases}1 & x>0 \\
0 & x<0\end{cases} \\
& \text { NONLINEAR } \\
& \begin{array}{l}
\text { AMPLLIVINES } \\
\text { OEYENDENT }
\end{array} \\
& k_{p}^{2}=k_{p o}^{2}-\frac{Q}{r_{b 0}^{2}} \\
& \text { fitegantey }
\end{aligned}
$$

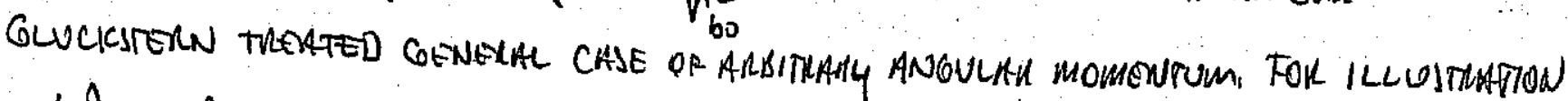
Use phase amplitude method HERE I SET ING, MOM $=0$.

$$
\begin{aligned}
& \frac{x}{r_{b_{0}}} \equiv A(s) \sin \psi(s) \\
& \frac{x^{\prime}}{r_{b_{0}}} \equiv k_{p} A(s) \cos \psi(s)
\end{aligned}
$$

$$
\psi \equiv k_{p} s+\alpha(s) \Rightarrow x=r
$$

Let $x^{\prime \prime}+k_{\beta}^{2} x=f(x, s)$

$$
=f\left(A, \psi, k_{B} s\right)
$$

Note

$$
\left.\begin{array}{rl}
\frac{x^{\prime}}{r_{b 0}} & =A^{\prime} \sin \psi+\left(k_{p}+\alpha^{\prime}\right) A \cos \psi \\
& =k_{p} A \cos \psi
\end{array}\right\} \Rightarrow A^{\prime} \sin \psi+\alpha^{\prime} A \cos \psi=0
$$




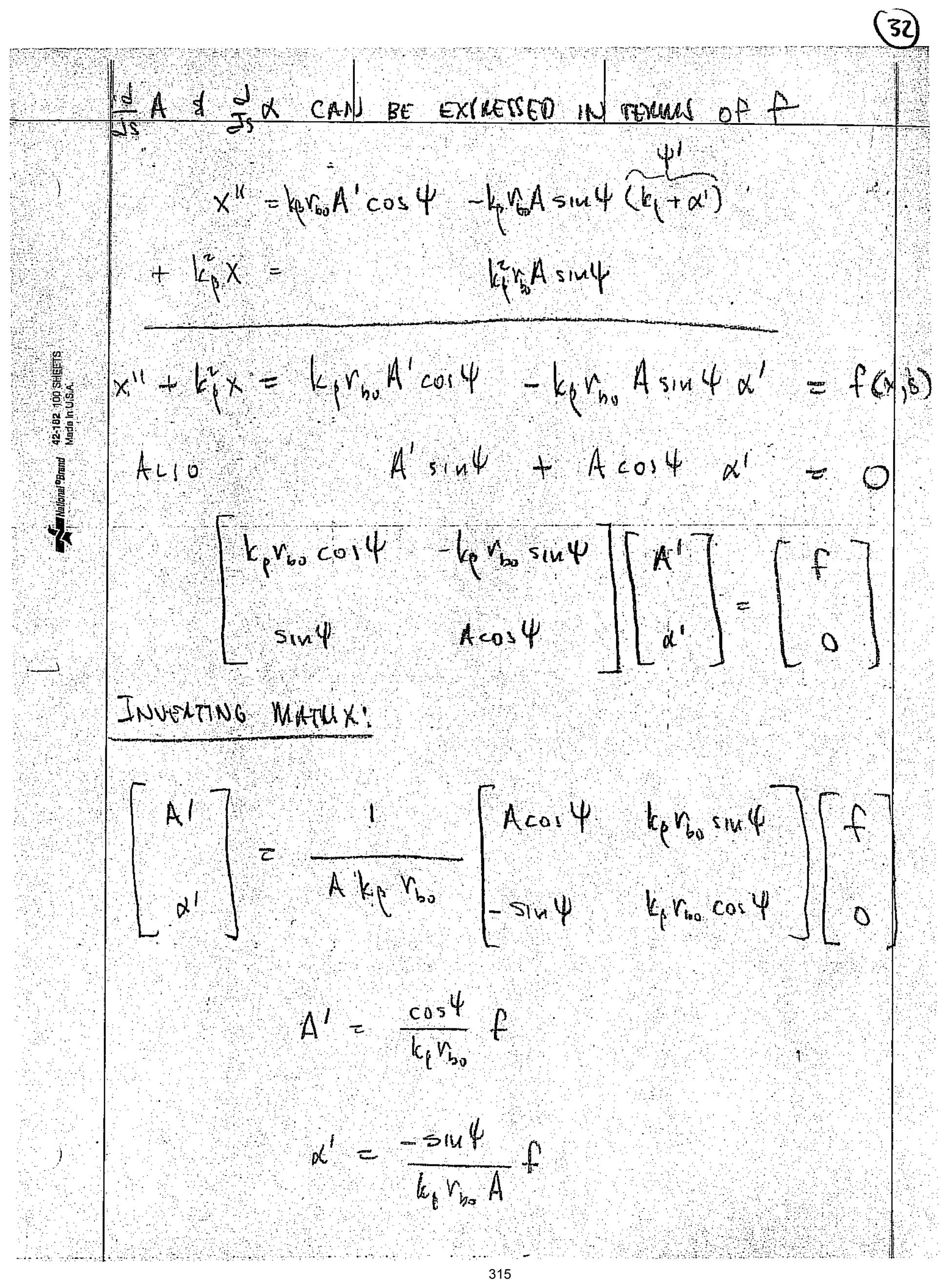


(33)

USIMG THESE DEFINITIONA THE EQUATION QR MOTION CAN BE EXYUESSET IS:

$$
\begin{aligned}
A^{\prime} & =\frac{1}{k_{\beta} r_{b_{0}}} f\left(A, \psi, k_{B} s\right) \cos \psi \\
\alpha^{\prime} & =\frac{-1}{k_{\beta} A r_{b_{0}}} f\left(A, \psi, k_{B} s\right) \sin \psi
\end{aligned}
$$

At resonance use expect $2 k_{p}-k_{B} \sim 0$,

so define $\Psi=2 \psi-k_{B} s=\left(2 k_{\beta}-k_{B}\right) s+2 \alpha$

$$
\Rightarrow \Psi^{\prime}=\left(2 k_{\beta}-k_{B}\right)+z_{\alpha^{\prime}}
$$

ELIMINATE, $k_{B} S$ in $f\left(A, \psi, k_{B} s\right)$ using

$$
k_{B S}=2 \psi-\Psi
$$

AUERAGE OUER ALL NON-RESONHNT FEQUENCY COMIONENTS; FOK RESONHNT YALTCLE EQUHTONI QP MOTION:

$$
\begin{aligned}
& A_{r}^{\prime}=\frac{1}{k_{\beta} r_{b_{0}}} \int_{-\pi}^{\pi} \frac{d \psi}{2 \pi} f\left(A_{r}, \Phi_{r}, \psi\right) \cos \psi \\
& \alpha_{v}^{\prime} \frac{-1}{r_{b_{0}} k_{\beta} A_{r}} \int_{-i \pi}^{\pi} \frac{d \psi}{2 \pi} f\left(A_{r}, \Psi_{r}, \psi\right) \sin \psi
\end{aligned}
$$

Ar \& $\Psi_{r}=\left(2 k_{\beta}-k_{j}\right)_{s}+2 \alpha$ are held fixed durin integlation.

316 
(34)

THE RESULT is

$$
\begin{aligned}
& A_{r}^{\prime}=F_{1}\left(A_{r}, \Psi_{r}\right) \\
& \Psi_{r}^{\prime}=\left(2 k_{\beta}-k_{z}\right)+2 F_{2}\left(A_{r}, \Psi_{r}\right)
\end{aligned}
$$

Let $\omega=A_{r}^{2}$

when $F_{1} \& F_{2}$ are exploit functions

$$
\begin{aligned}
& \omega^{\prime \prime}=2 A_{r} A_{r}^{\prime}=2 A_{r} F_{1}\left(A_{r}, \Psi_{r}\right)=2 \omega^{1 / 2} F_{1}\left(\omega^{\prime \prime}, \Psi_{r}\right) \\
& \Psi^{\prime}=\left(2 k_{\beta}-k_{B}\right)+2 F_{2}\left(A_{r}, \Psi_{n}\right)
\end{aligned}
$$

w \& I are conjugate variables, and the resonant particle equation of motion can be derived from an s-independent Hamiltonian $H$ as:

$$
\omega^{\prime}=\frac{\partial H}{\partial \Phi} \quad \Psi^{\prime}=-\frac{\partial H}{\partial \omega}
$$

Glucicstexn found a constant of the motion $H_{r}(\omega, \Psi)$ which satisfies Hamilton's EQuATION!

RESONANT PARTICLE WOULD STAY ON LINES OF CONSTANT H.

317 


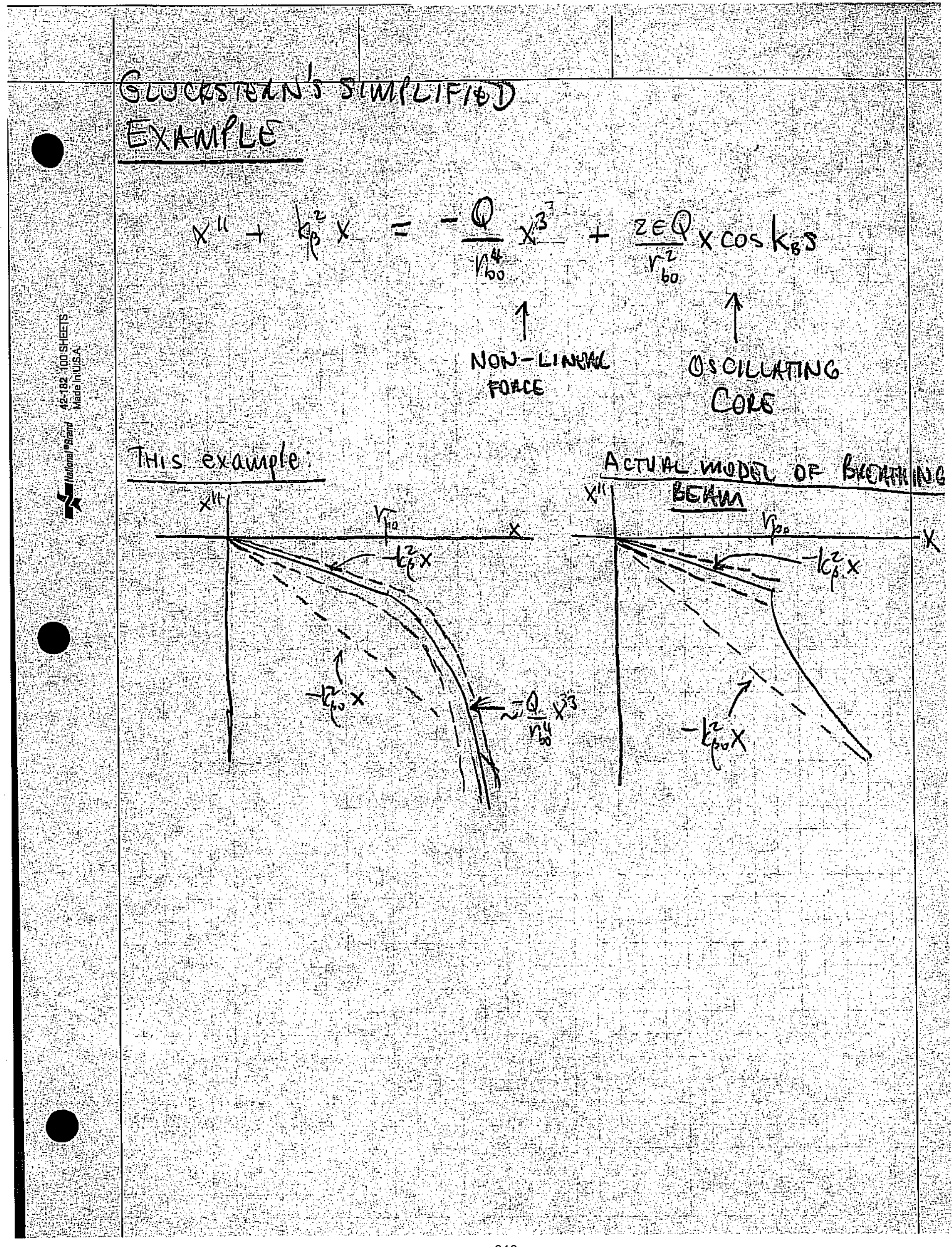


(36)

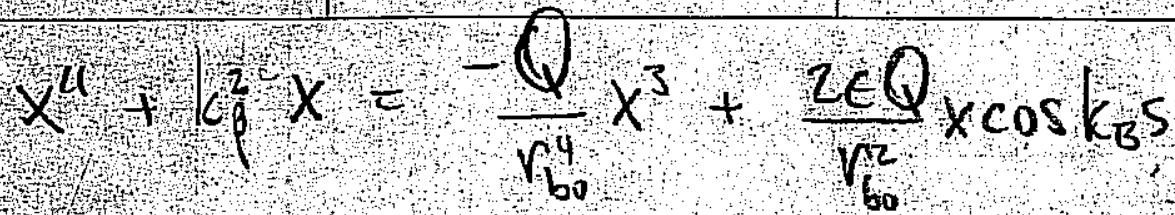

$$
\begin{aligned}
& =f(x, s)
\end{aligned}
$$

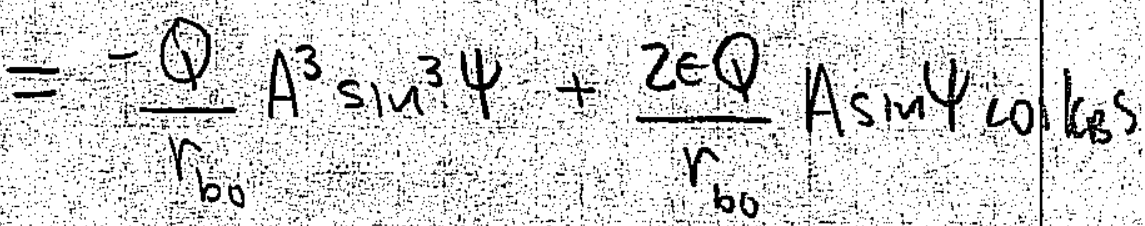

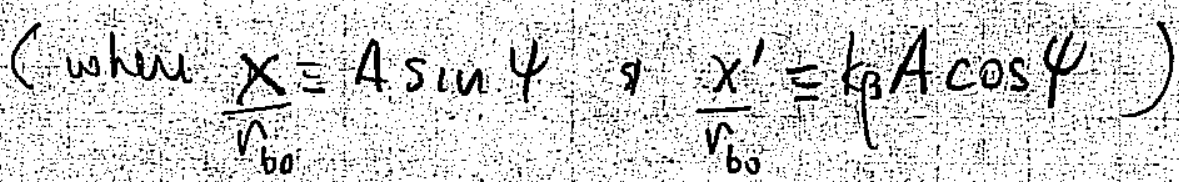

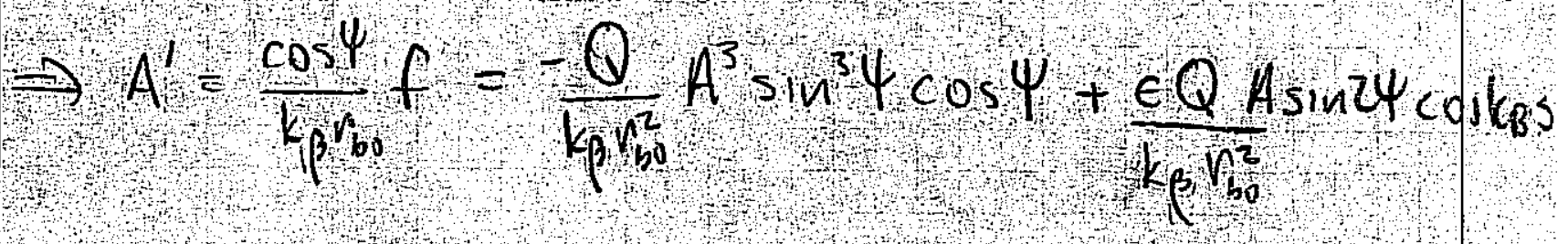

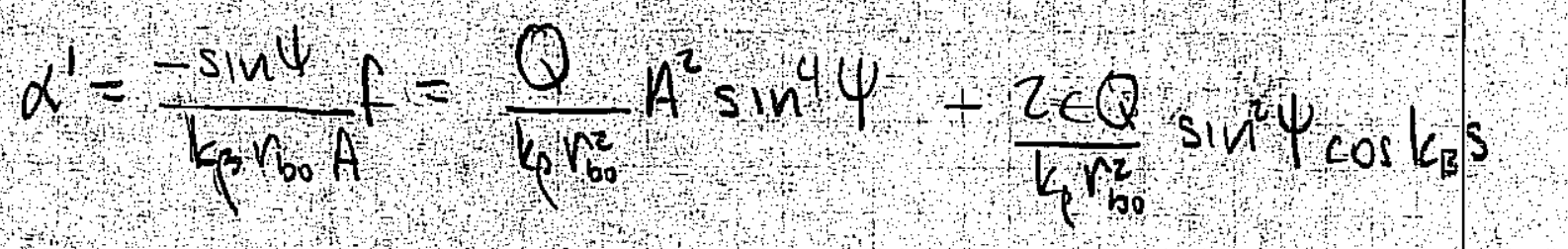

$\left.U \operatorname{sing} \Psi=2 \psi-l_{k} \mathrm{~s} \Rightarrow \cos k_{B} s=\cos [2 \psi]-\Phi\right]$

TMig loENT TES:

$$
=\cos [2 \psi] \cos [\Psi]+\sin [2 \psi] \sin [
$$

$$
\begin{aligned}
& \sin 24 \cos k_{s} s=\frac{1}{2} \sin \theta-\frac{1}{2} \cos 4 \sin \psi+\frac{1}{2} \sin 4 \theta \cos \theta \\
& \sin \psi=\frac{3}{8}-1 \frac{1}{2} \cos -\frac{1}{8} \cos 4 \psi \\
& \sin ^{2} \psi \cos l_{8} \rho=\cos 9\left[-\frac{1}{4}+\frac{1}{2} 24-\frac{1}{\cos } \varphi \psi\right] \\
& +\sin 4\left[\frac{1}{2} \sin 24-\frac{1}{4} \sin 44\right] \\
& \sin 4 \cos \psi=\frac{1}{4} \sin 2 \psi+\frac{1}{8} \sin 4 \varphi
\end{aligned}
$$


(37)

$$
\begin{aligned}
& \begin{array}{l}
\mathrm{O} \\
\mathrm{O}
\end{array} \mathrm{A}_{r}^{\prime}=\frac{\epsilon Q}{2 \mathrm{k}_{p} r_{b_{v}}^{2}} A_{r} \sin Q_{r} \\
& \Psi_{r}^{\prime}=\left(2 k_{\beta}-k_{\beta}\right)+2 \alpha_{r}^{\prime}
\end{aligned}
$$

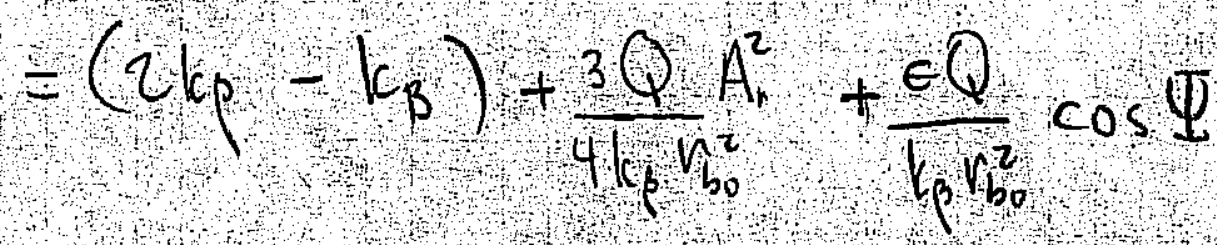

$$
\begin{aligned}
& \text { DEF INE } W=A_{r}^{2} \\
& w^{\prime}=2 A_{r} A_{r}^{\prime}=\frac{\epsilon Q}{C_{r} r_{b r}^{2}} w \sin Q_{r} \\
& q_{r}^{\prime}+\left(2 k_{p}-k_{B}\right)+\frac{30}{4 k_{p} n_{0}^{2}} \omega+\frac{\epsilon Q}{k_{p} r_{b_{0}}^{2}} \cos \theta
\end{aligned}
$$

A Hamilfonians can be found satisfyng Ham lton's equarems:

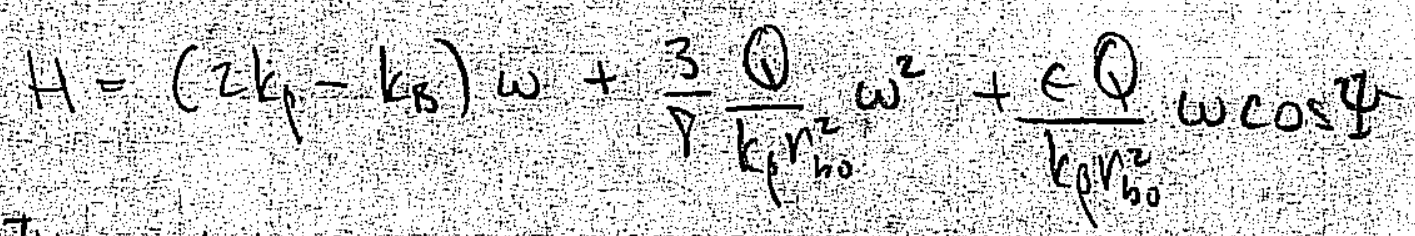

THIS CAN BE EXIKESSED AS:

$$
c \cos \varphi=\Delta=\frac{3}{5} \omega-\frac{C}{\omega}
$$

when $C$ is setermined by in fial condition and

$$
A=k_{j}\left(k_{B}-2 k_{l}\right) r_{b}^{2} / Q
$$

$\left(N_{01}\right)+K_{b}^{2}=4 K^{2}+2 Q / r_{h_{0}}$ for breathme mode $)$

320 


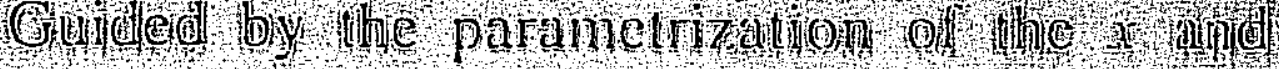

$\cos p]$

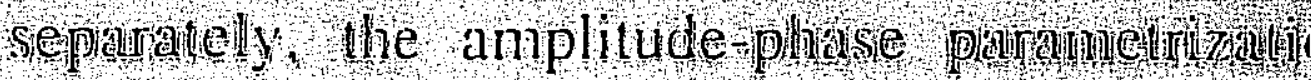
two dimensional oscillation is wittem as

$$
s=\frac{w^{2}+L^{2}}{2 w}-\frac{w^{2}-L^{2}}{2 w} \cos (2 q=+\gamma) \text {. }
$$

1.

Here $\|$ and $\gamma$ are slowly varying amplitude, iscillatory terms number $2 q-p$ parameters which would be constant if the rig Eq. (2.11) vanished. We now write

$$
s^{\prime}=q \frac{u^{2}-L^{2}}{w} \sin (2 q z+\gamma)
$$

and use Eq, (2.11) and the required connection $w^{\prime}$ and $\gamma^{\prime}$ implied by Eq. (2.13) to obtain explic

os $W$ sions for $n^{\prime}$ and $\gamma^{\prime}$. We then average over osed

of this resonant 1l of the motion

and where the he indial values pe equation we breathing mode,

d by the space

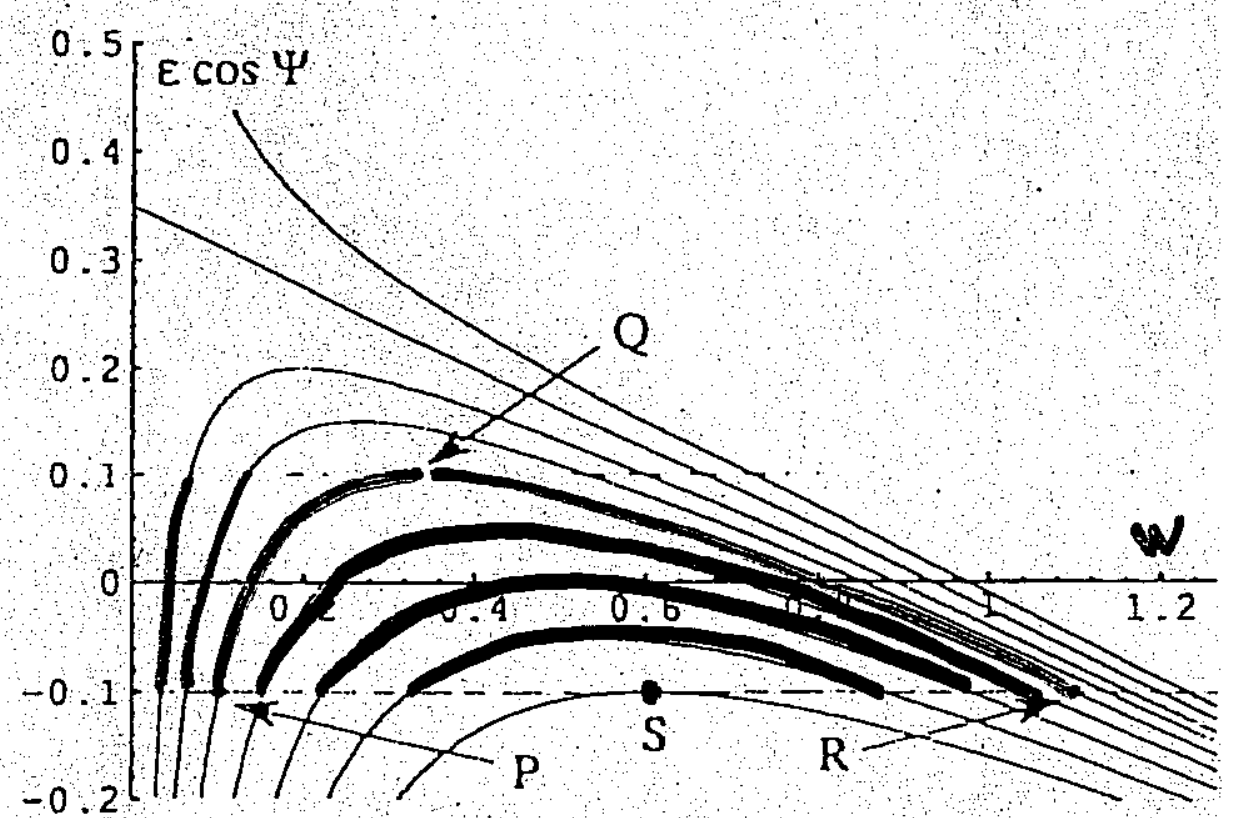

FIG. 1. Plot of $\epsilon \cos \Psi$ vs $u$ for the simplified $n$ \lrcorner$=0.35$ 


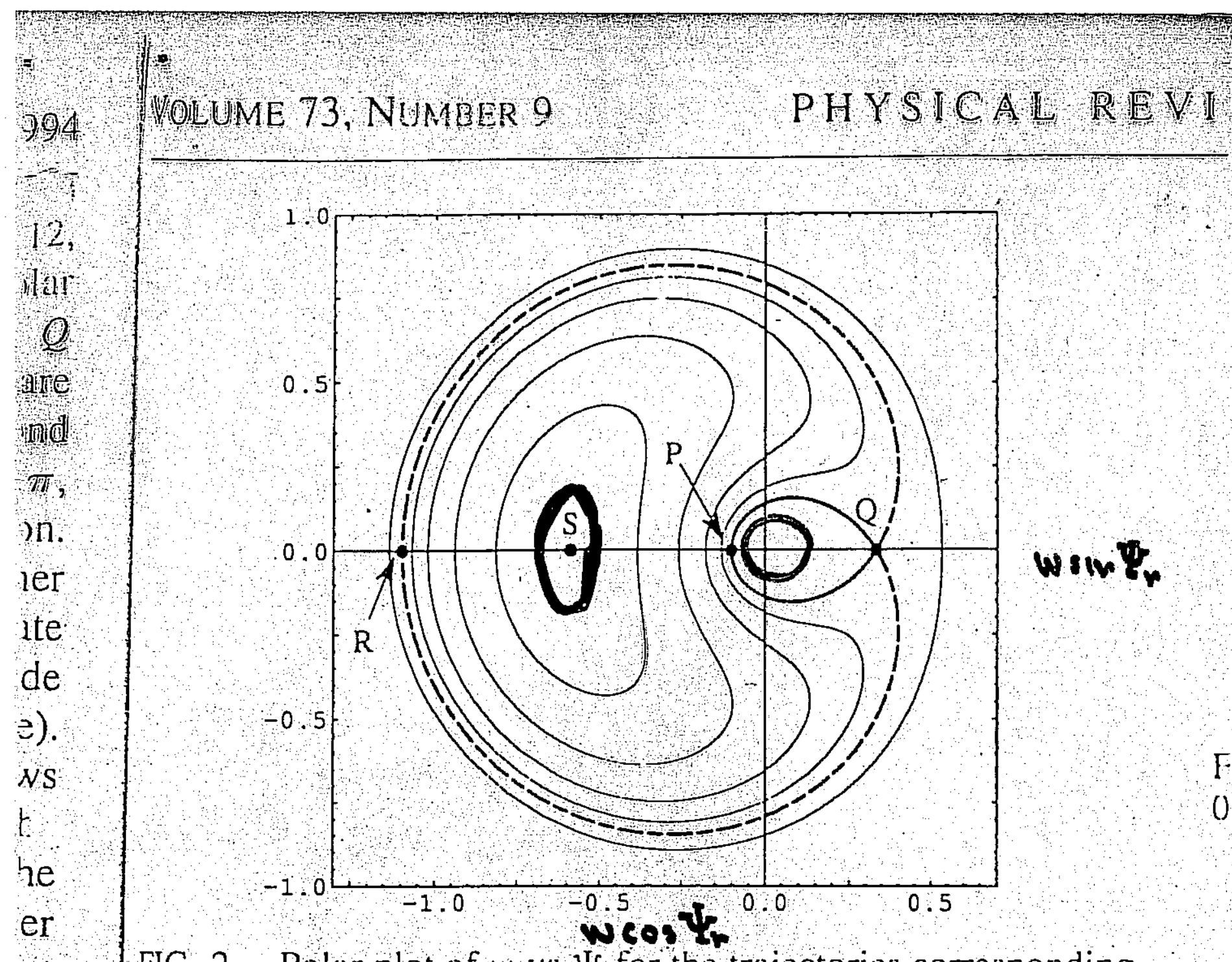

at 1 FIG. 2. Polar plot of $w$ vs Ir for the trajectories corresponding to the parametric resonance using $\Delta=0.35, \epsilon=0.1$, and the simplified nodel.

to

:d

7)

d all wave numbers except $2 q-p$, being careful to include 2 , 3 the step functions as we obtain these averages. The final equations for $w^{\prime}$ and $\Psi^{\prime}[\Psi=(2 q-p) z+\gamma]$ are similar lo Egs. $(2.7)$ and $(2.8)$ and again lead to an integral of the motion, which now is

$$
g(1-h) \epsilon \cos \Psi=f \Delta-t-C,
$$

Where $f(w)=\left(w^{2}+L^{2}\right) / 2 w, g(w)=\left(w^{2}-L\right) / 2 w$. 
Also

from $L=-1 / 2$ LO $L=12$

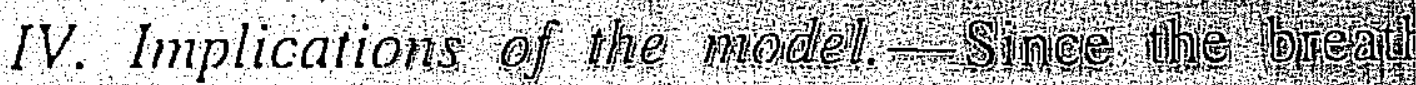

f)

$\mathrm{K}-\mathrm{V}$ beam is a solution of the Vlasov equation, pain within the core will continue to remain there, ever the presence of the resonant interaction. If howe

(2.16)

The arcquadrant. ependence ession for Eq. (2.6)

fect when trough the $1=0.35$

sy similar Fig. 4 for $\rightarrow$ model in cale for $w$ ming with en hy the

$2 m$ - The to

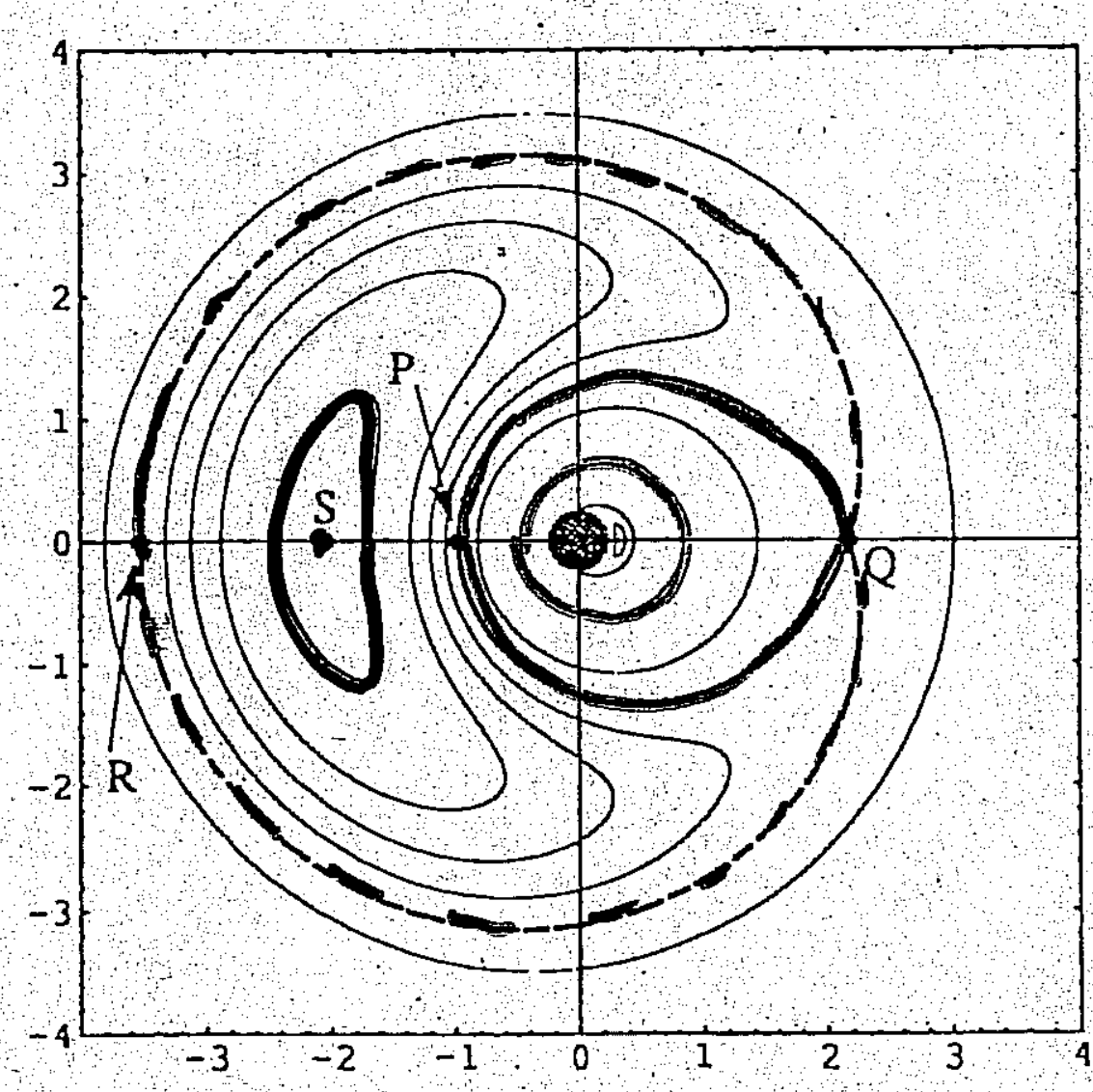

FIG. 4. Polar plot of $w$ vs $\Psi$ for the trajectories corresponi to the parametric resonance using $\Delta=0.35, L=0, \epsilon=$ and the exact nodel. 


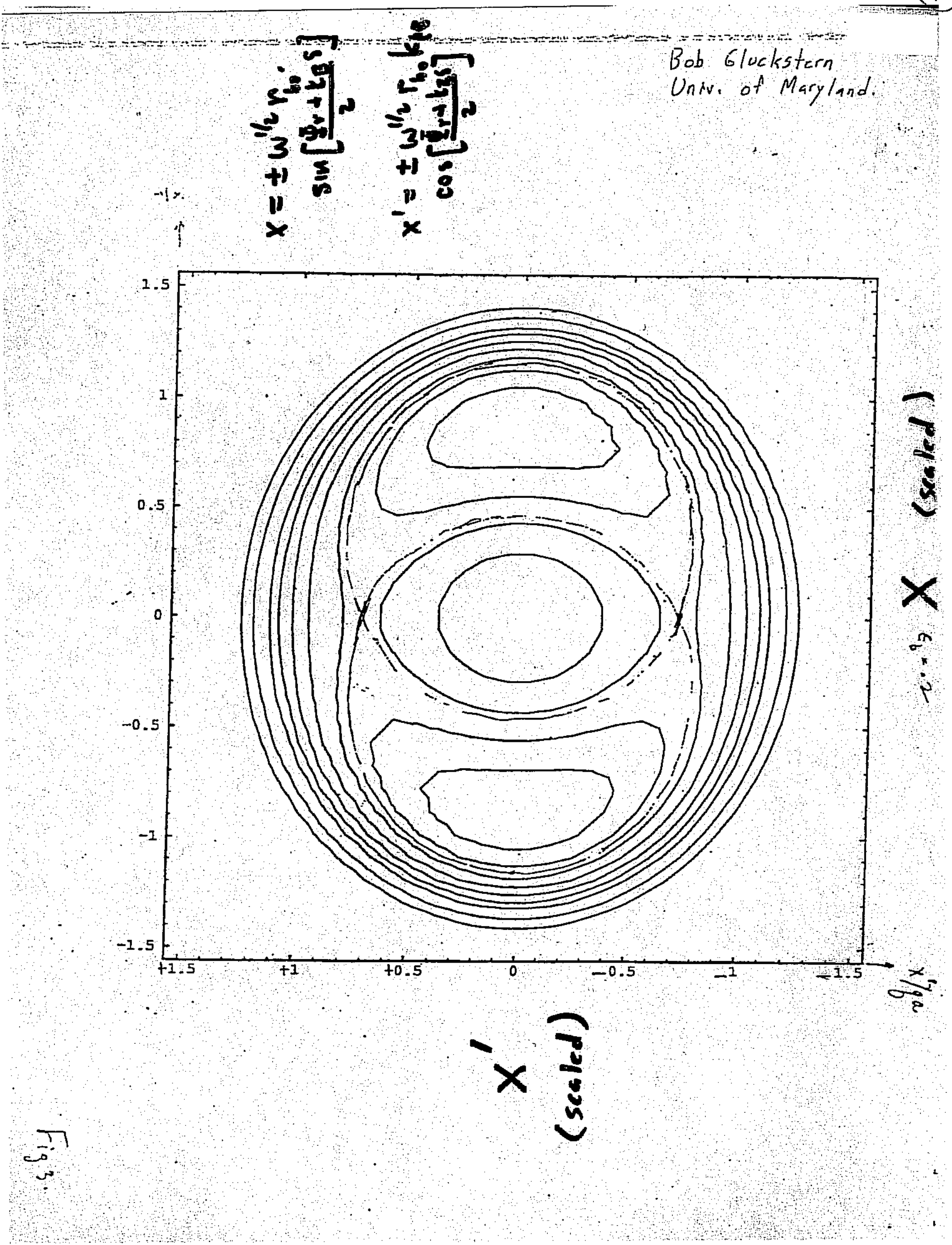




\section{Foom lom Wangler ${\text { Lalamor National } L_{a b}}$}

\section{Stroboscopic Map (The Peanut Diagram)}

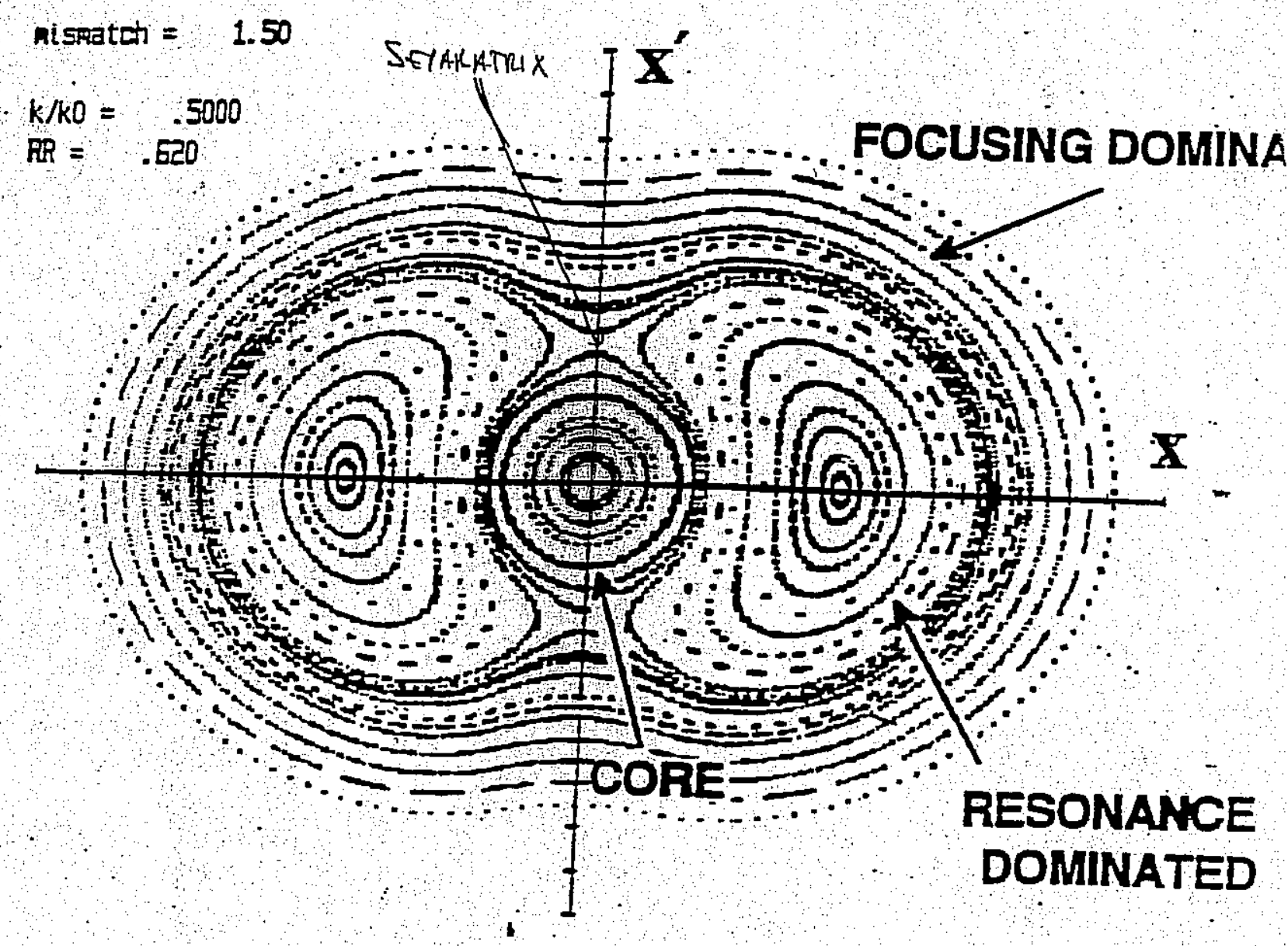

- Accumulate Many Snapshots of Prase Space Taken at Minimum Amplitude of Core Oscillation. (ox hay pderculath:

- Follow an Amay of Particles to Obtain a "Trajectory Field".

- Regutar rrajectories Appear as Smooth Curves.

- Chaotic Trajectories Appear as Stochastic Scatter.

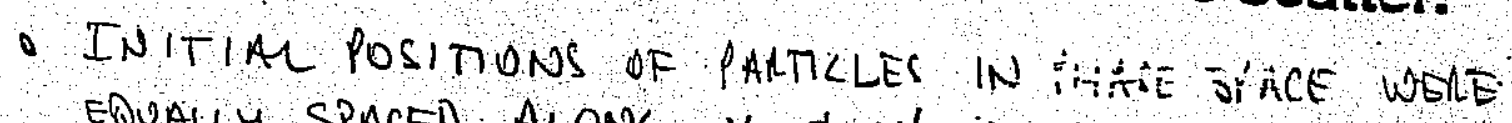
equaly spaced along $X$ \& $X^{\prime}$ aXes. 


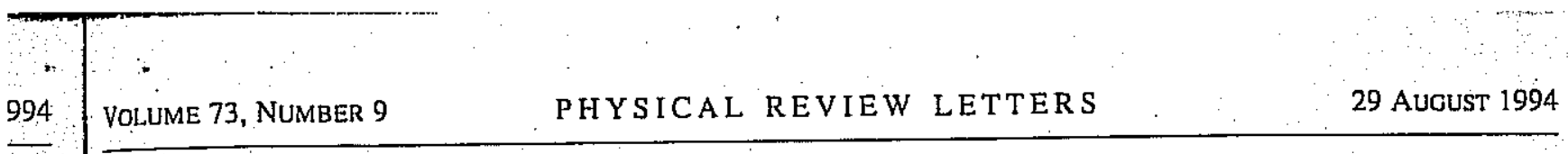

\title{
Analytic Model for Halo Formation in High Current Ion Linacs
}

\author{
Robert L. Gluckstern \\ Physics Department, University of Manland, College Park, Maryland 20742 \\ (Received 8 March 1994)
}

\begin{abstract}
We construct an azimuthally symmetric 2D model for halo formation in high current ion linacs. The Jriving term, a "bretthing" ostillation caused by a transverse mismatch along the linis, leads to growth of ion amplitudes in the core through the parametric resonance. As the ion amplitude grows, its wave number increases, enhancing the resonance. This leads to the formation of a halo surrounding the core. We explore the dependence of this mechanism on the tune depression and the size of the mismatch. The model agrees well with simulations at Los Alamos, but does not yet include the effects of chaos observed in the simulations as the tune depression becomes severe.
\end{abstract}

PACS numbers: $41.85,-p, 29.17 .+w, 29.27 . B d$

W:

lora,

248

H.J.

Een,

3.P.

..W.

raev

der

- 11

63);

Rev.

gen,

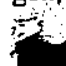

E. I.

ight

tion,

11) is

zven

, the

isica

4.P.

v. $\mathrm{A}$

near

rlog,

$2 \omega_{0}$

stent

srate

Rev:

lited

ress;

1. Introduction - High current, high duty factor ion linacs have become increasingly attractive in recent years. Among possible applications are heavy ion drivers for thermonuclear energy production, production of tritium, transmutation of radiouctive wastes, and production of radioactive isotopes for medical use.

Obviously, it is desirable to accelerate the maximum possible current in such linacs. Much work has been done to explore the optimum transverse phase space distribution in such beams. In particular, the KapchinskyVladimirsky (K-V) distribution [1] is simplest to analyze, since this projection into real space has a uniform density and therefore linear space charge forces. The stability of the $\mathrm{K}-\mathrm{V}$ distribution has been analyzed and approximately confirmed by numerical simulations. Nevertheless it appears that, particularly at high currents, the $\mathrm{K}-\mathrm{V}$ and other equilibrium distributions evolve to ones with rounded edges and tails. In many cases involving high peak current, the distribution spins off a cluster of particles in the form of a halo surrounding a dense core. This halo is seen in simulations as well as in actual linacs, such as LAMPF [2]. And efforts to remove the halo by collimation have been largely unsuccessful since the halos almost always regenerate.

It is clear that the halos will produce unacceptably high levels of radioactivity in high current, high duty factor linacs. For this reason considerable effort has recently been devoled to exploring their detailed structure and understunding the mechanism or mechunisns by which the halos are produced [3-6]. What has been leamed is that halos are most likely to be produced at transition locations, such as where there are discontinuities in frequency, structure geometry, transverse focusing pattem, accelerating gradient and phase, etc.

In the present paper, we propose an analytic model for halo formation which appears to reproduce the main features seen in simulations and in actual linacs. In particular we consider a circular $\mathrm{cw}$ beam with a $\mathrm{K}-\mathrm{V}$ core distribution and explore the motion of individual ions pussing through the core. Since energy transfer between ions and the core can lake place only if the core has a time dependent behavior, we consider the driving mechanism to be a "breathing" oscillation of the core. We then explore the resonant (parametric) interaction between the breathing core and the ions oscillating about and through the core. Of particular importance is the dependence of the frequency of each oscillating ion on its amplitude, which is related to the fraction of the oscillation for which the ion is within the core.

In spite of the fact that the actual distribution will have nonlinear fields, the use of a $\dot{K}-V$ distribution for the analysis leads us to a very likely mechanism for the development of the halo. In particular, the results provide an explanation for the low density region around the core which is surrounded by a somewhat higher density halo ring. This explanation will probably still apply for other self-consistent distributions.

II. Model.-We consider an azimuthally symmetric $\mathrm{K}-\mathrm{V}$ core of radius $a$ for which the equation of motion of an ion is

$$
x^{\prime \prime}+k^{2} x=x\left\{\begin{array}{ll}
\kappa / a^{2}, & r \leq a \\
\kappa / r^{2}, & r \geq a
\end{array}\right\}
$$

where the prime stands for $d / d z$, and $k$ is the wave number of the transverse motion in the absence of space charge. The perveance of the beam, $k=e I / 2 \pi \varepsilon_{0} m \nu^{3}$, is a dimensionless parameter proportional to the current $I$, where $e, m$, and $v$ are the charge, mass, and ion velocity, and $\varepsilon_{0}$ is the permittivily of free space. The equation for $y$ is identical to $\mathrm{Eq} .(2.1)$.

We now assume a core oscillation of wave number $p$ of the form $a \rightarrow a\left(1^{-}-\epsilon \cos p z\right)$ and expand $a^{-2}$ in Eq. (2.1) to first order in $\varepsilon$, the relative oscillation amplitude. After some algebra, Eq. (2.1) can be written as

$$
\begin{aligned}
x^{\prime \prime}+q^{2} x= & -\frac{\kappa}{a^{2}} x\left(1-\frac{a^{2}}{r^{2}}\right) \Theta(r-a) \\
& +\frac{2 \varepsilon \kappa}{a^{2}} x \cos p z \Theta(a-r),
\end{aligned}
$$

where $\Theta(u)=1,0$ for $u>0, u<0$ and where $q=$ $\sqrt{k^{2}-k / a^{2}}$ is the wave number of oscillations within the core. 
With the radial forces of Eq. (2.2), we see that the

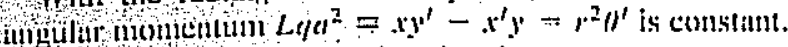
The equation for radial molion then becomes

$$
\begin{aligned}
& +r+q^{2}\left(r-\frac{L^{2} a^{4}}{r^{3}}\right)=-\frac{\kappa}{a^{2}} r\left(1-\frac{a^{2}}{r^{2}}\right) \Theta(r-a) \\
& +2 \frac{E \kappa}{a^{2}} r \cos p z \Theta(a-r) \text {. }
\end{aligned}
$$

The first term on the right makes the oscillation wave number depend on amplitude and the second allows for energy transfer between the core and the oscillating ion.

In order to understand the role of the different terms in Eq $(2.3)$, we coristrut a simplified molel by setting $L=$ 0 and invoking a pendulum model for the first term on the right side of Eq. (2.3) by replacing $r\left(1-a^{2} / r^{2}\right) \Theta(r-a)$ by $r^{3} / a^{2}$, corresponding to the cubic nonlinear term for a pendulum. (However, its sign is opposite from the conventional pendulum since, in our model, the wave number increases with increasing amplitude.) In addition, we extend the driving term to all values of $r$. The simplified equation for $x$ is therefore

$$
x^{\prime \prime}+q^{2} x=-\frac{\kappa}{a^{2}} \frac{x^{3}}{a^{2}}+2 \frac{E \kappa}{a^{2}} x \cos p z .
$$

We now use the phase-amplitude melhod by writing $x / a=A \sin \psi, x^{\prime} / a=q A \cos \psi$, where $\psi=q z+\alpha, \mathrm{im}-$ plying $A^{\prime} \sin \psi+A \alpha^{\prime} \cos \psi=0$. Here $A$ and $\alpha$ are taken to be the slowly varying amplitude and phase parameters of the ion oscillation. Substituting into Eq. (2.4) and solving for $A^{\prime}$ and $\alpha^{\prime}$ we obtain

$$
\begin{aligned}
A^{\prime} & =-\frac{\kappa A}{q a^{4}}\left[A^{2} \sin ^{3} \psi \cos \psi-\epsilon a^{2} \sin 2 \psi \cos p \varepsilon\right], \\
\alpha^{\prime} & =\frac{\kappa}{q a^{4}}\left[A^{2} \sin ^{4} \psi-2 \varepsilon a^{2} \sin ^{2} \psi \cos p z\right] .
\end{aligned}
$$

We now average over all rapidly varying oscillatory terms with the exception of the one with wave number $2 q-p$ (the paramelric resonance) and obtain

$$
\begin{aligned}
& A^{\prime}=\frac{\epsilon K}{2 q a^{2}} A \sin \Psi, \\
& \Psi^{\prime}=(2 q-p)+\frac{3 \kappa}{4 q a^{4}} A^{2}+\frac{E K}{q a^{2}} \cos \Psi,
\end{aligned}
$$

where $\Psi=(2 q-p) z+2 \alpha$ is the phase of this resonant interaction. One then finds that an integral of the motion exists, enabling us to write [7]

$$
\epsilon \cos \Psi=\Delta-\frac{3}{8} w-\frac{C}{w},
$$

where $w=A^{2} / a^{2}, \Delta=q(p-2 q) a^{2} / k$, and where the integration constant $C$ is determined by the initial values of $w$ and $2 \alpha$. By resorting to the envelope equation we can show that $p^{2}=4 q^{2}+2 \kappa / a^{2}$ for the breathing mode, so that

$$
\Delta=\frac{1}{1+\sqrt{\left(1+k^{2} / q^{2}\right) / 2}},
$$

where $q / k$ is the tune depression caused by the space charge. In Fig: I we plot $\epsilon \cos y r$ vs w [or $q / k=0.412$,

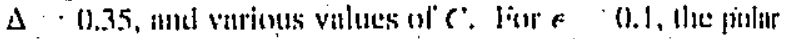
plol of 14 's $\Psi$ is shown in Fig. 2. It is clear that $Q$ is an unstable fixed point and that the origin and $S$ are stable fixed points. Figure 2 is equivalent to a "second order stroboscopic plol" for integral values of $\mathrm{mz} / 2 \pi$, and contains the main fealures of the resonant interaction. Specifically, all trajectories starting within the inner separatrix (thick solid curve) bounded by $P$ and $Q$ oscillate in stable orbits while any trajectory starting just outside will travel along the outer separatrix (thick dashed curve). For these particles, as the amplitude of motion grows the true oscillation wave number increases, enhancing the resconant lerin and locking in to the resumance. And the presence of a thin distribution of trajectories near the outer separatrix has the appearance of a halo in $x-y$ space at the radius corresponding to $R$ in Figs. 1 and 2 .

We now drop the simplified model and return to Eq. (2.3). Although the algebra is far more complicaled we eventually obtain a more accurate version of Eq. (2.9) with a very similar set of curves to those in Figs. 1 and 2. First we rewrite Eq. (2.3) for the variable $s=r^{2} / a^{2}$, obtaining

$$
\begin{aligned}
s^{\prime \prime}-\frac{\left(s^{\prime}\right)^{2}}{2 s}+2 q^{2}\left(s-\frac{L^{2}}{s}\right)= & -\frac{2 \kappa}{a^{2}}(s-1) \Theta(s-1) \\
& +4 \frac{E \kappa}{a^{2}} s \cos p z \Theta(1-s)
\end{aligned}
$$

Guided by the parametrization of the $x$ and $y$ motions separately, the amplitude-phase parametrization of the two-dimensional oscillation is written as

$$
s=\frac{w^{2}+L^{2}}{2 w}-\frac{w^{2}-L^{2}}{2 w} \cos (2 q z+\gamma) .
$$

Here $w$ and $\gamma$ are slowly varying amplitude and phase parameters which would be constant if the right side of Eq. (2.11) vanished. We now write

$$
s^{\prime}=q \frac{w^{2}-L^{2}}{w} \sin (2 q z+\gamma)
$$

and use Eq. (2.11) and the required conneclion between $w^{\prime}$ and $\gamma^{\prime}$ implied by Eq. (2.13) to obtain explicit expressions for $u^{\prime}$ und $\gamma^{\prime}$. We then' averinge over oscillations at

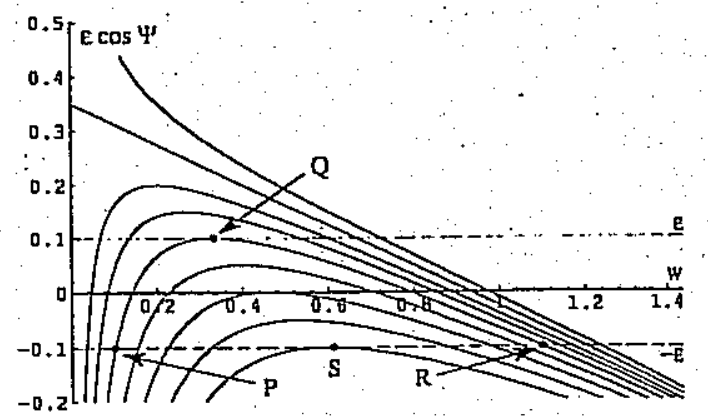

FIG. 1. Plot of $\varepsilon \cos \Psi$ vs $w$ for the simplified model with $\Delta=0.35$.
FIG.

to $\mathrm{tt}$

simp:

all $\mathrm{v}$

the :

equi

lar $\mathrm{t}$

the

whe

Her

for

for

tang

The

of 1

$b(n$

witl

$\mathrm{S}$

$L=$

cort

and

to 1

$E=$

Fig

is a

am]

$3 / 8$

I

dist 
0.412

: polar.

bat $Q$

ec

$z / 2 \pi$,

iction.

inner

sillate

utside

urve).

jrows

ig the

$d$ the

outer

ice at

in to

cated

(2.9)

1 and

$2 / a^{2}$

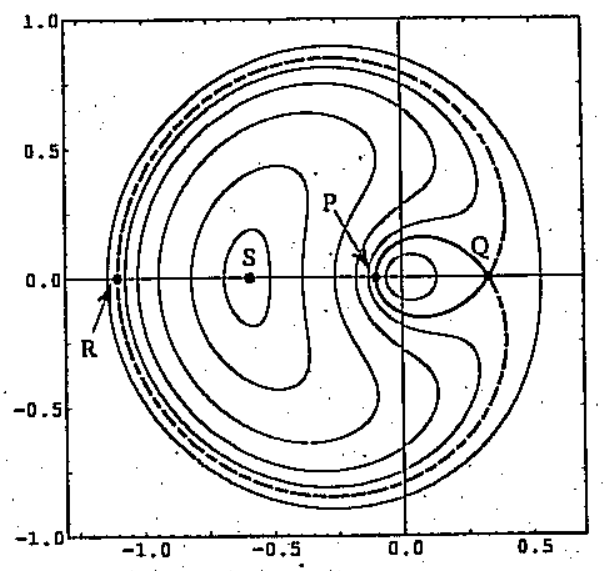

FIG. 2. Polur plot of $w$ vs I for the trajectories corresponding to the parametric resonunce using $\Delta=0.35, \epsilon=0.1$, and the simplified modsl.

all wave numbers except $2 q-\dot{p}$, being careful to include the step functions as we obtain these averages. The final equations for $w^{\prime}$ and $\Psi^{\prime}[\Psi r=(2 q-p) z+\gamma]$ are similar to Eqs. (2.7) and (2.8) and agiin leat to an integral of the motion, which now is

$$
g(1-h) \epsilon \cos \Psi=f \Delta-t-C,
$$

where $f(w)=\left(w^{2}+L^{2}\right) / 2 w, \quad g(w)=\left(w^{2}-L^{2}\right) / 2 w$ Here

$$
\pi h(w)=\tan ^{-1}[\ell /(1-f)]+\ell(1-f) / 2 g^{2}
$$

for $w \geq 1$ and $\ell(w)=\sqrt{(w-1)\left(w-L^{2}\right) / w}$. Also

$$
\begin{aligned}
t(w)= & \frac{1}{\pi} \int_{1}^{w} \frac{d w}{w g}\left[\left(g^{2}-f\right) \tan ^{-1}\left(\frac{\ell}{1-f}\right)\right. \\
& \left.+f \ell+L \tan ^{-1}\left(\frac{2 L \ell}{f-L^{2}}\right)\right],
\end{aligned}
$$

for $w \geq 1$, and $h(w)=t(w)=0$ for $w \leq 1$. The arctangents are taken to be in the first or second quadrant. The term in $b(w)$ comes from the amplitude dependence of the ion wave number. A more accurate expression for $b($ ii) can be obtained, if necessary, by solving Eq. (2.6) with $E=0$.

Since the resonance will have its greatest effect when $L=0$, corresponding to ion orbits which pass through the core center, we present a plot of $\epsilon \cos \psi$ vs $w$ for $\Delta=0.35$ and $L=0$ in Fig. 3. The pallern of curves is very similar to that in Fig. 1, and the $w, \Psi$ polar plot in Fig. 4 for $\epsilon=0.1$ has the same lopology as for the simple model in Fig. 2, as is also the case for $L \neq 0$. But the scale for $w$ is aboul 7 times larger, corresponding to a detuning with amplitude about 7 limes smaller than that given by the 3/8 factor in Eq. (2.14).

III. Distribution of $L$ in a symmetric $K-V$ beam. - The distribution in $L$ Tor a $\mathrm{K}-\mathrm{V}$ beam is proportional to

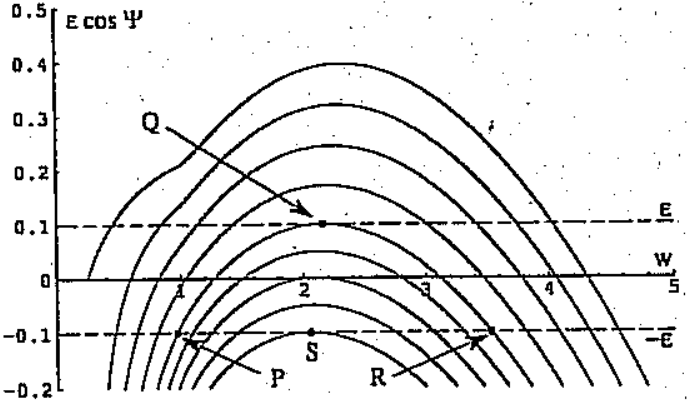

FIG. 3. Plot of $e \cos \mathrm{Yr}$ is $w$ for the exact model with $\Delta=$ $0.35, L=0$.

$$
\begin{aligned}
& f(L)-\iiint \int d x d y d x^{i} d y^{\prime} \delta\left(x^{2}+y^{2}\right. \\
&+\left.\frac{\left(x^{\prime}\right)^{2}}{q^{2}}+\frac{\left(y^{\prime}\right)^{2}}{q^{2}}-a^{2}\right) \delta\left(x^{\prime} y-x y^{\prime}-L q a^{2}\right)
\end{aligned}
$$

We first do $45^{\circ}$ rotations from the $x / a, y^{\prime} / q a$ space to the $u, u^{\prime}$ space and from the $y / a, x^{\prime} / q a$ space to the $\nu ; v^{\prime}$ space and follow this by integrating over the polar angles in the $u v^{\prime}$ and $v u^{\prime}$ spaces. This leads to

$$
\begin{aligned}
f(L)-\int_{0}^{\pi} d s \int_{0}^{\infty} d t \delta(s+t-1) \delta\left(\frac{s-t}{2}-L\right) \\
\quad=\left\{\begin{array}{ll}
1, & 2|L|<1 \\
0, & 2|L|>1
\end{array}\right]
\end{aligned}
$$

where $s=u^{2}+\left(v^{\prime}\right)^{2}$ and $t=u^{2}+\left(u^{\prime}\right)^{2}$ are both positive. Therefore the distribution in $L$ for a K-V beam is uniform from $L=-1 / 2$ to $L=1 / 2$ and vanishes for $|L|>1 / 2$.

IV. Implications of the model.-Since the breathing $\mathrm{K}-\mathrm{V}$ beam is a solution of the Vlasov equation, particles within the core will continue to remain there, even in the presence of the resonant interuction. If however,

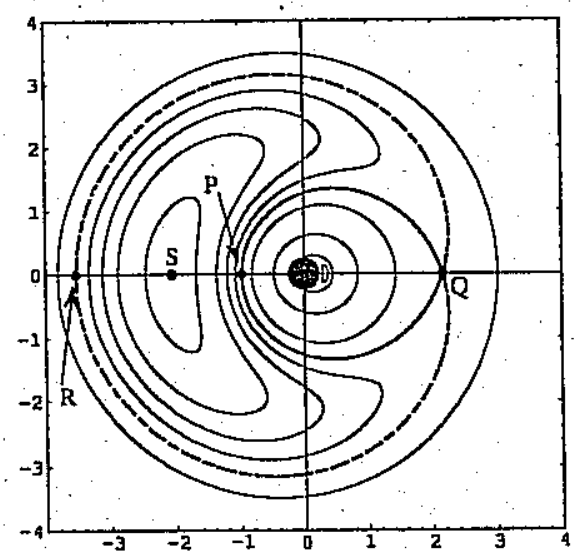

FIG. 4. Polar plot of $w$ vs $\Psi$ for the trajectories corresponding to the parametric resonance using $\Delta=0.35, L=0, E=0.1$, and the exact model. 
some other mechanism moves the particle outside the core, particularly to an oscillation amplitude exceeding that corresponding to the points $P$ or $Q$ in the figures, a halo will develop at a radius corresponding to point $R$ in the figures. The most likely mechanism to do this is an instability associaled with a nonlinear density perturbation. In addition, simulations show that chaotic motion develops near point $Q$ in the figures for a large amplitude breathing mode at high current, enabling particles in the core to populate the halo.

$V$. Summary and discussion. - We considered a symmetric $\mathrm{K}-\mathrm{V}$ beam undergoing a breathing mode and found that the parametric resonance $(2 q=p)$ is a vehicle for particles to leave the core of the beam and perform excursions to large amplitude, forming a distribution in real space in the form of a halo. In this calculation, we neglected the effect of high frequency terms, and the effect of other possible resonances and driving oscillations. Thus our model; which successfully describes a mechanism by which halos can and probably do form, is only an approximation to a much more complicated situation.

We have compared our predictions with some preliminary simulations performed for $L=0$ by Wangler [8]; and find that, for tune depressions from $q / k=1$ to 0.6 , the topology of the stroboscopic plot resembles Figs. 2 and 4 very closely. For tune depressions below 0.6, the stroboscopic plot shows the onset of chatic behavior in an ever widening band near the inner separatrix as the tune depression deepens. Particles inside but near to the inner separatrix are then able to move outside the inner separatrix and participate more easily in the development of the halo.

Wangler's simulations using a $\mathrm{K}-\mathrm{V}$ hetm $[8]$ conlirm that core ions always remuin within the inner separatrix. It is quite possible for core ions to lie outside the equivalent inner separatrix for nonuniform equilibrium charge density distributions. We therefore expect the halo mechanisms in the present model to apply to non-K-V beamis as well. Lagniel's simulations [6] give similar results, showing the onset of chaos for high space charge as well as the similarity with the three-body astronomical problem.
Our present model is unable to describe either diffusion or chaos in the $w$, Ir phase space. If we were to try to do so we would have to include the neglected higi frequency terms, as well as resonances other than the one corresponding to the purametric resomance. Integrals of the motion corresponding to Eq. (2.14) would no longer be valid. Descriptions of the growth of halos including the effects of chaos and diffusion will require further analysis and/or extensive numericnl simulations.

The iullyor would like to llauk Alex Dragt, Bub Jameson; Pierte Lapostolle, Ron Ruth, Rob, Ryne, Fred Skiff, and Tom Wangler for several helpful comments. $\mathrm{He}$ is also indebted to Dan Abell for performing the calculations leading to Figs. $1 \ddot{4}$.

[1] Sec I. M. Kapchinstiy. Themy of Resimemere Lintar

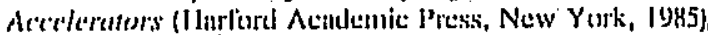
p. $247 \mathrm{fr}$.

[2] R. A. Jameson, Los Alamos Report No. LA-UR-93-1209 (unpublished). Jameson describes the early observations of emittance growth and halo production, with particular reference to LAMPF simulations and observations.

[3] M. Reiser, in Proceedings of the 1991 Particle Accelerator Conference, San Francisco, California, (unpublished), p. 2497.

[4] A. Cucchetti, M. Reiser, and T. Wangler, in Proceedings of the 1991 Particle Accelerator Conference (Ref. [3]), p. 251 .

[5] J.S, OC.mincil, T.P. Wimgler, R.S. Mills; and K.R. Crindall, in P'rucectings al' the lyes P'article Accelerator Conference, Washington, D.C. (unpublished), p. 3657.

[6] J. M. Lagniel, Nucl. Instrum. Methods Phys. Res., Sect. A 345,46 (1994).

(7) This result can also be obtained using a contact transformation, followed by neglecting rapidly oscillating terms. In fact, the transformed Hamiltonian in the canonical variables $w$ and $\Psi$ is $H=\left(\kappa / q a^{2}\right)[w \epsilon \cos y r-\Delta w+$ $\left.3 w^{2} / 8\right]$.

[8] Tom Wangler (private communication). 

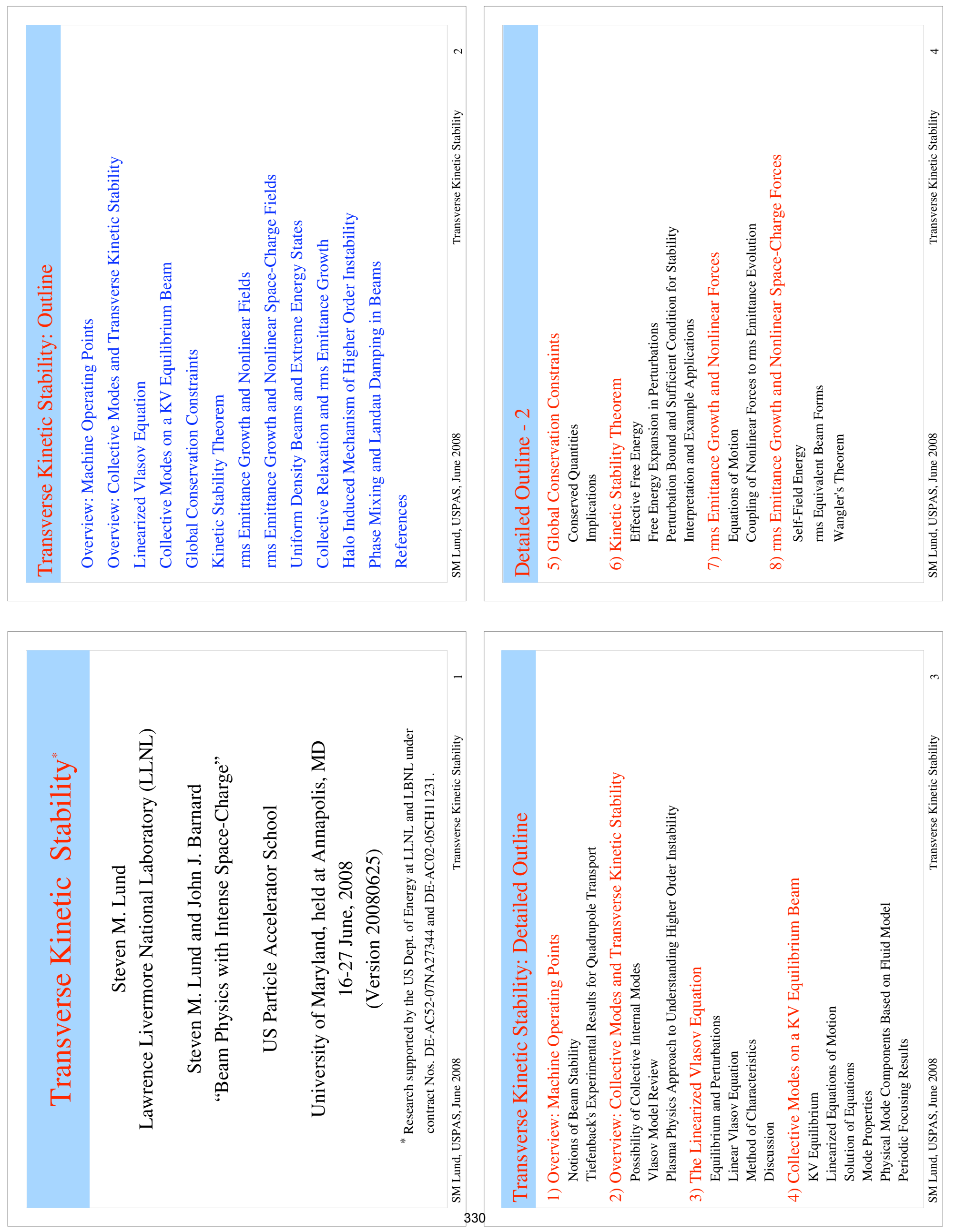


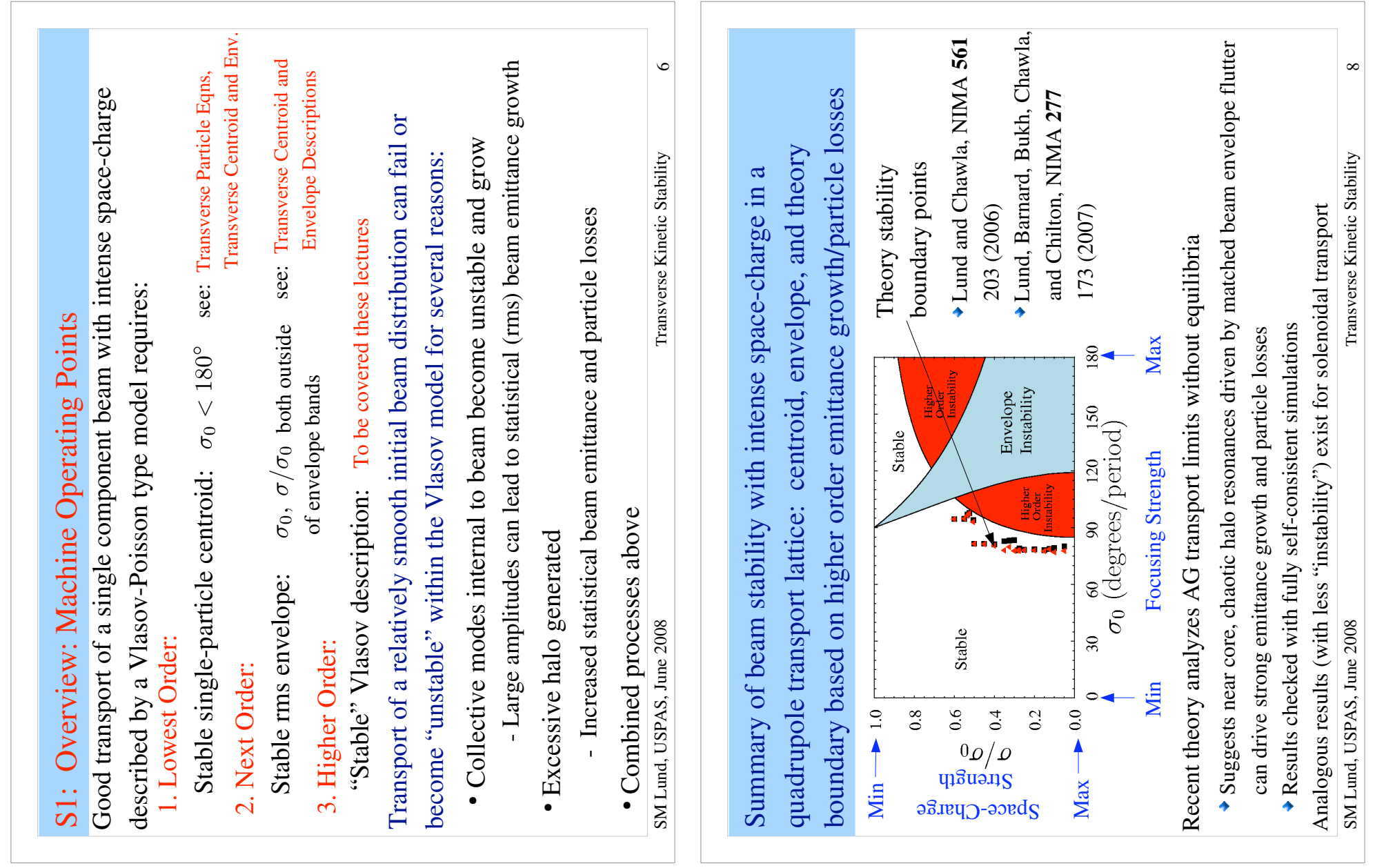

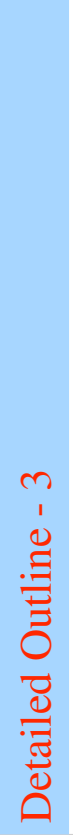
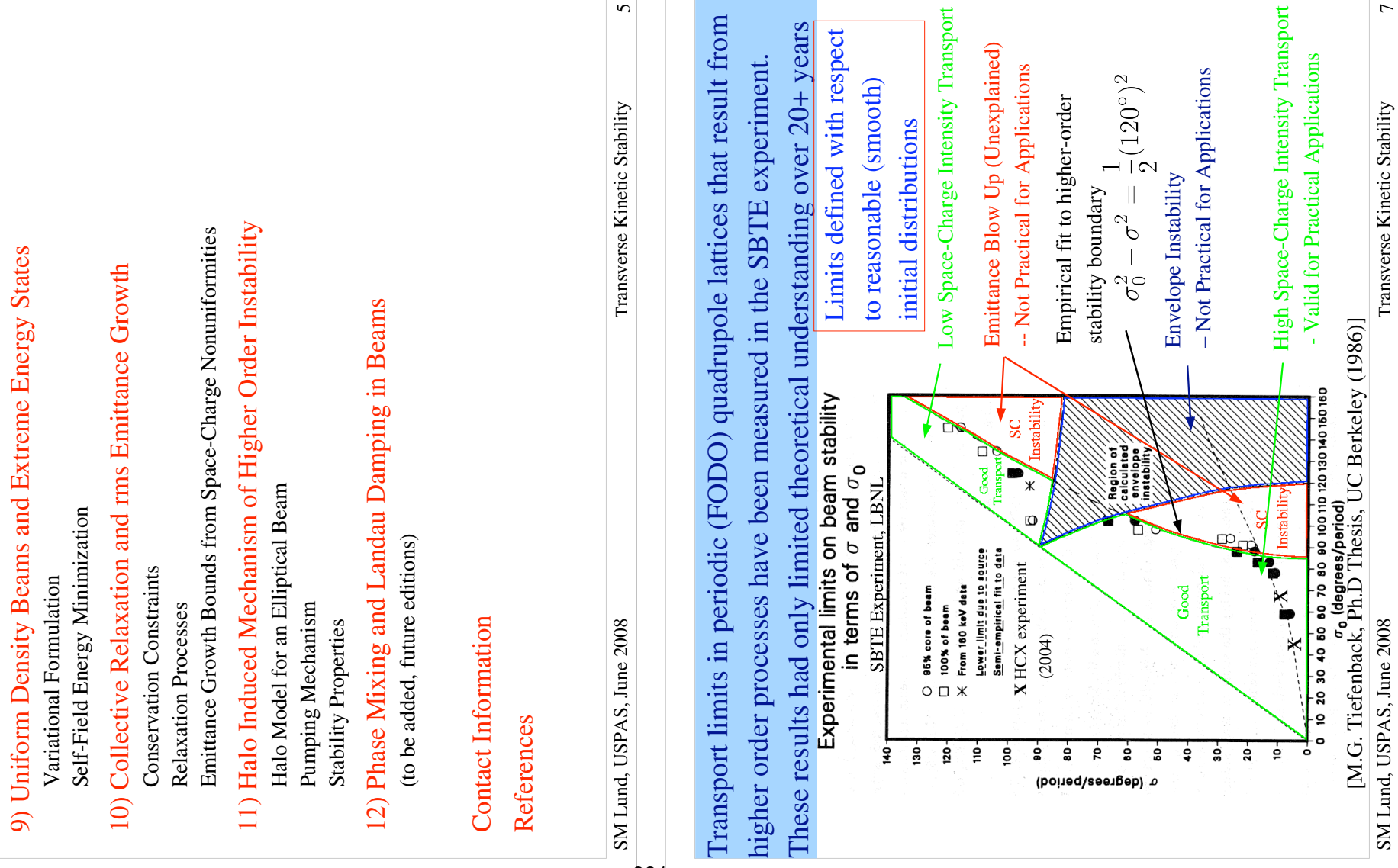

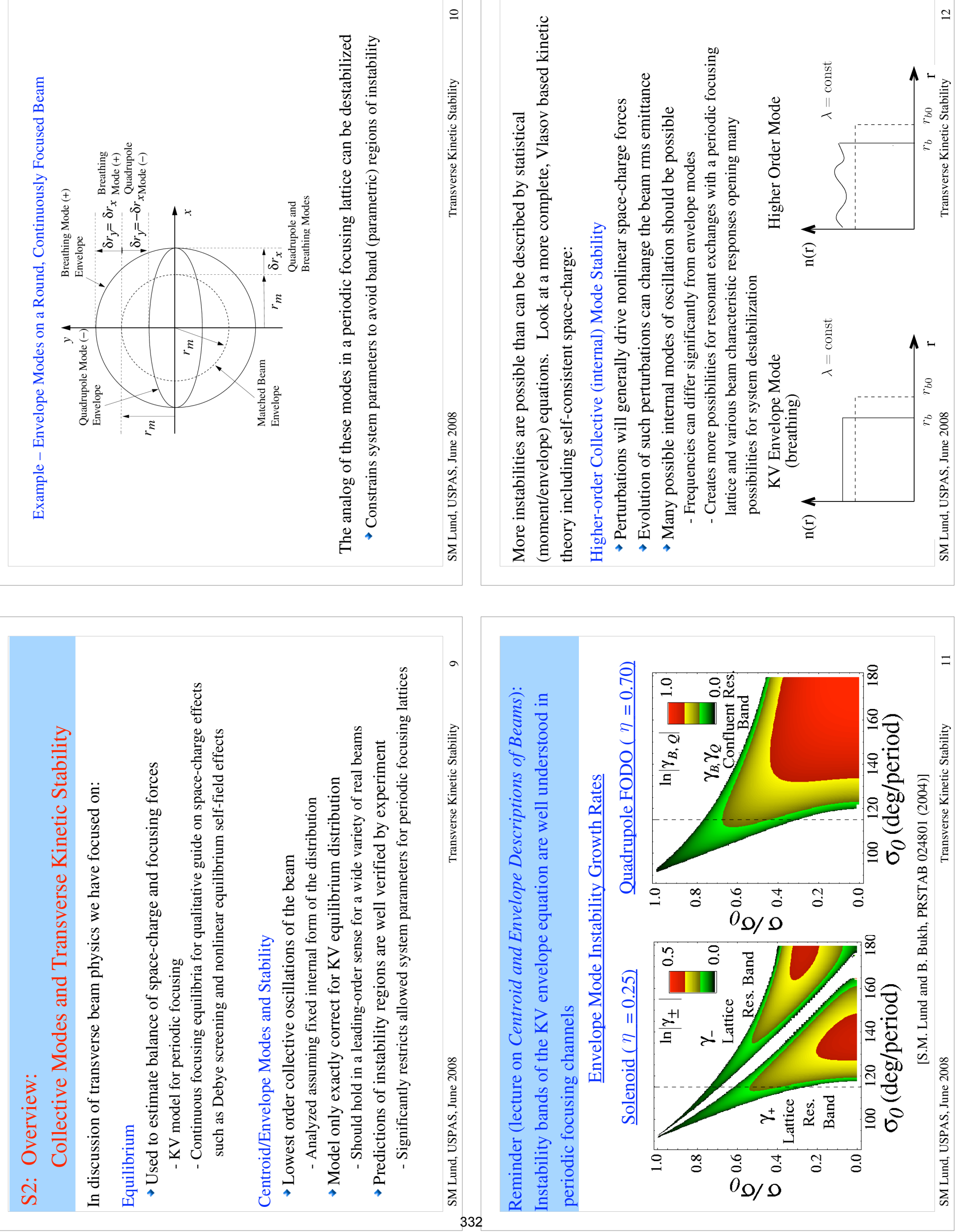

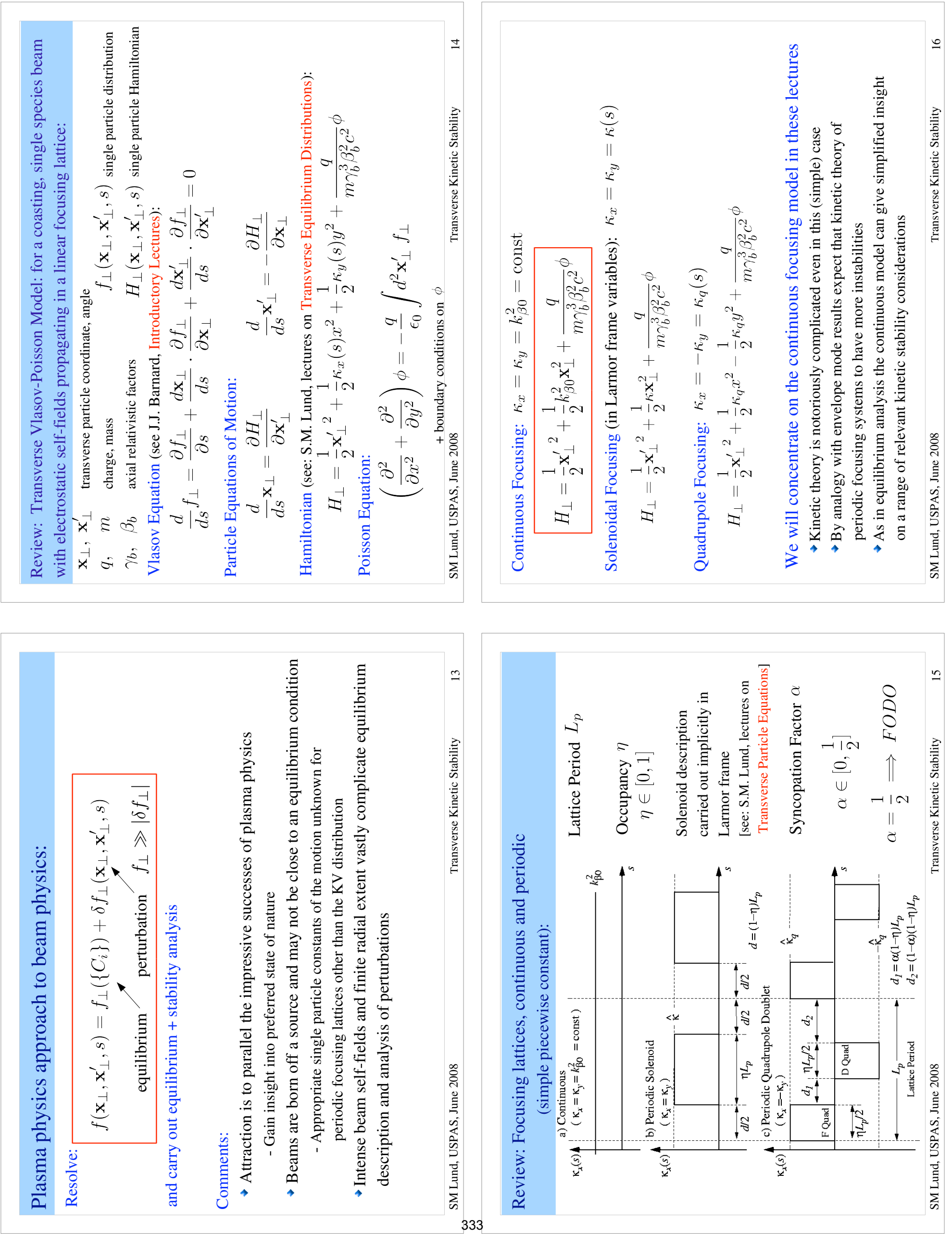


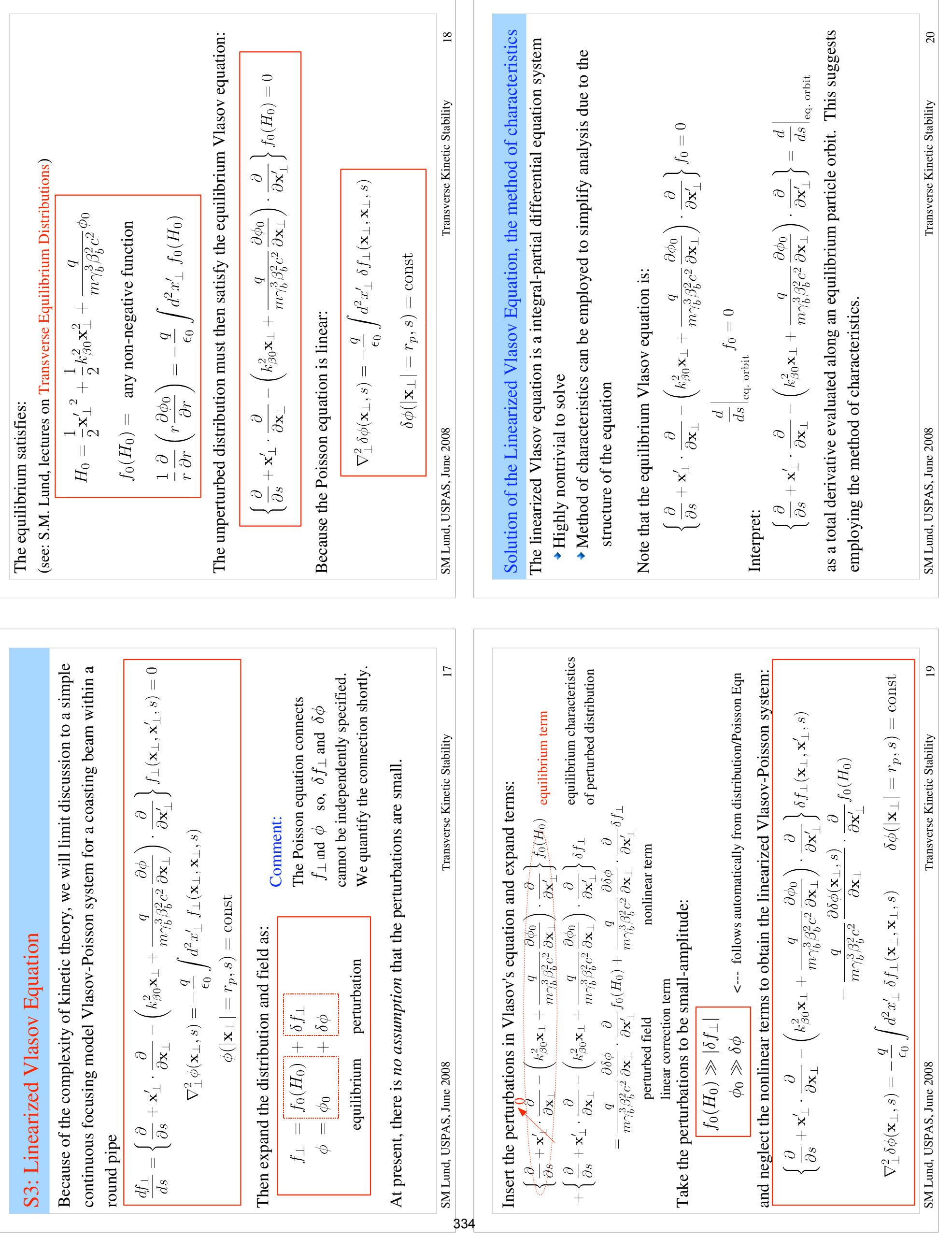



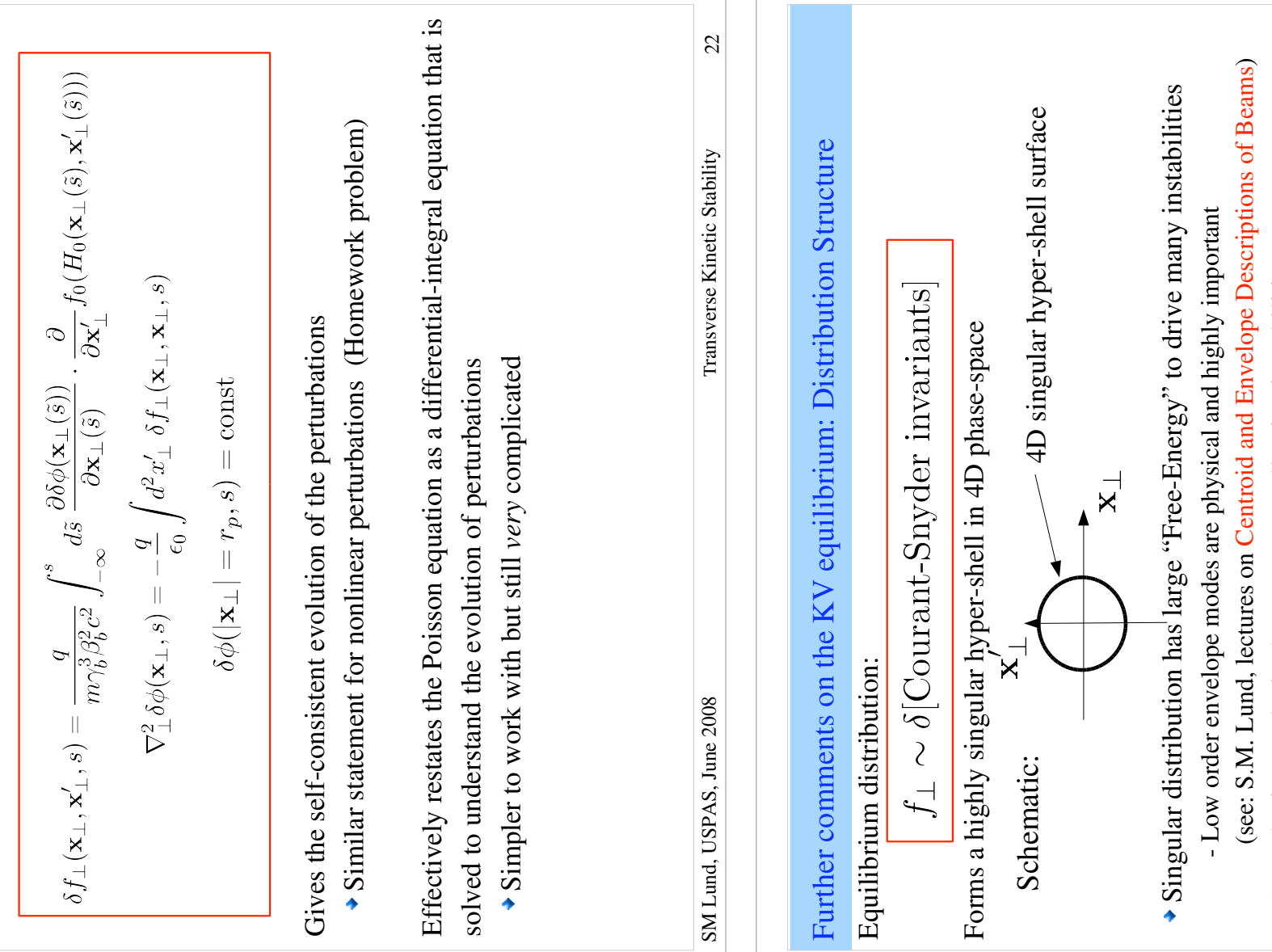

$\stackrel{4}{4}$

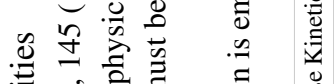

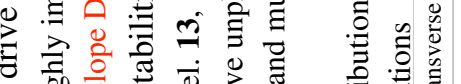

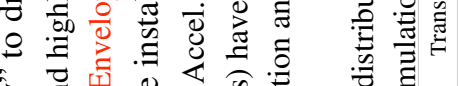

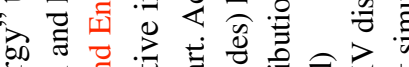

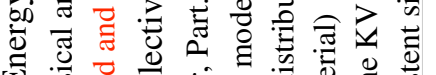

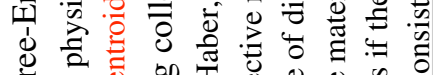

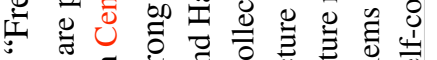
品 0 o

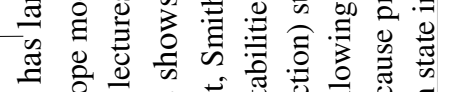

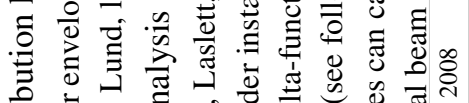

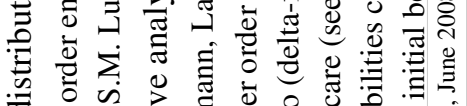

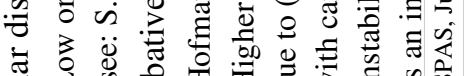

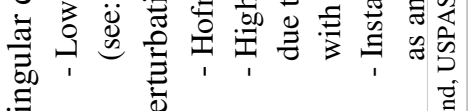

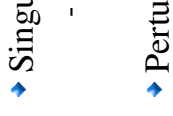
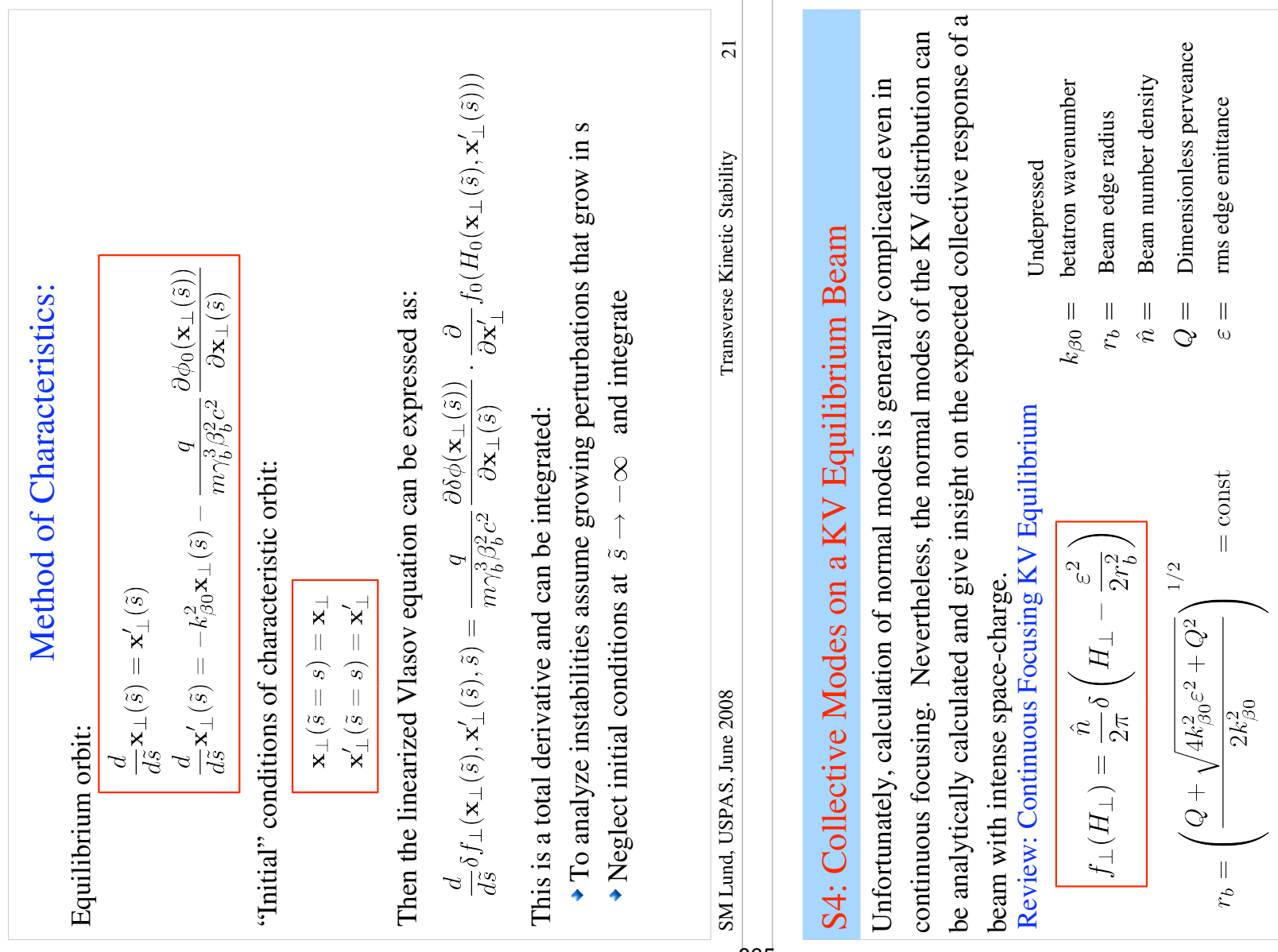

$\pi$ 

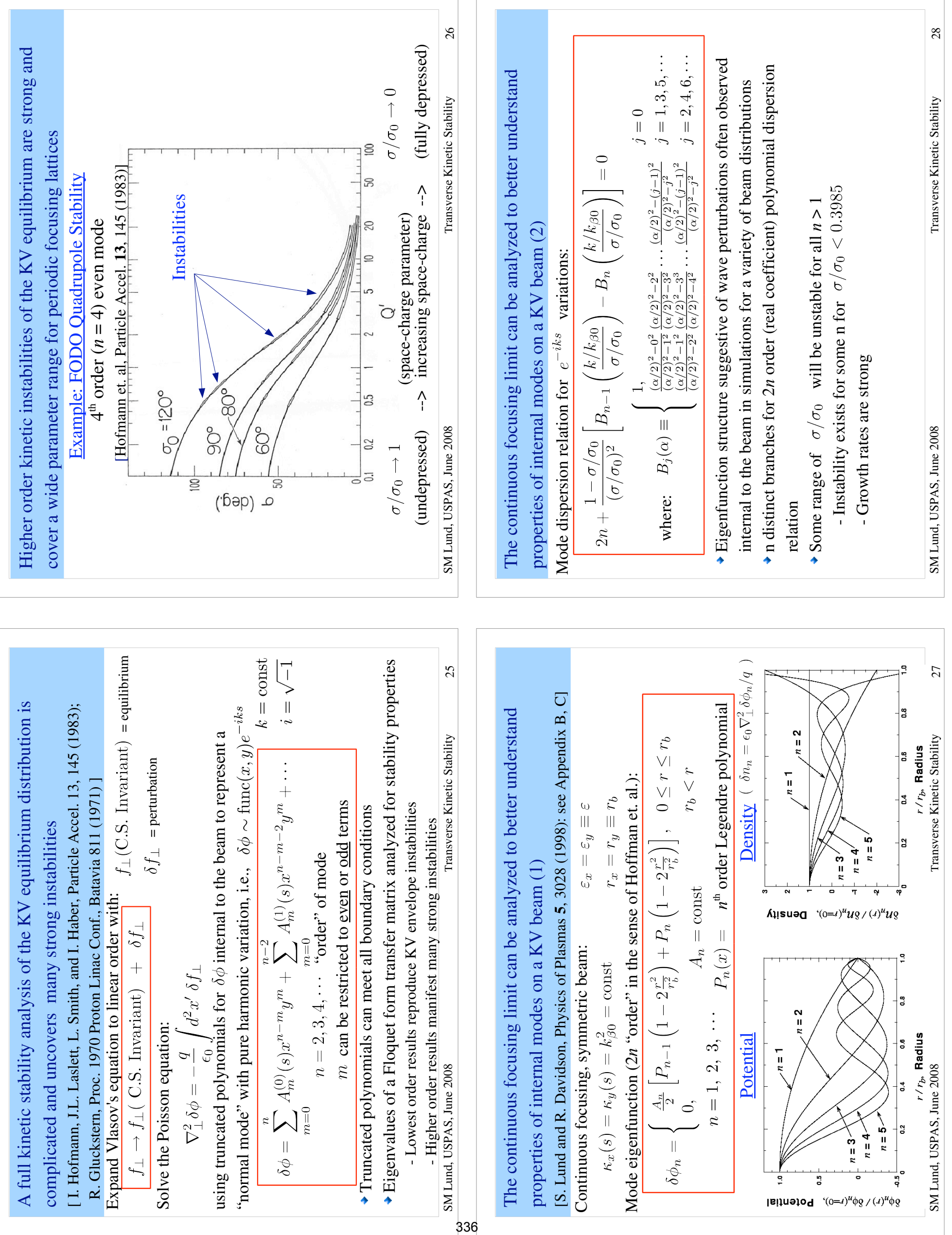

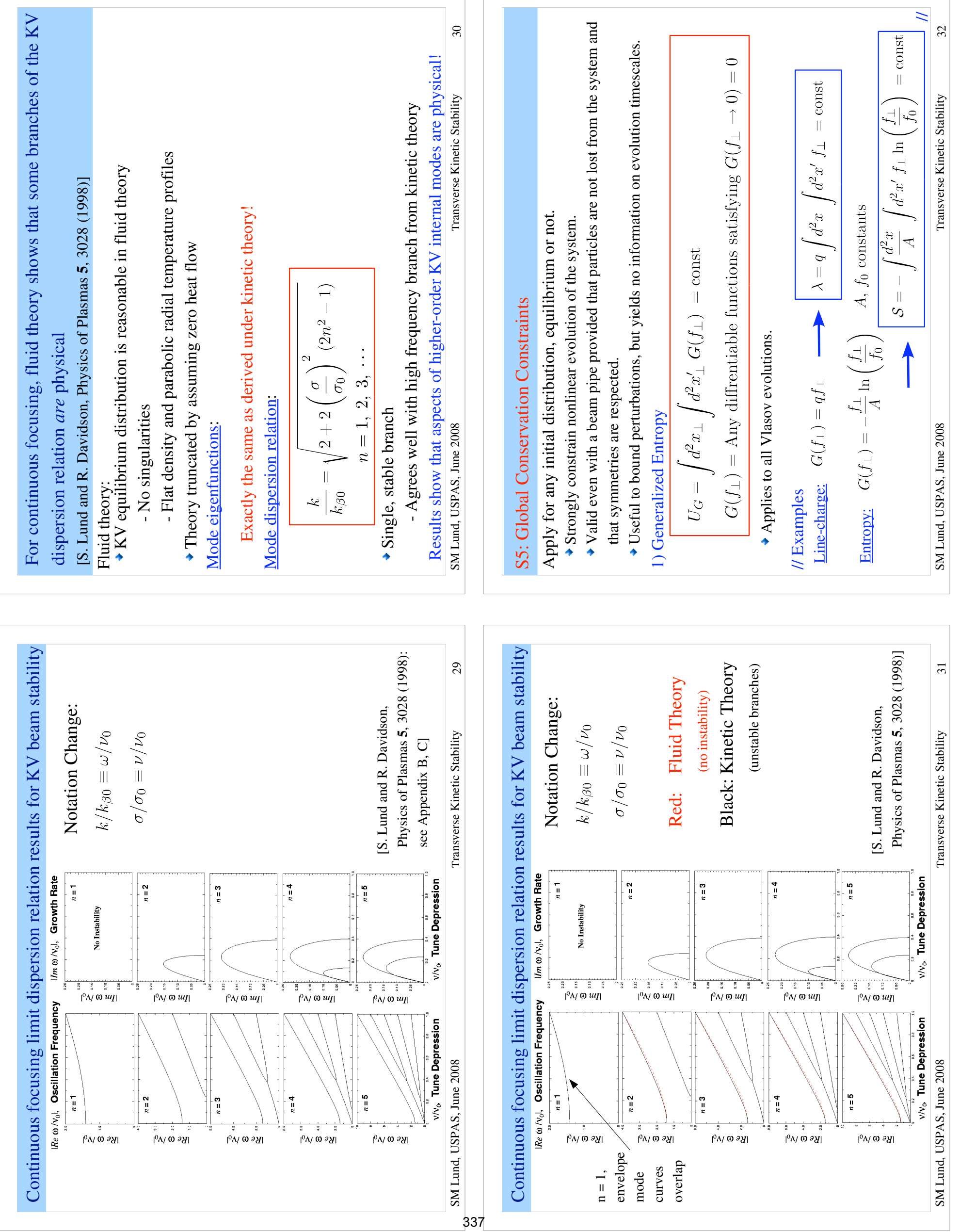


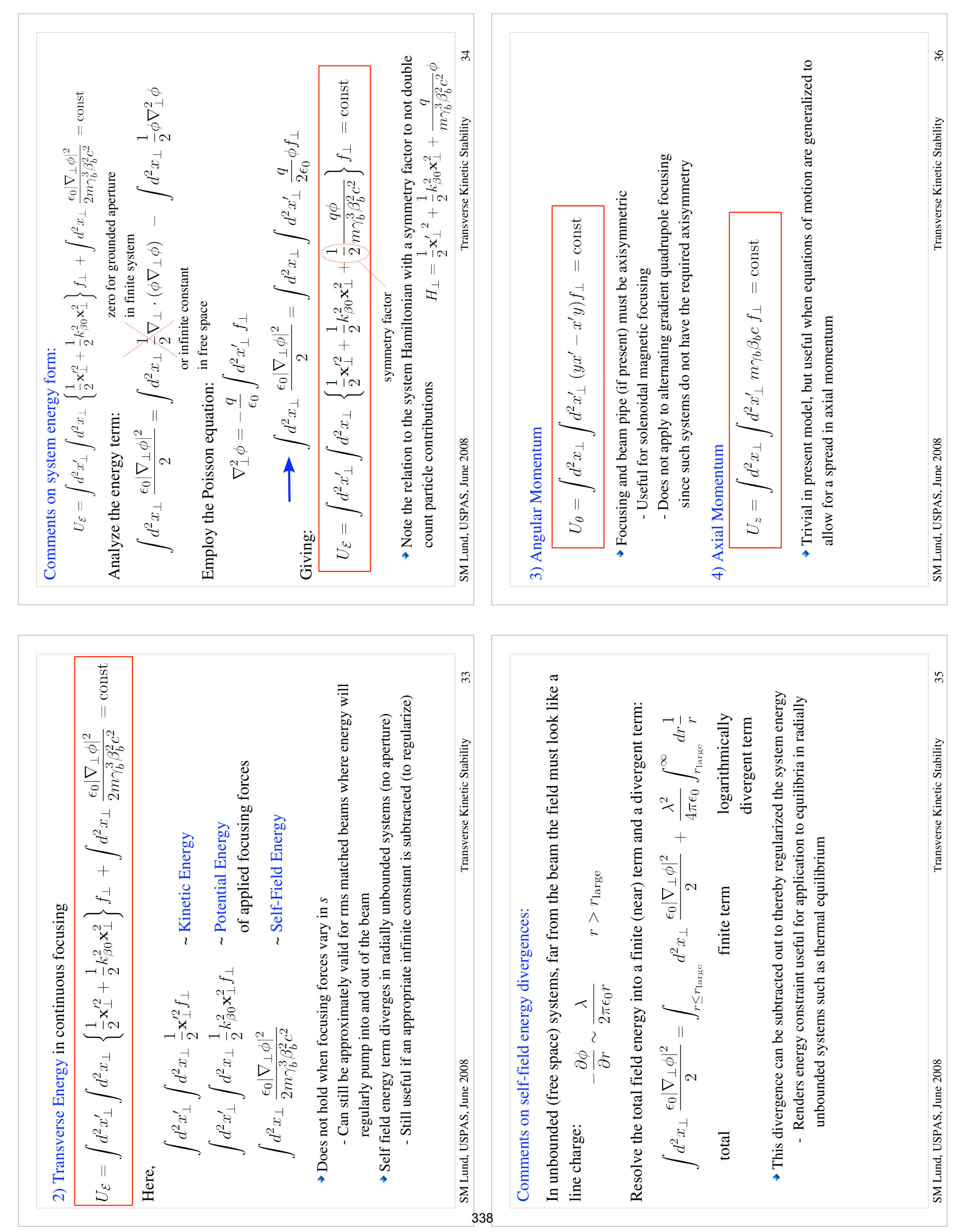




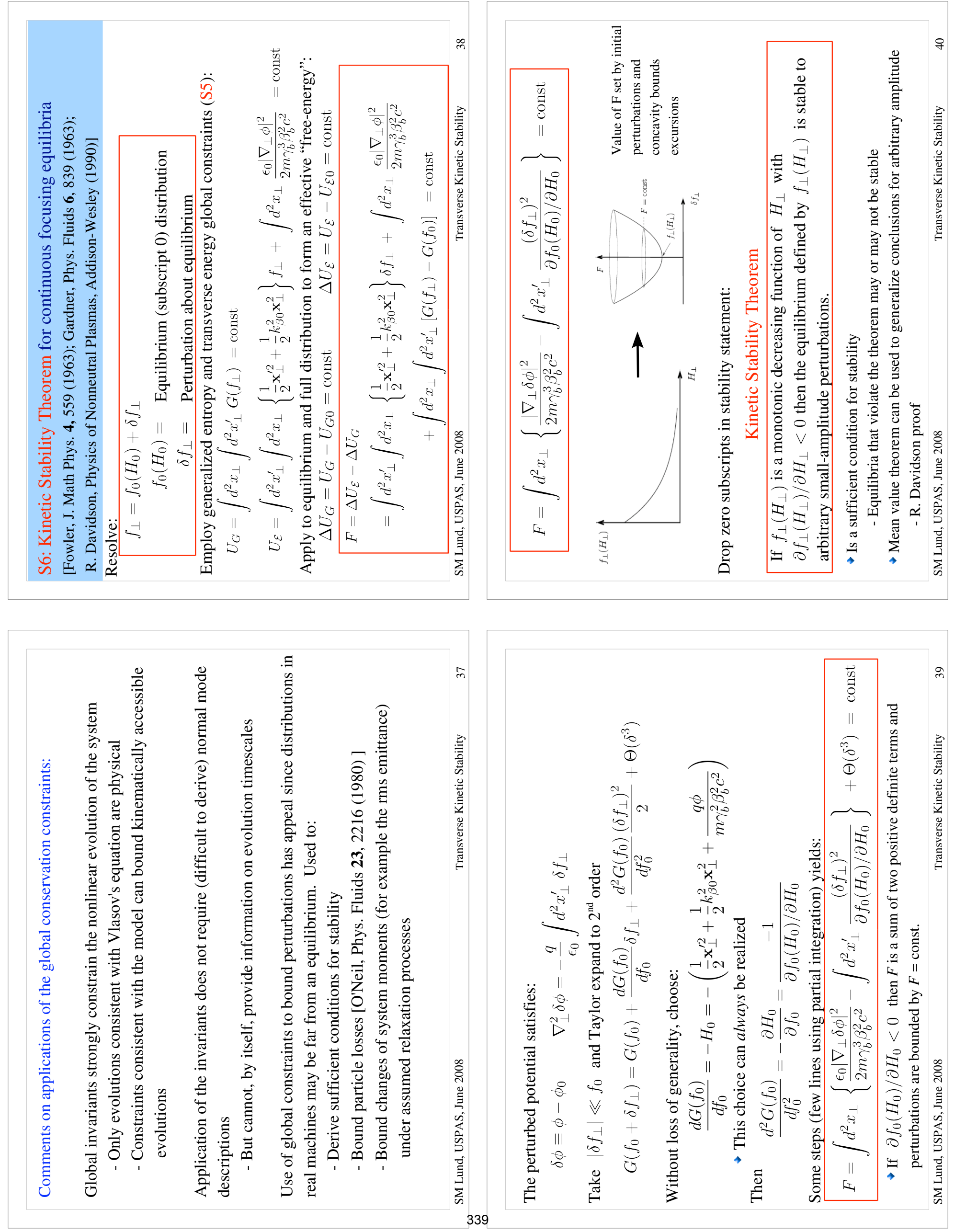



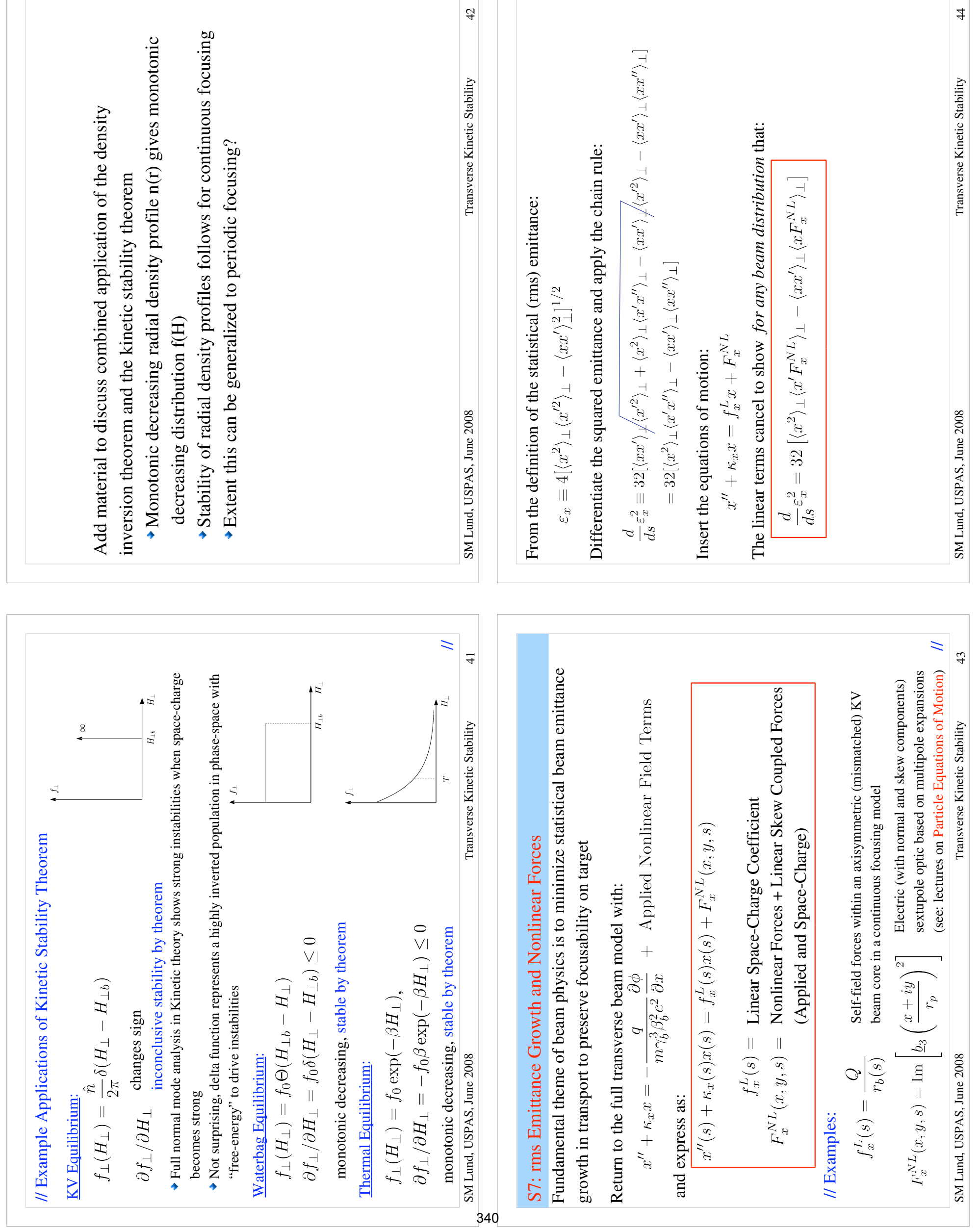


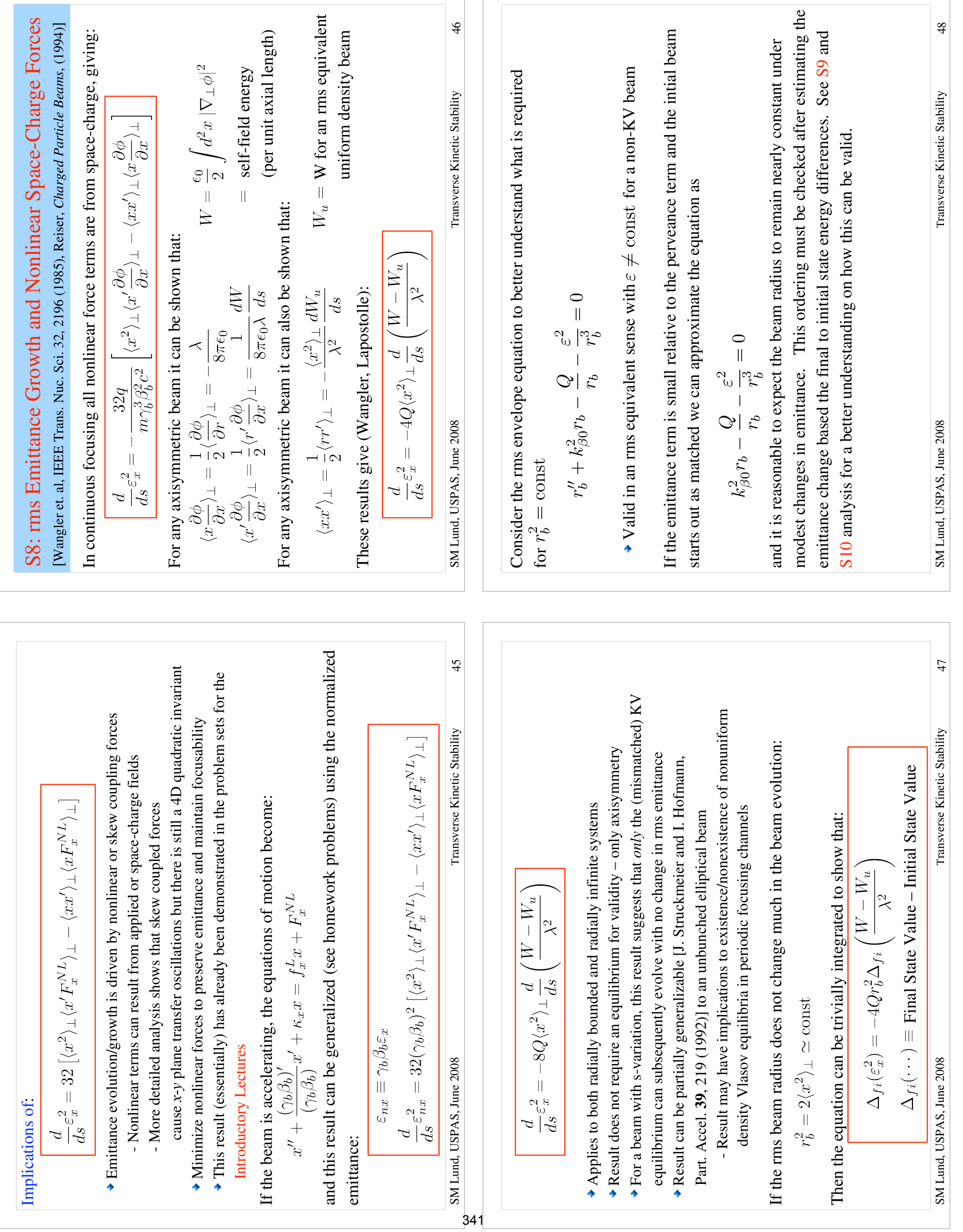



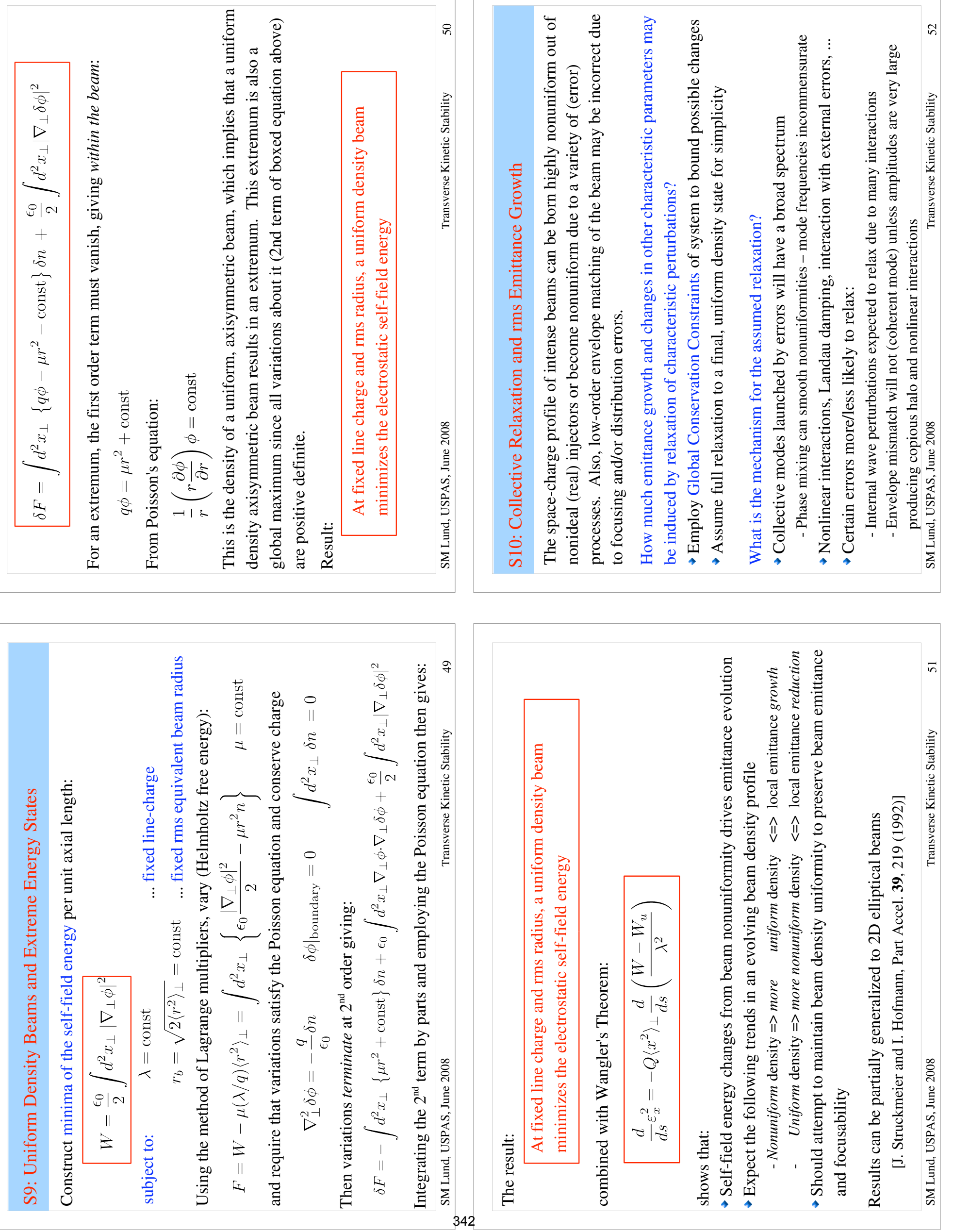

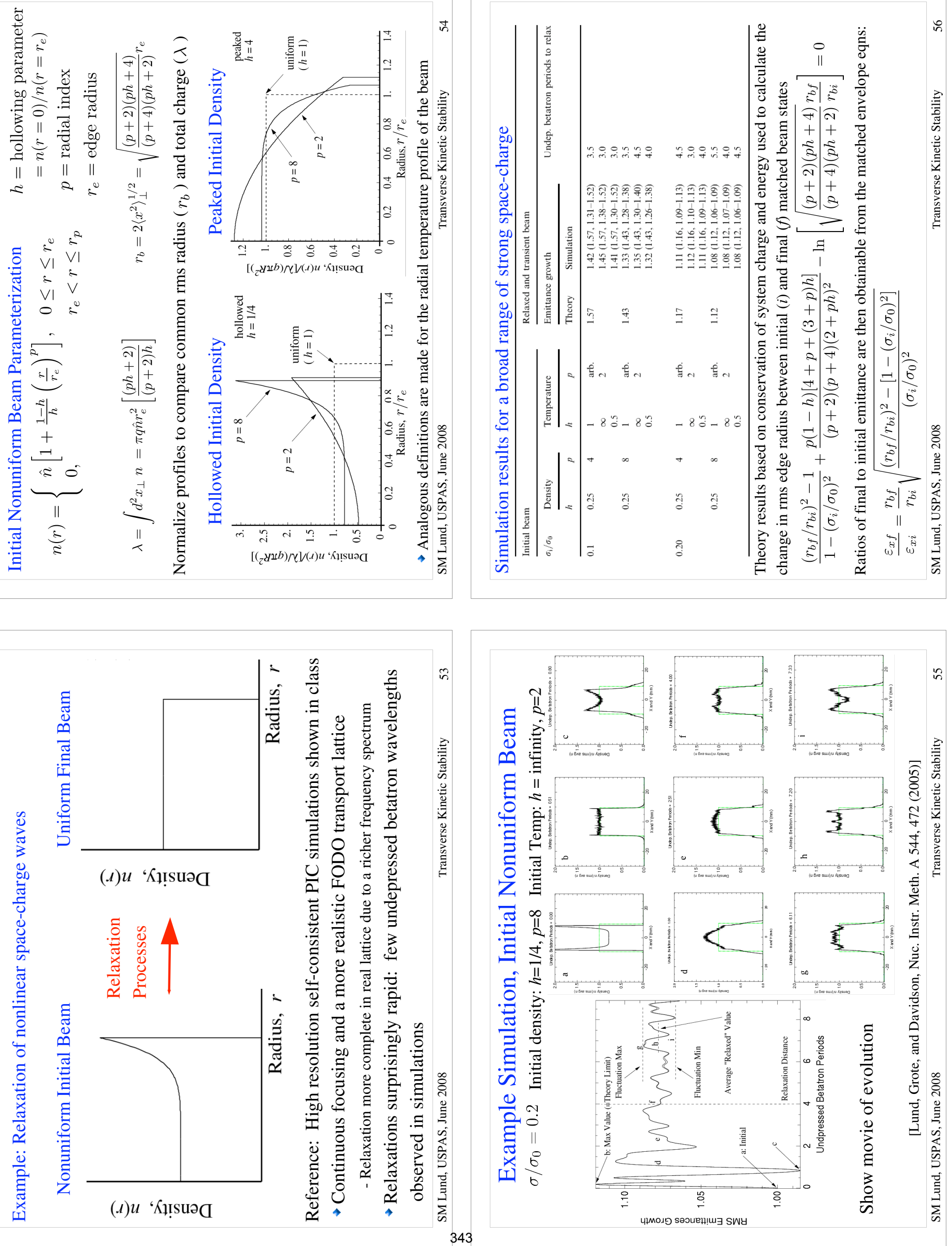

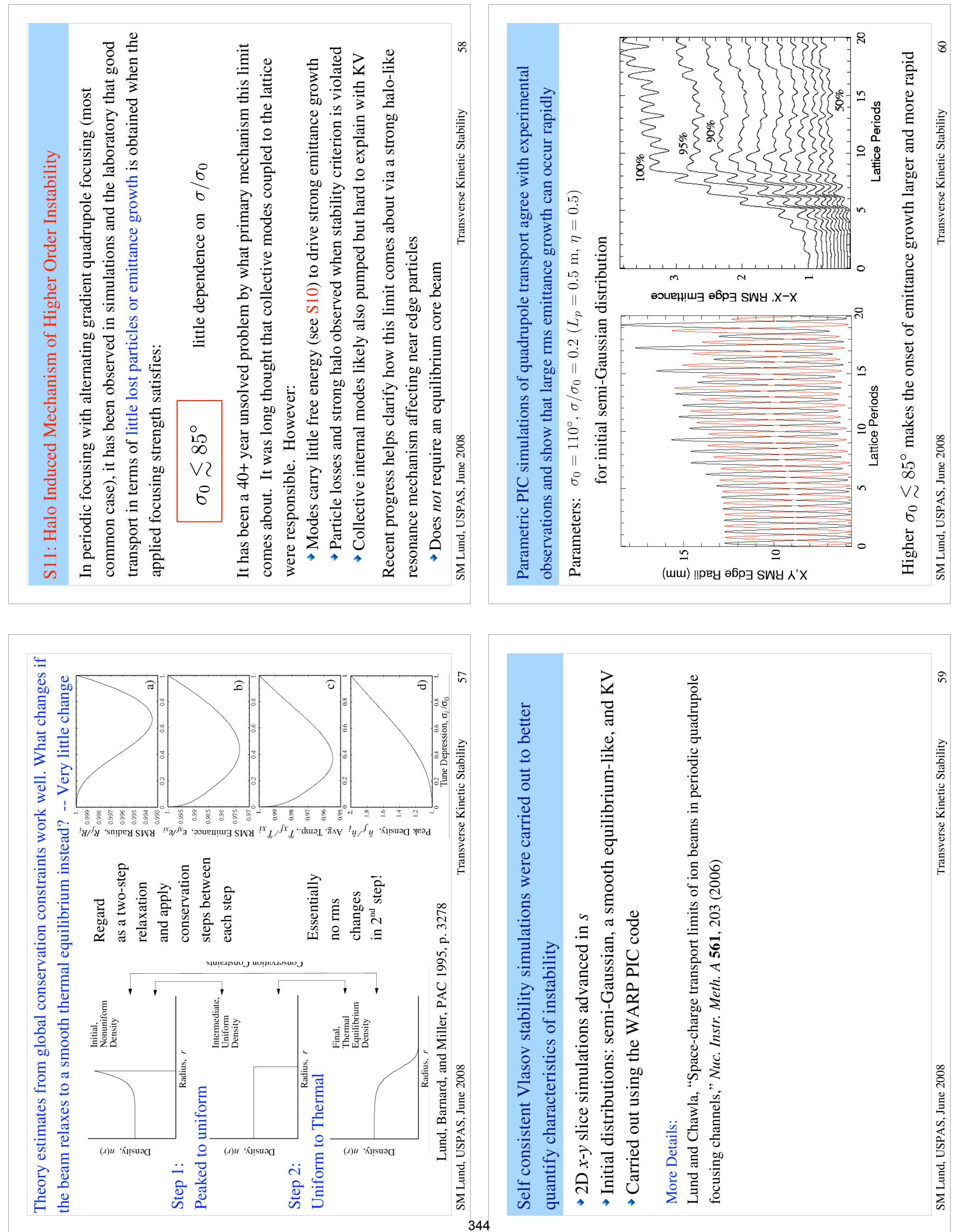

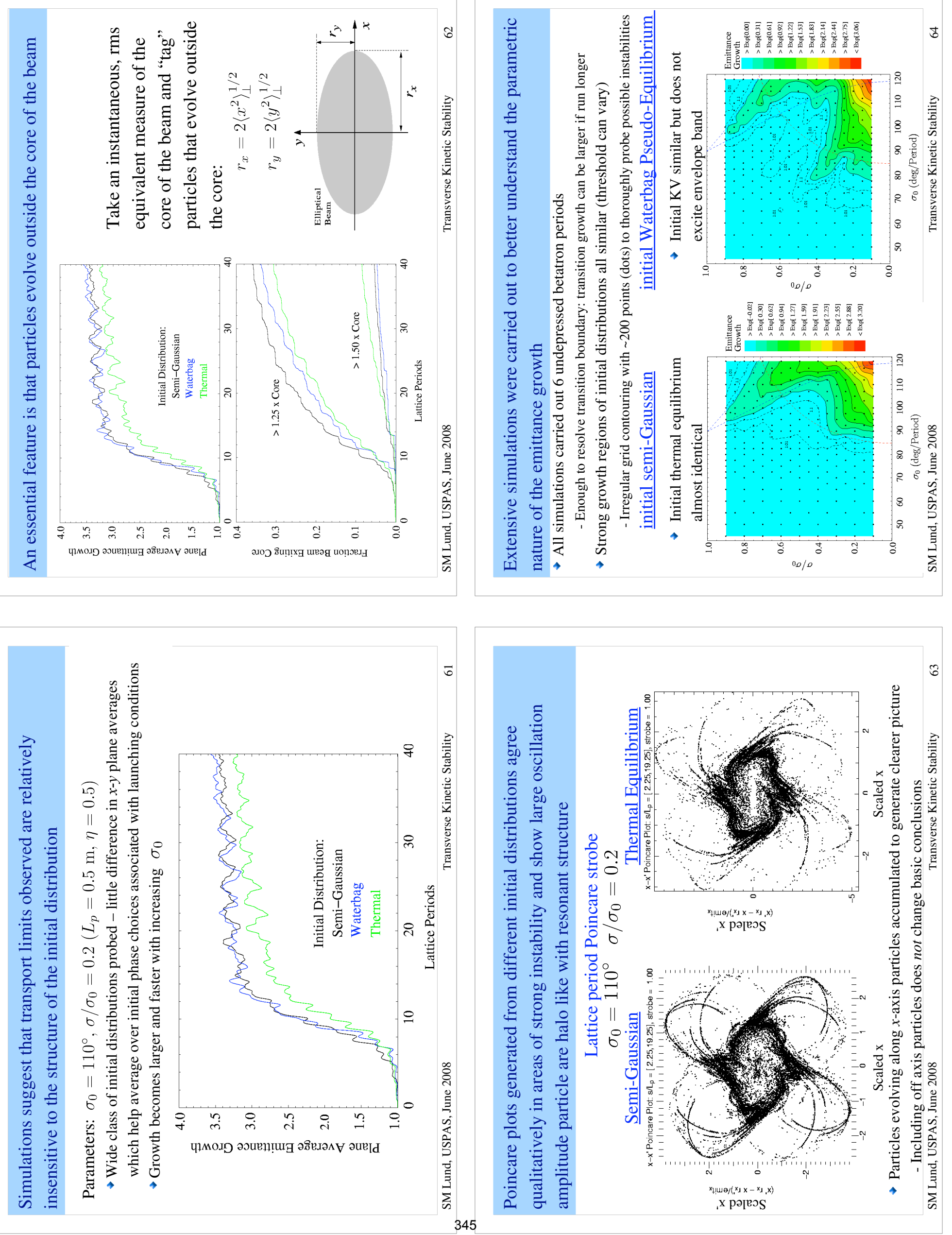

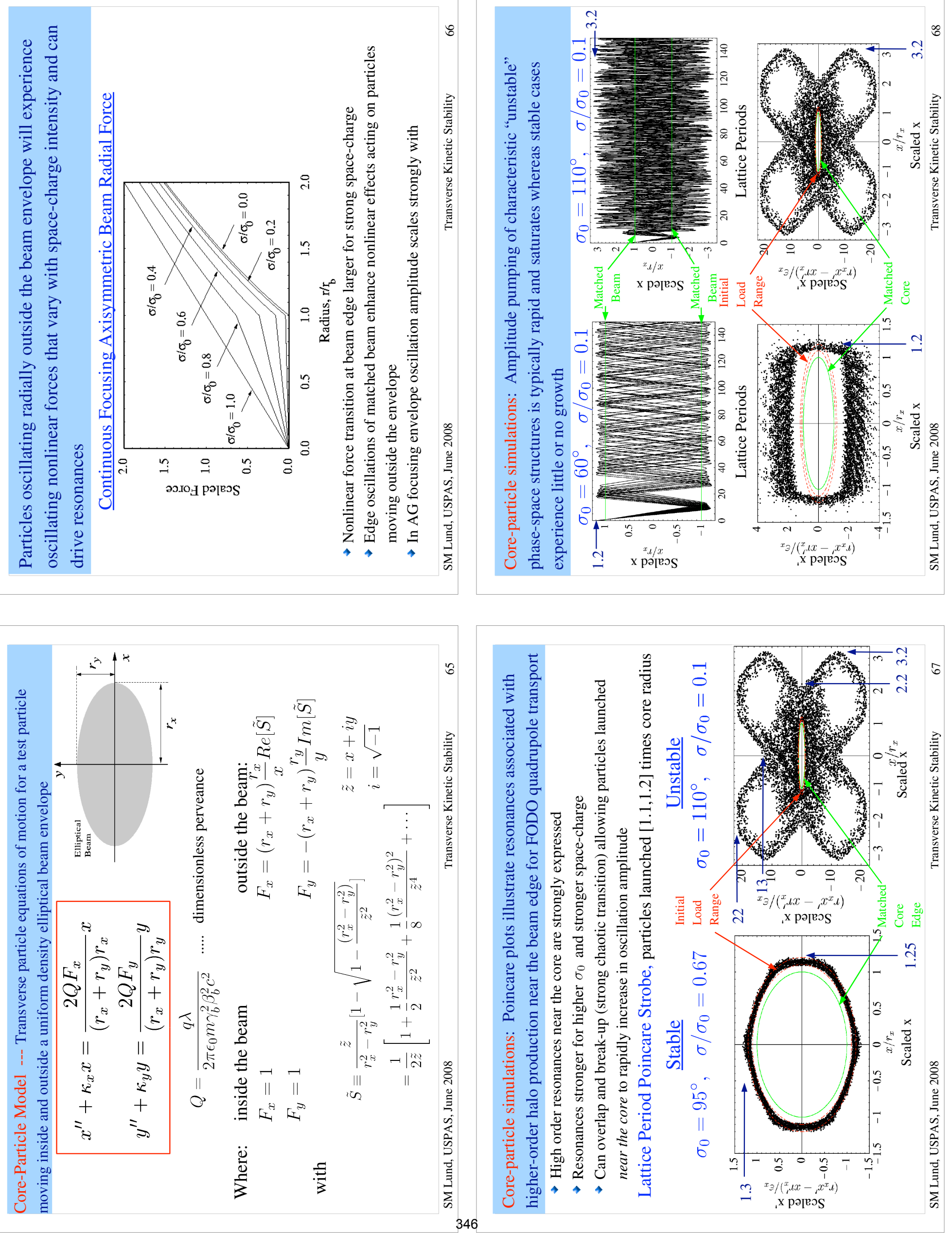

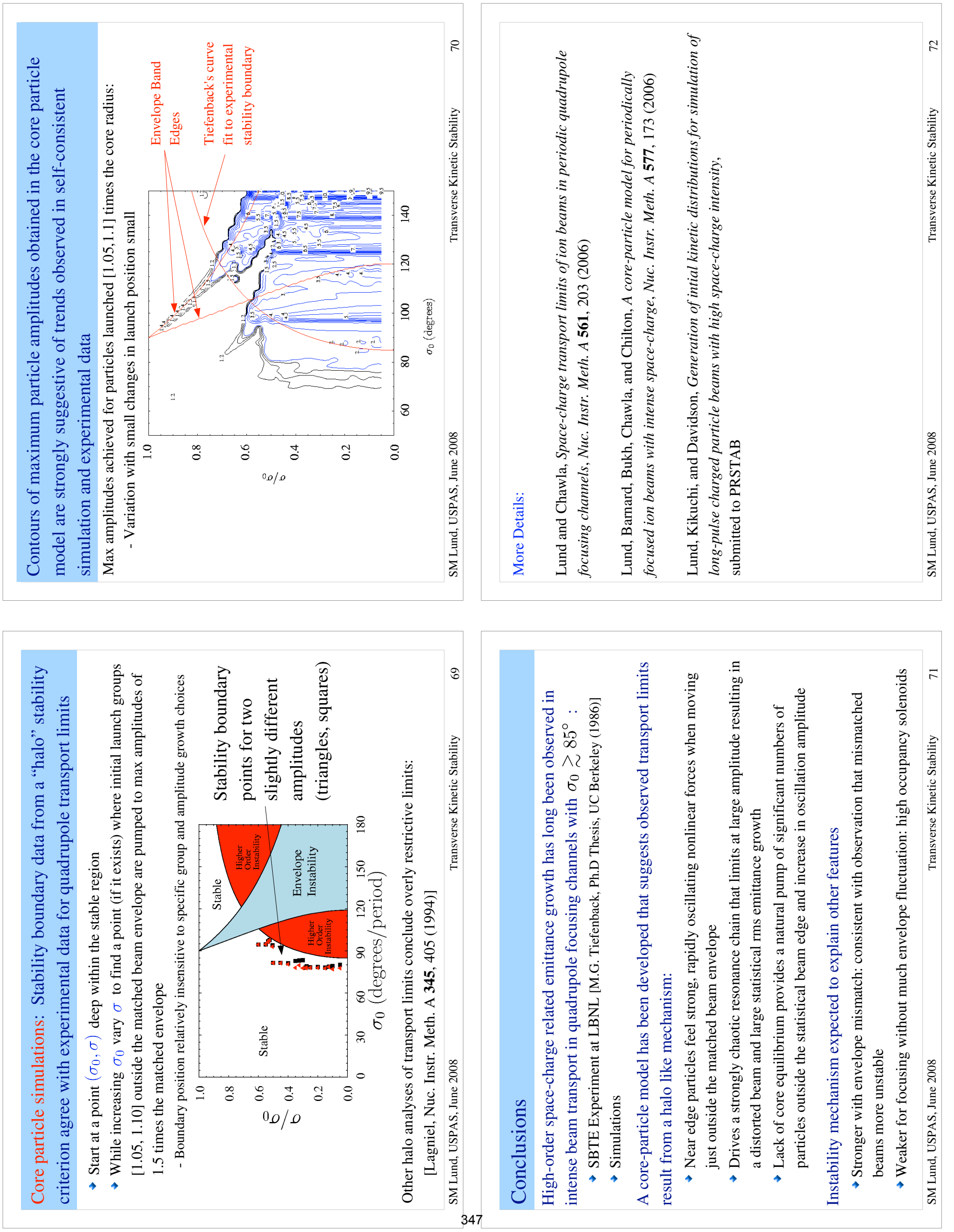

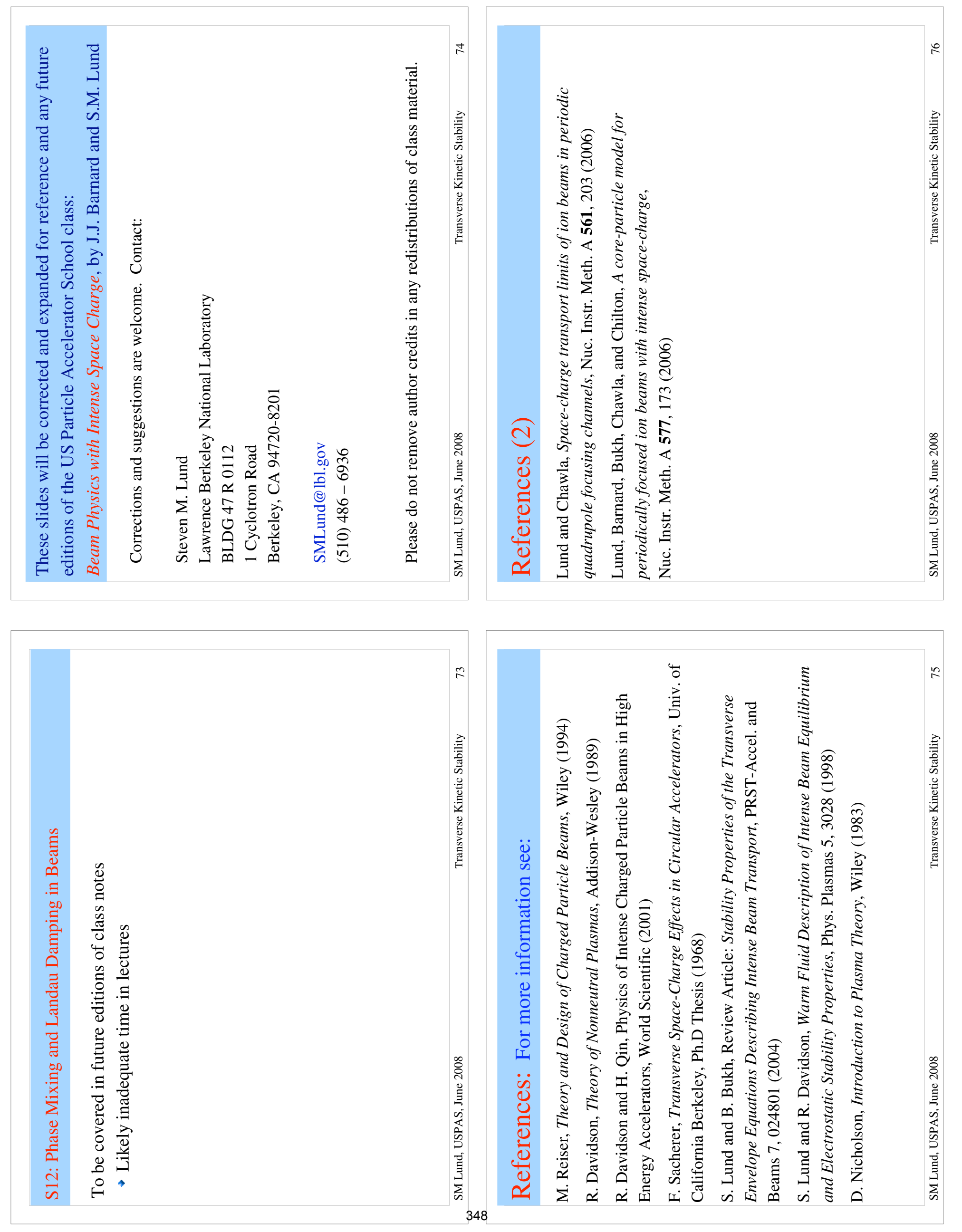
J.T Barnard and S.M. Lond

USPAS, June 2008

$\$ 4$ Collective Modes on a KV Equilibrium Beam

Here we take a $K V$ equilibrium distribution with

$$
f_{0}\left(H_{0}\right)=\frac{\hat{n}}{2 \pi} \delta\left[H_{0}-\frac{\varepsilon_{x}^{2}}{2 \Gamma_{0}^{2}}\right]
$$

$\hat{n}=$ constant density of $\mathrm{kV}$ equilibrium

$\varepsilon_{x}^{2}=x$-emittance.

$r_{b}=$ equilibrium beam radius.

$$
\begin{aligned}
& k_{\beta_{0}}^{2} \Gamma_{D}-\frac{Q}{\sqrt{b}}-\frac{\varepsilon_{x}^{2}}{\Gamma_{b}^{3}}=0 \\
& H_{0}=\frac{1}{2} \vec{x}_{1}^{\prime 2}+\frac{k_{\beta_{0}}^{2}}{2} \vec{x}_{1}^{2}+\frac{g}{\sum_{m} \gamma_{b}^{2} \beta_{b}^{2} c^{2}}
\end{aligned}
$$

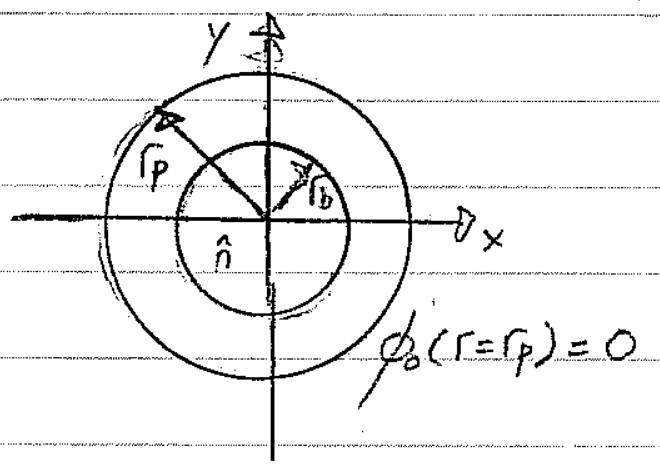

and assume small-amplitude axisyoumetric $(\partial / \partial \theta=0)$ perturbations with normal mode form:

$$
\begin{aligned}
& \delta f\left(\vec{x}_{1}, \vec{x}_{1}^{\prime}, s\right)=\delta f\left(r, \vec{x}_{1}^{\prime}, k\right) e^{-i k s} \\
& \delta \phi\left(\vec{x}_{4}, s\right)=\delta \phi(r, k) e^{-i k s}
\end{aligned}
$$

$l=$ cont (mode eigenfrequency)

349 
S.M. Lond

21

The equilibrium characteristics in the core of the kV beam can be expressed as:

$$
\begin{aligned}
r^{2}(\tilde{s})= & r^{2} \cos 2\left[k_{p}(\tilde{s}-s)\right]+\frac{r r^{\prime} \cos \psi \sin \left[2 k_{p}(\tilde{s}-s)\right]}{k_{\beta}} \\
& \quad \frac{r^{\prime 2}}{k_{k}^{2}} \sin ^{2}\left[k_{\beta}(\tilde{s}-s)\right] \\
x(\tilde{s}=s)=r \cos \theta \quad & ; x^{\prime}\left(\tilde{s}^{2}=s\right)=r^{\prime} \cos \theta_{p} \\
y(\tilde{s}=s)=r \sin \theta \quad & ; y^{\prime}(\tilde{s}=s)=r^{\prime} \sin \theta_{p}
\end{aligned}
$$$$
\psi \equiv \theta-\theta_{p}
$$

$$
k_{\beta}=\left(k_{\beta_{0}}^{2}-\frac{Q}{r_{b}^{2}}\right)^{1 / 2}=\frac{\varepsilon_{x}}{r_{b}^{2}}
$$

Depressed B-tron wavenumber of particle oscillations

These results can be inserted into the characteristic equation

$$
\delta f\left(\vec{x}_{\perp}, \vec{x}_{\perp}^{\prime}, s\right)=\frac{q}{m \gamma_{b}^{3} \beta_{b}^{2} c^{2}} \int_{-\infty}^{s} d \tilde{s} \frac{\partial \delta \phi\left(\vec{x}_{1}(\tilde{s})\right)}{\partial \vec{x}_{\perp}(\tilde{s})} \cdot \frac{\partial}{\partial \vec{x}_{\perp}^{\prime}(\tilde{s})} f_{0}\left(H_{0}\left(\vec{x}_{\perp}(\tilde{s}) ; \vec{x}_{\perp}^{\prime}(\tilde{s})\right)\right)
$$

to derive an expression for $\delta f\left(r, \vec{x}_{1}^{\prime}\right)$. This expression can then be inserted into the poison equation

$$
\frac{1}{r} \frac{\partial}{\partial r}\left(r \frac{\partial \delta \phi(r)}{\partial r}\right)=-\frac{g}{2 \varepsilon_{0}} \int d^{2} x^{\prime} \delta f\left(r, \vec{x}_{1}^{\prime}\right)
$$

to derive a lInear elgensalue equation for $\delta \phi(r)$ : 
S,M, Lond

3)

A signifrant amount of manipulation obtains the following form for the eigenvalue equation:

$$
\begin{aligned}
& \text { (1) } \\
& \left(\frac{\partial^{2}}{\partial r^{2}}+\frac{1}{r} \frac{\partial}{\partial r}\right) \delta \phi(r)=\frac{\hat{\theta}_{p}^{2}}{\gamma_{b} \beta_{b}^{2} c^{2}} \theta\left(r_{b}-r\right) \frac{1}{r_{\perp}^{\prime}} \frac{\partial}{\partial r_{\perp}^{\prime}} I_{o b}\left(r, r_{\perp}^{\prime}, k\right) \\
& \Gamma_{+}^{\prime 2}=\frac{\varepsilon_{x}^{2}}{\Gamma_{b}^{2}}\left(1-\frac{r^{2}}{\Gamma_{b}^{2}}\right) \\
& +\frac{\hat{\omega}_{p}^{2} /\left(b_{b} \beta_{b}^{2}\right)}{\varepsilon_{x}^{2} / r_{b}^{2}} \delta\left(r-r_{b}\right) \cdot\left[\delta \phi+I_{o r b}\left(r, r_{\perp}^{\prime}, k\right)\right] \\
& r_{\perp}^{\prime}=0
\end{aligned}
$$

Subject to:

where:

$$
\oint \phi\left(r=r_{b}\right)=0, \quad \hat{\omega}_{p}^{2}=\frac{q^{2} \hat{n}}{\varepsilon_{0} m}=\underset{\text { Freq. }}{\text { Plasma }}
$$

seq.

$$
\begin{aligned}
& \theta\left(r_{b}-r\right) \equiv\left\{\begin{array}{lll}
1 & r_{b}>r & \text { Heaviside } \\
0 & r_{b}<r & \text { step function }
\end{array}\right. \\
& \operatorname{Iorb}\left(r, r_{1}^{\prime}, k\right)=i k \int_{-\pi}^{\pi} \frac{d \psi}{2 \pi} \int_{-\infty}^{s} d \tilde{s} \delta \phi(r(\tilde{s}), k) e^{-i k(\tilde{s}-s)}
\end{aligned}
$$

Orbit integral.

Note:

- Term $(1) \propto C\left(\Gamma_{D}-r\right)$ is a body-nave perturbation existing only in the core $\left(r<r_{b}\right)$ of the equilibrium beam.

- Term (2) a $\delta\left(r-r_{b}\right)$ is a surface -wave perturbation existing only at the edge $\left(r=r_{b}\right)$ of the equilibrium beam.

- The orbit integral Forb $\left(r, r^{\prime} k\right)$ depends on both $\delta \phi$ and the exigent frequency $l$

The Poisson equation has become a linear integro-differential eigenvalue equation fixing the mode perturbed potential of and eigenfrequency $k$.

351 
Gluckstern Mode Solution

S.M. Land 4/

This eigenvalue equation is difficult, but it has been solved analytically

- A finite polynomial in $r^{2}$ expansion of $\delta \varnothing$ for $r<r_{b}$ can satisfy the equation (terms truncate)

- Expansions are inserted into the characteristic integrals and coefficients are identified powerby-power in $r^{2}$, and assembled.

Eigenfunction!

Solution (after much analysis)

$$
\begin{aligned}
& \delta \phi_{n}(r)=\left\{\begin{array}{ll}
\frac{A_{n}}{2}\left[P_{n-1}\left(1-2 r^{2} / r_{b}^{2}\right)+P_{n}\left(1-2 r^{2} / r_{b}^{2}\right)\right] & 0 \leq r<r_{b} \\
0 & j r_{b}<r \leq r_{p}
\end{array}\right] \\
& n=1,2,3, \ldots \text { radial mode Index } \\
& A_{n}=\text { constr. } \text { lInear mode amplitude. } \\
& P_{n}(x) n^{\prime} \text { th order Legendre Polynomial }
\end{aligned}
$$

Dispersion Relation:

Each n-labeled eigenfunction has in (degenerate) "eigentrequencies" \& satisfying an $n$th degree polynomial in $l^{2}$ dispersion relation.

$$
2_{n}+\frac{1-\left(\delta / \delta_{0}\right)^{2}}{\left(\delta / \delta_{0}\right)^{2}}\left[B_{n-1}\left(\frac{k / k_{\beta_{0}}}{\delta / \delta_{0}}\right)-B_{n}\left(\frac{k / k_{\beta 0}}{\delta / \delta_{0}}\right)\right]=0
$$

where: $\quad \frac{\delta}{\delta_{0}} \equiv \frac{k_{\beta}}{k_{\beta_{0}}}=\frac{\left(k_{\beta 0}^{2}-Q / \Gamma_{0}^{2}\right)^{1 / 2}}{k_{\beta 0}}$

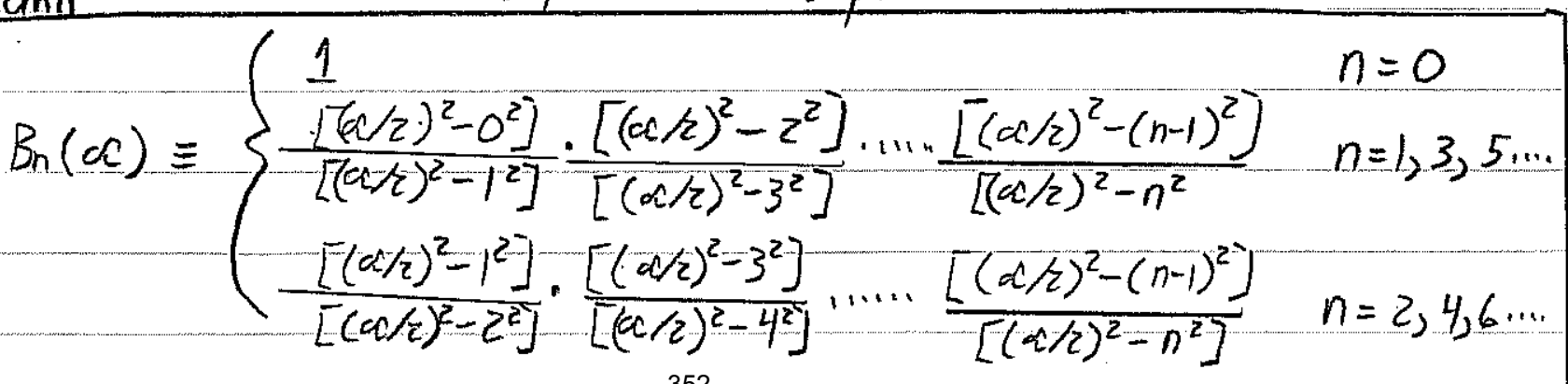


Properties:

S.M. Lond

5.

Radial Eigenfunction:

- Vanities outside the equilibrium beam edge $\left(r>r_{b}\right)$,

- Has nil nodes with $\&=0$ within the equilibrium beam $\left(r \angle r_{b}\right)$.

- Each $n$ labeled eigenfunction has Zn distinct frequencies

Corresponding perturbed density can be calculated from Poisson equation:

$$
\begin{aligned}
\delta n_{n} & =\delta n_{n}(r) e^{-i \delta_{s}} \\
\delta n_{n}(r) & =-\frac{\varepsilon_{0} \frac{\partial}{\partial}}{2 r}\left(r \frac{\partial \delta \phi_{n}}{\partial r}\right)
\end{aligned}
$$

- Find that the perturbed density of the mode is more larger near the outer (r it) edge of the beam for larger $n$.

Eigenfunction Form

Mode number $n$

$$
1
$$

$$
2
$$

$$
3
$$

$\delta \phi_{n} / A_{n}$ (potential) $1-\tilde{r}^{2}$

$1-4 \tilde{r}^{2}+3 \tilde{r}^{4}$

$1-9 \tilde{r}^{2}+18 \tilde{r}^{4}-10 \tilde{r}^{6}$

$1-16 \tilde{r}^{2}+60 \tilde{r}^{4}-80 \tilde{r}^{6}+35 \tilde{r}^{8}$ $\delta n_{n}$ (density, scaled wits)

$$
1
$$

$4\left(1-3 \tilde{r}^{2}\right)$

$9\left(1-8 \tilde{r}^{2}+10 \tilde{r}^{4}\right)$

$16\left(1-15 \tilde{r}^{2}+45 \tilde{r}^{4}-35 \tilde{r}^{6}\right)$

$$
\tilde{\Gamma} \equiv r / \Gamma_{b}
$$


S.M. Lund 6)

\section{Radial Eigen function}
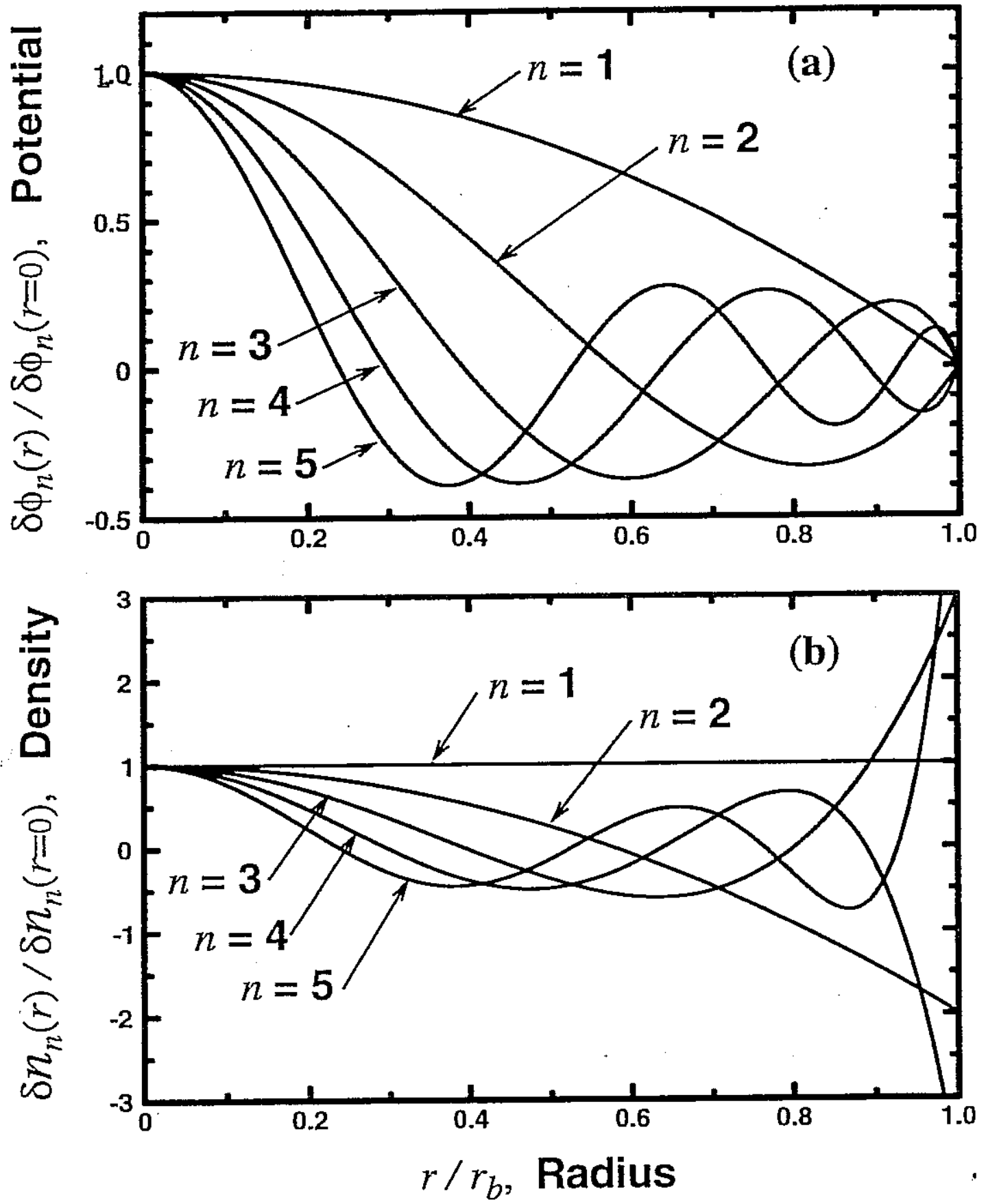
S.M. Lond

Perturbations should introduce no net charge in to the system:

$$
2 \pi \int_{0}^{r_{p}} d r \delta n(r)=0
$$

The $r<r_{b}$ component of the perturbations are not the only terms present For the $r<r_{b}$ eigenfunction calculated. $\int_{0}^{i d} d r r \delta n_{n}(r) \neq 0$. A more detailed analysis shows that:

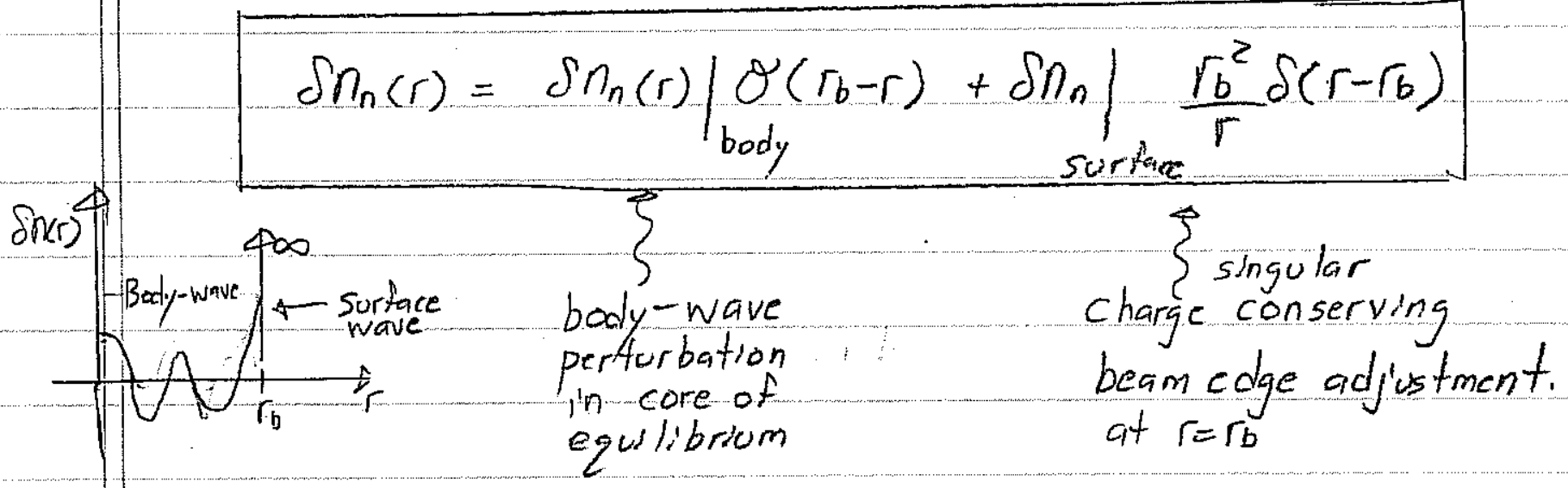

where:

$$
\begin{aligned}
& \left.\delta n_{n}\right|_{\text {body }}=-\frac{\varepsilon_{0}}{\xi} \frac{1}{r} \frac{\partial}{\partial r}\left(r \frac{\partial \delta \phi_{n}}{\partial r}\right) \\
& \left.\delta n_{n}\right|_{\text {surface }}=\text { canst } \times(-1)^{n} n A_{n}
\end{aligned}
$$

To lInear order this is equivalent to:

$$
\begin{aligned}
& n(r)=\left[\hat{n}+\left.\delta n_{n}(r)\right|_{\text {body }}\right] \theta\left[\Gamma_{b}+\delta \Gamma_{b}-r\right] \\
& \delta r_{b}=\operatorname{const} \times(-1)^{n} n A_{n}
\end{aligned}
$$


S.M. Lond

Dispersion Relation

- Polynomial in $k^{2} \Rightarrow \pm k$ solutions and therefore there will be unstable growing perturbations if $k$ is complex:

$$
\begin{array}{ll}
\delta \phi \sim \delta \phi_{n}(r) e^{-i k s} & \\
k=k_{r} \pm i k_{I} \quad k_{I}=\text { real part } \\
k_{I}=\text { imaginary part }
\end{array}
$$

For the unstable branch:

$$
\delta \phi \sim \delta \phi_{n}(r) e^{-i k r s} \cdot e^{\left|k_{1}\right| s} \Rightarrow \underset{\substack{\text { expowth. } \\ \text { frontal }}}{\text { expo ns }}
$$

- $k$ is a function of $n$ and $\sigma / \delta_{0}$ only.

$$
\begin{gathered}
0 \leqslant \delta / \delta_{0} \leq 1 \\
\uparrow \quad+ \\
\text { strongest, zero space-charge, } \\
\text { possible space } \\
\text { charge. }
\end{gathered}
$$

- Instabilities will occur over a range of $\delta / \delta_{0}$ and will turn off for $\delta / \delta_{0}$ large enough (weak space-charge). $\mathrm{kV}$ beam is always stable for zero space-charge since.

Dispersion Relations:

$$
\begin{array}{cl}
\frac{\text { Mode number } n}{1} & \frac{\text { Dispersion relation }}{2} \\
2 & \left(k / k_{p o}\right)^{2}-2\left(1+\delta^{2} / \delta_{0}^{2}\right)=0 \\
& \left.\left(k / k_{p o}\right)^{4}-2\left(1+9 \delta^{2} / \delta_{0}^{2}\right)\left(k / k_{p o}\right)^{2}-4 \delta^{2} / \delta_{0}\right)\left(1-1 \delta^{2} / \sigma_{0}^{2}\right)=0
\end{array}
$$

Rapidly more complicated? 
the Oscillation Frequency $\left|R e k_{20}\right|_{00} \mid$
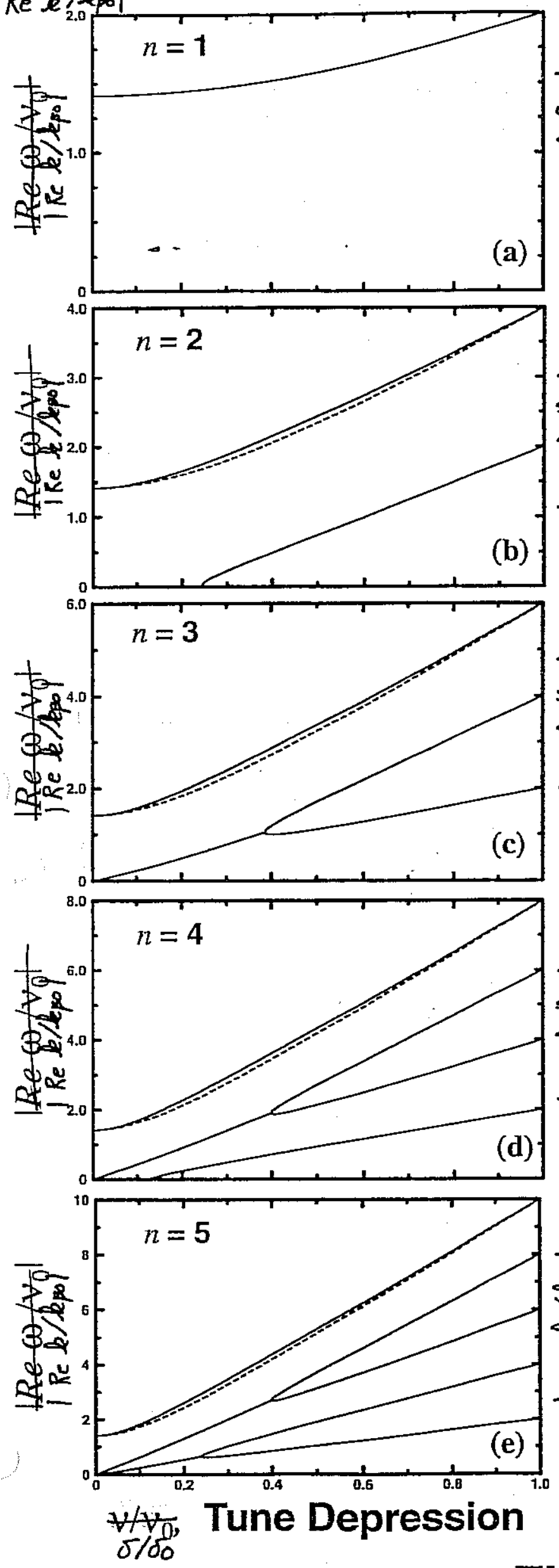

Fia $_{-35} 8$
Im oft, Growth Rate

9
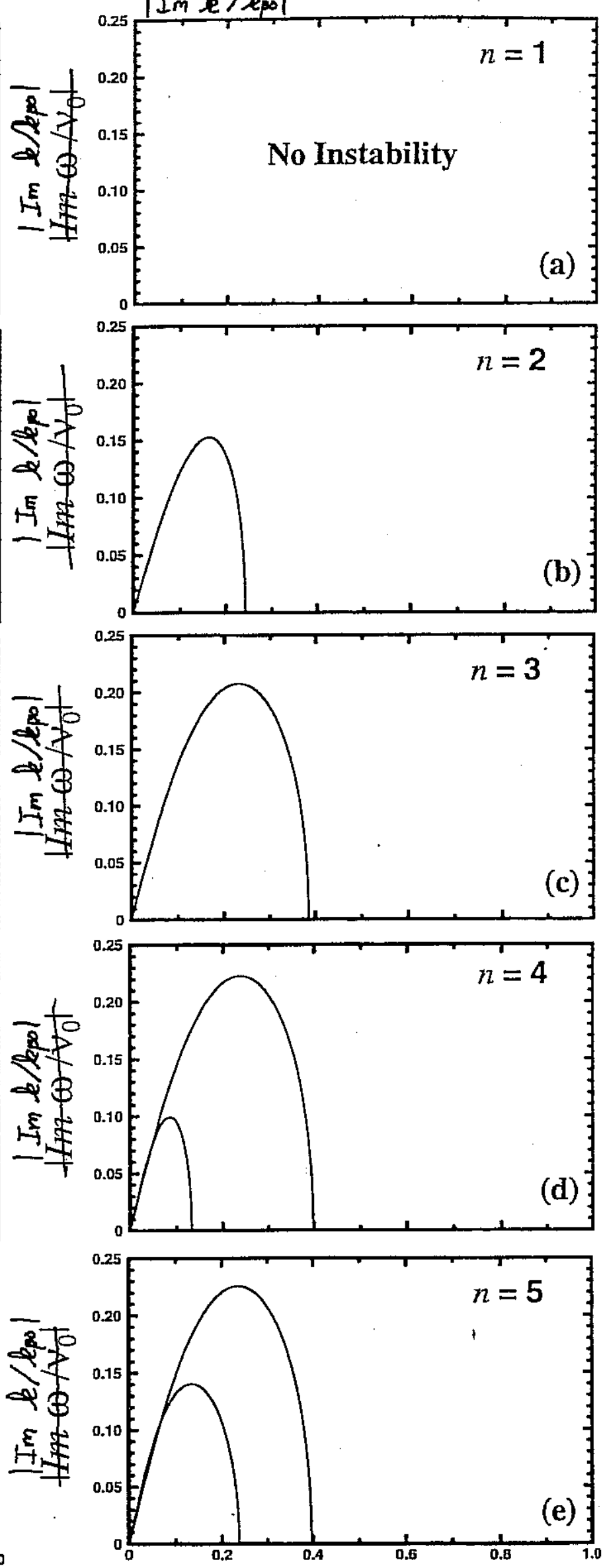

W/4 Tune Depression $\delta / \delta 0$ 

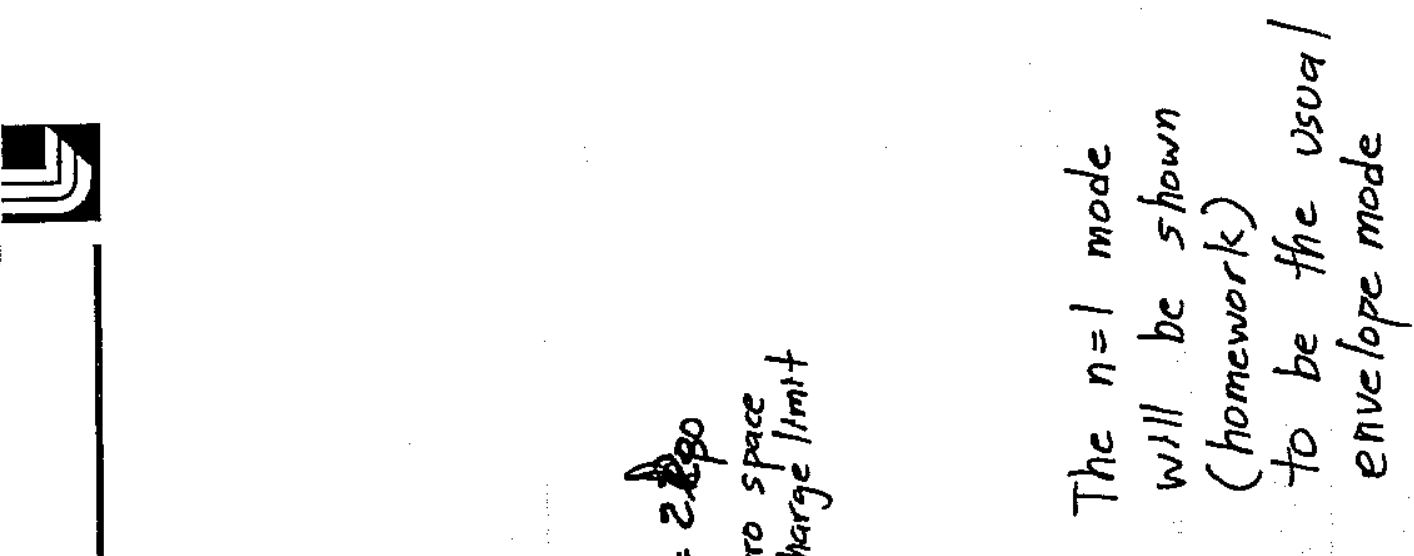

ठ) $\varepsilon^{\infty} \varepsilon^{2}$

तu

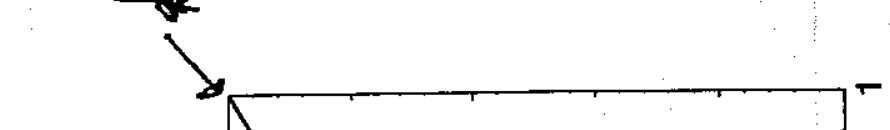

6

.

列 0

$\frac{18}{18}$

10

กำ

है

$E$

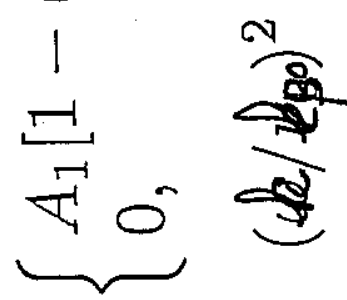

11

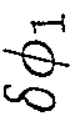

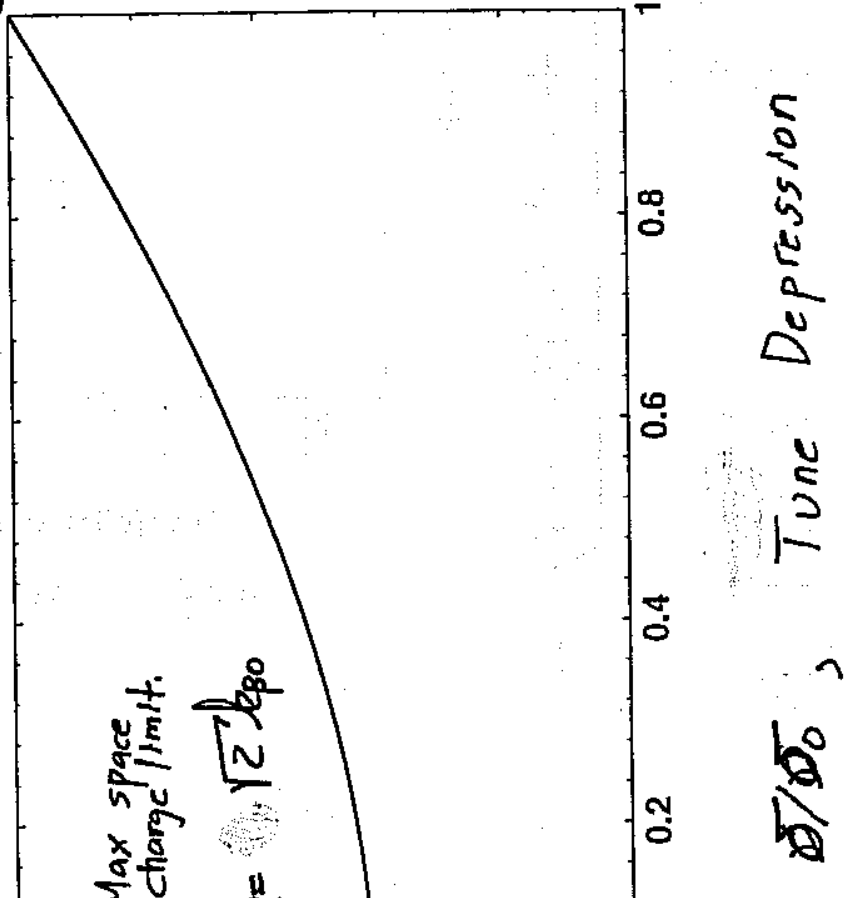

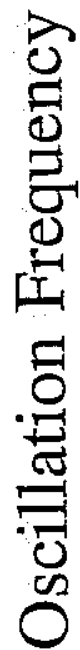



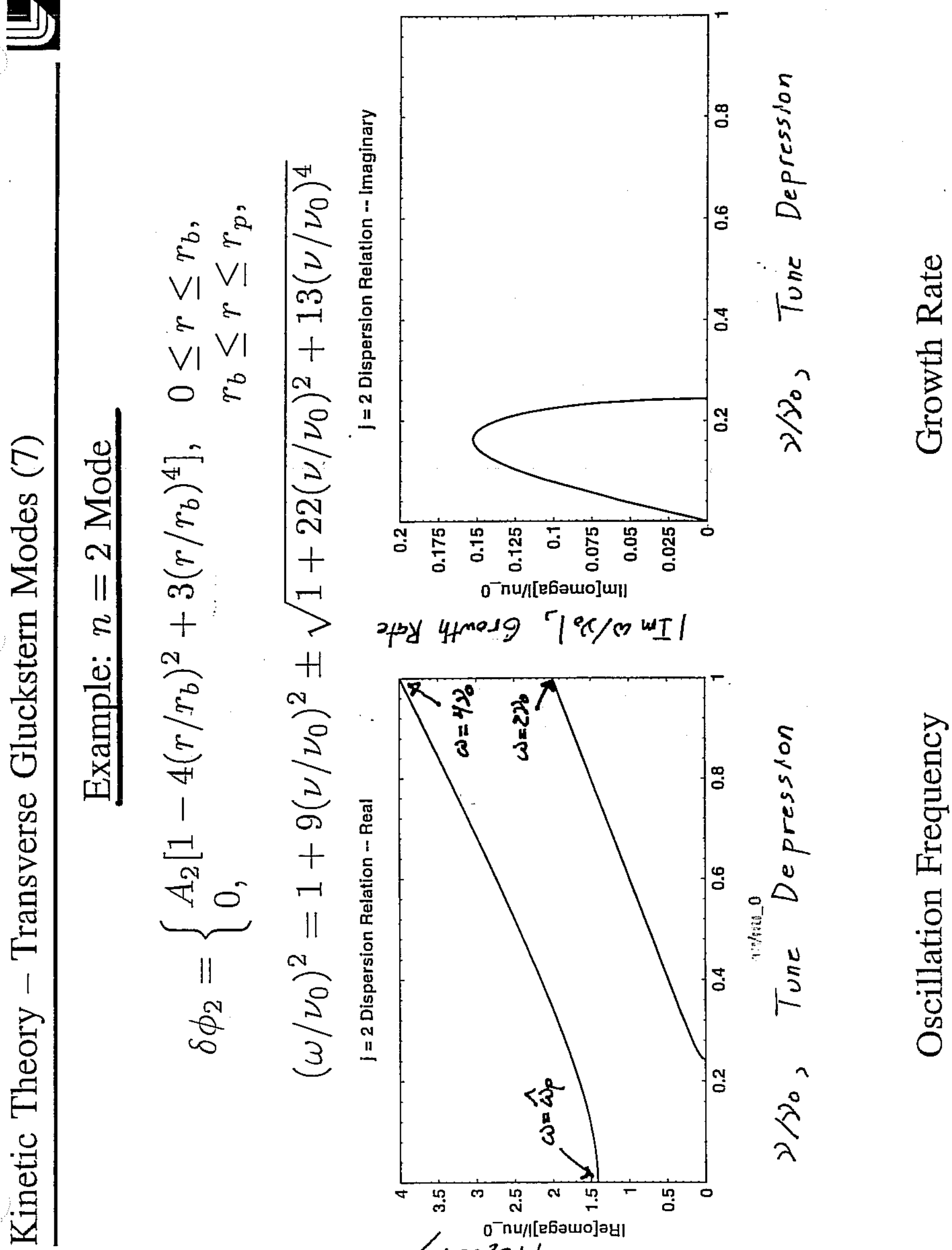

apby yfmesg $\mathrm{r} / \mathrm{ec} / \mathrm{m}$ wI

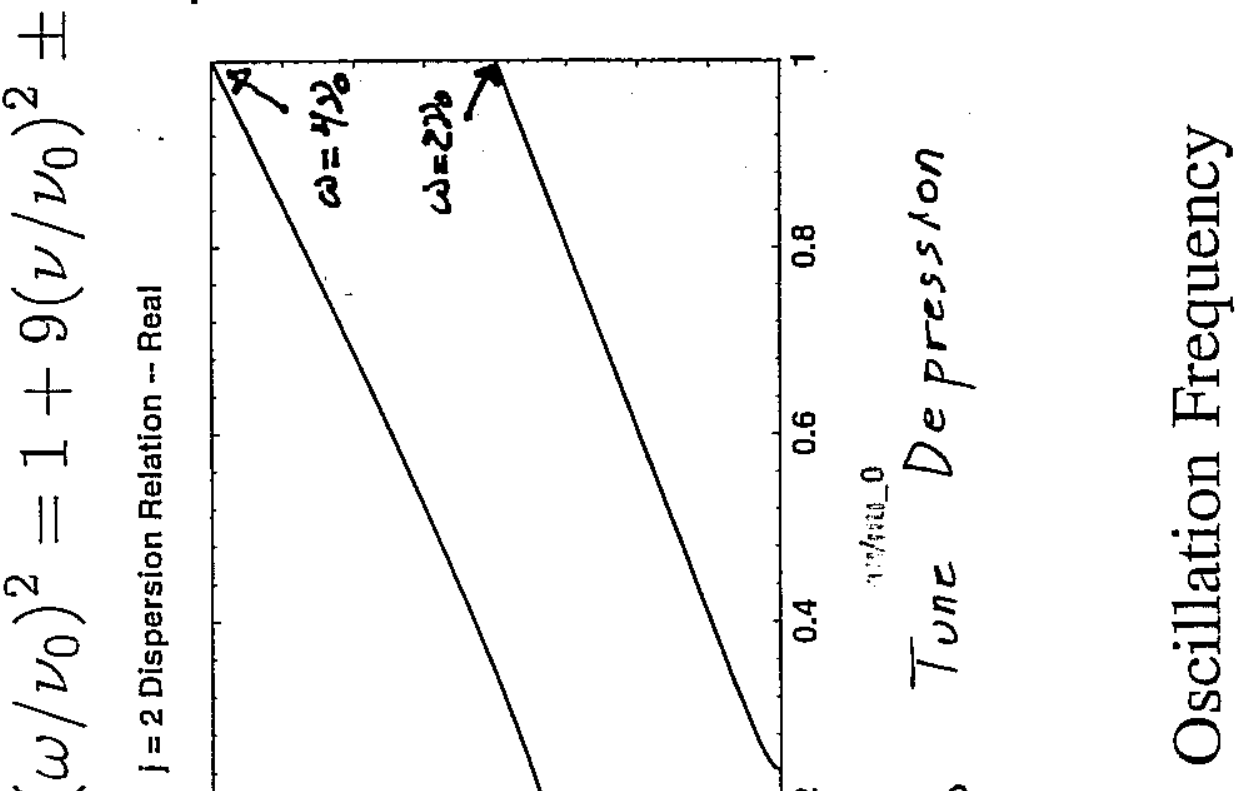


Discussion:

S.M. Lond

12)

As might be expected on physical grounds the singular $\mathrm{KV}$ distribution drives numerous, strong, collective instabilities. This implies that the $\mathrm{KV}$ model is suspect since real beams ave often transported where the $\mathrm{kV}$ model would predict strong instability. However:

- Low-order kV features (envelope modes) are correct and well verified.

- Higher order collective modes observed on intense beam cores often look similar to the KV model predictions in density potential etc but are not unstable.

How is this situation resolved? A partial answer was suggested by a fluid model developed by Lind and Davidson. In this model:

- Density and tempeture profiles (lie.) low order features) of the $\mathrm{kV}$ model were preserved.

- The singular phase-space structures wire eliminated. A stability analysis obtained:

Mode

Eigenfunction $\left(r<r_{b}\right)$

$$
\delta \phi_{n}=\frac{A_{n}}{2}\left[P_{n-1}\left(1-2 \frac{r^{2}}{r^{2}}\right)+P_{n}\left(1-2 \frac{r^{2}}{b^{2}}\right)\right]
$$

Mode Dispersion:

$$
\begin{gathered}
\left(\frac{k}{h_{30}}\right)^{2}=2+2\left(\frac{\delta}{\delta_{0}}\right)^{2}\left(2 n^{2}-1\right) \\
n=1,2,3, \ldots \ldots
\end{gathered}
$$


Features of fluid model:

S.M. Lond 13/

- Identical radial eigenfunction to the pull kinetic theory

- Fluid mode dispersion relation predicts stability for all modes and closely tracks the (stable) high frequency branch of the $\mathrm{KV}$ dispersion relation for the full range of space charge strength $0 \leq \delta / \delta_{0} \leq 1$

- Fluid mode dispersion relation plotted dashed on $\mathrm{KV}$ mode plots.

- The $n=1$ fluid envelope mode is identical to the $\mathrm{KV}$ envelope mode.

Since the fluid model reproduces the coarse macroscopic features of the $k V$ model. - which can be a good approximation at high space-charge intensities. this implies:

- KV model made eigenfunction should roughly model those of intense beams with smooth distributions.

- Oscillation frequencies may be close to the (stable) high frequency $k V$ mode branch

- May be other. lower frequency branches that are also physical.

- Many. high-order KV instabilities may be of little relevance to real beams.

- Low order (envelope and maybe others) can be relevant.

361 
$S, M$, Lond

The real issue for high intensity collective modes may not be higher order $\mathrm{KV}$ instabilities but if low-order collective modes can:

- Be driven unstable by periodic-(s-varying) focusing structures in machine lattices, errors in rings, etc.

- Drive the production of beam halo, etc.

References:

Material on the Kinetic stability of $\mathrm{KV}$ beams is found mostly in journals.

Original references

Gluckstern, Prow 1970 Proton Linac Conference, Nat. Accel. Lab .s pg. 811 - First $\mathrm{kV}$ mode analysis.

T.F. Wang and Li Smith, Part. Accel, 12, 247 (1982). Simplified (closed form) mode eigenfunction and dispersion relation.

Interpretation. of Branches, Mode structure, KV Fluid Stability S.M. Lond and R. C. Davidson, Physics of Plasmas 5; 3028 (1998). Detailed analysis of eigenfunction, dispersion relations, etc. in appendices. Fluid mode analysis and interpretations of $k V$ modes.

Other papers by Hoffman, Gluckstern, and others. Hoffman et al. analyzed $\mathrm{KV}$ in periodic foe using lattices. 


\section{John Barnard Steven Lund USPAS June 2008}

\section{Intrabeam collisions, gas and electron effects in intense beams}

1. Beam/beam coulomb collisions

2. Beam/gas scattering

3. Charge changing processes

4. Gas pressure instability

5. Electron cloud processes

6. Electron-ion instability 
Gas and electron effects

-Effects are quite different depending on $q, m$ of species being accelerated

-Circular accelerators vs. Linacs

( $\mathrm{t}_{\text {residence }} \sim \mathrm{ms}$ to days vs. 10's of $\mu \mathrm{s}$ )

-Long pulse vs. short pulse

( $t_{\text {pulse }} \sim 10$ 's of $\mu$ s vs. 10's of ns) 


\begin{tabular}{|c|c|c|}
\hline${ }^{+}$Heavy ion & $\mathcal{O}_{\text {molecule }}^{\text {Residual gas }}$ & $\mathrm{e}^{-}$electron \\
\hline
\end{tabular}

\section{Processes:}

1. Coulomb collisons (intra-beam)

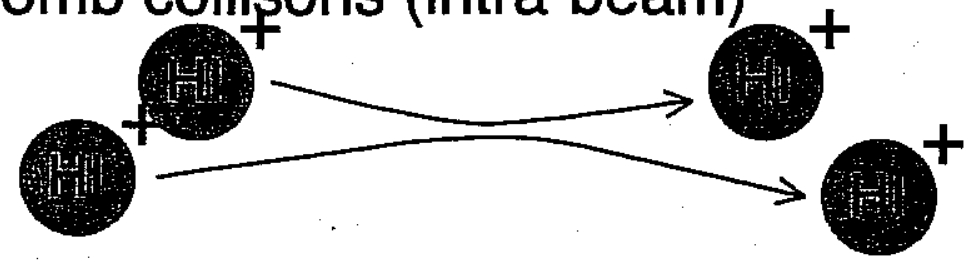

2. Coulomb collisions with residual gas

3. Charge exchange

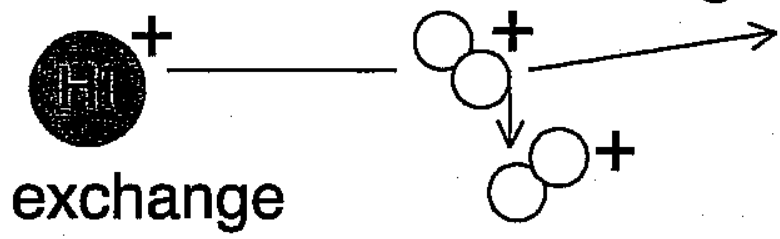

4. Stripping
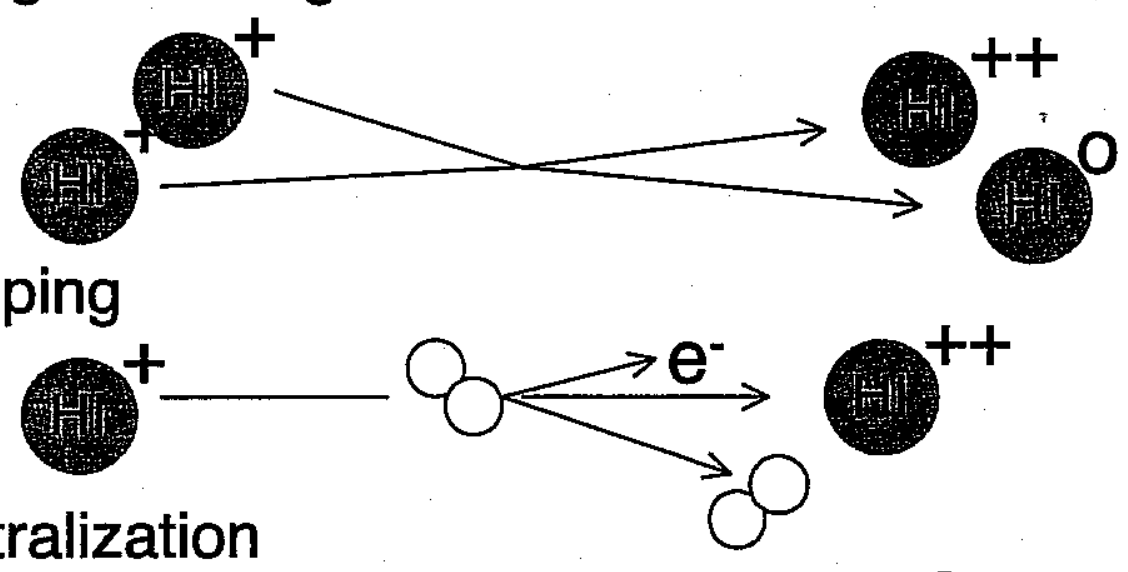

5. Neutralization
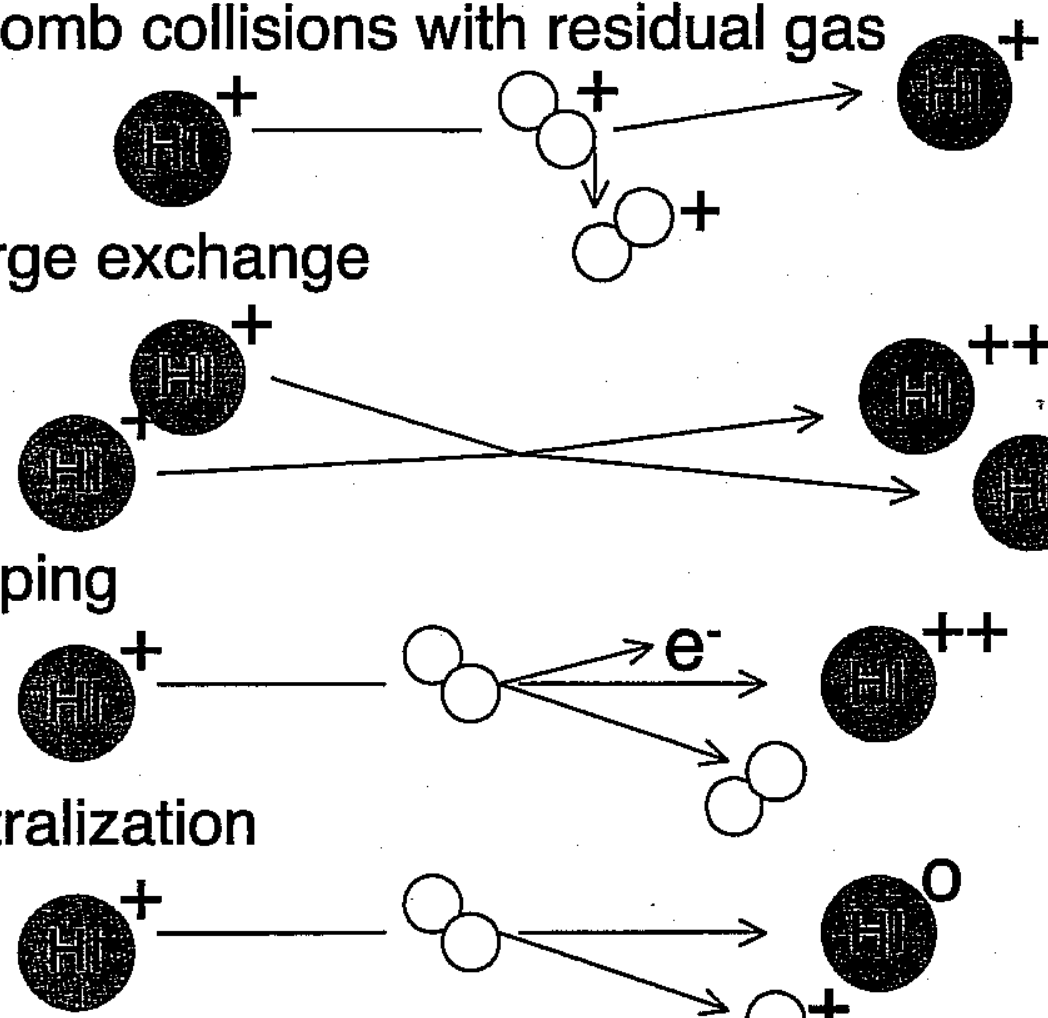

6. Gas lonization
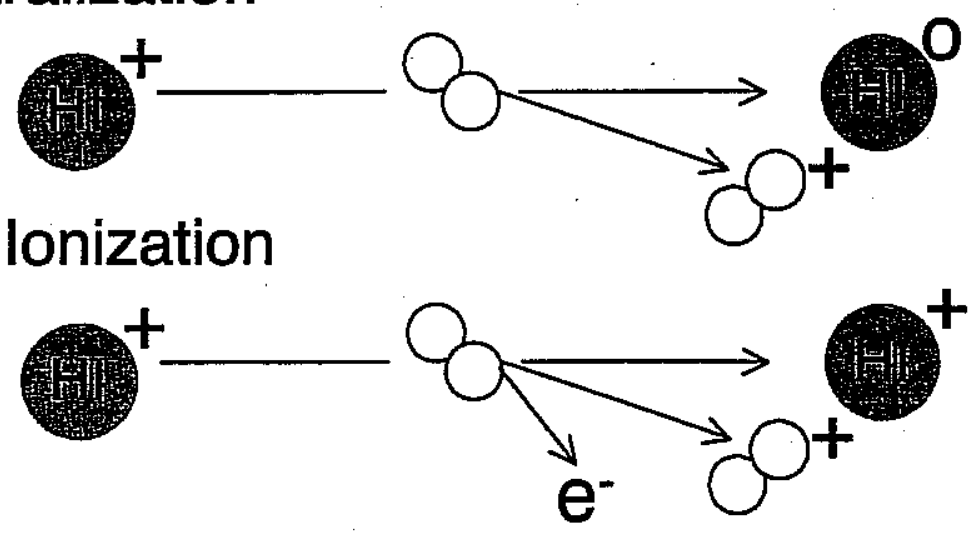


\section{Wall interactions}

desorption \&

electron emission $0^{\text {sputtering }}$

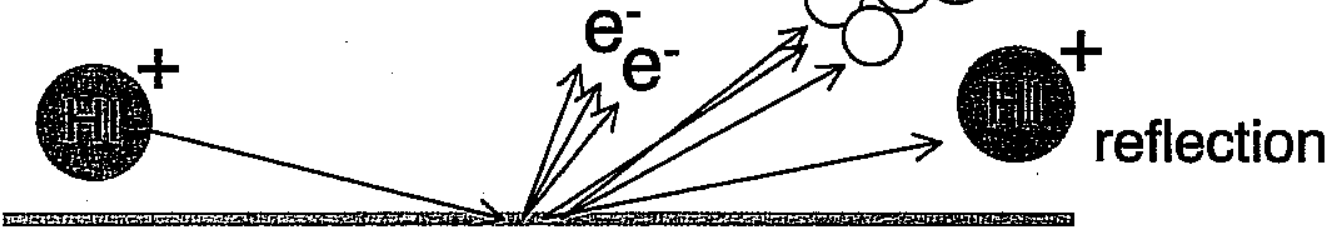

- desorption \&

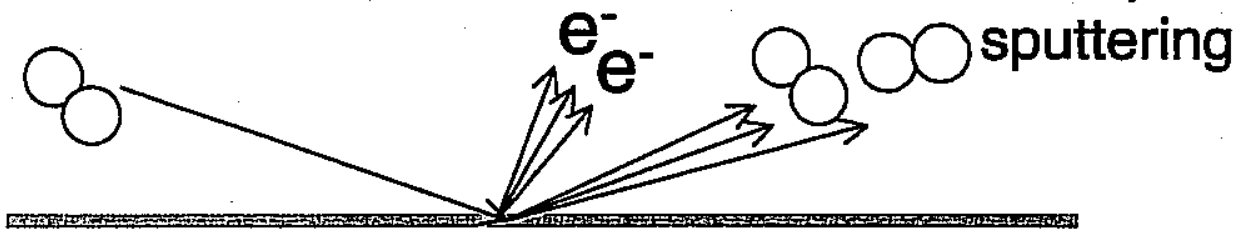

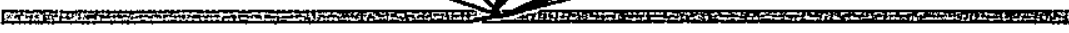
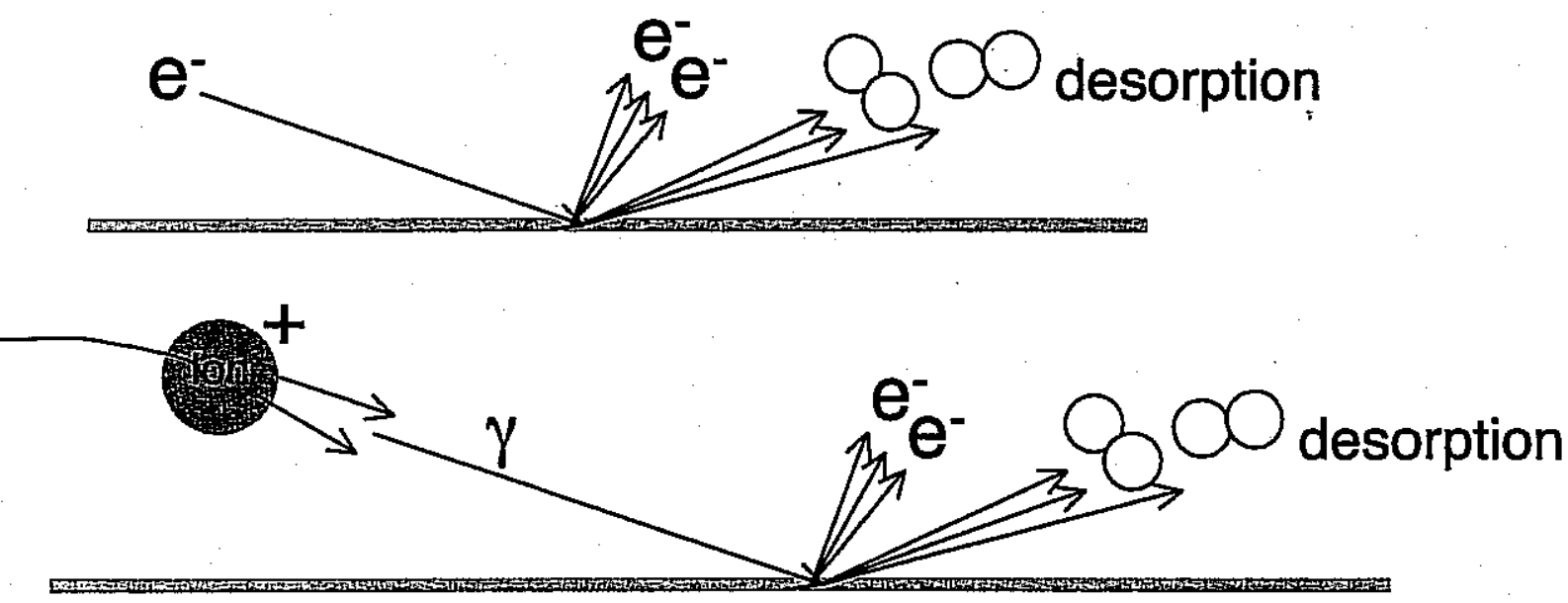

$\gamma$ synchrotron photon

John Barnard and Steven Lund USPAS 


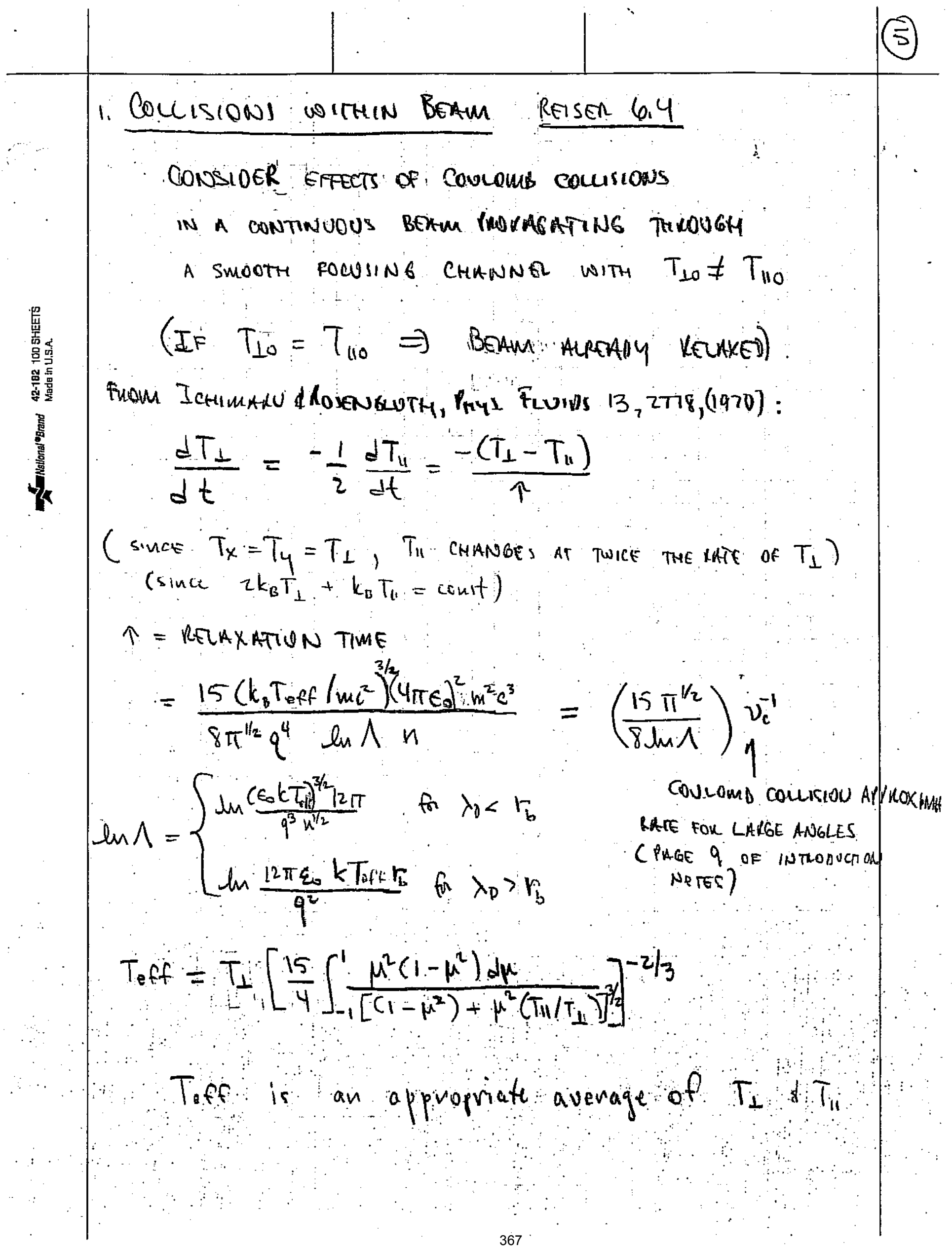




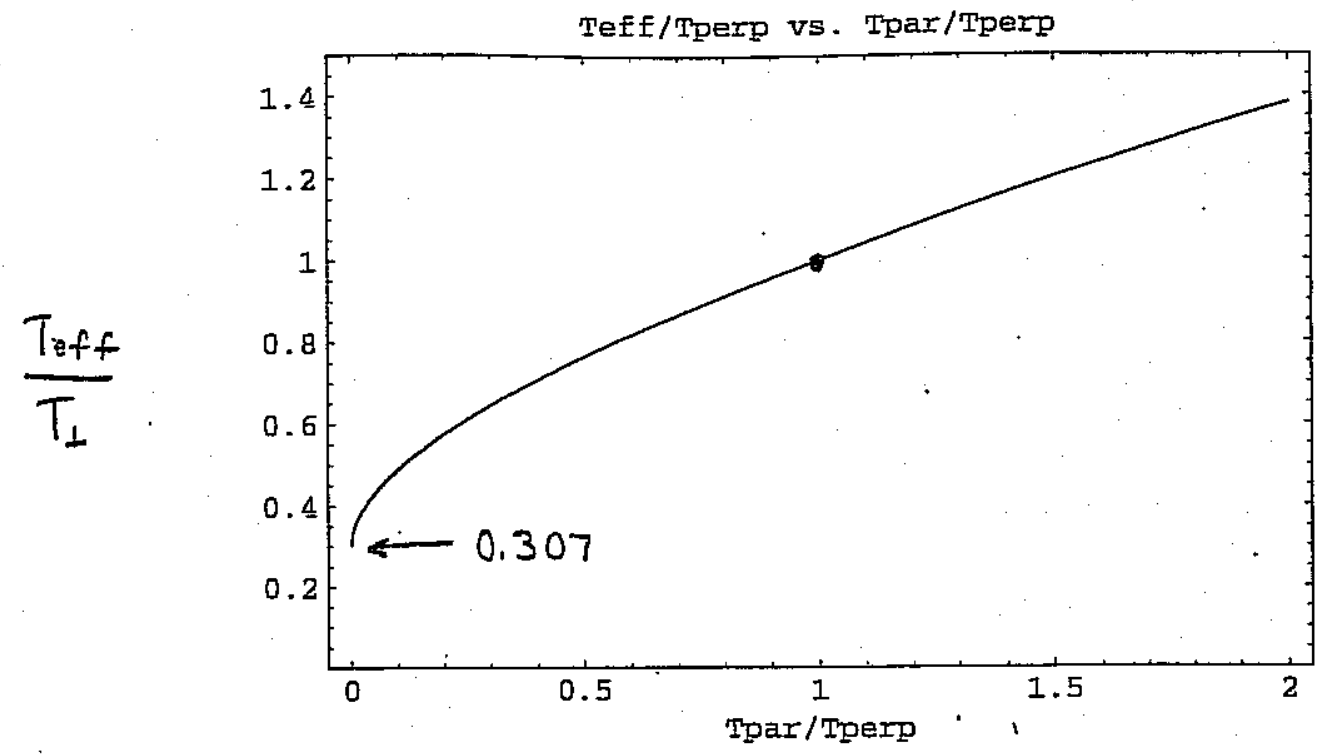

For $T_{i 1}=0$

$$
\begin{aligned}
& T_{\perp}=\frac{2}{3} T_{\perp 0}\left(1+\frac{1}{2} e^{-3 t / \tau_{\mathrm{eff}}}\right) \\
& T_{\| l}=\frac{2}{3} T_{\perp 0}\left(1-e^{-3 t / \tau_{\mathrm{eff}}}\right)
\end{aligned}
$$

(AYHOYIMATE SOLUTONA)

(6.156b)

$P_{\mathrm{uff}}=0.42 \uparrow_{\mathrm{eq}}$

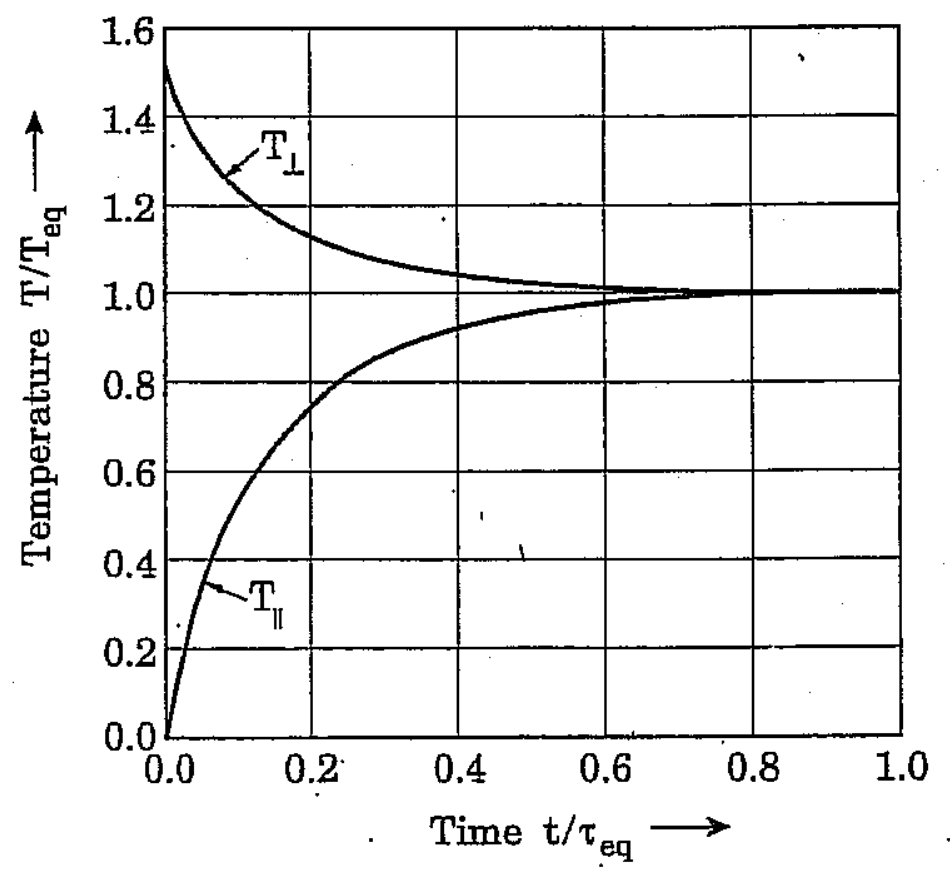

From

REISER $p .527$

$$
A_{e q} \simeq \uparrow\left(T_{e q}\right)
$$




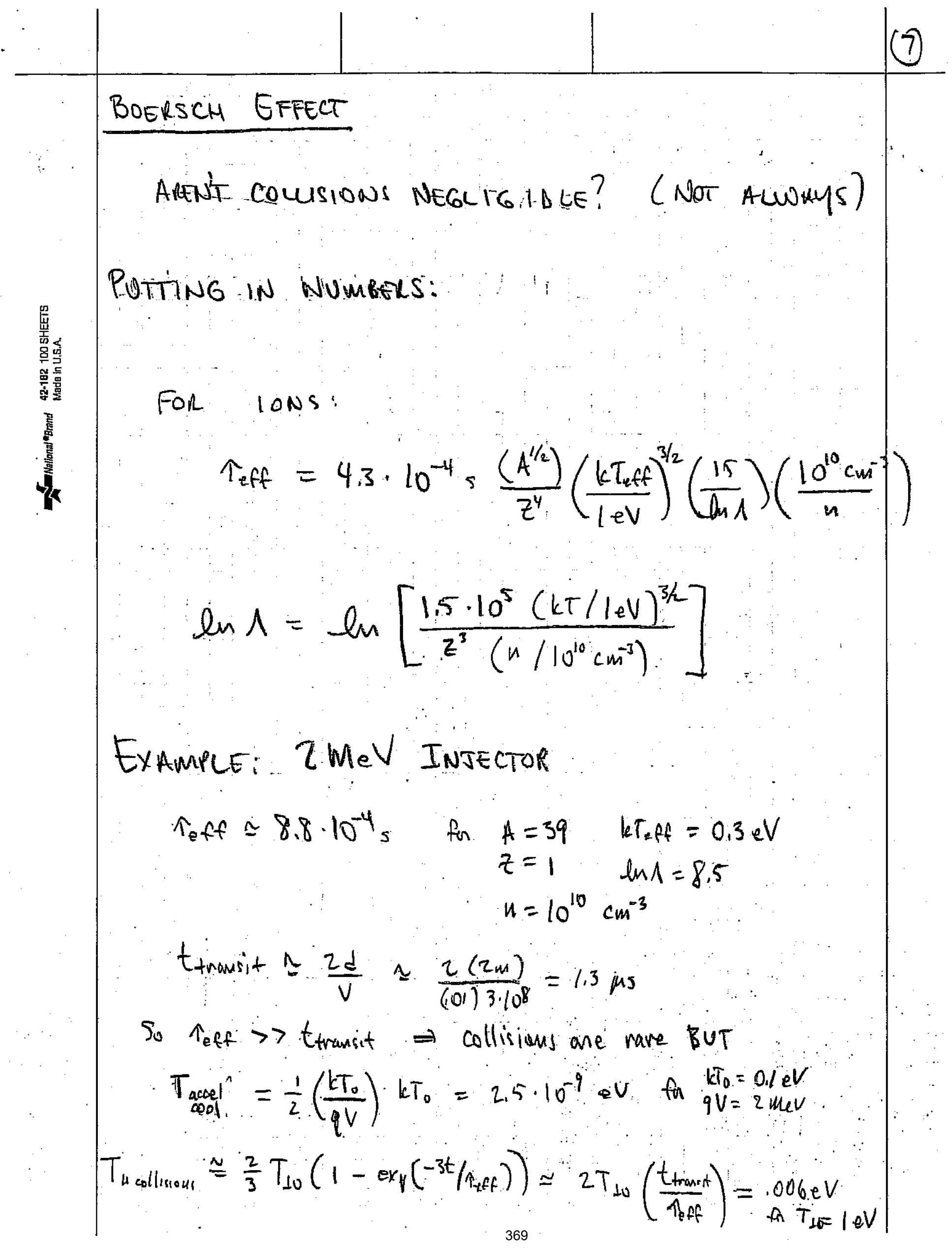




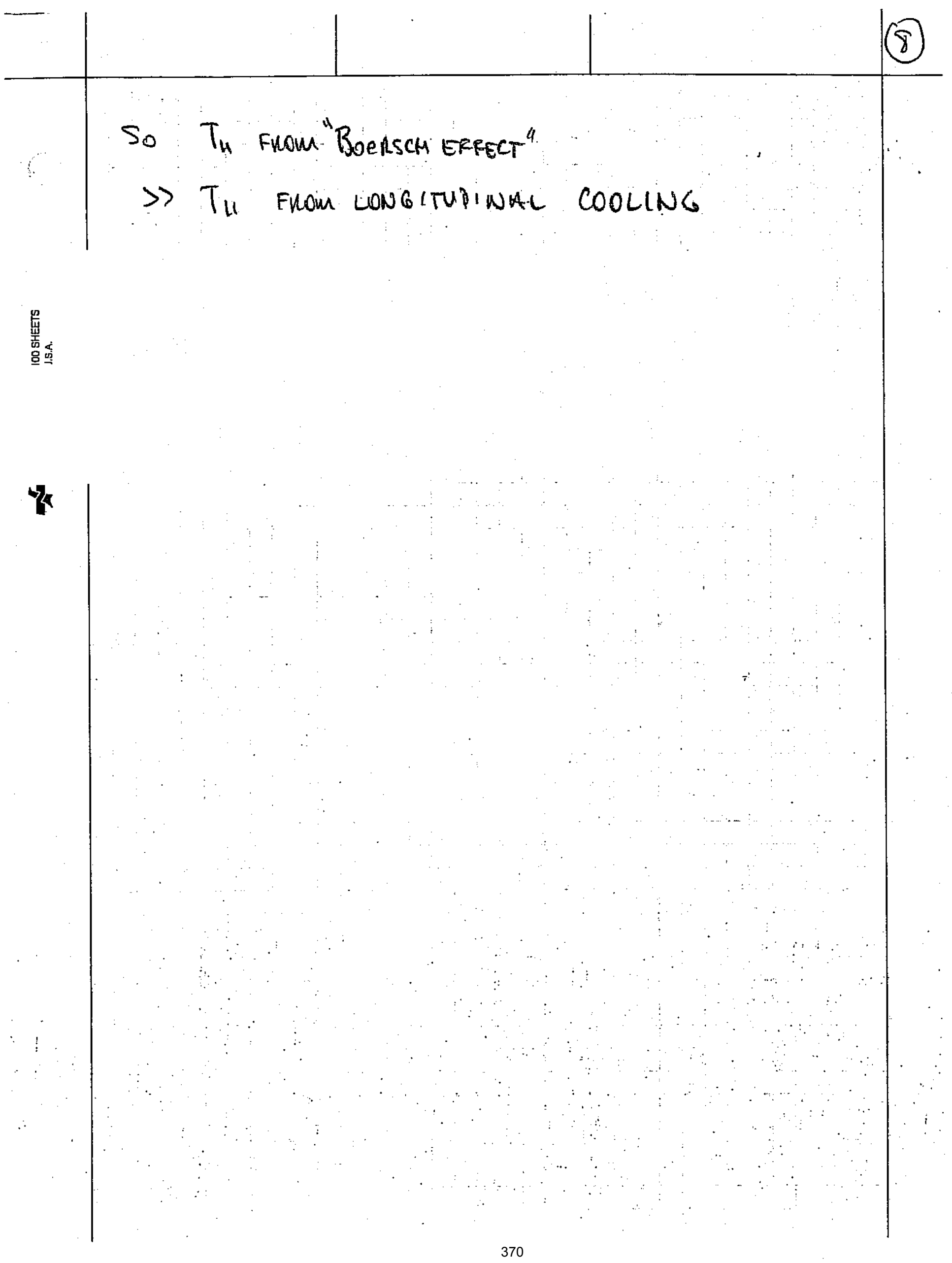




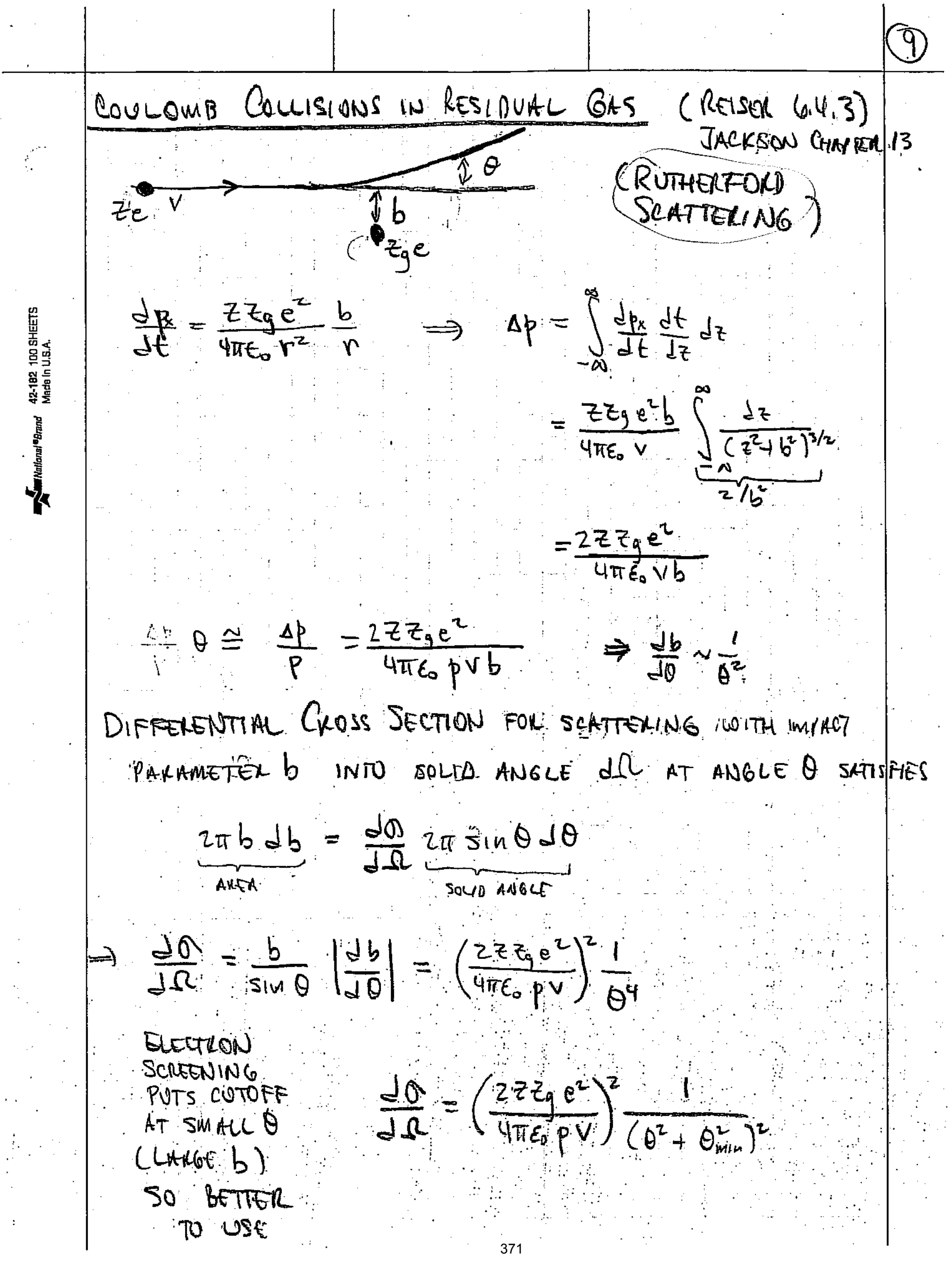




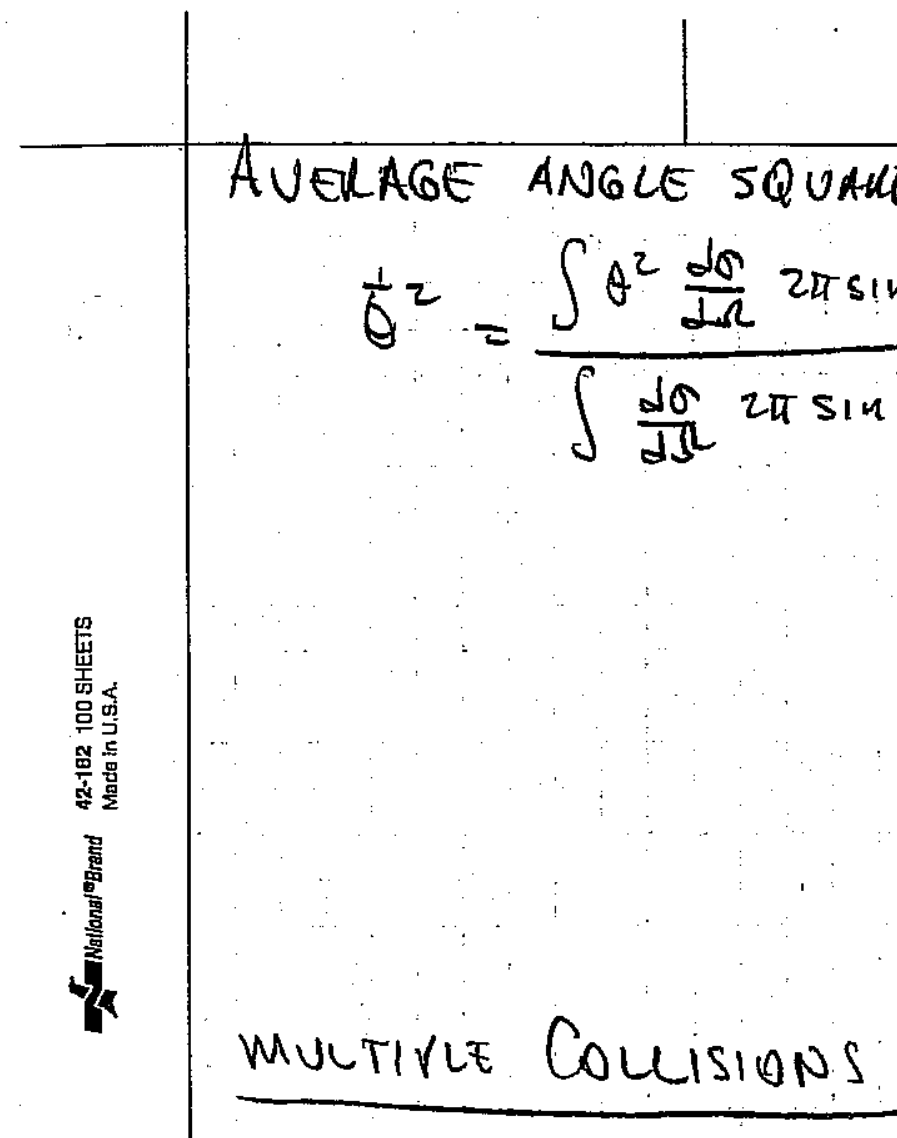

After traversing distance 5 , AND UNDERGOING NS COLLISIONS, THE

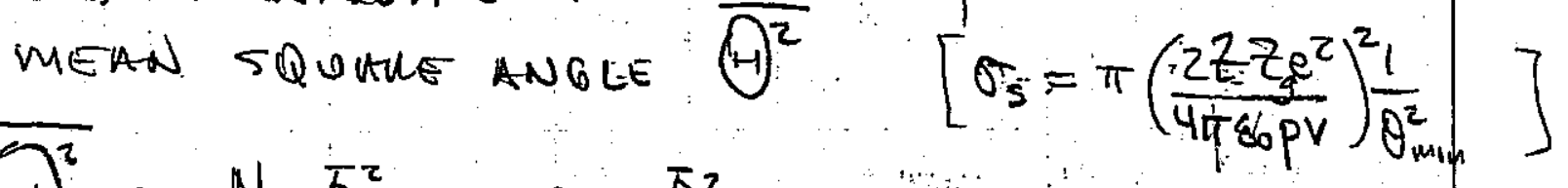

$$
\begin{aligned}
\overline{H^{2}} & =N_{s} \bar{\theta}^{2}=n_{g} \sigma_{s} s \theta^{2} \\
& =16 \pi n_{g}\left(\frac{z z g e^{2}}{4 \pi \epsilon_{0} m c^{2} \gamma p^{2}}\right)^{2} \ln \left(\frac{\theta_{\text {max }}}{\theta_{\text {min }}}\right) s
\end{aligned}
$$

Jackson argues $\theta_{\text {may }}$ arises from distributed NATURE OF NUCLEUS (NOT POINT CHANGE)

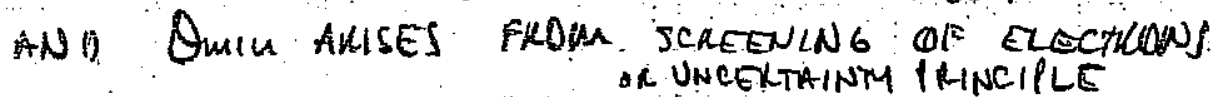

$$
\ln \frac{\theta_{\text {max }}}{\theta_{\text {man }}} \approx \ln \left(20 \% z_{g}^{-1 / 5}\right)
$$

372 


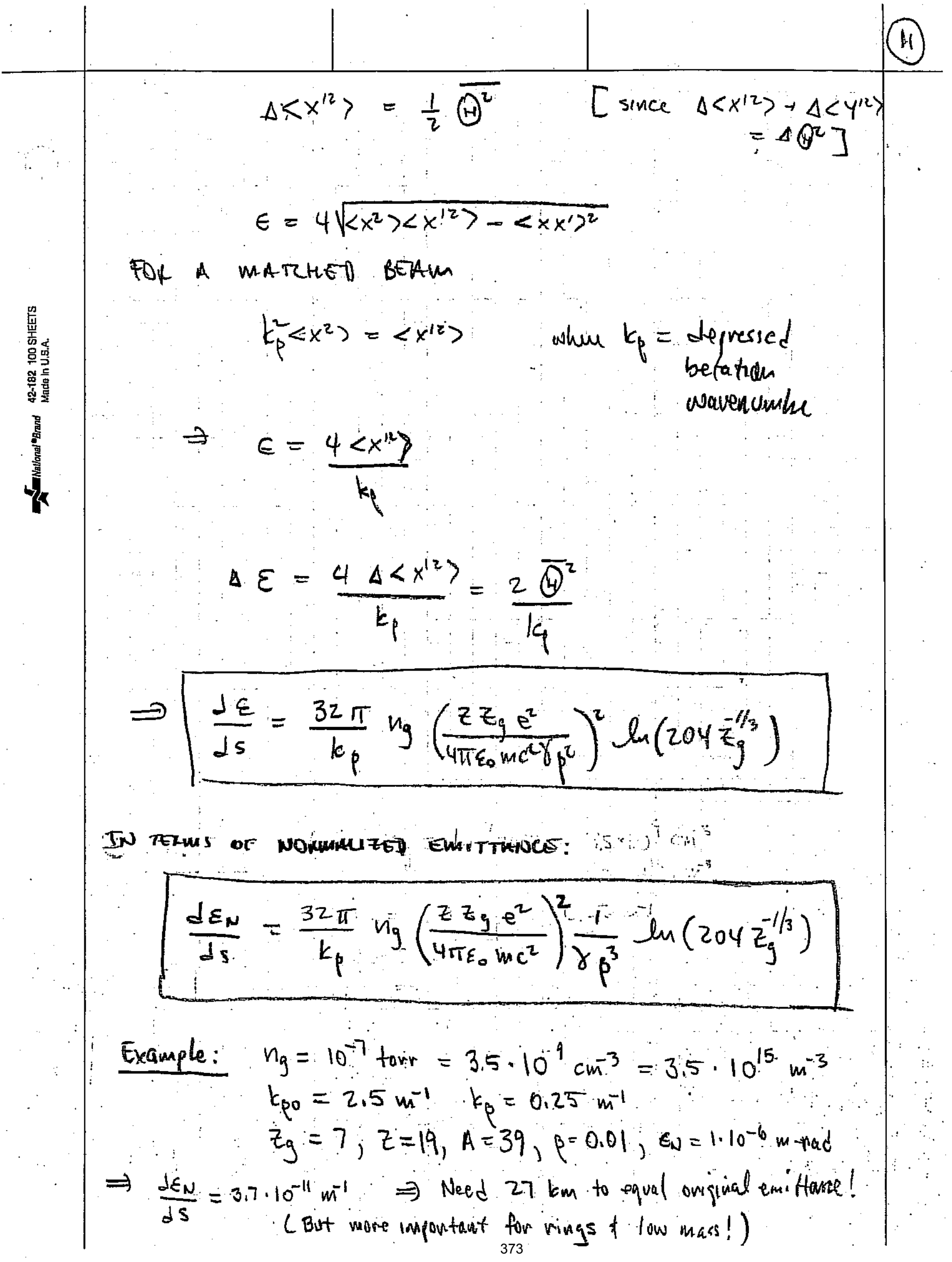




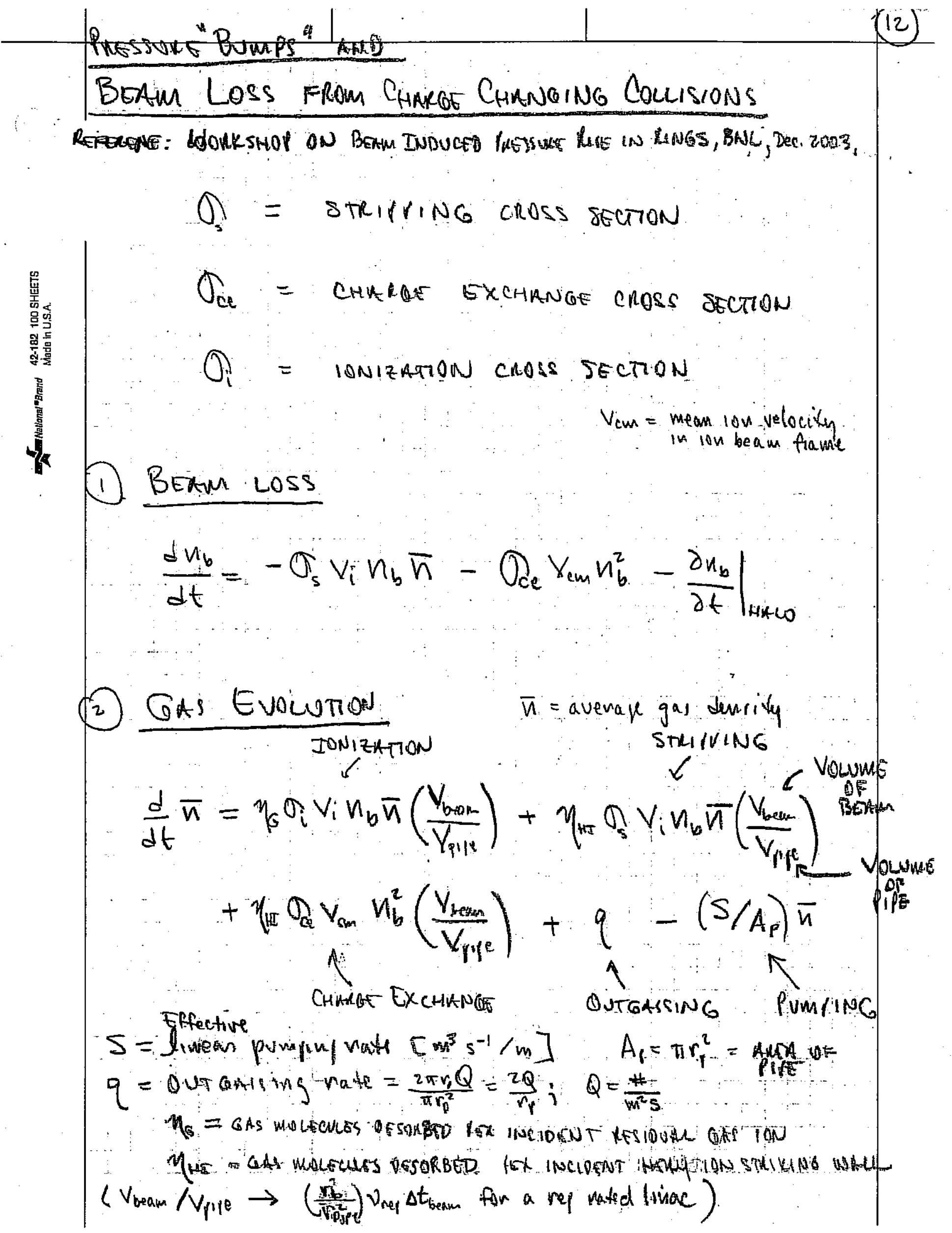

374 


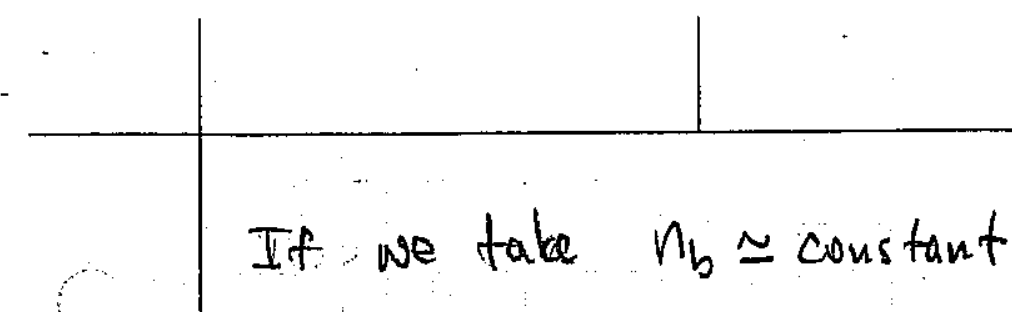

(13)

then we may express gas evolution equation as:

$$
\frac{d \bar{n}}{d t}=\frac{\bar{n}}{\pi}+q_{\text {eff }}
$$

with solution:

$$
\bar{u}=\left(\bar{n}_{0}+1 q_{\text {eff }}\right) \exp [t / \uparrow]-\uparrow q_{\text {eff }}
$$

$$
\begin{aligned}
& \text { HERE } \uparrow=\frac{1}{\left(n_{g} \sigma_{i}+\eta_{H_{I}} \sigma_{S}\right)\left(\frac{V_{\text {bank }}}{V_{\mu^{\prime}}}\right) n_{b} V_{i}-S / A_{p}} \\
& q_{\text {eff }}=q+\eta_{H I} \sigma_{C E} V_{c m} n_{b}^{2}\left(\frac{V_{\text {beam }}}{V_{\text {pf }}}\right)
\end{aligned}
$$

EQUILIBRIUM REACMOO IF: $\uparrow<0$ Che pumping exceeds desorption).

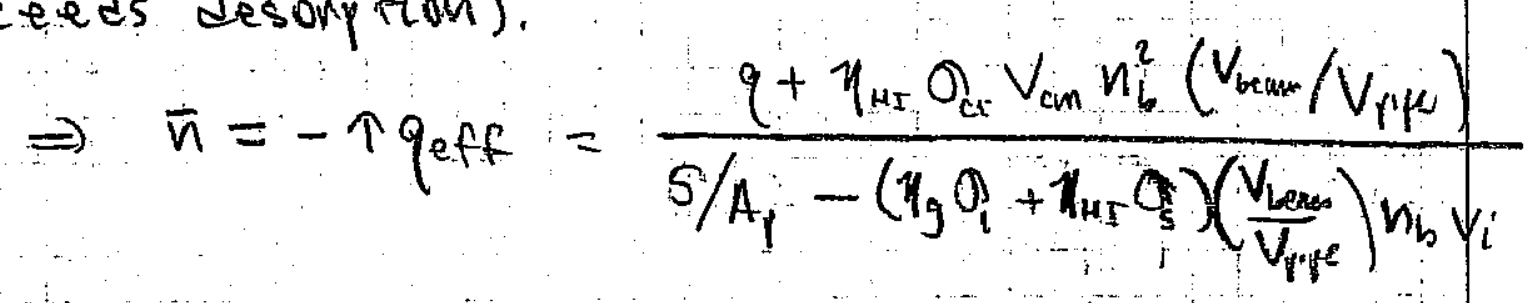

INSTABILITY IR

$$
n_{b} v_{i}=\frac{\frac{S}{A_{t}}\left(\frac{V_{\text {inf }}}{V_{\text {bean }}}\right)}{\eta_{g} \sigma_{i}+\eta_{\text {HE }} \sigma_{s}}
$$

375 
$\mid \frac{\mid}{\text { Instability finst observed on the Isi proton stonage }}$ ring, limiting corrent in mugy in 1970's.

If $l_{\text {beam }}=l_{\text {pipe }}$

14

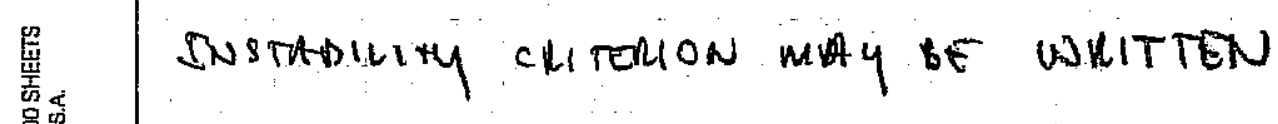

$$
I>\frac{z_{e} s}{\eta_{g} \alpha_{t}+\eta_{H I} \eta_{s}}
$$

EXAMPL: If $s=100 \ell^{-1} \mathrm{~m}^{-1}=0.1 \mathrm{~m}^{3} \mathrm{~s}^{-1} \mathrm{~m}^{-1}$

ISR

$$
\begin{aligned}
& y_{g}=4 \\
& \theta_{l}=10^{-22} \mathrm{~m}^{2}=10^{-16} \mathrm{~cm}^{2} ; \quad \sigma_{s}=0 \\
& z=1 \quad \text { (puatous) }
\end{aligned}
$$

$\Rightarrow I \leqslant 40$ Amperes

(Pressure RUNRonsys wele QBSGUEO ON the ISR AT 14-18A, (BENUENUTI etal, IEEE trous. an NUL. Sei. NS-24, 1773, 1979)

SEe "BeAM IndONGe Prossune Rise in Rings" $13^{\text {th }}$ ICFA BeAMI DY NAMICS MINI WOAKSHOI; BNL, Dec, 9-12,2003. webslte: $h++p: / /$ www.c-ad.bnl.gov/icfa

376 


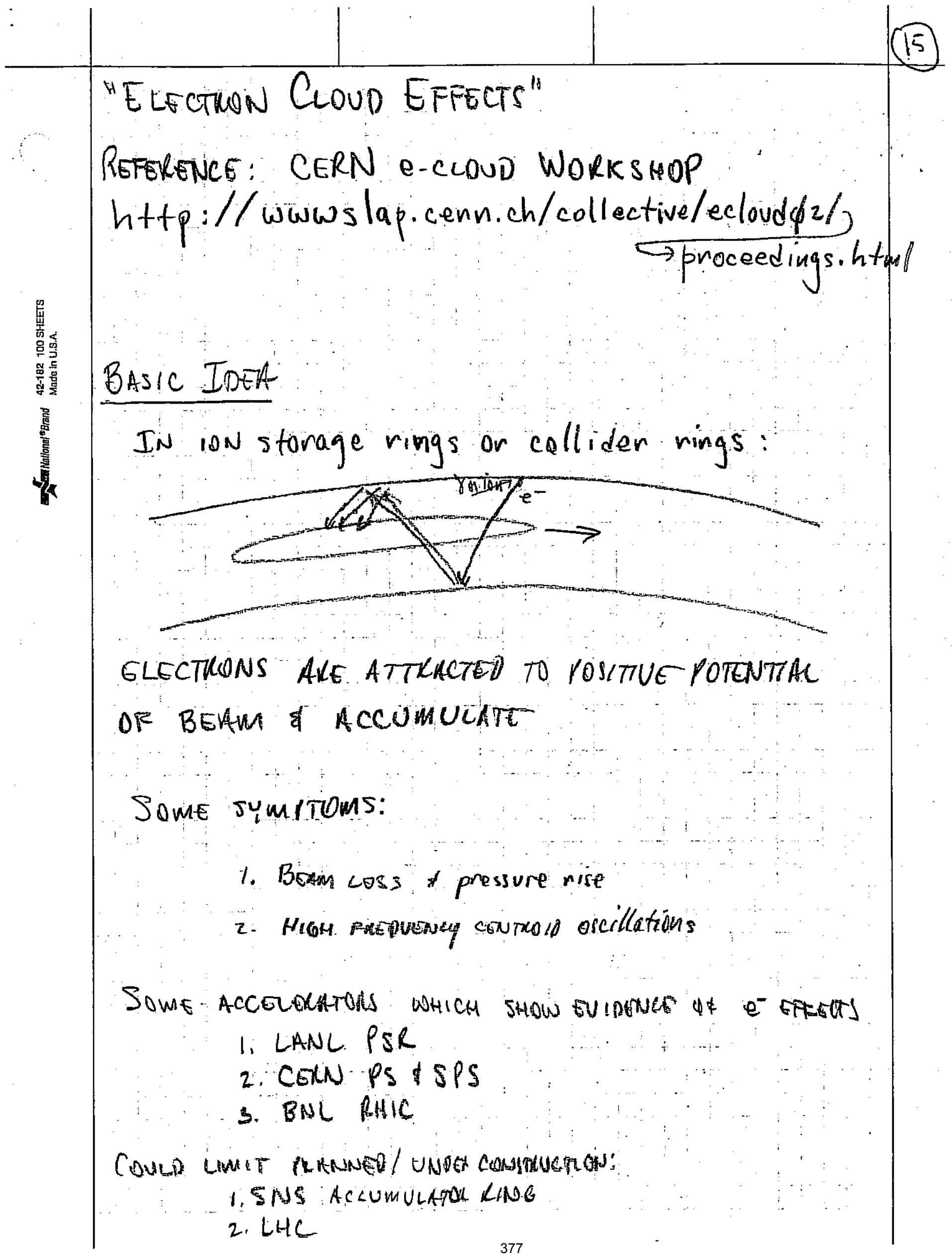




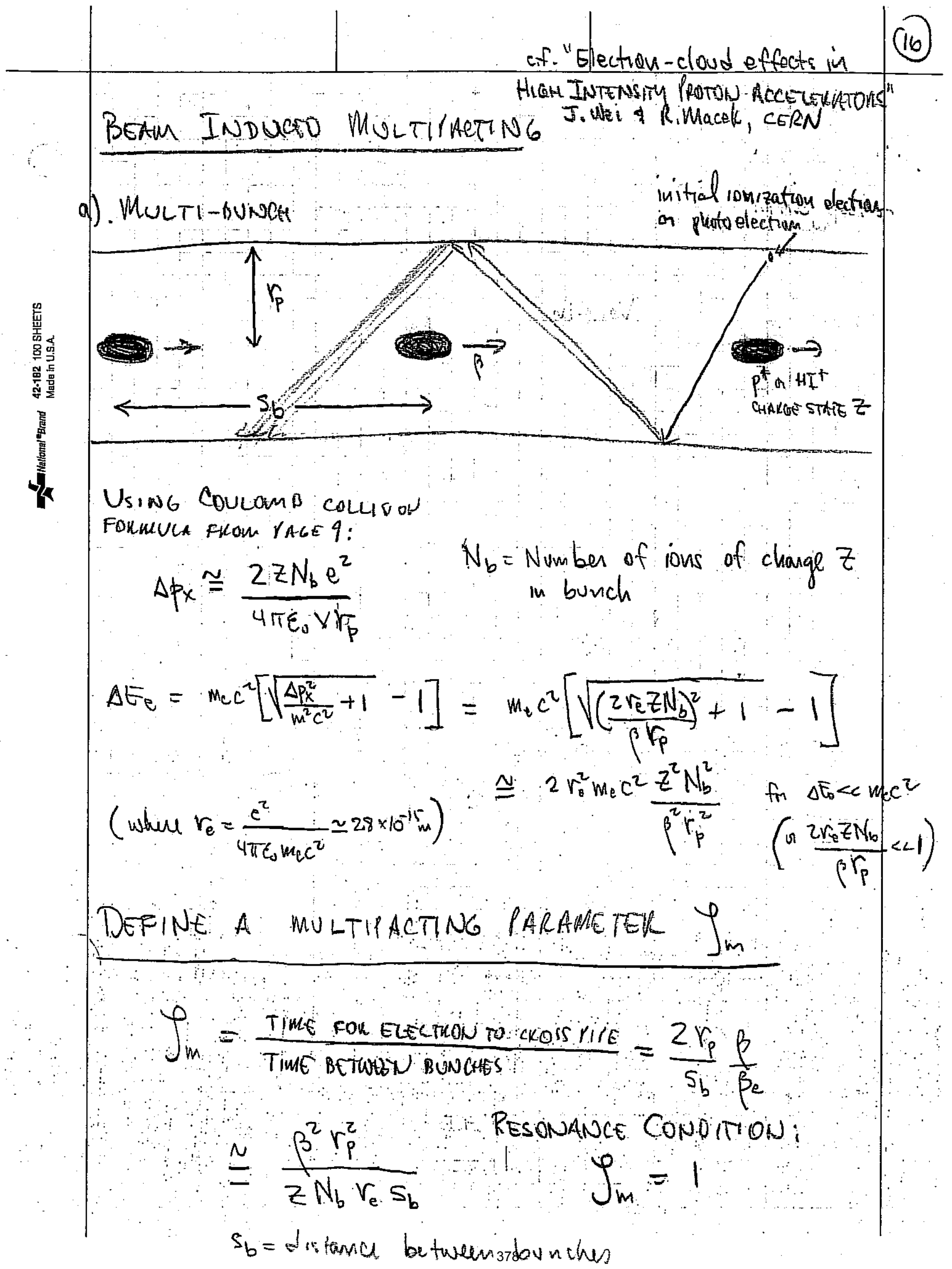




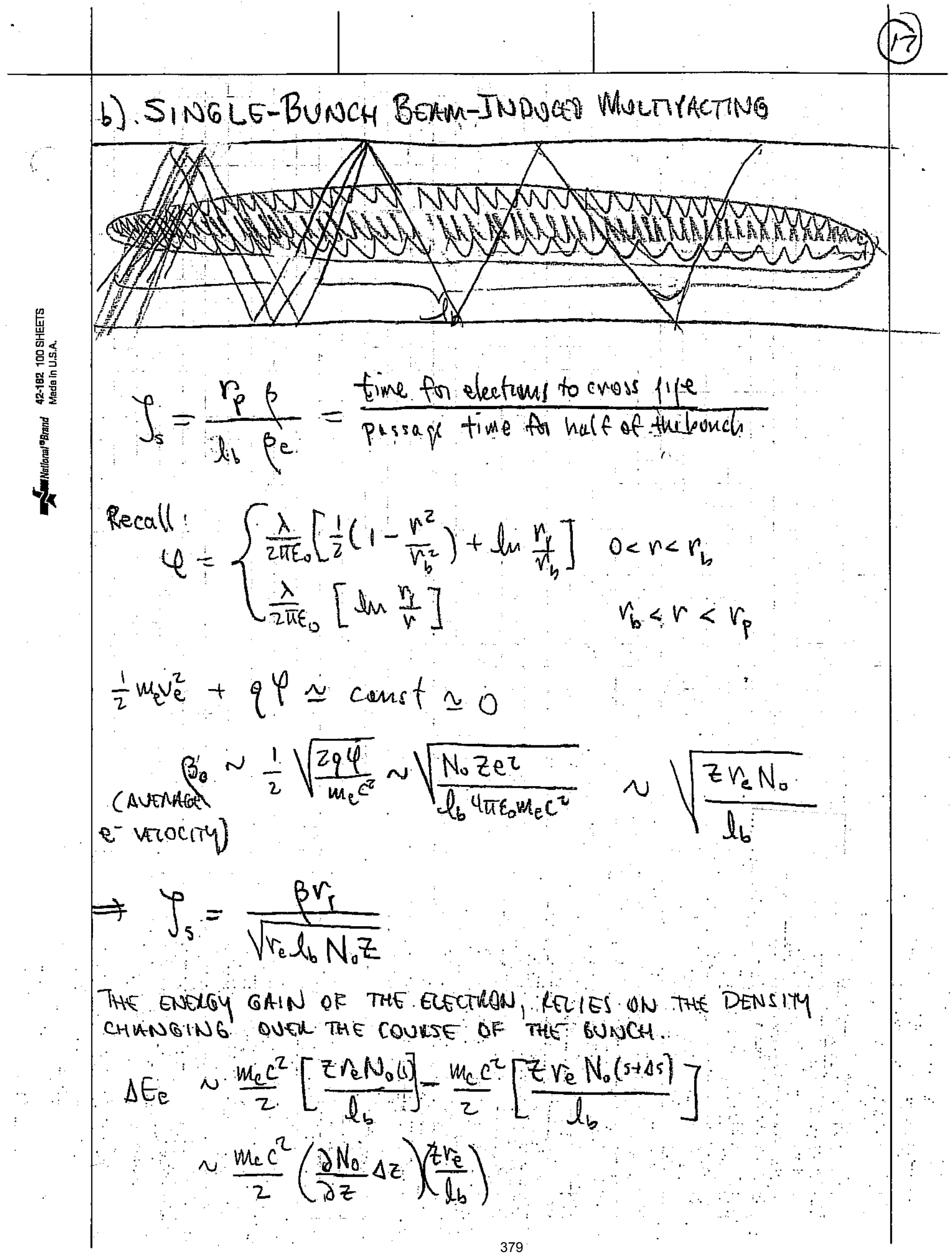




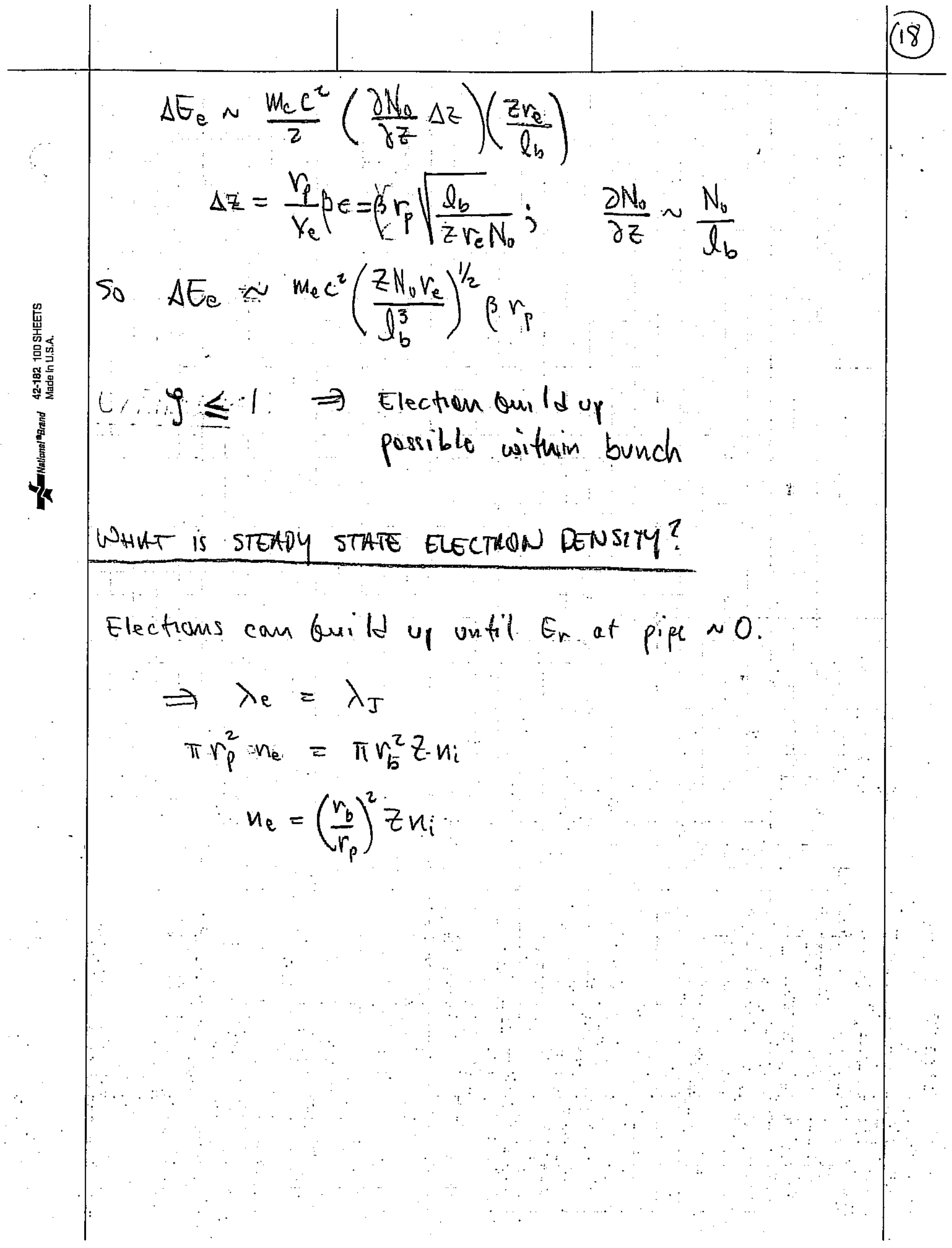




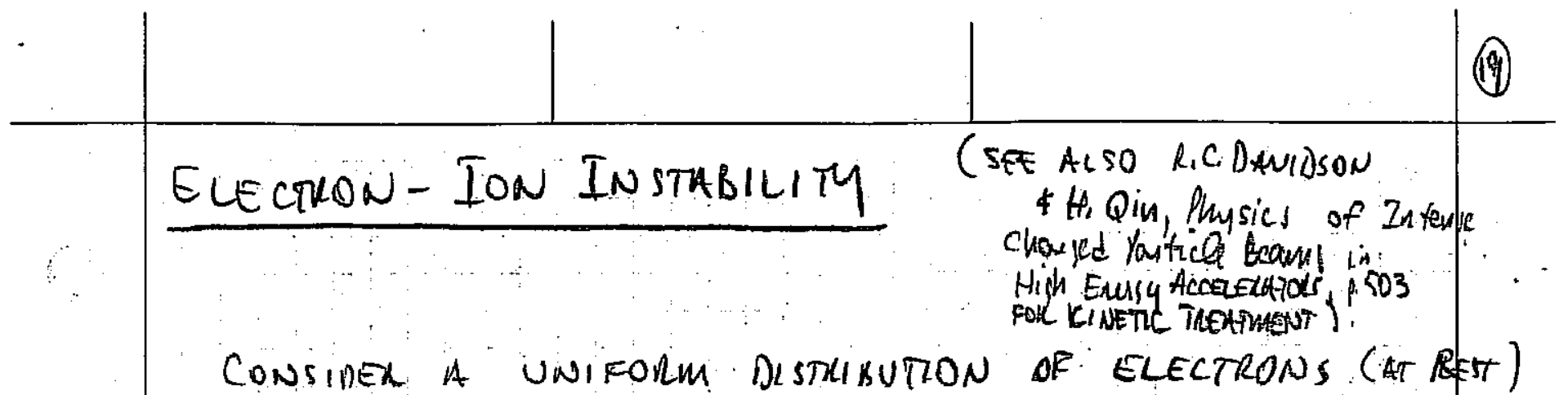
WHICH HAS THE SAME ARIUS (OK SHEATH SMALLER RADIUS) AI A UNIFORM DENSITY ION BEAM, THAT K MOVING AT VERITY

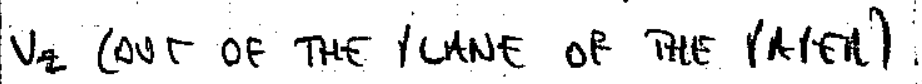
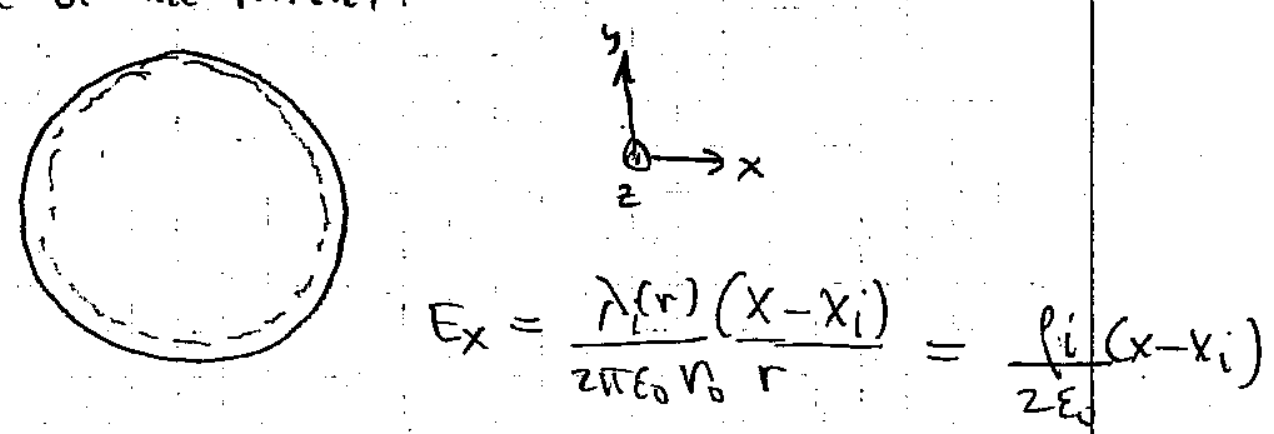

THE equation of motion fOL THE CENTLOLI DR the ELECTRONS IS OBTAINED FROM

$$
m_{e} \ddot{x}=\frac{-e \rho_{i}^{\prime}}{2 \varepsilon_{0}}\left(x-x_{i}\right)+\frac{e \rho_{e}}{2 \varepsilon_{u}}\left(x-x_{e}\right)
$$

or $\quad \frac{d^{2} \dot{x}_{e}}{d t^{2}}=-\frac{w_{p i}^{2}}{2}\left(\frac{m_{i}}{q^{i}} \frac{e}{m_{i}}\right)\left(x_{e}-x_{i}\right)$.

here $\omega_{i}^{2}=\frac{q^{2} n_{i}}{\epsilon_{0} m_{i}}=\frac{q p_{i}^{\prime}}{\epsilon_{0} m_{i}}$

(THE CENTE of oscillation for the elections is the center of the ion beam):

$x_{e}=$ centroid of election bean

$x_{i}=$ centroid of ion beam

381 


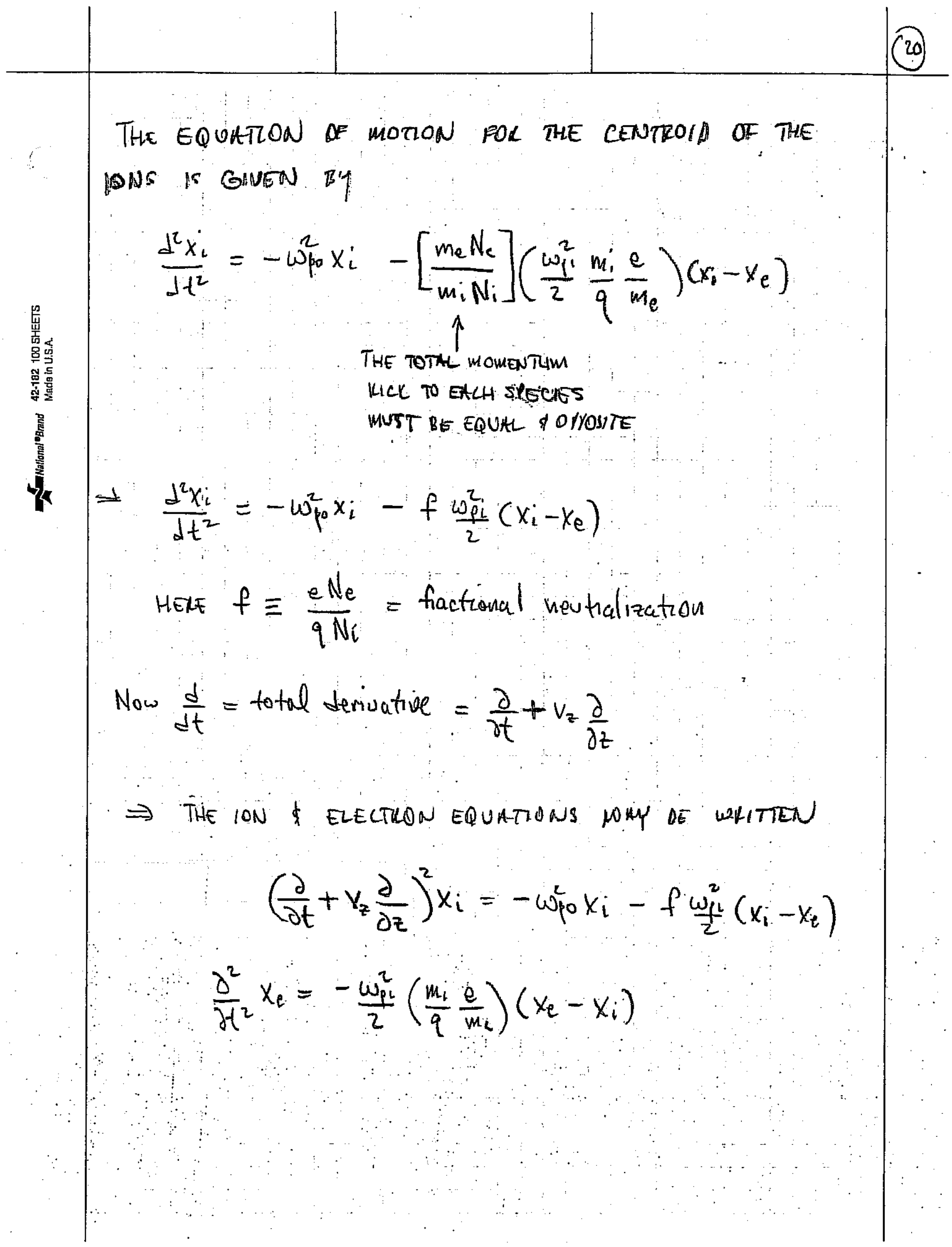

382 


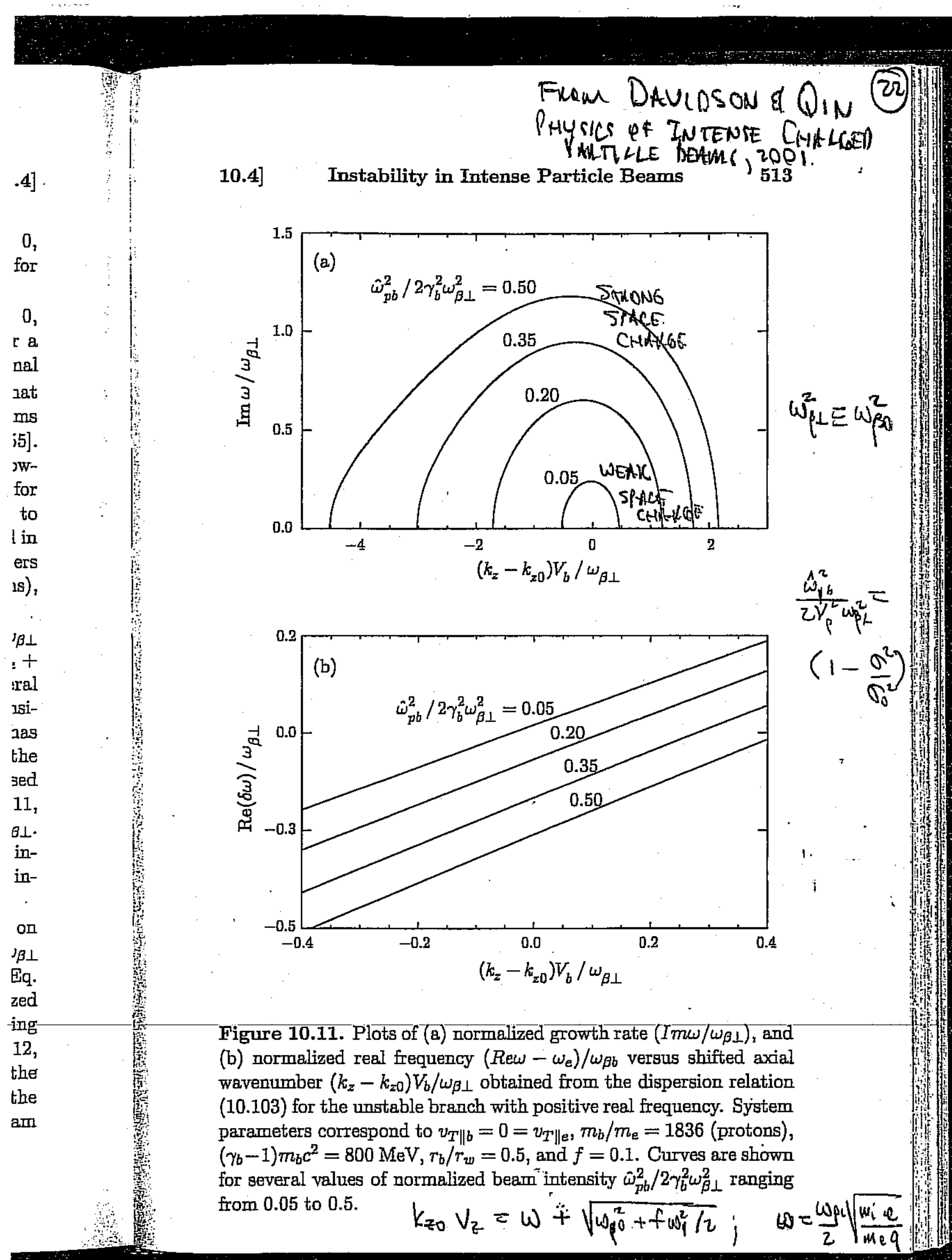




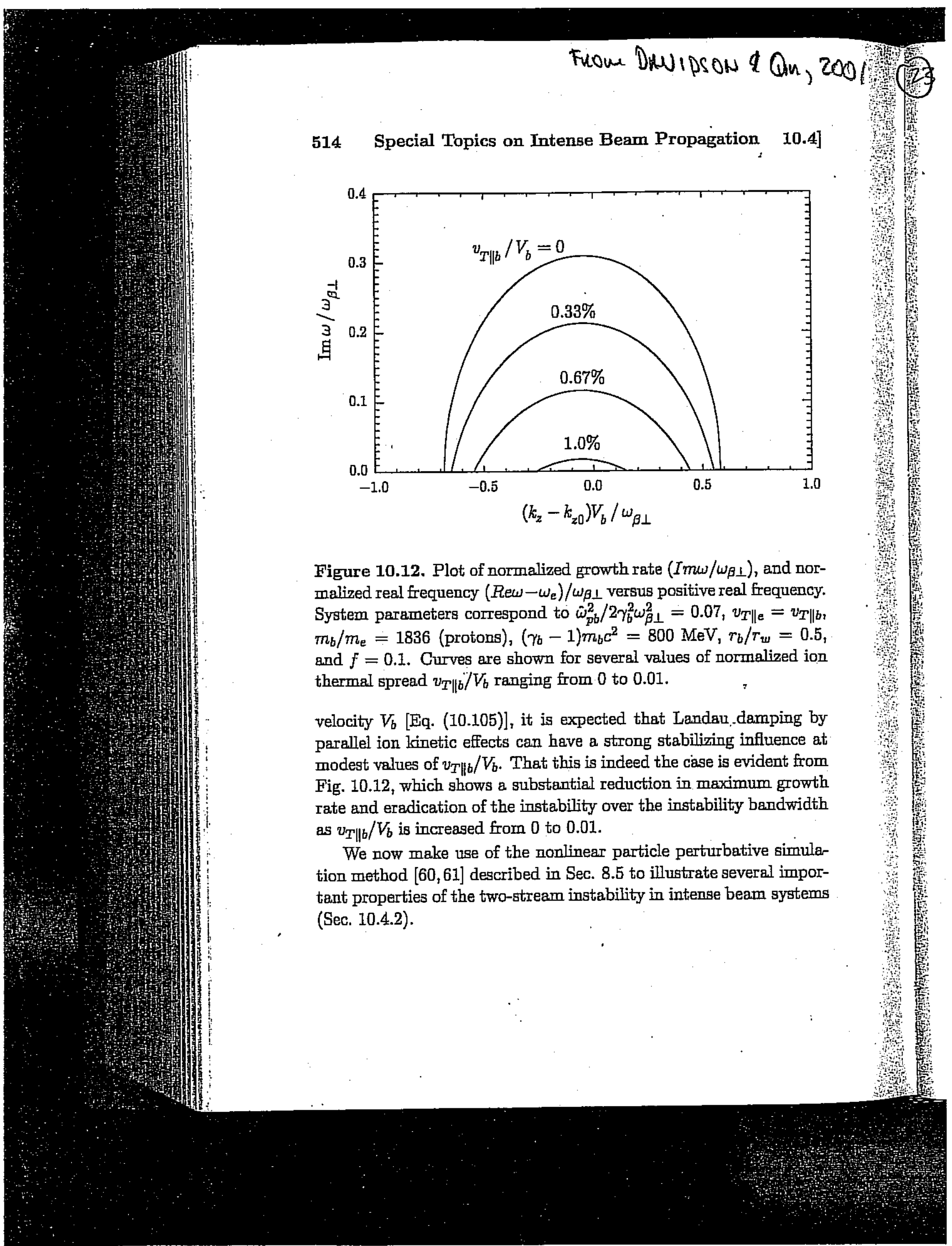




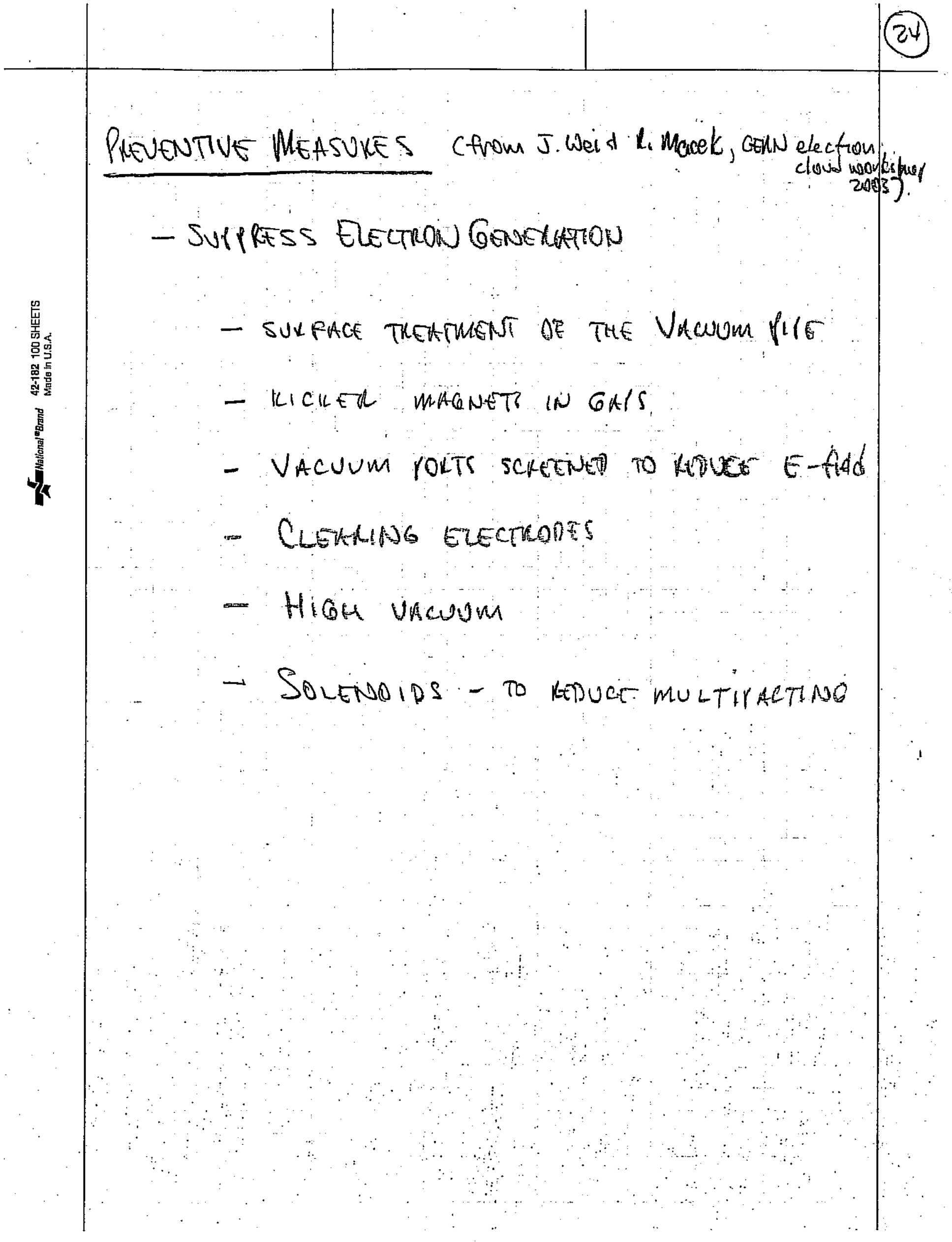




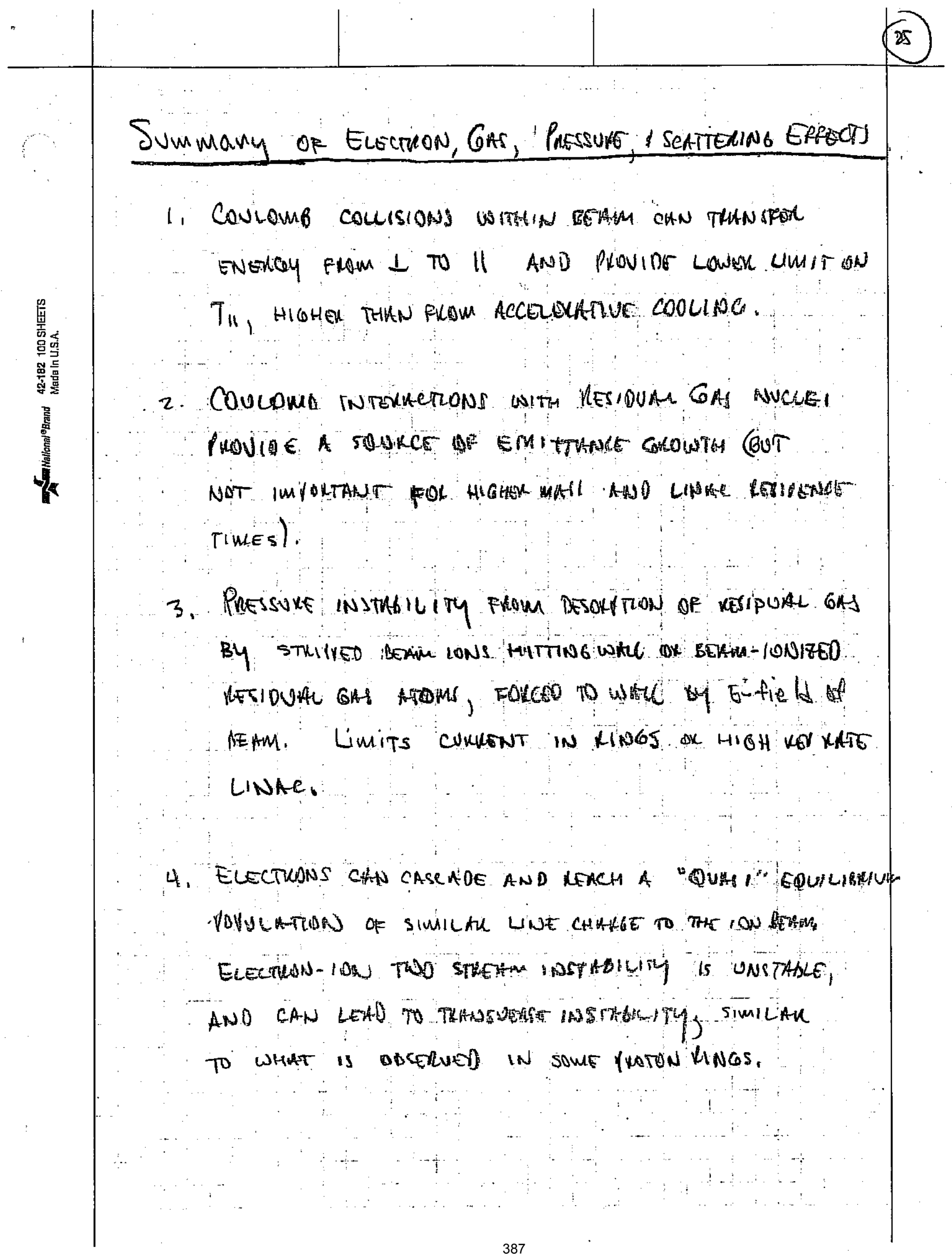




\section{John Barnard Steven Lund USPAS June 2008}

\section{An application of intense beams}

1. Heavy-ion fusion

A. Requirements

B. Targets for ICF

C. Accelerator

D. Drift compression

E. Final focus

F. Experiments 
(2)

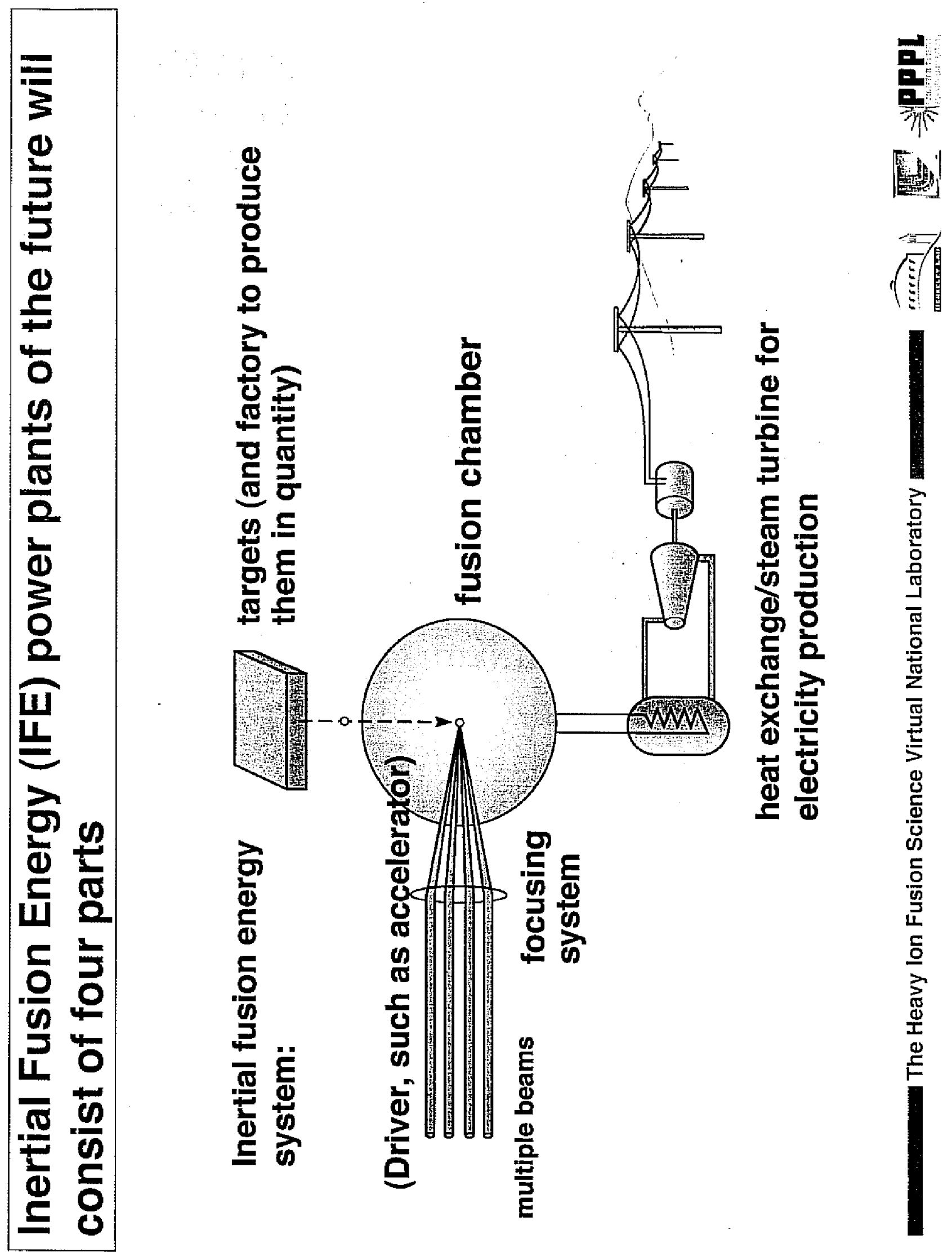




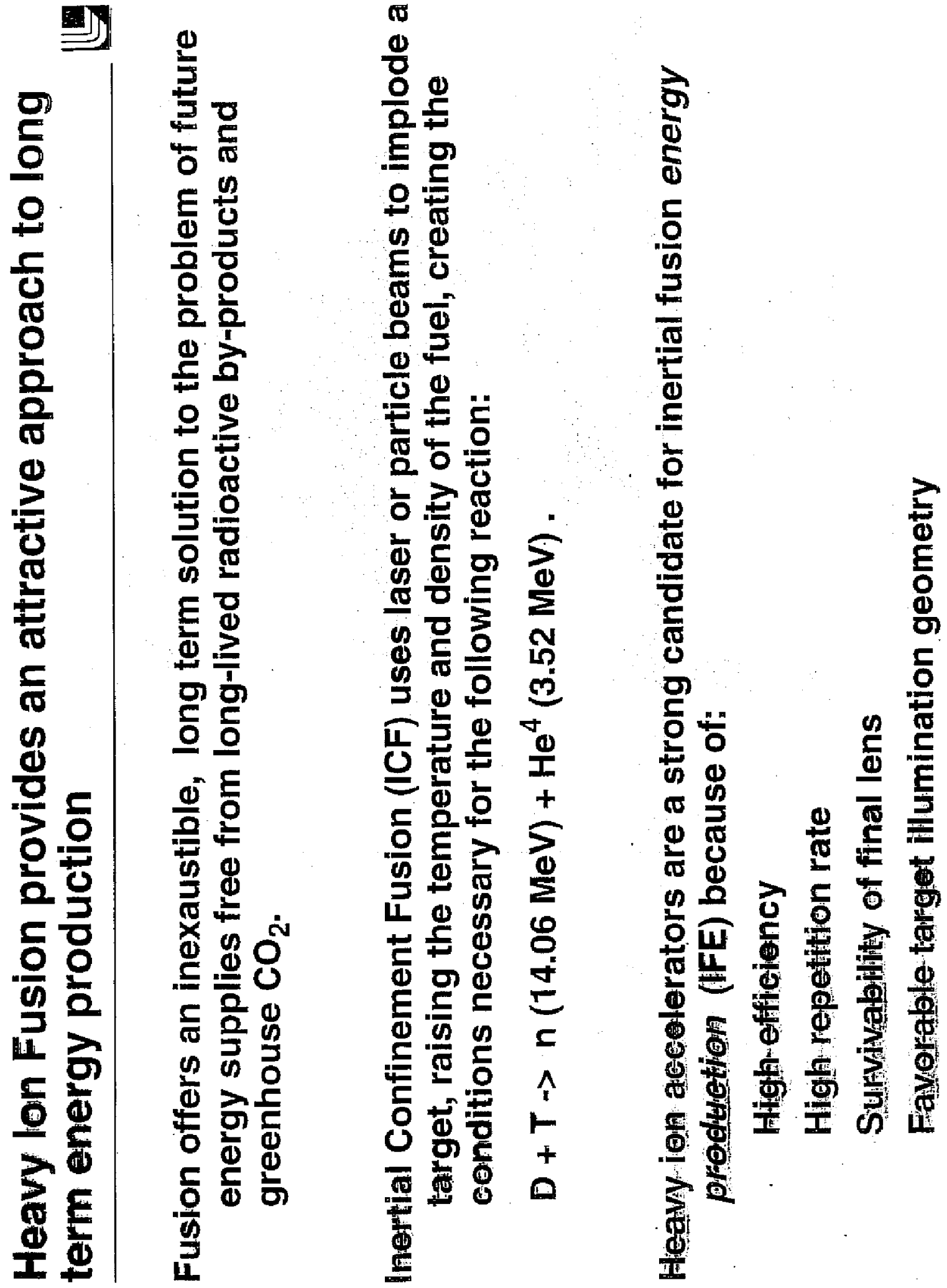




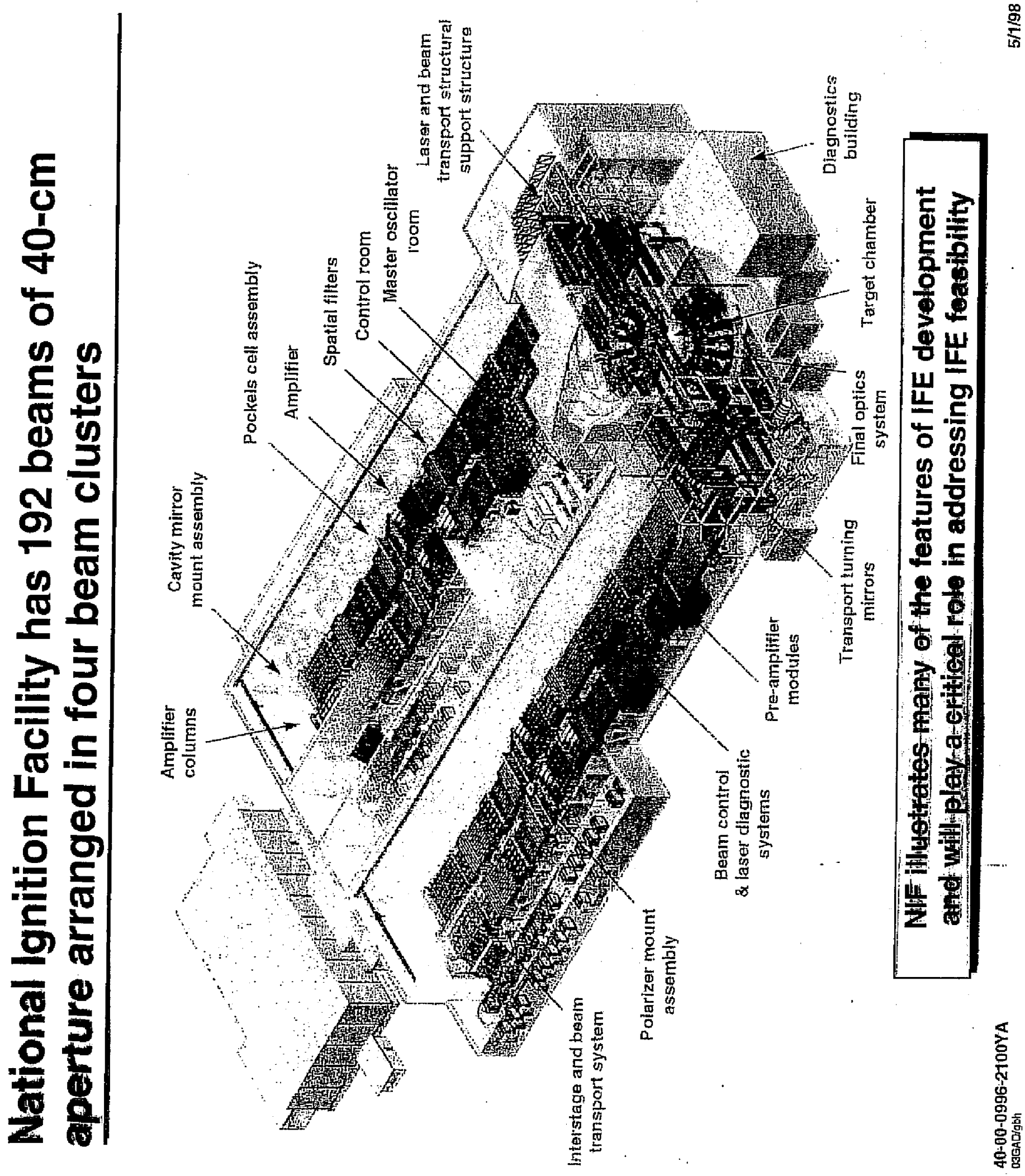

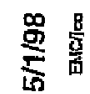



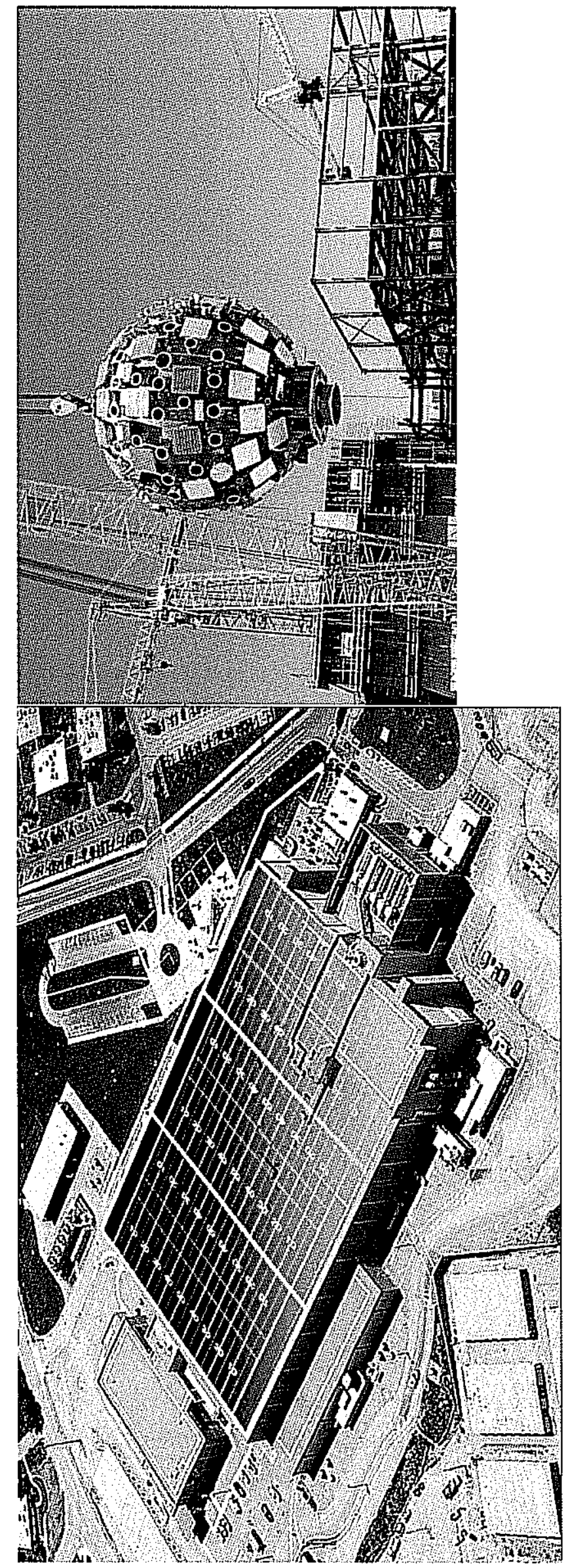
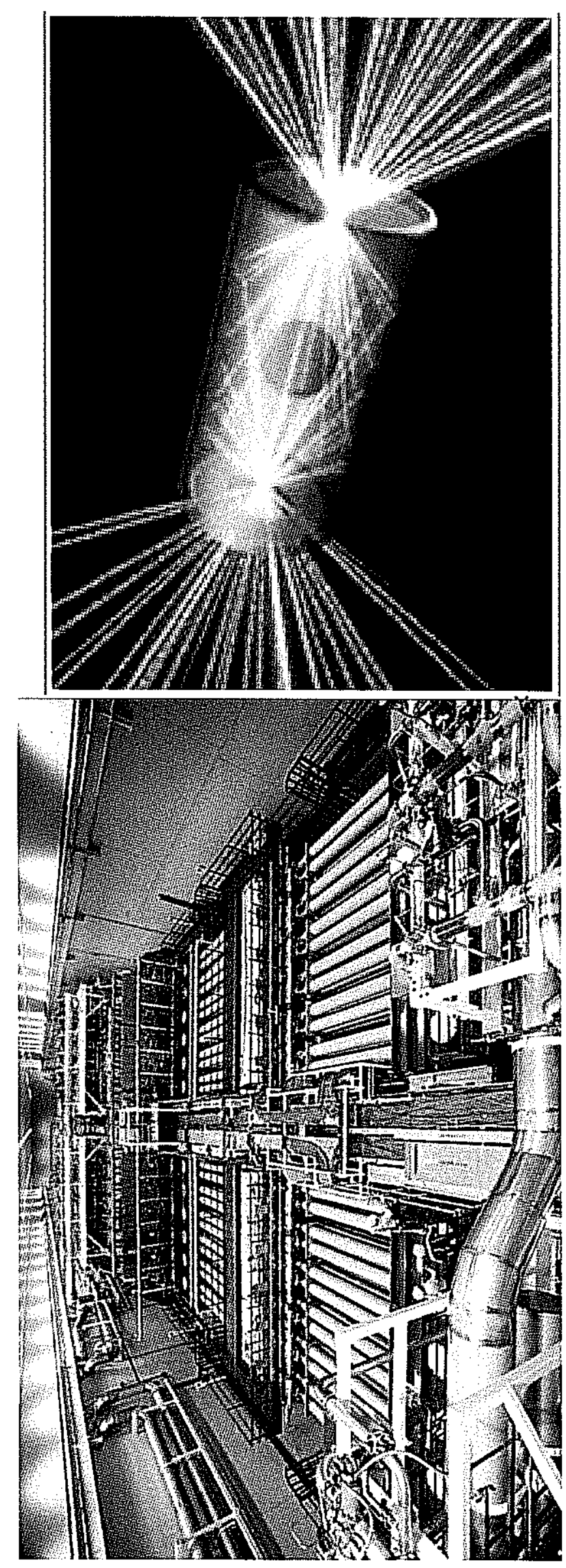


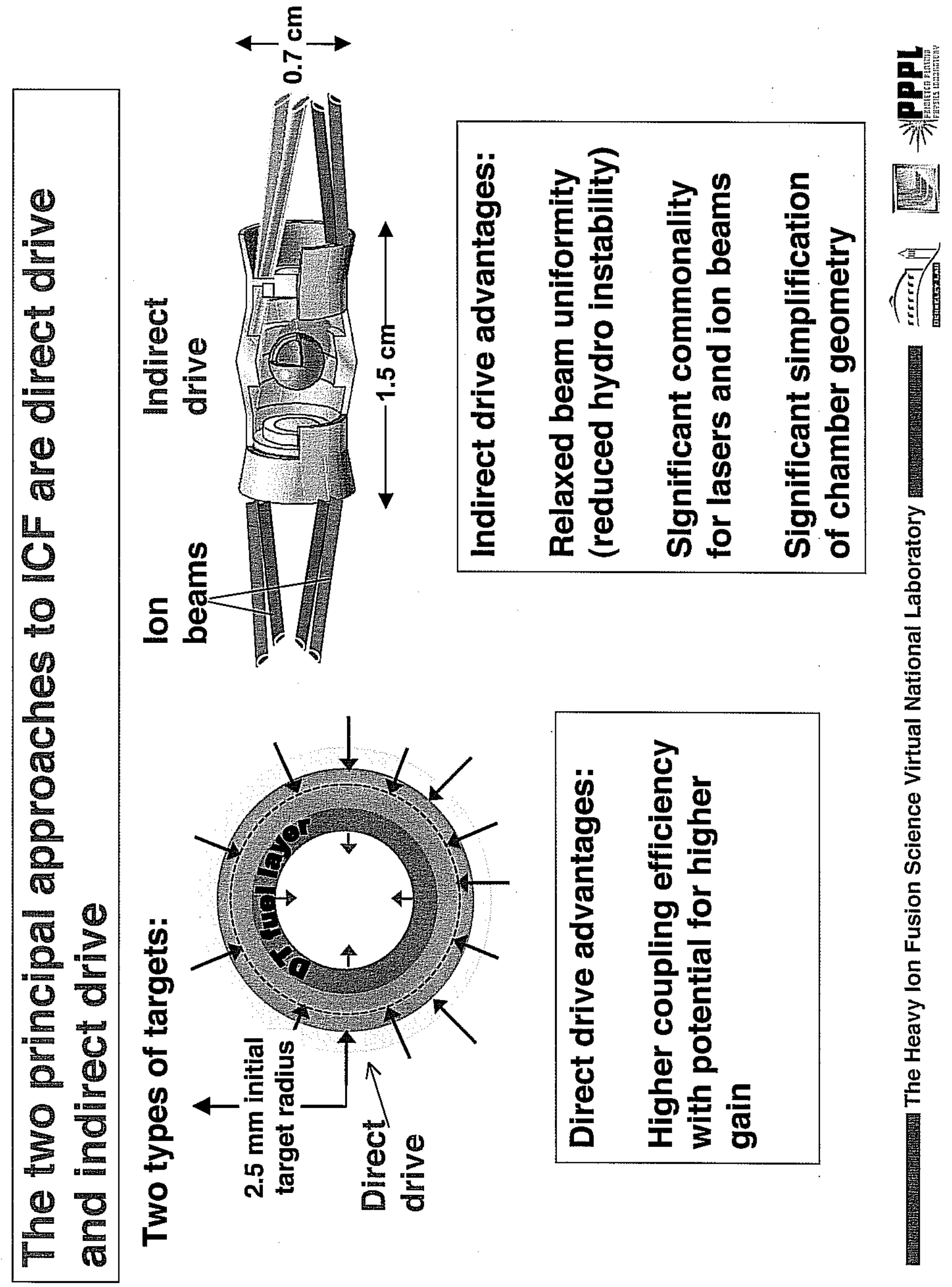




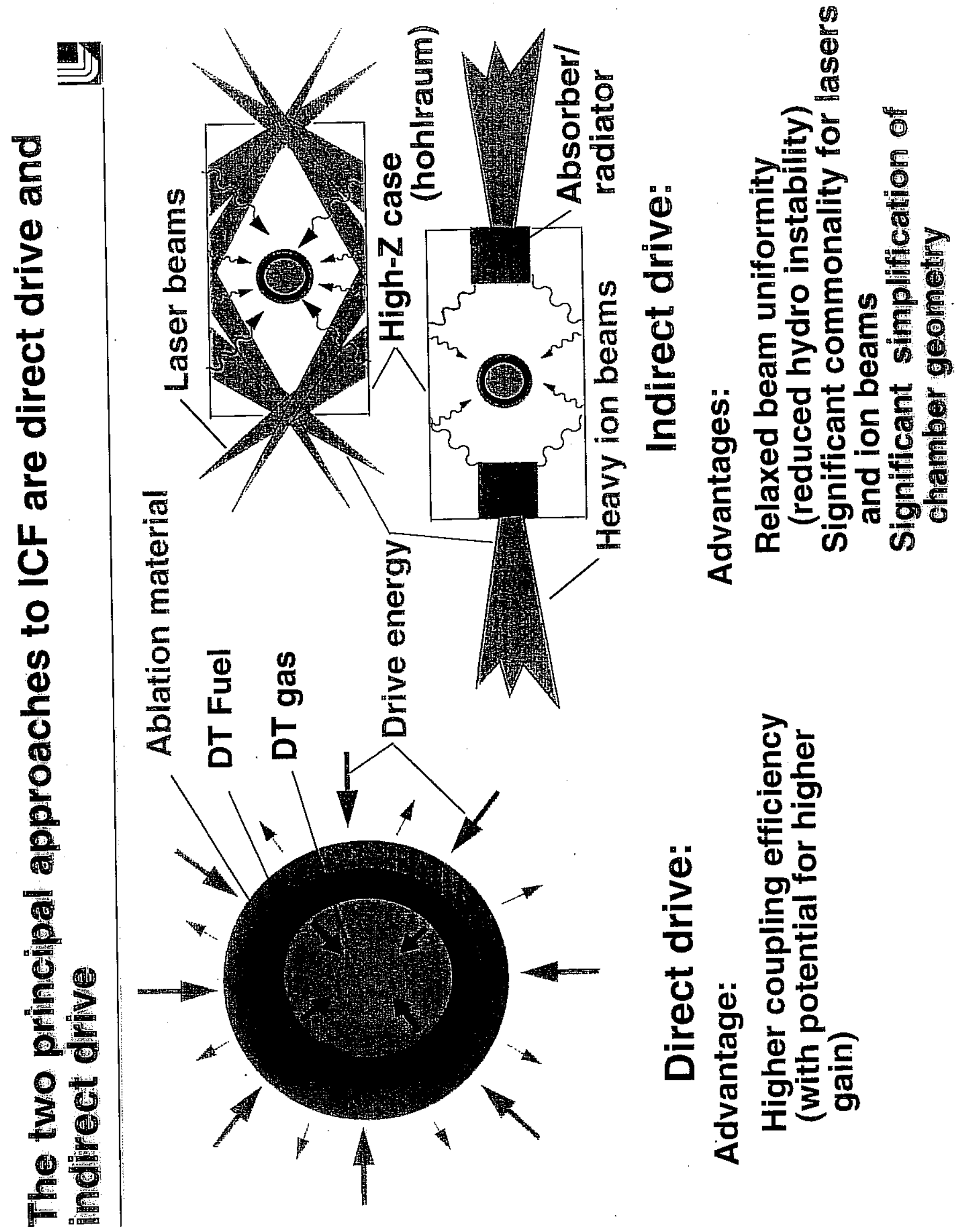




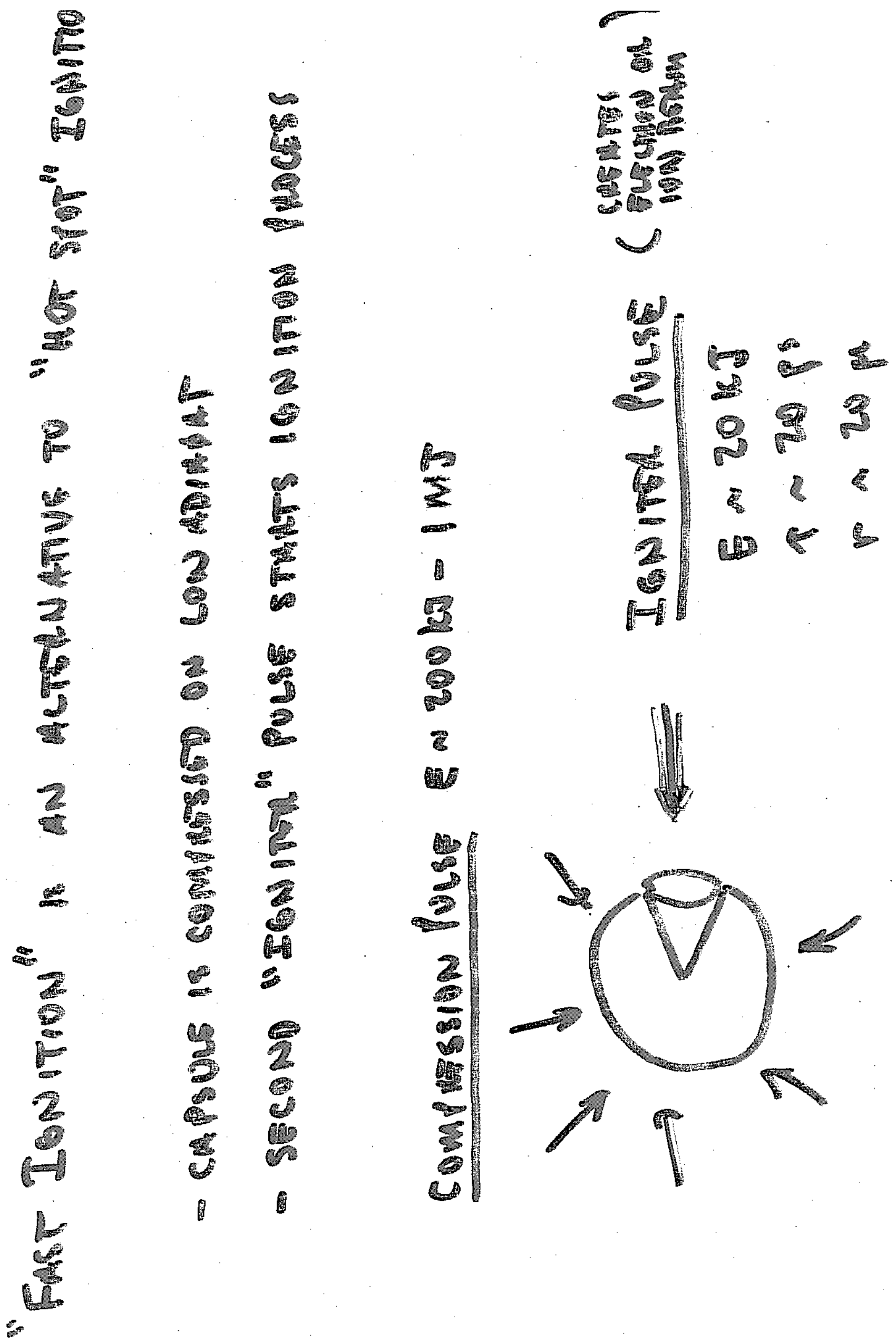



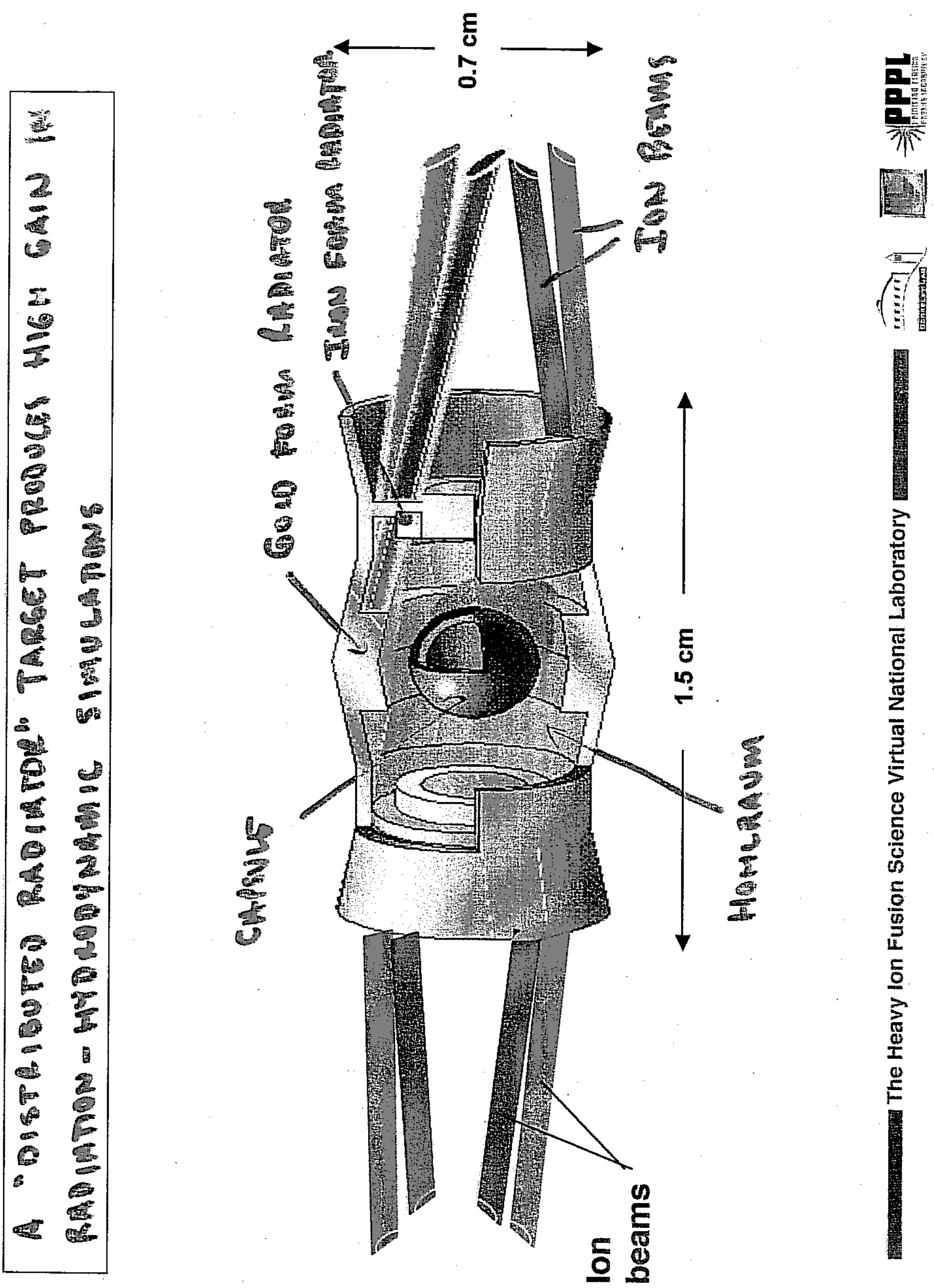

을

总

등

要

톤 


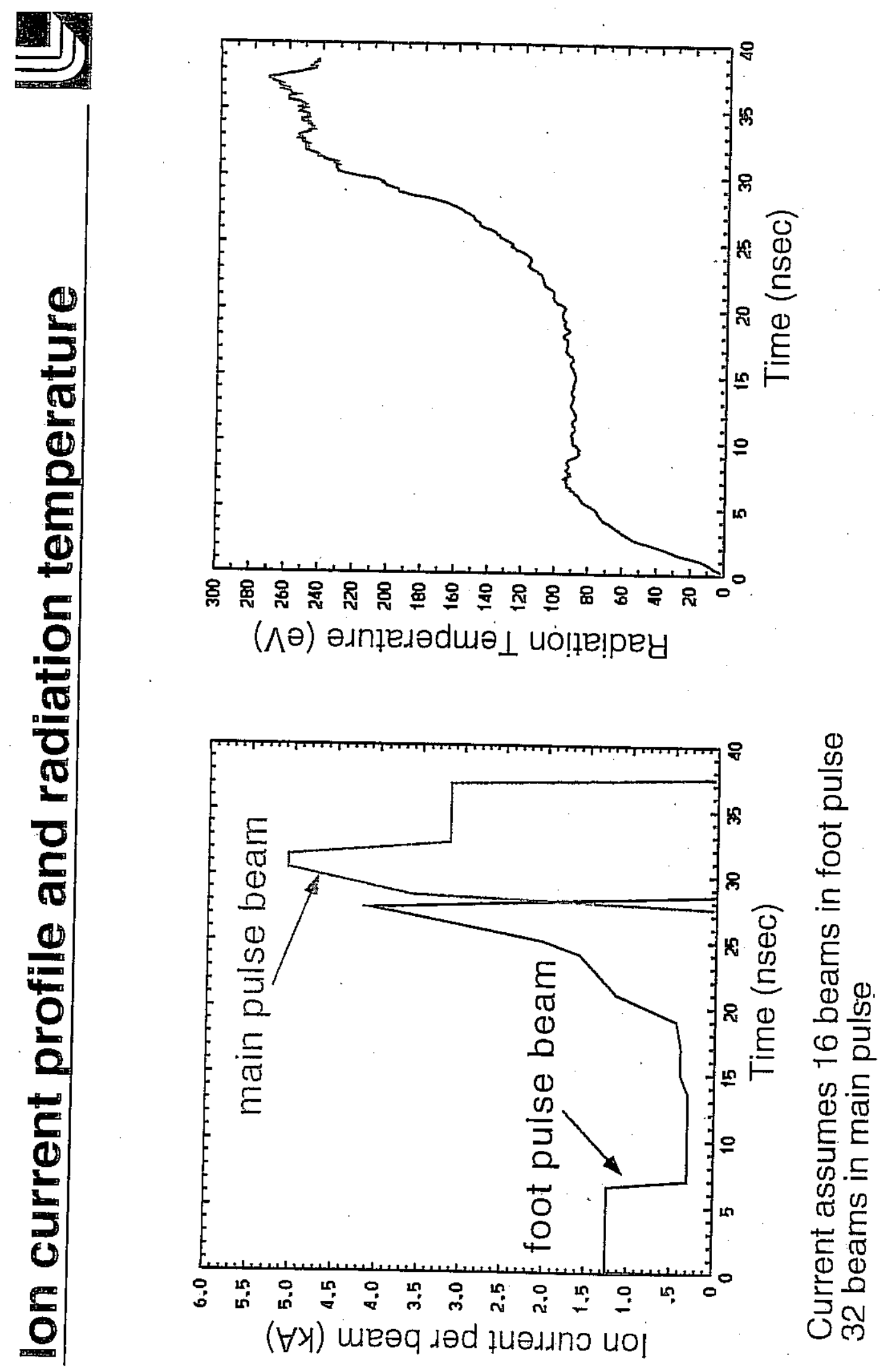

点 


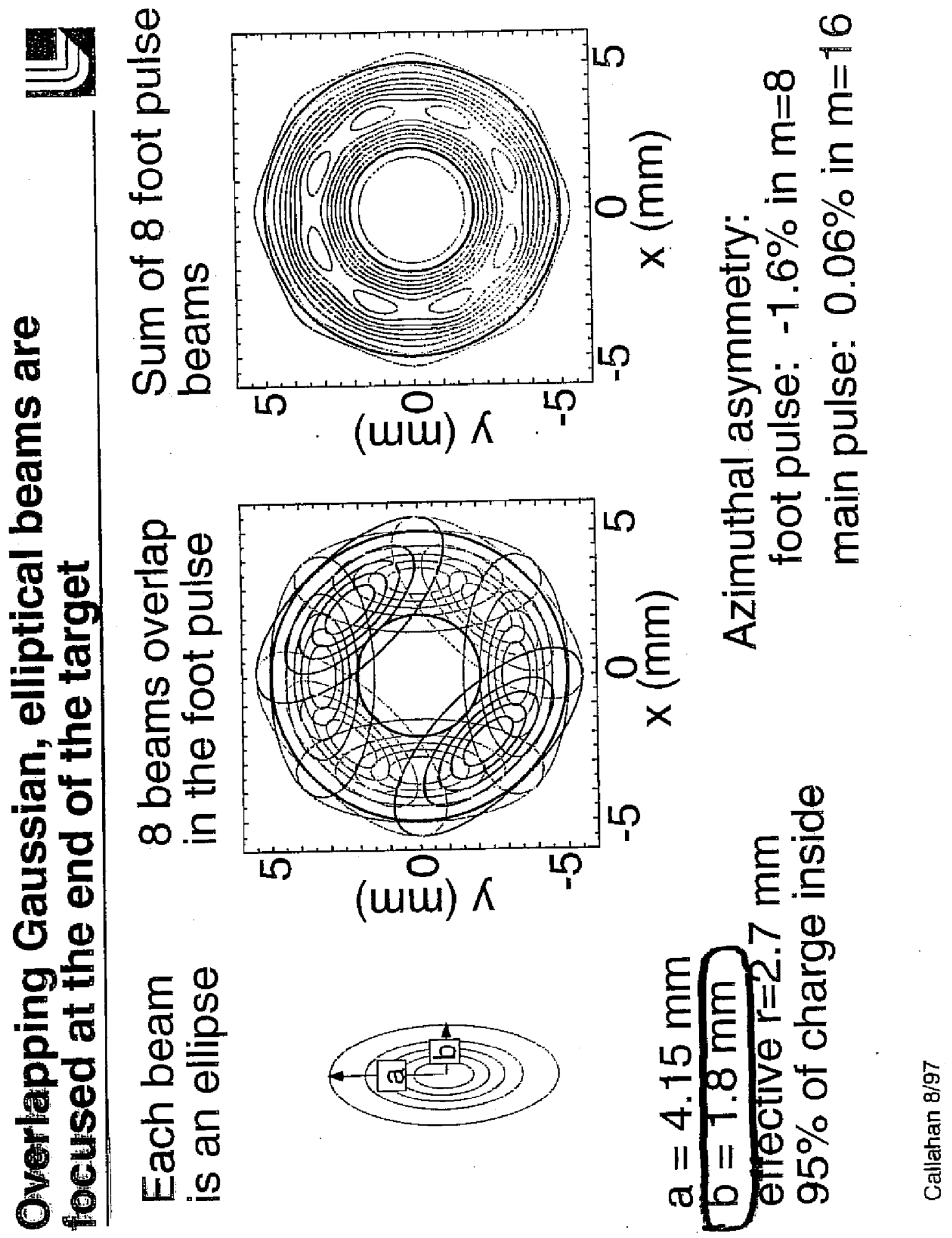



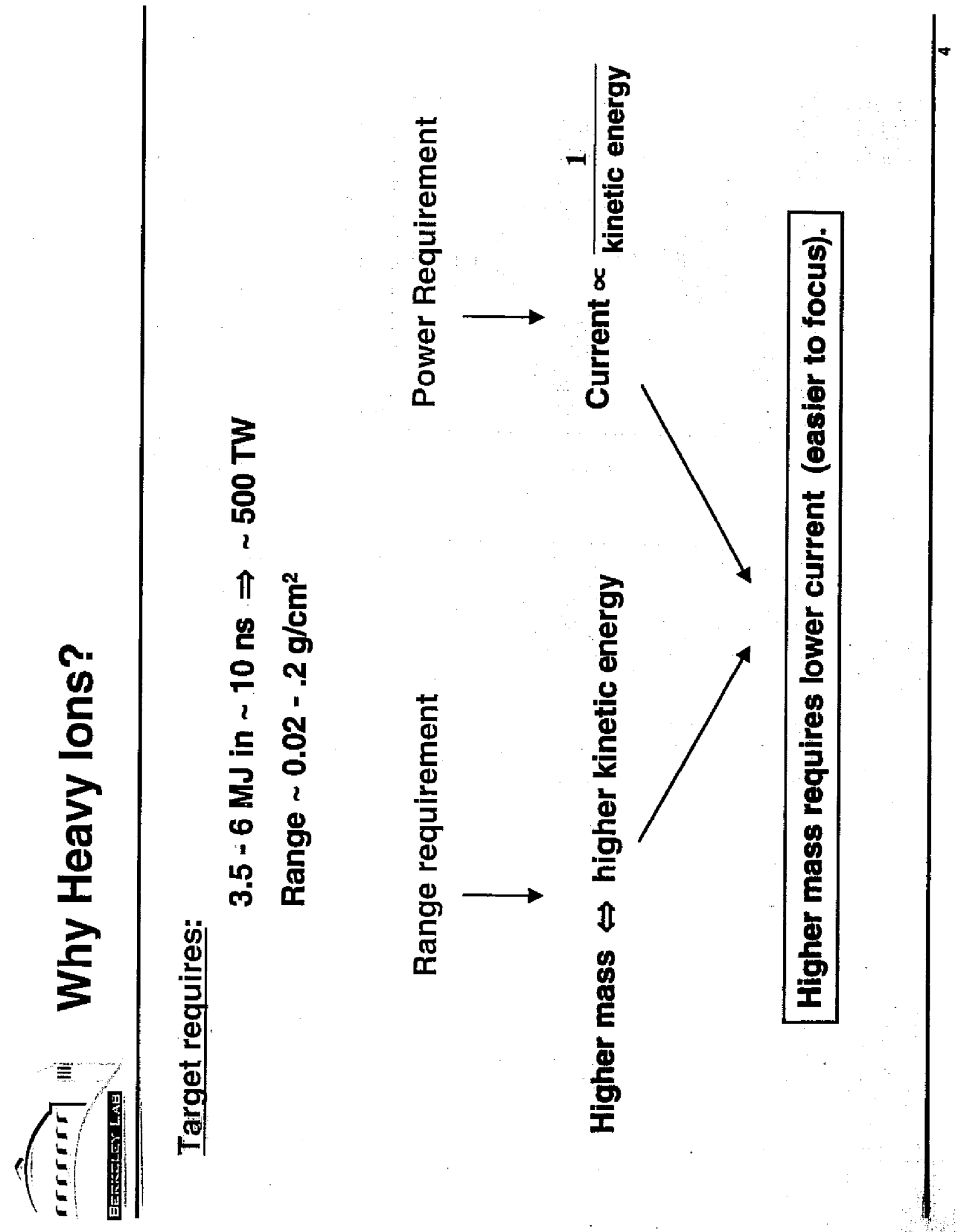


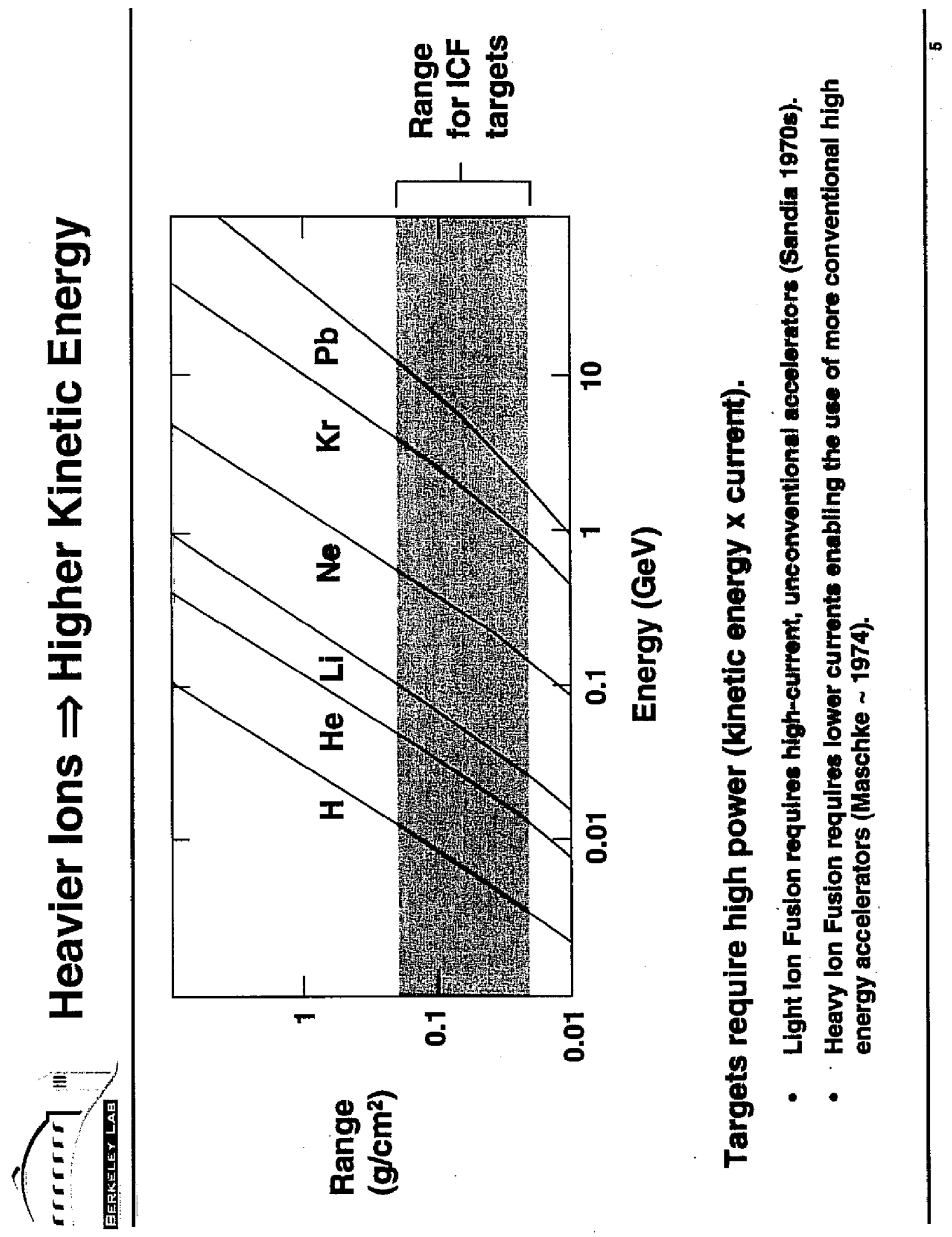




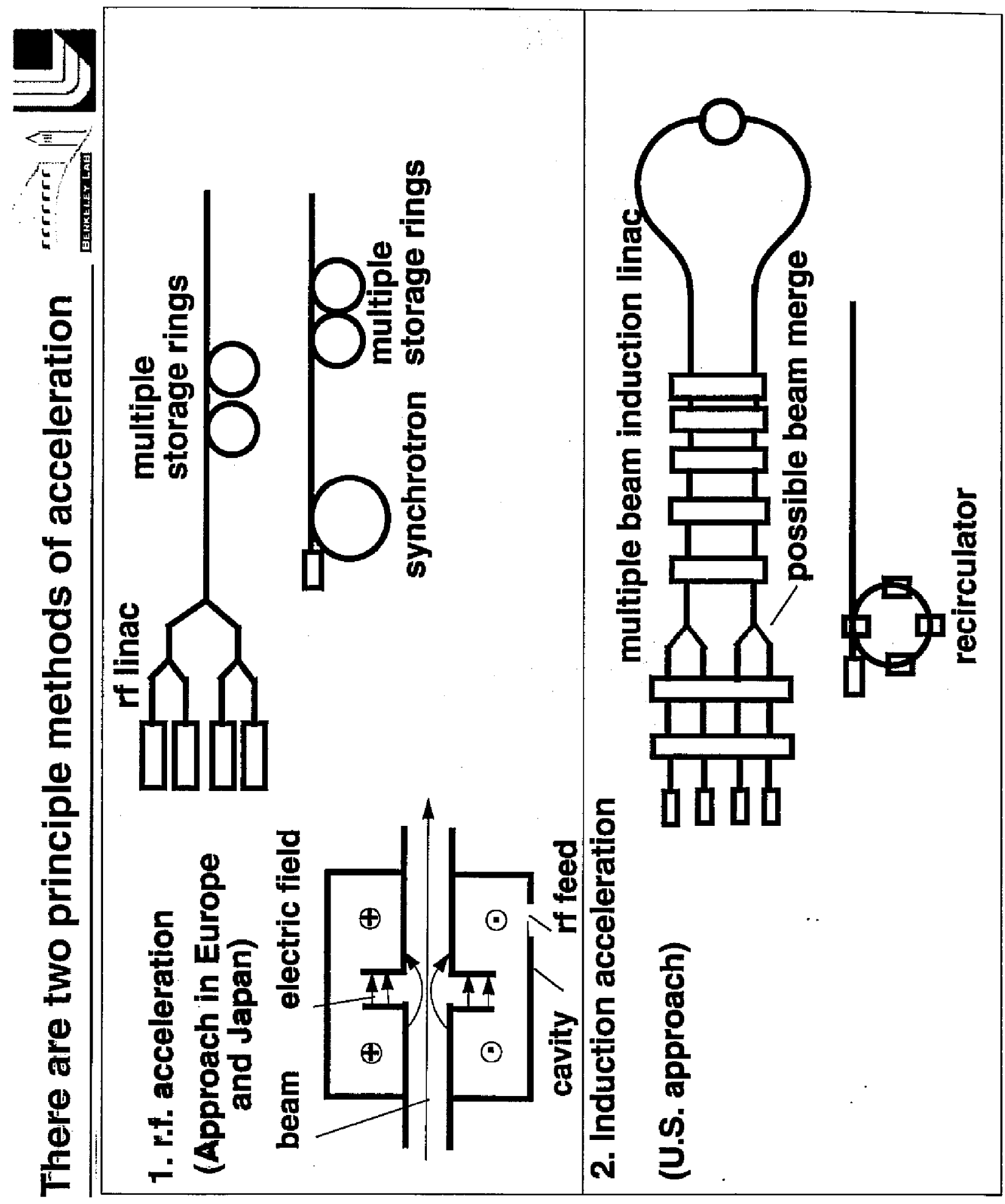




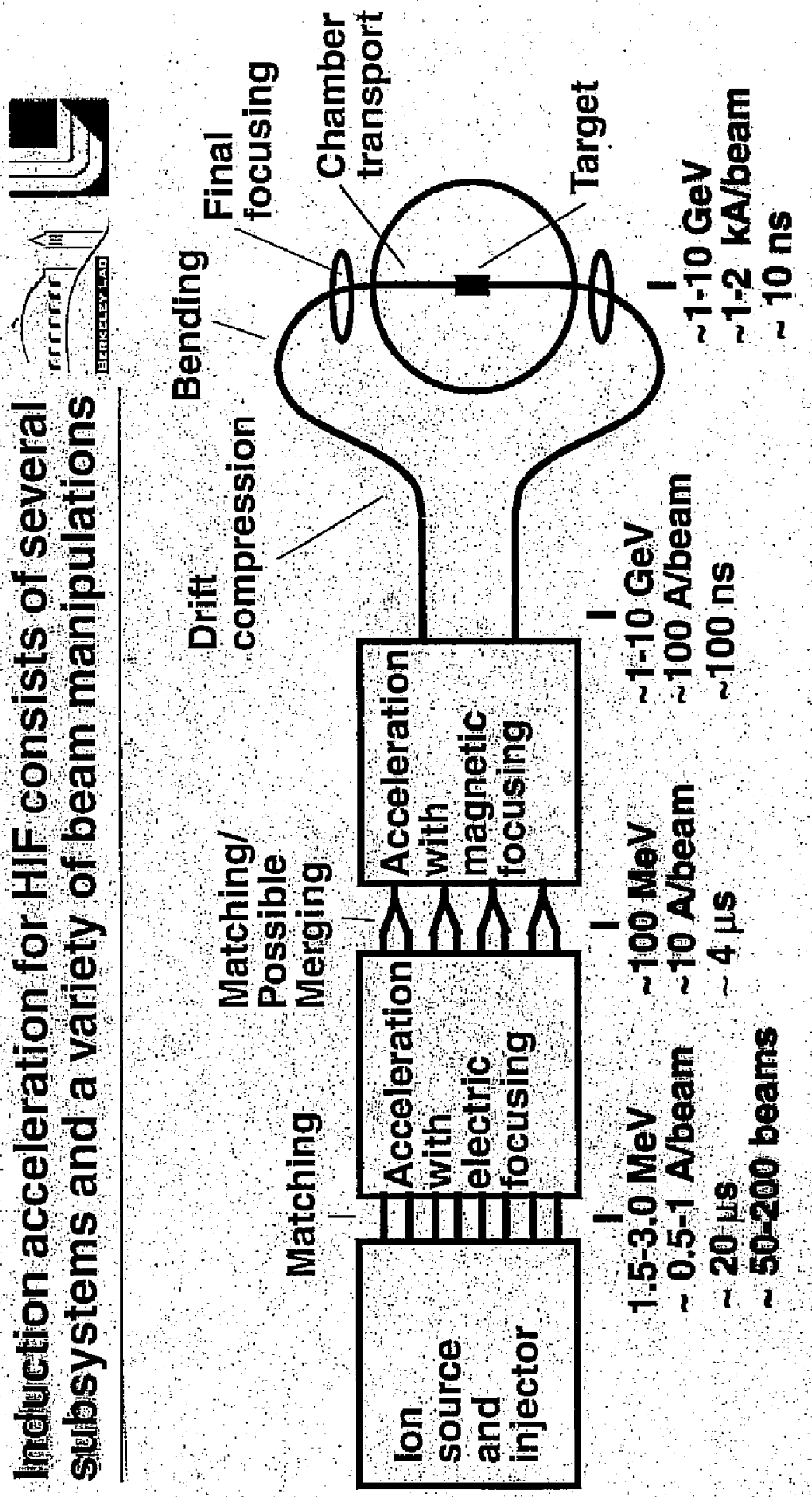



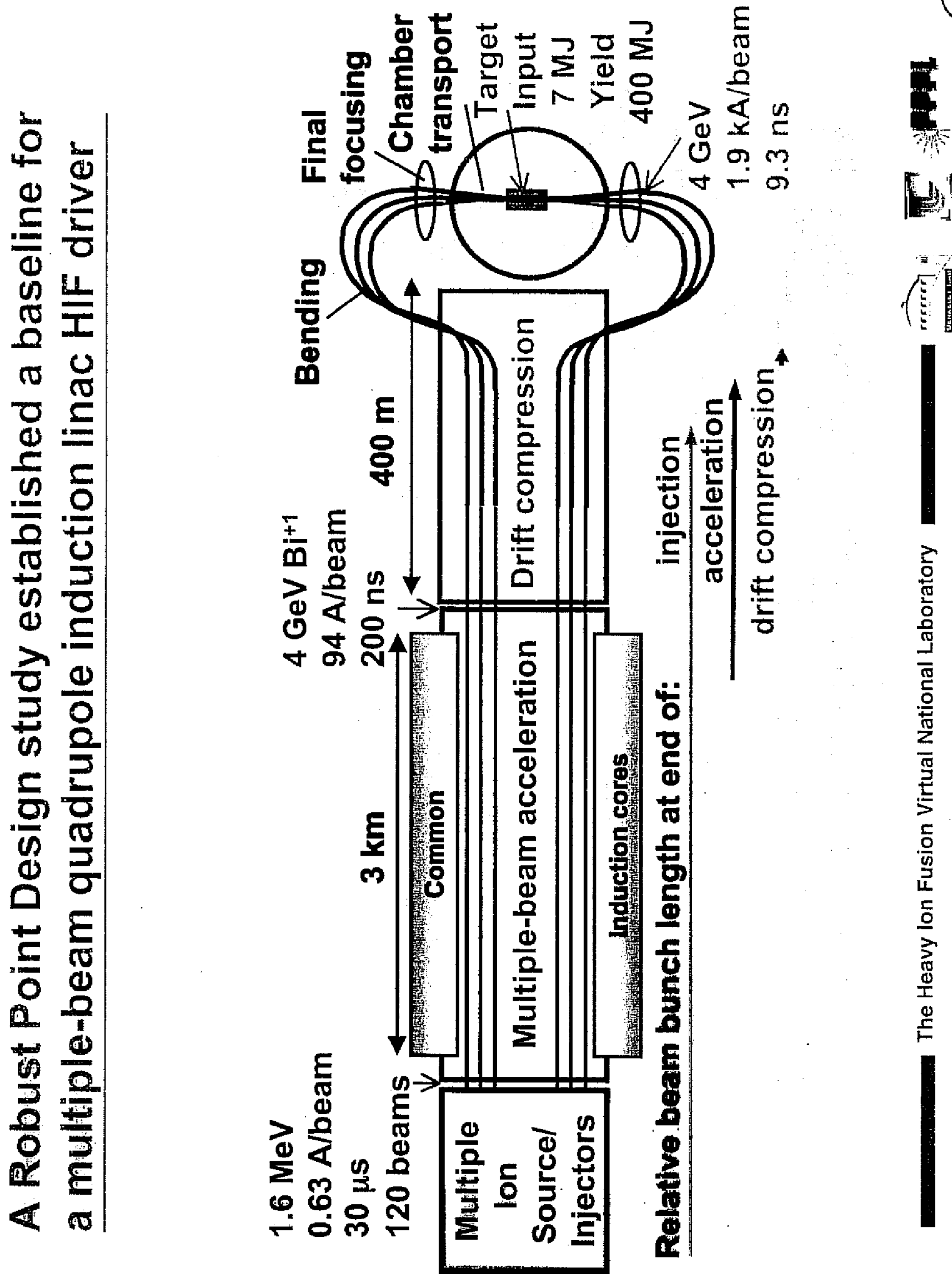

G!

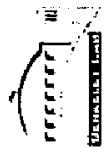

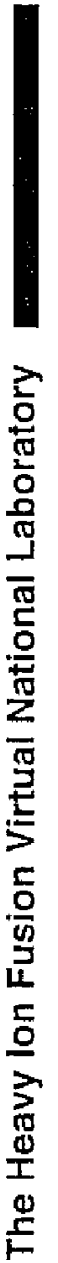




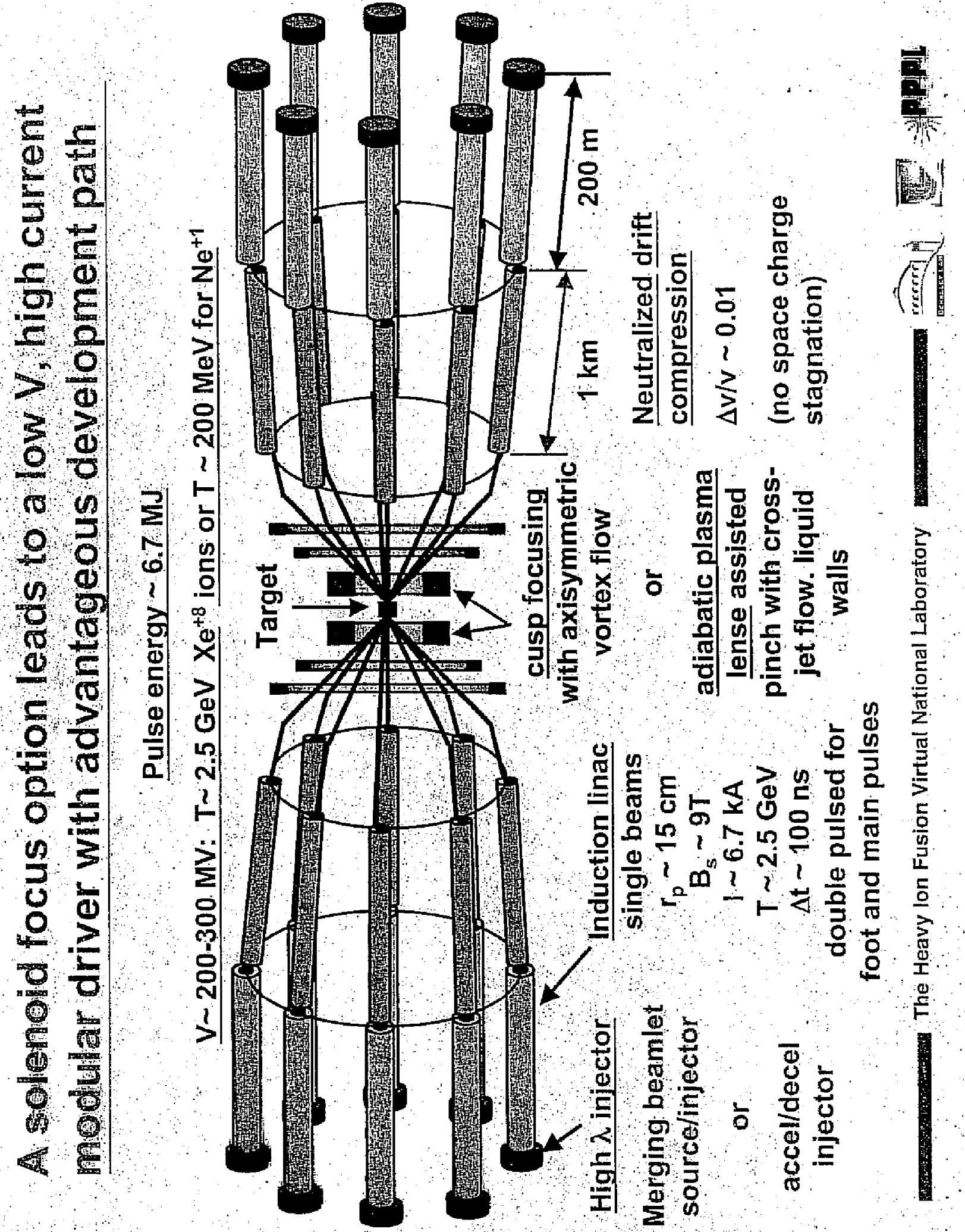




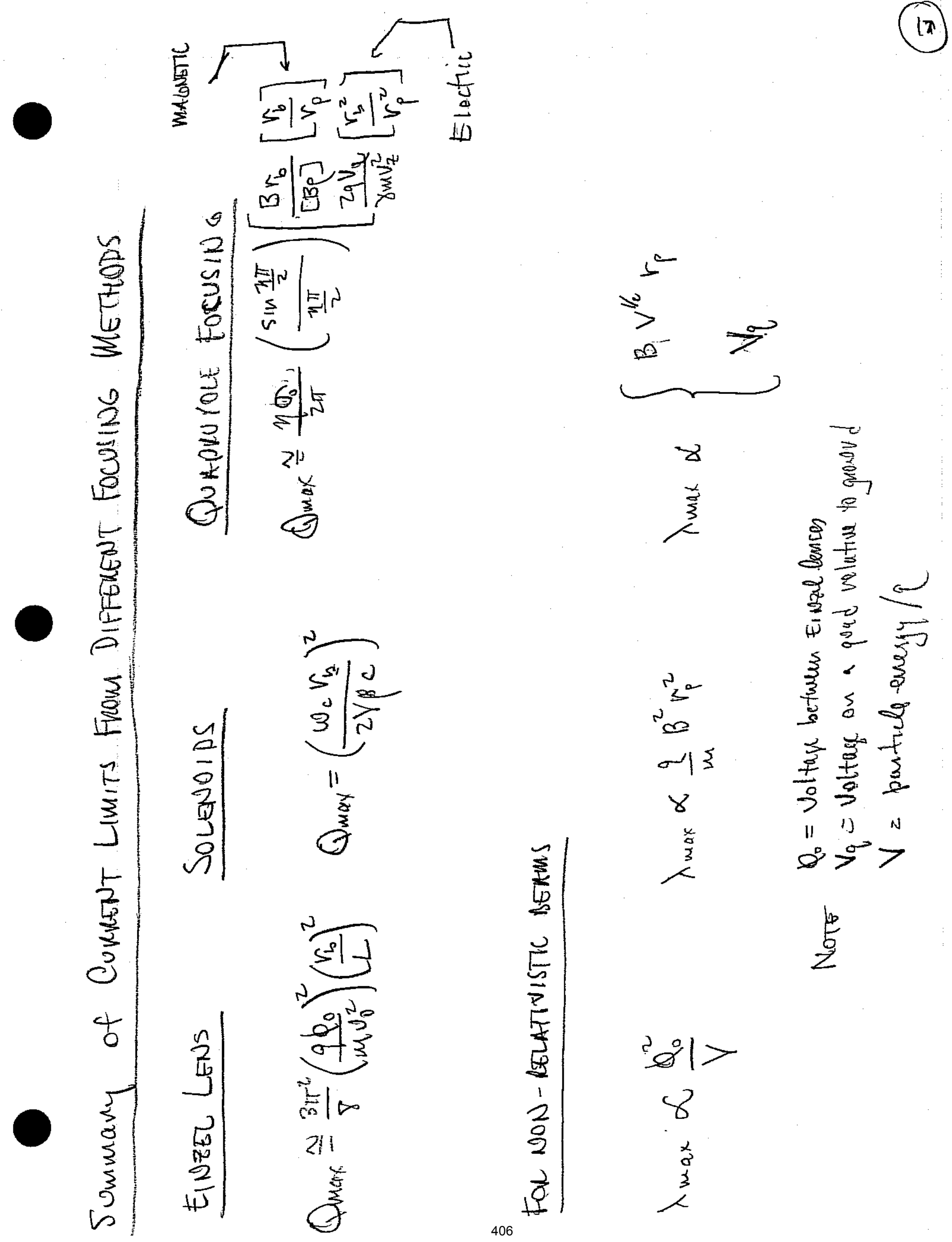




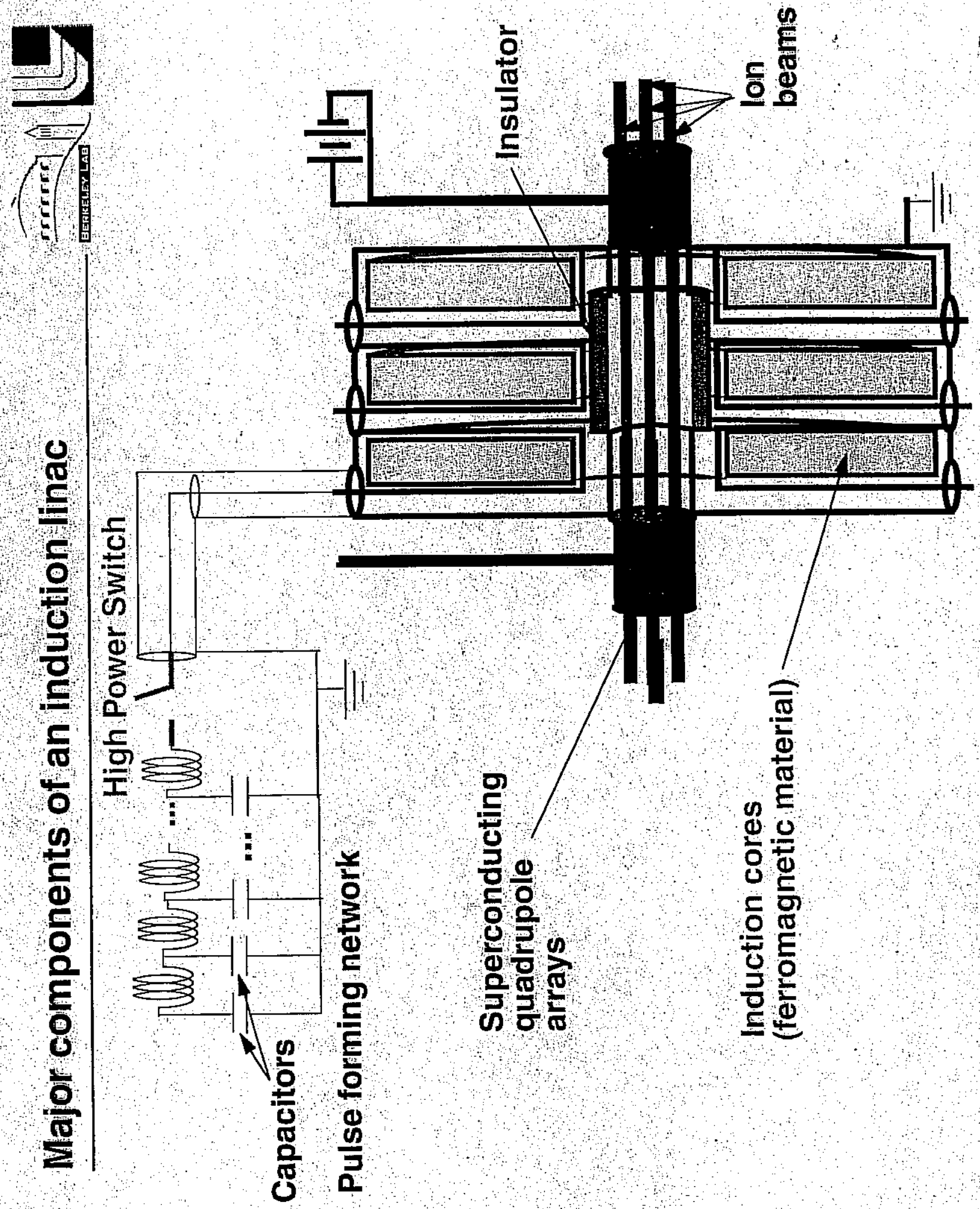




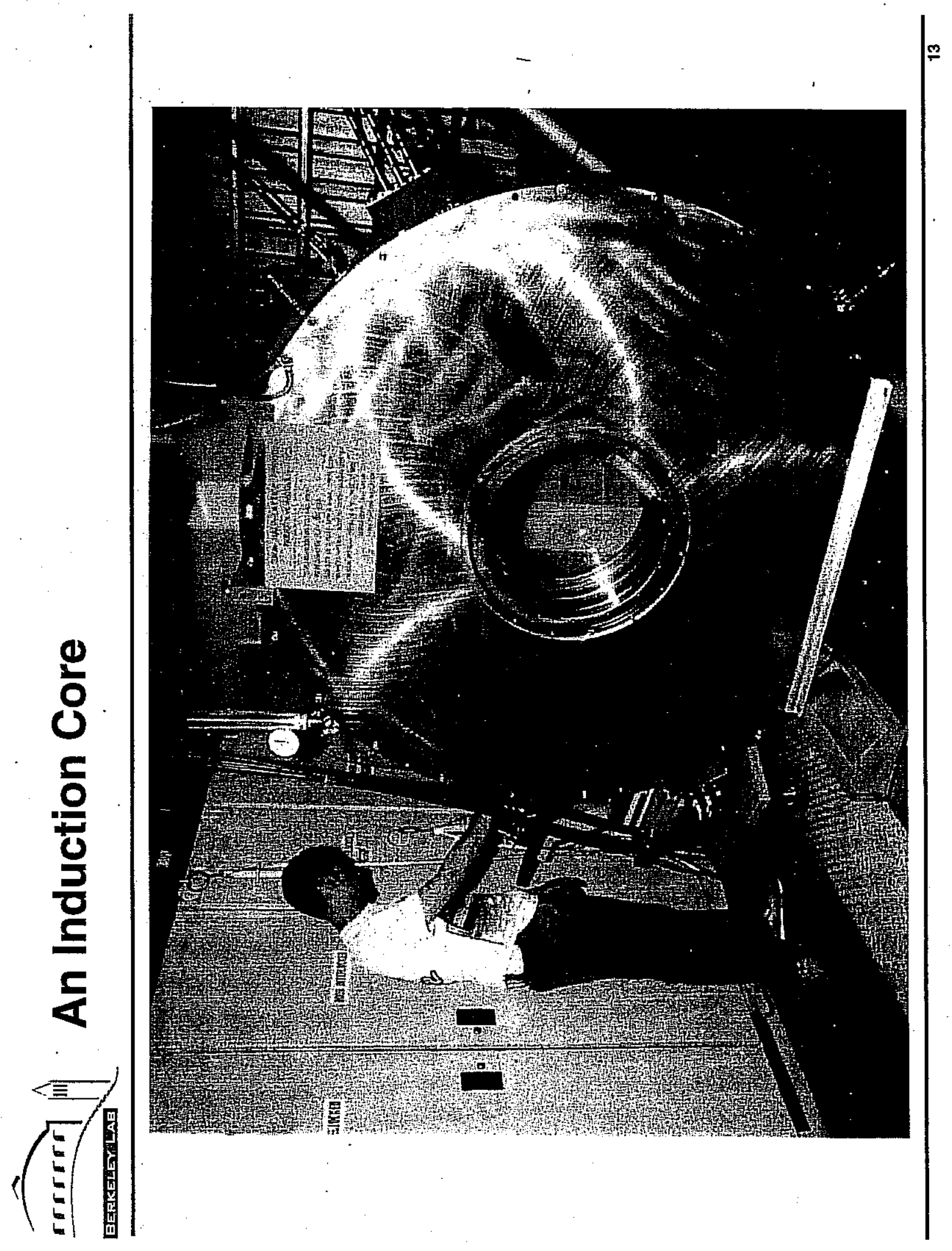




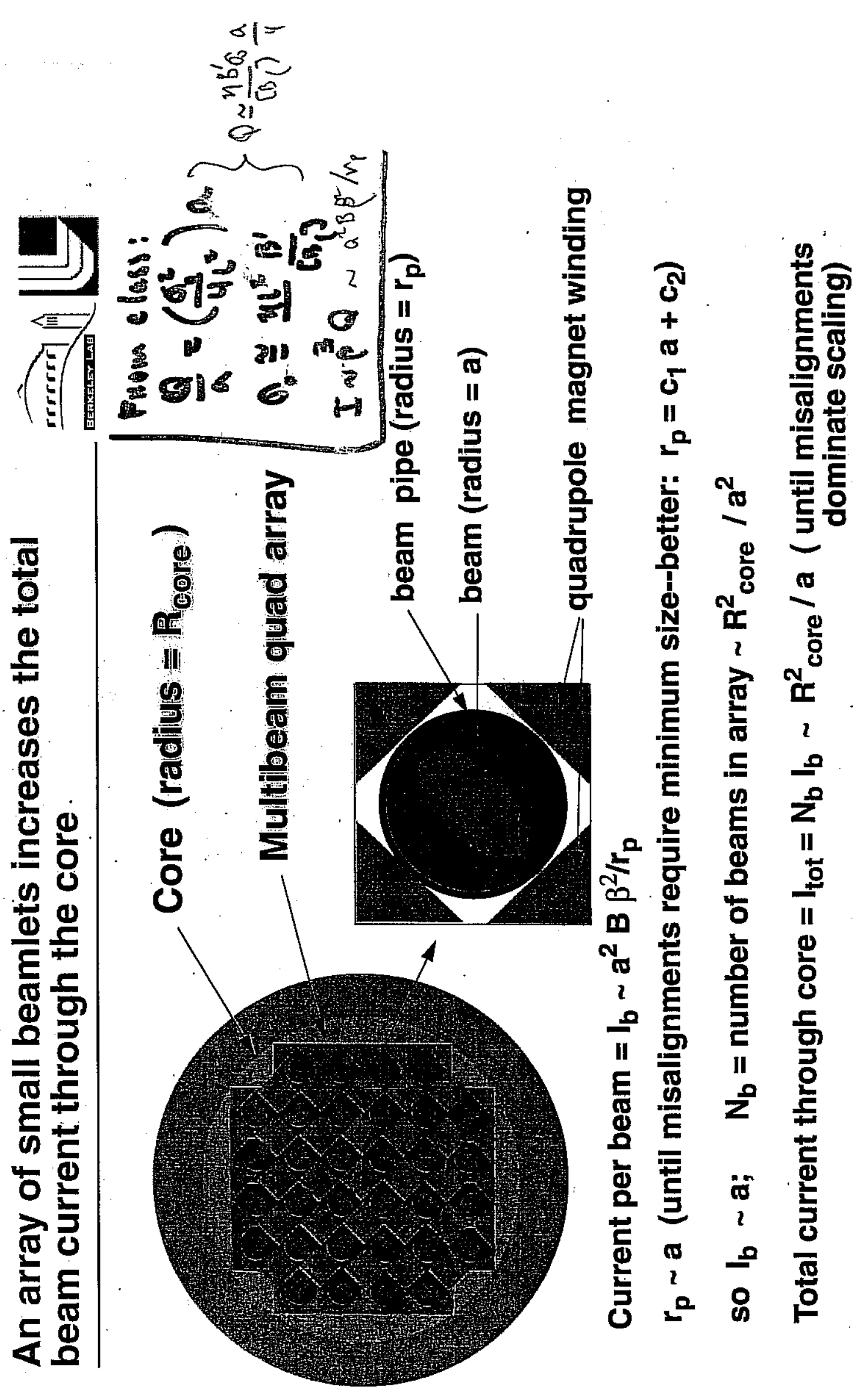




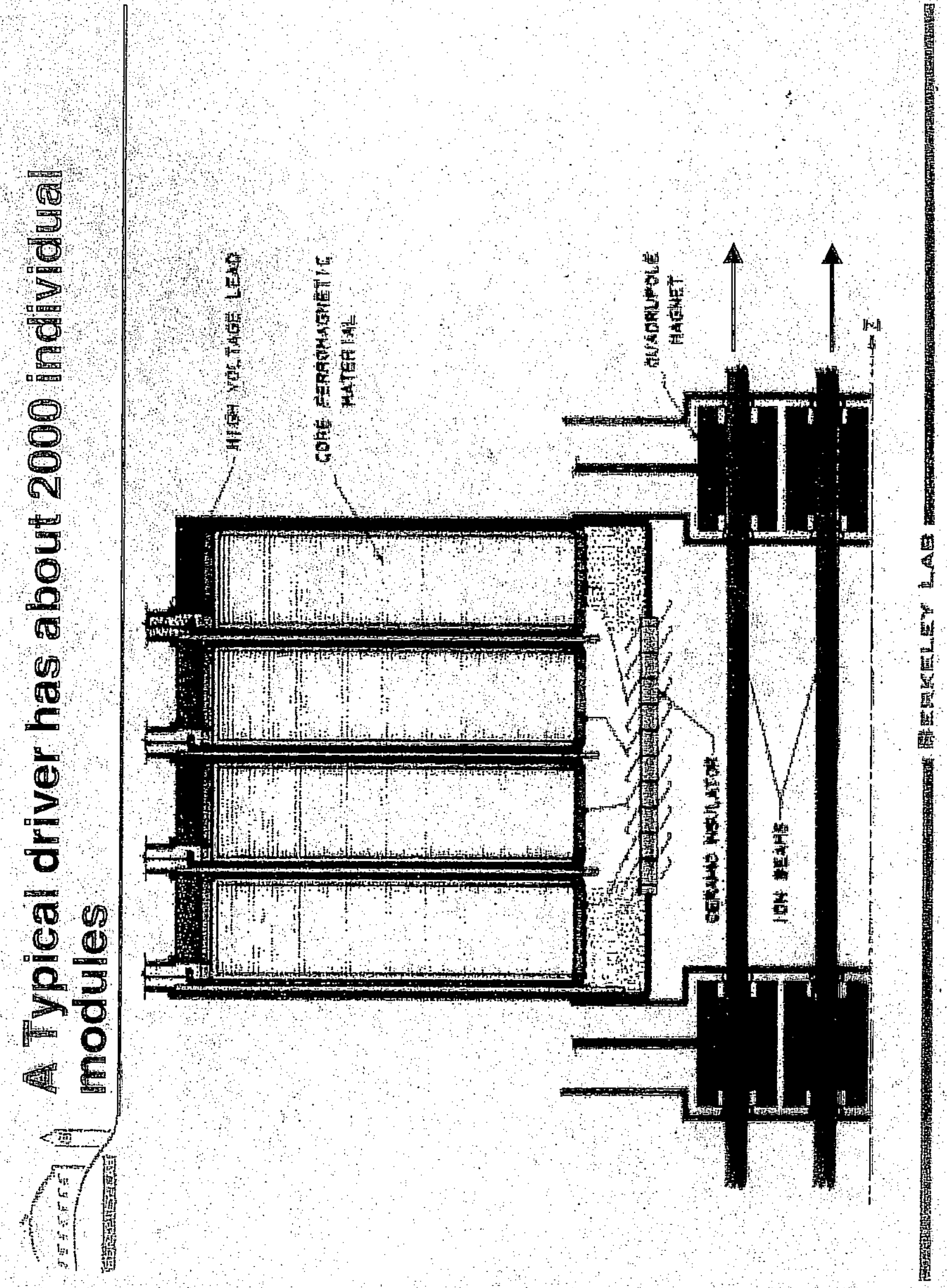


(4)

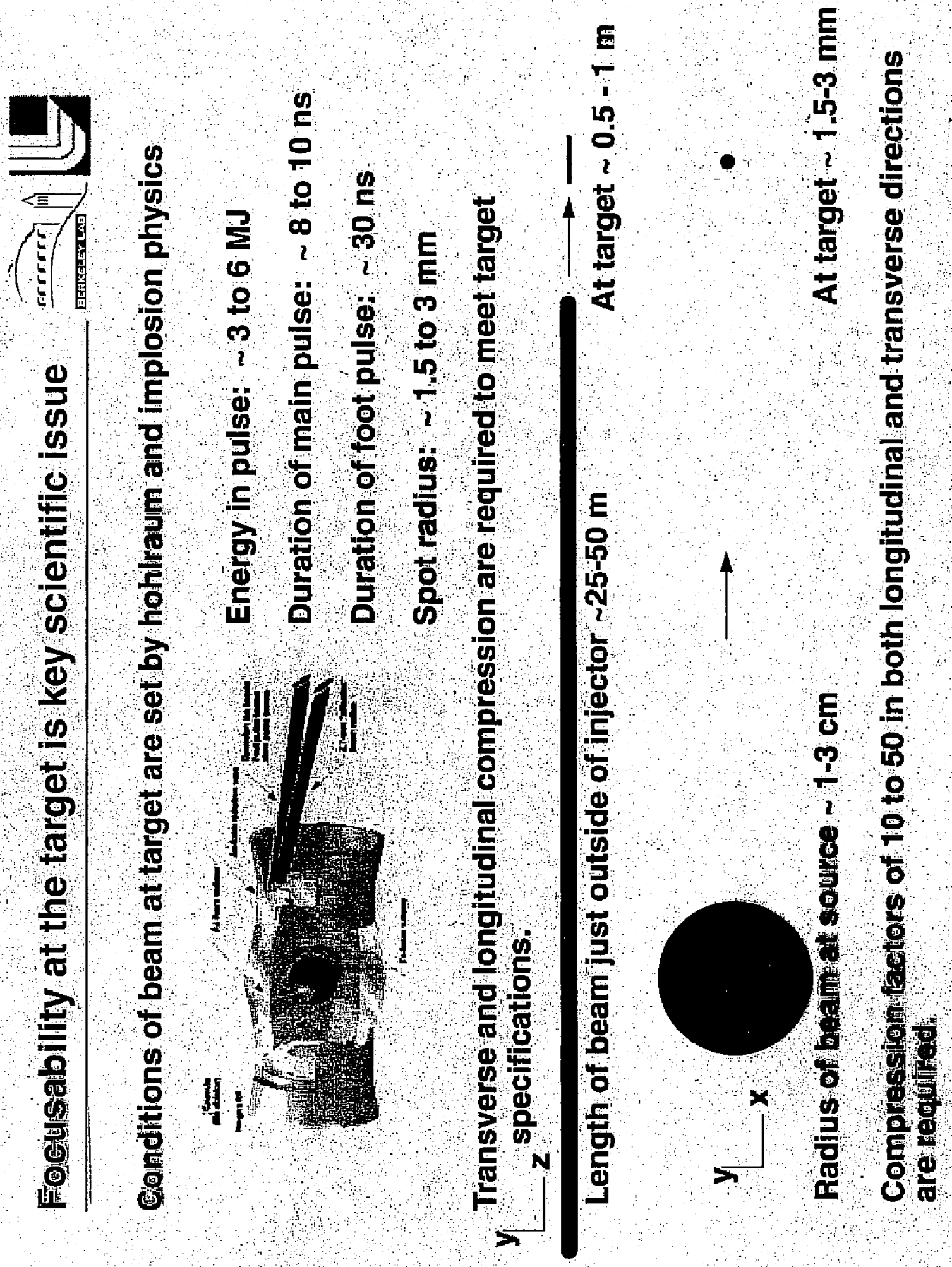




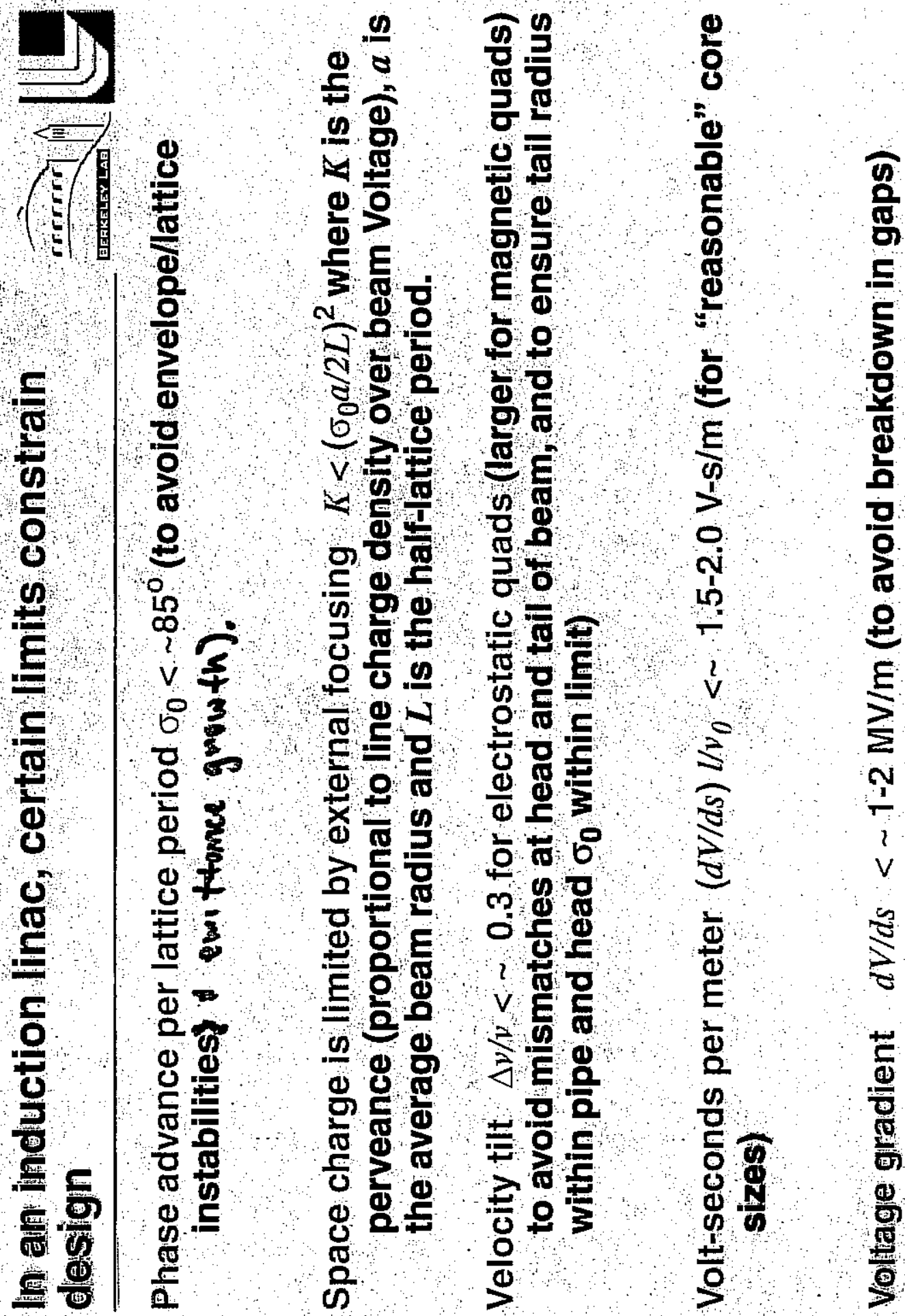



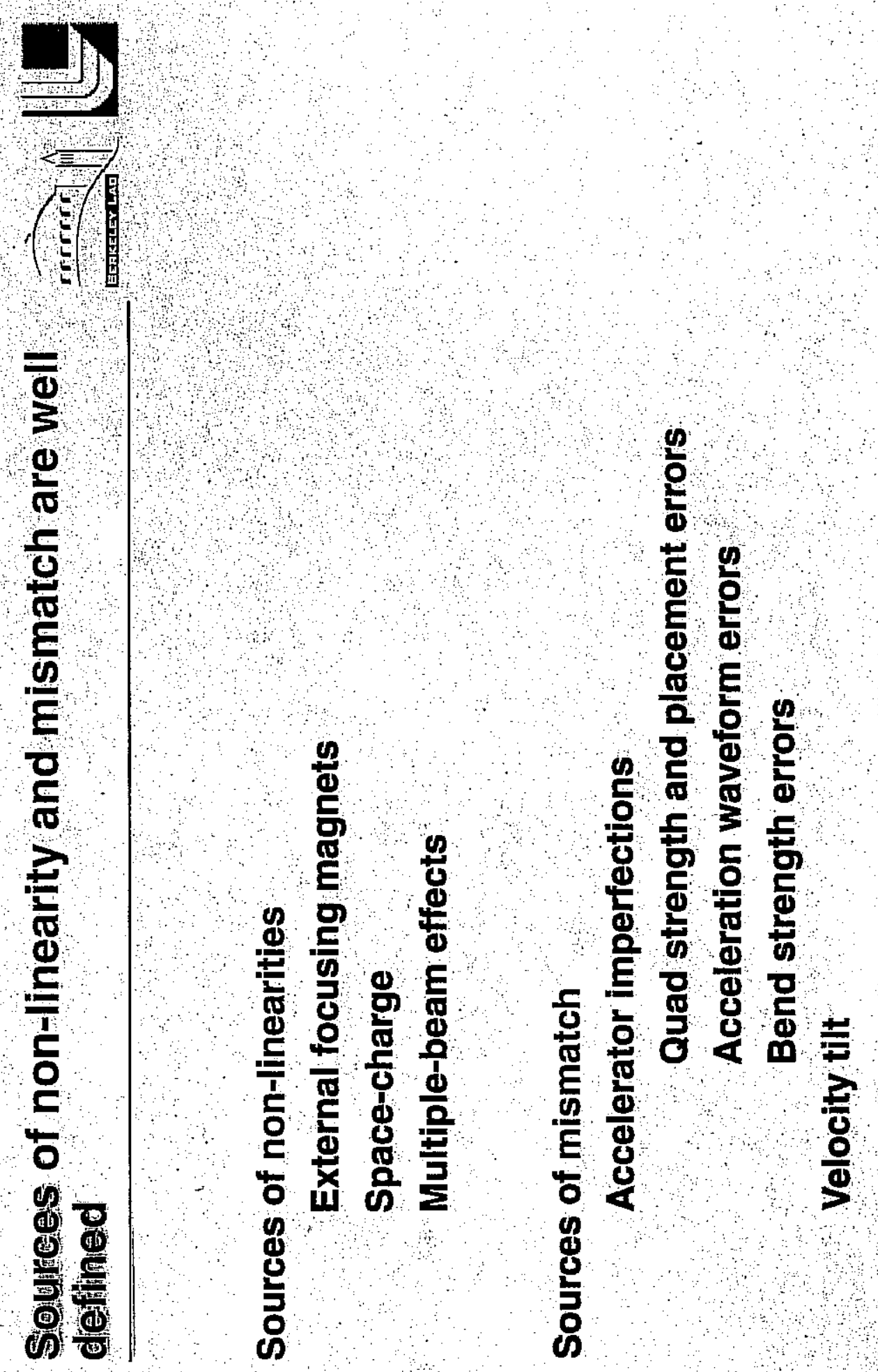

क

E

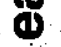

둥

g

$\$$ 


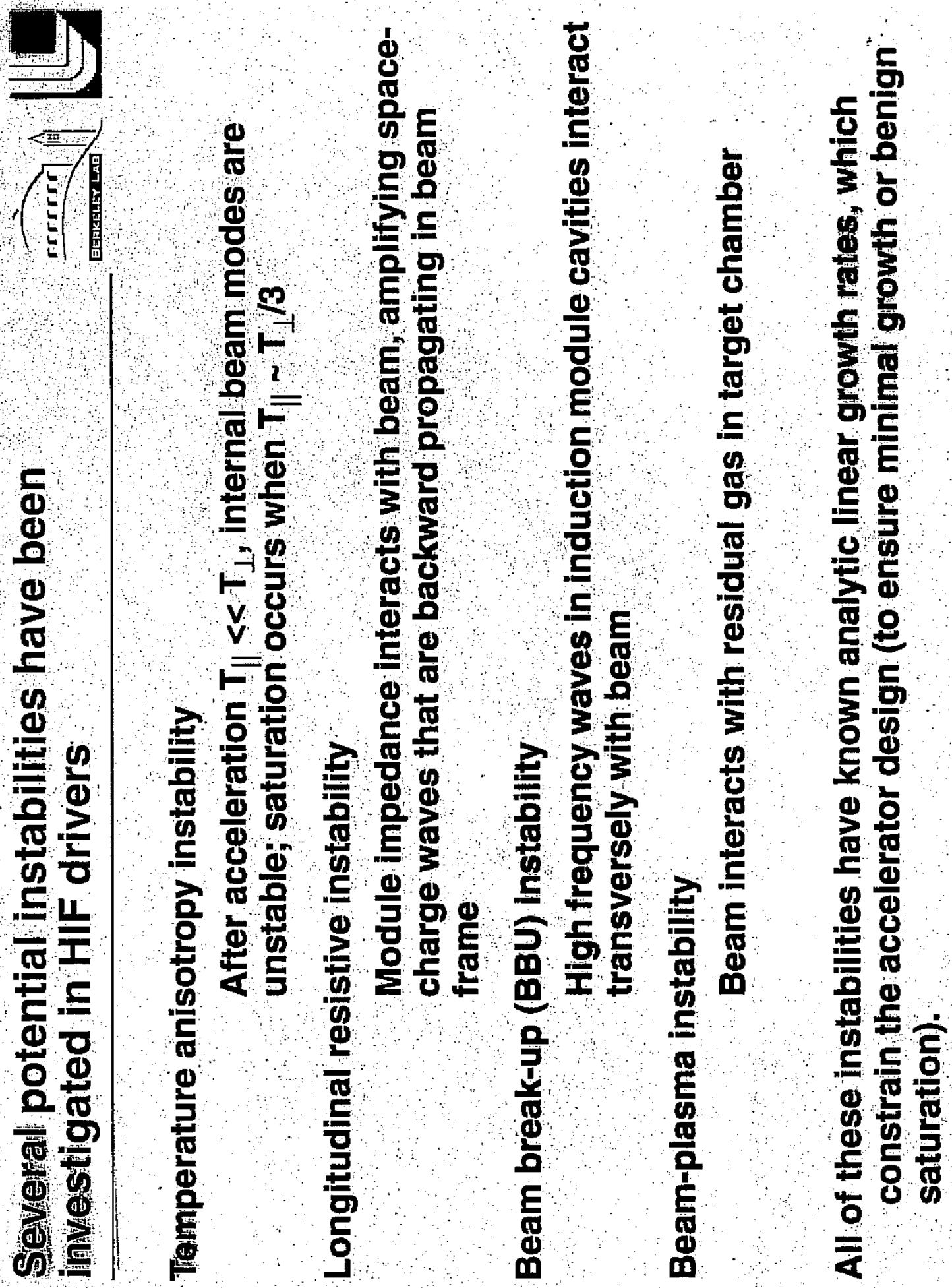




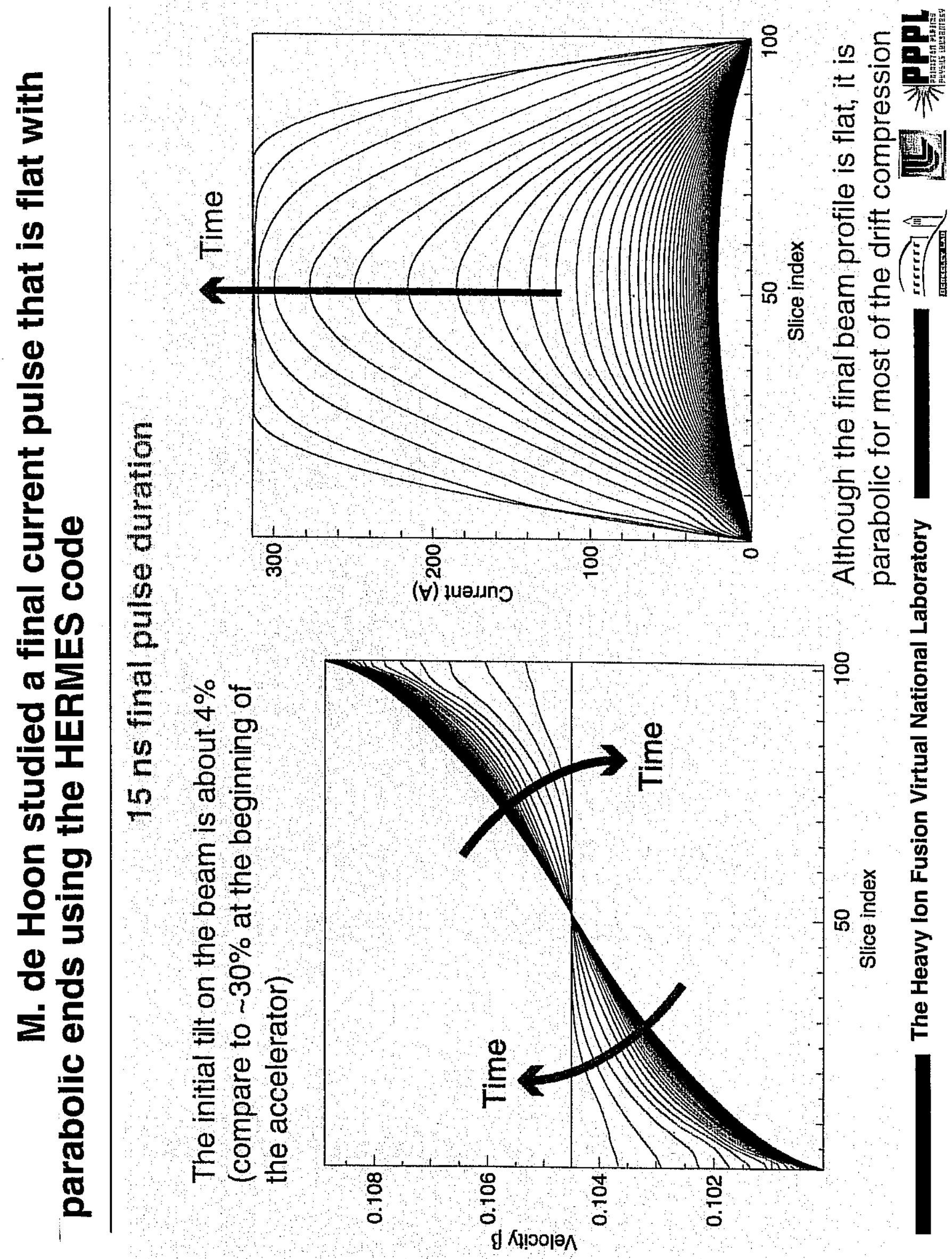




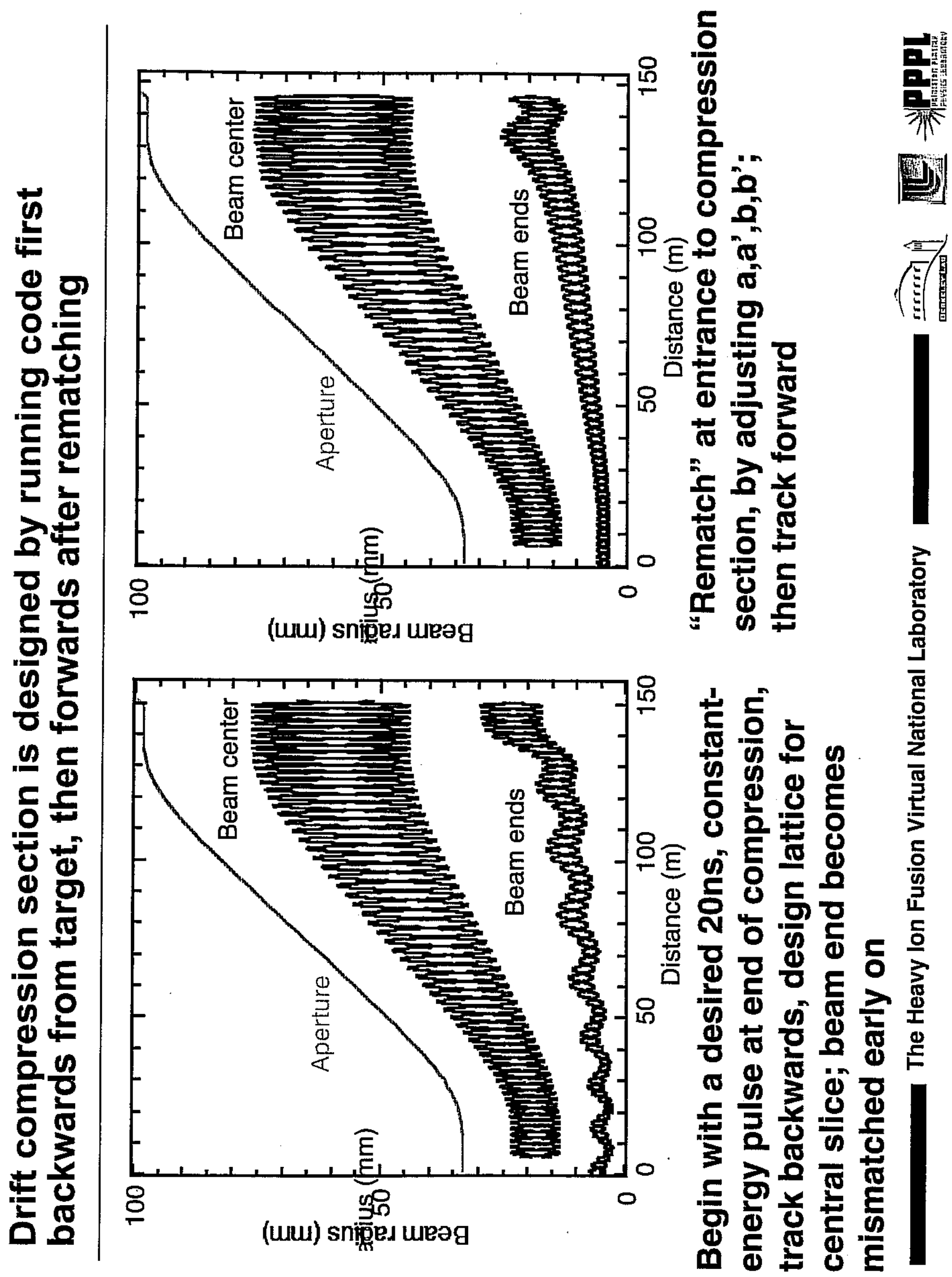


(26)

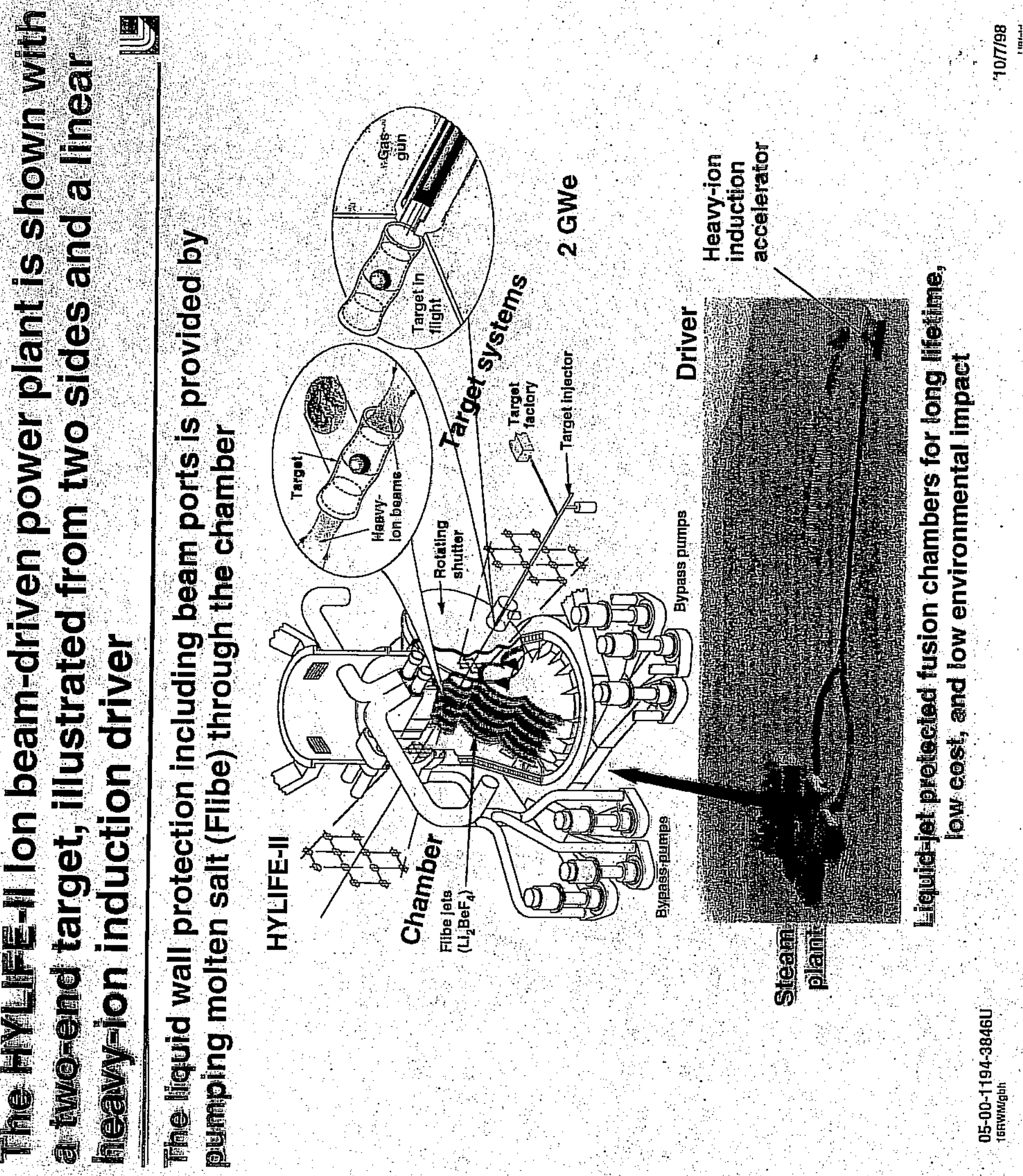



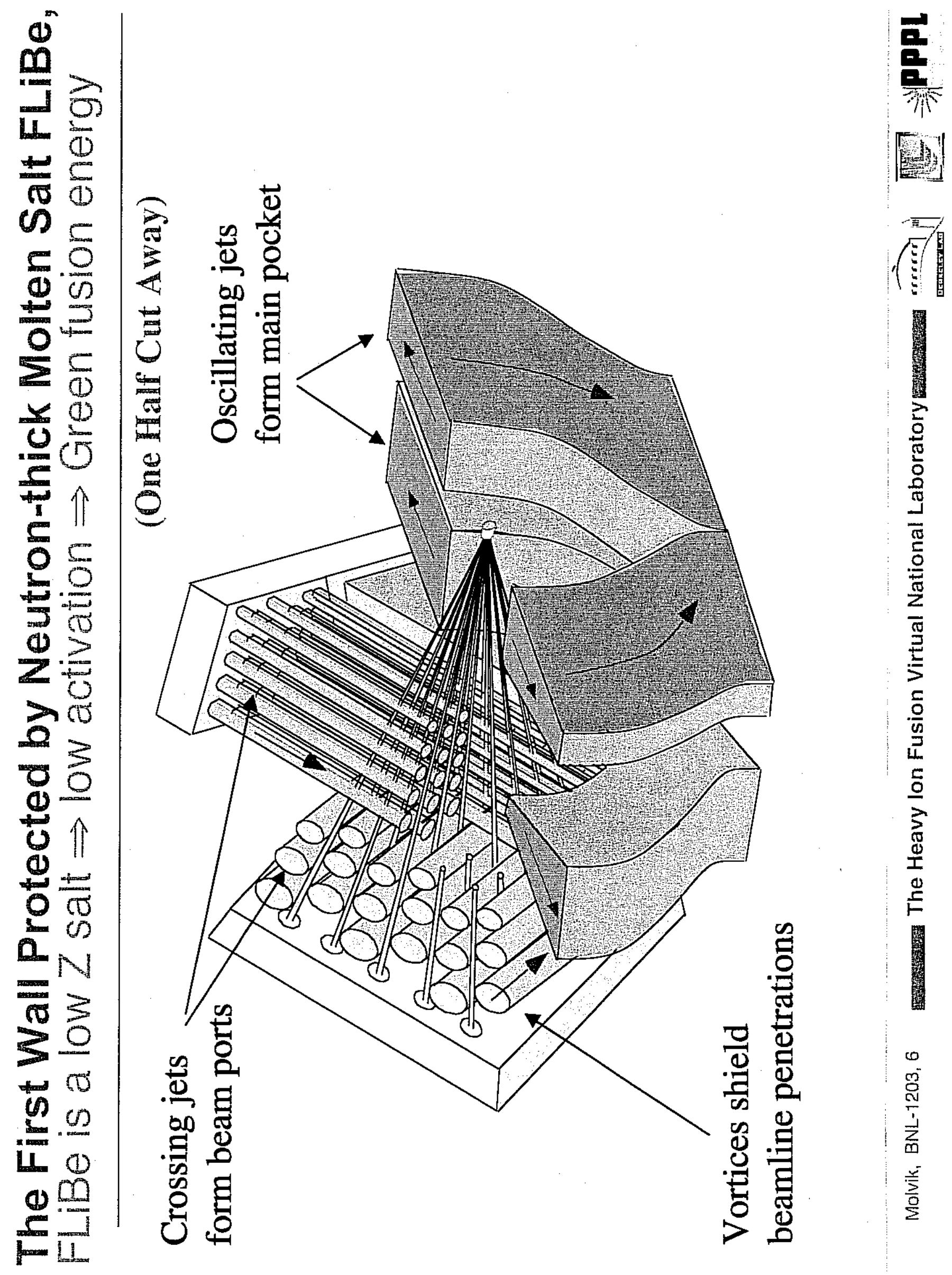


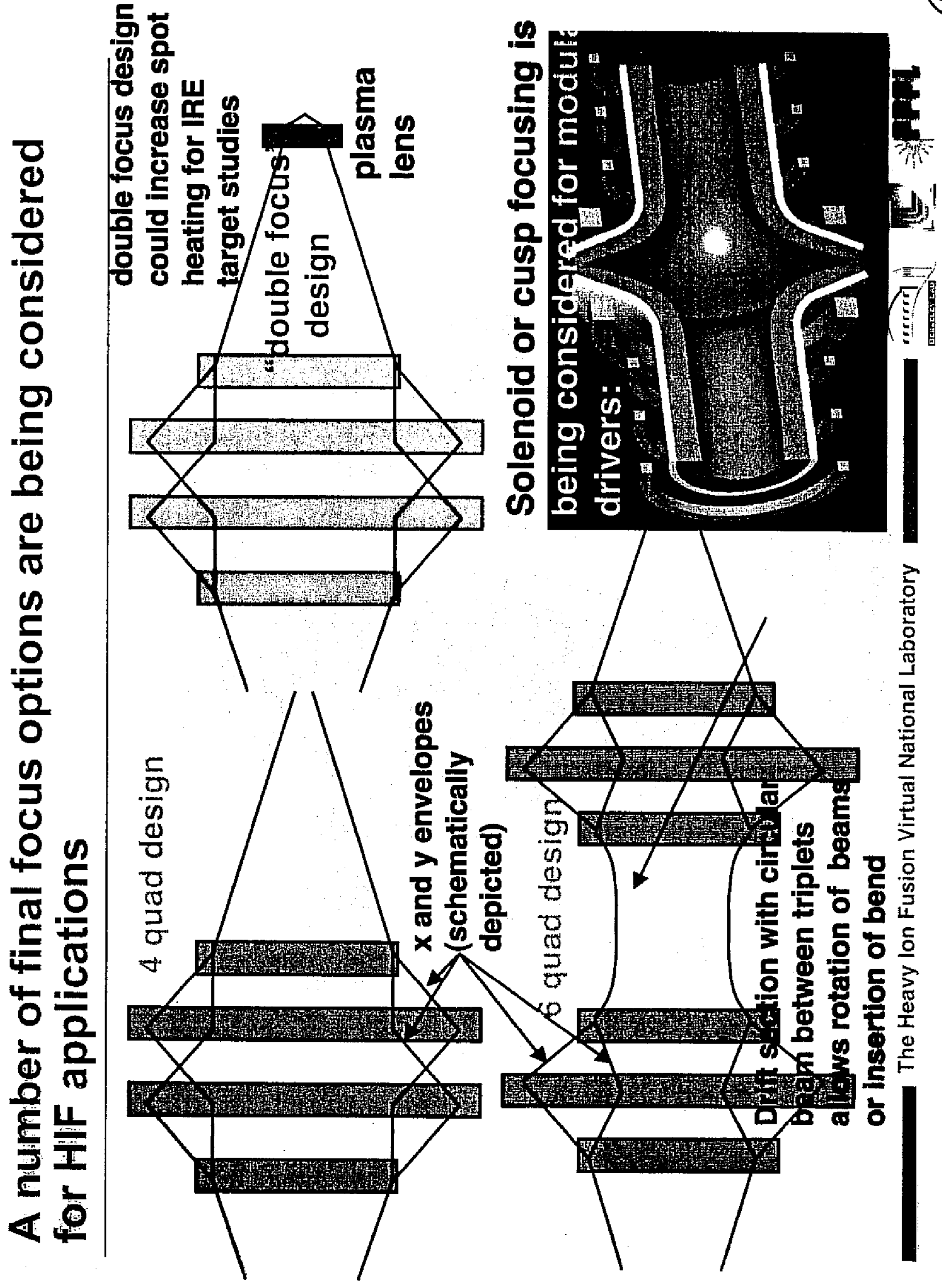


BAANATL

(29)

ESTIMATING SIOT SIZE

$$
\begin{aligned}
& r_{x}^{\prime}+\frac{\left.\left(\gamma_{p}\right)_{y}\right)}{\gamma_{p} r_{x}} r_{x}+k_{x}-\frac{2 Q}{r_{x}+r_{y}}-\frac{\varepsilon_{x}^{2}}{r_{x}^{3}}=0 \\
& r_{y}^{\prime}+\frac{\left(q_{p}\right)}{r_{p} p_{b}} r_{y}+k_{y} r_{y}-\frac{2 Q}{r_{x}+r_{y}}-\frac{\varepsilon_{y}^{2}}{r_{y}^{3}}=0
\end{aligned}
$$

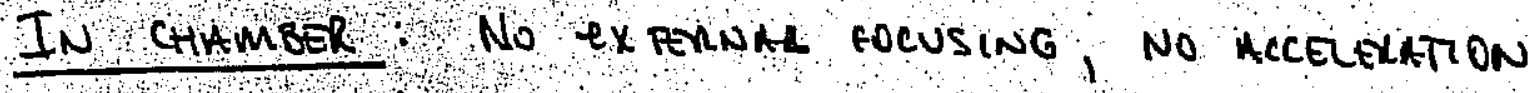
NOO BEAW IS OFTEN CINCULAR (BY DESION)

$$
\rightarrow k_{x}=k_{y}=\left(v_{k}\left(b_{b}\right)=0 \quad \& v_{x}=v_{y}=n_{b}\right.
$$

$\Rightarrow$ ENUTOHE EQUATON IS:

$$
r_{b}^{\prime}=\frac{Q}{r_{b}}+\frac{\epsilon^{2}}{r_{b}^{3}}
$$

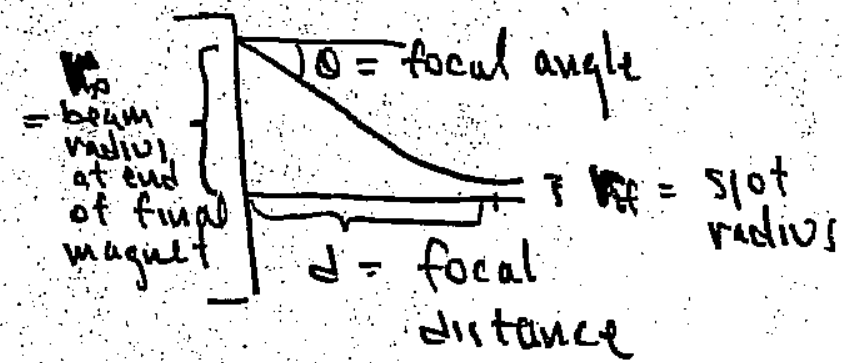

MULRLYING BY $V_{b}^{\prime}$ I INTERATNG $\Rightarrow$

$$
\frac{r_{b}^{1}}{2}-\frac{r_{b 0}^{2}}{2}=Q \ln \frac{r_{b f}}{r_{b_{0}}}+\frac{\varepsilon^{2}}{2 r_{b_{0}}^{2}}-\frac{\varepsilon^{2}}{2 r_{b f}^{2}}
$$

Now $r_{i \theta}=\theta$

$r_{\text {bf }}=$ spot vadius

$r_{b f}^{\prime}=0 \quad r_{b o} \bumpeq d \theta$ $r_{b f} \ll r_{b 0}$

$$
\Rightarrow \theta^{2} \cong 2 Q \ln \left(\frac{\theta L}{r_{L f}}\right)+\frac{\epsilon^{2}}{r_{b f}^{2}}
$$

FOR EMITHANC DOMINATED SPOT: $\quad r_{b f}=\frac{\epsilon}{\theta}$ 


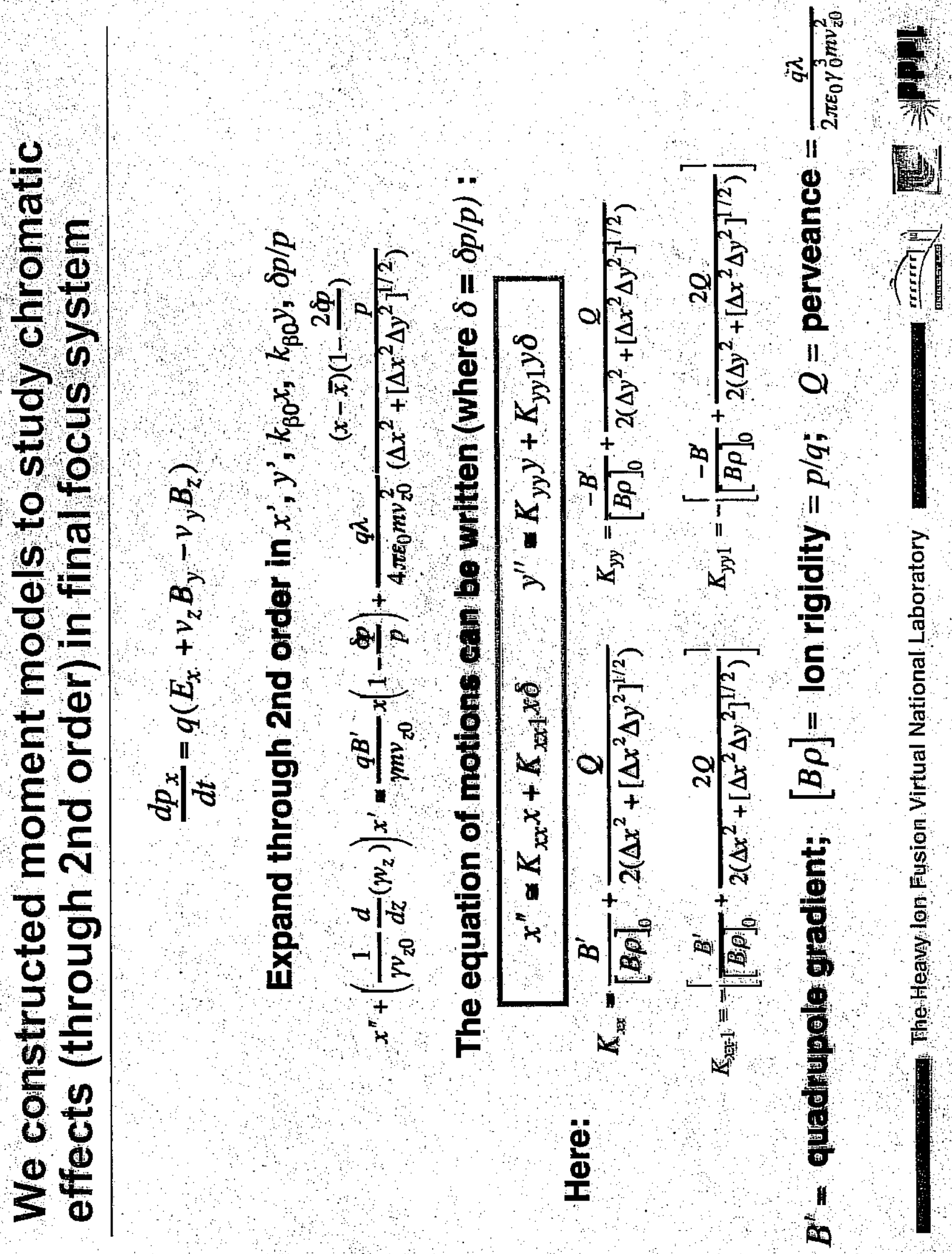




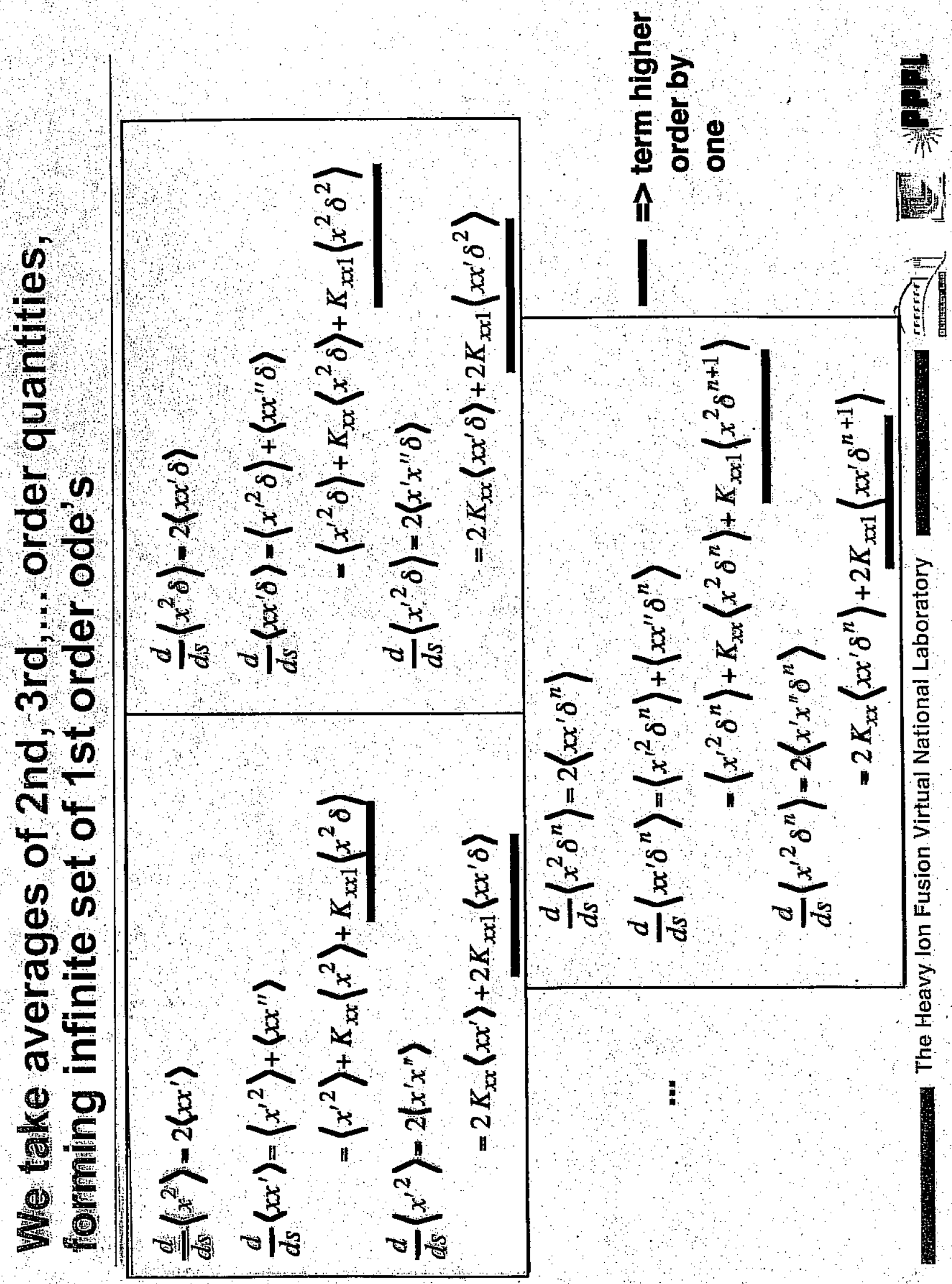




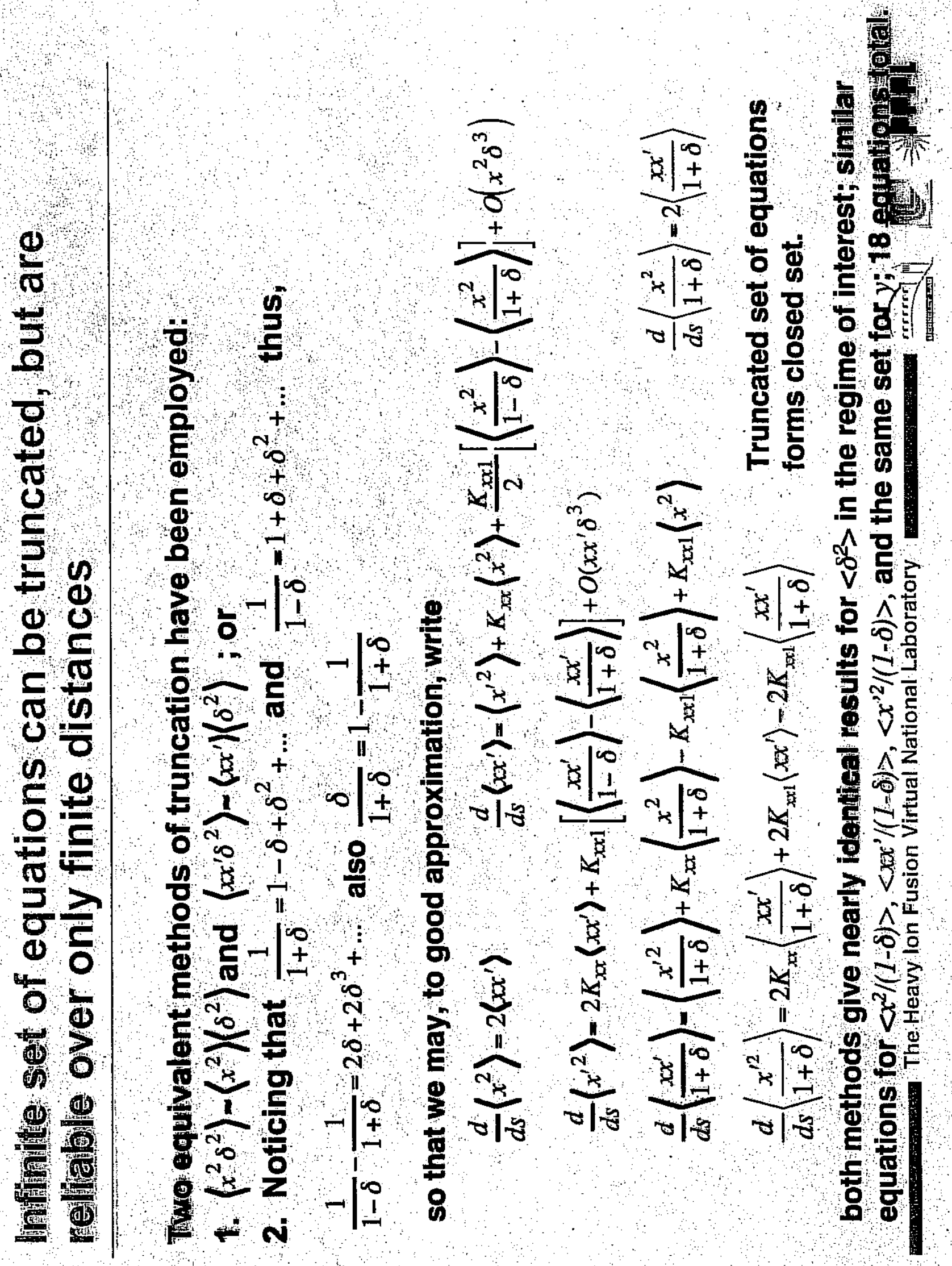


(39)

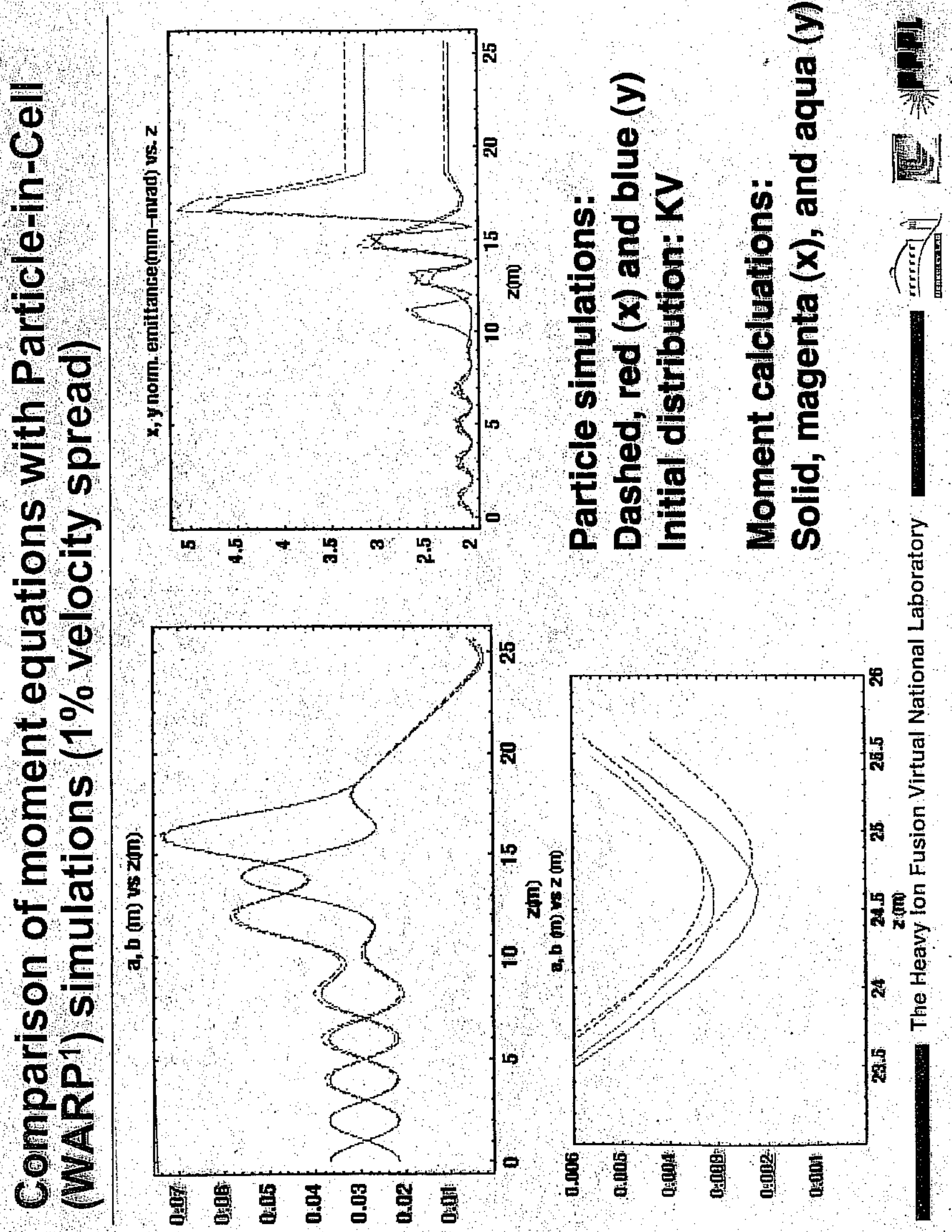


(3)

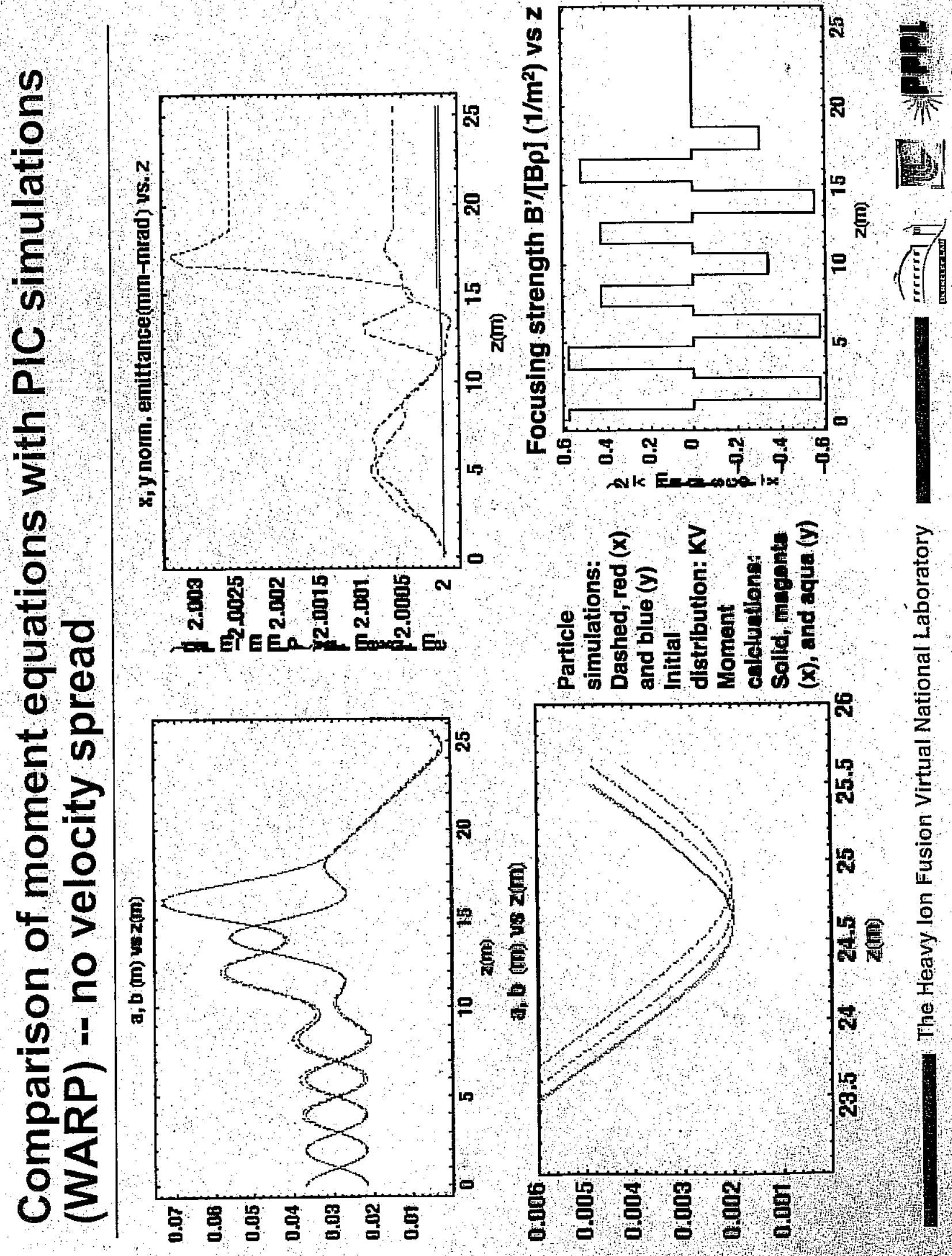




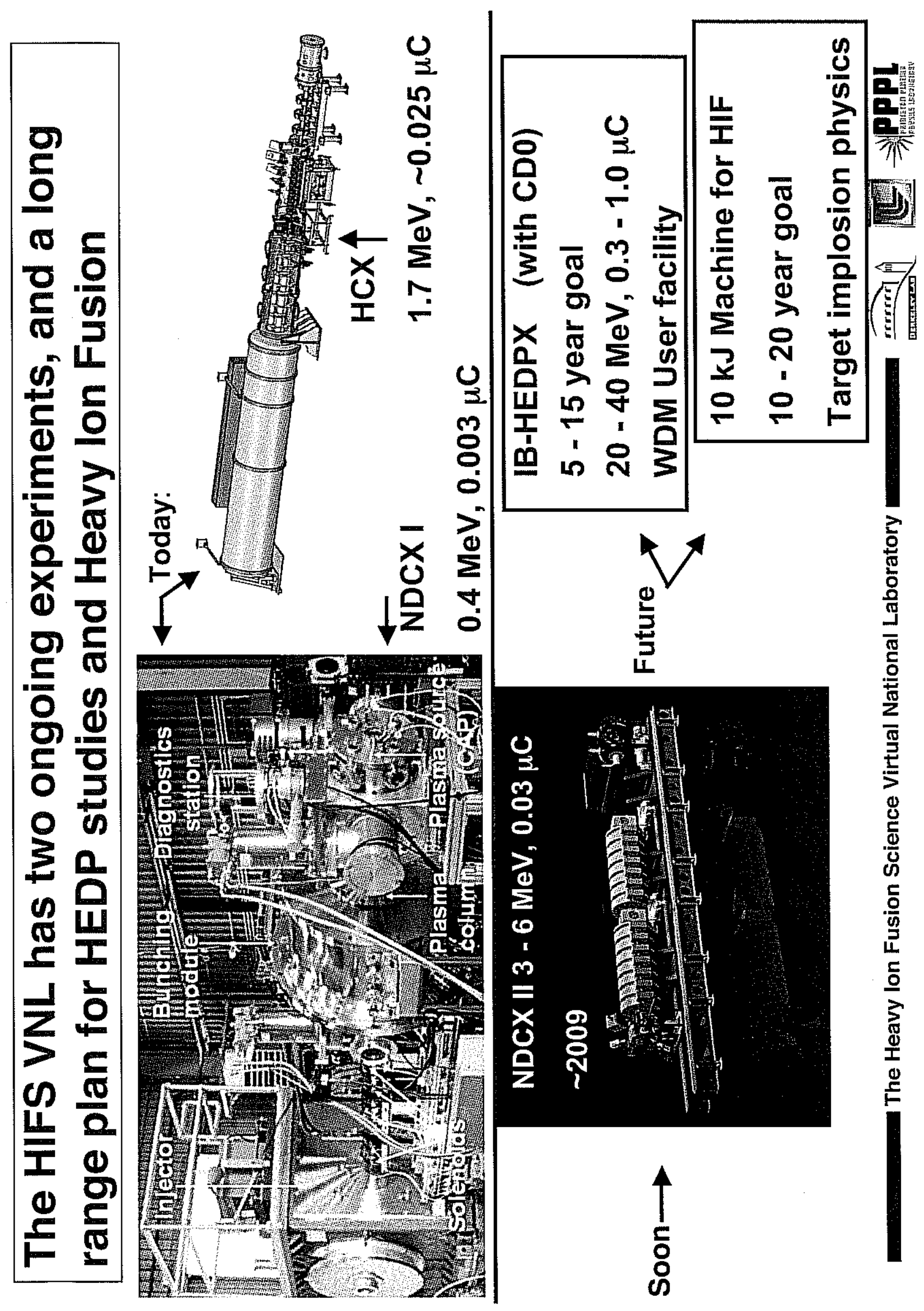




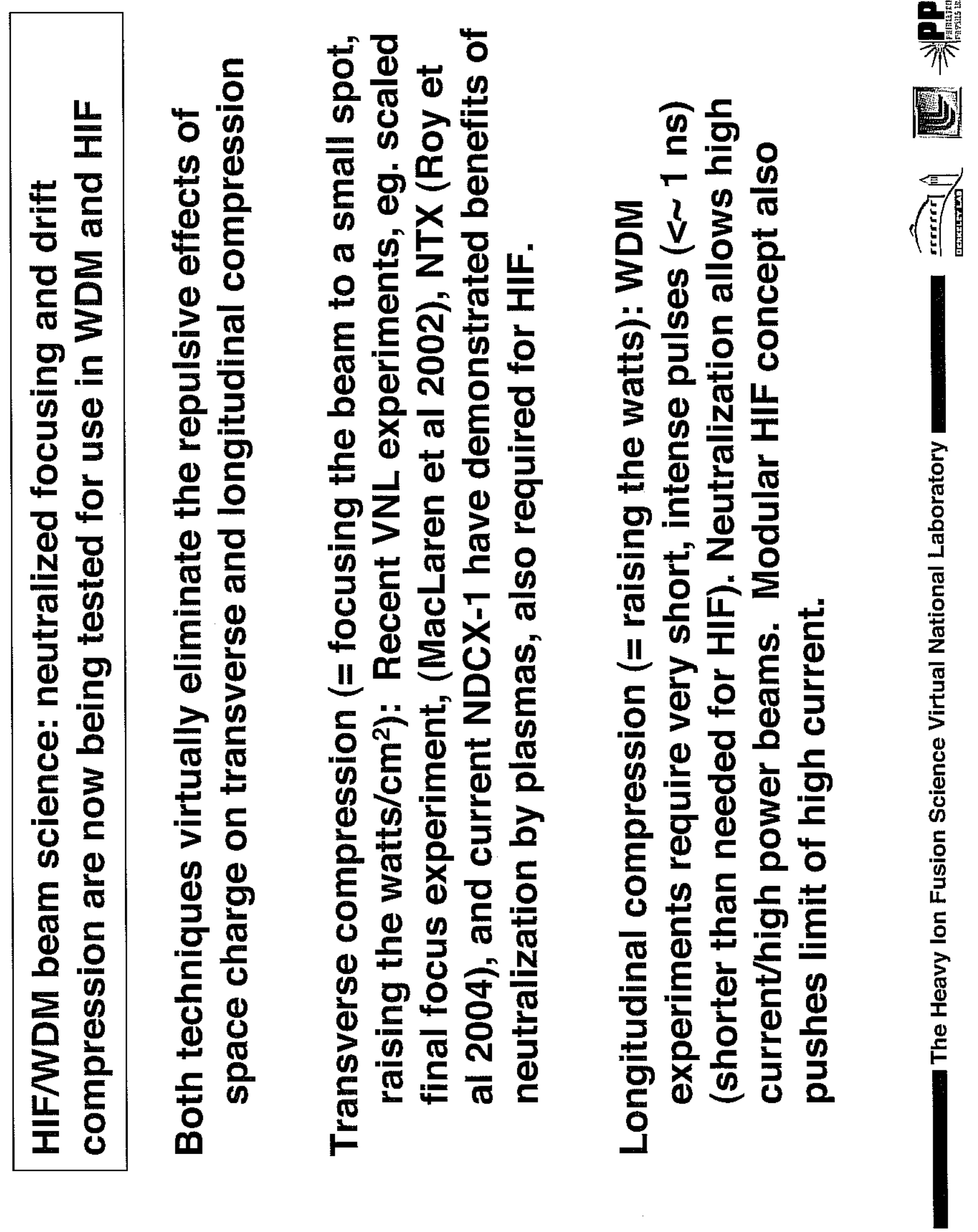




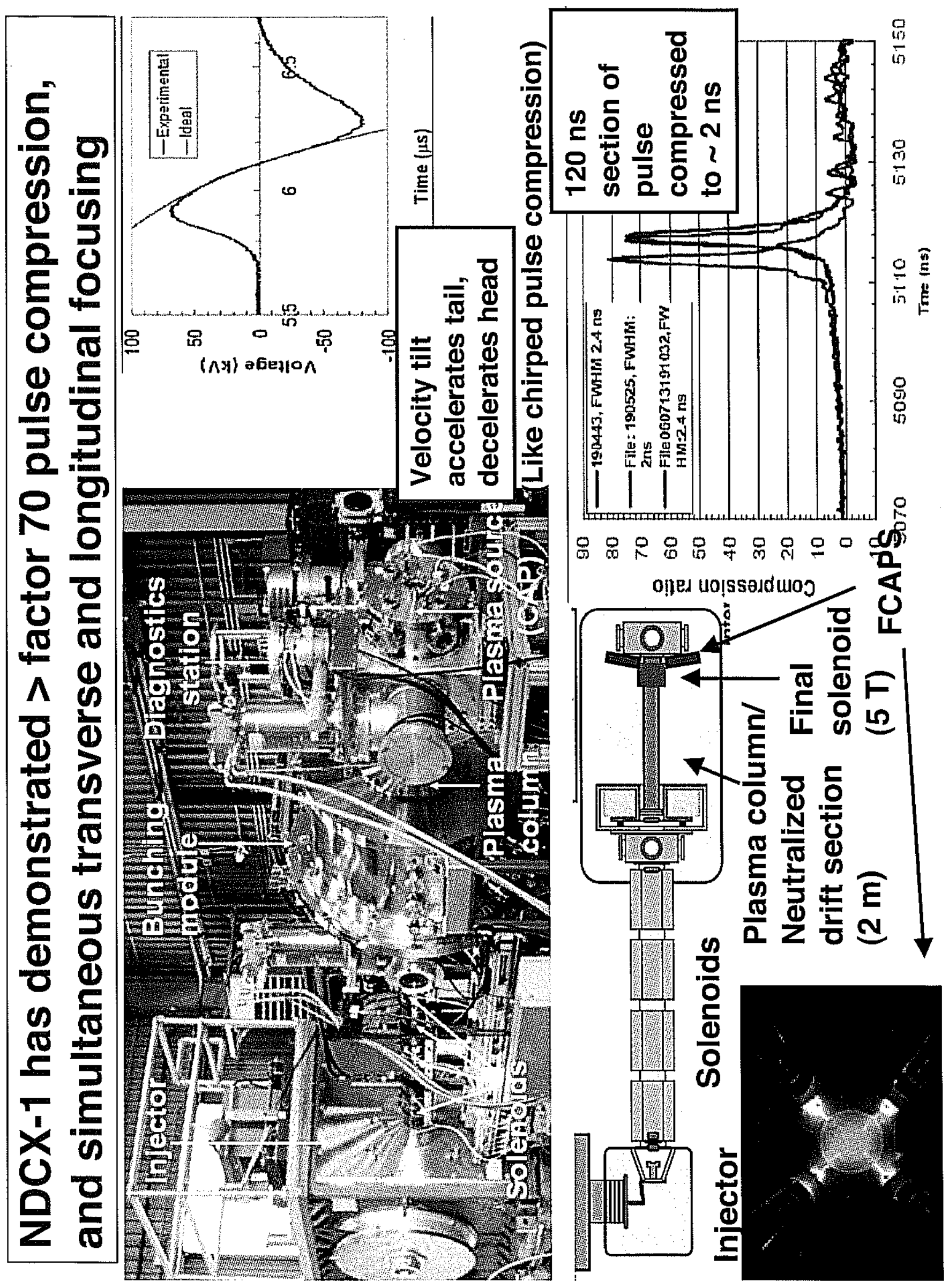




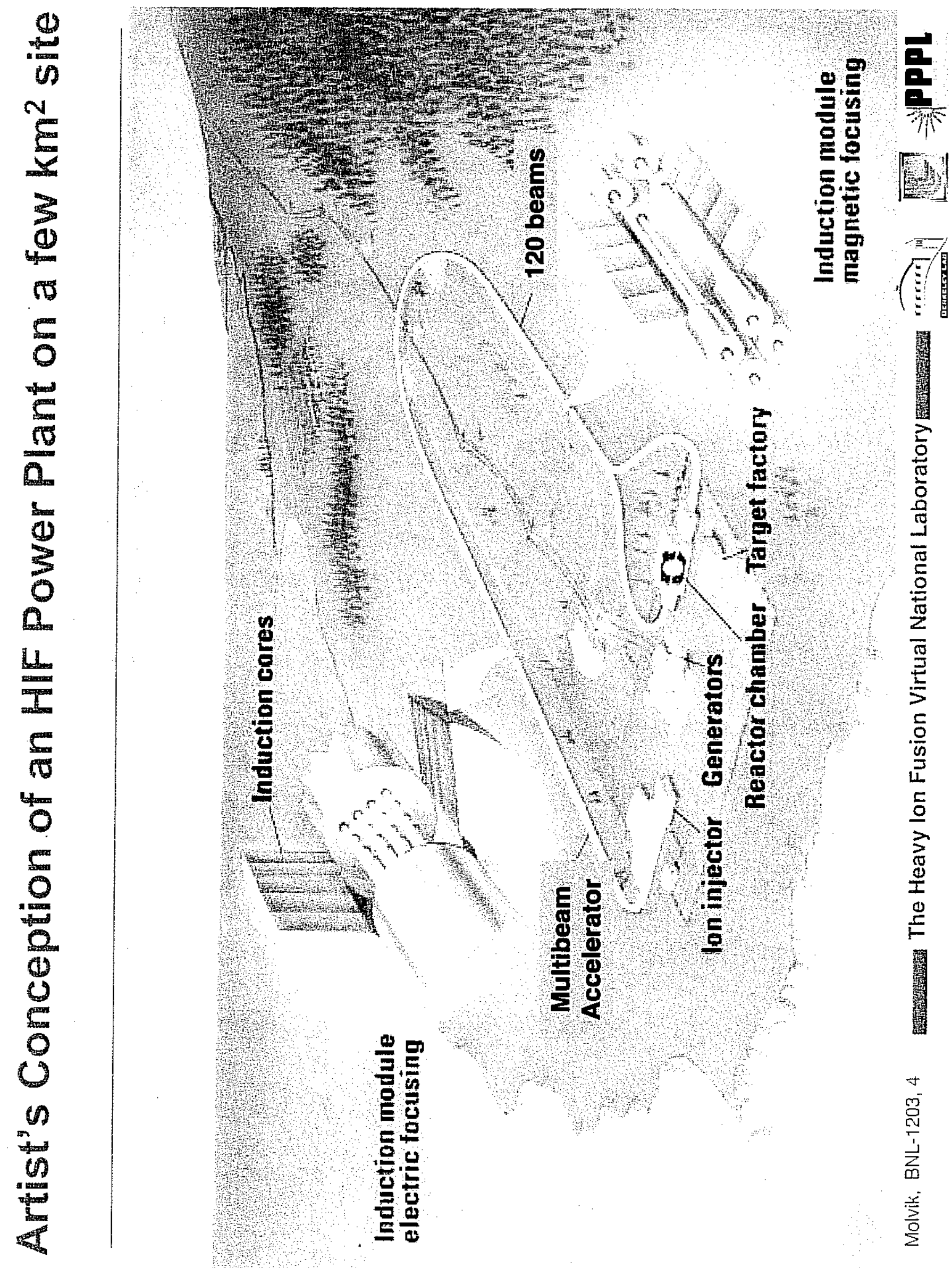




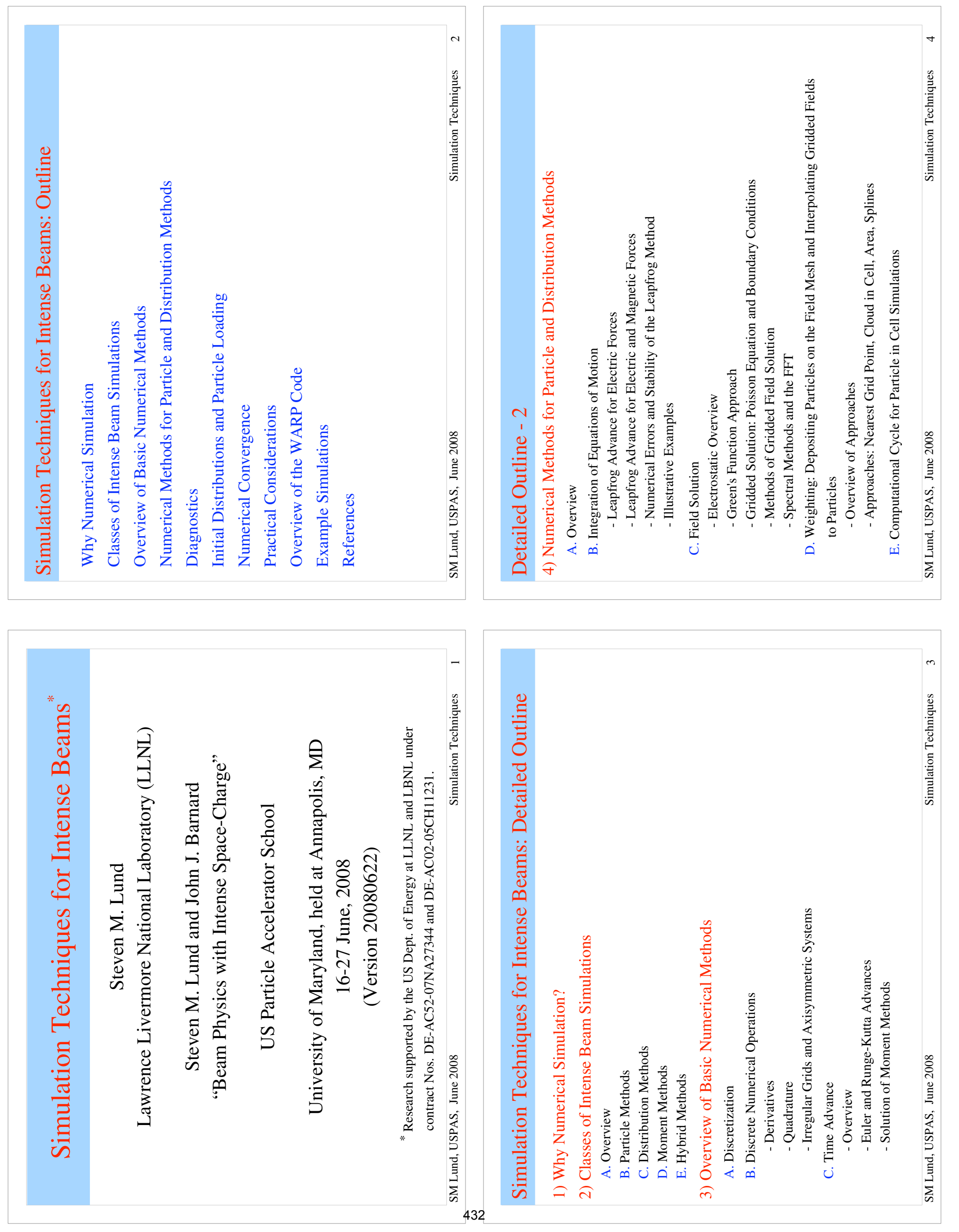




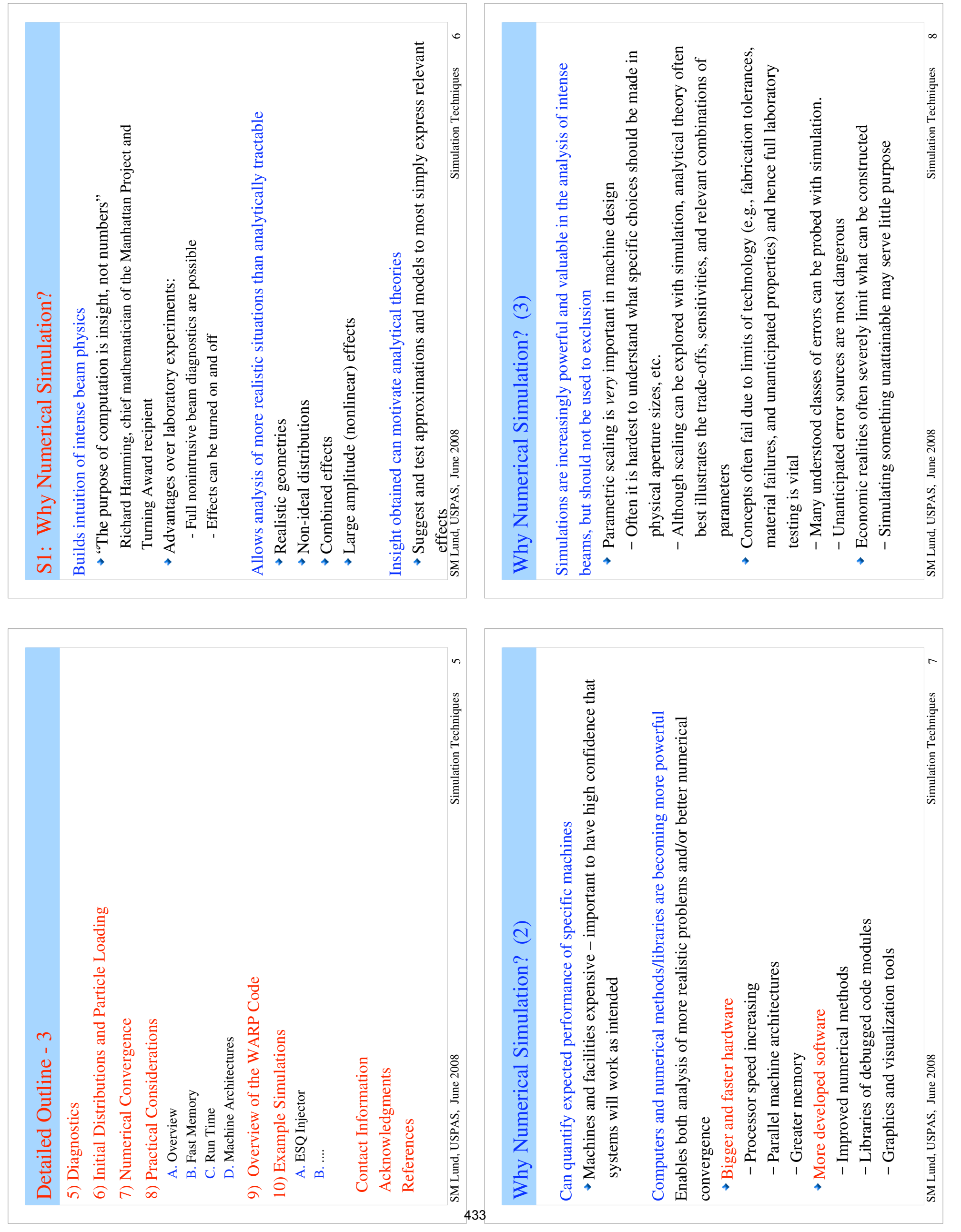



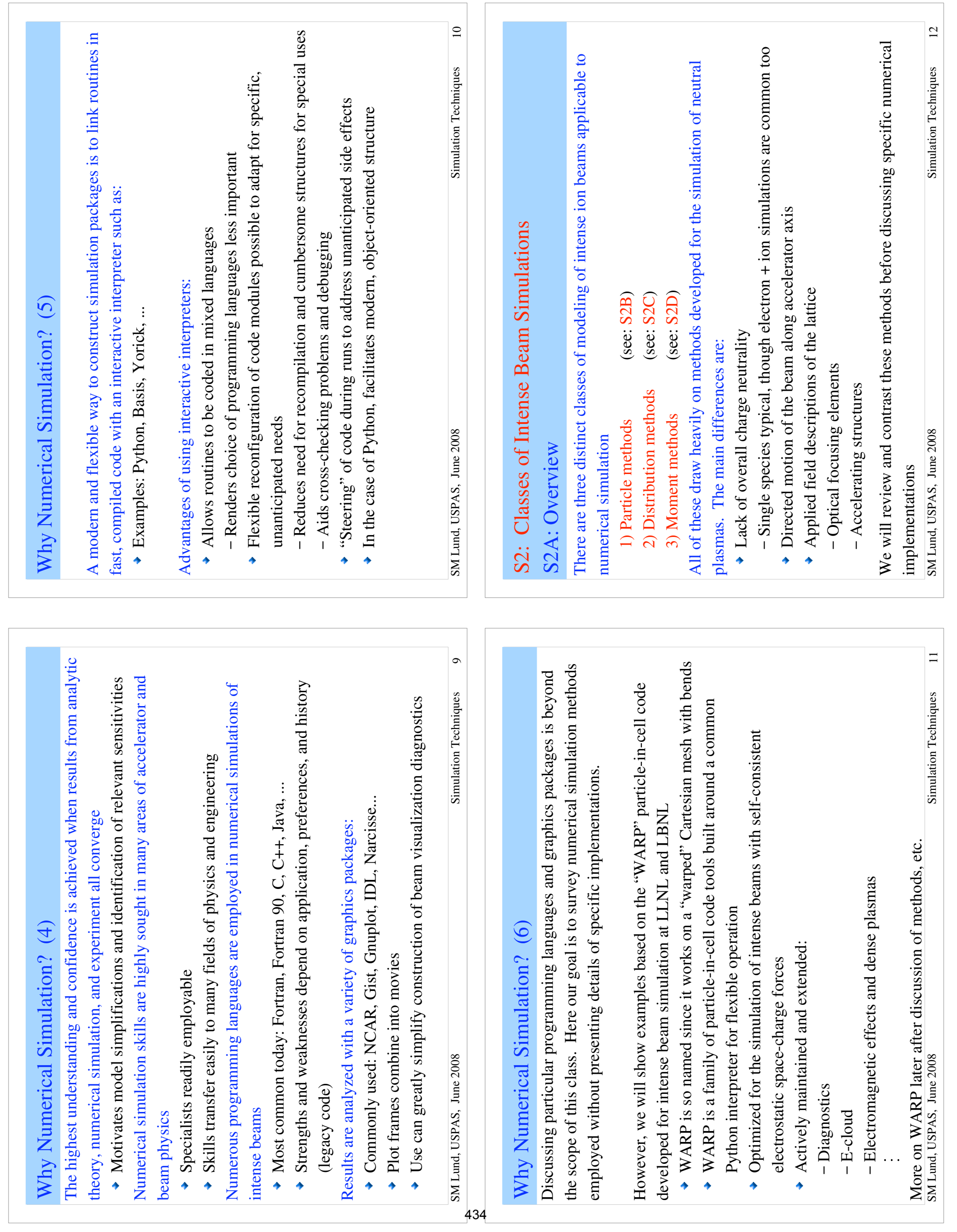

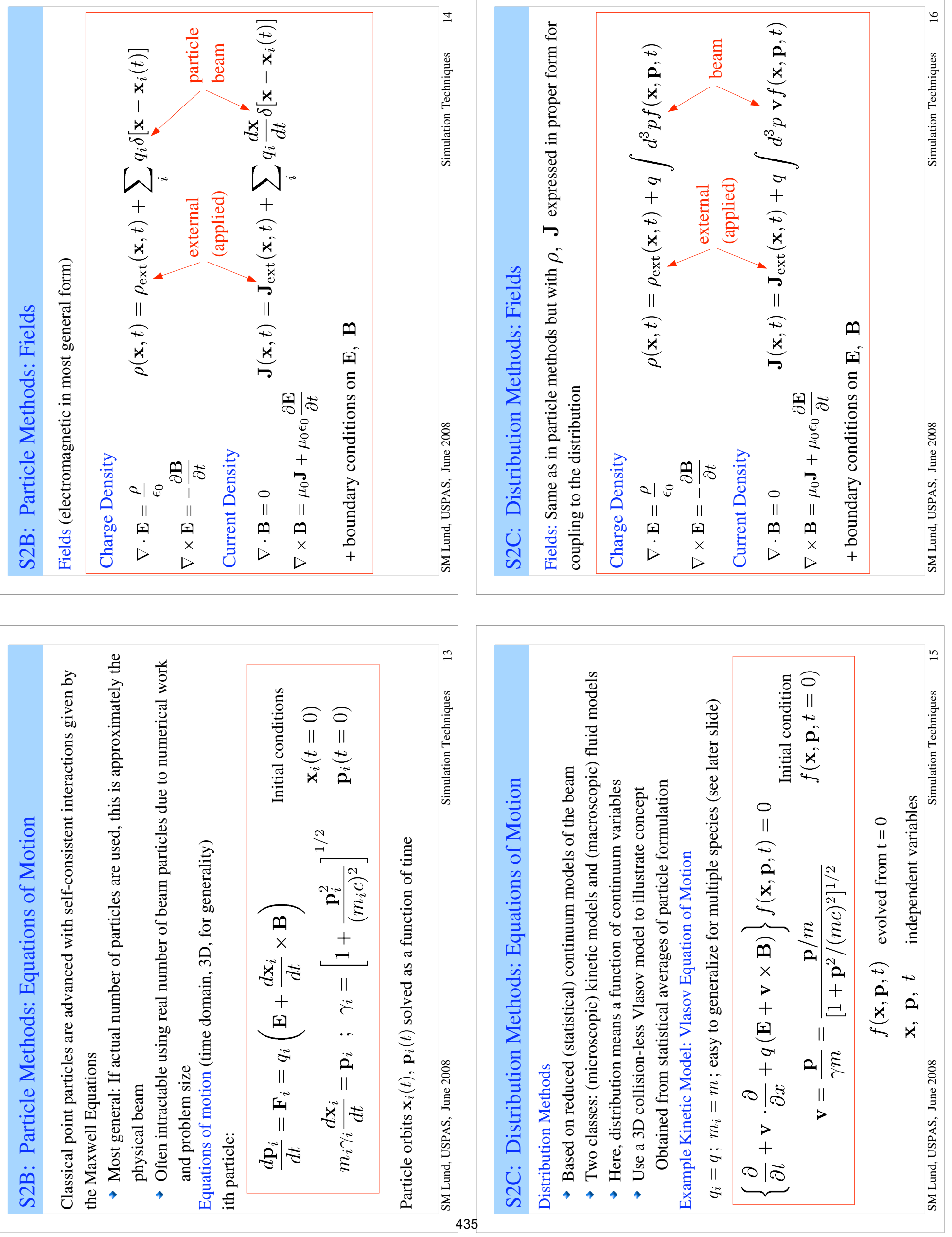

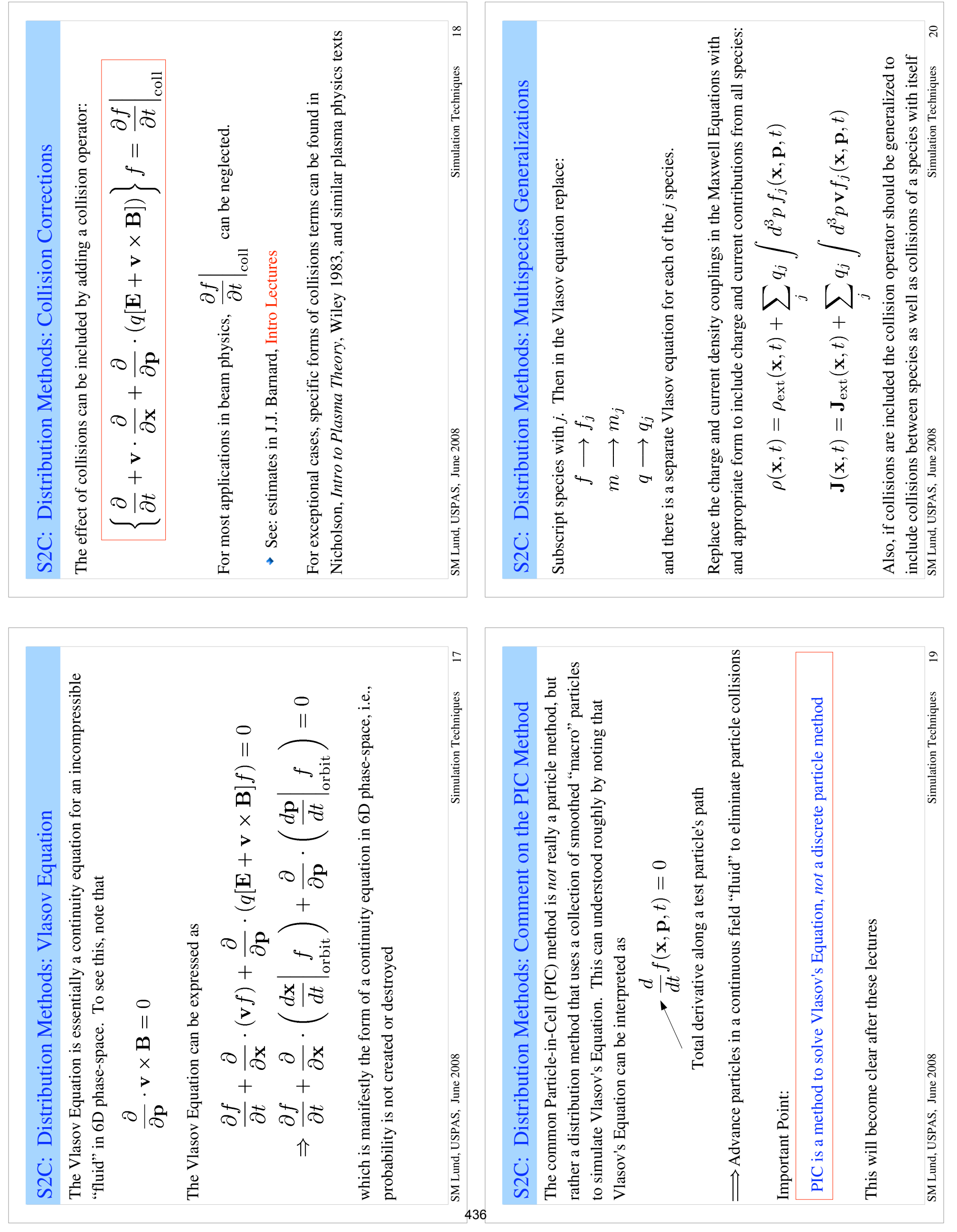

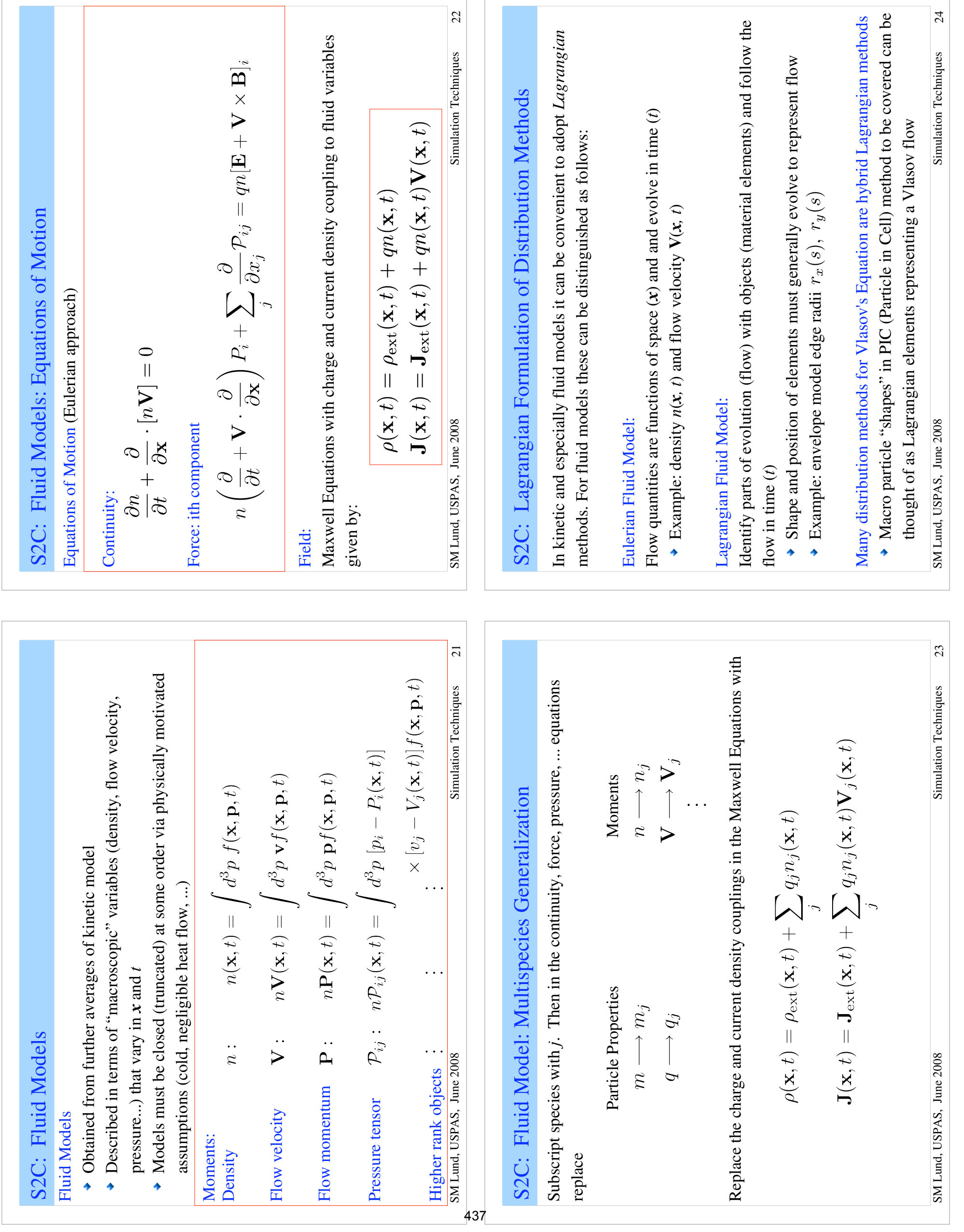


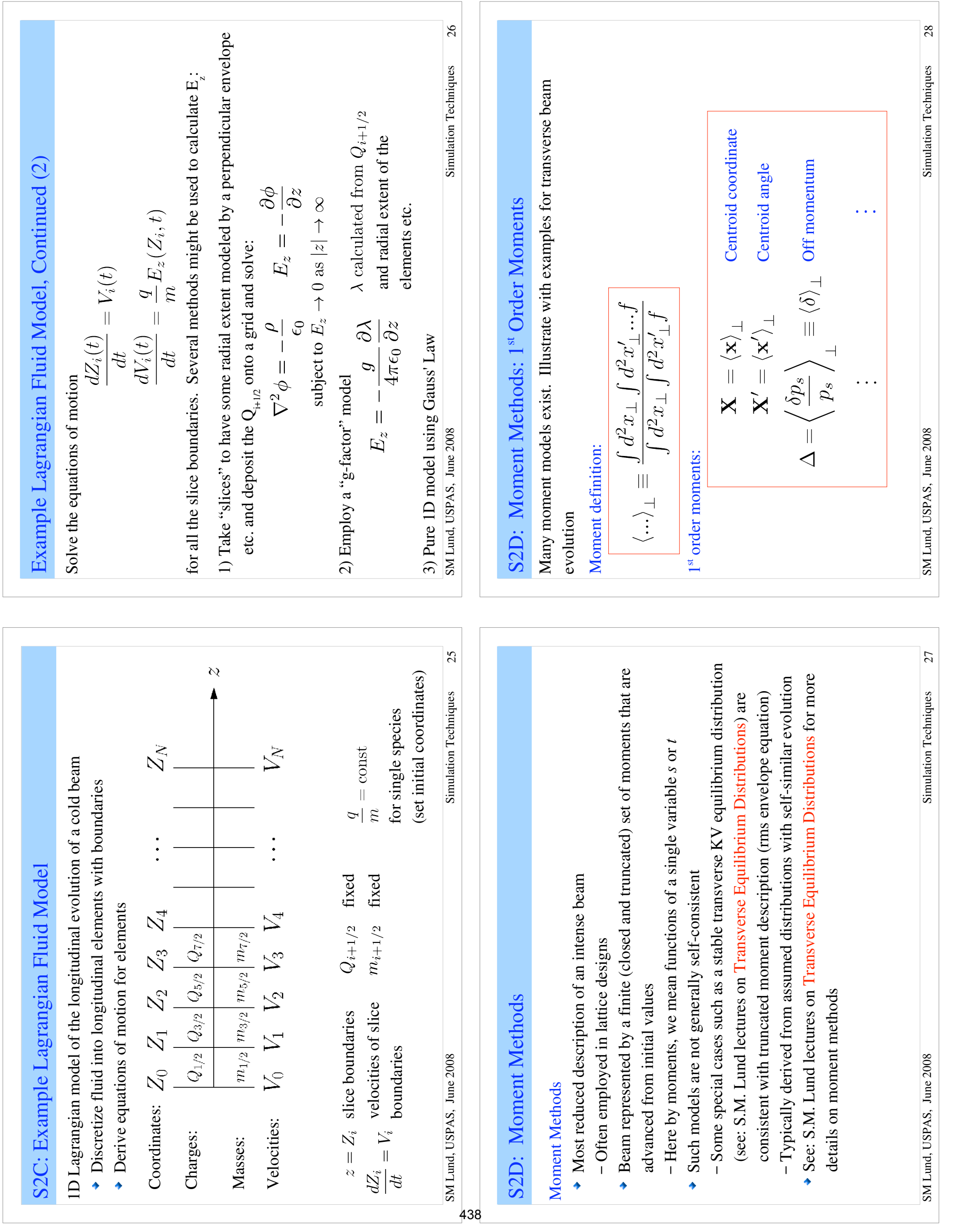



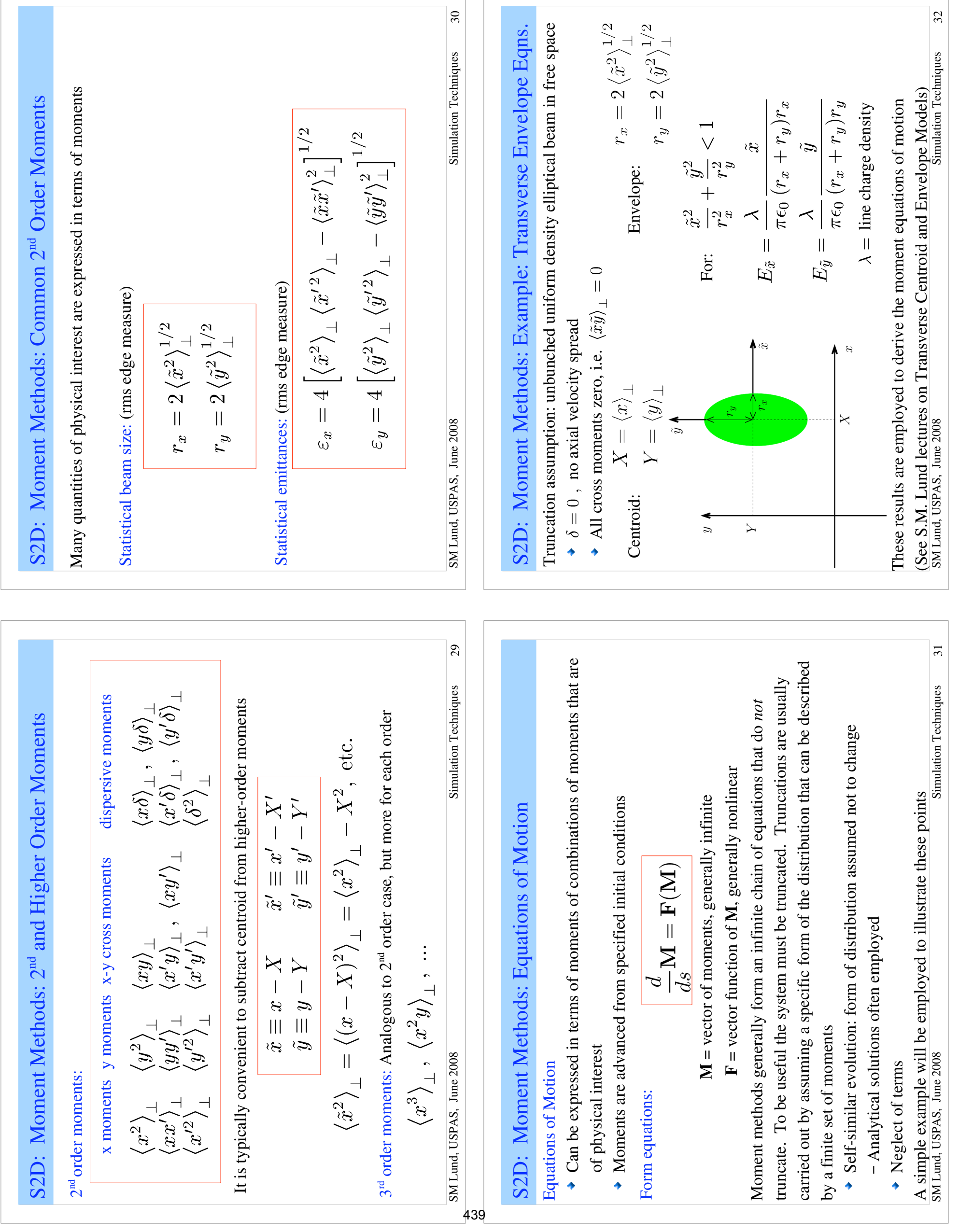


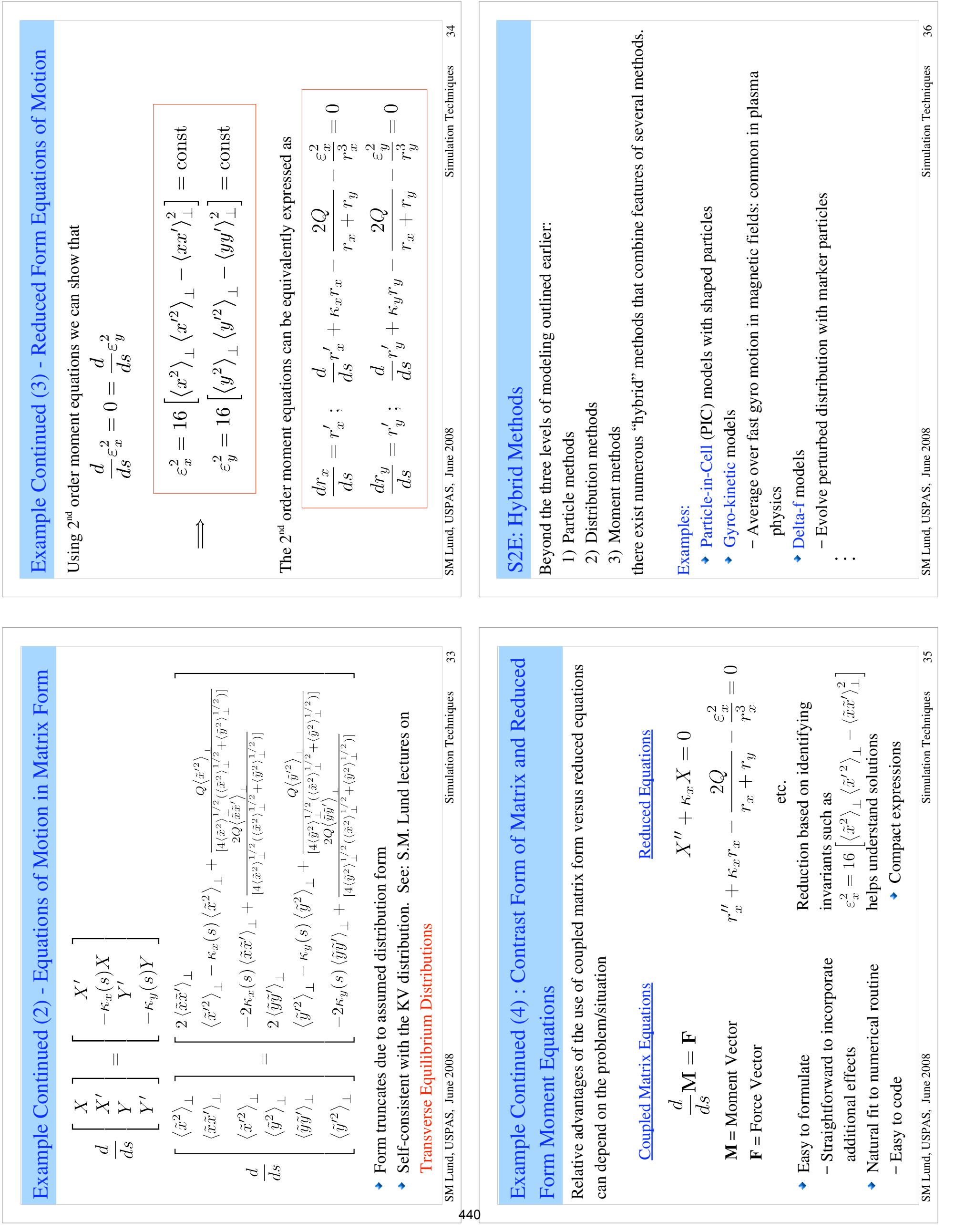




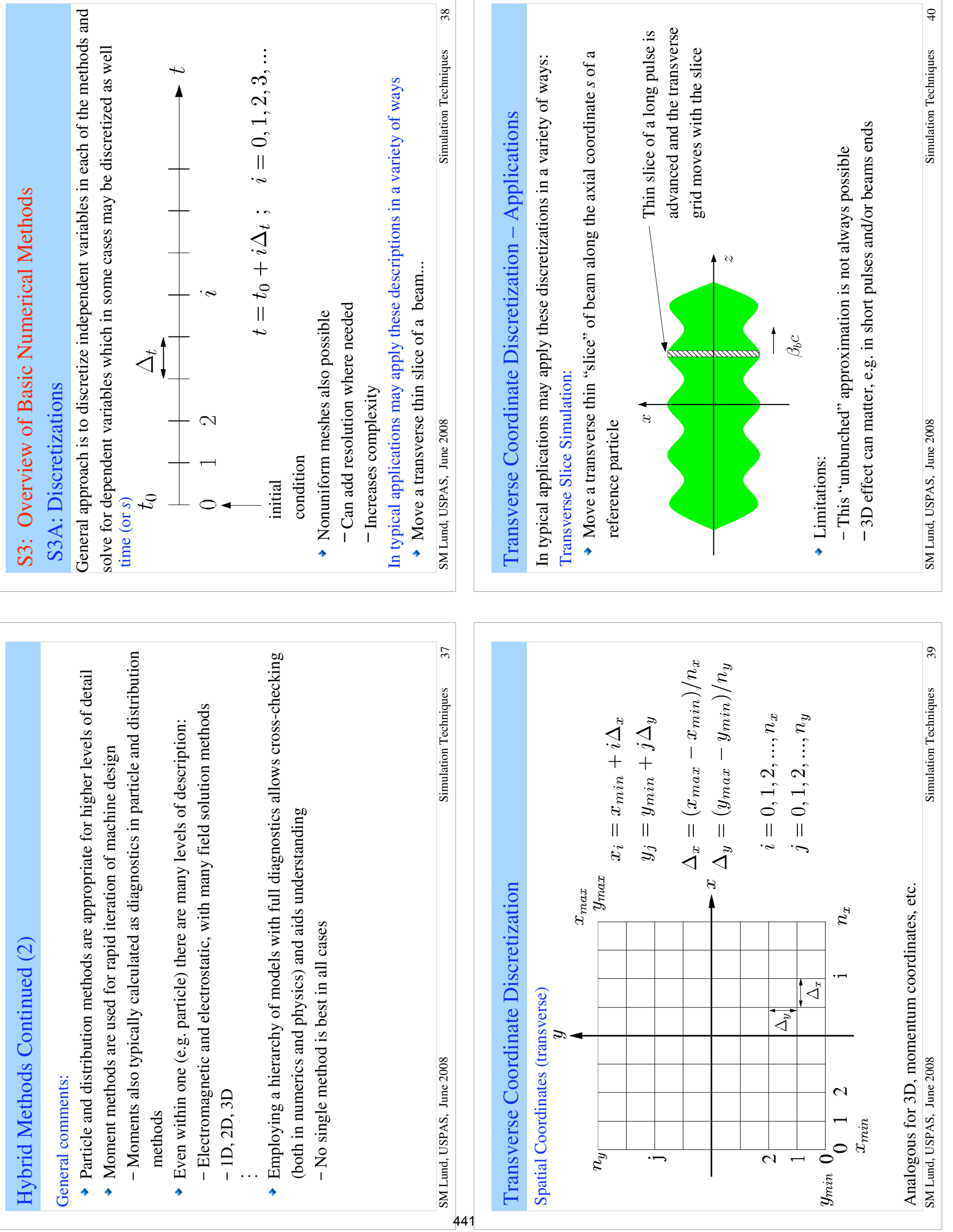




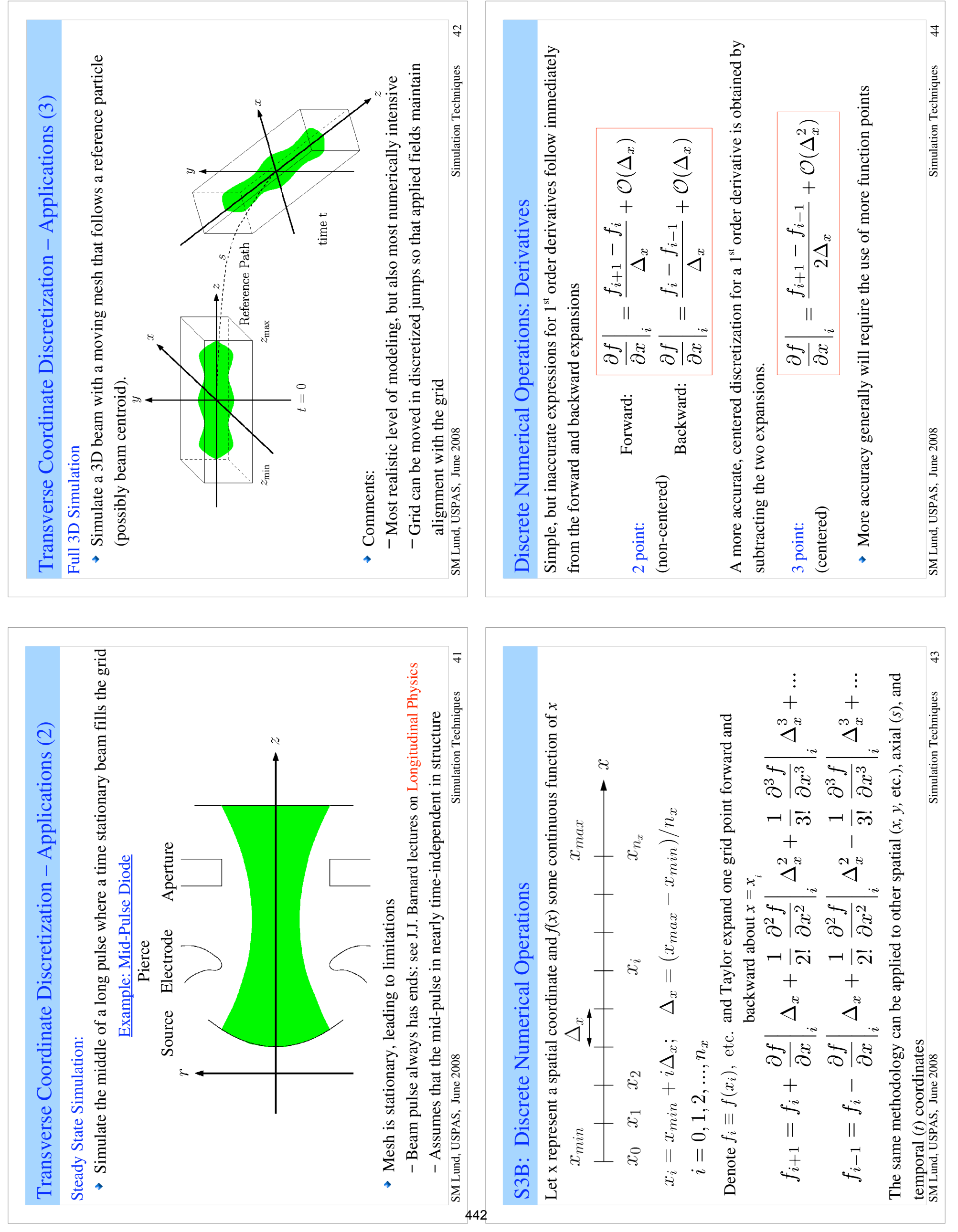



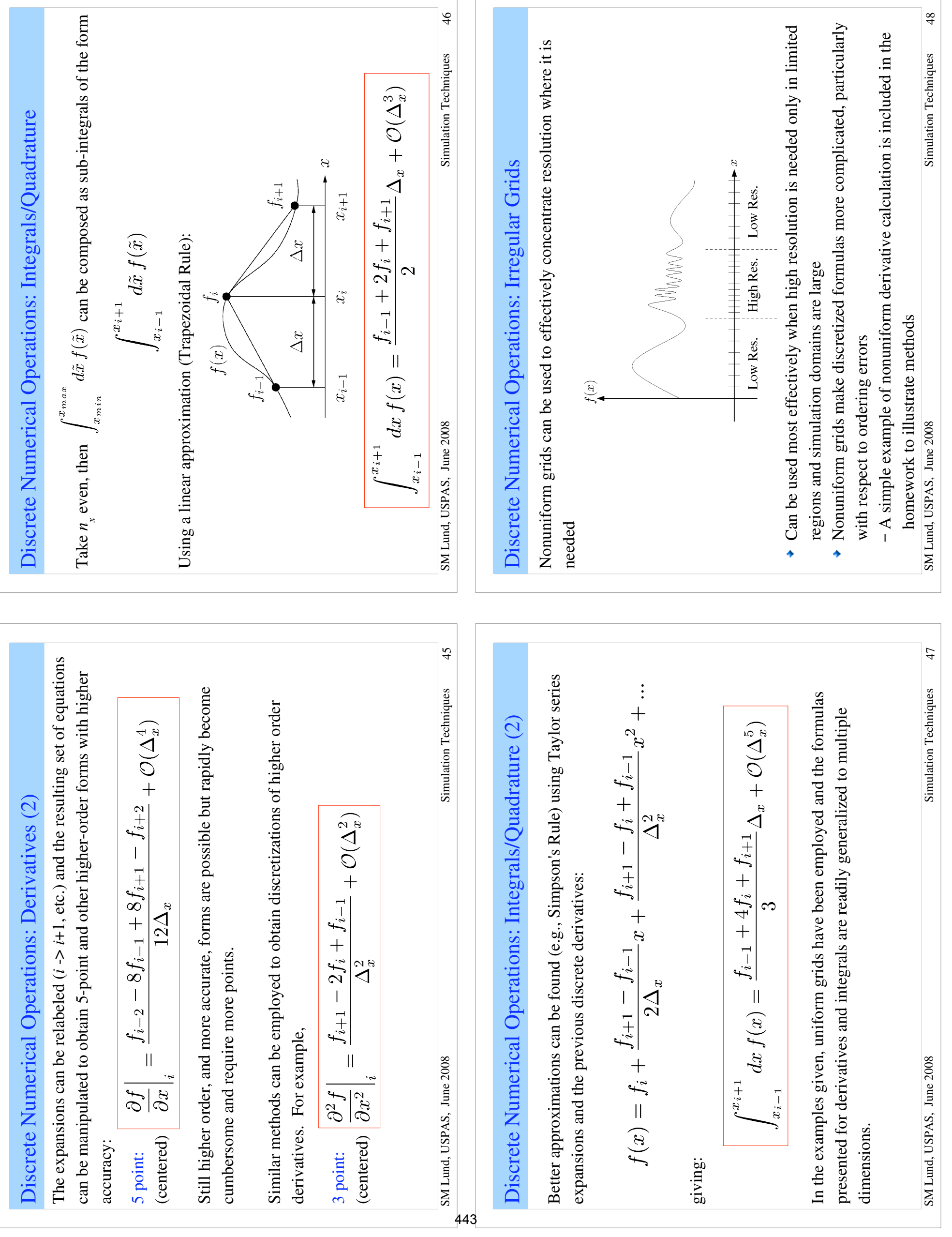


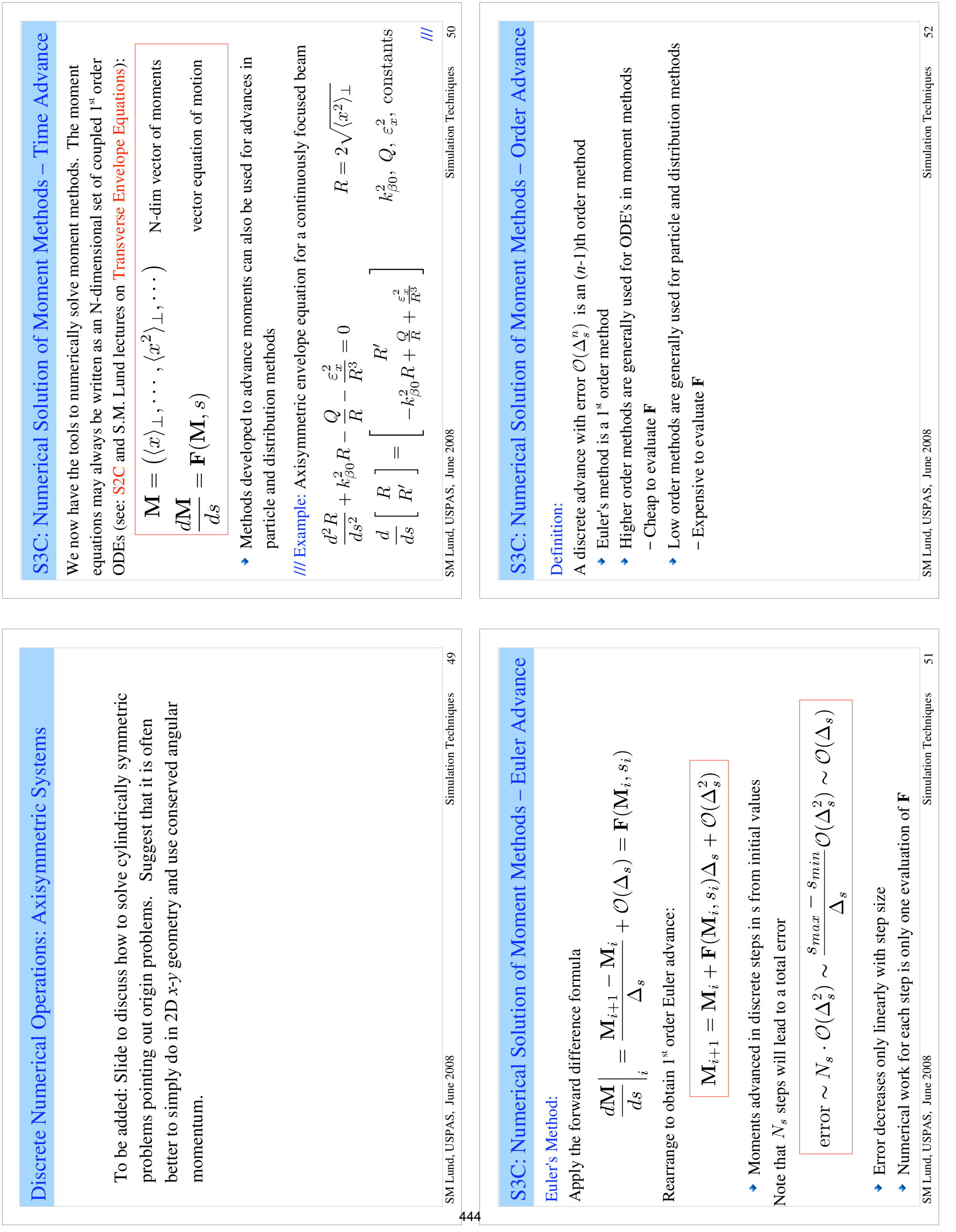




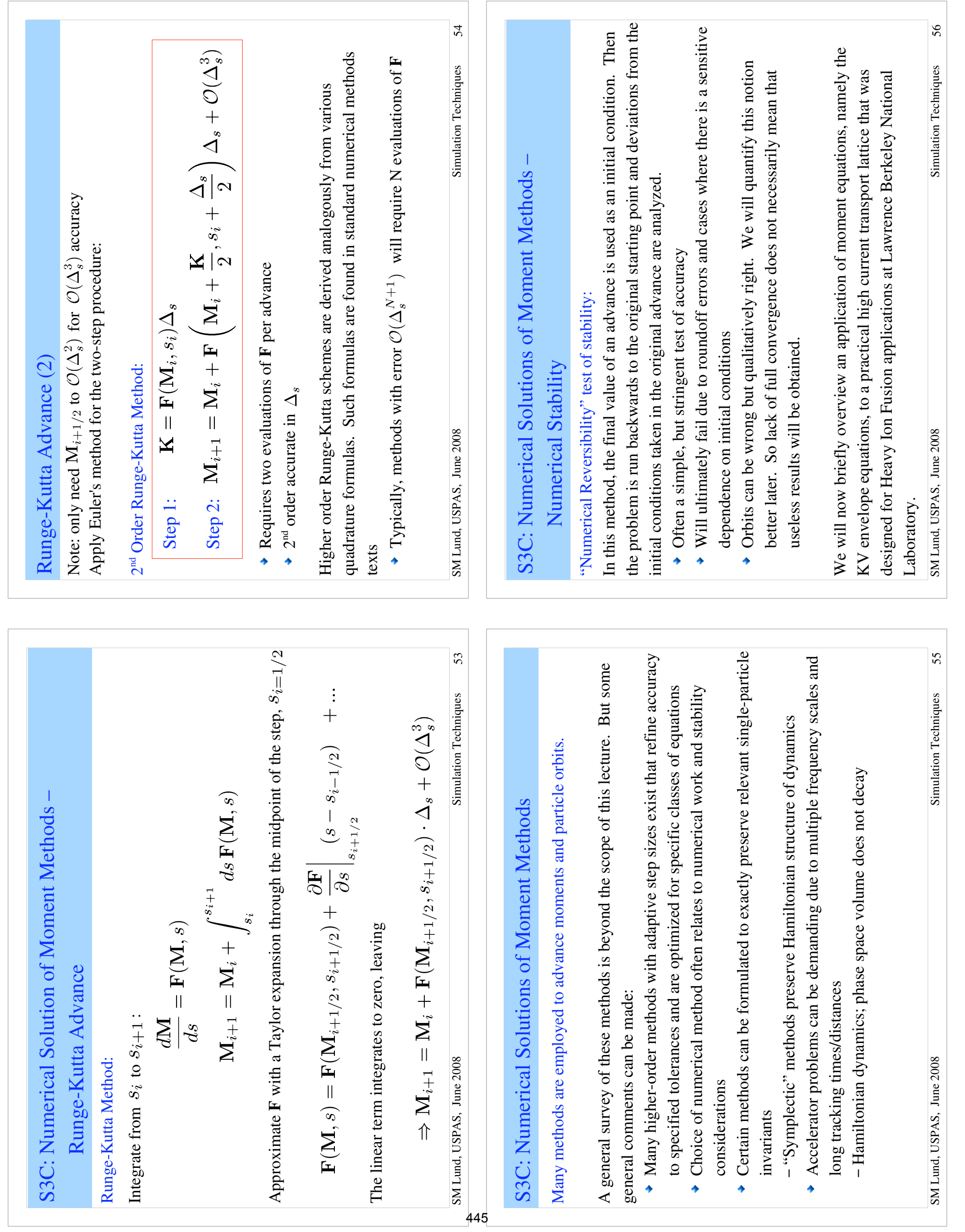



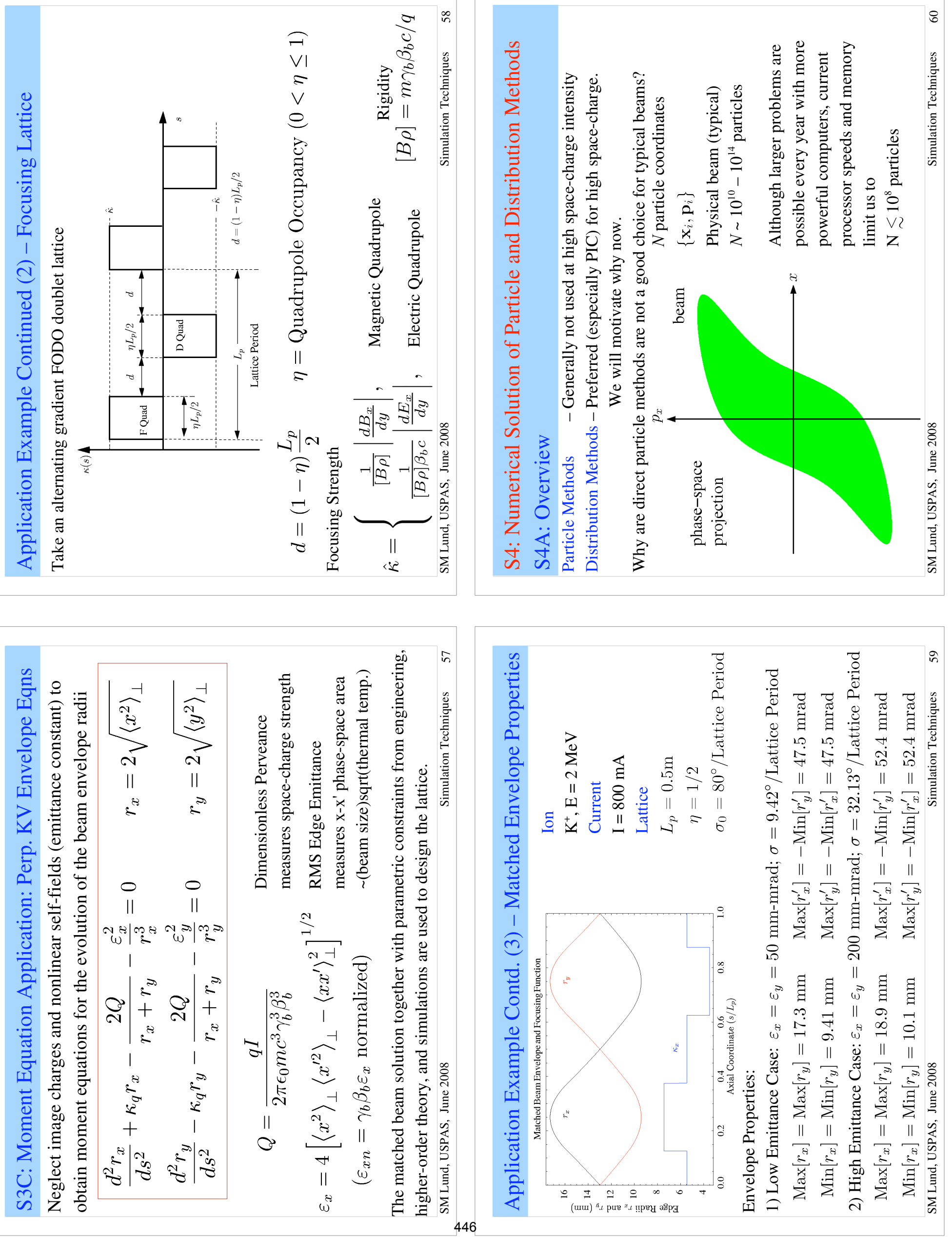

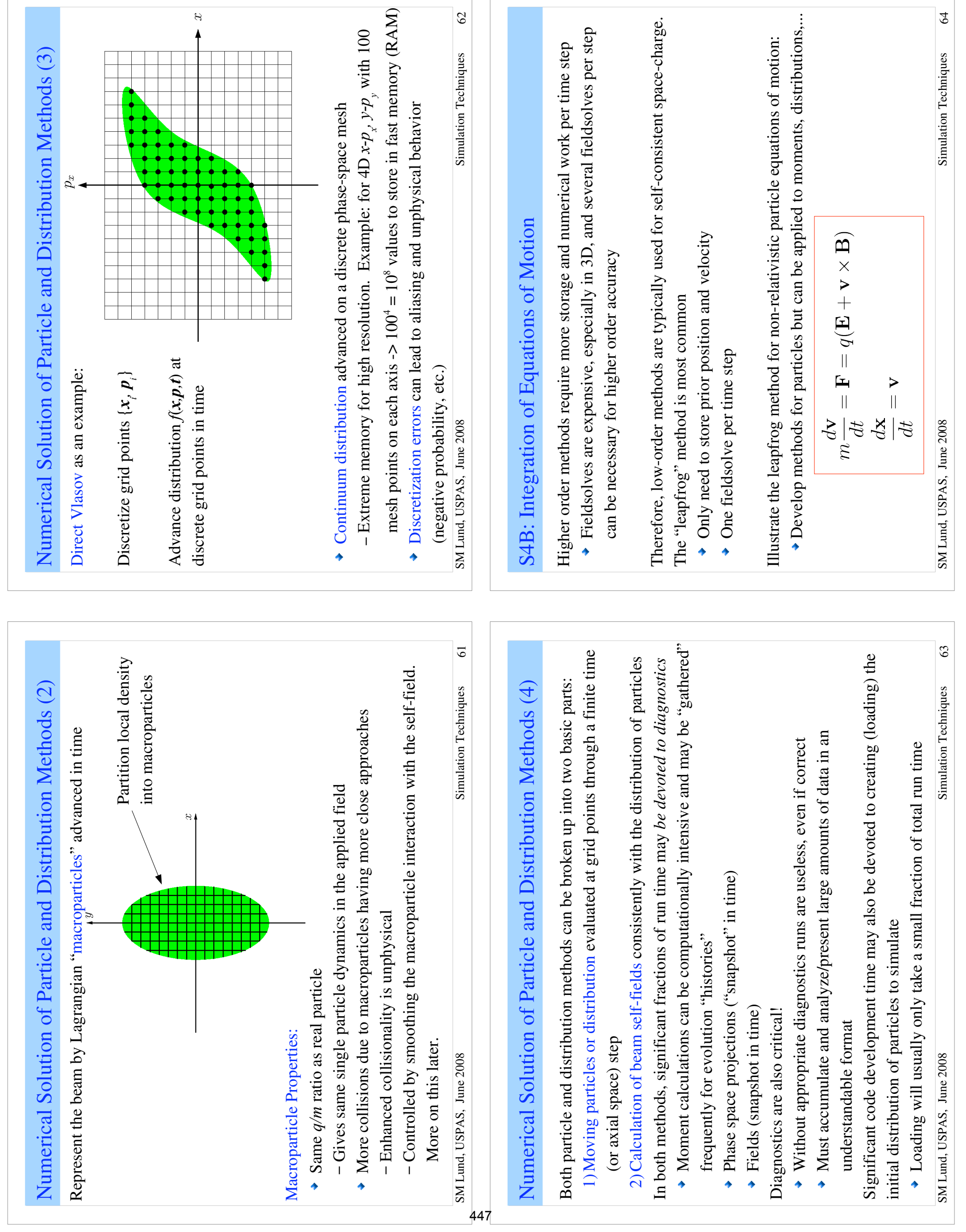

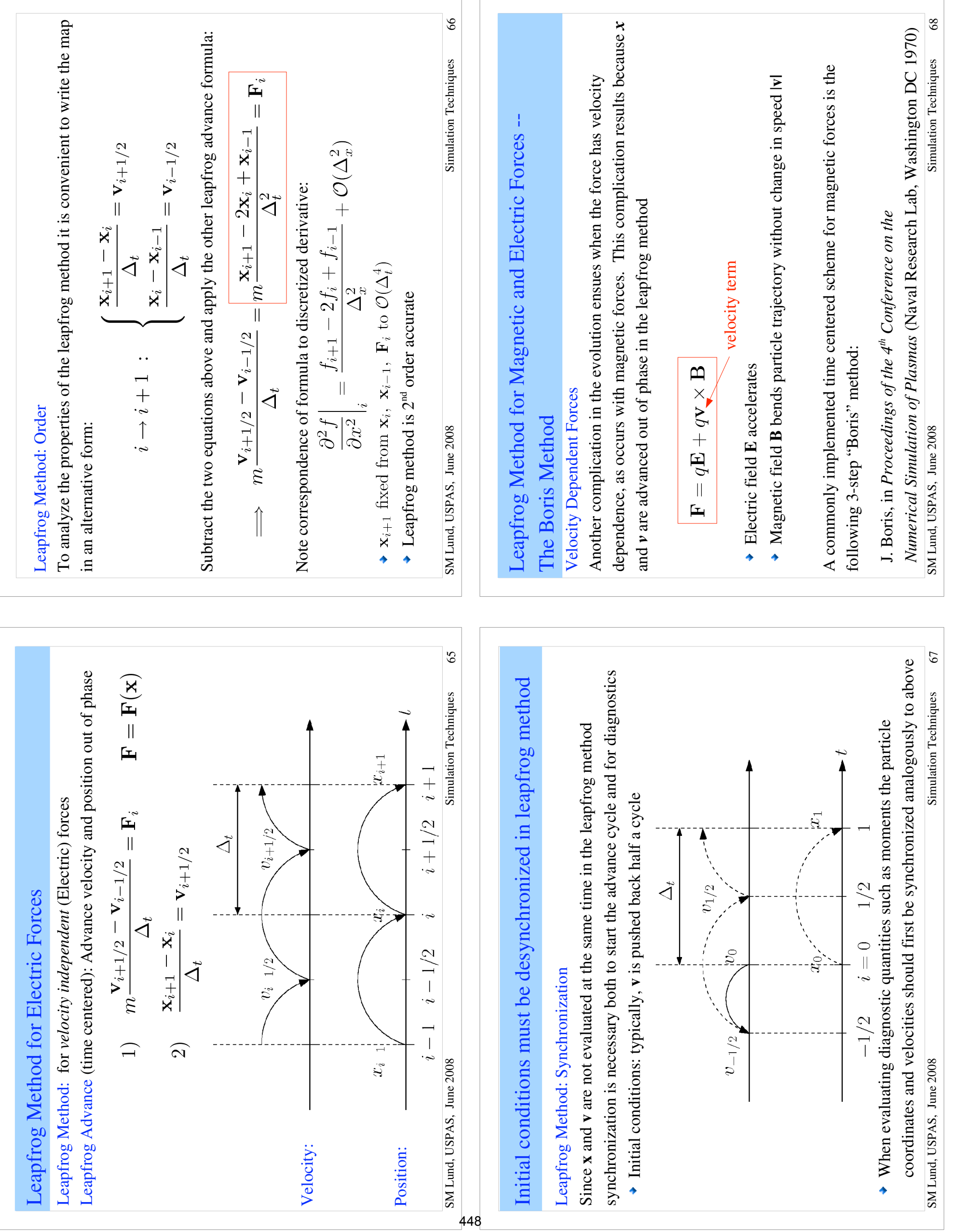


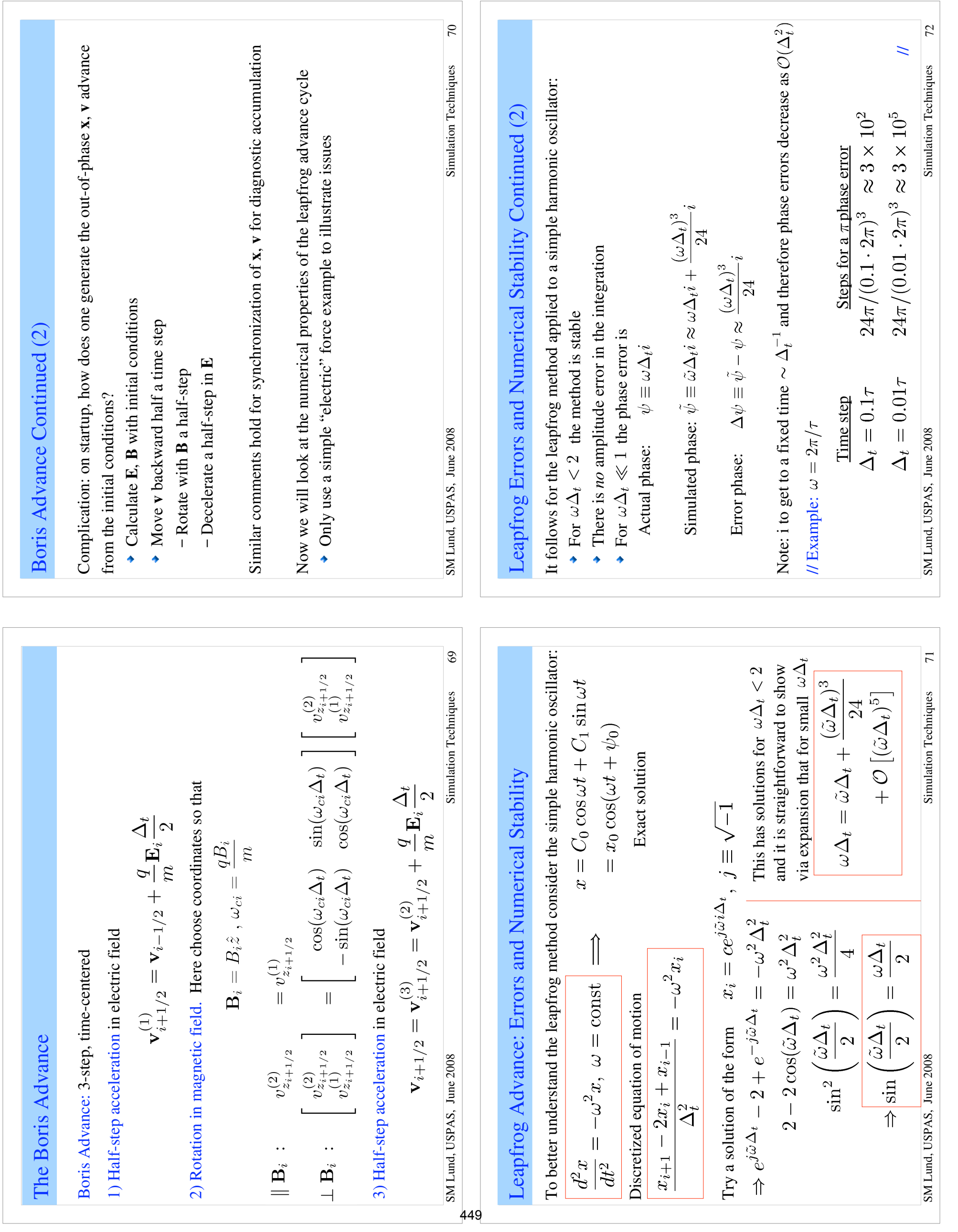



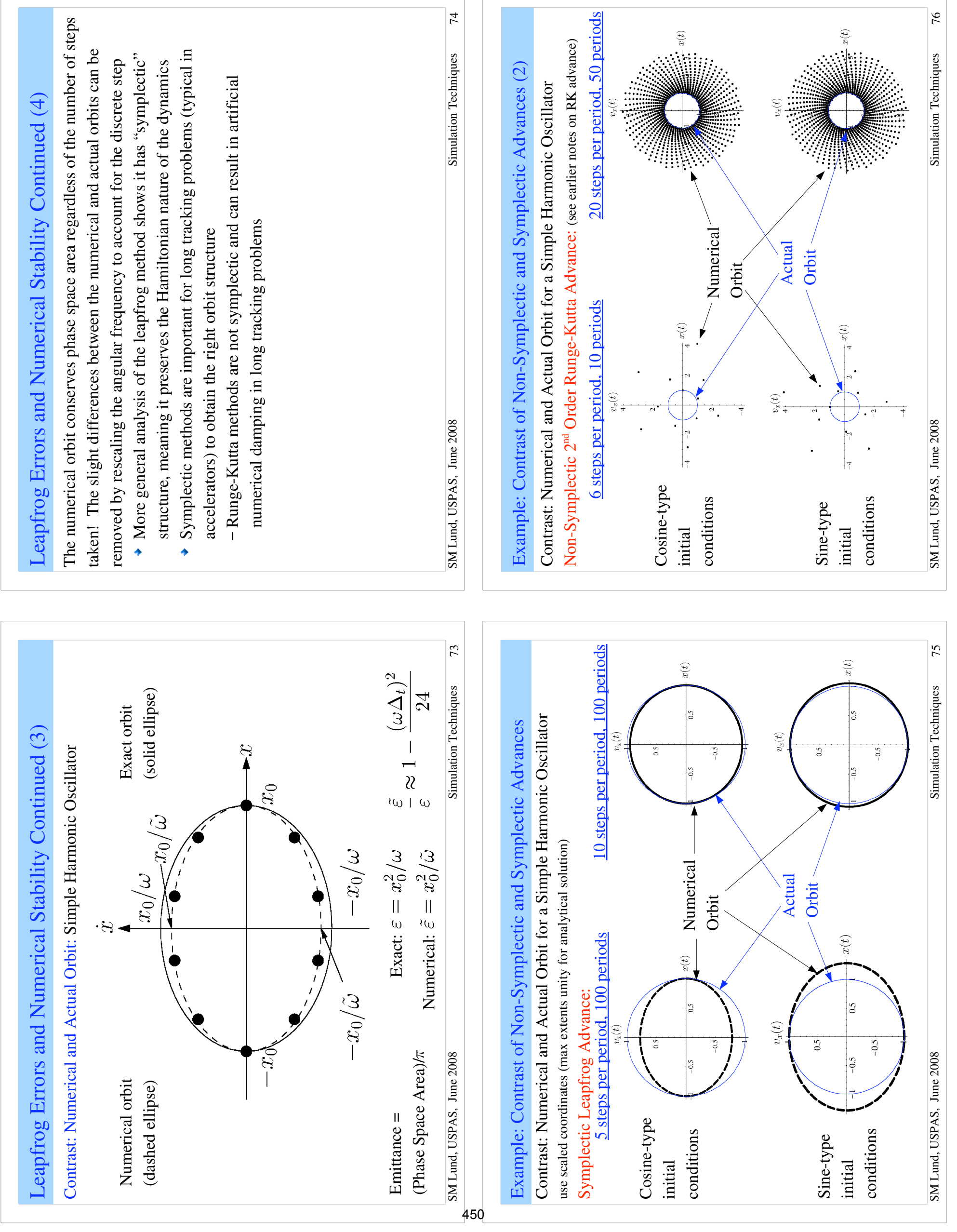


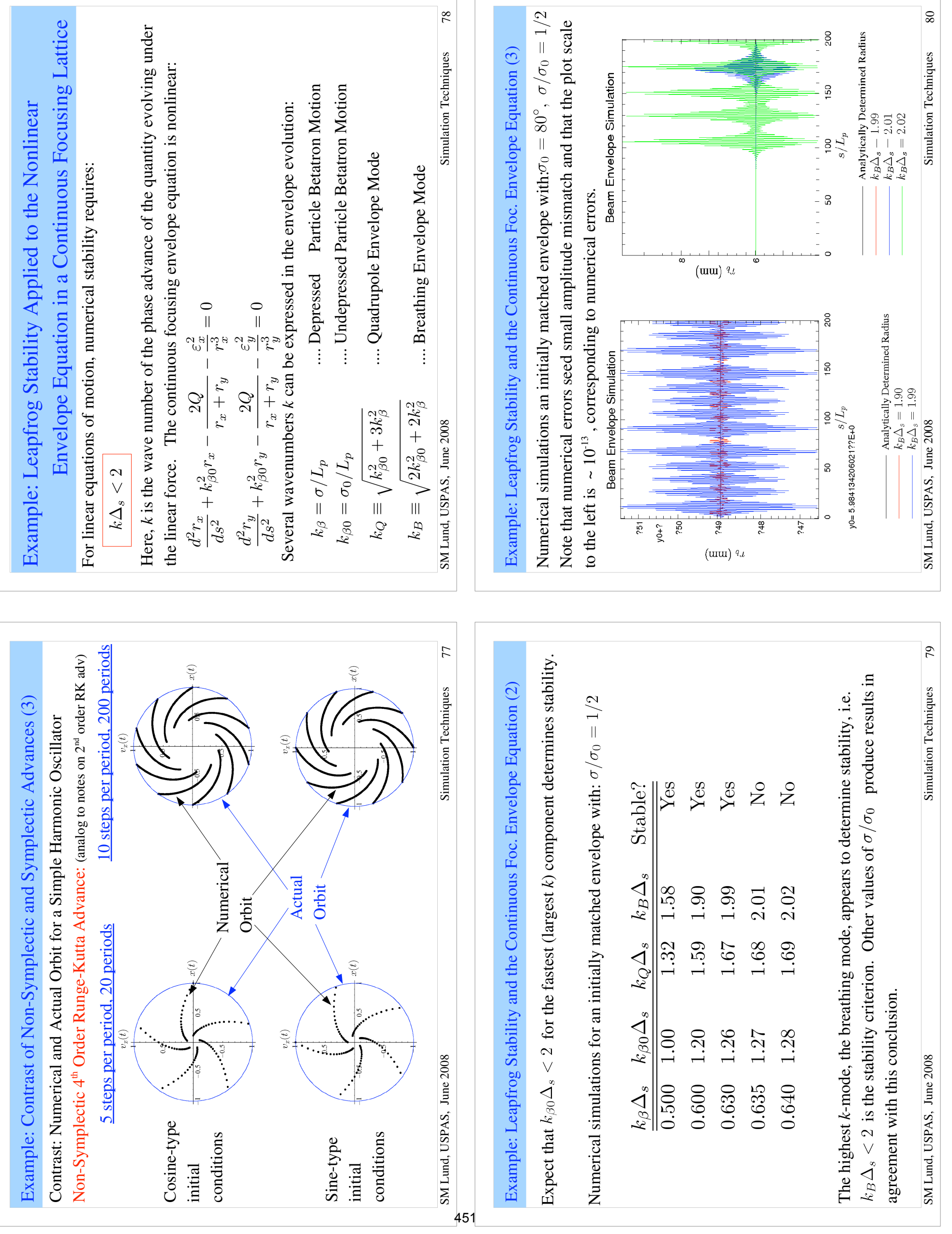




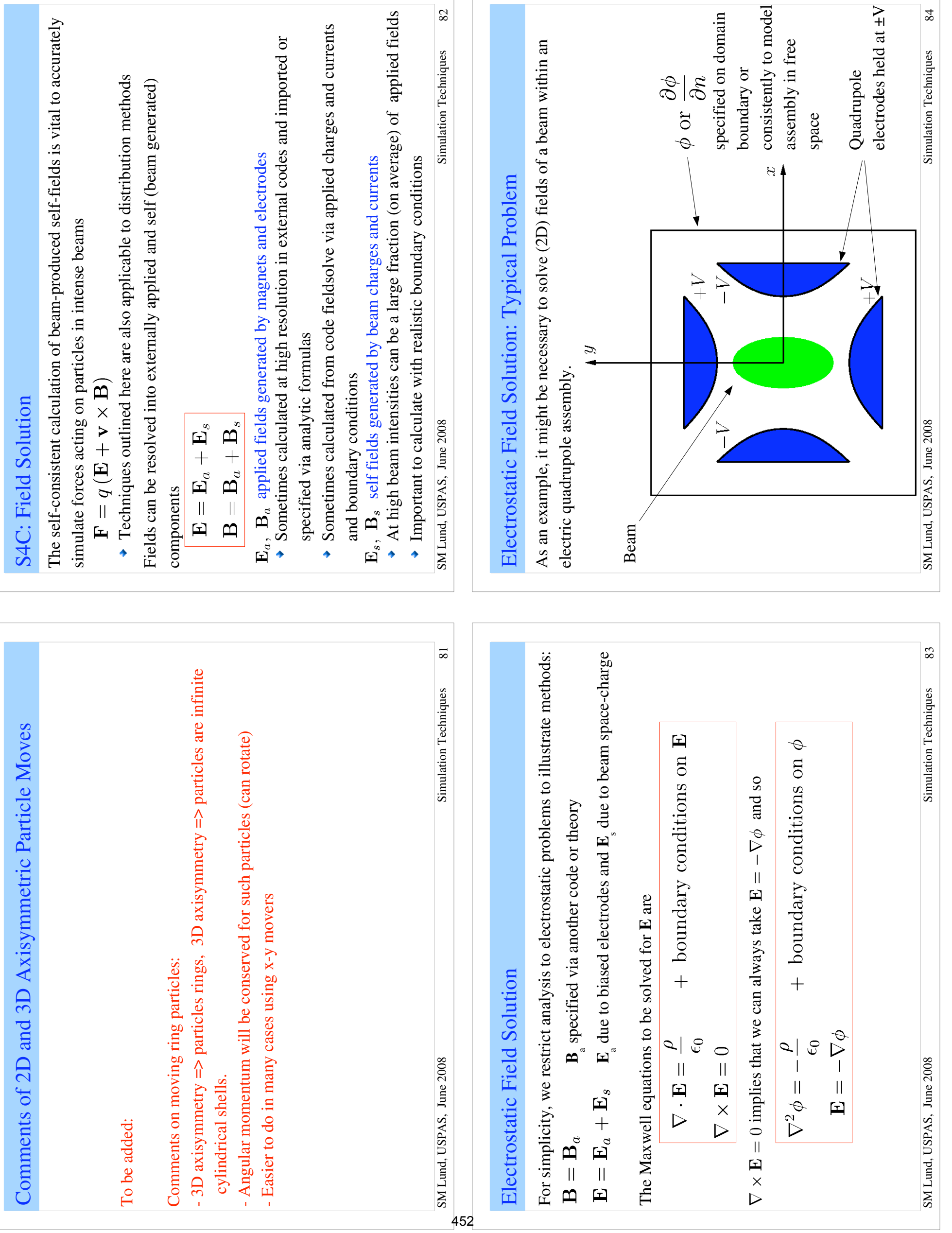



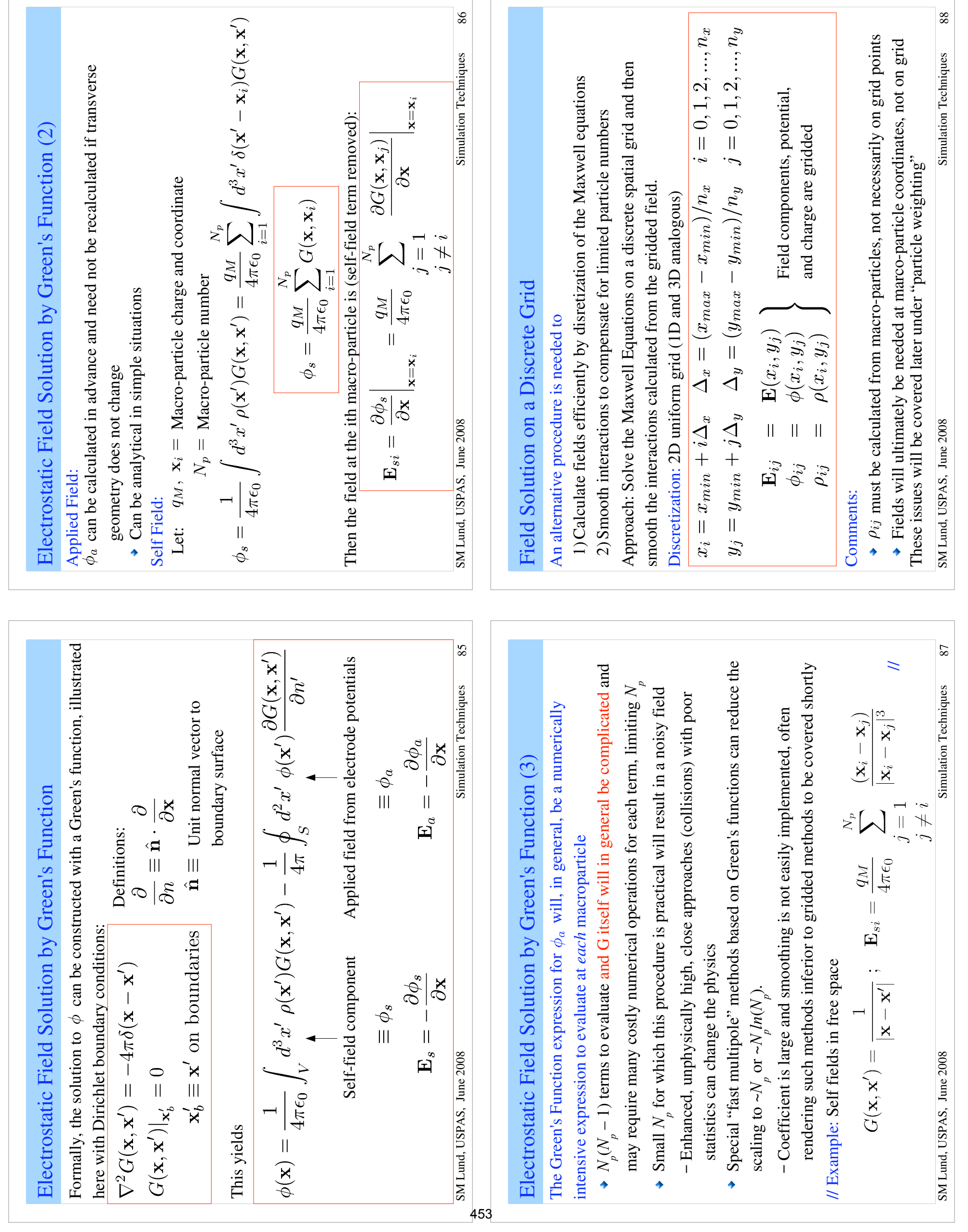


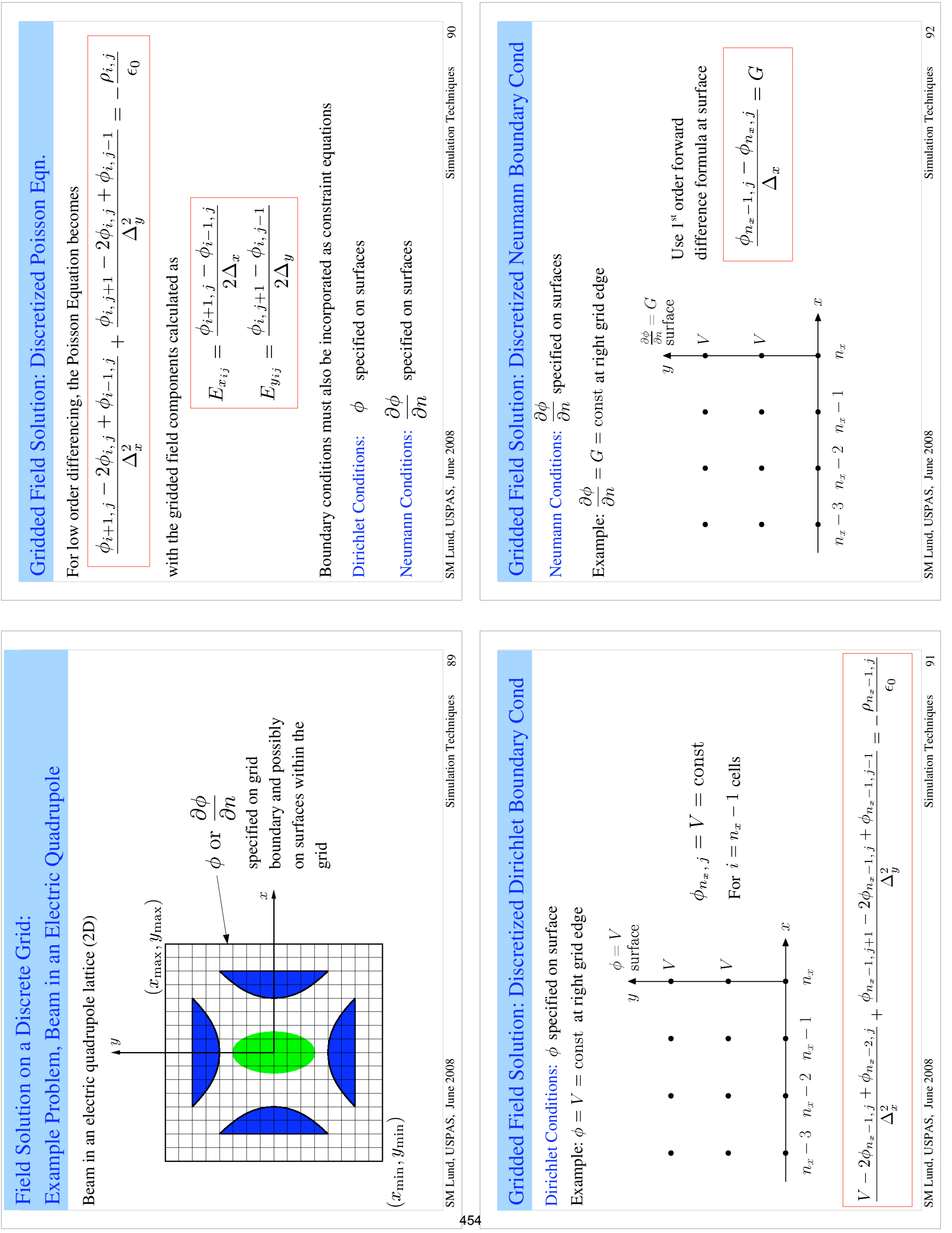




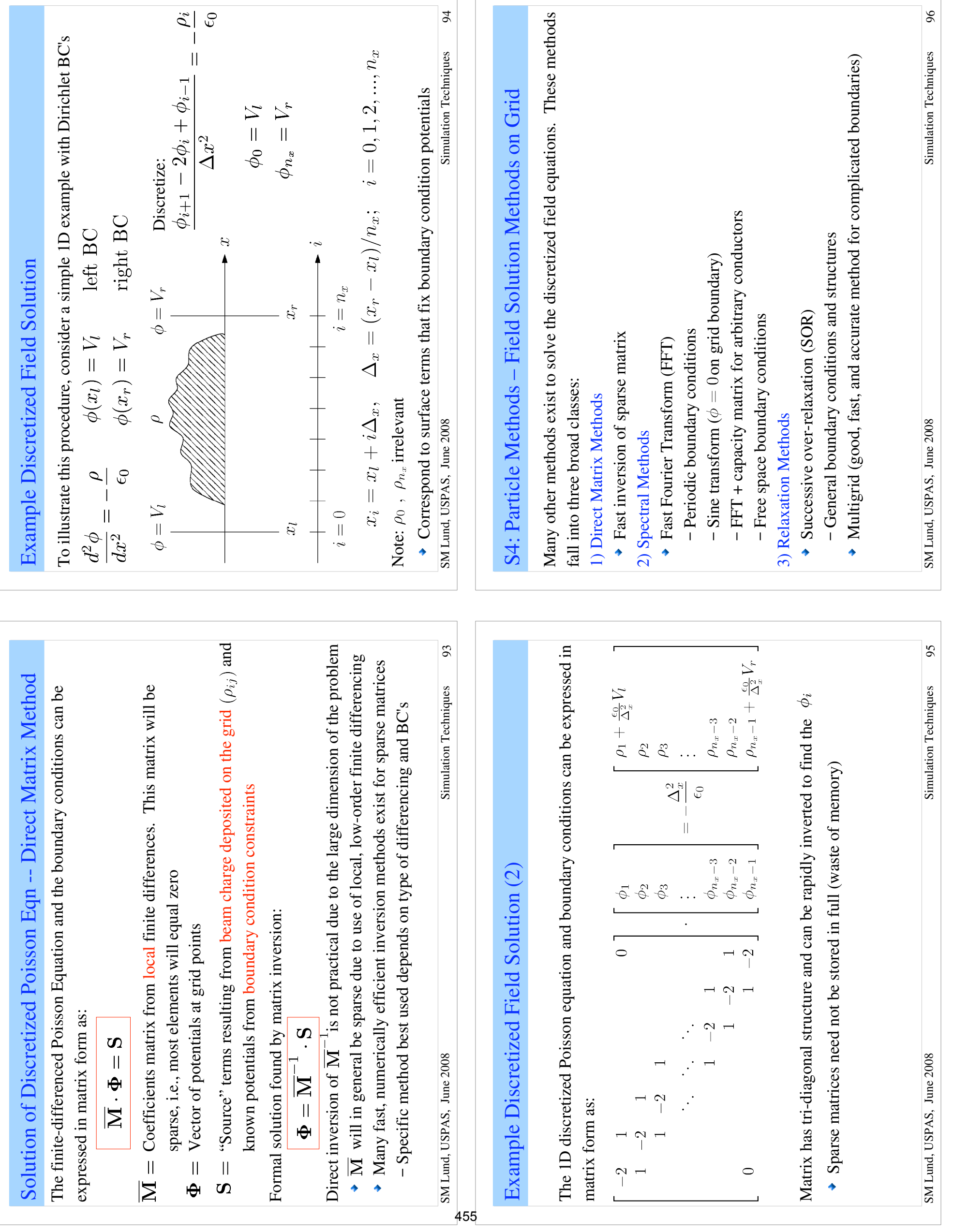



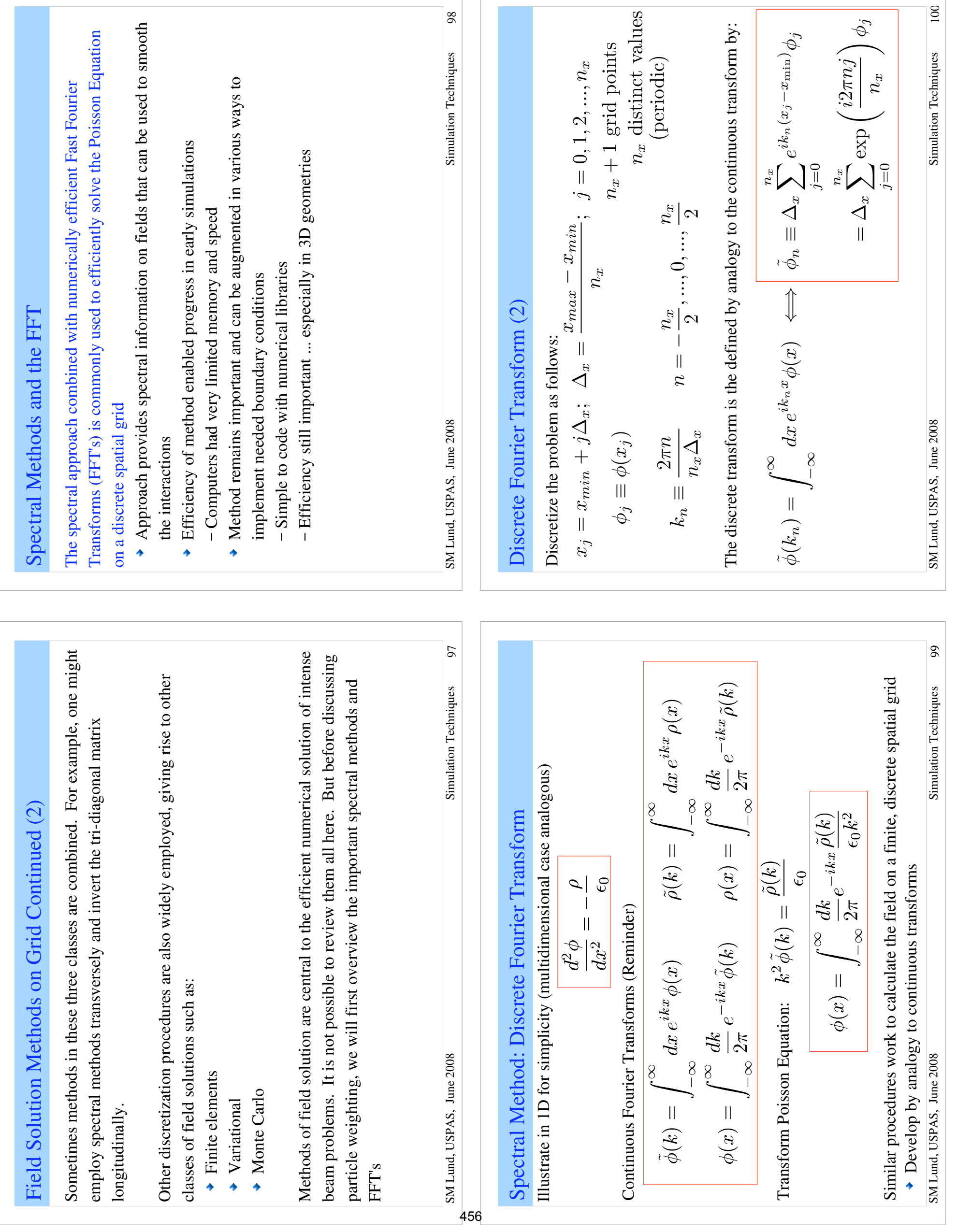


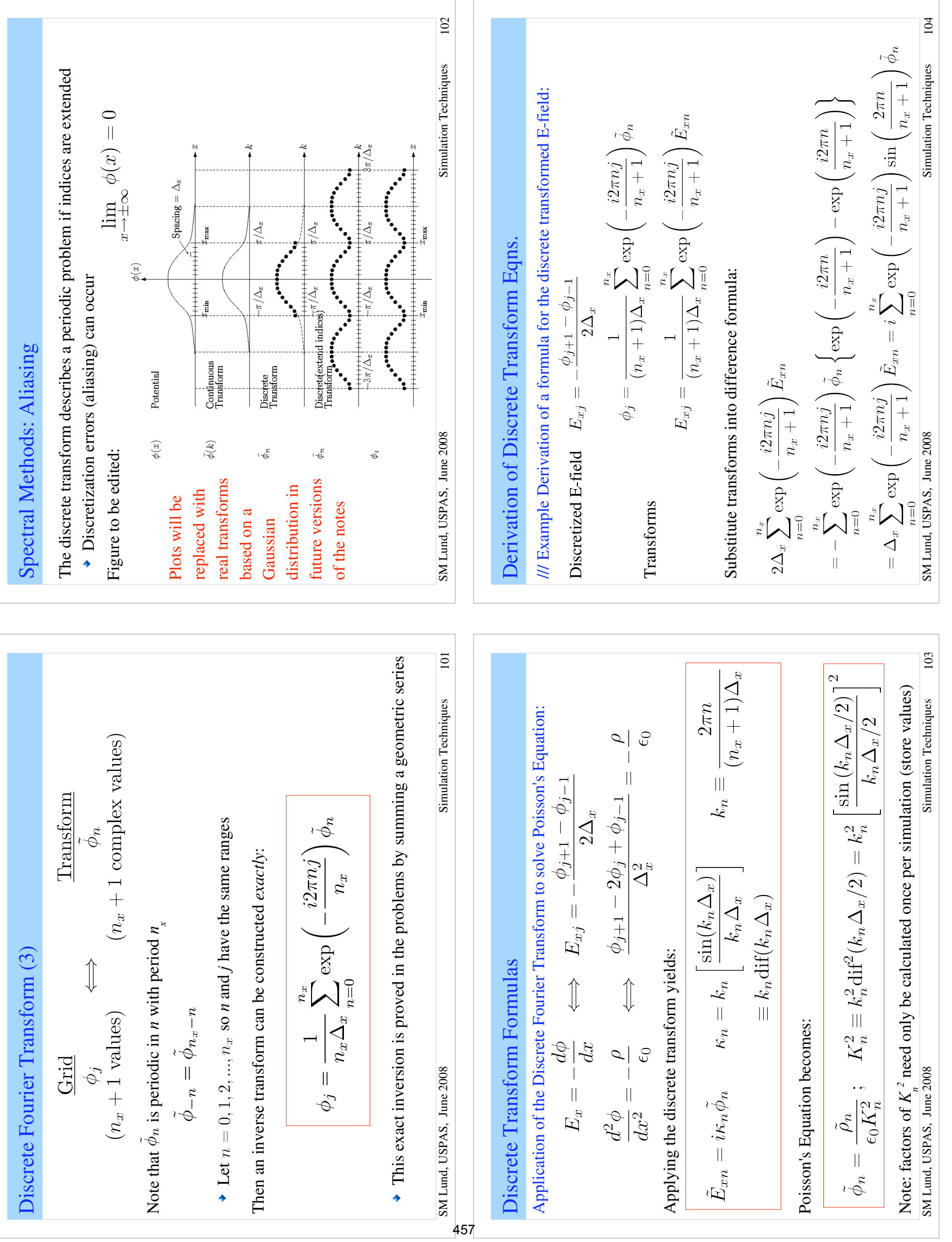



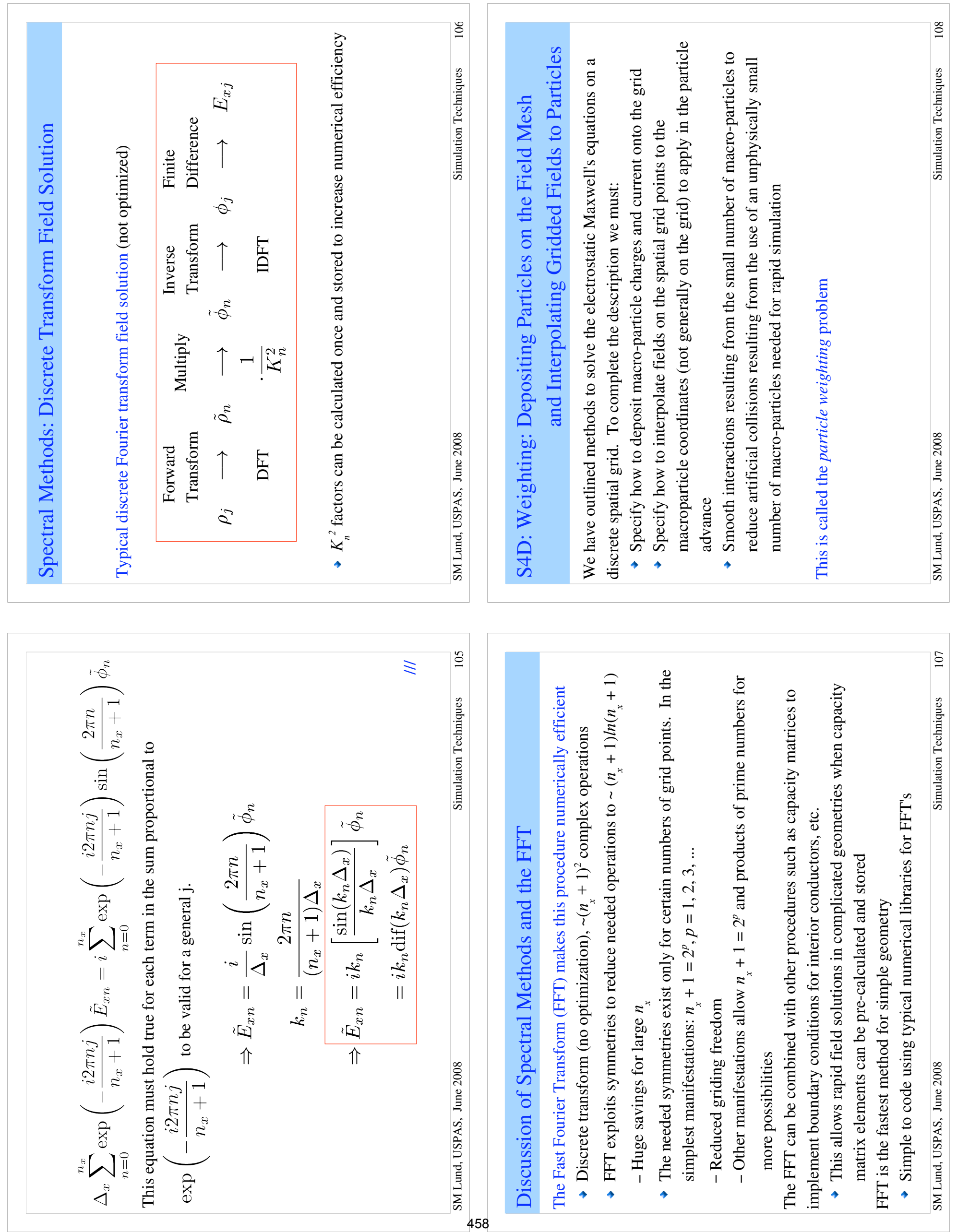

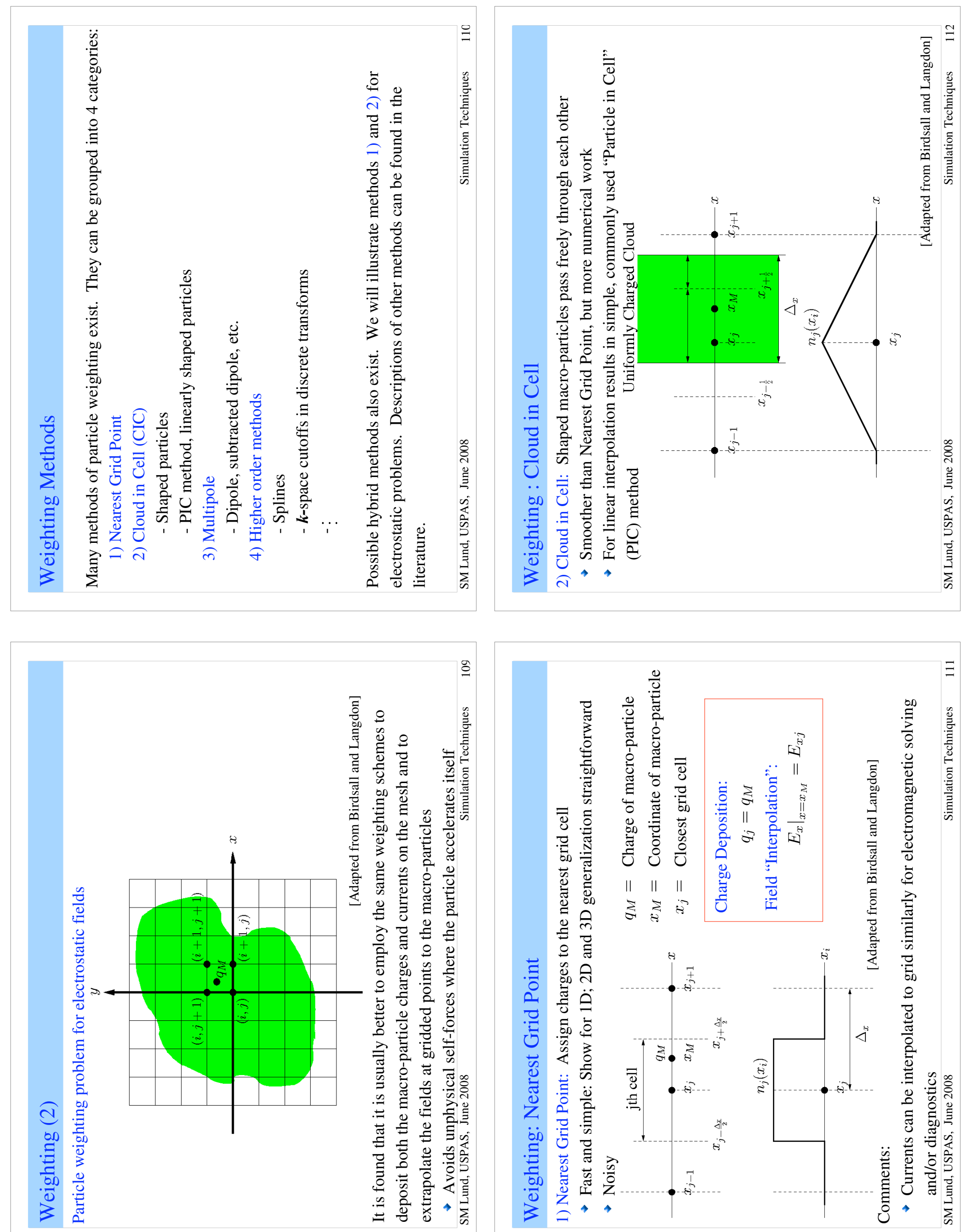

$\stackrel{9}{9}$

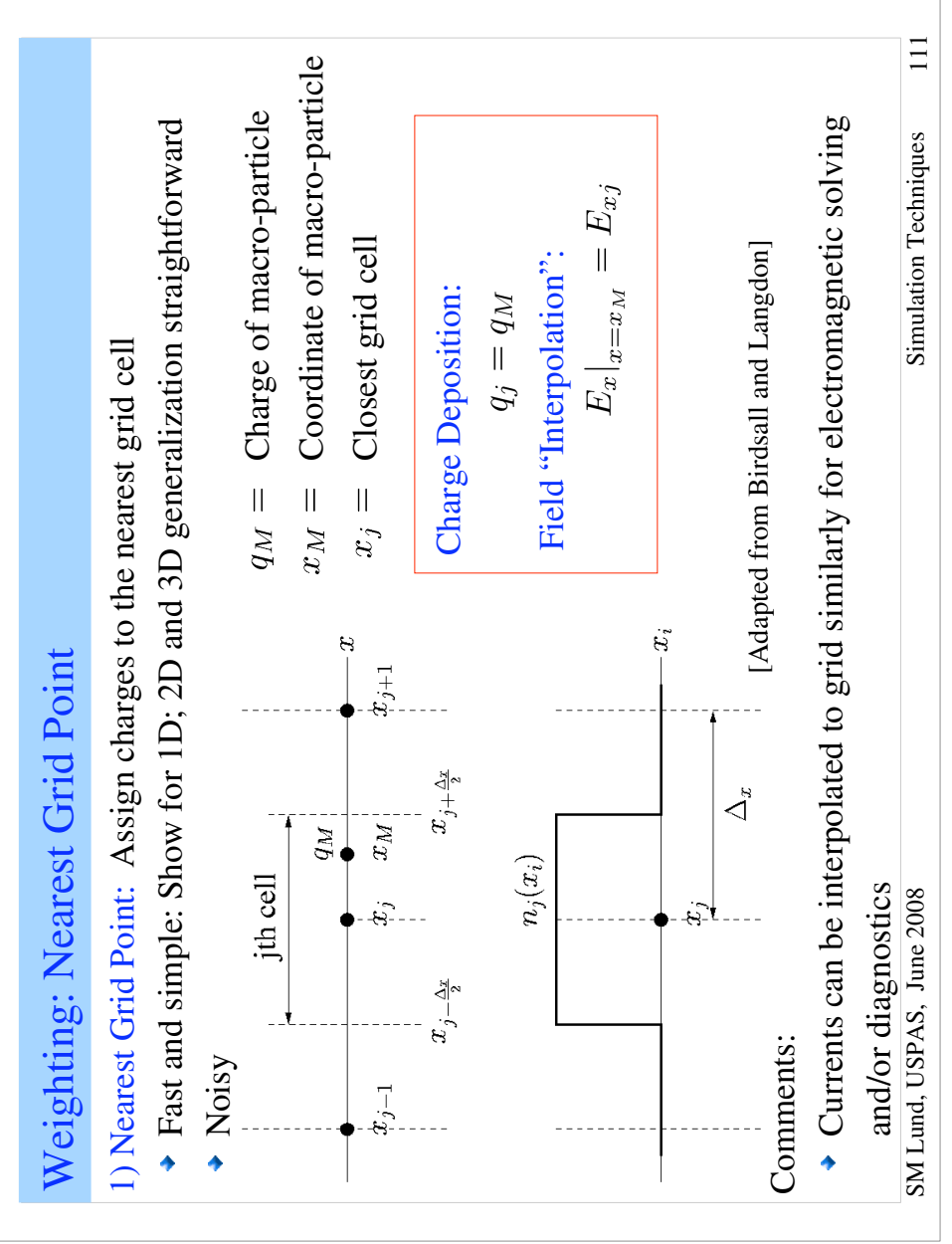



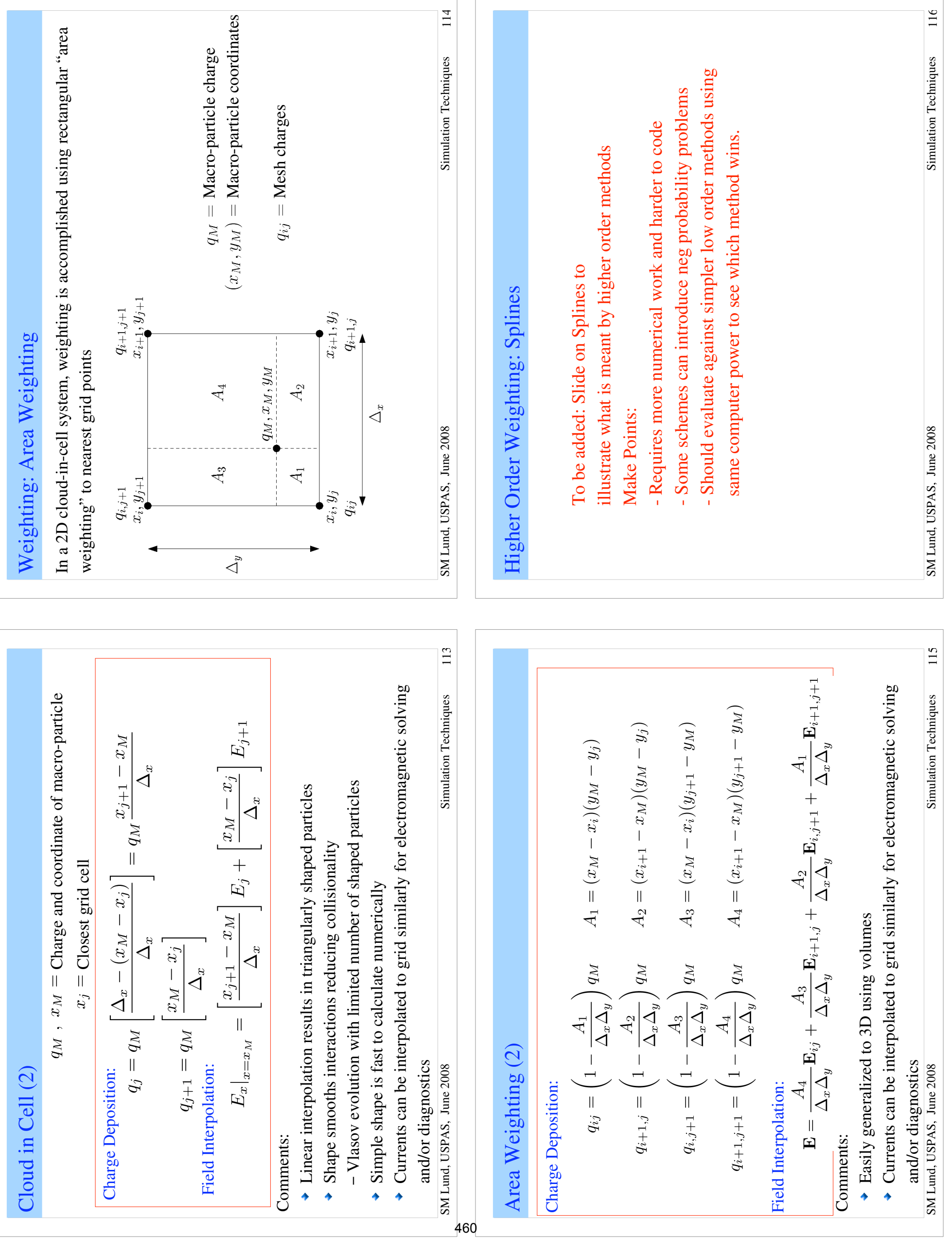

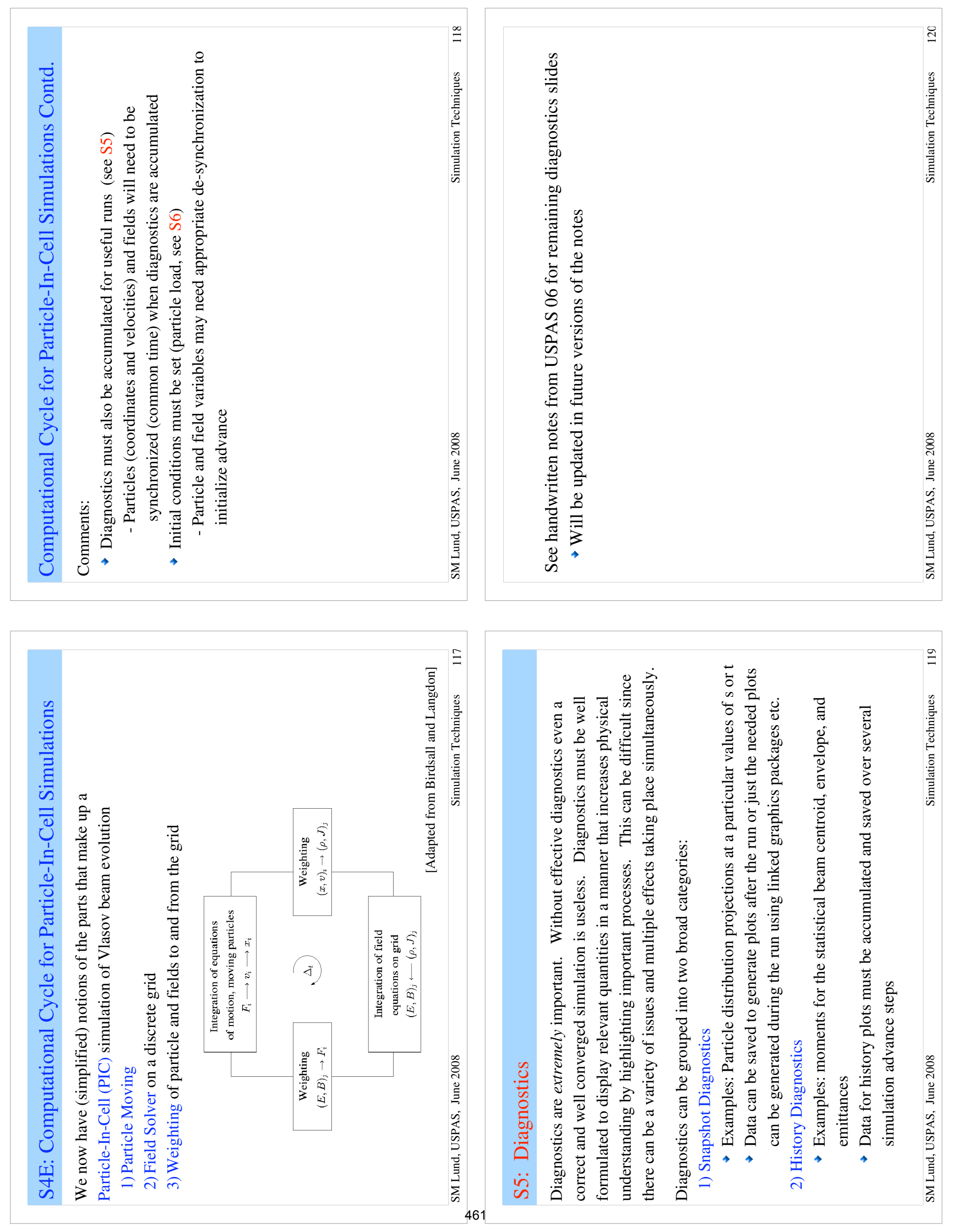

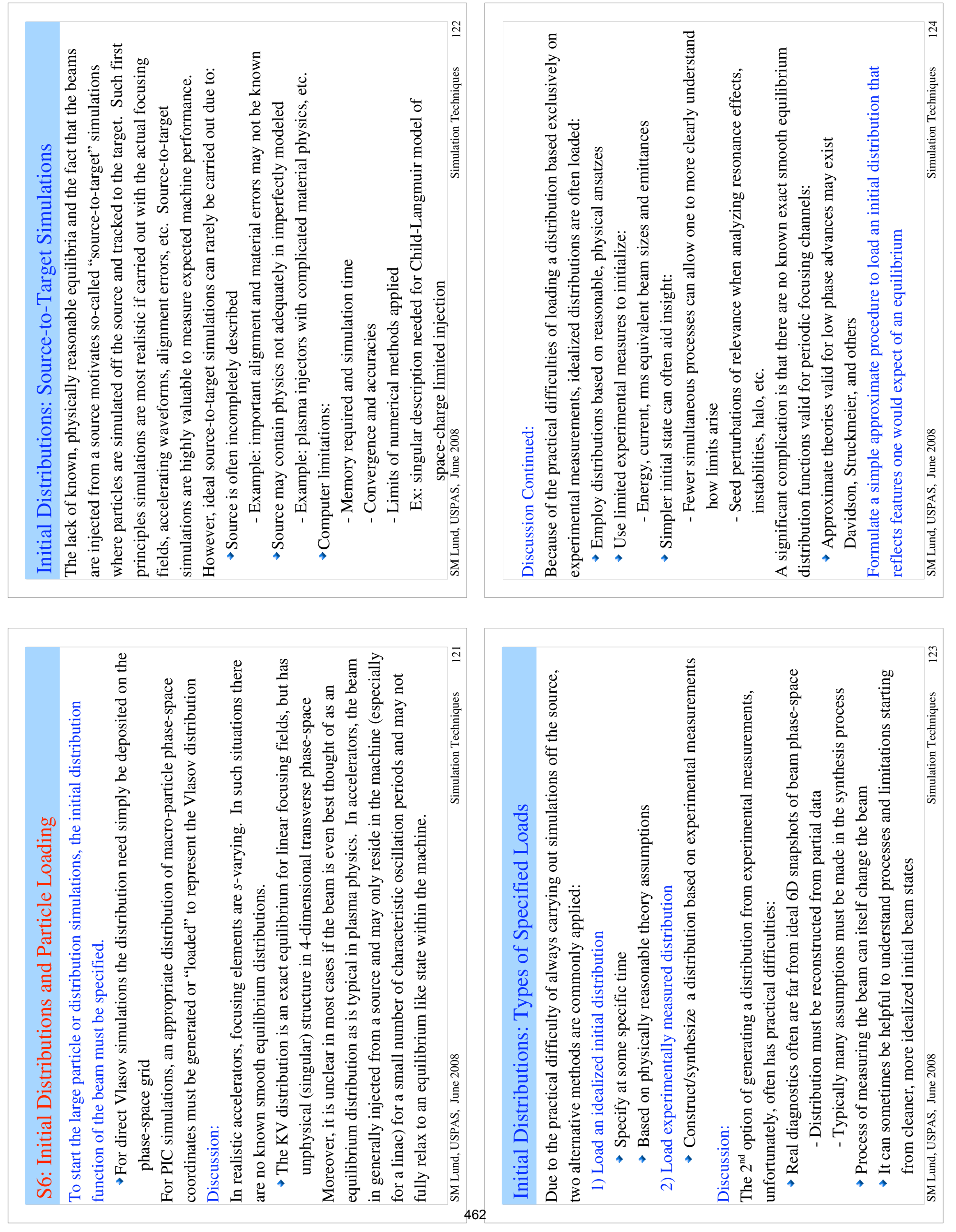


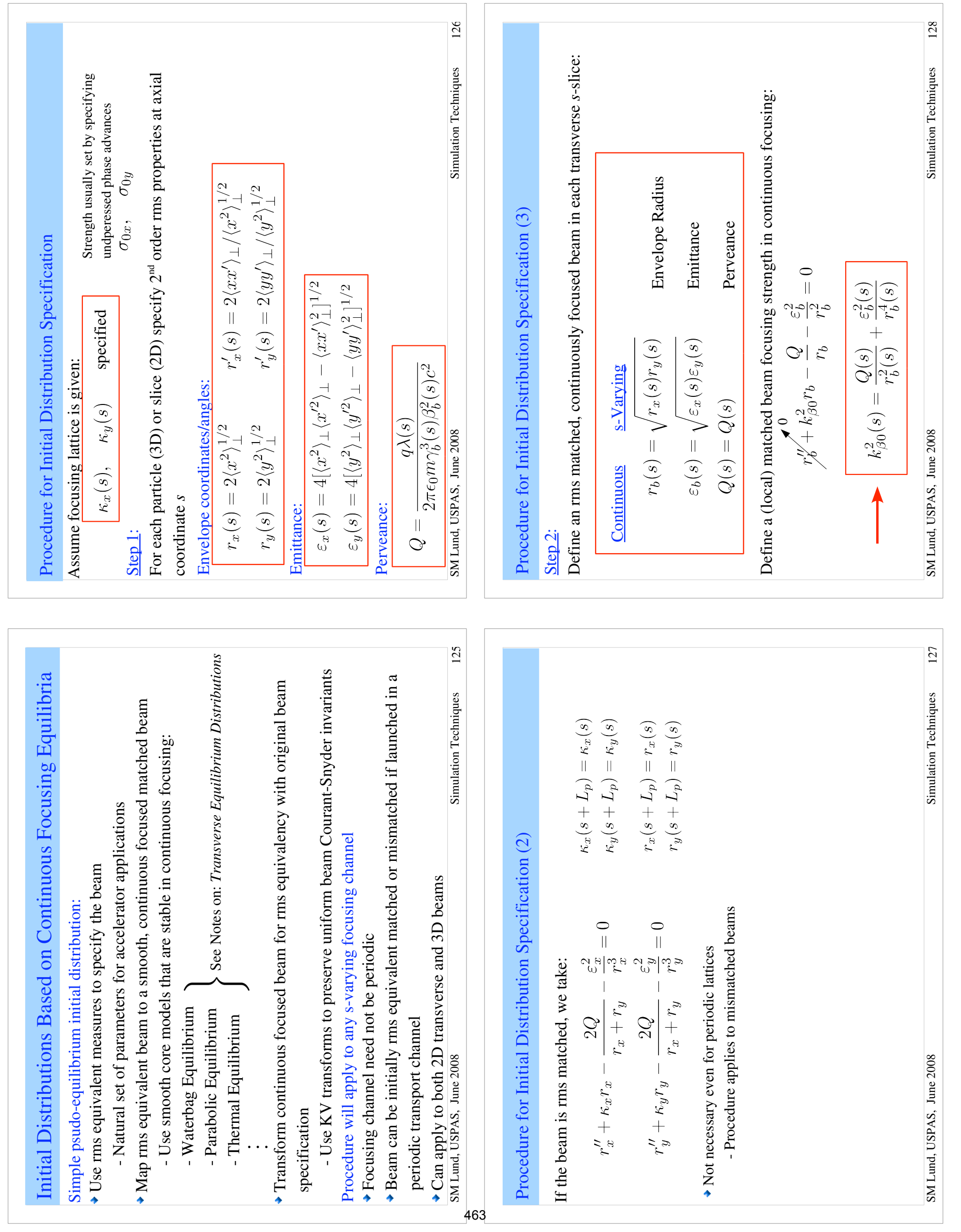



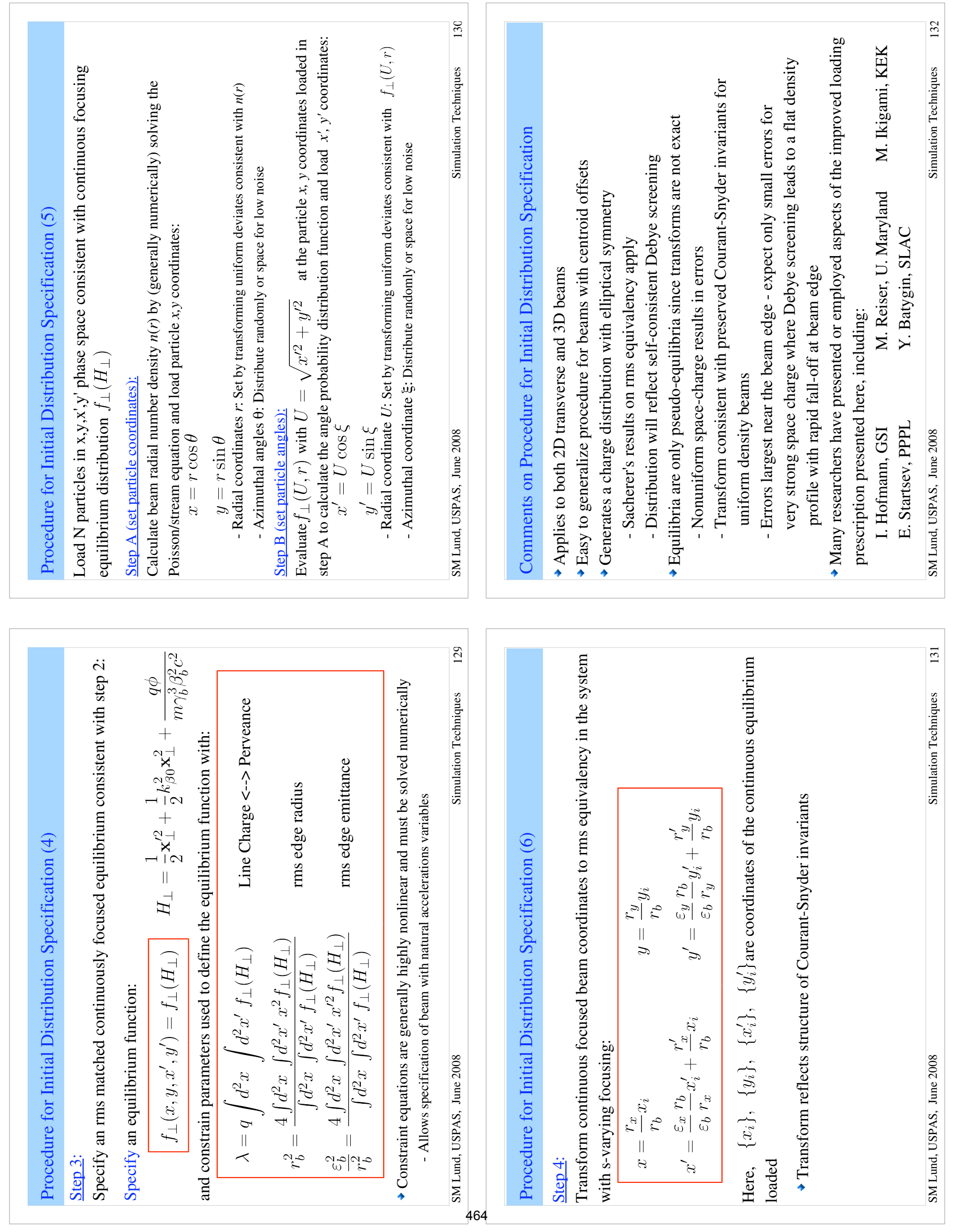

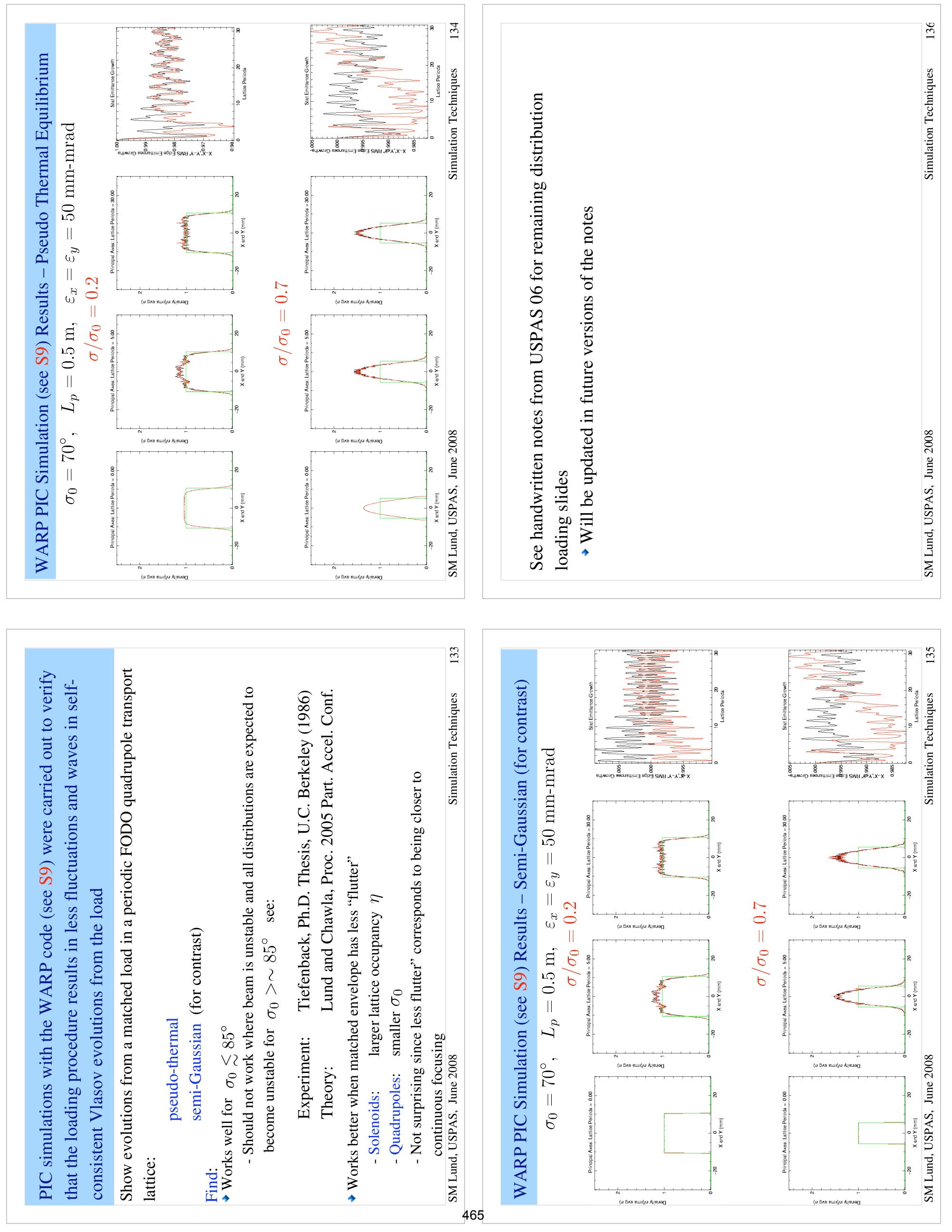

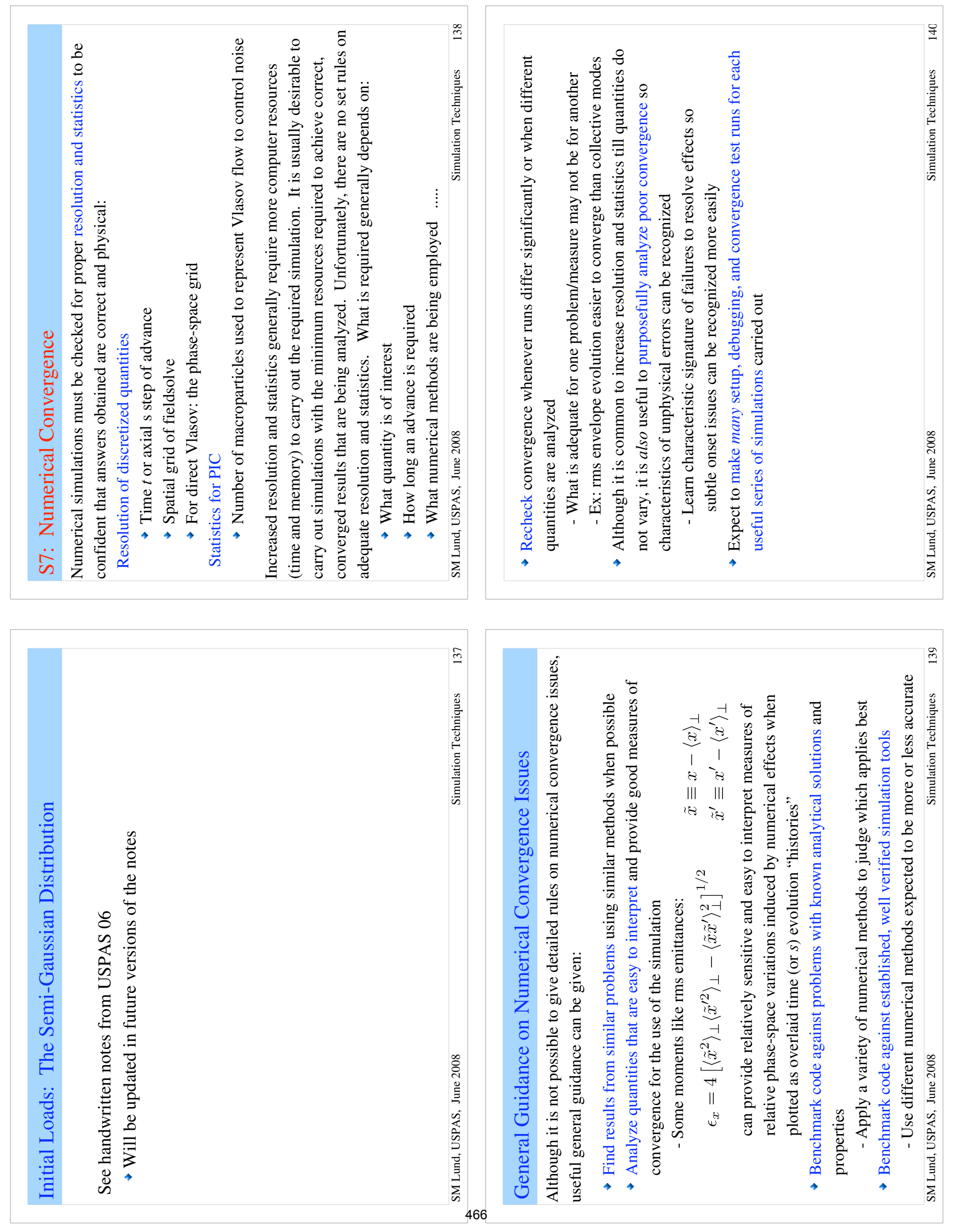

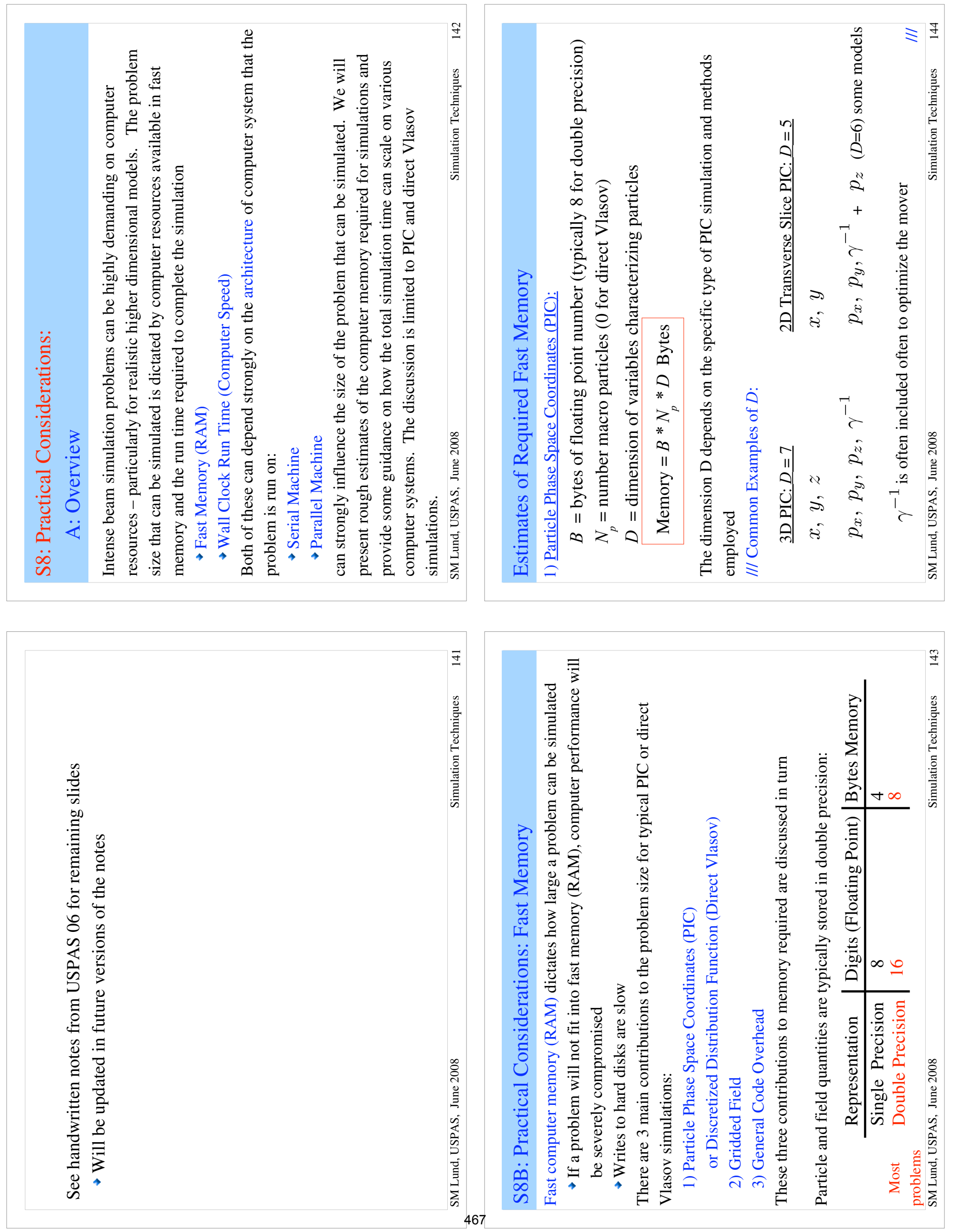

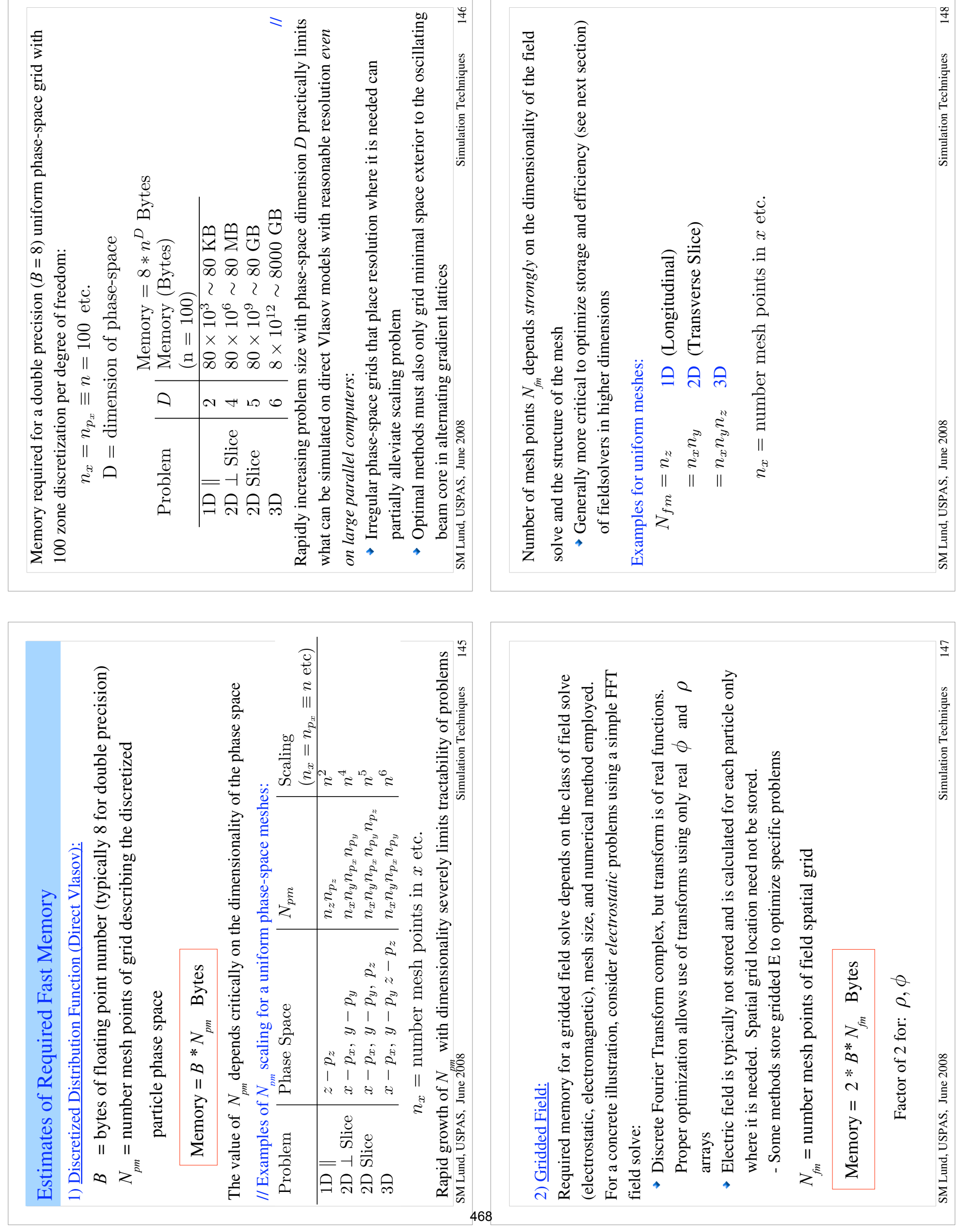


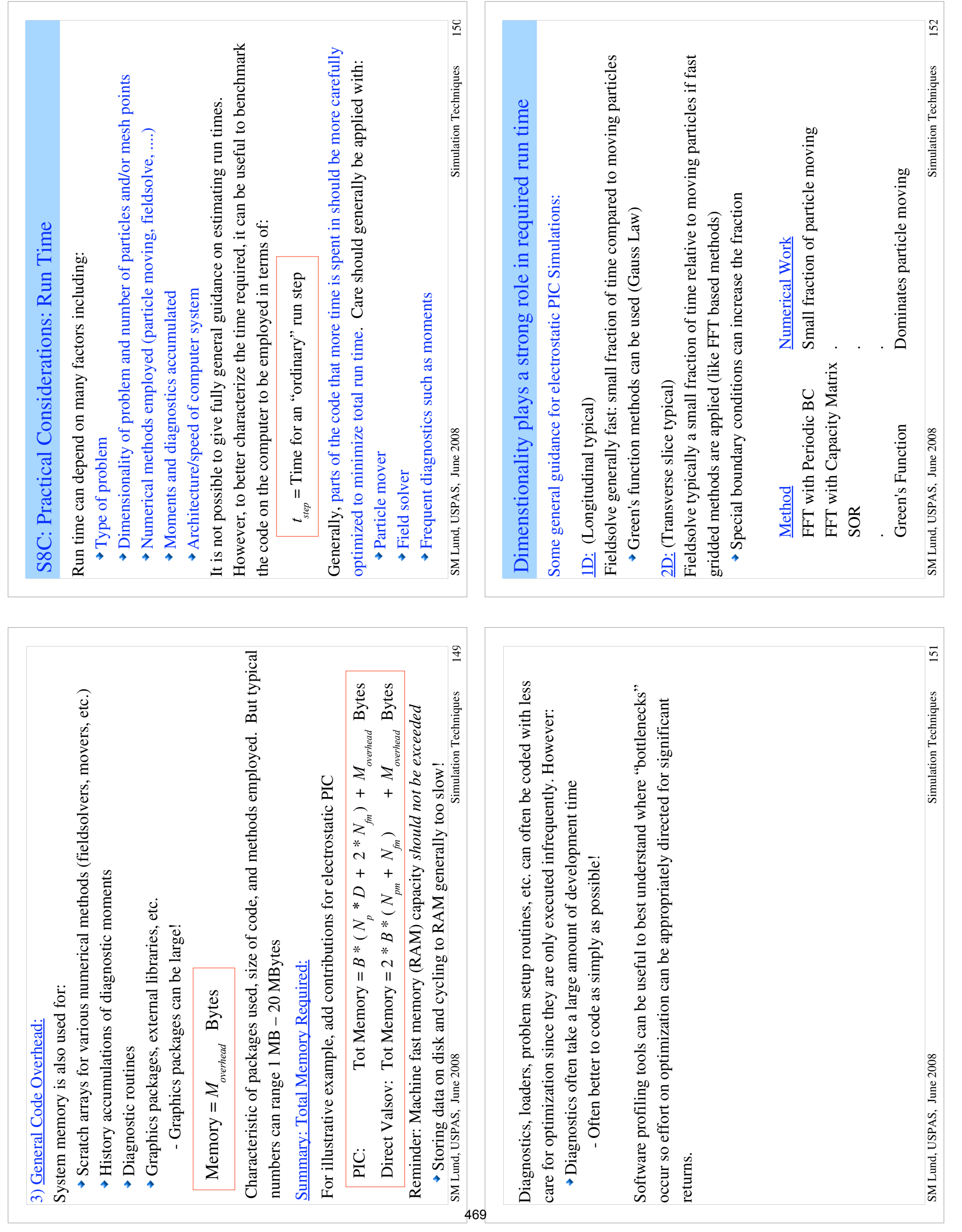



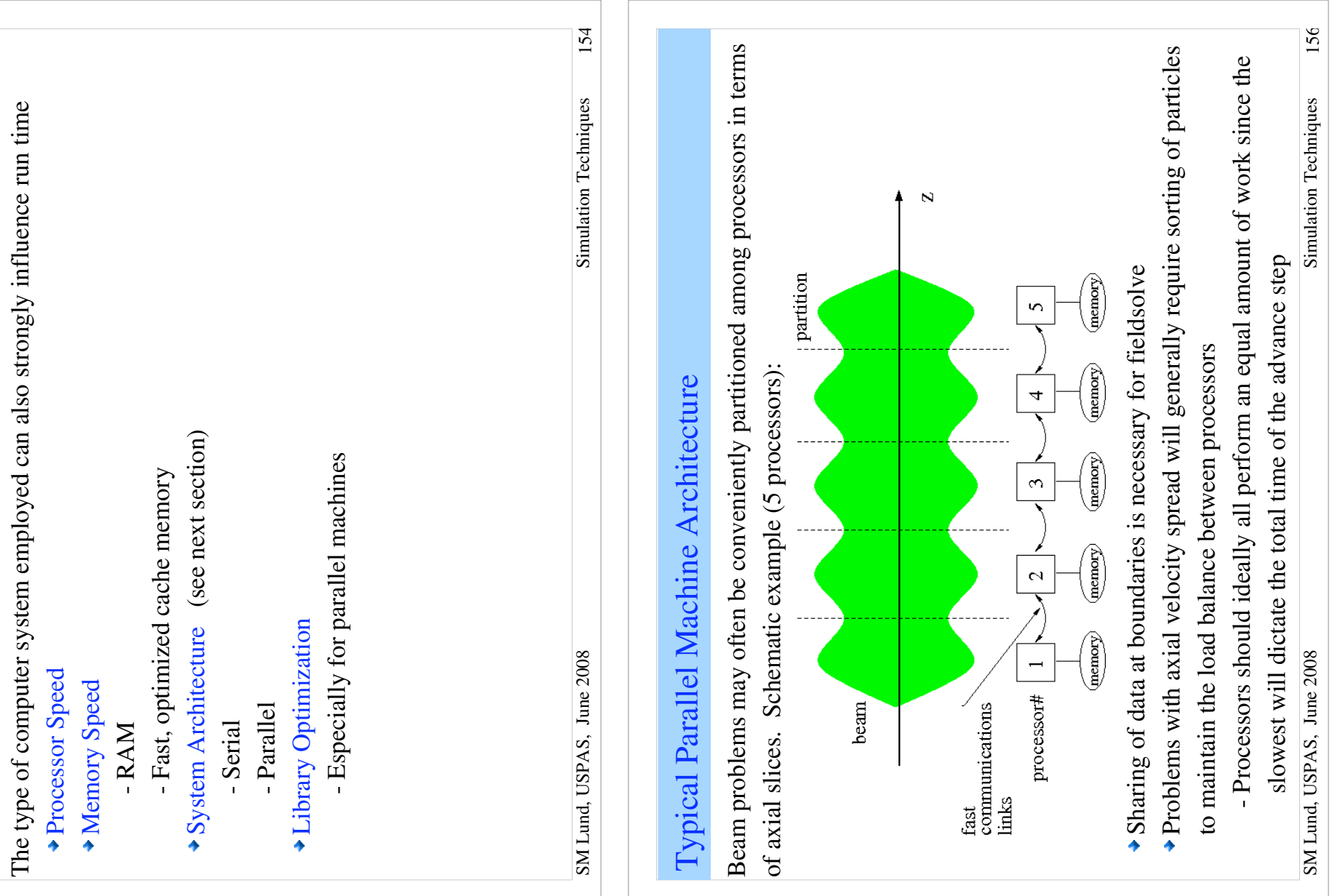

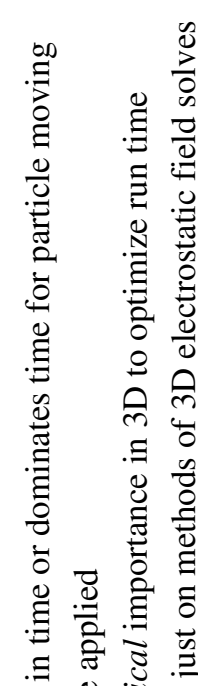

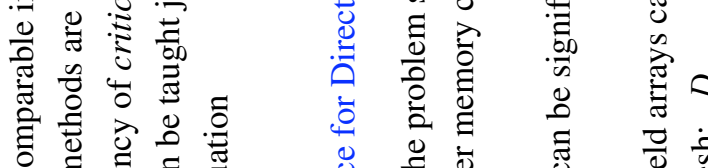

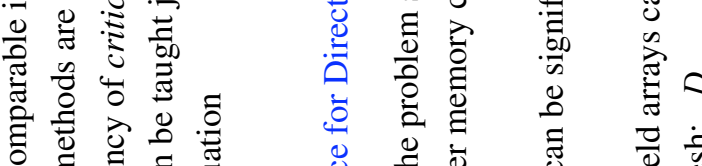

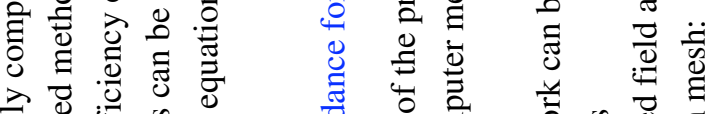

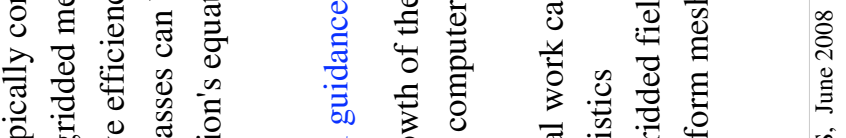

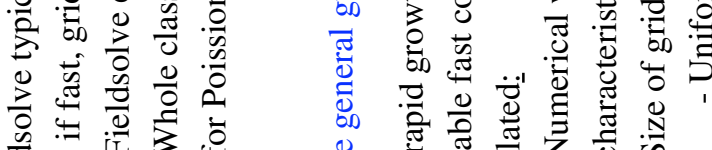

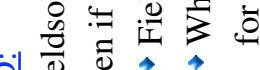

लो

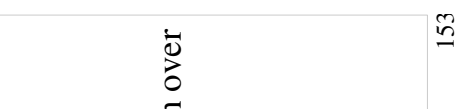

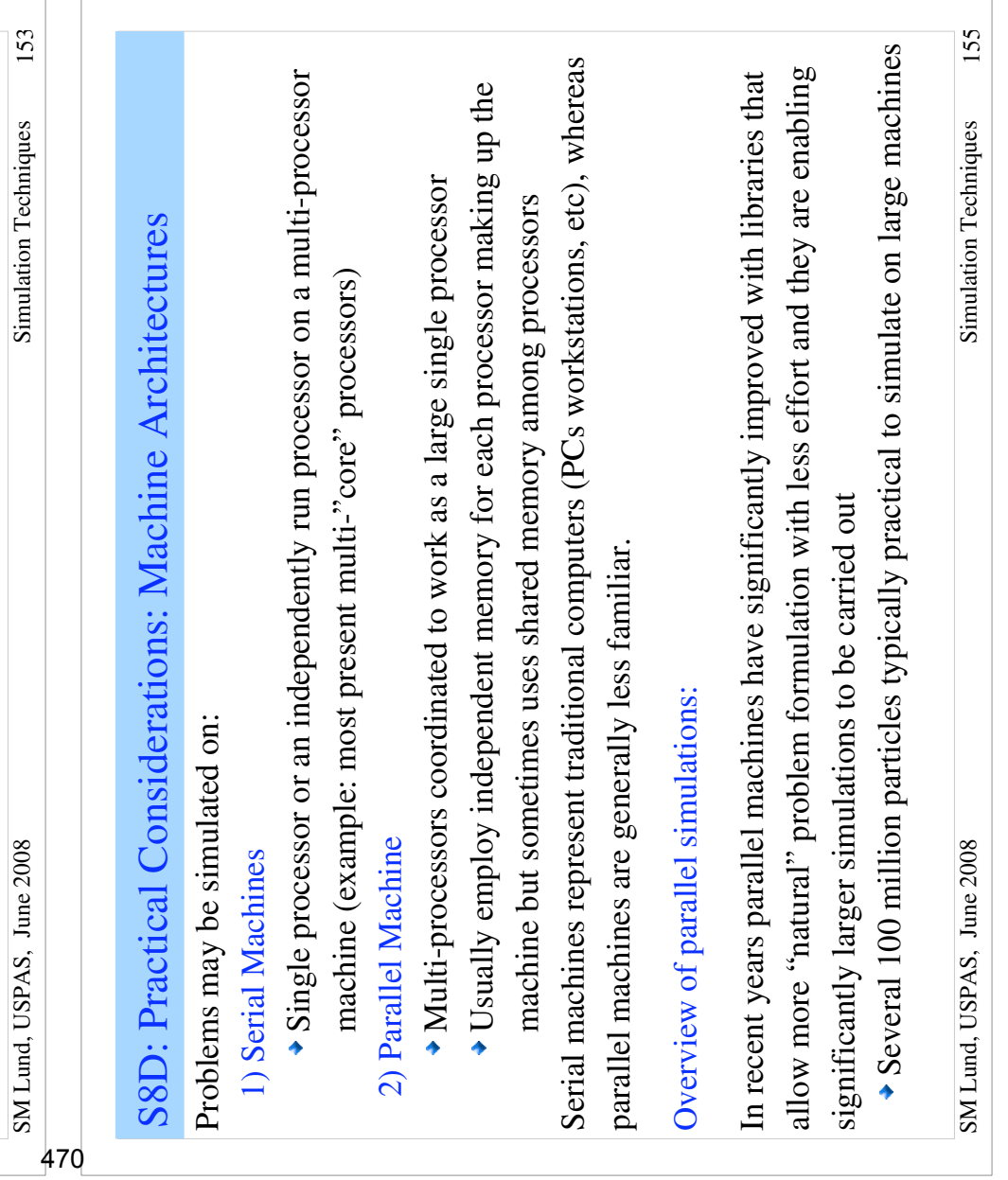




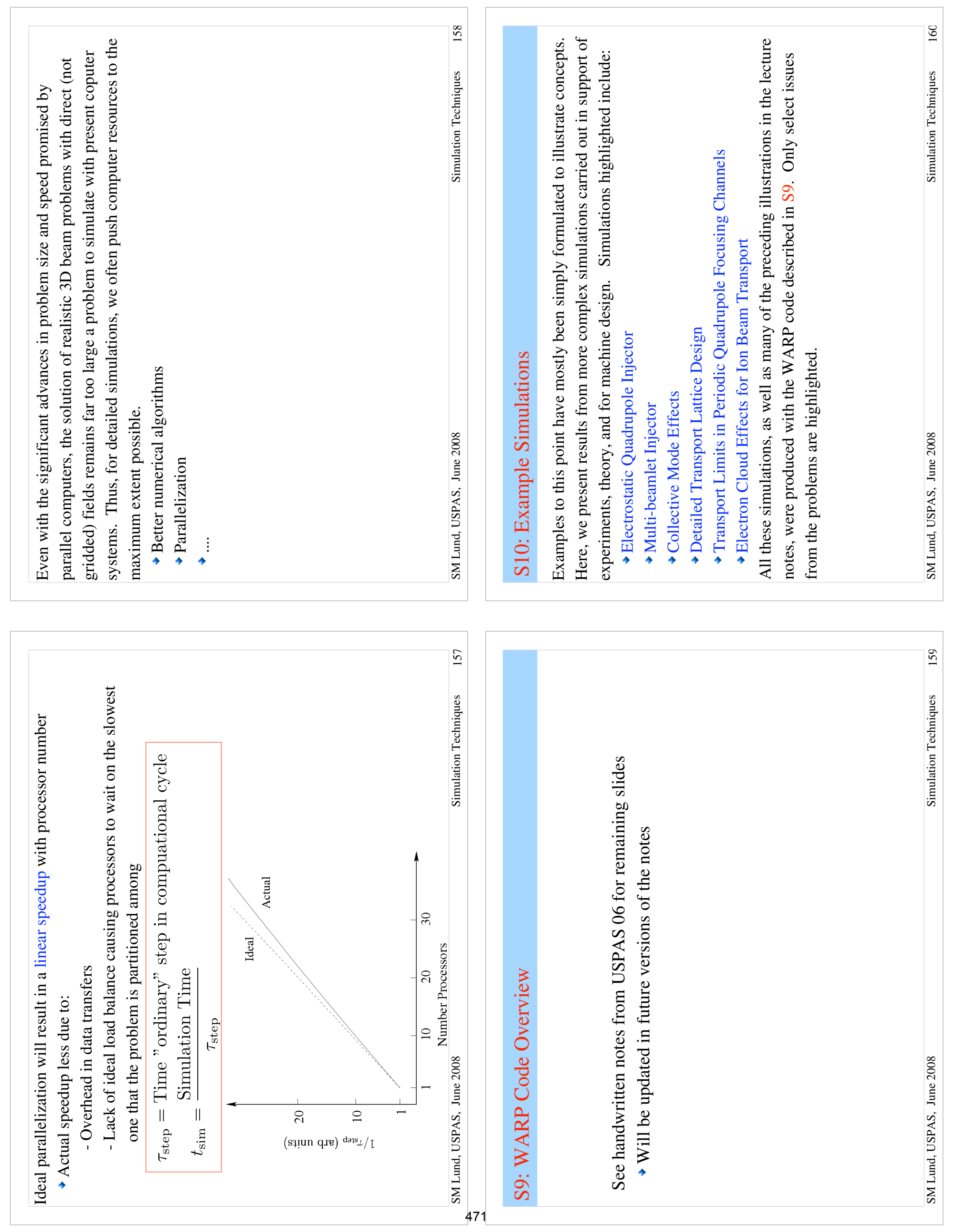




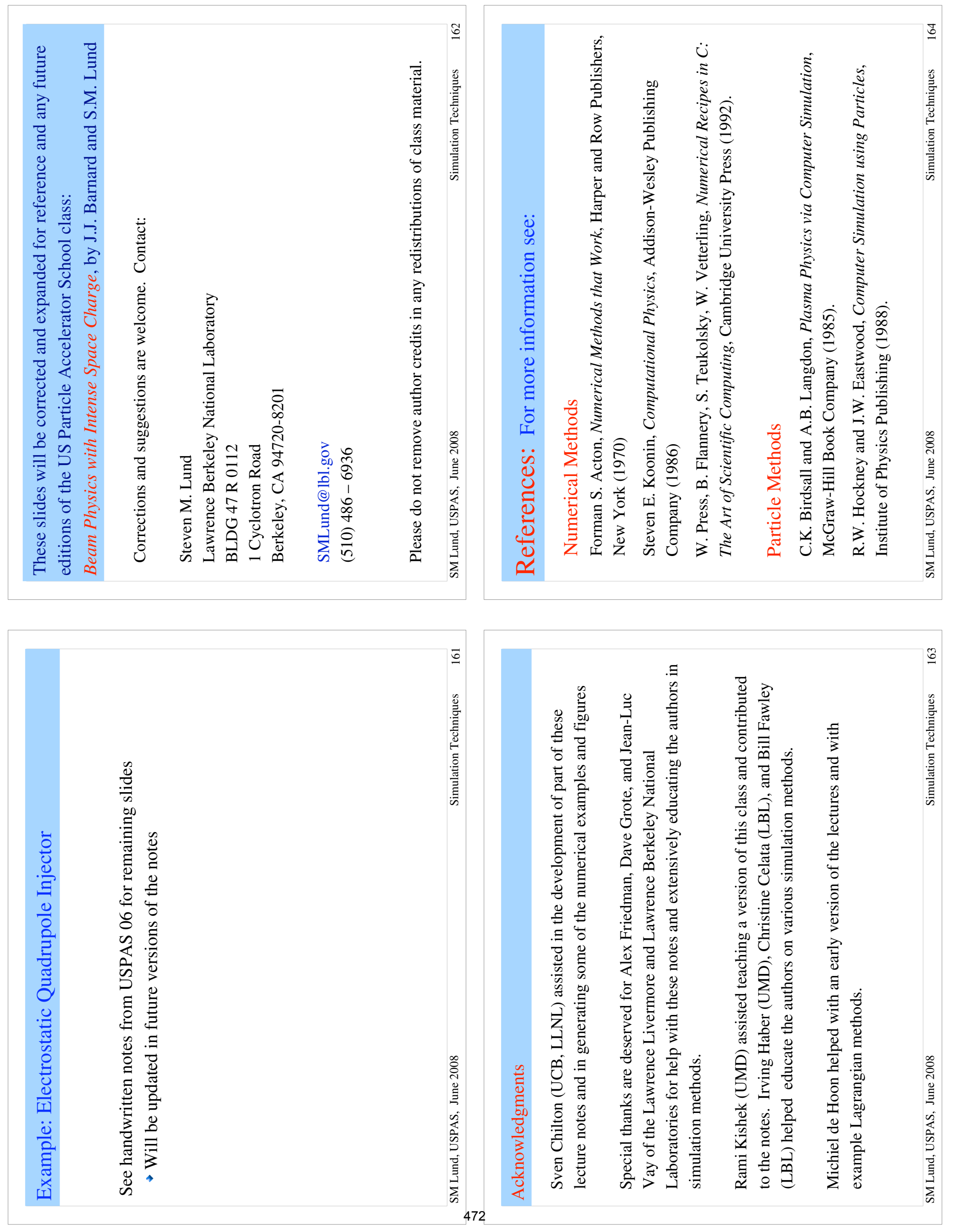


US Particle Accelerator School 2008

S.M. Land

28

\$5 Diagnostics: simulation Techniques - Supplement.

Depend on what is analyzed. Typical choices:

- Moments - statistical sums over the particle distribution (slices and foll beam), often plotted as time histories as the beam evolves in the accelerator:

centroid: $\quad x_{c}=\langle x\rangle$.......

RMS Widths: Beam Size $r_{x}=2\left\langle\left(x-x_{c}\right)^{2}\right\rangle^{1 / 2}$, w.... etc.

Emittance: $\quad \varepsilon_{x}=16\left[\left\langle\left(x-x_{c}\right)^{2}\right\rangle\left\langle\left(x^{\prime}-x_{c}^{\prime}\right)^{2}\right\rangle\right.$

$$
\left.-\left\langle\left(x-x_{c}\right)\left(x^{\prime}-x_{c}^{\prime}\right)\right\rangle^{2}\right]^{1 / 2}
$$

Here; $<-\cdots+\cdots\rangle_{1}=\frac{1}{N_{s}} \sum_{i}^{\text {stree }}\{\ldots \ldots \ldots\}_{i}$

etc,

Slice remittance

"history"

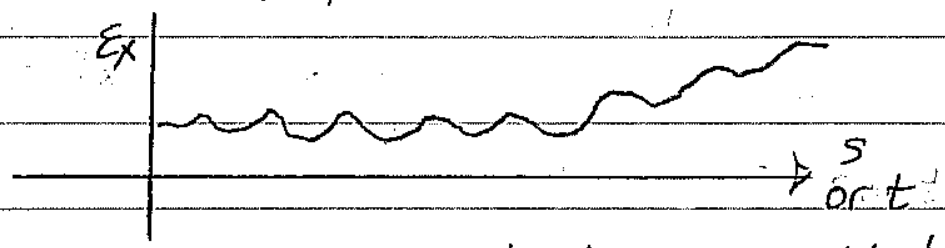

- Particle phase - space projections plotted as snapshots in time $\left(x-x^{\prime}, y-y^{\prime}, x-y, x^{\prime}-y^{\prime}, \ldots \ldots, \ldots\right)$.

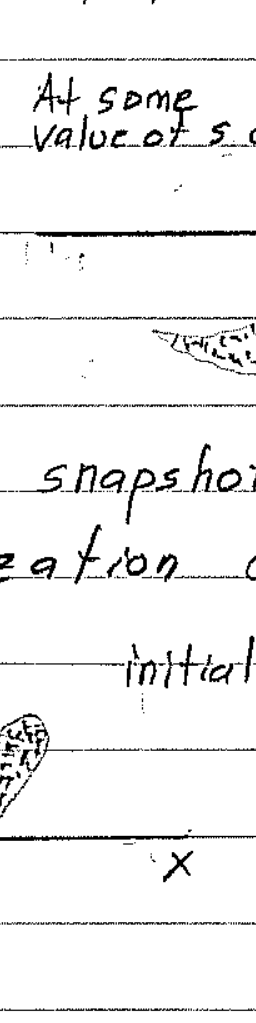

$4 x^{\prime}$

scatter plot of enough macro -particles to Visualize the phase -volume and structure of the beam.

Plotting snapshots at periodic locations allows visualization of the evolution of beam distortions:
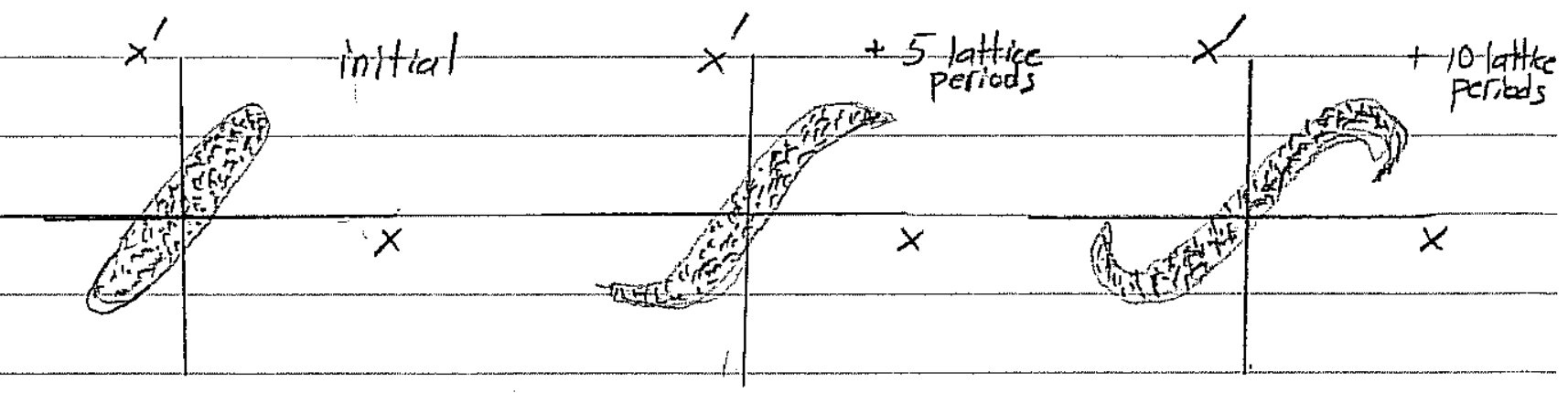

time 
S.M. Lond

129

- Field Diagnostics

Contours of self and applied fields illustrate fled structure. Examples:

1) Total potential cantors of $\phi$ (set and applied) of an elliptical beam in in electric quadrupole formed by 4 biased rods.

same of contours with
beam particles. plotted
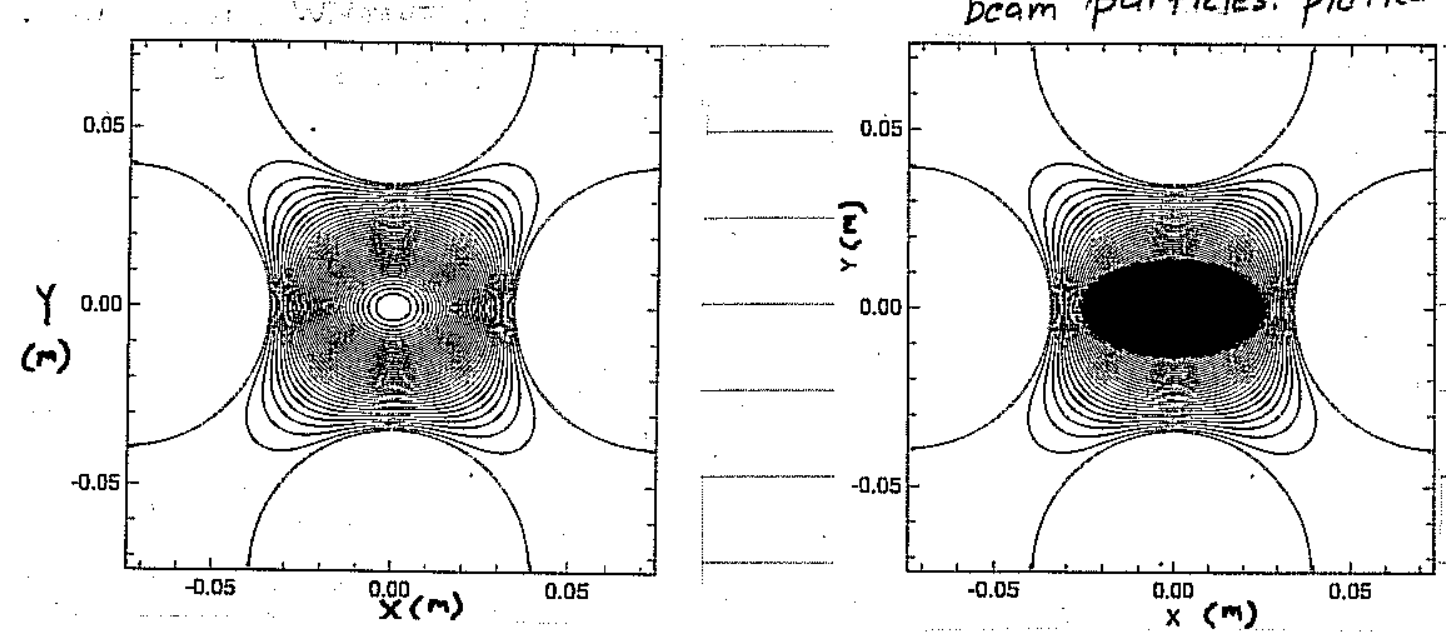

2) Applied Field Scalar magnetic potential contours of a permanent magnet lease Illustrate the structure of applied field nonlinearities:

8 Block

Permanent

Magnet

Quadrupole.

Permanent

Magnet $\mathrm{y}$

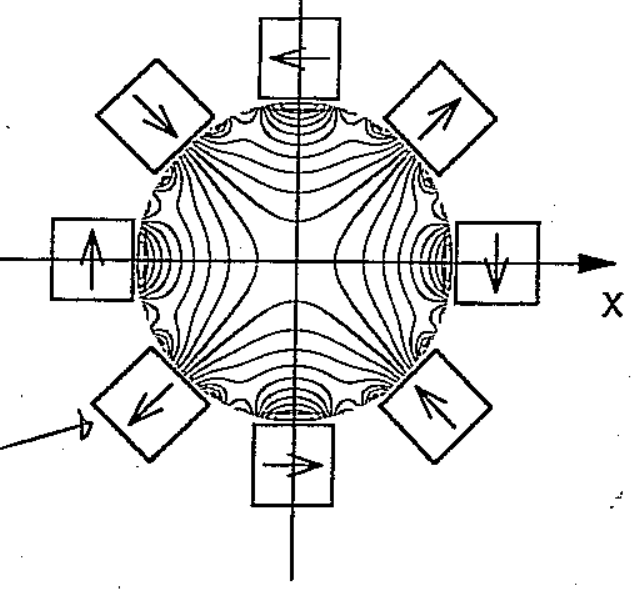

(a)

Note:

contours deviate from hyperbolic near apeture edge.

Numerous other field diagnostics are possible:

- Field energy ,s multiple moments,

474 
S.M. Lond $30 /$

$\$ 6$ Particle Loads

To start the simulation, one must specify and "load" the initial distribution function.

In realistic accelerators, focusing elements are s-varying, In such situations there are'ingeneral no known equilibrium distribution functions. Moreover, it is unclear in most cases if the beam is even best thought of as an equilibrium - perturbations as is Typical in plasms physics. Rather, in accelerators, the beam is injected from a source and may only reside in the machine (especially a lina) for a' small number of characteristic oscillation periods and may not fully relax to an equilibrium =like state. In such situations, so-called "source-to-target" simulations where the particles are simulated off the source and tracked to the target can be most realistic it carried out with realistic focusing fields, accelerating waveforms, alignment errors, etc.

Unfortunately, such idealized source-to-target simulations can rarely be carried out due to computer Limitations.

- Memory limits

- Numerical convergence and accuracy .... 
S.M. Lond

31

Two ways around this I imitation:

1) Load experimentally measured distribution at some point in the machine and advance as an initial condition.

2) Load an idealized initial distribution.

The list option can have practical difficulties: - Diagnostics often are far from an ideal GD "snapshot" of the beam phase - space. - Much information typically lost.

- Process of measuring the beam can itself change the beam.

Most commonly some experimental measures such as:

- rms beam sizes res, ry $\rightarrow r_{x}^{\prime}, r_{y}^{\prime}$

- rims remittances $\varepsilon_{x}$, $\varepsilon_{y}$

are loaded in the form of idealized distributions.

476 
$S, M$, Lond

32

It can be insightful to initialize the beam in a simplified manner

- Fever simultaneous processes can allow one to more clearly see how limits arise.

- Seed perturbations of relevance when analyzing resonance effects, instabilities, halo, eta.

In these situations it is often useful to load a round, continuously focused distribution such as i,

- Thermal Equilibrium

- Waterbag

- $k V$

and then transform the particle coordinates to "match" the local focusing structure of the lattice using a local $\mathrm{kV}$ envelope solution:

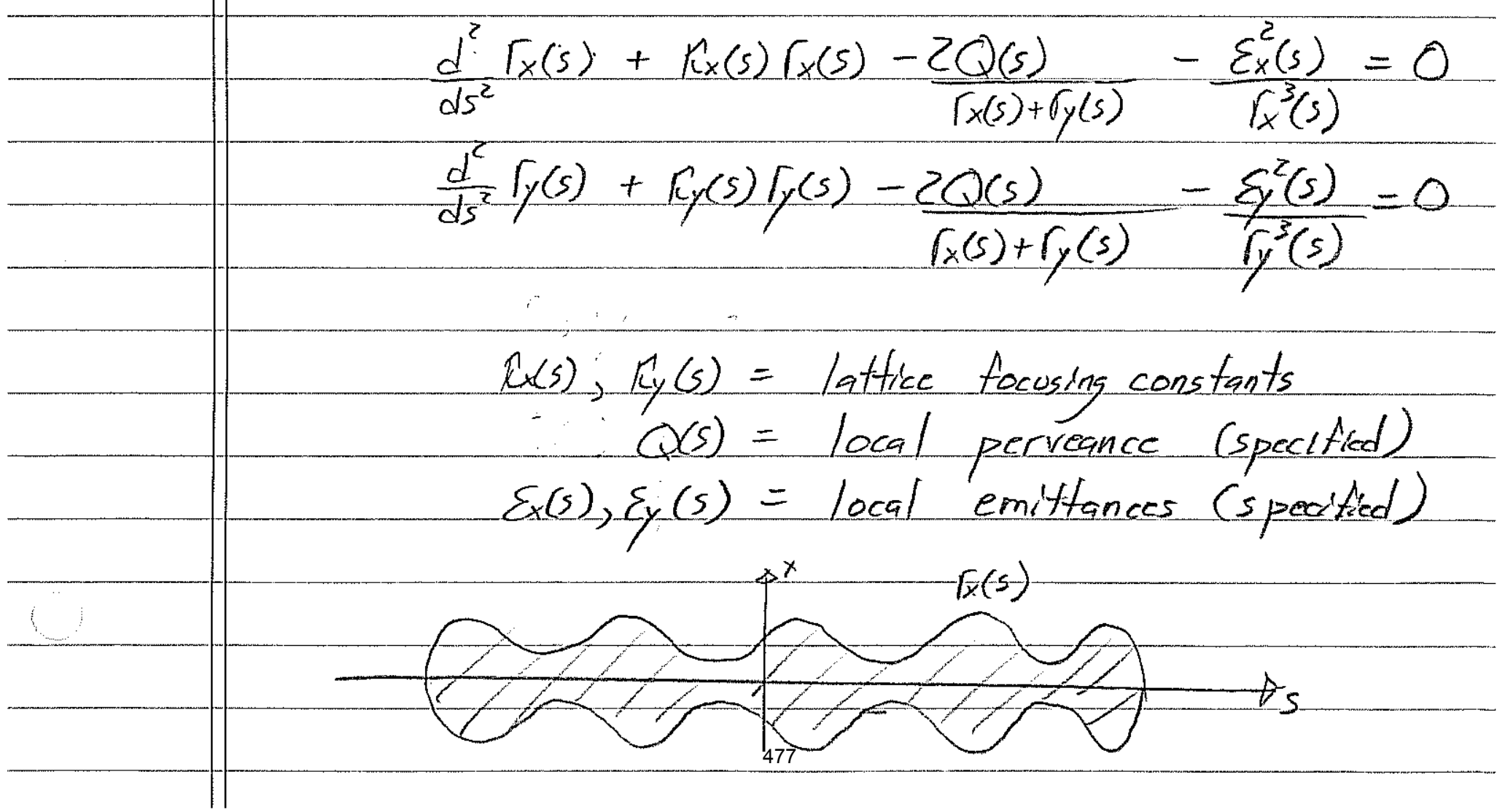


S.M. Lond

33

Procedure:

st

Load in each 1 slice round, continuous distribution:

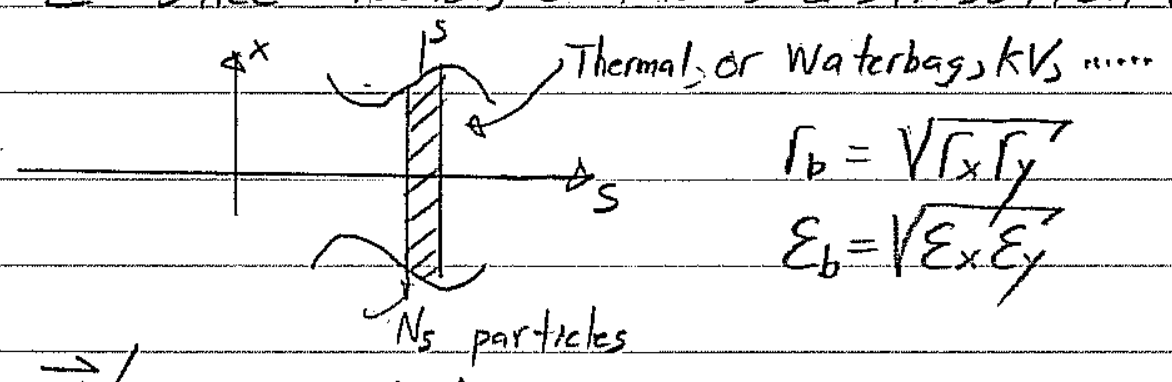

$$
\Rightarrow \quad \vec{x}_{i}, \vec{x}_{i}^{\prime} \text { specified }
$$

Ind

elliptical

Transform the spatial coordinates to match the envelope structure

$3 r d$

$$
\begin{aligned}
& x_{i} \longrightarrow \frac{\sqrt{x}}{\sqrt{b}} x_{i} \\
& y_{i} \longrightarrow \frac{\sqrt{y}}{\sqrt{b}} y_{i}
\end{aligned}
$$

Transform the local thermal velocity spreads to obtain the right average thermal force.

$$
\begin{aligned}
& x_{1}^{\prime} \longrightarrow \frac{\varepsilon_{x}}{\varepsilon_{b}} \frac{\sqrt{b}}{\sqrt{x}} x_{i}^{\prime} \\
& y_{i}^{\prime} \longrightarrow \frac{\varepsilon_{y}}{\varepsilon_{b}} \frac{\sqrt{b}}{r_{y}} y_{i}^{\prime}
\end{aligned}
$$

th

Add the correct coherent velocity to match the needed envelope angle,

$$
\begin{aligned}
& x_{i}^{\prime} \longrightarrow x_{i}^{\prime}+r_{x}^{\prime} \frac{x_{i}}{r_{x}} \\
& y_{i}^{\prime} \longrightarrow y_{i 48}^{\prime}+r_{y}^{\prime} \frac{y_{i}}{r_{x}}
\end{aligned}
$$


S.M. Lond 34

I/ Aside - The semi-Gaussian Distribution

It is not necessary to always load something based on a transformation of an equilibrium distribution to get a good quiescent load. Note that for high space -charge intensities:

- Beam space charge will be more or less uniform out to the edge, where the density will rapidly fall to zero.

- If the beam is injected off a uniform tempeture source or has relaxed, one expects roughly uniform thermal velocity spread across the cross-section of the beam,

This suggests the so-called "sem i-Gaussian" load specified as follows:

- Uniform density within an elliptical bean envelope.

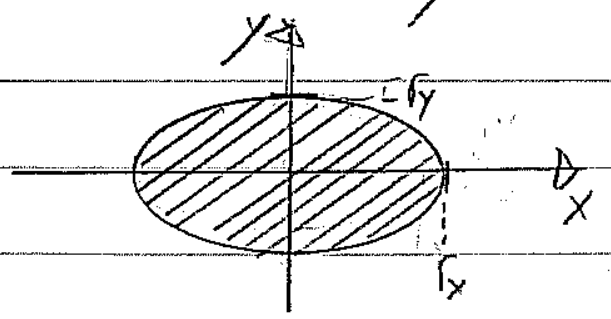

$x_{i}, y_{i}$ uniformly distributed for $\left(x / x_{x}^{\prime}\right)^{2}+\left(y / / y_{y}\right)^{2} \leq 1$

- Gaussian distributed thermal velocity spread with the correct coherent velocity to match the needed envelope angles

$$
\begin{aligned}
& x_{p}^{\prime}=\Gamma_{x}^{\prime} \frac{x_{i}}{\sqrt{x}}+\frac{\varepsilon_{x}}{2 \sqrt{x}}{\tilde{x_{x}}}_{y_{i}} \quad \tilde{\bar{x}}_{x_{y}} \text { Gaussian } \\
& y_{i}^{\prime}=\Gamma_{y}^{\prime} \frac{y_{i}^{\prime}}{\sqrt{y}}+\frac{\varepsilon y}{4 \sqrt{4} r_{y}} \tilde{F}_{i}
\end{aligned}
$$




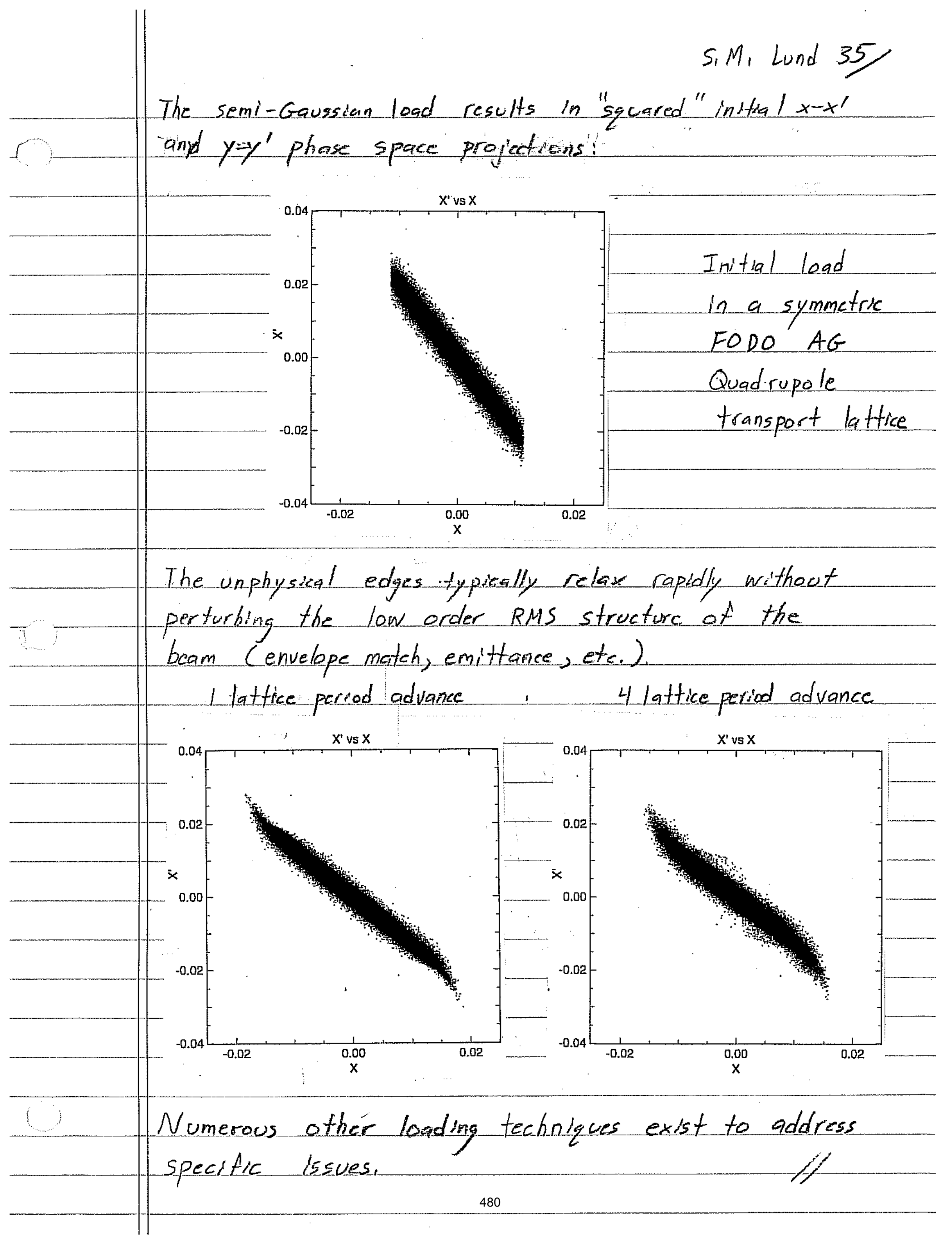


S, M, Lond

36

I"7 Numerical Convergence

Numerical simulations must be checked for proper resolution and statistics to be confident that answers obtained are correct and physical.

Resolution on i discritized quantities

- Time step in particle advance

- Spatial grid for fields

Statistics (number of macroparticles) to control noise.

More resolution and statistics require more computer time and memory, To be practical, one generally wishes to solve problems with the minimum resources required to achieve correct, converged answers. Unfortunately, there are no set rules on what resolution and statistics are required. This depends. On what one is examining w how long the simulations are run, what numerical methods a re employed,....

Some general guidance:

- The statistical rms emittances:

$$
\begin{aligned}
& \varepsilon_{x}=4\left[\left\langle x^{2}\right\rangle_{1}\left\langle x^{\prime 2}\right\rangle-\left\langle x x^{\prime}\right\rangle_{+}^{2}\right]^{1 / 2} \\
& \varepsilon_{y}=4\left[\left\langle y^{2}\right\rangle_{\perp}\left\langle y^{\prime \prime}\right\rangle_{1}-\left\langle y y^{\prime}\right\rangle_{1}\right]_{\text {and easy }}^{1 / 2}
\end{aligned}
$$

often prove to be sensitive and easy to interpret differences when plotted as our bid tine numerical

- picks up ismail phase space distortions induced 
S.M1 Lond 37

general guidance continued ....

- To get started find results from similar problems using similar methods.

- Benchmark code and methods against problems with known analytical solutions, established codes using both' similar and different numerical methods,...

- Recheck numerical convergence whenever runs differ significantly or when differing quantities are analyzed.

- What is adequate for one measure of the beam (say linage charge structure) may not be for another (say collective modes).

- Although it is common to increase resolution and statistics. till relevant quantities do not vary, it is also useful to purposefully analyze poor resolution and statistics regimes so the characteristics of unphysical numerical errors can be recognized.

- Expect to make many setup, convergence, and debugging runs for each useful series of simulations carried out.

482 
Specific Numerical Convergence

S.M. Lind

38

Comments:

Time Resolution,

These comments are applicable to both spatial or time steps of the particle advance, we will frame estimates in terms of timesteps.

- Particle coordinates should not move through more than one cell in a single step, This is a standard "Courant" condition:

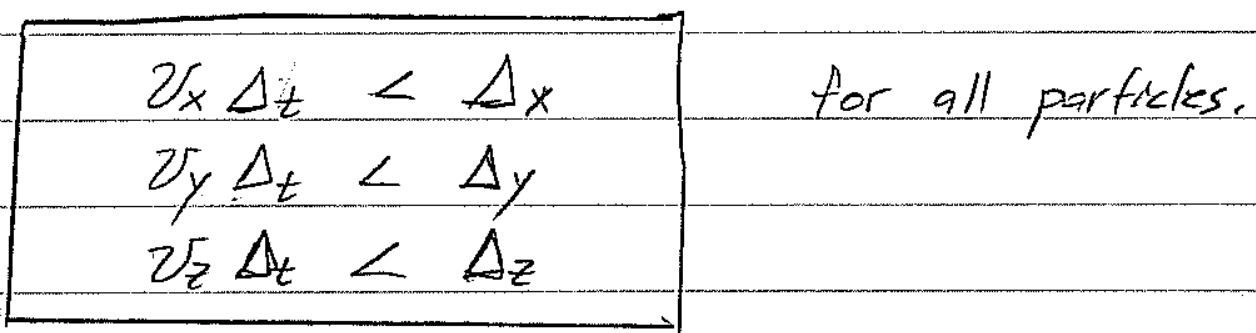

- Enough steps should be taken to resolve variations in applied field structures. ki

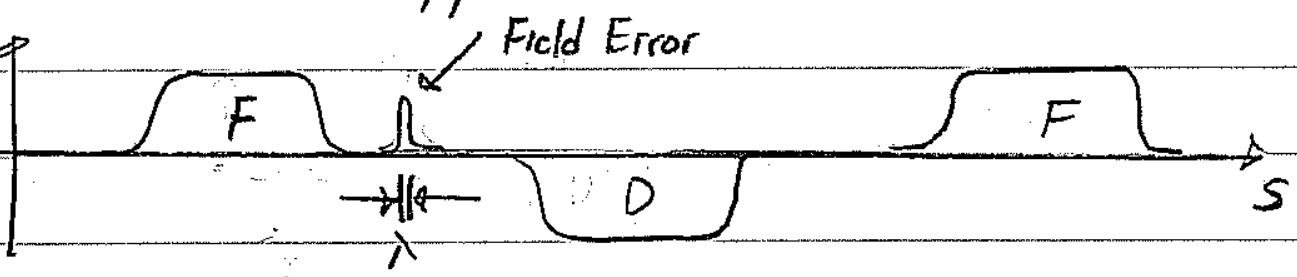

$$
\begin{aligned}
& v_{s} \Delta_{t} \leq \lambda \quad i \quad \lambda=\text { shortest wavelength of } \\
& \text { field structures to be } \\
& \text { modeled }
\end{aligned}
$$

- Phase variations in collective. waves (if of interest). should be resolved, for a leap-frog mover this requires:

$\omega=$ mode frequency component

$$
\frac{\omega \cdot \dot{\Delta}_{t}}{2 \pi}<\frac{1}{2}
$$


S.M L Lind

39.

Spatial Resolution

For cold beams. the beam edge can be sharp for most reasonable disfolbution functions:

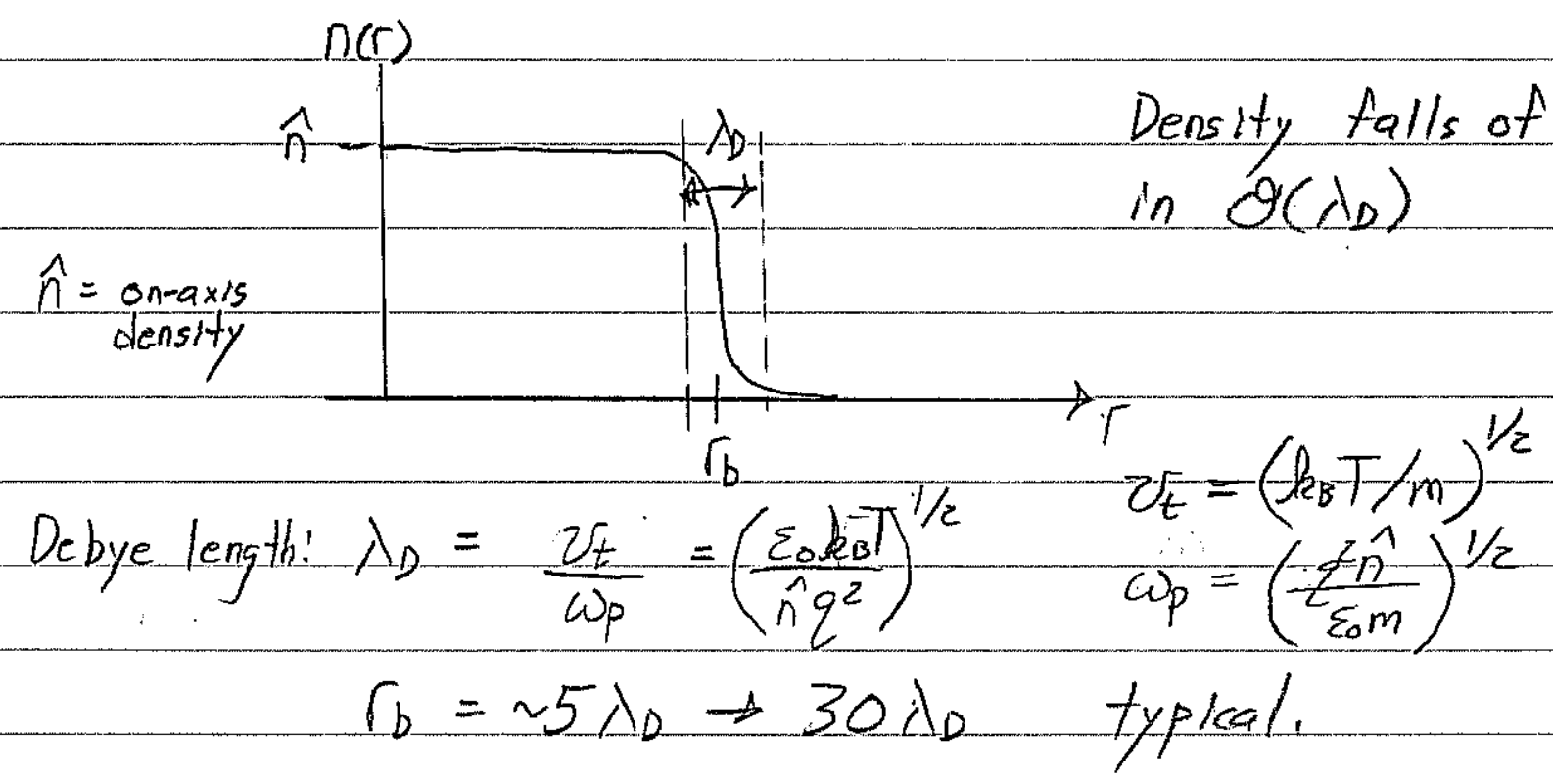

To resolve edge physics, the mesh should have several cells across the rapid density variation near the edge of the beam.

$$
d x, d y<\frac{\lambda D x}{2} y
$$

$$
\begin{aligned}
& \lambda_{D x_{y y}}=\left(\frac{k_{B} T_{x y y}}{m}\right)^{1 / 2} \\
& T_{x y}=\text { local kinetic } \\
& \text { temperature }
\end{aligned}
$$

The mesh should also resolve relevant spatial scales associated with processes of interest

- If applied electrostatic fields are calculated from biased conductors, the mesh should resolve conductor structures or special corrections should

- Self-fied fluctuations induced by collective modes should bet resolved. 
S.M. Lond

41

Statistics.

Collective effects typically require.

having a significant number of particles $N_{D}$ within the characteristic screening radius characterized by the Debye length

CD:

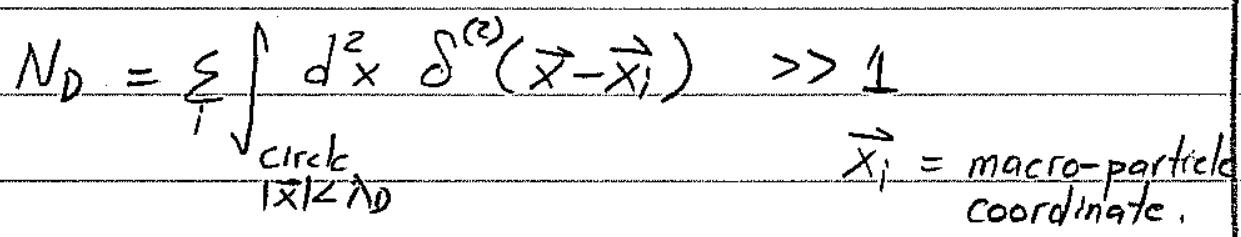

SD!

$$
N_{D}=\sum_{i} \int_{\substack{s p h e r c \\|\vec{x}|<\lambda_{D}}} d^{3} x \delta^{(3)}\left(\vec{x}-\vec{x}_{i}\right) \gg 1
$$

where:

$$
\begin{aligned}
& \lambda_{D}=\frac{v_{t}}{\omega_{p}}=\left(\frac{\varepsilon_{0} k_{B} T}{\hat{n}_{q}^{2}}\right)^{1 / 2} \quad v_{t}=\left(k_{B} T / m\right)^{1 / 2} \\
& \sum_{i} \Rightarrow \text { sum over all macro particles. }
\end{aligned}
$$

In simulations of higher order collective modes it may also be necessary to have a significant number of particles per cell on a mesh that resolves the relevant spatial variations of mode induced self -field fluctuations.

ID: $\quad N_{\text {cell }}=\sum_{i} \int_{c \text { ell }} d_{x}^{2} \delta^{(2)}\left(\vec{x}-\vec{x}_{i}\right) \quad \gg 1$

SDi $\quad N_{\text {cell }}=\sum_{i} \int_{\text {cell }} d^{3} x \delta^{(3)}\left(\vec{x}-\vec{x}_{i}\right) \gg 1$

- Larger Nell prevents local self fields from being noise dominated. - Larger Neal leases to larger No typically 
S.M. Lond

42

Good statistics are only needed in the beam core with the possible exception of certain beam -halo problems and near the beam edge.

- Most beams will only occupy a fraction of the foll grid.

Boundary structures for Electric Quadrupole.

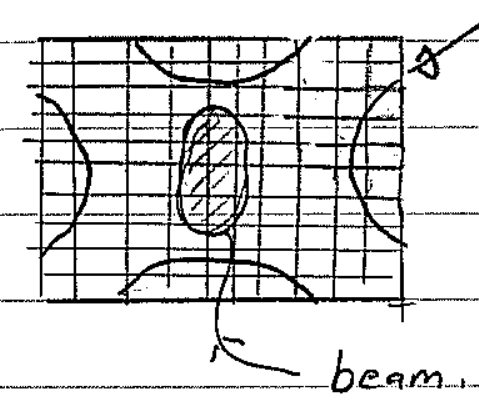

statistics should be evaluated in the cells that the beam occupies rather than average grid measures.

No comprehensive rules exits for how good the statistics must be. Individual problems must be checked and verified. Some general comments:

- What is adequate will typically depend on what is analyzed

- Image fields may be resolved with few particles

- Collective waves may take many particles if low nose (interpretable) diagnostic projections as e necked.

- Longer runs generally require increased statistics

- Poor statistics result in un physical collislonally that is often characterized by a linear rise in beam emittanezs with simulation time. 
S.M. Lind

$43 /$

Classes of Particle Simulations.

How important is smoothing?

3D Beam: $\quad N \sim 10^{10}-10^{14}$ particles typical

Simulations: $\quad N \approx 10_{\substack{\text { (modern parallel) } \\ \text { computers }}} ;$ typical $10^{3}-10^{6}$

Each simulation particle may represent: $10^{3} \rightarrow 10^{\prime \prime}$ particles in the real beam" for 3D simulations.

- Smoothing involved with particle weightings are key to obtaining physical answers. and limiting collisionality.

Is the situation really this bad?

- Lower dimensional models typically simulated.

3D Model

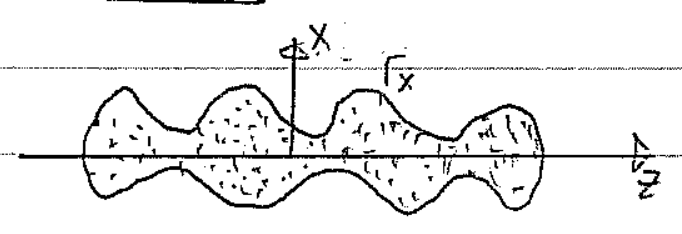

Physical charge - point charges

$$
p=\sum_{i} g \delta\left(x-x_{i}\right) \delta\left(y-y_{i}\right) \delta\left(z-z_{i}\right)
$$

N point particles with smoothed interactions.

Phase Space:

GD

Smoothed charge

$$
\begin{aligned}
& p=\sum_{i} q_{M} f\left(x-x_{i}, y-y_{i}, z-z_{i}\right) \\
& q_{M}: \begin{array}{l}
\text { Macro } \\
\text { particle } \\
\text { charge }
\end{array}
\end{aligned}
$$

488 
S.M. Lond 44/

ID 1 Thin Slice Model

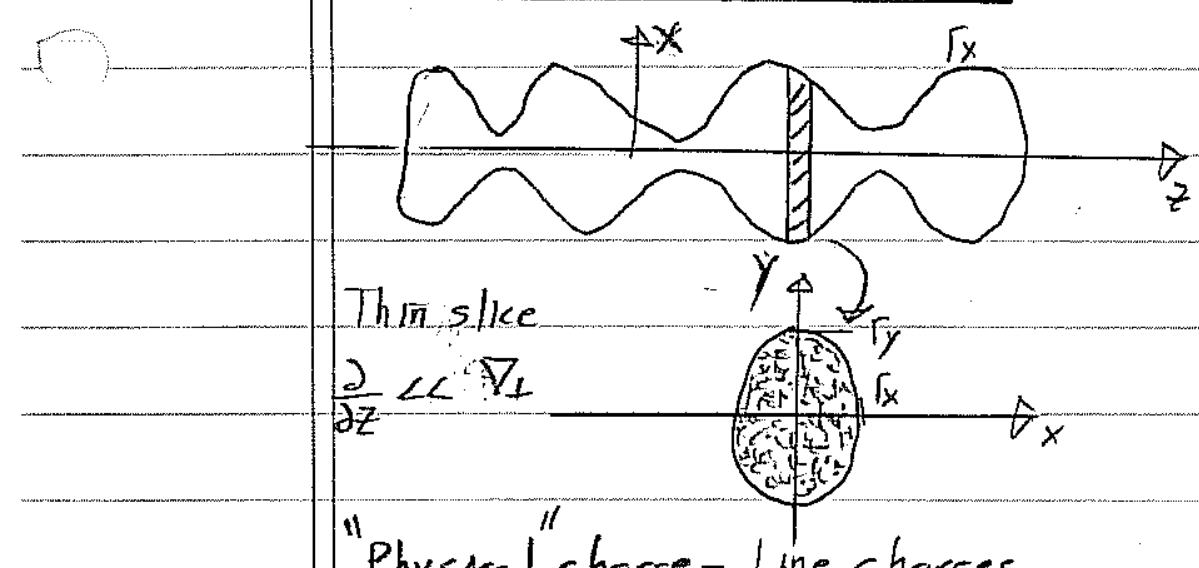

"Physical" charge -1 tine charges

$$
p=\sum_{i} \lambda_{p} \delta\left(x-x_{i}\right) \delta\left(y-y_{i}\right)
$$

N line charges with smoothed interactions.

Smoothed charge- $\quad \lambda_{M}$ : Macropartick

$\rho=\sum_{1} \lambda_{M} f\left(x-x_{i}, y-y_{i}\right)$ if: smoothing function

The slice must be tracked in $s$ with each particle mount the same increment ins with each step so that a slice maps to a slice.

- If $p_{z}$ is included the velocity distribution must be assumed "frozen in".

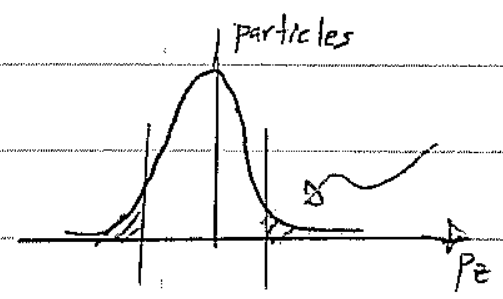

parts that would leave assumed replenished by particles from adjacent slices.

- Response to acceleration may be modeled with

- "Thick" slice models also possible with periodic boundary conditions. on the "slice" to try to recover some 3D effects of a long pulse in a periodic lattice.

489 
S.M. Lond 45\%

ZD r-z Model

$N$ charged Rings with smoothed interactions

Phase Space.

r, $z\} 4 D+$ possible

$1 D$

physical charge - cylindrical rings

$$
\rho=\sum_{i} \frac{Q_{i}}{2 \pi} \frac{\delta\left(r-r_{i}\right)}{\pi_{i}} \delta\left(z-z_{i}\right)
$$$$
\text { prs } p z .\}
$$

Po

(angular mom.)

Smoothed charge 4 or $5 D$.

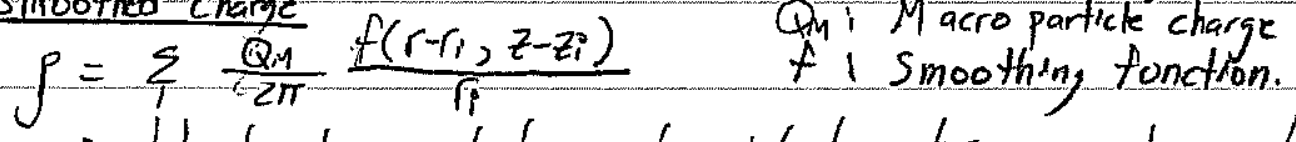

- Used to model solenoidal transport of an initial axisymmetric beam.

- Sometimes used to model AG beans with an approximately equivalent, 5 -dependant focusing force, $\quad \vec{x}_{\perp}^{\prime \prime}=k_{p_{0}}^{2}(s) \vec{x}_{\perp}+\cdots \cdot \cdot$

10 Axisymmetric Model

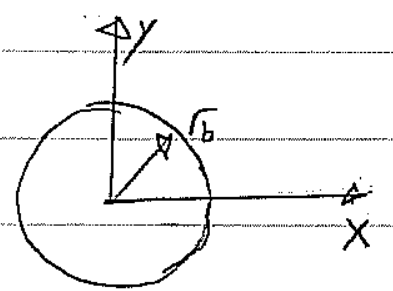

$\partial / \partial \theta=0$

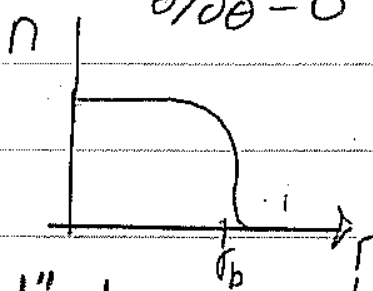

$N$ charged cylinders with smoothed interactions

Phase - Space

$r \geqslant R D+$ possible

$\mathrm{Pr}$

$P_{\theta,} ; p_{z}$

2D to 4D

"physical" charge - cylendricalshect smoothed charge

$$
\rho=\sum_{p} \frac{Q_{1}}{2 \pi} \frac{\delta\left(r-r_{i}\right)}{r_{i}} \quad \rho=\sum_{i} \frac{Q_{M}}{2 \pi} \frac{f\left(r-r_{i}\right)}{r_{i}}
$$

Qm: Macro change

$f:$ Smoothing function

- Simple model for continuously fac used, axisymmetric beams 
S.M. Lond 46

ID slab Model

N charged slabs with

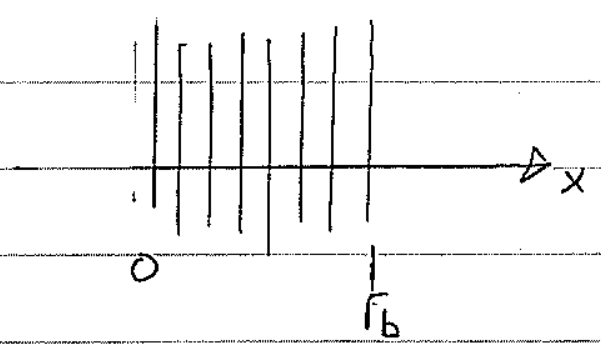
smoothed interactions.

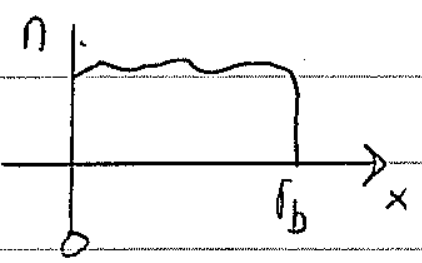

Phase - Space

$x\{Z D+$ possible

$p_{x}\left\{\quad p_{y}, p_{z}\right.$

ZD to $4 D$

"physical" charge - sheets

$$
p=\sum_{i} \delta \delta\left(x-x_{i}\right)
$$

smoothed charge

$$
p=\sum_{i} \delta_{M} f\left(x-x_{i}\right)
$$

$\sigma_{M}:$ Macro paitrek charge

- Most simple model, but slab geometry is least physical.

It is not immediately clear how such different models "can in many cases represent qualitatively similar collective interactions since force laws can change form with dimension. For example, in free space, we find that:

Mode $\mid$ Field due to th "particle"

BD $\quad \vec{E}=\frac{q \cdot\left(\vec{x}-\overrightarrow{x_{i}}\right)}{4 \varepsilon_{0}\left|\vec{x}-\overrightarrow{x_{i}}\right|^{3}}$

$20 \quad \vec{E}=\frac{\lambda_{i}\left(\vec{x}-\overrightarrow{x_{i}}\right)}{2 \pi \varepsilon_{0}\left|\vec{x}-\vec{x}_{1}\right|^{2}} \quad \lambda_{i}=$ line-charge

ID

$$
E_{x}=\frac{\delta_{i}}{2 \varepsilon_{0}} \frac{\left(x-x_{i}\right)}{\left|x-x_{i}\right|} \quad \delta_{i}=\frac{\text { sheet charge }}{\text { "partake" }}
$$

491 
S,M, Lond

47

The reason these radically different interactions can give similar physics is that the screening associated with collective interactions is found to be similar:

- Debye screening has similar characteristics in each dimension.

Showed $2 D$

form, in class.

W HIll show in final

that the

$$
\begin{array}{ll}
\lambda_{D}=\frac{v_{\text {thermal }}}{\omega_{p}}=\left(\frac{\varepsilon_{0} k_{B} T}{\hat{n}^{2}}\right)^{1 / 2} \quad v_{t}=\left(\underline{k}_{B} T / m\right)^{1 / 2} \\
\omega_{p}=\left(\frac{g^{2} \hat{n}}{\varepsilon_{\varepsilon_{0} m}}\right)^{1 / 2}
\end{array}
$$

scaling obtains

the same. Debye intiongth

- It is much easier to have a

significant number of particles within the characteristic screening distances for lower dimensional problems.

- Lower dimensional simulations can more easily resolve collective effects?

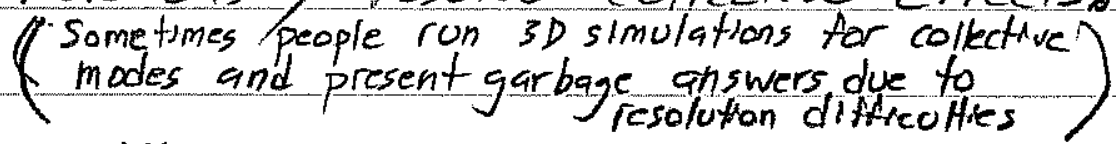

Example

$$
\begin{aligned}
& q=z e \\
& \text { ions } \\
& e=1.6 \times 10^{-19} \mathrm{C}
\end{aligned}
$$

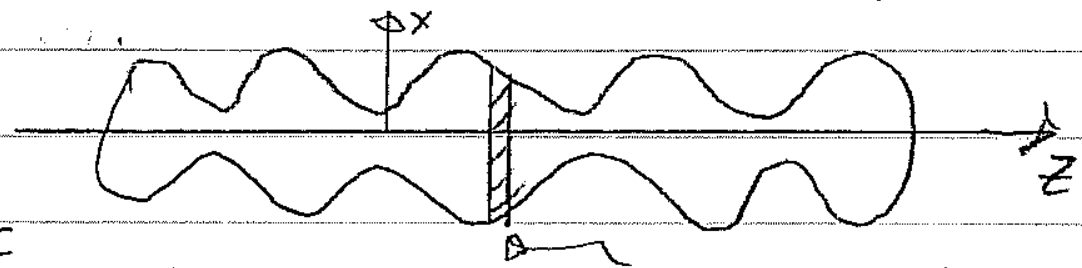

ID $\perp$ thin slice

$$
\lambda \sim 10^{-13} \rightarrow 10^{-7} \mathrm{c} / \mathrm{m}
$$

$$
\text { * particles }=\frac{\lambda}{\mathrm{cm}^{\prime}} \sim \frac{10^{4}}{Z} \rightarrow \frac{10^{10}}{Z}
$$

typical for intense beams

$$
q \equiv z^{z e}
$$

- Smoothing still important in lower dimensions and real beam is 3D

492 
89

S. W. Land Clot

WARP Code CNirryen

Electrostatic Multidimensional PIC code

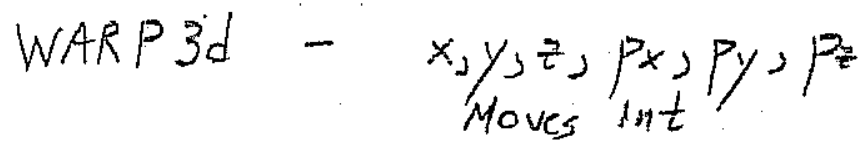

Many Fieldsolvers!

SOR, Moltigrad, FFT,

$F F T+$ Tridiag; FFT + Cap $M_{a}$

WARP XI - $x, y, p_{\text {Moves ins }}$, Pye

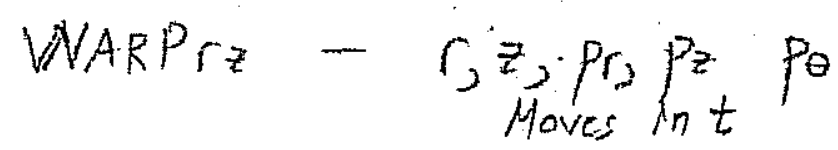

WARPer - envelope solver used to seed/ load PIC $r_{10}\left[r^{\prime}, r_{x}^{\prime}, r_{y}^{\prime}\right.$
Advances ins

Hermes - Fluid 11 t prided.

Space Charge Field

- Common diagnostic tools built around gist.

- Run with python in terpreter

Example Script

"ag-slice.py"

493 
¿10 Example PIC Simulations S.M. Lund 54/

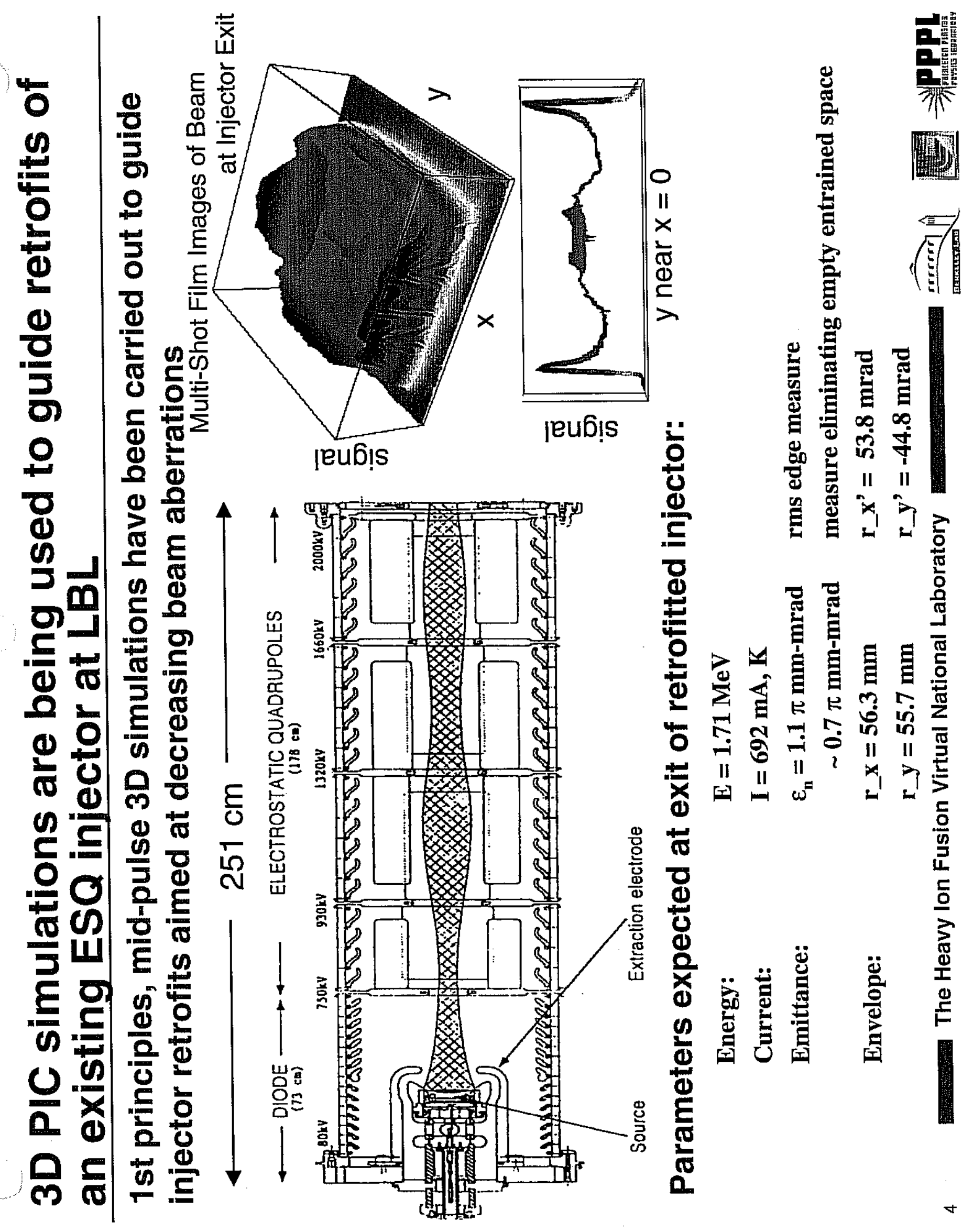




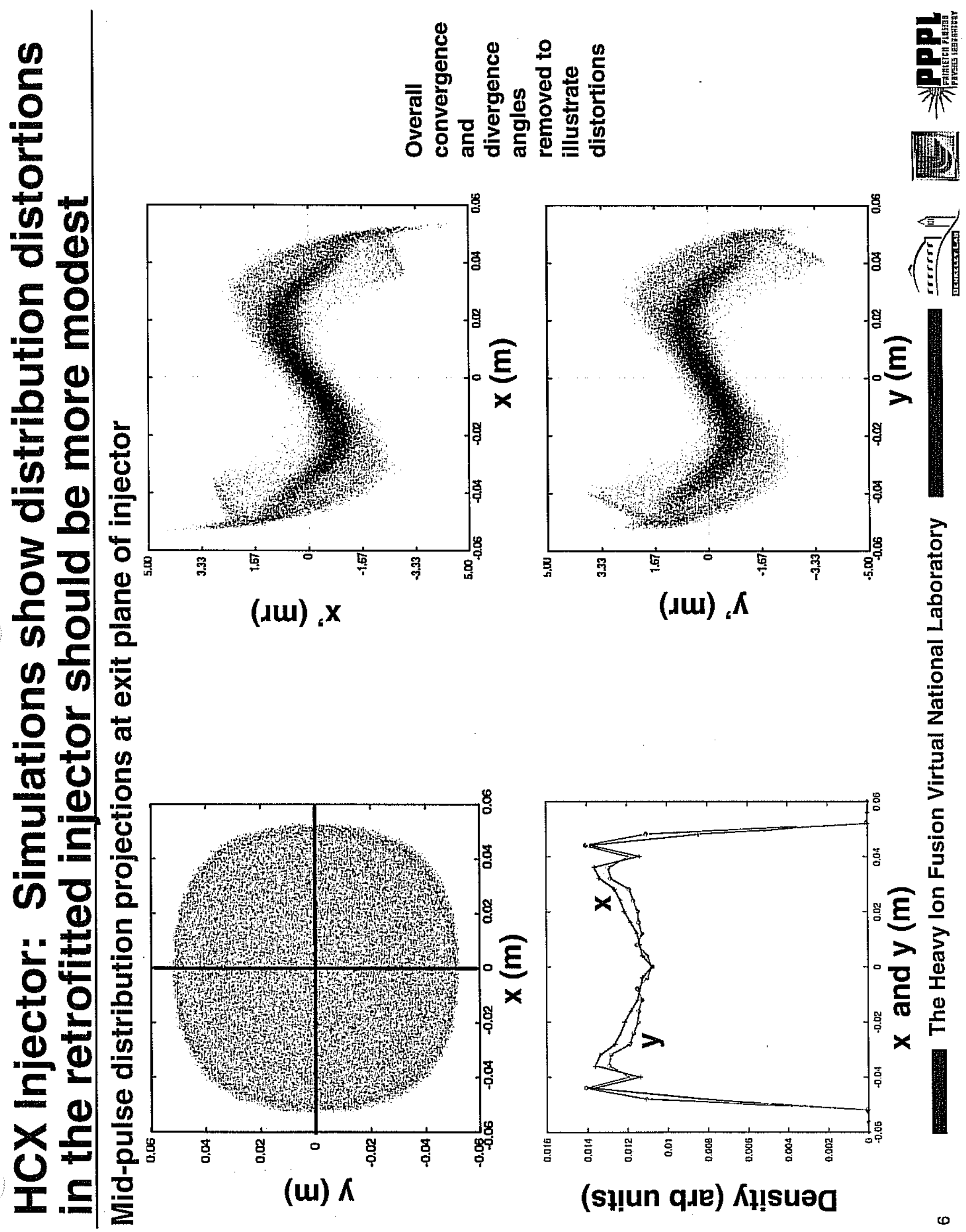




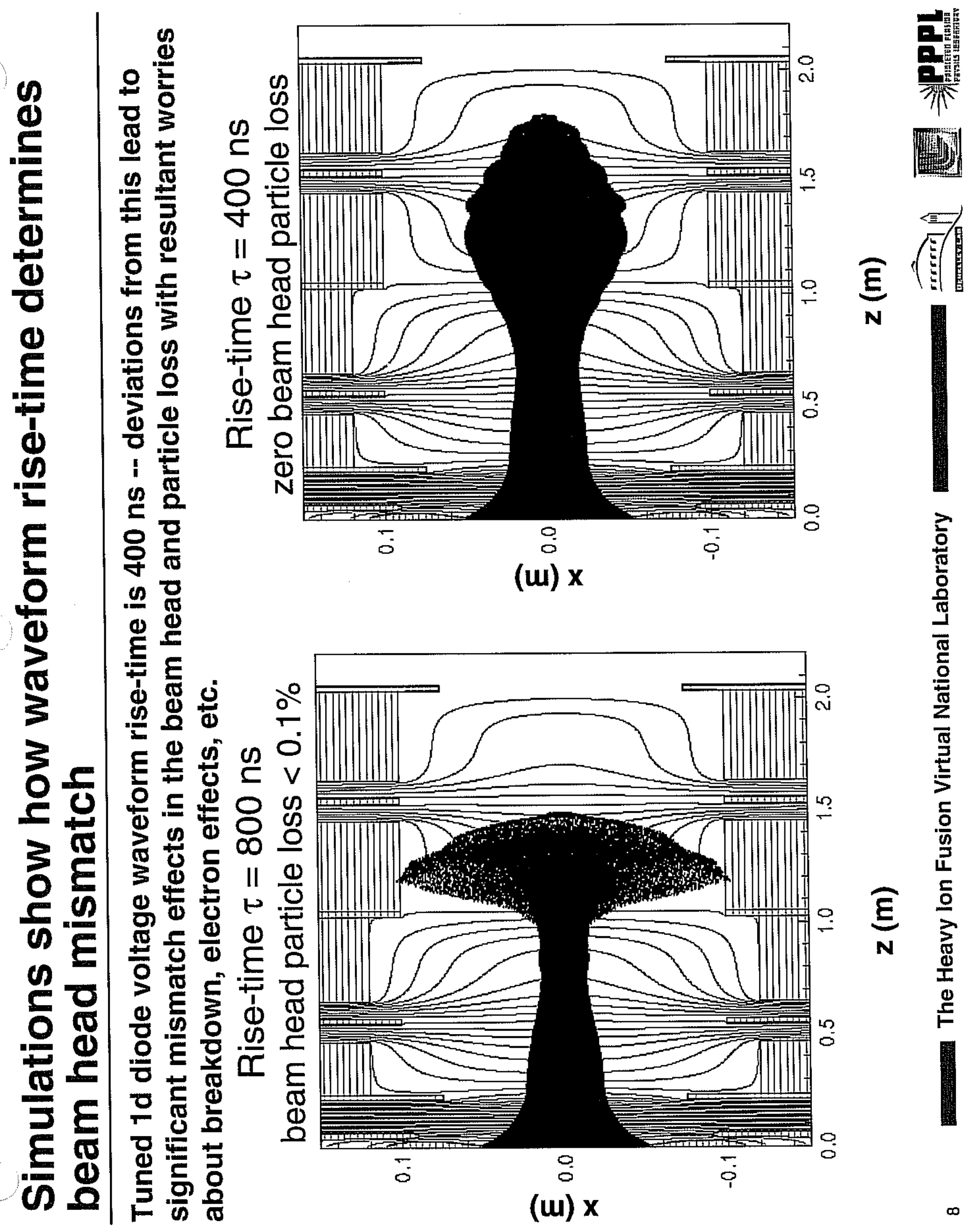




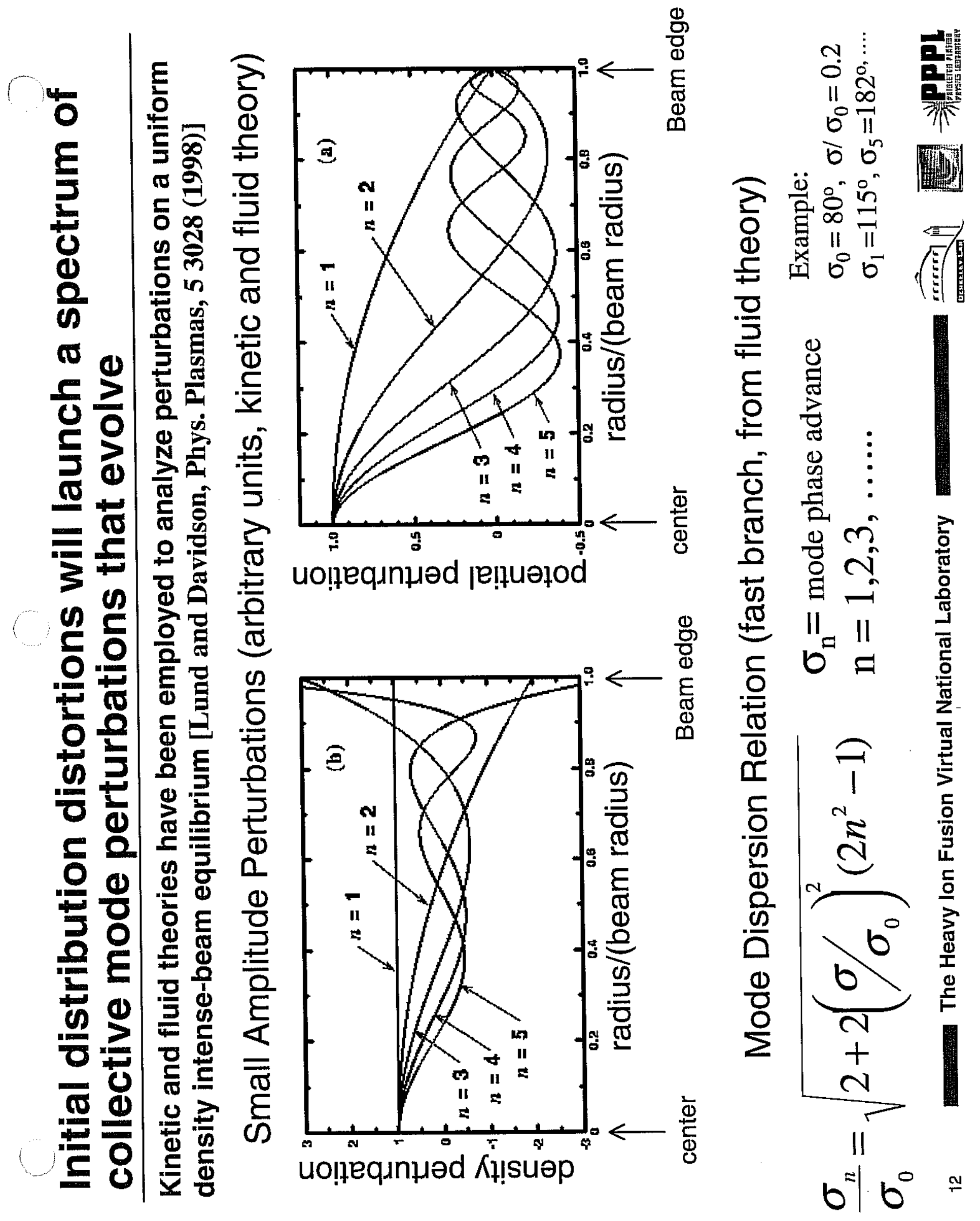



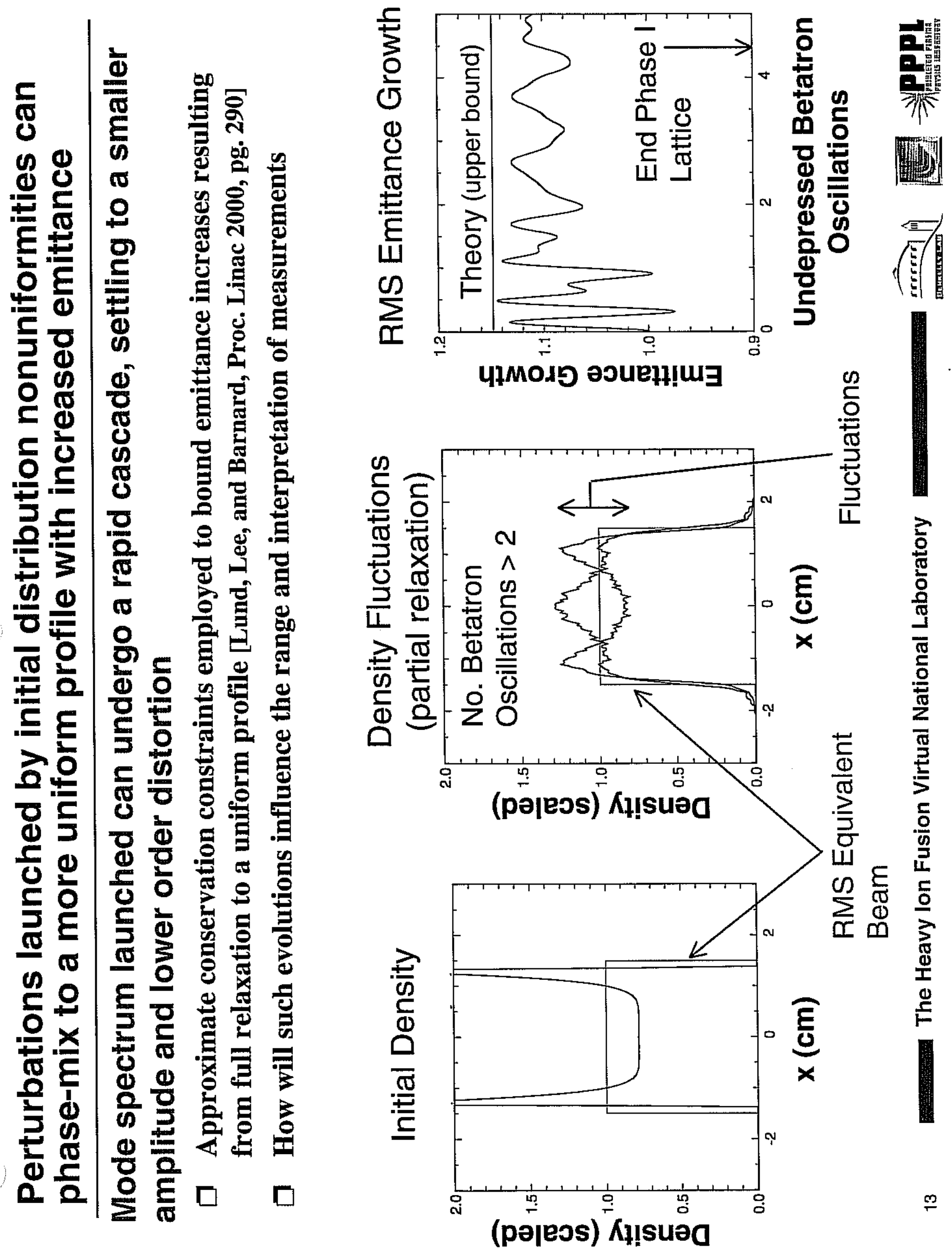
S.M. Lund 58/

达

ब

둥

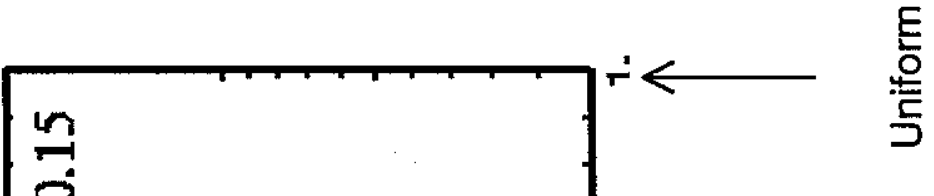

통

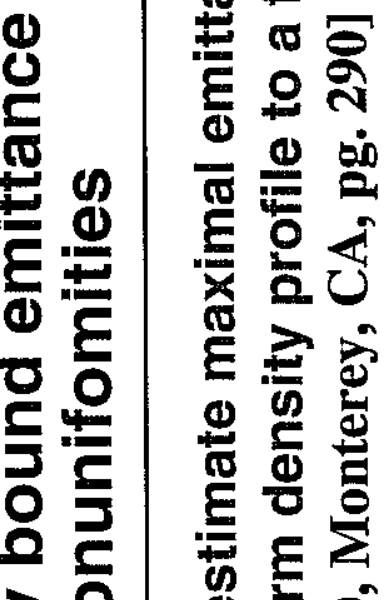

$\geq 0$

(1)

\$웅

을

60

호

$\Phi$ 당

d) 다 을

E

के

을 똥

티욜

10

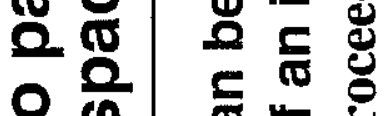

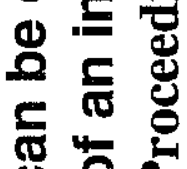

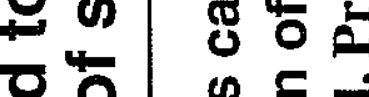

d)

o

둥

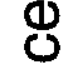

ธ్ర

$\frac{E}{E}$

5

(1) $x$

d

0

(1) 1

등 홍

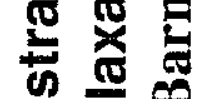

동 흔

○

퉁 통

E

의

논 율

ద들

(5)

잉

잉효

언

통

둥

은 혼 든

(1)

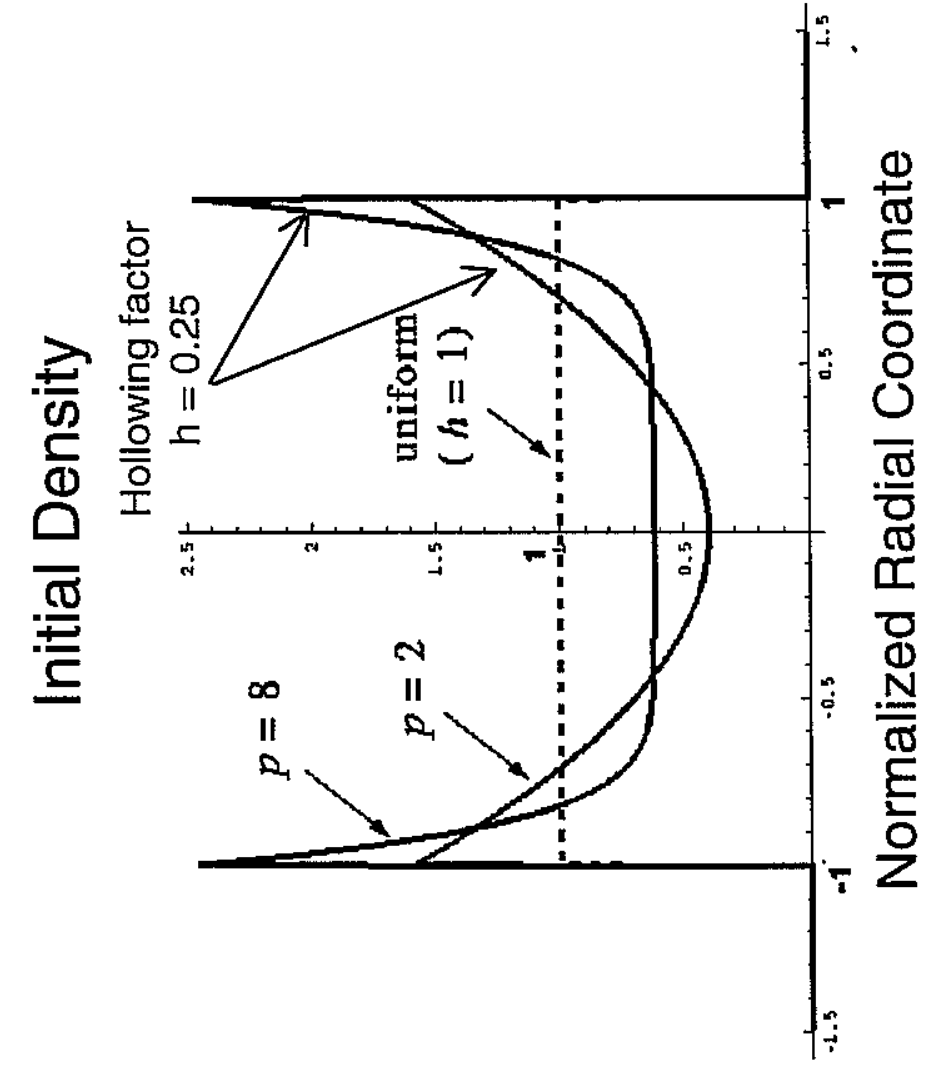

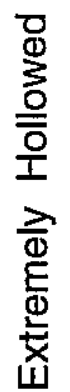

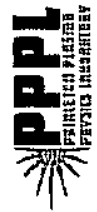

$\frac{0}{6}$
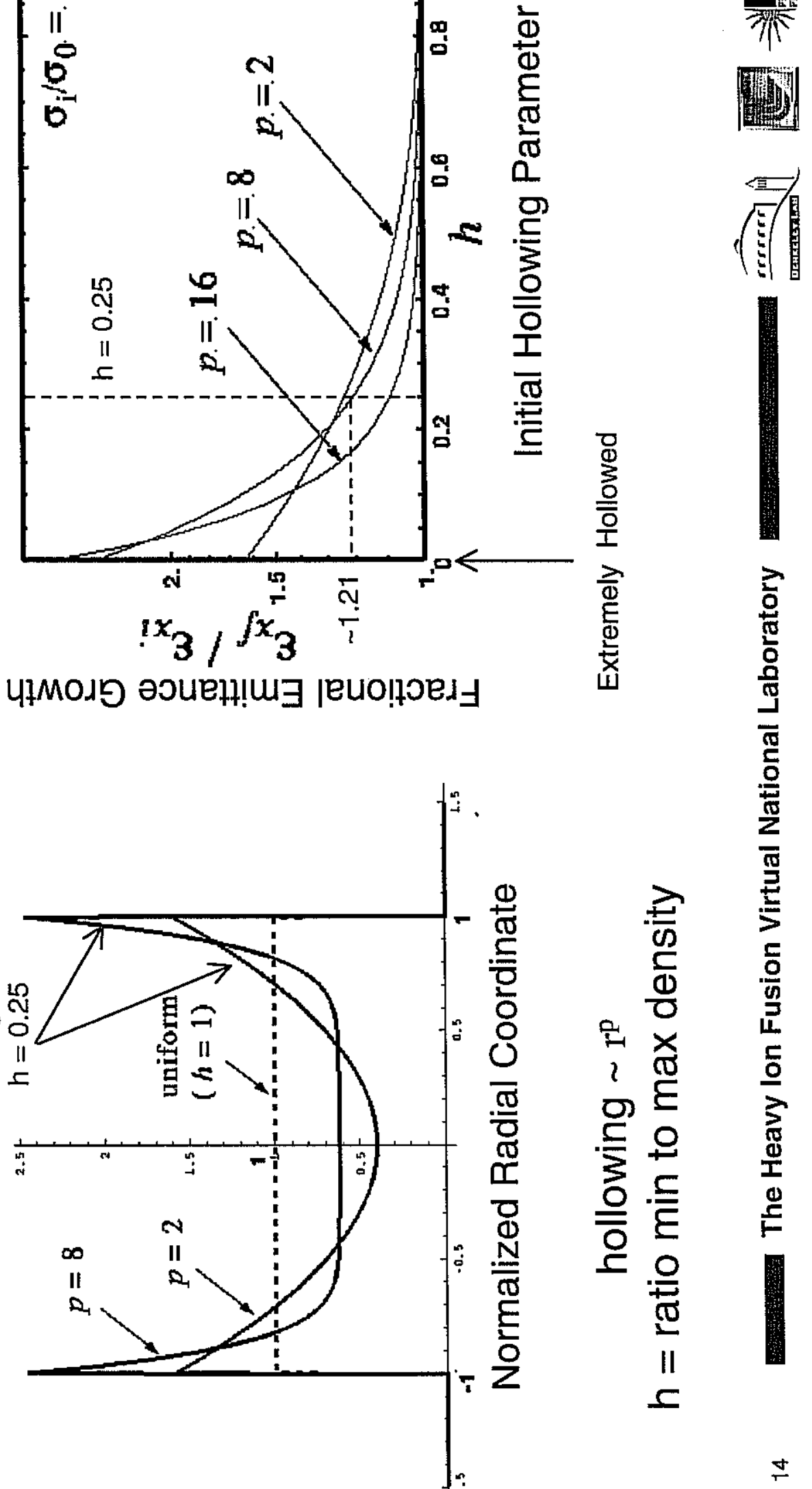
S.M. Lund 60/

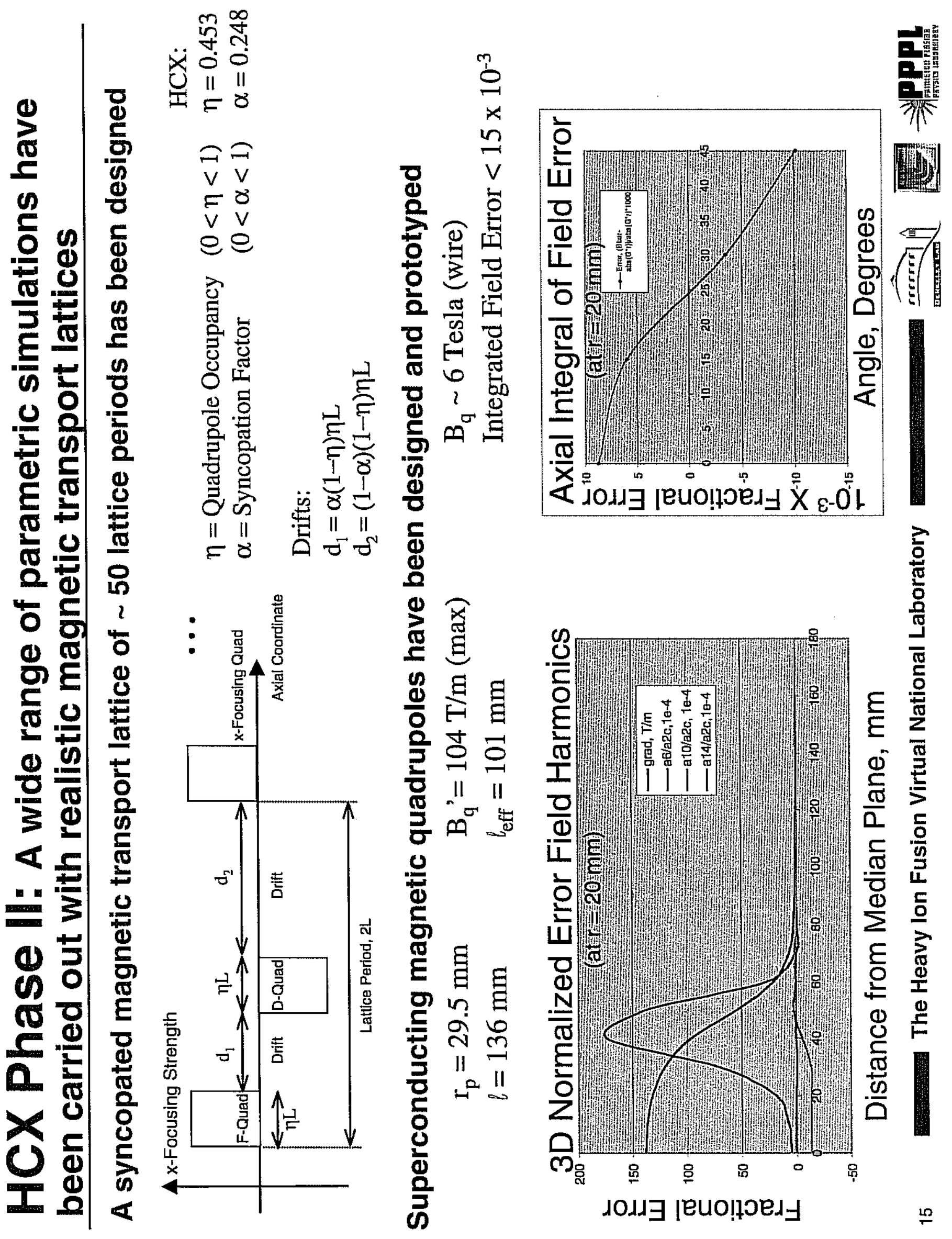


S.M. Lond 6.1\%

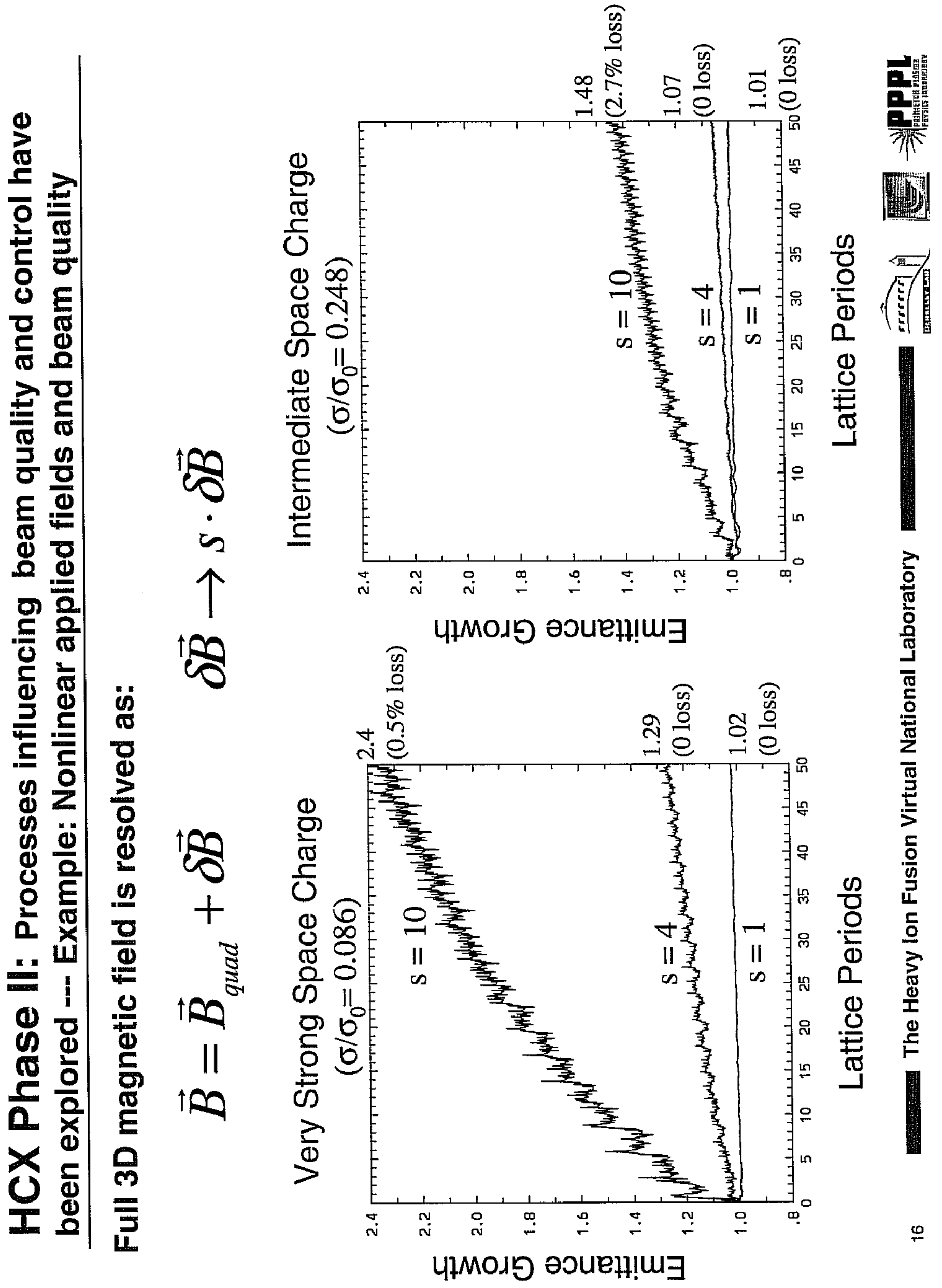




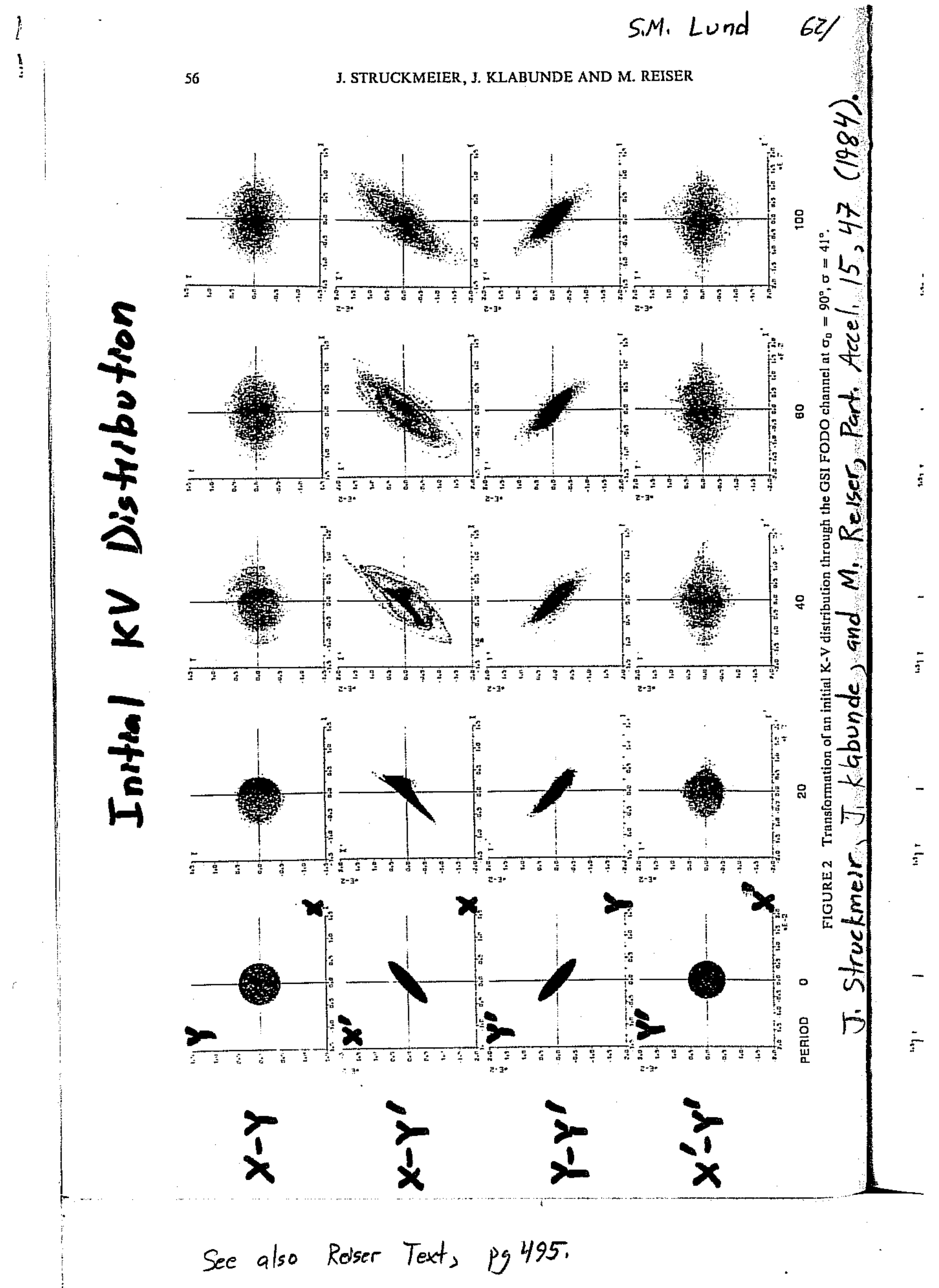




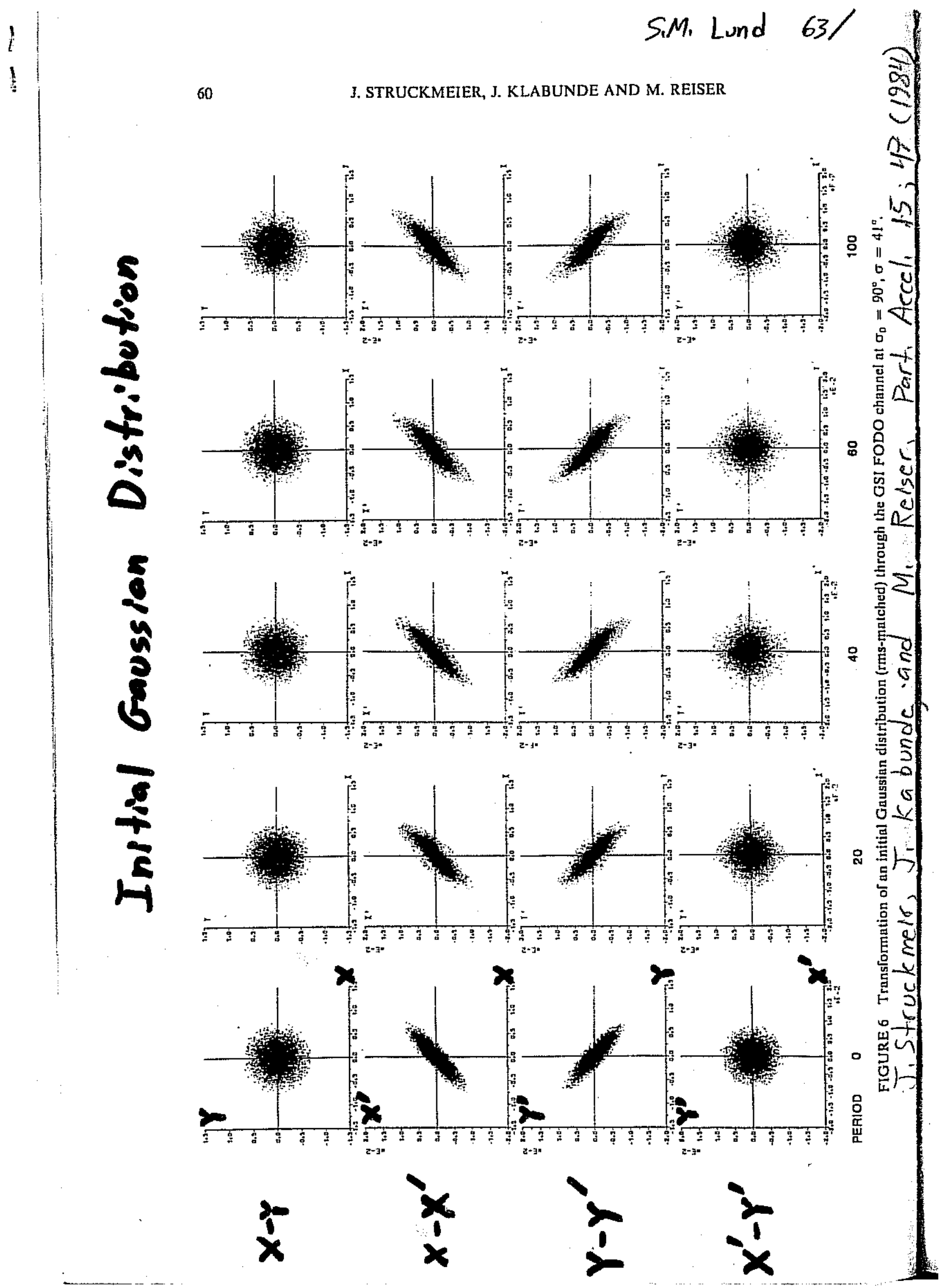


S.M. Lund 64/ 
SiM. Lond 65/

Distribution Methods - Direct Gridded

Solution of Vlasov's Equation.

Consider the Vlasov equation as an example.

$$
p \times s
$$

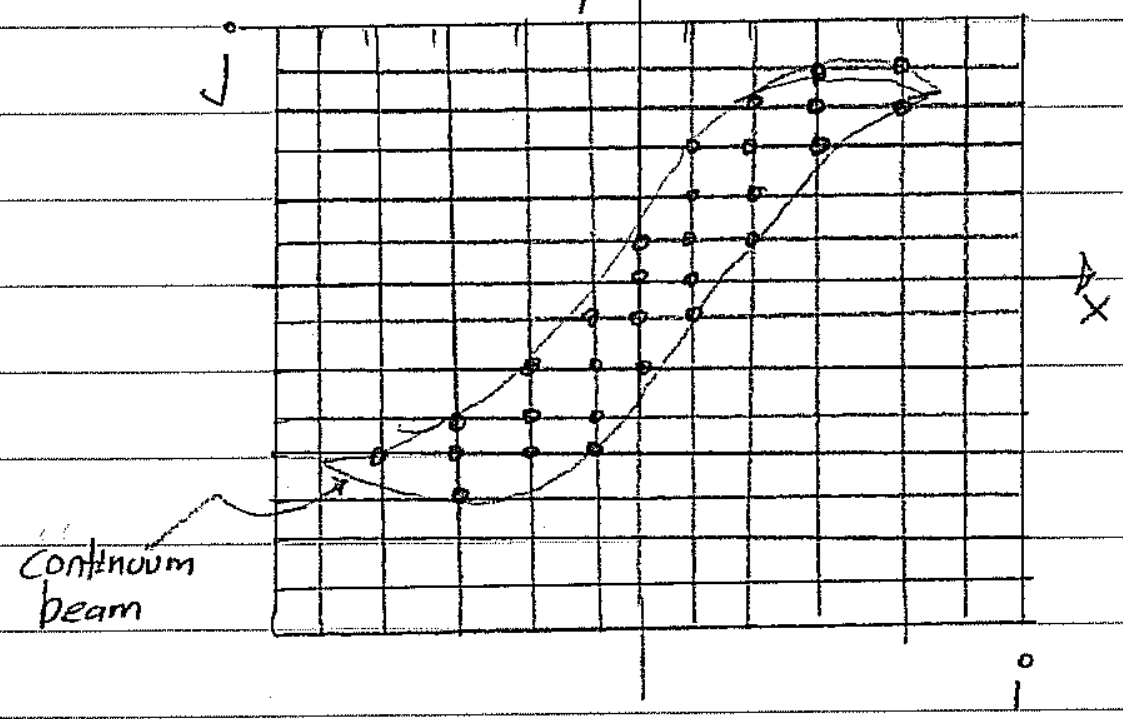

$$
f\left(x_{i,}, p_{j,}, t\right)=f_{i j}(t)
$$

The distribution is advanced at discrete grid points in time.

- Fields are typically solved using a discrete spatial mesh as for the particle methods described before,

- Deposition on mesh is typically straightforward in Vlasov care (sum over momentum variable.

- The distribution advance cycle is different than for particle methods.

- Numerical stability is key.

- Characteristics and "semi-Lagrangian" methods can be employed.

- Methods for solving for characteristics are familiars from dynamics l plasma physics. 
Direct Vlasov Methods

"Pros"

S.M. Lond 66/

Reasons for Vlasov simulations:

- Low noise - only discretization effects without statistical effects

- Allows clear analysis of collective effects and tenuous dis tribution components.

"Cons"

Reasons why Vlasor simulations are presently employed less than PIC:

- Extreme memory requirements for needed grid resolution in multidimensional phase space.

- Beams often have sharp edges in phase space that move in response to varying applied focusing forces.

- Numerical stability tends to be more difficult than in particle methods.

No time to illustrate Vlasov and other distribution methods. in this introductory course. We hope to cover these methods and other: numerical fieldsolve techniques in a later expanded version of this class.

However..... it is easy to generalize from what we have learned? 


\section{John Barnard Steven Lund USPAS June 2008}

\section{Summary of JB lectures}


STAKT WITH MI CKOSCOVIC PHHE SHACE DENSITY

$N(\underline{x}, \underline{\underline{v}}, t)=\sum_{i=1}^{N} \delta\left(\underline{x}-\underline{x}_{i}(t)\right) \delta\left(\underline{v}-\underline{V}_{i}(t)\right)$ Klimovitoviteh beweris

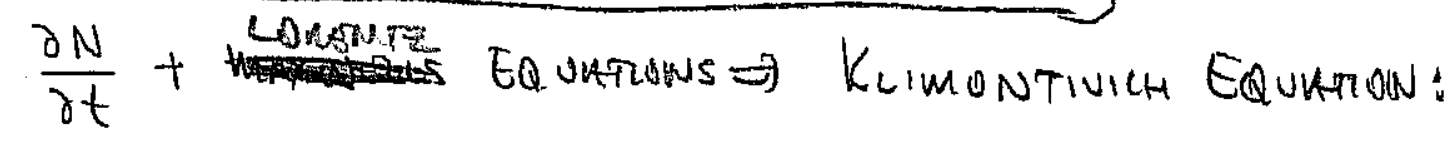

$$
\begin{aligned}
& \frac{\partial N}{\partial t}+v \cdot \nabla_{x} \cdot N(\underline{x}, \underline{v}, t)-\frac{q}{m}\left(\epsilon^{m}+v x B^{m}\right) \cdot \nabla_{v} N(\underline{x}, \underline{v}, t)=0
\end{aligned}
$$

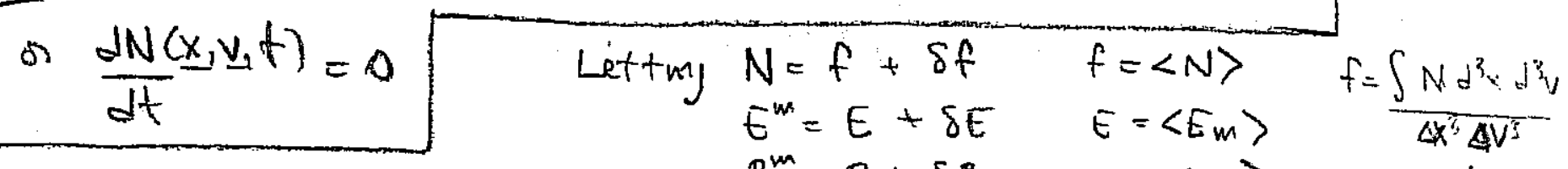

$$
\begin{aligned}
& B^{m}=B+\delta B \quad B=\left\langle B_{m}\right\rangle \quad n^{-1 / 3} \ll \Delta x \ll \lambda
\end{aligned}
$$

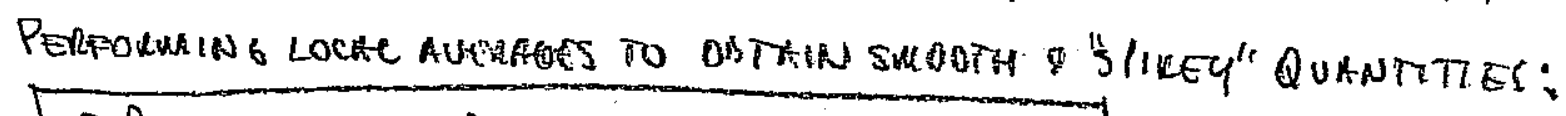

$$
\frac{\partial f}{\partial t}+\underline{v} \cdot \frac{\partial f}{\partial \cdot \underline{x}}+\frac{d v}{d t} \cdot \frac{\partial f}{\partial \underline{v}}=\frac{\partial f}{\partial t_{c}} \sim \frac{f}{\tau_{c}}
$$

We estimated $\left|\partial f / \partial t_{c}\right|$

$$
\begin{aligned}
& \left|\frac{\partial E \cdot \partial f / \partial \underline{v}}{m}\right| \sim \frac{1}{16 \lambda_{0}^{3} n_{0}} \ll 1 \\
& \lambda_{p}=V_{T H} / w_{p} \\
& v_{t h} \equiv \sqrt{\frac{k T}{m}} \quad w_{p} \equiv \sqrt{\frac{q^{2} n}{\varepsilon_{0} m}} \\
& \frac{\partial f}{\partial t}+\underline{v} \cdot \frac{\partial f}{\partial x}+\frac{d p}{d t} \cdot \frac{\partial f}{\partial p}=0 \quad: \quad \dot{p}=-\frac{\partial H}{\partial \underline{x}} ; \dot{x}=\frac{\partial H}{\partial p}
\end{aligned}
$$

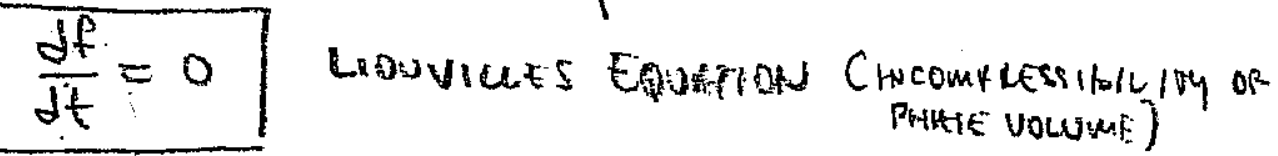

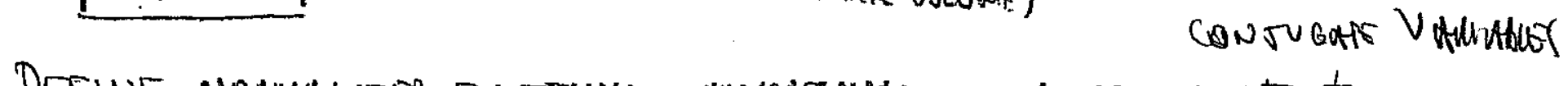

Define nonmalized emittaness roportional to $\underset{\Delta p_{z} \Delta z}{\Delta p_{x}} \propto \Delta E \Delta t$

SO THAT

$$
\Delta p_{y} \Delta y
$$

$$
\varepsilon_{N X}^{2}=\gamma_{\beta}^{2}\left(\left\langle x^{2}\right\rangle\left\langle x^{\prime}\right\rangle-\left\langle x x^{\prime}\right\rangle^{2}\right) \text { is }
$$

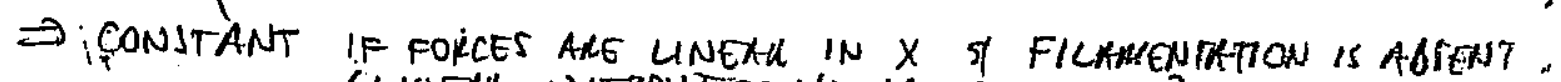
CLINEAR WITAOUTCOUVLFGG TO $Z$. OK 4 ?. 


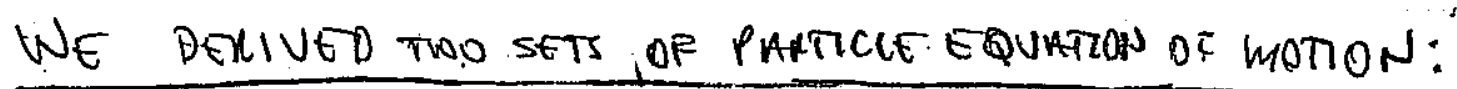

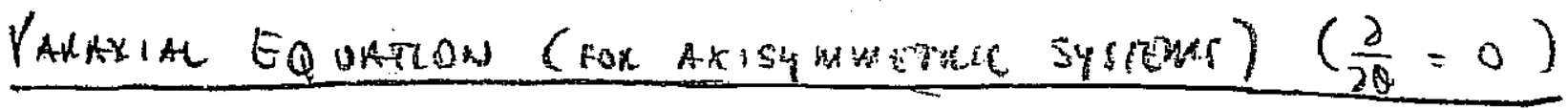

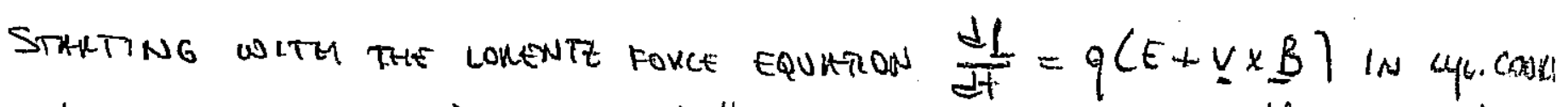

$$
\frac{d}{d t}\left(\gamma_{m} \dot{r}\right)-\gamma_{m} r \dot{\theta}^{2}=q\left(\frac{V^{\prime \prime}}{2} r+r \dot{\theta} B\right)+q\left(E_{r}^{\text {std }}+v_{z} B_{l}^{\text {seff }}\right) .
$$

$\uparrow 1$

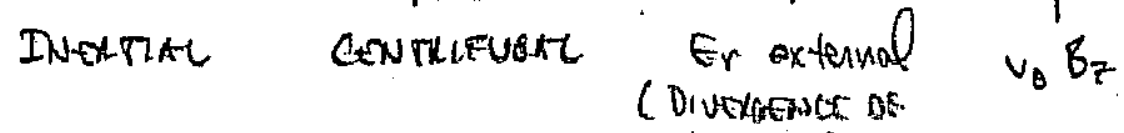

SALF - RIEUT

8-component: $E=0)$

$$
\dot{r} \equiv \frac{d r}{d t} ; r^{\prime}=\frac{d r}{d s}=\frac{\dot{r}}{\beta c}
$$

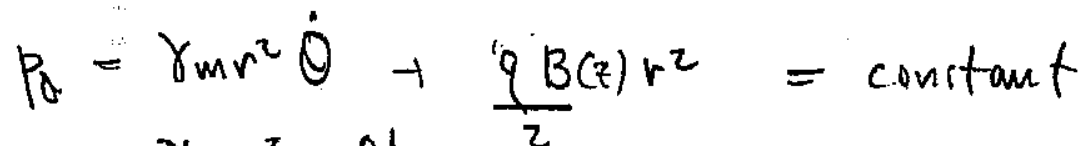

$$
\begin{aligned}
& =\gamma_{m} r^{2} \beta c \theta^{\prime}+\frac{q^{2} r^{2}}{2}=\text { conitant } \\
& r^{\prime \prime}+\frac{\gamma^{\prime}}{\beta^{2} \gamma} r^{\prime}+\frac{\gamma^{\prime \prime}}{2 \beta^{2} \gamma} r+\left(\frac{\omega_{c}}{2 \gamma \beta c}\right)^{2} r-\left(\frac{p_{\theta}}{\gamma \beta m c}\right)^{2} \frac{1}{r}-\frac{q}{\gamma^{2} m v_{e}^{2}} \frac{\lambda(r)}{2 \pi \varepsilon_{0} r}=0
\end{aligned}
$$

STATTSTTCAC AUERAGE OR THIS EQUARON

$$
\begin{aligned}
r_{b}^{\prime \prime}+\frac{\gamma^{\prime}}{\beta^{2} \gamma} r_{b}^{\prime}+\frac{\gamma^{\prime \prime}}{2 \beta^{2} \gamma_{b}}+\left(\frac{\omega_{c}}{2 \gamma_{p c}}\right)^{2} r_{b}-\frac{4\left\langle r_{b}\right\rangle^{2}}{\left(\gamma_{m p c}\right)^{2} r_{b}^{3}}-\frac{\varepsilon_{r}^{2}}{r_{b}^{3}}-\frac{Q}{r_{b}}=0 \\
\varepsilon_{r}^{2} \equiv 4\left(\left\langle r^{2}\right\rangle\left\langle r^{\prime 2}\right\rangle-\left\langle r r^{\prime}\right\rangle^{2}+\left\langle r^{2}\right\rangle\left\langle r^{2} \theta^{\prime}\right\rangle-\left\langle r^{2} \theta^{\prime}\right\rangle^{2}\right) ; \quad Q=\frac{q \lambda}{2 \pi \epsilon_{0} \gamma^{3} \beta^{2} m c^{2}} \\
=\varepsilon_{x}^{2}-4\left\langle r^{2} \theta^{\prime}\right\rangle^{2} \quad \text { (if } \rho=\rho(r) \text { ouly) }
\end{aligned}
$$

509 


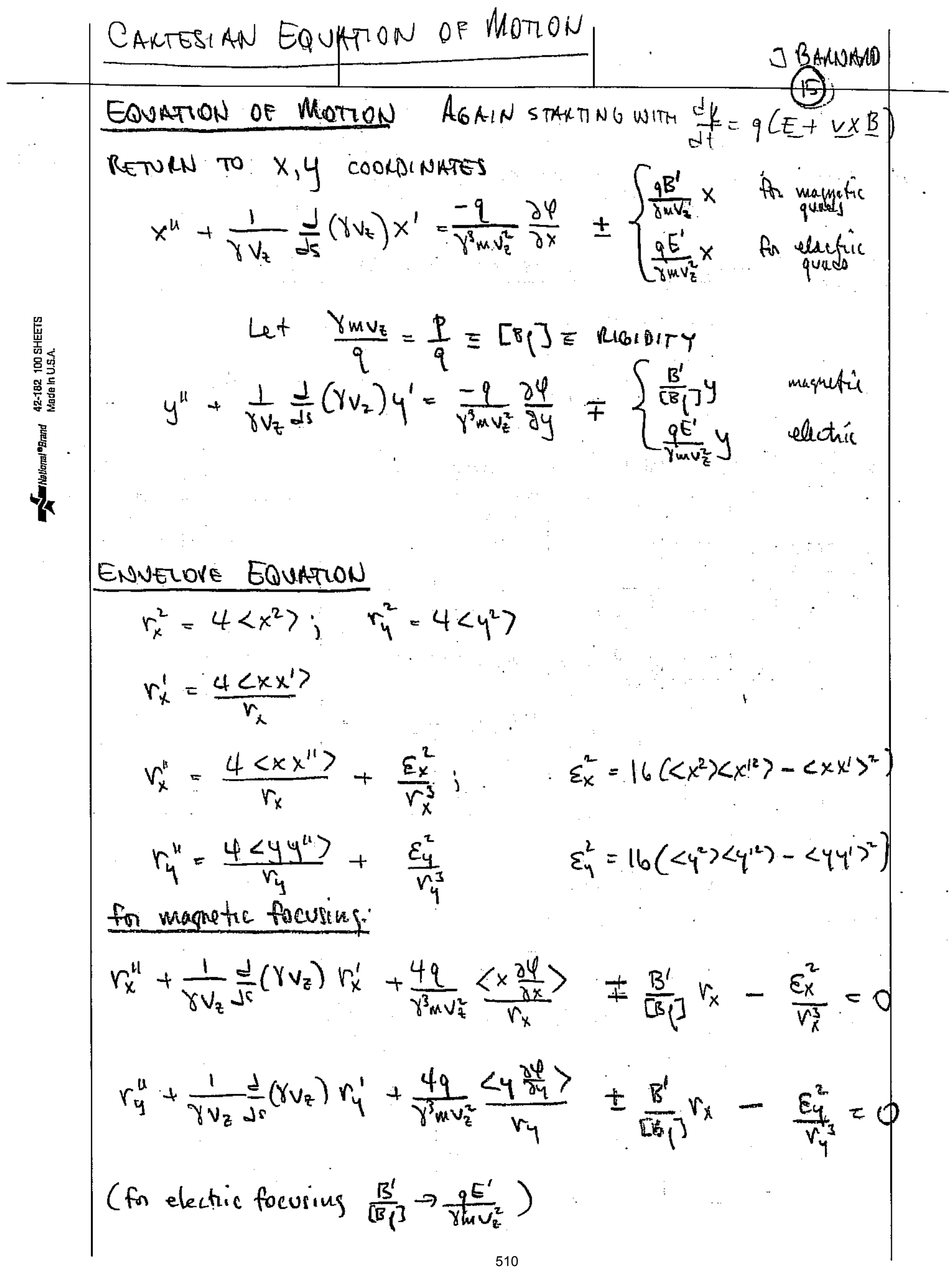


(16)

SPAGE CHAKGE TERM WITH ELLIITTCAL SYMHETHY

Now defocusing in ONE Direction and FOCUSING in THE OTHEX $\Rightarrow$ Rapial SYMmETRY SHOULD OE REPLCED \$4. Ellintil symmetrey: $\rho=\rho\left(\frac{x^{2}}{r_{x}^{2}}+\frac{y^{2}}{r_{y}^{2}}\right)$

CAN BE SHOWN THET

$$
\begin{aligned}
& \left\langle x \frac{\partial \phi}{\partial x}\right\rangle=\frac{-\lambda}{4 \pi \epsilon_{a}} \frac{r_{x}}{r_{x}+r_{y}} \\
& \left\langle y \frac{\partial \phi}{\partial y}\right\rangle=\frac{-\lambda}{4 \varepsilon_{0}} \frac{r_{y}}{r_{x}+r_{y}}
\end{aligned}
$$

USE, $Q(x, y)=\frac{-n_{x} r_{y}}{4 \varepsilon_{0}} \int_{0}^{\infty y} \frac{y(x) d s}{\sqrt{r_{x}^{2}+s} \sqrt{r_{y}^{2}+s}}$ to prove, where $\hat{p}(x)=\frac{d y}{d x}$

$$
\begin{aligned}
& p(x, y)=\left.\hat{\rho}(1)\right|_{s=0} \\
& \text { DEFINING } Q=\frac{2 \lambda q}{4 \pi \varepsilon_{0} \gamma^{3} m v_{z}^{2}} \\
& x=\frac{x^{2}}{v_{x}^{2}+5}+\frac{y^{2}}{v_{y}^{2}+5} \\
& \Rightarrow \begin{array}{l}
r_{x}^{\prime \prime}+\frac{1}{\gamma v_{z}} \frac{\partial}{d s}\left(\gamma v_{z}\right) r_{x}^{\prime}-\frac{2 Q}{r_{x}+r_{y}} \mp \frac{B^{\prime}}{[B p]} r_{x}-\frac{\varepsilon_{x}^{2}}{r_{x}^{3}}=0 \\
r_{y}^{\prime \prime}+\frac{1}{\gamma v_{z} d s} d\left(\gamma_{v_{z}}\right) r_{y}^{\prime}-\frac{2 Q}{r_{x}+r_{y}} \pm \frac{B^{\prime}}{[B \rho]} r_{y}-\frac{\varepsilon_{y}^{2}}{r_{y}^{3}}
\end{array}
\end{aligned}
$$

(for Electric Focosing $\frac{B^{\prime}}{[B]}+\frac{g E^{\prime}}{20 v_{z}^{\prime}}$ ).

(anAlogue to cikcular beAm:

$$
\left\langle r \frac{\partial \phi}{\partial r}\right\rangle=\frac{-\lambda}{4 \pi \varepsilon_{0}} \quad \text { ProveO in HOWEWolic) }
$$


ENVECOLE EQUATLNS DERIVET

rWS CENVELORS DEFINED IN TELWS OF RMS QUHNTTIES EMITTAWLE 1. PAKAXIAL $r_{b} ; \rho(r)$ NOT QUHHANTESO TO DE CONIELVEDT.

2. ELUITTCAL $r_{x}, r_{y} ; \quad \rho\left(\frac{x^{2}}{r_{x}^{2}}+\frac{y^{2}}{r_{y}^{2}}\right)$

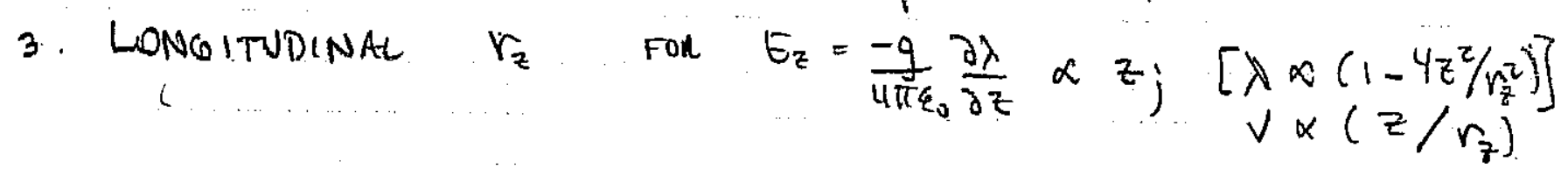

4. ELLISOIDAC of BUNCHES $r_{1}, r_{z}$ (Also $r_{x}, r_{y}, r_{z}$. ( $\rho$ conITANT) ...... $\quad \begin{array}{r}1, r_{z} \\ \text { r.f. Wanglen, section } 9.9) \text {. }\end{array}$

5. Ellitical with Images $r_{x}, r_{y} \ldots \quad\left(\frac{x^{2}}{r_{x}^{2}}+\frac{y^{2}}{r_{y}^{2}}\right)$

6. Larmon recane (continuos folono ids or

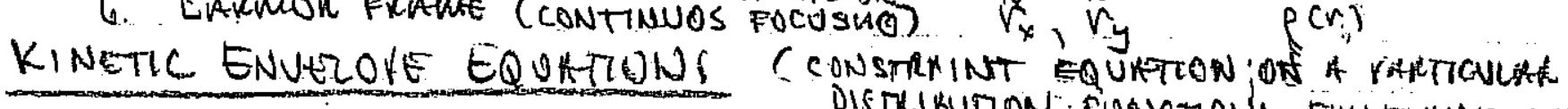

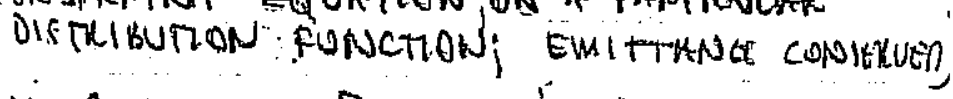

1. KV FOL ELUITCA NNIFORM BEAMS $f\left(x, x^{\prime}, y^{\prime}, y^{\prime}\right)$ [IDENTICAL TO \#2 AOQVE]

2. Neufren DisTLIBUTION for 1D $f\left(z, z^{\prime}\right)$

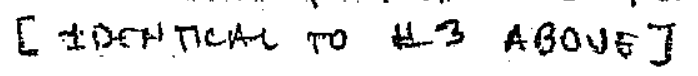

MOMENT EQUATONON

1. Transucuse with Chromatic EfFects $\left\langle x^{2}\right\rangle,\left\langle x^{12}\right\rangle,\left\langle x x^{\prime}\right\rangle,\left\langle x^{2} \delta\right\rangle,\left\langle x^{12} \delta\right\rangle,\left\langle x x^{\prime} \delta\right\rangle, \cdots$

512 


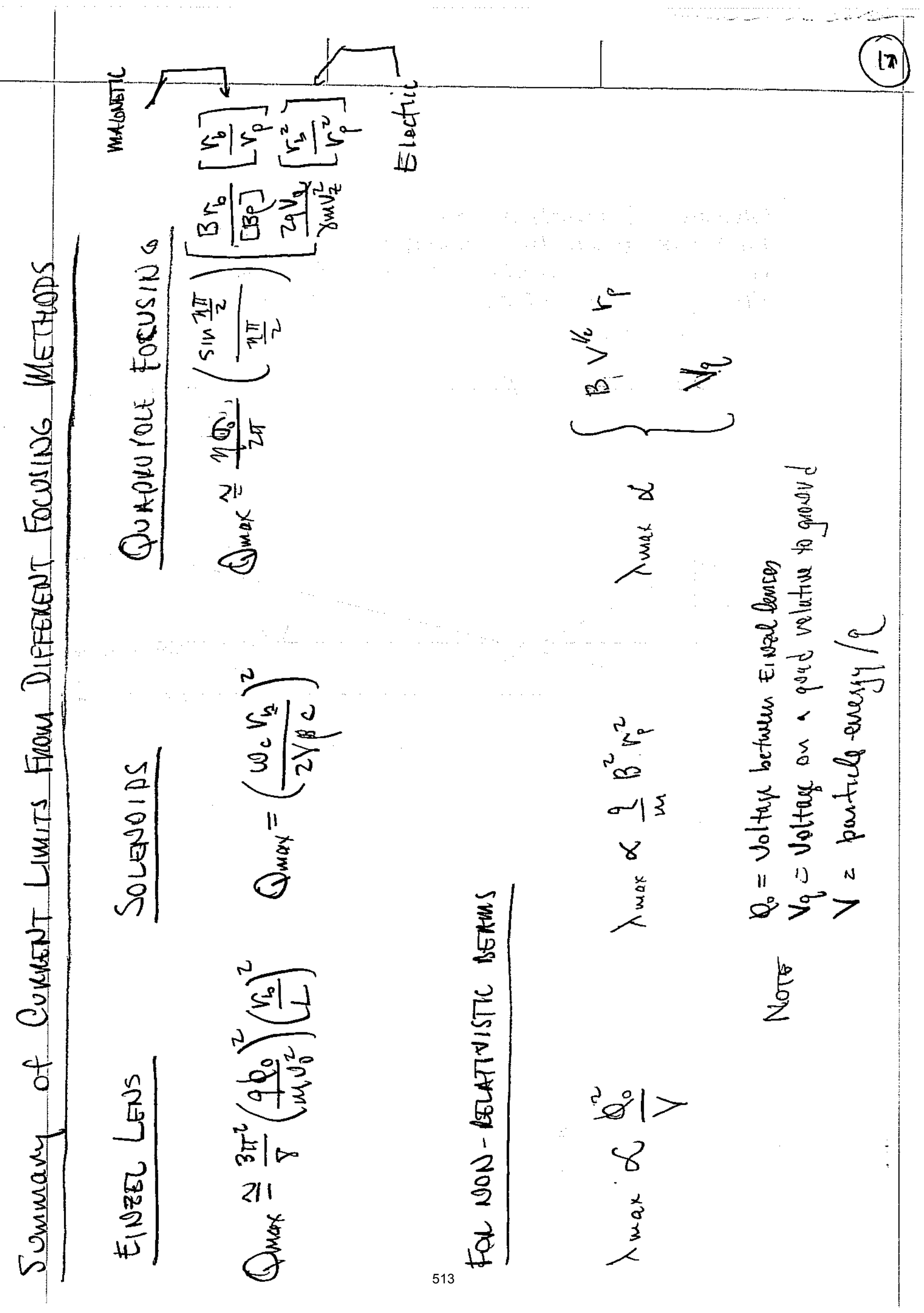


Solenoids: $\log \left\{\lambda\left[\mathrm{Cm}^{-1}\right]\right\}=-7.0$

Electrostatic quads: $\log \left\{\lambda\left[\mathrm{Cm}^{-1}\right]\right\}=-5,8$

Magnetic quads: $\log \left\{\lambda\left[\mathrm{Cm}^{-1}\right]\right\}=-8.9+\frac{1}{2} \log \left\{E_{\text {kin }}[\mathrm{eV}]\right\}$

Einzel lenses: $\log \left\{\lambda\left[\mathrm{Cm}^{-1}\right]\right\}=0.01-\log \left\{E_{\text {hin }}[\mathrm{eV}]\right\}$

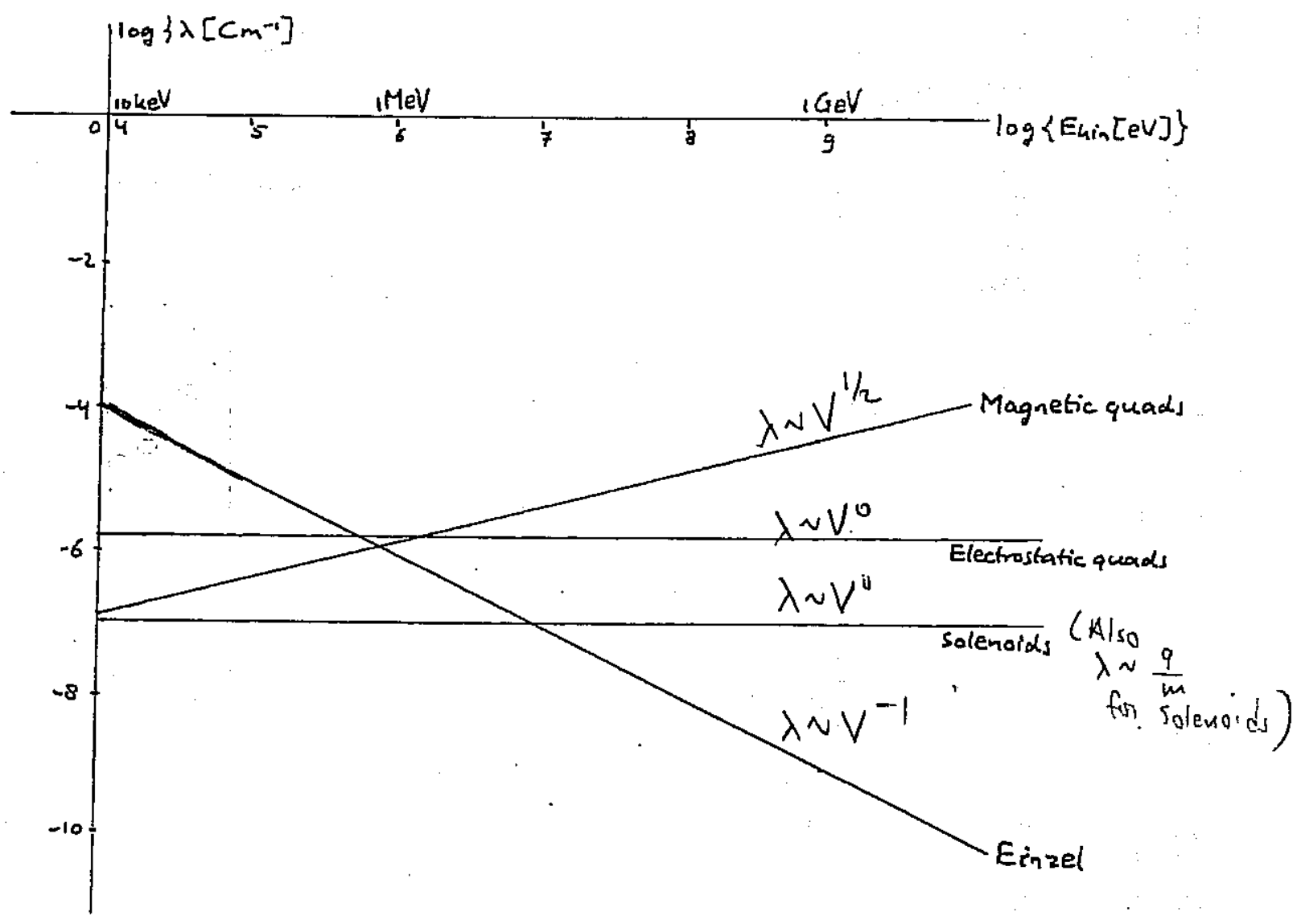


Estimating SHot Size

DARWIN

33

$$
\begin{aligned}
& r_{x}^{\prime \prime}+\frac{\left(\gamma_{b} p_{y}\right)^{\prime}}{\gamma_{p} p_{b}} r_{x}+k_{x} r_{x}-\frac{2 Q}{r_{x}+r_{y}}-\frac{\varepsilon_{x}^{2}}{r_{x}^{3}}=0 \\
& r_{y}^{\prime \prime}+\frac{\left(\gamma_{p} p_{y}^{\prime}\right.}{\gamma_{p} p_{b}} r_{y}+k_{y} r_{y}-\frac{2 Q}{r_{x}+r_{y}}-\frac{\varepsilon_{y}^{2}}{r_{y}^{3}}=0
\end{aligned}
$$

In chamber: No external focusing; no nccelelktion AND BEAM IS OFTEN CIRCULAR (BY DESIGN)

$$
\Rightarrow k_{x}=k_{y}=\left(v_{y} \beta_{b}\right)^{\prime}=0 \quad \& r_{x}=r_{y}=r_{b}
$$

$\Rightarrow$ ENJTZORE EQUATION IS:

$$
r_{b}^{\prime \prime}=\frac{Q}{r_{b}}+\frac{\epsilon^{2}}{r_{b}^{3}}
$$

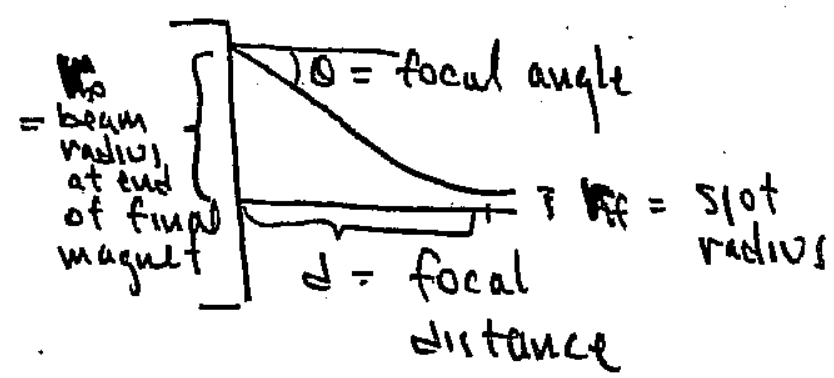

MULTRLYING BY $r_{b}^{\prime}+$ INTEGHATNG $\Rightarrow$

$$
\frac{r_{b f}^{\prime 2}}{2}-\frac{r_{b 0}^{\prime 2}}{2}=Q \ln \frac{r_{b f}}{r_{b_{0}}}+\frac{\varepsilon^{2}}{2 r_{b 0}^{2}}-\frac{\varepsilon^{2}}{2 r_{b f}^{2}}
$$

Now $\quad r_{b \theta}^{\prime} \cong \theta \quad r_{b f}=$ spot radius

$$
r_{b f} \ll r_{b 0}
$$

WHEN QR 0

$$
\begin{aligned}
& r_{b f}^{\prime}=0 \quad r_{b 0} \cong d \theta
\end{aligned}
$$

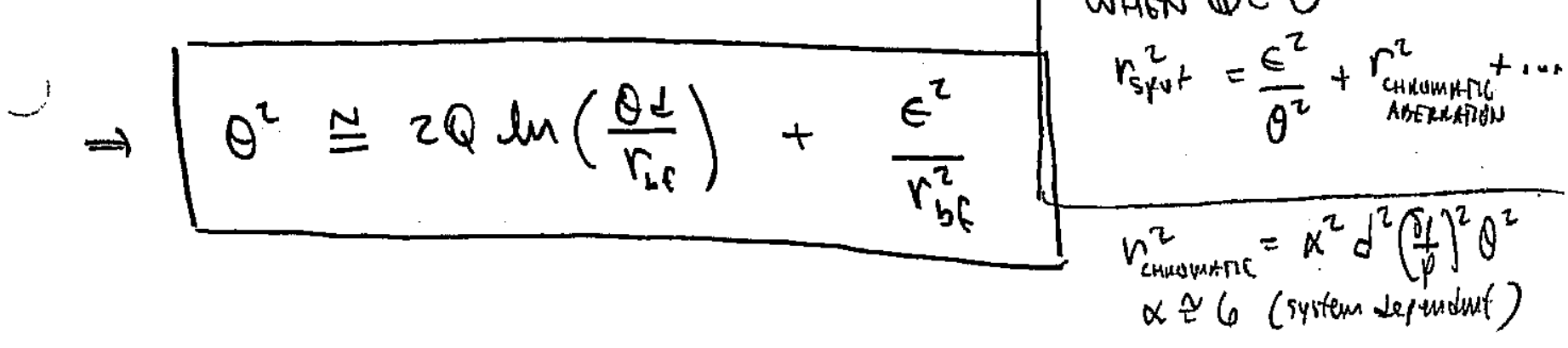

515 
Longitudinar Dinamics Summary

ID Vlasov Equation

$$
\begin{aligned}
& \frac{\partial \tilde{f}}{\partial s}+z^{\prime} \frac{\partial \tilde{f}}{\partial z}+z^{\prime \prime} \frac{\partial \tilde{f}}{\partial z^{\prime}}=0 \\
& E_{z}=\frac{-9}{\operatorname{lit\varepsilon } \varepsilon_{0}} \frac{\partial \lambda}{\gamma z} \\
& z^{\prime \prime}=\frac{q E_{z}}{m v_{0}^{2}} ; \quad \frac{\partial^{2} \phi}{\partial z^{2}}+\frac{1}{n} \frac{\partial}{\partial r}\left(r \frac{\partial \phi}{\partial r}\right)=-\frac{f}{\varepsilon_{0}} \\
& \text { 佂 } \\
& \text { CHILD-Langmuir in } \\
& \text { 1-D. PIOPE }
\end{aligned}
$$

"g- fector" model.

LEARS TO FLULO EQUHTIONS $\int($ Vla.sov Equation $) d z^{\prime}$

Ellifoectar Bunch

$$
\frac{\partial \lambda}{\partial s}+\frac{\partial}{\partial z}\left(\lambda \bar{z}^{\prime}\right)=0
$$

$$
\begin{aligned}
& \frac{\partial \bar{z}^{\prime}}{\partial s}+\bar{z}^{\prime} \frac{\partial \bar{z}^{\prime}}{\partial z}+\frac{1}{\lambda} \frac{\partial}{\partial z}\left(\lambda \Delta z^{\prime z}\right)=\frac{q E_{z}}{m v_{0}^{\prime}}
\end{aligned}
$$

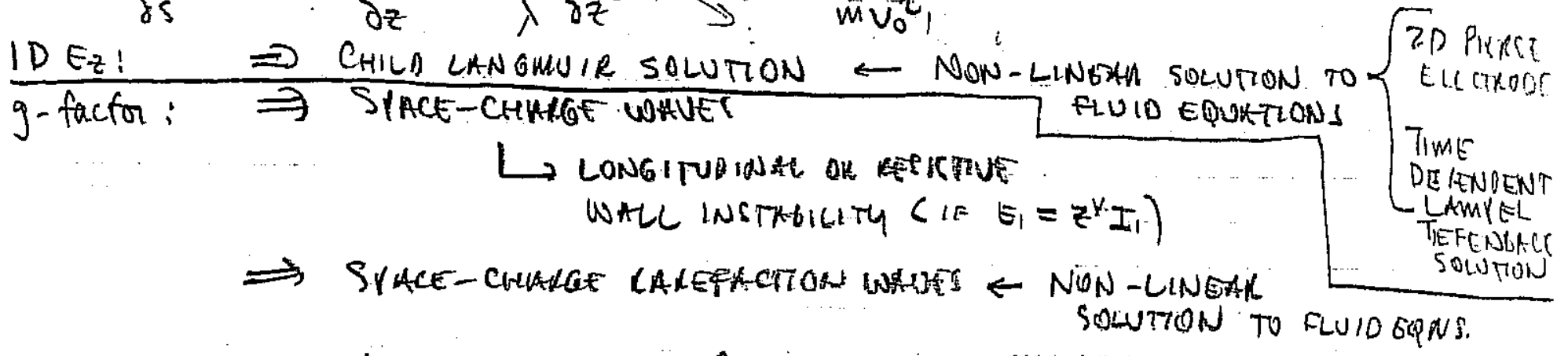

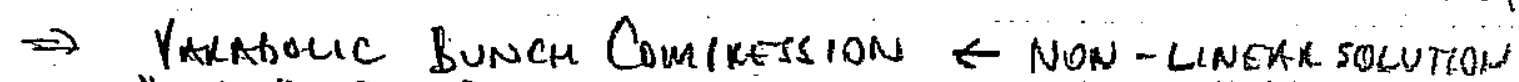

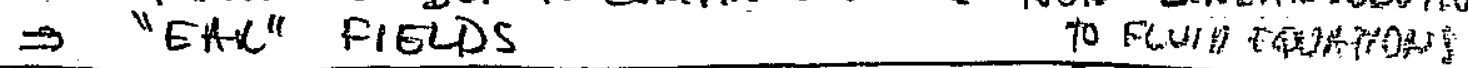

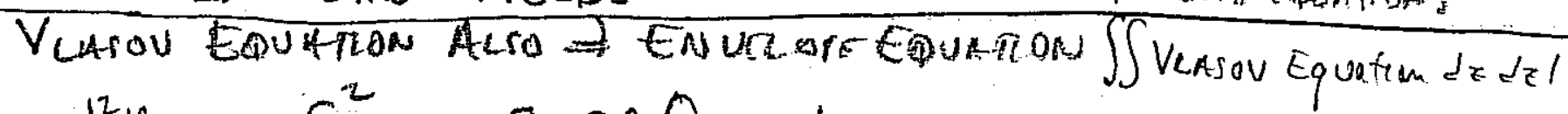

$$
\frac{d^{2} r_{z}}{d s^{2}}=\frac{\varepsilon_{z}^{2}}{r_{z}^{3}}+\frac{3}{2} \frac{9 q Q_{c}}{4 \pi \varepsilon_{u} m v^{2}} \frac{1}{r_{z}^{2}}-k(r) r_{z}
$$

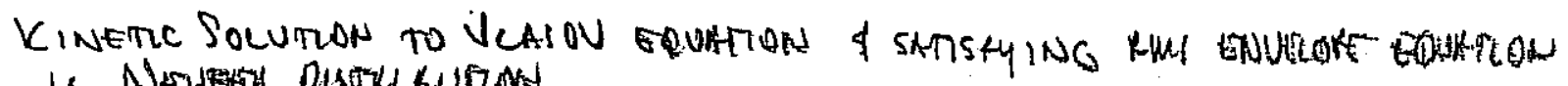

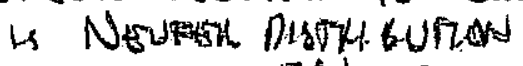

$$
f\left(z, z^{\prime}\right)=\frac{3 N}{2: \pi^{\prime} z_{z}} \sqrt{1-\frac{z^{2}}{r_{1}^{2}}-\frac{r_{z}^{2}\left(z^{\prime}-r_{z}^{\prime} z / r_{z}\right)^{2}}{5 \frac{2}{516}}}
$$


Normate MODE's

LONGITUDINAL

Siace-Chakge WhUES (RLUid)

$$
\begin{aligned}
& \omega= \pm e_{S} k \text { [IN BEAMUING FRME] }
\end{aligned}
$$

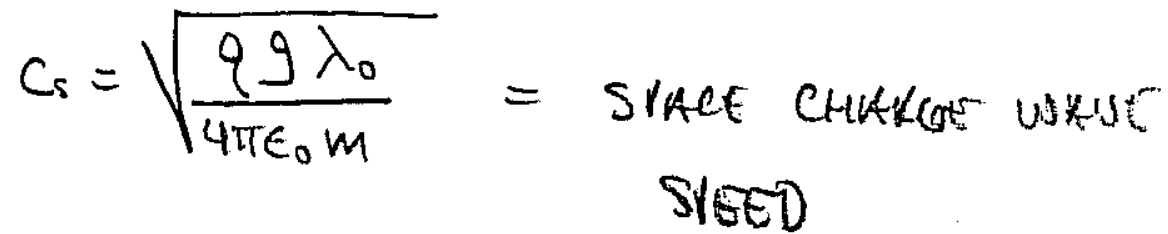

Transuerse

GN VEZONE MOPES

CONTINUOUS FOCUSING (LONG BUNCMEI)

BREATHING: $\quad k_{B}^{2}=2 k_{p_{0}}^{2}+2 k_{\beta}^{2}$

Quadruple $k_{Q}^{2}=k_{\beta O}^{2}+3 k_{\beta}^{2}$

CHexte $\left.t_{\beta}^{2} \equiv k_{p 0}^{2}-\frac{Q}{r_{b}^{2}}\right)$

(ANALOgOUS MODE IN BUNCHED BEAMS)

STEUE LOOKED hT mORES IN PEMIDDiC SYSTOMS (A CONTINIOUN + kinerte Mades (Gluckstzkas mados) FocvienG

+ Fluld modes

517 
INSTARILITEES

1. LONGITUDNAL (RESISTVE WAL) INBTAGHUA (FLUID InSTRSILITY)

2. ELECTRON-ION INSTKBILITY (CENTROID INSTHALITY)

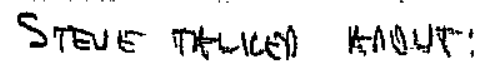

3. ENUELOL INATHLTEO

SrevE THeLEO ADOUT:

4. KINETTC INITRAILTTLES

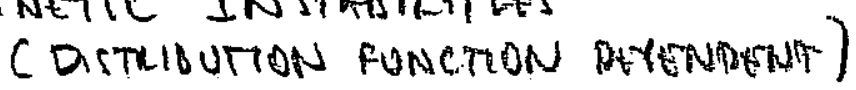

S. SINGLE PARTILE RESONHAT INITAALLIMES

- halo

- Ring resonances

518 
HALO:

Cove tost particle mopg:

$$
\begin{aligned}
& x^{\prime \prime}= \begin{cases}-\left[k_{00}^{2}-\frac{Q}{r_{b}^{2}}\right] x & \text { for } r<r_{b} \\
-\left[k_{b 0}^{2}-\frac{Q}{r^{2}}\right] x & \text { for } r>r_{b}\end{cases} \\
& r_{b}=r_{b 0}+\delta r_{b} \cos \left(k_{b} s+\phi\right)
\end{aligned}
$$

Guckstern's phase amplitude analyses:

$$
x^{11}+\left[k_{\beta O}^{2}-\frac{Q}{r_{b 0}^{2}}\right] x=\underset{\uparrow}{f}(x)
$$

Non linean + forking post

$$
x=A \sin \psi \quad x^{\prime}=k_{\beta} A \cos \psi \quad \leftarrow \text { PHALE/AMPLITUDE }
$$

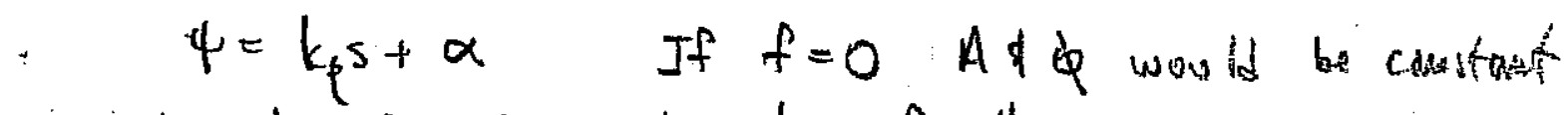

$$
\Rightarrow A^{\prime}=\frac{1}{E_{p} r_{b O}} f \cos \psi \quad \alpha^{\prime}=\frac{-1}{k_{p} r_{b 0} A} f \sin \psi
$$

DERING RESUNANT PHASE. $\Psi_{r}=2 \psi-k_{B} s$

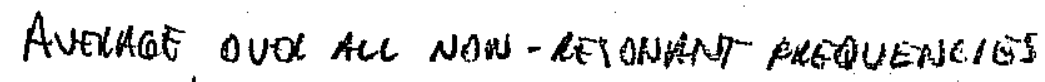

$$
\begin{aligned}
& A_{r}^{\prime}=\frac{1}{k_{p} r_{b o}} \int_{-\pi}^{t} f \cot \psi \frac{\psi d i}{2 \pi} j \quad \alpha_{r}^{\prime}=-\frac{1}{k_{1} A_{r}} \int_{-\pi}^{\pi} \frac{d u}{2 \pi} f \sin \psi \\
& \rightarrow A_{r}^{\prime}, \Psi_{r}^{\prime} \rightarrow \omega^{\prime}, \Phi_{r}^{\prime} \rightarrow H\left(\omega, \Phi_{r}\right) \rightarrow \text { GANE RESONHNR } \\
& \text { PARTCLE TARATCROM } \\
& \$ \text { SERAKATRX }
\end{aligned}
$$

519 


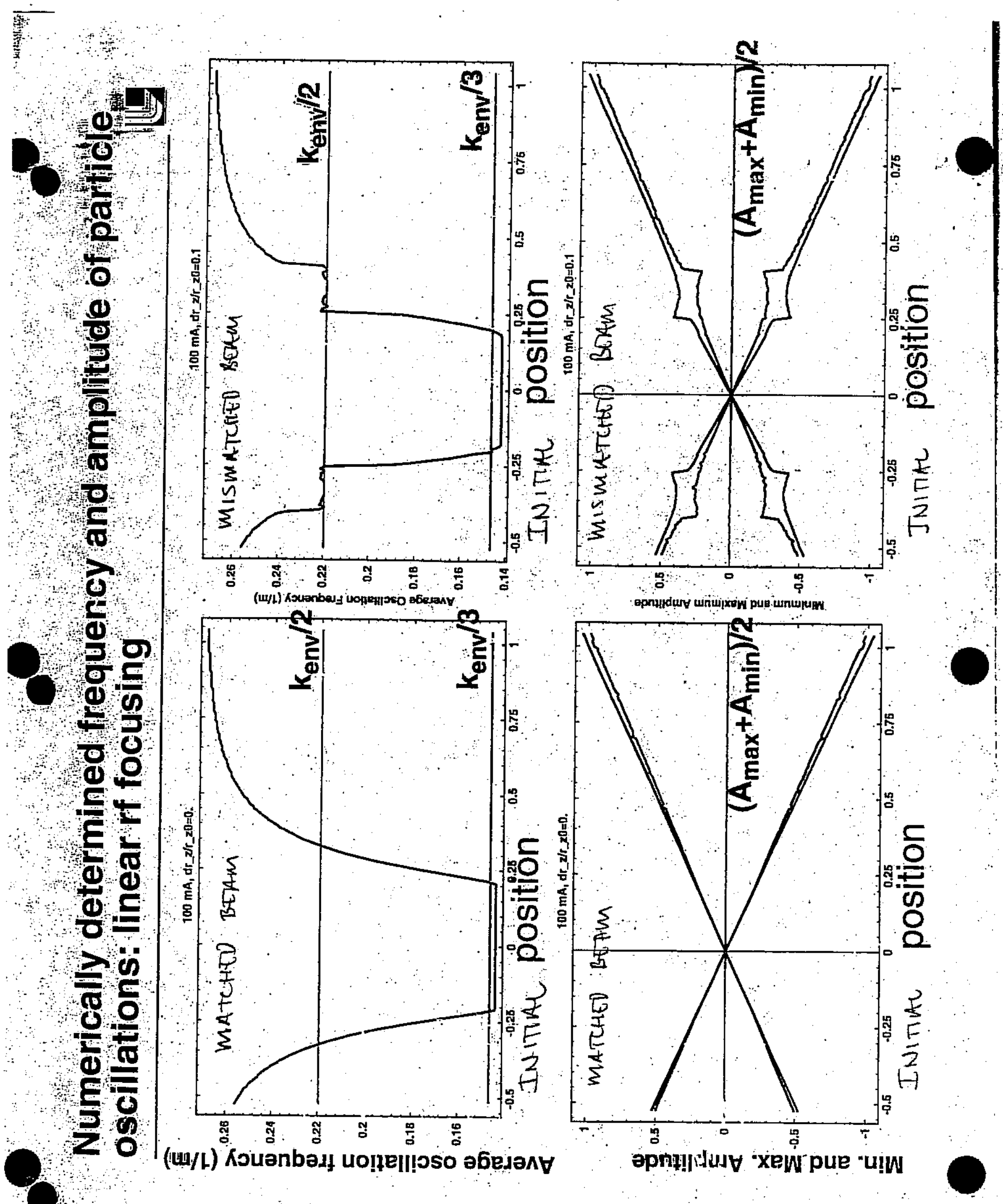




\begin{tabular}{|c|l|l|}
\hline Th $^{+}$Heavy ion & $O_{\text {molecule }}^{\text {Residual gas }}$ & $\mathrm{e}^{-}$electron \\
\hline
\end{tabular}

\section{Processes:}

1. Coulomb collisons (intra-beam)

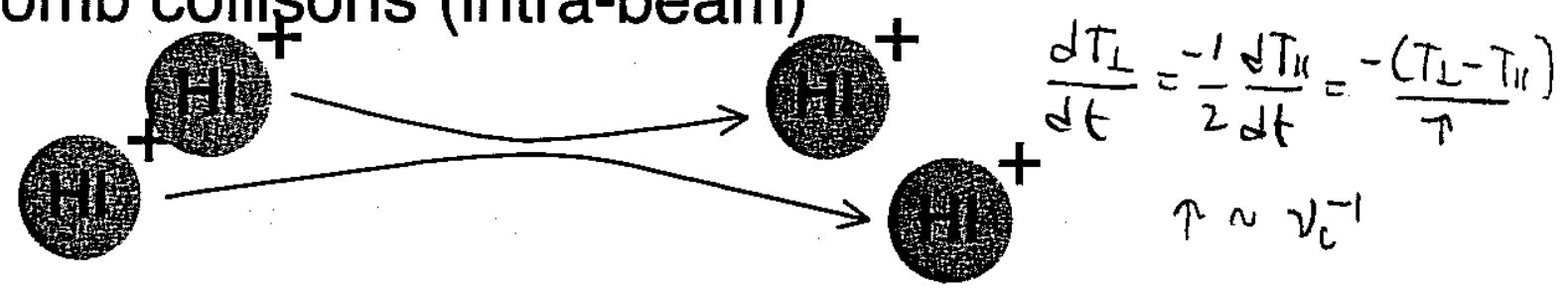

2. Coulomb collisions with residual gas

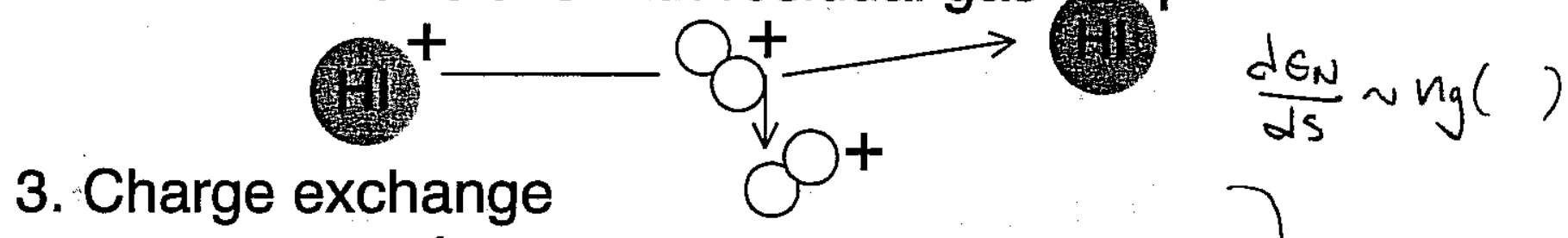

4. Stripping

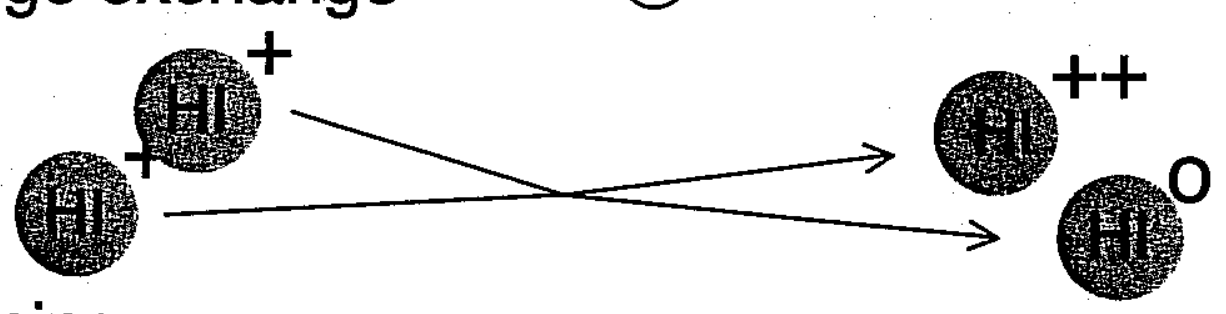

CHARES

CHANGING COLLSIONS

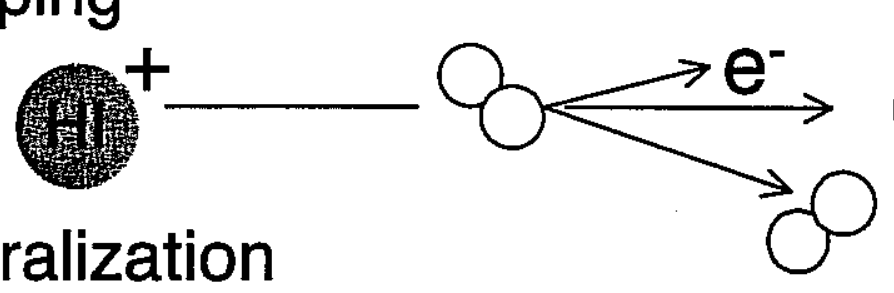

5. Neutralization
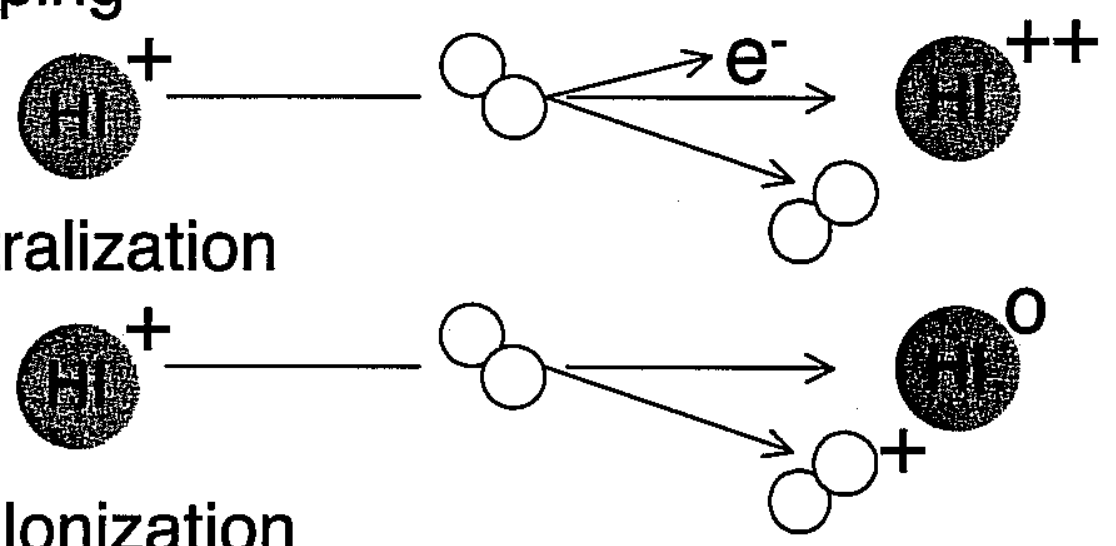

6. Gas Ionization

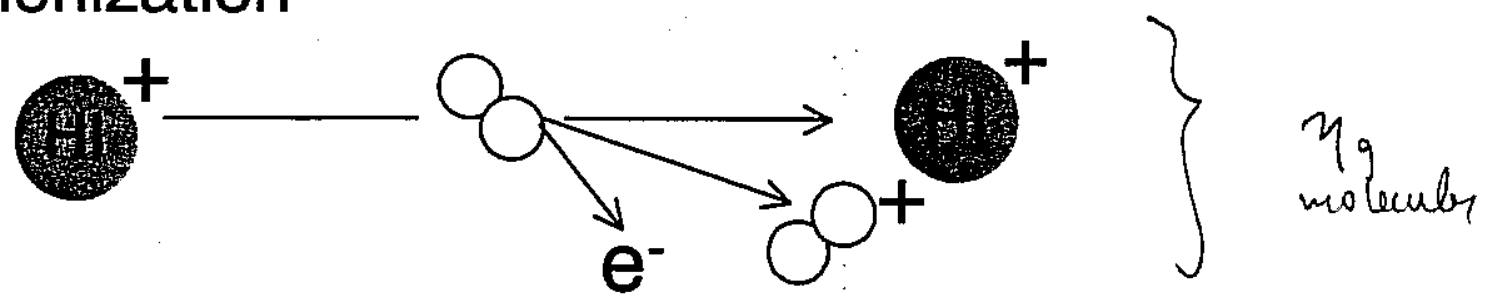




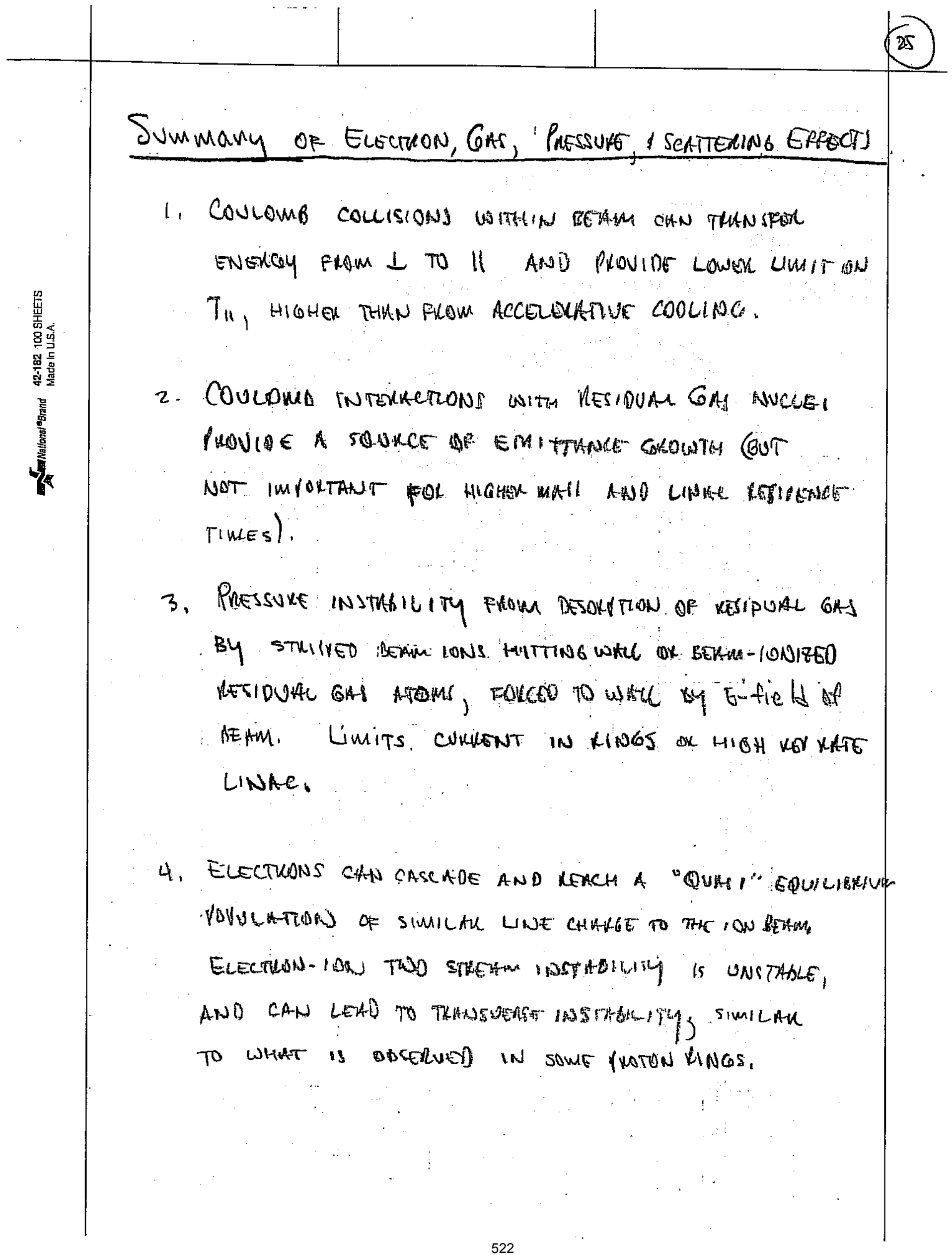




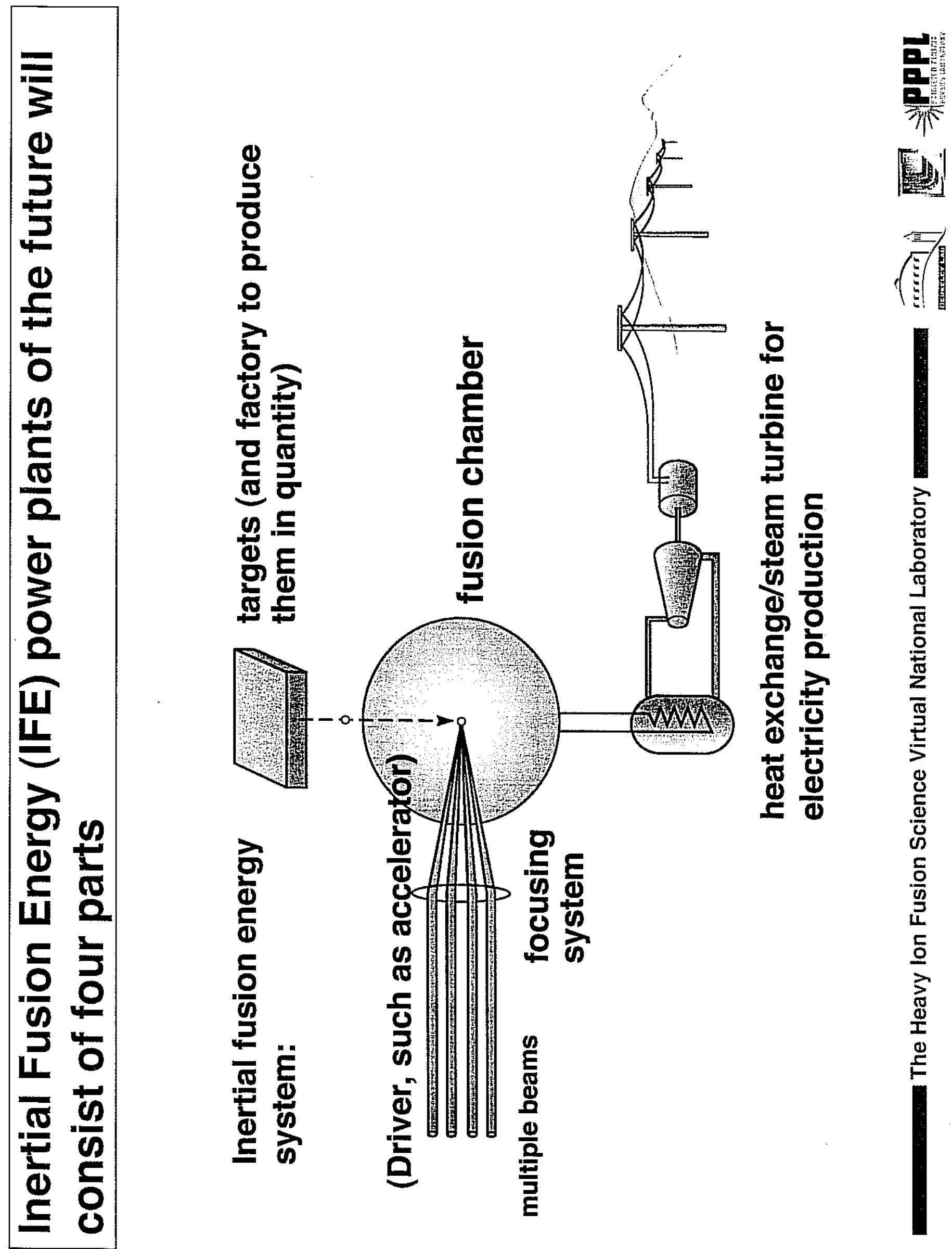

